Document downloaded from:

http://hdl.handle.net/10251/171426

This paper must be cited as:

Zarid, M.; García-Carpintero, V.; Esteras Gómez, C.; Esteva, J.; Bueso, MC.; Cañizares Sales, J.; Picó Sirvent, MB.... (2021). Transcriptomic analysis of a near-isogenic line of melon with high fruit flesh firmness during ripening. Journal of the Science of Food and Agriculture. 101(2):754-777. https://doi.org/10.1002/jsfa.10688

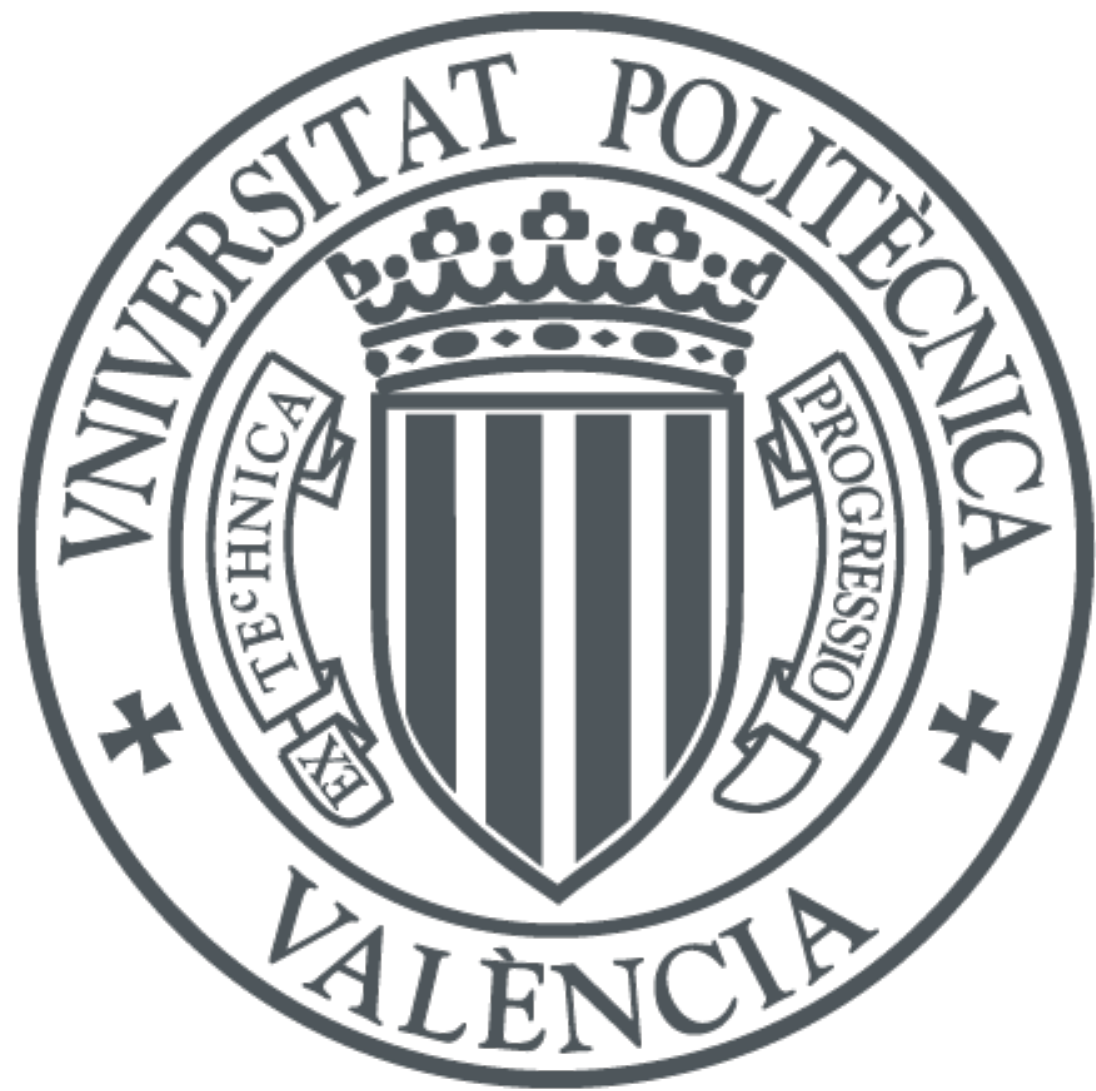

The final publication is available at

https://doi.org/10.1002/jsfa.10688

Copyright John Wiley \& Sons

Additional Information

This is the peer reviewed version of the following article: Zarid, M., García-Carpintero, V., Esteras, C., Esteva, J., Bueso, M.C., Cañizares, J., Picó, M.B., Monforte, A.J. and Fernández-Trujillo, J.P. (2021), Transcriptomic analysis of a near-isogenic line of melon with high fruit flesh firmness during ripening. J Sci Food Agric, 101: 754-777, which has been published in final form at https://doi.org/10.1002/jsfa.10688. This article may be used for non-commercial purposes in accordance with Wiley Terms and Conditions for SelfArchiving. 


\section{Transcriptomic analysis of a near-isogenic line of melon with high fruit flesh firmness during ripening}

\begin{tabular}{|r|l|}
\hline Journal: & Journal of the Science of Food and Agriculture \\
\hline Manuscript ID & JSFA-18-3688.R1 \\
\hline Wiley - Manuscript type: & Research Article \\
\hline Author: & n/a \\
\hline Complete List of Authors: & $\begin{array}{l}\text { ZARID, Mohamed; Technical University of Cartagena, Department of } \\
\text { Agricultural and Food Engineering } \\
\text { García-Carpintero, Victor; Universitat Politècnica de València (UPV), } \\
\text { Centro de Conservación y Mejora de la Agrodiversidad Valenciana } \\
\text { (COMAV) } \\
\text { ESTERAS, CRISTINA; Universitat Politecnica de Valencia, COMAV } \\
\text { Esteva, Juan; Technical University of Cartagena, Department of Plant } \\
\text { Production } \\
\text { Bueso, María C.; Technical University of Cartagena, Department of } \\
\text { Mathematics and Applied Statistics } \\
\text { Cañizares, Joaquín; Universitat Politècnica de València (UPV), Centro de } \\
\text { Conservación y Mejora de la Agrodiversidad Valenciana (COMAV) } \\
\text { PICO, MARIA BELEN; Universidad Politécnica de Valencia, Biotecnología } \\
\text { Monforte, Antonio; CSIC/Universidad Politécnica de Valencia, Instituto de } \\
\text { Biología Molecular y Celular de Plantas (IBMCP) } \\
\text { Fernandez-Trujillo, Juan; Universiad Polietcnica de Cartagena (UPCT), } \\
\text { Agricultural and Food Engineering }\end{array}$ \\
\hline Key Words: & $\begin{array}{l}\text { Cucumis melo L., Fruit quality traits, Postharvest quality, Fruis } \\
\text { senescence, RNA-seq, Fruit texture }\end{array}$ \\
\hline & \\
\hline
\end{tabular}




\section{Transcriptomic analysis of a near-isogenic line of}

\section{2 melon with high fruit flesh firmness during ripening}

3 Mohamed Zarid ${ }^{a}$, Victor García-Carpintero ${ }^{\text {b }}$, Cristina Esteras ${ }^{b}$, Juan Esteva ${ }^{a}$, María C.

$4 \quad$ Bueso $^{\mathbf{c}}$, Joaquín Cañizares ${ }^{\mathrm{b}}$, María B. Picób ${ }^{\text {, Antonio J. Monforte }}$, J. Pablo Fernández-

$5 \quad$ Trujillo

$6{ }^{\text {a }}$ Department of Agronomical Engineering, Regional Campus of International Excellence

7 “Campus Mare Nostrum” (CMN), Technical University of Cartagena (UPCT), Paseo Alfonso

8 XIII, 48, ETSIA, E-30203 Cartagena, Murcia, Spain.

9 b Universitat Politècnica de València (UPV). Centro de Conservación y Mejora de la 10 Agrodiversidad Valenciana (COMAV). Ciudad Politécnica de la Innovación, Edificio 8E, 11 Ingeniero Fausto Elio s/n, E-46022 Valencia, Spain.

${ }^{c}$ Department of Applied Mathematics and Statistics, CMN, UPCT, Doctor Fleming s/n, ETSII, 13 E-30202 Cartagena, Murcia, Spain.

14 d Instituto de Biología Molecular y Celular de Plantas (IBMCP). CSIC/Universidad Politécnica 15 de Valencia. Ciudad Politécnica de la Innovación, Edificio 8E, Ingeniero Fausto Elio s/n, E1646022 Valencia, Spain.

17 e Institute of Plant Biotechnology, CMN, UPCT, Campus Muralla del Mar, Plaza del Hospital 18 s/n, E-30202 Cartagena, Murcia, Spain

19 Runnig Title: Transcriptomics of melon during ripening

$20 *$ Correspondence to: Juan Pablo Fernández-Trujillo. Department of Agronomical

21 Engineering. Technical University of Cartagena (UPCT). Campus Alfonso XIII, Paseo 
22 Alfonso XIII, 48. ETSIA. E-30203 Cartagena (Murcia), Spain. Tel. +34 9683254 36. Fax $23+34968325732$.

24 E-mail address: juanp.fdez@upct.es

25 


\section{Transcriptomic analysis of a near-isogenic line of}

\section{melon with high fruit flesh firmness during ripening}

Mohamed Zarida ${ }^{a}$, Victor García-Carpintero ${ }^{b}$, Cristina Esteras $^{b}$, Juan Estevac, María C. Bueso $^{d}$, Joaquín Cañizares ${ }^{b}$, María B. Picób, Antonio J. Monforte ${ }^{\mathrm{e}}$, J. Pablo FernándezTrujillo $^{\mathrm{a}, \mathrm{f} *}$

BACKGROUND: A near-isogenic line (NIL) of melon (SC10-2) with introgression in linkage group $\mathrm{X}$ was studied from harvest (at firm-ripe stage of maturity) until day 18 of postharvest storage at $20^{\circ} \mathrm{C}$ together with its parental control ('Piel de Sapo', PS).

RESULTS: SC10-2 showed higher flesh firmness and whole fruit hardness but lower juiciness than its parental. SC10-2 showed a decrease in respiration rate accompanied by a decrease in ethylene production during ripening, both of which fell to a greater extent than in PS. The introgression affected eleven VOCs, the levels of which during ripening were generally higher in SC10-2 than in PS. Transcriptomic analysis from RNA-seq revealed differentially expressed genes (DEGs) associated with the effects studied. For example, 909 DEGs were exclusive to the introgression, and only 23 DEGs were exclusive to postharvest ripening time. Major functions of the DEGs associated with introgression or ripening time were identified by cluster analysis. About thirty-seven genes directly and/or indirectly affected the delay in ripening of SC10-2 compared with PS in general and, more particularly, the physiological and quality traits measured and, probably, the differential non-climacteric response. Of the former genes, we studied in more detail at least five that mapped in the introgression in linkage group (LG) $X$, and thirty-two outside it. 
CONCLUSION: There is an apparent control of textural changes, VOCs and fruit ripening by an eQTL located in LG X together with a direct control on them due to genes presented in the introgression (CmTrpD , CmNADH1, $C m$ TCP15, $C m$ GDSL esterase/lipase and $C m$ NAC18).

Keywords: Cucumis melo L., fruit quality traits, fruit senescence, postharvest quality, RNAseq, texture.

\section{INTRODUCTION}

Fruit ripening in melon (Cucumis melo L.) is characterized by a series of physiological, biochemical and organoleptic changes, as a consequence of a genetic program involving a coordinated cascade of responses that start in the placental tissue of the fruit and end in the rest of the mesocarp, with noticeable differences between climacteric and non-climacteric types. ${ }^{1,2,3}$ Non-climacteric melon fruit ripening is still poorly understood, hindering the application of postharvest techniques to delay ethylene-independent processes such as flesh softening or the production of certain aromas., ${ }^{4,5}$

New genetic and genomic tools are available in melon for studying fruit ripening including collections of near-isogenic lines, tilling platforms, saturated genetic maps, the genome sequence, and many Quantitative Trait Loci (QTLs) and eQTLs positioned on the genetic map, gene expression atlas, etc. ${ }^{6,7,8}$ However, this species still presents a challenge because of the size of its fruit and the difficulties in establishing the exact degree of maturity for harvesting.

Several collections of near-isogenic lines have recently been developed in this species.

One of them was obtained by crossing the Charentais type Védrantais (C. melo L. subsp. melo, Cantalupensis Group) with the Japanese Ginsen makuwa cultivar (C. melo L. subsp. agrestis, Makuwa group), ${ }^{9}$ several QTLs related to quality traits were reported in introgressions in 
73 linkage group (LG) X. Another involved crossing 'Piel de Sapo' (C. melo L. subsp. melo, 74 Ibericus Group; based on a recent classification ${ }^{10}$ with dudaim (C. melo sp. agrestis, Dudaim 75 group). ${ }^{11}$ Recently, Pereira et al. ${ }^{12}$ obtained two QTLs more in LG X. However, the most widely 76 studied collection for flesh firmness (at least at harvest) was developed using exotic non77 climacteric accession PI161375 (ssp. agrestis) as donor and the non-climacteric Spanish 78 cultivar PS as recurrent parental. ${ }^{13,14}$

79 RNA-Seq technology has also been applied for transcriptomic analysis during melon 80 fruit ripening and to determine QTLs, expression QTLs (eQTLs) and candidate genes related to 81 fruit quality. ${ }^{15,16,17}$ These authors concluded that the upregulation and downregulation of 82 multiple set of genes act coordinately during fruit ripening, although environmental and 83 seasonal effects need to be taken into account. ${ }^{4,18}$ Also, genes of $\beta$-D-xylosidase, glyoxysomal 84 malate synthase, chloroplastic anthranilate phosphoribosyltransferase (MELO3C011963), and 85 histidine kinase (MELO3C020055) have been associated with flesh firmness. ${ }^{19}$

The aroma profile together with textural traits are efficient traits for discriminating 87 climacteric NILs from non-climacteric ones at harvest or during postharvest, ${ }^{20,21,22}$ but little 88 information is available about aroma formation in non-climacteric melons, particularly during 89 postharvest ripening ${ }^{23}$ and in non-climacteric NILs during ripening.

One near-isogenic line (NIL) developed by Eduardo et al. ${ }^{13}$ (SC10-2) contains QTLs that affect flesh properties: for example PI161375 allele (vs. PS) increases flesh firmness and 92 flesh proportion vs. placental tissue at harvest ${ }^{14,24}$ and reduces flesh juiciness, extractable juice, 93 juice density and titratable acidity but increases $\mathrm{pH}$, dry matter and the flesh lightness colour 94 parameter. ${ }^{25} \mathrm{SC10}-2$ also shows differential volatile organic compounds (VOCs) at harvest, 95 lacking some compounds that are present in the PS parental, a pattern which is associated with 96 delayed ripening at harvest. ${ }^{24}$ Finally, $\mathrm{SC} 10-2$ in fresh-cut cubes also showed reduced juice 
97 leakage compared with PS during postharvest cold storage, ${ }^{26}$ but a certain risk of cracking 98 during preharvest. ${ }^{27}$

The aim of this study was to analyze and compare the transcriptomes of the NIL SC10-2

100

101

102

103

104

105

106

107

108

109

110

and its PS parental during ripening to reveal the genes associated with the introgression during melon postharvest ripening (particularly those associated with textural traits and VOCs), and also to provide further insights for identifying key potential pathways and regulators.

\section{MATERIAL AND METHODS}

\section{Plant material, crop management experimental design and sampling}

Fruits obtained from plants of the inbred parental C. melo 'Piel de sapo' (PS), inodorus type, and the NIL SC10-2 from the collection developed by Eduardo et al. ${ }^{13}$ were analysed. SC10-2 contains an introgression in homozygosis that covers the entire LG X from the Korean accession 'Shongwan Charmi' (PI 161375) in a PS genetic background (Eduardo et al., 2005). 13

Soil preparation, fertigation, plant protection, and other growing practices were those commonly used for melon cultivation in the Mediterranean conditions in Torre Pacheco (Murcia, Spain). ${ }^{20,22}$ To reduce fruit cracking, the measures reported by Fernández-Trujillo et al. ${ }^{27}$ were followed. The experiment was conducted in the "Centro Experimental de Formación y Experiencias Agrarias" (CIFEA) located in Torre Pacheco (Murcia, Spain). The field was divided into rows $2 \mathrm{~m}$ apart, in which each replicate consisted of six plants $1.5 \mathrm{~m}$ apart, in parallel rows. Twelve replicates of PS and seven of SC10-2 were planted. The plantation was surrounded by a border of the cultivar 'Nicolás' (Syngenta Seeds).

The fruit harvest and maturity indexes were slightly modified versions of those reported for non-climacteric melons. ${ }^{4}$ Minimum harvest indexes were the presence of a well formed and 
121 defect-free fruit, firm, well healed and dry epidermis with lignified netting, high density, 122 absence of trichomes, dark-green skin color, withering of the stem and leaf close to the fruit 123 peduncle, peduncle suberization, and light yellowing of the ground spot. The most common 124 harvest indices for both SC10-2 and PS were the rind netting and, in some fruit, an annular ring 125 or yellow color around a partly suberized peduncle, ${ }^{27}$ light yellowing of the ground spot 126 (particularly in PS), and a slight suberized crack around the peduncle, and dark blue-green skin 127 color (in SC10-2).

The harvest season lasted about 2 weeks in both lines, but all the fruit for this experiment 129 (three different biological replicates per line and/or storage time for transcriptomic analysis; more fruit for the study of physiological traits) were collected on the same day. PS was harvested about one month later than SC10-2 (7 July for PS; 9 August for SC10-2), due to the later ripening of this $\mathrm{NIL}^{24}$ and to the rain that fell during flowering, which provoked flower and fruit abscission in May. Fruits were harvested by an expert over a period of two weeks during morning hours $\left(05.45 \mathrm{~h}\right.$ to $10.00 \mathrm{~h}$, at a usual temperature of $21-26^{\circ} \mathrm{C}$. Total soluble solids and flesh firmness were used to assess the differences in maturity at harvest. ${ }^{25,28}$ Fruit in the firm-ripe stage of maturity were used in this experiment after storing fruit for 18 days at $20.5 \pm 0.6^{\circ} \mathrm{C}$ and a relative humidity of $88.2 \pm 5.4 \%$ (means $\pm \mathrm{SD}, \mathrm{n}=3$ biological replicates).

Fruits were immediately analysed after their arrival in the laboratory or during the postharvest storage. Flesh sampling was carried out following the methodology previously reported. ${ }^{25}$ After storage, samples lyophilised according to Dos-Santos et al. ${ }^{29}$

\section{Respiration rate, ethylene production}

142 Both physiological measurements were made in another lot of individual fruit ( $\mathrm{n}=6$ of different 143 biological replicates in firm-ripe stage of maturity) during postharvest ripening of $18 \mathrm{~d}$ at 20.5 
$144{ }^{\circ} \mathrm{C}$ and $88 \%$ relative humidity. The static method ${ }^{30}$ was followed for gas sampling and analysis

145 by gas chromatography of carbon dioxide and ethylene.

\section{Textural traits}

147 Whole fruit hardness (WFH) was determined at the equator (lateral hardness) as previously 148 reported for measuring the compression force (in N) to achieve $2 \mathrm{~mm}$ deformation. ${ }^{24}$ Flesh 149 firmness (FF), flesh juiciness (FJ) and juice density (JD) were measured according to the 150 methodology previously reported. ${ }^{25,28}$ The results were expressed in $\mathrm{N}$, grams of juice per $\mathrm{kg}$ 151 fresh weight or $\mathrm{kg}$ juice per $\mathrm{m}^{3}$ juice, respectively.

152 Volatile organic compounds (VOCs)

153 To analyze volatile organic compounds, the juice extracted from melons was filtered 154 through a four-layer cheesecloth. After $3 \mathrm{~min}$ at $23{ }^{\circ} \mathrm{C}$, the mixture containing $28.6 \% v / v$ of 155 saturated $\mathrm{CaCl}_{2}$ solution and the rest of the juice was poured into sterile polypropylene vials, 156 which were stored at $-80{ }^{\circ} \mathrm{C}$ until solid-phase microextraction and analysis by gas 157 chromatography mass spectrometry. 4,31

\section{$158 \quad$ RNA extraction}

The RNA extraction was performed using the TRI Reagent RNA isolation protocol (1 mL per 20-30 mg of lyophilized tissue per extraction) and treated with DNAse.

\section{mRNA library preparation and sequencing}

The library from DNA free total RNA was constructed following the TruSeq ${ }^{\mathrm{TM}}$ Stranded mRNA Sample Preparation kit protocol (Illumina Inc., Redwood. CA, USA).

The libraries were sequenced using TruSeq SBS Kit v3-HS, in paired end mode with a read length ranging from 35 to $76 \mathrm{bp}$ by the National Centre of Genomics Analysis (CNAG) in 
167 system (Illumina, Inc.) was used to generate 6-12 million Paired End reads Passing Filter for 168 each sample.

A quality read assessment was performed with FastQC package. ${ }^{32}$ These reads were 170 mapped against the C. melo genome CM3.5.1 (http://melonomics.cragenomica.es/) using the 171 Hisat2 mapper, ${ }^{33}$ counting the number of reads mapped to transcripts with StringTie. ${ }^{34}$ This 172 counting was guided using the genome annotation, and a unified set of transcripts was created 173 for the samples analyzed. Then, a Fragments Per Kilobase of transcript per Million (FPKM) 174 table with gene expression for each sample was generated and used to assess the expression 175 profiles of each sample by principal components analysis (PCA). Expression profiles showed 176 high differences between samples due to ribosomal genes expression, so this were removed 177 from further analysis steps. Differential expression analyses of both lines over time were run 178 using the Bioconductor package Ballgown, ${ }^{35}$ using FPKMs as measurement of gene expression. 179 Tables of DEGs, considering the effect of the introgression or the postharvest ripening time, 180 were obtained separately. Mev (RNA-Seq analysis in $\mathrm{MeV}^{36,37}$ was used to run PCA analyses 181 on DEGs and separate those genes into expression profile clusters. These clusters were 182 calculated by k-means algorithm with Pearson correlation as metric and FPKMs as 183 measurement. GO terms enrichment analyses were run for each one with the Blast2GO 184 package. ${ }^{38}$

185 Assessment of introgression effects on textural traits and VOCs

The experiment was analyzed by a two-way ANOVA using introgression ( $\mathrm{I}=\mathrm{SC10}-2$ or 187 PS) and postharvest ripening time (RT=0, 4, 8, 12, $18 \mathrm{~d})$ as factors followed by an LSD test at $188 \mathrm{p}=0.05$ for the main effects or I $\mathrm{x}$ RT interaction. For the DEGs, the significant effects of the 189 introgression (effect I alone) or the combined effect of postharvest ripening time and the 
190 interaction I x RT were calculated. The main introgression effects of SC10-2 were grouped into

191 effects above or below the PS.

192 Association between quality traits and DEGs

193 A two-way hierarchical clustering (HC), using Euclidean distance as the similarity measure and

194 Ward's linkage, was performed for analysing similarities between thirty-seven DEGs (Table 1)

195 and the quality traits measured (textural traits and VOCs) over postharvest ripening time. Scaled

196 and log-transformed data were presented in a heatmap (where columns are samples and rows

197 are variables) including two dendrograms, for the samples and variables, to visualize the results

198 of the clustering. The similarities between samples were explained by similarities with respect

199 to the values of variables.

200

201 RESULTS

202 Physiological behaviour and textural traits

203 The depletion in respiration rate and ethylene production were most pronounced during 204 the first four days of postharvest ripening and the rates of SC10-2, particularly of ethylene 205 production, were generally below those of PS (Fig. 1). Early or late harvested fruits showed, 206 respectively, higher or lower respiration and ethylene production rates irrespective of the line 207 (data not shown). SC10-2 could be stored for more than $40 \mathrm{~d}$ and PS for around $30 \mathrm{~d}$. The main 208 problem of extending fruit storage further, particularly at high relative humidity levels, is flesh 209 sugar loss and fungal decay (Fusarium sp.), particularly at the peduncle (data not shown).

210 Flesh firmness and whole fruit hardness were always higher in SC10-2 than in PS (fig.

211 2). The firm-ripe fruit of the NIL SC10-2 was slower to ripen postharvest than PS, as revealed 212 by its slower softening (higher whole fruit hardness and flesh firmness during ripening and 
213 reduced juiciness (introgression and postharvest ripening time significant at $\mathrm{P}<0.05$; Fig. 2).

214 Juice density (overall $1017 \pm 4 \mathrm{~kg} \cdot \mathrm{m}^{-3}$ ) did not change significantly in either line.

\section{Volatile organic compounds}

During postharvest storage (Fig. 3) two significant introgression $\mathrm{x}$ ripening time interactions $(\mathrm{P}<0.05)$ were observed for some groups of VOCs. After $4 \mathrm{~d}$, the relative levels of acetate esters were higher in PS than in SC10-2. The same was true for non-acetate esters but after $8 \mathrm{~d}$, while the concentration of other VOCs abruptly also increased after $12 \mathrm{~d}$ of storage in SC10-2 compared with PS levels. The relative levels of aldehydes, alcohols, acids and terpenes showed generally higher levels in $\mathrm{SC} 10-2$ than in PS (introgression significant, $\mathrm{P}<0.05$; Fig. 3). Aldehydes, alcohols and terpenes followed a similar pattern during ripening, with a maximum after 4-8 days and decreasing thereafter (time significant, $\mathrm{P}<0.05$; Fig. 3).

A total of thirteen individual VOCs showed significant effects for the factors studied during storage (Fig. 4), all of them belonging to four compound classes (aldehydes, ketones, alcohols and sulphur-derived compounds). Two compounds (1-phenylethanone and dodecanal) showed significant introgression $\mathrm{x}$ ripening time effects $(\mathrm{P}<0.05)$, because of the differences in their respective patterns of ripening in both lines (convex for SC10-2; concave for PS). A total of eleven individual VOCs of different classes showed a significant introgression effect according to the two-way ANOVA (Fig. 4), most of them alcohols or aldehydes with lower levels in PS than in SC10-2 during ripening (methanethiol, S-methyl ethanethioate, hexanal, octanal, (Z)-non-6-enal, nonanal, decanal, 4-isopropylcyclohexanol (isomer 1), 2-ethylhexan1-ol, nonan-1-ol, (E)-4-phenylbut-3-en-2-one). Decanal and hexanal also showed a significant time effect together with nonanal but decreased with the ripening time $(\mathrm{P}<0.05)$.

\section{Effect of LG X introgression on textural traits and VOCs during ripening}

Negative effects on the mean respiration rate and ethylene production rates and flesh juiciness were found in PS, while flesh firmness and whole fruit hardness showed an opposite 
trend. As regards the VOCs and considering the effects reported in the previous section, the introgression affected aldehydes (hexanal, octanal, (Z)-non-6-enal, nonanal, decanal); alcohols (4-isopropylcyclohexanol (isomer 1); 2-ethylhexan-1-ol; nonan-1-ol), ketones ((E)-4phenylbut-3-en-2-one) and sulphur-derived compounds (methanethiol, and S-methyl ethanethioate). Two time-dependent effects were observed for 1-phenylethanone and dodecanal.

\section{Differential expression analysis}

PCA analysis showed expression differences between groups and low variability within groups, so further steps in the analysis could be done without any concern about samples' expression profiles. As expected, the PCA based on a comparison between lines with time separated SC10-2 from PS better than the other PCA involving ripening time (Supplementary Figs. 1 and 2, respectively).

A total of 2954 DEGs were found by comparing both lines (i.e. considering the introgression factor or the interaction introgression $\mathrm{x}$ ripening time; Supplementary Table 1). A total of 2068 DEGs changed during postharvest ripening in both lines (Supplementary Tables 2 and 3), while 2045 of the formers were also DEGs, as seen from the comparison between lines over time. Therefore, 23 DEGs were exclusive to the postharvest ripening time effect and with no introgression or introgression x ripening time effects (Supplementary Table 3). Only 909 of the 2954 DEGs found for introgression showed a significant effect for introgression but did not appear in the DEGs when the factor ripening time was analyzed (Supplementary Table 4), making them the most interesting ones for this study.

During postharvest ripening, most of the identified DEGs were downregulated to a greater degree in SC10-2 than in PS but accounted for a smaller proportion of the total DEGs than in PS (Fig. 5). Comparing lines during ripening time (introgression effect), most of the upregulated DEGs in almost all of the functional categories were more abundant in SC10-2 than 
263 in PS (Fig. 6), but, in contrast to the first factor studied (ripening time effect), some DEGs were 264 upregulated in some biological processes (such as the negative regulation of cell growth, the 265 biosynthesis of indole-containing compound, mRNA 3'-end processing, jasmonic acid mediated introgression were as follows: DNA strand elongation involved in DNA replication; zinc ion binding; RNA binding; chloroplast stroma; oxidoreductase activity, acting on paired donors, with incorporation or reduction of molecular oxygen; hydrolase activity, acting on glycosylic bonds; monooxygenase activity; positive regulation of growth rate; transcription from RNA polymerase II promoter (Fig. 6).

Cluster analysis. Seven clusters were found when the introgression effect was studied in a comparison of lines over time (Fig. 7; Supplementary Table 1). However, considering the expression patterns during postharvest ripening time, we classified the DEGs into six main clusters (Fig. 8; Supplementary Table 2).

As regards the study of clusters involving introgression differences over time (Fig. 7), the most noticeable differences were the presence of $30 \%$ of total DEGs with upregulation of SC10-2 vs PS (clusters 1.1, 1.3 and 1.6) and 34.3\% with minor differences but the same trend except at harvest and after 12 days of ripening (cluster 1.2). These clusters were associated with the mRNA metabolic process, the response to abscisic acid, kinase activity, carbohydrate derivative biosynthetic processes, etc. (Supplementary Fig. 4). By contrast, only 7.3\% of DEGs (cluster 1.7) were clearly upregulated in PS compared with SC10-2, which was associated with 
288 the nucleolus, small molecule metabolic processes and lyase activity. However, $28.5 \%$ of DEGs 289 (clusters 1.4 and 1.5) showed the same trend but only from harvest up to 4 or $8 \mathrm{~d}$, probably due 290 to different responses between lines to oxidative stress, the polysaccharide metabolic process 291 or zinc ion binding, etc. (Supplementary Fig. 4). Clusters 1.2, 1.4 and 1.5 showed a noticeable 292 introgression $\mathrm{x}$ ripening time interaction (Fig. 7).

293 Generally, the genes expressed in the clusters were more abundant in PS than in SC102942 considering both factors (Supplementary. Figs. 3 and 4). In general, the study of clusters 295 referring to postharvest ripening time revealed that most of the DEGs related with this factor 296 were upregulated from harvest levels, peaking after 4,8 or $12 \mathrm{~d}$ of postharvest ripening (clusters $2972.2,2.3$, and 2.4; 47.1\% DEGs; Fig. 8). Also, clusters 2.5 and 2.6 represent genes highly 298 expressed after $4 \mathrm{~d}$ of ripening and peaking after $18 \mathrm{~d}$ (17.6\% DEGs; Fig. 8). In all the clusters 299 except cluster 2.1 (35.3\% of total DEGs, downregulated since harvest), slight introgression $\mathrm{x}$ 300 ripening time interactions were evident (the lines during ripening tending to cross). Cluster 2.1 301 reflects downregulation of the mRNA metabolic process, the RNA biosynthetic process or 302 cellular macromolecule biosynthetic process, among others (Supplementary Table 2). By 303 contrast, the rest of the clusters mostly represent upregulated genes associated with processes 304 such as transferase of hexosyl groups, binding (ATP, metal ion, RNA, zinc ion, coenzyme), 305 mRNA metabolic process, glycolytic process, cell wall, gluconeogenesis, etc. (Supplementary 306 Fig. 3).

Selected DEGs study. Overall, we selected and studied a total of thirty-seven 308 differentially expressed genes (DEGs) as a result of the introgression and/or postharvest 309 ripening time factors (nineteen of the selected ones) that were involved in different pathways 310 and functions (Table 1; Supplementary Tables 4 and 5). Among them, two selected DEGs 311 involved in primary metabolism, ATP-citrate synthase alpha chain protein (CmACLA-1), 312 which is involved in ligase and catalytic activity, Succinate--CoA ligase [ADP-forming] subunit 
313 alpha, mitochondrial ( $\mathrm{CmSCOA}$ ) involved in pyruvate metabolism and Citric Acid (TCA) 314 cycle, were generally upregulated in SC10-2 compared with PS (Fig. 9). However, some slight 315 differences in expression between NIL SC10-2 and PS, particularly as regards postharvest 316 ripening time, were found in four DEGs ( $C m$ NADP, $C m$ ACLB-2, $C m$ SDHA).

An additional DEGs were selected considering the traits studied and postharvest fruit ripening: $C m$ LOX18, CmAOS, CmOPR2, CmSWEET7, CmDFR4, CmPAL, CmWRKY33, CmGATA5, $C m$ TCP15, CmGDSL esterase/lipase, $C m$ ERF027, CmGDE4 and $C m$ ATC, and $C m \operatorname{TrpD}$ were generally upregulated in SC10-2 compared with PS (Fig. 10) with some exceptions in the expression of $\mathrm{Cm} \mathrm{GDE} 4$ and $C m \mathrm{ATC}$. In contrast, the genes of $\mathrm{CmACO} 2$, CmADH1, CmCAD1, CmNADH1 and $C m$ NAC18 were downregulated (Fig. 10). Only $C m$ NADH1, $C m$ TCP15 and $C m$ GDSL esterase/lipase were located in LG (Table 1), while for the other differentially expressed genes, some eQTLs could be found within LG X). Two genes involved in aminoacid metabolism (CmIIL1 and CmFAH were downregulated in SC10-2 vs. PS, while CmIPMI3 was upregulated (Fig. 10). Another two selected DEGs ( $C m$ L-GalDH and CmGME) involved in ascorbic acid metabolism were generally upregulated in SC10-2 vs. PS (Fig. 10).

Fourteen selected DEGs were significant for the introgression: CmMADS-box, $C m$ NAC18, $C m$ CAD1, $C m$ NADP, $C m$ ACLB-2, $C m I I L 1$ and $C m A C O 2$, which were generally downregulated in SC10-2 vs. PS (Fig. 11) with some exceptions in CmNADP, CmACLB-2 and CmIIL1 and some introgression x ripening time interactions (levels of SC10-2 increased over time and remained constant in PS) such as in $C m$ NAC18 (Fig. 11). As regards $C m$ WRKY33, CmACLA-1, CmFAH, CmSDHA, CmIPMI3, CmSCOA, CmL-GalDH and CmLOX18, they were generally upregulated in SC10-2 vs. PS (Fig. 11) with some exceptions in CmACLA-1, 
Some evident introgression $\mathrm{x}$ ripening time interactions were identified in some DEGs using ANOVA: $C m$ IIL1, $C m$ ADH1, $C m$ CAD1, $C m$ AOS, $C m$ SWEET7, $C m$ DFR4, $C m$ WRK33, CmGDSL esterase/lipase, CmERF027, CmGDPDL4, CmNAC18, CmACT, $C m L G a l D H$ and CmACLB-2 (Figs. 9, 10 and 11).

About 23 DEGs changed as time progressed and followed the typical trend to decrease from high harvest levels, or the contrary after 4-8 d of ripening (Table 1; Supplementary Tables 1 and 4; Fig. 12).

\section{Association between selected DEGs and quality traits (textural traits, VOCs).}

The analysis of the heatmap of the hierarchical clustering reveals a clear separation between SC10-2 and PS, and also from harvest to postharvest ripening time particularly in PS), and DEGs and quality traits could be grouped into eight clusters $(\mathrm{Cx}, \mathrm{x}=1-8)$ according to their values in the NIL and PS (Fig. 13; Supplementary Table 6). In general, in most of the clusters and cluster subgroups ( $\mathrm{Gx}, \mathrm{x}=1-8)$ studied with the values above 0 represents PS samples while the values below 0 represented the NIL SC10-2, with some exceptions for example in C3-G1 and C4-G2. Some associations were apparently unexpected according with the DEG function of Table 1. Certain association among the selected DEGs and textural traits were found within two of the eight clusters (C4, subgroup G2; C8, subgroup G8). In C4, the subgroup G2 associated CmWRKY33 (DNA binding transcription factor), textural traits (whole fruit hardness and flesh firmness) and eight VOCs. In C8, two acetate esters were associated with CmHK4-like gene and two textural traits (density and juiciness) (G8). In the former cases, PS levels were above SC10-2.

Of particular interest for postharvest ripening is the association detected in clusters $\mathrm{C} 7$ and $\mathrm{C} 8$ between some genes associated with aroma production or ripening with ester compounds (acetate, non-acetate or thioesters). For example, in C8 C8, two subgroups 
associated acetate and a sulfur-derived compound with $C m$ L-GalLDH (G6) or several thioester compounds, two ketones and isopropyl propionate with $C m \mathrm{NAC} 18$ (G7). The main differences 364 in both lines at harvest (caused mostly by the introgression) were due to nine DEGs (CmACLB2, CmFAH, CmIIL1, CmALT-2, CmNADP, CmACLA-1, CmSCOA, CmSDHA, CmIPMI3), 366 and eight VOCs (2-methylbutyl acetate, ethyl 2-methylpropanoate, methyl acetate, ethyl 2367 methylbutanoate, (E)-pent-2-enal, unidentified (24.64), acetone, and 1-methyl-4-prop-1-en-2368 ylcyclohexene) present in C7. Most of the previous DEGs at harvest (with the exception of 369 CmALT-2; Fig. 4) were involved in the Kreb's cycle (i.e., a clear association with differences 370 in respiration rate between lines; Fig. 1). The main differences in both lines at postharvest 371 ripening times 8, 12 and 18 were due to the four ester VOCs (2-methylbutyl acetate, ethyl 2372 methylpropanoate, methyl acetate and ethyl 2-methylbutanoate and the DEG CmACLB-2.

The subgroup G4 of C7 was associating several volatiles and two important genes of 374 secondary metabolism (ethylene biosynthesis such as $\mathrm{CmACO}$ ) and a transcription factor 375 (CmMADS-box) with higher levels in PS than in SC10-2. In C1, VOCs from 2-(2,2,3376 trimethylcyclopent-3-en-1-yl) acetaldehyde up to tetradecanal showed differences due to the 377 introgression but postharvest-ripening time dependent particularly with certain downregulation 378 in time 12 in SC10-2 vs PS. In C2, the same situation that in C1 was true with other time379 dependent trends depending on the introgression effect with volatile overproduction in SC10-2 380 particularly during the first days of ripening. In C3 the subgroup G1 was detected with certain 381 association between CmLOX18 (lipoxygenase activity) and methanethiol. In C7, the subgroup 382 G5 associated two volatiles (1-tridecanol, isobutyl-benzoate with a cinnamyl-alcohol 383 dehydrogenase (CmACLB-2). 


\section{Quality and physiological traits vs. gene expression}

Primary metabolic changes vs gene expression. As is known, fruit ripening is classified as climacteric and non-climacteric, depending on the presence or absence of a transient rise in the respiration rate and the production of autocatalytic ethylene..$^{5,21,39}$ Because the respiration rate was lower in SC10-2 than in PS (Fig. 1), it was thought to be of interest to check some genes of mitochondrial enzymatic activities, transporters that are involved in metabolic changes during fruit development, ${ }^{40}$ and genes of the tricarboxylic acid (TCA) cycle. ${ }^{41}$ In fact, these differences between the NIL and PS in DEG of the TCA cycle were confirmed by using a heatmap (Fig. 13; Supplementary Table 6). In particular, NADH:quinone oxidoreductase CmNADH1 (MELO3C012476) expression, the largest complex of the respiratory chain ${ }^{42}$, was higher in PS than in SC10-2 throughout the experimental time (Fig. 10), which would partly explain the higher respiration rate of PS (Fig. 1). This difference could also be attributed to several genes that codify for proteins that act as enzymes of the aerobic respiratory chains. ${ }^{43,44}$ For example, isocitrate dehydrogenase ( $C m$ NADP; MELO3C021563) expression was generally higher in PS than in NIL SC10-2 throughout the postharvest ripening time (Fig. 9). However, the mitochondrial succinate dehydrogenase ( $\mathrm{CmSDHA}$ ) first decreased before increasing during the last few days of ripening, but differences between lines were negligible when both factors (introgression and ripening time) were considered (Fig. 11).

For ATP-citrate synthase, two genes were differentially expressed in our experiment: ATP-citrate synthase alpha chain protein (CmACLA-1; MELO3C010675) and ATP-citrate synthase beta chain protein 2-like (CmACLB-2; MELO3C011482) (Fig. 9). After decreasing from harvest to 4 d CmACLA-1 was higher in the NIL SC10-2 than in PS. By contrast, CmACLB-2 expression was higher in PS (Fig. 11), probably because of the contribution of ACL, which is necessary for the generation of the cytosolic pool of acetyl-CoA. ${ }^{45,46}$ In fact, 
410 CmACLB-2 could be associated with formation of two acetate esters and perhaps two non411 acetate esters (Fig. 13; Supplementary Table 6).

The expression of succinate--CoA ligase [ADP-forming] subunit alpha, mitochondrial 413 gene ( $\mathrm{CmSCOA}$; MELO3C002167) associated with GTP binding, ATP binding, succinate-CoA 414 ligase (GDP-forming) activity, succinate-CoA ligase (ADP-forming) activity and ATP citrate 415 synthase activity, ${ }^{47}$ was not significantly different between PS and SC10-2 but the respiration 416 rate followed a similar pattern (Figs. 1 and 9).

417 Postharvest ripening delay and textural traits vs gene expression. This paper confirms 418 the effect of the long introgression covering the entire LG $\mathrm{X}^{13}$ that contained the previously 419 reported QTL ff10.2 of flesh firmness and textural traits at harvest. ${ }^{14,24,25}$ The introgression also 420 delayed fruit ripening in general, as seen from by physiological traits (respiration rate, ethylene 421 production, volatile production; Figs.1-4). Perpiñá et al. ${ }^{9}$ reported QTLs affecting fruit ripening 422 in a climacteric melon genetic background, including ff.10 for flesh firmness at harvest,_al.10 423 for the suppression of the formation of abscission layer, ar.10 that produced less aroma at 424 harvest, or QTLs affecting soluble solids and sugar content. Due to the length of these 425 introgressions in LG X, the effects on the aroma volatiles and textural traits could be due to 426 independent genes, or to pleiotropic effects due to a unique gene. ${ }^{24}$ In fact, the heatmap detected 427 association between CmWRKY33, textural traits and two VOCs (Fig. 13; Supplementary Table 428 6).

429 The candidate genes for $f f 10.2$ were $C m X T H 5$ and $C m E X P 3^{14}$, but during postharvest 430 ripening these genes did not show differential expression. Other authors ${ }^{19}$ map candidate genes 431 on LG X at harvest such anthranilate phosphoribosyltransferase: (CmTrpD; MELO3C011963) 432 o histidine kinase 4-like (CmHK4-like; MELO3C020055). The gene CmTrpD, with higher 433 levels in SC10-2 than in PS, could be a putative candidate gene during postharvest and 434 explained the differences due to the introgression in textural traits (Fig. 2). CmTrpD plays a role 
435 in cell wall metabolism but in the presence of ethylene. ${ }^{19}$ In tomato, QTLs affecting texture at 436 harvest are distinguished from those affected softening and other ripening-associated textural 437 traits during postharvest. ${ }^{48}$

The histidine kinase 4-like ( $\mathrm{CmHK} 4-\mathrm{like})$ gene, due to the association with juice density 439 and flesh juiciness affected by the introgression in LG X (Fig.13; Suppl. Table 4) and other 440 traits important from fruit ripening point of view, would require further attention. Histidine 441 Kinase 4 (HK4) and other plant hormones play essential and crucial roles in various aspects of 442 Arabidopsis and generally plant growth and development ${ }^{49}$ which maybe the case for CmHK4443 like in melon. HK4 positively regulates the cytokinin-signaling pathway as a direct receptor 444 molecule, knowing that cytokinins are a class of phytohormones that promote cytokinesis in 445 cell division in plant roots and shoots, and also in cell growth and differentiation. ${ }^{49}$ Cytokinins 446 also affect apical dominance, axillary bud growth, and leaf senescence. ${ }^{50}$

Several potential candidate genes have been proposed as being associated with melon 448 textural traits during ripening (mostly firmness, ffr 10.2, whole fruit hardness $w f h r 10.2$, or flesh 449 juiciness fjr10.2). This is common, as observed by the negative correlation between flesh 450 firmness and juiciness, ${ }^{25}$, because firmer fruit are usually less juicy. ${ }^{25}$ Moreno et al. ${ }^{14}$ reported $451 \quad f f 10.2$ at harvest and the unpublished results of these experiments ${ }^{25}$ would also confirm $w f h 10.2$. 452 However, juice density was not affected during ripening in contrast with the results of Obando 453 et al. ${ }^{25}$ who also reported the effect of the QTL of flesh firmness $f j 10.2$ but only at harvest time. 454 The expression of GDSL esterase/lipase (MELO3C011939) located in LG X was higher 455 in SC10-2 than in PS (Fig. 10), and SC10-2 also showed lower ethylene production (Fig. 1). 456 The expression of this gene could be associated with a delay or partial inhibition of some 457 ethylene-dependent flesh or whole fruit softening in SC10-2 compared with PS, because GDSL 458 expression in apple correlated negatively with ethylene production ${ }^{51}$ and in tomato it was 459 expressed in the epidermis of developing fruit. ${ }^{52}$ 
460 Of the potential candidate genes responsible for the increase in textural traits in SC10-2 461 compared with PS (Fig. 2), the glycerophosphoryl diester phosphodiesterase (CmGDE1; 462 MELO3C013101) gene has been found to play a role in plant cell wall remodelling. ${ }^{53,54}$ 463 464

DEGs exclusive to postharvest ripening time. Of the 23 genes that changed over time 465 (Table 1; Supplementary Tables 1 and 4; Fig. 12), we found typical ones in melon ripening 466 changes such as polygalacturonase ( $\mathrm{CmDHL}$ 92; MELO3C009970) (Table 1; Supplementary 467 Tables 1 and 4; Fig. 12) associated with cell-wall disassembly ${ }^{55}$ or other ongoing processes 468 typical of maintaining homeostasis or hydrolase activity and hydrolyzing O-glycosyl 469 compounds. For example, processes such as providing feed for diminished mitochondrial 470 respiration during postharvest ripening ( $C m \mathrm{MPC} 1$; MELO3C007517) (Table 1; Supplementary 471 Tables 1 and 4; Fig. 12), or increased protein detoxification (CmDTX; MELO3C017343) (Table 472 1; Supplementary Tables 1 and 4; Fig. 12). Some of these genes, such as the one that is 473 responsible for the increase of ABA receptors such as abscisic acid-insensitive 5-like protein 4 474 isoform X1 (CmABREX; MELO3C014384) (Table 1; Supplementary Tables 1 and 4; Fig. 12), 475 or the gene that decreases in auxin-responsive protein (CmIAA71; MELO3C000885) (Table 1; 476 Supplementary Tables 1 and 4; Fig. 12), would deserve further investigation due to the possible 477 similarity of non-climacteric ripening with the antagonist interaction of auxin and abscisic acid 478 with ethylene during ripening in other non-climacteric and climacteric fruits. ${ }^{56,57}$

479 Physiological traits and gene expression. The expression of CmACO2 480 (MELO3C019735) increased during ripening, a trend that was more pronounced in PS than in 481 SC10-2 (Fig. 10) (Fig. 11), which agrees with the faster ripening of PS (including higher level 482 of respiration, ethylene production, and the synthesis of some ethylene-dependent VOCs, such 483 as acetate esters and non-acetate esters; Figs. 1 and 4) in PS. CmACO2 and CmMADS-box 484 expression generally downregulated in SC10-2 vs PS (Figs. 10 and 11) also clustered with the 
485 level of four compounds unexpected that were generally more abundant in PS than in SC10-2 486 (C7, subgroup G4 of Fig. 13; Supplementary Table 6). The melon 1-aminocyclopropane-1487 carboxylic acid oxidase gene $(\mathrm{Cm} \mathrm{ACO})$ is an ethylene-responsive gene that catalyses the 488 conversion of ACC to ethylene during fruit ripening and is of greater importance in climacteric 489 fruit. ${ }^{2,58}$ However, CmACO2 has been mapped in LG VIII, ${ }^{14}$ and consequently some eQTLs 490 upstream of $\mathrm{CmACO} 2$ but affecting its expression should be mapping in LG X. On the other 491 hand, other genes with no differential expression in our non-climacteric experiment such as 492 CmACO1 have been associated with ethylene biosynthesis, together with CmACS1 and 493 CmACS5 in climacteric melon fruit ripening. ${ }^{3}$

494 More than four transcription factors were identified in our experiment; $C m$ WRKY33 495 (MELO3C009127), CmTCP15 (MELO3C01208), CmGATA5 (MELO3C023350) and 496 CmERF027 (MELO3C005630) (Table 1). Almost all of them increased in SC10-2 more than 497 in PS during the ripening time and therefore could be partially responsible for the delay of 498 ripening in the NIL SC10-2 compared with PS.

The expression of CmWRKY33 (ethylene-responsive gene during climacteric 500 ripening, ${ }^{59} \mathrm{Cm}$ GATA5 (transcriptional regulators involved in metabolism regulation), ${ }^{60}$ and $501 C m$ ERF027 (ethylene-inducible genes during fruit ripening) $61,62,63$ decreased from their highest 502 level of expression before $4 \mathrm{~d}$ of ripening but remained higher in SC10-2 than in PS throughout 503 the postharvest ripening time (Figs. 10 and 11). The behaviour of these genes was apparently 504 opposite to that of ethylene production, particularly during the first 4 days of non-climacteric 505 melon ripening of both lines (Fig. 1), and so they could be associated with the ripening 506 differences between climacteric and non-climacteric melon fruit. ${ }^{21}$ Particularly CmWRKY33 507 was found in the heatmap with association with textural traits (Fig. 13, Supplementary Table 508 6). CmGATA5 could interact with cis-acting elements involved in the light regulation of nuclear 509 genes encoding chloroplast glyceraldehyde-3-phosphate dehydrogenase (GAPDH) and 
510 therefore glycolysis ${ }^{64}$ or other processes such as ribosome biogenesis, mRNA metabolism or 511 lipid A biosynthesis. ${ }^{65,66}$

512 Glycerophosphodiester phosphodiesterases (CmGDPDL4; MELO3C013101) showed

513 similar gene expression to GATA5 in our experiment, with higher levels after $8 \mathrm{~d}$ of ripening 514 in SC10-2 than in PS (Fig. 10). This gene could be involved in fatty acid metabolism to further 515 provide glycerol phosphate and alcohol resulting from the hydrolysis of deacylated 516 glycerophospholipids. ${ }^{67}$

CmNAC18 (MELO3C016540) expression was stable in SC10-2 but was present at

518 higher levels than in PS, where it increased after harvest to reach levels similar to that of SC10-2 519 (Fig. 10). This $C m N A C-N O R$ domain transcription factor was expressed in the flesh of both 520 climacteric and non-climacteric melon types, but is probably transcriptionally regulated in non521 climacteric types because it has been considered a candidate gene for the QTL ETHQV6.3 of 522 climacteric fruit ripening. ${ }^{3}$ NAC transcription factor is a positive regulator of fruit ripening, 523 affecting ethylene synthesis and carotenoid accumulation, 3,68 but NAC also interacts with other 524 genes in the complex regulatory network of fruit ripening in tomato. ${ }^{68}$ All the above information 525 suggests a potential association of higher NAC levels with the delayed fruit ripening of the NIL 526 SC10-2 compared with PS.

527 In our experiment, a MADS-box transcription factor was identified among the DEGs 528 with higher relative expression in PS than in SC10-2 and decreasing with ripening time (Fig. 529 11). Some MADS-box genes are involved in the development and ripening of non-climacteric 530 fruits such as bilberry ${ }^{69}$ and strawberry. ${ }^{56,70}$

531 Postharvest VOCs vs gene expression. In previous investigations, noticeable 532 differences were found between the aroma profile of NIL SC10-2 and the control PS at 533 harvest. ${ }^{24,71}$ Some VOCs were lacking from the NIL SC10-2 compared with PS [some ketones 534 and alcohols, and one acid (e.g. 2-phenylpropanoic acid)], but also the concentration of 
535 aldehydes (e.g. 2,4-dimethylbenzaldehyde, 2-methylpropanal and 2-methylbutanal) were 536 higher in the NIL. ${ }^{24}$ In our experiment, too, the levels of many aldehydes (C6-up to C12) 537 remained high during ripening (Fig. 4), in agreement with the above results at harvest, but 538 ketone levels were similar, and acids were hardly present (data not shown). The NIL SC10-2 539 was characterized by a lower ketone content than PS. ${ }^{24}$ In our experiment, aldehydes, alcohols, 540 acids and terpenes were higher in SC10-2 than in PS during postharvest ripening, while the 541 opposite trend was seen for acetate and non-acetate esters (Fig. 3). As regards other VOCs, they 542 increased in SC10-2 and were higher than in PS at the end of the experiment (Fig. 3), which 543 could be due to senescence processes. ${ }^{22}$ Interestingly, the association among selected DEGs 544 linked to DNA-binding, ATP-binding, or metal-ion binding (and in general the end of non545 climacteric ripening) were more frequent with ester VOCs (see C7 and C8; Fig. 13; 546 Supplementary Table 6).

As regards other metabolic pathways associated with the differences between PS and

548 SC10-2 QTLs and volatiles, these were related in part to the free L-amino acid metabolism, in 549 which phenylalanine, valine, isoleucine and leucine ${ }^{24}$ are considered aroma precursors. ${ }^{23}$ The 550 higher aldehyde content was typical of the NIL SC10-2 and associated with its high flesh 551 firmness at harvest compared with PS, ${ }^{24}$ in agreement with our results (Figs. 2 and 3 ). 552 Aldehydes are key-flavor compounds synthesized from $\alpha$-keto acids particularly in melon fruit. $553 \quad 23$

The expression of GDPDL4 (MELO3C013101) in our experiment also was higher in 555 SC10-2 than in PS from harvest to $8 \mathrm{~d}$ of postharvest ripening (Fig. 10), which might explain 556 the abundance of alcohols in this NIL (Fig. 3), as explained above for CmGDPDL4 gene. In 557 this case, glycerophosphodiester phosphodiesterase (GDPD) hydrolyzes the 558 glycerophosphodiesters into sn-glycerol-3-phosphate (G-3-P) and the corresponding alcohols, ${ }^{72}$ 559 particularly in fruit tissue. ${ }^{73}$ GDPD have also been seen to be involved in the generation of 
560 phosphatidic acid, a precursor of diacylglycerol and inorganic phosphate by phosphatidate 561 phosphatase, ${ }^{74,75}$ or in the degradation of phospholipids into diacylglycerol and inorganic 562 phosphate. $^{76}$

CmLOX18 (MELO3C024348) was highly expressed in the NIL SC10-2 compared with PS, which had higher levels of aldehydes, terpenes, alcohols and other VOCs than PS (Figs. 3; 10 and 11). Certain association between $C m L O X 18$ and methanethiol production was found (Fig. 13; Supplementary Table 6), perhaps because of its aminoacid precursor degradation such as methionine or phenylalanine (see aminoacid metabolism below). $\mathrm{Cm}$ LOX genes are involved in generating fruit aroma in melon. ${ }^{77,78}$ Our results support that this is the LOX family gene responsible for producing LOX enzyme during ripening to form C6 VOCs such as hexanal, (Z)570 3-hexenal, and (Z)-3-hexenol in fruit, and is also possibly involved in the formation of straight571 chain ester. ${ }^{78}$

The enzymatic reaction of LOX causes polyunsaturated fatty acids, such as linoleic acid and linolenic acid, to generate hydroperoxide (HPO). ${ }^{79,80}$ HPO is catalyzed by hydroperoxide 574 lyase (HPL) to produce aldehydes, which are catalyzed by alcohol dehydrogenases (ADH) to 575 produce alcohols. ${ }^{77,81}$ The final step in ester biosynthesis is catalyzed by alcohol acyltransferase (AAT). ${ }^{82,83}$ Apparently, the higher expression of CmADH1 (MELO3C019548) in

577 PS than in SC10-2 would explain the differences in aroma production between lines because in 578 PS the former volatile metabolism would be more advanced and produce alcohols as precursors 579 of the esters (Fig. 3). In fact, Chen et al..$^{85}$ observed the upregulation of CmADH1 after the 580 addition of aldehydes, which indicated its potential function in aroma volatile or ester synthesis. 581 Also, in climacteric tomato fruit, Moummou et al. ${ }^{85}$ suggested a function of ADH1 of supplying 582 C5 and C6 volatiles from the lipoxygenase pathway. Alcohol acetyl transferases (CmAAT1 and 583 CmAAT2) showed similar relative expression in both lines in our experiment (data not shown), 
584 and it is known that the expression of AAT in PS is very low. ${ }^{23}$ Therefore, we conclude that

$585 C m A D H 1$ is a key enzyme, particularly in PS aroma formation.

586

587

588

589

590

591

592

593
594 (Fig. 11), which might have some influence in the conversion of cinnamyl aldehydes into 595 alcohols, using NADPH (quinone oxidoreductase) as a cofactor. ${ }^{87}$

596

597

598 599 600 601 602 603 604 605 of ethylene production by $C m$ AOS during the first $8 \mathrm{~d}$ of ripening was not evident (Figs. 1 and $60610)$

607

608

12-oxophytodienoate reductase 2 (CmOPR2; MELO3C008075) expression was also higher in SC10-2 than in PS (Fig. 10). Therefore, CmOPR2 may increase the activity of the $\beta$ oxidation pathway because the oxophytodienoate reductase family (OPR) is expressed throughout the plant and co-localises with enzymes involved in the $\beta$-oxidation of very long fatty acids in peroxisomes. ${ }^{86}$ In fact, the levels of most of the aldehydes in SC10-2 were higher than in PS (Fig. 3), and, consequently, alcohol dehydrogenase 1 (CmADH1; MELO3C019548) and cinnamyl alcohol dehydrogenase 1 (CmCAD1; MELO3C018492) genes showed lower expression in SC10-2 than in PS. CmCAD1 expression was also lower in SC10-2 than in PS In our experiment, the expression of allene oxide synthase gene (CmAOS; MELO3C010910) was higher in SC10-2 than in PS (Fig. 10). CmAOS could be involved in the jasmonic acid metabolism from hydroperoxides because AOS and allene oxide cyclase mediated in the branch of $\alpha$-linolenic acid metabolism for the formation of jasmonic acids. ${ }^{2,88}$ Two AOS isozymes identified in oriental melon fruit catalysed 10-OPDA and 12-OPDA formation, and other AOS isozymes, identified in different ripening stages, are differently regulated by genetic and environmental factors. ${ }^{2}$ Concomitant with jasmonic acid biosynthesis and ethylene production, AOS was downregulated during fruit development but increased again in the last stage of peach fruit development. ${ }^{89}$ However, in SC10-2 the hypothetical stimulation

Aminoacid metabolism. The 3-isopropylmalate dehydratase small subunit 3-like (CmIPMI3; MELO3C005518) was generally higher in the NIL SC10-2 than in PS, except at 12 
609 d (Fig. 10 and 11), in contrast to the behaviour of 3-isopropylmalate dehydratase large subunit 610 (CmIIL1; MELO3C003344) at harvest and in senescence (Figs. 9 and 11). Generally, 3611 isopropylmalate dehydratase catalyses the stereo-specific isomerisation of 2-isopropylmalate 612 and 3-isopropylmalate, via the formation of 2-isopropylmaleate, which is the second step in the 613 biosynthesis of the aminoacid leucine, ${ }^{90}$ a potential precursor of some VOCs of interest here, 614 such as 2-ethylhexan-1-ol, nonanol, or 4-Isopropylhexanol (isomer 1) (Fig. 4; Table 1).

By contrast, fumarylacetoacetase $(\mathrm{CmFAH}$; MELO3C007433) expression and alanine aminotransferase (CmALT-2; MELO3C010686) expression were always higher in PS than in NIL SC10-2 (Figs. 10 and 11). FAH is involved in the metabolism of amino acids 618 (phenylalanine and tyrosine) and the catabolism of its derivatives, ${ }^{90}$ Phe being of interest as a 619 potential precursor of some VOCs of interest here such as methanetiol or S-methyl 620

Gene expression and other postharvest changes. Dihydroflavonol 4-reductase (CmDFR4; MELO3C020508), the first committed enzyme of the flavonoid biosynthetic 624 pathway including VOC regulation, ${ }^{93}$ showed higher expression in SC10-2 compared with PS 625 particularly after 4 days of postharvest ripening (Fig. 10).

Apparently in SC10-2, CmPAL (MELO3C017811) gene expression is a response to late 627 fruit ripening, which contrast with the lack of changes during ripening in PS (Fig. 10). Melon 628 fruit phenylalanine ammonia-lyase (PAL), the first enzyme of phenylpropanoid biosynthesis, is 629 transcriptionally induced in response to fruit ripening and CmPAL gene expression follows the 630 631 expression kinetics of the ethylene biosynthetic genes during fruit development. ${ }^{94}$

CmACT (MELO3C005591) regulatory domain expression showed higher levels in 632 SC10-2 than PS during the first 8 days of postharvest ripening (Fig. 10), and the ACT was 
633 generally found in enzymes involved in amino acid and purine metabolism, ${ }^{95,96}$ some of them 634 probably associated with the formation of volatile precursors.

10). TCP proteins are involved in biological processes of potential interest for future studies in

637 others aspects of factors affecting melon fruit ripening, such as hormone pathways, ${ }^{97}$ mitochondrial biogenesis, ${ }^{98}$ and regulation of the circadian clock. ${ }^{99}$

The higher expression of the bidirectional sugar transporter CmSWEET7 (MELO3C016259) in SC10-2 compared with PS after 4 days of ripening (Fig. 10) could be related with fruit senescence, environmental adaptation, the sugar accumulation pattern, or other essential fruit biological processes. ${ }^{100,101,102}$ Some QTLs in LG X associated with the soluble solids content and individual sugars (sucrose, fructose) in other genetic backgrounds have also been mapped ${ }^{9}$. Although sugars are not discussed here, SC10-2 is less sweet than PS at harvest. In fact, several QTLs that negatively affect sensory traits have been mapped at harvest in LG X (sweetness, taste and global sensorial quality appreciation) compared with PS, due to lower sucrose, total sugars and sucrose equivalents. ${ }^{103}$ One hypothesis for the mechanism of $C m$ SWEET7 gene would be to delay ripening in order to supply additional sugar resources to the fruit metabolism in SC10-2. This hypothesis would agree with results in tomato, where SWEET transporter was also upregulated in the lines with a high fructose-to-glucose ratio. ${ }^{102}$

GalLDH catalyses the oxidation of the last precursor, L-galactono- $\gamma$-lactone, to ascorbic acid. ${ }^{104}$ In our experiment, evidence of differences in ascorbate metabolism would include lower L-galactono-1, 4-lactone dehydrogenase ( $C m L-G a l L D H ; ~ M E L O 3 C 018576)$ relative expression with higher expression in PS than in SC10-2 until $8 \mathrm{~d}$ (Fig. 9 and 11). Other evidence is the report on one QTL that reduced levels of ascorbic at harvest in LG X. ${ }^{103}$

As regards GDP-mannose-3',5'-epimerase (CmGME; MELO3C004377), in our experiment it showed higher expression in NIL SC10-2 than in PS but it decreased after $12 \mathrm{~d}$ 
658 (Fig. 9). The GME, which converts GDP-d-mannose to GDP-1-galactose, is generally 659 considered to be a central enzyme of the major ascorbate biosynthesis pathway in higher 660 plants. ${ }^{105}$ The formation of GDP-d-mannose is the initial step in the pathway of ascorbate 661 biosynthesis, and GDP-d-mannose is also a known precursor for the synthesis of d-mannose, 662 1-fucose and l-galactose, and therefore for hemicelluloses such as (galacto)glucomannans and 663 for the pectin rhamnogalacturonan II. ${ }^{106}$ This biosynthesis could be of importance for 664 maintaining melon flesh firmness during ripening (Fig. 2).

665 General processes deduced from transcriptome analysis

666 As regards the comparison between lines, many of the genes downregulated in cluster

6671.1 (Supplementary Table 1) were related with regulation of the cellular macromolecule 668 biosynthetic process, the RNA biosynthetic process and other genes depending to the 669 photosynthesis processes. The genes related to photosynthesis during fruit ripening are also 670 downregulated in non-climacteric grape. ${ }^{107}$

671 As regards the postharvest ripening time effect (Supplementary Table 2), clusters 2.2 672 and 2.4 were the most closely associated with this effect (Fig. 8), a sharp rise in the expression 673 of DEGs being evident in the two non-climacteric genotypes. The same is true for clusters 1.4, 6741.5 and 1.6, considering the comparison between lines (Fig. 8; Supplementary Table 1). Most 675 genes of both group of clusters were related to biosynthetic processes (Supplementary Tables 1 676 and 2), for example those related to ethylene-regulated and ripening-related genes, ${ }^{108}$ including 677 those involved in ethylene synthesis, fruit texture, and aroma volatile production. ${ }^{5}$

678 Considering the postharvest ripening time effect, most DEGs of clusters 2.3, 2.5 and 2.6 679 showed higher expression in PS than in SC10-2 and a trend to increase (Fig. 8). The same is 680 true for clusters 1.5 and 1.6, but considering the comparison between lines over time (Fig. 7). 681 The DEGs of the above-mentioned clusters were generally related to molecular function and 682 cellular components (Supplementary Tables 1 and 2), and these genes affected in one way or 
683 another to flesh firmness and whole fruit hardness because they showed the opposite trend and 684 led to fruit softening. As regards other non-climacteric NILs (i.e. SC7-2) with firm flesh and 685 other climacteric melons and fruits in general, it has been reported that flesh textural changes 686 are accompanied by loss of neutral sugars, solubilisation and depolymerisation of the 687 polysaccharides of the cell wall, and rearrangements of their associations, as the result of the 688 combined action of several cell wall-modifying enzymes such as polygalacturonase and others 689 (see above), acting in both pectic and hemicellulosic fractions. ${ }^{28,55}$ Consequently, it is these 690 changes that probably make the PS fruit texture less resistant and juicier than SC10-2.

\section{Implications for the future}

692

693

694

695

696

697

698

699

700

701

702

703

704

705

706

707

The genetic changes described not only explain new aspects of the non-climacteric fruit ripening - such as the control of non-climacteric ripening, respiration and ethylene biosynthesis and action, texture, or aroma production - but also provide a few new ideas about the changes in different metabolic pathways. These could help growers and researchers to develop new melon varieties with good quality traits with a long shelf life, for example by manipulating genes such as CmNAC18 or CmSWEET7.

To better understand the effect of introgressions in LG $\mathrm{X}$ and the transcriptional regulation of metabolic genes during fruit ripening, other specific development and textural transcriptome analyses are necessary, probably using NILs with shorter introgressions such as SC10-1, SC10-3 or SC10-4, or the development of NILs with shorter introgressions that are more useful for melon breeding. ${ }^{109,110}$ Recently, Perpiñá et al. ${ }^{111}$ developed a breeding line with a short introgression (between markers CMPSNP172 and CMPSNP65) in LG X from the cultivar Ginsen Makuwa (closely related to SC), ${ }^{112}$ that also extended fruit shelf-life and maintained flesh firmness but in the Charentais genetic background (a climacteric type). This would confirm the presence of genes involved in fruit ripening in LG $\mathrm{X}$ from different germplasm sources which effects are stable in different genetic backgrounds and, therefore, 
708 with a potential for breeding. Unfortunately, the DEGs studied here with more detail that 709 mapped in LG X (MELO3C012476, MELO3C012086 or MELO3C011939) were not within 710 the LG X region flanked by the above-mentioned SNPs).

\section{CONCLUSIONS}

The introgressions in LG X delayed non-climacteric fruit ripening as demonstrated by physiological and textural traits: reduced ethylene production and respiration rates, and, consequently delayed softening and loss of whole fruit hardness and an increase in flesh juiciness. The ripening delay consequently delayed senescence processes, which impacted on volatile production in the NIL SC10-2 compared with PS, as evidenced by ester levels (acetate non-acetate thioester) in this experiment. The clusters of the DEGs associated with the factor postharvest ripening time show that most genes are upregulated over time, while the comparison between lines showed noticeable gene upregulation in SC10-2 compared with PS at harvest or after $8 \mathrm{~d}$ of ripening) and downregulation in $29 \%$ of the DEGs.

The transcriptome of the melon NIL SC10-2 and its parental PS suggest that the genes involved in the ripening pathways are regulated dynamically to activate the expression of some genes during ripening time (e.g. CmLOX18, CmAOS, CmDFR4, CmOPR2, CmGATA5, CmTCP15 and CmGDSL esterase/lipase process genes) and to delay the expression of others (genes related to cellular component such as $C m \mathrm{NAC} 18, C m \mathrm{NADH} 1, C m \mathrm{CAD} 1, C m \mathrm{ACO} 2$ and CmADH1) during fruit ripening. Certain association of several DEGs with textural traits and volatiles were detected by heatmap and hierarchical clustering.

The identified genes differentially expressed during the ripening time and located in different linkage group (other than LG $\mathrm{X}$ in the case of $C m$ NADH1, $C m$ TCP15 and $C m$ GDSL esterase/lipase) might be controlled by an expression QTL (eQTL) located in LG X that would 
733 contribute to, or be involved in, crosstalk with other QTLs associated with differential textural 734 traits and aroma volatile production in NIL SC10-2 and PS during postharvest ripening. 735 Generally, all the genes identified in this experiment (whether or not located in the LG X) were 736 affected by the introgression in LG X.

\section{ACKNOWLEDGEMENTS}

739

740

741

742

743

744

745

746

747

748

749

750

751

752

753

754

755

756

This work was funded by grants 11784/PI/09 (Seneca Foundation, Region of Murcia) and

Ministry of Economy and Innovation (AGL2010-20858). M. Zarid acknowledges an UEErasmus predoctoral fellowship, a program coordinated by the University of Murcia in the framework of CMN. Thanks are due to Semillas Fitó S.A. (Barcelona, Spain), for providing seeds of PS melons and IRTA-CRAG for the seeds of SC10-2. We acknowledge the assistance of P. Varó and his team in CIFEA-Torre Pacheco for crop management, to N. Dos-Santos, M. Medina, M. García-Gutiérrez, A. Hakmaoui, E. Cuadros, I. Canales and A.A. Escudero (UPCT) for sampling and technical assistance, to SAIT-UPCT for GC-MS analysis, to A.G. Sifres (COMAV) for RNA extraction, and to CNAG (Barcelona) for professional assistance in RNAseq. The authors declare that the research was conducted in the absence of any commercial or financial relationships that could be construed as a potential conflict of interest.

\section{REFERENCES}

1 Ríos P, Argyris J, Vegas J, Leida C, Kenigswald M, Tzuri G, et al., ETHQV6.3 is involved in melon climacteric fruit ripening and is encoded by a NAC domain transcription factor. Plant J 91:671-683 (2017). DOI: 10.1111/tpj.13596

2 Ezura $\mathrm{H}$ and Owino WO, Melon, an alternative model plant for elucidating fruit ripening. Plant Sci 175:121-129 (2008). DOI: 10.1016/j.plantsci.2008.02.004 
7573 Guo X, Xu J, Cui X, Chen $\mathrm{H}$ and Qi H, iTRAQ-based protein profiling and fruit quality 758 changes at different development stages of oriental melon. BMC Plant Biol 17:28 (2017). 759 DOI: $10.1186 / \mathrm{s} 12870-017-0977-7$

7604 Chaparro-Torres LA. Bueso MC and Fernández-Trujillo JP, Aroma volatiles obtained at 761 harvest by HS-SPME/GC-MS and INDEX/MS-E-nose fingerprint discriminate climacteric 762 behavior in melon fruit. J Sci Food Agric 96:2352-2365 (2016). DOI: 10.1002/jsfa.7350

7635 Pech JC, Bouzayen M and Latché A, Climacteric fruit ripening: ethylene-dependent and 764 independent regulation of ripening pathways in melon fruit. Plant Sci 175:114-120 (2008). 765 DOI: $10.1111 /$ tpj.13596

7666 Dahmani-Mardas F, Troadec C, Boualem A, Lévêque S, Alsadon AA, Aldoss AA, et al., 767 Engineering melon plants with improved fruit shelf life using the TILLING approach. PLoS 768 ONE 5:e15776 (2010). DOI: 10.1371/journal.pone.0015776

7697 González M, Xu M, Esteras C, Roig C, Monforte AJ, Troadec C, et al., Towards a TILLING 770 platform for functional genomics in 'Piel de Sapo' melons. BMC Res Notes 4:289 (2011). 771 DOI: $10.1186 / 1756-0500-4-289$.

7728 Yano R, Nonaka S and Ezura H, Melonet-DB, a grand RNA-seq gene expression atlas in 773 melon (Cucumis melo L.). Plant Cell Physiol 59:e4 (2018). DOI: 10.1093/pcp/pcx193

7749 Perpiñá G, Esteras C, Gibon Y, Monforte AJ and Picó B, A new genomic library of melon 775 introgression lines in a cantaloupe genetic background for dissecting desirable agronomical 776 traits. BMC Plant Biol 16:154 (2016). DOI: 10.1186/s12870-016-0842-0

77710 Pitrat, M. Melon genetic resources: phenotypic diversity and horticultural taxonomy. In: 778 Grumet R, Katzir N and Garcia-Mas J (Eds) Genetics and genomics of the Cucurbitaceae. 779 Springer, New York. pp 409-415 (2017). https://dx.doi.org/10.1007/7397_2017_1 
78011 Castro GE, Perpiñá G, Esteras C, Monforte AJ and Picó MB, A new introgression line 781 collection to improve 'Piel de Sapo' melons. Acta Hort 1151:81-86 (2016). DOI: $782 \quad 10.17660 /$ ActaHortic.2017.1151.14

78312 Pereira L, Ruggieri V, Pérez S, Alexiou KG, Fernández M, Jahrmann M, et al., QTL 784 mapping of melon fruit quality traits using a high-density GBS-based genetic map. BMC $785 \quad$ Plant Biol 18:324 (2018). DOI: 10.1186/s12870-018-1537-5

78613 Eduardo I, Arús P and Monforte AJ, Development of a genomic library of near isogenic 787 lines (NILs) in melon (Cucumis melo L.) from the exotic accession PI 161375. Theor Appl $788 \quad$ Genet 112:139-148 (2005). DOI: 10.1007/s00122-005-0116-y

78914 Moreno E, Obando J, Dos-Santos N, Fernández-Trujillo JP. Monforte AJ and Garcia-Mas J, 790 Candidate genes and QTLs for fruit ripening and softening in melon. Theor Appl Genet 791 116:589-602 (2008). DOI:10.1007/s00122-007-0694-y

79215 Galpaz N, Gonda I, Shem-Tov D, Barad O, Tzuri G, Lev S, et al., Deciphering genetic 793 factors that determine melon fruit-quality traits using RNA-seq-based high-resolution QTL $794 \quad$ and eQTL mapping. The Plant J 94:169-191 (2018). DOI: 10.1111/tpj.13838

79516 Saladié M, Cañizares J, Phillips MA, Rodriguez-Concepcion M, Larrigaudière C, Gibon Y, 796 et al., Comparative transcriptional profiling analysis of developing melon (Cucumis melo L.) 797 fruit from climacteric and non-climacteric varieties. BMC Genom 16:440 (2015). DOI: $798 \quad 10.1186 / \mathrm{s} 12864-015-1649-3$

79917 Zhang H, Wang H, Yi H, Zhai W, Wang W and Fu Q, Transcriptome profiling of Cucumis 800 melo fruit development and ripening. Hort Res 3:16014 (2016). DOI: 801 10.1038/hortres.2016.14

80218 Eduardo I, Arús P, Monforte AJ, Monforte AJ, Obando J, Fernández-Trujillo JP, et al., 803 Estimating the genetic architecture of fruit quality traits in melon using a genomic library of 
804 near isogenic lines. $J$ Am Soc Hort Sci 132:80-89 (2007). 805 http://journal.ashspublications.org/content/132/1/80.full

80619 Nimmakayala P, Tomason YR, Abburi VL, Alvarado A, Saminathan T, Vajja VG, et al., 807 Genome-wide differentiation of various melon horticultural groups for use in GWAS for 808 fruit firmness and construction of a high resolution genetic map. Front Plant Sci 7:1437 809 (2016). DOI: 10.3389/fpls.2016.01437

81020 Fernández-Trujillo JP, Fernández-Talavera M, Ruiz-León MT, Roca MJ and Dos-Santos N, 811 Aroma volatiles during whole melon ripening in a climacteric near-isogenic line and its 812 inbred non-climacteric parents. Acta Hort 934:951-958 (2012). DOI: $813 \quad 10.17660 /$ ActaHortic.2012.934.127

81421 Obando-Ulloa JM, Moreno E, García-Mas J, Nicolai B, Lammertyn J, Monforte AJ, et al., 815 Climacteric or non-climacteric behavior in melon fruit 1. Aroma volatiles. Postharvest Biol 816 Technol 49:27-37 (2008). DOI: 10.1016/j.postharvbio.2007.11.004

81722 Obando-Ulloa JM, Nicolai B, Lammertyn J, Bueso MC, Monforte AJ and Fernández818 Trujillo JP, Aroma volatiles associated with the senescence of climacteric or non-climacteric 819 melon fruit. Postharvest Biol Technol 52:146-155 (2009b). DOI: $820 \quad 10.1016 /$ j.postharvbio.2008.11.007

82123 Gonda I, Bar E, Portnoy V, Lev S, Burger J, Schaffer AA, et al., Branched-chain and 822 aromatic amino acid catabolism into aroma volatiles in Cucumis melo L. fruit. J Exp Bot 823 61:1111-1123 (2010). DOI: 10.1093/jxb/erp390

82424 Dos-Santos N, Bueso MC and Fernández-Trujillo JP, Aroma volatiles as biomarkers of 825 textural differences at harvest in non-climacteric near-isogenic lines of melon. Food Res Int 826 54:1801-1812 (2013). DOI: 10.1021/jf201155a. 
82725 Obando J, Fernández-Trujillo JP, Martínez JA, Alarcón AL, Eduardo I, Arús P, et al., 828 Identification of quantitative trait loci of melon fruit quality traits. J Amer Soc Hort Sci 829 133:139-151 (2008). http://journal.ashspublications.org/content/133/1/139.full.pdf + html

83026 Gomes H, Fundo J, Obando-Ulloa JM, Almeida DPF and Fernández-Trujillo JP, The genetic 831 background of quality and cell wall changes in fresh-cut melons. Acta Hort 877:1011-1018 832 (2010). DOI: 10.17660/ActaHortic.2010.877.136

83327 Fernández-Trujillo JP, Lester G, Dos-Santos N, Martínez JA, Esteva J, Jifon JL, et al., Pre834 and postharvest muskmelon fruit cracking: causes and potential remedies. HortTechnol 23:266-275 (2013). http://horttech.ashspublications.org/content/23/3/266.full.pdf + html

28 Dos-Santos N, Jiménez-Araujo A, Rodríguez-Arcos R and Fernández-Trujillo JP, Cell wall polysaccharides of near-isogenic lines of melon and their inbred parentals which show differential flesh firmness and physiological behavior. J Agric Food Chem 59:7773-7784 (2011). DOI: $10.1021 / \mathrm{jf201155a}$

29 Dos-Santos N, Obando J, Ostos I, Melgarejo LM, Moreno E, Monforte AJ, et al., Optimización de la extracción de ácidos orgánicos en pulpa liofilizada de melón y análisis por cromatografía líquida de alta eficiencia. In: Rabasseda J and Martín de la Hinojosa MI (coord.). Congreso IV IBEROLAB, Ministerio de Cultura, Pesca y Alimentación, Madrid, pp.

$$
\text { 203-211 }
$$
http:/www.iberolab.org/opencms/export/sites/Iberolab/comunicaciones/Comunicaciones/d ocumentos_comunicaciones/Requisitos_Tecnicos_0039.pdf

30 Fernández-Trujillo JP, Obando-Ulloa JM, Martínez JA, Moreno E, García-Mas J and Monforte AJ, Climacteric or non-climacteric behavior in melon fruit 2. Linking climacteric pattern and main postharvest disorders and decay in a set of near-isogenic lines. Postharvest Biol Technol 50:125-134 (2008). DOI: 10.1016/j.postharvbio.2008.04.007 
85131 Fernández-Trujillo JP, Zarid M and Bueso MC, Methodology to remove strong outliers of 852 non-climacteric melon fruit aroma at harvest obtained by HS-SPME GC-MS analysis. $853 \quad$ Separations 5:30 (2018). DOI:10.3390/separations5020030

85432 Andrews S, FastQC: a quality control tool for high throughput sequence data. Babraham 855 Bioinf. (2010). Available online at: $\underline{\text { http://www.bioinformatics.babraham.ac.uk/projects/fastqc }}$

33 Kim D, Langmead B and Salzberg SL, HISAT: a fast spliced aligner with low memory 858 requirements. Nat Meth 12:357-360 (2015). DOI: 10.1038/nmeth.3317

34 Pertea M, Pertea GM, Antonescu CM, Chang TC, Mendell JT, and Salzberg SL, StringTie 860 861 862 enables improved reconstruction of a transcriptome from RNA-seq reads. Nat Biotechnol 33:290-295 (2015). DOI: 10.1038/nbt.3122

35 Fu J, Frazee AC, Collado-Torres L, Jaffe AE and Leek JT, Ballgown: Flexible, isoformlevel differential expression analysis. R package version 2.12 .0 (2018).

36 Howe EA, Sinha R, Schlauch D and Quackenbush J, RNA-Seq analysis in MeV. Bioinformat 27:3209-3210 (2011). DOI: 10.1093/bioinformatics/btr490

37 MEV, Multi experiment viewer. TM4 MeV stand-alone client (2018). http://mev.tm4.org/\#/welcome. Accesed 5 october (2018). Download at: https://sourceforge.net/projects/mev-tm4/

86938 Conesa A, Götz S, García-Gómez JM, Terol J, Talón M and Robles M, Blast2GO: a 870 universal tool for annotation, visualization and analysis in functional genomics research. 871 Bioinformat 21:3674-3676 (2005). DOI: 10.1093/bioinformatics/bti610

87239 Paul V, Pandey R and Srivastava GC, The fading distinctions between classical patterns of 873 ripening in climacteric and non-climacteric fruit and the ubiquity of ethylene. An overview. J Food Sci Technol 49:1-21 (2012). DOI: 10.1007/s13197-011-0293-4 
87540 Perotti VE, Moreno AS and Podestá FE, Physiological aspects of fruit ripening: The

876

877

878

879

880

881

882

883

884

885

886

887

888

889

890

891

892

893

894

895

896

897

898 mitochondrial connection. Mitochondrion 17:1-6 (2014). DOI: 10.1016/j.mito.2014.04.010

41 Araújo WL, Nunes-Nesi A, Nikoloski Z, Sweetlove LJ and Fernie AR, Metabolic control and regulation of the tricarboxylic acid cycle in photosynthetic and heterotrophic plant tissues. Plant Cell Environ 35:1-21 (2012). DOI: 10.1111/j.1365-3040.2011.02332.x

42 Melo AMP, Bandeiras TM and Teixeira M, New Insights into Type II NAD(P)H:Quinone Oxidoreductases. Microbiol Mol Biol Rev 68:603-616 (2004). DOI: 10.1128/MMBR.68.4.603-616.2004

43 Moller IM, Plant mitochondria and oxidative stress: Electron Transport, NADPH turnover, and metabolism of reactive oxygen species. Plant Physiol Plant Mol Biol 52:561-591 (2001). DOI: 10.1146/annurev.arplant.52.1.561

44 Yagi T, Di Bernardo S, Nakamuro-Ogiso E, Kao MC, Seo BB and Matsuno-Yagi A, NADH dehydrogenases (NADH:quinone oxidoreductase). In: Zannoni D (ed), Respiration in archaea and bacteria, vol. 1, pp. 15-40 (2004). Kluwer Pub., Dordrecht, The Netherlands.

45 Fatland BL, Ke J, Anderson MD, Mentzen WI, Cui LW, Allred CC, et al., Molecular characterization of a heteromeric ATP-citrate lyase that generates cytosolic acetyl-coenzyme A in Arabidopsis. Plant Physiol 130:740-756 (2002). DOI: 10.1104/pp.008110

46 Sánchez LB, Galperin M and Muller M, Acetyl-CoA synthetase from the amitochondriate eukaryote Giardia lamblia belongs to the newly recognized superfamily of acyl-CoA synthetases (nucleoside diphosphate-forming). J Biol Chem 275:5794-5803 (2000). DOI: $10.1074 / j b c .275 .8 .5794$

47 Fraser ME, James MNG, Bridger WA, and Wolodko WT, A detailed structural description of Escherichia coli succinyl-CoA synthetase1. J Mol Biol 285:1633-1653 (1999). DOI: 
89948 Causse M, Genetic background of flavour: the case of the tomato. In: Bruckner B and Wyllie 900 SG (Eds), Fruit and vegetable flavour: Recent advances and future prospects. 12:229-253 901 (2008). Woodhead Pub. Ltd., Abington, UK.

90249 Nishimura C, Ohashi Y, Sato S, Kato T, Tabata S, and Ueguchi C, Histidine kinase homologs 903 that act as cytokinin receptors possess overlapping functions in the regulation of shoot and 904 root growth in Arabidopsis. Plant Cell 6: 1365-1377 (2004). DOI: 10.1105/tpc.021477

90550 Kieber JJ, Tribute to Folke Skoog: Recent advances in our understanding of cytokinin 906 biology. J Plant Growth Regul 1:1-2 (2002). DOI: 10.1007/s003440010059

90751 Zhang Z, Jiang S, Wang N, Li M, Ji X, Sun S, et al., Identification of differentially expressed 908 genes associated with apple fruit ripening and softening by suppression subtractive 909 hybridization. PLoS ONE 10:e0146061 (2015b). DOI:10.1371/journal.pone.0146061

91052 Girard AL, Mounet F, Lemaire-Chamley M, Gaillard C, Elmorjani K, Vivancos J, et al., 911 Tomato GDSL1 is required for cutin deposition in the fruit cuticle. Plant Cell 24:3119-3134 912 (2012). DOI: $10.1105 /$ tpc. 112.101055

91353 Hayashi S, Ishii T, Matsunaga T, Tominaga R, Kuromori T, Wada T, et al., The 914 glycerophosphoryl diester phosphodiesterase-like proteins SHV3 and its homologs play 915 important roles in cell wall organization. Plant Cell Physiol 49:1522-1535 (2008). DOI: $916 \quad 10.1093 / \mathrm{pcp} / \mathrm{pcn} 120$.

91754 Ge W, Song Y, Zhang C, Zhang Y, Burlingame AL and Guo Y, Proteomic analyses of 918 apoplastic proteins from germinating Arabidopsis thaliana pollen. Biochim Biophys Acta 919 1814:1964-1973 (2011). DOI: 10.1016/j.bbapap.2011.07.013.

92055 Rose JKC, Hadfield KA, Labavitch JM and Bennett AB, Temporal sequence of cell wall 921 disassembly in rapidly ripening melon fruit. Plant Physiol 117:345-361 (1998). DOI: $922 \quad 10.1104 /$ pp.117.2.345 
92356 Daminato M, Guzzo F and Casadoro G, A SHATTERPROOF-like gene controls ripening in 924 non-climacteric strawberries, and auxin and abscisic acid antagonistically affect its $925 \quad$ expression. $J$ Exp Bot 64:3775-3786 (2013). DOI: $10.1093 / \mathrm{jxb} / \mathrm{ert} 214$

$92657 \mathrm{Lu} \mathrm{W}$, Mao L, Chen J, Han X, Ren X, Ying T, et al., Interaction of abscisic acid and auxin 927 on gene expression involved in banana ripening. Acta Physiol Plant 40:46 (2018). DOI: $928 \quad 10.1007 / \mathrm{s} 11738-018-2621-\mathrm{z}$

92958 Barry CS, Llop-Tous MI and Grierson D, The regulation of 1-aminocyclopropane-1930 carboxylic acid synthase gene expression during the transition from system-1 to system-2 931 ethylene synthesis in tomato. Plant Physiol 123:979-986 (2000). DOI: $932 \quad 10.1104 /$ pp.123.3.979

93359 Wang L, Zhang X, Wang L, Tian Y, Jia N, Chen S, et al., Regulation of ethylene-responsive 934 SlWRKYs involved in color change during tomato fruit ripening. Sci Rep 7:16674 (2017). 935 DOI: $10.1038 / \mathrm{s} 41598-017-16851-y$

93660 Zhang C, Hou Y, Hao Q, Chen H, Chen L, Yuan S, et al., Genome-wide survey of the 937 soybean GATA transcription factor gene family and expression analysis under low nitrogen $938 \quad$ stress. PLoS ONE 10:e0125174 (2015a). DOI: 10.1371/journal.pone.0125174

93961 Sharma MK, Kumar R, Solanke AU, Sharma R, Tyagi AK, and Sharma AK, Identification, 940 phylogeny, and transcript profiling of ERF family genes during development and abiotic 941 stress treatments in tomato. Mol Genet Genom 284:455-475 (2010). DOI: 10.1007/s00438$942 \quad 010-0580-1$

94362 Pirrello J, Prasad BC, Zhang W, Chen K, Mila I, Zouine M, et al., Functional analysis and 944 binding affinity of tomato ethylene response factors provide insight on the molecular bases 945 of plant differential responses to ethylene. BMC Plant Biol 12:190 (2011). DOI: $946 \quad 10.1186 / 1471-2229-12-190$ 
94763 Liu M, Diretto G, Pirrello J, Roustan JP, Li Z, Giuliano G, et al., The chimeric repressor 948 version of an Ethylene Response Factor (ERF) family member, S1-ERF.B3, shows 949 contrasting effects on tomato fruit ripening. New Phytol 201:203:206-218 (2014). DOI: $950 \quad 10.1111 /$ nph.12771.

95164 Jeong MJ and Shih MC, Interaction of a GATA factor with cis-acting elements involved in 952 light regulation of nuclear genes encoding chloroplast glyceraldehyde-3-phosphate 953 dehydrogenase in Arabidopsis. Biochem Biophys Res Comm 300:555-562 (2003). DOI: $954 \quad 10.1016 / \mathrm{S} 0006-291 \mathrm{X}(02) 02892-9$

95565 Tarze A, Deniaud A, Le Bras M, Maillier E, Molle D, Larochette N, et al., GAPDH, a novel 956 regulator of the pro-apoptotic mitochondrial membrane permeabilization. Oncogene 957 26:2606-2620 (2007). DOI: 10.1038/sj.onc.1210074. PMID 17072346.

95866 Zala D, Hinckelmann MV, Yu H, Lyra da Cunha MM, Liot G, Cordelières FP, et al., 959 Vesicular glycolysis provides on-board energy for fast axonal transport. Cell 152:479-491 960 (2013). DOI: 10.1016/j.cell.2012.12.029

96167 Yanaka N, Mammalian glycerophosphodiester phosphodiesterases. Biosci Biotechnol 962 Biochem 71:1811-1818 (2007). DOI: 10.1271/bbb.70062

96368 Zhu M, Chen G, Zhou S, Tu Y, Wang Y, Dong T., et al., A new tomato NAC 964 (NAM/ATAF1/2/CUC2) transcription factor, SINAC4, functions as a positive regulator of 965 fruit ripening and carotenoid accumulation. Plant Cell Physiol 55:119-135. DOI: $966 \quad 10.1093 / \mathrm{pcp} / \mathrm{pct} 162$

96769 Jaakola L, Poole M, Jones MO, Kämäräinen-Karppinen T, Koskimäki, JJ, Hohtola A, et al., 968 A SQUAMOSA MADS box gene involved in the regulation of anthocyanin accumulation 969 in bilberry fruits. Plant Physiol 153:1619-1629 (2010). DOI: 10.1104/pp.110.158279

97070 Seymour GB, Ryder CD, Cevik V, Hammond JP, Popovich A, King GJ, et al., A 971 SEPALLATA gene is involved in the development and ripening of strawberry (Fragariax 
972

973

974

975

976

977

978

979

980

981

982

983

984

985

986

987

988

989

990

991

992

993

994

995

996

ananassa Duch.) fruit, a non-climacteric tissue. J Exp Bot 62:1179-1188 (2011). DOI: 10.1093/jxb/erq360

71 Obando-Ulloa JM, Ruiz J, Monforte AJ and Fernández-Trujillo JP, Aroma profile of a collection of near-isogenic lines of melon. Food Chem 118:815-822 (2010). DOI: 10.1016/j.foodchem.2009.05.068

72 Gaude N, Nakamura Y, Scheible WR, Ohta H, and Dormann P, Phospholipase C5 (NPC5) is involved in galactolipid accumulation during phosphate limitation in leaves of Arabidopsis. Plant J 56:28-39 (2008). DOI: 10.1111/j.1365-313X.2008.03582.x

73 Cheng Y, Zhou W, El Sheery NI, Peters C, Li M, Wang X, et al., Characterization of the Arabidopsis glycerophosphodiester phosphodiesterase (GDPD) family reveals a role of the plastid-localized AtGDPD1 in maintaining cellular phosphate homeostasis under phosphate starvation. Plant J 67:746-746 (2011). DOI: 10.1111/j.1365-313X.2011.04538.x

74 Li M, Qin C, Welti R, and Wang X, Double knockouts of phospholipases Dzeta1 and Dzeta2 in Arabidopsis affect root elongation during phosphate-limited growth but do not affect root hair patterning. Plant Physiol 140:761-770. DOI: 10.1104/pp.105.070995

75 Nakamura Y, Koizumi R, Shui G, Shimojima M, Wenk M, Ito T, et al., Arabidopsis lipins mediate eukaryotic pathway of lipid metabolism and cope critically with phosphate starvation. Proc Natl Acad Sci USA 106:20978-20983 (2009). DOI: 10.1073/pnas.0907173106

76 Nakamura Y, Awai K, Masuda T, Yoshioka Y, Tkamiya K and Ohta H, A novel phosphatidylcholine-hydrolyzing phospholipase $\mathrm{C}$ induced by phosphate starvation in Arabidopsis. J Biol Chem 280:7469-7476 (2005). DOI: 10.1074/jbc.M408799200

77 Tang Y, Zhang C, Cao S, Wang X and Qi H, The Effect of CmLOXs on the production of volatile organic compounds in four aroma types of melon (Cucumis melo). PLoS ONE 10:e0143567 (2015). DOI: 10.1371/journal.pone.0143567 
99778 Zhang C, Cao S, Jin Y, Ju L, Chen Q, Xing Q, et al., Melon 13-lipoxygenase CmLOX18 998 may be involved in C6 volatiles biosynthesis in fruit. Sci Rep 7:2816 (2017). DOI: $999 \quad 10.1038 / \mathrm{s} 41598-017-02559-6$

100079 Liavonchanka A and Feussner I, Lipoxygenases: occurrence, functions and catalysis. J Plant 1001 Physiol 163:348-357 (2006). DOI: 10.1016/j.jplph.2005.11.006

100280 Andreou A and Feussner I, Lipoxygenases-structure and reaction mechanism. Phytochem 1003 70:1504-1510 (2009). DOI: 10.1016/j.phytochem.2009.05.008.

100481 Jin Y, Zhang C, Liu W, Tang Y, Qi H, Chen H, et al., The alcohol dehydrogenase gene 1005 family in melon (Cucumis melo L.): Bioinformatic analysis and expression patterns. Front 1006 Plant Sci 7:670 (2016). DOI: 10.3389/fpls.2016.00670

100782 Tijet N, Schneider C, Muller BL and Brash AR, Biogenesis of volatile aldehydes from fatty 1008 acid hydroperoxides: molecular cloning of a hydroperoxide lyase (CYP74C) with specificity 1009 for both the 9-and 13-hydroperoxides of linoleic and linolenic acids. Arch Biochem Biophys 1010 386:281-289 (2001). DOI: 10.1006/abbi.2000.2218

101183 Manríquez D, El-Sharkawy I, Flores FB, El-Yahyaoui F, Regad F, Bouzayen M, et al., Two 1012 highly divergent alcohol dehydrogenases of melon exhibit fruit ripening-specific expression 1013 and distinct biochemical characteristics. Plant Mol Biol 61:675-685 (2006). DOI: $1014 \quad 10.1007 / \mathrm{s} 11103-006-0040-9$

101584 Chen H, Cao S, Jin Y, Tang Y and Qi H, The relationship between CmADHs and the 1016 diversity of volatile organic compounds of three aroma types of melon (Cucumis melo). 1017 Front Physiol 7:254 (2016). DOI: 10.3389/fphys.2016.00254

101885 Moummou H, Tonfack LB, Chervin C, Benichou M, Youmbi E, Ginies C, et al., Functional 1019 characterization of SlscADH1, a fruit-ripening-associated short-chain alcohol 1020 dehydrogenase of tomato. $J$ Plant Physiol 169:1435-1444 (2012). DOI: $1021 \quad 10.1016 / j . j p l p h .2012 .06 .007$ 
102286 Strassner J, Schaller F, Frick UB, Howe GA, Weiler EW, Amrhein N, et al., Characterization 1023 and cDNA-microarray expression analysis of 12-oxophytodienoate reductases reveals 1024 differential roles for octadecanoid biosynthesis in the local versus the systemic wound response. Plant J 32:585-601 (2002). DOI: 10.1046/j.1365-313X.2002.01449.x

1026

1027

1028

1029

1030

1031

1032

1033

1034

1035

1036

1037

1038

1039

1040

1041

1042

1043

1044

1045

1046

87 Sattler SE, Funnell-Harris DL and Pedersen JF, Brown midrib mutations and their importance to the utilization of maize, sorghum, and pearl millet lignocellulosic tissues. Plant Sci 178:229-238 (2010). DOI: 10.1016/j.plantsci.2010.01.001

88 Wasternack C and Hause B, Jasmonates and octadecanoids: signals in plant stress responses and development. Prog Nucleic Acid Res Mol Biol 72:165-221 (2002). DOI: 10.1016/S00796603(02)72070-9

89 Torrigiani P, Fregola F, Ziosi V, Ruiz KB, Kondo S, and Costa G, Differential expression of allene oxide synthase (AOS), and jasmonate relationship with ethylene biosynthesis in seed and mesocarp of developing peach fruit. Postharvest Biol Technol 63:67-73 (2012). DOI: $10.1016 /$ j.postharvbio.2011.08.008

90 Gruer MJ, Artymiuk PJ and Guest JR, The aconitase family: three structural variations on a common theme. Trends Biochem Sci 22:3-6 (1997). DOI: 10.1016/S0968-0004(96)10069-4

91 Han C, Ren C, Zhi T, Zhou Z, Liu Y, Chen F, et al., Disruption of fumarylacetoacetate hydrolase causes spontaneous cell death under short-day conditions in Arabidopsis. Plant Physiol 162:1956-1964 (2013). DOI: 10.1104/pp.113.216804

92 Freilich S, Lev S, Gonda I, Reuveni E, Portnoy V, Oren E, et al., Systems approach for exploring the intricate associations between sweetness, color and aroma in melon fruits. BMC Plant Biol 15:71 (2015). DOI: 10.1186/s12870-015-0449-x

93 Cheng H, Li L, Cheng S, Cao F, Xu F, Yuan H, et al., Molecular cloning and characterization of three genes encoding dihydroflavonol-4-reductase from Ginkgo biloba in anthocyanin biosynthetic pathway. PLoS ONE 8:e72017 (2013). DOI: 10.1371/journal.pone.0072017 

104794 Diallinas $\mathrm{G}$ and Kanellis AK, A phenylalanine ammonia-lyase gene from melon fruit: cDNA 1048 cloning, sequence and expression in response to development and wounding. Plant Mol Biol 1049 26:473-479 (1994). DOI: 10.1007/BF00039557
105095 Aravind L and Koonin EV, Gleaning non-trivial structural, functional and evolutionary 1051 information about proteins by iterative database searches. J Mol Biol 287:1023-1040 (1999). 1052 DOI: $10.1006 /$ jmbi.1999.2653

105396 Chipman DM and Shaanan B, The ACT domain family. Curr Opin Struct Biol 11:694-700 1054 (2001). DOI: 10.1016/S0959-440X(01)00272-X

105597 Aguilar-Martinez JA, Poza-Carrión C and Cubas P, Arabidopsis BRANCHED1 acts as an 1056 integrator of branching signals within axillary buds. Plant Cell 19:458-472 (2007). DOI: 1057 https://doi.org/10.1105/tpc. 106.048934

105898 Hammani K, Gobert A, Hleibieh K, Choulier L, Small I and Giege, P, An Arabidopsis dual1059 localized pentatricopeptide repeat protein interacts with nuclear proteins involved in gene 1060 expression regulation. Plant Cell 23:730-740 (2011). DOI: 10.1105/tpc.110.081638

106199 Giraud E, Ng S, Carrie C, Duncan O, Low J, Lee CP, et al., TCP transcription factors link 1062 the regulation of genes encoding mitochondrial proteins with the circadian clock in 1063 Arabidopsis thaliana. Plant Cell 22:3921-3934 (2010). DOI: 10.1105/tpc.110.074518

1064100 Zhou Y, Liu L, Huang W, Yuan M, Li Z, Zhou F, et al., Overexpression of OsSWEET5 in 1065 rice causes growth retardation and precocious senescence. PLoS ONE 9:e94210 (2014). DOI: $1066 \quad$ 10.1371/journal.pone.0094210

1067101 Guo WJ, Nagy R, Chen HY, Pfrunder S, Yu YC, Santelia D, et al., SWEET17, a facilitative 1068 transporter, mediates fructose transport across the tonoplast of Arabidopsis roots and leaves. 1069 Plant Physiol 164:777-789 (2014). DOI: 10.1104/pp.113.232751.

1070102 Shammai A, Petreikov M, Yeselson Y, Faigenboim A, Moy-Komemi, M, et al., Natural 1071 genetic variation for expression of a SWEET transporter among wild species of Solanum 
1072

1073

1074

1075

1076

1077

1078

1079

1080

1081

1082

1083

1084

1085

1086

1087

1088

1089

1090

1091

1092

1093

1094

1095

1096

lycopersicum (tomato) determines the hexose composition of ripening tomato fruit. Plant $J$ 96:343-357 (2018). DOI: 10.1111/tpj.14035

103 Obando-Ulloa JM, Eduardo I, Monforte AJ and Fernández-Trujillo JP, Identification of QTLs related to sugar and organic acid composition in melon using near-isogenic lines. Scientia Hort 121:425-433 (2009a). DOI: 10.1016/j.scienta.2009.02.023

104 Hancock RD and Viola R, Biosynthesis and catabolism of L-ascorbic acid in plants. Crit Rev Plant Sci 24:167-188 (2005). DOI: 10.1080/07352680591002165

105 Gilbert L, Alhagdow M, Nunes-Nesi A, Quemener B, Guillon F, Bouchet B, et al., GDPD-mannose 3,5-epimerase (GME) plays a key role at the intersection of ascorbate and noncellulosic cell-wall biosynthesis in tomato. Plant J 60:499-508 (2009). DOI: 10.1111/j.1365313X.2009.03972.x.

106 Reiter WD and Vauzin GF, Molecular genetics of nucleotide sugar interconversion pathways in plants. Plant Mol Biol 47:95-113 (2001). DOI: 10.1023/A:1010671129803

107 Pilati S, Perazzolli M, Malossini A, Cestaro A, Demattè L, Fontana P, et al., Genome-wide transcriptional analysis of grapevine berry ripening reveals a set of genes similarly modulated during three seasons and the occurrence of an oxidative burst at veraison. $B M C$ Genom 8:428 (2007). DOI: 10.1186/1471-2164-8-428

108 Alexander L and Grierson D, Ethylene biosynthesis and action in tomato: a model for climacteric fruit ripening. J Exp Bot 53:2039-2055 (2002). DOI: 10.1093/jxb/erf072

109 Argyris JM, Pujol M, Martin-Hernandez AM and Garcia-Mas J, Combined use of genetic and genomics resources to understand virus resistance and fruit quality traits in melon. Physiol Plant 155:4-11 (2015a). DOI: 10.1111/ppl.12323

110 Argyris JM, Ruiz-Herrera A, Madriz-Masis P, Sansaverino W, Morata J, Pujol M, et al., Use of targeted SNP selection for an improved anchoring of the melon (Cucumis melo L.) scaffold genome assembly. BMC Genom 16:4 (2015b). DOI: 10.1186/s12864-014-1196-3 
1097111 Perpiñá G, Cebolla-Cornejo J, Esteras C, Monforte AJ and Picó B, ‘MAK-10’: A long 1098 shelf-life Charentais breeding line developed by introgression of a genomic region from 1099 Makuwa melon. HortSci 52:1633-1638 (2017). DOI: 10.21273/HORTSCI12068-17

1100112 Monforte AJ, Garcia-Mas J and Arús P, Cucumis melo L. intraspecific classification based $1101 \quad$ on microsatellite variation. Plant Breed 122:153-157 (2003). 


\section{Captions for Figures}

1104 Fig. 1 - Respiration rate and ethylene production of individual fruit, the near-isogenic line 1105 SC10-2 and its parental line 'Piel de Sapo' (PS), harvested in firm-ripe stage of maturity during $110618 \mathrm{~d}$ of postharvest ripening at $20.5{ }^{\circ} \mathrm{C}$ and $88 \%$ relative humidity (mean $\pm \mathrm{SE}, \mathrm{n}=6$ ).

Fig. 2 - Textural traits of melon fruit, the near-isogenic line SC10-2 and its parental line 'Piel de Sapo' (PS) harvested in firm-ripe stage of maturity during $18 \mathrm{~d}$ of ripening at $20.5{ }^{\circ} \mathrm{C}$ and $88 \%$ relative humidity (mean $\pm \mathrm{SE}, \mathrm{n}=3$ ). A. Flesh firmness $\mathrm{B}$. Whole fruit hardness C. Flesh 1111 juiciness.

Fig. 3 - Main compound classes identified by static headspace solid-phase micro-extraction and gas chromatography-mass spectrometry (HS-SPME/GC-MS) in melon fruit of the nearisogenic line (NIL) SC10-2 and its parental line 'Piel de Sapo' (PS) harvested in firm-ripe stage of maturity and subjected to storage at $20.5{ }^{\circ} \mathrm{C}$ and $88 \%$ relative humidity for $18 \mathrm{~d}$. Data are expressed as percentage of each compound class with match quality above 50 with respect to the sum of areas of the identified compounds without considering exogenous ones (mean $\pm \mathrm{SE}$, $\mathrm{n}=3$ ). LSD were calculated after a two-way ANOVA with introgression and ripening time as 1120 factors.

Fig. 4 - Main compound classes identified by the static headspace solid-phase micro-extraction and gas chromatography-mass spectrometry (HS-SPME/GC-MS) technique in melon fruits of 1124 the near-isogenic line (NIL) SC10-2 and its parental line 'Piel de Sapo' (PS) (n= 3) subjected 1125 to storage at $20.5{ }^{\circ} \mathrm{C}$ and $88 \%$ relative humidity for $18 \mathrm{~d}$. The mean concentration was expressed 1126 in $\mathrm{ng} \mathrm{g}^{-1}$ equivalent of the internal standard (1-phenylethanol) on a fresh weight basis. 


\begin{abstract}
1127 (transformed into $\log 2$ ). Means were statistically significant according to two-way ANOVA 1128 with introgression and ripening time as factors.
\end{abstract}

1129 1133 for $18 \mathrm{~d}$.

Fig. 5 - Gene Ontology (GO) of differentially expressed genes only considering the ripening time factor in melon fruit of the near-isogenic line NIL SC10-2 and its parental 'Piel de Sapo' (PS) harvested in firm-ripe stage of maturity and stored at $20.5{ }^{\circ} \mathrm{C}$ and $88 \%$ relative humidity

Fig. 6 - Gene Ontology (GO) of differentially expressed genes only considering the introgression factor in melon fruit of the near-isogenic line NIL SC10-2 and its parental 'Piel de Sapo' (PS) harvested in firm-ripe stage of maturity and stored at $20.5{ }^{\circ} \mathrm{C}$ and $88 \%$ relative humidity for $18 \mathrm{~d}$.

1139 during fruit ripening $\left(0,4,8,12\right.$ and $18 \mathrm{~d}$ at $20.5{ }^{\circ} \mathrm{C}$ and $88 \%$ relative humidity).

Fig. 8 - Cluster analysis of the differentially expressed genes significant in the comparison 1146 between lines over time in fruit of the melon near-isogenic line SC10-2 and its parental control 1147 'Piel de Sapo' (PS). Differentially expressed genes were categorized into seven clusters 1148 depending on their expression during fruit ripening $\left(0,4,8,12\right.$ and $18 \mathrm{~d}$ at $20.5{ }^{\circ} \mathrm{C}$ and $88 \%$ 1149 relative humidity). 
1151 Fig. 9 - Relative expression of selective genes only considering the ripening time with 1152 differential expression involved in primary metabolism or ascorbic acid metabolism in fruit of 1153 the melon near-isogenic line SC10-2 and its parental control PS harvested in firm-ripe stage of 1154 maturity and stored during $18 \mathrm{~d}$ of ripening at $20.5^{\circ} \mathrm{C}$ and $88 \%$ relative humidity. $\mathrm{CmSDHA}$ : 1155 Succinate dehydrogenase [ubiquinone] flavoprotein subunit, mitochondrial/ CmNADP: 1156 isocitrate dehydrogenase [NADP]/ CmL-GalDH: L-galactose dehydrogenase/ CmGME: GDP1157 mannose-3',5'-epimerase/ CmACLA-1: ATP-citrate synthase alpha chain protein/ CmACLB-2: 1158 ATP-citrate synthase beta chain protein 2-like/ CmSCOA: Succinate--CoA ligase [ADP1159 forming] subunit alpha, mitochondrial/ CmIIL1: 3-isopropylmalate dehydratase large subunit/ $1160 C m$ NADH1: NADPH:quinone oxidoreductase-like.

Fig. 10 - Relative expression of selective genes only considering the ripening time involved in quality and physiological process in fruit of the melon near-isogenic line SC10-2 and its parental control PS harvested in firm-ripe stage of maturity and stored during $18 \mathrm{~d}$ of ripening at $20.5^{\circ} \mathrm{C}$ and $88 \%$ relative humidity. $C m L O X 18$ : Fruit ripening/ $C m \mathrm{ACO} 2$ : Ethylene biosynthesis/ CmADH1 and CmCAD1: Phenylpropanoid biosynthesis/ CmAOS and CmOPR2: Oxylipin biosynthesis/ $C m$ SWEET7: Sugar transporter activity/ CmDFR4: Flavonoid biosynthesis/ CmNADH1: Oxidoreductase activity/ CmPAL: Phenylpropanoid metabolism/ CmWRKY33, CmGATA5, CmTCP15, CmNAC18: Transcription factor activity/ CmERF027: Ethylene 1170 transcription factor activity/ CmGDSL: Esterase and lipase activity/ CmGDE4: Glycerol 1171 metabolism/ CmTAC: Amino acid activity/ CmIPMI3: 3-isopropylmalate dehydratase small 1172 subunit 3-like/ CmALT-2: alanine aminotransferase.

1174 Fig. 11 - Relative expression of selective genes only mainly considering the comparison 1175 between lines over time and involved in different process in fruit of the melon near-isogenic 
1176 line SC10-2 and its parental control PS harvested in firm-ripe stage of maturity and stored 1177 during $18 \mathrm{~d}$ of ripening at $20.5 \mathrm{oC}$ and $88 \%$ relative humidity. $C m$ NAC18: Transcription factor 1178 activity/ CmWRKY33: Transcription factor activity/ CmCAD1: Phenylpropanoid biosynthesis/ 1179 CmMADS-box: MADS-box transcription factor/ CmACLA-1: ATP-citrate synthase alpha 1180 chain protein/ CmIIL1: 3-isopropylmalate dehydratase large subunit/ CmFAH: 1181 fumarylacetoacetase/ $\mathrm{CmACO}$ : Ethylene biosynthesis/ CmSDHA: Succinate dehydrogenase 1182 [ubiquinone] flavoprotein subunit, mitochondrial/ CmIPMI3: 3-isopropylmalate dehydratase 1183 small subunit 3-like/ CmSCOA: Succinate--CoA ligase [ADP-forming] subunit alpha, 1184 mitochondrial/ CmL-GalDH: L-galactose dehydrogenase/ CmLOX18: Fruit ripening/ 1185 CmNADP: isocitrate dehydrogenase [NADP]/ CmACLB-2: ATP-citrate synthase beta chain 1186 protein 2-like. CmTrpD: tryptophan biosynthetic process, transferase activity, transferring 1187 glycosyl groups/ CmHK4-like: cytokinin receptor activity, two-component response regulator 1188 activity, two-component sensor activity.

Fig. 12 - Relative expression of selective genes only considering the postharvest ripening time factor and involved in different process in fruit of the melon near-isogenic line SC10-2 and its parental control PS harvested in firm-ripe stage of maturity and stored during $18 \mathrm{~d}$ of ripening 1193 at $20.5^{\circ} \mathrm{C}$ and $88 \%$ relative humidity. CmDHL92: Polygalacturonase At1g48100/ CmMPC1: Mitochondrial pyruvate carrier/ CmDTX: Protein detoxificatoin/ CmABREX1: Abscisic acidinsensitive 5-like protein 4 isoform X1/ CmIAA71: auxin-responsive protein SAUR71-like.

Fig. 13. Heatmap and hierarchical clustering (using Euclidean distance as the similarity 1198 measure and Ward's linkage) applied to thirty-seven differentially expressed genes (Table 1) 1199 and the quality traits measured (textural traits and VOCs) over postharvest ripening time 1200 (columns represent the samples, and rows the variables). Original data were scaled and log2- 
1201 transformed. The eight clusters (C) found were numbered from one to eight, and the subcluster 1202 group's $(G)$ outlined were numbered also from one to eight. 
1204 Table 1. Differentially expressed genes (thirty-seven) transcripts from RNA-seq of the near-isogenic line (NIL) SC10-2 fruit $(n=3)$ of melon and its parental control 'Piel de Sapo' (PS) during 18 d postharvest ripening at $20.5{ }^{\circ} \mathrm{C}$ and $88 \%$ relative humidity. Effect of postharvest ripening time (RT) or the introgression (I) alone or combined with RT. All the information comes from Melonomics version 4.0 www.melonomics.net, including the chromosome (LG) location except when unavailable (LG 0 ).

\begin{tabular}{|c|c|c|c|c|c|c|c|}
\hline Gene & Transcript name & LG & $\begin{array}{c}\text { Locus } \\
\text { start }\end{array}$ & $\begin{array}{c}\text { Locus } \\
\text { end }\end{array}$ & Gene description & Code & Factor \\
\hline MELO3C024348 & MELO3C024348.2.1 & 1 & 36244644 & 36249009 & Lipoxygenase & CmLOX18 & $\mathrm{RT} / \mathrm{I}$ \\
\hline MELO3C019735 & MELO3C019735.2.1 & 11 & 23244951 & 23246562 & $\begin{array}{l}\text { 1-aminocyclopropane-1-carboxylate } \\
\text { oxidase }\end{array}$ & $\mathrm{CmACO} 2$ & RT/ I \\
\hline MELO3C019548 & MELO3C019548.2.1 & 6 & 12928441 & 12930407 & Cinnamyl alcohol dehydrogenase & $\mathrm{CmADH} 1$ & RT \\
\hline MELO3C018492 & MELO3C018492.2.1 & 1 & 614743 & 617080 & Cinnamyl alcohol dehydrogenase & $C m \mathrm{CAD} 1$ & $\mathrm{RT} / \mathrm{I}$ \\
\hline MELO3C010910 & MELO3C010910.2.1 & 3 & 30073454 & 30075374 & Allene oxide synthase & $C m$ AOS & RT \\
\hline MELO3C008075 & MELO3C008075.2.1 & 3 & 159591 & 162626 & $\begin{array}{l}\text { 12-oxophytodienoate reductase-like } \\
\text { protein }\end{array}$ & $\mathrm{Cm} \mathrm{OPR} 2$ & RT \\
\hline MELO3C020508 & MELO3C020508.2.1 & 12 & 399308 & 402564 & $\begin{array}{l}\text { Cinnamoyl-CoA reductase 1/ } \\
\text { Bifunctional dihydroflavonol 4- } \\
\text { reductase }\end{array}$ & CmDFR4 & RT \\
\hline MELO3C012476 & MELO3C012476.2.1 & 10 & 214914 & 216424 & NADPH:quinone oxidoreductase-like & $C m$ NADH1 & $\mathrm{RT}$ \\
\hline MELO3C005630 & MELO3C005630.2.1 & 9 & 23110939 & 23111708 & $\begin{array}{l}\text { Ethylene-responsive transcription } \\
\text { factor ERF027 }\end{array}$ & $\mathrm{CmERF027}$ & RT \\
\hline MELO3C012086 & MELO3C012086.2.1 & 10 & 2703625 & 2705396 & Transcription factor TCP15 & $\mathrm{Cm} \mathrm{TCP} 15$ & RT \\
\hline MELO3C011939 & MELO3C011939.2.1 & 10 & 3788375 & 792148 & GDSL esterase/lipase At3g26430 & $\begin{array}{l}\text { CmGDSL } \\
\text { esterase/ } \\
\text { lipase }\end{array}$ & RT \\
\hline MELO3C013101 & MELO3C013101.2.1 & 1 & 12324577 & 12332243 & $\begin{array}{l}\text { Glycerophosphodiester } \\
\text { phosphodiesterase GDPDL4-like }\end{array}$ & CmGDPDL4 & RT \\
\hline MELO3C016540 & MELO3C016540.2.1 & 6 & 27663292 & 27665351 & NAC domain protein & $C m$ NAC18 & $\mathrm{RT} / \mathrm{I}$ \\
\hline MELO3C005591 & MELO3C005591.2.1 & 9 & 22744057 & 22747399 & ACT domain-containing family protein & $C m \mathrm{ACT}$ & $\mathrm{RT}$ \\
\hline MELO3C017560 & MELO3C017560.2.1 & 7 & 25124825 & 25133417 & $\begin{array}{l}\text { Succinate dehydrogenase flavoprotein } \\
\text { subunit, mitochondrial }\end{array}$ & $\mathrm{CmSDHA}$ & $\mathrm{RT} / \mathrm{I}$ \\
\hline MELO3C005518 & MELO3C005518.2.1 & 9 & 22123330 & 22124520 & $\begin{array}{l}\text { 3-isopropylmalate dehydratase small } \\
\text { subunit 3-like }\end{array}$ & CmIPMI3 & $\mathrm{RT} / \mathrm{I}$ \\
\hline MELO3C003344 & MELO3C003344.2.1 & 4 & 456132 & 464549 & $\begin{array}{l}\text { 3-isopropylmalate dehydratase large } \\
\text { subunit }\end{array}$ & CmIIL1 & $\mathrm{RT} / \mathrm{I}$ \\
\hline MELO3C007433 & MELO3C007433.2.1 & 8 & 2763982 & 2767562 & Fumarylacetoacetase & $\mathrm{CmFAH}$ & I \\
\hline MELO3C009970 & MELO3C009970.2.1 & 4 & 27614894 & 27618780 & Polygalacturonase At1g48100 & CmDHL92 & $\mathrm{RT}$ \\
\hline MELO3C007517 & MELO3C007517.2.1 & 8 & 3320368 & 3322511 & Mitochondrial pyruvate carrier & $\mathrm{CmMPC1}$ & RT \\
\hline MELO3C017343 & MELO3C017343.2.1 & 2 & 24148159 & 24152142 & Protein DETOXIFICATION & $C m$ DTX & RT \\
\hline MELO3C014384 & MELO3C014384.2.1 & 5 & 2954394 & 2961074 & $\begin{array}{l}\text { ABSCISIC ACID-INSENSITIVE 5- } \\
\text { like protein } 4 \text { isoform X1 }\end{array}$ & $C m$ ABREX & $\mathrm{RT}$ \\
\hline MELO3C000885 & MELO3C000885.2.1 & 0 & 20311284 & 20311754 & auxin-responsive protein SAUR71-like & CmIAA71 & RT \\
\hline MELO3C024001 & MELO3C024001.2.1 & 4 & 23817884 & 23822674 & MADS-box transcription factor & $\begin{array}{l}C m \text { MADS- } \\
\text { box }\end{array}$ & I \\
\hline MELO3C010686 & MELO3C010686.2.1 & 3 & 31468671 & 31472617 & Alanine aminotransferase 2 & CmALT-2 & I \\
\hline MELO3C011963 & MELO3C011963.2.1 & 10 & 3557598 & 3564518 & Anthranilate phosphoribosyltransferase & $C m \operatorname{TrpD}$ & $\mathrm{RT}$ \\
\hline MELO3C020055 & MELO3C020055.2.1 & 10 & 10840410 & 10848396 & Histidine kinase 4-like & CmHK4-like & $\mathrm{RT} / \mathrm{I}$ \\
\hline
\end{tabular}


2
3
4
5
6
7
8
9

10

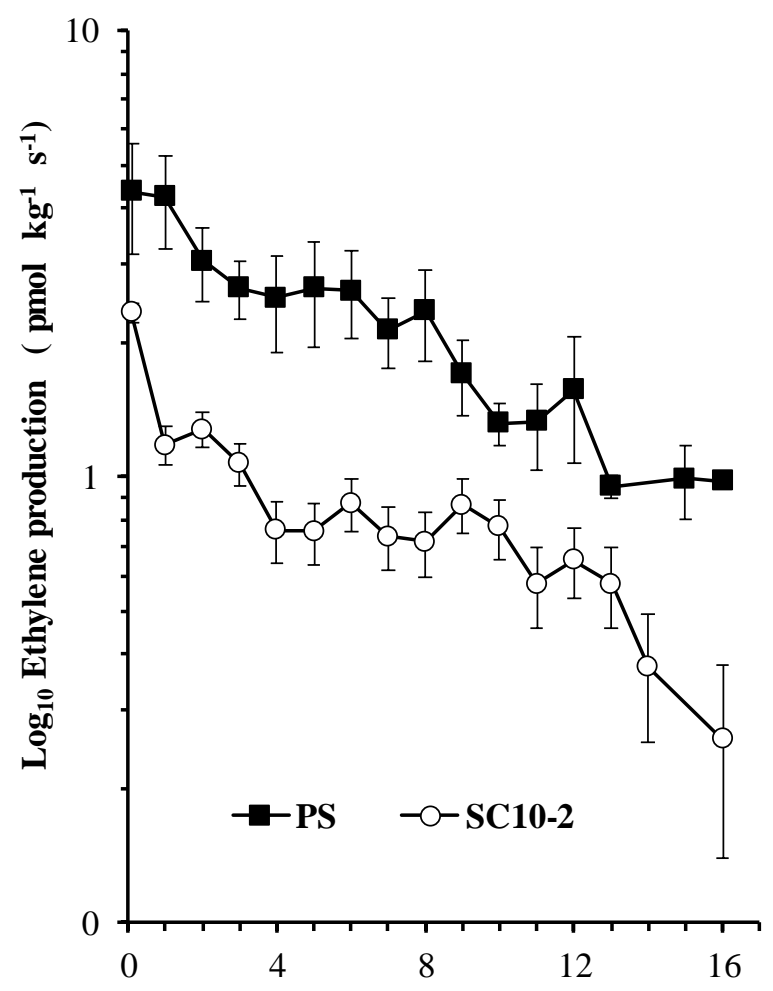

Time (days)

Fig. 1 


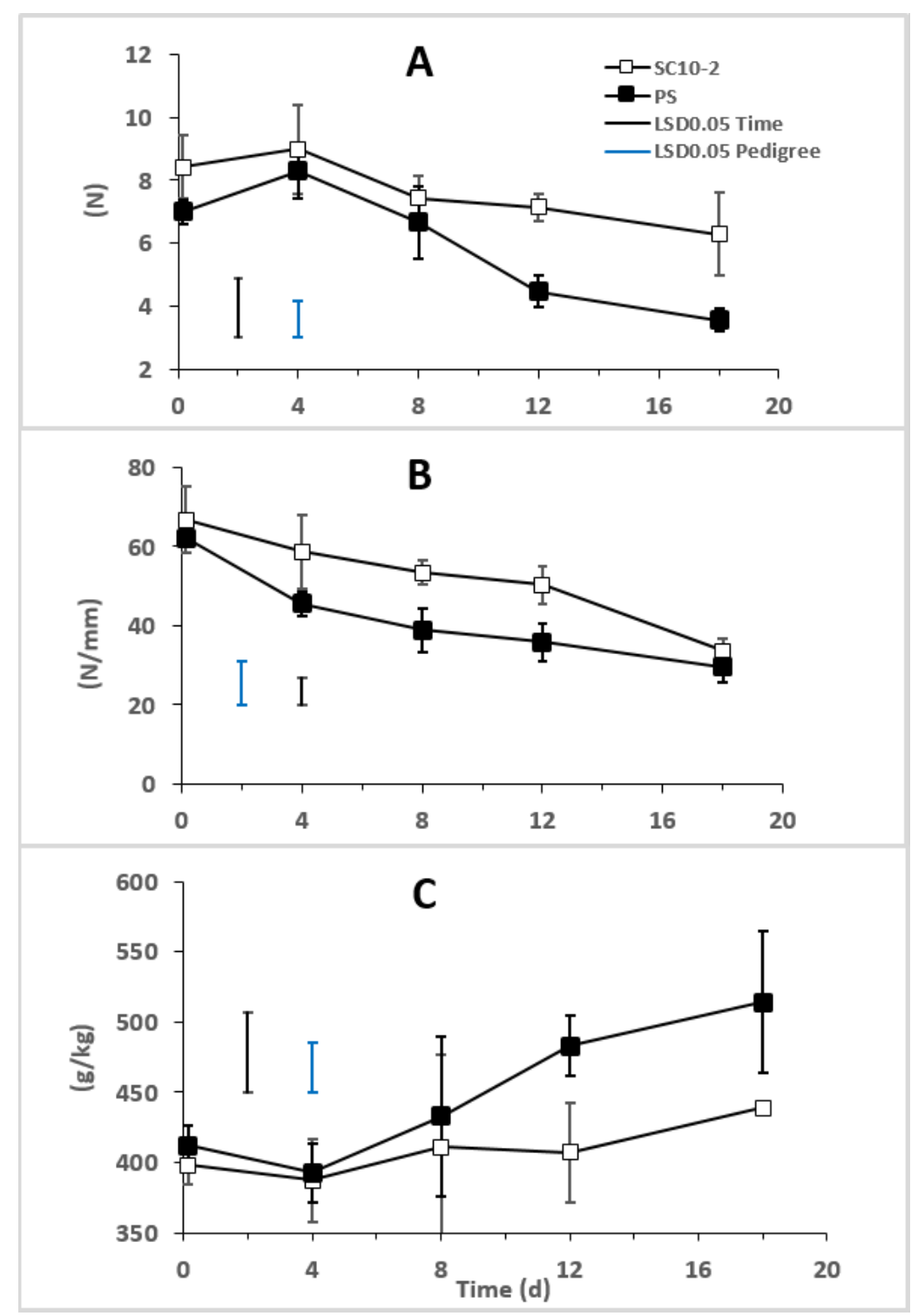

Figure 2 
Acetate esters
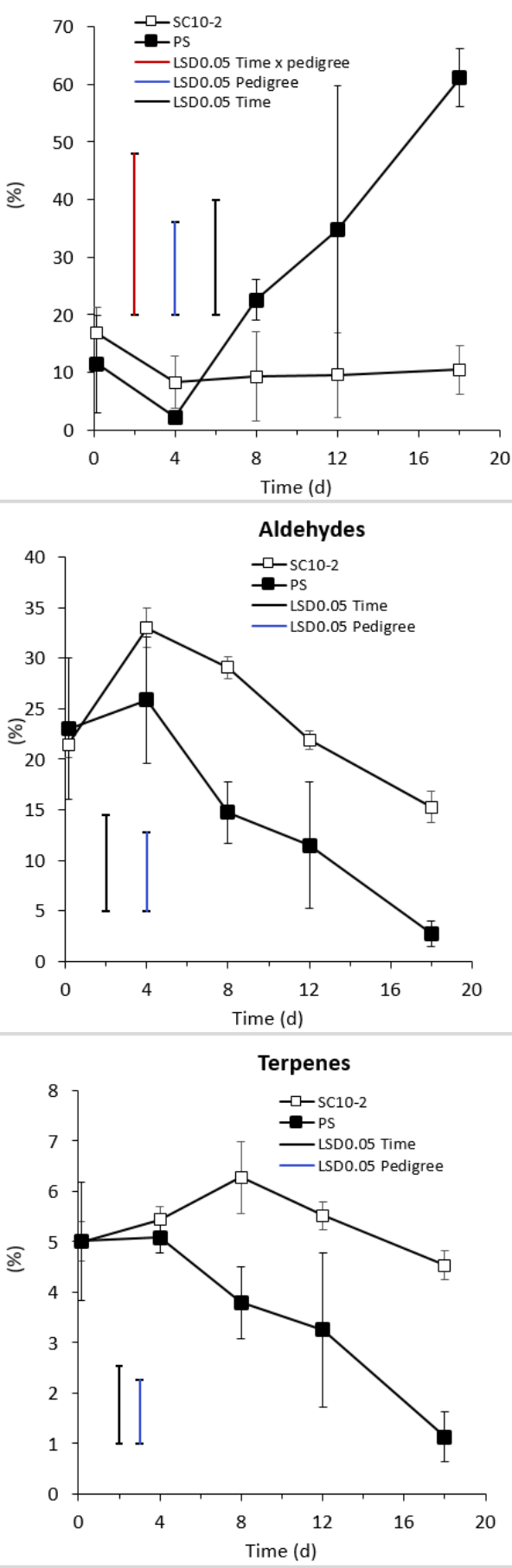

\section{Non-acetate esters}

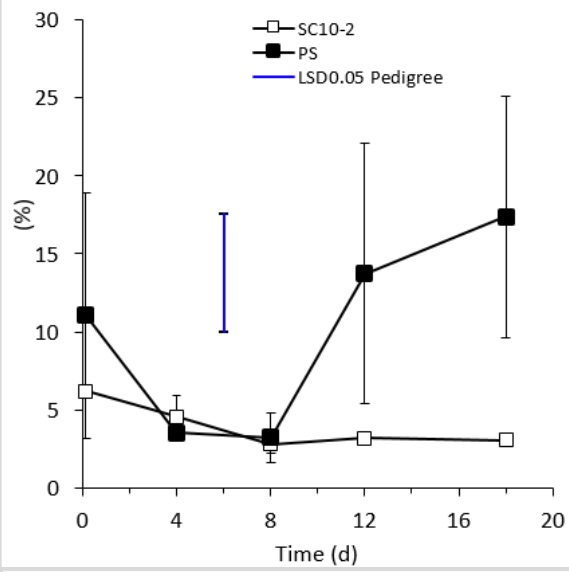

Alcohols
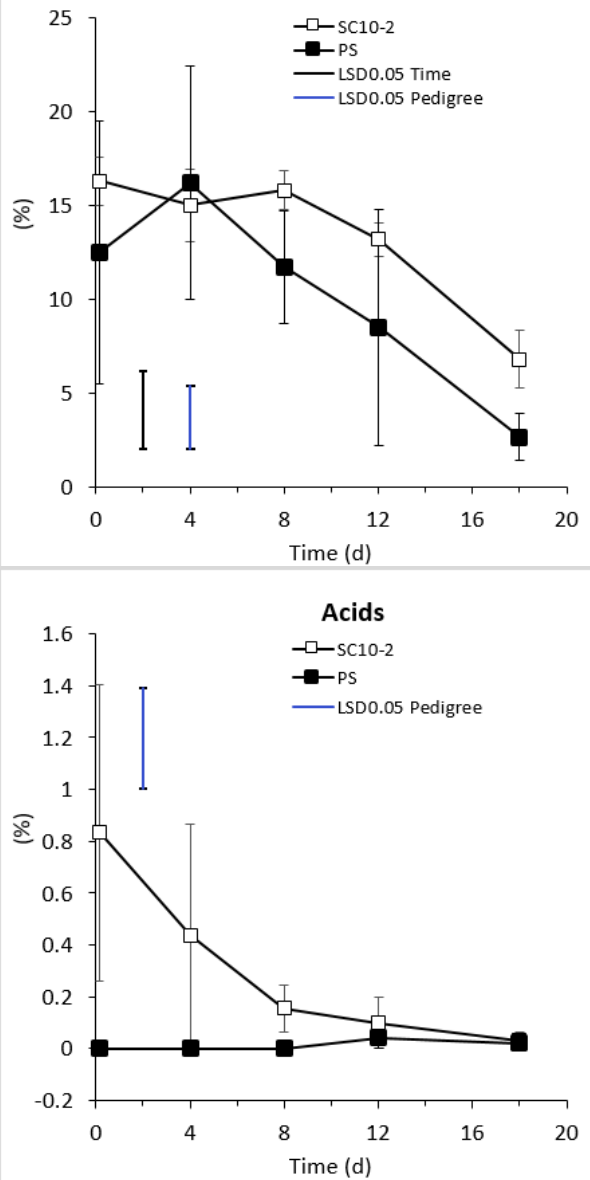

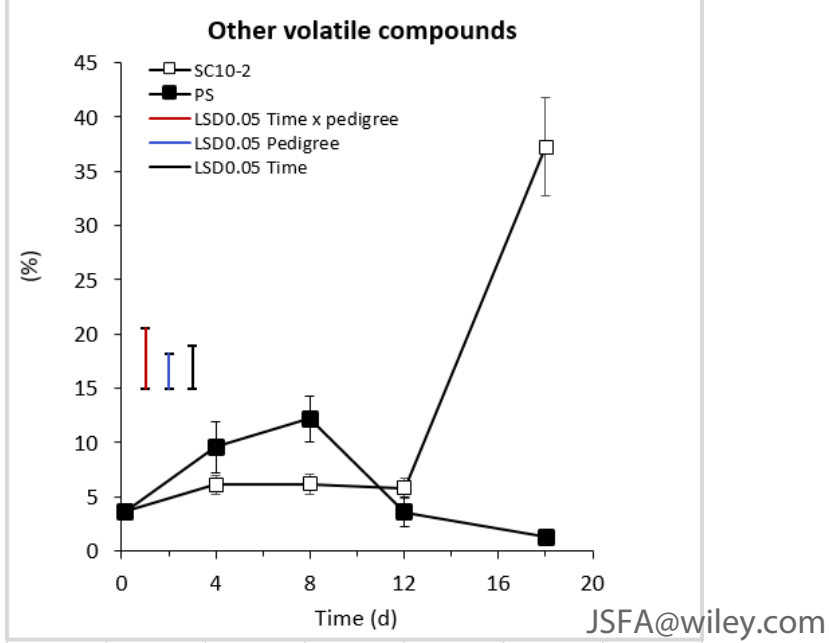

Fig. 3 

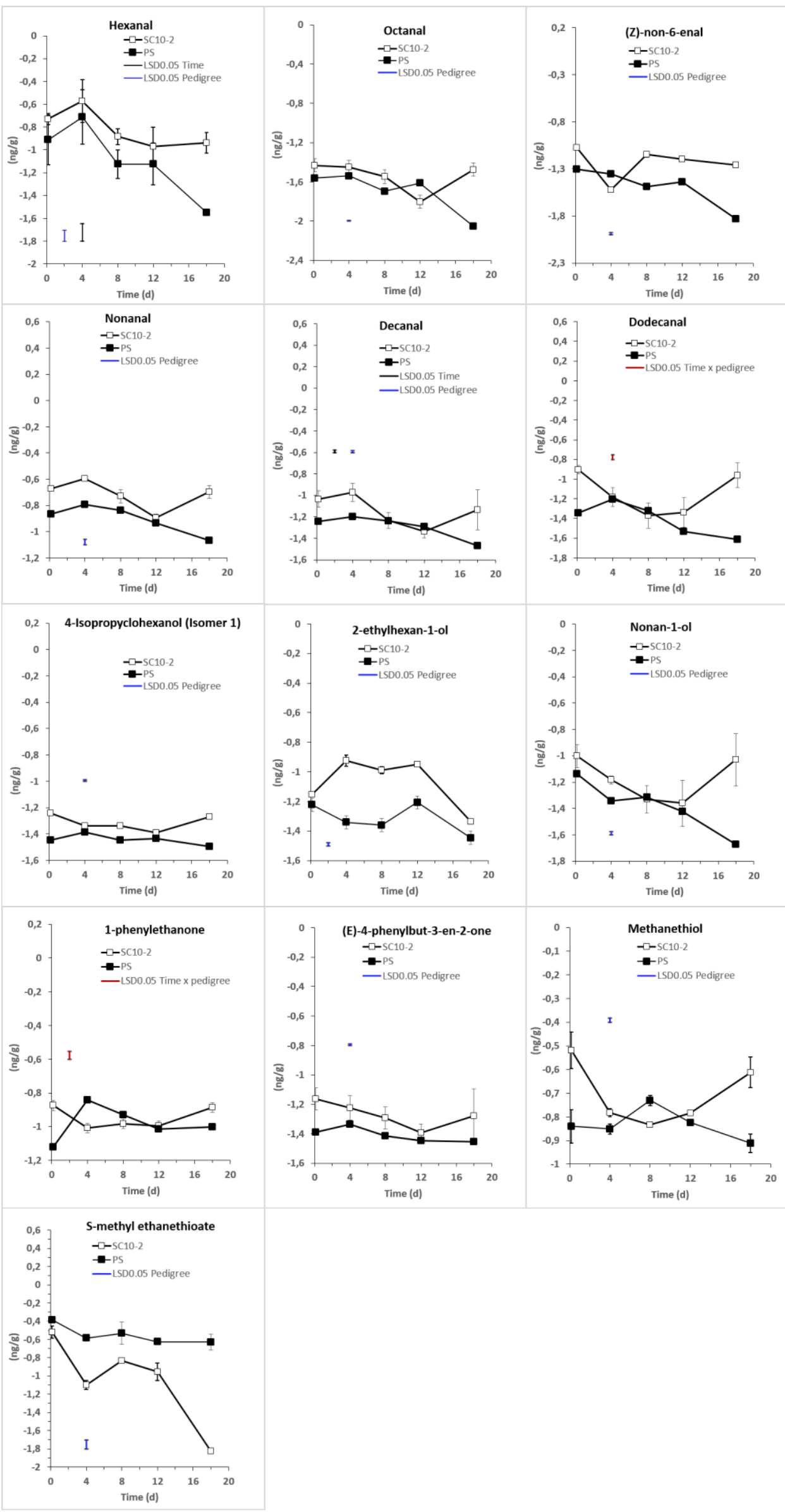


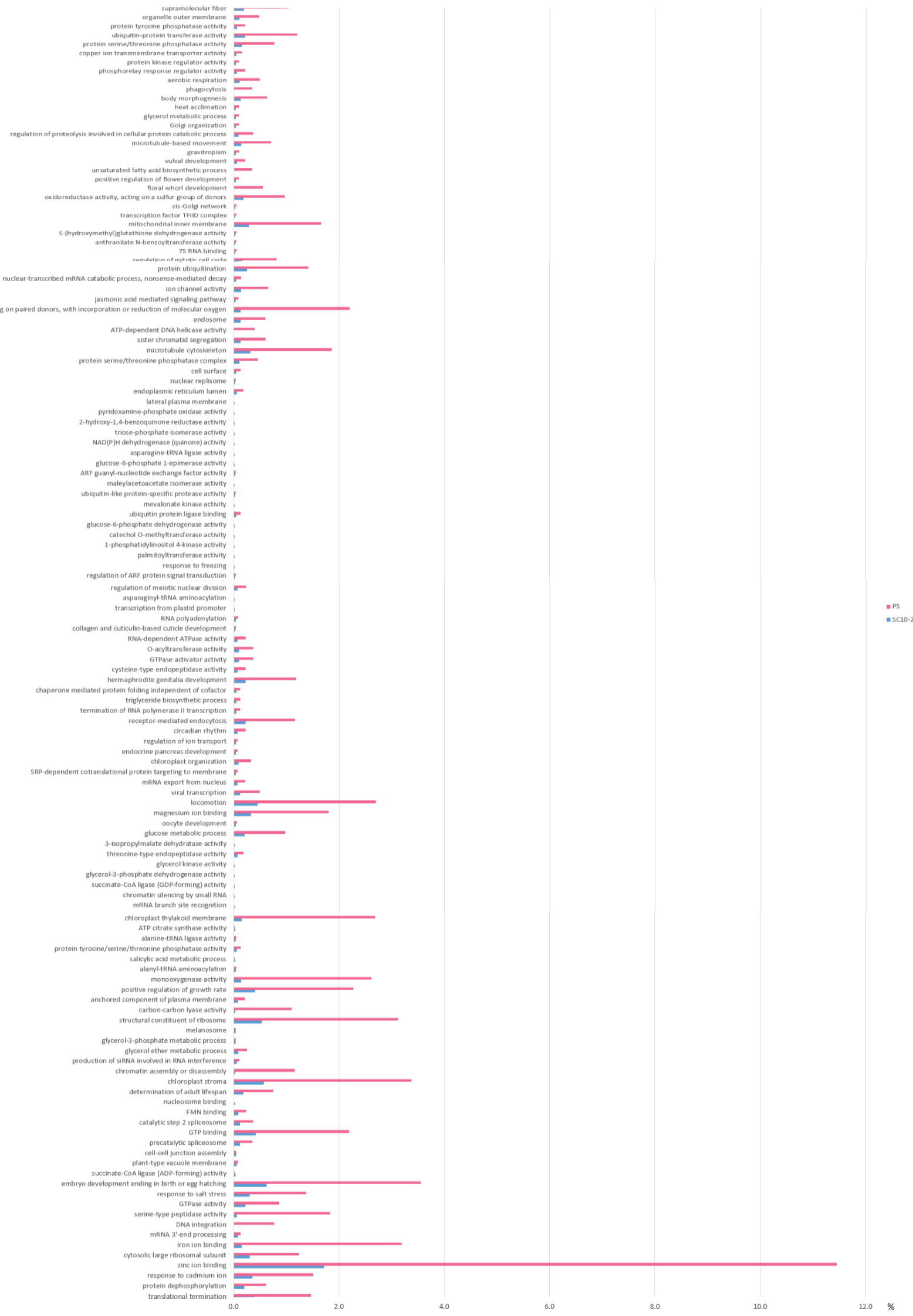


Differential GO-term Distribution

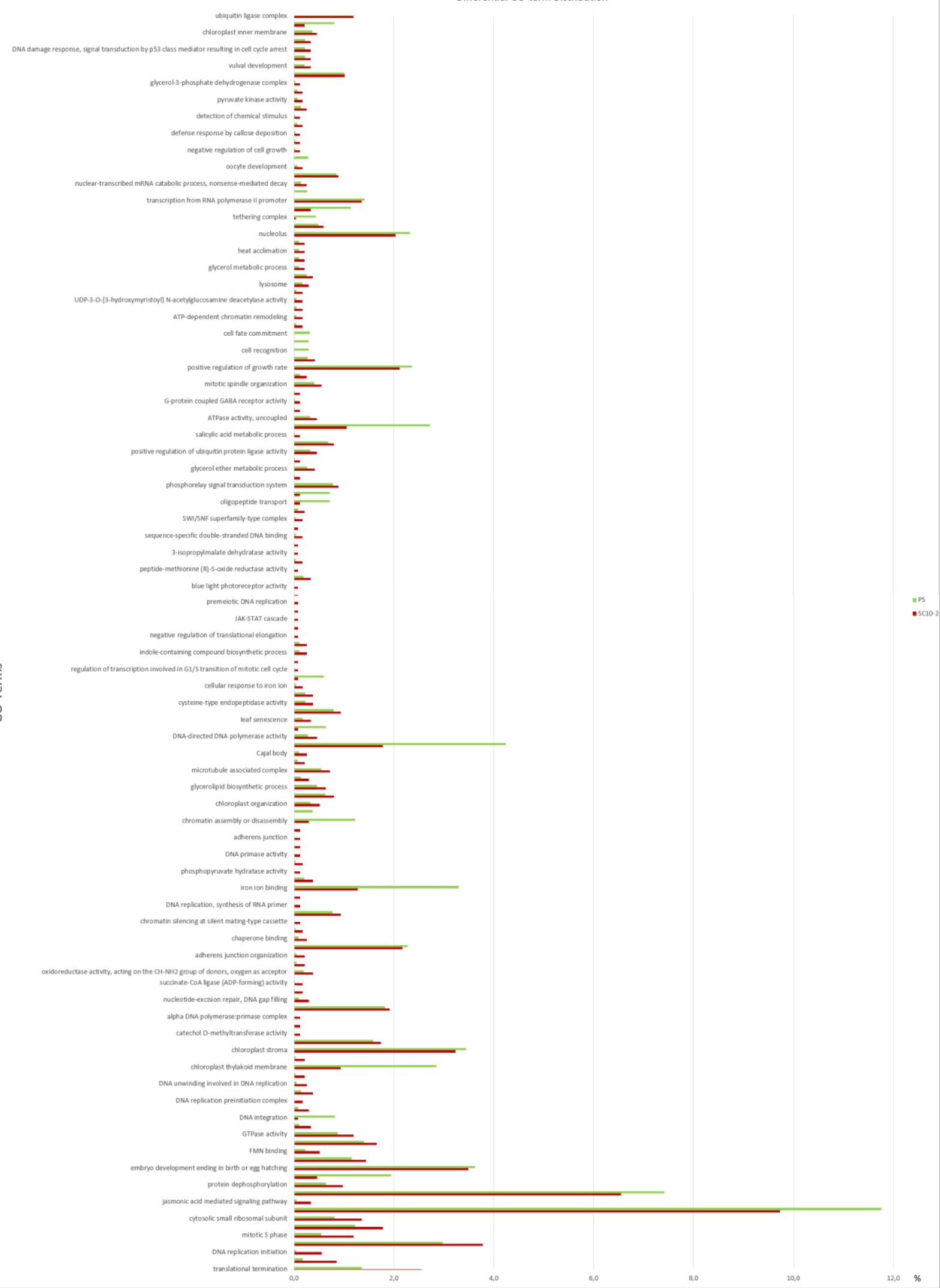



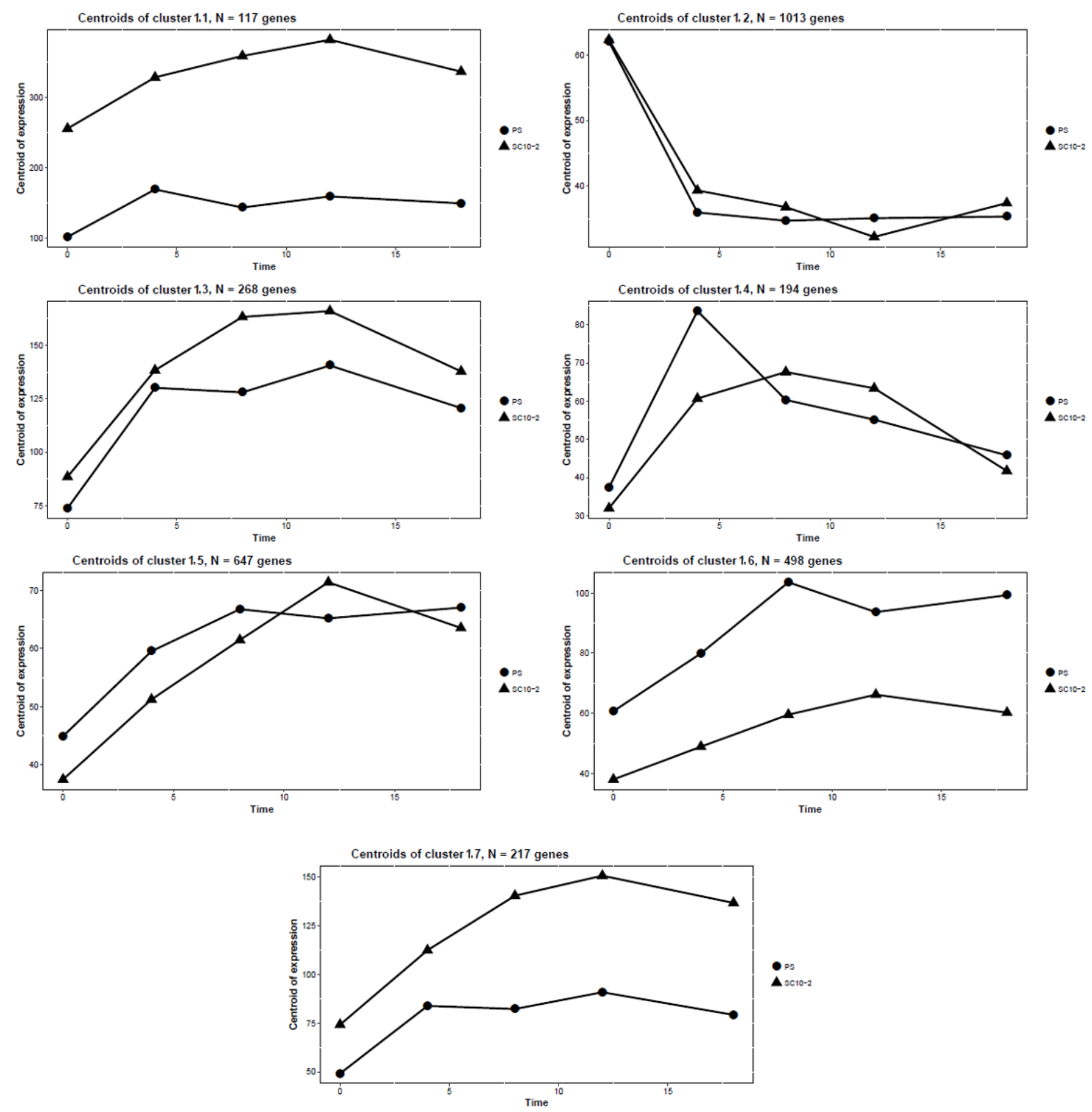

Fig. 7 

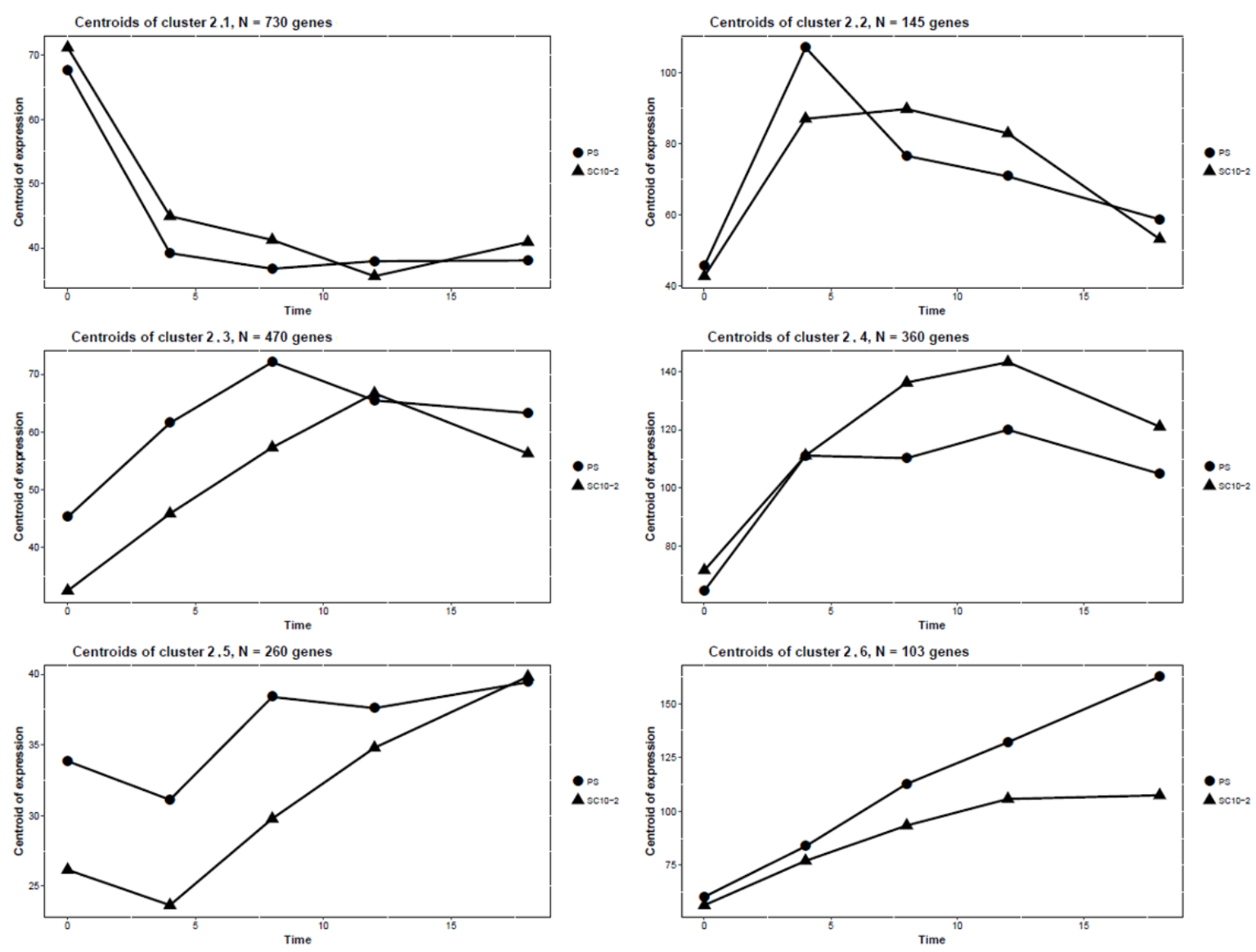

Figure 8 

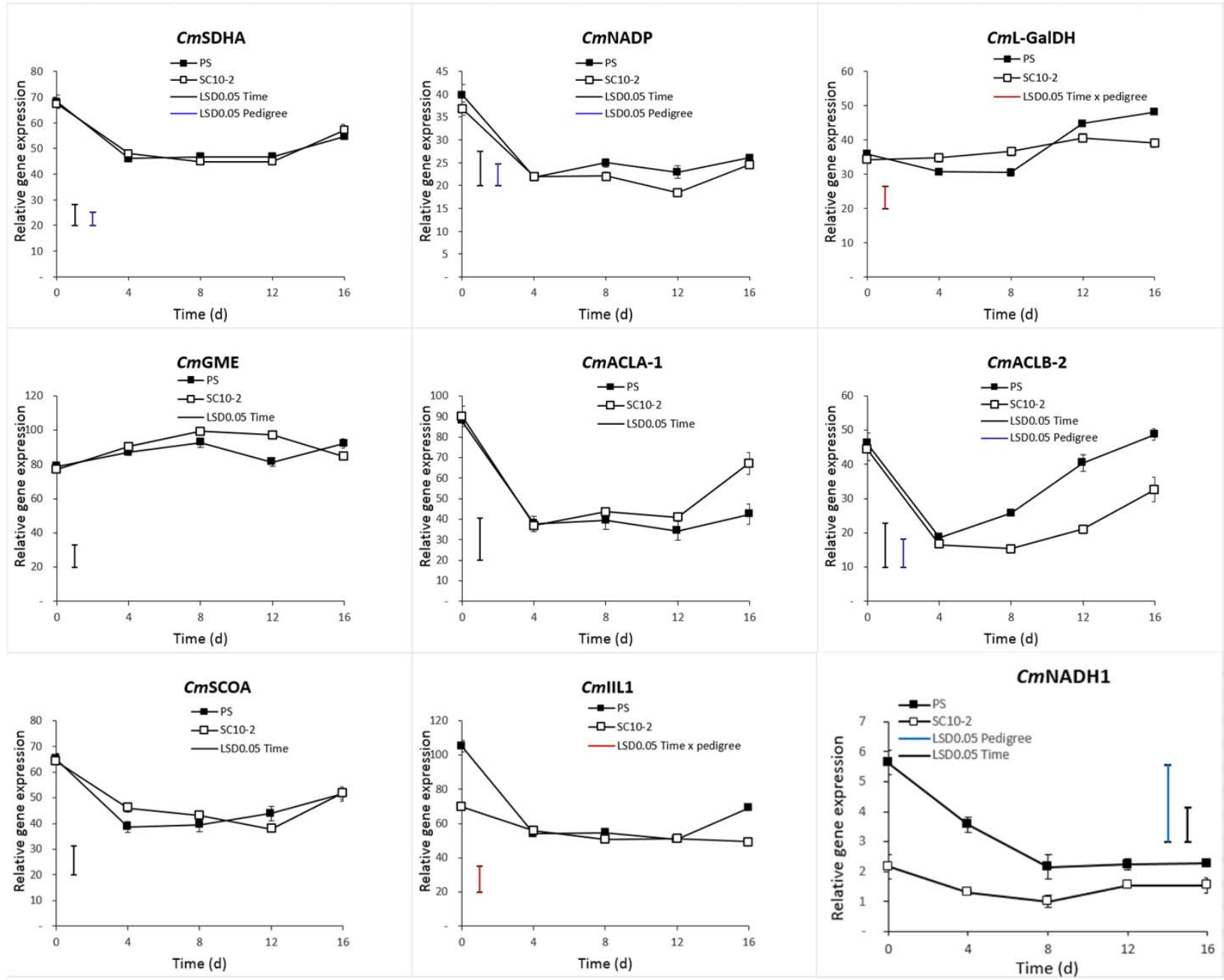

Fig. 9 

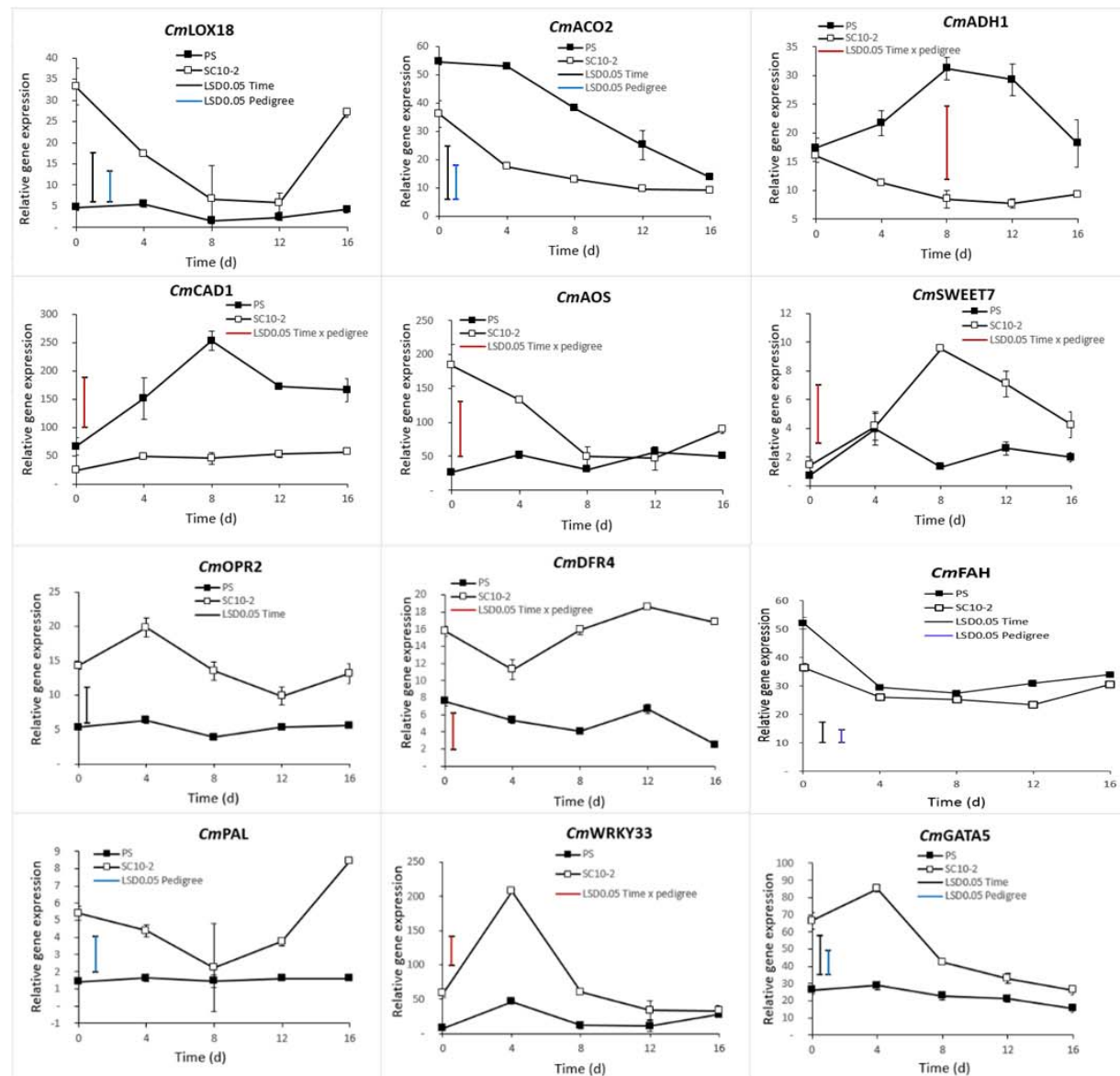

Fig. 10
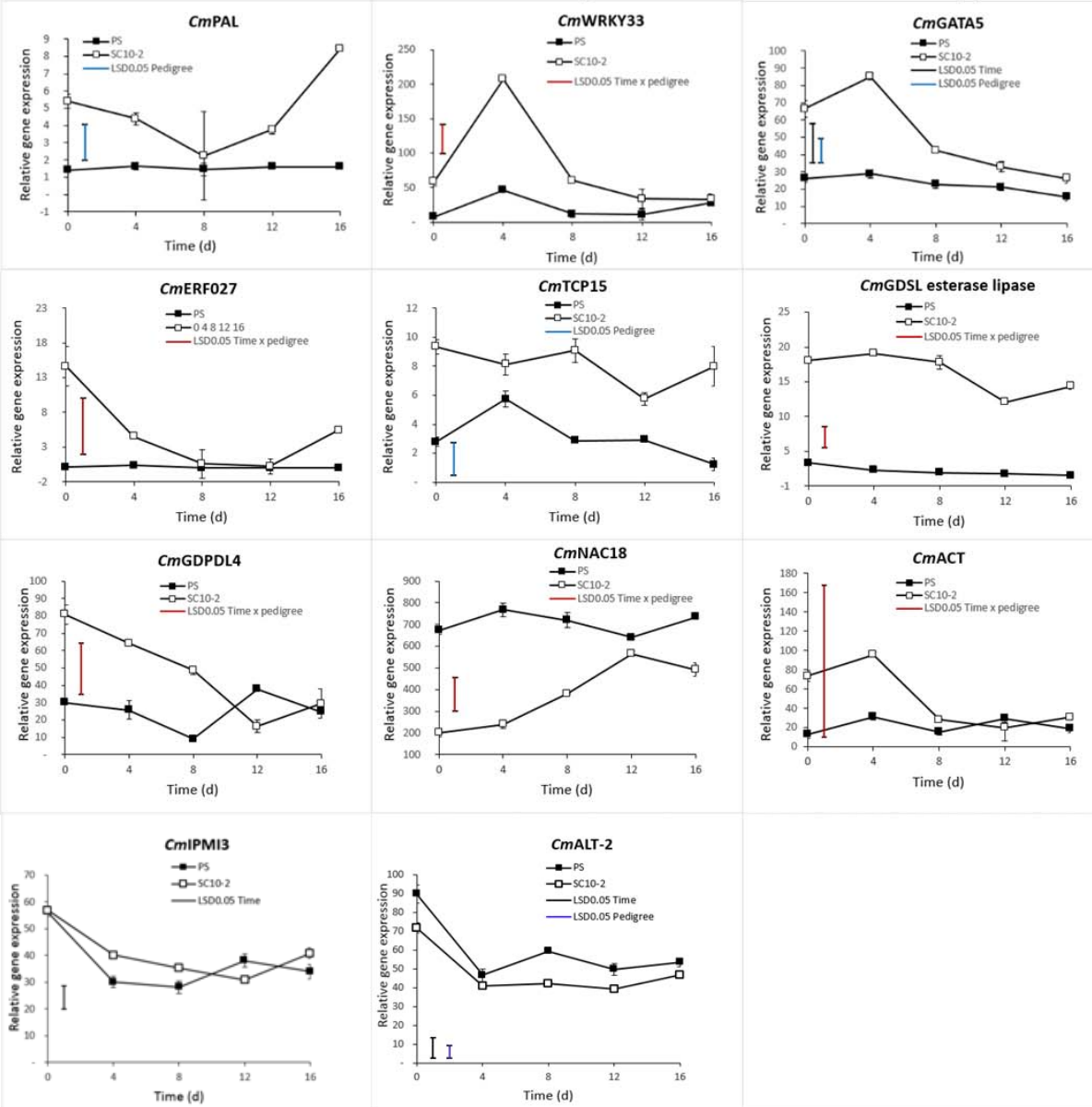

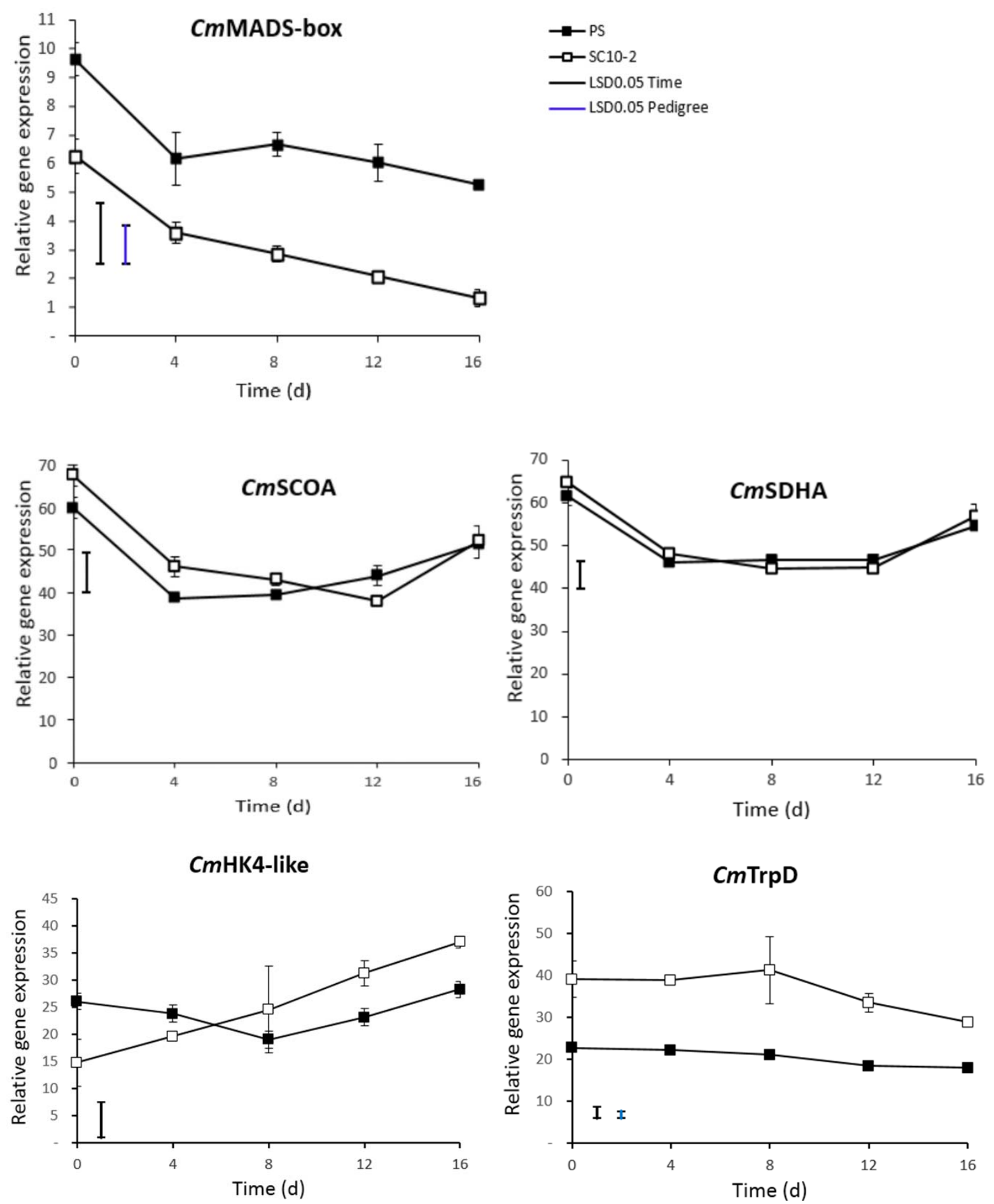

Fig. 11 

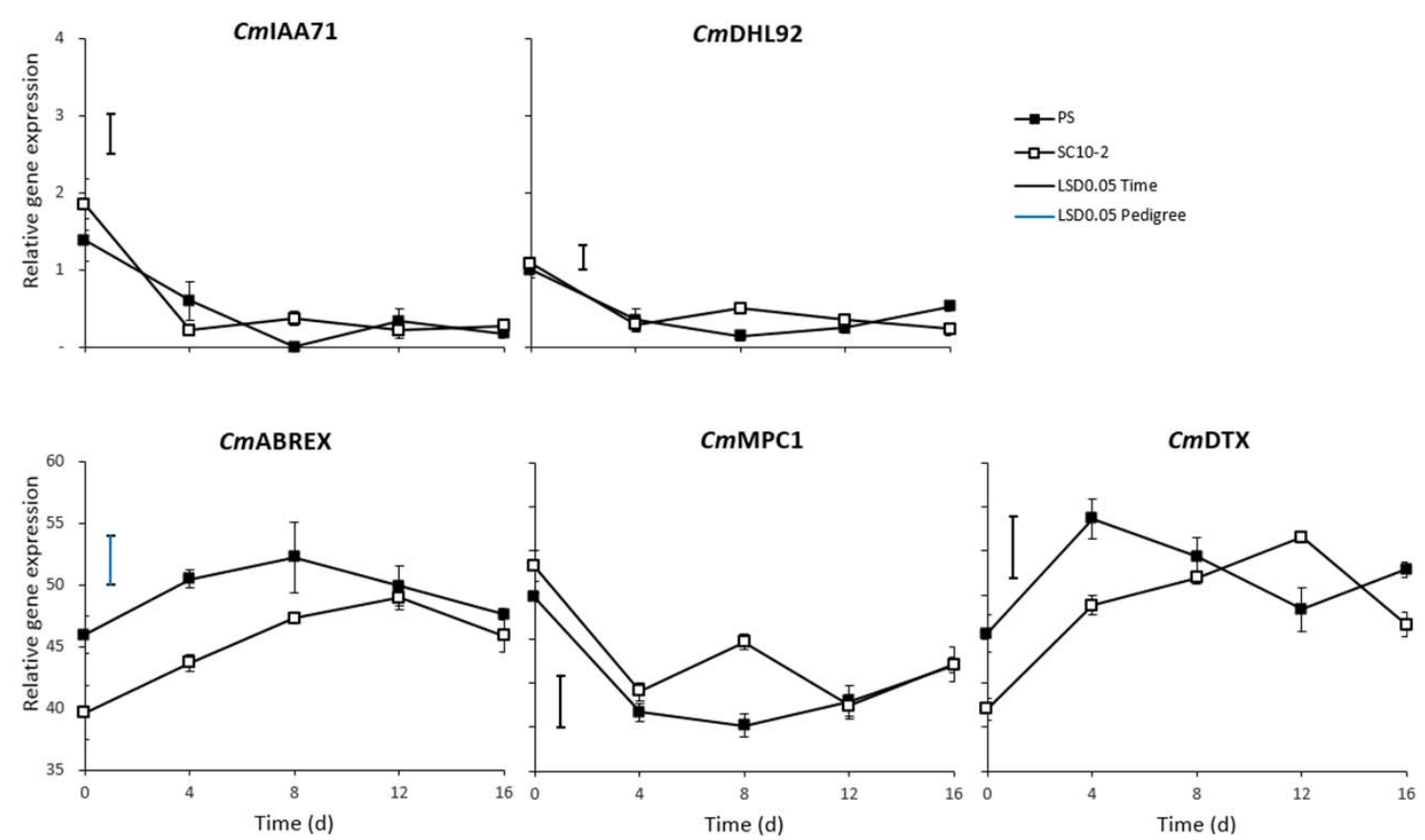

Fig. 12 

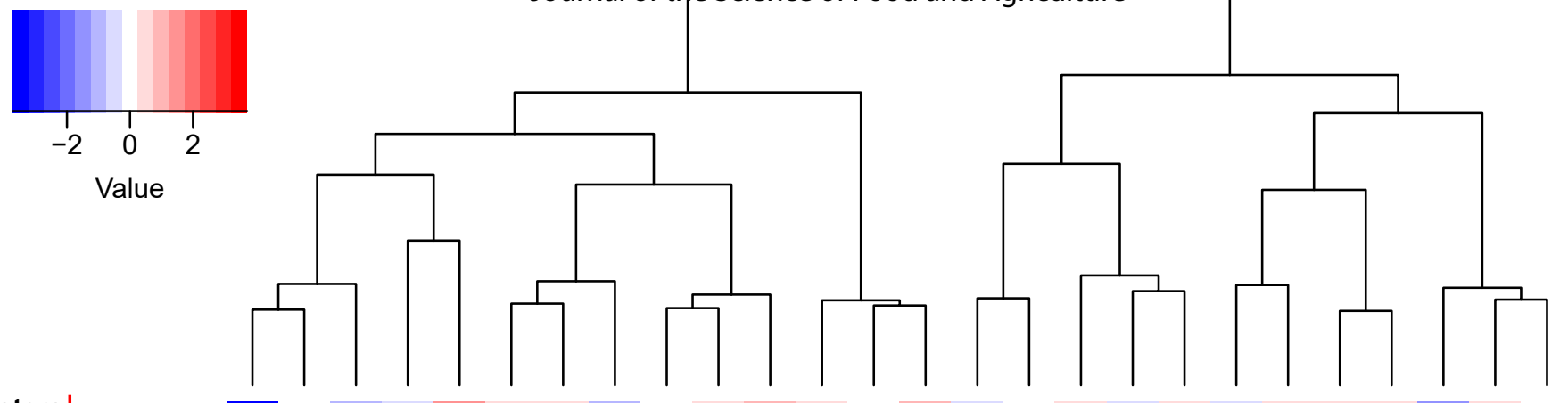

Clusters:

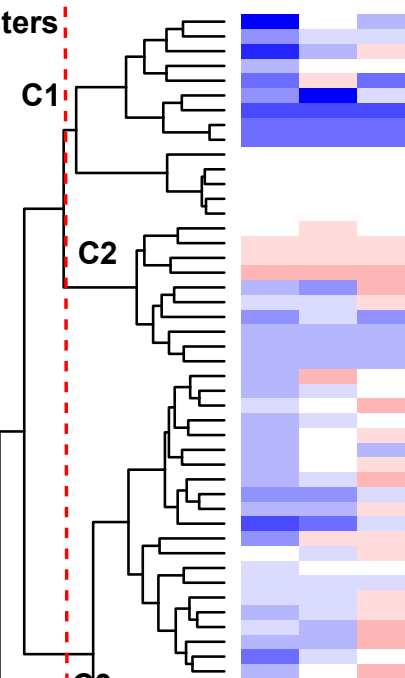

C3

드

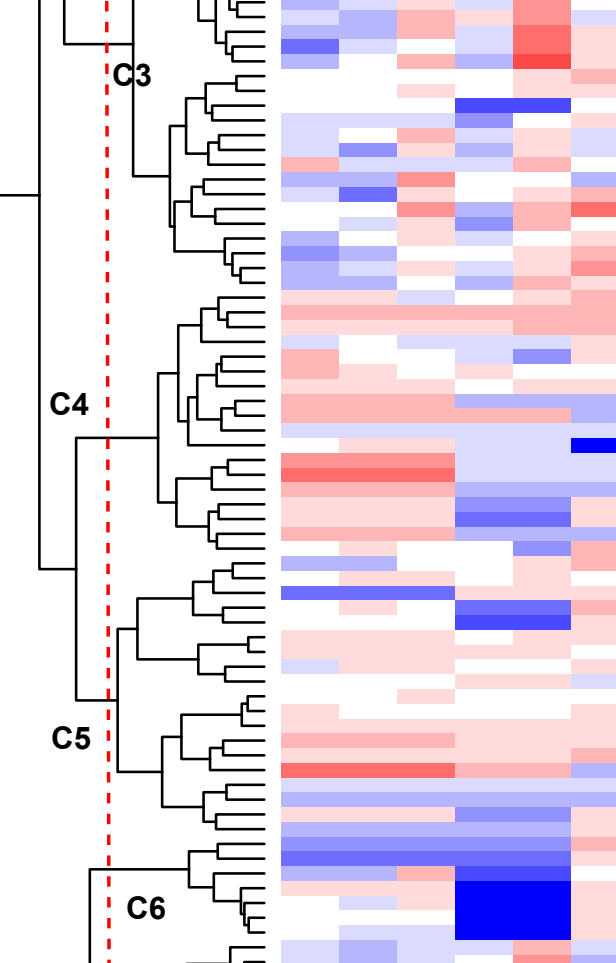

47
48
50
51
52
53
55
5
57
58
59

C7:

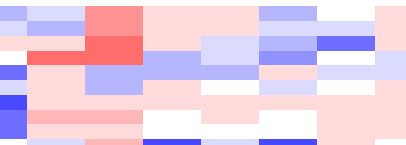<smiles>[CH]</smiles>
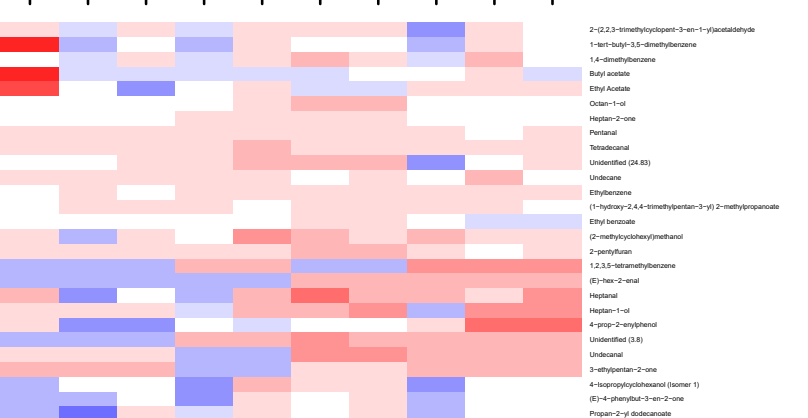

G1

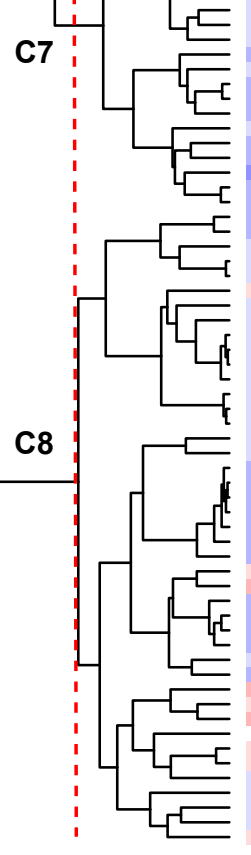

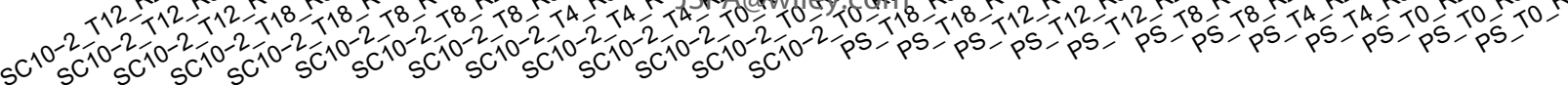


1

2
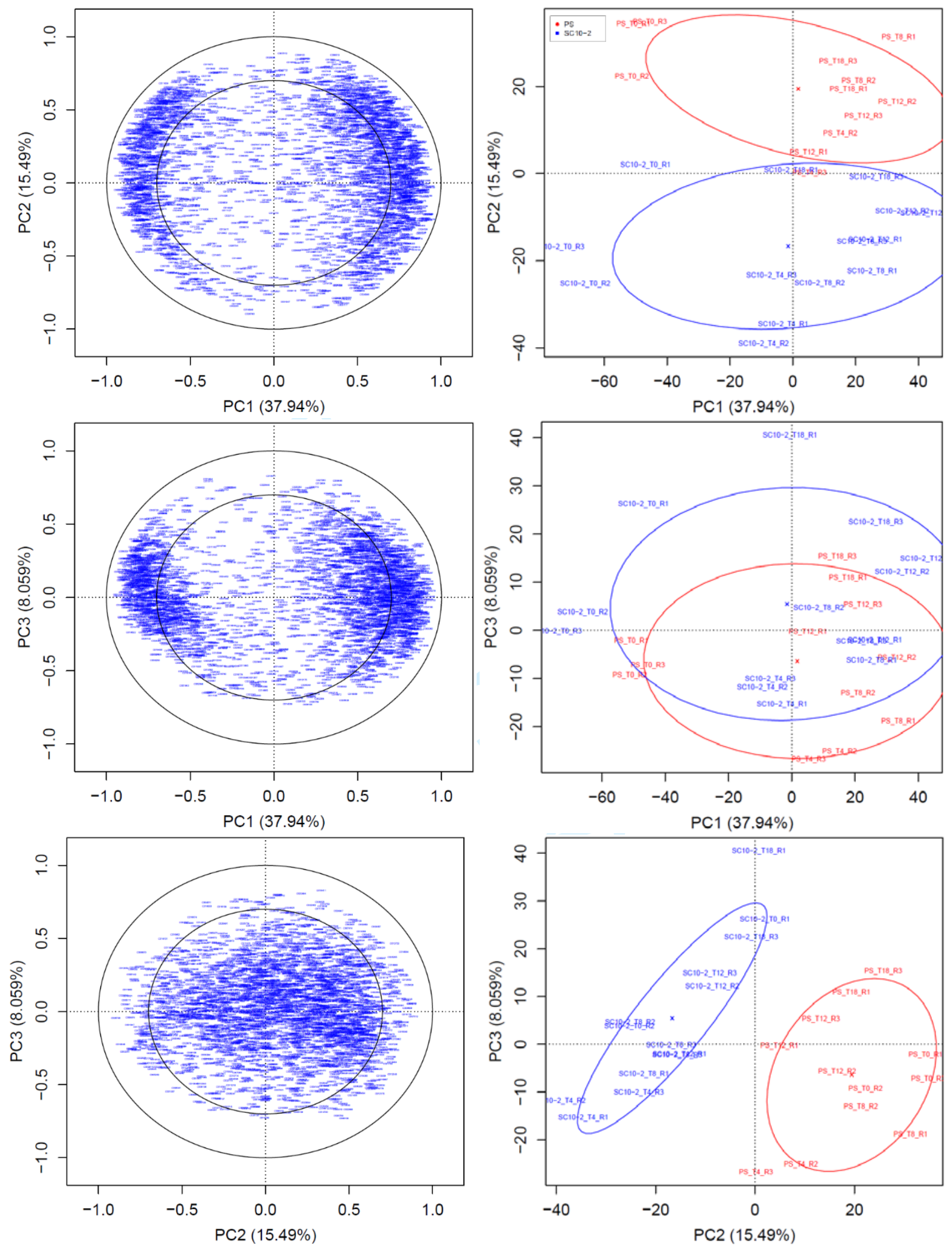

Supplementary Figure 1. Plot of the centroids of scores and loading plots of the Principal Component Analysis (PCA) of the expressed genes significant in introgression and identified in the near-isogenic line (NIL) SC10-2 and the parental control 'Piel de

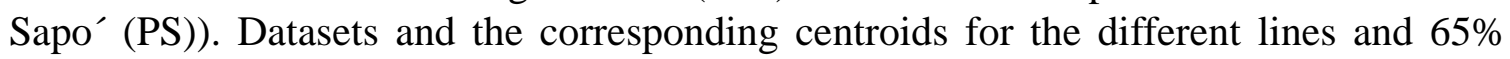
confidence ellipses. Tx $(x=0,4,8,12,18)$ indicates the postharvest ripening time in days and $\mathrm{Rn}(\mathrm{n}=1-3)$ the corresponding replicate 

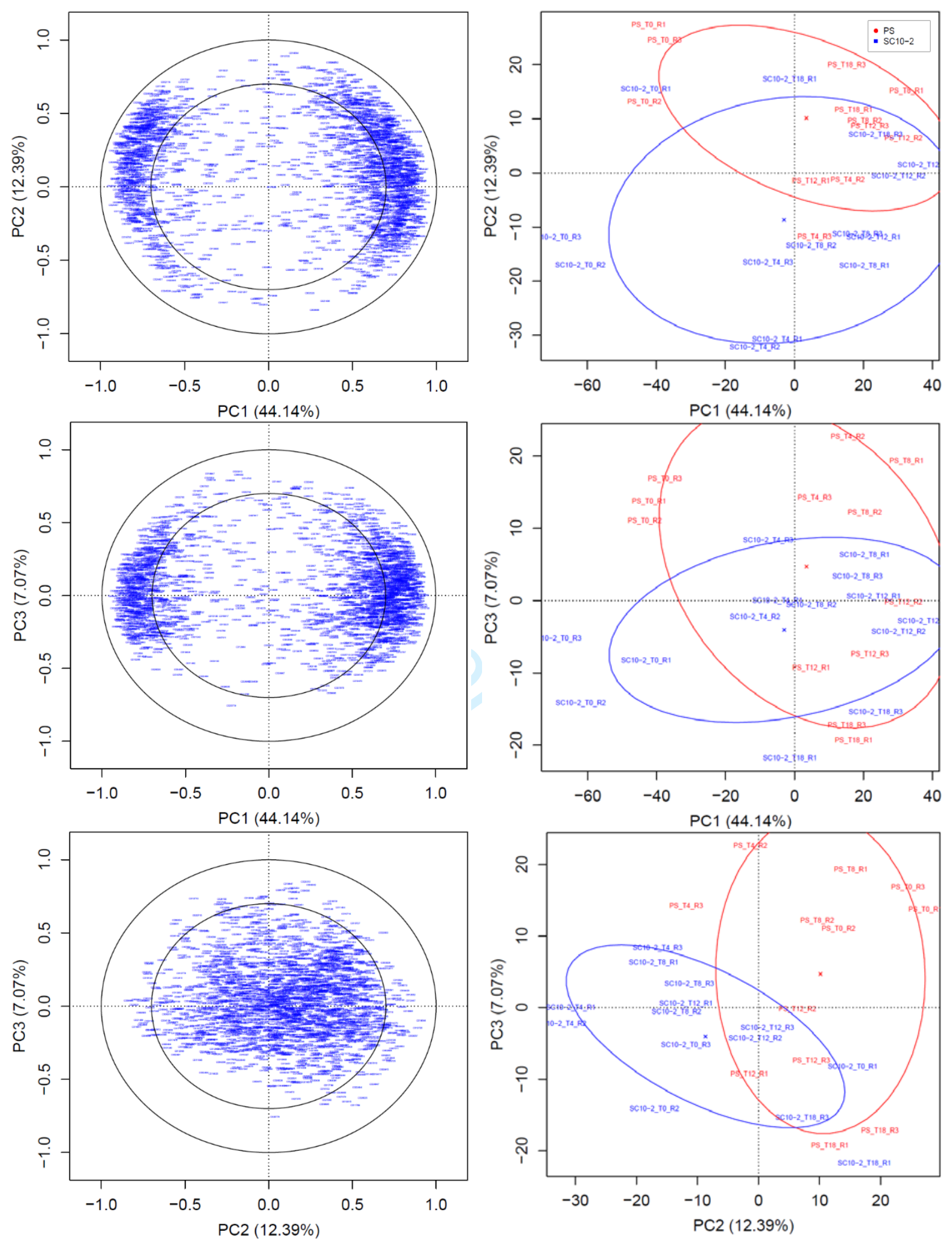

Supplementary Figure 2. Plot of the centroids of scores and loading plots of the Principal Component Analysis (PCA) of the expressed genes significant in ripening time identified in the near-isogenic line (NIL) SC10-2 and the parental control 'Piel de Sapo' (PS). Datasets and the corresponding centroids for the different lines and 65\% confidence ellipses. Tx $(x=0,4,8,12,18)$ indicates the postharvest ripening time in days and $\mathrm{Rn}(\mathrm{n}=1-3)$ the corresponding replicate. 
Supplementary Figure 3. Gene Ontology (GO) of the different clusters obtained using differentially expressed genes, only considering the postharvest ripening factor in melon fruit of the near-isogenic line NIL SC10-2 and its parental 'Piel de Sapo' (PS) harvested in firm-ripe stage of maturity and stored at $20.5^{\circ} \mathrm{C}$ and $88 \%$ relative humidity for $18 \mathrm{~d}$. 


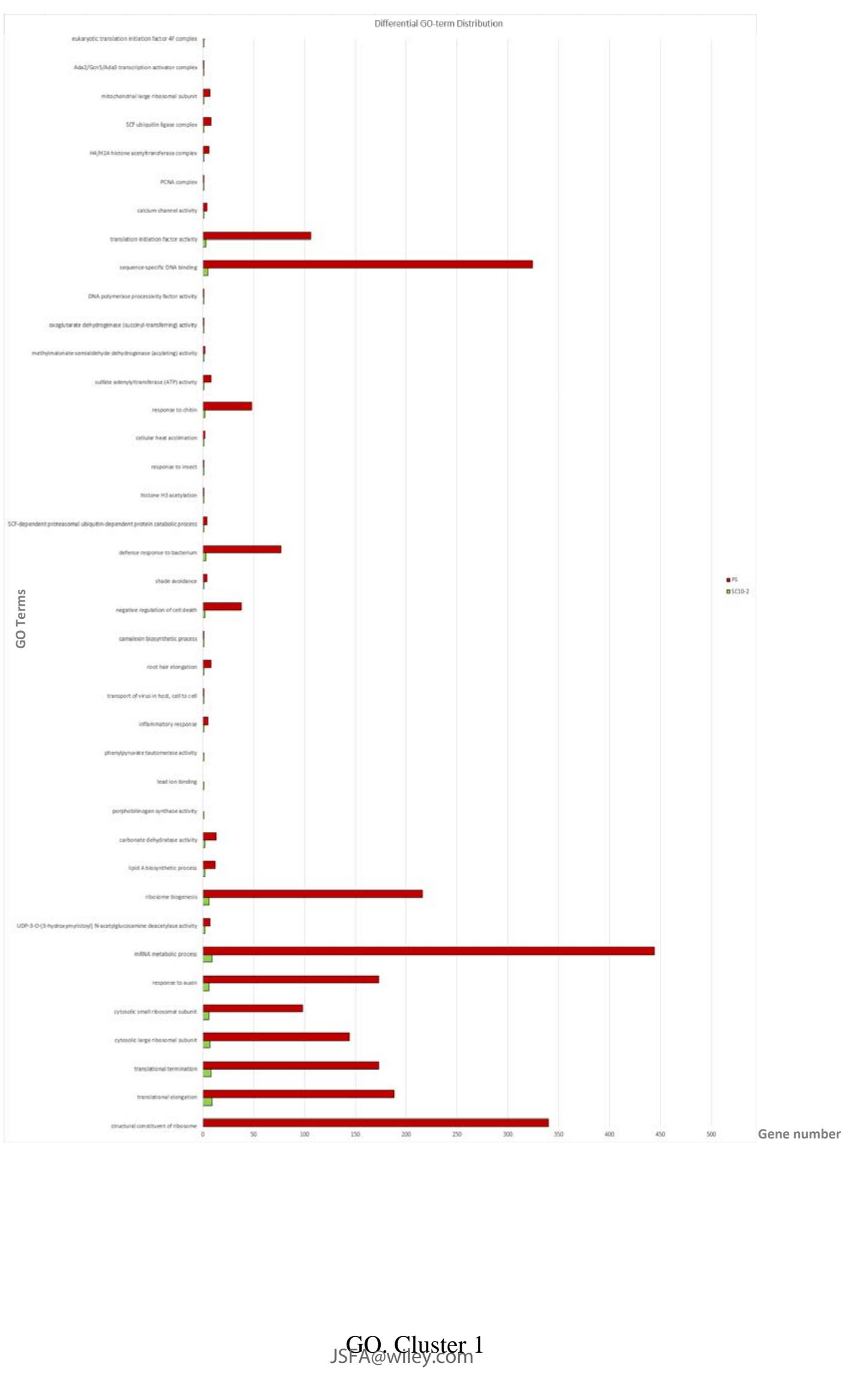




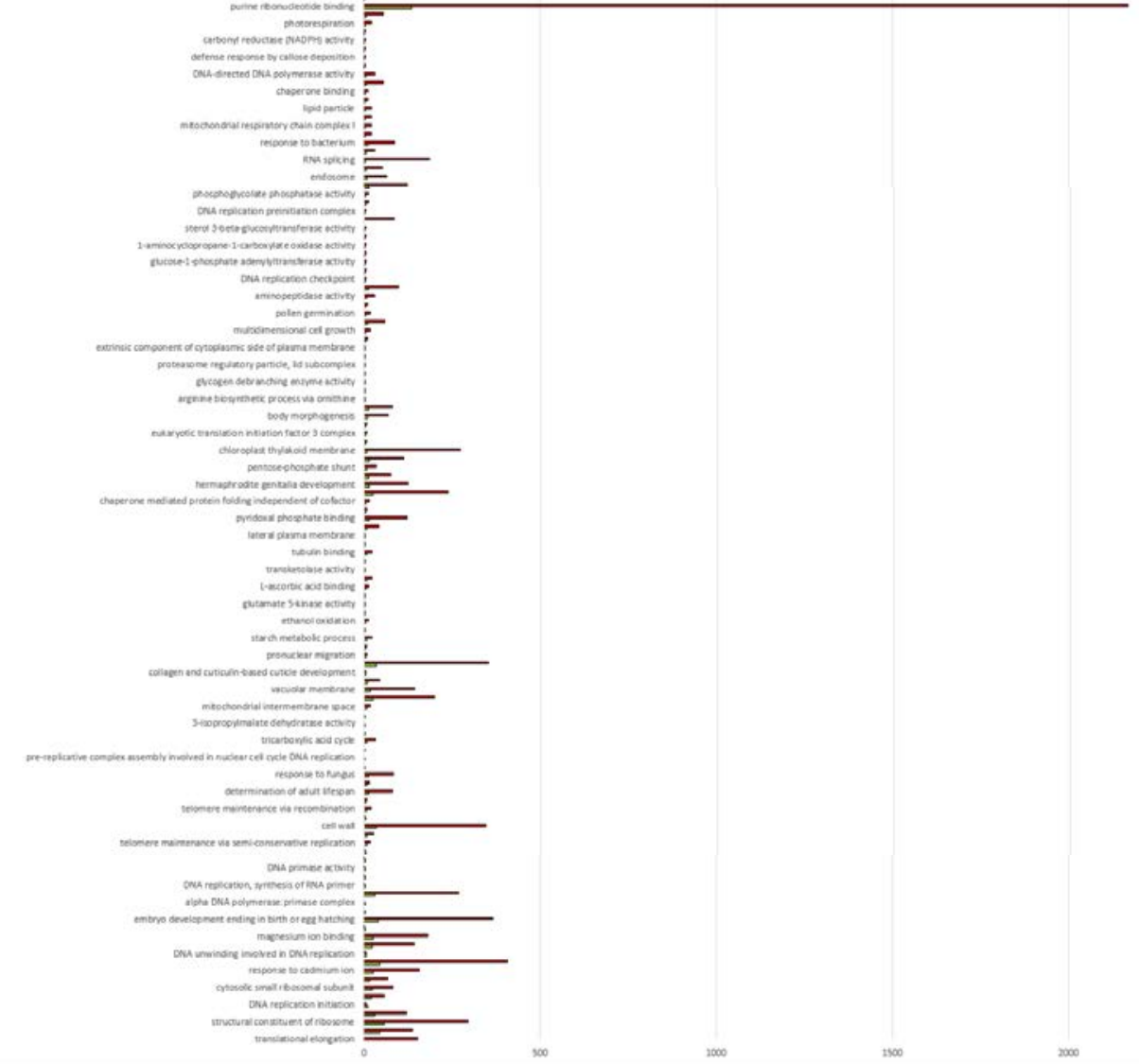



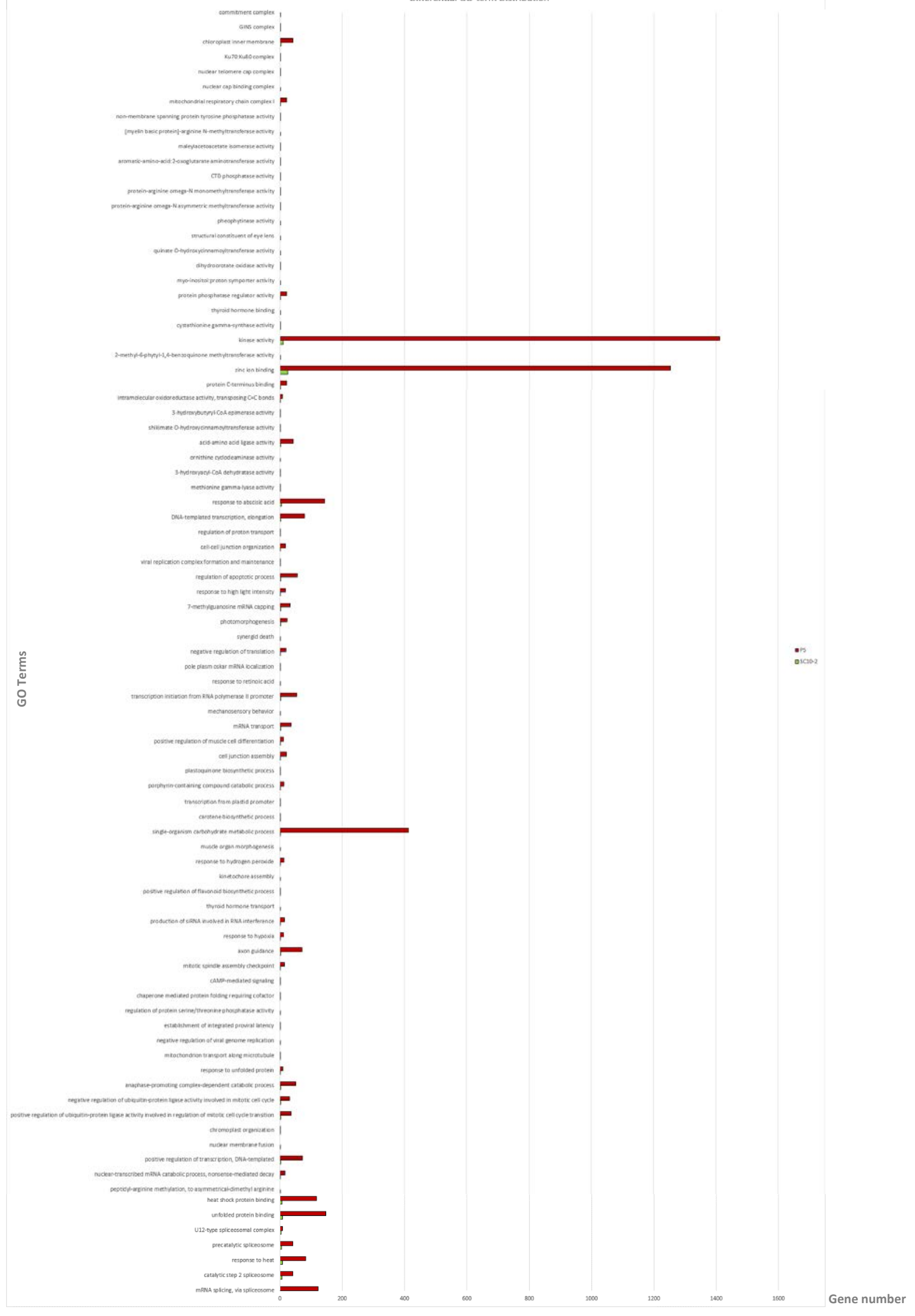


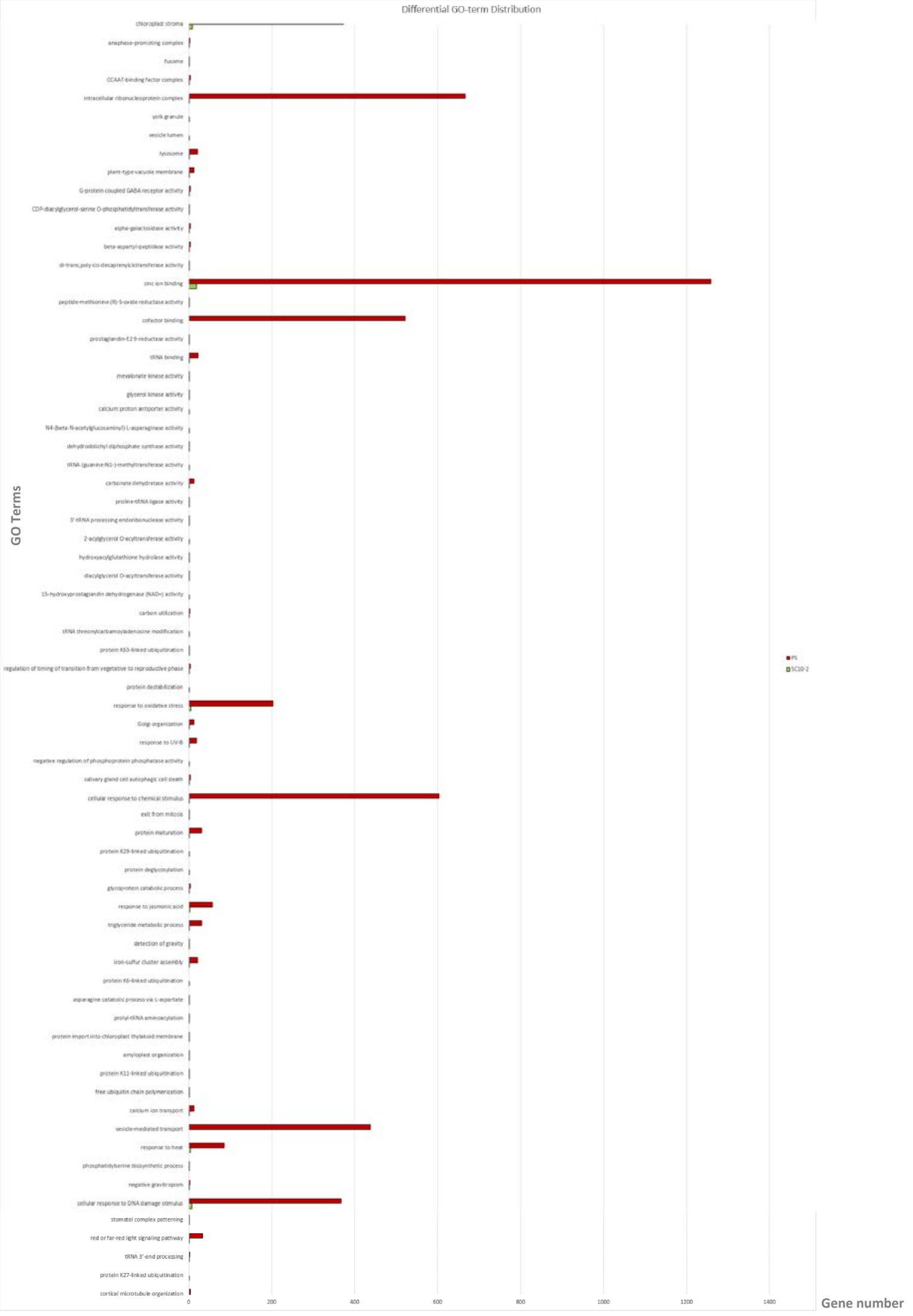




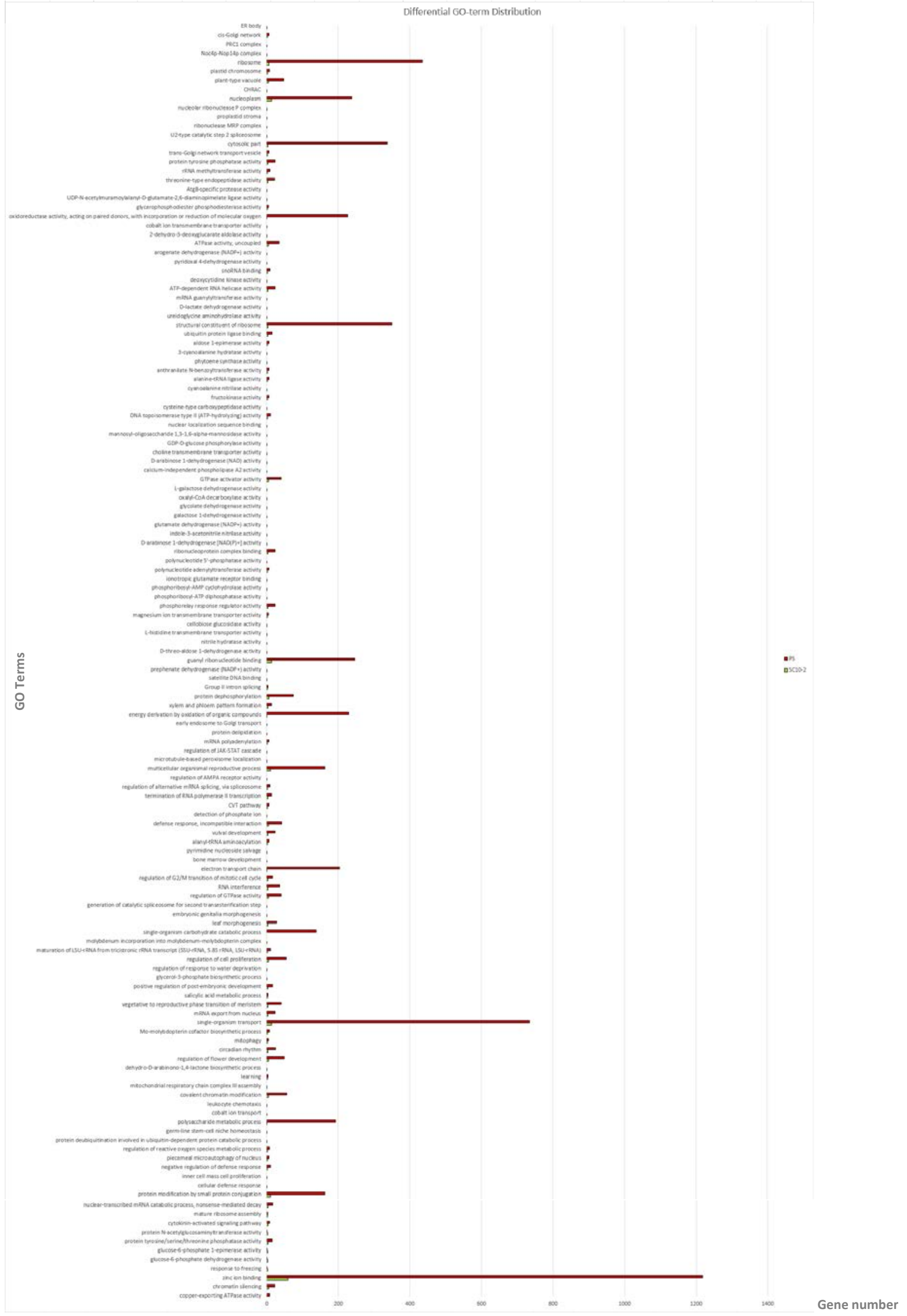




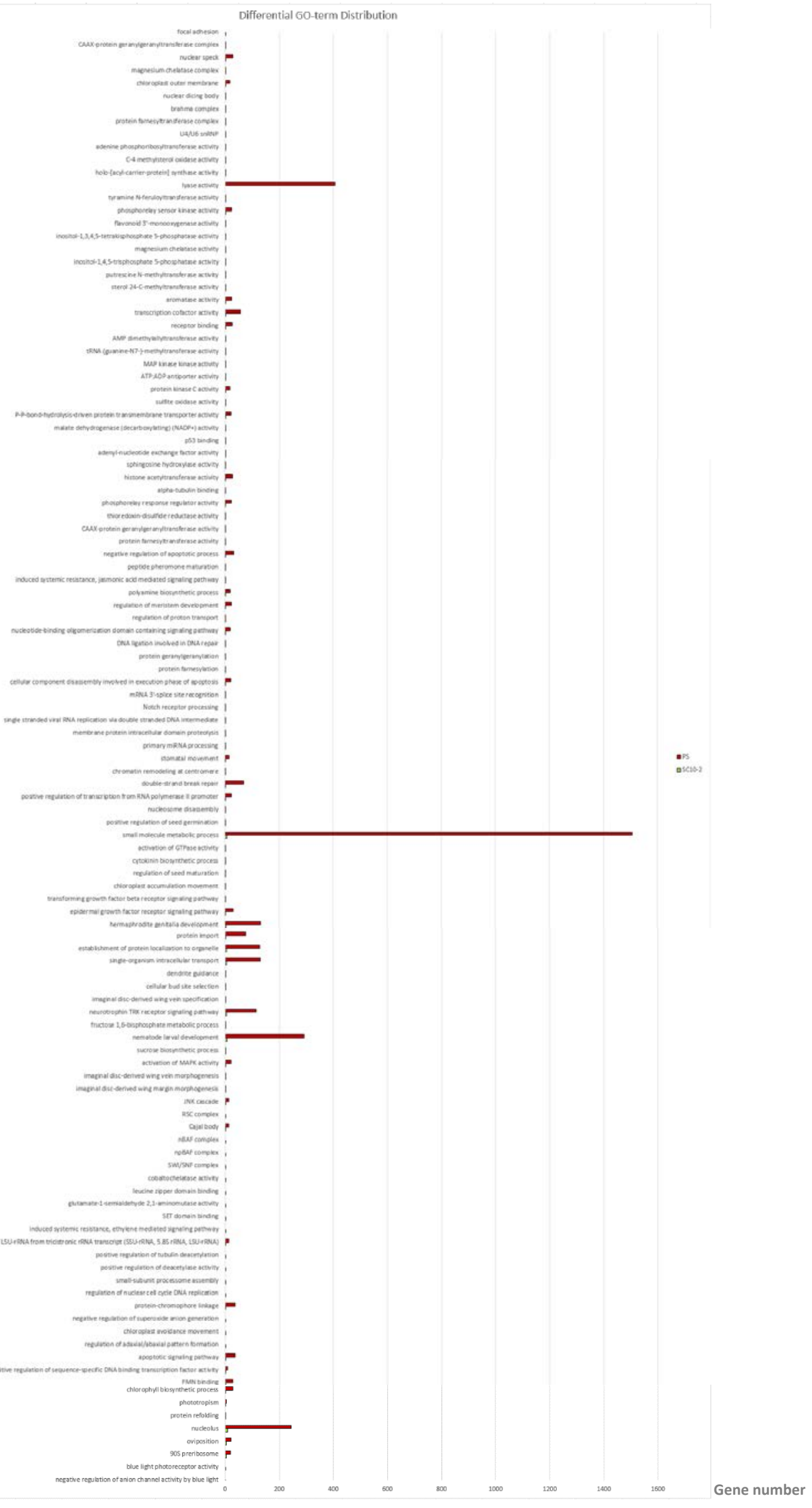


Supplementary Figure 4. Gene Ontology (GO) of the different clusters obtained using differentially expressed genes, only considering the introgression differences over time in melon fruit of the near-isogenic line NIL SC10-2 and its parental 'Piel de Sapo' (PS) harvested in firm-ripe stage of maturity and stored at $20.5^{\circ} \mathrm{C}$ and $88 \%$ relative humidity for18 $\mathrm{d}$. 
Differential Go-term Distribution 


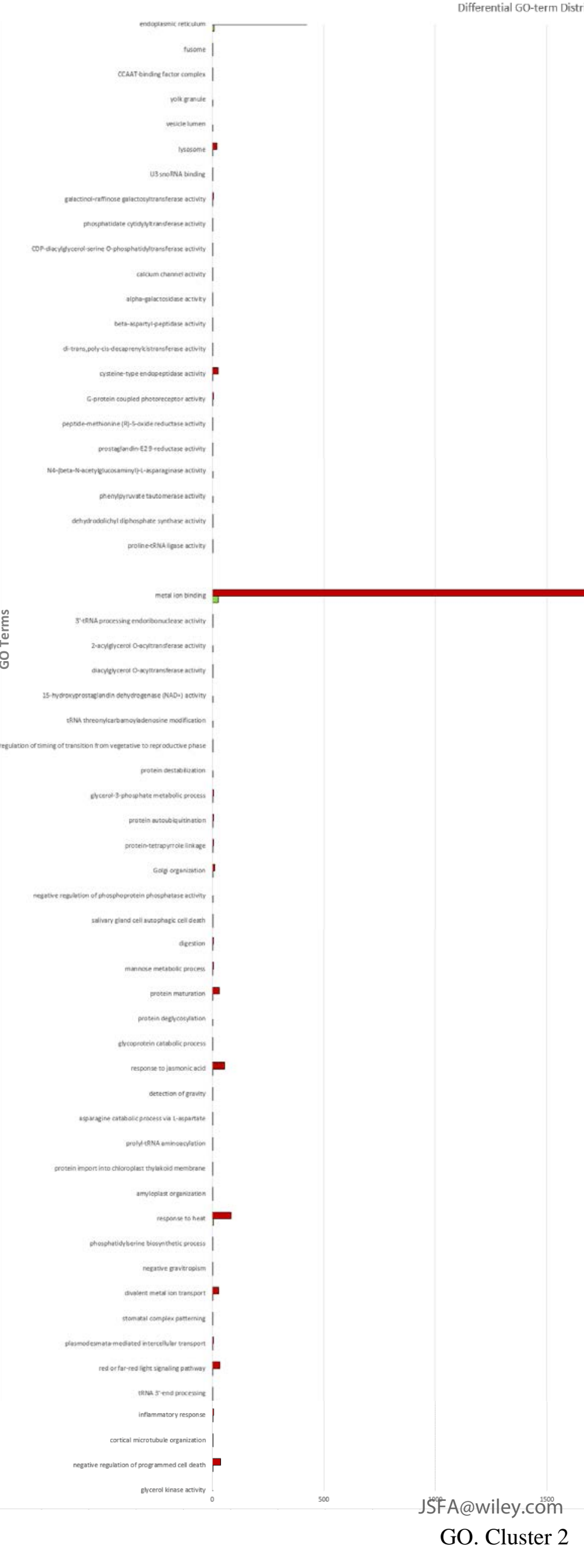




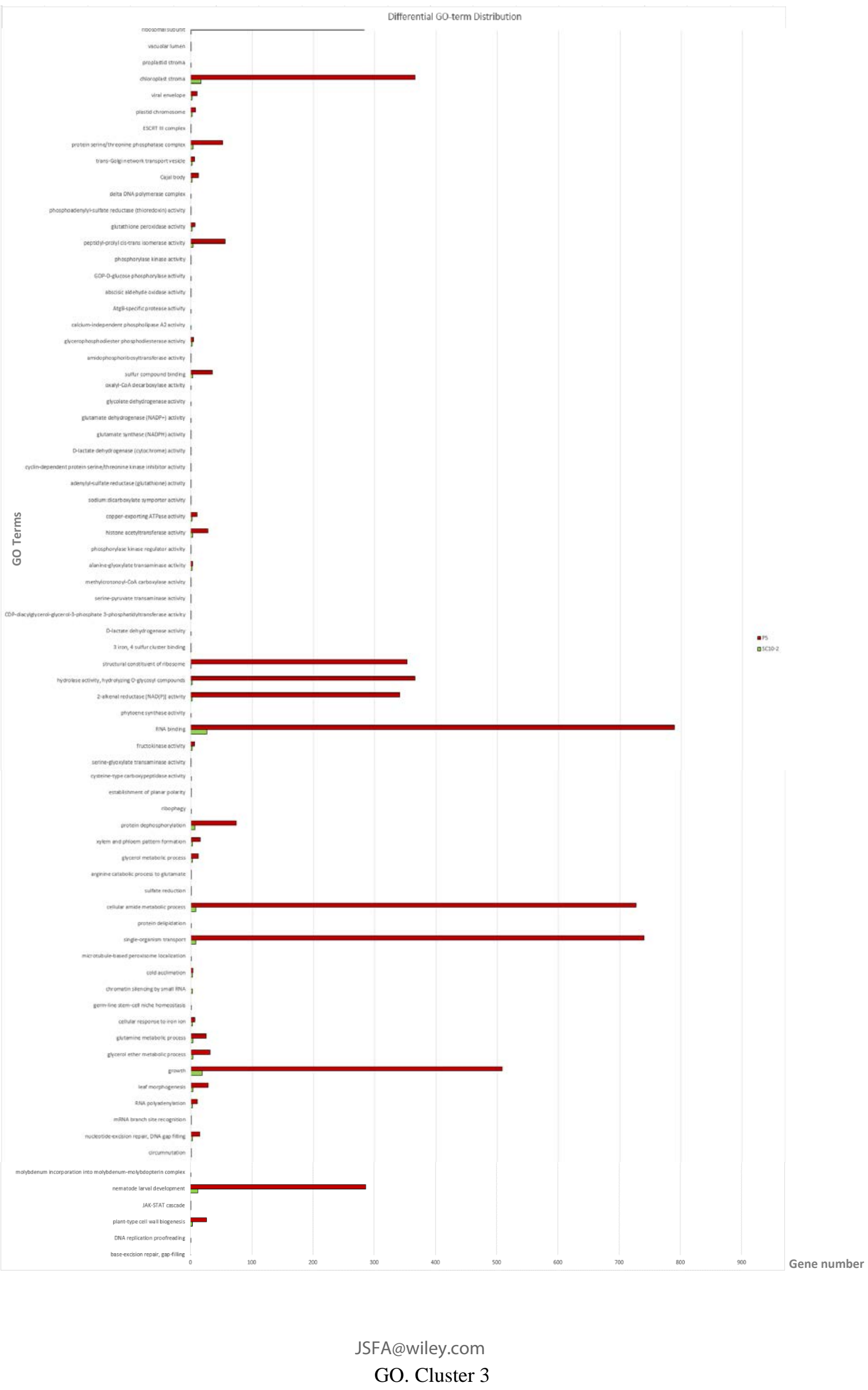


Differential GO-term Distribution

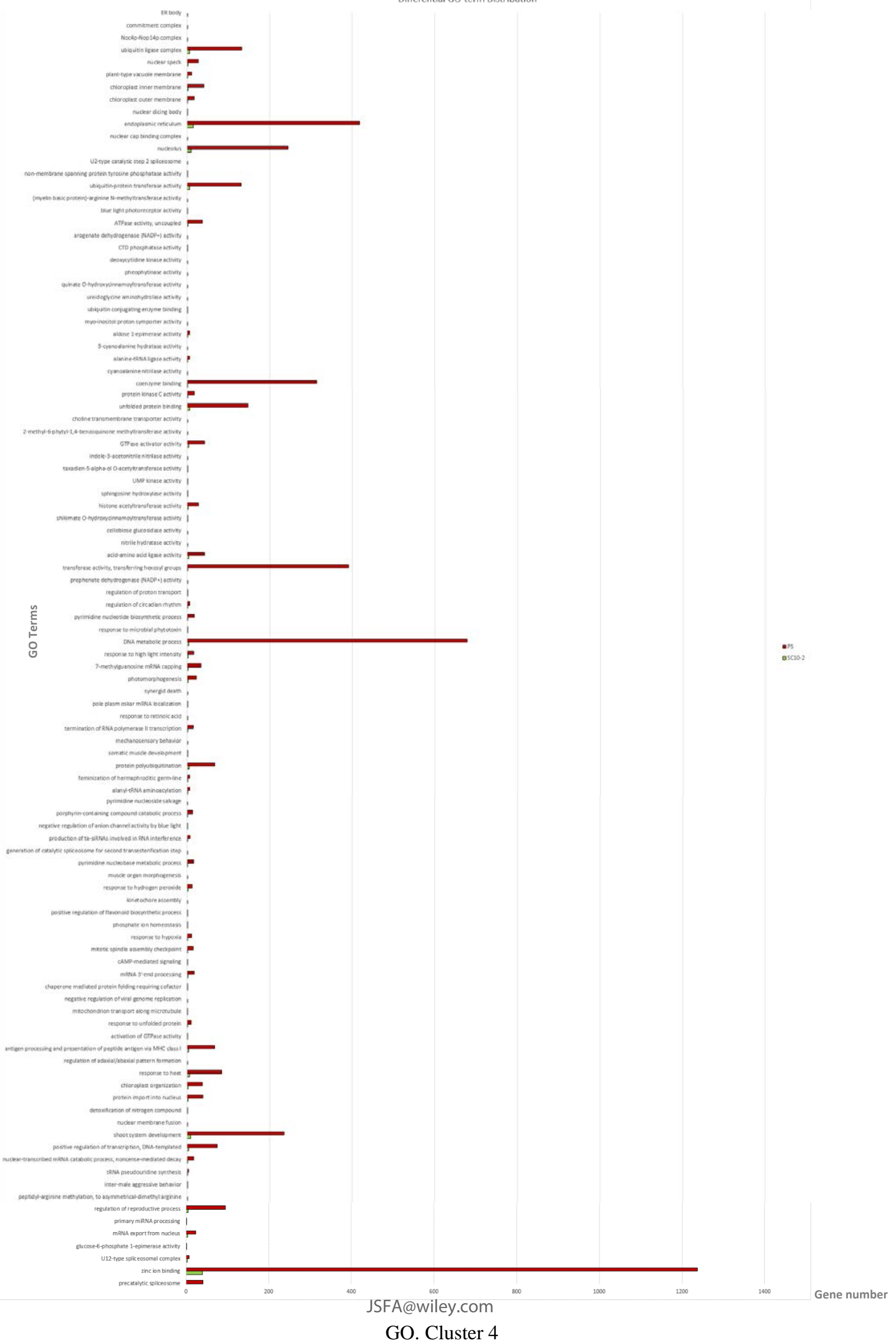




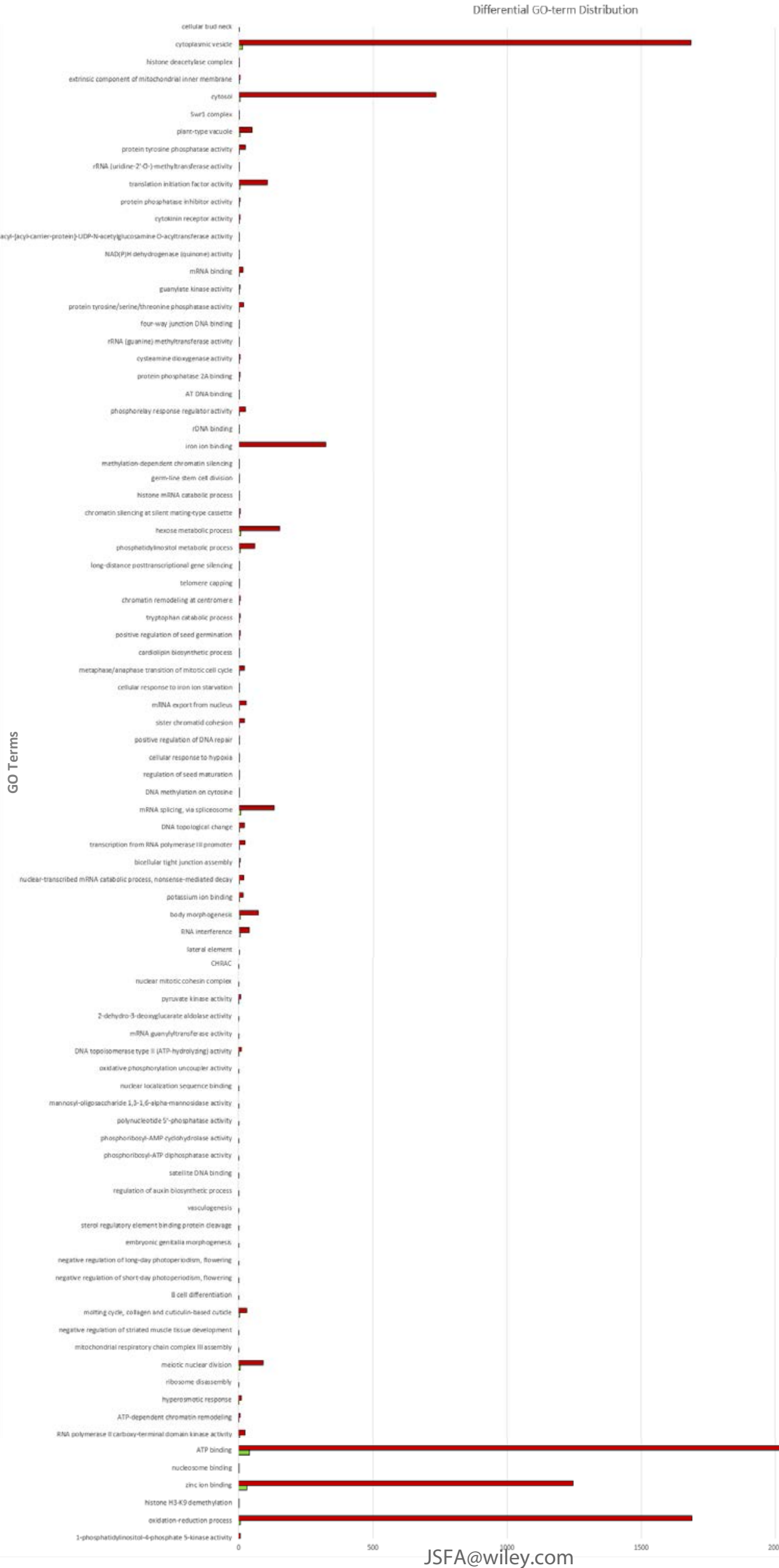


Supplementary Table 1. List of total Differentially Expressed Genes (2954 DEGs) only considering the comparison between lines over time fruit during postharvest storage at $20.5^{\circ} \mathrm{C}$ and $88 \%$ relative humidity for $18 \mathrm{~d}$ of the near-isogenic line SC10-2 and its parental control 'Piel de Sapo' (PS). 1 Presence 0 Absence in the list of DEGs during postharvest ripening time. Gene Ontology terms (GO terms) of the seven different clusters (named 1.x) obtained according to the differentially expressed gene pattern.

\begin{tabular}{|c|c|c|c|c|}
\hline Gene ID & pval & qval & Description & \\
\hline MELO3C010748 & $2.12940776123105 \mathrm{e}-12$ & $4.2053673876552 \mathrm{e}-08$ & Heat shock transcription factor & 1 \\
\hline MELO3C023889 & $1.64774860422767 \mathrm{e}-11$ & $1.62706935924462 \mathrm{e}-07$ & Ultraviolet-B receptor UVR8 & 1 \\
\hline MELO3C005540 & $7.05828728797542 \mathrm{e}-11$ & 4.64647052167422e-07 & $14 \mathrm{kDa}$ proline-rich protein dc 2.15 & 1 \\
\hline MELO3C026975 & $4.80710693473441 \mathrm{e}-10$ & $1.99977760837466 \mathrm{e}-06$ & 40S ribosomal protein S15a & 1 \\
\hline MELO3C012728 & $5.06298447611186 \mathrm{e}-10$ & $1.99977760837466 \mathrm{e}-06$ & Vacuolar sorting-associated protein 2-like protein & 1 \\
\hline MELO3C003298 & $1.04465369688 \mathrm{e}-09$ & $3.43847764328054 \mathrm{e}-06$ & high mobility group B protein 1 & 1 \\
\hline MELO3C013974 & $1.49749790345766 \mathrm{e}-09$ & $4.22486944219789 \mathrm{e}-06$ & Hsp70-Hsp90 organizing protein 3 & 1 \\
\hline MELO3C026279 & $2.1098163216493 e-09$ & $5.20834531703152 \mathrm{e}-06$ & cell division cycle protein 48 homolog & 1 \\
\hline MELO3C018265 & $2.94331214956145 \mathrm{e}-09$ & $5.28431560378992 \mathrm{e}-06$ & ADP-ribosylation factor GTPase-activating protein & 1 \\
\hline MELO3C020533 & $2.81429302084746 \mathrm{e}-09$ & $5.28431560378992 \mathrm{e}-06$ & Ran-binding protein 1 & 1 \\
\hline MELO3C026613 & $2.4889622585178 \mathrm{e}-09$ & $5.28431560378992 \mathrm{e}-06$ & Tubulin alpha chain & 1 \\
\hline MELO3C015733 & 4.18112777733626e-09 & $6.88109103955115 \mathrm{e}-06$ & Pre-mRNA-splicing factor ATP-dependent RNA helicase & 1 \\
\hline MELO3C016101 & $4.9076187558228 \mathrm{e}-09$ & $6.92289734348174 \mathrm{e}-06$ & UBP1-associated protein 2C & 1 \\
\hline MELO3C022036 & $4.56549242855431 \mathrm{e}-09$ & $6.92289734348174 \mathrm{e}-06$ & protein LONGIFOLIA 2 & 1 \\
\hline MELO3C019941 & $6.56920629005242 \mathrm{e}-09$ & $8.62635084597496 \mathrm{e}-06$ & T-complex protein 11 & 1 \\
\hline MELO3C018099 & $6.98878999116914 \mathrm{e}-09$ & $8.62635084597496 \mathrm{e}-06$ & No data found & 0 \\
\hline MELO3C023417 & $1.0827110208389 \mathrm{e}-08$ & $1.25779176179691 \mathrm{e}-05$ & DUF4050 family protein & 1 \\
\hline MELO3C002468 & $1.31388873114702 \mathrm{e}-08$ & $1.44155491952347 \mathrm{e}-05$ & Tobamovirus multiplication protein 1 & 1 \\
\hline MELO3C003282 & $1.38912555902593 e-08$ & $1.44388635080016 \mathrm{e}-05$ & cleft lip and palate transmembrane protein 1 homolog & 1 \\
\hline MELO3C024028 & $1.65297714405099 \mathrm{e}-08$ & $1.63223228089315 \mathrm{e}-05$ & DEAD-box ATP-dependent RNA helicase & 1 \\
\hline MELO3C014090 & $1.74401538766134 \mathrm{e}-08$ & $1.6401218995678 \mathrm{e}-05$ & No data found & 1 \\
\hline MELO3C008669 & $2.53079915912835 \mathrm{e}-08$ & $2.11626693401623 e-05$ & RING-type E3 ubiquitin transferase & 1 \\
\hline MELO3C018038 & $2.64090955770513 e-08$ & 2.11626693401623e-05 & proline iminopeptidase & 1 \\
\hline MELO3C001317 & $2.67895454708622 \mathrm{e}-08$ & $2.11626693401623 e-05$ & Coiled-coil protein (DUF572) & 1 \\
\hline MELO3C003752 & $2.55143854932527 \mathrm{e}-08$ & 2.11626693401623e-05 & gibberellin-regulated protein 11-like & 1 \\
\hline MELO3C010731 & $2.89443125023325 \mathrm{e}-08$ & $2.19854318310987 \mathrm{e}-05$ & cyclin-dependent kinase G-2 isoform X1 & 1 \\
\hline MELO3C008209 & 3.06606515732e-08 & $2.24265632562639 \mathrm{e}-05$ & Ankyrin & 1 \\
\hline MELO3C013868 & $3.65972404603454 \mathrm{e}-08$ & $2.41181691777337 \mathrm{e}-05$ & Cytochrome P450 family ent-kaurenoic acid oxidase & 1 \\
\hline MELO3C019616 & $3.66370487281387 \mathrm{e}-08$ & $2.41181691777337 \mathrm{e}-05$ & E3 ubiquitin-protein ligase RGLG2 & 1 \\
\hline MELO3C003444 & $3.45134805179725 \mathrm{e}-08$ & $2.41181691777337 \mathrm{e}-05$ & transcription factor IWS1 & 1 \\
\hline MELO3C004023 & 4.57532089992441e-08 & $2.82368788914397 \mathrm{e}-05$ & NADPH--cytochrome P450 reductase & 1 \\
\hline MELO3C019369 & 4.44710406277693e-08 & 2.82368788914397e-05 & 50S ribosomal protein L9 & 1 \\
\hline MELO3C015789 & 4.94272753837066e-08 & $2.84560916498596 \mathrm{e}-05$ & sucrose-binding protein-like & 1 \\
\hline MELO3C024775 & $4.86891227335917 \mathrm{e}-08$ & $2.84560916498596 \mathrm{e}-05$ & 10 & 1 \\
\hline MELO3C022533 & $5.04310703197675 \mathrm{e}-08$ & $2.84560916498596 \mathrm{e}-05$ & shaggy-related protein kinase theta & 1 \\
\hline MELO3C018195 & 6.19554454406313e-08 & 3.30691376218115e-05 & Arf GTPase activating protein & 1 \\
\hline MELO3C026898 & 6.14242697816181e-08 & 3.30691376218115e-05 & Cathepsin B-like cysteine protease & 1 \\
\hline MELO3C004204 & 6.59174723693923e-08 & $3.42580042585034 \mathrm{e}-05$ & Importin subunit alpha & 1 \\
\hline MELO3C002485 & 7.36338064966091e-08 & 3.63548511125383e-05 & Transmembrane protein, putative & 1 \\
\hline MELO3C003812 & 7.18805542865297e-08 & 3.63548511125383e-05 & Glucose-1-phosphate adenylyltransferase & 1 \\
\hline MELO3C021514 & $8.24147254885332 \mathrm{e}-08$ & $3.77341577131167 e-05$ & No data found & 0 \\
\hline MELO3C023342 & $8.40702283344541 \mathrm{e}-08$ & 3.77341577131167e-05 & kinesin-related protein 11-like & 1 \\
\hline MELO3C002104 & 8.08121399797912e-08 & $3.77341577131167 e-05$ & Receptor-like kinase & 1 \\
\hline MELO3C015197 & $8.2265061651654 \mathrm{e}-08$ & 3.77341577131167e-05 & Translocation protein Sec62 & 1 \\
\hline MELO3C000452 & $9.44282844139721 \mathrm{e}-08$ & $4.14414264198119 \mathrm{e}-05$ & Coiled-coil protein (DUF572) & 1 \\
\hline MELO3C016073 & $9.75029128547078 \mathrm{e}-08$ & $4.18605440427744 \mathrm{e}-05$ & Tudor/PWWP/MBT superfamily protein & 1 \\
\hline MELO3C006028 & $1.07972809137991 \mathrm{e}-07$ & $4.53692554822591 \mathrm{e}-05$ & cyclic nucleotide-gated ion channel 1 & 1 \\
\hline MELO3C007022 & $1.11002842029251 \mathrm{e}-07$ & 4.56707318174099e-05 & UDP-N-acetylglucosamine diphosphorylase 2-like & 1 \\
\hline MELO3C003570 & $1.13579167182465 \mathrm{e}-07$ & $4.57770402589081 \mathrm{e}-05$ & Protein ROOT PRIMORDIUM DEFECTIVE 1 & 1 \\
\hline MELO3C007499 & $1.18439269947146 \mathrm{e}-07$ & $4.58638655330624 \mathrm{e}-05$ & protein DA1-related 1-like & 1 \\
\hline MELO3C017271 & $1.17156354062509 \mathrm{e}-07$ & $4.58638655330624 \mathrm{e}-05$ & Acetyl-coenzyme A synthetase & 1 \\
\hline MELO3C014089 & $1.3594595926758 \mathrm{e}-07$ & $5.16307067226047 \mathrm{e}-05$ & caffeic acid 3-O-methyltransferase 1-like & 1 \\
\hline MELO3C012331 & $1.44328094586754 \mathrm{e}-07$ & $5.37799158489398 \mathrm{e}-05$ & dnaJ protein homolog & 0 \\
\hline MELO3C002508 & $1.4915760315759 \mathrm{e}-07$ & $5.45502500881343 e-05$ & thioredoxin-like protein CXXS1 & 1 \\
\hline MELO3C003905 & $1.80851489561817 \mathrm{e}-07$ & $6.39831774649798 \mathrm{e}-05$ & glycine--tRNA ligase, mitochondrial 1 & 1 \\
\hline MELO3C021108 & $1.84669660008296 \mathrm{e}-07$ & $6.39831774649798 \mathrm{e}-05$ & Peptidylprolyl isomerase & 1 \\
\hline MELO3C005293 & $1.82100714729572 \mathrm{e}-07$ & $6.39831774649798 \mathrm{e}-05$ & Phosphoglucomutase, putative & 1 \\
\hline MELO3C017963 & $1.99110337106845 \mathrm{e}-07$ & $6.77970697848806 \mathrm{e}-05$ & Lycopene beta-cyclase & 1 \\
\hline MELO3C016475 & $2.08360193654222 \mathrm{e}-07$ & $6.97441604148684 \mathrm{e}-05$ & Chloride channel protein & 1 \\
\hline MELO3C019973 & $2.2593505843993 e-07$ & 7.4366524485503e-05 & 2-C-methyl-D-erythritol 4-phosphate cytidylyltransferase & 1 \\
\hline
\end{tabular}


|MELO3C013123 2.50657820832778e-07 7.98426016713957e-05 Syntaxin-51

$\begin{array}{lll}\text { MELO3C017095 2.50064974283148e-07 } & 7.98426016713957 \mathrm{e}-05 & \text { CASP-like protein }\end{array}$

MELO3C007204 2.70307014549331e-07 8.47348131799166e-05 60S ribosomal protein L11-like

MELO3C022213 2.95504305247718e-07 8.82741693322043e-05 Myb family transcription factor family protein

$\begin{array}{lll}\text { MELO3C025783 } 2.88477511212193 e-07 & 8.82741693322043 e-05 & \text { receptor-like serine/threonine-protein kinase NCRK }\end{array}$

MELO3C003875 2.90642418132592e-07 8.82741693322043e-05 Protein phosphatase 2c, putative

MELO3C012788 $2.99476902387852 \mathrm{e}-07 \quad 8.82741693322043 \mathrm{e}-05$ Transcription initiation factor IIF alpha subunit family protein

MELO3C002464 3.08444173202638e-07 8.82820866170854e-05 polyadenylate-binding protein RBP45-like

MELO3C003563 3.07599423821081e-07 8.82820866170854e-05 No data found

MELO3C002736 3.17503783264783e-07 8.957688879566e-05 Calcyclin-binding protein

MELO3C026304 3.34660603895642e-07 9.30874967089442e-05 nuclear cap-binding protein subunit 2

MELO3C023110 3.42064934821806e-07 9.37785766616996e-05 Alkaline alpha galactosidase

MELO3C020989 3.46642163973065e-07 9.37785766616996e-05 phosphatidylinositol 4-kinase alpha 1

MELO3C017380 3.55206742130854e-07 9.4796999328949e-05 zinc finger CCHC domain-containing protein 7 isoform X3

MELO3C014061 3.64245731243606e-07 9.59131859510665e-05 WPP domain interacting protein, putative

MELO3C012356 3.74511264888966e-07 9.73187232933183e-05 Aspartyl aminopeptidase

MELO3C023146 4.04118919106189e-07 0.000102319801710617 villin-2-like

MELO3C023449 4.03846042607015e-07 0.000102319801710617 UPF0481 plant-like protein

MELO3C024278 4.11229531316337e-07 0.000102802177391979 Mitochondrial carrier protein

$\begin{array}{llll}\text { MELO3C015720 } & 4.28536336860574 \mathrm{e}-07 & 0.000105789551458244 & \text { Smr (Small MutS-related) domain protein }\end{array}$

MELO3C004433 4.57582358448505e-07 0.000111565357987648 calcium uptake protein 1, mitochondrial-like isoform X1

MELO3C010244 5.04511900589755e-07 0.000121507384448135 Temperature-induced lipocalin

MELO3C024910 5.28152974021978e-07 0.000125668591373013 FRIGIDA-like protein

MELO3C023537 5.4337361676815e-07 0.00012691733086263 zinc finger A20 and AN1 domain-containing stress-associated protein 8-like

MELO3C015590 5.46254145694647e-07 0.00012691733086263 protochlorophyllide-dependent translocon component 52, chloroplastic-like

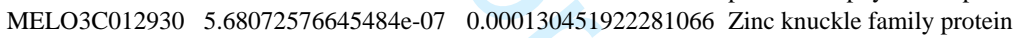

MELO3C011379 5.84145015025683e-07 0.00013260091841083 Chaperone protein dnaJ

MELO3C017213 5.98506860738368e-07 0.000134317181735478 UDP-glucose 6-dehydrogenase

MELO3C014359 $6.34542943744876 \mathrm{e}-07 \quad 0.000140804366247388$ Calcium-binding EF-hand family protein

MELO3C011117 $6.51958590447421 \mathrm{e}-07 \quad 0.00014284228791607$ receptor-like protein kinase HSL1

$\begin{array}{llll}\text { MELO3C003581 } 6.58192728764107 \mathrm{e}-07 & 0.00014284228791607 & \text { signal peptide peptidase }\end{array}$

MELO3C006120 7.02155050880116e-07 0.000148453468702257 MYB-related transcription factor

$\begin{array}{llll}\text { MELO3C011085 } 6.97631587276426 \mathrm{e}-07 & 0.000148453468702257 & \text { Argininosuccinate lyase }\end{array}$

MELO3C003506 7.06599121880203e-07 0.000148453468702257 Rhamnogalacturonate lyase family protein

$\begin{array}{lllll}\text { MELO3C012146 } 7.17645318282223 \mathrm{e}-07 & 0.000149187130429007 & 14-3-3 & \text { protein, putative }\end{array}$

MELO3C023559 7.44244884831602e-07 0.000153105127401451 40S ribosomal protein S27

MELO3C011089 $7.54406601521929 \mathrm{e}-07 \quad 0.000153595628592336$ O-fucosyltransferase family protein

MELO3C016717 $7.90376939896476 \mathrm{e}-07 \quad 0.00015927708353077$ COP1-interacting protein, putative

MELO3C007177 8.23092384139024e-07 0.000164194459539006 Transcription factor GTE12

MELO3C011324 8.36300357853759e-07 0.000165160957672539 No data found

MELO3C004799 8.70907565730938e-07 0.000170292609065548 splicing factor U2af small subunit B-like

MELO3C006297 9.06615200468863e-07 0.000172160996096727 RNA recognition motif (RRM) containing protein

MELO3C007072 9.03693317066967e-07 0.000172160996096727 Caffeoylshikimate esterase

$\begin{array}{lll}\text { MELO3C025061 } 9.00795385505582 \mathrm{e}-07 & 0.000172160996096727 & \text { V-type proton ATPase subunit C }\end{array}$

MELO3C005262 9.43659170360434e-07 0.000177488809099507 PLATZ transcription factor family protein, putative

MELO3C004194 9.58587341726158e-07 0.000178595673695754 calreticulin

MELO3C022416 9.74627697658548e-07 0.000179887125243539 Ubiquitin family protein

MELO3C021903 $1.01411382180672 \mathrm{e}-06 \quad 0.000183740677677623$ Alpha/beta hydrolase-3

MELO3C015327 $1.01389569406862 \mathrm{e}-06 \quad 0.000183740677677623$ isoaspartyl peptidase/L-asparaginase

MELO3C013136 $1.03190995592861 \mathrm{e}-06 \quad 0.000185265361087583$ GDP-L-galactose phosphorylase 1

MELO3C003448 $1.2097755952567 \mathrm{e}-06 \quad 0.000215241966042564$ Methyltransferase-related family protein

MELO3C024862 $1.29411787552769 \mathrm{e}-06 \quad 0.000228192267176753$ Plant peroxidase

MELO3C019120 $1.32913722605821 \mathrm{e}-06 \quad 0.00023229319537543$ R3H domain-containing protein 1-like isoform X1

MELO3C021462 $1.37274241240881 \mathrm{e}-06 \quad 0.000237809560549663$ DDT domain-containing protein PTM

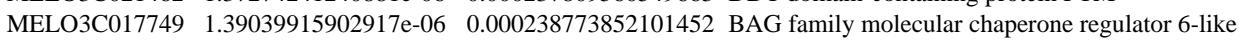

MELO3C015067 1.43038817013164e-06 0.000243523585964911 transcription factor LHW

MELO3C016754 $1.51495070621621 \mathrm{e}-06 \quad 0.000253548826246305$ Cold regulated gene 27, putative isoform 3

MELO3C005731 $1.50559889966484 \mathrm{e}-06 \quad 0.000253548826246305$ Intracellular protein transport protein USO1-like protein

MELO3C017884 $1.52819748255872 \mathrm{e}-06 \quad 0.000253616572126489$ DUF4050 family protein

MELO3C013622 $1.60432891793683 \mathrm{e}-06 \quad 0.000264032431669453$ mannan endo-1,4-beta-mannosidase 2

MELO3C022408 $1.62486392341066 \mathrm{e}-06 \quad 0.000265201963830059$ UV-stimulated scaffold protein A homolog

MELO3C024626 $1.7288799445403 \mathrm{e}-06 \quad 0.000279865983809232$ Protein LIKE COV 1

MELO3C009967 $1.75992938844516 \mathrm{e}-06 \quad 0.000282575979613036$ cyclin-dependent kinase C-2-like

MELO3C020570 $1.778215225956 \mathrm{e}-06 \quad 0.000283209455624235$ Maternal effect embryo arrest protein

MELO3C022066 $1.8187769972311 \mathrm{e}-06 \quad 0.000287352215346536$ Zinc finger CCHC domain-containing protein 8

MELO3C013988 $1.86327363427274 \mathrm{e}-06 \quad 0.000292045960343273$ Myelodysplasia-myeloid leukemia factor 1-interacting protein

MELO3C027330 $1.90953358347734 \mathrm{e}-06 \quad 0.000294620146406984$ Caffeic acid O-methyltransferase

MELO3C001987 $1.90165617108828 \mathrm{e}-06 \quad 0.000294620146406984$ cyclic dof factor 3 
|MELO3C006997 $1.975027581258 \mathrm{e}-06 \quad 0.000302362943428406$ galactan beta-1,4-galactosyltransferase GALS3-like MELO3C018525 2.01982641567788e-06 0.000306842706794019 Myosin heavy chain-like protein MELO3C021940 2.06217394693731e-06 0.00031088452884019 Cold acclimation protein

MELO3C025488 2.10223959085098e-06 0.000312158869772302 transcription initiation factor TFIID subunit 1-like $\begin{array}{lll}\text { MELO3C020880 2.09783662086238e-06 } & 0.000312158869772302 & \text { Pathogenesis-related protein } 1\end{array}$ MELO3C009091 2.15805440451078e-06 0.000318055346527488 50S ribosomal protein L17

$\begin{array}{llll}\text { MELO3C007139 } 2.23241586572165 e-06 & 0.000324832030554499 & \text { la-related protein 6A }\end{array}$

MELO3C012149 2.23693129552949e-06 0.000324832030554499 PLASMODESMATA CALLOSE-BINDING PROTEIN 3

MELO3C007547 2.28760654796822e-06 0.000327376389245105 AT3g50560/T20E23_160

MELO3C007735 2.28658548895311e-06 0.000327376389245105 E3 ubiquitin-protein ligase RING1

MELO3C002689 2.36888940319258e-06 0.000336569761321224 serine/threonine-protein kinase STY8-like

MELO3C010985 2.3905490020848e-06 0.00033722108744409 B-box zinc finger protein 20-like

MELO3C009579 2.41978410275845e-06 0.000338924228690614 Myosin-binding protein 7

MELO3C003975 $2.4639976213825 \mathrm{e}-06 \quad 0.000342686542427345$ protein CHLOROPLAST IMPORT APPARATUS 2 isoform X2

MELO3C003331 2.48646479494852e-06 0.000343392959688379 BAX inhibitor-1

MELO3C021782 2.59632473731397e-06 0.000356075119702872 alpha-glucosidase

MELO3C014555 2.65481477645935e-06 0.000359109157673258 splicing factor 3B subunit 6-like protein

MELO3C022488 $2.65025887680181 \mathrm{e}-06 \quad 0.000359109157673258$ tubulin-folding cofactor D

MELO3C026045 2.71888340064308e-06 0.000365273661763947 alanine--glyoxylate aminotransferase 2 homolog 2, mitochondrial-like

MELO3C006929 2.74598986926833e-06 0.000366422661676893 BAHD acyltransferase DCR

MELO3C024188 $2.78370137918316 \mathrm{e}-06 \quad 0.000368961869379115$ ribulose-1,5 bisphosphate carboxylase/oxygenase large subunit N-

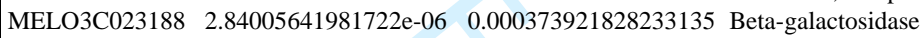

MELO3C013183 $2.90970862626416 \mathrm{e}-06 \quad 0.000379146148603654$ elongation factor-like GTPase 1

MELO3C026090 2.91813330233204e-06 0.000379146148603654 Sec14p-like phosphatidylinositol transfer family protein

$\begin{array}{llll}\text { MELO3C012737 } 2.98116016383965 e-06 & 0.00037983827145593 & \text { F21O3.15 protein }\end{array}$

MELO3C021100 $2.94284546042967 \mathrm{e}-06 \quad 0.00037983827145593$ Heat shock $70 \mathrm{kDa}$ protein

MELO3C018644 $2.96391440057775 \mathrm{e}-06 \quad 0.00037983827145593$ serine/arginine-rich splicing factor SC35

MELO3C022819 3.01520986245585e-06 0.000381713971625901 Myosin heavy chain-like protein, putative

$\begin{array}{llll}\text { MELO3C006921 3.11710684453015e-06 } & 0.000389618627041936 \text { No data found }\end{array}$

MELO3C018149 3.10182773133327e-06 0.000389618627041936 Beta-glucosidase, putative

MELO3C008069 $3.14379035937229 \mathrm{e}-06 \quad 0.000390482489353732$ Phosphoinositide phosphatase family protein

MELO3C013712 3.18040612723447e-06 0.00039256150379221 Fact complex subunit spt16

$\begin{array}{llll}\text { MELO3C022511 } 3.22910355765593 e-06 & 0.000396096684224516 & \text { synaptotagmin-5 }\end{array}$

MELO3C026068 3.27213557271833e-06 0.000398338562635824 Transcriptional adapter ADA2b-like protein

MELO3C010675 3.28772017366141e-06 0.000398338562635824 ATP-citrate synthase alpha chain protein

MELO3C009296 3.38391772602975 e-06 0.000405601118255557 Energy-coupling factor transporter ATP-binding EcfA 1

MELO3C010235 3.38873788607863e-06 0.000405601118255557 No data found

MELO3C002056 3.41335219067318e-06 0.000406086098877136 O-fucosyltransferase family protein

MELO3C007008 3.47097234532079e-06 0.000408989535555527 cytochrome c6, chloroplastic

MELO3C022204 3.47917575438395e-06 0.000408989535555527 SART-1 family protein DOT2 isoform X2

MELO3C019002 3.51510799601051e-06 0.000410768448598885 Annexin

MELO3C002674 3.53997063085032e-06 0.000411240470521547 BnaA04g24650D protein

MELO3C008598 3.67564570480372e-06 0.000424504836398647 Lecithin:cholesterol acyltransferase family protein

MELO3C012074 3.70429519958027e-06 0.000425326313351806 Ubiquitin-conjugating enzyme, E2

MELO3C012400 3.73601575998084e-06 0.000426488874241974 pentatricopeptide repeat-containing protein At2g22070

MELO3C012597 $3.76446955963061 \mathrm{e}-06 \quad 0.000427267295018074$ Defective in cullin neddylation protein

MELO3C017897 $3.79392199290063 \mathrm{e}-06 \quad 0.000428149516787397$ Plant/protein

MELO3C005069 4.07248831202356e-06 0.000456974839057689 GEM-like protein 4

MELO3C013128 4.09613167873246e-06 0.000457031099001624 Golgin family A protein

MELO3C004439 4.20919038268686e-06 0.000467007308245409 Protein phosphatase 2c, putative

MELO3C007827 4.27775306732769e-06 0.00047169877077526 pyridine nucleotide-disulfide oxidoreductase domain-containing protein 2-like

MELO3C026614 4.29924445488616e-06 0.00047169877077526 WD repeat-containing protein 55

MELO3C013870 $4.40428128078985 \mathrm{e}-06 \quad 0.000477912917661092$ Chloroplast $40 \mathrm{kDa}$ outer membrane envelope protein

MELO3C015804 4.40195694373369e-06 0.000477912917661092 heat stress transcription factor A-6b-like isoform X1

MELO3C010295 4.43891109647154e-06 0.000478586239136045 Armadillo-like helical

MELO3C013000 4.45895326350865e-06 0.000478586239136045 Malic enzyme

MELO3C006926 4.53250933496463e-06 0.000480700689972296 Divalent ion symporter

MELO3C016805 4.5614759661694e-06 0.000480700689972296 No data found

MELO3C017908 4.58576324779969e-06 0.000480700689972296 No data found

MELO3C015891 4.59599066471306e-06 0.000480700689972296 U1 small nuclear ribonucleoprotein C

MELO3C004732 $4.62469649575858 \mathrm{e}-06 \quad 0.000480700689972296$ Alpha/beta-Hydrolases superfamily protein

MELO3C001976 4.62163323611264e-06 0.000480700689972296 Pentatricopeptide repeat-containing family protein

MELO3C015764 4.69261976665525e-06 0.000482679936310805 F-box/LRR-repeat protein 17

MELO3C000673 4.68826114552456e-06 0.000482679936310805 ATP-dependent zinc metalloprotease FtsH

MELO3C014178 4.90082299253736e-06 0.00049501531360691 Trigger factor

MELO3C016366 $4.84117746490931 \mathrm{e}-06 \quad 0.00049501531360691$ Phosphatidylcholine transfer protein

MELO3C022210 4.87671299553849e-06 0.00049501531360691 regulator of nonsense transcripts UPF3-like

MELO3C011196 4.91280578596154e-06 0.00049501531360691 ABC1 family protein, expressed 
|MELO3C002469 4.96222207591046e-06 0.000497456465873887 BnaC01g13120D protein

MELO3C025257 $5.17156136781161 \mathrm{e}-06 \quad 0.000515824067943997 \mathrm{KH}$ domain-containing family protein MELO3C021534 5.25036197840389e-06 0.000517501450917762 homeobox-leucine zipper protein HAT5-like MELO3C023408 5.26699031011546e-06 0.000517501450917762 Lactoylglutathione lyase MELO3C025798 5.24348350627868e-06 0.000517501450917762 cytochrome P450 71A1-like

MELO3C015929 5.35053621086856e-06 0.000523107621922986 GTP-binding protein SAR1A

$\begin{array}{llll}\text { MELO3C017521 } 5.38464579769027 \mathrm{e}-06 & 0.000523849112603868 & \text { No data found }\end{array}$

MELO3C006836 5.46398631773304e-06 0.000526381784336145 Activating transcription factor 7-interacting 2

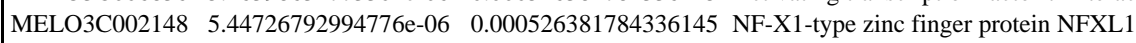

MELO3C023857 5.61354392358826e-06 0.000538164460907498 GATA zinc finger domain-containing protein 10-like isoform X2

MELO3C024270 5.74599306712997e-06 0.000548201048708936 Terpene cyclase/mutase family member

MELO3C017104 5.97652550948791e-06 0.000567453857148446 Trehalose-6-phosphate synthase, putative

$\begin{array}{llll}\text { MELO3C005382 } 6.01113701315104 \mathrm{e}-06 & 0.0005680093056111 & \text { luc7-like protein } 3\end{array}$

MELO3C023409 6.14485843719592e-06 0.000576681512004752 BEL1-like homeodomain protein 7

MELO3C020612 6.16131444797219e-06 0.000576681512004752 Unknown protein

MELO3C024316 6.22303370301225e-06 0.000579710814154665 Late embryogenesis abundant protein, LEA-14

MELO3C018948 6.26667399727943e-06 0.000581035421466063 Alpha-1,4 glucan phosphorylase

$\begin{array}{lll}\text { MELO3C009879 } 6.36776455420485 \mathrm{e}-06 & 0.000587649449443886 & \text { Cullin family protein }\end{array}$

MELO3C026738 6.6257926438773e-06 0.000608617576390385 Acyl-CoA N-acyltransferase (NAT) superfamily protein

$\begin{array}{lll}\text { MELO3C007207 } 6.76358441276115 e-06 & 0.000615548518744792 & \text { Kinase family protein }\end{array}$

MELO3C024545 $6.75704541253097 \mathrm{e}-06 \quad 0.000615548518744792$ Transmembrane 9 superfamily member

MELO3C016298 $7.03896222753464 \mathrm{e}-06 \quad 0.000634760114299459$ Ribosome maturation factor

MELO3C010136 $7.03790217027045 \mathrm{e}-06 \quad 0.000634760114299459$ Glycogenin-1

MELO3C016877 7.08417712036447e-06 0.000635933699773081 Beta-fructofuranosidase, insoluble isoenzyme CWINV1

MELO3C013630 7.27915942988488e-06 0.000650480179098627 switch 2 isoform X1

MELO3C009482 7.35514913974278e-06 0.000651375965743409 Rho GTPase-activating protein

MELO3C004305 7.32999088659536e-06 0.000651375965743409 Pre-mRNA-splicing factor SLU7

MELO3C002767 7.40044993308153e-06 0.00065246198985905 Nuclear-interacting partner of ALK

MELO3C011284 $7.49379739761746 \mathrm{e}-06 \quad 0.000657755576913543$ Aspartate aminotransferase

MELO3C004591 7.82543966471039e-06 0.0006773432768121 Phosphotransferase

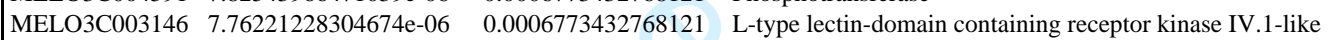

MELO3C022065 7.89187488892118e-06 0.0006773432768121 Nuclear matrix constituent protein 1

$\begin{array}{llll}\text { MELO3C023513 } 7.92274530070358 \mathrm{e}-06 & 0.0006773432768121 & \text { Metal tolerance protein C2 }\end{array}$

MELO3C011963 7.81226541701763 -06 0.0006773432768121 anthranilate phosphoribosyltransferase

$\begin{array}{lllll}\text { MELO3C017093 } 7.90303656583724 \mathrm{e}-06 & 0.0006773432768121 & \text { Kinesin light chain }\end{array}$

MELO3C025813 8.03564289664216e-06 0.000684034101576664 Polyadenylate-binding protein-interacting protein 3

MELO3C006383 8.19423848996692e-06 0.000685711931942189 acid phosphatase 1-like

MELO3C005211 8.1839472017009e-06 0.000685711931942189 Zinc finger, CCCH-type

MELO3C011984 8.09410918967135e-06 0.000685711931942189 pyrrolidone-carboxylate peptidase

MELO3C020001 8.17250552875848e-06 0.000685711931942189 Mini-chromosome maintenance complex-binding protein

MELO3C026410 8.27649747914272e-06 0.000689673201331601 transcription factor PIF4-like

MELO3C026629 8.48234927142144e-06 0.000703856788913034 potassium channel AKT1

MELO3C005952 8.62960587788564e-06 0.000713079859758843 60S acidic ribosomal protein P1-like

MELO3C026239 8.8767811139423e-06 0.000730448125913527 Protein tipD, putative

MELO3C020244 9.03752558945037e-06 0.000737638866316949 UTP--glucose-1-phosphate uridylyltransferase

MELO3C014083 9.07089525736815e-06 0.000737638866316949 Transmembrane protein, putative

MELO3C002302 9.07621877133113e-06 0.000737638866316949 Kinase family protein

MELO3C017657 9.15025518799517e-06 0.000738148417531329 branchpoint-bridging protein

MELO3C025502 9.15724149552766e-06 0.000738148417531329 Pentatricopeptide repeat-containing protein

MELO3C009423 9.29697871865365e-06 0.000746365986645085 Uridylate kinase

MELO3C005252 9.39636883812156e-06 0.000751291045279606 Ankyrin repeat-containing protein, putative

MELO3C020444 9.46083891961713e-06 0.000753301154100758 Purple acid phosphatase

MELO3C005301 9.59131909827882e-06 0.000753301154100758 At1g70780

MELO3C027060 9.50844332436862e-06 0.000753301154100758 Pectinesterase

MELO3C015322 9.61222800310857e-06 0.000753301154100758 cyclin-L1-1

MELO3C017346 9.60276551809081e-06 0.000753301154100758 alpha-dioxygenase 2

$\begin{array}{lll}\text { MELO3C014575 } 9.6661640416329 \mathrm{e}-06 & 0.000754533887977107 & \text { Saccharopine dehydrogenase }\end{array}$

MELO3C013703 1.01872779812995 -05 0.000788974717069347 diacylglycerol O-acyltransferase 2-like

MELO3C026689 $1.01759043160676 \mathrm{e}-05 \quad 0.000788974717069347$ LRR receptor-like kinase, putative

MELO3C009339 $1.05713260298845 \mathrm{e}-05 \quad 0.000815519991266366$ Glycosyl transferase, family 31

$\begin{array}{llll}\text { MELO3C017385 } \quad 1.078928932019 \mathrm{e}-05 & 0.000829096010834364 \text { pollen receptor-like kinase } 1\end{array}$

MELO3C008596 1.10425164834282e-05 0.000845266116400095 S-adenosyl-L-methionine-dependent methyltransferase

MELO3C024844 $1.11280763014365 \mathrm{e}-05 \quad 0.000848526559370927$ At5g24610

MELO3C013792 $1.13111690041023 e-05 \quad 0.000859170294853912$ Ribosomal protein L28

MELO3C012136 $1.13801551119286 \mathrm{e}-05 \quad 0.000861098403469263$ Nodulin-related protein 1

MELO3C009461 $1.15575520555078 \mathrm{e}-05 \quad 0.00086458369524327$ Protein LITTLE ZIPPER 4

MELO3C007614 $1.15006113516891 \mathrm{e}-05 \quad 0.00086458369524327$ Leucine-rich repeat family protein

MELO3C010714 1.15230725494797e-05 0.00086458369524327 two-component response regulator ARR11 
MELO3C014019 1.16203737828835e-05 0.000866002874861004 Rhomboid-like protein MELO3C026749 $1.20811670227816 \mathrm{e}-05 \quad 0.000896958524559827$ DNA helicase

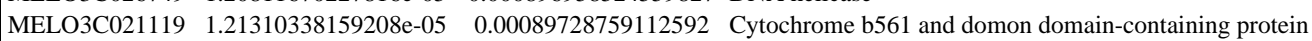

MELO3C011747 $1.22550755131678 \mathrm{e}-05 \quad 0.000903080172796835$ Protein FAR1-RELATED SEQUENCE 5

$\begin{array}{llll}\text { MELO3C009315 } 1.23509541338773 \mathrm{e}-05 & 0.00090491340308527 & \text { Ribosomal protein S12 }\end{array}$

MELO3C014678 $1.2388353868964 \mathrm{e}-05 \quad 0.00090491340308527$ Kelch repeat-containing F-box family protein

MELO3C016855 $1.24174151722167 \mathrm{e}-05 \quad 0.00090491340308527 \quad$ CRG16

MELO3C002700 1.24733027745405 -05 0.000905644325347061 Serine/threonine-protein kinase PLK4

$\begin{array}{llll}\text { MELO3C014174 } 1.25786625135849 \mathrm{e}-05 & 0.000909948739856364 & \text { (S)-ureidoglycine aminohydrolase }\end{array}$

MELO3C003916 $1.26667633769673 \mathrm{e}-05 \quad 0.000910771796448124$ Cellulose synthase

MELO3C023590 $1.2682274749265 \mathrm{e}-05 \quad 0.000910771796448124$ Pheophytinase, chloroplastic

MELO3C017364 $1.27819750068703 e-05 \quad 0.000914605885545946$ Ribosomal protein L19

MELO3C007033 $1.28479493655131 \mathrm{e}-05 \quad 0.000916007769023535$ protein SUPPRESSOR OF FRI 4 isoform X2

MELO3C014749 $1.29109006289374 \mathrm{e}-05 \quad 0.000917184807629082$ Acetyl-coenzyme A synthetase

MELO3C006107 $1.32783063222508 \mathrm{e}-05 \quad 0.000939904199133086$ RNA polymerase I-specific transcription initiation factor RRN3

MELO3C008910 $1.34152483453054 \mathrm{e}-05 \quad 0.000942838930859203$ transcription termination factor MTEF18, mitochondrial-like

MELO3C023986 $1.33894728500117 \mathrm{e}-05 \quad 0.000942838930859203$ DNA topoisomerase 2

MELO3C007432 $1.35509775082143 \mathrm{e}-05 \quad 0.000949000903580584$ Phosphatidate cytidylyltransferase

MELO3C003915 $1.36025675472329 \mathrm{e}-05 \quad 0.000949247726114142$ Pentatricopeptide repeat-containing protein

MELO3C022707 $1.38477429754147 \mathrm{e}-05 \quad 0.000962954493033326$ Ubiquitin-conjugating enzyme family protein

MELO3C009222 1.39431327602635 -05 0.000963333195559096 Protein RETICULATA, chloroplastic

MELO3C025451 $1.39726341720214 \mathrm{e}-05 \quad 0.000963333195559096$ Actin-depolymerizing factor family protein

MELO3C016016 $1.39995254000436 \mathrm{e}-05 \quad 0.000963333195559096$ xyloglucan 6-xylosyltransferase 1

MELO3C025977 $1.4120841748122 \mathrm{e}-05 \quad 0.000968307304457158$ OBERON-like protein

MELO3C020875 $1.4385688624774 \mathrm{e}-05 \quad 0.000983055241005747$ CLK4-associating serine/arginine-rich protein

MELO3C009276 $1.46222781025607 \mathrm{e}-05 \quad 0.000995777138784382$ Acyl-CoA N-acyltransferase with RING/FYVE/PHD-type zinc finger domain-

MELO3C022342 $1.4755856923232 \mathrm{e}-05 \quad 0.00100142068170759$ NAC domain-containing protein 83

MELO3C006313 $1.50251507858856 \mathrm{e}-05 \quad 0.00101325938561605$ Myb family transcription factor APL

MELO3C024262 $1.5032913058155 \mathrm{e}-05 \quad 0.00101325938561605$ activating signal cointegrator 1 complex subunit 1

MELO3C014825 $1.52073123453889 \mathrm{e}-05 \quad 0.00102152793030301$ Steroid nuclear receptor, ligand-binding

$\begin{array}{llll}\text { MELO3C004636 } 1.56355188315471 \mathrm{e}-05 & 0.00103625657153415 & \text { T-complex protein } 1 \text { subunit beta }\end{array}$

MELO3C016380 $1.55814578131075 \mathrm{e}-05 \quad 0.00103625657153415$ Laccase

MELO3C024447 $1.56364604950721 \mathrm{e}-05 \quad 0.00103625657153415$ Protein SEC13 like

MELO3C011160 $1.54835320682611 \mathrm{e}-05 \quad 0.00103625657153415$ 2-on-2 hemoglobin

MELO3C017720 1.5829999835848 -05 0.00104557413631492 protein farnesyltransferase/geranylgeranyltransferase type-1 subunit alpha

MELO3C006959 $1.59256922638296 \mathrm{e}-05$

MELO3C009591 1.61324199619006e-05

MELO3C004553 1.60189519178289e-05

MELO3C025526 1.62295206489471e-05

MELO3C003813 $1.61832411422758 \mathrm{e}-05$

MELO3C013093 $1.62023783316645 \mathrm{e}-05$

MELO3C013925 $1.63110244950637 \mathrm{e}-05$

MELO3C011731 $1.65099639795052 \mathrm{e}-05$

MELO3C006948 1.66519377275964e-05

MELO3C025282 $1.67030612752139 \mathrm{e}-05$

MELO3C025799 $1.66160070578236 \mathrm{e}-05$

MELO3C001954 1.67788745651931e-05

MELO3C021588 1.71895920806353e-05

MELO3C021279 1.70987463855576e-05

MELO3C008321 1.71502866039841e-05

MELO3C012083 $1.73075270275547 \mathrm{e}-05$

MELO3C024407 1.74182752812335e-05

MELO3C015627 1.76483058498267e-05

MELO3C018053 1.80475750266584e-05

MELO3C006602 $1.86668661633638 \mathrm{e}-05$

MELO3C017677 1.86747600382509e-05

MELO3C019451 1.89781614502449e-05

MELO3C008933 1.9062906662648e-05

MELO3C021107 1.93475282579403e-05

MELO3C023442 $1.95211350888513 \mathrm{e}-05$

MELO3C004593 $1.95826094848695 \mathrm{e}-05$

MELO3C020394 1.97117608555786e-05

MELO3C012088 $1.97023626935522 \mathrm{e}-05$

MELO3C014649 2.00030745243218e-05

MELO3C007069 2.00138907577863e-05

MELO3C012243 2.02363198715805e-05

MELO3C025855 2.11718078086953e-05

0.0010483883217279 Reticulon-like protein

0.0010508747649051 IQ domain-containing protein IQM6

0.0010508747649051 multiprotein-bridging factor 1c

0.0010508747649051 Pollen Ole e 1 allergen/extensin

0.0010508747649051 Mannan endo-1,4-beta-mannosidase-like protein

0.0010508747649051 glyoxylate/succinic semialdehyde reductase 2, chloroplastic-like

0.00105270072795102 Myb transcription factor

0.00106206931150244 Ubiquitin carboxyl-terminal hydrolase

0.00106409276491677 Protein SGT1 homolog

0.00106409276491677 SCARECROW

0.00106409276491677 Alpha-ketoglutarate-dependent dioxygenase AlkB

0.00106548551057234 Glycosyltransferase

0.00108113775159384 CRS1/YhbY (CRM) domain protein

0.00108113775159384 GTP-binding protein SAR1A-like

0.00108113775159384 Biotin carboxyl carrier protein of acetyl-CoA carboxylase

0.00108509952783231 Actin-binding LIM protein 1, putative

0.00108858708395279 Charged multivesicular body 1

0.00109948388715529 serrate RNA effector molecule

0.00112082251321219 Costars family protein At4g33640

0.00115252448748568 serine/threonine-protein kinase RUNKEL

0.00115252448748568 chitinase-like protein 1

0.00116760034417722 Unknown protein

0.00116917187478458 Zinc finger family protein

0.00118295459927574 Peptidylprolyl isomerase

0.00118988548416582 glycerol kinase

0.00118995986066673 OXS3

0.00119048185057132 Phospholipid-transporting ATPase

0.00119048185057132 sphinganine C4-monooxygenase 2

0.00120138093791952 Metal tolerance protein C2

0.00120138093791952 Protein phosphatase 2c, putative

0.00121105176104195 tetraketide alpha-pyrone reductase 2-like

0.00125940371209013 Alkyl transferase

MELO3C020589 2.11652532021711e-05 $0.00125940371209013 \quad 17.5 \mathrm{kDa}$ class I heat shock protein 
MELO3C011138 2.13686692861481e-05 MELO3C019527 2.14406552635493e-05 MELO3C015263 2.16246135184006e-05 MELO3C017683 2.17107562363594e-05 MELO3C011825 2.18256773739656e-05 MELO3C008690 2.20750108673728e-05 MELO3C022786 2.23215132971744e-05 MELO3C007075 2.28317060992911e-05 MELO3C009187 2.32460548075242e-05 MELO3C010761 2.32330067020392e-05 MELO3C024385 2.38134500429865e-05 MELO3C012378 2.39378786565103e-05 MELO3C019633 2.39288368943935e-05 MELO3C014114 2.42431211936456e-05 MELO3C011271 2.4241787024426e-05 MELO3C025111 2.43574415158543e-05 MELO3C007673 2.49104628767816e-05 MELO3C005467 2.49680630366456e-05 MELO3C008520 2.63403040063892e-05 MELO3C013369 2.6235044301437e-05 MELO3C007265 2.60273344152573e-05 MELO3C019056 2.61549670041994e-05 MELO3C023560 2.58082812095717e-05 MELO3C012196 2.62297140761758e-05 MELO3C025859 2.62944593677483e-05 MELO3C021670 2.5745984559733e-05 MELO3C010119 2.58387265157367e-05 MELO3C009886 2.60319997607006e-05 MELO3C006493 2.65023384483642e-05 MELO3C016552 2.6816575519617e-05 MELO3C017856 2.66462100604326e-05 MELO3C025079 2.6770532013165e-05 MELO3C022436 2.67748264992651e-05 MELO3C010042 2.68630872994891e-05 MELO3C024214 2.70940042867984e-05 MELO3C004278 2.78234834507618e-05 MELO3C006705 2.79177989914725e-05 MELO3C005490 2.77615447602519e-05 MELO3C020109 2.78415158727618e-05 MELO3C026470 2.79308280978219e-05 MELO3C000817 2.77390788183673e-05 MELO3C002677 2.7819366417603e-05 MELO3C002044 2.78036725491759e-05 MELO3C021398 2.8164374234807e-05 MELO3C014530 2.84013662230631e-05 MELO3C012748 2.83333883075443e-05 MELO3C014507 2.84858782809705e-05 MELO3C006260 2.87159989742491e-05 MELO3C025345 2.88485624249102e-05 MELO3C010686 2.88107005061988e-05 MELO3C013022 2.88535809854773e-05 MELO3C016541 2.93398141502088e-05 MELO3C017836 2.96642891901566e-05 MELO3C003313 2.99724595917983e-05 MELO3C020701 3.0080522178122e-05 MELO3C016074 3.02469208611766e-05 MELO3C024292 3.02042248422341e-05 MELO3C008367 3.03231984830621e-05 MELO3C013104 3.07867267796968e-05 MELO3C007083 3.07940069153023e-05 MELO3C022499 3.08502797945476e-05 MELO3C012967 3.09977807957384e-05 MELO3C009171 3.14944790682503e-05 MELO3C023731 3.17116579537924e-05 MELO3C017474 3.17265623519525e-05 MELO3C007767 3.23184251792563e-05 MELO3C026235 3.25468898165759e-05 MELO3C013383 3.32537247063058e-05
$0.00126729684604246 \quad$ Ultraviolet-B receptor UVR8

0.00126775898443065 CBS domain-containing protein CBSX1, chloroplastic 0.00127481938022356 Werner syndrome-like exonuclease 0.00127608846699959 Clathrin interactor EPSIN 2 0.00127903650581141 nifU-like protein 2, chloroplastic 0.00128982067934836 splicing factor 3B subunit 2 0.00130037630119733 Phd finger protein, putative 0.001326186363985 ADP-ribosylation factor GTPase-activating protein AGD12 0.00134235770875379 Transferase family protein 0.00134235770875379 far upstream element-binding protein 1 0.00137028743648528 protein indeterminate-domain 7 0.00137028743648528 phosphoglucan phosphatase DSP4, amyloplastic 0.00137028743648528 Glyceraldehyde-3-phosphate dehydrogenase 0.00137976196096054 RPM1-interacting protein 4 $0.0013797619609605414 \mathrm{kDa}$ proline-rich protein DC2.15 0.00138228480602473 CoA ligase

0.00140884079117347 tubulin alpha chain-like 0.00140884079117347 At5g07590

0.00144498517728383 Cysteine protease 0.00144498517728383 Methyltransferase-like protein 0.00144498517728383 Protein DEHYDRATION-INDUCED 19 0.00144498517728383 two-component response regulator ARR8-like 0.00144498517728383 PsaB RNA-binding protein 0.00144498517728383 Phospholipid-transporting ATPase 0.00144498517728383 zinc finger CCCH domain-containing protein 25 0.00144498517728383 Nuclear inhibitor of protein phosphatase 1 $0.00144498517728383 \mathrm{U} 11 / \mathrm{U} 12$ small nuclear ribonucleoprotein $48 \mathrm{kDa}$ protein 0.00144498517728383 plant cysteine oxidase 2 0.00144950576797161 ATP sulfurylase 0.00144950576797161 Fiber expressed protein 0.00144950576797161 extensin-like 0.00144950576797161 universal stress protein A-like protein 0.00144950576797161 Amaranthin-like lectin 0.00144950576797161 endoribonuclease Dicer homolog 2 isoform X2 0.0014579822633787 Cytochrome b561 and domon domain-containing protein 0.00147094913094369 mevalonate kinase-like

0.00147094913094369 Binding protein

0.00147094913094369 U-box domain-containing protein 35-like isoform X2 0.00147094913094369 Nop53 protein 0.00147094913094369 Potassium transporter

0.00147094913094369 Magnesium transporter MRS2-like protein 0.00147094913094369 NAD(P)-binding Rossmann-fold superfamily protein 0.00147094913094369 DNA polymerase alpha subunit B 0.00147930379458299 serine incorporator 3 0.00148385868132083 Nuclear transcription factor Y subunit C8 0.00148385868132083 Lysine-specific demethylase 0.00148434725638756 F-box family protein 0.0014878051459065 Dynamin, putative 0.0014878051459065 Polyol transporter 5-like protein 0.0014878051459065 Alanine aminotransferase 2 0.0014878051459065 Serine/threonine-protein kinase ATM

0.00150893747305332 Peroxisomal membrane $22 \mathrm{kDa}$ (Mpv17/PMP22) family protein 0.00152166246030235 UPF0326 protein 0.00153348731730162 Membrane steroid-binding protein 0.00153503936045408 Myb transcription factor 0.00153552012010767 No data found 0.00153552012010767 O-fucosyltransferase family protein 0.00153552012010767 serine/threonine-protein kinase STY8-like 0.00155028543425578 Upstream activation factor subunit spp27 0.00155028543425578 187-kDa microtubule-associated protein AIR9 0.00155028543425578 Myeloid leukemia factor 0.00155374409374375 At5g57230 0.00157464422055412 Mitochondrial carrier protein 0.00157825662440481 phosphatidylcholine transfer protein-like isoform X1 0.00157825662440481 Zinc finger protein 0.00160365974589229 Lipoxygenase y domain-containing protein 1 0.00161094868919188 haloacid dehalogenase-like hydrolase domain-containing protein At3g48420 0.00163772521003699 Calcium-binding EF hand family protein 
MELO3C005849 3.3214611836252e-05 MELO3C023526 3.33581295046681e-05 MELO3C009996 3.39940585575293e-05 MELO3C016253 3.42100392104472e-05 MELO3C013470 3.44618684419151e-05 MELO3C022144 3.4767194971419e-05 MELO3C015277 3.55894665726719e-05 MELO3C020830 3.59045646862732e-05 MELO3C004542 3.60642823011847e-05 MELO3C004366 3.62660963766359e-05 MELO3C009495 3.67311633746592e-05 MELO3C013248 3.66059451775147e-05 MELO3C014150 3.65614978239392e-05 MELO3C024450 3.69654147589493e-05 MELO3C023306 3.68811505284672e-05 MELO3C013664 3.66656130084131e-05 MELO3C022386 3.69238571342745e-05 MELO3C025256 3.74632503545858e-05 MELO3C005565 3.75027854699894e-05 MELO3C003911 3.78070399631447e-05 MELO3C007825 3.79500651352949e-05 MELO3C004492 3.83150140621513e-05 MELO3C024005 3.84547735918783e-05 MELO3C017898 3.89913539624587e-05 MELO3C024586 3.89204117599018e-05 MELO3C002154 3.88440754720554e-05 MELO3C021113 3.92616078019881e-05 MELO3C001175 3.92192487606025e-05 MELO3C018502 3.92965738810203e-05 MELO3C013891 4.00972913509046e-05 MELO3C020824 4.01866788451999e-05 MELO3C024596 4.07119270215972e-05 MELO3C002115 4.08557565708678e-05 MELO3C014175 4.15915338907169e-05 MELO3C014009 4.16693491427367e-05 MELO3C013366 4.18519287170849e-05 MELO3C024435 4.20256696651577e-05 MELO3C006736 4.24021129614705e-05 MELO3C015860 4.25403537831492e-05 MELO3C020132 4.28311459950148e-05 MELO3C002226 4.29674849788952e-05 MELO3C010275 4.36787795317084e-05 MELO3C017620 4.38789811226803e-05 MELO3C020780 4.38459148834891e-05 MELO3C008330 4.42680489831648e-05 MELO3C004641 4.4476178534758e-05 MELO3C009595 4.45835594083954e-05 MELO3C013455 4.49710978889239e-05 MELO3C010297 4.49064579863823e-05 MELO3C003471 4.50313240207834e-05 MELO3C010997 4.52420342280657e-05 MELO3C018394 4.6087660756533e-05 MELO3C004669 4.6537154970494e-05 MELO3C026502 4.65858718168288e-05 MELO3C015043 4.71004272699638e-05 MELO3C025034 4.73219853005524e-05 MELO3C022721 4.73412372383741e-05 MELO3C005245 4.79549976355464e-05 MELO3C024885 4.83734309704165e-05 MELO3C025391 4.83084956899305e-05 MELO3C025056 4.8402534760128e-05 MELO3C023753 4.85878972154019e-05 MELO3C025784 4.88195283186998e-05 MELO3C024376 4.90235715564502e-05 MELO3C024863 4.93566951739188e-05 MELO3C021587 5.05070902130367e-05 MELO3C011705 5.07150635979947e-05 MELO3C021999 5.12860701999962e-05
0.00163772521003699 p-loop containing nucleoside triphosphate hydrolases superfamily protein 0.00163878034723306 chaperone protein dnaJ GFA2, mitochondrial 0.00166587757432419 dnaJ protein homolog 0.00167231204051268 Zinc finger protein, putative 0.00168046281446761 Ankyrin repeat domain-containing 2-like protein 0.0016911756982526 TMV resistance protein N-like 0.00172691984113931 GATA transcription factor 16-like 0.00173793933330689 BZIP transcription factor, putative (DUF630 and DUF632) 0.00174140222778997 tRNA (guanine(37)-N1)-methyltransferase 0.00174687594473703 Prenylyltransferase superfamily protein 0.00175067140545441 CASP-like protein 0.00175067140545441 nucleolar complex protein 4 homolog 0.00175067140545441 Fimbrin, putative 0.00175067140545441 nuclear pore complex protein NUP35 0.00175067140545441 adoMet-dependent rRNA methyltransferase spb1 isoform X1 0.00175067140545441 Transcriptional elongation regulator MINIYO 0.00175067140545441 50S ribosomal protein L14 0.00176764322254611 protein RETICULATA-RELATED 3, chloroplastic-like 0.00176764322254611 Elongation factor G, chloroplastic 0.00177774102912415 protein BONZAI 3 0.0017802276398027 random slug protein 5-like

0.00179308818178537 methyl-CpG-binding domain-containing protein 11-like 0.00179537428762649 Acyl-CoA--sterol O-acyltransferase 1 0.00180760621925962 F-box family protein 0.00180760621925962 Ribosomal protein S8e/ribosomal biogenesis NSA2 0.00180760621925962 Monoglyceride lipase 0.00180901640460669 asparagine--tRNA ligase, cytoplasmic 2 0.00180901640460669 Glutathione s-transferase 0.00180901640460669 MAR-binding filament-like protein 0.00184140770420847 AT5g47090/K14A3_4 0.00184140770420847 Pre-mRNA-splicing factor syf2 0.00186115705266093 DNA polymerase 0.0018634187910348 Poly(A) polymerase 0.00189178845108025 Phospho-2-dehydro-3-deoxyheptonate aldolase 0.00189178845108025 PGR5-like protein 1A, chloroplastic 0.00189571958769199 Protein nuclear fusion defective 4 0.00189923329569153 Poly(A) polymerase 0.00191187061387233 Beta-1,3-galactosyltransferase-like protein 0.00191373450310573 Calcium permeable stress-gated cation channel 1 0.00192243705058079 Potassium transporter 0.00192418335793243 polynucleotide 5'-hydroxyl-kinase NOL9 0.00195161135061473 Serine-rich protein-like protein 0.00195172522115273 BnaA10g17500D protein 0.00195172522115273 Triosephosphate isomerase 0.00196460606599668 S-acyltransferase 0.00196941715220389 Protein BREVIS RADIX 0.00196975551399642 serine/arginine-rich SC35-like splicing factor SCL33 0.00197627470685878 Calcium ion-binding protein 0.00197627470685878 Purple acid phosphatase 0.00197627470685878 protein SAWADEE HOMEODOMAIN HOMOLOG 1-like isoform X2 0.00198111958751678 Protein IQ-DOMAIN 32 0.00201368409796631 Abscisic acid receptor 0.00202648542403205 Pentatricopeptide repeat-containing protein 0.00202648542403205 Phytochrome 0.00204436557836157 DnaJ-like protein 0.00204582515146751 peroxiredoxin Q, chloroplastic 0.00204582515146751 Ninja-family protein AFP3 0.00206782368625416 Xyloglucan endotransglucosylase/hydrolase 0.00207353939040731 ATP-dependent DNA helicase 0.00207353939040731 No data found 0.00207353939040731 zinc finger CCCH domain-containing protein 53-like 0.00207697485304539 BnaC06g27400D protein 0.0020823690383715 Zinc finger (C3HC4-type RING finger) family protein 0.00208656576437141 Eukaryotic initiation factor 4A (ATP-dependent RNA helicase eIF4A) 0.00209622660858005 No data found 0.00214048181248339 NAC domain-containing protein 8 isoform X1 0.00214469334260556 Pyruvate dehydrogenase E1 component subunit alpha 0.00215959189846423 Expansin protein 
MELO3C025308 5.12768922132922e-05 MELO3C025887 5.14003217724213e-05 MELO3C023727 5.20365869023376e-05 MELO3C022647 5.23411990736022e-05 MELO3C023808 5.24853113137436e-05 MELO3C024514 5.27174142808118e-05 MELO3C026107 5.30162818748181e-05 MELO3C006189 5.33192345207878e-05 MELO3C007609 5.33345582096612e-05 MELO3C009292 5.42517615724769e-05 MELO3C023475 5.42682466746491e-05 MELO3C011843 5.43007606186308e-05 MELO3C020783 5.43366113658905e-05 MELO3C011285 5.41233647706196e-05 MELO3C016696 5.44683324148476e-05 MELO3C007010 5.52589988974672e-05 MELO3C011003 5.59736594196725e-05 MELO3C022693 5.60978884305285e-05 MELO3C016002 5.719334841503e-05 MELO3C020957 5.70871739773438e-05 MELO3C007653 5.75656860861695e-05 MELO3C026835 5.75725188270981e-05 MELO3C019411 5.82043706141011e-05 MELO3C007562 5.83480571787476e-05 MELO3C006145 5.87198712955761e-05 MELO3C009338 5.94246730396542e-05 MELO3C006854 5.93200212051226e-05 MELO3C019522 5.92040129191451e-05 MELO3C016186 5.91282394308834e-05 MELO3C002384 5.94135676414975e-05 MELO3C018749 5.95836233487912e-05 MELO3C004273 6.01342544248595e-05 MELO3C021332 6.05753663890374e-05 MELO3C015339 6.06364340023369e-05 MELO3C014260 6.11461584065776e-05 MELO3C003278 6.17486979386239e-05 MELO3C013736 6.21357963599412e-05 MELO3C021207 6.30709811876295e-05 MELO3C005992 6.3408310252755e-05 MELO3C015734 6.34573361908419e-05 MELO3C015439 6.32324988869559e-05 MELO3C022162 6.40116656055456e-05 MELO3C011408 6.39667592298165e-05 MELO3C013738 6.49744440790467e-05 MELO3C010439 6.49361610987054e-05 MELO3C027375 6.50197840010858e-05 MELO3C011144 6.49593068970944e-05 MELO3C017813 6.58591244822038e-05 MELO3C025582 6.5766423634761e-05 MELO3C011422 6.60698899755952e-05 MELO3C020255 6.65873213148149e-05 MELO3C016660 6.74206308142189e-05 MELO3C008046 6.73044786470012e-05 MELO3C012391 6.73894846392464e-05 MELO3C008513 6.82229065330775e-05 MELO3C022074 6.86128717086731e-05 MELO3C016019 6.84870669735993e-05 MELO3C013946 6.88829284867243e-05 MELO3C021771 6.96161904623072e-05 MELO3C005703 6.97949721109037e-05 MELO3C011011 7.00407553777538e-05 MELO3C019135 7.0205236232912e-05 MELO3C024138 7.04960768541651e-05 MELO3C020860 7.10014079137178e-05 MELO3C016468 7.31467793237961e-05 MELO3C014658 7.36569729663517e-05 MELO3C005581 7.39243833014491e-05 MELO3C017384 7.39395658053699e-05
0.00215959189846423 auxin-responsive protein IAA16-like 0.00215979777592244 No data found 0.00218189077438273 CDT1-like protein a, chloroplastic 0.0021900134332724 UDP-glycosyltransferase 89A2-like 0.00219140045060279 SAC3/GANP/Nin1/mts3/eIF-3 p25 family isoform 1 0.00219644771019357 enolase 0.00220424958051744 No data found 0.00220818488486918 Glutathione S-transferase 0.00220818488486918 Subtilisin-like protease 0.00222633555573646 ABC transporter G family member 20 0.00222633555573646 pre-mRNA-splicing factor ISY1 homolog 0.00222633555573646 No data found

0.00222633555573646 protein XAP5 CIRCADIAN TIMEKEEPER 0.00222633555573646 60S ribosomal protein L39

0.00222711200178225 PROTON PUMP INTERACTOR 1 family protein

0.00225477266369025 phosphatidate cytidylyltransferase, mitochondrial isoform X1 0.00227922432964765 transcription factor E2FC isoform X3 0.00227958271319858 GDSL esterase/lipase 5 0.00231457261854186 Arginine/serine-rich splicing factor, putative 0.00231457261854186 GDSL esterase/lipase 5-like 0.00232040749860482 Protein PHLOEM PROTEIN 2-LIKE A10

0.00232040749860482 Pentatricopeptide repeat-containing protein At2g30100, chloroplastic 0.00234109595775536 Kinase superfamily protein 0.00234210524638839 Lipase 0.00235224896189925 F-box protein PP2-A13 0.00235658206397616 60S ribosomal protein L12 0.00235658206397616 transcription factor bHLH118-like 0.00235658206397616 Exocyst complex component 0.00235658206397616 No data found 0.00235658206397616 Coiled-coil domain-containing protein SCD2 0.00235815025554164 Phosphatase 2C family protein 0.0023751827812731 Mitochondrial Rho GTPase 0.00238547596635887 40S ribosomal protein S21 0.00238547596635887 UDP-N-acetylglucosamine transferase subunit ALG13 homolog 0.00240074648582803 Interactor of constitutive active ROPs-like protein 0.0024195933245831 PHD finger protein At1g33420 0.00242994028180689 basic endochitinase C 0.00246163795943576 Signal peptide peptidase-like protein 0.00246211971008436 homeobox-leucine zipper protein ATHB-7 0.00246211971008436 No data found 0.00246211971008436 Protein PLASTID MOVEMENT IMPAIRED 1-RELATED 1 0.00247390681808986 mannan endo-1,4-beta-mannosidase 1-like 0.00247390681808986 nucleolar protein 14 isoform X2 0.00249335090143193 Ganglioside-induced differentiation-associated protein 2 0.00249335090143193 structure-specific endonuclease subunit SLX4-like isoform X2 0.00249335090143193 Pectinesterase 0.00249335090143193 Translation initiation factor IF-2 0.00251576760038499 ADP-ribosylation factor GTPase-activating protein AGD3 0.00251576760038499 Ribosomal protein S5/S7

0.0025189464423321 Pentatricopeptide repeat-containing protein 0.00253378229026258 3-phosphoinositide-dependent protein kinase-1 0.00255074719913795 Flavin-binding kelch domain F box protein 0.00255074719913795 zinc finger CCCH domain-containing protein 38 isoform X1 0.00255074719913795 NAC domain-containing protein 16 0.00257616478225956 FAST kinase domain-containing 3 0.00258102019690397 Tryptophan synthase-related 0.00258102019690397 heat shock factor-binding protein 1 0.00258625276555954 Small heat shock protein, chloroplastic 0.00260882380538919 exocyst complex component EXO84C 0.00261056989435272 Beta-carotene 3-hydroxylase

0.00261481073337478 Transducin/WD40 repeat-like superfamily protein 0.00261600605729015 4-alpha-glucanotransferase DPE2 0.00262189646288683 Auxin response factor 0.00263572707685717 DNA polymerase 0.00271027344252467 No data found 0.00271924112679749 Peroxidase 0.00271924112679749 Ankyrin repeat/KH domain protein (DUF1442) 0.00271924112679749 tRNA pseudouridine synthase 
MELO3C025026 7.38424094659518e-05 MELO3C014419 7.56717115111272e-05 MELO3C014309 7.59720866843061e-05 MELO3C004308 7.65458843351441e-05 MELO3C014027 7.6019752883516e-05 MELO3C021979 7.63281862419252e-05 MELO3C012242 7.65118129815168e-05 MELO3C015202 7.64393255101581e-05 MELO3C020003 7.64967470061606e-05 MELO3C013084 7.63203350274688e-05 MELO3C009827 7.66422901630204e-05 MELO3C004186 7.71375798698237e-05 MELO3C016917 7.71474370981906e-05 MELO3C002821 7.79612487069592e-05 MELO3C015603 7.8575539132153e-05 MELO3C011173 7.89147137463253e-05 MELO3C006158 7.91940140983938e-05 MELO3C010737 7.96416118012955e-05 MELO3C025265 8.02798553996009e-05 MELO3C015594 8.04513144398999e-05 MELO3C008076 8.00977753115939e-05 MELO3C003393 8.0430383471497e-05 MELO3C019730 8.11006363776734e-05 MELO3C009560 8.21626649760709e-05 MELO3C023161 8.22211586961608e-05 MELO3C023018 8.26704742665774e-05 MELO3C021786 8.280598956667e-05 MELO3C015704 8.32907324128662e-05 MELO3C025307 8.33075462164379e-05 MELO3C016262 8.34670928354653e-05 MELO3C025654 8.41080056708243e-05 MELO3C010913 8.4125360631182e-05 MELO3C023419 8.4525555592907e-05 MELO3C022998 8.50718977087883e-05 MELO3C003977 8.50368989402384e-05 MELO3C008197 8.52787411298461e-05 MELO3C016126 8.64520687487991e-05 MELO3C005754 8.61995591473486e-05 MELO3C005828 8.65793669142523e-05 MELO3C025956 8.6286284875392e-05 MELO3C021232 8.63064375860034e-05 MELO3C007483 8.75097762822463e-05 MELO3C008879 8.77609174688709e-05 MELO3C025073 8.93035963320798e-05 MELO3C005872 8.92453339496058e-05 MELO3C019225 8.99096474886729e-05 MELO3C003659 8.9996260208558e-05 MELO3C014565 9.04686218434758e-05 MELO3C022494 9.03951638639988e-05 MELO3C010877 9.02100101682901e-05 MELO3C004610 9.15000192608373e-05 MELO3C022068 9.14387798359728e-05 MELO3C021228 9.16269800550484e-05 MELO3C003638 9.1278293153696e-05 MELO3C006670 9.20264952706251e-05 MELO3C026234 9.21463782431742e-05 MELO3C025779 9.2346093012563e-05 MELO3C007571 9.30114872104149e-05 MELO3C024850 9.32171318644981e-05 MELO3C012943 9.46720039655125e-05 MELO3C013810 9.56120904125335e-05 MELO3C021436 9.61451448351358e-05 MELO3C010021 9.6358700537813e-05 MELO3C022901 9.65210274401773e-05 MELO3C021923 9.72721588077263e-05 MELO3C017755 9.79998273007432e-05 MELO3C009312 9.82774985767154e-05 MELO3C017946 $9.85155856012554 \mathrm{e}-05$
0.00271924112679749 abscisic stress-ripening protein 2-like 0.0027671089367998 Unknown protein 0.0027671089367998 Magnesium transporter MRS2-like protein 0.0027671089367998 Photosystem II reaction center PsbP family protein 0.0027671089367998 Cytochrome P450 family protein 0.0027671089367998 Glutaredoxin family protein, putative 0.0027671089367998 ethylene-responsive transcription factor ERF118 0.0027671089367998 zinc finger A20 and AN1 domain-containing stress-associated protein 4-like 0.0027671089367998 40S ribosomal protein S26 0.0027671089367998 Aminopeptidase 0.0027671089367998 No data found 0.00277519988206223 Unknown protein 0.00277519988206223 Damaged dna-binding 2, putative isoform 1 0.00279937581947952 Unknown protein 0.00281631274468401 E3 ubiquitin-protein ligase RING1-like 0.00282334543800032 Gamma-glutamylcyclotransferase 0.00282821443838911 lysophospholipid acyltransferase LPEAT2 isoform X1 0.00283906532755196 RING-type E3 ubiquitin transferase 0.00284737098364442 Unknown protein 0.00284737098364442 No data found 0.00284737098364442 No data found 0.00284737098364442 Plant intracellular ras group-related LRR protein 0.00286521729485272 zinc finger CCCH domain-containing protein 3-like 0.00289444859730923 Polyketide cyclase/dehydrase/lipid transport superfamily protein 0.00289444859730923 nuclear transcription factor Y subunit A-7-like 0.00290468115089194 splicing factor U2af large subunit B isoform X1 0.00290468115089194 Cyclin-T1-like protein

0.00291193049597952 Protein trigalactosyldiacylglycerol 2, chloroplastic 0.00291193049597952 Transmembrane 19

0.00291235267916538 26S proteasome non-ATPase regulatory subunit 8 0.00292498547025566 Transmembrane protein 0.00292498547025566 Protein DA1-related 1 0.00293373496907613 methyl-CpG-binding domain-containing protein 11 0.00294235535525545 Malate dehydrogenase 0.00294235535525545 Microtubule-associated family protein 0.00294435289960373 Chloride channel protein 0.00296335514244293 Pathogen-related protein 0.00296335514244293 PHD finger family protein 0.00296335514244293 SKP1-like protein 21 0.00296335514244293 No data found 0.00296335514244293 Novel plant snare, putative 0.0029900182903081 ABC transporter family protein 0.00299342030931387 Triosephosphate isomerase 0.00303555374175946 GRIP and coiled-coil domain-containing protein 1 0.00303555374175946 Polypyrimidine tract binding protein, putative 0.00304860401862575 Formin-like protein 0.00304860401862575 Glucan endo-1,3-beta-glucosidase, putative 0.00304891606277612 Homeobox domain-containing protein 0.00304891606277612 No data found 0.00304891606277612 Phosphatidate phosphatase PAH2 0.003067019032385 zinc finger CCCH domain-containing protein 20-like 0.003067019032385 proline-rich receptor-like protein kinase PERK8 0.003067019032385 tRNA pseudouridine synthase 0.003067019032385 replication protein A $32 \mathrm{kDa}$ subunit A-like 0.00307398449987238 Translationally-controlled tumor protein homolog 0.00307398449987238 elongator complex protein 3 0.00307545192395465 Nucleotide/sugar transporter family protein 0.00309239707225334 OTU domain-containing protein 0.00309402544065878 60S ribosomal protein L9-like 0.00313704262804514 ADP,ATP carrier protein 0.00316288638786788 At3g49990 0.00317520144707207 60S acidic ribosomal protein P2B-like 0.00317694153075337 ER membrane protein complex subunit 10 0.00317698961819344 Alpha/beta-Hydrolases superfamily protein, putative 0.00319638579749382 CCG-binding protein 1 0.00321494782286109 zinc finger protein CONSTANS-LIKE 15-like 0.00321871031408218 Pentatricopeptide repeat-containing protein At3g09650, chloroplastic 0.00322116605966754 bifunctional epoxide hydrolase 2-like 
MELO3C016303 9.87452484355611e-05 MELO3C002041 9.90924691245398e-05 MELO3C009572 0.00010010530796678 MELO3C006316 9.98384531540886e-05 MELO3C020557 0.000100154739182123 MELO3C023996 0.000100250959649495 MELO3C024366 0.000100516259118888 MELO3C017255 0.000101150217111434 MELO3C017754 0.000102643919310808 MELO3C025278 0.00010184182706352 MELO3C005405 0.000102359121522122 MELO3C021901 0.000102486479856445 MELO3C025903 0.000102095232691002 MELO3C012956 0.00010262148165241 MELO3C003145 0.000103066071958313 MELO3C006853 0.000103656565781596 MELO3C013261 0.000104022481609345 MELO3C013440 0.000103896359409772 MELO3C021116 0.000104315721652704 MELO3C015563 0.000103478026248882 MELO3C008332 0.000104296975344309 MELO3C025812 0.000103525632135115 MELO3C024396 0.000104622452385073 MELO3C003906 0.000107750928353134 MELO3C021258 0.00010778944155021 MELO3C015373 0.000108200386231605 MELO3C009776 0.000108425898432851 MELO3C013031 0.000108816587797156 MELO3C005869 0.000108994104927262 MELO3C009354 0.000109747032851715 MELO3C016198 0.000109404821318382 MELO3C009076 0.000109755888868523 MELO3C022658 0.000109846504569466 MELO3C007433 0.000111052139491363 MELO3C007572 0.000110860800119461 MELO3C005299 0.000110942650853096 MELO3C011955 0.000111068147271243 MELO3C008752 0.00011157295959352 MELO3C014730 0.000111888076964051 MELO3C025853 0.000112716329796925 MELO3C002085 0.000113224239913556 MELO3C020294 0.000113888702424569 MELO3C023310 0.000114261528182169 MELO3C022773 0.00011559372525527 MELO3C012529 0.000116333438386462 MELO3C002885 0.000116374025256993 MELO3C024324 0.000116198570528248 MELO3C019844 0.000116328959425371 MELO3C022837 0.000116753774780598 MELO3C010776 0.000116715602386908 MELO3C010102 0.000117010176775678 MELO3C019470 0.000118662693332494 MELO3C005094 0.000118738009081243 MELO3C012180 0.000118248526876918 MELO3C003658 0.000118704100653622 MELO3C025049 0.000119458467457201 MELO3C005331 0.000120522869021755 MELO3C001323 0.000120652579684855 MELO3C015024 0.000121409478646695 MELO3C014719 0.00012160994411281 MELO3C012622 0.000122331082063387 MELO3C019012 0.000123020613497338 MELO3C011773 0.00012378596496454 MELO3C011099 0.000124519937175549 MELO3C001165 0.000124820210286147 MELO3C005945 0.000125956652920811 MELO3C014016 0.000125606445577908 MELO3C016924 0.000125729655771067
0.00322333869645272 ATP-dependent zinc metalloprotease FtsH 0.00322933526854874 RHOMBOID-like protein 12, mitochondrial 0.00324566590511127 ABC transporter family protein 0.00324566590511127 cytochrome b5 0.00324566590511127 Tetratricopeptide repeat (TPR)-like superfamily protein 0.00324566590511127 RNA polymerase sigma factor sigD, chloroplastic 0.00324892897109479 Mitochondrial carrier protein 0.00326407783943417 BSD domain-containing protein 0.00328012097486915 NAC domain-containing protein $0.00328012097486915 \mathrm{Emp24/gp25L/p24} \mathrm{family/GOLD} \mathrm{family} \mathrm{protein}$ 0.00328012097486915 CDGSH iron-sulfur domain-containing protein NEET 0.00328012097486915 Alpha/beta hydrolase-3

0.00328012097486915 At5g59460 0.00328012097486915 Protein MARD1 0.00328829055751975 Cyclin d, putative 0.00329094438804991 receptor-like serine/threonine-protein kinase isoform X2 0.00329094438804991 No data found 0.00329094438804991 PHD domain-containing protein/BAH domain-containing protein 0.00329094438804991 No data found 0.00329094438804991 TATA-box-binding protein

0.00329094438804991 5-formyltetrahydrofolate cyclo-ligase-like protein COG021_

0.00329094438804991 ATP-dependent zinc metalloprotease FtsH 0.00329535695718149 Cytochrome p450 0.00338431427849777 Ethylene receptor 0.00338431427849777 flowering time control protein FCA isoform X2 0.0033918244883936 40S ribosomal protein S25 0.00339350723954101 DNA-directed RNA polymerase II, putative 0.00340034619051587 Glutamate dehydrogenase, putative 0.00340051276178279 Bidirectional sugar transporter SWEET 0.00340558652863798 Glutathione gamma-glutamylcysteinyltransferase 1 0.00340558652863798 No data found 0.00340558652863798 Nucleolar protein gar2-like protein 0.00340558652863798 Ubiquitin-specific protease family C19 protein 0.00342197323004645 fumarylacetoacetase 0.00342197323004645 AP2-like ethylene-responsive transcription factor TOE3 0.00342197323004645 C2 domain-containing protein 0.00342197323004645 Tetratricopeptide-like helical 0.0034321719299259 TATA box-binding protein-associated factor RNA polymerase I subunit B $0.00343651264690986 \quad \mathrm{~N}$ utilization substance B 0.00345657577198675 protein DAMAGED DNA-BINDING 2 0.00346676823884159 ethylene-responsive transcription factor ERF071 0.00348171514579384 DNA-directed RNA polymerase subunit beta 0.00348771394137505 Glucose-1-phosphate adenylyltransferase 0.00352293283960852 Transmembrane protein, putative 0.00352495494601283 Ras-related Rab7 0.00352495494601283 transcription factor GTE1 0.00352495494601283 Chaperone protein DnaJ 0.00352495494601283 ras-related protein RABC2a-like 0.00352564265770953 Cysteine-rich repeat secretory protein 60 0.00352564265770953 Branched-chain-amino-acid aminotransferase 0.00352799081090514 O-linked n-acetylglucosamine transferase, ogt, putative 0.00355835651190511 glutelin type-A 2-like 0.00355835651190511 histidine biosynthesis bifunctional protein hisIE, chloroplastic 0.00355835651190511 ruBisCO large subunit-binding protein subunit beta, chloroplastic-like 0.00355835651190511 Protein MARD1 0.00357452314213979 transcription factor TCP21-like 0.00359934712416343 Acyl-[acyl-carrier-protein] hydrolase 0.00359934712416343 transmembrane protein 184C 0.00361646424403254 RING-type E3 ubiquitin transferase 0.00361698009982514 Aldehyde oxidase, putative 0.00363295720251103 peptidyl-prolyl cis-trans isomerase CYP59 0.00364794909303142 peptidyl-prolyl cis-trans isomerase CYP59 0.00366514096264573 Oxidoreductase/transition metal ion-binding protein (DUF3531) 0.00368135365161664 Universal stress protein A-like protein 0.00368471499692246 eukaryotic translation initiation factor 3 subunit M 0.00369128273352684 Dihydroorotate dehydrogenase (DUF3598) 0.00369128273352684 Phospholipase D 0.00369128273352684 pentatricopeptide repeat-containing protein At4g33170-like 
MELO3C011550 0.0001259772425133980 .00369128273352684 transcription factor HBP-1b(C38) isoform X2 
MELO3C011402 0.000153953444997246

MEl $0.00410313979116141 \quad$ D-aminoacyl-tRNA deacylase

$\begin{array}{lllll}\text { MELO3C008974 } & 0.000155525458351624 & 0.00413387924224256 & \text { 60S ribosomal protein L35a }\end{array}$

MELO3C023979 0.0001563789117935420 .00415097732393906 Cellulose synthase

MELO3C021064 $0.000157428826421557 \quad 0.00417323744026756$ malonyl-CoA:anthocyanidin 5-O-glucoside-6"-O-malonyltransferase MELO3C006645 $0.000158263172499673 \quad 0.00417852860119792$ No data found

MELO3C003147 $0.000158254093796217 \quad 0.00417852860119792$ LOW QUALITY PROTEIN: L-type lectin-domain containing receptor kinase MELO3C001121 0.00015812671487514 MELO3C010760 0.000158563073288098 MELO3C024871 $0.000160253718284764-0.004219805225187$ Pollen specific protein sf2

$\begin{array}{llll}\text { MELO3C017653 } 0.000160928051555986 & 0.00422261443857199 & \text { Isoaspartyl peptidase/L-asparaginase }\end{array}$

$\begin{array}{llll}\text { MELO3C008785 } 0.000160986249053918 & 0.00422261443857199 & \mathrm{BTB} / \mathrm{POZ} \text { domain protein }\end{array}$

MELO3C024222 $0.000161002008822964 \quad 0.00422261443857199$ aldo-keto reductase family 4 member C9-like

MELO3C025594 $0.000161236287363531 \quad 0.00422315044979094 \quad$ C2 domain-containing protein

MELO3C019722 $0.000162089692921086 \quad 0.00423791057130512$ Protein disulfide-isomerase like 2-2

MELO3C015230 $0.000162228993463298 \quad 0.00423791057130512$ Pectin acetylesterase

MELO3C018510 0.0001625854144093890 .00424161076508721 somatic embryogenesis receptor kinase 1-like

$\begin{array}{llll}\text { MELO3C013260 } & 0.000162887802810818 & 0.00424389342705917 & \text { protein LAZ1 isoform X1 }\end{array}$

MELO3C009203 0.0001654373360133740 .00426283287985112 tubulin beta chain-like

MELO3C023685 $0.000165296367253043 \quad 0.00426283287985112$ alcohol dehydrogenase-like

MELO3C006088 $0.000165137396803905 \quad 0.00426283287985112$ 50S ribosomal protein L5, chloroplastic

$\begin{array}{llll}\text { MELO3C006974 } 0.000165557386138326 & 0.00426283287985112 & \text { Plastid division protein PDV1 }\end{array}$

MELO3C023255 $0.000164791051848967 \quad 0.00426283287985112$ DNA-directed RNA polymerase subunit beta-beta protein, putative (DUF506)

MELO3C002817 $0.000164407991422699 \quad 0.00426283287985112$ No data found

$\begin{array}{llll}\text { MELO3C002163 } 0.000164730021437798 & 0.00426283287985112 & \text { E3 ubiquitin-protein ligase RING1-like }\end{array}$

MELO3C010165 $0.000165256170291617 \quad 0.00426283287985112$ Cyclic nucleotide-gated ion channel-like protein

MELO3C026141 0.0001651349128699580 .00426283287985112 ADP-ribosylation factor-like

MELO3C014128 $0.000165992853658037 \quad 0.00426848029543303$ Calcium-dependent protein kinase

MELO3C012572 $0.000166407907161714 \quad 0.00427358876272652$ CTP synthase

MELO3C003973 $0.000167132576897933 \quad 0.00427592256812336$ SNW/SKI-interacting protein-like

MELO3C017838 0.0001669392059696630 .00427592256812336 Ubiquitin system component Cue

$\begin{array}{llll}\text { MELO3C014217 } & 0.000167148322577915 & 0.00427592256812336 & \text { Telomere repeat-binding protein } 4\end{array}$

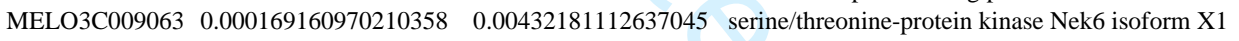

MELO3C018306 $0.000169535131114396 \quad 0.00432577429506227$ Mitogen-activated protein kinase

MELO3C005001 0.0001705625451323690 .00434637381137955 dof zinc finger protein DOF5.3-like

MELO3C005882 0.0001716377613670830 .00436813679025582 Eukaryotic translation initiation factor 3 subunit 10

$\begin{array}{llll}\text { MELO3C015374 } 0.000172882858217371 & 0.00439416160480678 & \text { Superoxide dismutase [Cu-Zn] }\end{array}$

MELO3C012201 $0.000173729943490741 \quad 0.0044017019494474$ Isoprenylcysteine alpha-carbonyl methylesterase ICME proteir

MELO3C020583 $0.000173737441671706 \quad 0.0044017019494474$ Neutral ceramidase

MELO3C002566 $0.000173848170569091 \quad 0.0044017019494474$ Katanin p80 WD40 repeat-containing subunit B1 homolog

MELO3C014799 $0.000175285251514445 \quad 0.00442673712552274$ TRANSPORT INHIBITOR RESPONSE 1 protein, putative

MELO3C015185 0.0001750883117238590 .00442673712552274 MP domain-containing protein

MELO3C022562 $0.00017556921672901 \quad 0.00442824579972059$ protein CHROMATIN REMODELING 19 isoform X1

$\begin{array}{llll}\text { MELO3C010421 } & 0.000176144776798615 & 0.00443709591453551 & \text { Random slug protein } 5\end{array}$

MELO3C014379 0.0001775151422507280 .00446023733372727 Protein RETICULATA-RELATED 1, chloroplastic

MELO3C001948 $0.000177457027202066 \quad 0.00446023733372727$ Short-chain dehydrogenase, putative

MELO3C003508 $0.000178471292703875 \quad 0.00447856360814335$ UDP-glycosyltransferase 91C1

MELO3C005673 0.0001835149225359880 .00459928452431881 tubulin beta chain-like

MELO3C009561 $0.000183781133722527 \quad 0.00460011864370873$ iron-sulfur cluster co-chaperone protein HscB, mitochondrial

$\begin{array}{llll}\text { MELO3C006651 } 0.000184318937710204 & 0.00460191491888599 & \text { No data found }\end{array}$

MELO3C017358 0.000184207700662187

MELO3C013346 0.000185552097884845

MELO3C015111 0.000188240908695514

MELO3C010245 0.000189301601007941

0.00460191491888599 C2 domain-containing protein

0.0046268540165755 Phospholipase-like protein (PEARLI 4) family protein

0.00468798197456205 rho GTPase-activating protein 7

MELO3C012076 $0.00019072837700751 \quad 0.00473798077675636$ UDP-galactose/UDP-glucose transporter family protein

MELO3C025468 $0.000191221524523888 \quad 0.00474349870973502$ ABC transporter B family protein

$\begin{array}{lll}\text { MELO3C004833 } 0.000191430881141263 & 0.00474349870973502 & \text { crooked neck-like protein } 1\end{array}$

MELO3C015350 $0.000191817108435632 \quad 0.0047471128753074$ Sugar transporter ERD6-like protein

$\begin{array}{llll}\text { MELO3C006239 } 0.000194745937404539 & 0.0048075468972528 & \text { Receptor protein kinase, putative }\end{array}$

MELO3C022684 $0.000194616340531306 \quad 0.0048075468972528$ peroxisomal (S)-2-hydroxy-acid oxidase GLO4-like

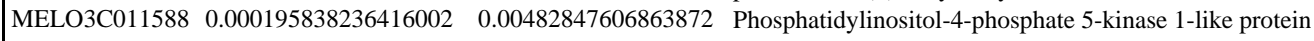

MELO3C003733 0.0001962444461800490 .00483245831372793 ribonuclease P protein subunit p25-like protein isoform X1

MELO3C000826 $0.000197316742958864 \quad 0.00484075572260199$ Transcription factor

MELO3C021280 0.0001970736551358820 .00484075572260199 Calmodulin-binding transcription activator

MELO3C018455 0.0001970934594621720 .00484075572260199 thioredoxin M3, chloroplastic

MELO3C024902 $0.000197626941677576 \quad 0.00484235046053404$ ABC subfamily C transporter

$\begin{array}{llll}\text { MELO3C005808 } & 0.000198080417585844 & 0.00484539295425683 & \text { No data found }\end{array}$

MELO3C024344 0.0001982418100683340 .00484539295425683 DUF1677 family protein (DUF1677) 
MELO3C025606 0.000198581595278524 MELO3C016360 0.00019959105733458 MELO3C019026 0.000200262784349703 MELO3C019564 0.000201417398875803 MELO3C000994 0.000201282242801692 MELO3C013841 0.000206757258935486 MELO3C003817 0.00020674784003627 MELO3C004551 0.000207150930850553 MELO3C005035 0.000208333784670112 MELO3C011395 0.000212015636750662 MELO3C009566 0.000212899672441647 MELO3C007624 0.00021680183247641 MELO3C005520 0.000216989391112588 MELO3C023503 0.000216126270384365 MELO3C022540 0.000216005163480082 MELO3C013615 0.00021629852461702 MELO3C003842 0.000216935765092741 MELO3C026764 0.000217488779697295 MELO3C020601 0.000218758247361506 MELO3C017432 0.000219003420802943 MELO3C019629 0.000220238525441285 MELO3C004999 0.000222439350828108 MELO3C010189 0.00022317153203999 MELO3C017165 0.000223376505001038 MELO3C009914 0.000223519853685517 MELO3C021308 0.00022430380657712 MELO3C019921 0.000224478914295334 MELO3C019139 0.000225618022833496 MELO3C026027 0.000225677263039903 MELO3C012869 0.000228239696927757 MELO3C013214 0.000232109115587242 MELO3C017043 0.000233289549711801 MELO3C011682 0.00023316957210473 MELO3C009429 0.000234027845987472 MELO3C005111 0.00023446163867713 MELO3C005745 0.000234311765738404 MELO3C023464 0.000234798570744266 MELO3C021339 0.000234900900137558 MELO3C014614 0.000235860026803869 MELO3C010705 0.000235766264270243 MELO3C019435 0.000236976943461809 MELO3C015852 0.000236938477880977 MELO3C002886 0.000237461224858571 MELO3C017008 0.000239417539566356 MELO3C026804 0.000239213874166588 MELO3C000201 0.000239277857855269 MELO3C021461 0.000239571693509077 MELO3C021742 0.000238546835302778 MELO3C024688 0.000240582824529545 MELO3C009127 0.000241609568591317 MELO3C004441 0.000242392300670469 MELO3C016707 0.000243440104488402 MELO3C012173 0.00024337823626408 MELO3C011044 0.000243145665177247 MELO3C003120 0.000244375341807102 MELO3C012004 0.000244992421164048 MELO3C018437 0.000245175579068335 MELO3C002075 0.00024584132238592 MELO3C011507 0.000245627075923016 MELO3C024192 0.000247316007776055 MELO3C007425 0.000247283160818346 MELO3C015496 0.000251336419125447 MELO3C021292 0.000251956426508548 MELO3C011986 0.000252668788317534 MELO3C013600 0.000256143100017092 MELO3C005797 0.000255289098429867 MELO3C012033 0.000255521627540833 MELO3C022354 0.000254944927874368 0.00486632566827238 Transferring glycosyl group transferase 0.00487668277203734 Phosphate transporter 0.00489273334612328 High chlorophyll fluorescence phenotype 173 0.00489273334612328 wall-associated receptor kinase 2-like 0.00501012160333364 nucleolar protein 12

0.00501012160333364 DNA polymerase epsilon catalytic subunit A 0.00501350947716613 ARF guanine-nucleotide exchange factor GNL2 0.00503596562233786 E3 SUMO-protein ligase SIZ1

0.00511870025695455 Hydroxyproline O-arabinosyltransferase 1 0.0051337675592797 Protein WVD2-like 4

0.00519433149706969 Acyl-CoA N-acyltransferases (NAT) superfamily proteir 0.00519433149706969 Ribosomal protein L15 0.00519433149706969 Unknown protein

0.00519433149706969 NADH dehydrogenase [ubiquinone] 1 beta subcomplex subunit 7 0.00519433149706969 E3 ubiquitin-protein ligase RMA1H1 0.00519433149706969 Dead box ATP-dependent RNA helicase, putative 0.00519998294218144 Magnesium transporter MRS2-like protein 0.00522354898241223 Protein phosphatase 2C 0.00522354898241223 At1g16840/F17F16.27 0.00524667145831115 ALA-interacting subunit 3-like

0.00529271655361963 nicotinamide adenine dinucleotide transporter 1 , chloroplastic-like 0.00529927201732925 lactation elevated protein 1 0.00529927201732925 HVA22-like protein 0.00529927201732925 Alpha-mannosidase 0.00530926236936354 Multiple organellar RNA editing factor 8, chloroplastic/mitochondrial 0.00530926236936354 Phospholipid-transporting ATPase

0.00532485097703111 Nascent polypeptide-associated complex subunit alpha-like protein

0.00532485097703111 Macrophage migration inhibitory factor family protein

0.00537888517258504 Adaptin ear-binding coat-associated protein, putative

0.00546355533221984 DNA damage-inducible protein 1

0.00547828218461161 Glutamate receptor

0.00547828218461161 Fructose-1,6-bisphosphatase

0.00548351994895583 peroxiredoxin-2E-2, chloroplastic

0.00548351994895583 Coiled-coil protein

0.00548351994895583 Abhydrolase_5 domain-containing protein

0.00548351994895583 sister chromatid cohesion protein PDS5 homolog B-B-like 0.00548351994895583 60S ribosomal protein L13a, putative

0.00549292413838397 Leucine-rich repeat receptor-like protein kinase family

0.00549292413838397 T-complex protein 1 subunit epsilon

$0.00550595018403207 \quad$ MLO-like protein 4

0.00550595018403207 adenylate isopentenyltransferase 3, chloroplastic

0.00551071883634772 Alpha-taxilin

0.00552722123260604 Ubiquitin family protein

0.00552722123260604 histone-lysine N-methyltransferase, H3 lysine-9 specific SUVH1-like 0.00552722123260604 dof zinc finger protein DOF1.5-like 0.00552722123260604 No data found

0.00552722123260604 Glycine cleavage system $\mathrm{H}$, mitochondrial

0.00554407258066976 TATA-binding protein-associated factor $2 \mathrm{~N}$

0.00556124402110712 WRKY family transcription factor family protein

0.00557276547839475 RING-H2 finger protein ATL16

0.00557737659343555 Choline transporter-like protein 2

0.00557737659343555 DNA-directed RNA polymerase

0.00557737659343555 cytochrome c oxidase subunit 6b-1-like

0.00559231590422763 SLT1

0.00559765608210468 Xyloglucan endotransglucosylase/hydrolase

0.00559765608210468 Pentatricopeptide repeat-containing protein At5g39710 0.00559990804590489 No data found

0.00559990804590489 Vesicle transport protein GOT1

0.0056205337601488 Fasciclin-like arabinogalactan protein

0.0056205337601488 1-aminocyclopropane-1-carboxylate oxidase 1

0.00570533671414766 Plant/F27B13-30 protein

0.00571284439393493 Protein DEHYDRATION-INDUCED 19

0.00572242649137956 Polygalacturonase

0.00574837509345177 O-methyltransferase, putative

0.00574837509345177 Unknown protein

0.00574837509345177 Neurofilament heavy protein

0.00574837509345177 Light-regulated protein, putative 
MELO3C017242 0.00025602726417806 MELO3C026522 0.000255690699527245 MELO3C011105 0.000255669095747146 MELO3C009782 0.000254722393082774 MELO3C026803 0.000258450915686992 MELO3C022442 0.000258437787819199

\section{MELO3C002351 0.000259527766881318} MELO3C008058 0.000261626098192913 MELO3C022196 0.000261549622935453 MELO3C010214 0.000261610083673203

MELO3C026201 0.000260842116481808

MELO3C011601 0.000260986200993574

MELO3C011107 0.000261609531341023

MELO3C005967 0.000264984834755788

MELO3C022114 0.000265447627879833

MELO3C003186 0.000266588800294176

MELO3C011622 0.000266085187327958

MELO3C010958 0.000266339972744611

MELO3C011482 0.000266994123112263

MELO3C016601 0.000268047574068397

MELO3C002470 0.000268233120904071

MELO3C003294 0.000268967860220348

MELO3C017993 0.000271532165540656

MELO3C025255 0.000273271096768335

MELO3C006594 0.000275652840892704

MELO3C022014 0.000274733521762549

MELO3C005588 0.000275761756498127

MELO3C002223 0.000275887427115262

MELO3C024975 0.000275516874583381

MELO3C013766 0.000277047881957371

MELO3C017544 0.000276862046831305

MELO3C018441 0.00027722236907457

MELO3C022679 0.000277183104936407

MELO3C013188 0.000277872280472269

MELO3C002118 0.000277836043142798

MELO3C013046 0.000278180830887376

MELO3C005002 0.000281040859585691

MELO3C012349 0.000282115086382206

MELO3C008097 0.000282891183413736

MELO3C022353 0.000283476774613156

$\begin{array}{ll}M E L O 3 C 008236 & 0.000283857813457633 \\ \text { MELO3C007082 } & 0.000285601472570951\end{array}$

MELO3C012218 0.000285696896113241

MELO3C009779 0.000287063425019052

MELO3C020850 0.000287415273795277

MELO3C009539 0.000288935313469407

MELO3C008986 0.000288021661580151

MELO3C023484 0.000288852383731109

MELO3C010053 0.000289607650206358

MELO3C017226 0.000289611239794763

MELO3C010630 0.000289461750767162

MELO3C003771 0.000289095891857327

MELO3C007084 0.000290601122250878

MELO3C008295 0.000291253199451558

MELO3C023251 0.000292832830838363

MELO3C025232 0.000294396155300802

MELO3C016288 0.000296833075818848

MELO3C003487 0.0002971194290049

MELO3C006785 0.00030098724067229

MELO3C016573 0.000300781649135162

MELO3C012335 0.00030093175099899

MELO3C002298 0.00030226452811033

MELO3C008469 0.00030238428484719

MELO3C015385 0.000304250502092929

MELO3C019777 0.000304243646278235

MELO3C025091 0.000304781112087205
0.00574837509345177 protein COBRA-like

0.00574837509345177 monosaccharide-sensing protein 2-like

0.00574837509345177 Chaperone protein dnaJ

00574837509345177 5-methyltetrahydropteroyltriglutamate-- homocysteine methyltransferase

0.00578701489104581 S-acyltransferase

00578701489104581 At1g08760

0.00580454571703188 Receptor protein kinase-like protein

0.00581198404185809 mitogen-activated protein kinase kinase kinase YODA isoform X1

0.00581198404185809 Kinase family protein

0.00581198404185809 Nucleoid-associated protein At2g24020, chloroplastic

0.00581198404185809 non-specific phospholipase C6

0.00581198404185809 No data found

0.00581198404185809 Serine hydroxymethyltransferase

0.005879983709654 At3g15351

00588364220314122 RmlC-like cupins superfamily protein

0.00588910762529048 Proteasome inhibitor-related

0.00588910762529048 Receptor-like kinase

0.00588910762529048 Gamma carbonic anhydrase 1, mitochondrial

0.00589147143837328 ATP-citrate synthase beta chain protein 2-like

0.00590561416358361 No data found

00590561416358361 Patatin

0.00591519629342055 vacuolar cation/proton exchanger 3

0.00596494853978021 40S ribosomal protein S29

0.00599647876675316 Zinc transporter

0.00602044287082797 Inosine-uridine preferring nucleoside hydrolase

0.00602044287082797 WRKY transcription factor, putative

0.00602044287082797 No data found

0.00602044287082797 hydroxyproline O-galactosyltransferase GALT6

0.00602044287082797 DUF3511 domain protein

0.00602295331887094 Translocase of chloroplast

0.00602295331887094 DNA-directed RNA polymerase III subunit RPC5

0.00602295331887094 presequence protease 1, chloroplastic/mitochondrial-like

0.00602295331887094 oligouridylate-binding protein 1B-like

0.00602381961256513 Pentatricopeptide repeat-containing protein

0.00602381961256513 Elongation factor 1 alpha

0.0060238960846434 Kinase family protein

0.00607916312810274 Interactor of constitutive active ROPs-like protein

0.00609572302074638 replication protein A $14 \mathrm{kDa}$ subunit B-like

0.00610581200135287 Polyprotein

0.0061117716395581 tRNA dimethylallyltransferase

S-adenosyl-L-methionine-dependent methyltransferases superfamily protein

0.00611331293127022 isoform 1

0.00613952992528879 No data found

0.00613952992528879 Two-component response regulator

0.00616219084858832 S-adenosyl-L-methionine-dependent methyltransferases superfamily protein

0.00616304477978603 Filament-like plant protein 7

0.00616328919688229 NC domain-containing protein

0.00616328919688229 No data found

0.00616328919688229 AP2-like ethylene-responsive transcription factor At1g16060

0.00616328919688229 Isopentenyl diphosphate isomerase

0.00616328919688229 glycine-rich RNA-binding protein 2, mitochondrial-like

0.00616328919688229 40S ribosomal protein S8

0.00616328919688229 Mitochondrial 39S ribosomal protein L53

0.00617769813060558 BTB/POZ domain-containing protein

0.0061849026193213 ABC1-like protein

0.00621176753622646 callose synthase 5

0.00623822926076775 Ubiquitin-conjugating enzyme, E2

0.00628245353684985 F-box/LRR-repeat protein 14

0.00628245353684985 receptor-like protein kinase HAIKU2

$0.00634386020921778 \mathrm{U} 11 / \mathrm{U} 12$ small nuclear ribonucleoprotein $35 \mathrm{kDa}$ protein

0.00634386020921778 Serine/threonine-protein kinase

0.00634386020921778 Uricase

0.00635973082156247 Methylthioribose-1-phosphate isomerase

0.00635973082156247 Kinase family protein

0.00638538062256456 Pentatricopeptide repeat-containing protein

0.00638538062256456 U4/U6 small nuclear ribonucleoprotein PRP4-like protein

0.0063897263085034 protein REVEILLE 1-like isoform X1 
|MELO3C017939 $0.000306345373478512 \quad 0.00641571026598847 \quad$ Ribosomal protein L19

$\begin{array}{llll}\text { MELO3C007011 } 0.000307586424169948 & 0.00642904807225306 & \text { No data found }\end{array}$

$\begin{array}{llll}\text { MELO3C007611 } 0.000307633319574618 & 0.00642904807225306 & \text { Prolyl 4-hydroxylase alpha-like protein }\end{array}$

MELO3C013883 0.0003081626880317990 .00643330330437632 BOI-related E3 ubiquitin-protein ligase 1

$\begin{array}{llll}\text { MELO3C022514 } 0.000309803504096484 & 0.00645779523213418 & \text { UTP:RNA uridylyltransferase } 1\end{array}$

MELO3C002328 $0.000310299011578308 \quad 0.00645779523213418$ zinc finger CCCH domain-containing protein 43

$\begin{array}{lllll}\text { MELO3C011090 } 0.000310316860362314 & 0.00645779523213418 & \text { Unknown protein }\end{array}$

MELO3C008440 $0.000311127156138014 \quad 0.00646784232270487$ wall-associated receptor kinase 2-like

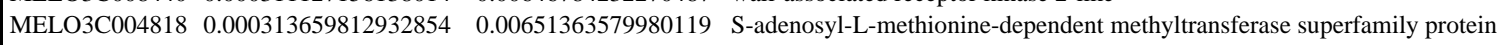

MELO3C014557 $0.000316734499387072 \quad 0.00655680254548773$ Peptidyl-prolyl cis-trans isomerase

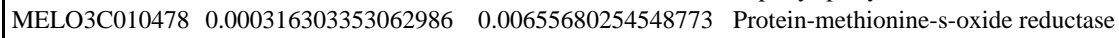

MELO3C013527 $0.000316579852200904 \quad 0.00655680254548773$ 1D-myo-inositol 2-amino-2-deoxy-alpha-D-glucopyranoside ligase

$\begin{array}{llll}\text { MELO3C001849 } 0.000317464593990291 & 0.00656503483425577 & \text { No data found }\end{array}$

MELO3C025628 0.0003196300514306530 .00660290155408364 Aspartyl/glutamyl-tRNA (Asn/Gln) amidotransferase subunit B

MELO3C017799 $0.000320760795404995 \quad 0.00661242687729984$ F2P16.20 protein, putative isoform 1

MELO3C011882 $0.000320479602688328 \quad 0.00661242687729984$ RING-type E3 ubiquitin transferase

MELO3C019053 $0.000321423875051297 \quad 0.00661859054599197$ Ectonucleotide pyrophosphatase/phosphodiesterase family member 3

MELO3C021608 $0.00032173005844105 \quad 0.00661859054599197$ zinc finger protein CONSTANS-LIKE 6

MELO3C022345 $0.000322137578457116 \quad 0.00662007808215357$ somatic embryogenesis receptor kinase 2-like

$\begin{array}{lll}\text { MELO3C011110 } 0.000324177713222018 & 0.00665507864700794 & \text { transcription factor bHLH93-like }\end{array}$

$\begin{array}{llll}\text { MELO3C008732 } & 0.000326177477364187 & 0.00668529463580516 & \text { No data found }\end{array}$

$\begin{array}{lllll}\text { MELO3C016284 } 0.000326326600279314 & 0.00668529463580516 & \text { BnaC06g13730D protein }\end{array}$

MELO3C013949 $0.000326936864283978 \quad 0.00669085609610806$ Ribosomal protein L20

$\begin{array}{llll}\text { MELO3C025848 } 0.000329847364902602 & 0.00674343230793115 & \text { Peptidyl-prolyl cis-trans isomerase }\end{array}$

$\begin{array}{llll}\text { MELO3C016213 } 0.00033459521642587 & 0.00683342391850518 \text { Beta-amylase }\end{array}$

MELO3C018956 0.0003357071538918620 .00683492843526844 Ubiquitin carboxyl-terminal hydrolase, putative

MELO3C007152 $0.000335088922765014 \quad 0.00683492843526844$ Leucine-rich receptor-like protein kinase family protein

MELO3C010769 $0.000335478511820808 \quad 0.00683492843526844$ Protein EARLY FLOWERING 3

$\begin{array}{llll}\text { MELO3C015450 } & 0.000340086584732724 & 0.00691696185570192 & \text { Pollen-specific protein SF21 }\end{array}$

$\begin{array}{lll}\text { MELO3C006413 } 0.000342652819556477 & 0.00696198614549472 & \text { Clathrin interactor EPSIN } 2\end{array}$

$\begin{array}{lll}\text { MELO3C017698 } 0.00034384648671526 & 0.00697905885523091 & \text { Vesicle transport protein GOT1 }\end{array}$

MELO3C018478 $0.000344872724779233 \quad 0.00698552968375904$ kynurenine formamidase

$\begin{array}{llll}\text { MELO3C011104 } 0.00034473878406005 & 0.00698552968375904 & \text { Glucose-6-phosphate isomerase }\end{array}$

$\begin{array}{lllll}\text { MELO3C021510 } & 0.000345680560706607 & 0.00699471864077334 & \text { Ferredoxin-thioredoxin reductase catalytic chain }\end{array}$

MELO3C011212 0.0003510430496577180 .00709595617982627 Photosystem II reaction center PsbP family protein

MELO3C006983 0.0003534012054771110 .00713631943452705 Protein phosphatase 2C

$\begin{array}{llll}\text { MELO3C014307 } 0.000354028631387227 & 0.00714168686544059 & \text { PRA1 family protein }\end{array}$

MELO3C026030 0.0003554780016079560 .00716360719770972 Protein phosphatase-2c, putative

MELO3C019462 $0.000356006879700388 \quad 0.00716695195433533$ Membralin, putative

MELO3C010774 $0.000360111028847587 \quad 0.00724219216773014$ Zinc finger CCCH domain-containing protein

MELO3C010329 $0.000361489058516851 \quad 0.0072625100881478$ methyl-CpG-binding domain-containing protein 11-like

MELO3C007086 $0.000362295746433317 \quad 0.00727131981332476$ ABC transporter C family member 12-like

MELO3C014306 0.0003629839321159790 .00727773571102382 signal recognition particle $9 \mathrm{kDa}$ protein

$\begin{array}{llll}\text { MELO3C007119 } 0.000364741286312031 & 0.00730555341113216 \quad \text { E3 ubiquitin-protein ligase arkadia-A, putative }\end{array}$

MELO3C022212 0.0003693320251839660 .00739000827290592 Deoxycytidine kinase

MELO3C002722 $0.0003700889872421120 .00739765932089521 \quad 3$-oxoacyl-[acyl-carrier-protein] synthase

MELO3C002607 $0.000370819442000903 \quad 0.00740476558147202$ Sulfate/thiosulfate import ATP-binding protein cysA, putative

$\begin{array}{llll}\text { MELO3C014272 } 0.000376517115107222 & 0.00750665144381306 & \text { Pyruvate kinase }\end{array}$

MELO3C017784 0.0003766819373547390 .00750665144381306 Pentatricopeptide repeat-containing family protein

MELO3C026155 $0.000378623549355916 \quad 0.00753773838329637$ (+)-neomenthol dehydrogenase

MELO3C006598 0.0003806724964283690 .00757089741386089 serine/arginine-rich SC35-like splicing factor SCL28

MELO3C008040 0.0003819089181892330 .00758784630313799 RING-type E3 ubiquitin transferase

MELO3C012525 $0.000384064071674795 \quad 0.00762299633317137$ Receptor-like kinase

MELO3C026919 $0.000385875008708902 \quad 0.00765125054918887$ RuBisCO large subunit-binding protein subunit alpha, chloroplastic

MELO3C003242 $0.000388061894538216 \quad 0.0076868950403563$ Clathrin light chain

MELO3C006096 0.0003888697478163780 .00769517900764094 DNA replication complex GINS protein SLD5

MELO3C008925 $0.000389715645112587 \quad 0.00770419847380229$ GTPase LSG1-2-like

$\begin{array}{llll}\text { MELO3C007691 } 0.000390499220882301 & 0.00771196911320456 & \text { Auxin-responsive protein }\end{array}$

$\begin{array}{llll}\text { MELO3C018720 } 0.000391460927158693 & 0.00772323861184519 & \text { F-box protein MAX2 }\end{array}$

MELO3C006726 0.0003936130473605190 .00775021343202681 Galactokinase

$\begin{array}{llll}\text { MELO3C023350 } 0.000393498277305726 & 0.00775021343202681 & \text { GATA transcription factor }\end{array}$

$\begin{array}{lll}\text { MELO3C017960 } 0.00039484077823515 & 0.00775296869722632 & \text { NADH dehydrogenase [ubiquinone] iron-sulfur protein 5-B }\end{array}$

$\begin{array}{lll}\text { MELO3C007833 } 0.000394930705828633 & 0.00775296869722632 & \text { ATPase family AAA domain-containing protein } 3\end{array}$

$\begin{array}{llll}\text { MELO3C005861 } 0.000394326202309614 & 0.00775296869722632 & \text { Hexosyltransferase }\end{array}$

MELO3C019503 $0.000397224747742952 \quad 0.00776020694699844$ zinc-binding alcohol dehydrogenase domain-containing protein 2

MELO3C027373 0.0003983848922810560 .00776020694699844 ADP-ribosylation factor GTPase-activating protein AGD12-like

MELO3C022007 0.0003970715383118110 .00776020694699844 transmembrane emp24 domain-containing protein p24delta9 
MELO3C024444 0.000395871151194438 MELO3C012447 0.00039769110672716 MELO3C020610 0.000397760016506599 MELO3C002609 $0.000398442951251021 \quad 0.00776020694699844$ serine/threonine-protein kinase HT1-like $\begin{array}{llll}\text { MELO3C024644 } 0.000397462342182631 & 0.00776020694699844 & 50 \text { S ribosomal protein L18 }\end{array}$ $\begin{array}{llll}\text { MELO3C019816 } 0.000399693237937981 & 0.00777688842959329 & \text { Amino acid transporter family protein }\end{array}$ MELO3C009758 $0.000402735654175146 \quad 0.00780045318224247$ Amino acid transporter family protein MELO3C009408 $0.000402832046479062 \quad 0.00780045318224247$ Tetratricopeptide repeat (TPR)-like superfamily protein MELO3C016817 $0.000402351344261254 \quad 0.00780045318224247$ Calcium-transporting ATPase

MELO3C026025 $0.000402879246842236 \quad 0.00780045318224247$ Dehydration responsive element binding transcription factor

$\begin{array}{lllll}\text { MELO3C017480 } & 0.000401515371484806 & 0.00780045318224247 & \text { Xyloglucan endotransglucosylase/hydrolase }\end{array}$

MELO3C024544 0.0004041086711787930 .00781659367983349 Cell division FtsZ-1, chloroplastic-like protein

$\begin{array}{lll}\text { MELO3C005517 } 0.0004077385948561 & 0.00787908953993455 & \text { Tryptophan RNA-binding attenuator protein-like }\end{array}$

MELO3C007043 $0.000408454688791027 \quad 0.00788521177803909$ UDP-glucose 4-epimerase, putative

MELO3C013773 $0.000410427524175638 \quad 0.00790881113427016$ Mediator of RNA polymerase II transcription subunit

MELO3C003426 $0.000410478070415055 \quad 0.00790881113427016$ Inositol-tetrakisphosphate 1-kinase

$\begin{array}{llll}\text { MELO3C014619 } 0.000416573957624733 & 0.00798238170586701 & \text { IRK-interacting protein-like }\end{array}$

$\begin{array}{lll}\text { MELO3C021139 } 0.000416728063664884 & 0.00798238170586701 & \text { No data found }\end{array}$

MELO3C005875 $0.000415414077330256 \quad 0.00798238170586701$ Curved DNA-binding protein

$\begin{array}{llll}\text { MELO3C012171 } 0.000417125825128095 & 0.00798238170586701 & \text { phosphatidylinositol 4-kinase gamma } 5\end{array}$

$\begin{array}{lll}\text { MELO3C025940 } 0.000416422648866988 & 0.00798238170586701 & \text { CONSTANS-like zinc finger protein }\end{array}$

$\begin{array}{lll}\text { MELO3C002382 } 0.000417101743036552 & 0.00798238170586701 & \text { Kinase family protein }\end{array}$

MELO3C017207 $0.000416952793157011 \quad 0.00798238170586701 \quad$ LYR motif-containing protein 4

MELO3C002312 $0.000421192848875096 \quad 0.00805240810497025$ cytochrome P450 71A1-like

MELO3C026966 $0.00042238849588494 \quad 0.00806745687159737$ 2-oxoglutarate (2OG) and Fe(II)-dependent oxygenase superfamily protein

MELO3C017319 $0.000423412418095515 \quad 0.00807919985021093$ BnaA06g03540D protein

MELO3C023667 $0.000425142227014508 \quad 0.00809656108130136$ Tetratricopeptide repeat-containing protein

$\begin{array}{llll}\text { MELO3C008086 } & 0.00042505111070712 & 0.00809656108130136 & \text { Delta-aminolevulinic acid dehydratase }\end{array}$

MELO3C010565 $0.000429306516313455 \quad 0.00815720266161301$ No data found

MELO3C007254 $0.000428996821239314 \quad 0.00815720266161301$ guanylate-binding protein 2

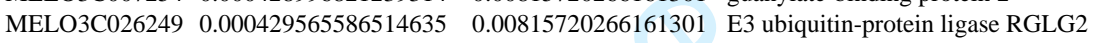

MELO3C022021 $0.00043037785930633 \quad 0.00816477650666735$ Lysine--trna ligase

$\begin{array}{llll}\text { MELO3C016868 } 0.00043226379708261 & 0.00819268496025381 & \text { serine/threonine-protein kinase EDR1 }\end{array}$

$\begin{array}{llll}\text { MELO3C020924 } 0.000436769436912066 & 0.00827014344158809 & \text { zinc finger protein } 830 \text { isoform X1 }\end{array}$

MELO3C022005 0.0004380061770528080 .00828561684924894 Phosphate transporter PHO1-like protein

MELO3C025753 $0.000438646630847916 \quad 0.0082897916867134$ elongation factor 1-alpha

MELO3C022975 0.0004403780762596290 .00830959014615144 At1g04330

MELO3C018718 $0.000440535768039929 \quad 0.00830959014615144$ At2g42760

$\begin{array}{llll}\text { MELO3C005757 } 0.000442811253232867 & 0.00834454145047317 & \text { Luminal binding heat shock protein } 70\end{array}$

MELO3C006289 0.0004463320493871550 .00840287096601232 Unknown protein

MELO3C020049 0.0004480145259926350 .00842651321317005 Ribosomal protein S5/S7

MELO3C007494 $0.000451721422672913 \quad 0.00848815069112022$ Hexosyltransferase

$\begin{array}{llll}\text { MELO3C023370 } 0.000452977173029057 & 0.00850365607428787 & \text { No data found }\end{array}$

MELO3C000425 0.0004559365420644390 .00855108335159602 alanine--tRNA ligase-like

MELO3C023876 $0.000456434521640348 \quad 0.00855230110804101$ glycerol-3-phosphate acyltransferase 5-like

MELO3C016308 $0.000457945081585698 \quad 0.00857247148458383$ myosin-11-like

$\begin{array}{llll}\text { MELO3C009542 } & 0.00046017704841339 & 0.00859657502875971 & \text { BEL1-like homeodomain protein } 1\end{array}$

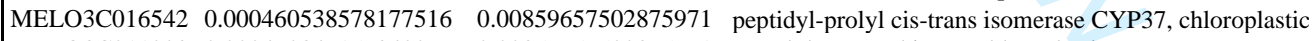

MELO3C011208 0.0004603471568400690 .00859657502875971 acetylglutamate kinase, chloroplastic

$\begin{array}{llll}\text { MELO3C006160 } 0.000462267098868541 & 0.00861871471817833 & \text { Histone deacetylase complex subunit SAP18 }\end{array}$

MELO3C020721 0.0004625974784175920 .00861871471817833 triacylglycerol lipase SDP1

$\begin{array}{llll}\text { MELO3C007106 } 0.000465408661012523 & 0.00864826228616565 & \text { Remorin family protein }\end{array}$

$\begin{array}{llll}\text { MELO3C015502 } 0.000465497129484738 & 0.00864826228616565 & \text { Integral membrane protein }\end{array}$

MELO3C025336 $0.000465391273717142 \quad 0.00864826228616565$ BnaC03g47530D protein

$\begin{array}{llll}\text { MELO3C009550 } 0.000471294734155636 & 0.0087265283090437 & 33 \mathrm{kDa} \text { ribonucleoprotein, chloroplastic }\end{array}$

MELO3C005109 0.000470509180352852

MELO3C022406 0.000471916705171882

MELO3C019873 0.000471726154005148

MELO3C003825 0.00047191919763323

MELO3C000237 0.000472801986159843

0.0087265283090437 alpha-mannosidase

0.0087265283090437 phosphatidylinositol 4-kinase gamma 4

$0.0087265283090437 \quad 3$-oxoacyl-[acyl-carrier-protein] synthase-like protein

0.0087265283090437 Cysteine/Histidine-rich C1 domain family protein, putative

0.00873467392392025 Major facilitator superfamily domain-containing protein 12

$\begin{array}{lllll}\text { MELO3C007821 } 0.000476474174394781 & 0.00879428828983414 & \text { U1 small nuclear ribonucleoprotein } 70 \mathrm{kDa}\end{array}$

$\begin{array}{llll}\text { MELO3C009490 } 0.000477551177120339 & 0.00880593669182967 & \text { Ribosomal protein S11 }\end{array}$

$\begin{array}{lllll}\text { MELO3C026433 } & 0.000478847432524576 & 0.00882160256056703 & \text { U4/U6 small nuclear ribonucleoprotein PRP4-like protein }\end{array}$

MELO3C026780 $0.000482363906618488 \quad 0.00886692143153668$ Ribosomal protein S19

$\begin{array}{llll}\text { MELO3C010512 } & 0.000482211167430746 & 0.00886692143153668 & \text { Plastid transcriptionally active } 6\end{array}$

$\begin{array}{llll}\text { MELO3C015867 } & 0.00048265433889827 & 0.00886692143153668 & \text { E3 ubiquitin ligase-like protein }\end{array}$

MELO3C007421 $0.000484638644706603 \quad 0.00888684177744727$ pyrophosphate--fructose 6-phosphate 1-phosphotransferase subunit beta

MELO3C019813 $0.000484275792564381 \quad 0.00888684177744727$ Lysine-specific demethylase REF6 
MELO3C018368 0.0004853611481934190 .00889183424459354 omega-hydroxypalmitate O-feruloyl transferase

$\begin{array}{lll}\text { MELO3C021408 } 0.000486240439558361 & 0.00889968715554965 & \text { Protein trichome birefringence-like } 10\end{array}$

$\begin{array}{llll}\text { MELO3C007181 } 0.00048750090623062 & 0.00891449573810048 & \text { MADS box transcription factor AGAMOUS }\end{array}$

MELO3C013476 0.0004892730298566850 .00893862448347796 phototropin-2

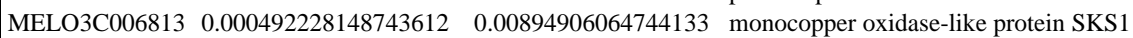

MELO3C023222 $0.000492403978258671 \quad 0.00894906064744133$ ABC transporter-like protein

MELO3C019735 $0.000492040262321525 \quad 0.00894906064744133$ 1-aminocyclopropane-1-carboxylate oxidase

MELO3C021759 $0.000492563113259847 \quad 0.00894906064744133$ lecithin-cholesterol acyltransferase-like 4

$\begin{array}{llll}\text { MELO3C011484 } & 0.000491054220355669 & 0.00894906064744133 & \text { Spermidine synthase }\end{array}$

MELO3C026705 0.0004903052748583820 .00894906064744133 Vesicle-associated membrane protein, putative

MELO3C012651 $0.00049485375550895 \quad 0.00898241435436238$ FRIGIDA-like protein

MELO3C021368 $0.000495830343627213 \quad 0.00899187645206044$ DnaJ subfamily B member 14

MELO3C009670 $0.000496738100771443 \quad 0.00900007408452774$ PHD-finger protein

MELO3C020386 $0.000500316681290025 \quad 0.00902351976145818$ E3 ubiquitin-protein ligase LAP

MELO3C013938 0.0004994192430350930 .00902351976145818 Protein transport protein Sec16B

MELO3C009005 0.000499727849437859

MELO3C005238 0.000499204461091307

MELO3C011070 0.000500007962388027

MELO3C006420 0.000501794482363316

MELO3C013436 0.000502109941717843

MELO3C012073 0.000503847273066516

MELO3C006280 0.000504505272682865

MELO3C008245 0.000504765109731187

MELO3C014007 0.000506074895368025

MELO3C026398 0.000506910045428688

MELO3C023998 0.000507430810900544

MELO3C009506 0.000508124357512574

MELO3C003541 0.000510134406861762

MELO3C019404 0.000510767191047368

MELO3C021846 0.00051267436100022

MELO3C019688 0.000512648137834137

MELO3C006430 0.000517174720864766

MELO3C008866 0.000517893399529346

MELO3C020562 0.000520045834638383

MELO3C009261 0.000521943976709904

MELO3C004505 0.000521802144234362

MELO3C025989 0.000522128633384078

MELO3C023163 0.000524847951735463

MELO3C026263 0.000524589257258512

MELO3C014273 0.000526136935624533

MELO3C003695 0.00052583296602382

MELO3C022609 0.000527154911452721

MELO3C000279 0.000528395036966334

MELO3C023830 0.000528124502867655

MELO3C007909 0.000529760023340375

MELO3C005720 0.000530338114349549

MELO3C008491 0.000531797074916351

MELO3C016775 0.000532384973778277

MELO3C003292 0.000535229738756193

MELO3C015375 0.000536261199456822

MELO3C009346 0.000538853443754816

MELO3C009994 0.000538889907489848

MELO3C007391 0.000540671539647652

MELO3C002332 0.000541447285320018

MELO3C024016 0.000542761100591038

MELO3C017902 0.000543462838672237

MELO3C006175 0.000544486571854463

MELO3C025062 0.000544142102876677

MELO3C003567 0.000545821213981501

MELO3C011576 0.000546754914670933

MELO3C012178 0.000548063155305423

MELO3C016015 0.000549275667963922

MELO3C022260 0.000550317736865757

0.00902351976145818 Unknown protein

0.00902351976145818 GAGA-binding transcriptional activator

0.00902351976145818 Rhamnogalacturonate lyase

0.00903935208658677 Cotton fiber protein

0.00903935208658677 DNA helicase

0.00906236775572916 SNF1-related kinase regulatory subunit gamma 1

0.00906236922916473 60S ribosomal protein L37a

0.00906236922916473 Delta-1-pyrroline-5-carboxylate synthetase

0.00907763225124716 Superoxide dismutase 1

0.00908436160360359 APO protein 1, chloroplastic

0.00908544975927003 Protein RETICULATA-RELATED 4, chloroplastic

0.00908962675409042 secoisolariciresinol dehydrogenase-like

$0.00911732524987596 \mathrm{NAD}(\mathrm{P}) \mathrm{H}$-hydrate epimerase

0.00912038088245431 phytosulfokine receptor 2

0.00913791151208787 cytochrome P450 89A2-like

0.00913791151208787 Peroxisomal and mitochondrial division factor 2

0.00920981385244208 ethylene-responsive transcription factor 1B

0.00921430337595049 Plasma-membrane choline transporter family protein

0.00924427109655574 No data found

0.0092563001622102 Peptide transporter family protein

0.0092563001622102 Ribosomal protein L31

0.0092563001622102 Kinase superfamily protein

0.00928783351149074 Kinase superfamily protein

0.00928783351149074 DEAD-box ATP-dependent RNA helicase-like protein

0.00929398778322799 Pentatricopeptide repeat-containing protein family

0.00929398778322799 ethylene-responsive transcription factor SHINE 2-like

0.00930364820936531 No data found

0.00930889704286184 Late cornified envelope protein 1E

0.00930889704286184 protein-tyrosine-phosphatase MKP1 isoform X1

0.00932462629318099 Armadillo repeat only protein

0.0093264892433564 Late embryogenesis abundant protein

0.00934382600758276 Zinc finger protein CONSTANS

0.00934584075301973 Leucine-rich repeat receptor-like protein kinase family

0.00938743526704801 protein N-methyltransferase NNT1 isoform X1

0.00939718050405748 Sodium/hydrogen exchanger

0.00942651619399203 translin

0.00942651619399203 MD-2-related lipid recognition domain-containing protein / ML domain-

0.00944931171371813 Glycosyl transferase, family 31

0.00945450259751108 Telomere length regulation TEL2

0.00946907153319118 Metal-dependent phosphohydrolase

0.00947294580841837 monothiol glutaredoxin-S15, mitochondrial

0.00947406635026766 ankyrin repeat and zinc finger domain-containing protein 1

0.00947406635026766 transcription initiation factor TFIID subunit 11-like

0.00948892883355692 Glycosyltransferase

0.00949680106406003 zinc finger protein CONSTANS-LIKE 6

0.00951115927427662 protein PHYLLO, chloroplastic isoform X1

0.00952383245532879 AAA-type ATPase family protein

0.00952517527200863 CDPK-related kinase 3 isoform X1

MELO3C026636 0.0005502737235472120 .00952517527200863 Protein arginine N-methyltransferase

$\begin{array}{llll}\text { MELO3C008806 } 0.000551102874571718 & 0.00953041214528622 & \text { Echinoderm microtubule-associated protein-like } 6\end{array}$

MELO3C023776 $0.000553621720863151 \quad 0.00955723371094962$ Laminin subunit gamma-1

MELO3C026221 0.0005533042879410920 .00955723371094962 protein PLASTID TRANSCRIPTIONALLY ACTIVE 10-like

MELO3C023047 0.0005553573762036020 .00957882342589078 glutamyl-tRNA(Gln) amidotransferase subunit B, chloroplastic/mitochondrial 
MELO3C021456 $0.000557011378721106 \quad 0.00959059958008991$ histone deacetylase HDT1-like MELO3C002180 $0.000556955335141218 \quad 0.00959059958008991$ mRNA splicing factor, Cwf18

MELO3C015290 $0.000557828007353156 \quad 0.00959629383032881$ peptidyl-prolyl cis-trans isomerase G

MELO3C019497 0.0005589428593389960 .00960710402879532 Tubby-like F-box protein

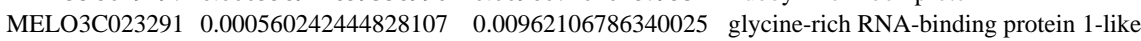

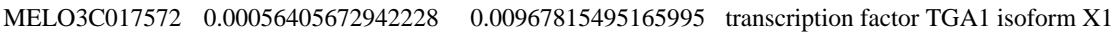

$\begin{array}{llll}\text { MELO3C006950 } 0.000566029637463883 & 0.0097035757901686 & \text { Pentatricopeptide repeat-containing protein At4g21190 }\end{array}$

MELO3C014394 0.0005672512876685460 .00971608471827071 Ribosomal N-lysine methyltransferase 5

$\begin{array}{llll}\text { MELO3C005706 } 0.000567796577401691 & 0.00971699705988388 & \text { 40S ribosomal protein S10-1 }\end{array}$

MELO3C027308 $0.000574051466060821 \quad 0.00981006784135699$ alanine--tRNA ligase-like

MELO3C021404 $0.000574228488764428 \quad 0.00981006784135699$ Heavy metal-associated isoprenylated plant protein 21

MELO3C012284 0.0005787777589547630 .00987924110769024 Zinc finger, B-box

MELO3C002714 0.0005797023262978930 .00988647775652598 60S ribosomal protein L18a-like protein

$\begin{array}{llll}\text { MELO3C022359 } 0.000581911996475948 & 0.00991559967075366 & \text { F3H9.20 protein }\end{array}$

MELO3C024951 $0.000582445935391473 \quad 0.00991614205003984$ cellulose synthase-like protein E6

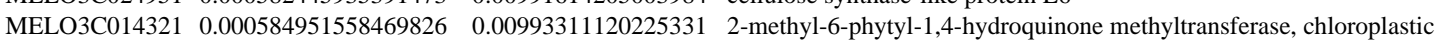

MELO3C017623 $0.000584098236359187 \quad 0.00993311120225331$ Metacaspase-1

$\begin{array}{llll}\text { MELO3C023413 } 0.000584468800800653 & 0.00993311120225331 & \text { Signal peptidase I }\end{array}$

MELO3C004105 0.0005885047491036580 .00995066805654806 Cytochrome c oxidase subunit

MELO3C013315 $0.000587711132197466 \quad 0.00995066805654806 \quad$ O-methyltransferase, putative

MELO3C016140 0.0005883644682191940 .00995066805654806 Solanesyl diphosphate synthase, putative

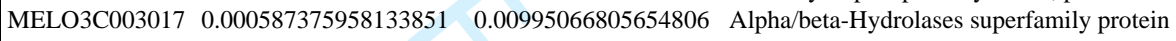

MELO3C025102 0.0005876839656748790 .00995066805654806 Phytoene synthase

$\begin{array}{llll}\text { MELO3C008018 } 0.000589155695845944 & 0.0099531529831151 & \text { Like-COV protein }\end{array}$

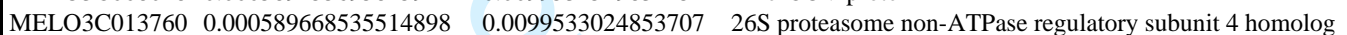

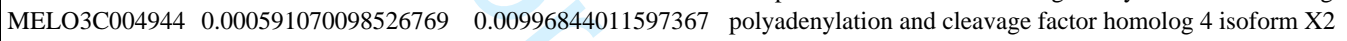

MELO3C024760 0.0005921639100592290 .00997836609194515 RNA polymerase sigma factor sigA

MELO3C011805 0.0005928198056628360 .00998090225237456 No data found

MELO3C014315 0.0005951526276681830 .00998400707837636 Bifunctional DNA-directed RNA polymerase subunit beta-beta

MELO3C013921 0.0005955319427984880 .00998400707837636 Protein-tyrosine phosphatase mitochondrial 1-like protein

MELO3C010524 $0.000594398368218396 \quad 0.00998400707837636$ arabinosyltransferase XEG113

MELO3C002757 0.0005948565628118850 .00998400707837636 signal recognition particle $14 \mathrm{kDa}$ protein

$\begin{array}{llll}\text { MELO3C018461 } 0.00059493992894244 & 0.00998400707837636 & \text { E3 ubiquitin-protein ligase MARCH6 }\end{array}$

MELO3C020276 0.000599313743151031

MELO3C012960 0.000599614992799591

MELO3C011787 0.000601120982774539

MELO3C018462 0.000600683577282934

MELO3C016314 0.00060235623438154

MELO3C006412 0.00060289070656494

MELO3C007492 0.000606809347387105

MELO3C005899 0.000607220982714818

MELO3C025346 0.00060757358539465

MELO3C010928 0.000609112966384107

MELO3C025449 0.000611945427998251

MELO3C024474 0.000615373567698807

MELO3C005518 0.000616382245133273

MELO3C010055 0.000619950377860445

MELO3C006418 0.000621568353065705

MELO3C011873 0.000621061425400415

MELO3C024108 0.000623760788199368

MELO3C004100 0.000624466041786231

MELO3C006875 0.000627322932097107

MELO3C006051 0.000629690258800331

MELO3C014161 0.000629786395247356

MELO3C017030 0.000630415357957714

MELO3C012411 0.000630978450257835

MELO3C015514 0.000632477107366913

MELO3C017300 0.000634855988458249

MELO3C018406 0.000637480217704711

MELO3C006154 0.000639515485286957

MELO3C013710 0.000641723438790986

MELO3C015270 0.000642051019905554

MELO3C003726 0.000640546691929922

MELO3C009966 0.000641231456532054

MELO3C011640 0.00064292610395178

MELO3C018027 0.000645454282273139

MELO3C007441 0.000645005876675375

0.0100354207566094 Tobamovirus multiplication 1

0.0100354207566094 BZIP protein, putative

0.0100436026132101 No data found

0.0100436026132101 Polyadenylate-binding protein

0.0100557339584117 Aldose 1-epimerase, putative

0.0100561558817154 60S ribosomal protein L14, putative

0.0101086526857278 elongation of fatty acids protein 3-like

0.0101086526857278 Protein yippee-like

0.0101086526857278 Sugar/inositol transporter

0.0101257339841075 PPPDE putative thiol peptidase family protein

0.0101642643040685 Zinc finger C3H1 domain-containing protein, putative

0.0102126156205746 zinc finger protein 593

$0.0102207665483938 \quad 3$-isopropylmalate dehydratase small subunit 3-like

0.0102713087352063 4-hydroxy-4-methyl-2-oxoglutarate aldolase

0.0102808654980692 Ankyrin repeat-containing protein

$0.0102808654980692 \quad 60$ S ribosomal protein L17

0.0103084952352714 OBERON-like protein

0.010311521621435 WD-repeat protein, putative

0.0103500422606397 BAG family molecular chaperone regulator 1-like

$0.0103733540615013 \quad$ BTB/POZ domain-containing protein POB1

0.0103733540615013 aspartic proteinase A1-like

0.0103750607535891 Bromodomain-containing protein

0.0103756814439151 pumilio homolog 4

0.0103916725402572 12-oxophytodienoate reductase 3

0.0104220872120216 AT-rich interactive domain-containing protein 5A, putative

0.0104564757636631 Cytochrome c biogenesis protein

0.0104811546215204 Cyclin-D-binding Myb-like transcription factor 1

0.0104878954442637 Auxin efflux carrier

0.0104878954442637 single-stranded DNA-binding protein, mitochondrial

0.0104878954442637 Methyltransferase

0.0104878954442637 ADP-ribosylation factor-like

0.0104935104354907 Zinc finger, C6HC-type

0.0105173899510002 Pyruvate kinase

0.0105173899510002 RING-type E3 ubiquitin transferase

MELO3C013429 $0.000649935677337288 \quad 0.0105752863095764$ kinesin-related protein 11 
MELO3C002144 0.00065007836243991 MELO3C006005 0.000651967117786256 MELO3C011675 0.000653292394574789 MELO3C015151 0.000654376429938264 MELO3C024457 0.000656618608750104 MELO3C021845 0.000659542009912029 MELO3C019630 0.000661812702282605 MELO3C009148 0.000666792796674809 MELO3C014722 0.000668654842860272 MELO3C012452 0.000667682946013937 MELO3C001971 0.000668754302383112 MELO3C007934 0.000669504598248771 MELO3C019163 0.000671672363601994 MELO3C009771 0.000672773809379912 MELO3C004434 0.000674552868131673 MELO3C007012 0.000677563644158052 MELO3C019820 0.000678838654992631 MELO3C014297 0.000679949464106544 MELO3C002872 0.000681526249681097 MELO3C008099 0.00068197637830214 MELO3C012283 0.000684239705449396 MELO3C017651 0.000689957443399614 MELO3C017711 0.000692222789745456 MELO3C009624 0.000694814321152259 MELO3C008494 0.000693792651051739 MELO3C005367 0.000694218350740128 MELO3C023990 0.000694276492963164 MELO3C000881 0.000697534212565021 MELO3C020587 0.000701975152042733 MELO3C007103 0.000705517478176043 MELO3C022868 0.000706077302177532 MELO3C005147 0.000706388678327285 MELO3C013007 0.000705481661930962 MELO3C008775 0.000711395060906339 MELO3C018120 0.000710958527238903 MELO3C025349 0.000711969357716224 MELO3C005942 0.000713428603669519 MELO3C013779 0.000715958032653785 MELO3C008959 0.000715790240196523 MELO3C003078 0.000715397659275085 MELO3C017880 $\quad 0.0007166424359909$ MELO3C007667 0.000719402723322826 MELO3C013343 0.000720118690283433 MELO3C009128 0.000720765197783857 MELO3C019871 0.00072164473946712 MELO3C014358 0.000722535218217302 MELO3C003394 0.000724782540440172 MELO3C012722 0.000725794860899187 MELO3C018052 0.000727486395143639 MELO3C007899 0.000728120600503868 MELO3C010223 0.00072914126891510 MELO3C019498 0.000730368821527216 MELO3C007248 0.000730214662693074 MELO3C003418 0.000731189926678533 MELO3C018487 0.000731828295116532 MELO3C009133 0.0007353585541372 MELO3C001997 0.000735912792879545 MELO3C014062 0.000737302663945227 MELO3C004589 0.000738241873302403 MELO3C014459 0.000741853945029902 MELO3C013245 0.000740135031843447 MELO3C017561 0.000741241733906883 MELO3C015750 0.000739717212356328 MELO3C001996 0.000741526118682501 MELO3C017968 0.000742732357898568 MELO3C003678 0.000746920851982136 MELO3C009747 0.000752031580646517 MELO3C003086 0.000755050317633899
0.0105752863095764 Receptor-like kinase 1

0.0105972828058936 Bifunctional fucokinase/fucose pyrophosphorylase 0.0106100916944552 Cmp-sialic acid transporter, putative 0.0106189647615865 Alanine:glyoxylate aminotransferase 0.010646601727591 ATP-dependent zinc metalloprotease FtsH 0.0106852298226027 Cytochrome P450

0.0107132287355567 ribosomal RNA large subunit methyltransferase I 0.0107850048661186 Methionine aminopeptidase,related 0.0107902195406569 ethylene-responsive transcription factor RAP2-11 0.0107902195406569 CSC1-like protein isoform X1

0.0107902195406569 HVA22-like protein

0.010793507192502 methyltransferase-like protein 23 isoform X3

0.0108196227640912 ABC transporter F family-like protein

0.0108285329759119 Ectonucleotide pyrophosphatase/phosphodiesterase family member 3

0.0108483262155801 Serine-rich protein-like protein

0.0108878799092574 DNA polymerase eta

0.0108994996727231 Glycerol-3-phosphate dehydrogenase

0.0109084662604713 type I inositol polyphosphate 5-phosphatase 4

0.0109232372222944 Short-chain dehydrogenase/reductase family protein

0.0109232372222944 eukaryotic translation initiation factor 2D

0.0109506077333226 Small ubiquitin-related modifier

0.0110331737244526 Receptor-like kinase

0.0110604432643066 Proline--tRNA ligase

0.0110660387326097 Actin family protein

$0.0110660387326097 \quad$ E3 ubiquitin-protein ligase

0.0110660387326097 dehydration-responsive element-binding protein 1D-like

0.0110660387326097 Methyltransferase

0.0111004054504002 Unknown protein

0.0111620831543413 No data found

0.0111962038589772 Protein plastid transcriptionally active 12

0.0111962038589772 No data found

0.0111962038589772 NADPH:QUINONE OXIDOREDUCTASE family protein

0.0111962038589772 profilin-like

0.0112574848219866 Elongation factor 4

0.0112574848219866 Purple acid phosphatase

0.0112575523182848 subtilisin-like protease SBT6.1

0.0112716011950955 Kinase family protein

$0.0112844813941577 \quad$ 2-keto-3-deoxy-L-rhamnonate aldolase

0.0112844813941577 1-acyl-sn-glycerol-3-phosphate acyltransferase

0.0112844813941577 Protein MODIFIER OF SNC1 11

0.0112862611390624 Glucan endo-1,3-beta-glucosidase

0.0113207046875717 Epidermal patterning factor-like protein

0.0113229490560569 FAD-dependent urate hydroxylase-like

0.0113240985608858 F-box/LRR-repeat protein 15

0.0113289045784866 Phytol kinase

0.0113338745230925 transcription initiation factor TFIID subunit 6-like

0.011360103485042 Protein LSD1

0.0113669490149866 Pentatricopeptide repeat-containing family protein

0.0113844126923072 dynamin-related protein 3A-like

0.0113853157081163 WD repeat-containing protein 82

0.0113922554745287 WRKY transcription factor

0.0113934074694637 Tubby-like F-box protein

0.0113934074694637 Transcription factor VOZ1

0.0113972137821423 PLATZ transcription factor family protein

0.0113981679812748 Lipase

0.0114437336587229 Receptor-like kinase

0.0114437336587229 Galactoside 2-alpha-L-fucosyltransferase

0.0114563259718759 Tir-nbs resistance protein

0.0114619015376173 Mitochondrial import inner membrane translocase subunit Tim17-like protein

0.0114728845422048 Serine/threonine phosphatase

0.0114728845422048 40S ribosomal protein S11-like

0.0114728845422048 Gras family transcription factor

0.0114728845422048 No data found

0.0114728845422048 No data found

0.0114774814836767 LOW QUALITY PROTEIN: translocase of chloroplast 159, chloroplastic-like

0.0115331820999181 DNA-directed RNA polymerase subunit beta

0.0116030247548344 Zinc finger (C3HC4-type RING finger) family protein, putative 0.0116405064191662 protein GPR107 
MELO3C002741 0.000764731490141668 MELO3C021326 0.000766461351612402 MELO3C015233 0.000766447720925711 MELO3C015304 0.00076794260535884 MELO3C014991 0.000770621360316692 MELO3C025645 0.000773924241273871 MELO3C015686 0.000774471299118229 MELO3C024241 0.00077368783366849 MELO3C013645 0.000774280158386098 MELO3C011793 0.000775832531782594 MELO3C019253 0.000776921270584152 MELO3C023481 0.000777841519056355 MELO3C020808 0.000778407489006927 MELO3C009250 0.000782324663164635 MELO3C005971 0.000782531579639922 MELO3C002662 0.00078275615172041 MELO3C023644 0.000784187854190543

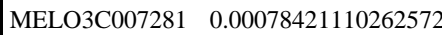
MELO3C016152 0.000786489841811733 MELO3C016884 0.000792002683039694 MELO3C007328 0.000791713477300116 MELO3C025585 0.000791107901455534 MELO3C023114 0.000793652645098342 MELO3C021548 0.000793655547673966 MELO3C021170 0.00079612896994774 MELO3C015844 0.000797345327828602 MELO3C025549 0.00079926856065926 MELO3C011847 0.000801855921626515 MELO3C024982 0.000801587460503073 MELO3C013076 0.000801616547371209 MELO3C013761 0.000803412442861307 MELO3C007931 0.000803749000804377 MELO3C021620 0.000804655961069711 MELO3C012670 0.000806556187156526 MELO3C026224 0.000806198958957616 MELO3C009797 0.000806319229198271 MELO3C023412 0.000810590269652245 MELO3C019815 0.000810636612879723 MELO3C011018 0.000812005118265402 MELO3C012730 0.00081283392472975 MELO3C016522 0.000815966088231534 MELO3C005332 0.000815992277250377 MELO3C002819 0.000815110446897416 MELO3C006925 0.000817431595872442 MELO3C017131 0.000818103995706476 MELO3C007143 0.000819606583127408 MELO3C024558 0.000823867542946388 MELO3C005650 0.000828101492612809 MELO3C019948 0.000830095547025289 MELO3C007495 0.000833697338175732 MELO3C005360 0.000834453426250992 MELO3C013541 0.000834727841994676 MELO3C018010 0.0008354022801359 MELO3C009687 0.000836070000848177 MELO3C003222 0.000839084099028331 MELO3C001947 0.000841859856859473 MELO3C025499 0.00084299621236239 MELO3C006241 0.000846579502224443 MELO3C013173 0.000846446048688998 MELO3C017320 0.000846271674169286 MELO3C003945 0.00085012626266634 MELO3C022639 0.000849764070270709 MELO3C016408 0.000849878202600962 MELO3C015796 0.000854500203095521 MELO3C018591 0.00085408519847574 MELO3C013338 0.000857578253785185 MELO3C024287 0.000858057790619982 MELO3C022319 0.000857819419544437
0.0117805633376036 Protein TIFY 5A

0.0117888202749169 Peroxisomal fatty acid beta-oxidation multifunctional protein 0.0117888202749169 ABC1-like kinase

$0.0118024112943438 \quad 60$ S ribosomal protein L22-2

$0.0118343711080049 \quad$ MLP-like protein 28

0.0118566152606867 gamma-glutamyl hydrolase 2-like

0.0118566152606867 Lectin receptor kinase

$0.0118566152606867 \quad$ Kinesin-like protein

0.0118566152606867 Alanine--tRNA ligase

0.0118682545857277 transcription termination factor MTERF9, chloroplastic-like

0.0118757106600359 thioredoxin domain-containing protein 9 homolog

0.011880038253785 nudix hydrolase 3-like

0.011880038253785 Choline transporter-related family protein

0.0119187750503673 Laccase

0.0119187750503673 E3 ubiquitin-protein ligase BRE1-like 1

0.0119187750503673 Receptor kinase, putative

0.0119225443154391 subtilisin-like protease SBT3.17

0.0119225443154391 Transmembrane protein, putative

0.0119479906814922 Gibberellin 2-oxidase

0.0120040375958181 Methyltransferase

0.0120040375958181 Wound-responsive family protein

0.0120040375958181 bifunctional nitrilase/nitrile hydratase NIT4A

0.0120106539547994 Cellulose synthase

$0.0120106539547994 \quad$ BnaC05g50840D protein

$0.0120388598985436 \quad \mathrm{BTB} / \mathrm{POZ}$ domain-containing protein POB1

0.012048028216746 DNA repair helicase

0.0120678553550915 vesicle-fusing ATPase

0.0120792163205203 60S ribosomal protein L5

0.0120792163205203 Farnesyl diphosphate synthase

0.0120792163205203 casein kinase I

0.0120892909496463 MLO-like protein

0.0120892909496463 Protein preY, mitochondrial, putative

0.0120937218989085 transcription initiation factor TFIID subunit 7

0.0120946682916889 Ribosomal protein S5/S7

0.0120946682916889 Aspartyl aminopeptidase family protein

0.0120946682916889 HSP20-like chaperones superfamily protein

$0.0121374241605471 \quad$ O-fucosyltransferase family protein

0.0121374241605471 Receptor-like protein kinase

0.0121487038489571 Thaumatin-like protein 1

0.0121518979405661 Structural maintenance of chromosomes protein

0.0121714739300738 Unknown protein

0.0121714739300738 CDP-diacylglycerol--serine O-phosphatidyltransferase 1

0.0121714739300738 eukaryotic translation initiation factor 3 subunit A-like

0.0121837408202905 mitogen-activated protein kinase kinase kinase YODA-like

0.0121845669767777 General negative regulator of transcription subunit 4

$0.0121977471063928 \quad \mathrm{NAD}(\mathrm{P})$-binding domain-containing protein

0.0122519277903978 sorting nexin 1

0.0123056255662983 Dead box ATP-dependent RNA helicase, putative

0.0123259826753402 Oxidoreductase family protein

0.0123668718316225 protein LYK5-like

0.0123668718316225 mediator of RNA polymerase II transcription subunit 36a-like

0.0123668718316225 No data found

0.012367585929838 At3g19650

0.012368199585581 No data found

0.012403496909963 mRNA-capping enzyme

0.0124352208774254 eukaryotic translation initiation factor 3 subunit G-like

$0.0124426996995103 \quad$ Gb|AAD43168.1

0.0124676350405895 protein NRT1/ PTR FAMILY 7.3-like

0.0124676350405895 NAC domain-containing protein 17-like

0.0124676350405895 Zinc finger protein, putative

0.0124919222927065 Protein trichome birefringence

0.0124919222927065 No data found

0.0124919222927065 D-2-hydroxyglutarate dehydrogenase, mitochondrial-like

0.0125375367837544 Acetyl-CoA carboxylase, putative

0.0125375367837544 Transcription factor, putative

0.0125439869644173 RNA-binding protein 39 isoform X1

0.0125439869644173 GATA transcription factor 24-like

0.0125439869644173 DNA primase large subunit 
MELO3C020710 0.000855996771340739 MELO3C011139 0.000858115671118931 MELO3C013139 0.000862543291056284 MELO3C003439 0.000864308651512991 MELO3C013707 0.000869260816383277 MELO3C026026 0.000872361768709862 MELO3C014888 0.000874915018815425 MELO3C026198 0.000877911808357279 MELO3C002816 0.000879193317881222 MELO3C011944 0.000880749748052967 MELO3C016558 0.000881863372467206 MELO3C006320 0.000883864838401527 MELO3C015098 0.000890296667866552 MELO3C018689 0.000892082156492835 MELO3C010693 0.000893079815306175 MELO3C010903 0.00089679269942633 MELO3C012634 0.000897600518375397 MELO3C020806 0.000898152359463777 MELO3C013437 0.000905985501302276 MELO3C007398 0.000902959644092149 MELO3C007472 0.000905497264532817 MELO3C003057 0.00090625881451678 MELO3C016033 0.000904166343776214 MELO3C024333 0.000905840775861422 MELO3C025720 0.00090037014311295 MELO3C015198 0.000903708398408365 MELO3C010183 0.000904336723108301 MELO3C017091 0.000906532461513176 MELO3C003312 0.000901569386704937 MELO3C013403 0.000911652577039623 MELO3C026102 0.000911617325186298 MELO3C003139 0.000913198703413887 MELO3C014849 0.000914437911399668 MELO3C020172 0.000915802238327545 MELO3C014258 0.000917959386003142 MELO3C004142 0.000918487265869183 MELO3C007144 0.000921378557700914 MELO3C019858 0.000923438376700947 MELO3C018046 0.000926412561525591 MELO3C021858 0.000925765305583526 MELO3C025347 0.000928175455473412 MELO3C009770 0.000931788216151919 MELO3C005244 0.000931247853704242 MELO3C002159 0.000933740659770033 MELO3C015926 0.000935266432297022 MELO3C008149 0.000936996534496304 MELO3C017347 0.000938758613047153 MELO3C007917 0.000939995495577994 MELO3C025885 0.000940506159318399 MELO3C004539 0.000946307413841607 MELO3C006569 0.000945446689776475 MELO3C013757 0.000947021349863686 MELO3C017581 0.000945962882874185 MELO3C005046 0.000947141386938188 MELO3C024673 0.000950133026605071 MELO3C001994 0.000950855162021425 MELO3C016464 0.00095330453726683 MELO3C007646 0.000954499819987076 MELO3C020749 0.000958357604638915 MELO3C002897 0.000957057417631413 MELO3C012906 0.000957862608659155 MELO3C004480 0.000959813634533235 MELO3C011759 0.000962693217067989 MELO3C026443 0.000961972935031419 MELO3C002092 0.000963011258913182 MELO3C020835 0.000965408283639557 MELO3C005939 0.000969123816900619 MELO3C027064 0.000971938273088613
0.0125439869644173 THO complex subunit 4A 0.0125439869644173 Nuclear pore complex protein NUP85 0.0125993842123303 prohibitin-1, mitochondrial $0.0126158400286253 \mathrm{NAD}(\mathrm{P})$-binding rossmann-fold protein 0.0126787532221221 Thioredoxin reductase 0.0127145923027683 PRA1 family protein 0.012742401701022 yrdC domain-containing protein, mitochondrial isoform X2 0.0127766251313544 Gamma carbonic anhydrase-like 1, mitochondrial 0.0127858533393492 obg-like ATPase 1

0.0127990631157454 Beta-1,4-mannosyl-glycoprotein 4-beta-N-acetylglucosaminyltransferase 0.0128058233403345 Cyclic nucleotide-gated ion channel, putative 0.0128254567917647 Type 1 membrane protein, putative isoform 1 0.012909301684065 Tubulin beta chain 0.0129257010334387 nuclear transcription factor Y subunit C-2 0.0129306695546053 Lysine ketoglutarate reductase trans-splicing protein (DUF707) 0.0129749150336781 thylakoidal processing peptidase 1, chloroplastic-like 0.0129755749429774 Receptor-like protein kinase 0.0129755749429774 Peptidyl-prolyl cis-trans isomerase 0.0129920969393496 V-type proton ATPase subunit a $0.0129920969393496 \quad 5$-adenylylsulfate reductase 0.0129920969393496 LRR receptor-like serine/threonine-protein kinase GSO1 isoform X1 0.0129920969393496 Phosphatidylinositol-4-phosphate 5-kinase, putative 0.0129920969393496 glutathione S-transferase-like 0.0129920969393496 DNA-binding protein, putative 0.0129920969393496 phototropin-1

0.0129920969393496 Pentatricopeptide repeat-containing protein 0.0129920969393496 glycine-rich cell wall structural protein 2-like 0.0129920969393496 Pleckstrin homology domain-containing family M member 3 0.0129920969393496 Protein kinase superfamily protein 0.0130465411188083 auxin-responsive protein SAUR36-like 0.0130465411188083 Rhodanese-like domain-containing protein 6 0.013059204340131 ATP-dependent zinc metalloprotease FtsH 0.0130674633228886 Alpha/beta hydrolase-3 0.0130774970388508 Hydroxyacylglutathione hydrolase 0.0130968989268235 IQ-domain 1 0.0130968989268235 Unknown protein 0.0131286472842968 DNA helicase 0.0131485108157657 No data found 0.0131718658585809 Zinc transport ZntB 0.0131718658585809 WEB family protein At3g02930, chloroplastic 0.0131874367411111 Short-chain dehydrogenase/reductase 0.0132197453166553 two-component response regulator ORR9-like 0.0132197453166553 ABC transporter F family member 3 0.013237935599281 Nascent polypeptide-associated complex subunit beta 0.0132500550727646 DNA helicase

0.0132650498636326 LEAF RUST 10 DISEASE-RESISTANCE LOCUS RECEPTOR-LIKE 0.0132804755365818 Actin-interacting protein 1-2 0.013286163190543 thiosulfate sulfurtransferase 18 isoform X1 0.013286163190543 ras-related protein Rab11C 0.0133322132934015 serine/threonine protein phosphatase 2A $57 \mathrm{kDa}$ regulatory subunit B' iota 0.0133322132934015 Cytochrome P450 family protein 0.0133322132934015 Proteasome subunit alpha type 0.0133322132934015 inositol-3-phosphate synthase 0.0133322132934015 protein indeterminate-domain 2 0.0133647985344897 Trihelix transcription factor 0.0133654367222499 thioredoxin-like 1-2, chloroplastic 0.0133903352108696 No data found 0.0133975955543175 heptahelical transmembrane protein 4-like

0.0134231236411446 amidophosphoribosyltransferase, chloroplastic-like 0.0134231236411446 Nuclear receptor corepressor 1 0.0134231236411446 CDT1-like protein a, chloroplastic 0.0134339897012026 No data found 0.0134501480567726 transcription factor DIVARICATA 0.0134501480567726 No data found 0.0134501480567726 Receptor-like kinase 0.0134740976633199 Unknown protein 0.0135164027259677 nucleoside diphosphate kinas 0.0135460895943733 Pentatricopeptide repeat-containing protein 
2
MELO3C008088 0.000973514744218917 MELO3C003880 0.000975348030759493 MELO3C012016 0.00097897348711995 MELO3C016915 0.000983430844025079 MELO3C007613 0.000983096242711756 MELO3C017416 0.000982464041033859

MELO3C005915 0.000986201687010468 MELO3C025944 0.000985917354598187 MELO3C010599 0.000985933718972154 MELO3C016811 0.000987087449194402 MELO3C012695 0.000991906014960131 MELO3C005439 0.000993351291647127 MELO3C009735 0.000998956915092108 MELO3C009562 0.00100183372192109 MELO3C013175 0.00100051618365293 MELO3C014176 0.000996507629712262 MELO3C016139 0.00099980979828429 MELO3C017878 0.00100181581724934 MELO3C018042 0.000996419011577765 MELO3C015770 0.000999619597627954 MELO3C017467 0.000996740137645613 MELO3C010964 0.000998038684778546 MELO3C012873 0.000995473042618045 MELO3C009961 0.000997510371846033 MELO3C003951 0.00100473081766883 MELO3C007467 0.00100905049094524 MELO3C015523 0.00101538983608185 \begin{tabular}{ll} 
MELO3C007316 0.00101829843398527 \\
\hline
\end{tabular} MELO3C010601 0.00101839561951511 MELO3C025590 0.00102358772268074 MELO3C010273 0.0010234211073139 MELO3C015727 0.00102505641861605 MELO3C001962 0.00102517306062677 MELO3C013125 0.00103621741953086 MELO3C007127 0.00103536298460694 MELO3C005201 0.00103600206925747 MELO3C001943 0.00103568118847042 MELO3C010755 0.00103635927060819 MELO3C009038 0.00103983524063711 MELO3C024239 0.0010406075041931 MELO3C018758 0.00104031520097592 MELO3C010959 0.00104181627537914 MELO3C002016 0.00104357250065368 MELO3C004597 0.0010513769558067 MELO3C014116 0.0010524633137019 MELO3C007647 0.0010600013683566 MELO3C021598 0.0010608705730426 MELO3C005286 0.00106186121305873 MELO3C021366 0.00106142095838846 MELO3C010052 0.00106101733938158 MELO3C011488 0.00106087293661083 MELO3C010821 0.00106381826751312 MELO3C013824 0.00106573213139216 MELO3C005300 0.0010652510900182 MELO3C000254 0.00107265318236205 MELO3C013964 0.00107467152470697 MELO3C019125 0.00107877740647

MELO3C013406 0.00108795323877697 MELO3C017833 0.00109169981020196 MELO3C002134 0.00109141215498465 MELO3C018732 0.00109122951858942 MELO3C002171 0.00109283685930461 MELO3C008124 0.0010950939938712 MELO3C020616 0.00110160610411703 MELO3C009334 0.00111024230362466 MELO3C016951 0.00110990724649795
0.0135584927246681 phospholipase SGR2 0.0135744526141432 Histone deacetylase complex subunit 0.0136153150684027 MLP-like protein 423

0.0136484720580824 copper-transporting ATPase PAA2, chloroplastic isoform X1

0.0136484720580824 acyl-CoA-binding domain-containing protein 3

0.0136484720580824 Cyclin-dependent protein kinase inhibitor SMR15

Mitochondrial import inner membrane translocase subunit

0.0136581326204556 Tim17/Tim22/Tim23 family protein

0.0136581326204556 ABC1 family protein

0.0136581326204556 Gamma-glutamylcyclotransferase

0.0136608199258166 Ubiquitin system component Cue

0.0137178934800053 la-related protein 6B isoform X1

$0.0137282677807831 \quad$ NO-associated protein 1, chloroplastic/mitochondrial

$0.0137301972062592 \quad$ NF-kappa-B-activating protein

0.0137301972062592 Pentatricopeptide repeat-containing protein

0.0137301972062592 Pentatricopeptide repeat-containing family protein

$0.0137301972062592 \quad 30$ S ribosomal protein S6

$0.0137301972062592 \quad$ Mitogen-activated protein kinase

0.0137301972062592 Protein LURP-one-related 17

0.0137301972062592 Phosphoglycerate mutase-like protein 1

0.0137301972062592 phospholipase A1-Igamma1, chloroplastic-like isoform X1

0.0137301972062592 Syntaxin/T-SNARE family protein

0.0137301972062592 Cyclin-T1-like protein

0.0137301972062592 NAC domain protein

0.0137301972062592 At1g23710

0.0137603529252023 No data found

0.0138099363448909 No data found

$0.0138870733191 \quad$ Cysteine protease

0.0139089177661161 Transmembrane protein, putative

0.0139089177661161 LysM domain-containing GPI-anchored protein 1

0.0139605206734958 NF-kappa-B inhibitor-like protein 2 isoform 2

0.0139605206734958 Transmembrane protein

0.0139628570857366 Maternal effect embryo arrest 60

0.0139628570857366 protein REVEILLE 2-like

0.0140667073781726 Lipid-binding serum glycoprotein family protein, putative

0.0140667073781726 9-cis-epoxycarotenoid dioxygenase

0.0140667073781726 multicopper oxidase LPR1-like

0.0140667073781726 17. class I heat shock protein

0.0140667073781726 At5g11810

0.0140953069960971 adenylosuccinate synthetase, chloroplastic

0.0140953069960971 Mitochondrial carrier protein

0.0140953069960971 Secretory carrier-associated membrane protein

0.014102007966047 Adenosine kinase, putative

0.0141161050105544 zinc finger protein ZAT4-like

0.0142119394252064 DUF1005 family protein (DUF1005)

0.0142168932847461 poly(A)-specific ribonuclease PARN

0.0142852160059244 xyloglucan 6-xylosyltransferase 1

0.0142852160059244 pre-rRNA-processing protein ESF2

0.0142852160059244 Mediator of RNA polymerase II transcription subunit 23

0.0142852160059244 Acyl carrier protein

0.0142852160059244 Basic leucine zipper/W2 domain protein

0.0142852160059244 ylmG homolog protein 2, chloroplastic

0.0143018018823121 Glutamyl-tRNA (Gln) amidotransferase subunit A

0.0143080515723071 inactive poly [ADP-ribose] polymerase RCD1-like

$0.0143080515723071 \quad$ BnaC07g20870D protein

0.014391187295155 Auxin response factor

0.0144084778964277 Flavin-containing monooxygenase

0.0144537143828874 WAT1-related protein At5g07050-like

0.0145667718729535 tRNA-dihydrouridine $(47)$ synthase $[\mathrm{NAD}(\mathrm{P})(+)]$

0.0145872662731249 Golgi SNAP receptor complex member 1-1

0.0145872662731249 protection of telomeres protein 1a-like isoform X1

0.0145872662731249 Histone-lysine N-methyltransferase, H3 lysine-36 specific

0.0145925862977734 LOW QUALITY PROTEIN: probable metal-nicotianamine transporter YSL5

0.0146128454628124 SNARE associated Golgi protein family

0.0146898169819089 Haloacid dehalogenase-like hydrolase

0.0147850136576423 Methyltransferase type 11

0.0147850136576423 Glutathione peroxidase 
MELO3C019907 0.00111354912043438 MELO3C013702 0.00111548535806738 \begin{tabular}{ll} 
MELO3C021370 0.00111564575125667 \\
\hline
\end{tabular} MELO3C004713 0.00111704118586142 MELO3C020825 0.00111940322596316 MELO3C012628 0.00112110321793302 MELO3C019140 0.00112109638180102 MELO3C009380 0.00112906693300263 MELO3C013859 0.00112932514486075 MELO3C006995 0.00112951941137085 MELO3C007743 0.00112985006801136 MELO3C008318 $\quad 0.00113812614307651$ MELO3C008052 0.00114026869495676 MELO3C023492 0.00114758708393703 MELO3C020963 0.00114885976832013 MELO3C008233 0.00115122999267281 MELO3C024505 0.00115519001740017 MELO3C022000 0.00115848232083415 MELO3C025511 0.00116227454348072 MELO3C005511 0.00116090537302926 MELO3C023317 0.00116296655211834 MELO3C003305 0.00116254711149832 MELO3C003420 $\quad 0.0011601994899415$ \begin{tabular}{ll} 
MELO3C018616 0.0011655704891641 \\
\hline
\end{tabular} MELO3C010482 0.00117143822600851 MELO3C017259 0.0011720880653634 MELO3C024554 0.00117317342071144 MELO3C004437 0.0011762151255299 MELO3C018981 0.00117475910333109 MELO3C026230 0.00117574360508321 MELO3C023200 0.00117863846732424 MELO3C017757 0.00118580073769048 MELO3C010651 0.00118541624822299 MELO3C016562 0.00118807502290619 MELO3C006802 0.00118904758520466 MELO3C025917 0.00119861897500195 MELO3C009101 0.00120197975122283 MELO3C018950 $\quad 0.00120877376552109$ MELO3C011752 0.00121308127110931 MELO3C026886 0.00121509032211076 MELO3C002651 0.00121668506400485 \begin{tabular}{ll} 
MELO3C022930 0.0012198997375259 \\
\hline
\end{tabular} MELO3C002363 0.00121933620530013 MELO3C017254 0.00121947301870651 MELO3C014732 0.00122174468221292 MELO3C006268 0.00122376359873888 MELO3C007914 0.00122356487409925 MELO3C017945 0.00122821280365626 MELO3C018617 0.00122760195676008 MELO3C023725 0.00123619916806539 MELO3C007724 0.00123610608361802 MELO3C007986 0.00123446337349731 MELO3C010353 0.00123739501059106 MELO3C003675 0.00123674154707298 MELO3C003249 0.00124444883017238 MELO3C024088 0.00124664245485939 MELO3C014877 0.00124810334608227 MELO3C013822 0.0012520996871519 MELO3C025637 0.00125177019058254 MELO3C022009 0.00125751310639199 MELO3C010375 0.00126258066098428 MELO3C026013 0.00126215947958241 MELO3C012713 0.00126675355311634 MELO3C008252 0.00126698624011023 MELO3C014398 0.00127170983656311 MELO3C019504 0.00127161606417181 MELO3C002271 0.00127212561074663 MELO3C008801 0.00127339795929893
0.0148190576680987 40S ribosomal protein S17 0.0148269770804629 kinesin-like calmodulin-binding protein 0.0148269770804629 PAX-interacting protein 1 0.0148355389237238 Ribosomal protein 0.0148569182187812 Phospholipase-like protein 0.0148595083563484 BOI-related E3 ubiquitin-protein ligase 1-like

0.0148595083563484 Nascent polypeptide-associated complex subunit alpha-like protein 0.0149353473849775 ras-related protein RABC2a 0.0149353473849775 CAAX amino terminal protease 0.0149353473849775 DNA helicase

0.0149353473849775 Polyadenylate-binding 2

0.0150346844144602 Dehydration-responsive element-binding protein 2C 0.0150529187544793 No data found

0.0151394103678506 Receptor kinase

0.0151460824863513 WRKY family transcription factor

0.0151672055538995 Thylakoid lumenal $15 \mathrm{kDa}$ protein 1, chloroplastic

0.0152092317690906 SRSF protein kinase 1-like

0.0152424166250191 KH domain-containing protein

0.0152506151645319 elongation factor 1-alpha-like

0.0152506151645319 palmitoyl-protein thioesterase 1-like

0.0152506151645319 phosphatidylinositol/phosphatidylcholine transfer protein SFH8-like

0.0152506151645319 Protein CDI

0.0152506151645319 Ring finger protein, putative

0.0152746195026555 Alkyl transferase

0.0153396734280064 F-box protein 7

0.0153396734280064 Protoheme IX farnesyltransferase, mitochondrial

0.0153437098580332 Chaperone dnaJ-like protein

0.0153529891038268 Methyltransferase-related family protein

0.0153529891038268 UNC93-like protein

0.0153529891038268 Ribonuclease J

0.015374459109106 Receptor-like protein kinase

0.0154474793988452 Inositol transporter 1

0.0154474793988452 No data found

0.0154669041709784 At4g33560

0.0154693680897278 Protein DEHYDRATION-INDUCED 19

0.0155836248435244 CAX-interacting protein 4

0.0156170382282234 B3 domain-containing transcription repressor VAL2

0.015694985598472 Chaperone protein DNAj, putative

0.0157405663752547 E3 ubiquitin ligase BIG BROTHER-related

0.0157562828439694 Chaperone DnaJ

0.0157666097959526 protein SCAR3

0.0157772101613615 mitotic spindle checkpoint protein MAD1

0.0157772101613615 TITAN-like protein

0.0157772101613615 Sterile alpha motif domain-containing family protein

0.0157907301891512 Pyruvate kinase

$0.015796148569604 \quad 10 \mathrm{kDa}$ chaperonin isoform X1

0.015796148569604 calmodulin-lysine N-methyltransferase isoform X1

0.015832881631467 bifunctional epoxide hydrolase 2-like

0.015832881631467 Pentatricopeptide repeat-containing protein

0.0158993585323115 Protein ROOT PRIMORDIUM DEFECTIVE 1

0.0158993585323115 Pesticidal crystal cry8Ba protein

0.0158993585323115 Histidine phosphatase family (Branch 1) protein

0.0158993585323115 protein NRT1/ PTR FAMILY 6.2-like

0.0158993585323115 Protein TIFY 8

0.0159795968446518 target of Myb protein 1 isoform X1

0.0159973631195699 F-box family protein, putative

0.0160057097284278 DNA/RNA-binding protein KIN17

0.0160361327636594 Unknown protein

0.0160361327636594 Mannosyltransferase

0.0160950267907553 Vesicle transport v-SNARE family protein

0.0161389679441932 DNA ligase

0.0161389679441932 Plectin-like protein

0.016174344703256 Zinc finger, CCCH-type

0.016174344703256 Protease inhibitor/seed storage/lipid transfer family protein

0.0162085217333131 Myosin-binding protein 1

0.0162085217333131 Meiosis arrest female protein 1-like protein

0.0162085217333131 F-box protein SKIP16

0.0162142722747869 H/ACA ribonucleoprotein complex subunit 
2
MELO3C002687 0.00127622712446374 MELO3C012470 0.00127972386650055 MELO3C007690 0.00128234955404771 MELO3C015518 0.00128401665288447 MELO3C016882 0.00128643995485178 MELO3C023961 0.00128738672658923 MELO3C011040 0.00129103487176252 MELO3C023304 0.00129224801413341 $\begin{array}{lll}\text { MELO3C006507 } & 0.00129529568976838\end{array}$ MELO3C015862 0.00129679606732103 MELO3C011193 0.00130134505932378 MELO3C014190 0.00130600334466013 MELO3C016890 0.00131018435614794 MELO3C017808 0.00131120432915433 MELO3C018360 0.00131240394258225 MELO3C016300 0.00132248461135576 MELO3C012715 0.00132243173147539 MELO3C005294 0.00132249068593726 MELO3C021556 0.00132370263701453 MELO3C006249 0.00132556843054021 MELO3C009467 0.001327686515185 MELO3C016114 0.00132956872636503 MELO3C012296 0.00133013796895898 MELO3C026175 0.00132851382917032 MELO3C002646 0.00133518613930561 MELO3C011088 0.00133656832394879 MELO3C004475 0.00133894602167994 MELO3C012100 0.00133814393390308 MELO3C015409 0.00134065781250059 MELO3C018106 0.0013404763400835 MELO3C014018 0.00134463174948718 MELO3C003846 0.00135074087660314 MELO3C020978 0.00135752330090511 MELO3C011952 0.00136365991062004 MELO3C009873 0.00136459747634987 $\begin{array}{ll}\text { MELO3C004461 } & 0.00136807981374421\end{array}$ MELO3C015398 0.00136911537600959 MELO3C018573 0.00137461012268614 MELO3C009172 0.00137678127435881 MELO3C016608 0.00137878915949885 MELO3C003926 0.00138073092069713

MELO3C005199 0.00138077415568361 MELO3C008375 0.00138251768956899

MELO3C008114 0.00138405655359919 MELO3C017272 0.00138518236716068 MELO3C019561 0.00138619118409045 $\begin{array}{ll}\text { MELO3C006587 } & 0.0013871485080218\end{array}$ MELO3C006136 0.00139306498415936 MELO3C019011 0.00139215182628716 MELO3C005696 0.00139171797478665 MELO3C021247 0.0013904593458578 MELO3C018688 0.00139270363874489 MELO3C006754 0.0013989936942238 MELO3C027346 0.00139863809916196 MELO3C015791 0.0013969561102013 MELO3C022388 0.00139981762664432 MELO3C026711 0.00139804378035702 MELO3C016623 0.00140078931774978 MELO3C016965 0.00140646424613533 MELO3C015422 0.00140579592416201 MELO3C021047 0.00140828800908288 MELO3C009663 0.00141290977451258 MELO3C004467 0.00141258546588374 MELO3C013359 0.00141108727208694
0.0162398256965427 dynamin-related protein 4C-like 0.0162738355695553 two-component response regulator ORR9-like 0.0162967318808805 No data found

0.0163074243587237 Acyl-CoA N-acyltransferase (NAT) superfamily protein

0.0163277009436811 Magnesium transporter NIPA

0.0163292231621135 ras-related protein RABA4c isoform X1

0.0163649856755058 40S ribosomal protein S6-like

0.0163698563381147 Isoflavone reductase like

$0.0163979452418178 \quad 40$ S ribosomal protein S3a

0.0164064225070615 Unknown protein

0.0164534337878267 COP9 signalosome complex subunit 5b

0.0165017658692853 DUF4408 domain protein

0.0165440094946072 aminopeptidase M1

0.0165463094546126 Protein NEOXANTHIN-DEFICIENT 1

0.0165508719425651 No data found

0.0166461877352294 UDP-glucose 4-epimerase family protein

0.0166461877352294 mediator of RNA polymerase II transcription subunit 19a-like

0.0166461877352294 Myosin-binding protein 2

0.0166508301773249 ADP-ribosylation factor-like

0.0166636861456007 No data found

0.0166786633326799 Alpha/beta-Hydrolases superfamily protein

0.0166786633326799 Protein ROOT INITIATION DEFECTIVE 3

0.0166786633326799 Unknown protein

0.0166786633326799 Long chain base biosynthesis protein

0.0167313395083417 Transcription initiation factor IIB

0.0167380392071431 mechanosensitive ion channel protein 3, chloroplastic-like

0.016746576936135 arogenate dehydrogenase 1, chloroplastic-like

0.016746576936135 ribonuclease 3-like

0.0167467749140254 ABC transporter B family protein

$0.0167467749140254 \quad$ ABC transporter B family protein

0.0167857979902796 Pentatricopeptide repeat-containing protein family

0.0168514097106983 Glucan endo-1,3-beta-glucosidase, putative

0.0169253331247317 Adenylyl cyclase-associated protein

0.0169911164510001 UDP-galactose:fucoside alpha-3-galactosyltransferase

0.0169920779069569 zinc-finger homeodomain protein 9-like

0.017024705886348 protein TOPLESS

0.017026863703283 cysteine synthase-like

0.017084440096242 Inorganic pyrophosphatase

0.0171006625077434 RING finger protein

0.0171148379075693 Transmembrane protein, putative

0.0171179590713092 No data found

0.0171179590713092 charged multivesicular body protein 7 isoform X1

0.0171288217385809 Unknown protein

$$
\text { (2) }
$$

TSA: Wollemia nobilis Ref_Wollemi_Transcript_28887_1569 transcribed

0.017137136600019 RNA sequence

0.0171403299304863 Ethylene-responsive transcription factor

0.0171420724449607 At1g78995

0.0171431763985747 Endonuclease/exonuclease/phosphatase family protein

0.0171625953662903 Signal recognition particle $54 \mathrm{kDa}$ protein

0.0171625953662903 copper-transporting ATPase PAA1, chloroplastic isoform X1

$0.0171625953662903 \quad$ E3 ubiquitin-protein ligase RHA1B

0.0171625953662903 Dihydrolipoyl dehydrogenase

0.0171625953662903 dynamin-like protein ARC5

0.0171921631272379 SWI/SNF-related matrix-associated actin-dependent regulator of chromatin

0.0171921631272379 Indole-3-acetic acid-amido synthetase GH3.3

0.0171921631272379 Pentatricopeptide repeat-containing protein

0.0171921631272379 Pre-mRNA-processing factor 39

0.0171921631272379 Protein FAM32A-like

$0.0171934047459543 \quad \mathrm{NAD}(\mathrm{P}) \mathrm{H}$ dehydrogenase (Quinone)

0.0172416278069067 Formin-like protein

0.0172416278069067 Arginine--tRNA ligase

0.0172532753668597 nucleolin-like

0.0172561584703465 1-acyl-sn-glycerol-3-phosphate acyltransferase-like protein

0.0172561584703465 Exostosin family protein

0.0172561584703465 Ubiquitin-conjugating enzyme, E2 
MELO3C005079 0.00141241328020525 MELO3C021384 0.00141376598334197 MELO3C026760 0.00141223060883444 MELO3C017133 0.00141800169024686 MELO3C020927 0.00141984372965775 MELO3C014895 0.00142136942562132 MELO3C017703 0.00142356391852405 MELO3C008096 0.00142424626433679 MELO3C016382 0.00142850587618693 MELO3C022448 0.00142880345963026 \begin{tabular}{ll} 
MELO3C019524 0.0014304129339574 \\
\hline
\end{tabular} MELO3C023309 0.00143156903792496 MELO3C011834 0.00143661881258605 MELO3C025593 0.00144052700680608 MELO3C013587 0.00144121378209339 MELO3C003416 0.00144044124356046 MELO3C016383 0.00144678432164969 MELO3C017844 0.00144724600566726 MELO3C014454 0.00145093122867479 MELO3C007047 0.0014515386926125 MELO3C023367 0.00145318824654184 MELO3C004576 0.00145859722393693 MELO3C022932 0.00145937989094569 MELO3C021360 0.00146048979136648 MELO3C015477 0.00146228225489542 MELO3C003375 0.00146380420207692 MELO3C014281 0.0014702360035872 MELO3C005421 0.00146899146702206 MELO3C021914 0.00146823354847903 MELO3C018768 0.00146951190071176 MELO3C023985 0.00147349210060765 MELO3C007497 0.0014780779626572 MELO3C002227 0.00147953668231926

MELO3C014008 0.00148146067848454 MELO3C020591 0.00148245648757772 MELO3C002416 0.00148622342396076 MELO3C004577 0.00148880872396417 MELO3C018494 0.00150424303608299 MELO3C000075 0.0015064080353504 MELO3C027219 0.00150762899745338 MELO3C020942 0.00150894782427158 MELO3C022059 0.00151061562675903 MELO3C005651 0.00151252537705282 MELO3C009588 0.00151414543658712 MELO3C022783 0.00151463788301731 MELO3C021536 0.00151639341105281 MELO3C015130 0.00152015005154871 MELO3C025354 0.00151869336680555 MELO3C008285 0.00151933283763561 MELO3C003457 0.00152415156525021 MELO3C003826 0.00152506826935495 MELO3C014604 0.0015283132911843 MELO3C016826 0.00153179476841669 MELO3C021148 0.00153136859350866 MELO3C025035 0.00153071881752509 MELO3C020427 0.00153376154089113 MELO3C021563 0.00153757354735573 MELO3C022392 0.00153951311588807 MELO3C011439 0.00154415748059011 MELO3C006294 0.00154543307012311 MELO3C013965 0.00154666283116955 MELO3C014230 0.00155245603648058 MELO3C017115 0.00155312632818672 MELO3C004279 0.00156216413039512 MELO3C010197 0.00157227802397697
0.0172561584703465 protein ASPARTIC PROTEASE IN GUARD CELL 2 0.0172561584703465 Cox19-like CHCH family protein 0.0172561584703465 Arabinogalactan protein 0.0172971682400774 No data found 0.0173089468006241 Molybdenum cofactor sulfurase 0.0173168567468202 squamosa promoter-binding-like protein 13A 0.0173305233976508 disease resistance protein RGA2-like 0.0173305233976508 Galactose-binding domain-like protein 0.0173645781687618 small nuclear ribonucleoprotein Sm D2-like 0.0173645781687618 Metal tolerance protein $0.017373447129597 \quad 40$ S ribosomal protein S16

0.0173768020466995 SNF1-related protein kinase regulatory subunit gamma-1-like 0.0174273863204926 Protein EI24 like

0.0174509693332693 Pentatricopeptide repeat-containing family protein 0.0174509693332693 No data found

0.0174509693332693 Unknown protein

0.0175025482951149 RING-type E3 ubiquitin transferase

0.0175025482951149 calcineurin subunit B

0.0175329893825102 Receptor-like protein kinase

0.0175329893825102 Protein kinase

0.0175421850128085 DNA repair protein XRCC1

0.0175954172565851 sugar transport protein 14-like

0.0175954172565851 Auxin response factor

0.0175980554543604 Pentatricopeptide repeat-containing family protein

0.0176089099097132 No data found

0.0176164955434596 Two-component response regulator-like protein APRR2

0.0176508758874429 Aldo/keto reductase, putative

0.0176508758874429 GTF2H2

0.0176508758874429 peroxidase 10-like

0.0176508758874429 Wound-responsive family protein

0.0176792196202312 RNA/RNP complex-1-interacting phosphatase, putative

0.0177234740039569 Transcription termination factor family protein 0.0177302002057786 Unknown protein

0.0177424905636089 Adenosine monophosphate-protein transferase and cysteine protease ibpA

$\begin{array}{cl}0.0177436564685893 & \text { Glycosyltransferase } \\ 0.017777968746094 & \text { Ankyrin repeat family protein }\end{array}$

0.0177981134924749 CRS2-associated factor 1, chloroplastic

0.0179717457468862 Protein TIC 21, chloroplastic

0.0179867305260793 Myosin heavy chain-like protein, putative

0.0179904320668923 Hydroxycinnamoyl-CoA shikimate/quinate hydroxycinnamoyltransferase

0.0179952962448909 PQ-loop repeat family protein / transmembrane family protein

0.0180043138279203 Pentatricopeptide repeat-containing protein

0.0180162024556189 30S ribosomal S1

0.0180196286456078 Downstream neighbor of Son

0.0180196286456078 transmembrane protein 50A

0.0180296529048055 Quinone oxidoreductase-like protein

0.0180417327932905 polygalacturonase-like

0.0180417327932905 Late embryogenesis abundant protein

0.0180417327932905 eukaryotic translation initiation factor 3 subunit F

0.018078359917193 protein EMBRYONIC FLOWER 1-like isoform X3

0.0180783753010149 No data found

$0.0181059743176957 \quad$ F16F4.11 protein

0.0181146196894978 At4g33800

0.0181146196894978 Autophagy-related protein

0.0181146196894978 lecithin-cholesterol acyltransferase-like 1

0.018127023740909 DEAD-box ATP-dependent RNA helicase 42-like

0.0181612081260337 isocitrate dehydrogenase [NADP]

0.018173248371592 Cytochrome oxidase complex assembly protein

$0.0182171840407253 \quad 6,7-$ dimethyl-8-ribityllumazine synthase

0.0182213478817083 Dna repair helicase xpb1

0.0182249667379281 Flavin-containing monooxygenase

0.0182793157660068 Ethylene insensitive 2

0.0182793157660068 succinate dehydrogenase subunit 7B, mitochondrial-like

0.0183747346105856 RING zinc finger family protein

0.0184785490735071 Tetratricopeptide repeat (TPR)-like superfamily protein 


\section{MELO3C026288 0.00157286146096336}

MELO3C010403 0.00157447526897514 MELO3C016483 0.00158023620980208 MELO3C008947 0.00158137311774076 MELO3C024313 0.00158260126212806 MELO3C002034 0.00158329498422793 MELO3C010600 0.00158218164950008 MELO3C013750 0.00158688511649507 MELO3C015747 0.00158659415425944 MELO3C005214 0.00158559844227557 MELO3C020906 0.00158710874420764 MELO3C014944 0.00158975633025693 MELO3C016193 0.00158896575333467 MELO3C022042 0.00159143253219463 MELO3C021348 0.00159087168485317 MELO3C010688 0.00159627418691699 MELO3C018476 0.0016042694552616 MELO3C019491 0.00160887864625414 MELO3C007099 0.00160852551941759 MELO3C020159 0.00160703147890584 MELO3C023469 0.00161398184714412 MELO3C012604 0.00161829168735006 MELO3C008773 0.00161989152750242 MELO3C013829 0.00162029178298806 MELO3C003734 0.00162208649782969 MELO3C009234 0.00162371681969942 MELO3C020789 0.00162576706990036 MELO3C010960 0.0016263958322309 MELO3C023338 0.00163648326242916 MELO3C010456 0.00163788891852379 MELO3C023852 0.00164327417852994 MELO3C014850 0.00164799342509903 MELO3C015748 0.00164874579002283 MELO3C005404 0.00164747201365656 MELO3C005918 0.00164998656602089 MELO3C008092 0.00165607824610392 MELO3C014611 0.00166499764807648 MELO3C009455 0.00166599357714137 MELO3C015946 0.00166733441386024 MELO3C004391 0.00167241694262599 MELO3C013753 0.00168358970314475 MELO3C002314 0.00168227509520191 MELO3C002117 0.00168396710795249 MELO3C008313 0.0016862201830351 MELO3C024001 0.00169134873987065 MELO3C011109 0.00169484210432513 MELO3C018434 0.00169761795586676 $\begin{array}{ll}\text { MELO3C003770 } & 0.0016973381454457\end{array}$ MELO3C026046 0.00170144999900546 MELO3C014346 0.00170775748623742 MELO3C020369 0.00170878788052986 MELO3C005759 0.00170806862709461 MELO3C026518 0.00171160126017267 MELO3C005711 0.00171342810463715 MELO3C019488 0.00171945922624073 MELO3C007617 0.00171996224191284 MELO3C003852 0.00172185560495652 MELO3C004139 0.0017260170705593 MELO3C009090 0.00173121000396637 MELO3C004645 0.00173460385515178 MELO3C006409 0.0017350513931752 MELO3C019490 0.00173728752931601 MELO3C008815 0.00173564949107596 MELO3C005455 0.00173406833228484 MELO3C017223 0.00173331296437929 MELO3C008195 0.00173319093407187
0.0184785490735071 Serine/threonine-protein phosphatase 2A 55 kDa regulatory subunit B LOW QUALITY PROTEIN: LEAF RUST 10 DISEASE-RESISTANCE

0.0184865113477943 LOCUS RECEPTOR-LIKE PROTEIN KINASE-like 1.4 0.0185349689647406 phosphoribosylamine--glycine ligase 0.0185349689647406 CBS domain-containing protein 0.0185349689647406 AT-rich interactive domain-containing protein 4-like 0.0185349689647406 ninja-family protein 6-like isoform X2 0.0185349689647406 DNA ligase 1 isoform X3 0.0185356656353381 SKP1-like protein 1B 0.0185356656353381 At3g60850 0.0185356656353381 Defensin 0.0185356656353381 60S ribosomal protein L26-1-like 0.0185423015211278 homeobox-leucine zipper protein HAT22-like 0.0185423015211278 Lysine-specific histone demethylase 1 0.0185423015211278 DUF2039 family protein 0.0185423015211278 E3 ubiquitin-protein ligase Topors 0.0185877470031979 F-box protein At1g70590 0.0186698394059878 cyclin-D3-3 0.0186904378734547 Nucleolar complex protein 2 homolog 0.0186904378734547 Nucleoporin protein Ndc1-Nup 0.0186904378734547 transmembrane protein 87A 0.0187386992940912 Protein LOW PSII ACCUMULATION 3, chloroplastic 0.018777698315791 Phosphoglycerate kinase 0.0187788394496662 Succinate dehydrogenase assembly factor 2, mitochondrial 0.0187788394496662 Calmodulin family protein 0.0187886136338056 T4O12.20 0.0187964733131558 SUPERMAN-like zinc finger protein 0.0188054398657658 Nucleoporin NUP188 like 0.0188054398657658 Proteasome subunit alpha type 0.018911005236813 cysteine proteinase RD19a-like 0.0189161802642844 Phosphatidylinositol-4-phosphate 5-kinase, putative 0.0189672833148964 Actin family protein 0.0189971298758232 No data found 0.0189971298758232 transcription factor EMB1444 0.0189971298758232 LOW QUALITY PROTEIN: xyloglucan galactosyltransferase XLT2 0.0190003409284819 NPL4 family protein 0.01905937603864 50S ribosomal protein L14, putative 0.0191508669492501 Pre-mRNA-splicing factor CWC22 0.0191511683090599 BZIP transcription factor family protein 0.0191554318437033 GATA transcription factor-like protein 0.0192026524418143 PRA1 family protein 0.0193016055803562 Vacuolar protein sorting-associated protein 35 0.0193016055803562 cytochrome P450 71A1-like 0.0193016055803562 T-complex protein 1 subunit delta 0.0193162194865199 UTP--glucose-1-phosphate uridylyltransferase 3, chloroplastic 0.0193637369644669 MADS-box transcription factor 0.0193924894080632 Octicosapeptide/Phox/Bem1p 0.019401769103248 Armadillo repeat-containing protein, putative 0.019401769103248 TLD-domain nucleolar protein

0.0194343181205082 alpha-ketoglutarate-dependent dioxygenase AlkB-like 0.0194843255499909 protein PALE CRESS, chloroplastic 0.0194843255499909 60S ribosomal protein L37a 0.0194843255499909 30S ribosomal protein S6 alpha, chloroplastic 0.0195051432701385 Photosystem II reaction center PsbP family protein 0.0195147010602532 heavy metal-associated isoprenylated plant protein 3-like 0.0195665520250788 Pentatricopeptide repeat-containing protein 0.0195665520250788 At5g67390 0.0195768142442639 DNA ligase-like protein 0.0196128372419308 Ubiquitin carboxyl-terminal hydrolase, putative 0.0196392051611116 Kinase family protein 0.0196392051611116 40S ribosomal S3-like protein 0.0196392051611116 Glycolipid transfer protein domain-containing protein 0.0196392051611116 40S ribosomal protein S16 0.0196392051611116 nucleolar MIF4G domain-containing protein 1 0.0196392051611116 origin of replication complex subunit 3 0.0196392051611116 Mitochondrial carrier protein, putative 0.0196392051611116 Katanin p60 atpase-containing subunit a1 
MELO3C018180 0.00173648133509596 MELO3C007601 0.00174263062211799 MELO3C016494 0.00174848962342988 MELO3C007269 0.00174774945217715 MELO3C023451 0.00174626345788564 MELO3C017294 0.00174730515676302 \begin{tabular}{ll} 
MELO3C003671 0.0017542994104538 \\
\hline
\end{tabular} MELO3C015470 0.00175565135908395 MELO3C013769 0.0017643629105647 MELO3C005913 0.00176561299034728 \begin{tabular}{ll} 
MELO3C002390 0.001768224463224 \\
\hline
\end{tabular} MELO3C015695 0.00176927873858901 MELO3C021264 0.00177097699103468 MELO3C017440 0.00177258734143249 MELO3C008115 0.0017806901857147 MELO3C011496 0.00178431101807353 MELO3C021452 0.00179794402675915 MELO3C015692 0.00179684828190319 MELO3C018459 0.00179830009973014 MELO3C026143 0.00179937326870028 MELO3C009032 0.00180618680062949 MELO3C025142 0.00180918499272109 MELO3C017149 $\quad 0.00181586970268244$ MELO3C007800 0.00181959699758183 MELO3C013929 0.0018284576743629 MELO3C002604 0.00183522225955302 MELO3C019168 0.00184600708229754 MELO3C008786 0.00185244710486099 MELO3C016694 0.00186271795353732 MELO3C007935 0.0018647188806673 MELO3C005389 0.00186395168082631 MELO3C026593 0.00186878067212692 MELO3C012493 0.00187295993871484 MELO3C012420 0.00187233804376197 MELO3C003456 0.00187113290658125 MELO3C016923 0.00187914670450451 MELO3C026058 0.00188077657974373 MELO3C012852 0.00188039613797097 MELO3C015177 0.00188263980533365 MELO3C008032 0.00188467890401967 MELO3C019226 0.00188936224392533 MELO3C018413 0.00188895876601003 MELO3C015664 0.00188866569845136 MELO3C020988 0.00189735927898682 MELO3C014447 0.00190474460798895 MELO3C023862 0.0019068491527422 MELO3C025744 0.00190635644741377 MELO3C002219 0.00190789234408573 MELO3C025651 0.00193151944245629 MELO3C026194 0.00193480153015102 MELO3C004459 0.0019419027908818 MELO3C014099 0.00194294651315607 MELO3C017167 0.00194967540488111 MELO3C009583 0.00195706642749194 MELO3C023799 0.00195756025201366 MELO3C014091 0.00195604462548404 MELO3C022124 0.00195823538333095 MELO3C024926 0.00196315683099746 MELO3C020588 0.00196327140921926 MELO3C008516 0.00196685604928237 MELO3C024337 0.00196598778055268 MELO3C017730 0.0019724064548432 MELO3C025272 0.00197489363397907 MELO3C008116 $\quad 0.00197505523169139$ MELO3C014488 0.00198659047530292 MELO3C022477 0.00198647332954294 MELO3C016469 0.00198908132816278 MELO3C023622 0.00199024853339758
0.0196392051611116 tRNA (guanine(37)-N1)-methyltransferase 0.0196883364738033 Lysine-rich arabinogalactan protein 18 0.019709430121642 Polygalacturonase 0.019709430121642 Auxin repressed protein 0.019709430121642 BRCA1-associated protein 0.019709430121642 Carbon catabolite repressor protein 4 like 3 0.0197636389372801 SPOC domain/transcription elongation factor S-II, putative 0.0197675933241442 beta-galactosidase 0.0198543607525597 Seven transmembrane receptor 0.0198571132951984 Auxin-responsive family protein 0.0198751650109338 At5g44650 0.0198757029626816 Expansin

0.0198834704922933 Ubiquitin-conjugating enzyme, E2 0.0198902428442899 U3 small nucleolar RNA-associated protein 25-like $0.01996981855632 \quad 5 '-3$ ' exonuclease

0.0199990682723803 BRCT domain DNA repair protein 0.0201216026456491 No data found

0.0201216026456491 Brefeldin A-inhibited guanine nucleotide-exchange protein 0.0201216026456491 40S ribosomal protein S16

0.0201222099000916 Unknown protein

0.020186974038275 fructokinase-like 2, chloroplastic

0.0202090466183534 Proliferating cell nuclear antigen

0.0202722502873236 Pentatricopeptide repeat-containing protein

0.0203023848052223 Receptor protein kinase, putative

0.0203897293116844 Unknown protein

0.0204536142234269 DUF2921 family protein

0.0205622074835274 Metacaspase-9

0.0206223099627394 Rhomboid protein, putative

0.0207238791076526 VQ motif-containing protein

0.0207238791076526 Peroxidase

0.0207238791076526 NADH dehydrogenase [ubiquinone] 1 alpha subcomplex subunit 12 0.0207573394228541 Surfeit locus protein 2 (SURF2)

0.0207687174787643 Choline/ethanolamine kinase

0.0207687174787643 No data found

0.0207687174787643 Alpha/beta-Hydrolases superfamily protein

0.0208203232473985 No data found

0.0208203232473985 Non-specific serine/threonine protein kinase

0.0208203232473985 Protein BREAST CANCER SUSCEPTIBILITY 1-like protein

0.0208292736781705 60S ribosomal protein L13a, putative

0.020840158832858 Actin

0.0208569116575077 transcription factor ILR3-like

0.0208569116575077 Allene oxide synthase

0.0208569116575077 RING-type E3 ubiquitin transferase

0.020933490726654 Unknown protein

0.0210027680620675 Nuclear ribonuclease Z

0.0210027680620675 Factor of DNA methylation 1

0.0210027680620675 Zinc finger family protein, putative

0.0210027680620675 HIPL1 protein-like isoform X1

0.0212510180886179 Intracellular protein transport protein USO1, putative

0.0212752758457419 No data found

0.0213410737977304 actin-related protein 6

0.0213410737977304 BnaC03g71690D protein

0.0214030792501374 Transmembrane protein

0.0214493569525252 E3 ubiquitin-protein ligase BAH1-like protein, putative

0.0214493569525252 12-oxophytodienoate reductase 3

0.0214493569525252 caffeic acid 3-O-methyltransferase-like

0.0214493569525252 Glycerophosphodiester phosphodiesterase GDPD4

0.0214806908923386 two-pore potassium channel 1 isoform X1

$0.021480690892338617 .5 \mathrm{kDa}$ class I heat shock protein

0.0214960930366782 Guanosine nucleotide diphosphate dissociation inhibitor

0.0214960930366782 transmembrane protein 87B isoform X2

0.0215448313477314 Pentatricopeptide repeat-containing family protein

0.021549925840151 AT3g54190/F24B22_150

0.021549925840151 Magnesium transporter NIPA

0.0216518627465548 Cyclin T1 family protein

0.0216518627465548 Protein nuclear fusion defective 4

0.0216670530335834 zinc finger CCCH domain-containing protein 5

0.0216678160342165 Short-chain dehydrogenase/reductase 
MELO3C005260 0.00200197408497349 MELO3C021345 0.00200657619838918 MELO3C006870 0.0020155939407539 MELO3C020834 0.00201564352309913 MELO3C002717 0.00201365744032933 MELO3C019814 0.00201274196770884 MELO3C008766 0.00202108742744311 MELO3C006934 0.00202201822291359 MELO3C018999 0.00202098577629739 MELO3C007228 0.00202308834498344 MELO3C022845 0.00201915646106055 MELO3C021622 0.00202499337392292 MELO3C013411 0.0020279124270397 MELO3C014706 0.00203201183773705 MELO3C026657 0.00203570078237614 MELO3C027092 0.00204005247370298 MELO3C014417 0.00204144586045041 MELO3C023473 0.00204448889464182 MELO3C019899 0.00204636292220117 MELO3C009149 0.00205119616259886 MELO3C011654 0.00205279144184656 MELO3C018781 0.0020542658700694 MELO3C012131 0.00207255568294284 MELO3C003423 0.00207648623644074 MELO3C016550 0.00207952457836447 MELO3C011972 0.00208045004987989 MELO3C015616 0.00208012082145714 MELO3C009119 0.00208260743603428 MELO3C006314 0.00208855728757984 MELO3C019818 0.00209071289150375 MELO3C009600 0.00209252401614957 MELO3C013539 0.00209583349110565 MELO3C010198 0.00210011636188545 MELO3C002372 0.00211176063197727 MELO3C002268 0.00211346018252012 MELO3C026887 0.00212116551042296 MELO3C004015 0.00212540410728268 MELO3C011763 0.0021263565296451 MELO3C021153 0.00213199409674547 MELO3C005926 0.00213685711803191 MELO3C006092 0.00213681237477381 MELO3C012052 0.00213774633341712 MELO3C009872 0.0021356274309372 MELO3C025491 0.00215106029913348 MELO3C023221 0.00215059543241081 MELO3C001656 0.00215343807777302 MELO3C015457 0.00216212621458034 MELO3C020836 0.00216873957663433 MELO3C025710 0.00216856106267871 MELO3C002598 0.00217096035433062 MELO3C005704 0.00217420806065527 MELO3C004533 0.00218101748222876 MELO3C005821 0.0021861903058622 MELO3C009489 0.0021885277242305

MELO3C024916 0.00219277810808238 MELO3C010727 0.00219323526137982 MELO3C003496 0.00219345078663657 MELO3C021777 0.00219600458758573 MELO3C011042 0.00220196138837392 MELO3C019361 0.00220806422194508 MELO3C016149 0.00220997904778486 MELO3C009308 0.00221549861231163 MELO3C006759 0.00221808743540164 MELO3C007340 0.00222103371849125 MELO3C006212 0.00222542135216774 MELO3C009757 0.00222821468022771
0.0217834634733562 Vacuolar iron transporter $1 \quad 1$ 0.0218215161574823 cullin-1

0.0218719472185081 ethylene-responsive transcription factor ERF027 0.0218719472185081 Unknown protein

0.0218719472185081 prefoldin subunit 6

0.0218719472185081 ABC transporter $\mathrm{G}$ family member

0.021892587246618 sister chromatid cohesion 1 protein 4 isoform X1 0.021892587246618 protein REVEILLE 6-like isoform X2

0.021892587246618 Transmembrane protein, putative 0.021892587246618 GDSL esterase/lipase

0.021892587246618 RING-type E3 ubiquitin transferase 0.021901201610955 Elicitor-responsive protein 1

0.0219207676637149 NADP dependent sorbitol 6-phosphate dehydrogenase family protein 0.0219530644329699 Argonaute

0.0219808937950499 Glycine-rich protein

0.0220158449744045 DExH-box ATP-dependent RNA helicase DExH12-like 0.0220188499716194 sugar transporter ERD6-like 7

0.0220396349237343 Phosphatase 2C family protein 0.0220478021552378 AMMECR1 family

0.0220878260715184 Copper chaperone SCO1/SenC 0.0220929581389797 RING zinc finger protein-like

0.0220967846775603 Leucine-rich repeat family protein

0.0222813838772119 eukaryotic translation initiation factor 5A-like

0.0223114943870883 Zinc finger CCCH domain protein

0.0223176578137305 Unknown protein

0.0223176578137305 Alpha-glucan water dikinase, chloroplastic

0.0223176578137305 BnaA04g07840D protein

0.0223286722335727 Protein LURP-one-related 15

0.0223803135498721 60S ribosomal protein L13a, putative

0.0223912629578675 DVL8

0.022398513167988 60S ribosomal protein L21

0.0224217852740224 vesicle-associated protein 4-2-like

0.0224554401899706 DNA-directed RNA polymerase subunit beta

0.022567727662835 heavy metal-associated isoprenylated plant protein 3-like

0.0225736750376365 Iron-sulfur cluster assembly protein

0.022643728467753 Tryptophan synthase, alpha chain

0.0226746301857241 Pentatricopeptide repeat-containing family protein

0.0226746301857241 LRR receptor-like kinase

0.0227224778287244 Arginine--tRNA ligase

0.0227347077752584 protein BONZAI 1

0.0227347077752584 Glycoside hydrolase family 28 protein/polygalacturonase family protein

0.0227347077752584 dnaJ homolog subfamily B member 1

0.0227347077752584 Gibberellin-regulated family protein

0.0228516889981641 transcription initiation factor TFIID subunit 1-like

0.0228516889981641 DNA cross-link repair family protein

0.0228646497838383 Unknown protein

$0.0229445623921263 \mathrm{~K}(+)$ efflux antiporter

0.0229900364460286 transportin-1

0.0229900364460286 Nuclear factor related to kappa-B-binding protein

0.0230012317798688 E3 ubiquitin-protein ligase RMA1H1-like

0.0230232895388101 Beta-carotene hydroxylase

0.0230830194300835 GDP-mannose transporter, putative

0.0231253735139114 mRNA, clone: RTFL01-46-D12

0.0231377055812785 Ribosomal protein S11

Agenet domain-containing protein / bromo-adjacent domain-containing

0.0231525705960907 protein, putative

0.0231525705960907 3-oxoacyl-[acyl-carrier-protein] reductase 4

0.0231525705960907 Phosphoglycerate mutase-like protein 1

0.0231671445514052 Vesicle-associated membrane protein, putative

0.0232175843347552 VQ motif-containing protein 9

0.0232695092418321 fasciclin-like arabinogalactan protein 7

0.0232772673145084 Cationic amino acid transporter, putative

0.023322964869159 LRR receptor-like kinase

0.023337777710041 Ca2+-activated RelA/SpoT-like protein

0.023356333816019 NAD(P)H dehydrogenase (Quinone) FQR1-like

0.0233900193102506 tRNA (Guanine-N(7)-)-methyltransferase

0.0234069211275623 Amino acid transporter family protein 
MELO3C008970 0.00223264451449334 MELO3C015292 0.00225207974747366 MELO3C020002 0.00225192014674969 \begin{tabular}{ll} 
MELO3C005976 0.00225640094494073 \\
\hline
\end{tabular} MELO3C007466 0.00225882808818756 MELO3C003532 0.00225770592718466 MELO3C007270 0.00226366852216031 MELO3C026053 0.00226283308011266 \begin{tabular}{ll} 
MELO3C010806 0.0022690529645768 \\
\hline
\end{tabular} $\begin{array}{ll}\text { MELO3C013780 } & 0.00227310793406177\end{array}$ \begin{tabular}{ll} 
MELO3C025943 0.00227386513337247 \\
\hline
\end{tabular} MELO3C014588 0.00227553039129758 MELO3C018569 0.00229013798537503 MELO3C026897 0.00229123193674452 MELO3C011885 0.00229709778793552

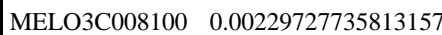
MELO3C006202 0.00230065370872889 MELO3C024312 0.00229967510877782 MELO3C019333 0.00230343015017287 MELO3C008037 0.00230496063997476 MELO3C013489 0.00230717829032479 MELO3C022165 0.00231035379912992 MELO3C018492 0.00231232472788601 \begin{tabular}{ll} 
MELO3C016031 0.0023175934401175 \\
\hline
\end{tabular} MELO3C006106 $\quad 0.00232061450614851$ MELO3C011216 0.00232275821897354 MELO3C012445 0.0023251385175671 MELO3C018264 0.00232641112745924 MELO3C009318 0.00234066344919559 MELO3C016952 0.00234128339180883 MELO3C023445 0.00234094296969889 MELO3C015103 0.00233825502701834 MELO3C021217 0.00234540104667236 MELO3C007793 0.00235058569176971 MELO3C010517 0.00235527000820146 MELO3C023224 0.00235334258523257 MELO3C026247 0.00235451557099309 MELO3C019947 0.00236105137720877 MELO3C011167 0.00236258553874436 MELO3C005136 0.00236472222389739 MELO3C017817 0.0023669444389276 MELO3C003722 0.00237262336257049 MELO3C020532 0.0023744538022763 MELO3C009984 0.00237666292726679 MELO3C017462 0.00238451283574159 MELO3C013034 0.00238546539224549 MELO3C004281 0.00239189379534643 MELO3C019725 0.00239709679529321 MELO3C023404 0.00240231684114978 MELO3C004084 0.00240477987021892 MELO3C021521 0.00240698686863405 MELO3C003593 0.00240673840906169 MELO3C016970 0.00240841038837392 MELO3C026286 0.00240990356136972 MELO3C008847 0.00241118052862543 MELO3C019724 0.00241443701091759 MELO3C023540 0.00242100412623403 MELO3C027184 0.00242276758035198 MELO3C021295 0.00243412618990746 MELO3C004761 0.00243983236026302 MELO3C021783 0.00244000503742026 MELO3C018797 0.00243837045809947 MELO3C022094 0.00244379917129034 MELO3C009908 0.00245124923270912 MELO3C009019 0.00245329237328473 MELO3C015210 0.00245634052673682 MELO3C011264 0.00245758550501518 MELO3C009639 0.00246576674180576
0.023440986983907 vacuolar protein sorting-associated protein 20 homolog 2-like 0.0236199272081026 Epoxide hydrolase 2 0.0236199272081026 Protein IDA 0.0236526869753898 Protein AATF

0.0236530201026597 RNA cytidine acetyltransferase 0.0236530201026597 Pesticidal crystal cry8Ba protein 0.023678596209822 Ring finger protein, putative 0.023678596209822 WEB family protein At2g38370 0.0237223541542759 DNA excision repair protein ERCC-8 0.0237475211628624 Early nodulin-like protein 1 0.0237475211628624 RPM1-interacting protein 4 0.0237523518486976 Calcium-dependent protein kinase 0.0238909923541539 peptide methionine sulfoxide reductase B5-like 0.0238909923541539 thioredoxin-like 1-1, chloroplastic 0.0239287608363609 Lipoxygenase

0.0239287608363609 gibberellin receptor GID1B

0.0239386776046823 Peptidase_M22 domain-containing protein 0.0239386776046823 At1g53885 0.0239549457797599 DUF679 domain membrane protein

0.0239582461467693 Ubiquinol-cytochrome C chaperone family protein 0.0239686817757097 monosaccharide-sensing protein 2

0.0239890521445934 Actin-binding LIM protein 1, putative 0.0239969001844565 Cinnamyl alcohol dehydrogenase 0.0240389458239919 glutathione S-transferase-like 0.0240576461322451 U-box domain-containing protein kinase family protein, putative 0.0240672361314315 Photosynthetic NDH subcomplex B 3 0.0240792661685541 ATP synthase subunit d, mitochondrial 0.0240798183208556 No data found 0.0241830573770045 Ribonuclease P protein subunit p29 0.0241830573770045 eukaryotic translation initiation factor-like 0.0241830573770045 F-box/LRR-repeat protein 14 0.0241830573770045 Histone deacetylase

0.0242129248670844 MACPF domain-containing CAD1-like protein 0.0242537705468966 cytochrome P450 CYP736A12-like 0.0242640727135997 WAT1-related protein 0.0242640727135997 Glutathione-S-transferase 0.0242640727135997 Raffinose synthase 0.0243109508073493 Oxidoreductase family protein 0.0243140707684535 basic leucine zipper 9 0.0243233849998695 Protein EARLY RESPONSIVE TO DEHYDRATION 15 0.0243335688310157 transaldolase

0.0243792605553614 serine/threonine-protein kinase STY46-like isoform X1 0.0243853812486504 Potassium transporter

0.0243953826146527 Zinc finger CCCH domain-containing protein 41 0.0244603094659689 (DL)-glycerol-3-phosphatase 2 0.0244603094659689 Charged multivesicular body 1 0.0245134979576008 mevalonate kinase-like

0.0245540791546917 ethylene-responsive transcription factor 3-like 0.0245947927920513 DNA replication complex GINS protein PSF1 0.0246043393730093 No data found 0.0246043393730093 WAT1-related protein

0.0246043393730093 Bromo-adjacent-like (BAH) domain protein 0.0246061545576805 Chaperone protein 0.0246086791279682 Protein MICRORCHIDIA 6

0.0246089944495213 M50 family peptidase

0.0246295023391588 Triacylglycerol lipase 2, putative 0.024683743153844 Unknown protein 0.0246889767514816 DNA primase

0.0247919330193308 Transducin family protein/WD-40 repeat protein 0.0248134188898108 CBS domain-containing protein CBSX1, chloroplastic 0.0248134188898108 Peroxisome biogenesis protein 1 0.0248134188898108 Cysteine-rich receptor-kinase-like protein 0.024839212472369 T-complex protein 1 subunit zeta 1 0.0249021199057471 Type I inositol polyphosphate 5-phosphatase, putative 0.0249100622519281 Rhodanese-like domain-containing family protein 0.0249280206155854 ETHYLENE INSENSITIVE 3-like 3 protein 0.0249280206155854 Histone acetyltransferase 0.0249496524561083 Squamosa promoter binding protein 
MELO3C013353 0.00246544497319956 MELO3C010493 0.00246180150676134 MELO3C012545 0.0024685614916824 MELO3C021185 0.00246257535260708 MELO3C019827 0.00246720498745034 MELO3C010794 0.00246827361479818 MELO3C017333 0.00247027858353721 MELO3C002785 0.00247438794712673 MELO3C012428 0.0024886778630655 MELO3C019500 0.00249021021586082 MELO3C018683 0.00249307436724266 MELO3C011084 0.00250020007177665 MELO3C009268 0.00250588454406664 MELO3C013149 0.00250643639339809 MELO3C006957 0.0025065557324131 MELO3C009290 0.00251076561525454 MELO3C004381 0.00251245552795387 MELO3C016067 0.00252103326446729 MELO3C023844 0.00252223327356038 \begin{tabular}{lll} 
MELO3C019704 0.00252200355091303 \\
\hline
\end{tabular} MELO3C013745 0.00252732137778466 MELO3C022231 0.00252743946445322 MELO3C004564 0.00253284738789272 MELO3C018460 0.00253286875232273 MELO3C005779 0.00253841918105957 MELO3C025725 0.00253729953931148 MELO3C004423 0.00253995268287976 MELO3C018716 0.00254246520730694 MELO3C019673 0.00254436052110441 MELO3C004321 0.00255025674306286 \begin{tabular}{ll} 
MELO3C016014 0.0025571448957098 \\
\hline
\end{tabular} MELO3C003874 0.00255880335336345 MELO3C010640 0.00256492543970355 MELO3C015459 0.0025681230640956 MELO3C010716 0.00256975248916946 MELO3C002167 0.0025805609976346 MELO3C002589 0.00258385469115019 MELO3C024357 0.0025853839593345 \begin{tabular}{ll} 
MELO3C002277 0.0025908406468802 \\
\hline
\end{tabular} MELO3C022399 0.00259592798474784 MELO3C016434 0.00260306171692859 MELO3C015825 0.00260506410889971 MELO3C026572 0.00260563742446895 MELO3C008389 0.0026054713783954 MELO3C019810 0.00260464821668416 MELO3C003818 0.00260420294403418 MELO3C007660 0.00260875272791905 MELO3C019661 0.00260850482660091 MELO3C023969 0.00261005344261589 MELO3C024244 0.00261508509481012 MELO3C009587 0.00261788420547349 MELO3C000251 0.00262134113062928 MELO3C018344 0.00262491390901554 MELO3C017158 0.00262413446791454 MELO3C007785 0.00263134950686683 MELO3C011498 0.00263254371742017 MELO3C009532 0.0026377958393965 \begin{tabular}{lll} 
MELO3C021618 0.00263523784937714 \\
\hline
\end{tabular} MELO3C027119 0.00263408267344634 MELO3C026901 0.00263783859288103 MELO3C026222 0.00264225576370025 MELO3C004385 0.00265207979831772 MELO3C006055 0.00265102172481946 MELO3C010522 0.00264977766937746 MELO3C018365 0.00265274515385761 MELO3C021604 0.00265591567654666 MELO3C024086 0.00266368909276027 MELO3C017481 0.00266485371328151
0.0249496524561083 heavy metal-associated isoprenylated plant protein 26-like 0.0249496524561083 Aldehyde dehydrogenase 0.0249496524561083 phosphatidylinositol 4-kinase alpha 1-like 0.0249496524561083 Peptidase M48

0.0249496524561083 MEF2BNB-like protein 0.0249496524561083 No data found 0.0249542361873536 BnaC05g35140D protein 0.0249829691041952 epimerase family protein SDR39U1 homolog, chloroplastic-like 0.0251144093600821 mRNA-decapping enzyme subunit 2-like

0.0251170385868414 Protein-protein interaction regulator family protein 0.0251330912091247 Actin

0.0251920669477128 Ribosomal RNA small subunit methyltransferase A 0.0252175084867174 SLT1 0.0252175084867174 UPF0505 protein C16orf62 homolog isoform X1 0.0252175084867174 DUF21 domain-containing protein 0.0252470010874042 Phosphatidate phosphatase PAH1 0.025251137008428 Auxin-responsive protein 0.0253107646948902 Mitochondrial processing peptidase beta subunit 0.0253107646948902 2-oxoglutarate dehydrogenase E1 component family protein 0.0253107646948902 Serine/threonine protein phosphatase 7 long form 0.0253372598900947 Phosphatase 2C family protein

0.0253372598900947 Corepressor 0.0253659355931144 protein NUCLEAR FUSION DEFECTIVE 6, chloroplastic/mitochondrial-like 0.0253659355931144 O-acyltransferase WSD1-like 0.025395765150327 Metal-dependent protein hydrolase 0.025395765150327 RNA binding (RRM/RBD/RNP motifs) family protein 0.0253982407768063 protease 2 0.025410498673636 ras-related protein Rab11D

0.0254165786197728 BOI-related E3 ubiquitin-protein ligase 1-like 0.0254625987961317 Vat protein

0.0255184712205016 thiamine pyrophosphokinase 1 0.0255221249624115 cytochrome P450 734A1-like

0.0255702738559845 zinc finger CCCH domain-containing protein 1 0.0255892343051584 Cytidine deaminase 0.0255925576947089 Protein HHL1, chloroplastic 0.0256872475515553 Succinate--CoA ligase [ADP-forming] subunit alpha, mitochondrial 0.0257070762194081 At2g47960/T9J23.10 0.0257093392814184 transcription initiation factor TFIID subunit 12b 0.0257506350957408 Glutathione transport system permease protein gsiD 0.0257882202066323 alcohol dehydrogenase-like 0.0258067871092464 protein STICHEL 0.0258067871092464 F-box protein, putative 0.0258067871092464 Proline transporter 0.0258067871092464 RNA-binding protein 2 0.0258067871092464 Adenosine kinase 0.0258067871092464 Tubby-like F-box protein 0.0258116902544923 DUF21 domain-containing-like protein 0.0258116902544923 No data found 0.0258116902544923 Unknown protein

0.0258485062749775 Ribosomal protein L37

0.0258632292015487 S-adenosyl-L-methionine-dependent methyltransferases superfamily protein 0.0258844329943989 Protein LAZ1 0.0258938185759979 No data found 0.0258938185759979 Serine/threonine-protein kinase atr 0.0259431666044565 RING-H2 zinc finger protein RHA2a 0.0259431666044565 Splicing factor u2af large subunit, putative 0.025943562933669 NDR1/HIN1-like protein 12 0.025943562933669 ribosomal RNA large subunit methyltransferase E-like 0.025943562933669 1-aminocyclopropane-1-carboxylate oxidase 2 0.025943562933669 HNH endonuclease

0.0259740712181763 Transcription factor GTE10

0.0260253671353869 pathogenesis-related protein PR-4-like 0.0260253671353869 Aldose 1-epimerase 0.0260253671353869 Zinc knuckle family protein 0.0260253671353869 GDP-Man:Man(3)GlcNAc(2)-PP-Dol alpha-1,2-mannosyltransferase 0.0260435346058193 glycerol-3-phosphate dehydrogenase [NAD $(+)]$ $0.026105255944244370 \mathrm{kDa}$ heat shock protein 0.0261052559442443 Xyloglucan endotransglucosylase/hydrolase 
MELO3C004519 0.00267496625574049 MELO3C007081 0.00267470348746146 MELO3C014687 0.00269195405579215 MELO3C023385 0.00270256906142263 MELO3C023358 0.00270407856419341 MELO3C006439 0.00270856493483707 MELO3C022497 0.00270918512667995 MELO3C015093 0.00271896315900721 MELO3C026723 0.00271827361883736 MELO3C010744 0.00272509819331868 MELO3C014925 0.00272905227228559 MELO3C007405 0.00272984986989211 MELO3C023569 0.00272901561183836 MELO3C005103 0.00273416426217743 MELO3C023599 0.00273464015593183 MELO3C011717 0.00273572632368102

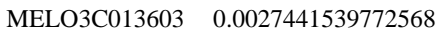
\begin{tabular}{ll} 
MELO3C006153 0.00275443894018501 \\
\hline
\end{tabular} MELO3C011263 0.00276183244145933 MELO3C019501 0.00275780114814639 MELO3C013331 0.0027606036489658 MELO3C015131 0.00276010714569663 MELO3C003861 0.00275803858939361 MELO3C008214 0.00276361792622737 MELO3C017228 0.00276531793823609 MELO3C007857 0.00277499196625031 MELO3C022228 0.00277292080975666 MELO3C015861 0.00277506452761478 MELO3C012418 0.00277589428653835 MELO3C025494 0.00278404522379472 MELO3C013665 0.00278843488334646 MELO3C018985 0.00280648064981326 MELO3C006949 0.0028143816981141 MELO3C020733 0.00282827819139519 MELO3C024984 0.00282776638965032 MELO3C006681 0.00283034097739443 MELO3C015682 0.00283388627394909 MELO3C014199 0.00283809781829736 MELO3C010350 0.00283887711442787 MELO3C019326 $\quad 0.00284198844840122$ MELO3C011716 0.00285347093160948 MELO3C012175 0.00285815822128543 MELO3C025490 0.00286245136842622 MELO3C009188 0.00286979849156177 MELO3C006858 0.00286606413668511 MELO3C016931 0.00286763125209966 MELO3C020157 0.00286972839969413 MELO3C018408 0.00286762071520663 MELO3C006576 $\quad 0.00287199260083404$ MELO3C008248 0.00287463929746568 MELO3C013852 $\quad 0.00287625232888011$ MELO3C007689 0.00289790596181549 MELO3C008074 0.00289864968973574

MELO3C012268 0.00290928855179373 MELO3C011953 0.00290852105031103 MELO3C022246 0.00291399362481704 MELO3C002232 0.00292148187912489 MELO3C009949 0.00292063003617526 MELO3C026782 0.0029315704215952 MELO3C025699 0.00293717560620022 MELO3C015807 0.00294721274408638 MELO3C023406 0.00294286704306013 MELO3C025872 0.00294290350336335 MELO3C025854 0.00294734785833395 MELO3C022411 0.00294748959767621 MELO3C008481 0.00294454167237668
0.0261783491499598 NADH-cytochrome b5 reductase-like protein 0.0261783491499598 SIT4 phosphatase-associated family protein 0.0263315505932833 Structural maintenance of chromosomes protein 0.0264222952445721 molybdate transporter 2

0.0264239720753368 Ubiquitin fusion degradation 1 protein

0.0264476999835899 1-aminocyclopropane-1-carboxylate oxidase homolog 1-like 0.0264476999835899 40S ribosomal protein S13

0.0265169399640659 Fasciclin-like arabinogalactan protein 16

0.0265169399640659 Plastidal glycolate/glycerate translocator 1, chloroplastic 0.0265636546001237 CTD small phosphatase-like protein 2

0.026570628428043 Glycerophosphodiester phosphodiesterase, putative 0.026570628428043 peroxisomal membrane protein PMP22

0.026570628428043 Alpha-N-acetylglucosaminidase

0.0265885133692798 Cytochrome b-c1 complex subunit Rieske, mitochondrial 0.0265885133692798 Glutamine synthetase 0.0265885133692798 Protein kinase-like protein 0.0266573029497514 Plant/protein (DUF789)

0.0267440583233598 UDP-galactose/UDP-glucose transporter 2-like 0.0267500877324082 EEIG1/EHBP1 protein amino-terminal domain protein 0.0267500877324082 Transmembrane 53 0.0267500877324082 hippocampus abundant transcript-like protein 1 0.0267500877324082 Pentatricopeptide repeat-containing family protein 0.0267500877324082 H/ACA ribonucleoprotein complex non-core subunit NAF1-like isoform X1 0.0267542600122864 Emb|CAB62340.1

0.0267576011573859 Replication protein A 70 kDa DNA-binding subunit

0.0268074015965016 LOW QUALITY PROTEIN: protein HYPER-SENSITIVITY-RELATED 40.0268074015965016 Dienelactone hydrolase 0.0268074015965016 isoamylase 2, chloroplastic

0.0268074015965016 Tyramine N-feruloyltransferase 4/11 0.0268729761117898 leukotriene A-4 hydrolase homolog 0.0269021985887685 mechanosensitive ion channel protein 6-like 0.027063079274004960 S acidic ribosomal protein P1

$0.0271260244783091 \mathrm{NADH}$-ubiquinone oxidoreductase-related 0.0272333817659014 Hexosyltransferase

0.0272333817659014 12-oxophytodienoate reductase 3 0.0272399629447186 bifunctional epoxide hydrolase 2-like

0.0272607988427767 Zinc finger BED domain-containing protein DAYSLEEPER 0.0272822307215747 E3 ubiquitin-protein ligase RING1-like 0.0272822307215747 heme oxygenase 1 , chloroplastic 0.0272988472118073 Glutaredoxin

0.0273958179039162 TBC1 domain family member 8B

0.0274274862546968 Thioredoxin-like protein aaed1, chloroplastic

0.0274553434070177 Protein TONNEAU 1b

0.0274591329505104 transcription factor DIVARICATA-like 0.0274591329505104 At5g51840

0.0274591329505104 Hydroxyproline-rich glycoprotein 0.0274591329505104 Glycerol-3-phosphate dehydrogenase [NAD(+)] 0.0274591329505104 methyltransferase-like protein 13 0.0274668193093808 ARM repeat superfamily protein 0.0274788245332283 GRF1-interacting factor 3 0.0274809420624351 Plant/protein

0.0276681646798411 protein RMD5 homolog A 0.0276681646798411 No data found

LEAF RUST 10 DISEASE-RESISTANCE LOCUS RECEPTOR-LIKE

0.0277428969625178 PROTEIN KINASE-like 1.2 isoform X4 0.0277428969625178 Unknown protein 0.0277743533284323 cytochrome P450 78A9-like

0.0278188744603845 serine/threonine-protein kinase fray2

0.0278188744603845 Pentatricopeptide repeat-containing protein

0.027901486388474 Protein WVD2-like 3

0.0279413685196763 Mitochondrial carrier protein

0.0279586801462572 2-oxoglutarate (2OG) and Fe(II)-dependent oxygenase superfamily protein 0.0279586801462572 Polygalacturonase

0.0279586801462572 protein NRT1/ PTR FAMILY 8.1-like

0.0279586801462572 No data found

0.0279586801462572 No data found

0.0279586801462572 Glutamate synthase 1 [nadh], chloroplastic 
MELO3C017624 0.0029620275572394 MELO3C021565 0.00296685498420146 MELO3C022694 0.00296775156879869 MELO3C012527 0.00297250118494685 MELO3C019044 0.00298063279371774 MELO3C002058 0.00298196540003237 MELO3C021929 0.00299072573288184 MELO3C026077 0.0029907075838328 MELO3C009278 0.00299360468042398 MELO3C019004 0.00299954481518294 MELO3C022500 0.00300012319494214 MELO3C018495 0.00299853266939398 MELO3C016801 0.00300623854141413 MELO3C007413 0.00301537223321668 MELO3C026638 0.0030153881996442 \begin{tabular}{ll} 
MELO3C006943 0.00303125387801784 \\
\hline
\end{tabular} MELO3C017687 0.00303163409125018 MELO3C002719 0.00303345100982744 MELO3C010135 0.00303274871299286 MELO3C007950 0.00304051349157075 MELO3C022482 0.00303891540561274 MELO3C011123 0.00303966505635012 MELO3C006635 0.00304572063851438 MELO3C017786 0.00304667114337587 MELO3C007580 0.003050768630666 MELO3C012211 0.00304978606683126 MELO3C007841 0.00305590903747888 MELO3C009328 0.00306327293243192 MELO3C013275 0.00307285130009638 MELO3C004134 0.00307469793348203 MELO3C025897 0.00307432554173359 MELO3C026715 0.00307034640401294 MELO3C019546 0.00308152563759556 MELO3C006945 0.00308380034190114 MELO3C026015 0.00309308236949102 MELO3C015954 0.0030947715945715 MELO3C023201 0.0030977794094238 MELO3C025916 0.00309672394330129 MELO3C013879 0.00310242322052934 MELO3C006833 0.00310508976483725 MELO3C019779 0.00310717541311201 MELO3C003821 0.00310708875855792 MELO3C006596 0.00312259521677238 MELO3C016899 0.00312150091819829 MELO3C016315 0.00312509809007677 MELO3C024172 0.00313617279548883 MELO3C012716 0.00313511425606883 MELO3C006546 0.00313962609965124 MELO3C007711 0.0031447472936853 MELO3C011172 0.00314734549239759 MELO3C020628 0.00314969349944749 MELO3C003368 0.0031518431083446 MELO3C018128 0.00315436270314007 MELO3C026935 0.00315856654564306 \begin{tabular}{ll} 
MELO3C011323 0.0031636598807594 \\
\hline
\end{tabular} MELO3C003790 0.00316375085180232 MELO3C010057 0.00317500236112433 MELO3C016435 0.00318004727588184 MELO3C026949 0.00318437434441865 MELO3C004179 0.0031931976062749 MELO3C017665 0.00319763962111685 MELO3C020958 0.0032001673855715 MELO3C026019 0.00321334455151734 MELO3C027349 0.00321673340123285 MELO3C009275 0.00322209073351121 MELO3C025912 0.00322317336174127 MELO3C013566 0.00323029250749773 MELO3C006900 0.00323377434712158
0.0280830927642443 Superoxide dismutase 0.0281103720538155 DExH-box ATP-dependent RNA helicase DExH17-like 0.0281103720538155 Ethylene-responsive transcription factor

0.0281418628482816 Guanine nucleotide-binding protein beta subunit-like protein 0.0282044227419728 Tubulin folding cofactor B

0.0282044227419728 PHD and RING finger domain-containing protein 1

0.0282602117218581 B3 domain-containing transcription factor VRN1-like 0.0282602117218581 leucine-rich repeat extensin-like protein 3

0.0282738875340474 Pectin acetylesterase

0.0282948581551635 ENTH/ANTH/VHS superfamily protein, putative

0.0282948581551635 Vacuolar protein sorting 26

0.0282948581551635 Carbohydrate esterase, putative (DUF303)

0.028338999978228 aspartic proteinase-like protein 2

0.0283981409417135 Core-2/I-branching beta-1,6-N-acetylglucosaminyltransferase family protein 0.0283981409417135 acyl-protein thioesterase 2

0.0285138619671975 Keratin-associated protein, putative (DUF819)

0.0285138619671975 solute carrier family 35 member F1

0.0285138619671975 60S ribosomal protein L18a

0.0285138619671975 Kelch repeat-containing protein family

0.0285394966468777 Thiol-disulfide oxidoreductase LTO1

0.0285394966468777 SWI/SNF complex component SNF12 homolog

0.0285394966468777 oleosin 1-like

0.0285701369470703 transcription termination factor MTEF1, chloroplastic-like

0.0285701369470703 GPI-anchored protein

0.028581418257601 phosphatidylinositol 3,4,5-trisphosphate 3-phosphatase and protein-tyrosine-

0.028581418257601 Coiled-coil domain-containing protein 130

0.0286160016980419 Bacteriophage N4 adsorption B

0.0286713635746909 Lectin receptor kinase-like protein

0.0287238455479359 Eukaryotic translation initiation factor 3 subunit A, putative

0.0287238455479359 leucine aminopeptidase 1-like

0.0287238455479359 pumilio homolog 1-like

0.0287238455479359 Tetratricopeptide repeat (TPR)-like superfamily protein

0.0287740188259455 PRKR-interacting protein 1

0.0287816507335566 mediator of RNA polymerase II transcription subunit 36a-like

0.0288546451181285 cyclin-dependent kinase 11B-like

0.0288567725312524 No data found

0.0288575686588257 No data found

0.0288575686588257 Triosephosphate isomerase, putative

0.0288872023490023 Regulator of nonsense transcripts 1-like protein

0.0288905872097688 AT-hook motif nuclear-localized protein 1

$0.0288905872097688 \quad$ U4/U6 small nuclear ribonucleoprotein PRP4-like protein

0.0288905872097688 agglutinin-like

0.0290066476651166 squamosa promoter-binding-like protein 7

0.0290066476651166 Mitochondrial carrier protein

0.0290162492623066 Unknown protein

0.0290917221879328 Histone deacetylase

0.0290917221879328 ribonuclease 2-like

0.0291100825549354 BRI1 kinase inhibitor 1-like

0.0291438828263684 U-box domain-containing protein kinase family protein, putative

0.0291542805484803 Proteasome subunit beta type

0.0291623520490335 Protein kinase

0.0291685799187898 Intron maturase, type II family protein

0.0291782243673598 Pentatricopeptide repeat-containing protein

0.0292034319802925 Homeobox-leucine zipper HOX24

0.0292240016708344 No data found

0.0292240016708344 subtilisin-like protease SBT3.17 isoform X1

0.0293142223608436 WRKY transcription factor SUSIBA2-like isoform X2

0.0293470811455095 PHD finger alfin-like protein

0.0293732876823559 DNA ligase

0.0294409241486102 RNA polymerase II-associated protein 3

0.0294681217346882 Ubiquitin-protein ligase, putative

0.0294776612395762 Shikimate kinase

0.0295852408148792 Abscisic acid receptor

0.0296026411653996 MACPF domain NSL1-like protein

0.0296342880451715 No data found

0.0296342880451715 Protein kinase

0.0296859221640636 Indole-3-acetic acid-amido synthetase GH3.3

0.0297040974796763 No data found
1 
MELO3C008985 0.00323792539730006 MELO3C011141 0.00324029178816565 MELO3C020113 0.00324422109289979 MELO3C023885 0.00324576723815473 MELO3C010695 0.00325920601822216 MELO3C011641 0.00326082986585996 MELO3C015999 0.00327258479556591 MELO3C002240 0.00327636508104368 MELO3C014270 0.00328035162177276 MELO3C017682 0.00329068344277839 MELO3C011626 0.00329055575005754 MELO3C010882 0.00328785247888019 MELO3C015845 0.00329570830198256 MELO3C006367 0.00329600978397937 MELO3C016702 0.00330136386296176 MELO3C018564 0.00330194105096393 MELO3C017142 0.00330403547657232 MELO3C014696 0.00330563892729141 MELO3C026387 0.00331292201755107 MELO3C024476 0.00332258243518369 MELO3C011977 0.00332273570783626 MELO3C006104 0.00332623952523958 MELO3C011450 0.00333358893825408 MELO3C016379 0.00333695453228211 MELO3C013981 0.00335602505490162 MELO3C024538 0.00335382112544536 MELO3C005084 0.00335625046193033 MELO3C021429 0.00335193882671869 MELO3C004988 0.00335093209097537 MELO3C001354 0.00335814086479347 MELO3C012642 0.00336533823483554

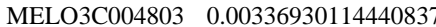
MELO3C014283 0.00337200910851509 MELO3C007612 0.00337643607475258 MELO3C022717 0.00338495688318097 MELO3C012390 0.00339255872463551 MELO3C003646 0.00339160336545463 MELO3C007740 0.00339467117104308 MELO3C012160 0.00339732236416479 MELO3C015670 0.00339843699782583 MELO3C009826 0.0033996930658422 MELO3C020709 0.00340145935932612 MELO3C003618 0.00340400756003645 MELO3C003537 0.00340619799978614 MELO3C005597 0.00341005728531718 MELO3C012457 0.00341130840210702 MELO3C005446 0.00342424757612758 MELO3C024964 0.00343821174470194 MELO3C012627 0.00344637204195242 MELO3C008749 0.00344966036879268 MELO3C025053 0.00345273664238088 MELO3C017141 0.00345887223368579 MELO3C005776 0.00346706651856055 MELO3C011443 0.00346914766687134 MELO3C009159 0.00347745214263795 MELO3C019807 0.00348962571938949 MELO3C004651 0.00349339114977409 MELO3C016321 0.00351307924928468 MELO3C021455 0.00351239881555254 MELO3C002644 0.00351114986297452 MELO3C013068 0.00352314090533479 MELO3C007737 0.00352347083815385 MELO3C003545 0.00352514241961388 MELO3C008002 0.0035312711957558 MELO3C013051 0.00353085892452476 MELO3C009284 0.00353465922443519 MELO3C006293 0.00354341023305915 MELO3C006679 0.00354132679588104
0.0297284001261175 Glucose-6-phosphate 1-dehydrogenase 0.0297363022883287 Transcriptional regulatory plant protein, putative 0.029758533378392 ADP,ATP carrier protein

0.0297588937726638 Tropinone reductase family protein 0.0298682411386865 No data found 0.0298692620690484 gamma-secretase subunit APH1-like 0.0299630399293608 60S ribosomal protein L22-2 0.0299837506883835 RING-type E3 ubiquitin transferase 0.0300063289385781 No data found 0.0300590690617162 extensin-2

0.0300590690617162 Ribosomal protein S8

0.0300590690617162 Origin recognition complex subunit 2 family protein

0.030079897053516 basic 7S globulin 2

0.030079897053516 histone-lysine N-methyltransferase family member SUVH9

0.0301062021308803 translation initiation factor IF-1, chloroplastic

0.0301062021308803 Protein trichome birefringence-like 12

0.0301113966898139 tRNA (Ile)-lysidine synthase

0.0301121140106449 Glycosyltransferase

0.0301645444557935 Pentatricopeptide repeat-containing protein At3g59040

0.0302260283252226 alpha carbonic anhydrase 7-like

0.0302260283252226 polygalacturonase At1g48100

0.0302439707108455 Atg8-interacting protein 1

0.0302968467287528 Kelch repeat-containing F-box family protein

0.0303134843873226 Ultraviolet-B receptor UVR8

0.030418811552392 40S ribosomal protein S13

0.030418811552392 zinc finger A20 and AN1 domain-containing stress-associated protein 4

$0.030418811552392 \quad$ E3 ubiquitin-protein ligase

0.030418811552392 Ribosomal protein

0.030418811552392 Protein SIEL

0.030421983458168 Kinase family protein

0.0304732071525754 Glucan endo-1,3-beta-glucosidase-like protein 1

0.0304951092121544 BnaC01g17260D protein

0.0305056380595807 BIG SEEDS 1

0.0305317014836486 molybdate-anion transporter

0.0305947430141606 FHA domain-containing protein FHA2

0.030635410266496 NAC domain-containing protein 53-like

0.030635410266496 guanine nucleotide-binding protein subunit gamma 2-like

0.0306404757572806 40S ribosomal protein S6-like

0.0306437874748141 Protein BIG GRAIN 1-like E

0.0306437874748141 Phosphatidylinositol N-acetylglucosaminyltransferase subunit A

0.0306437874748141 Carboxypeptidase

0.0306457212077242 K-stimulated pyrophosphate-energized sodium pump protein

0.0306546946206839 Unknown protein

0.0306604395158507 WD-40 repeat-containing protein MSI1

0.0306784743320635 1-aminocyclopropane-1-carboxylate synthase

0.0306784743320635 Annexin

0.0307808217482675 60S ribosomal protein L13

$0.03089228559878 \quad$ E3 ubiquitin-protein ligase

0.0309515240820911 KRR1 small subunit processome component homolog

0.0309669739196758 CBS domain-containing protein CBSX6

0.0309805070197092 lysine-specific demethylase JMJ25

0.03102146582337 Dynein light chain

0.0310808427939411 Plant UBX domain-containing protein 10

0.0310853889623603 Glycosyltransferase

0.0311456700067832 ALC-interacting protein 1

0.0312405341487865 Zinc finger (C3HC4-type RING finger) family protein

0.0312600733198408 DENN (AEX-3) domain-containing protein

0.0313935756082005 multiple RNA-binding domain-containing protein 1

0.0313935756082005 BnaC04g30420D protein

0.0313935756082005 Transcription factor

0.0314579681657778 No data found

0.0314579681657778 No data found

$0.031458670422482828 \mathrm{kDa}$ ribonucleoprotein

0.0314849096365604 serine/threonine-protein kinase D6PKL2

0.0314849096365604 Dead box ATP-dependent RNA helicase, putative

0.0314952662272863 Protein SLOW GREEN 1, chloroplastic

0.0314952662272863 Werner Syndrome-like exonuclease

0.0314952662272863 Dihydrolipoamide acetyltransferase component of pyruvate dehydrogenase 
2

3

4

5

6

7

8

9
MELO3C016574 0.00353635905852601 MELO3C017979 0.00354359621029066 MELO3C018634 0.00353843304329915 MELO3C012787 0.00354012354896582 MELO3C002187 0.00354604168811656 MELO3C013699 0.00354782004554266 MELO3C002921 0.00354961794420117 MELO3C011266 0.00355690370295292 MELO3C010886 0.00355680004882486 MELO3C009118 0.00356566194604402 MELO3C003452 0.00357083843738115 MELO3C027277 0.00357454085905751 MELO3C006224 0.00357531010014744 MELO3C006942 0.00357863116017443 MELO3C012303 0.00357875902153149 MELO3C006470 0.00359422249341379 MELO3C013634 0.00359595157280856 MELO3C016056 0.00360431063231181 MELO3C008871 0.00360628755649928 MELO3C020139 0.00361941064351767 MELO3C018700 0.00361828856415125 MELO3C009973 0.00361824768775709 MELO3C019010 0.00362104834108679 MELO3C010566 0.00362510291757867 MELO3C011769 0.00362608309377821 MELO3C016706 0.00362792550019198 MELO3C012844 0.00363671067198779 MELO3C017291 0.00364457998267875 MELO3C020055 0.00365494902297803 MELO3C026594 0.00365773426356975 MELO3C004333 0.00366799011256524 MELO3C003135 0.0036685906279057 MELO3C026250 $\quad 0.00366806256004015$ MELO3C014100 0.00367353119137726 MELO3C019954 0.00367499471328736 MELO3C018086 0.00367393222298185 MELO3C002364 0.00368322380190222 MELO3C007651 0.00368605120965426 MELO3C017041 0.00369325977155832 MELO3C005067 0.00369064033854793 MELO3C011580 0.00369339856859607 MELO3C011306 0.00370901783915945 MELO3C013737 0.00371380927877951 MELO3C009047 0.00371812788353043 MELO3C009362 0.00372330748990279 MELO3C021694 0.003723971676308 MELO3C018849 0.0037317716935662

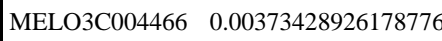
MELO3C012147 0.00373986616904487 MELO3C022715 0.00374086553989794 MELO3C018156 0.00374297430987069 MELO3C020756 0.00374575962541046 MELO3C009313 0.00374905481299304 MELO3C021441 0.00375323054063759 MELO3C019256 0.00375711455194661 MELO3C017659 0.00376166987941229 MELO3C023561 0.00376053621892014 MELO3C025595 0.00377304170472659 MELO3C024527 0.00377219600226342 MELO3C021072 0.00376902665796097 MELO3C022135 0.00377269415701442 MELO3C003511 0.00377406417538706 MELO3C009659 0.00378297423157448 MELO3C012087 0.00378286641513303 MELO3C025844 0.00378430346700454 MELO3C005590 0.00378620329661872 MELO3C017128 0.00379795715646769 MELO3C023131 0.00380245973358373
0.0314952662272863 Serine/threonine-protein kinase 0.0314952662272863 Bifunctional protein FolD 0.0314952662272863 7-deoxyloganetin glucosyltransferase-like 0.0314952662272863 Sterol 3-beta-glucosyltransferase 0.0315028237960477 Plant/protein (DUF789) 0.0315044505752797 Pectinesterase 0.0315062493393388 Cysteine proteinase inhibitor 0.0315425645395677 Histone acetyltransferase 0.0315425645395677 Succinate dehydrogenase subunit 5, mitochondrial 0.0316060402928291 TOM1-like protein 2

0.0316377246746704 Synaptonemal complex protein 1 0.031648946287679 Polygalacturonase

0.031648946287679 HOPM interactor 7 0.0316511025151031 Receptor-like protein kinase, putative 0.0316511025151031 Trichome birefringence-like protein 0.0317736347459395 p-loop nucleoside triphosphate hydrolase superfamily protein 0.0317746969178507 DNA-directed RNA polymerase subunit beta 0.0318343160454052 monothiol glutaredoxin-S11 0.0318375381999572 Chaperone protein dnaJ, putative 0.0319105985709065 No data found 0.0319105985709065 UDP-glycosyltransferase 74F2-like 0.0319105985709065 Dead box ATP-dependent RNA helicase, putative 0.0319107914717193 No data found

0.0319266674182015 Topoisomerase II-associated protein PAT1 0.0319266674182015 Pyruvate kinase

0.031928654502358 E3 ubiquitin-protein ligase RMA3

0.0319917145038248 Glutathione S-transferase family protein

0.0320466652172407 40S ribosomal protein S28

0.0321235372740512 histidine kinase 4-like

$0.0321337161793767 \quad$ E3 ubiquitin-protein ligase RGLG2

0.0321861378545134 DCD (Development and Cell Death) domain protein

0.0321861378545134 Ribonuclease II, chloroplastic/mitochondrial

0.0321861378545134 Rhodanese-like domain-containing protein 11, chloroplastic

0.0321994102008483 Heavy metal-associated isoprenylated plant protein 21

0.0321994102008483 NAC domain-containing protein 17-like

0.0321994102008483 No data found

0.0322572003830452 glucuronoxylan 4-O-methyltransferase 1

0.0322676530760027 BSD domain-containing protein

0.032289034232494 Protein canopy 1-like

0.032289034232494 dihydroorotate dehydrogenase (Quinone), mitochondrial-like

0.032289034232494 Selenoprotein K

0.0324112359759115 U2 snRNP-associated SURP motif-containing protein

0.032438752519512 Eukaryotic translation initiation factor 3 subunit A, putative

0.0324621165215926 Endonuclease/exonuclease/phosphatase family protein

0.032484415475003 4-coumarate--CoA ligase-like 7

0.032484415475003 equilibrative nucleotide transporter 3-like

0.0325380835215183 Glycosyltransferase

0.0325456657683347 Alpha-galactosidase

0.0325742299591907 Chromatin modification-related protein EAF7

0.0325742299591907 E3 ubiquitin-protein ligase RNF4-like isoform X1

0.0325782281382267 HEAT repeat-containing 8

0.032588108741071 auxin-responsive protein SAUR24-like

0.0326024145758694 MCM domain-containing protein

0.0326243617724699 Low molecular weight protein-tyrosine-phosphatase, putative

0.0326437550754041 Glutamate receptor

0.03265460151583 Ribosomal protein L15

0.03265460151583 Zinc finger, PHD-type

0.0326903479823329 Hydroxyacylglutathione hydrolase 3, mitochondrial-like protein

0.0326903479823329 Monodehydroascorbate reductase family protein

0.0326903479823329 p-loop containing nucleoside triphosphate hydrolases superfamily protein,

0.0326903479823329 Dynamin homolog

0.0326903479823329 No data found

0.0327359654708158 WD-repeat protein, putative

0.0327359654708158 At1g69680/T6C23 12

0.0327359654708158 zinc finger $\mathrm{CCCH}$ domain-containing protein 66

0.0327380599408595 AT-hook motif nuclear-localized protein 1

0.0328253198613043 two-component response regulator ARR5-like

0.0328498588270101 Magnesium chelatase subunit 
MELO3C022307 0.00380791780284617 MELO3C015877 0.00381685302485724 MELO3C018872 0.00382096715178948 MELO3C007206 0.00382538871009896 \begin{tabular}{ll} 
MELO3C007781 0.0038398486676372 \\
\hline
\end{tabular} MELO3C007981 0.00384716409883801 MELO3C026665 0.0038477634826739 MELO3C011206 0.00384958612008623 MELO3C016629 0.00385657378659299 MELO3C004813 0.00385700668977274 MELO3C002951 0.00386183647055627 MELO3C024701 0.00386218060951637 MELO3C022756 0.00385939753056552 MELO3C006362 0.00387559388006831 MELO3C016972 0.00388047519379775 MELO3C026799 0.00387701403635354 MELO3C005658 0.00387972951477389 $\begin{array}{lll}\text { MELO3C002698 } & 0.00388183824560484\end{array}$ MELO3C017496 0.00387421851442116 MELO3C007029 0.00388454470007149 MELO3C026403 0.00388878465702924

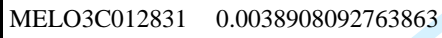
MELO3C014561 0.00389493667687124 MELO3C017322 0.00389583227761392 MELO3C004334 0.00390896641762595 MELO3C006528 0.00391035791241645 MELO3C000756 0.00390717971422494 MELO3C026504 0.00390827463156984 MELO3C013878 0.00391222575567463 MELO3C025139 0.00392025166600019 MELO3C002556 0.0039218049617562 MELO3C008158 0.00393031276265376 MELO3C019983 0.00392989468547922 MELO3C016939 0.00394970593609312 MELO3C008944 0.00394846519055947 MELO3C011989 0.00395059384120378 MELO3C026495 0.00394489756670136 MELO3C013044 0.00394792703568192 MELO3C004163 0.00395312441178808 MELO3C007919 0.00395536602137492 MELO3C022017 0.00395691777946994 MELO3C011127 0.00395892010502719 MELO3C002789 0.00396747730644487 MELO3C013221 0.00397472788079656 MELO3C007232 0.00397436879822932 MELO3C021281 0.00397525249759023 MELO3C018648 0.00397329808233615 MELO3C009755 0.00399223218178957 MELO3C006187 0.00398992103329143 MELO3C006954 0.00399297475803051 MELO3C026373 0.0039887085281346 MELO3C007648 0.00398694127513544 MELO3C019798 0.00398754437598248 MELO3C011236 0.00398898589143037 MELO3C018126 0.00399118870471526 MELO3C006817 0.003994972976333 MELO3C009105 0.0040092906562702 MELO3C022425 0.00400986824679883 MELO3C026103 0.00401371508088511 MELO3C016780 0.00401640311312934 MELO3C006678 0.00402069563885754 MELO3C011227 0.00402447276814755 MELO3C023474 0.00402423910770855 MELO3C015941 0.00403168091934258 MELO3C011726 0.00404263312343378 MELO3C017221 0.00404275392874143 MELO3C026210 0.00404664750198225 $\begin{array}{ll}\text { MELO3C006574 } & 0.00405142086150934\end{array}$
0.0328826273233096 Pentatricopeptide repeat-containing family protein 0.0329453804142944 Autophagy-related protein 18g 0.0329664833030539 eukaryotic translation initiation factor 1A-like 0.0329902190549102 Receptor-like kinase

0.0331004676286194 C2 domain-containing family protein 0.0331397649451927 Plant intracellular ras group-related LRR protein 0.0331397649451927 BTB/POZ domain-containing protein At5g48800 0.0331410097147267 dentin sialophosphoprotein-like 0.0331759691273178 integrator complex subunit 9 homolog isoform X1 0.0331759691273178 No data found

0.0331771225999734 Peptidyl-prolyl cis-trans isomerase

0.0331771225999734 Elongation factor Ts, mitochondrial

0.0331771225999734 No data found

0.0332591859056182 Beta-amylase

0.0332591859056182 stromal cell-derived factor 2-like protein

0.0332591859056182 Kinase family protein

0.0332591859056182 O-glucosyltransferase rumi homolog

0.0332591859056182 Eukaryotic translation initiation factor 4E

0.0332591859056182 pathogenesis-related protein PR-1

0.0332679415792332 glycine-rich RNA-binding protein 4, mitochondrial

$0.033289817161539 \quad 60$ S ribosomal protein L27

0.0332927176773627 WD repeat-containing protein 26

0.0333068362123798 Hydroxyproline O-arabinosyltransferase 1

0.0333068362123798 LEAF RUST 10 DISEASE-RESISTANCE LOCUS RECEPTOR-LIKE

0.0333732318117167 ERAD-associated E3 ubiquitin-protein ligase HRD1B-like

0.0333732318117167 At1g05410/T25N20_5

0.0333732318117167 Alanine--tRNA ligase

0.0333732318117167 centromere protein $\mathrm{V}$ isoform $\mathrm{X} 1$

0.0333747500858826 haloacid dehalogenase-like hydrolase domain-containing protein Sgpp 0.0334275900689354 No data found

0.0334275900689354 heterogeneous nuclear ribonucleoprotein U-like protein 1

0.0334712146397797 Protein PLASTID MOVEMENT IMPAIRED 2

0.0334712146397797 Wound-responsive family protein

0.0335715480937751 Protein phosphatase 2c, putative

0.0335715480937751 ultraviolet-B receptor UVR8 isoform X2 0.0335715480937751 Unknown protein

0.0335715480937751 cytochrome P450 CYP82D47-like

0.0335715480937751 Serine/threonine-protein phosphatase 2A $55 \mathrm{kDa}$ regulatory subunit B

0.0335786038745818 trafficking protein particle complex subunit 4

0.0335819377854541 alpha-mannosidase

0.0335819377854541 Ribosomal protein L18/L5

0.0335844987775695 receptor-like protein kinase HSL1

0.0336426403284584 BnaCnng71930D protein

0.0336507765001755 KH domain-containing protein

0.0336507765001755 spermidine coumaroyl-CoA acyltransferase

0.0336507765001755 Beta-D-xylosidase family protein

0.0336507765001755 Annexin

0.0336852876960037 Sigma factor binding protein 1, chloroplastic

0.0336852876960037 Proteasome subunit beta type

0.0336852876960037 Ornithine cyclodeaminase/mu-crystallin

0.0336852876960037 Glycosyltransferase

0.0336852876960037 RuvB-like helicase

0.0336852876960037 Acyl-CoA N-acyltransferase domain protein

0.0336852876960037 Peptidyl-prolyl cis-trans isomerase

0.0336852876960037 Autophagy-related protein 18f

0.0336877546155424 pectinesterase-like

0.0337845085349958 Receptor-like kinase plant-like protein

$0.0337845085349958 \quad 15 \mathrm{kDa}$ selenoprotein

0.0338024985639232 DUF538 family protein

0.0338107182784277 ethylene-responsive transcription factor ERF011-like

0.0338324321141021 DUF581 family protein, putative (DUF581)

0.0338353821618331 zeaxanthin epoxidase, chloroplastic-like

0.0338353821618331 Hydroxycinnamoyl-CoA shikimate/quinate hydroxycinnamoyltransferase

0.0338815602025943 Long cell-linked locus protein, putative, expressed

0.0339457259093174 Nuclear transcription factor Y subunit B

0.0339457259093174 Histone deacetylase

0.0339639785451115 SNF1-related protein kinase regulatory subunit gamma-1

0.0339895966839201 guanylate kinase 3, chloroplastic-like 
|MELO3C006891 0.00405529113468006 MELO3C002258 0.00406375095139522 MELO3C007940 0.00406921847048769 MELO3C009601 0.00408256489346259 MELO3C006406 0.00409087920567841 MELO3C008489 0.00408119611396818 MELO3C018935 0.00409080955661678 MELO3C024610 0.004090155111938 MELO3C011869 0.00408858935710987 MELO3C019649 0.00408633114963042 MELO3C016540 0.00409820484929724 MELO3C024466 $\quad 0.00409776864969524$ MELO3C015915 0.00409647381032929 MELO3C013444 0.00410871929360257 MELO3C011320 0.00411998198635644 MELO3C009370 0.00412796035380381 MELO3C024404 0.00412659958826311 MELO3C015314 0.00412612017060354 MELO3C022568 0.00413550035043642 MELO3C018374 0.00413637456871707 MELO3C024936 0.00413994951308028 MELO3C005710 0.00414841548622957 MELO3C005504 0.00415565745621238 MELO3C021144 0.00415874744190292 MELO3C014591 0.00417094646782634 MELO3C022382 0.00417453238090104 MELO3C010664 0.00417184497662493 MELO3C003323 0.00417463696282061 MELO3C021407 0.00418955137017907 MELO3C004316 0.00419185506808106 MELO3C017094 0.00419379751734894 MELO3C011908 0.00419762735150164 MELO3C002084 0.00420015070006774 MELO3C017520 0.00420329844082712 MELO3C027370 0.00420544322376737 MELO3C011799 0.00420786589404742 MELO3C021944 0.00421779613002127 MELO3C013419 0.00423115977641308 MELO3C012361 0.00422999190468754 MELO3C027137 0.00423176386381408 MELO3C004239 0.00424349429512305 MELO3C016852 0.00424135737752129 MELO3C007071 0.00424254602009433 MELO3C006348 0.00425488104939142 MELO3C009713 0.00425803339606046 MELO3C019295 0.00426894623574081 MELO3C013835 0.00427577875347029 MELO3C017571 0.00428197580447165 MELO3C017989 0.00430088886261248 MELO3C021636 0.00429798729381359 MELO3C025550 0.00429966579888252 MELO3C004637 0.00431383370119853 MELO3C009787 0.00431780204327548 MELO3C002515 0.00433020588612543 MELO3C024896 0.00433539021546703 MELO3C004382 0.00433957988353706 MELO3C005089 0.00434194979060565 MELO3C003793 0.0043420036534445 MELO3C015857 0.00434471017132754 MELO3C017187 0.00435977689092781 MELO3C007635 0.00435740828139641 MELO3C008977 0.00435899313719323 MELO3C022236 0.00435478022543923 MELO3C019833 0.00436921025270542 MELO3C025454 0.00438074020671109 MELO3C013892 0.00438636358545552 MELO3C007055 0.00439030907538485 MELO3C017245 0.004395158508533
0.0340076197956673 heat stress transcription factor B-2b-like 0.0340640991252564 No data found 0.0340954584529747 Protein phosphatase 2c, putative 0.034175454074849 Dirigent protein

0.034175454074849 Transducin/WD40 domain-like protein, putative

0.034175454074849 Serine/threonine-protein kinase stt7, chloroplastic 0.034175454074849 Unknown protein

0.034175454074849 Iron-sulfur binding oxidoreductase

0.034175454074849 cytochrome P450 78A5-like

0.034175454074849 auxin-responsive protein SAUR71

0.0341932604853279 NAC domain protein

0.0341932604853279 heavy metal-associated isoprenylated plant protein 3-like isoform X2

0.0341932604853279 Heme binding

0.0342665106965191 No data found

0.0343459367870634 NAD(P)-binding rossmann-fold protein

0.0343689245477535 ACT domain-containing protein

0.0343689245477535 Tudor/PWWP/MBT superfamily protein

0.0343689245477535 protein SRC2-like

0.0344099668734598 protein indeterminate-domain 5, chloroplastic

0.0344099668734598 DUF248-1

0.03442520544582 Cleavage and polyadenylation specificity factor subunit

0.0344810847801127 heavy metal-associated isoprenylated plant protein 3

0.0345267476242063 Disease resistance family protein

0.0345378903406816 squamosa promoter-binding-like protein 8

0.0346116311413704 cyclin-dependent protein kinase inhibitor SMR3-like

0.0346116311413704 Succinate--CoA ligase [ADP-forming] subunit beta, mitochondrial

0.0346116311413704 F28C11.19

0.0346116311413704 Myosin heavy chain-like protein

0.0347207091941529 Stem-specific protein TSJT1

0.0347252289175893 Prefoldin chaperone subunit family protein, putative

\subsection{No data found}

0.0347438988117376 Heat shock protein HSP26

0.0347502204338659 Protein NEGATIVE REGULATOR OF RESISTANCE

0.0347617005476946 L-allo-threonine aldolase

0.0347648799607291 O-methyltransferase, putative

0.0347703529462521 Cytochrome P450

0.0348378317740652 Flavoprotein wrbA

0.0349094003953484 30S ribosomal protein S31, mitochondrial

0.0349094003953484 cell division control protein 2 homolog A

0.0349094003953484 Gibberellin receptor GID1A

0.0349623566267773 Zinc finger protein VAR3, chloroplastic

0.0349623566267773 zinc finger CCCH domain-containing protein 20-like

0.0349623566267773 WPP domain associated protein

0.0350415537299546 PHD finger protein ING

0.0350528976818666 translation factor GUF1 homolog, chloroplastic

0.0351280913373522 Cellulose synthase family protein

0.0351696603924552 Heat shock transcription factor family protein

0.0352059700926356 alanine--glyoxylate aminotransferase 2 homolog 1, mitochondria]

0.0353173613919892 PXMP2/4 family protein 4

0.0353173613919892 No data found

0.0353173613919892 No data found

0.0354089367269201 pre-mRNA cleavage factor Im $25 \mathrm{kDa}$ subunit 1

0.0354267854394048 Protein SRC1

0.0355138023443069 Eukaryotic translation initiation factor-like protein

0.035541561380348 Calcium-dependent lipid-binding (CaLB domain) family protein

0.035551505038091 Auxin-responsive protein

0.035551505038091 40S ribosomal protein S27

0.035551505038091 GTP-binding protein hflx, putative

0.0355589229894519 No data found

0.0356231832101503 Pectinesterase inhibitor

0.0356231832101503 F-box protein, putative

0.0356231832101503 Kinesin-like protein

0.0356231832101503 Cation/H(+) antiporter 11

0.0356854976346896 Glycine-rich RNA-binding family protein

0.0357648773635127 2-oxoglutarate dehydrogenase E1 component family protein

0.0357959894418021 Calcineurin B-like protein

0.0358133886533562 reticuline oxidase-like protein

0.0358381442547557 squamosa promoter-binding-like protein 6
0

0 
MELO3C020908 0.00439808294774702 \begin{tabular}{ll} 
MELO3C017365 0.004401984544528 \\
\hline
\end{tabular} MELO3C011106 0.00440441154588012 MELO3C012982 0.00440400022067899 MELO3C006555 0.00441530169934157 MELO3C017756 0.0044125107954931 MELO3C022535 0.00441084542698378 MELO3C011419 0.00441372812875063 MELO3C011719 0.00441889278353991 MELO3C027061 0.00442033027194499 MELO3C025492 0.00442331481104608 MELO3C014781 0.00443181098567713 MELO3C013364 0.00443433490235257 MELO3C007317 0.00443550104810231 MELO3C027082 0.0044357731607878 MELO3C006341 0.00444064861850657 MELO3C026519 0.00444300260802799 MELO3C013004 0.00444391127554433 MELO3C007001 0.00444609229565951

MELO3C012080 0.00444939745034345 MELO3C016733 0.00445369614312496 MELO3C017857 0.00447660665509919 MELO3C025196 0.00448195650987304 MELO3C006795 0.00448602272104126 MELO3C002811 0.00448958909934571 MELO3C009686 0.00449453289572133 MELO3C018333 0.00451435304406078 MELO3C009569 0.00452389862146674 MELO3C017023 0.00452304563686967 MELO3C023402 0.00452320197757028 MELO3C018884 0.00453160110978612 MELO3C024747 0.00453016901239933 MELO3C002054 0.00454481945338836 MELO3C002292 0.00454712501382337 MELO3C014381 0.00455362792746339 MELO3C017274 0.00455931809361776 MELO3C005627 0.00457680273778194 $\begin{array}{ll}\text { MELO3C011270 } & 0.0045788351918522\end{array}$ MELO3C021242 0.00458194269778134 MELO3C022180 0.00458428047942672 MELO3C007661 0.00458836570581656 MELO3C009581 0.00459335511739778 MELO3C021302 0.00459515873505412 MELO3C002611 0.00459421197278376 MELO3C022766 0.00459622213447552 MELO3C013309 0.00461226279515592 MELO3C007896 0.00461243236161057 MELO3C022208 0.00461235112080338 MELO3C002241 0.00460760544686867 MELO3C022174 0.00461874366324821 MELO3C021458 0.00461777456584467 MELO3C014961 0.00462684788511114 MELO3C007108 0.00462896316894279 MELO3C024729 0.0046335805594675 MELO3C025893 0.00464191527612789 MELO3C010631 0.00465069359190351 MELO3C018500 0.00466513527941126 MELO3C007936 0.00466850680925801 MELO3C014298 0.00468816507599967 MELO3C006119 0.00467883824833293 MELO3C025122 0.00469406507564141 MELO3C024352 0.00469137170648004 MELO3C012231 0.00468490302386826 MELO3C011942 0.0046793778862565 MELO3C002254 0.00469046994419164 MELO3C010334 0.00469594567999265
0.0358471894903243 Glycosyl hydrolase family 43 protein 0.0358543790682549 DEAD-box ATP-dependent RNA helicase 42 0.0358543790682549 Pentatricopeptide repeat-containing protein 0.0358543790682549 Bet1-like SNARE 1-1 0.0358838655392167 NEDD8-activating enzyme E1 catalytic subunit 0.0358838655392167 calcium-dependent protein kinase 24 0.0358838655392167 Methionine adenosyltransferase 2 subunit beta 0.0358838655392167 Purple acid phosphatase 0.0358951901894086 Adenylate kinase 0.0358951901894086 PLATZ transcription factor family protein 0.0359046626400941 transcription initiation factor TFIID subunit 1-like 0.0359466902553953 AT5g13970/MAC12_6

0.0359466902553953 MYB-related transcription factor 0.0359466902553953 Transmembrane protein, putative 0.0359466902553953 transcription factor bHLH149-like 0.0359683622052152 Holliday junction resolvase 0.0359683622052152 AT-hook motif nuclear-localized protein 10 0.0359683622052152 protein IQ-DOMAIN 14-like 0.035971272735346 Peptidylprolyl isomerase

0.03598327200935 binding partner of ACD11 1 0.0360032931357245 Estradiol 17-beta-dehydrogenase 1 0.0361736926479353 Unknown protein

0.0362021100668641 CTD small phosphatase-like protein 0.0362201401135911 F-box protein SKIP16 0.0362341214233668 SWIM zinc finger family protein 0.0362592034957518 Pleiotropic drug resistance ABC transporter 0.0364042295905089 Six-bladed beta-propeller, TolB-like protein $0.0364365717273029 \quad$ Gb|AAF02136.1

0.0364365717273029 Catalase

0.0364365717273029 Biotin carboxyl carrier protein of acetyl-CoA carboxylase 0.0364688632099291 No data found 0.0364688632099291 30S ribosomal protein S5, putative 0.0365603419083367 Movement protein binding protein 2C 0.0365639950724747 transcription factor MYB86-like 0.0366013829627491 Arginine/glutamate-rich 1 protein $0.0366322103461583 \quad \mathrm{G}$ patch domain-containing protein TGH 0.0367577378074239 UDP-galactose/UDP-glucose transporter 2 0.0367591122780037 Adenine phosphoribosyltransferase, putative 0.0367691126934107 YTH domain family protein 2 0.0367729306207141 Protein DETOXIFICATION 0.036790756932266 Transmembrane protein, putative $0.036793997135694 \quad$ D-lactate dehydrogenase, putative 0.036793997135694 Phosphatase 2C (PP2C)-like protein 0.036793997135694 VQ motif-containing protein $0.036793997135694 \quad 50$ S ribosomal protein L35 0.0368639930026092 Coiled-coil protein 0.0368639930026092 DNA ligase 3 0.0368639930026092 Transmembrane protein, putative 0.0368639930026092 Protein ROS1 0.0368845809160894 molybdopterin synthase sulfur carrier subunit 0.0368845809160894 glucomannan 4-beta-mannosyltransferase 9-like 0.0369343649486903 No data found 0.0369363206559399 Auxilin-related protein 2 0.0369582320149126 NADH dehydrogenase [ubiquinone] iron-sulfur protein 6, mitochondrial 0.0370097637417238 chaperone protein DnaJ 0.0370647892439477 replication factor C subunit 5 0.0371648877100012 TIP41-like family protein 0.0371767503935631 aspartyl protease family protein 1-like 0.0372301209290144 CST complex subunit STN1 0.0372301209290144 Remorin 0.0372301209290144 Transmembrane protein, putative 0.0372301209290144 Transcription initiation factor TFIID subunit 9 0.0372301209290144 Homologous-pairing protein 2-like protein $0.0372301209290144 \quad$ E3 ubiquitin-protein ligase 0.0372301209290144 No data found 0.0372301209290144 Non-specific serine/threonine protein kinase 
2

3

4

5

6

7

8

9
MELO3C018609 0.00468423052131772 MELO3C018637 0.00469303610003224 MELO3C010982 0.00469150142351815 MELO3C010869 0.00470255932622954 MELO3C008126 0.00471205326936386 $\begin{array}{ll}\text { MELO3C024467 } & 0.0047202723733617\end{array}$ MELO3C005850 0.00472341949742638 MELO3C011045 0.00471896157234275 MELO3C003619 0.00472073883878732 MELO3C018767 0.00472407531450247 MELO3C006522 0.00474301631824137 MELO3C020620 0.00474164902427332 MELO3C003924 0.00475797865690586 MELO3C007388 0.00476193222621446 MELO3C022903 0.00476195972279325 MELO3C017526 0.00476558186294107 MELO3C010860 0.00476961101908036 MELO3C015779 0.00477524685384889 MELO3C006867 0.00478082614067543 MELO3C024282 0.00477892180585116 MELO3C021352 0.00478487268238359 MELO3C025264 0.00480324345966632 MELO3C026332 0.00480409452460129 MELO3C024359 0.00479821584987294 MELO3C021766 0.00480031640730605 MELO3C010205 0.00480220260665098 MELO3C026379 0.00481892308236753 MELO3C004128 0.00482450500558018 MELO3C006557 0.00482848374613742 MELO3C014503 0.00484227068846665 MELO3C004504 0.004853594233164 MELO3C009653 0.00486423822417426 MELO3C019979 0.00486300975469733 MELO3C006846 0.00486680411514517 MELO3C023373 0.00488233112164127 MELO3C020848 0.00488021440735875 MELO3C021380 0.00487514380395182 MELO3C011261 0.0048818429137143 MELO3C009865 0.00488061309291565 MELO3C017766 0.0048895206154731 MELO3C024047 0.00489669867223619 MELO3C023098 0.00490672223331468 MELO3C014607 0.00491102235340035 MELO3C011936 0.00491136386101831 MELO3C014848 0.00492368631958517 MELO3C004616 0.00492100162867981 MELO3C013809 0.00492199748758904 MELO3C023525 0.00493771673994492 MELO3C024980 0.00493883004315743 MELO3C005683 0.0049435184544383 MELO3C026427 0.00494570982192477 MELO3C014560 0.00496315380380685 MELO3C024468 0.00496279709271841 MELO3C014666 0.00496701622528295 MELO3C022914 0.00496848275061024 MELO3C002200 0.00497295451208024 MELO3C005243 0.00498172170978672 MELO3C004555 0.00498570584906988 MELO3C019021 0.00499094402569167 MELO3C026734 0.00499084688857632 MELO3C024947 0.00499678096282596 MELO3C016831 0.00499389552060658 MELO3C024452 0.00499963757377242 MELO3C018867 0.00499926959078445 MELO3C017478 0.00500308520310322 MELO3C020617 0.00500844294115677 MELO3C002030 0.00501632420632747 MELO3C009465 0.00502843552261645
0.0372301209290144 nicotinamidase 1-like 0.0372301209290144 outer envelope protein 64, mitochondrial 0.0372301209290144 cysteine proteinase RD19a-like 0.0372675939541361 Calcium-binding EF-hand

0.0373278539978607 receptor homology region, transmembrane domain- and RING domain0.037348183901565 No data found

0.037348183901565 S-type anion channel SLAH2 0.037348183901565 Histone $\mathrm{H} 3$

0.037348183901565 serine/arginine-rich splicing factor RSZ22A

0.037348183901565 Wound-responsive family protein

0.0374679317075795 Acyl-CoA N-acyltransferases (NAT) superfamily protein, putative 0.0374679317075795 telomere repeat-binding protein 5-like

0.0375710997581903 Nuclear speckle splicing regulatory-like protein (DUF2040)

0.0375724900381318 calcium-dependent protein kinase-like

0.0375724900381318 No data found

0.0375860528000093 No data found

0.0376028135791688 thioredoxin-like 4, chloroplastic

0.0376322227121555 peptidyl-prolyl cis-trans isomerase CYP63

0.0376461465120411 ATP-dependent Clp protease ATP-binding subunit clpX

0.0376461465120411 DUF3820 family protein

0.0376629934652824 No data found

0.0377390862236877 Auxin efflux carrier

0.0377390862236877 DNA helicase INO80-like protein

$0.0377390862236877 \quad 40$ S ribosomal S3-like protein

0.0377390862236877 Cysteine proteinase inhibitor

$0.0377390862236877 \quad$ Myosin-binding protein 7

0.0378405216515612 choline-phosphate cytidylyltransferase 2-like

0.0378692962461061 hepatoma-derived growth factor-related protein 2-like isoform X1

0.0378854690117076 ATP-dependent (S)-NAD(P)H-hydrate dehydratase

0.0379785559279301 Nuclear transcription factor Y subunit B

0.0380522558597681 SPX domain-containing protein 1

0.0381054504915579 transcription factor bHLH48

$0.0381054504915579 \quad 40$ S ribosomal protein S18

0.038110433969073 Mitochondrial metalloendopeptidase OMA1

0.0381563740883631 Aspartate aminotransferase

0.0381563740883631 AP2-like ethylene-responsive transcription factor TOE3 isoform X1

0.0381563740883631 Prolyl 4-hydroxylase alpha subunit, putative

0.0381563740883631 Peroxidase

0.0381563740883631 No data found

0.0381974456625705 Ras family

0.0382383954440461 Kelch repeat-containing F-box family protein

0.0383015246583919 No data found

0.038307474285644 Zinc transporter ZTP29

0.038307474285644 protein-tyrosine-phosphatase MKP1-like

0.0383581385110405 Zinc finger, RING-type

0.0383581385110405 No data found

0.0383581385110405 calcium permeable stress-gated cation channel 1-like

$0.0384457842027261 \quad 60$ S ribosomal protein L6

0.0384457842027261 No data found

0.0384671181862498 UDP-N-acetylmuramoyl-L-alanyl-D-glutamate--2, 6-diaminopimelate ligase

0.0384690127109855 B-cell receptor-associated 31-like protein

0.0385743110867302 Phospholipase A2

0.0385743110867302 Thioredoxin, putative

0.0385853581760918 Dirigent protein

$0.0385853581760918 \quad 50$ S ribosomal protein L15

0.0386049051332833 ABC transporter A family protein

0.0386577689770444 purple acid phosphatase 4-like

0.0386734897145644 At3g13227

0.0386837337375921 Endoplasmic reticulum-Golgi intermediate compartment 3

0.0386837337375921 lamin-like protein

0.0386903771333979 Inner centromere protein, ARK-binding region protein

0.0386903771333979 RING-type E3 ubiquitin transferase

0.0386903771333979 No data found

$0.0386903771333979 \quad$ E6-like protein

0.0387018917650159 Xyloglucan endotransglucosylase/hydrolase

0.0387281674412314 Haloacid dehalogenase-like hydrolase

0.0387739282781844 Glycine-rich RNA-binding protein

0.0388523369077278 Terminal flower 1 
MELO3C018257 0.00503800739884341 MELO3C002891 0.00504688773087425 MELO3C006299 0.00505397492352333

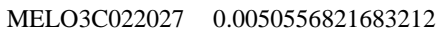
\begin{tabular}{ll} 
MELO3C021713 0.00505438158441507 \\
\hline
\end{tabular} MELO3C012854 0.00505464637579456 MELO3C010569 0.00506299294919321 MELO3C008932 0.00506938195028461 MELO3C009748 0.00507356902590583 MELO3C020574 0.00507969128465335 MELO3C017092 0.00509335428043201 MELO3C018572 0.00509558321440995 MELO3C010865 0.00509674037241725 MELO3C002653 0.00510434896997825 MELO3C002679 0.00510933008744363 MELO3C017563 0.00512088786410558 MELO3C013472 0.0051198180085511 MELO3C019542 0.00513228821363398 MELO3C016667 0.00514137660805702 MELO3C012583 0.00513599158009104 MELO3C021691 0.00514153474524282 MELO3C015299 0.00514189399425591 MELO3C018610 0.0051432171743353 \begin{tabular}{ll} 
MELO3C014936 0.0051599163782603 \\
\hline
\end{tabular} MELO3C016335 0.00515823944960314 MELO3C022426 0.0051671505635198 MELO3C015455 0.00517018623505283 MELO3C012084 0.00517341272002758 MELO3C007190 0.00518428034658769 MELO3C024869 0.0051870663759751 MELO3C005803 0.00519167594605396 MELO3C014288 0.00520148075471183 MELO3C016221 0.00520810517208448 MELO3C010745 0.00520874436567942 MELO3C020579 0.00521804986638397 MELO3C013820 0.00522492446332201 MELO3C022417 0.00522320791153241 MELO3C004917 0.00522269622442229 MELO3C006182 0.00523154057570219 MELO3C012683 0.00524867626788583 MELO3C022310 0.00524944366594116 MELO3C005215 0.0052570899040556 MELO3C019137 0.00526112589863548 MELO3C017906 0.00526885628831797 MELO3C007409 0.00527492254906536 MELO3C006221 0.00527834117750325 MELO3C006760 0.00527934253288698 MELO3C007195 0.00528427713697965 MELO3C010941 0.00528496183711913 MELO3C008118 0.0052918874227319 MELO3C025023 0.00529572304770753 MELO3C003627 0.00529445457556355 MELO3C003690 0.00529425199930755 MELO3C011062 0.00531329000863789 MELO3C007688 0.00531587170426806 MELO3C011704 0.00532175202668261 MELO3C020748 0.00533361546979561 MELO3C001920 0.00533117758364088 MELO3C015481 0.00533354470146574 MELO3C011279 0.00532757903706893 MELO3C005634 0.00533638245875689 MELO3C012881 0.00533881960791294 MELO3C023004 0.00534674243286837 MELO3C004825 0.00534772241914294 MELO3C026640 0.00534644158281883 MELO3C007000 0.0053577184510808 MELO3C012295 0.00536354190955324 MELO3C012912 0.00536579815994709
0.038911070832913 No data found

0.0389644197799201 Pseudouridine synthase, RsuA/RluB/C/D/E/F

0.0389713767143541 RNA-directed DNA polymerase (Reverse transcriptase)-related family protein 0.0389713767143541 40S ribosomal protein S19

0.0389713767143541 Ubiquinol oxidase

0.0389713767143541 Long-chain-alcohol oxidase

0.0390125040006308 Obg-like ATPase 1

0.0390464992730775 thioredoxin-like 3-1, chloroplastic

0.0390635145000446 Pentatricopeptide repeat-containing protein At1g80270, mitochondrial 0.0390954104367183 No data found

0.0391808196243162 diphthine methyltransferase

0.0391808196243162 glutamate-1-semialdehyde 2,1-aminomutase 2, chloroplastic-like

0.0391808196243162 Calcium-binding EF-hand family protein, putative

0.0392240419486772 No data found

0.0392470478012152 heat stress transcription factor A-4c

0.0393052524011742 Protein AE7

0.0393052524011742 Histone deacetylase

0.0393774514106673 D-3-phosphoglycerate dehydrogenase

0.0393847987498828 Random slug protein 5

0.0393847987498828 nuclear-pore anchor-like

0.0393847987498828 F-box protein family

0.0393847987498828 serine/arginine-rich splicing factor SR30

0.0393847987498828 Fructose-1,6-bisphosphatase

0.0394820567819693 BRO1 domain-containing protein

$0.0394820567819693 \quad$ F22C12.19

0.0395220977842573 Something about silencing protein

0.0395300069516293 Acyl--UDP-N-acetylglucosamine O-acyltransferase

0.0395393683466814 40S ribosomal protein S9, putative

0.0396070996382051 Splicing factor 3B, subunit 5

0.0396130602703528 No data found

0.0396329370926246 WAT1-related protein

0.0396924433635255 VAN3-binding protein

0.0397171785628582 ABC transporter G family member 14

0.0397171785628582 mitochondrial import receptor subunit TOM20-like

0.0397727776191497 Enolase

0.0397791184372191 bZIP transcription factor 16

0.0397791184372191 RNA-binding protein 24-B

0.0397791184372191 sulfite oxidase-like

0.0398141405894191 Pentatricopeptide repeat-containing protein At1g01970

0.0399196237807747 Pentatricopeptide repeat-containing protein, mitochondrial

0.0399196237807747 Histidine kinase

0.0399623820304827 defensin Ec-AMP-D2-like

0.0399776742486156 Plant/MNJ7-17 protein, putative

0.0400210164761506 acyl-protein thioesterase 2

0.0400516898967673 WRKY family transcription factor

0.0400544508958836 Glutathione s-transferase, putative

0.0400544508958836 Chromatin structure-remodeling complex protein BSH

0.040066299931388 ER lumen protein-retaining receptor

0.040066299931388 3-ketoacyl-CoA synthase

0.0400863298080399 Inner membrane protein oxaA

0.0400863298080399 thioredoxin-like protein slr0233

0.0400863298080399 Transcriptional adapter 1

0.0400863298080399 adenine nucleotide transporter BT1, chloroplastic/mitochondrial-like

0.0402038943986934 Serine hydrolase FSH

0.0402080238558368 tRNA-dihydrouridine synthase

0.0402370906489108 RAS-related protein RABC1

0.0402651268780556 Receptor-like protein kinase

0.0402651268780556 No data found

0.0402651268780556 Chloroplastic group IIA intron splicing facilitator CRS1

0.0402651268780556 Hydroxyproline O-arabinosyltransferase 1

0.0402706217722545 phosphatidylinositol glycan anchor biosynthesis class U protein-like

0.0402736243073616 rho-N domain-containing protein 1, chloroplastic isoform X3

0.040294608949124 casein kinase I-like isoform X1

0.040294608949124 Ser/thr-rich protein T10 in DGCR region-like protein

0.040294608949124 Mitogen-activated protein kinase kinase kinase 1 isoform 1

0.0403545315371452 Katanin p80 WD40 repeat-containing subunit B1 homolog

0.0403829924406279 Tetratricopeptide repeat-like superfamily protein

0.0403845837884127 Chlorophyll a-b binding protein, chloroplastic 
MELO3C002072 0.00536839161606895 MELO3C014951 0.00538851756489089 MELO3C020489 0.00538680953554949 MELO3C005380 0.00539842275126157 MELO3C009151 0.00540405465087668 MELO3C023173 0.00540260609983467 MELO3C016700 0.00541295604689029 MELO3C024248 0.00541360366330046 MELO3C002313 0.00541541475618779 MELO3C015113 0.00542243994015512 MELO3C007235 0.00543615744316983 MELO3C017551 0.00544379027512432 MELO3C017169 0.0054419971112214 MELO3C002293 0.00544667677743771 MELO3C009170 0.00545100219701766 MELO3C016881 0.00546445639647308 MELO3C010023 0.00548021836409496 MELO3C022605 0.00548519315338203 MELO3C006643 0.00548775039087324 MELO3C019156 0.00549336446814186 MELO3C022885 0.00549564783216616 MELO3C015423 0.00549259467745444 MELO3C026577 0.00549584987134866 MELO3C022961 0.00549927721920196 MELO3C009329 0.00550540936501021 MELO3C024348 0.00550384303802409 MELO3C005298 0.00551011550099556 MELO3C003554 0.00551021715769784 MELO3C009549 0.00551926640667522 MELO3C017925 0.00552454836960814 MELO3C022530 0.00552368037194162 MELO3C011897 0.00553231331154147 MELO3C003453 0.00554160058650854 MELO3C023308 0.0055442020245402 MELO3C006093 0.00554875124716214 MELO3C006553 0.00555487297960233 MELO3C022742 0.00556230935646029 MELO3C009753 0.00556782329136662 MELO3C005559 0.00556759058409906 MELO3C021715 0.00558441460505543 MELO3C026293 0.00561249329691038 MELO3C021818 0.00561839821362631 MELO3C013030 0.00562152618538336 MELO3C017430 0.0056314445582144 MELO3C007563 0.00564058020111158 MELO3C026567 0.00563811290088667 MELO3C013581 0.00563795363412223 MELO3C008152 0.00563998177954761 MELO3C022309 0.00564333704074471 MELO3C019429 0.00564600559118555 MELO3C014876 0.00565538385941067 MELO3C016778 0.00565933442381517 MELO3C004117 0.00566929710292929 MELO3C007636 0.00567263519002581 MELO3C020997 0.00568704454460378 MELO3C026499 0.00568866895933029 MELO3C013692 0.00569296709952316 MELO3C002261 0.00570743449773126 MELO3C009193 0.0057141543213195 MELO3C019997 0.0057189492179438 MELO3C014390 0.00572324775500266 MELO3C026392 0.0057372614827349 MELO3C006151 0.0057406825704176 MELO3C013951 0.00574760326577217 MELO3C016676 0.00574342477656464 MELO3C003123 0.00574566882149075 MELO3C022650 0.00574909690102454 MELO3C025152 0.00576102443116722
0.0403887108669507 Adenine nucleotide alpha hydrolases-like superfamily protein 0.0405092628051124 tRNA/rRNA methyltransferase family protein 0.0405092628051124 WRKY transcription factor 1 0.0405682842141038 protein phosphatase inhibitor 2 0.0405797244487314 phosphoinositide phosphatase SAC8 0.0405797244487314 sister chromatid cohesion 1 protein 3 0.0406186957918544 Inner membrane protein oxaA 0.0406186957918544 Dynein light chain family protein 0.0406186957918544 Serine/threonine-protein kinase AFC2 0.0406559477517553 Protein SMG7

0.0407433295427556 Protein IQ-DOMAIN 1 0.0407695920149526 Pentatricopeptide repeat-containing protein 0.0407695920149526 60S ribosomal protein L34 0.0407757466556548 Armadillo/beta-catenin repeat family protein 0.0407926647930662 vacuolar cation/proton exchanger 3-like 0.0408778596113435 Cytokinin riboside 5'-monophosphate phosphoribohydrolase 0.0409802470550971 Ycf20-like protein

0.0410019226291225 thiamine biosynthetic bifunctional enzyme TH1, chloroplastic-like $0.0410039815297562 \quad$ E3 ubiquitin-protein ligase 0.0410039815297562 protein GrpE 0.0410039815297562 methyl-CpG-binding domain protein 4-like protein 0.0410039815297562 Damaged dna-binding 2, putative isoform 1 0.0410039815297562 mitogen-activated protein kinase kinase 3 0.0410140580823336 4-hydroxyphenylpyruvate dioxygenase 0.0410288036036176 Peroxidase 0.0410288036036176 Lipoxygenase 0.0410336646483313 DELLA protein GAI 0.0410336646483313 homeobox-leucine zipper protein ATHB-6-like 0.0410855605975985 No data found 0.0410939004713338 Myb transcription factor

0.0410939004713338 Armadillo/beta-catenin repeat family protein 0.0411361655081448 F-box protein SKIP14

0.041189713956702 eukaryotic translation initiation factor 3 subunit L-like 0.0411935461936209 F-box protein At2g16365

0.0412118421888699 UDP-glycosyltransferase 91A1-like 0.0412417994263784 3-dehydrosphinganine reductase TSC10A isoform X1 0.0412814909735943 ATP-dependent DNA helicase, RecQ family protein, expressed 0.0412913789640253 Transcription factor PERIANTHIA 0.0412913789640253 transaldolase 0.0413988753886035 E3 ubiquitin-protein ligase COP1-like 0.041591418431776 DUF1645 family protein 0.0416195597602798 Protein LIGHT-DEPENDENT SHORT HYPOCOTYLS 10 0.0416271168485699 Tudor/PWWP/MBT superfamily protein 0.0416849320015653 Fiber protein Fb15 0.0416900517933206 Lipase

0.0416900517933206 Pentatricopeptide repeat-containing protein At4g18975, chloroplastic 0.0416900517933206 shaggy-related protein kinase eta

0.0416900517933206 Class I glutamine amidotransferase-like superfamily protein 0.0416948235008108 Ectonucleotide pyrophosphatase/phosphodiesterase family member 3 0.0416989395737934 Basic-leucine zipper (BZIP) transcription factor family protein 0.041752589098879 Kelch repeat-containing F-box family protein $0.0417661418295686 \mathrm{SPla} / \mathrm{RYanodine}$ receptor (SPRY) domain protein 0.0418240375367018 Carboxypeptidase

0.0418330367318221 Calcium-transporting ATPase 0.0419199713723186 Sterol 3-beta-glucosyltransferase 0.0419199713723186 Zinc finger HIT domain-containing protein 3 0.0419359967357266 Transmembrane protein, putative 0.0420268918328466 serine/threonine-protein kinase HT1-like 0.0420606909026235 Transmembrane protein 0.0420803010824039 Histone H4 0.0420962457778575 mavicyanin-like 0.042183610209431 RING/U-box superfamily protein 0.0421920901889014 protein-ribulosamine 3-kinase, chloroplastic 0.0421920901889014 NADH dehydrogenase [ubiquinone] 1 beta subcomplex subunit 8 , 0.0421920901889014 Peptide methionine sulfoxide reductase 0.0421920901889014 E3 ubiquitin-protein ligase XB3-like 0.0421920901889014 At1g16840/F17F16.27 0.0422639195732249 Coronatine-insensitive 1 
MELO3C009325 0.00577467309870794 MELO3C014035 0.00577498523058584 MELO3C016723 0.00577229566189108

MELO3C012351 0.00577787071656299 MELO3C013215 0.00578362349766959 MELO3C016017 0.00578589705892163 MELO3C002023 0.00580928505066813 MELO3C007814 0.00582928347011036 \begin{tabular}{ll} 
MELO3C021695 0.0058292778713428 \\
\hline
\end{tabular} MELO3C022301 0.00583286609252087 MELO3C014353 0.00584390830887993 MELO3C018739 0.00584621381637895 MELO3C011890 0.00585525734603176 MELO3C025877 0.00585523358807216 MELO3C007133 0.00586456153890169 MELO3C020666 0.0058657145241845 MELO3C004534 0.00587048715416727 MELO3C024909 0.005876180212674 MELO3C006765 0.00587699496132954 MELO3C015718 0.00587510034530903 MELO3C018802 0.00587443787186281 MELO3C012708 0.00588417269765351 MELO3C004011 0.00590548212715403 MELO3C006191 0.00590581813556534 MELO3C010772 0.00590688392788552 MELO3C009201 0.00591891723251758 MELO3C006188 0.00592525493769747 MELO3C013243 0.00591825048021155 MELO3C016108 0.0059234494023328 MELO3C008056 0.0059159671652691 MELO3C019145 0.00591396721573012 MELO3C002690 0.00591329059347701 MELO3C003396 0.00592368884294037 MELO3C010378 0.00592824461531416 MELO3C024777 0.00593479565849098 MELO3C009446 $\quad 0.00593804692995192$ MELO3C026748 0.0059455675826553 MELO3C014562 0.00599556157265702 MELO3C003917 0.00599045408550714 MELO3C016296 0.00599404152910388 MELO3C002981 0.00599647789055124 MELO3C012239 0.00599508591673292 MELO3C018576 0.006001066828351 MELO3C007947 0.00601022964576781 MELO3C000960 $\quad 0.00601217131829979$ MELO3C012636 0.00604022024987105

MELO3C007305 0.00604570718491382 MELO3C002619 0.00604427870695745 MELO3C017434 0.00605024264847209 MELO3C024960 0.00605807790577961 MELO3C011968 0.00606024825596196 MELO3C020484 0.0060595841623905 MELO3C019453 0.00606717259072709 MELO3C004524 0.00608588071467309 MELO3C012929 0.00608677022585591 MELO3C021977 0.00610215935596248 MELO3C005419 0.00610228848337935 MELO3C008275 0.00609738000015136 MELO3C018119 0.00610157934889466 MELO3C012855 0.00610856561304385 MELO3C018729 0.00611147585383698 MELO3C004028 0.0061196128433687 MELO3C024761 0.00611839938520808
0.0423191774837995 POLAR LOCALIZATION DURING ASYMMETRIC DIVISION AND 0.0423191774837995 No data found 0.0423191774837995 serpin-ZX-like

L-aminoadipate-semialdehyde dehydrogenase-phosphopantetheinyl transferase

0.0423246175005202 like

0.0423510494829354 Chaperone DnaJ domain protein

0.0423519944464949 translocator protein homolog

0.0425074362599648 DExH-box ATP-dependent RNA helicase DExH14

0.0426221840989298 protein indeterminate-domain 2-like

0.0426221840989298 Phosphatidate cytidylyltransferase

0.0426325952854162 serine/threonine-protein kinase ATG1a isoform X4

0.0426975009959562 Transmembrane protein, putative

0.0426985490605281 GPI-anchored protein LORELEI

0.0427329923602296 DNA-binding storekeeper protein-related

0.0427329923602296 Glucose-6-phosphate isomerase

0.0427776942902953 Transmembrane protein

0.0427776942902953 ATP-dependent clp protease

0.0427809706934379 Glycine-rich RNA-binding protein, putative

0.0427809706934379 Mitochondrial transcription termination factor family protein

0.0427809706934379 DEAD-box ATP-dependent RNA helicase 50

0.0427809706934379 ATP binding protein

0.0427809706934379 Peroxisome biogenesis protein 22

0.042817437953559 Pentatricopeptide repeat-containing protein

0.0429352413293379 No data found

0.0429352413293379 Katanin p80 WD40 repeat-containing subunit B1 homolog

0.0429352413293379 clathrin interactor EPSIN 1

0.0429423338585641 Immediate early response 3-interacting protein 1

0.0429423338585641 ATP-dependent DNA helicase 2 subunit KU80

0.0429423338585641 TOM1-like protein 2

0.0429423338585641 Receptor-like protein kinase

0.0429423338585641 NAC domain-containing protein 82

0.0429423338585641 ER membrane protein complex subunit 8/9 homolog

0.0429423338585641 Myosin heavy chain kinase B

0.0429423338585641 Phospholipid hydroperoxide glutathione peroxidase

0.0429482402449888 No data found

0.0429799337952102 ABC transporter B family protein

0.0429877158429694 Serine/threonine-protein phosphatase PP1

0.043026388490238 Enolase

0.0433154505707741 E3 ubiquitin-protein ligase ICP0

0.0433154505707741 Heat shock $70 \mathrm{kDa}$ protein

0.0433154505707741 Chlororespiratory reduction31

0.0433154505707741 transmembrane emp24 domain-containing protein p24delta9-like

0.0433154505707741 Bowman-Birk type bran trypsin inhibitor

0.0433327491016833 L-galactose dehydrogenase

0.0433812098520652 Ankyrin repeat domain-containing protein, chloroplastic

0.0433812098520652 Peptidase_M22 domain-containing protein

0.0435676806846981 Unknown protein

SNF2 domain-containing protein / helicase domain-containing protein / zinc

0.0435754274433807 finger protein-like protein

0.0435754274433807 Mitochondrial carrier protein, expressed

0.0435922079768973 Oxysterol-binding protein-related protein 4C

0.0436165607897204 pentatricopeptide repeat-containing protein At5g66520-like

0.0436165607897204 Lipase

0.0436165607897204 B3 domain-containing transcription repressor VAL2-like isoform X1

0.0436504887046518 Unknown protein

0.0437596014526496 zinc finger CCCH domain-containing protein 39-like

0.0437596014526496 Transmembrane protein, putative

0.0438073774112173 Translation factor GUF1 homolog, mitochondrial

0.0438073774112173 AT-hook motif nuclear-localized protein 6-like

0.0438073774112173 Translation initiation factor 2 subunit gamma

0.0438073774112173 CRS2-associated factor 2, mitochondrial

0.043836505193315 Thioredoxin family protein

0.0438414590037873 F21O3.6 protein

0.0438679615403588 Syntaxin/T-SNARE family protein

0.0438679615403588 GDSL esterase/lipase At2g30310-like 
2
|MELO3C024178 0.00612424666004419 MELO3C023608 0.00613091710727787 MELO3C025210 0.00613790062530528 MELO3C021934 0.00613720039845989 MELO3C006919 0.00614558306381441 MELO3C018549 $\quad 0.0061508796175187$ MELO3C002746 0.00616727372711723 MELO3C011357 0.00619072089896822 MELO3C024739 0.00619905310426472 MELO3C014540 0.00620616913018546 MELO3C002036 0.00622651950250241 MELO3C016913 0.0062308640362656 MELO3C007762 0.00623705673597619 MELO3C021058 0.00623574434668006 MELO3C015155 0.00623862458801827 MELO3C010763 0.00623461804273584 MELO3C027004 0.00624716628925381 MELO3C026199 0.0062499307886954 MELO3C018765 0.00625106892234339 MELO3C003173 0.00627159763267748 MELO3C011375 0.00627310801694059 MELO3C022302 0.00627652237112752 \begin{tabular}{ll} 
MELO3C020793 0.00628178082278441 \\
\hline
\end{tabular} MELO3C008503 0.00629332806158112 MELO3C012054 0.00629326949914211 MELO3C013884 0.00630182195868945 MELO3C024563 0.00631506277400962 MELO3C014872 0.00632247490555671 MELO3C007513 0.00632190735505112 MELO3C005964 0.00632905007685847 MELO3C013087 0.00633709589246012 MELO3C012701 0.00636922311907195 MELO3C018356 0.00636756099341584 MELO3C021329 0.00636951373363392 MELO3C021253 0.00637146288046764 MELO3C026388 0.00637537403507316 MELO3C010153 0.00637634889135241 MELO3C007784 0.00638303272564489 MELO3C009381 0.00639888795602483 MELO3C016674 0.00642353608518753 MELO3C018174 0.00642233138082371 MELO3C004357 0.00642826459136869 MELO3C016498 0.00643136952453571 MELO3C018412 0.00643238898121734 MELO3C016846 0.00644461626837156 MELO3C025627 0.00644076471782506 MELO3C025613 0.00644431749613228 MELO3C022004 0.00644429970006122 MELO3C006050 0.00644986291475913 MELO3C006253 0.00646031208615239 MELO3C010427 0.00645475405348239 MELO3C010381 0.00645743435607238 MELO3C016083 0.00645283144289732 MELO3C002209 0.00645126342186264 MELO3C003341 0.00646141419979185 MELO3C009195 0.00646569829267873 MELO3C014214 0.00646880382342496 MELO3C010810 0.00646732888793111 MELO3C013814 0.00648131394496065 MELO3C002275 0.00649370496098678 MELO3C022225 0.00649818775899091 MELO3C004648 0.00650643547640417 MELO3C014127 0.00651810160220068 MELO3C013774 0.00651526241483036 MELO3C010338 0.00651877464855244 MELO3C017394 0.00652352679417967 MELO3C011399 0.00652419783353908 MELO3C009480 0.00653901503596366
0.0438852493792499 Histidine--tRNA ligase 0.0439171135116542 Zinc finger family protein 0.0439352662012157 gibberellin 2-beta-dioxygenase 2-like 0.0439352662012157 Unknown protein

0.0439743188142285 cleavage and polyadenylation specificity factor subunit 2 0.0439962772786588 DUF21 domain-containing protein

0.0440975701798835 Pyruvate dehydrogenase E1 component subunit beta

0.0442492026904536 serine/arginine-rich splicing factor SC35-like 0.0442927278423024 growth-regulating factor 4-like 0.0443275349555272 blue copper protein-like

0.0444568089858714 DUF1118-domain-containing protein 0.0444628643048621 No data found

0.0444628643048621 Mediator of RNA polymerase II transcription subunit 26, putative

0.0444628643048621 cell division control protein 45 homolog

0.0444628643048621 Scarecrow-like 3

0.0444628643048621 vacuolar-processing enzyme-like

0.0445033742420186 poly(A)-specific ribonuclease PARN-like

0.0445033742420186 Transmembrane protein, putative

0.0445033742420186 AT4g28240/F26K10_120

0.0446281016666281 Proteasome subunit alpha type

0.0446281016666281 BPS1-like protein

0.044636312678213 protein LHY-like isoform X1

0.0446576275986931 Amidase, putative

0.0447075308950235 Protein ABIL2

0.0447075308950235 Glycine-rich protein

$0.0447517734132175 \quad$ E3 ubiquitin-protein ligase LAP

0.0448296817842976 O-linked $\mathrm{N}$-acetylglucosamine transferase, putative

0.0448500563612929 Lipase

0.0448500563612929 No data found

$0.044880578085414 \quad$ Late cornified envelope protein 1E

$0.0449215027926041 \quad$ 5'/3'-nucleotidase SurE

0.0451003657442134 Cysteine proteinase inhibitor

0.0451003657442134 Protein WVD2-like 7

0.0451003657442134 ATP-binding cassette sub-family C member 11

0.0451003657442134 Beta-glucosidase

$0.0451026197189537 \quad \mathrm{~N}$-acetyl-D-glucosamine kinase-like

$0.0451026197189537 \quad$ CTP-dependent diacylglycerol kinase 1

0.0451337319365417 Unknown protein

0.0452296486197332 splicing factor 3A subunit 2

0.0453713927562119 cyclin-SDS-like isoform X1

0.0453713927562119 zinc finger protein CONSTANS-LIKE 4

0.0453852268631873 No data found

0.0453852268631873 MACPF domain-containing protein At4g24290

0.0453852268631873 allene oxide synthase 3-like

0.0454066095911773 Phosphoribosyl-AMP cyclohydrolase

0.0454066095911773 Sodium/calcium exchanger family protein

0.0454066095911773 Tyrosine aminotransferase

0.0454066095911773 SPX domain protein

0.0454115548155478 Phosphatidylinositol-4-phosphate 5-kinase family protein

0.0454115548155478 protein ECERIFERUM 1-like

0.0454115548155478 aspartate-semialdehyde dehydrogenase

$0.0454115548155478 \quad$ 2-hydroxyacyl-CoA lyase

0.0454115548155478 Ubiquitin

0.0454115548155478 Homeobox leucine zipper family protein

0.0454115548155478 Casein kinase II subunit beta

0.0454150041623959 OTU domain-containing protein

0.0454150041623959 ABC transporter G family-like protein

0.0454150041623959 Sigma factor sigb regulation protein rsbq

$0.0454866627928315 \quad 26 \mathrm{~S}$ proteasome non-ATPase regulatory subunit 13

0.0455574349110223 anthranilate phosphoribosyltransferase, chloroplastic

0.0455726953310765 protein NRT1/ PTR FAMILY 5.10-like

0.045614339447464 Stress-response A/B barrel domain-containing protein UP3

0.0456522271398093 Actin-related protein 8

0.0456522271398093 Cystathionine gamma-synthase

0.0456522271398093 Outward-rectifying potassium channel 4-like protein

0.0456578253063655 No data found

0.0456578253063655 LOW QUALITY PROTEIN: transcription factor BIM2

$0.0457291104621977 \quad$ ABC transporter family protein 
MELO3C009226 0.00653689188363704 MELO3C006727 0.00654713755056613 MELO3C014592 0.00655080438696443 MELO3C025386 0.00655608608069769 MELO3C002931 0.00656237554505812 MELO3C003629 0.00657160924631839 MELO3C025085 0.00657351860449662 MELO3C017111 0.00658733845633164 MELO3C000210 0.00659371367780071 MELO3C002863 0.00659694398511679 MELO3C010710 0.00660311013512804 MELO3C006770 0.00661343968116468 MELO3C014101 0.00661437329406644 MELO3C005578 0.00660977458179657 MELO3C020940 0.00661550757875262 MELO3C011317 0.00660728708017455 MELO3C003039 0.00663998790077891 MELO3C023313 0.00665769860589904 MELO3C002412 0.00666469864478825 MELO3C021730 0.00666911679857307 MELO3C007344 0.0066785985161617 MELO3C019691 0.00668174178677394 MELO3C023315 0.00668561297068715 MELO3C010430 0.00670618584904936 MELO3C010243 0.00671615745963616 MELO3C016714 0.00672515339221591 MELO3C024515 0.00672876682696899 MELO3C002102 0.00673479236241348 MELO3C006703 0.00674630940843268 MELO3C005600 0.0067735201512592 MELO3C022928 0.00677352836102607 MELO3C015597 0.00677974577639429 MELO3C018604 0.00677908066685062 MELO3C026067 0.00678676319927907 MELO3C007325 0.00679902181931435 MELO3C023845 0.00680785721675858 MELO3C018418 0.00681891427759185 MELO3C010532 0.00684685289919851 MELO3C026628 0.00686069658808242

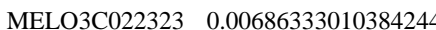
MELO3C007871 0.00687289009524761 MELO3C002405 0.00687058154327702 MELO3C022636 0.00688113418024483 MELO3C003447 0.00688547634453296 MELO3C015424 0.00690715562065036 MELO3C016795 0.00692639658527194 MELO3C026546 0.00693441822564089 MELO3C014262 0.00696264092237953 MELO3C020440 0.00696854568206295 MELO3C004152 0.00696272408374665 MELO3C006100 0.00695734585909258 MELO3C014870 0.00695033903996145 MELO3C010475 0.00694843697490344 MELO3C018008 0.0069448281081157 MELO3C007024 0.00695655689989738 MELO3C025262 0.00696807649833175 MELO3C003085 0.00696185243230085 MELO3C022024 0.00693627676482245 MELO3C020840 0.00696181741775093 MELO3C025883 0.00696229192516018 MELO3C025870 0.0069539047423004 MELO3C002552 0.00693441431194941 MELO3C008234 0.00696379313695883 MELO3C005532 0.00697466168591299 MELO3C021323 0.00699292881807612 MELO3C011016 0.00701159249660366 MELO3C011386 0.00701788286723926 MELO3C010709 0.0070296723169897
0.0457291104621977 serine/threonine-protein kinase ATG1c 0.0457697060127895 Neutral/alkaline invertase 0.0457791351161219 heterogeneous nuclear ribonucleoprotein F 0.04579983870099 Polyadenylate-binding protein-interacting protein 4 0.0458275652897287 Paired amphipathic helix SIN3-like protein 0.0458729395477752 Plasma membrane ATPase

0.0458729395477752 Class I heat shock protein

0.0459531427672531 39S ribosomal protein L47, mitochondrial 0.0459813740900022 Unknown protein 0.0459876621115677 patellin-4

0.046014404396134 Short-chain dehydrogenase/reductase 0.0460196052035173 At1g08580

0.0460196052035173 Unknown protein

0.0460196052035173 Amine oxidase

0.0460196052035173 Pentatricopeptide repeat-containing family protein

0.0460196052035173 zinc finger protein CONSTANS-LIKE 4

0.0461736341734094 No data found

0.0462804962224218 Transmembrane protein, putative

0.0463128548683755 Ankyrin repeat family protein

0.0463272555944494 calcium permeable stress-gated cation channel 1-like 0.0463768080505195 No data found

0.0463823263785584 Hexosyltransferase

0.0463928919740339 diaminopimelate decarboxylase 2, chloroplastic-like

0.0465193060529947 At1g78110

0.046572118564029 VAMP-like protein YKT61

0.0466181306924788 protochlorophyllide reductase, chloroplastic

0.0466268126546704 splicing factor U2af small subunit B-like

0.046652197251948 Receptor-like kinase

0.0467155906406511 CoA ligase

0.046871202383288 Phosphate translocator-related family protein

0.046871202383288 No data found

0.0468813723172307 Calcium ion binding

0.0468813723172307 Ubiquitin-like-specific protease 2

0.0469134709214429 tRNA (Guanine(9)-N1)-methyltransferase-like

0.046981764139132 Sugar transporter, putative

0.0470263631247867 serine/arginine-rich SC35-like splicing factor SCL30

0.0470862720518047 No data found

0.0472626696631497 Structural constituent of ribosome, putative

0.0473416830601117 Zinc finger protein

0.0473433133848356 proton pump-interactor 1-like

0.0473761628240995 rRNA adenine N(6)-methyltransferase

0.0473761628240995 50S ribosomal protein L7/L12

0.0474164406579397 No data found

0.04742981246187 Major facilitator superfamily transporter

0.0475625580028675 Glucan endo-1,3-beta-glucosidase, putative

0.04767842668614 Clavata3/ESR (CLE) gene family member MtCLE20

0.0476860043919131 DNA-directed RNA polymerase subunit beta

0.0476860043919131 60S ribosomal protein L4-like

0.0476860043919131 UBX domain-containing protein 1

0.0476860043919131 40S ribosomal protein S23-1

0.0476860043919131 Ubiquitin-conjugating enzyme, E2

0.0476860043919131 DSR6

0.0476860043919131 UDP-glucuronosyltransferase 2B17

0.0476860043919131 Glycyl-tRNA synthetase beta subunit

0.0476860043919131 Phenylalanine--tRNA ligase

0.0476860043919131 AAA + ATPase domain-containing protein

0.0476860043919131 Flagellin N-methylase

0.0476860043919131 Hexosyltransferase

0.0476860043919131 Formin-like protein

0.0476860043919131 Gb|AAC95187.1

0.0476860043919131 Transcription factor

0.0476860043919131 Wound-responsive family protein

0.0476860043919131 Transferring glycosyl group transferase

0.0477113244319694 Pectinesterase

0.0478197199543578 condensin-2 complex subunit D3

0.0479307511995243 tubulin beta chain

0.0479571518149163 DNA gyrase subunit A

0.048021099477077 telomere-associated protein RIF1-like isoform X2 
2
MELO3C026341 0.00704211024903056 MELO3C006306 0.00704624370381213 MELO3C024211 0.00708416893756425 MELO3C021434 0.00709534752098828 MELO3C022219 0.00711140982249026 MELO3C007597 0.00712542430296725 MELO3C017791 0.00713147184175345 MELO3C008811 0.007139438811121 MELO3C024179 0.00715903843773669 MELO3C018056 0.00715686435559881 MELO3C011234 0.00715969969949182 MELO3C024853 0.00717709886898354 MELO3C018841 0.00717850423405153 MELO3C022802 0.00718337005107827 MELO3C009638 0.00720397515405002 MELO3C014057 0.00719121069185757 MELO3C017745 0.00720673118743775 MELO3C014215 0.00719953826615072 MELO3C003108 0.00720218464493627 MELO3C025150 0.00720791735611159 MELO3C021391 0.00719687947412662 MELO3C021274 0.00720832830460949 MELO3C018769 0.00720953787301104 MELO3C012904 0.00719324434856972 MELO3C019780 0.00721681585490708 MELO3C010939 0.00722100799204228 MELO3C026824 0.00723804249067217 MELO3C021328 0.00724702951271161 MELO3C022348 0.00724762287890601 MELO3C004223 0.00725452501643375 MELO3C017562 0.00725386990279486 MELO3C008420 $\quad 0.0072686274147804$ MELO3C009316 0.0072779413909807 MELO3C013370 0.00728490803364623 MELO3C021385 0.00728849085706462 MELO3C009552 0.00729901228807617 MELO3C020458 0.00729730268308693 MELO3C016448 0.00729807390629156 MELO3C019257 0.00729310653565496 MELO3C005288 0.00730395227585312 MELO3C018966 0.00730701020630065 MELO3C006035 0.00731675729151005 MELO3C004603 0.00732905066766798 MELO3C012007 0.00732642441238118 MELO3C025324 0.00732887618986666 MELO3C010766 0.00733014592886683 MELO3C019260 0.00733326272891488 MELO3C017705 0.00734710733815036 MELO3C012421 0.0073514013120205 MELO3C010584 0.00736507254652774 MELO3C002071 0.00737535584584981 MELO3C005980 0.00738295761679109 MELO3C020839 0.00739116720083399 MELO3C026554 0.007393470127247 MELO3C008219 0.0074119726130466 MELO3C004992 0.00742737438247398 MELO3C008610 0.00743112678605029 MELO3C012753 0.00743754746958103 MELO3C021306 0.00743815264091596 MELO3C017009 $\quad 0.00744176012404185$ MELO3C007097 0.00744929403958527 MELO3C022759 0.00746050443032131 MELO3C016549 0.00746559537964697
0.0480894312960251 ubiquitin-conjugating enzyme E2 22 0.048101025546694 beta-mannosyltransferase 1 0.0483432109011598 BTB/POZ domain-containing protein At5g48130 0.0484027696690838 DNA polymerase epsilon catalytic subunit A $0.0484955913620028 \mathrm{SPla} / \mathrm{RY}$ anodine receptor (SPRY) domain-containing protein 0.0485743888710046 Indole-3-acetic acid-amido synthetase GH3.3 0.048598839683502 No data found 0.0486363494587198 importin-5

0.0487239522278649 zinc finger protein CONSTANS-LIKE 9-like 0.0487239522278649 Carbonic anhydrase

0.0487239522278649 GTP-binding nuclear protein 0.0488182782776459 GTP-binding protein, chloroplastic 0.0488182782776459 Beta-adaptin-like protein 0.0488345525434578 Sulfate transporter, putative 0.0488443099327942 UBP1-associated protein 2C-like 0.0488443099327942 Plant UBX domain-containing protein 8 $0.0488443099327942 \quad$ F12F1.11 0.0488443099327942 No data found 0.0488443099327942 Histone H2A 0.0488443099327942 No data found 0.0488443099327942 Protein ABCI12, chloroplastic

0.0488443099327942 Outer envelope pore protein 16-2, chloroplastic 0.0488443099327942 Wound-responsive family protein 0.0488443099327942 Autophagy-related protein 9 0.0488768505893552 protein kinase 2B, chloroplastic-like 0.0488884768031687 FK506-binding protein 4-like 0.048987012045334 cytochrome P450 714A1-like 0.0490182548751763 ubiquitin-like-specific protease ESD4 0.0490182548751763 Protein TIFY 9 0.0490313533708249 ATP-dependent Clp protease proteolytic subunit 0.0490313533708249 Random slug protein 5 0.0491098606960309 Transcriptional corepressor SEUSS 0.0491559728216409 Interactor of constitutive active ROPs-like protein 0.0491862047030699 translocase of chloroplast 120, chloroplastic-like 0.0491935768749724 Phosphatase 2C family protein 0.0491973357260124 Mads box protein, putative 0.0491973357260124 cyclin-dependent kinase inhibitor 5-like 0.0491973357260124 methionine S-methyltransferase 0.0491973357260124 CDT1-like protein a, chloroplastic 0.0492138360613522 F-box family protein 0.0492176482142672 ATP-dependent 6-phosphofructokinase 0.0492664983805087 Rhodanese-like domain-containing protein 4, chloroplastic 0.0492894286514099 Protein-tyrosine phosphatase mitochondrial 1 0.0492894286514099 spermidine synthase 0.0492894286514099 Leucine-rich repeat extensin-like protein 4 0.0492894286514099 UPF0587 protein C1orf123 homolog 0.0492936030065827 Glutamate receptor 0.0493698614566626 Transducin/WD40 repeat-like superfamily protein 0.0493819130990112 Cytochrome c oxidase assembly protein COX16 0.0494569254407944 ribosome maturation protein SBDS 0.0495091443234833 single-stranded DNA-binding protein, mitochondrial 0.0495433333245012 Protein ARABIDILLO 1 0.0495801838855691 glutamine--tRNA ligase-like 0.0495801838855691 alcohol dehydrogenase-like 7 0.0496873887084377 glyceraldehyde-3-phosphate dehydrogenase 0.049773741662531 Protein nuclear fusion defective 4 0.0497819955555316 No data found 0.0497952801713388 Unknown protein 0.0497952801713388 ethylene-responsive transcription factor RAP2-3-like 0.0498025485224339 4-coumarate--CoA ligase family protein 0.0498360799416564 F-box protein SKIP19 0.0498941760902186 MOB kinase activator-like 1A $0.0499113213109844 \quad$ U3 small nucleolar RNA-associated protein 14 B isoform 3 
Supplementary Table 1.B Gene Ontology terms (GO terms) of the cluster 1.1 obtained considering the comparison between lines over time fruit during storage at $20.5^{\circ} \mathrm{C}$ and $88 \%$ relative humidity for $18 \mathrm{~d}$ the near-isogenic line SC10-2 and its parental "Piel de Sapo" obtained according to the differentially expressed gene pattern (Supl. Table 1.A)

\begin{tabular}{|c|c|c|c|c|c|c|c|c|c|c|c|}
\hline Tags & GO ID & GO Name & GO Category & FDR & P-Value & Nr Test & Nr Reference & \begin{tabular}{|l} 
Non Annot \\
Test \\
\end{tabular} & \begin{tabular}{|l|}
$\begin{array}{l}\text { Non Annot } \\
\text { Reference }\end{array}$ \\
\end{tabular} & \begin{tabular}{|l} 
TestSet \\
Sequences
\end{tabular} & RefSet Sequences \\
\hline [OVER] & GO:0003735 & $\begin{array}{l}\text { structural } \\
\text { constituent } \\
\text { of ribosome }\end{array}$ & $\begin{array}{l}\text { MOLECULAR } \\
\text { _FUNCTION }\end{array}$ & $\begin{array}{l}4.094870144 \\
646738 \mathrm{E}-5\end{array}$ & $\begin{array}{l}1.192136587 \\
6400271 \mathrm{E}-8\end{array}$ & 14 & 340 & 83 & 16691 & $\begin{array}{l}\text { MELO3C020906; } \\
\text { MELO3CO11873; } \\
\text { MELO3CO17939; } \\
\text { MELO3CO09315; } \\
\text { MELO3CO08974; } \\
\text { MELO3CO21332; } \\
\text { MELO3COO6507; } \\
\text { MELO3CO17111; } \\
\text { MELO3CO15177; } \\
\text { MELO3CO19524; } \\
\text { MELO3C026780; } \\
\text { MELO3CO17169; } \\
\text { MELO3CO12967; } \\
\text { MELO3C018459 }\end{array}$ & $\begin{array}{l}\text { MELO3C011310; } \\
\text { MELO3C020704; } \\
\text { MELO3C006088; } \\
\text { MELO3C002164; } \\
\text { MELO3C012084; } \\
\text { MELO3C014262; } \\
\text { MELO3C024506; } \\
\text { MELO3C020945; } \\
\text { MELO3CO06400; } \\
\text { MELO3CO03258; } \\
\text { MELO3CO06766; } \\
\text { MELO3CO24747; } \\
\text { MELO3CO20141; } \\
\text { MELO3C022441; } \\
\text { MELO3CO03811; } \\
\text { MELO3CO01514; } \\
\text { MELO3C025276; } \\
\text { MELO3C016201; } \\
\text { MELO3C025030; } \\
\text { MELO3C018985; } \\
\text { MELO3C016688; } \\
\text { MELO3C014824; } \\
\text { MELO3C017659; } \\
\text { MELO3C020836; } \\
\text { MELO3CO07740; } \\
\text { MELO3CO13981; } \\
\text { MELO3CO09489; }\end{array}$ \\
\hline [OVER] & GO:0006414 & \begin{tabular}{|l|} 
translational \\
elongation
\end{tabular} & \begin{tabular}{|l} 
BIOLOGICAL \\
PROCESS
\end{tabular} & $\begin{array}{l}0.001273370 \\
6759199884\end{array}$ & $\begin{array}{l}1.752097670 \\
0846581 E-6\end{array}$ & 9 & 188 & 88 & 16843 & $\begin{array}{l}\text { MELO3C020906; } \\
\text { MELO3C011873; } \\
\text { MELO3CO06507; } \\
\text { MELO3C025511; } \\
\text { MELO3C017939; } \\
\text { MELO3C026780; } \\
\text { MELO3C017169; } \\
\text { MELO3C012967; } \\
\text { MELO3C008974 }\end{array}$ & $\begin{array}{l}\text { MELO3C008660; } \\
\text { MELO3C015999; } \\
\text { MELO3C011276; } \\
\text { MELO3C010222; } \\
\text { MELO3C006088; } \\
\text { MELO3C014262; } \\
\text { MELO3C024506; } \\
\text { MELO3C006245; } \\
\text { MELO3CO03258; } \\
\text { MELO3CO05952; } \\
\text { MELO3C022601; } \\
\text { MELO3CO26403; } \\
\text { MELO3CO20141; } \\
\text { MELO3CO22441; } \\
\text { MELO3C020023; } \\
\text { MELO3C023012; } \\
\text { MELO3C010867; } \\
\text { MELO3C016201; } \\
\text { MELO3C025030; } \\
\text { MELO3C018985; } \\
\text { MELO3C011956; } \\
\text { MELO3C016963; } \\
\text { MELO3C014306; } \\
\text { MELO3C017659; } \\
\text { MELO3C010630; } \\
\text { MELO3CO07740; } \\
\text { MELO3C013981; }\end{array}$ \\
\hline
\end{tabular}




\begin{tabular}{|c|c|c|c|c|c|c|c|c|c|c|c|}
\hline [OVER] & GO:0006415 & \begin{tabular}{|l|l} 
translational \\
termination
\end{tabular} & \begin{tabular}{|l} 
BIOLOGICAL \\
PROCESS
\end{tabular} & \begin{tabular}{|l|}
0.004535439 \\
996171705
\end{tabular} & \begin{tabular}{|l|}
8.640762058 \\
$752189 \mathrm{E}-6$
\end{tabular} & 8 & 173 & 89 & 16858 & $\begin{array}{l}\text { MELO3C020906; } \\
\text { MELO3C011873; } \\
\text { MELO3C006507; } \\
\text { MELO3C017939; } \\
\text { MELO3C026780; } \\
\text { MELO3C017169; } \\
\text { MELO3C012967; } \\
\text { MELO3C008974 }\end{array}$ & $\begin{array}{l}\text { MELO3C015999; } \\
\text { MELO3C011276; } \\
\text { MELO3C010222; } \\
\text { MELO3CO06088; } \\
\text { MELO3C014262; } \\
\text { MELO3CO24506; } \\
\text { MELO3CO06245; } \\
\text { MELO3CO03258; } \\
\text { MELO3CO05952; } \\
\text { MELO3CO22601; } \\
\text { MELO3CO26403; } \\
\text { MELO3CO20141; } \\
\text { MELO3CO22441; } \\
\text { MELO3CO23012; } \\
\text { MELO3C010867; } \\
\text { MELO3C016201; } \\
\text { MELO3C016049; } \\
\text { MELO3C025030; } \\
\text { MELO3C011956; } \\
\text { MELO3C016963; } \\
\text { MELO3C017659; } \\
\text { MELO3C013984; } \\
\text { MELO3C010630; } \\
\text { MELO3C007740; } \\
\text { MELO3C013981; } \\
\text { MELO3CO02294; } \\
\text { MELO3C012131; }\end{array}$ \\
\hline$[$ OVER] & GO:0022625 & $\begin{array}{l}\text { lytosolic } \\
\text { large } \\
\text { ribosomal } \\
\text { subunit }\end{array}$ & $\begin{array}{l}\text { CELLULAR_C } \\
\text { OMPONENT }\end{array}$ & \begin{tabular}{|l|}
0.010233764 \\
854266155
\end{tabular} & $\begin{array}{l}2.382968107 \\
4709507 E-5\end{array}$ & 7 & 144 & 90 & $\mid 16887$ & $\begin{array}{l}\text { MELO3C020906; } \\
\text { MELO3C011873; } \\
\text { MELO3C017939; } \\
\text { MELO3C015177; } \\
\text { MELO3C017169; } \\
\text { MELO3C012967; } \\
\text { MELO3C008974 }\end{array}$ & 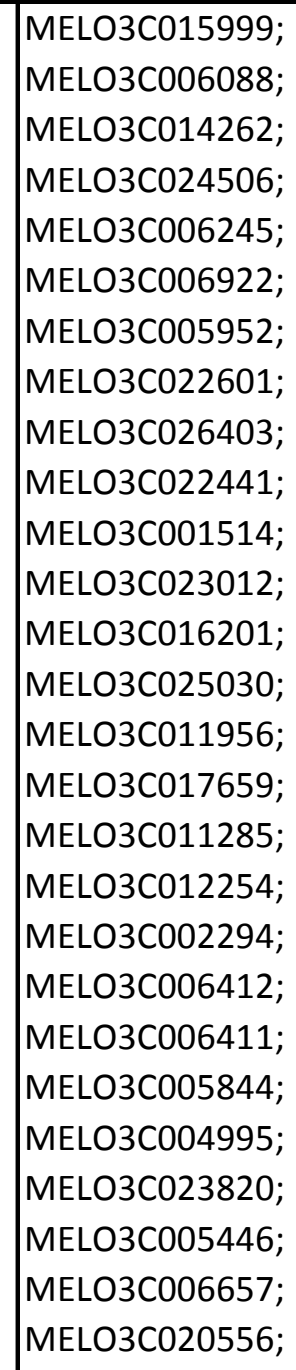 \\
\hline $\mid$ [OVER] & GO:0022627 & $\begin{array}{l}\text { cytosolic } \\
\text { small } \\
\text { ribosomal } \\
\text { subunit }\end{array}$ & $\begin{array}{l}\text { CELLULAR_C } \\
\text { OMPONENT }\end{array}$ & \begin{tabular}{|l|}
0.011249454 \\
162474009
\end{tabular} & \begin{tabular}{|l|}
2.738541974 \\
$3533255 E-5$
\end{tabular} & 6 & 98 & 91 & 16933 & $\begin{array}{l}\text { MELO3C019524; } \\
\text { MELO3C026780; } \\
\text { MELO3C021332; } \\
\text { MELO3C009315; } \\
\text { MELO3C006507; } \\
\text { MELO3C018459 }\end{array}$ & 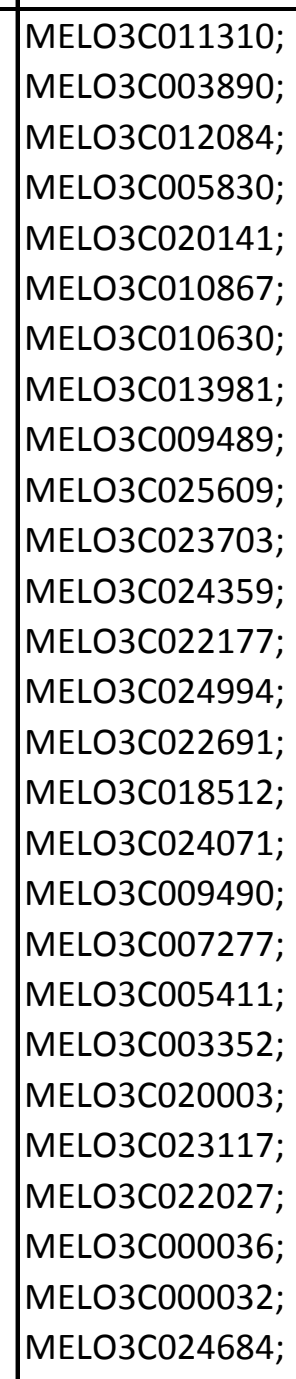 \\
\hline
\end{tabular}




\begin{tabular}{|c|c|c|c|c|c|c|c|c|c|c|c|}
\hline [] & GO:0009733 & $\begin{array}{l}\text { response to } \\
\text { auxin }\end{array}$ & \begin{tabular}{|l} 
BIOLOGICAL \\
PROCESS
\end{tabular} & \begin{tabular}{|l|}
0.145554739 \\
62843424
\end{tabular} & \begin{tabular}{|l|}
5.392057458 \\
$71634 \mathrm{E}-4$
\end{tabular} & 6 & \begin{tabular}{|l|}
173 \\
\end{tabular} & 91 & 16858 & $\begin{array}{l}\text { MELO3C008032; } \\
\text { MELO3C013566; } \\
\text { MELO3C020756; } \\
\text { MELO3C027346; } \\
\text { MELO3C019649; } \\
\text { MELO3C022932 }\end{array}$ & 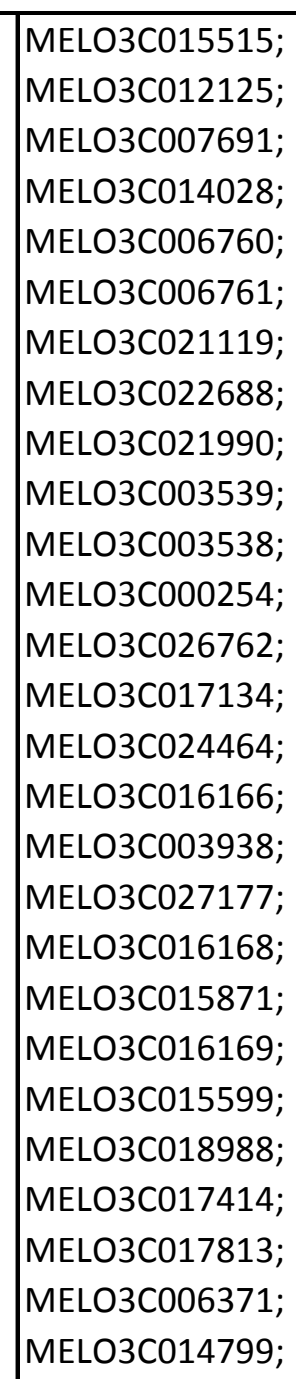 \\
\hline [] & GO:0016071 & \begin{tabular}{|l} 
mRNA \\
metabolic \\
process
\end{tabular} & \begin{tabular}{|l|} 
BIOLOGICAL \\
PROCESS
\end{tabular} & $\begin{array}{l}0.258287212 \\
351632\end{array}$ & \begin{tabular}{|l|}
0.001068411 \\
4237850173
\end{tabular} & \begin{tabular}{l|l}
9 \\
\end{tabular} & 444 & 88 & $\mid 16587$ & $\begin{array}{l}\text { MELO3C020906; } \\
\text { MELO3C011873; } \\
\text { MELO3C006507; } \\
\text { MELO3C01793; } \\
\text { MELO3C026780; } \\
\text { MELO3C012967; } \\
\text { MELO3C008974; } \\
\text { MELO3C016952; } \\
\text { MELO3C007190 }\end{array}$ & $\begin{array}{l}\text { MELO3C020705; } \\
\text { MELO3C004580; } \\
\text { MELO3C014262; } \\
\text { MELO3C013051; } \\
\text { MELO3C002045; } \\
\text { MELO3C020824; } \\
\text { MELO3CO03258; } \\
\text { MELO3CO07978; } \\
\text { MELO3CO06767; } \\
\text { MELO3CO26246; } \\
\text { MELO3C017771; } \\
\text { MELO3CO16201; } \\
\text { MELO3C015113; } \\
\text { MELO3C025030; } \\
\text { MELO3C017775; } \\
\text { MELO3C019837; } \\
\text { MELO3C017659; } \\
\text { MELO3C020836; } \\
\text { MELO3C007740; } \\
\text { MELO3C002294; } \\
\text { MELO3C002173; } \\
\text { MELO3C020718; } \\
\text { MELO3CO06412; } \\
\text { MELO3CO06411; } \\
\text { MELO3CO06416; } \\
\text { MELO3CO05446; } \\
\text { MELO3C008956; }\end{array}$ \\
\hline [] & GO:0008759 & \begin{tabular}{|l|} 
UDP-3-O-[3- \\
hydroxymyris \\
toyl] N- \\
acetylglucosa \\
mine \\
deacetylase \\
activity
\end{tabular} & $\begin{array}{l}\text { MOLECULAR } \\
\text { _FUNCTION }\end{array}$ & $\begin{array}{l}.258287212 \\
351632\end{array}$ & \begin{tabular}{|l|}
0.001113546 \\
9572003578 \\
\end{tabular} & 2 & 7 & 95 & $\mid 17024$ & $\begin{array}{l}\text { MELO3C019524; } \\
\text { MELO3C018459 }\end{array}$ & $\begin{array}{l}\text { MELO3C012357; } \\
\text { MELO3CO06726; } \\
\text { MELO3C003890; } \\
\text { MELO3C005830; } \\
\text { MELO3C019490; } \\
\text { MELO3C016655; } \\
\text { MELO3C016656 }\end{array}$ \\
\hline
\end{tabular}




\begin{tabular}{|c|c|c|c|c|c|c|c|c|c|c|c|}
\hline [] & GO:0042254 & $\begin{array}{l}\text { ribosome } \\
\text { biogenesis }\end{array}$ & \begin{tabular}{|l|} 
BIOLOGICAL_ \\
PROCESS
\end{tabular} & $\begin{array}{l}0.360951956 \\
0299322\end{array}$ & \begin{tabular}{l|}
0.001642774 \\
567028692
\end{tabular} & 6 & 216 & 91 & 16815 & $\begin{array}{l}\text { MELO3C017169; } \\
\text { MELO3CO05360; } \\
\text { MELO3C021332; } \\
\text { MELO3C012967; } \\
\text { MELO3C008974; } \\
\text { MELO3C017939 }\end{array}$ & 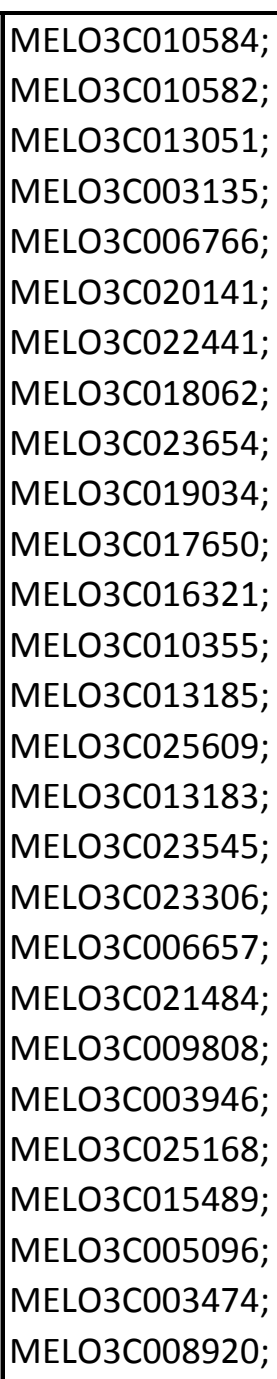 \\
\hline [] & GO:0009245 & \begin{tabular}{|l} 
lipid A \\
biosynthetic \\
process
\end{tabular} & \begin{tabular}{|l|} 
BIOLOGICAL_ \\
PROCESS
\end{tabular} & \begin{tabular}{|l|}
0.502075949 \\
4333862
\end{tabular} & \begin{tabular}{|l|}
0.002763330 \\
7970508134
\end{tabular} & 2 & 12 & 95 & \begin{tabular}{|l|}
17019 \\
\end{tabular} & $\begin{array}{l}\text { MELO3C019524; } \\
\text { MELO3C018459 }\end{array}$ & $\begin{array}{l}\text { MELO3C019719; } \\
\text { MELO3C012357; } \\
\text { MELO3CO06726; } \\
\text { MELO3C025412; } \\
\text { MELO3C017583; } \\
\text { MELO3C013155; } \\
\text { MELO3C00389;; } \\
\text { MELO3C007369; } \\
\text { MELO3C005830; } \\
\text { MELO3C019490; } \\
\text { MELO3C016655; } \\
\text { MELO3C016656 }\end{array}$ \\
\hline [] & GO:0004089 & $\begin{array}{l}\text { carbonate } \\
\text { dehydratase } \\
\text { activity }\end{array}$ & $\begin{array}{l}\text { MOLECULAR } \\
\text { FUNCTION }\end{array}$ & $\begin{array}{l}0.555809609 \\
9709452\end{array}$ & \begin{tabular}{|l|}
0.003176727 \\
237344522
\end{tabular} & 2 & 13 & 95 & 17018 & $\begin{array}{l}\text { MELO3C016817; } \\
\text { MELO3C024476 }\end{array}$ & 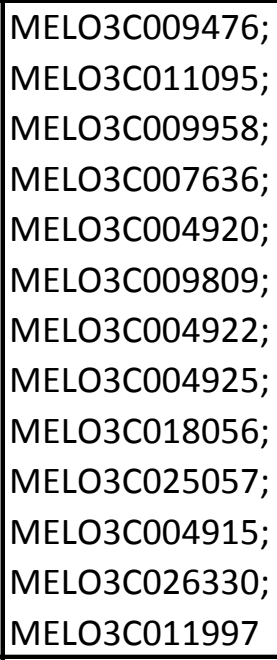 \\
\hline [] & GO:0004655 & \begin{tabular}{|l} 
porphobilino \\
gen synthase \\
activity
\end{tabular} & $\begin{array}{l}\text { MOLECULAR } \\
\text {-FUNCTION }\end{array}$ & \begin{tabular}{|l|}
0.849306435 \\
9928953
\end{tabular} & \begin{tabular}{|l|}
0.005663241 \\
476243904
\end{tabular} & 1 & 0 & 96 & 17031 & MELO3C008086 & \\
\hline [] & GO:0032791 & \begin{tabular}{|l|} 
lead ion \\
binding
\end{tabular} & $\begin{array}{l}\text { MOLECULAR } \\
\text { FUNCTION }\end{array}$ & \begin{tabular}{|l|}
0.849306435 \\
9928953
\end{tabular} & \begin{tabular}{|l|}
0.005663241 \\
476243904
\end{tabular} & 1 & 0 & 96 & 17031 & MELO3C008086 & \\
\hline [] & GO:0050178 & $\begin{array}{l}\text { phenylpyruv } \\
\text { ate } \\
\text { tautomerase } \\
\text { activity }\end{array}$ & $\begin{array}{l}\text { MOLECULAR } \\
\text { FUNCTION }\end{array}$ & \begin{tabular}{|l|}
0.849306435 \\
9928953
\end{tabular} & \begin{tabular}{|l|}
0.005663241 \\
476243904
\end{tabular} & 1 & 0 & 96 & 17031 & MELO3C026027 & \\
\hline [] & GO:0006954 & $\begin{array}{l}\text { inflammator } \\
\text { y response }\end{array}$ & $\begin{array}{l}\text { BIOLOGICAL } \\
\text { PROCESS }\end{array}$ & 1.0 & \begin{tabular}{|l|}
0.033506803 \\
36738886
\end{tabular} & 1 & 5 & 96 & $\begin{array}{l}17026 \\
\end{array}$ & MELO3C026027 & $\begin{array}{l}\text { MELO3C026028; } \\
\text { MELO3C015103; } \\
\text { MELO3C017426; } \\
\text { MELO3C018756; } \\
\text { MELO3C022821 }\end{array}$ \\
\hline [] & \begin{tabular}{|l|l}
$G O: 0046740$ \\
\end{tabular} & $\begin{array}{l}\text { transport of } \\
\text { virus in host, } \\
\text { cell to cell }\end{array}$ & \begin{tabular}{|l|} 
BIOLOGICAL \\
PROCESS
\end{tabular} & 1.0 & \begin{tabular}{|l|}
0.011294739 \\
437078653
\end{tabular} & 1 & 1 & 96 & 17030 & MELO3C016952 & MELO3C010207 \\
\hline [] & GO:0048767 & \begin{tabular}{|l|} 
root hair \\
elongation
\end{tabular} & \begin{tabular}{|l|} 
BIOLOGICAL_ \\
PROCESS
\end{tabular} & 1.0 & $\begin{array}{l}0.049841076 \\
76659685\end{array}$ & 1 & 8 & 96 & 17023 & MELO3C008032 & $\begin{array}{l}\text { MELO3CO06684; } \\
\text { MELO3C023264; } \\
\text { MELO3C002781; } \\
\text { MELO3C026629; } \\
\text { MELO3C021704; } \\
\text { MELO3C015501; } \\
\text { MELO3C019823; } \\
\text { MELO3C018182 }\end{array}$ \\
\hline [] & GO:0010120 & $\begin{array}{l}\text { camalexin } \\
\text { biosynthetic } \\
\text { process }\end{array}$ & \begin{tabular}{|l} 
BIOLOGICAL_ \\
PROCESS
\end{tabular} & 1.0 & \begin{tabular}{|l|}
0.011294739 \\
437078653
\end{tabular} & 1 & 1 & 96 & 17030 & MELO3C009127 & MELO3C020718 \\
\hline
\end{tabular}




\begin{tabular}{|c|c|c|c|c|c|c|c|c|c|c|c|}
\hline [] & GO:0060548 & $\begin{array}{l}\text { negative } \\
\text { regulation of } \\
\text { cell death }\end{array}$ & $\begin{array}{l}\text { BIOLOGICAL_ } \\
\text { PROCESS }\end{array}$ & 1.0 & $\begin{array}{l}0.021532204 \\
623148688\end{array}$ & 2 & 38 & 95 & 16993 & $\begin{array}{l}\text { MELO3C003911; } \\
\text { MELO3C017623 }\end{array}$ & $\begin{array}{l}\text { MELO3C002481; } \\
\text { MELO3C011470; } \\
\text { MELO3C006121; } \\
\text { MELO3C003331; } \\
\text { MELO3C007237; } \\
\text { MELO3C003332; } \\
\text { MELO3C005976; } \\
\text { MELO3C004622; } \\
\text { MELO3C025417; } \\
\text { MELO3C022346; } \\
\text { MELO3C005757; } \\
\text { MELO3C025413; } \\
\text { MELO3C016083; } \\
\text { MELO3C005912; } \\
\text { MELO3C025752; } \\
\text { MELO3C021057; } \\
\text { MELO3C023972; } \\
\text { MELO3C009939; } \\
\text { MELO3C004827; } \\
\text { MELO3C024340; } \\
\text { MELO3C025355; } \\
\text { MELO3C005718; } \\
\text { MELO3C011710; } \\
\text { MELO3C013522; } \\
\text { MELO3C011983; } \\
\text { MELO3C012376; } \\
\text { MELO3C011021; }\end{array}$ \\
\hline [] & GO:0009641 & $\begin{array}{l}\text { shade } \\
\text { avoidance }\end{array}$ & \begin{tabular}{|l} 
BIOLOGICAL \\
PROCESS
\end{tabular} & 1.0 & \begin{tabular}{|l|}
0.028000528 \\
254957892
\end{tabular} & 1 & 4 & 96 & 17027 & MELO3C025152 & $\begin{array}{l}\text { MELO3C015549; } \\
\text { MELO3C019731; } \\
\text { MELO3C016115; } \\
\text { MELO3C013923 }\end{array}$ \\
\hline [] & GO:0042742 & \begin{tabular}{|l} 
defense \\
response to \\
bacterium
\end{tabular} & \begin{tabular}{|l} 
BIOLOGICAL \\
PROCESS
\end{tabular} & 1.0 & \begin{tabular}{|l|}
0.010560261 \\
351637559
\end{tabular} & 3 & 77 & 94 & 16954 & $\begin{array}{l}\text { MELO3C025152; } \\
\text { MELO3C006035; } \\
\text { MELO3C009127 }\end{array}$ & $\begin{array}{l}\text { MELO3C021918; } \\
\text { MELO3C006046; } \\
\text { MELO3C014386; } \\
\text { MELO3C004385; } \\
\text { MELO3C002120; } \\
\text { MELO3C004387; } \\
\text { MELO3C004386; } \\
\text { MELO3C005674; } \\
\text { MELO3C009633; } \\
\text { MELO3C009952; } \\
\text { MELO3C003137; } \\
\text { MELO3C023217; } \\
\text { MELO3C026722; } \\
\text { MELO3C003536; } \\
\text { MELO3C004867; } \\
\text { MELO3C023453; } \\
\text { MELO3C021553; } \\
\text { MELO3C025076; } \\
\text { MELO3C014706; } \\
\text { MELO3C011997; } \\
\text { MELO3C016008; } \\
\text { MELO3C013811; } \\
\text { MELO3C013621; } \\
\text { MELO3C016939; } \\
\text { MELO3C014230; } \\
\text { MELO3C020235; } \\
\text { MELO3C014391; }\end{array}$ \\
\hline[] & GO:0031146 & \begin{tabular}{|l|} 
SCF- \\
dependent \\
proteasomal \\
ubiquitin- \\
dependent \\
protein \\
catabolic \\
process
\end{tabular} & $\begin{array}{l}\text { BIOLOGICAL } \\
\text { PROCESS }\end{array}$ & 1.0 & \begin{tabular}{|l|}
0.028000528 \\
254957892
\end{tabular} & 1 & 4 & 96 & 17027 & MELO3C025152 & $\begin{array}{l}\text { MELO3C015764; } \\
\text { MELO3C016791; } \\
\text { MELO3C002401; } \\
\text { MELO3C010832 }\end{array}$ \\
\hline [] & GO:0043966 & $\begin{array}{l}\text { histone } \mathrm{H} 3 \\
\text { acetylation }\end{array}$ & \begin{tabular}{|l|} 
BIOLOGICAL_ \\
PROCESS
\end{tabular} & 1.0 & \begin{tabular}{|l|}
0.011294739 \\
437078653 \\
\end{tabular} & 1 & 1 & 96 & 17030 & MELO3C014503 & MELO3C015732 \\
\hline [] & GO:0009625 & \begin{tabular}{|l} 
response to \\
insect
\end{tabular} & \begin{tabular}{|l|} 
BIOLOGICAL_ \\
PROCESS
\end{tabular} & 1.0 & \begin{tabular}{|l|}
0.011294739 \\
437078653 \\
\end{tabular} & 1 & 1 & 96 & 17030 & MELO3C025152 & MELO3C003906 \\
\hline [] & GO:0070370 & $\begin{array}{l}\text { cellular heat } \\
\text { acclimation }\end{array}$ & $\begin{array}{l}\text { BIOLOGICAL } \\
\text { PROCESS }\end{array}$ & 1.0 & \begin{tabular}{|l|}
0.016894669 \\
96843291
\end{tabular} & 1 & 2 & 96 & 17029 & MELO3C009127 & $\begin{array}{l}\text { MELO3C025686; } \\
\text { MELO3C016019 }\end{array}$ \\
\hline
\end{tabular}




\begin{tabular}{|c|c|c|c|c|c|c|c|c|c|c|c|}
\hline[] & GO:0010200 & $\begin{array}{l}\text { response to } \\
\text { chitin }\end{array}$ & \begin{tabular}{|l} 
BIOLOGICAL \\
PROCESS
\end{tabular} & 1.0 & \begin{tabular}{|l|}
0.032608717 \\
776354594
\end{tabular} & 2 & 48 & 95 & 16983 & $\begin{array}{l}\text { MELO3C006891; } \\
\text { MELO3C009127 }\end{array}$ & $\begin{array}{l}\text { MELO3C013454; } \\
\text { MELO3C007030; } \\
\text { MELO3CO04286; } \\
\text { MELO3CO09376; } \\
\text { MELO3C021416; } \\
\text { MELO3CO06300; } \\
\text { MELO3CO24989; } \\
\text { MELO3CO02644; } \\
\text { MELO3CO05639; } \\
\text { MELO3CO19231; } \\
\text { MELO3CO04627; } \\
\text { MELO3CO26740; } \\
\text { MELO3CO16285; } \\
\text { MELO3C010748; } \\
\text { MELO3C013679; } \\
\text { MELO3CO04093; } \\
\text { MELO3CO06431; } \\
\text { MELO3C013287; } \\
\text { MELO3CO08014; } \\
\text { MELO3C016819; } \\
\text { MELO3C020718; } \\
\text { MELO3C005466; } \\
\text { MELO3C022715; } \\
\text { MELO3C005465; } \\
\text { MELO3C007444; } \\
\text { MELO3C025904; } \\
\text { MELO3C020971; }\end{array}$ \\
\hline [] & GO:0004781 & \begin{tabular}{|l} 
sulfate \\
adenylyltrans \\
ferase (ATP) \\
activity
\end{tabular} & $\begin{array}{l}\text { MOLECULAR } \\
\text { FUNCTION }\end{array}$ & 1.0 & \begin{tabular}{|l|}
0.049841076 \\
76659685 \\
\\
\\
\\
\end{tabular} & 1 & 8 & \begin{tabular}{|c|}
96 \\
\end{tabular} & 17023 & MELO3C025511 & $\begin{array}{l}\text { MELO3C006493; } \\
\text { MELO3C002118; } \\
\text { MELO3C025510; } \\
\text { MELO3C002878; } \\
\text { MELO3C026259; } \\
\text { MELO3C003637; } \\
\text { MELO3C003762; } \\
\text { MELO3C014280 }\end{array}$ \\
\hline [] & GO:0004491 & \begin{tabular}{|l} 
methylmalon \\
ate- \\
semialdehyd \\
e \\
dehydrogena \\
se (acylating) \\
activity
\end{tabular} & $\begin{array}{l}\text { MOLECULAR } \\
\text { FUNCTION }\end{array}$ & 1.0 & \begin{tabular}{|l|}
0.016894669 \\
96843291
\end{tabular} & 1 & 2 & 96 & 17029 & MELO3C014951 & $\begin{array}{l}\text { MELO3C018583; } \\
\text { MELO3C007705 }\end{array}$ \\
\hline [] & GO:0004591 & $\begin{array}{l}\text { oxoglutarate } \\
\text { dehydrogena } \\
\text { se (succinyl- } \\
\text { transferring) } \\
\text { activity }\end{array}$ & $\begin{array}{l}\text { MOLECULAR } \\
\text { FUNCTION }\end{array}$ & 1.0 & \begin{tabular}{|l|}
0.011294739 \\
437078653 \\
\end{tabular} & 1 & 1 & 96 & 17030 & MELO3C023844 & MELO3C025454 \\
\hline [] & GO:0030337 & \begin{tabular}{|l|} 
DNA \\
polymerase \\
processivity \\
factor \\
activity \\
\end{tabular} & $\begin{array}{l}\text { MOLECULAR } \\
\text {-FUNCTION }\end{array}$ & 1.0 & \begin{tabular}{|l|}
0.011294739 \\
437078653
\end{tabular} & 1 & 1 & 96 & 17030 & MELO3C025142 & \begin{tabular}{|l|} 
MELO3C025879 \\
\end{tabular} \\
\hline [] & GO:0043565 & $\begin{array}{l}\text { sequence- } \\
\text { specific DNA } \\
\text { binding }\end{array}$ & $\begin{array}{l}\text { MOLECULAR } \\
\text { FUNCTION }\end{array}$ & 1.0 & \begin{tabular}{|l|}
0.039077991 \\
14803682
\end{tabular} & 5 & 324 & 92 & \begin{tabular}{|c|}
16707 \\
\end{tabular} & $\begin{array}{l}\text { MELO3C009127; } \\
\text { MELO3C02335; } \\
\text { MELO3C020489; } \\
\text { MELO3C006891; } \\
\text { MELO3C014503 }\end{array}$ & $\begin{array}{l}\text { MELO3C011432; } \\
\text { MELO3C010223; } \\
\text { MELO3C011550; } \\
\text { MELO3CO05791; } \\
\text { MELO3C014384; } \\
\text { MELO3C024507; } \\
\text { MELO3CO08942; } \\
\text { MELO3C013173; } \\
\text { MELO3C022209; } \\
\text { MELO3CO01991; } \\
\text { MELO3CO25714; } \\
\text { MELO3CO07736; } \\
\text { MELO3COO6887; } \\
\text { MELO3CO22205; } \\
\text { MELO3CO02723; } \\
\text { MELO3CO25951; } \\
\text { MELO3CO20145; } \\
\text { MELO3C018187; } \\
\text { MELO3C017415; } \\
\text { MELO3C019715; } \\
\text { MELO3CO07181; } \\
\text { MELO3C023092; } \\
\text { MELO3C011797; } \\
\text { MELO3C015239; } \\
\text { MELO3C017429; } \\
\text { MELO3CO03140; } \\
\text { MELO3C002050; }\end{array}$ \\
\hline
\end{tabular}




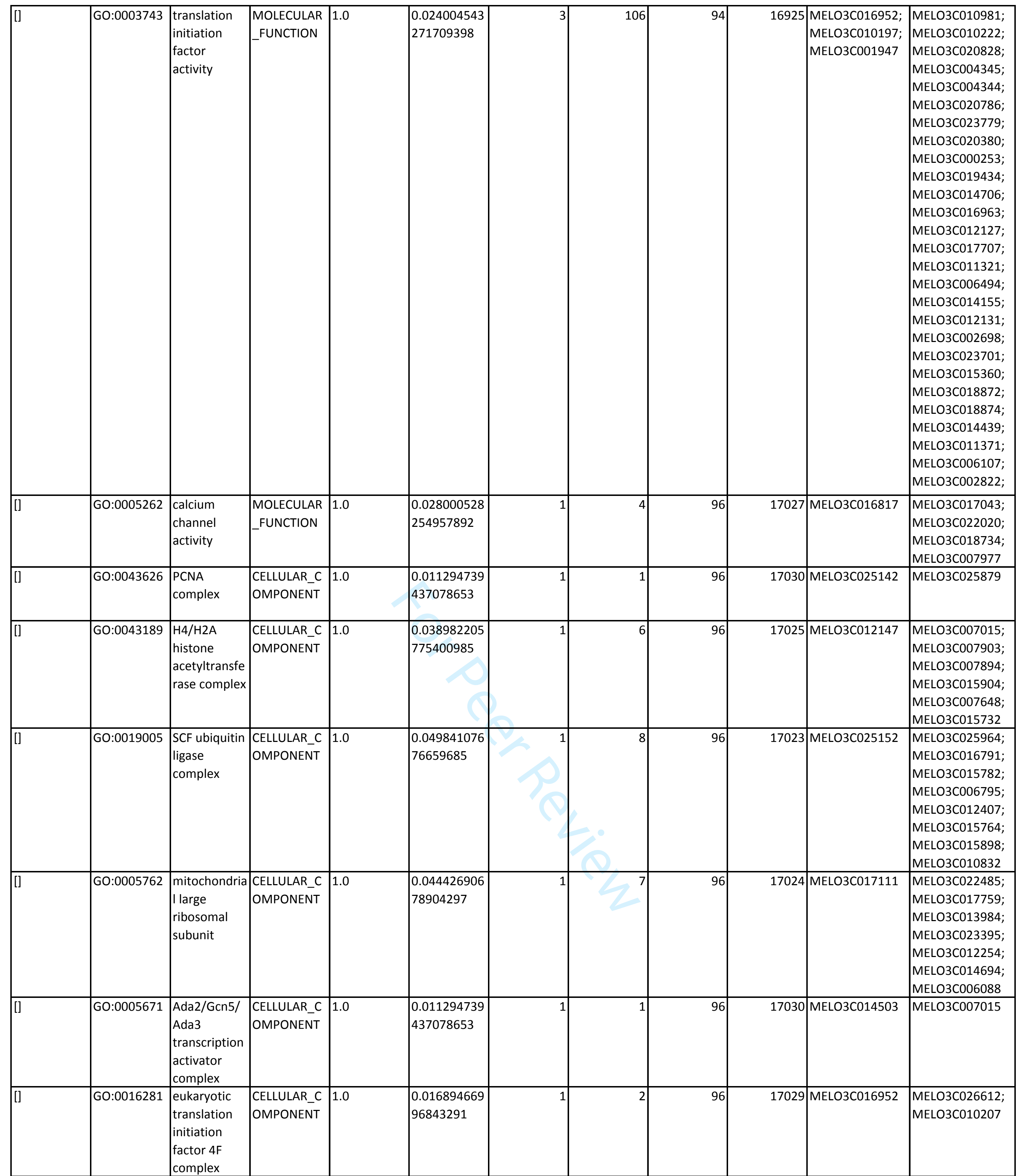


Supplementary Table 1.C Gene Ontology terms (GO terms) of the cluster 1.2 obtained considering the comparison between lines over time fruit during storage at $20.5^{\circ} \mathrm{C}$ and $88 \%$ relative humidity for $18 \mathrm{~d}$ the near-isogenic line SC10-2 and its parental "Piel de Sapo" obtained according to the differentially expressed gene pattern (Supl. Table 1.A)

\begin{tabular}{|c|c|c|c|c|c|c|c|c|c|c|c|}
\hline Tags & GO ID & GO Name & GO Category & FDR & P-Value & Nr Test & Nr Reference & \begin{tabular}{|l} 
Non Annot \\
Test
\end{tabular} & \begin{tabular}{|l} 
Non Annot \\
Reference
\end{tabular} & \begin{tabular}{|l}
$\begin{array}{l}\text { TestSet } \\
\text { Sequences }\end{array}$ \\
\end{tabular} & $\begin{array}{l}\begin{array}{l}\text { RefSet } \\
\text { Sequences }\end{array} \\
\end{array}$ \\
\hline [OVER] & GO:0006414 & $\begin{array}{l}\text { translational } \\
\text { elongation }\end{array}$ & \begin{tabular}{|l|} 
BIOLOGICAL_ \\
PROCESS
\end{tabular} & $\begin{array}{l}1.370890716 \\
6724634 \mathrm{E}-15\end{array}$ & \begin{tabular}{|l|}
6.524615516 \\
$124876 \mathrm{E}-19$
\end{tabular} \mid & 46 & 151 & 809 & 16122 & 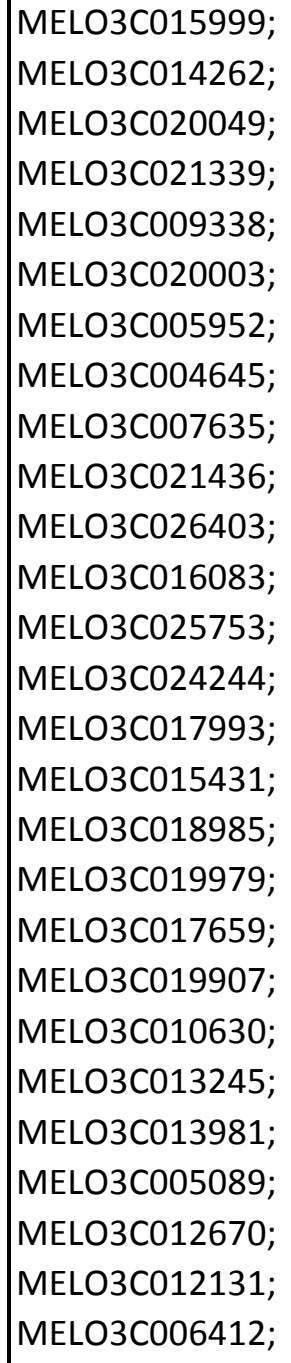 & 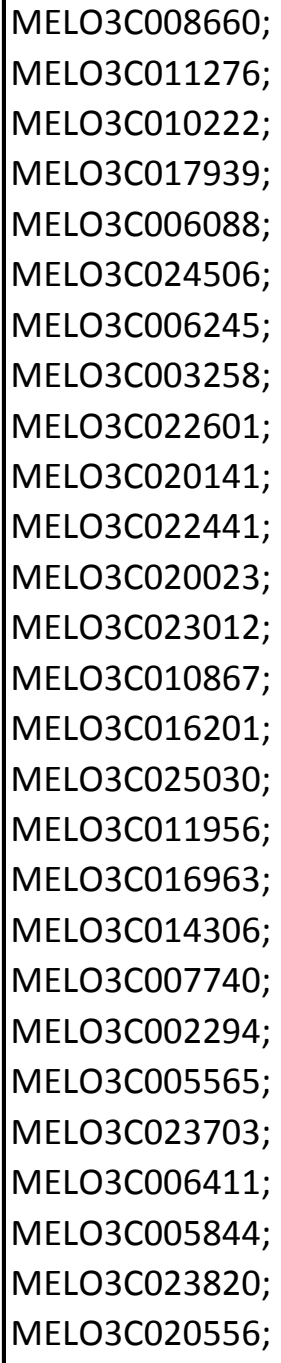 \\
\hline [OVER] & GO:0006415 & \begin{tabular}{|l|} 
translational \\
termination
\end{tabular} & \begin{tabular}{|l|} 
BIOLOGICAL \\
PROCESS
\end{tabular} & $\begin{array}{l}1.370890716 \\
6724634 \mathrm{E}-15\end{array}$ & $\begin{array}{l}7.254925469 \\
265789 \mathrm{E}-19\end{array}$ & 44 & 137 & 811 & 16136 & 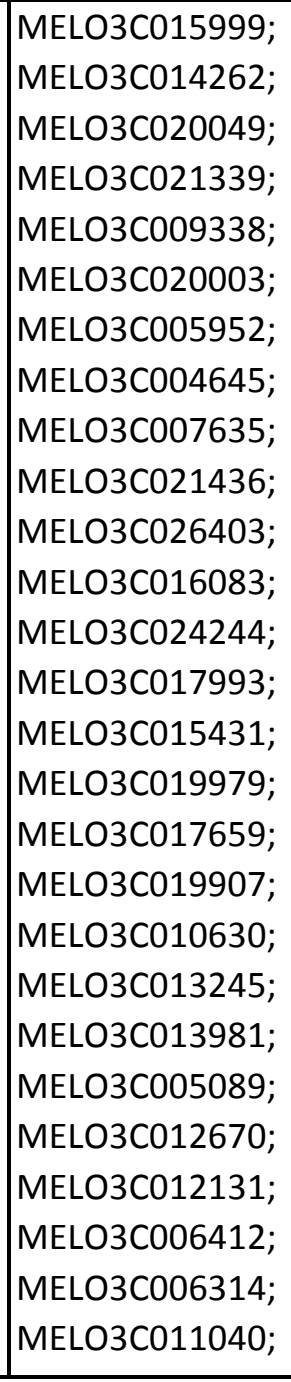 & 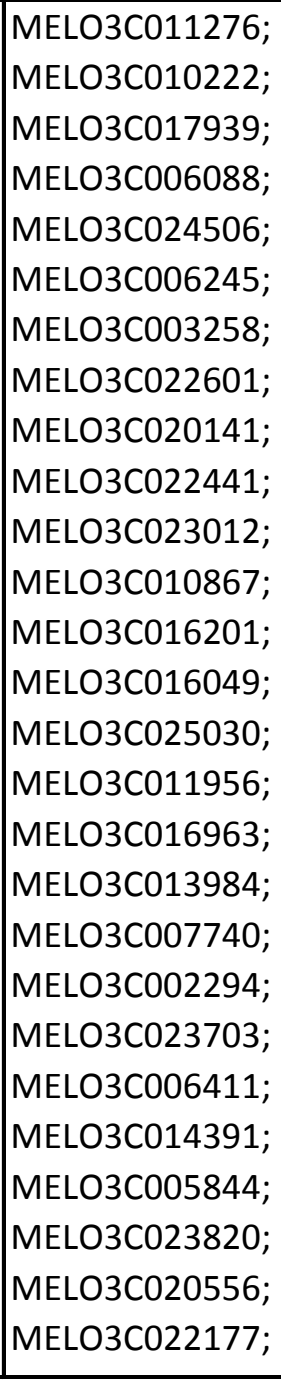 \\
\hline
\end{tabular}




\begin{tabular}{|c|c|c|c|c|c|c|c|c|c|c|c|}
\hline [OVER] & GO:0003735 & $\begin{array}{l}\text { structural } \\
\text { constituent of } \\
\text { ribosome }\end{array}$ & $\begin{array}{l}\text { MOLECULAR } \\
\text { FUNCTION }\end{array}$ & \begin{tabular}{|l|}
6.244379144 \\
$367021 E-13$
\end{tabular} & \begin{tabular}{|l|}
8.464621208 \\
$697664 \mathrm{E}-16$
\end{tabular} & 58 & 296 & 797 & 15977 & $\begin{array}{l}\text { MELO3C015999; } \\
\text { MELO3C012084; } \\
\text { MELO3C014262; } \\
\text { MELO3C005952; } \\
\text { MELO3C022766; } \\
\text { MELO3C021436; } \\
\text { MELO3C026403; } \\
\text { MELO3C004505; } \\
\text { MELO3C002405; } \\
\text { MELO3C005759; } \\
\text { MELO3C015431; } \\
\text { MELO3C018985; } \\
\text { MELO3C017659; } \\
\text { MELO3C010630; } \\
\text { MELO3C013981; } \\
\text { MELO3C009489; } \\
\text { MELO3C011285; } \\
\text { MELO3C005089; } \\
\text { MELO3C006412; } \\
\text { MELO3C011040; } \\
\text { MELO3C005520; } \\
\text { MELO3C009600; } \\
\text { MELO3C019490; } \\
\text { MELO3C005446; } \\
\text { MELO3C022497; } \\
\text { MELO3C004713; } \\
\text { MELO3C011847; }\end{array}$ & $\begin{array}{l}\text { MELO3C011310; } \\
\text { MELO3C020704; } \\
\text { MELO3C006088; } \\
\text { MELO3C002164; } \\
\text { MELO3C024506; } \\
\text { MELO3C020945; } \\
\text { MELO3C006400; } \\
\text { MELO3C003258; } \\
\text { MELO3C006766; } \\
\text { MELO3C024747; } \\
\text { MELO3C020141; } \\
\text { MELO3C022441; } \\
\text { MELO3C003811; } \\
\text { MELO3C001514; } \\
\text { MELO3C025276; } \\
\text { MELO3C016201; } \\
\text { MELO3C025030; } \\
\text { MELO3C016688; } \\
\text { MELO3C014824; } \\
\text { MELO3C020836; } \\
\text { MELO3C007740; } \\
\text { MELO3C002294; } \\
\text { MELO3C025609; } \\
\text { MELO3C006411; } \\
\text { MELO3C007869; } \\
\text { MELO3C023788; } \\
\text { MELO3C025845; }\end{array}$ \\
\hline [OVER] & GO:0022625 & $\begin{array}{l}\text { cytosolic large } \\
\text { ribosomal } \\
\text { subunit }\end{array}$ & $\begin{array}{l}\text { CELLULAR_C } \\
\text { OMPONENT }\end{array}$ & \begin{tabular}{|l|}
.687624034 \\
$4621164 \mathrm{E}-9$
\end{tabular} & $\begin{array}{l}1.324383242 \\
571619 \mathrm{E}-11\end{array}$ & 31 & 120 & 824 & 16153 & $\begin{array}{l}\text { MELO3C015999; } \\
\text { MELO3C014262; } \\
\text { MELO3C021339; } \\
\text { MELO3C009338; } \\
\text { MELO3C005952; } \\
\text { MELO3C007635; } \\
\text { MELO3C020369; } \\
\text { MELO3C021436; } \\
\text { MELO3C026403; } \\
\text { MELO3C022386; } \\
\text { MELO3C016083; } \\
\text { MELO3C024244; } \\
\text { MELO3C017659; } \\
\text { MELO3C011285; } \\
\text { MELO3C006412; } \\
\text { MELO3C006314; } \\
\text { MELO3C005520; } \\
\text { MELO3C009600; } \\
\text { MELO3C021429; } \\
\text { MELO3C005446; } \\
\text { MELO3C023525; } \\
\text { MELO3C023201; } \\
\text { MELO3C024850; } \\
\text { MELO3C004713; } \\
\text { MELO3C004616; } \\
\text { MELO3C016198; } \\
\text { MELO3C002719; }\end{array}$ & $\begin{array}{l}\text { MELO3C017939; } \\
\text { MELO3C006088; } \\
\text { MELO3C024506; } \\
\text { MELO3C006245; } \\
\text { MELO3C006922; } \\
\text { MELO3C022601; } \\
\text { MELO3C022441; } \\
\text { MELO3C001514; } \\
\text { MELO3C023012; } \\
\text { MELO3C016201; } \\
\text { MELO3C025030; } \\
\text { MELO3C011956; } \\
\text { MELO3C012254; } \\
\text { MELO3C002294; } \\
\text { MELO3C006411; } \\
\text { MELO3C005844; } \\
\text { MELO3C004995; } \\
\text { MELO3C023820; } \\
\text { MELO3C006657; } \\
\text { MELO3C020556; } \\
\text { MELO3C025168; } \\
\text { MELO3C000241; } \\
\text { MELO3C023260; } \\
\text { MELO3C011448; } \\
\text { MELO3C016459; } \\
\text { MELO3C011720; } \\
\text { MELO3C002668; }\end{array}$ \\
\hline [OVER] & GO:0006270 & $\begin{array}{l}\text { DNA } \\
\text { replication } \\
\text { initiation }\end{array}$ & $\begin{array}{l}\text { BIOLOGICAL } \\
\text { PROCESS }\end{array}$ & \begin{tabular}{|l|}
1.555434373 \\
$5842954 \mathrm{E}-8$
\end{tabular} & \begin{tabular}{|l|}
4.230010148 \\
$584021 \mathrm{E}-11$ \\
\end{tabular} & 11 & 6 & 844 & 16267 & $\begin{array}{l}\text { MELO3C010882; } \\
\text { MELO3C020860; } \\
\text { MELO3C021058; } \\
\text { MELO3C015926; } \\
\text { MELO3C007144; } \\
\text { MELO3C006995; } \\
\text { MELO3C022319; } \\
\text { MELO3C027184; } \\
\text { MELO3C026749; } \\
\text { MELO3C004448; } \\
\text { MELO3C013436 }\end{array}$ & $\begin{array}{l}\text { MELO3C006096; } \\
\text { MELO3C012616; } \\
\text { MELO3C016227; } \\
\text { MELO3C021204; } \\
\text { MELO3C001937; } \\
\text { MELO3C023404 }\end{array}$ \\
\hline
\end{tabular}




\begin{tabular}{|c|c|c|c|c|c|c|c|c|c|c|c|}
\hline [OVER] & GO:0000084 & $\begin{array}{l}\text { mitotic S } \\
\text { phase }\end{array}$ & 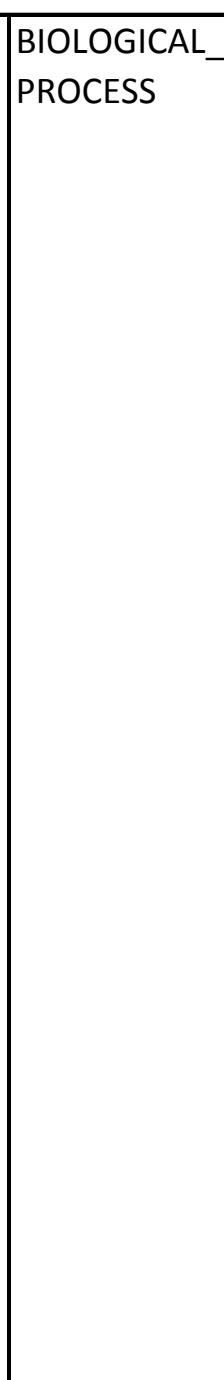 & $\begin{array}{l}1.820760600 \\
4169125 E-7\end{array}$ & $\begin{array}{l}7.323126885 \\
673442 E-10\end{array}$ & 20 & 57 & 835 & 16216 & 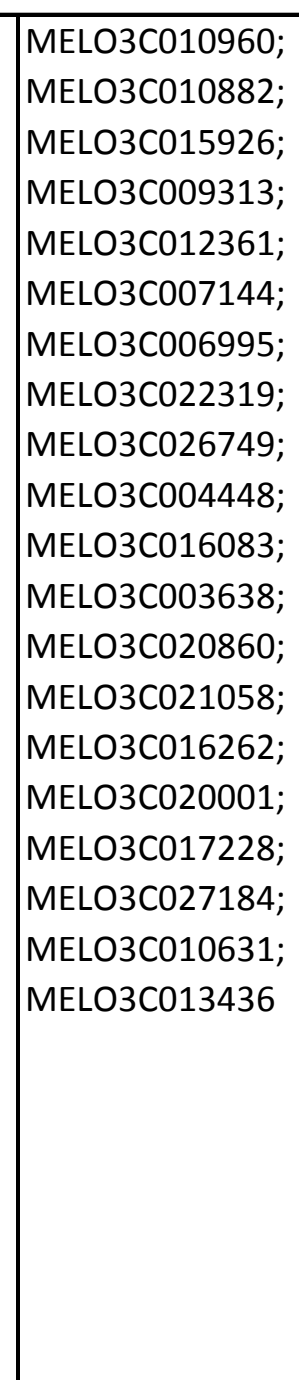 & 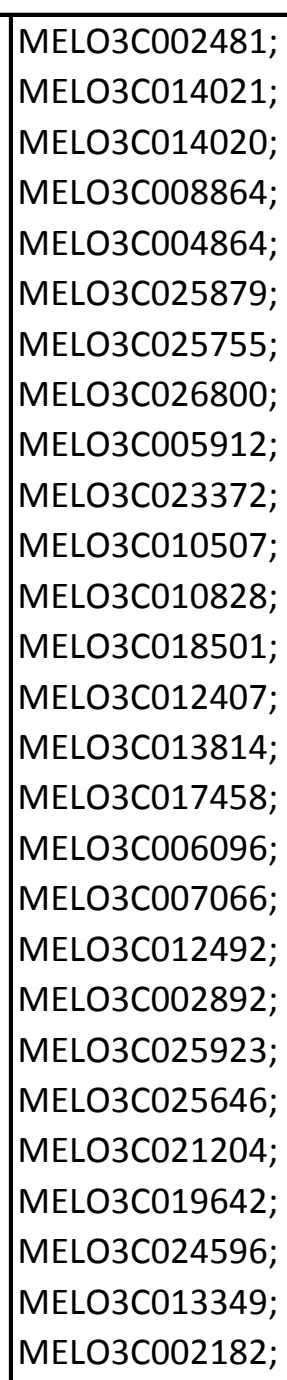 \\
\hline [OVER] & GO:0022627 & $\begin{array}{l}\begin{array}{l}\text { cytososolic small } \\
\text { ribosomal } \\
\text { subunit }\end{array} \\
\end{array}$ & 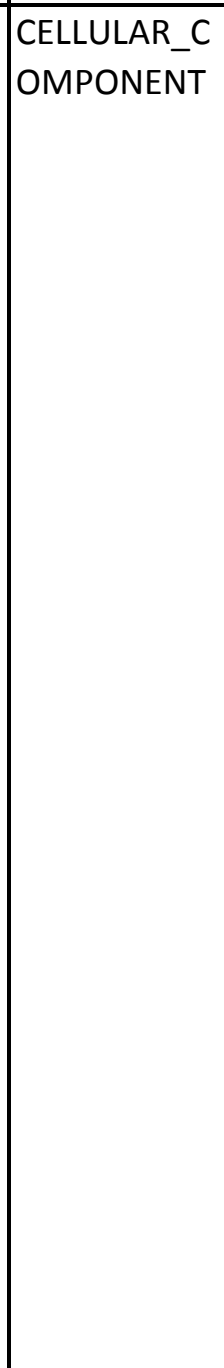 & $\begin{array}{l}1.387321900 \\
8327823 \mathrm{E}-6\end{array}$ & $\begin{array}{l}6.901368473 \\
6601155-9\end{array}$ & 22 & 82 & 833 & 16191 & 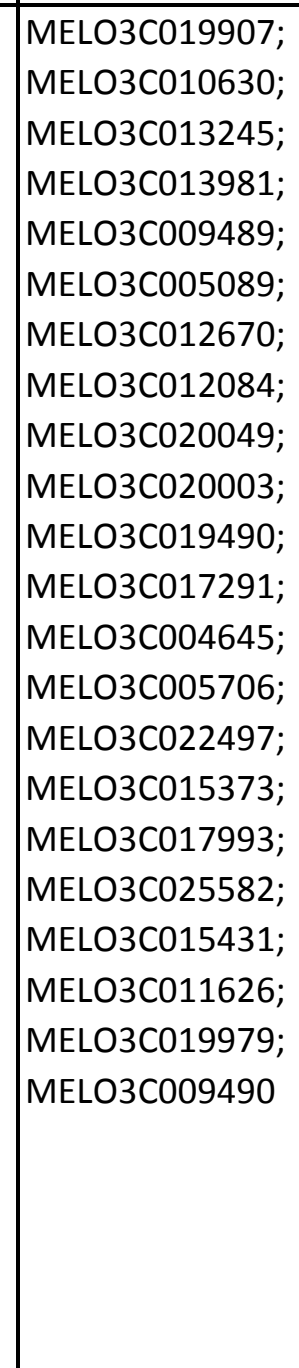 & 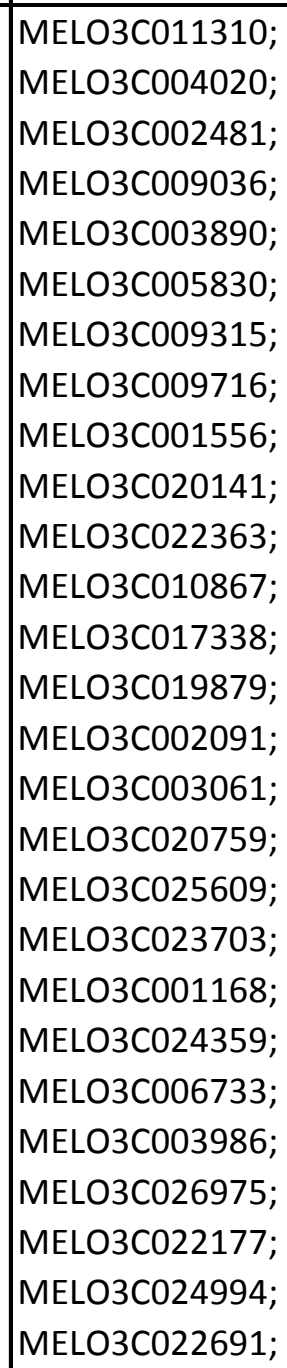 \\
\hline [OVER] & GO:0006096 & 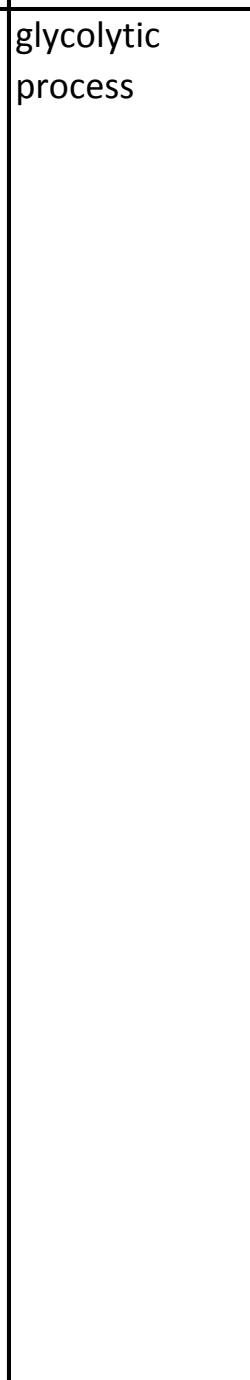 & $\begin{array}{l}\text { BIOLOGICAL } \\
\text { PROCESS }\end{array}$ & $\begin{array}{l}4.0050000977 \\
0361411 E-4\end{array}$ & $\begin{array}{ll}3.391193985 \\
6360213 E-6\end{array}$ & 16 & 68 & 839 & 16205 & 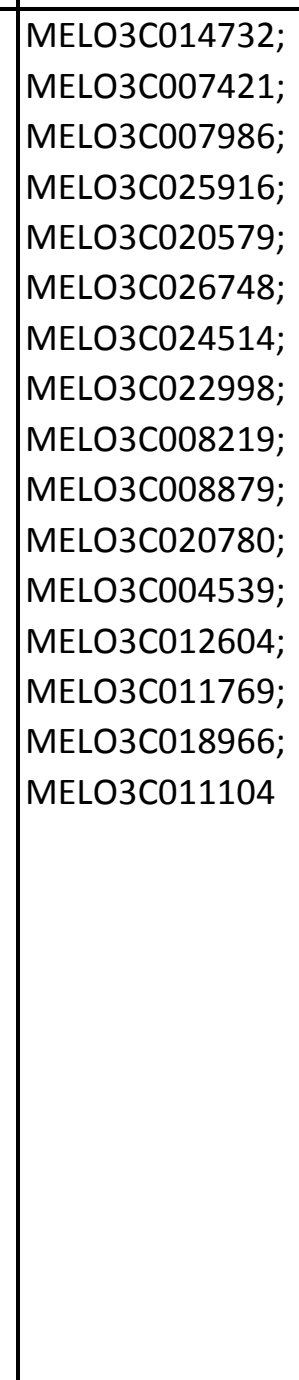 & 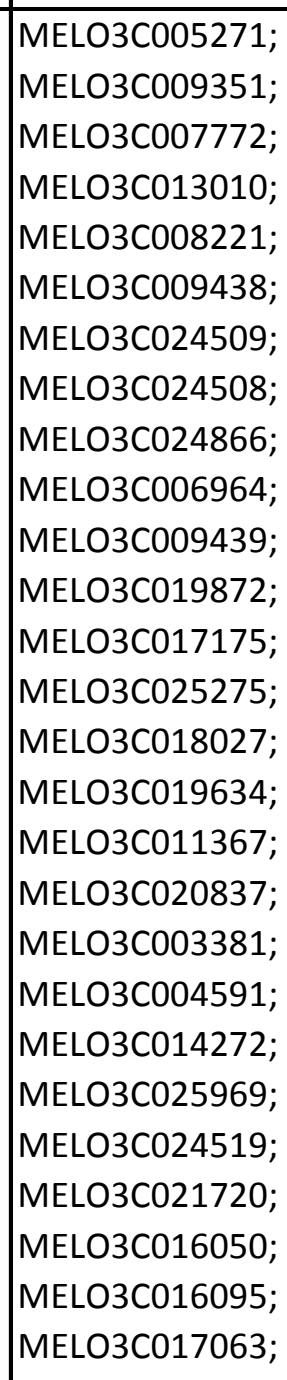 \\
\hline
\end{tabular}




\begin{tabular}{|c|c|c|c|c|c|c|c|c|c|c|c|}
\hline [OVER] & GO:0046686 & $\begin{array}{l}\text { response to } \\
\text { cadmium ion }\end{array}$ & \begin{tabular}{|l|} 
BIOLOGICAL_ \\
PROCESS
\end{tabular} & \begin{tabular}{|l|}
4.817428058 \\
$505066 \mathrm{E}-4$
\end{tabular} & \begin{tabular}{|l|}
4.181087010 \\
$980265 \mathrm{E}-6$
\end{tabular} & 25 & 157 & 830 & 16116 & $\begin{array}{l}\text { MELO3C005293; } \\
\text { MELO3C011256; } \\
\text { MELO3C013214; } \\
\text { MELO3C017817; } \\
\text { MELO3C013411; } \\
\text { MELO3C009038; } \\
\text { MELO3C003173; } \\
\text { MELO3C006241; } \\
\text { MELO3C025916; } \\
\text { MELO3C005757; } \\
\text { MELO3C022382; } \\
\text { MELO3C026224; } \\
\text { MELO3C004194; } \\
\text { MELO3C009203; } \\
\text { MELO3C011284; } \\
\text { MELO3C003344; } \\
\text { MELO3C024514; } \\
\text { MELO3C021247; } \\
\text { MELO3C008879; } \\
\text { MELO3C002117; } \\
\text { MELO3C018573; } \\
\text { MELO3C002816; } \\
\text { MELO3C015385; } \\
\text { MELO3C010599; } \\
\text { MELO3C011107 }\end{array}$ & $\begin{array}{l}\text { MELO3C013974; } \\
\text { MELO3C006086; } \\
\text { MELO3C021919; } \\
\text { MELO3C020429; } \\
\text { MELO3C003491; } \\
\text { MELO3C003370; } \\
\text { MELO3C008541; } \\
\text { MELO3C005395; } \\
\text { MELO3C004980; } \\
\text { MELO3C009910; } \\
\text { MELO3C007698; } \\
\text { MELO3C005674; } \\
\text { MELO3C009633; } \\
\text { MELO3C009915; } \\
\text { MELO3C002444; } \\
\text { MELO3C019040; } \\
\text { MELO3C003536; } \\
\text { MELO3C026800; } \\
\text { MELO3C021113; } \\
\text { MELO3C024461; } \\
\text { MELO3C017134; } \\
\text { MELO3C018623; } \\
\text { MELO3C026360; } \\
\text { MELO3C011712; } \\
\text { MELO3C019318; } \\
\text { MELO3C006493; } \\
\text { MELO3C006095; }\end{array}$ \\
\hline [OVER] & GO:0016071 & $\begin{array}{l}\text { mRNA } \\
\text { metabolic } \\
\text { process }\end{array}$ & \begin{tabular}{|l|} 
BIOLOGICAL_ \\
PROCESS
\end{tabular} & \begin{tabular}{|l|}
5.366657722 \\
$896626 \mathrm{E}-4$
\end{tabular} & \begin{tabular}{|l|}
4.941778385 \\
$817173 \mathrm{E}-6$
\end{tabular} & 46 & 407 & 809 & 15866 & $\begin{array}{l}\text { MELO3C015999; } \\
\text { MELO3C011256; } \\
\text { MELO3C010960; } \\
\text { MELO3C014262; } \\
\text { MELO3C020049; } \\
\text { MELO3C021339; } \\
\text { MELO3C009338; } \\
\text { MELO3C020003; } \\
\text { MELO3C005952; } \\
\text { MELO3C004645; } \\
\text { MELO3C007635; } \\
\text { MELO3C021436; } \\
\text { MELO3C026403; } \\
\text { MELO3C016083; } \\
\text { MELO3C016382; } \\
\text { MELO3C016262; } \\
\text { MELO3C024244; } \\
\text { MELO3C017993; } \\
\text { MELO3C017659; } \\
\text { MELO3C019907; } \\
\text { MELO3C010630; } \\
\text { MELO3C013245; } \\
\text { MELO3C013981; } \\
\text { MELO3C005089; } \\
\text { MELO3C008332; } \\
\text { MELO3C012670; } \\
\text { MELO3C006412; }\end{array}$ & $\begin{array}{l}\text { MELO3C020705; } \\
\text { MELO3C004580; } \\
\text { MELO3C013051; } \\
\text { MELO3C002045; } \\
\text { MELO3C020824; } \\
\text { MELO3C003258; } \\
\text { MELO3C007978; } \\
\text { MELO3C006767; } \\
\text { MELO3C026246; } \\
\text { MELO3C017771; } \\
\text { MELO3C016201; } \\
\text { MELO3C015113; } \\
\text { MELO3C025030; } \\
\text { MELO3C017775; } \\
\text { MELO3C019837; } \\
\text { MELO3C020836; } \\
\text { MELO3C007740; } \\
\text { MELO3C002294; } \\
\text { MELO3C002173; } \\
\text { MELO3C020718; } \\
\text { MELO3C006411; } \\
\text { MELO3C006416; } \\
\text { MELO3C008956; } \\
\text { MELO3C008955; } \\
\text { MELO3C019964; } \\
\text { MELO3C016459; } \\
\text { MELO3C014122; }\end{array}$ \\
\hline [OVER] & GO:0006268 & $\begin{array}{l}\text { DNA } \\
\text { unwinding } \\
\text { involved in } \\
\text { DNA } \\
\text { replication }\end{array}$ & \begin{tabular}{|l|} 
BIOLOGICAL \\
PROCESS
\end{tabular} & $\begin{array}{l}5.812401463 \\
16843 E-4\end{array}$ & \begin{tabular}{|l|}
5.659832076 \\
$751646 \mathrm{E}-6$
\end{tabular} & 6 & 5 & 849 & 16268 & $\begin{array}{l}\text { MELO3C006995; } \\
\text { MELO3C015926; } \\
\text { MELO3C026749; } \\
\text { MELO3C004448; } \\
\text { MELO3C007144; } \\
\text { MELO3C013436 }\end{array}$ & $\begin{array}{l}\text { MELO3C014153; } \\
\text { MELO3C00968; } \\
\text { MELO3C003609; } \\
\text { MELO3C006788; } \\
\text { MELO3C003608 }\end{array}$ \\
\hline
\end{tabular}




\begin{tabular}{|c|c|c|c|c|c|c|c|c|c|c|c|}
\hline [OVER] & GO:0009651 & $\begin{array}{l}\text { response to } \\
\text { salt stress }\end{array}$ & \begin{tabular}{|l|} 
BIOLOGICAL_ \\
PROCESS
\end{tabular} & \begin{tabular}{|l|}
6.888800376 \\
$818608 \mathrm{E}-4$
\end{tabular} & $\begin{array}{l}7.072540607 \\
021645 \mathrm{E}-6\end{array}$ & 23 & 141 & 832 & 16132 & $\begin{array}{l}\text { MELO3C012136; } \\
\text { MELO3C012445; } \\
\text { MELO3C004194; } \\
\text { MELO3C009203; } \\
\text { MELO3C011044; } \\
\text { MELO3C025916; } \\
\text { MELO3C005147; } \\
\text { MELO3C011172; } \\
\text { MELO3C026629; } \\
\text { MELO3C004105; } \\
\text { MELO3C024514; } \\
\text { MELO3C008879; } \\
\text { MELO3C027349; } \\
\text { MELO3C017242; } \\
\text { MELO3C018573; } \\
\text { MELO3C004519; } \\
\text { MELO3C003906; } \\
\text { MELO3C015098; } \\
\text { MELO3C016033; } \\
\text { MELO3C015186; } \\
\text { MELO3C025595; } \\
\text { MELO3C015374; } \\
\text { MELO3C016017 }\end{array}$ & $\begin{array}{l}\text { MELO3C021918; } \\
\text { MELO3C006086; } \\
\text { MELO3C017935; } \\
\text { MELO3C013454; } \\
\text { MELO3C00458;; } \\
\text { MELO3C012085; } \\
\text { MELO3C022725; } \\
\text { MELO3C008666; } \\
\text { MELO3C006648; } \\
\text { MELO3C002203; } \\
\text { MELO3C026009; } \\
\text { MELO3C024345; } \\
\text { MELO3C007739; } \\
\text { MELO3C026245; } \\
\text { MELO3C017134; } \\
\text { MELO3C024222; } \\
\text { MELO3C019039; } \\
\text { MELO3C010625; } \\
\text { MELO3C012925; } \\
\text { MELO3C026482; } \\
\text { MELO3C016738; } \\
\text { MELO3C002170; } \\
\text { MELO3C012099; } \\
\text { MELO3C012097; } \\
\text { MELO3C007067; } \\
\text { MELO3C009123; } \\
\text { MELO3C005444; }\end{array}$ \\
\hline [OVER] & GO:0000287 & $\begin{array}{l}\text { magnesium } \\
\text { ion binding }\end{array}$ & \begin{tabular}{|l|} 
MOLECULAR \\
_FUNCTION
\end{tabular} & \begin{tabular}{|l|}
0.004724750 \\
385781459
\end{tabular} & \begin{tabular}{|l|}
6.301000726 \\
$169176 \mathrm{E}-5$
\end{tabular} & 25 & 182 & 830 & 16091 & $\begin{array}{l}\text { MELO3C005293; } \\
\text { MELO3C010286; } \\
\text { MELO3C011972; } \\
\text { MELO3C007153; } \\
\text { MELO3C009038; } \\
\text { MELO3C026748; } \\
\text { MELO3C024982; } \\
\text { MELO3C020244; } \\
\text { MELO3C005939; } \\
\text { MELO3C014732; } \\
\text { MELO3C012178; } \\
\text { MELO3C010794; } \\
\text { MELO3C012196; } \\
\text { MELO3C020579; } \\
\text { MELO3C024514; } \\
\text { MELO3C006736; } \\
\text { MELO3C004439; } \\
\text { MELO3C021385; } \\
\text { MELO3C021563; } \\
\text { MELO3C018573; } \\
\text { MELO3C022494; } \\
\text { MELO3C020394; } \\
\text { MELO3C011704; } \\
\text { MELO3C011769; } \\
\text { MELO3C013745 }\end{array}$ & $\begin{array}{l}\text { MELO3C005271; } \\
\text { MELO3C020829; } \\
\text { MELO3C013336; } \\
\text { MELO3C018628; } \\
\text { MELO3C018507; } \\
\text { MELO3C005396; } \\
\text { MELO3C008426; } \\
\text { MELO3C024509; } \\
\text { MELO3C022605; } \\
\text { MELO3C024508; } \\
\text { MELO3C001595; } \\
\text { MELO3C004226; } \\
\text { MELO3C003379; } \\
\text { MELO3C026007; } \\
\text { MELO3C002448; } \\
\text { MELO3C026005; } \\
\text { MELO3C026006; } \\
\text { MELO3C025552; } \\
\text { MELO3C026004; } \\
\text { MELO3C002727; } \\
\text { MELO3C015076; } \\
\text { MELO3C000372; } \\
\text { MELO3C000130; } \\
\text { MELO3C011713; } \\
\text { MELO3C019677; } \\
\text { MELO3C013218; } \\
\text { MELO3C014943; }\end{array}$ \\
\hline [OVER] & GO:0004775 & $\begin{array}{l}\text { succinate-CoA } \\
\text { ligase (ADP- } \\
\text { forming) } \\
\text { activity }\end{array}$ & \begin{tabular}{|l|} 
MOLECULAR \\
FUNCTION
\end{tabular} & \begin{tabular}{|l|}
0.005883572 \\
491923695
\end{tabular} & \begin{tabular}{|l|}
8.531429206 \\
$112895 \mathrm{E}-5$
\end{tabular} & 4 & 2 & 851 & 16271 & $\begin{array}{l}\text { MELO3C002167; } \\
\text { MELO3C022382; } \\
\text { MELO3C010675; } \\
\text { MELO3C011482 }\end{array}$ & $\begin{array}{l}\text { MELO3C021268; } \\
\text { MELO3C015245 }\end{array}$ \\
\hline
\end{tabular}




\begin{tabular}{|c|c|c|c|c|c|c|c|c|c|c|c|}
\hline$[$ OVER] & GO:0009792 & $\begin{array}{l}\text { embryo } \\
\text { development } \\
\text { ending in birth } \\
\text { or egg } \\
\text { hatching }\end{array}$ & $\begin{array}{l}\text { BIOLOGICAL } \\
\text { PROCESS }\end{array}$ & $\begin{array}{l}0.007257974 \\
306578477\end{array}$ & $\begin{array}{l}1.067801046 \\
374268 \mathrm{E}-4\end{array}$ & 39 & 366 & 816 & 15907 & $\begin{array}{l}\text { MELO3C010243; } \\
\text { MELO3C011256; } \\
\text { MELO3C013753; } \\
\text { MELO3C008285; } \\
\text { MELO3C010960; } \\
\text { MELO3C002180; } \\
\text { MELO3C010882; } \\
\text { MELO3C012361; } \\
\text { MELO3C011193; } \\
\text { MELO3C009338; } \\
\text { MELO3C007433; } \\
\text { MELO3C005679; } \\
\text { MELO3C020369; } \\
\text { MELO3C022540; } \\
\text { MELO3C006949; } \\
\text { MELO3C019253; } \\
\text { MELO3C022488; } \\
\text { MELO3C020860; } \\
\text { MELO3C021058; } \\
\text { MELO3C019997; } \\
\text { MELO3C017993; } \\
\text { MELO3C011285; } \\
\text { MELO3C022319; } \\
\text { MELO3C009600; } \\
\text { MELO3C005421; } \\
\text { MELO3C004278; } \\
\text { MELO3C025549; }\end{array}$ & $\begin{array}{l}\text { MELO3C010584; } \\
\text { MELO3C011795; } \\
\text { MELO3C012522; } \\
\text { MELO3C009592; } \\
\text { MELO3C006760; } \\
\text { MELO3C002166; } \\
\text { MELO3C002045; } \\
\text { MELO3C004344; } \\
\text { MELO3C019031; } \\
\text { MELO3C022562; } \\
\text { MELO3C018062; } \\
\text { MELO3C026800; } \\
\text { MELO3C026245; } \\
\text { MELO3C018187; } \\
\text { MELO3C019034; } \\
\text { MELO3C025275; } \\
\text { MELO3C014708; } \\
\text { MELO3C015235; } \\
\text { MELO3C015599; } \\
\text { MELO3C019956; } \\
\text { MELO3C006096; } \\
\text { MELO3C008151; } \\
\text { MELO3C020836; } \\
\text { MELO3C008157; } \\
\text { MELO3C002173; } \\
\text { MELO3C004473; } \\
\text { MELO3C014153; }\end{array}$ \\
\hline$[$ OVER] & GO:0004807 & \begin{tabular}{|l} 
triose- \\
phosphate \\
isomerase \\
activity \\
\end{tabular} & $\begin{array}{l}\text { MOLECULAR } \\
\text { FUNCTION }\end{array}$ & $\begin{array}{l}0.008134044 \\
001450522\end{array}$ & $\begin{array}{l}1.239735749 \\
5860236 \mathrm{E}-4\end{array}$ & 3 & 0 & 852 & 16273 & $\begin{array}{l}\text { MELO3C020780; } \\
\text { MELO3C025916; } \\
\text { MELO3C008879 }\end{array}$ & \\
\hline [OVER] & GO:0005658 & $\begin{array}{l}\text { alpha DNA } \\
\text { polymerase:pri } \\
\text { mase complex }\end{array}$ & $\begin{array}{l}\text { CELLULAR_C } \\
\text { OMPONENT }\end{array}$ & $\begin{array}{l}0.008134044 \\
001450522\end{array}$ & $\begin{array}{l}1.239735749 \\
5860236 \mathrm{E}-4\end{array}$ & 3 & 0 & 852 & 16273 & $\begin{array}{l}\text { MELO3C027184; } \\
\text { MELO3C022319; } \\
\text { MELO3C020860 }\end{array}$ & \\
\hline$[$ OVER] & GO:0002119 & \begin{tabular}{|l} 
nematode \\
larval \\
development
\end{tabular} & $\begin{array}{l}\text { BIOLOGICAL } \\
\text { PROCESS }\end{array}$ & $\begin{array}{l}0.015229400 \\
702566667\end{array}$ & $\begin{array}{l}2.417876910 \\
8647335 \mathrm{E}-4\end{array}$ & 30 & 268 & 825 & 16005 & $\begin{array}{l}\text { MELO3C010243; } \\
\text { MELO3C011256; } \\
\text { MELO3C008285; } \\
\text { MELO3C010960; } \\
\text { MELO3C010882; } \\
\text { MELO3C016707; } \\
\text { MELO3C011193; } \\
\text { MELO3C009338; } \\
\text { MELO3C007433; } \\
\text { MELO3C020369; } \\
\text { MELO3C022540; } \\
\text { MELO3C006949; } \\
\text { MELO3C022488; } \\
\text { MELO3C016262; } \\
\text { MELO3C019997; } \\
\text { MELO3C017993; } \\
\text { MELO3C011285; } \\
\text { MELO3C009600; } \\
\text { MELO3C005421; } \\
\text { MELO3C004278; } \\
\text { MELO3C025549; } \\
\text { MELO3C004459; } \\
\text { MELO3C023201; } \\
\text { MELO3C002118; } \\
\text { MELO3C002717; } \\
\text { MELO3C003905; } \\
\text { MELO3C022094; }\end{array}$ & $\begin{array}{l}\text { MELO3C011795; } \\
\text { MELO3C012522; } \\
\text { MELO3C006760; } \\
\text { MELO3C002166; } \\
\text { MELO3C002045; } \\
\text { MELO3C004344; } \\
\text { MELO3C019031; } \\
\text { MELO3C026800; } \\
\text { MELO3C019034; } \\
\text { MELO3C025275; } \\
\text { MELO3C016321; } \\
\text { MELO3C014708; } \\
\text { MELO3C015235; } \\
\text { MELO3C019956; } \\
\text { MELO3C020836; } \\
\text { MELO3C008157; } \\
\text { MELO3C002173; } \\
\text { MELO3C004473; } \\
\text { MELO3C014153; } \\
\text { MELO3C013185; } \\
\text { MELO3C023545; } \\
\text { MELO3C008835; } \\
\text { MELO3C023306; } \\
\text { MELO3C023427; } \\
\text { MELO3C006657; } \\
\text { MELO3C024757; } \\
\text { MELO3C003703; }\end{array}$ \\
\hline [OVER] & GO:0006269 & \begin{tabular}{|l} 
DNA \\
replication, \\
synthesis of \\
RNA primer \\
\end{tabular} & $\begin{array}{l}\text { BIOLOGICAL } \\
\text { PROCESS }\end{array}$ & $\begin{array}{l}0.026531682 \\
698092328\end{array}$ & $\begin{array}{l}4.773905650 \\
588162 \mathrm{E}-4\end{array}$ & 3 & 1 & 852 & 16272 & $\begin{array}{l}\text { MELO3C027184; } \\
\text { MELO3C022319; } \\
\text { MELO3C020860 }\end{array}$ & MELO3C006956 \\
\hline [OVER] & GO:0004634 & $\begin{array}{l}\text { phosphopyruv } \\
\text { ate hydratase } \\
\text { activity }\end{array}$ & $\begin{array}{l}\text { MOLECULAR } \\
\text { FUNCTION }\end{array}$ & $\begin{array}{l}0.026531682 \\
698092328\end{array}$ & $\begin{array}{l}4.773905650 \\
588162 \mathrm{E}-4\end{array}$ & 3 & 1 & 852 & 16272 & $\begin{array}{l}\text { MELO3C020579; } \\
\text { MELO3C026748; } \\
\text { MELO3C024514 }\end{array}$ & MELO3C017268 \\
\hline [OVER] & GO:0003896 & $\begin{array}{l}\text { DNA primase } \\
\text { activity }\end{array}$ & $\begin{array}{l}\text { MOLECULAR } \\
\text { FUNCTION }\end{array}$ & $\begin{array}{l}0.026531682 \\
698092328\end{array}$ & $\begin{array}{l}4.773905650 \\
588162 E-4\end{array}$ & 3 & 1 & 852 & 16272 & $\begin{array}{l}\text { MELO3C027184; } \\
\text { MELO3C022319; } \\
\text { MELO3C020860 }\end{array}$ & MELO3C006956 \\
\hline [OVER] & GO:0000015 & $\begin{array}{l}\text { phosphopyruv } \\
\text { ate hydratase } \\
\text { complex }\end{array}$ & $\begin{array}{l}\text { CELLULAR_C } \\
\text { OMPONENT }\end{array}$ & $\begin{array}{l}0.026531682 \\
698092328\end{array}$ & \begin{tabular}{|l|}
4.773905650 \\
$588162 \mathrm{E}-4$
\end{tabular} & 3 & 1 & 852 & 16272 & $\begin{array}{l}\text { MELO3C020579; } \\
\text { MELO3C026748; } \\
\text { MELO3C024514 }\end{array}$ & MELO3C017268 \\
\hline
\end{tabular}




\begin{tabular}{|c|c|c|c|c|c|c|c|c|c|c|c|}
\hline [OVER] & GO:0032201 & $\begin{array}{l}\text { telomere } \\
\text { maintenance } \\
\text { via semi- } \\
\text { conservative } \\
\text { replication }\end{array}$ & $\begin{array}{l}\text { BIOLOGICAL } \\
\text { PROCESS }\end{array}$ & \begin{tabular}{|l|}
0.030742106 \\
527155904
\end{tabular} & \begin{tabular}{|l|}
5.694187809 \\
$327141 \mathrm{E}-4$
\end{tabular} & 6 & 16 & 849 & 16257 & $\begin{array}{l}\text { MELO3C003638; } \\
\text { MELO3C022319; } \\
\text { MELO3C020860; } \\
\text { MELO3C017228; } \\
\text { MELO3C027184; } \\
\text { MELO3C010631 }\end{array}$ & $\begin{array}{l}\text { MELO3C005384; } \\
\text { MELO3C014021; } \\
\text { MELO3C014020; } \\
\text { MELO3C008864; } \\
\text { MELO3C002892; } \\
\text { MELO3C026949; } \\
\text { MELO3C025879; } \\
\text { MELO3C025646; } \\
\text { MELO3C010507; } \\
\text { MELO3C024596; } \\
\text { MELO3C001937; } \\
\text { MELO3C025142; } \\
\text { MELO3C014529; } \\
\text { MELO3C015225; } \\
\text { MELO3C014528; } \\
\text { MELO3C017458 }\end{array}$ \\
\hline [OVER] & GO:0010181 & FMN binding & $\begin{array}{l}\text { MOLECULAR } \\
\text { _FUNCTION }\end{array}$ & \begin{tabular}{|l|}
0.043259870 \\
25490484
\end{tabular} & $\begin{array}{l}8.470656220 \\
530689 E-4\end{array}$ & 7 & 25 & 848 & 16248 & $\begin{array}{l}\text { MELO3C007340; } \\
\text { MELO3C022684; } \\
\text { MELO3C011062; } \\
\text { MELO3C003541; } \\
\text { MELO3C023799; } \\
\text { MELO3C016623; } \\
\text { MELO3C015514; }\end{array}$ & $\begin{array}{l}\text { MELO3C008482; } \\
\text { MELO3C013476; } \\
\text { MELO3C008481; } \\
\text { MELO3C004023; } \\
\text { MELO3C009759; } \\
\text { MELO3C022683; } \\
\text { MELO3C008805; } \\
\text { MELO3C022681; } \\
\text { MELO3C024281; } \\
\text { MELO3C018625; } \\
\text { MELO3C018626; } \\
\text { MELO3C010940; } \\
\text { MELO3C017636; } \\
\text { MELO3C008075; } \\
\text { MELO3C007124; } \\
\text { MELO3C006296; } \\
\text { MELO3C006011; } \\
\text { MELO3C009702; } \\
\text { MELO3C021944; } \\
\text { MELO3C005229; } \\
\text { MELO3C003644; } \\
\text { MELO3C025720; } \\
\text { MELO3C017461; } \\
\text { MELO3C025861; } \\
\text { MELO3C007808 }\end{array}$ \\
\hline [OVER] & GO:0005618 & cell wall & $\begin{array}{l}\text { CELLULAR_C } \\
\text { OMPONENT }\end{array}$ & \begin{tabular}{|l|}
0.052751843 \\
14947438
\end{tabular} & $\begin{array}{l}0.001099927 \\
2968296415\end{array}$ & 34 & 345 & 821 & 15928 & $\begin{array}{l}\text { MELO3C012004; } \\
\text { MELO3C013699; } \\
\text { MELO3C011256; } \\
\text { MELO3C009133; } \\
\text { MELO3C012100; } \\
\text { MELO3C025916; } \\
\text { MELO3C020049; } \\
\text { MELO3C005757; } \\
\text { MELO3C020840; } \\
\text { MELO3C016067; } \\
\text { MELO3C017478; } \\
\text { MELO3C019979; } \\
\text { MELO3C010686; } \\
\text { MELO3C009203; } \\
\text { MELO3C005089; } \\
\text { MELO3C012670; } \\
\text { MELO3C021404; } \\
\text { MELO3C023406; } \\
\text { MELO3C005201; } \\
\text { MELO3C004597; } \\
\text { MELO3C009429; } \\
\text { MELO3C008879; } \\
\text { MELO3C017480; } \\
\text { MELO3C007609; } \\
\text { MELO3C021782; } \\
\text { MELO3C025645; } \\
\text { MELO3C025324; }\end{array}$ & $\begin{array}{l}\text { MELO3C015914; } \\
\text { MELO3C020704; } \\
\text { MELO3C007057; } \\
\text { MELO3C013055; } \\
\text { MELO3C026806; } \\
\text { MELO3C005316; } \\
\text { MELO3C002047; } \\
\text { MELO3C008946; } \\
\text { MELO3C003810; } \\
\text { MELO3C021595; } \\
\text { MELO3C021471; } \\
\text { MELO3C019039; } \\
\text { MELO3C018862; } \\
\text { MELO3C018743; } \\
\text { MELO3C008032; } \\
\text { MELO3C009120; } \\
\text { MELO3C005561; } \\
\text { MELO3C012099; } \\
\text { MELO3C009488; } \\
\text { MELO3C009367; } \\
\text { MELO3C012097; } \\
\text { MELO3C005560; } \\
\text { MELO3C012096; } \\
\text { MELO3C004477; } \\
\text { MELO3C008159; } \\
\text { MELO3C009924; } \\
\text { MELO3C010919; }\end{array}$ \\
\hline [OVER] & GO:0003878 & $\begin{array}{l}\text { ATP citrate } \\
\text { synthase } \\
\text { activity }\end{array}$ & $\begin{array}{l}\text { MOLECULAR } \\
\text { FUNCTION }\end{array}$ & \begin{tabular}{|l|}
0.054554186 \\
35648744
\end{tabular} & $\begin{array}{l}0.001149056 \\
2113612404\end{array}$ & 3 & 2 & 852 & 16271 & $\begin{array}{l}\text { MELO3C010675; } \\
\text { MELO3C011482; } \\
\text { MELO3C002167 }\end{array}$ & $\begin{array}{l}\text { MELO3C021268; } \\
\text { MELO3C015245 }\end{array}$ \\
\hline
\end{tabular}




\begin{tabular}{|c|c|c|c|c|c|c|c|c|c|c|c|}
\hline [OVER] & GO:0000722 & \begin{tabular}{|l|} 
telomere \\
maintenance \\
via \\
recombination
\end{tabular} & $\begin{array}{l}\text { BIOLOGICAL } \\
\text { PROCESS }\end{array}$ & \begin{tabular}{|l|}
0.055611060 \\
82724685
\end{tabular} & \begin{tabular}{|l|}
0.001188974 \\
839871281 \\
\end{tabular} & 6 & 19 & 849 & 16254 & $\begin{array}{l}\text { MELO3C003638; } \\
\text { MELO3C022319; } \\
\text { MELO3C020860; } \\
\text { MELO3C017228; } \\
\text { MELO3C027184; } \\
\text { MELO3C010631 }\end{array}$ & $\begin{array}{l}\text { MELO3C013324; } \\
\text { MELO3C006292; } \\
\text { MELO3C005384; } \\
\text { MELO3C014021; } \\
\text { MELO3C014020; } \\
\text { MELO3C008864; } \\
\text { MELO3C002892; } \\
\text { MELO3C026949; } \\
\text { MELO3C025879; } \\
\text { MELO3C025646; } \\
\text { MELO3C019510; } \\
\text { MELO3C010507; } \\
\text { MELO3C024596; } \\
\text { MELO3C001937; } \\
\text { MELO3C025142; } \\
\text { MELO3C014529; } \\
\text { MELO3C015225; } \\
\text { MELO3C014528; } \\
\text { MELO3C017458 }\end{array}$ \\
\hline [OVER] & GO:0031018 & \begin{tabular}{|l|} 
endocrine \\
pancreas \\
development
\end{tabular} & $\begin{array}{l}\text { BIOLOGICAL } \\
\text { PROCESS }\end{array}$ & \begin{tabular}{|l|}
0.069037243 \\
51414402
\end{tabular} & $\begin{array}{l}0.001534485 \\
7258647593\end{array}$ & 4 & 7 & 851 & 16266 & $\begin{array}{l}\text { MELO3C023201; } \\
\text { MELO3C017993; } \\
\text { MELO3C009338; } \\
\text { MELO3C017291 }\end{array}$ & $\begin{array}{l}\text { MELO3C014237; } \\
\text { MELO3C017184; } \\
\text { MELO3C006662; } \\
\text { MELO3C017169; } \\
\text { MELO3C018512; } \\
\text { MELO3C023127; } \\
\text { MELO3C017290 }\end{array}$ \\
\hline [OVER] & GO:0008340 & $\begin{array}{l}\text { determination } \\
\text { of adult } \\
\text { lifespan }\end{array}$ & $\begin{array}{l}\text { BIOLOGICAL_ } \\
\text { PROCESS }\end{array}$ & \begin{tabular}{|l|}
0.082143398 \\
29049341
\end{tabular} & \begin{tabular}{|l|}
0.001851878 \\
0520612932
\end{tabular} & 12 & 79 & 843 & 16194 & $\begin{array}{l}\text { MELO3C014007; } \\
\text { MELO3C011256; } \\
\text { MELO3C023201; } \\
\text { MELO3C022540; } \\
\text { MELO3C006949; } \\
\text { MELO3C008285; } \\
\text { MELO3C010960; } \\
\text { MELO3C020860; } \\
\text { MELO3C016262; } \\
\text { MELO3C022094; } \\
\text { MELO3C004278; } \\
\text { MELO3C011104 }\end{array}$ & $\begin{array}{l}\text { MELO3C016329; } \\
\text { MELO3C010584; } \\
\text { MELO3C012522; } \\
\text { MELO3C014221; } \\
\text { MELO3C006121; } \\
\text { MELO3C005753; } \\
\text { MELO3C007214; } \\
\text { MELO3C024822; } \\
\text { MELO3C008306; } \\
\text { MELO3C004945; } \\
\text { MELO3C025755; } \\
\text { MELO3C025796; } \\
\text { MELO3C020783; } \\
\text { MELO3C004827; } \\
\text { MELO3C025275; } \\
\text { MELO3C015199; } \\
\text { MELO3C019712; } \\
\text { MELO3C022280; } \\
\text { MELO3C027174; } \\
\text { MELO3C013814; } \\
\text { MELO3C011830; } \\
\text { MELO3C017627; } \\
\text { MELO3C010795; } \\
\text { MELO3C009840; } \\
\text { MELO3C015928; } \\
\text { MELO3C014153; } \\
\text { MELO3C022219; }\end{array}$ \\
\hline $\begin{array}{l}\text { [OVER] } \\
\end{array}$ & GO:0000022 & $\begin{array}{l}\text { mitotic spindle } \\
\text { elongation }\end{array}$ & $\begin{array}{l}\text { BIOLOGICAL_ } \\
\text { PROCESS }\end{array}$ & \begin{tabular}{|l|}
0.087111452 \\
99844858
\end{tabular} & $\begin{array}{l}0.001982400 \\
2986259916\end{array}$ & 5 & 14 & 850 & 16259 & $\begin{array}{l}\text { MELO3C009338; } \\
\text { MELO3C009600; } \\
\text { MELO3C011285; } \\
\text { MELO3C006280; } \\
\text { MELO3C020369 }\end{array}$ & $\begin{array}{l}\text { MELO3C014237; } \\
\text { MELO3C007036; } \\
\text { MELO3C003342; } \\
\text { MELO3C025755; } \\
\text { MELO3C004807; } \\
\text { MELO3C023594; } \\
\text { MELO3C024782; } \\
\text { MELO3C016981; } \\
\text { MELO3C024150; } \\
\text { MELO3C024020; } \\
\text { MELO3C026580; } \\
\text { MELO3C015269; } \\
\text { MELO3C017744; } \\
\text { MELO3C012667 }\end{array}$ \\
\hline
\end{tabular}




\begin{tabular}{|c|c|c|c|c|c|c|c|c|c|c|c|}
\hline [OVER] & GO:0009620 & $\begin{array}{l}\text { response to } \\
\text { fungus }\end{array}$ & $\begin{array}{l}\text { BIOLOGICAL } \\
\text { PROCESS }\end{array}$ & \begin{tabular}{|l|}
0.096341971 \\
58665671
\end{tabular} & \begin{tabular}{|l|}
0.002233159 \\
5869472714
\end{tabular} & 12 & 81 & 843 & 16192 & $\begin{array}{l}\text { MELO3C011972; } \\
\text { MELO3C006870; } \\
\text { MELO3C020628; } \\
\text { MELO3C019983; } \\
\text { MELO3C002470; } \\
\text { MELO3C006187; } \\
\text { MELO3C005214; } \\
\text { MELO3C016972; } \\
\text { MELO3C012527; } \\
\text { MELO3C005215; } \\
\text { MELO3C019735; } \\
\text { MELO3C015514 }\end{array}$ & $\begin{array}{l}\text { MELO3C005550; } \\
\text { MELO3C007375; } \\
\text { MELO3C004385; } \\
\text { MELO3C005312; } \\
\text { MELO3C002120; } \\
\text { MELO3C003133; } \\
\text { MELO3C004387; } \\
\text { MELO3C006765; } \\
\text { MELO3C004386; } \\
\text { MELO3C025914; } \\
\text { MELO3C005674; } \\
\text { MELO3C005630; } \\
\text { MELO3C025912; } \\
\text { MELO3C009838; } \\
\text { MELO3C005633; } \\
\text { MELO3C024989; } \\
\text { MELO3C019595; } \\
\text { MELO3C002608; } \\
\text { MELO3C025152; } \\
\text { MELO3C018623; } \\
\text { MELO3C009441; } \\
\text { MELO3C013621; } \\
\text { MELO3C014230; } \\
\text { MELO3C005642; } \\
\text { MELO3C005643; } \\
\text { MELO3C024319; } \\
\text { MELO3C020956; }\end{array}$ \\
\hline [OVER] & GO:0000083 & $\begin{array}{l}\text { regulation of } \\
\text { transcription } \\
\text { involved in } \\
\text { G1/S transition } \\
\text { of mitotic cell } \\
\text { cycle }\end{array}$ & $\begin{array}{l}\text { BIOLOGICAL_ } \\
\text { PROCESS }\end{array}$ & $\begin{array}{l}0.098809558 \\
99413238\end{array}$ & $\begin{array}{l}0.002489063 \\
827328906\end{array}$ & 2 & 0 & 853 & 16273 & $\begin{array}{l}\text { MELO3C020860; } \\
\text { MELO3C021058 }\end{array}$ & \\
\hline [OVER] & GO:0006267 & $\begin{array}{l}\text { pre-replicative } \\
\text { complex } \\
\text { assembly } \\
\text { involved in } \\
\text { nuclear cell } \\
\text { cycle DNA } \\
\text { replication }\end{array}$ & $\begin{array}{l}\text { BIOLOGICAL } \\
\text { PROCESS }\end{array}$ & \begin{tabular}{|l|}
0.098809558 \\
99413238
\end{tabular} & \begin{tabular}{|l|}
0.002489063 \\
827328906
\end{tabular} & 2 & 0 & 853 & 16273 & $\begin{array}{l}\text { MELO3C010882; } \\
\text { MELO3C021058 }\end{array}$ & \\
\hline [OVER] & GO:0006279 & \begin{tabular}{|l} 
premeiotic \\
DNA \\
replication \\
\end{tabular} & \begin{tabular}{|l} 
BIOLOGICAL_ \\
PROCESS
\end{tabular} & \begin{tabular}{|l|}
0.098809558 \\
99413238
\end{tabular} & \begin{tabular}{|l|}
0.002489063 \\
827328906
\end{tabular} & 2 & 0 & 853 & 16273 & $\begin{array}{l}\text { MELO3C020860; } \\
\text { MELO3C021058 }\end{array}$ & \\
\hline [OVER] & GO:0006099 & $\begin{array}{l}\text { tricarboxylic } \\
\text { acid cycle }\end{array}$ & \begin{tabular}{|l} 
BIOLOGICAL_ \\
PROCESS
\end{tabular} & \begin{tabular}{|l|}
0.098809558 \\
99413238
\end{tabular} & \begin{tabular}{|l|}
0.002448335 \\
174535359 \\
\end{tabular} & 7 & 31 & 848 & 16242 & $\begin{array}{l}\text { MELO3C021563; } \\
\text { MELO3C022382; } \\
\text { MELO3C017560; } \\
\text { MELO3C011482; } \\
\text { MELO3C002167; } \\
\text { MELO3C021247; } \\
\text { MELO3C022998 }\end{array}$ & $\begin{array}{l}\text { MELO3C012389; } \\
\text { MELO3C017938; } \\
\text { MELO3C007674; } \\
\text { MELO3C014221; } \\
\text { MELO3C013153; } \\
\text { MELO3C003577; } \\
\text { MELO3C025636; } \\
\text { MELO3C022343; } \\
\text { MELO3C005539; } \\
\text { MELO3C017175; } \\
\text { MELO3C019711; } \\
\text { MELO3C025076; } \\
\text { MELO3C018724; } \\
\text { MELO3C014824; } \\
\text { MELO3C019879; } \\
\text { MELO3C002350; } \\
\text { MELO3C023506; } \\
\text { MELO3C007968; } \\
\text { MELO3C021268; } \\
\text { MELO3C021545; } \\
\text { MELO3C006734; } \\
\text { MELO3C005348; } \\
\text { MELO3C016050; } \\
\text { MELO3C016095; } \\
\text { MELO3C005968; } \\
\text { MELO3C019300; } \\
\text { MELO3C019243; }\end{array}$ \\
\hline [OVER] & GO:0004776 & $\begin{array}{l}\text { succinate-CoA } \\
\text { ligase (GDP- } \\
\text { forming) } \\
\text { activity }\end{array}$ & $\begin{array}{l}\text { MOLECULAR } \\
\text { FUNCTION }\end{array}$ & \begin{tabular}{|l|}
0.098809558 \\
99413238
\end{tabular} & \begin{tabular}{|l|}
0.002489063 \\
827328906
\end{tabular} & 2 & 0 & 853 & 16273 & $\begin{array}{l}\text { MELO3C022382; } \\
\text { MELO3C002167 }\end{array}$ & \\
\hline [OVER] & GO:0003861 & $\begin{array}{l}\text { - } \\
\text { isopropylmalat } \\
\text { e dehydratase } \\
\text { activity }\end{array}$ & $\begin{array}{l}\text { MOLECULAR } \\
\text { FUNCTION }\end{array}$ & \begin{tabular}{|l|}
0.098809558 \\
99413238
\end{tabular} & \begin{tabular}{|l|}
0.002489063 \\
827328906
\end{tabular} & 2 & 0 & 853 & 16273 & $\begin{array}{l}\text { MELO3C005518; } \\
\text { MELO3C003344 }\end{array}$ & \\
\hline [OVER] & GO:0005656 & $\begin{array}{l}\text { nuclear pre- } \\
\text { replicative } \\
\text { complex }\end{array}$ & $\begin{array}{l}\text { CELLULAR_C } \\
\text { OMPONENT }\end{array}$ & \begin{tabular}{|l|}
0.098809558 \\
99413238
\end{tabular} & \begin{tabular}{|l|}
0.002489063 \\
827328906
\end{tabular} & 2 & 0 & 853 & 16273 & $\begin{array}{l}\text { MELO3C010882; } \\
\text { MELO3C021058 }\end{array}$ & \\
\hline
\end{tabular}




\begin{tabular}{|c|c|c|c|c|c|c|c|c|c|c|c|}
\hline[] & |GO:0005758 & \begin{tabular}{|l} 
mitochondrial \\
intermembran \\
e space
\end{tabular} & \begin{tabular}{|l} 
CELLULAR_C \\
OMPONENT
\end{tabular} & \begin{tabular}{|l|}
0.100242902 \\
5175407
\end{tabular} & $\begin{array}{l}0.002535780 \\
4510681868\end{array}$ & & 15 & 850 & 16258 & $\begin{array}{l}\text { MELO3C014007; } \\
\text { MELO3C022540; } \\
\text { MELO3C004519; } \\
\text { MELO3C007281; } \\
\text { MELO3C016067 }\end{array}$ & 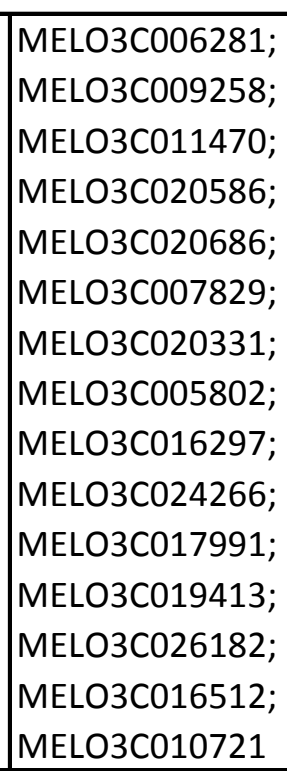 \\
\hline [1] & 60:0048046 & apoplast & \begin{tabular}{|l} 
CELLULAR_C \\
OMPONENT
\end{tabular} & \begin{tabular}{|l|}
0.100446648 \\
32028213
\end{tabular} & \begin{tabular}{|l}
0.002551566 \\
003055431
\end{tabular} & O & 199 & 833 & 16074 & 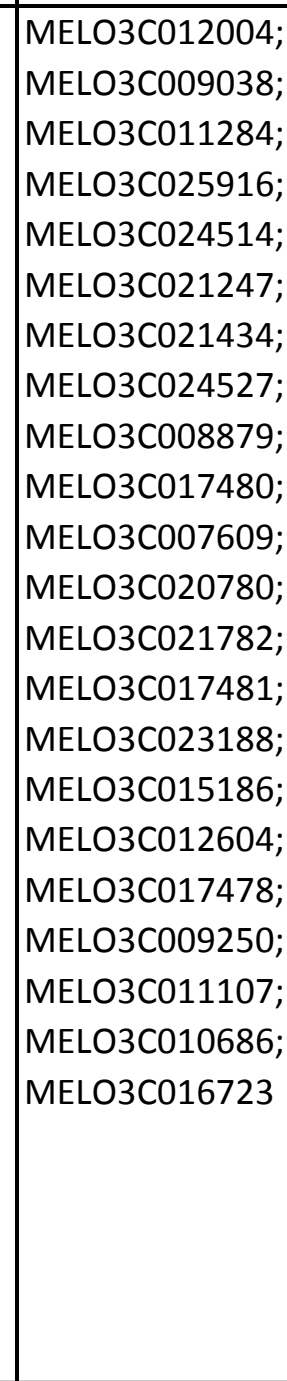 & 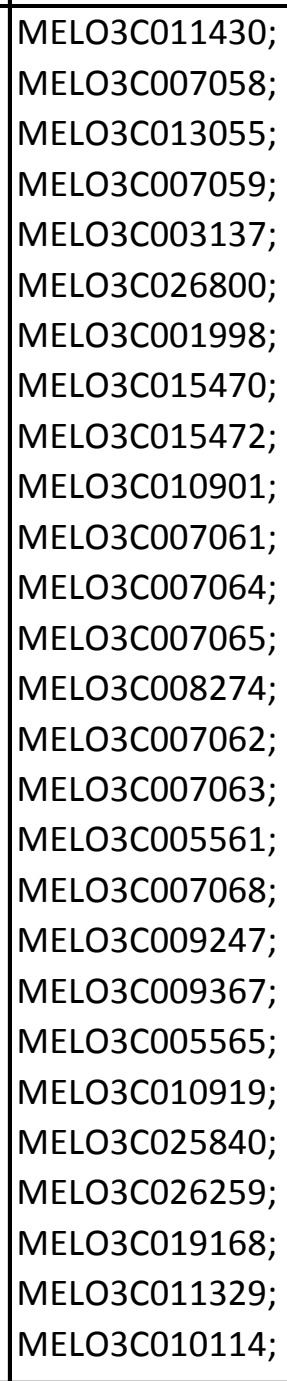 \\
\hline [] & 60:0005774 & \begin{tabular}{|l|} 
vacuolar \\
membrane
\end{tabular} & $\begin{array}{l}\text { CELLULAR_C } \\
\text { OMPONENT }\end{array}$ & \begin{tabular}{|l|}
0.108668297 \\
48299485
\end{tabular} & $\begin{array}{l}0.002794919 \\
1668467134\end{array}$ & 17 & 142 & 838 & 16131 & 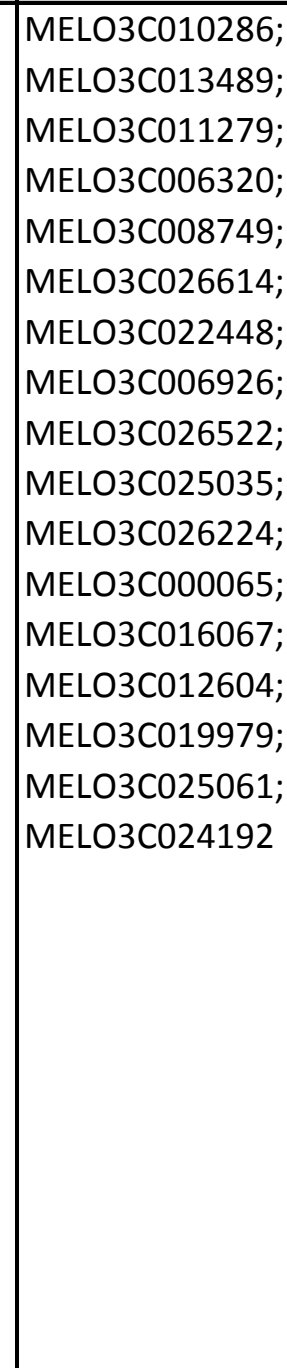 & 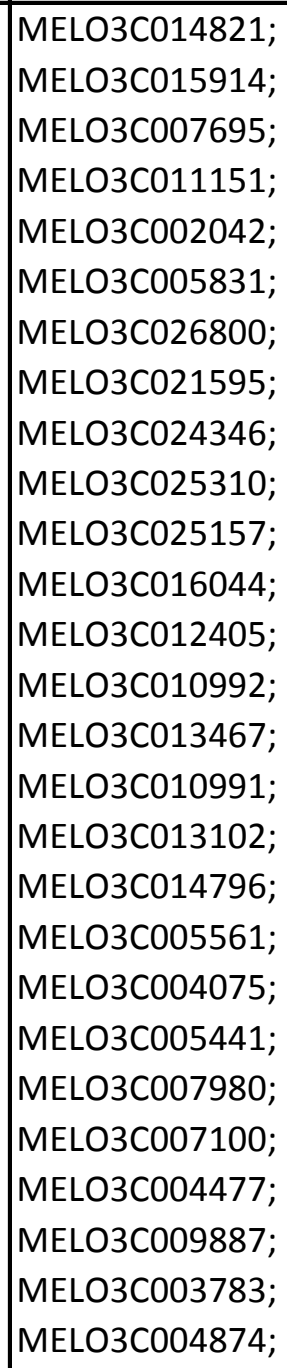 \\
\hline
\end{tabular}




\begin{tabular}{|c|c|c|c|c|c|c|c|c|c|c|c|}
\hline[] & GO:0006094 & \begin{tabular}{|l|} 
gluconeogenes \\
is
\end{tabular} & $\begin{array}{l}\text { BIOLOGICAL } \\
\text { PROCESS }\end{array}$ & \begin{tabular}{|l|}
0.118788715 \\
98209911
\end{tabular} & \begin{tabular}{|l|}
0.003105505 \\
1701501355
\end{tabular} & 8 & 42 & 847 & 16231 & $\begin{array}{l}\text { MELO3C020780; } \\
\text { MELO3CO11284; } \\
\text { MELO3CO25916; } \\
\text { MELO3C012604; } \\
\text { MELO3C021604; } \\
\text { MELO3C011104; } \\
\text { MELO3C022998; } \\
\text { MELO3C008879 }\end{array}$ & 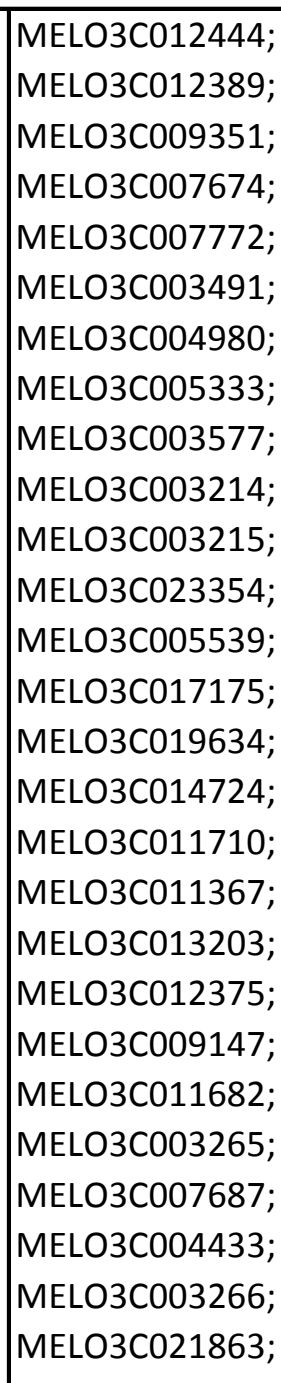 \\
\hline[] & GO:0040002 & \begin{tabular}{|l|} 
collagen and \\
cuticulin-based \\
cuticle \\
development
\end{tabular} & $\begin{array}{l}\text { BIOLOGICAL } \\
\text { PROCESS }\end{array}$ & \begin{tabular}{|l|}
0.139255769 \\
1939414
\end{tabular} & \begin{tabular}{|l|}
0.003729012 \\
4477209114 \\
\end{tabular} & 3 & 4 & 852 & 16269 & $\begin{array}{l}\text { MELO3C016707; } \\
\text { MELO3C019253; } \\
\text { MELO3C012361 }\end{array}$ & $\begin{array}{l}\text { MELO3C016321; } \\
\text { MELO3CO23052; } \\
\text { MELO3CO08151; } \\
\text { MELO3C015126 }\end{array}$ \\
\hline[] & GO:0009570 & $\begin{array}{l}\text { chloroplast } \\
\text { stroma }\end{array}$ & $\begin{array}{l}\text { CELLULAR_C } \\
\text { OMPONENT }\end{array}$ & \begin{tabular}{|l|}
0.153190735 \\
75706385
\end{tabular} & $\begin{array}{l}0.004150807 \\
404086404\end{array}$ & 32 & 351 & 823 & 15922 & 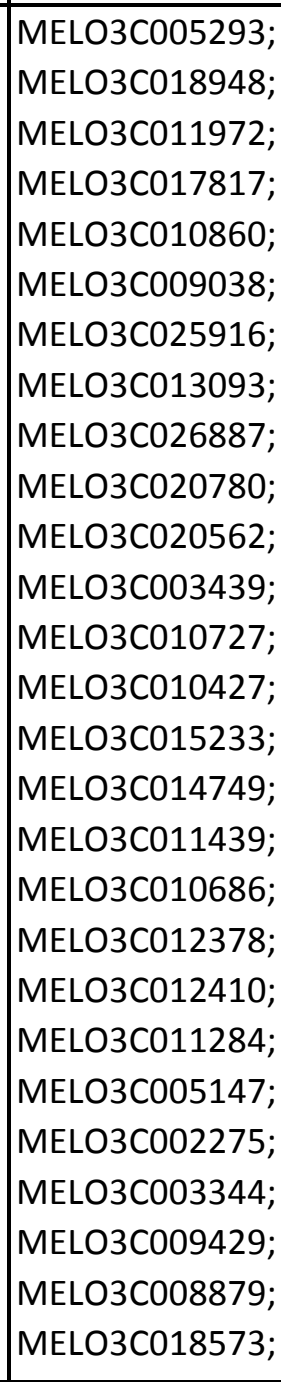 & 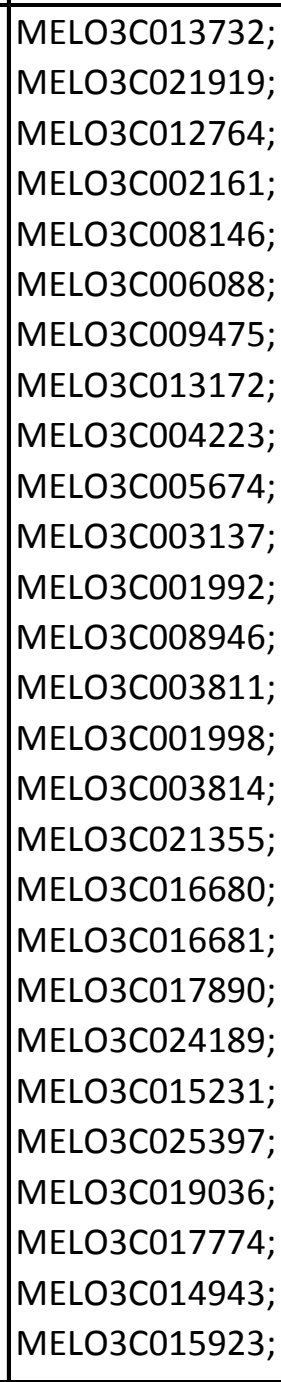 \\
\hline [] & GO:0035046 & \begin{tabular}{|l}
$\begin{array}{l}\text { pronuclear } \\
\text { migration }\end{array}$ \\
\end{tabular} & \begin{tabular}{|l} 
BIOLOGICAL \\
PROCESS
\end{tabular} & \begin{tabular}{|l|}
.200328266 \\
52597704
\end{tabular} & \begin{tabular}{|l|}
0.005746079 \\
617753998
\end{tabular} & 3 & 5 & 852 & 16268 & $\begin{array}{l}\text { MELO3C019253; } \\
\text { MELO3C012361; } \\
\text { MELO3C020860 }\end{array}$ & $\begin{array}{l}\text { MELO3C022016; } \\
\text { MELO3CO05064; } \\
\text { MELO3C019956; } \\
\text { MELO3C006723; } \\
\text { MELO3C004458 }\end{array}$ \\
\hline [] & GO:0051903 & \begin{tabular}{|l|} 
S- \\
(hydroxymeth \\
yl)glutathione \\
dehydrogenas \\
e activity
\end{tabular} & \begin{tabular}{|l|} 
MOLECULAR \\
_FUNCTION
\end{tabular} & \begin{tabular}{|l|}
0.200328266 \\
52597704
\end{tabular} & \begin{tabular}{|l|}
0.005746079 \\
617753998
\end{tabular} & 3 & 5 & 852 & 16268 & $\begin{array}{l}\text { MELO3C023685; } \\
\text { MELO3C026554; } \\
\text { MELO3C022399 }\end{array}$ & $\begin{array}{l}\text { MELO3CO02189; } \\
\text { MELO3CO05792; } \\
\text { MELO3C026553; } \\
\text { MELO3C027151; } \\
\text { MELO3C026552 }\end{array}$ \\
\hline
\end{tabular}




\begin{tabular}{|c|c|c|c|c|c|c|c|c|c|c|c|}
\hline[] & GO:0005982 & $\begin{array}{l}\text { starch } \\
\text { metabolic } \\
\text { process }\end{array}$ & \begin{tabular}{|l|} 
BIOLOGICAL_ \\
PROCESS
\end{tabular} & \begin{tabular}{|l|}
0.230430474 \\
49313317
\end{tabular} & \begin{tabular}{|l|}
0.007068005 \\
702295764
\end{tabular} & 5 & 20 & 850 & 16253 & $\begin{array}{l}\text { MELO3C005293; } \\
\text { MELO3C003812; } \\
\text { MELO3C011972; } \\
\text { MELO3C011104; } \\
\text { MELO3C019135 }\end{array}$ & $\begin{array}{l}\text { MELO3C009960; } \\
\text { MELO3C014105; } \\
\text { MELO3C005396; } \\
\text { MELO3C006585; } \\
\text { MELO3C006552; } \\
\text { MELO3C011682; } \\
\text { MELO3C020949; } \\
\text { MELO3C007155; } \\
\text { MELO3C012071; } \\
\text { MELO3C005795; } \\
\text { MELO3C002455; } \\
\text { MELO3C023105; } \\
\text { MELO3C021412; } \\
\text { MELO3C025149; } \\
\text { MELO3C026536; } \\
\text { MELO3C024754; } \\
\text { MELO3C024175; } \\
\text { MELO3C022275; } \\
\text { MELO3C017942; } \\
\text { MELO3C017735 }\end{array}$ \\
\hline [] & GO:0006277 & $\begin{array}{l}\text { DNA } \\
\text { amplification }\end{array}$ & \begin{tabular}{|l|} 
BIOLOGICAL_ \\
PROCESS
\end{tabular} & \begin{tabular}{|l|}
0.230430474 \\
49313317
\end{tabular} & \begin{tabular}{|l|}
0.007219244 \\
332130336
\end{tabular} & 2 & 1 & 853 & 16272 & $\begin{array}{l}\text { MELO3C010882; } \\
\text { MELO3C021058 }\end{array}$ & MELO3C019031 \\
\hline [] & GO:0006069 & $\begin{array}{l}\text { ethanol } \\
\text { oxidation }\end{array}$ & $\begin{array}{l}\text { BIOLOGICAL_ } \\
\text { PROCESS }\end{array}$ & \begin{tabular}{|l|}
0.230430474 \\
49313317
\end{tabular} & \begin{tabular}{|l|}
0.006931616 \\
846499603
\end{tabular} & 4 & 12 & 851 & 16261 & $\begin{array}{l}\text { MELO3C023685; } \\
\text { MELO3C022399; } \\
\text { MELO3C014749; } \\
\text { MELO3C026554 }\end{array}$ & $\begin{array}{l}\text { MELO3C023687; } \\
\text { MELO3C005792; } \\
\text { MELO3C004383; } \\
\text { MELO3C026553; } \\
\text { MELO3C017100; } \\
\text { MELO3C017125; } \\
\text { MELO3C004430; } \\
\text { MELO3C019622; } \\
\text { MELO3C002189; } \\
\text { MELO3C027151; } \\
\text { MELO3C005081; } \\
\text { MELO3C025328 }\end{array}$ \\
\hline [] & GO:0003688 & \begin{tabular}{|l|} 
DNA \\
replication \\
origin binding
\end{tabular} & $\begin{array}{l}\text { MOLECULAR } \\
\text { FUNCTION }\end{array}$ & \begin{tabular}{|l|}
0.230430474 \\
49313317
\end{tabular} & $\begin{array}{l}0.007219244 \\
332130336\end{array}$ & 2 & 1 & 853 & 16272 & $\begin{array}{l}\text { MELO3C010882; } \\
\text { MELO3C021058 }\end{array}$ & MELO3C021204 \\
\hline [] & GO:0004349 & $\begin{array}{l}\text { glutamate 5- } \\
\text { kinase activity }\end{array}$ & $\begin{array}{l}\text { MOLECULAR } \\
\text { FUNCTION }\end{array}$ & \begin{tabular}{|l|}
0.230430474 \\
49313317
\end{tabular} & $\begin{array}{l}0.007219244 \\
332130336\end{array}$ & 2 & 1 & 853 & 16272 & $\begin{array}{l}\text { MELO3C008245; } \\
\text { MELO3C011208 }\end{array}$ & MELO3C009229 \\
\hline [] & GO:0050486 & $\begin{array}{l}\text { intramolecular } \\
\text { transferase } \\
\text { activity, } \\
\text { transferring } \\
\text { hydroxy } \\
\text { groups }\end{array}$ & $\begin{array}{l}\text { MOLECULAR } \\
\text { FUNCTION }\end{array}$ & \begin{tabular}{|l|}
0.230430474 \\
49313317
\end{tabular} & \begin{tabular}{|l|}
0.007219244 \\
332130336
\end{tabular} & 2 & 1 & 853 & 16272 & $\begin{array}{l}\text { MELO3C012178; } \\
\text { MELO3C003344 }\end{array}$ & MELO3C017156 \\
\hline [] & GO:0031418 & $\begin{array}{l}\text { L-ascorbic acid } \\
\text { binding }\end{array}$ & $\begin{array}{l}\text { MOLECULAR } \\
\text { FUNCTION }\end{array}$ & \begin{tabular}{|l|}
0.230430474 \\
49313317
\end{tabular} & \begin{tabular}{|l|}
0.006931616 \\
846499603
\end{tabular} & 4 & 12 & 851 & 16261 & $\begin{array}{l}\text { MELO3C007425; } \\
\text { MELO3C007611; } \\
\text { MELO3C021380; } \\
\text { MELO3C019735 }\end{array}$ & $\begin{array}{l}\text { MELO3C006592; } \\
\text { MELO3C010508; } \\
\text { MELO3C021480; } \\
\text { MELO3C005334; } \\
\text { MELO3C017675; } \\
\text { MELO3C020028; } \\
\text { MELO3C005478; } \\
\text { MELO3C006861; } \\
\text { MELO3C006307; } \\
\text { MELO3C007943; } \\
\text { MELO3C014437; } \\
\text { MELO3C004414 }\end{array}$ \\
\hline[] & GO:0004298 & $\begin{array}{l}\text { threonine-type } \\
\text { endopeptidase } \\
\text { activity }\end{array}$ & $\begin{array}{l}\text { MOLECULAR } \\
\text { FUNCTION }\end{array}$ & \begin{tabular}{|l|}
0.230430474 \\
49313317
\end{tabular} & \begin{tabular}{|l|}
0.007068005 \\
702295764
\end{tabular} & 5 & 20 & 850 & 16253 & $\begin{array}{l}\text { MELO3C010960; } \\
\text { MELO3C011172; } \\
\text { MELO3C003173; } \\
\text { MELO3C013757; } \\
\text { MELO3C006187 }\end{array}$ & $\begin{array}{l}\text { MELO3C011880; } \\
\text { MELO3C006131; } \\
\text { MELO3C007920; } \\
\text { MELO3C021745; } \\
\text { MELO3C021746; } \\
\text { MELO3C000704; } \\
\text { MELO3C025735; } \\
\text { MELO3C023267; } \\
\text { MELO3C025755; } \\
\text { MELO3C02680; } \\
\text { MELO3C000762; } \\
\text { MELO3C006517; } \\
\text { MELO3C019011; } \\
\text { MELO3C019010; } \\
\text { MELO3C019012; } \\
\text { MELO3C027069; } \\
\text { MELO3C024343; } \\
\text { MELO3C010635; } \\
\text { MELO3C026360; } \\
\text { MELO3C011449 }\end{array}$ \\
\hline[] & GO:0004802 & $\begin{array}{l}\text { transketolase } \\
\text { activity }\end{array}$ & $\begin{array}{l}\text { MOLECULAR } \\
\text { FUNCTION }\end{array}$ & \begin{tabular}{|l|}
0.230430474 \\
49313317
\end{tabular} & $\begin{array}{l}0.007219244 \\
332130336\end{array}$ & 2 & 1 & 853 & 16272 & $\begin{array}{l}\text { MELO3C002746; } \\
\text { MELO3C022494 }\end{array}$ & MELO3C006200 \\
\hline
\end{tabular}




\begin{tabular}{|c|c|c|c|c|c|c|c|c|c|c|c|}
\hline [] & GO:0050625 & $\begin{array}{l}\text { 2-hydroxy-1,4- } \\
\text { benzoquinone } \\
\text { reductase } \\
\text { activity }\end{array}$ & $\begin{array}{l}\text { MOLECULAR } \\
\text { FUNCTION }\end{array}$ & \begin{tabular}{|l|}
0.230430474 \\
49313317
\end{tabular} & \begin{tabular}{|l|l}
0.007219244 \\
332130336
\end{tabular} & 2 & 1 & 853 & 16272 & $\begin{array}{l}\text { MELO3C007340; } \\
\text { MELO3C016623 }\end{array}$ & MELO3C025861 \\
\hline [] & GO:0015631 & tubulin binding & $\begin{array}{l}\text { MOLECULAR } \\
\text { FUNCTION }\end{array}$ & $\begin{array}{l}0.230430474 \\
49313317\end{array}$ & \begin{tabular}{|l|}
0.007068005 \\
702295764
\end{tabular} & 5 & 20 & 850 & 16253 & $\begin{array}{l}\text { MELO3C022488; } \\
\text { MELO3CO13702; } \\
\text { MELO3COO2717; } \\
\text { MELO3C017221; } \\
\text { MELO3C006602 }\end{array}$ & 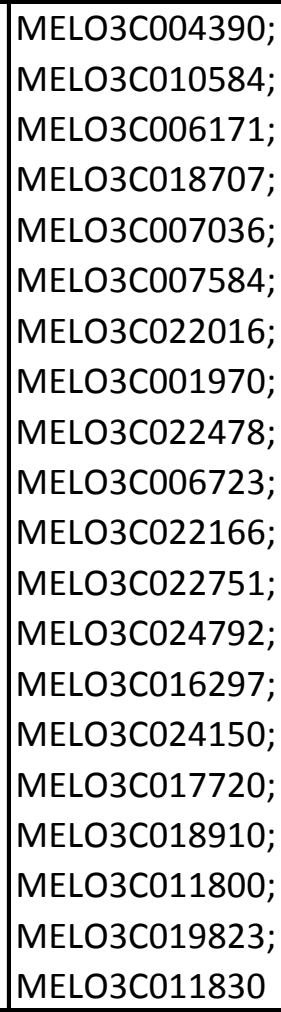 \\
\hline [] & GO:0004733 & $\begin{array}{l}\text { pyridoxamine- } \\
\text { phosphate } \\
\text { oxidase } \\
\text { activity }\end{array}$ & $\begin{array}{l}\text { MOLECULAR } \\
\text { FUNCTION }\end{array}$ & \begin{tabular}{|l|}
0.230430474 \\
49313317
\end{tabular} & \begin{tabular}{|l|}
0.007219244 \\
332130336
\end{tabular} & 2 & 1 & 853 & 16272 & $\begin{array}{l}\text { MELO3C011062; } \\
\text { MELO3C003541 }\end{array}$ & MELO3C006011 \\
\hline [] & GO:0016328 & $\begin{array}{l}\text { lateral plasma } \\
\text { membrane }\end{array}$ & \begin{tabular}{|l} 
CELLULAR_C \\
OMPONENT
\end{tabular} & \begin{tabular}{|l}
0.230430474 \\
49313317
\end{tabular} & \begin{tabular}{|l|}
0.007219244 \\
332130336
\end{tabular} & 2 & 1 & 853 & 16272 & $\begin{array}{l}\text { MELO3C017242; } \\
\text { MELO3C022488 }\end{array}$ & MELO3C007829 \\
\hline [] & GO:0005351 & $\begin{array}{l}\text { sugar:proton } \\
\text { symporter } \\
\text { activity }\end{array}$ & $\begin{array}{l}\text { MOLECULAR } \\
\text { FUNCTION }\end{array}$ & \begin{tabular}{|l|}
0.232427225 \\
77311475
\end{tabular} & \begin{tabular}{|l}
0.007355603 \\
35585958
\end{tabular} & 7 & 39 & 848 & 16234 & $\begin{array}{l}\text { MELO3C013489; } \\
\text { MELO3CO11675; } \\
\text { MELO3CO25345; } \\
\text { MELO3C026522; } \\
\text { MELO3C004576; } \\
\text { MELO3C016017; } \\
\text { MELO3C014417 }\end{array}$ & 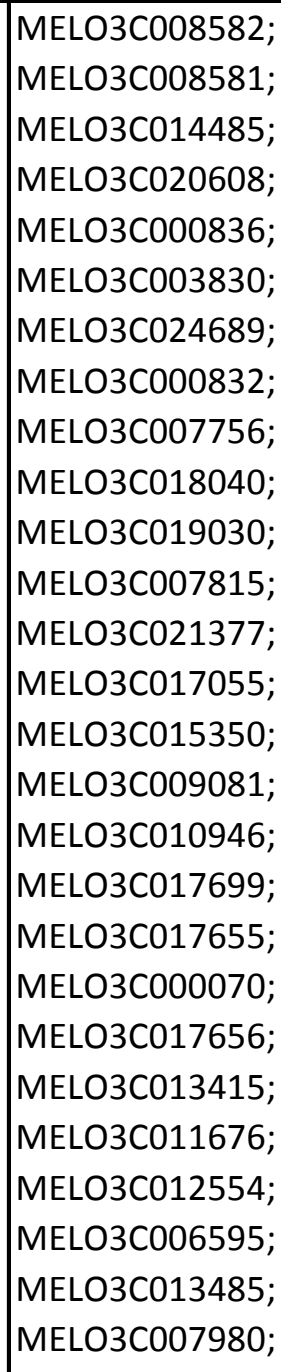 \\
\hline
\end{tabular}




\begin{tabular}{|c|c|c|c|c|c|c|c|c|c|c|c|}
\hline[] & GO:0030170 & \begin{tabular}{|l|} 
pyridoxal \\
phosphate \\
binding
\end{tabular} & $\begin{array}{l}\text { MOLECULAR } \\
\text { FUNCTION }\end{array}$ & \begin{tabular}{|l|}
.252097071 \\
37125503
\end{tabular} & \begin{tabular}{|l|}
0.008111506 \\
106780433
\end{tabular} & 14 & 120 & 841 & 16153 & $\begin{array}{l}\text { MELO3C018948; } \\
\text { MELO3CO20927; } \\
\text { MELO3CO11284; } \\
\text { MELO3CO01121; } \\
\text { MELO3C020444; } \\
\text { MELO3CO01323; } \\
\text { MELO3CO23373; } \\
\text { MELO3COOO251; } \\
\text { MELO3CO26045; } \\
\text { MELO3CO26175; } \\
\text { MELO3CO15398; } \\
\text { MELO3CO11107; } \\
\text { MELO3C010686; } \\
\text { MELO3C016448 }\end{array}$ & 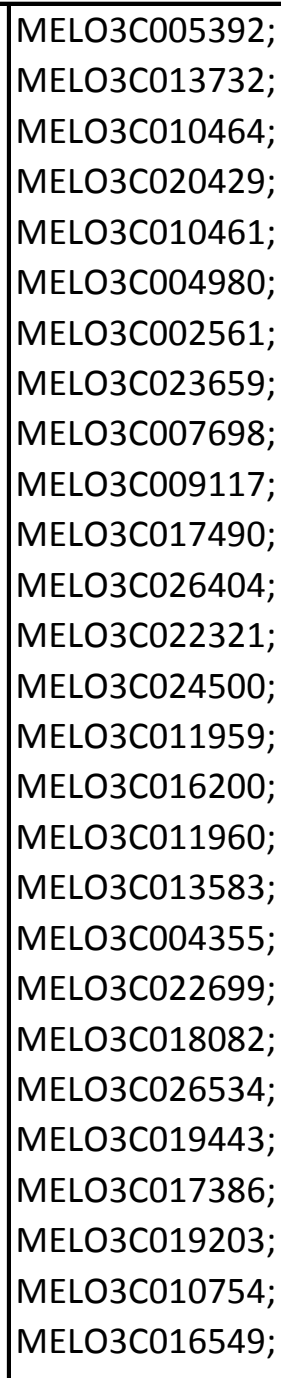 \\
\hline[] & GO:0004723 & \begin{tabular}{|l|} 
calcium- \\
dependent \\
protein \\
serine/threoni \\
ne \\
phosphatase \\
activity \\
\end{tabular} & $\begin{array}{l}\text { MOLECULAR } \\
\text { FUNCTION }\end{array}$ & \begin{tabular}{|l|}
0.252199617 \\
96018175
\end{tabular} & \begin{tabular}{|l|}
0.008301659 \\
7359882
\end{tabular} & 3 & 6 & 852 & 16267 & $\begin{array}{l}\text { MELO3C017844; } \\
\text { MELO3C013892; } \\
\text { MELO3C022260 }\end{array}$ & $\begin{array}{l}\text { MELO3C023443; } \\
\text { MELO3C019099; } \\
\text { MELO3CO18436; } \\
\text { MELO3C014069; } \\
\text { MELO3C026598; } \\
\text { MELO3CO07998 }\end{array}$ \\
\hline[] & GO:0051086 & \begin{tabular}{|l|} 
chaperone \\
mediated \\
protein folding \\
independent \\
of cofactor
\end{tabular} & \begin{tabular}{|l} 
BIOLOGICAL \\
PROCESS
\end{tabular} & \begin{tabular}{|l|}
0.255614581 \\
9474702
\end{tabular} & $\begin{array}{l}0.008711673 \\
940208024\end{array}$ & 4 & 13 & 851 & 16260 & $\begin{array}{l}\text { MELO3C002117; } \\
\text { MELO3CO09624; } \\
\text { MELO3C022094; } \\
\text { MELO3C010705 }\end{array}$ & $\begin{array}{l}\text { MELO3CO14127; } \\
\text { MELO3CO15615; } \\
\text { MELO3CO08567; } \\
\text { MELO3CO21633; } \\
\text { MELO3COO3128; } \\
\text { MELO3CO04458; } \\
\text { MELO3CO23558; } \\
\text { MELO3CO04636; } \\
\text { MELO3CO26888; } \\
\text { MELO3CO17295; } \\
\text { MELOOCO23852; } \\
\text { MELO3CO18683; } \\
\text { MELO3CO23264 }\end{array}$ \\
\hline[] & GO:0040010 & \begin{tabular}{|l|} 
positive \\
regulation of \\
growth rate
\end{tabular} & \begin{tabular}{|l} 
BIOLOGICAL \\
PROCESS
\end{tabular} & \begin{tabular}{|l|}
0.260820291 \\
43776764
\end{tabular} & \begin{tabular}{|l|}
0.008944302 \\
966324801
\end{tabular} & 23 & 238 & 832 & 16035 & 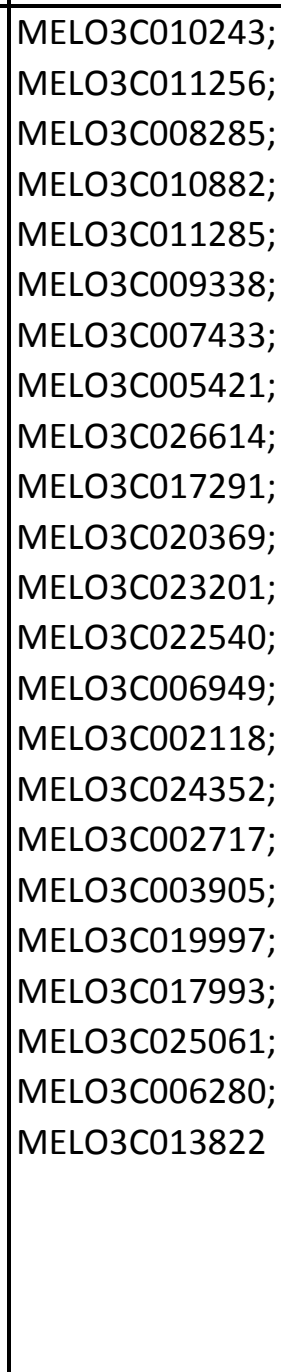 & 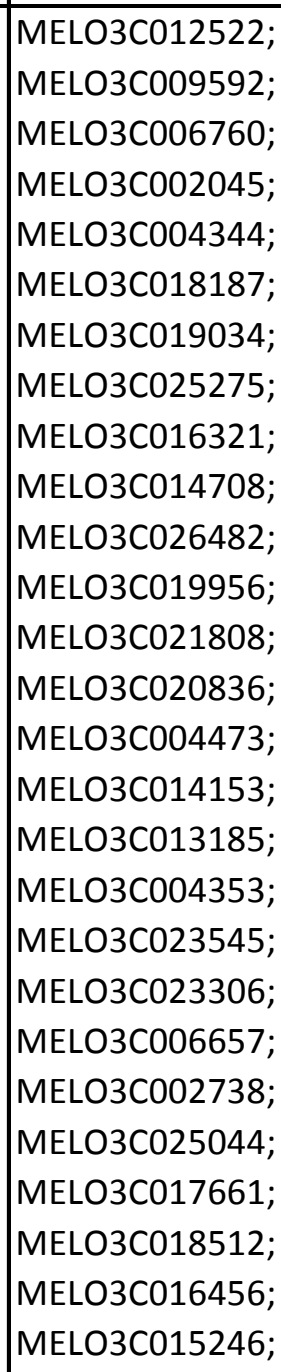 \\
\hline
\end{tabular}




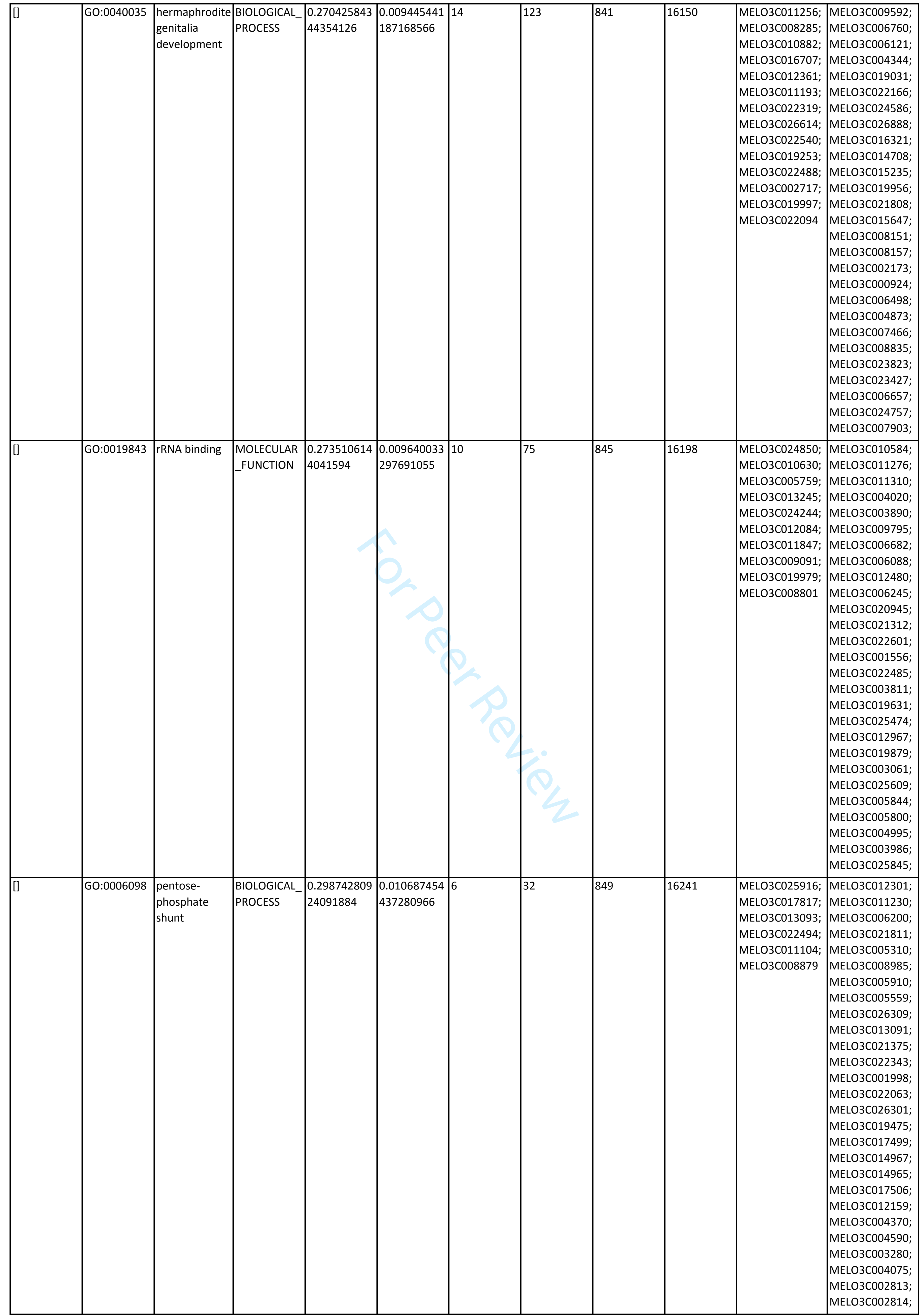




\begin{tabular}{|c|c|c|c|c|c|c|c|c|c|c|c|}
\hline [] & GO:0008237 & $\begin{array}{l}\text { metallopeptid } \\
\text { ase activity }\end{array}$ & $\begin{array}{l}\text { MOLECULAR } \\
\text { FUNCTION }\end{array}$ & $\begin{array}{l}0.298890668 \\
43941614\end{array}$ & $\begin{array}{l}0.010724379 \\
403150092\end{array}$ & 13 & 111 & 842 & 16162 & $\begin{array}{l}\text { MELO3C012356; } \\
\text { MELO3C008052; } \\
\text { MELO3C009148; } \\
\text { MELO3C011193; } \\
\text { MELO3C004134; } \\
\text { MELO3C006846; } \\
\text { MELO3C008847; } \\
\text { MELO3C003139; } \\
\text { MELO3C018441; } \\
\text { MELO3C026224; } \\
\text { MELO3C016890; } \\
\text { MELO3C016067; } \\
\text { MELO3C025494; }\end{array}$ & $\begin{array}{l}\text { MELO3C006089; } \\
\text { MELO3C014386; } \\
\text { MELO3C022843; } \\
\text { MELO3C019273; } \\
\text { MELO3C017011; } \\
\text { MELO3C026003; } \\
\text { MELO3C010623; } \\
\text { MELO3C016738; } \\
\text { MELO3C000768; } \\
\text { MELO3C005965; } \\
\text { MELO3C026539; } \\
\text { MELO3C023149; } \\
\text { MELO3C005688; } \\
\text { MELO3C015483; } \\
\text { MELO3C004918; } \\
\text { MELO3C023023; } \\
\text { MELO3C004919; } \\
\text { MELO3C013276; } \\
\text { MELO3C014882; } \\
\text { MELO3C003474; } \\
\text { MELO3C006466; } \\
\text { MELO3C025899; } \\
\text { MELO3C026225; } \\
\text { MELO3C000673; } \\
\text { MELO3C004605; } \\
\text { MELO3C025493; } \\
\text { MELO3C019536; }\end{array}$ \\
\hline[] & GO:0009535 & $\begin{array}{l}\text { chloroplast } \\
\text { thylakoid } \\
\text { membrane }\end{array}$ & $\begin{array}{l}\text { CELLULAR_C } \\
\text { OMPONENT }\end{array}$ & $\begin{array}{l}0.307464322 \\
89120923\end{array}$ & \begin{tabular}{|l|}
0.011227264 \\
119122268
\end{tabular} & 5 & 271 & 850 & 16002 & $\begin{array}{l}\text { MELO3C007153; } \\
\text { MELO3C011955; } \\
\text { MELO3C005759; } \\
\text { MELO3C022494; } \\
\text { MELO3C003139 }\end{array}$ & $\begin{array}{l}\text { MELO3C009475; } \\
\text { MELO3C005432; } \\
\text { MELO3C001992; } \\
\text { MELO3C026802; } \\
\text { MELO3C019395; } \\
\text { MELO3C025953; } \\
\text { MELO3C001998; } \\
\text { MELO3C025277; } \\
\text { MELO3C002727; } \\
\text { MELO3C022680; } \\
\text { MELO3C017774; } \\
\text { MELO3C018748; } \\
\text { MELO3C010469; } \\
\text { MELO3C009241; } \\
\text { MELO3C005562; } \\
\text { MELO3C014156; } \\
\text { MELO3C009245; } \\
\text { MELO3C006532; } \\
\text { MELO3C022335; } \\
\text { MELO3C021127; } \\
\text { MELO3C009809; } \\
\text { MELO3C027107; } \\
\text { MELO3C005329; } \\
\text { MELO3C006539; } \\
\text { MELO3C027106; } \\
\text { MELO3C007507; } \\
\text { MELO3C026259; }\end{array}$ \\
\hline [] & GO:0009695 & $\begin{array}{l}\text { jasmonic acid } \\
\text { biosynthetic } \\
\text { process }\end{array}$ & $\begin{array}{l}\text { BIOLOGICAL_ } \\
\text { PROCESS }\end{array}$ & $\begin{array}{l}0.309262993 \\
5311\end{array}$ & $\begin{array}{l}0.011423876 \\
454525179\end{array}$ & 3 & 7 & 852 & 16266 & $\begin{array}{l}\text { MELO3C009362; } \\
\text { MELO3C002470; } \\
\text { MELO3C015514 }\end{array}$ & $\begin{array}{l}\text { MELO3C004955; } \\
\text { MELO3C012388; } \\
\text { MELO3C010910; } \\
\text { MELO3C004565; } \\
\text { MELO3C008062; } \\
\text { MELO3C018734; } \\
\text { MELO3C002346 }\end{array}$ \\
\hline [] & GO:0005852 & $\begin{array}{l}\text { eukaryotic } \\
\text { translation } \\
\text { initiation } \\
\text { factor } 3 \\
\text { complex }\end{array}$ & $\begin{array}{l}\text { CELLULAR_C } \\
\text { OMPONENTT }\end{array}$ & $\begin{array}{l}0.309262993 \\
5311\end{array}$ & $\begin{array}{l}0.011423876 \\
454525179\end{array}$ & 3 & 7 & 852 & 16266 & $\begin{array}{l}\text { MELO3C011193; } \\
\text { MELO3C008285; } \\
\text { MELO3C003453 }\end{array}$ & $\begin{array}{l}\text { MELO3C026327; } \\
\text { MELO3C017707; } \\
\text { MELO3C009841; } \\
\text { MELO3C019434; } \\
\text { MELO3C01555; } \\
\text { MELO3C022038; } \\
\text { MELO3C007050 }\end{array}$ \\
\hline [] & GO:0019773 & $\begin{array}{l}\text { proteasome } \\
\text { core complex, } \\
\text { alpha-subunit } \\
\text { complex }\end{array}$ & $\begin{array}{l}\text { CELLULAR_C } \\
\text { OMPONENTT }\end{array}$ & $\begin{array}{l}0.309262993 \\
5311\end{array}$ & \begin{tabular}{|l|}
0.011423876 \\
454525179
\end{tabular} & 3 & 7 & 852 & 16266 & $\begin{array}{l}\text { MELO3C003173; } \\
\text { MELO3C010960; } \\
\text { MELO3C013757 }\end{array}$ & $\begin{array}{l}\text { MELO3C026800; } \\
\text { MELO3C024343; } \\
\text { MELO3C007920; } \\
\text { MELO3C021745; } \\
\text { MELO3C021746; } \\
\text { MELO3C02636; } \\
\text { MELO3C025735 }\end{array}$ \\
\hline
\end{tabular}




\begin{tabular}{|c|c|c|c|c|c|c|c|c|c|c|c|}
\hline [] & GO:0010171 & $\begin{array}{l}\text { body } \\
\text { morphogenesi } \\
\text { s }\end{array}$ & $\begin{array}{l}\text { BIOLOGICAL } \\
\text { PROCESS }\end{array}$ & $\begin{array}{l}0.323410779 \\
95372997\end{array}$ & $\begin{array}{l}0.012117635 \\
066005548\end{array}$ & 9 & 66 & 846 & 16207 & $\begin{array}{l}\text { MELO3C011256; } \\
\text { MELO3C008285; } \\
\text { MELO3C022488; } \\
\text { MELO3C010882; } \\
\text { MELO3C002717; } \\
\text { MELO3C019997; } \\
\text { MELO3C017993; } \\
\text { MELO3C005421; } \\
\text { MELO3C004459 }\end{array}$ & $\begin{array}{l}\text { MELO3C009592; } \\
\text { MELO3C014221; } \\
\text { MELO3C002120; } \\
\text { MELO3C004344; } \\
\text { MELO3C004622; } \\
\text { MELO3C007816; } \\
\text { MELO3C025310; } \\
\text { MELO3C019034; } \\
\text { MELO3C018102; } \\
\text { MELO3C025275; } \\
\text { MELO3C014708; } \\
\text { MELO3C019996; } \\
\text { MELO3C013814; } \\
\text { MELO3C017216; } \\
\text { MELO3C012449; } \\
\text { MELO3C019956; } \\
\text { MELO3C020836; } \\
\text { MELO3C014153; } \\
\text { MELO3C000924; } \\
\text { MELO3C003222; } \\
\text { MELO3C022016; } \\
\text { MELO3C023865; } \\
\text { MELO3C008835; } \\
\text { MELO3C007829; } \\
\text { MELO3C007903; } \\
\text { MELO3C023741; } \\
\text { MELO3C026971; }\end{array}$ \\
\hline [] & GO:1901617 & $\begin{array}{l}\text { organic } \\
\text { hydroxy } \\
\text { compound } \\
\text { biosynthetic } \\
\text { process }\end{array}$ & $\begin{array}{l}\text { BIOLOGICAL_ } \\
\text { PROCESS }\end{array}$ & $\begin{array}{l}0.324046881 \\
660428\end{array}$ & $\begin{array}{l}0.012175766 \\
616157064\end{array}$ & 10 & 78 & 845 & 16195 & $\begin{array}{l}\text { MELO3C024861; } \\
\text { MELO3C024982; } \\
\text { MELO3C003726; } \\
\text { MELO3C017581; } \\
\text { MELO3C004519; } \\
\text { MELO3C014175; } \\
\text { MELO3C011062; } \\
\text { MELO3C017963; } \\
\text { MELO3C003541; } \\
\text { MELO3C004278 }\end{array}$ & $\begin{array}{l}\text { MELO3C017815; } \\
\text { MELO3C013732; } \\
\text { MELO3C011037; } \\
\text { MELO3C010781; } \\
\text { MELO3C012288; } \\
\text { MELO3C012088; } \\
\text { MELO3C008543; } \\
\text { MELO3C009431; } \\
\text { MELO3C008942; } \\
\text { MELO3C011150; } \\
\text { MELO3C007774; } \\
\text { MELO3C005515; } \\
\text { MELO3C006525; } \\
\text { MELO3C020384; } \\
\text { MELO3C022961; } \\
\text { MELO3C019790; } \\
\text { MELO3C017176; } \\
\text { MELO3C017495; } \\
\text { MELO3C017057; } \\
\text { MELO3C027255; } \\
\text { MELO3C024061; } \\
\text { MELO3C016008; } \\
\text { MELO3C016845; } \\
\text { MELO3C007140; } \\
\text { MELO3C017829; } \\
\text { MELO3C009168; } \\
\text { MELO3C006011; }\end{array}$ \\
\hline [] & GO:0042450 & $\begin{array}{l}\text { arginine } \\
\text { biosynthetic } \\
\text { process via } \\
\text { ornithine }\end{array}$ & $\begin{array}{l}\text { BIOLOGICAL_ } \\
\text { PROCESS }\end{array}$ & \begin{tabular}{|l|}
0.344398054 \\
3808898
\end{tabular} & \begin{tabular}{|l|}
0.013961098 \\
097785857
\end{tabular} & 2 & 2 & 853 & 16271 & $\begin{array}{l}\text { MELO3C011085; } \\
\text { MELO3C011208 }\end{array}$ & $\begin{array}{l}\text { MELO3C023629; } \\
\text { MELO3C003523 }\end{array}$ \\
\hline [] & GO:0006272 & $\begin{array}{l}\text { leading strand } \\
\text { elongation }\end{array}$ & $\begin{array}{l}\text { BIOLOGICAL } \\
\text { PROCESS }\end{array}$ & \begin{tabular}{|l|}
0.344398054 \\
3808898
\end{tabular} & \begin{tabular}{|l|}
0.013961098 \\
097785857
\end{tabular} & 2 & 2 & 853 & 16271 & $\begin{array}{l}\text { MELO3C010631; } \\
\text { MELO3C020860 }\end{array}$ & $\begin{array}{l}\text { MELO3C014021; } \\
\text { MELO3C010507 }\end{array}$ \\
\hline [] & GO:0004133 & \begin{tabular}{|l} 
glycogen \\
debranching \\
enzyme \\
activity
\end{tabular} & $\begin{array}{l}\text { MOLECULAR } \\
\text { FUNCTION }\end{array}$ & \begin{tabular}{|l|}
0.344398054 \\
3808898
\end{tabular} & \begin{tabular}{|l|}
0.013961098 \\
097785857
\end{tabular} & 2 & 2 & 853 & 16271 & $\begin{array}{l}\text { MELO3C015861; } \\
\text { MELO3C019135 }\end{array}$ & \begin{tabular}{|l} 
MELO3C017391; \\
MELO3C020949
\end{tabular} \\
\hline [] & GO:0005664 & $\begin{array}{l}\text { nuclear origin } \\
\text { of replication } \\
\text { recognition } \\
\text { complex }\end{array}$ & $\begin{array}{l}\text { CELLULAR_C } \\
\text { OMPONENT }\end{array}$ & $\begin{array}{l}0.344398054 \\
3808898\end{array}$ & \begin{tabular}{|l|}
0.013961098 \\
097785857
\end{tabular} & 2 & 2 & 853 & 16271 & $\begin{array}{l}\text { MELO3C005455; } \\
\text { MELO3C010882 }\end{array}$ & $\begin{array}{l}\text { MELO3C008530; } \\
\text { MELO3C021204 }\end{array}$ \\
\hline [] & GO:0008541 & $\begin{array}{l}\text { proteasome } \\
\text { regulatory } \\
\text { particle, lid } \\
\text { subcomplex }\end{array}$ & $\begin{array}{l}\text { CELLULAR_C } \\
\text { OMPONENTT }\end{array}$ & \begin{tabular}{|l|}
0.344398054 \\
3808898
\end{tabular} & \begin{tabular}{|l|}
0.013961098 \\
097785857
\end{tabular} & 2 & 2 & 853 & 16271 & $\begin{array}{l}\text { MELO3C016262; } \\
\text { MELO3C011193 }\end{array}$ & $\begin{array}{l}\text { MELO3C025923; } \\
\text { MELO3C013814 }\end{array}$ \\
\hline [] & GO:0031307 & $\begin{array}{l}\text { integral } \\
\text { component of } \\
\text { mitochondrial } \\
\text { outer } \\
\text { membrane }\end{array}$ & $\begin{array}{l}\text { CELLULAR_C } \\
\text { OMPONENT }\end{array}$ & $\begin{array}{l}0.344398054 \\
3808898\end{array}$ & \begin{tabular}{|l|}
0.013961098 \\
097785857
\end{tabular} & 2 & 2 & 853 & 16271 & $\begin{array}{l}\text { MELO3C004519; } \\
\text { MELO3C019820 }\end{array}$ & \begin{tabular}{|l} 
MELO3C019821; \\
MELO3C013700
\end{tabular} \\
\hline
\end{tabular}




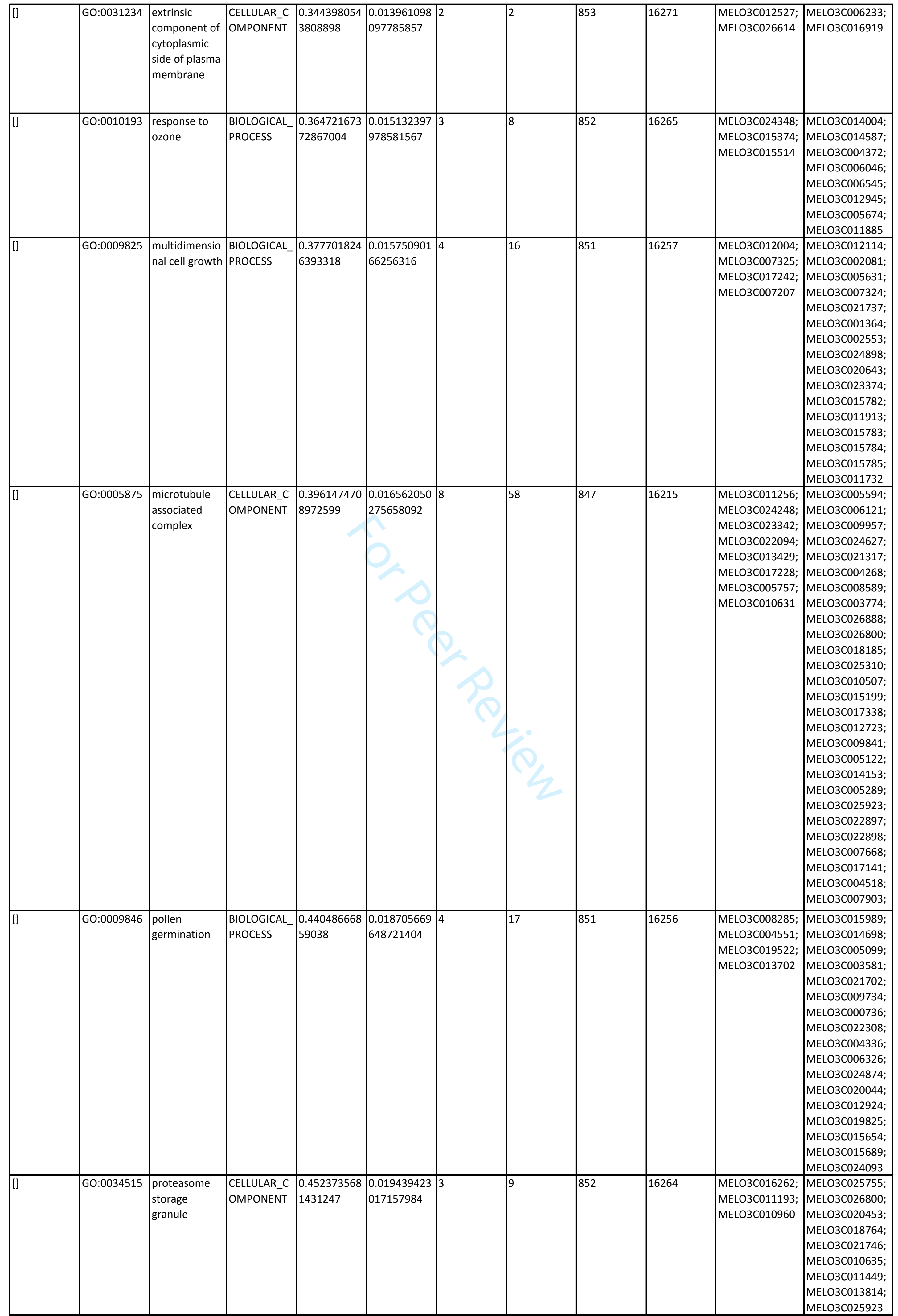




\begin{tabular}{|c|c|c|c|c|c|c|c|c|c|c|c|}
\hline[] & GO:0004177 & \begin{tabular}{|l|} 
aminopeptidas \\
e activity
\end{tabular} & $\begin{array}{l}\text { MOLECULAR } \\
\text { FUNCTION }\end{array}$ & \begin{tabular}{|l|}
0.464617069 \\
97455804
\end{tabular} & \begin{tabular}{|l|}
0.020113080 \\
188356714
\end{tabular} & 5 & 27 & 850 & 16246 & $\begin{array}{l}\text { MELO3C012356; } \\
\text { MELO3CO04134; } \\
\text { MELO3CO09148; } \\
\text { MELO3C026224; } \\
\text { MELO3C016890 }\end{array}$ & 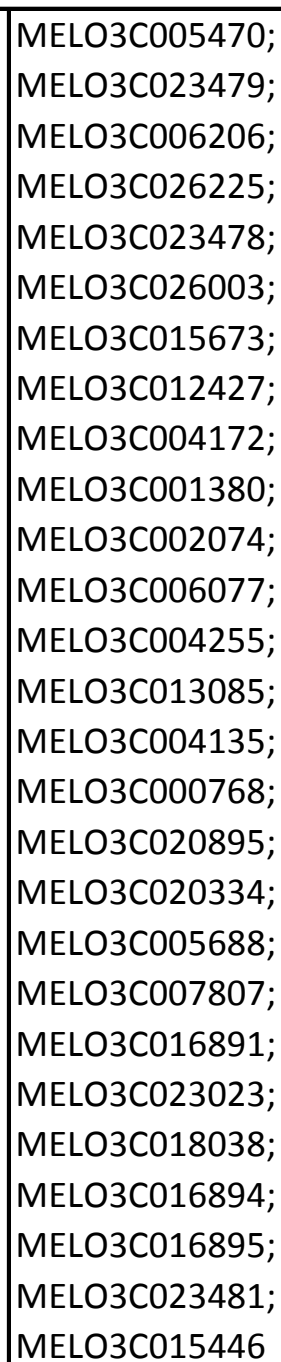 \\
\hline[] & GO:0003924 & \begin{tabular}{|l} 
GTPase \\
activity
\end{tabular} & $\begin{array}{l}\text { MOLECULAR } \\
\text { FUNCTION }\end{array}$ & \begin{tabular}{|l|}
.479601191 \\
6051134
\end{tabular} & \begin{tabular}{|l|}
0.021015547 \\
55763304
\end{tabular} & 11 & 95 & 844 & 16178 & $\begin{array}{l}\text { MELO3C011234; } \\
\text { MELO3COO2118; } \\
\text { MELO3CO25753; } \\
\text { MELO3CO22411; } \\
\text { MELO3CO24544; } \\
\text { MELO3CO25885; } \\
\text { MELO3CO09203; } \\
\text { MELO3COO7673; } \\
\text { MELO3C015098; } \\
\text { MELO3CO05673; } \\
\text { MELO3CO02687 }\end{array}$ & 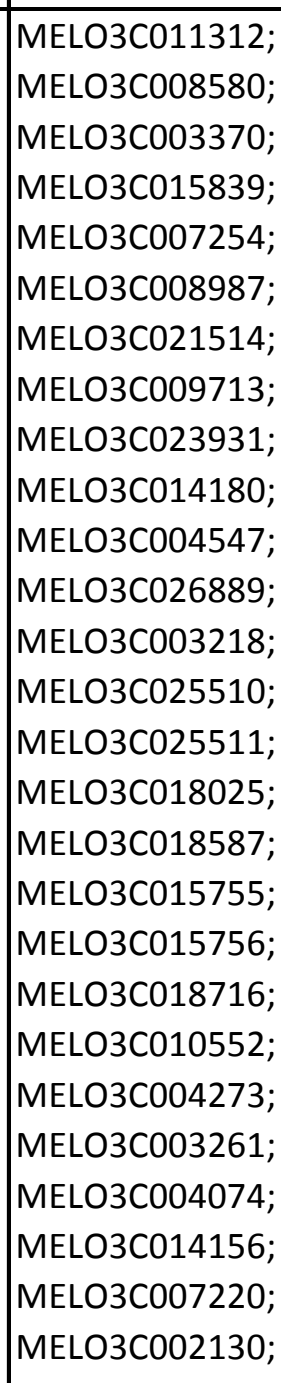 \\
\hline[] & GO:0000076 & \begin{tabular}{|l|} 
DNA \\
replication \\
checkpoint
\end{tabular} & \begin{tabular}{|l} 
BIOLOGICAL \\
PROCESS
\end{tabular} & \begin{tabular}{|l|}
0.494426130 \\
26338766
\end{tabular} & \begin{tabular}{|l|}
0.022502459 \\
357880686
\end{tabular} & 2 & 3 & 853 & 16270 & $\begin{array}{l}\text { MELO3C020860; } \\
\text { MELO3C021058 }\end{array}$ & $\begin{array}{l}\text { MELO3C016039; } \\
\text { MELO3C013324; } \\
\text { MELO3C007925 }\end{array}$ \\
\hline $\mid[]$ & GO:0016882 & $\begin{array}{l}\text { cyclo-ligase } \\
\text { activity }\end{array}$ & \begin{tabular}{|l|} 
MOLECULAR \\
_FUNCTION
\end{tabular} & \begin{tabular}{|l|}
0.494426130 \\
26338766
\end{tabular} & \begin{tabular}{|l|}
0.022502459 \\
357880686
\end{tabular} & 2 & 3 & 853 & 16270 & \begin{tabular}{|l} 
MELO3C016483; \\
MELO3C008332
\end{tabular} & $\begin{array}{l}\text { MELO3C005781; } \\
\text { MELO3C017138; } \\
\text { MELO3C017137 }\end{array}$ \\
\hline [] & GO:0008878 & \begin{tabular}{|l|} 
glucose-1- \\
phosphate \\
adenylyltransf \\
erase activity
\end{tabular} & $\begin{array}{l}\text { MOLECULAR } \\
\text { FUNCTION }\end{array}$ & \begin{tabular}{|l|}
0.494426130 \\
26338766
\end{tabular} & \begin{tabular}{|l|}
0.022502459 \\
357880686
\end{tabular} & 2 & 3 & 853 & 16270 & $\begin{array}{l}\text { MELO3C003812; } \\
\text { MELO3C023310 }\end{array}$ & $\begin{array}{l}\text { MELO3C006552; } \\
\text { MELO3C024175; } \\
\text { MELO3C021412 }\end{array}$ \\
\hline \begin{tabular}{|l}
{[]} \\
\end{tabular} & GO:0008963 & \begin{tabular}{|l|} 
phospho-N- \\
acetylmuramo \\
yl- \\
pentapeptide- \\
transferase \\
activity
\end{tabular} & $\begin{array}{l}\text { MOLECULAR } \\
\text {-FUNCTION }\end{array}$ & \begin{tabular}{|l|}
0.494426130 \\
26338766
\end{tabular} & \begin{tabular}{|l|}
0.022502459 \\
357880686
\end{tabular} & 2 & 3 & 853 & 16270 & \begin{tabular}{|l} 
MELO3C009489; \\
MELO3C009490
\end{tabular} & $\begin{array}{l}\text { MELO3C002715; } \\
\text { MELO3C002716; } \\
\text { MELO3C014202 }\end{array}$ \\
\hline [] & GO:0009815 & \begin{tabular}{|l|}
$1-$ \\
aminocyclopro \\
pane-1- \\
carboxylate \\
oxidase \\
activity
\end{tabular} & $\begin{array}{l}\text { MOLECULAR } \\
\text { FUNCTION }\end{array}$ & $\begin{array}{l}0.494426130 \\
26338766\end{array}$ & \begin{tabular}{|l|}
0.022502459 \\
357880686
\end{tabular} & 2 & 3 & 853 & 16270 & $\begin{array}{l}\text { MELO3C007425; } \\
\text { MELO3C019735 }\end{array}$ & $\begin{array}{l}\text { MELO3C010508; } \\
\text { MELO3C006437; } \\
\text { MELO3C014437 }\end{array}$ \\
\hline [] & \begin{tabular}{|l|} 
GO:0019781 \\
\end{tabular} & $\begin{array}{l}\text { NEDD8 } \\
\text { activating } \\
\text { enzyme } \\
\text { activity } \\
\end{array}$ & \begin{tabular}{|l|} 
MOLECULAR \\
_FUNCTION
\end{tabular} & \begin{tabular}{|l|}
0.494426130 \\
26338766
\end{tabular} & \begin{tabular}{|l|}
0.022502459 \\
357880686
\end{tabular} & 2 & 3 & 853 & 16270 & $\begin{array}{l}\text { MELO3C011193; } \\
\text { MELO3C006555 }\end{array}$ & $\begin{array}{l}\text { MELO3C008213; } \\
\text { MELO3C014386; } \\
\text { MELO3C013230 }\end{array}$ \\
\hline
\end{tabular}




\begin{tabular}{|c|c|c|c|c|c|c|c|c|c|c|c|}
\hline[] & GO:0016906 & \begin{tabular}{|l} 
sterol 3-beta- \\
glucosyltransfe \\
rase activity
\end{tabular} & $\begin{array}{l}\text { MOLECULAR } \\
\text { FUNCTION }\end{array}$ & $\begin{array}{l}0.494426130 \\
26338766\end{array}$ & \begin{tabular}{|l|}
0.022502459 \\
357880686
\end{tabular} & 2 & 3 & 853 & 16270 & $\begin{array}{l}\text { MELO3C020997; } \\
\text { MELO3C012787 }\end{array}$ & $\begin{array}{l}\text { MELO3C010080; } \\
\text { MELO3C010079; } \\
\text { MELO3C008339 }\end{array}$ \\
\hline[] & GO:0016709 & $\begin{array}{l}\text { oxidoreductas } \\
\text { e activity, } \\
\text { acting on } \\
\text { paired donors, } \\
\text { with } \\
\text { incorporation } \\
\text { or reduction of } \\
\text { molecular } \\
\text { oxygen, } \\
\text { NAD(P)H as } \\
\text { one donor, } \\
\text { and } \\
\text { incorporation } \\
\text { of one atom of } \\
\text { oxygen }\end{array}$ & $\begin{array}{l}\text { MOLECULAR } \\
\text { FUNCTION }\end{array}$ & \begin{tabular}{|l|}
0.494426130 \\
26338766
\end{tabular} & \begin{tabular}{|l|}
0.022296311 \\
975877454
\end{tabular} & 0 & 84 & 855 & 16189 & & 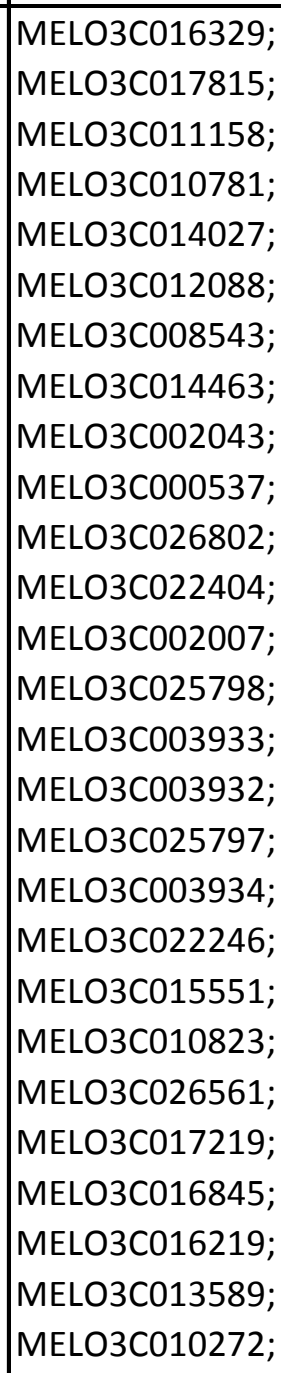 \\
\hline[] & GO:0031261 & \begin{tabular}{|l|} 
DNA \\
replication \\
preinitiation \\
complex
\end{tabular} & \begin{tabular}{|l|} 
CELLULAR_C \\
OMPONENT
\end{tabular} & \begin{tabular}{|l|}
0.494426130 \\
26338766
\end{tabular} & $\begin{array}{l}0.022502459 \\
357880686\end{array}$ & 2 & 3 & 853 & 16270 & $\begin{array}{l}\text { MELO3C010882; } \\
\text { MELO3C021058 }\end{array}$ & $\begin{array}{l}\text { MELO3C006096; } \\
\text { MELO3C024840; } \\
\text { MELO3C023404 }\end{array}$ \\
\hline[] & GO:0006553 & $\begin{array}{l}\text { lysine } \\
\text { metabolic } \\
\text { process }\end{array}$ & \begin{tabular}{|l} 
BIOLOGICAL \\
PROCESS
\end{tabular} & \begin{tabular}{|l|}
0.527670345 \\
5771554
\end{tabular} & \begin{tabular}{|l|}
0.024350579 \\
029597773
\end{tabular} & 3 & 10 & 852 & 16263 & $\begin{array}{l}\text { MELO3C010427; } \\
\text { MELO3C023315; } \\
\text { MELO3C021247 }\end{array}$ & $\begin{array}{l}\text { MELO3CO24345; } \\
\text { MELO3COO9153; } \\
\text { MELO3COO8136; } \\
\text { MELO3CO03100; } \\
\text { MELO3C018037; } \\
\text { MELO3COO6179; } \\
\text { MELO3C013171; } \\
\text { MELO3C015556; } \\
\text { MELO3CO06876; } \\
\text { MELO3CO06734 }\end{array}$ \\
\hline[] & \begin{tabular}{|l|l|} 
GO:0008967 \\
\end{tabular} & $\begin{array}{l}\text { phosphoglycol } \\
\text { ate } \\
\text { phosphatase } \\
\text { activity }\end{array}$ & $\begin{array}{l}\text { MOLECULAR } \\
\text { _FUNCTION }\end{array}$ & \begin{tabular}{|l|}
0.527670345 \\
5771554
\end{tabular} & \begin{tabular}{|l|}
0.024350579 \\
029597773
\end{tabular} & 3 & 10 & 852 & 16263 & $\begin{array}{l}\text { MELO3C017462; } \\
\text { MELO3C013878; } \\
\text { MELO3C007011 }\end{array}$ & $\begin{array}{l}\text { MELO3C018727; } \\
\text { MELO3CO02558; } \\
\text { MELO3C025302; } \\
\text { MELO3CO06364; } \\
\text { MELO3C016693; } \\
\text { MELO3C022462; } \\
\text { MELO3CO08753; } \\
\text { MELO3CO08533; } \\
\text { MELO3CO09765; } \\
\text { MELO3C019407 }\end{array}$ \\
\hline[] & GO:0006898 & $\begin{array}{l}\text { receptor- } \\
\text { mediated } \\
\text { endocytosis }\end{array}$ & \begin{tabular}{|l} 
BIOLOGICAL \\
PROCESS
\end{tabular} & \begin{tabular}{|l|}
.545390005 \\
5429007
\end{tabular} & \begin{tabular}{|l|}
0.025399195 \\
85508852
\end{tabular} & 13 & 122 & 842 & 16151 & 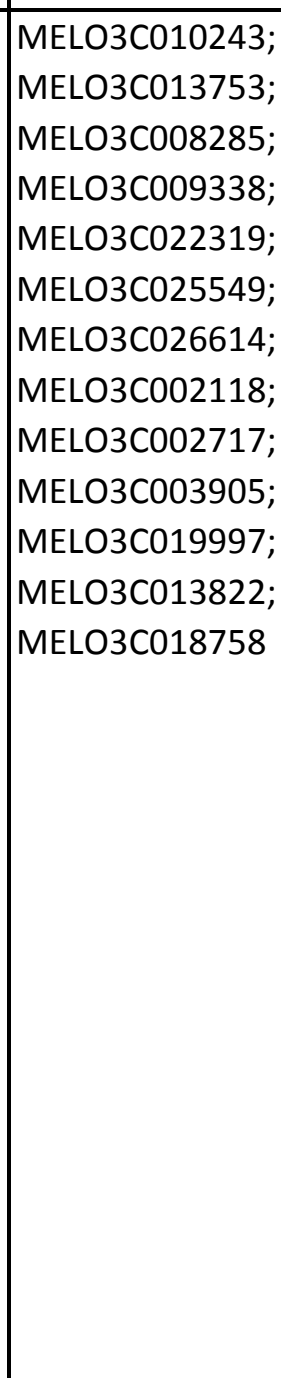 & 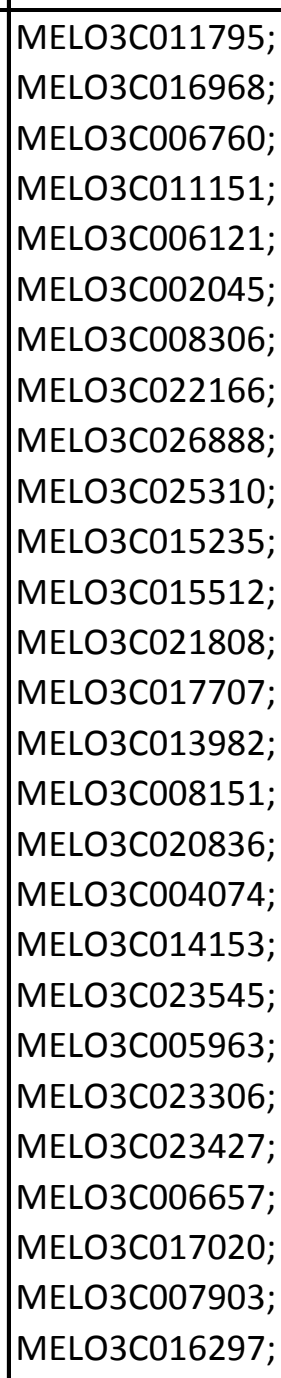 \\
\hline
\end{tabular}




\begin{tabular}{|c|c|c|c|c|c|c|c|c|c|c|}
\hline [] & |GO:0005768 & endosome & $\begin{array}{l}\text { CELLULAR_C } \\
\text { OMPONENT }\end{array}$ & $\begin{array}{l}0.565805720 \\
8745785\end{array}$ & \begin{tabular}{|l|}
0.026649401 \\
54415616
\end{tabular} & 64 & 847 & 16209 & $\begin{array}{l}\text { MELO3CO10243; } \\
\text { MELO3CO06926; } \\
\text { MELO3CO13753; } \\
\text { MELO3CO04391; } \\
\text { MELO3CO10772; } \\
\text { MELO3CO25783; } \\
\text { MELO3CO26614; } \\
\text { MELO3C018758 }\end{array}$ & 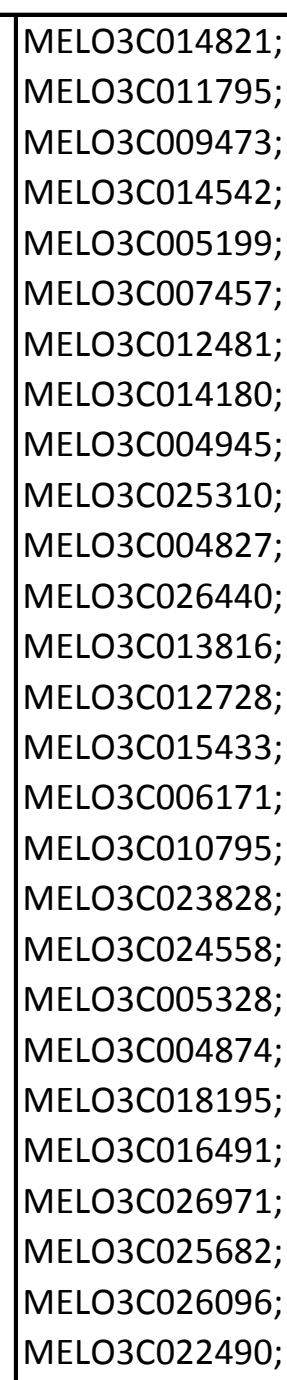 \\
\hline [1] & GO:0005543 & $\begin{array}{l}\text { phospholipid } \\
\text { binding }\end{array}$ & $\begin{array}{l}\text { MOLECULAR } \\
\text { FUNCTION }\end{array}$ & $\begin{array}{l}0.567870912 \\
6777027\end{array}$ & \begin{tabular}{|l|}
0.026926986 \\
5452594
\end{tabular} & 52 & 848 & 16221 & 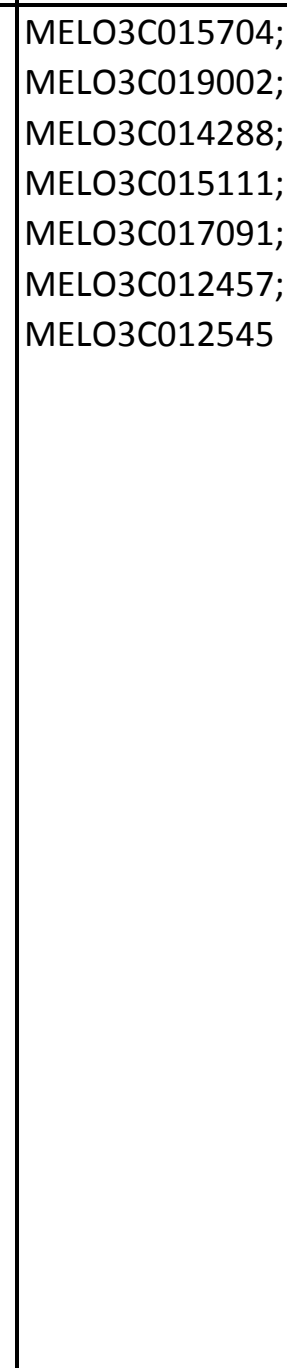 & 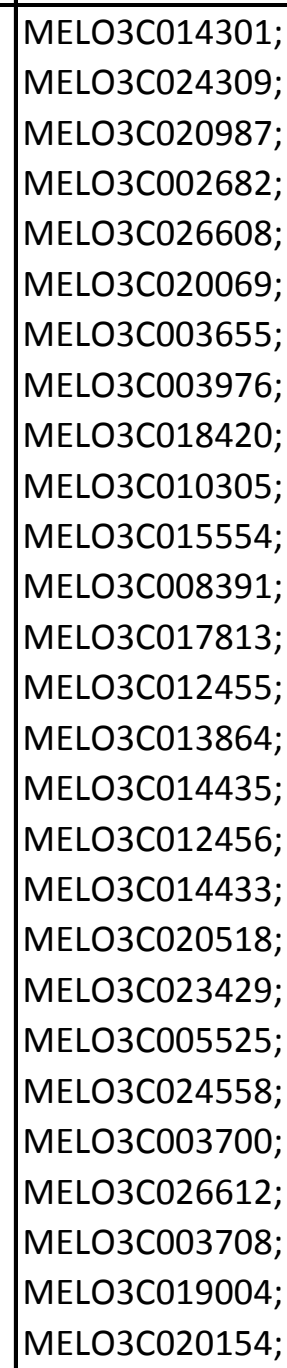 \\
\hline [] & GO:0008380 & RNA splicing & $\begin{array}{l}\text { BBOLOGICAL } \\
\text { PROCESS }\end{array}$ & \begin{tabular}{|l|l|}
0.570330694 \\
6368167
\end{tabular} & \begin{tabular}{|l|}
0.027116409 \\
352641695
\end{tabular} & 185 & 852 & 16088 & $\begin{array}{l}\text { MELO3CO11256; } \\
\text { MELO3CO08332; } \\
\text { MELOЗC016382 }\end{array}$ & 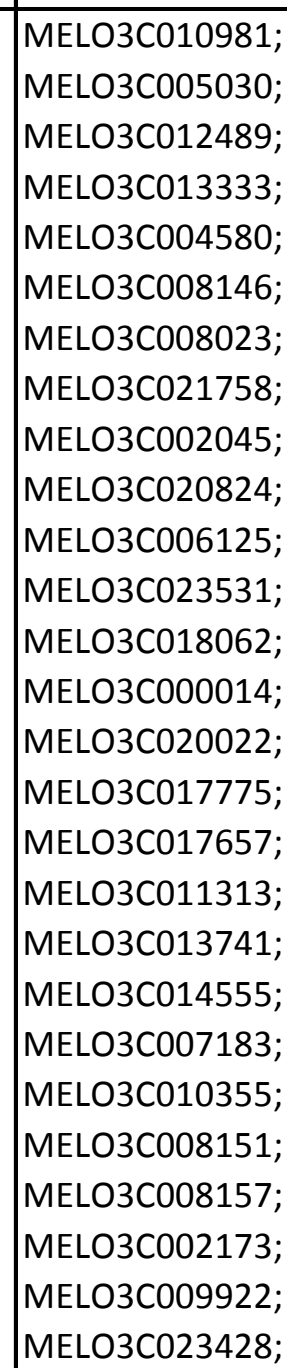 \\
\hline
\end{tabular}




\begin{tabular}{|c|c|c|c|c|c|c|c|c|c|c|c|}
\hline$[0]$ & GO:0000323 & 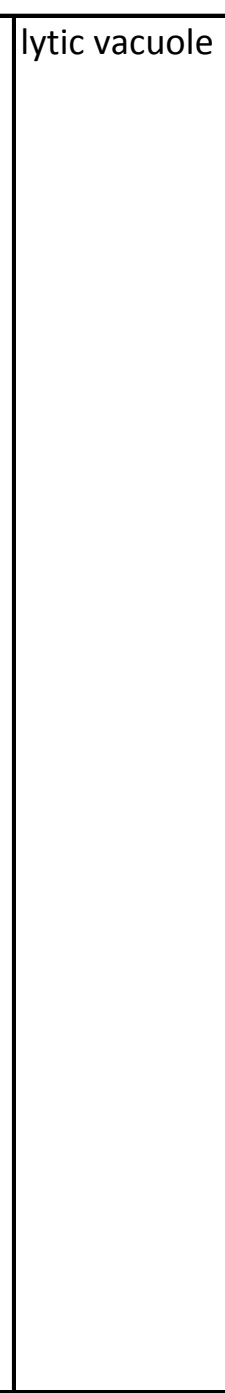 & $\begin{array}{l}\text { CELLULAR_C } \\
\text { OMPONENT }\end{array}$ & \begin{tabular}{|l|}
0.596008937 \\
9346575
\end{tabular} & \begin{tabular}{|l|}
0.028702801 \\
308241866
\end{tabular} & & 30 & 850 & 16243 & $\begin{array}{l}\text { MELO3C010243; } \\
\text { MELO3C006926; } \\
\text { MELO3C025645; } \\
\text { MELO3C025061; } \\
\text { MELO3C023569 }\end{array}$ & 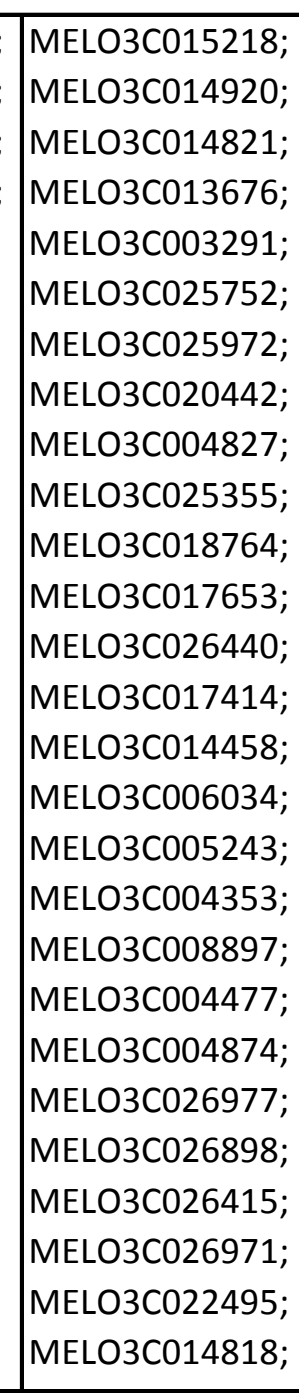 \\
\hline [] & |GO:0009617 & $\begin{array}{l}\text { response to } \\
\text { bacterium }\end{array}$ & $\begin{array}{l}\text { BIOLOGGICAL_ } \\
\text { PROCESS }\end{array}$ & $-\left[\begin{array}{l}.6033053389 \\
6150709\end{array}\right.$ & $\begin{array}{l}0.029676057 \\
561601084\end{array}$ & 10 & 87 & 845 & 16186 & $\begin{array}{l}\text { MELO3CO12136; } \\
\text { MELO3CO18573; } \\
\text { MELO3COO3906; } \\
\text { MELO3CO16033; } \\
\text { MELO3CO15374; } \\
\text { MELO3CCO05147; } \\
\text { MELO3CO16972; } \\
\text { MELO3CO13757; } \\
\text { MELO3CO24348; } \\
\text { MELO3CO09429 }\end{array}$ & 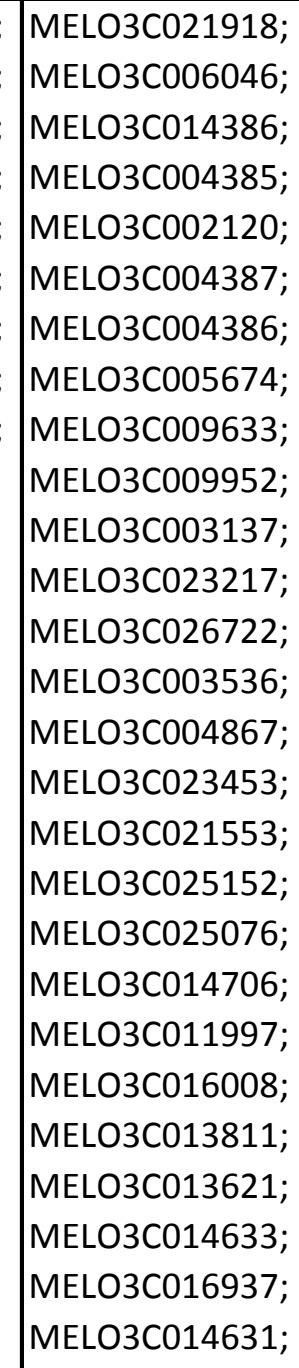 \\
\hline [] & GO:0009225 & \begin{tabular}{|l} 
nucleotide- \\
sugar \\
metabolic \\
process
\end{tabular} & $\begin{array}{l}\text { BBOLOGICAL } \\
\text { PROCESS }\end{array}$ & $\begin{array}{l}0.603305389 \\
6150709\end{array}$ & \begin{tabular}{|l|}
0.029524383 \\
7540197966
\end{tabular} & 4 & 20 & 851 & 16253 & $\begin{array}{l}\text { MELO3CO03812; } \\
\text { MELO3CO23310; } \\
\text { MELO3COO7043; } \\
\text { MELO3C020244 }\end{array}$ & 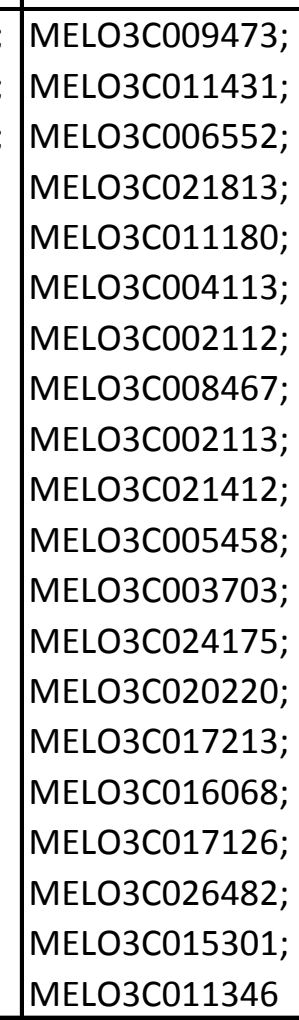 \\
\hline
\end{tabular}




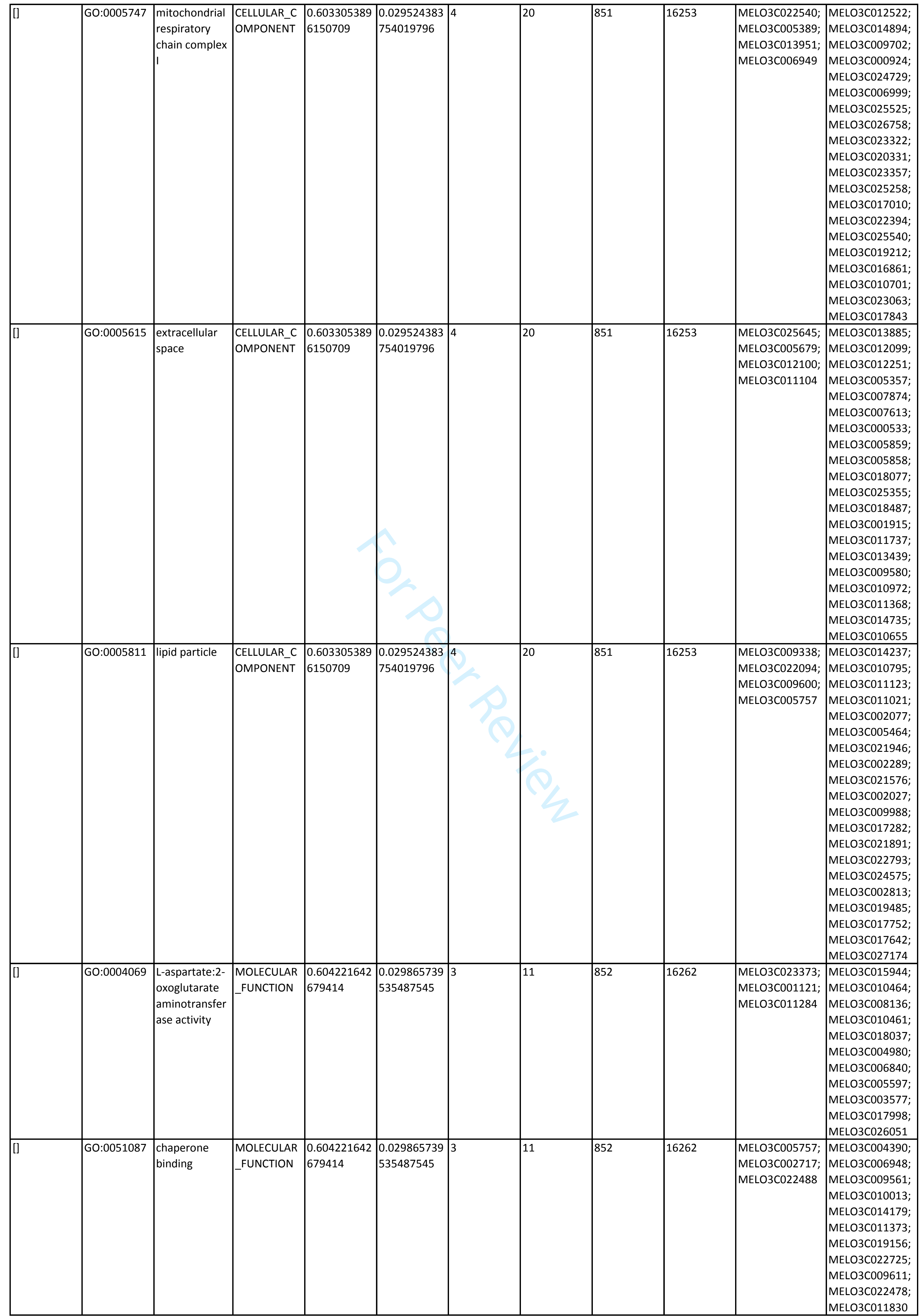




\begin{tabular}{|c|c|c|c|c|c|c|c|c|c|c|c|}
\hline[] & GO:0051287 & NAD binding & $\begin{array}{l}\text { MOLECULAR } \\
\text { FUNCTION }\end{array}$ & \begin{tabular}{|l|}
0.622789509 \\
948272
\end{tabular} & \begin{tabular}{|l|}
0.031640449 \\
277642944
\end{tabular} & 7 & 54 & 848 & 16219 & $\begin{array}{l}\text { MELO3C021563; } \\
\text { MELO3C019542; } \\
\text { MELO3C010727; } \\
\text { MELO3C010427; } \\
\text { MELO3C021604; } \\
\text { MELO3C019633; } \\
\text { MELO3C008219 }\end{array}$ & $\begin{array}{l}\text { MELO3C006085; } \\
\text { MELO3C021919; } \\
\text { MELO3C011431; } \\
\text { MELO3C006164; } \\
\text { MELO3C014221; } \\
\text { MELO3C009956; } \\
\text { MELO3C024942; } \\
\text { MELO3C005116; } \\
\text { MELO3C023217; } \\
\text { MELO3C017213; } \\
\text { MELO3C025076; } \\
\text { MELO3C025791; } \\
\text { MELO3C027054; } \\
\text { MELO3C017853; } \\
\text { MELO3C015407; } \\
\text { MELO3C014752; } \\
\text { MELO3C022537; } \\
\text { MELO3C022538; } \\
\text { MELO3C020158; } \\
\text { MELO3C020157; } \\
\text { MELO3C026337; } \\
\text { MELO3C024039; } \\
\text { MELO3C002813; } \\
\text { MELO3C027223; } \\
\text { MELO3C013505; } \\
\text { MELO3C011129; } \\
\text { MELO3C019405; }\end{array}$ \\
\hline [] & GO:0003887 & $\begin{array}{l}\text { DNA-directed } \\
\text { DNA } \\
\text { polymerase } \\
\text { activity }\end{array}$ & $\begin{array}{l}\text { MOLECULAR } \\
\text { FUNCTION }\end{array}$ & \begin{tabular}{|l|}
0.624981353 \\
74969
\end{tabular} & $\begin{array}{l}0.032009283 \\
040244606\end{array}$ & 5 & 31 & 850 & 16242 & $\begin{array}{l}\text { MELO3C002044; } \\
\text { MELO3C022319; } \\
\text { MELO3C020860; } \\
\text { MELO3C010631; } \\
\text { MELO3C021434 }\end{array}$ & $\begin{array}{l}\text { MELO3C011257; } \\
\text { MELO3C016705; } \\
\text { MELO3C007194; } \\
\text { MELO3C005496; } \\
\text { MELO3C007012; } \\
\text { MELO3C014021; } \\
\text { MELO3C014020; } \\
\text { MELO3C009536; } \\
\text { MELO3C021435; } \\
\text { MELO3C005956; } \\
\text { MELO3C005957; } \\
\text { MELO3C001935; } \\
\text { MELO3C010507; } \\
\text { MELO3C001936; } \\
\text { MELO3C025396; } \\
\text { MELO3C001937; } \\
\text { MELO3C018521; } \\
\text { MELO3C025395; } \\
\text { MELO3C014529; } \\
\text { MELO3C011159; } \\
\text { MELO3C014503; } \\
\text { MELO3C002892; } \\
\text { MELO3C004237; } \\
\text { MELO3C004239; } \\
\text { MELO3C004238; } \\
\text { MELO3C024597; } \\
\text { MELO3C018652; }\end{array}$ \\
\hline [] & GO:0009098 & $\begin{array}{l}\text { leucine } \\
\text { biosynthetic } \\
\text { process }\end{array}$ & $\begin{array}{l}\text { BIOLOGICAL_ } \\
\text { PROCESS }\end{array}$ & \begin{tabular}{|l|}
0.628210989 \\
0968163
\end{tabular} & \begin{tabular}{|l|}
0.032647289 \\
97105597
\end{tabular} & 2 & 4 & 853 & 16269 & $\begin{array}{l}\text { MELO3C005518; } \\
\text { MELO3C003344 }\end{array}$ & $\begin{array}{l}\text { MELO3C017853; } \\
\text { MELO3C024817; } \\
\text { MELO3C014221; } \\
\text { MELO3C020296 }\end{array}$ \\
\hline [] & GO:0052542 & $\begin{array}{l}\text { defense } \\
\text { response by } \\
\text { callose } \\
\text { deposition }\end{array}$ & $\begin{array}{l}\text { BIOLOGICAL_ } \\
\text { PROCESS }\end{array}$ & \begin{tabular}{|l|}
0.628210989 \\
0968163
\end{tabular} & \begin{tabular}{|l|}
.032647289 \\
97105597
\end{tabular} & 2 & 4 & 853 & 16269 & $\begin{array}{l}\text { MELO3C027349; } \\
\text { MELO3C003906 }\end{array}$ & $\begin{array}{l}\text { MELO3C014230; } \\
\text { MELO3C013621; } \\
\text { MELO3C005674; } \\
\text { MELO3C005718 }\end{array}$ \\
\hline [] & GO:0006103 & $\begin{array}{l}\text { 2-oxoglutarate } \\
\text { metabolic } \\
\text { process }\end{array}$ & $\begin{array}{l}\text { BIOLOGICAL_ } \\
\text { PROCESS }\end{array}$ & \begin{tabular}{|l|}
0.628210989 \\
0968163
\end{tabular} & \begin{tabular}{|l|}
0.032647289 \\
97105597
\end{tabular} & 2 & 4 & 853 & 16269 & $\begin{array}{l}\text { MELO3C021563; } \\
\text { MELO3C011284 }\end{array}$ & $\begin{array}{l}\text { MELO3C025076; } \\
\text { MELO3C004980; } \\
\text { MELO3C003577; } \\
\text { MELO3C019166 }\end{array}$ \\
\hline [] & GO:0004090 & $\begin{array}{l}\text { carbonyl } \\
\text { reductase } \\
\text { (NADPH) } \\
\text { activity }\end{array}$ & $\begin{array}{l}\text { MOLECULAR } \\
\text { FUNCTION }\end{array}$ & \begin{tabular}{|l|}
0.628210989 \\
0968163
\end{tabular} & \begin{tabular}{|l|}
0.032647289 \\
97105597
\end{tabular} & 2 & 4 & 853 & 16269 & $\begin{array}{l}\text { MELO3C002872; } \\
\text { MELO3C001948 }\end{array}$ & $\begin{array}{l}\text { MELO3C019918; } \\
\text { MELO3C014897; } \\
\text { MELO3C021704; } \\
\text { MELO3C020605 }\end{array}$ \\
\hline [] & GO:0009331 & $\begin{array}{l}\text { glycerol-3- } \\
\text { phosphate } \\
\text { dehydrogenas } \\
\text { e complex }\end{array}$ & $\begin{array}{l}\text { CELLULAR_C } \\
\text { OMPONENT }\end{array}$ & \begin{tabular}{|l|}
0.628210989 \\
0968163
\end{tabular} & \begin{tabular}{|l|}
.032647289 \\
97105597
\end{tabular} & 2 & 4 & 853 & 16269 & $\begin{array}{l}\text { MELO3C021604; } \\
\text { MELO3C019820 }\end{array}$ & $\begin{array}{l}\text { MELO3C026337; } \\
\text { MELO3C020158; } \\
\text { MELO3C020157; } \\
\text { MELO3C005116 }\end{array}$ \\
\hline
\end{tabular}




\begin{tabular}{|c|c|c|c|c|c|c|c|c|c|c|c|}
\hline[] & GO:0009853 & $\begin{array}{l}\text { photorespirati } \\
\text { on }\end{array}$ & \begin{tabular}{|l} 
BIOLOGICAL \\
PROCESS
\end{tabular} & \begin{tabular}{|l|}
0.645170309 \\
8531466
\end{tabular} & \begin{tabular}{|l|}
0.033801789 \\
095820024
\end{tabular} & 4 & 21 & 851 & 16252 & $\begin{array}{l}\text { MELO3C022540; } \\
\text { MELO3CO06949; } \\
\text { MELO3C017960; } \\
\text { MELO3C026045 }\end{array}$ & 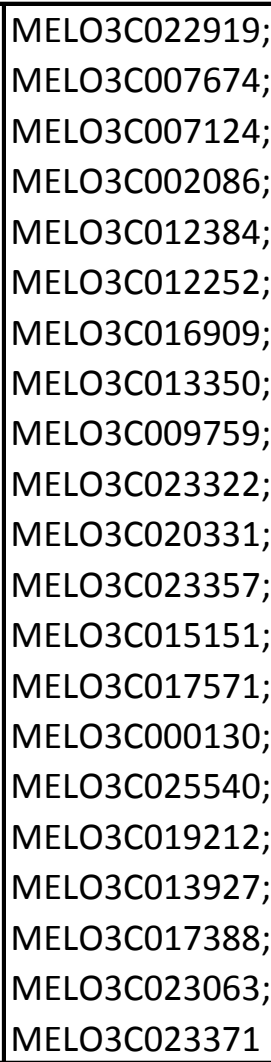 \\
\hline [] & GO:0018279 & \begin{tabular}{|l} 
protein $\mathrm{N}-$ \\
linked \\
glycosylation \\
via asparagine
\end{tabular} & \begin{tabular}{|l} 
BIOLOGICAL \\
PROCESS
\end{tabular} & \begin{tabular}{|l|}
0.646265517 \\
3874357
\end{tabular} & \begin{tabular}{|l|}
0.034191953 \\
73089502
\end{tabular} & 7 & 55 & 848 & 16218 & $\begin{array}{l}\text { MELO3C003812; } \\
\text { MELO3CO23310; } \\
\text { MELO3C011944; } \\
\text { MELO3CO25637; } \\
\text { MELO3CO21279; } \\
\text { MELO3CO24447; } \\
\text { MELO3C013822 }\end{array}$ & 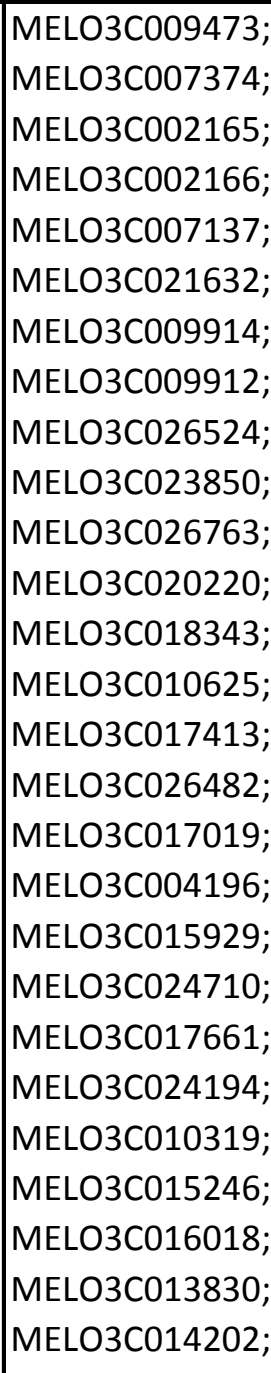 \\
\hline [] & GO:0032555 & $\begin{array}{l}\text { purine } \\
\text { ribonucleotide } \\
\text { binding }\end{array}$ & $\begin{array}{l}\text { MOLECULAR } \\
\text { FUNCTION }\end{array}$ & \begin{tabular}{|l|}
0.655899167 \\
5750125
\end{tabular} & \begin{tabular}{|l|}
0.034919271 \\
940117624
\end{tabular} & 136 & 2169 & 719 & 14104 & 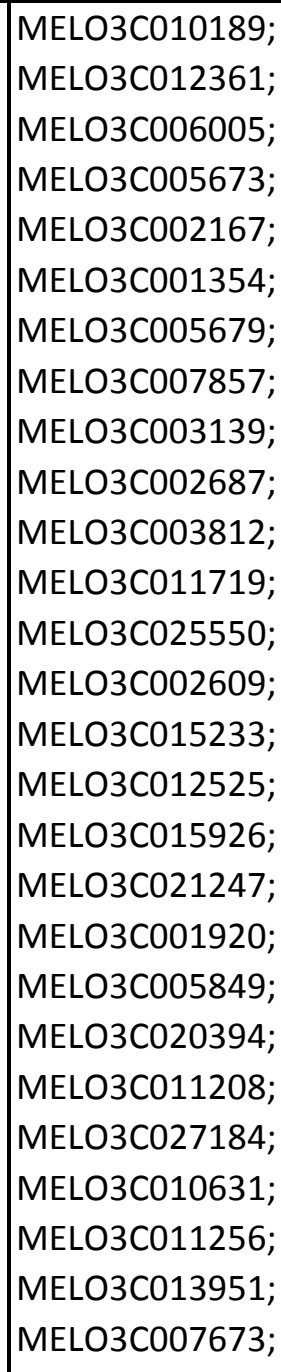 & 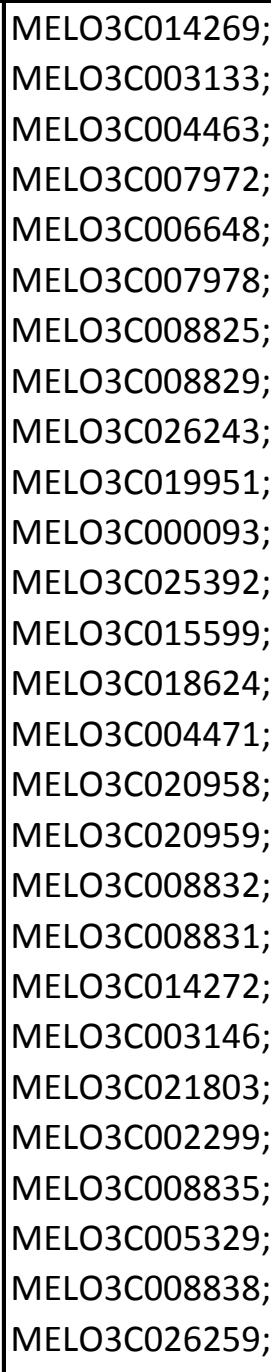 \\
\hline [] & GO:0042817 & \begin{tabular}{|l} 
pyridoxal \\
metabolic \\
process
\end{tabular} & \begin{tabular}{|l} 
BIOLOGICAL_ \\
PROCESS
\end{tabular} & \begin{tabular}{|l|}
0.658697966 \\
5414314
\end{tabular} & \begin{tabular}{|l|}
0.049918262 \\
49403735
\end{tabular} & 1 & 0 & 854 & 16273 & MELO3C003541 & \\
\hline \begin{tabular}{|l}
{[]} \\
\end{tabular} & GO:0048482 & $\begin{array}{l}\text { plant ovule } \\
\text { morphogenesi } \\
\text { s }\end{array}$ & $\begin{array}{l}\begin{array}{l}\text { BIOLOGICAL } \\
\text { PROCESS }\end{array} \\
\text { PRS }\end{array}$ & \begin{tabular}{|l|}
0.658697966 \\
5414314
\end{tabular} & \begin{tabular}{|l|}
0.049918262 \\
49403735
\end{tabular} & 1 & 0 & 854 & 16273 & MELO3C010913 & \\
\hline [] & GO:0015680 & $\begin{array}{l}\text { intracellular } \\
\text { copper ion } \\
\text { transport } \\
\end{array}$ & \begin{tabular}{|l} 
BIOLOGICAL \\
PROCESS
\end{tabular} & \begin{tabular}{|l|}
0.658697966 \\
5414314
\end{tabular} & \begin{tabular}{|l|}
0.049918262 \\
49403735
\end{tabular} & 1 & 0 & 854 & 16273 & MELO3C014007 & \\
\hline
\end{tabular}




\begin{tabular}{|c|c|c|c|c|c|c|c|c|c|c|c|}
\hline[] & GO:0046087 & \begin{tabular}{|l} 
cytidine \\
metabolic \\
process
\end{tabular} & $\begin{array}{l}\text { BIOLOGICAL_ } \\
\text { PROCESS }\end{array}$ & \begin{tabular}{|l|}
0.658697966 \\
5414314
\end{tabular} & $\begin{array}{l}0.049918262 \\
49403735\end{array}$ & 1 & 0 & 854 & 16273 & MELO3C015459 & \\
\hline[] & GO:0071398 & \begin{tabular}{|l} 
cellular \\
response to \\
fatty acid \\
\end{tabular} & $\begin{array}{l}\text { BIOLOGICAL_ } \\
\text { PROCESS }\end{array}$ & \begin{tabular}{|l|}
0.658697966 \\
5414314
\end{tabular} & $\begin{array}{l}0.049918262 \\
49403735\end{array}$ & 1 & 0 & 854 & 16273 & MELO3C019735 & \\
\hline[] & GO:0005978 & $\begin{array}{l}\text { glycogen } \\
\text { biosynthetic } \\
\text { process }\end{array}$ & $\begin{array}{l}\text { BIOLOGICAL_ } \\
\text { PROCESS }\end{array}$ & $\begin{array}{l}0.658697966 \\
5414314\end{array}$ & $\begin{array}{l}0.038420622 \\
02444051\end{array}$ & 4 & 22 & 851 & 16251 & $\begin{array}{l}\text { MELO3C005293; } \\
\text { MELO3C003812; } \\
\text { MELO3C023310; } \\
\text { MELO3C020244 }\end{array}$ & $\begin{array}{l}\text { MELO3C011991; } \\
\text { MELO3C013146; } \\
\text { MELO3C004270; } \\
\text { MELO3C005396; } \\
\text { MELO3C009445; } \\
\text { MELO3C006552; } \\
\text { MELO3C009861; } \\
\text { MELO3C007155; } \\
\text { MELO3C008045; } \\
\text { MELO3C005795; } \\
\text { MELO3C002455; } \\
\text { MELO3C021412; } \\
\text { MELO3C008407; } \\
\text { MELO3C008614; } \\
\text { MELO3C021249; } \\
\text { MELO3C026536; } \\
\text { MELO3C025521; } \\
\text { MELO3C024175; } \\
\text { MELO3C012925; } \\
\text { MELO3C018534; } \\
\text { MELO3C010862; } \\
\text { MELO3C011346 }\end{array}$ \\
\hline[] & GO:0006616 & \begin{tabular}{|l|} 
SRP- \\
dependent \\
cotranslational \\
protein \\
targeting to \\
membrane, \\
translocation \\
\end{tabular} & $\begin{array}{l}\text { BIOLOGICAL_ } \\
\text { PROCESS } \\
\\
\end{array}$ & $\begin{array}{l}0.658697966 \\
5414314\end{array}$ & $\begin{array}{l}0.049918262 \\
49403735\end{array}$ & 1 & 0 & 854 & 16273 & MELO3C005757 & \\
\hline[] & GO:0006977 & $\begin{array}{l}\text { DNA damage } \\
\text { response, } \\
\text { signal } \\
\text { transduction } \\
\text { by p53 class } \\
\text { mediator } \\
\text { resulting in cell } \\
\text { cycle arrest }\end{array}$ & $\begin{array}{l}\text { BIOLOGICAL_ } \\
\text { PROCESS }\end{array}$ & $\begin{array}{l}0.658697966 \\
5414314\end{array}$ & $\begin{array}{l}0.048685998 \\
78337037\end{array}$ & 4 & 24 & 851 & 16249 & $\begin{array}{l}\text { MELO3C016083; } \\
\text { MELO3C010960; } \\
\text { MELO3C016262; } \\
\text { MELO3C012361 } \\
\end{array}$ & $\begin{array}{l}\text { MELO3C011983; } \\
\text { MELO3C002481; } \\
\text { MELO3C011470; } \\
\text { MELO3C021746; } \\
\text { MELO3C014086; } \\
\text { MELO3C000704; } \\
\text { MELO3C004136; } \\
\text { MELO3C021715; } \\
\text { MELO3C025923; } \\
\text { MELO3C025417; } \\
\text { MELO3C005738; } \\
\text { MELO3C023476; } \\
\text { MELO3C026239; } \\
\text { MELO3C003316; } \\
\text { MELO3C025755; } \\
\text { MELO3C026800; } \\
\text { MELO3C005912; } \\
\text { MELO3C020453; } \\
\text { MELO3C018099; } \\
\text { MELO3C019642; } \\
\text { MELO3C019587; } \\
\text { MELO3C019731; } \\
\text { MELO3C013814; } \\
\text { MELO3C016699 }\end{array}$ \\
\hline [] & GO:0031938 & \begin{tabular}{|l} 
regulation of \\
chromatin \\
silencing at \\
telomere \\
\end{tabular} & $\begin{array}{l}\text { BIOLOGICAL_ } \\
\text { PROCESS }\end{array}$ & $\begin{array}{l}0.658697966 \\
5414314\end{array}$ & $\begin{array}{l}0.049918262 \\
49403735\end{array}$ & 1 & 0 & 854 & 16273 & MELO3C021058 & \\
\hline [] & GO:0030950 & \begin{tabular}{|l|} 
establishment \\
or \\
maintenance \\
of actin \\
cytoskeleton \\
polarity \\
\end{tabular} & $\begin{array}{l}\text { BIOLOGICAL_ } \\
\text { PROCESS }\end{array}$ & $\begin{array}{l}0.658697966 \\
5414314\end{array}$ & $\begin{array}{l}0.049918262 \\
49403735\end{array}$ & 1 & 0 & 854 & 16273 & MELO3C026614 & \\
\hline [] & GO:0046292 & \begin{tabular}{|l|} 
formaldehyde \\
metabolic \\
process
\end{tabular} & $\begin{array}{l}\text { BIOLOGICAL_ } \\
\text { PROCESS }\end{array}$ & $\begin{array}{l}0.658697966 \\
5414314\end{array}$ & $\begin{array}{l}0.049918262 \\
49403735\end{array}$ & 1 & 0 & 854 & 16273 & MELO3C022399 & \\
\hline[] & GO:0006561 & $\begin{array}{l}\text { proline } \\
\text { biosynthetic } \\
\text { process }\end{array}$ & $\begin{array}{l}\text { BIOLOGICAL_ } \\
\text { PROCESS }\end{array}$ & \begin{tabular}{|l|}
0.658697966 \\
5414314
\end{tabular} & $\begin{array}{l}0.044214600 \\
976853265\end{array}$ & 2 & 5 & 853 & 16268 & $\begin{array}{l}\text { MELO3C008245; } \\
\text { MELO3C011208 }\end{array}$ & $\begin{array}{l}\text { MELO3C019039; } \\
\text { MELO3C009229; } \\
\text { MELO3C005129; } \\
\text { MELO3C024239; } \\
\text { MELO3C002752 }\end{array}$ \\
\hline[] & GO:0010812 & $\begin{array}{l}\text { negative } \\
\text { regulation of } \\
\text { cell-substrate } \\
\text { adhesion }\end{array}$ & $\begin{array}{l}\text { BIOLOGICAL_ } \\
\text { PROCESS }\end{array}$ & $\begin{array}{l}0.658697966 \\
5414314\end{array}$ & $\begin{array}{l}0.049918262 \\
49403735\end{array}$ & 1 & 0 & 854 & 16273 & MELO3C022488 & \\
\hline[] & GO:0006420 & \begin{tabular}{|l|} 
arginyl-tRNA \\
aminoacylatio \\
$n$
\end{tabular} & $\begin{array}{l}\text { BIOLOGICAL_ } \\
\text { PROCESS }\end{array}$ & \begin{tabular}{|l|}
0.658697966 \\
5414314
\end{tabular} & \begin{tabular}{|l|}
0.049918262 \\
49403735
\end{tabular} & 1 & 0 & 854 & 16273 & MELO3C015422 & \\
\hline
\end{tabular}




\begin{tabular}{|c|c|c|c|c|c|c|c|c|c|c|c|}
\hline[] & GO:0040018 & \begin{tabular}{|l} 
positive \\
regulation of \\
multicellular \\
organism \\
growth
\end{tabular} & \begin{tabular}{|l|} 
BIOLOGICAL \\
PROCESS
\end{tabular} & \begin{tabular}{|l}
0.658697966 \\
5414314
\end{tabular} & \begin{tabular}{|l|}
0.039310242 \\
87564423
\end{tabular} & 5 & 33 & 850 & 16240 & $\begin{array}{l}\text { MELO3C013753; } \\
\text { MELO3CO02118; } \\
\text { MELO3C010882; } \\
\text { MELO3C011285; } \\
\text { MELO3C025061 }\end{array}$ & 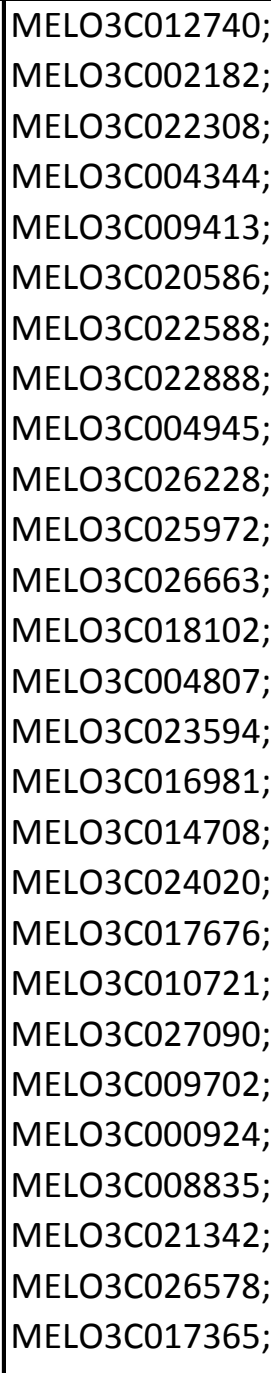 \\
\hline[] & GO:0042435 & \begin{tabular}{|l} 
indole- \\
containing \\
compound \\
biosynthetic \\
process
\end{tabular} & \begin{tabular}{|l} 
BIOLOGICAL \\
PROCESS
\end{tabular} & \begin{tabular}{|l}
0.658697966 \\
5414314
\end{tabular} & \begin{tabular}{|l|}
0.042683186 \\
08102429
\end{tabular} & 3 & 13 & 852 & 16260 & $\begin{array}{l}\text { MELO3C026887; } \\
\text { MELO3CO02275; } \\
\text { MELO3C013757 }\end{array}$ & $\begin{array}{l}\text { MELO3CO08294; } \\
\text { MELO3CO14311; } \\
\text { MELO3CO20718; } \\
\text { MELO3CO07920; } \\
\text { MELO3CO09018; } \\
\text { MELO3CO05674; } \\
\text { MELO3CO09127; } \\
\text { MELO3CO02476; } \\
\text { MELO3CO18082; } \\
\text { MELO3CO2678;; } \\
\text { MELO3CO22074; } \\
\text { MELO3CO24189; } \\
\text { MELO3CO11963 }\end{array}$ \\
\hline [] & GO:1901264 & \begin{tabular}{|l|} 
carbohydrate \\
derivative \\
transport
\end{tabular} & \begin{tabular}{|l} 
BIOLOGICAL_ \\
PROCESS
\end{tabular} & \begin{tabular}{|l}
0.658697966 \\
5414314
\end{tabular} & \begin{tabular}{|l|}
0.042683186 \\
08102429
\end{tabular} & 3 & 13 & 852 & 16260 & $\begin{array}{l}\text { MELO3C006409; } \\
\text { MELO3C011675; } \\
\text { MELO3C004533 }\end{array}$ & $\begin{array}{l}\text { MELO3CO10474; } \\
\text { MELO3C012236; } \\
\text { MELO3CO13485; } \\
\text { MELO3C013186; } \\
\text { MELO3CO08403; } \\
\text { MELO3CO2055; } \\
\text { MELO3CO07756; } \\
\text { MELO3CO06217; } \\
\text { MELO3CO21055; } \\
\text { MELO3C017661; } \\
\text { MELO3CO11559; } \\
\text { MELO3C011623; } \\
\text { MELO3CO11676 }\end{array}$ \\
\hline[] & GO:0006123 & \begin{tabular}{|l} 
mitochondrial \\
electron \\
transport, \\
cytochrome c \\
to oxygen
\end{tabular} & \begin{tabular}{|l|} 
BIOLOGICAL \\
PROCESS
\end{tabular} & \begin{tabular}{|l|}
0.658697966 \\
5414314
\end{tabular} & \begin{tabular}{|l|}
0.044214600 \\
976853265
\end{tabular} & 2 & 5 & 853 & 16268 & $\begin{array}{l}\text { MELO3C004105; } \\
\text { MELO3C011044 }\end{array}$ & $\begin{array}{l}\text { MELO3C015922; } \\
\text { MELO3CO0336; } \\
\text { MELO3C016120; } \\
\text { MELO3C018157; } \\
\text { MELO3C006242 }\end{array}$ \\
\hline[] & GO:0006121 & \begin{tabular}{|l|} 
mitochondrial \\
electron \\
transport, \\
succinate to \\
ubiquinone
\end{tabular} & \begin{tabular}{|l|} 
BIOLOGICAL \\
PROCESS
\end{tabular} & \begin{tabular}{|l|}
0.658697966 \\
5414314
\end{tabular} & \begin{tabular}{|l|}
0.049918262 \\
49403735
\end{tabular} & 1 & 0 & 854 & 16273 & MELO3C017560 & \\
\hline[] & GO:0006127 & \begin{tabular}{|l} 
glycerophosph \\
ate shuttle
\end{tabular} & $\begin{array}{l}\text { BIOLOGICAL } \\
\text { PROCESS }\end{array}$ & \begin{tabular}{|l|}
0.658697966 \\
5414314
\end{tabular} & \begin{tabular}{|l|}
0.049918262 \\
49403735
\end{tabular} & 1 & 0 & 854 & 16273 & MELO3C019820 & \\
\hline [] & GO:0009629 & \begin{tabular}{|l|} 
response to \\
gravity
\end{tabular} & \begin{tabular}{|l|} 
BIOLOGICAL \\
PROCESS
\end{tabular} & \begin{tabular}{|l|}
0.658697966 \\
5414314
\end{tabular} & \begin{tabular}{|l|}
0.042683186 \\
08102429
\end{tabular} & 3 & 13 & 852 & 16260 & $\begin{array}{l}\text { MELO3C005293; } \\
\text { MELO3CO08847; } \\
\text { MELO3C025282 }\end{array}$ & 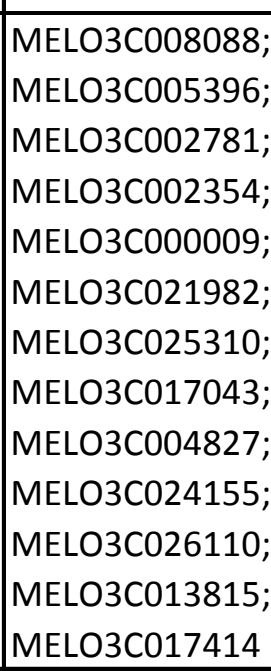 \\
\hline[] & GO:0019563 & \begin{tabular}{|l} 
glycerol \\
catabolic \\
process
\end{tabular} & \begin{tabular}{|l|} 
BIOLOGICAL \\
PROCESS
\end{tabular} & \begin{tabular}{|l|}
0.658697966 \\
5414314
\end{tabular} & \begin{tabular}{|l|}
0.049918262 \\
49403735
\end{tabular} & 1 & 0 & 854 & 16273 & MELO3C019820 & \\
\hline
\end{tabular}




\begin{tabular}{|c|c|c|c|c|c|c|c|c|c|c|c|}
\hline [] & GO:0009401 & \begin{tabular}{|l} 
phosphoenolp \\
yruvate- \\
dependent \\
sugar \\
phosphotransf \\
erase system
\end{tabular} & \begin{tabular}{|l} 
BIOLOGICAL \\
PROCESS
\end{tabular} & $\begin{array}{l}0.658697966 \\
5414314\end{array}$ & \begin{tabular}{|l|}
0.049918262 \\
49403735
\end{tabular} & 1 & 10 & 854 & 16273 & MELO3C016017 & \\
\hline [] & GO:0098542 & $\begin{array}{l}\text { defense } \\
\text { response to } \\
\text { other } \\
\text { organism }\end{array}$ & $\begin{array}{l}\text { BIOLOGICAL } \\
\text { PROCESS }\end{array}$ & $\begin{array}{l}0.658697966 \\
5414314\end{array}$ & \begin{tabular}{|l|}
0.040943794 \\
239169086
\end{tabular} & 16 & 173 & 839 & 16100 & 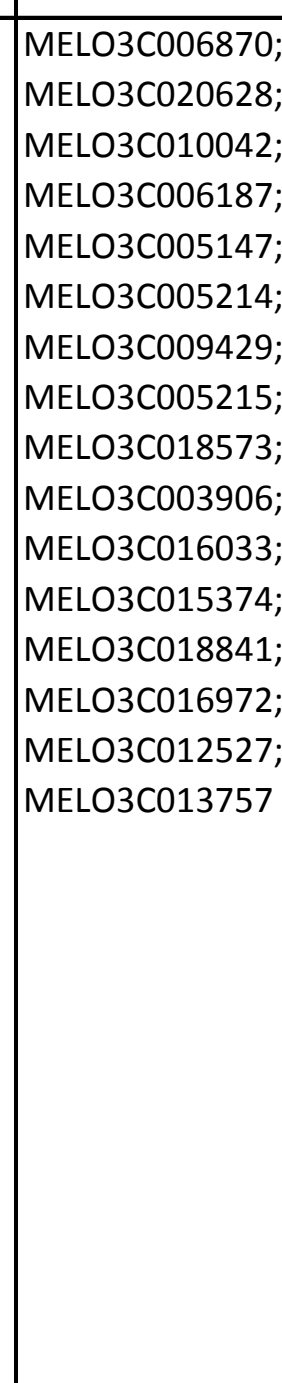 & 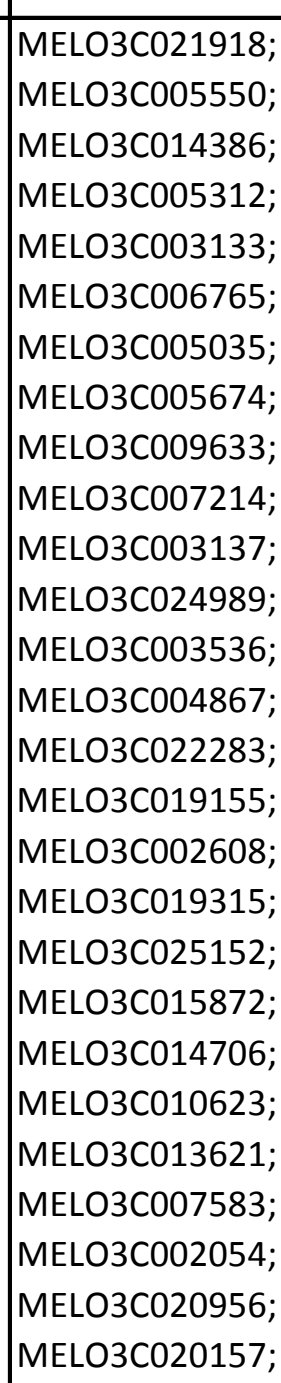 \\
\hline [] & GO:0007310 & \begin{tabular}{|l|} 
oocyte \\
dorsal/ventral \\
axis \\
specification
\end{tabular} & $\begin{array}{l}\text { BIOLOGICAL } \\
\text { PROCESS }\end{array}$ & $\begin{array}{l}0.658697966 \\
5414314\end{array}$ & $\begin{array}{l}0.049918262 \\
49403735\end{array}$ & 1 & To & 854 & 16273 & MELO3C011193 & \\
\hline [] & GO:0009727 & \begin{tabular}{|l} 
detection of \\
ethylene \\
stimulus \\
\end{tabular} & $\begin{array}{l}\text { BIOLOGICAL } \\
\text { PROCESS }\end{array}$ & $\begin{array}{l}0.658697966 \\
5414314\end{array}$ & \begin{tabular}{|l|}
0.049918262 \\
49403735 \\
\end{tabular} & 1 & 10 & 854 & 16273 & MELO3C003906 & \\
\hline [] & GO:0000738 & $\begin{array}{l}\text { DNA catabolic } \\
\text { process, } \\
\text { exonucleolytic }\end{array}$ & $\begin{array}{l}\text { BIOLOGICAL } \\
\text { PROCESS }\end{array}$ & $\begin{array}{l}0.658697966 \\
5414314\end{array}$ & \begin{tabular}{|l|}
0.049918262 \\
49403735
\end{tabular} & 1 & To & 854 & 16273 & MELO3C006293 & \\
\hline 1 & GO:0007018 & $\begin{array}{l}\text { microtubule- } \\
\text { based } \\
\text { movement }\end{array}$ & \begin{tabular}{|l} 
BIOLOGICAL \\
PROCESS
\end{tabular} & $\begin{array}{l}0.658697966 \\
5414314\end{array}$ & \begin{tabular}{|l|}
0.036469460 \\
81068046
\end{tabular} & 9 & 74 & 846 & 16199 & $\begin{array}{l}\text { MELO3C009203; } \\
\text { MELO3CO24241; } \\
\text { MELO3COO7673; } \\
\text { MELO3C02334; } \\
\text { MELO3C015098; } \\
\text { MELO3CO0567; } \\
\text { MELO3C01342; } \\
\text { MELO3C013702; } \\
\text { MELO3CO08977 }\end{array}$ & 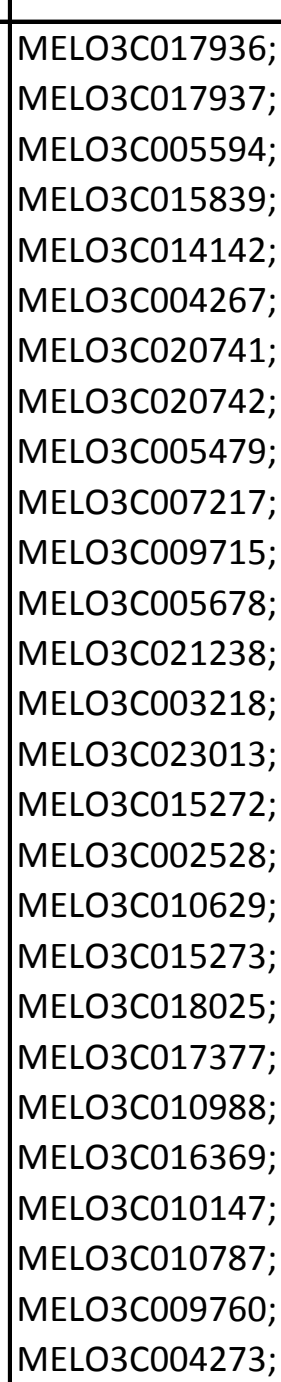 \\
\hline & GO:0008347 & \begin{tabular}{|l|l|} 
glial cell \\
migration
\end{tabular} & $\begin{array}{l}\text { BIOLOGICAL } \\
\text { PROCESS }\end{array}$ & $\begin{array}{l}0.658697966 \\
5414314\end{array}$ & \begin{tabular}{|l|}
0.049918262 \\
49403735
\end{tabular} & 1 & lo & 854 & 16273 & MELO3C011193 & \\
\hline
\end{tabular}




\begin{tabular}{|c|c|c|c|c|c|c|c|c|c|c|c|}
\hline[] & GO:0009308 & $\begin{array}{l}\text { amine } \\
\text { metabolic } \\
\text { process }\end{array}$ & $\begin{array}{l}\text { BIOLOGICAL_ } \\
\text { PROCESS }\end{array}$ & \begin{tabular}{|l|}
0.658697966 \\
5414314
\end{tabular} & $\begin{array}{l}0.047014335 \\
70500883\end{array}$ & 9 & 82 & 846 & 16191 & $\begin{array}{l}\text { MELO3C026887; } \\
\text { MELO3C010960; } \\
\text { MELO3C016262; } \\
\text { MELO3C011484; } \\
\text { MELO3C003906; } \\
\text { MELO3C016881; } \\
\text { MELO3C002275; } \\
\text { MELO3C023315; } \\
\text { MELO3C005578 }\end{array}$ & $\begin{array}{l}\text { MELO3C016208; } \\
\text { MELO3C012201; } \\
\text { MELO3C004185; } \\
\text { MELO3C004184; } \\
\text { MELO3C004187; } \\
\text { MELO3C010461; } \\
\text { MELO3C005153; } \\
\text { MELO3C005877; } \\
\text { MELO3C007417; } \\
\text { MELO3C004624; } \\
\text { MELO3C004626; } \\
\text { MELO3C025755; } \\
\text { MELO3C026800; } \\
\text { MELO3C023775; } \\
\text { MELO3C017173; } \\
\text { MELO3C026323; } \\
\text { MELO3C024189; } \\
\text { MELO3C015199; } \\
\text { MELO3C013814; } \\
\text { MELO3C015478; } \\
\text { MELO3C012007; } \\
\text { MELO3C014479; } \\
\text { MELO3C014478; } \\
\text { MELO3C014311; } \\
\text { MELO3C008477; } \\
\text { MELO3C002293; } \\
\text { MELO3C014035; }\end{array}$ \\
\hline[] & GO:0031115 & $\begin{array}{l}\text { negative } \\
\text { regulation of } \\
\text { microtubule } \\
\text { polymerization }\end{array}$ & $\begin{array}{l}\text { BIOLOGICAL } \\
\text { PROCESS }\end{array}$ & \begin{tabular}{|l|}
0.658697966 \\
5414314
\end{tabular} & $\begin{array}{l}0.049918262 \\
49403735\end{array}$ & 1 & 0 & 854 & 16273 & MELO3C022488 & \\
\hline$[$ [] & GO:0006297 & $\begin{array}{l}\text { nucleotide- } \\
\text { excision repair, } \\
\text { DNA gap filling }\end{array}$ & $\begin{array}{l}\text { BIOLOGICAL_ } \\
\text { PROCESS }\end{array}$ & $\begin{array}{l}0.658697966 \\
5414314\end{array}$ & \begin{tabular}{|l|}
0.042683186 \\
08102429
\end{tabular} & 3 & 13 & 852 & 16260 & $\begin{array}{l}\text { MELO3C017228; } \\
\text { MELO3C010631; } \\
\text { MELO3C003638 }\end{array}$ & $\begin{array}{l}\text { MELO3C014021; } \\
\text { MELO3C014020; } \\
\text { MELO3C008864; } \\
\text { MELO3C026949; } \\
\text { MELO3C025879; } \\
\text { MELO3C010507; } \\
\text { MELO3C024596; } \\
\text { MELO3C001937; } \\
\text { MELO3C018521; } \\
\text { MELO3C025142; } \\
\text { MELO3C014529; } \\
\text { MELO3C014528; } \\
\text { MELO3C017458 }\end{array}$ \\
\hline [] & GO:0042149 & \begin{tabular}{|l} 
cellular \\
response to \\
glucose \\
starvation \\
\end{tabular} & $\begin{array}{l}\text { BIOLOGICAL_ } \\
\text { PROCESS }\end{array}$ & \begin{tabular}{|l|}
0.658697966 \\
5414314
\end{tabular} & $\begin{array}{l}0.049918262 \\
49403735\end{array}$ & 1 & 0 & 854 & 16273 & MELO3C005757 & \\
\hline[] & GO:0035821 & $\begin{array}{l}\text { modification } \\
\text { of morphology } \\
\text { or physiology } \\
\text { of other } \\
\text { organism }\end{array}$ & $\begin{array}{l}\text { BIOLOGICAL_ } \\
\text { PROCESS }\end{array}$ & \begin{tabular}{|l|}
0.658697966 \\
5414314
\end{tabular} & \begin{tabular}{|l|}
0.042683186 \\
08102429
\end{tabular} & 3 & 13 & 852 & 16260 & $\begin{array}{l}\text { MELO3C005214; } \\
\text { MELO3C016972; } \\
\text { MELO3C005215 }\end{array}$ & $\begin{array}{l}\text { MELO3C012664; } \\
\text { MELO3C011257; } \\
\text { MELO3C010750; } \\
\text { MELO3C015938; } \\
\text { MELO3C007375; } \\
\text { MELO3C005642; } \\
\text { MELO3C005643; } \\
\text { MELO3C005641; } \\
\text { MELO3C021126; } \\
\text { MELO3C023207; } \\
\text { MELO3C023203; } \\
\text { MELO3C005929; } \\
\text { MELO3C001957 }\end{array}$ \\
\hline [] & GO:0007229 & $\begin{array}{l}\text { integrin- } \\
\text { mediated } \\
\text { signaling } \\
\text { pathway }\end{array}$ & $\begin{array}{l}\text { BIOLOGICAL_ } \\
\text { PROCESS }\end{array}$ & \begin{tabular}{|l|}
0.658697966 \\
5414314
\end{tabular} & \begin{tabular}{|l|}
0.049918262 \\
49403735
\end{tabular} & 1 & 0 & 854 & 16273 & MELO3C007690 & \\
\hline
\end{tabular}




\begin{tabular}{|c|c|c|c|c|c|c|c|c|c|c|c|}
\hline [] & GO:0009664 & $\begin{array}{l}\text { plant-type cell } \\
\text { wall } \\
\text { organization }\end{array}$ & \begin{tabular}{|l|} 
BIOLOGICAL_ \\
PROCESS
\end{tabular} & $\begin{array}{l}0.658697966 \\
5414314\end{array}$ & \begin{tabular}{|l|}
0.043310123 \\
129766544
\end{tabular} & 5 & 34 & 850 & 16239 & $\begin{array}{l}\text { MELO3C012004; } \\
\text { MELO3C021999; } \\
\text { MELO3C020626; } \\
\text { MELO3C017242; } \\
\text { MELO3C010524 }\end{array}$ & $\begin{array}{l}\text { MELO3C010062; } \\
\text { MELO3C021619; } \\
\text { MELO3C011350; } \\
\text { MELO3C020005; } \\
\text { MELO3C003134; } \\
\text { MELO3C005613; } \\
\text { MELO3C001993; } \\
\text { MELO3C017181; } \\
\text { MELO3C024488; } \\
\text { MELO3C025996; } \\
\text { MELO3C024487; } \\
\text { MELO3C016062; } \\
\text { MELO3C026188; } \\
\text { MELO3C020143; } \\
\text { MELO3C003916; } \\
\text { MELO3C015695; } \\
\text { MELO3C025095; } \\
\text { MELO3C012108; } \\
\text { MELO3C011732; } \\
\text { MELO3C016517; } \\
\text { MELO3C016916; } \\
\text { MELO3C014013; } \\
\text { MELO3C025907; } \\
\text { MELO3C008552; } \\
\text { MELO3C020810; } \\
\text { MELO3C005962; } \\
\text { MELO3C006318; }\end{array}$ \\
\hline[] & GO:0046827 & \begin{tabular}{|l} 
positive \\
regulation of \\
protein export \\
from nucleus
\end{tabular} & \begin{tabular}{|l|} 
BIOLOGICAL \\
PROCESS
\end{tabular} & \begin{tabular}{|l|}
0.658697966 \\
5414314
\end{tabular} & \begin{tabular}{|l|}
0.049918262 \\
49403735
\end{tabular} & 1 & 0 & 854 & 16273 & MELO3C007207 & \\
\hline$[$ [] & GO:0010342 & $\begin{array}{l}\text { endosperm } \\
\text { cellularization }\end{array}$ & \begin{tabular}{|l|} 
BIOLOGICAL \\
PROCESS
\end{tabular} & $\begin{array}{l}0.658697966 \\
5414314\end{array}$ & \begin{tabular}{|l|}
0.049918262 \\
49403735
\end{tabular} & 1 & 0 & 854 & 16273 & MELO3C006602 & \\
\hline [] & GO:0060904 & $\begin{array}{l}\text { regulation of } \\
\text { protein folding } \\
\text { in endoplasmic } \\
\text { reticulum }\end{array}$ & \begin{tabular}{|l|} 
BIOLOGICAL \\
PROCESS
\end{tabular} & \begin{tabular}{|l|}
0.658697966 \\
5414314
\end{tabular} & \begin{tabular}{|l|}
0.049918262 \\
49403735 \\
\end{tabular} & 1 & 0 & 854 & 16273 & MELO3C005757 & \\
\hline [] & GO:0010312 & $\begin{array}{l}\text { detoxification } \\
\text { of zinc ion }\end{array}$ & \begin{tabular}{|l|} 
BIOLOGICAL \\
PROCESS
\end{tabular} & \begin{tabular}{|l|}
0.658697966 \\
5414314 \\
\end{tabular} & \begin{tabular}{|l|}
0.049918262 \\
49403735 \\
\end{tabular} & 1 & 0 & 854 & 16273 & MELO3C022448 & \\
\hline [] & GO:0048759 & $\begin{array}{l}\text { xylem vessel } \\
\text { member cell } \\
\text { differentiation }\end{array}$ & \begin{tabular}{|l|} 
BIOLOGICAL_ \\
PROCESS
\end{tabular} & \begin{tabular}{|l|}
0.658697966 \\
5414314
\end{tabular} & \begin{tabular}{|l|}
0.049918262 \\
49403735
\end{tabular} & 1 & 0 & 854 & 16273 & MELO3C011484 & \\
\hline [] & GO:0001556 & $\begin{array}{l}\text { oocyte } \\
\text { maturation }\end{array}$ & \begin{tabular}{|l|} 
BIOLOGICAL \\
PROCESS
\end{tabular} & \begin{tabular}{|l|}
0.658697966 \\
5414314 \\
\end{tabular} & \begin{tabular}{|l|}
0.049918262 \\
49403735 \\
\end{tabular} & 1 & 0 & 854 & 16273 & MELO3C012361 & \\
\hline$[$ [] & GO:0046474 & $\begin{array}{l}\text { glycerophosph } \\
\text { olipid } \\
\text { biosynthetic } \\
\text { process }\end{array}$ & \begin{tabular}{|l|} 
BIOLOGICAL \\
PROCESS
\end{tabular} & \begin{tabular}{|l|}
0.658697966 \\
5414314
\end{tabular} & \begin{tabular}{|l|}
0.039310242 \\
87564423
\end{tabular} & 5 & 33 & 850 & 16240 & $\begin{array}{l}\text { MELO3C007931; } \\
\text { MELO3C008959; } \\
\text { MELO3C025637; } \\
\text { MELO3C015670; } \\
\text { MELO3C012545 }\end{array}$ & $\begin{array}{l}\text { MELO3C013830; } \\
\text { MELO3C007010; } \\
\text { MELO3C012286; } \\
\text { MELO3C020821; } \\
\text { MELO3C020987; } \\
\text { MELO3C005332; } \\
\text { MELO3C022308; } \\
\text { MELO3C020989; } \\
\text { MELO3C005634; } \\
\text { MELO3C021052; } \\
\text { MELO3C026440; } \\
\text { MELO3C017214; } \\
\text { MELO3C013319; } \\
\text { MELO3C017019; } \\
\text { MELO3C010621; } \\
\text { MELO3C011215; } \\
\text { MELO3C015647; } \\
\text { MELO3C011047; } \\
\text { MELO3C013842; } \\
\text { MELO3C009088; } \\
\text { MELO3C009186; } \\
\text { MELO3C013982; } \\
\text { MELO3C014212; } \\
\text { MELO3C008992; } \\
\text { MELO3C005285; } \\
\text { MELO3C006258; } \\
\text { MELO3C014054; }\end{array}$ \\
\hline [] & GO:0034333 & $\begin{array}{l}\text { adherens } \\
\text { junction } \\
\text { assembly }\end{array}$ & \begin{tabular}{|l|} 
BIOLOGICAL \\
PROCESS
\end{tabular} & \begin{tabular}{|l|}
0.658697966 \\
5414314
\end{tabular} & \begin{tabular}{|l|}
0.049918262 \\
49403735
\end{tabular} & 1 & 0 & 854 & 16273 & MELO3C022488 & \\
\hline [] & GO:0010266 & $\begin{array}{l}\text { response to } \\
\text { vitamin B1 }\end{array}$ & \begin{tabular}{|l|} 
BIOLOGICAL_ \\
PROCESS
\end{tabular} & \begin{tabular}{|l|}
0.658697966 \\
5414314
\end{tabular} & \begin{tabular}{|l|}
0.049918262 \\
49403735
\end{tabular} & 1 & 0 & 854 & 16273 & MELO3C020562 & \\
\hline [] & GO:0032012 & $\begin{array}{l}\text { regulation of } \\
\text { ARF protein } \\
\text { signal } \\
\text { transduction }\end{array}$ & \begin{tabular}{|l|} 
BIOLOGICAL \\
PROCESS
\end{tabular} & \begin{tabular}{|l|}
0.658697966 \\
5414314
\end{tabular} & \begin{tabular}{|l|}
0.044214600 \\
976853265
\end{tabular} & 2 & 5 & 853 & 16268 & $\begin{array}{l}\text { MELO3C004551; } \\
\text { MELO3C006224 }\end{array}$ & $\begin{array}{l}\text { MELO3C023883; } \\
\text { MELO3C012923; } \\
\text { MELO3C012924; } \\
\text { MELO3C006181; } \\
\text { MELO3C015692 }\end{array}$ \\
\hline
\end{tabular}




\begin{tabular}{|c|c|c|c|c|c|c|c|c|c|c|c|}
\hline[] & GO:0043154 & $\begin{array}{l}\text { negative } \\
\text { regulation of } \\
\text { cysteine-type } \\
\text { endopeptidase } \\
\text { activity } \\
\text { involved in } \\
\text { apoptotic } \\
\text { process }\end{array}$ & $\begin{array}{l}\text { BIOLOGICAL } \\
\text { PROCESS }\end{array}$ & $\begin{array}{l}0.658697966 \\
5414314\end{array}$ & $\begin{array}{l}0.049918262 \\
49403735\end{array}$ & 1 & 0 & 854 & 16273 & MELO3C005757 & \\
\hline [] & GO:0010299 & $\begin{array}{l}\text { detoxification } \\
\text { of cobalt ion }\end{array}$ & $\begin{array}{l}\text { BIOLOGICAL_ } \\
\text { PROCESS }\end{array}$ & $\begin{array}{l}0.658697966 \\
5414314\end{array}$ & $\begin{array}{l}0.049918262 \\
49403735\end{array}$ & 1 & 0 & 854 & 16273 & MELO3C022448 & \\
\hline[] & GO:0048730 & $\begin{array}{l}\text { epidermis } \\
\text { morphogenesi } \\
\mathrm{s}\end{array}$ & $\begin{array}{l}\text { BIOLOGICAL } \\
\text { PROCESS }\end{array}$ & $\begin{array}{l}0.658697966 \\
5414314\end{array}$ & $\begin{array}{l}0.049918262 \\
49403735\end{array}$ & 1 & 0 & 854 & 16273 & MELO3C006929 & \\
\hline[] & GO:0015966 & $\begin{array}{l}\text { diadenosine } \\
\text { tetraphosphat } \\
\text { e biosynthetic } \\
\text { process }\end{array}$ & $\begin{array}{l}\text { BIOLOGICAL } \\
\text { PROCESS }\end{array}$ & $\begin{array}{l}0.658697966 \\
5414314\end{array}$ & $\begin{array}{l}0.049918262 \\
49403735\end{array}$ & 1 & 0 & 854 & 16273 & MELO3C003905 & \\
\hline[] & GO:0034484 & \begin{tabular}{|l} 
raffinose \\
catabolic \\
process
\end{tabular} & $\begin{array}{l}\text { BIOLOGICAL_ } \\
\text { PROCESS }\end{array}$ & $\begin{array}{l}0.658697966 \\
5414314\end{array}$ & $\begin{array}{l}0.049918262 \\
49403735\end{array}$ & 1 & 0 & 854 & 16273 & MELO3C023110 & \\
\hline [] & GO:0006903 & \begin{tabular}{|l} 
vesicle \\
targeting
\end{tabular} & $\begin{array}{l}\text { BIOLOGICAL } \\
\text { PROCESS }\end{array}$ & $\begin{array}{l}0.658697966 \\
5414314\end{array}$ & $\begin{array}{l}0.039310242 \\
87564423\end{array}$ & 5 & 33 & 850 & 16240 & $\begin{array}{l}\text { MELO3C010243; } \\
\text { MELO3C004551; } \\
\text { MELO3C021279; } \\
\text { MELO3C024447; } \\
\text { MELO3C009966 }\end{array}$ & $\begin{array}{l}\text { MELO3C013434; } \\
\text { MELO3C006782; } \\
\text { MELO3C023339; } \\
\text { MELO3C021556; } \\
\text { MELO3C009912; } \\
\text { MELO3C023630; } \\
\text { MELO3C020980; } \\
\text { MELO3C025796; } \\
\text { MELO3C024225; } \\
\text { MELO3C026763; } \\
\text { MELO3C018343; } \\
\text { MELO3C012923; } \\
\text { MELO3C012528; } \\
\text { MELO3C019856; } \\
\text { MELO3C010666; } \\
\text { MELO3C007042; } \\
\text { MELO3C009240; } \\
\text { MELO3C004196; } \\
\text { MELO3C007300; } \\
\text { MELO3C009269; } \\
\text { MELO3C012991; } \\
\text { MELO3C007583; } \\
\text { MELO3C015929; } \\
\text { MELO3C005422; } \\
\text { MELO3C005423; } \\
\text { MELO3C009969; }\end{array}$ \\
\hline [] & GO:0004301 & \begin{tabular}{|l} 
epoxide \\
hydrolase \\
activity \\
\end{tabular} & $\begin{array}{l}\text { MOLECULAR } \\
\text { FUNCTION }\end{array}$ & $\begin{array}{l}0.658697966 \\
5414314\end{array}$ & $\begin{array}{l}0.049918262 \\
49403735\end{array}$ & 1 & 0 & 854 & 16273 & MELO3C017946 & \\
\hline [] & GO:0030942 & $\begin{array}{l}\text { endoplasmic } \\
\text { reticulum } \\
\text { signal peptide } \\
\text { binding }\end{array}$ & $\begin{array}{l}\text { MOLECULAR } \\
\text { FUNCTION }\end{array}$ & $\begin{array}{l}0.658697966 \\
5414314\end{array}$ & $\begin{array}{l}0.049918262 \\
49403735\end{array}$ & 1 & 0 & 854 & 16273 & MELO3C002757 & \\
\hline [] & GO:0050311 & \begin{tabular}{|l} 
sulfite \\
reductase \\
(ferredoxin) \\
activity \\
\end{tabular} & $\begin{array}{l}\text { MOLECULAR } \\
\text { FUNCTION }\end{array}$ & $\begin{array}{l}0.658697966 \\
5414314\end{array}$ & $\begin{array}{l}0.049918262 \\
49403735\end{array}$ & 1 & 0 & 854 & 16273 & MELO3C015186 & \\
\hline
\end{tabular}




\begin{tabular}{|c|c|c|c|c|c|c|c|c|c|c|c|}
\hline [] & GO:0005509 & $\begin{array}{l}\text { calcium ion } \\
\text { binding }\end{array}$ & $\begin{array}{l}\text { MOLECULAR } \\
\text { FUNCTION }\end{array}$ & \begin{tabular}{|l|}
0.658697966 \\
5414314
\end{tabular} & $\begin{array}{l}0.045998400 \\
92609351\end{array}$ & 18 & 209 & 837 & 16064 & $\begin{array}{l}\text { MELO3C014016; } \\
\text { MELO3C004194; } \\
\text { MELO3C014588; } \\
\text { MELO3C010794; } \\
\text { MELO3C013892; } \\
\text { MELO3C006158; } \\
\text { MELO3C014150; } \\
\text { MELO3C005757; } \\
\text { MELO3C026749; } \\
\text { MELO3C006759; } \\
\text { MELO3C019002; } \\
\text { MELO3C022494; } \\
\text { MELO3C022260; } \\
\text { MELO3C019820; } \\
\text { MELO3C017844; } \\
\text { MELO3C013702; } \\
\text { MELO3C014658; } \\
\text { MELO3C012457 }\end{array}$ & $\begin{array}{l}\text { MELO3C003372; } \\
\text { MELO3C004465; } \\
\text { MELO3C008705; } \\
\text { MELO3C024866; } \\
\text { MELO3C025712; } \\
\text { MELO3C023415; } \\
\text { MELO3C005319; } \\
\text { MELO3C002967; } \\
\text { MELO3C019035; } \\
\text { MELO3C019036; } \\
\text { MELO3C020263; } \\
\text { MELO3C015597; } \\
\text { MELO3C016569; } \\
\text { MELO3C010348; } \\
\text { MELO3C020719; } \\
\text { MELO3C014279; } \\
\text { MELO3C013982; } \\
\text { MELO3C023542; } \\
\text { MELO3C007507; } \\
\text { MELO3C020277; } \\
\text { MELO3C016330; } \\
\text { MELO3C016333; } \\
\text { MELO3C018876; } \\
\text { MELO3C018877; } \\
\text { MELO3C013504; } \\
\text { MELO3C018517; } \\
\text { MELO3C018879; }\end{array}$ \\
\hline [] & GO:0016532 & \begin{tabular}{|l} 
superoxide \\
dismutase \\
copper \\
chaperone \\
activity \\
\end{tabular} & $\begin{array}{l}\text { MOLECULAR } \\
\text { FUNCTION }\end{array}$ & $\begin{array}{l}0.658697966 \\
5414314\end{array}$ & $\begin{array}{l}0.049918262 \\
49403735\end{array}$ & 1 & 0 & 854 & 16273 & MELO3C014007 & \\
\hline[] & GO:0050201 & $\begin{array}{l}\text { fucokinase } \\
\text { activity }\end{array}$ & $\begin{array}{l}\text { MOLECULAR } \\
\text { FUNCTION }\end{array}$ & $\begin{array}{l}0.658697966 \\
5414314\end{array}$ & $\begin{array}{l}0.049918262 \\
49403735\end{array}$ & 1 & 0 & 854 & 16273 & MELO3C006005 & \\
\hline [] & GO:0008908 & $\begin{array}{l}\text { isochorismatas } \\
\text { e activity }\end{array}$ & $\begin{array}{l}\text { MOLECULAR } \\
\text { FUNCTION }\end{array}$ & $\begin{array}{l}0.658697966 \\
5414314\end{array}$ & $\begin{array}{l}0.049918262 \\
49403735\end{array}$ & 1 & 0 & 854 & 16273 & MELO3C011747 & \\
\hline[] & GO:0016762 & \begin{tabular}{|l|} 
xyloglucan:xyl \\
oglucosyl \\
transferase \\
activity
\end{tabular} & $\begin{array}{l}\text { MOLECULAR } \\
\text { FUNCTION }\end{array}$ & $\begin{array}{l}0.658697966 \\
5414314\end{array}$ & $\begin{array}{l}0.048685998 \\
78337037\end{array}$ & 4 & 24 & 851 & 16249 & $\begin{array}{l}\text { MELO3C012004; } \\
\text { MELO3C017480; } \\
\text { MELO3C017481; } \\
\text { MELO3C017478 }\end{array}$ & $\begin{array}{l}\text { MELO3C014469; } \\
\text { MELO3C014468; } \\
\text { MELO3C014467; } \\
\text { MELO3C014466; } \\
\text { MELO3C014465; } \\
\text { MELO3C002480; } \\
\text { MELO3C009367; } \\
\text { MELO3C004087; } \\
\text { MELO3C003441; } \\
\text { MELO3C024704; } \\
\text { MELO3C005245; } \\
\text { MELO3C021686; } \\
\text { MELO3C004941; } \\
\text { MELO3C018292; } \\
\text { MELO3C026945; } \\
\text { MELO3C001951; } \\
\text { MELO3C026755; } \\
\text { MELO3C017482; } \\
\text { MELO3C021685; } \\
\text { MELO3C018033; } \\
\text { MELO3C011706; } \\
\text { MELO3C017476; } \\
\text { MELO3C018785; } \\
\text { MELO3C017479 }\end{array}$ \\
\hline[] & GO:0004637 & $\begin{array}{l}\text { phosphoribosy } \\
\text { lamine-glycine } \\
\text { ligase activity }\end{array}$ & $\begin{array}{l}\text { MOLECULAR } \\
\text { FUNCTION }\end{array}$ & \begin{tabular}{|l|}
0.658697966 \\
5414314
\end{tabular} & $\begin{array}{l}0.049918262 \\
49403735\end{array}$ & 1 & 0 & 854 & 16273 & MELO3C016483 & \\
\hline [] & GO:0052636 & $\begin{array}{l}\text { arabinosyltran } \\
\text { sferase activity }\end{array}$ & $\begin{array}{l}\text { MOLECULAR } \\
\text { FUNCTION }\end{array}$ & $\begin{array}{l}0.658697966 \\
5414314\end{array}$ & $\begin{array}{l}0.049918262 \\
49403735\end{array}$ & 1 & 0 & 854 & 16273 & MELO3C010524 & \\
\hline[] & GO:0035259 & \begin{tabular}{|l|} 
glucocorticoid \\
receptor \\
binding
\end{tabular} & $\begin{array}{l}\text { MOLECULAR } \\
\text { FUNCTION }\end{array}$ & $\begin{array}{l}0.658697966 \\
5414314\end{array}$ & $\begin{array}{l}0.049918262 \\
49403735\end{array}$ & 1 & 0 & 854 & 16273 & MELO3C012231 & \\
\hline
\end{tabular}




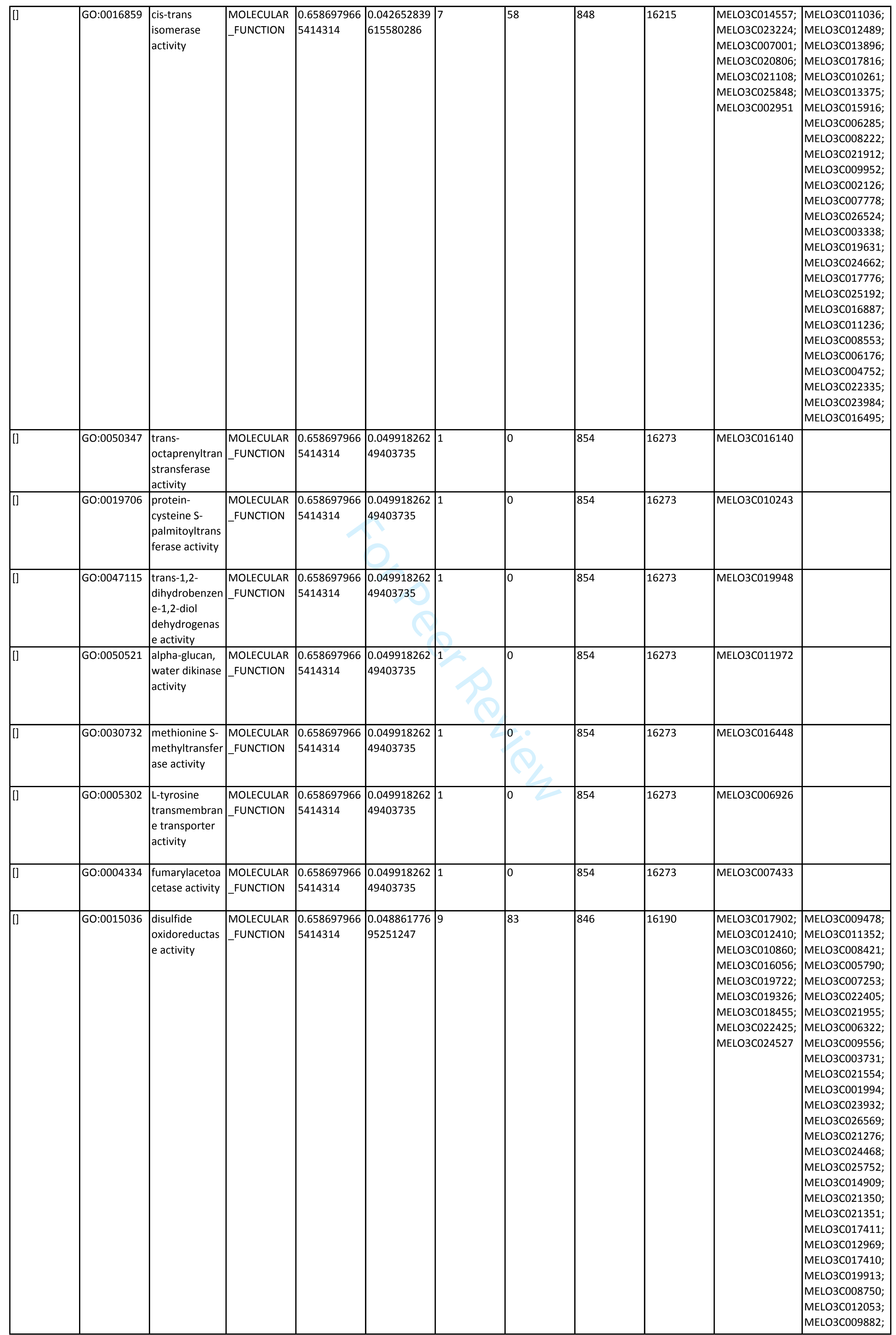




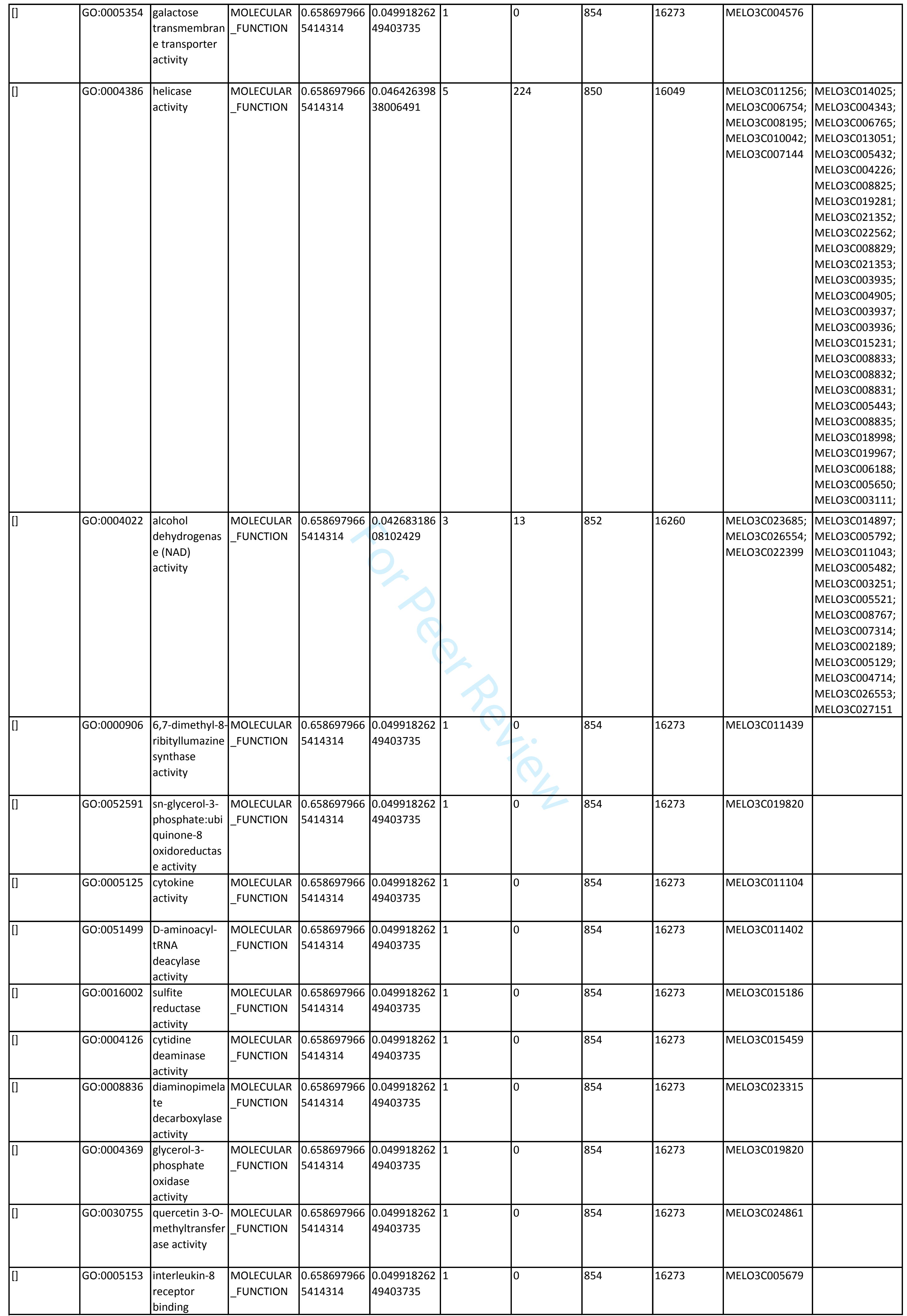




\begin{tabular}{|c|c|c|c|c|c|c|c|c|c|c|c|}
\hline[] & GO:0033858 & \begin{tabular}{|l|}
$\mathrm{N}-$ \\
acetylgalactos \\
amine kinase \\
activity
\end{tabular} & $\begin{array}{l}\text { MOLECULAR } \\
\text {-FUNCTION }\end{array}$ & \begin{tabular}{|l|}
0.658697966 \\
5414314
\end{tabular} & \begin{tabular}{|l|}
0.049918262 \\
49403735
\end{tabular} & 1 & 0 & 854 & 16273 & MELO3C006726 & \\
\hline[] & GO:0015088 & \begin{tabular}{|l|} 
copper uptake \\
transmembran \\
e transporter \\
activity
\end{tabular} & \begin{tabular}{|l|} 
MOLECULAR \\
_FUNCTION
\end{tabular} & \begin{tabular}{|l|}
0.658697966 \\
5414314
\end{tabular} & \begin{tabular}{|l|}
0.049918262 \\
49403735
\end{tabular} & 1 & 0 & 854 & 16273 & MELO3C018494 & \\
\hline [] & \begin{tabular}{|l|l|} 
GO:0004073 \\
\end{tabular} & \begin{tabular}{|l|} 
aspartate- \\
semialdehyde \\
dehydrogenas \\
e activity
\end{tabular} & $\begin{array}{l}\text { MOLECULAR } \\
\text {-FUNCTION }\end{array}$ & \begin{tabular}{|l|}
0.658697966 \\
5414314
\end{tabular} & \begin{tabular}{|l|}
0.049918262 \\
49403735
\end{tabular} & 1 & 0 & 854 & 16273 & MELO3C010427 & \\
\hline [] & \begin{tabular}{|l|l|} 
GO:0033961 \\
\end{tabular} & \begin{tabular}{|l} 
cis-stilbene- \\
oxide \\
hydrolase \\
activity
\end{tabular} & $\begin{array}{l}\text { MOLECULAR } \\
\text {-FUNCTION }\end{array}$ & \begin{tabular}{|l|}
0.658697966 \\
5414314
\end{tabular} & \begin{tabular}{|l|}
0.049918262 \\
49403735
\end{tabular} & 1 & 0 & 854 & 16273 & MELO3C015292 & \\
\hline[] & GO:0030331 & \begin{tabular}{|l} 
estrogen \\
receptor \\
binding
\end{tabular} & $\begin{array}{l}\text { MOLECULAR } \\
\text { FUNCTION }\end{array}$ & \begin{tabular}{|l|}
0.658697966 \\
5414314
\end{tabular} & \begin{tabular}{|l|}
0.049918262 \\
49403735
\end{tabular} & 1 & 0 & 854 & 16273 & MELO3C012231 & \\
\hline[] & GO:0004048 & \begin{tabular}{|l|} 
anthranilate \\
phosphoribosy \\
Itransferase \\
activity
\end{tabular} & $\begin{array}{l}\text { MOLECULAR } \\
\text { FUNCTION }\end{array}$ & \begin{tabular}{|l|}
0.658697966 \\
5414314
\end{tabular} & \begin{tabular}{|l|}
0.049918262 \\
49403735
\end{tabular} & 1 & 0 & 854 & 16273 & MELO3C002275 & \\
\hline[] & GO:0008893 & \begin{tabular}{|l|} 
guanosine-3', \\
bis(diphosphat \\
e) $3^{\prime}-$ \\
diphosphatase \\
activity
\end{tabular} & $\begin{array}{l}\text { MOLECULAR } \\
\text { FUNCTION }\end{array}$ & \begin{tabular}{|l|}
0.658697966 \\
5414314
\end{tabular} & \begin{tabular}{|l|}
0.049918262 \\
49403735
\end{tabular} & 1 & 0 & 854 & 16273 & MELO3C006759 & \\
\hline [] & \begin{tabular}{|l|l|} 
GO:0030797 \\
\end{tabular} & $\begin{array}{l}24- \\
\text { methylenester } \\
\text { ol C- } \\
\text { methyltransfer } \\
\text { ase activity }\end{array}$ & $\begin{array}{l}\text { MOLECULAR } \\
\text { _FUNCTION }\end{array}$ & \begin{tabular}{|l|}
0.658697966 \\
5414314
\end{tabular} & $\begin{array}{l}0.049918262 \\
49403735 \\
\end{array}$ & 1 & 0 & 854 & 16273 & MELO3C003726 & \\
\hline[] & GO:0047705 & $\begin{array}{l}\text { bilirubin } \\
\text { oxidase } \\
\text { activity }\end{array}$ & $\begin{array}{l}\text { MOLECULAR } \\
\text { FUNCTION }\end{array}$ & \begin{tabular}{|l|}
0.658697966 \\
5414314
\end{tabular} & \begin{tabular}{|l|}
0.049918262 \\
49403735
\end{tabular} & 1 & 0 & 854 & 16273 & MELO3C005201 & \\
\hline[] & GO:0032442 & \begin{tabular}{|l|} 
phenylcoumar \\
an benzylic \\
ether \\
reductase \\
activity
\end{tabular} & $\begin{array}{l}\text { MOLECULAR } \\
\text { FUNCTION }\end{array}$ & \begin{tabular}{|l|}
0.658697966 \\
5414314
\end{tabular} & \begin{tabular}{|l|}
0.049918262 \\
49403735
\end{tabular} & 1 & 0 & 854 & 16273 & MELO3C023304 & \\
\hline [] & GO:0030267 & \begin{tabular}{|l|} 
glyoxylate \\
reductase \\
(NADP) activity
\end{tabular} & \begin{tabular}{|l} 
MOLECULAR \\
FUNCTION
\end{tabular} & \begin{tabular}{|l|}
0.658697966 \\
5414314
\end{tabular} & \begin{tabular}{|l|}
0.049918262 \\
49403735
\end{tabular} & 1 & 0 & 854 & 16273 & MELO3C013093 & \\
\hline \begin{tabular}{|l}
] \\
\end{tabular} & GO:0005086 & \begin{tabular}{|l|} 
ARF guanyl- \\
nucleotide \\
exchange \\
factor activity
\end{tabular} & $\begin{array}{l}\text { MOLECULAR } \\
\text { _FUNCTION }\end{array}$ & \begin{tabular}{|l|}
0.658697966 \\
5414314
\end{tabular} & \begin{tabular}{|l|}
0.044214600 \\
976853265
\end{tabular} & 2 & 5 & 853 & 16268 & $\begin{array}{l}\text { MELO3C004551; } \\
\text { MELO3C006224 }\end{array}$ & $\begin{array}{l}\text { MELO3C023883; } \\
\text { MELO3C012923; } \\
\text { MELO3C012924; } \\
\text { MELO3C006181; } \\
\text { MELO3C015692 }\end{array}$ \\
\hline [] & GO:0008236 & $\begin{array}{l}\text { serine-type } \\
\text { peptidase } \\
\text { activity }\end{array}$ & $\begin{array}{l}\text { MOLECULAR } \\
\text { FUNCTION }\end{array}$ & \begin{tabular}{|l|}
0.658697966 \\
5414314
\end{tabular} & \begin{tabular}{|l|}
0.038263953 \\
28303021
\end{tabular} & 3 & 181 & 852 & 16092 & $\begin{array}{l}\text { MELO3C007609; } \\
\text { MELO3C00455; } \\
\text { MELO3C004117 }\end{array}$ & 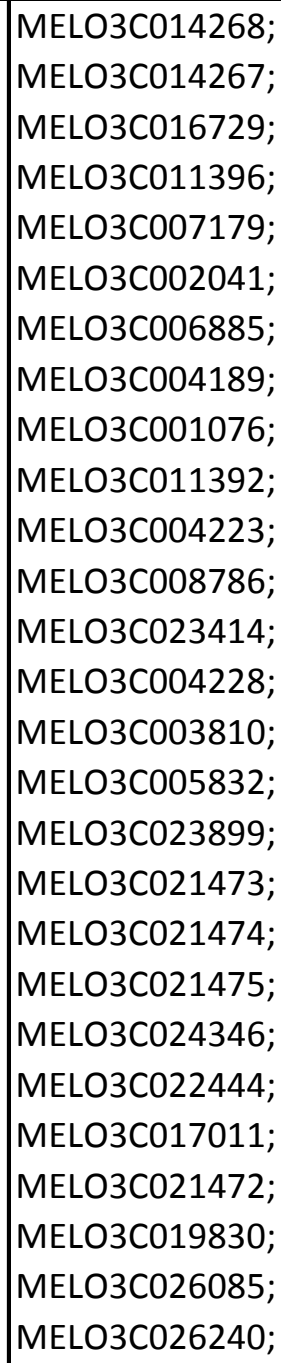 \\
\hline
\end{tabular}




\begin{tabular}{|c|c|c|c|c|c|c|c|c|c|c|c|}
\hline[] & GO:0047837 & \begin{tabular}{|l|} 
D-xylose 1- \\
dehydrogenas \\
e (NADP+) \\
activity
\end{tabular} & $\begin{array}{l}\text { MOLECULAR } \\
\text { FUNCTION }\end{array}$ & \begin{tabular}{|l|}
0.658697966 \\
5414314
\end{tabular} & $\begin{array}{l}0.049918262 \\
49403735\end{array}$ & 1 & 0 & 854 & 16273 & MELO3C019948 & \\
\hline [] & GO:0034618 & $\begin{array}{l}\text { arginine } \\
\text { binding }\end{array}$ & $\begin{array}{l}\text { MOLECULAR } \\
\text { FUNCTION }\end{array}$ & \begin{tabular}{|l|}
0.658697966 \\
5414314
\end{tabular} & $\begin{array}{l}0.049918262 \\
49403735\end{array}$ & 1 & 0 & 854 & 16273 & MELO3C011208 & \\
\hline [] & GO:0032550 & $\begin{array}{l}\text { purine } \\
\text { ribonucleoside } \\
\text { binding }\end{array}$ & $\begin{array}{l}\text { MOLECULAR } \\
\text { FUNCTION }\end{array}$ & \begin{tabular}{|l|}
0.658697966 \\
5414314
\end{tabular} & $\begin{array}{l}0.039434437 \\
36037198\end{array}$ & 135 & 2163 & 720 & 14110 & $\begin{array}{l}\text { MELO3C010189; } \\
\text { MELO3C012361; } \\
\text { MELO3C006005; } \\
\text { MELO3C005673; } \\
\text { MELO3C002167; } \\
\text { MELO3C001354; } \\
\text { MELO3C005679; } \\
\text { MELO3C007857; } \\
\text { MELO3C003139; } \\
\text { MELO3C002687; } \\
\text { MELO3C003812; } \\
\text { MELO3C011719; } \\
\text { MELO3C025550; } \\
\text { MELO3C002609; } \\
\text { MELO3C015233; } \\
\text { MELO3C012525; } \\
\text { MELO3C015926; } \\
\text { MELO3C021247; } \\
\text { MELO3C001920; } \\
\text { MELO3C005849; } \\
\text { MELO3C020394; } \\
\text { MELO3C011208; } \\
\text { MELO3C027184; } \\
\text { MELO3C010631; } \\
\text { MELO3C011256; } \\
\text { MELO3C013951; } \\
\text { MELO3C007673; }\end{array}$ & $\begin{array}{l}\text { MELO3C014269; } \\
\text { MELO3C003133; } \\
\text { MELO3C004463; } \\
\text { MELO3C007972; } \\
\text { MELO3C006648; } \\
\text { MELO3C007978; } \\
\text { MELO3C008825; } \\
\text { MELO3C008829; } \\
\text { MELO3C026243; } \\
\text { MELO3C019951; } \\
\text { MELO3C000093; } \\
\text { MELO3C025392; } \\
\text { MELO3C015599; } \\
\text { MELO3C018624; } \\
\text { MELO3C004471; } \\
\text { MELO3C020958; } \\
\text { MELO3C020959; } \\
\text { MELO3C008832; } \\
\text { MELO3C008831; } \\
\text { MELO3C014272; } \\
\text { MELO3C003146; } \\
\text { MELO3C021803; } \\
\text { MELO3C002299; } \\
\text { MELO3C008835; } \\
\text { MELO3C005329; } \\
\text { MELO3C008838; } \\
\text { MELO3C026259; }\end{array}$ \\
\hline [] & GO:0034722 & $\begin{array}{l}\text { gamma- } \\
\text { glutamyl- } \\
\text { peptidase } \\
\text { activity } \\
\end{array}$ & $\begin{array}{l}\text { MOLECULAR } \\
\text { FUNCTION }\end{array}$ & \begin{tabular}{|l|}
0.658697966 \\
5414314
\end{tabular} & \begin{tabular}{|l}
0.049918262 \\
49403735 \\
\end{tabular} & 1 & 0 & 854 & 16273 & MELO3C025645 & \\
\hline [] & GO:0033799 & $\begin{array}{l}\text { myricetin 3'-O- } \\
\text { methyltransfer } \\
\text { ase activity }\end{array}$ & $\begin{array}{l}\text { MOLECULAR } \\
\text { FUNCTION }\end{array}$ & \begin{tabular}{|l|}
0.658697966 \\
5414314
\end{tabular} & $\begin{array}{l}0.049918262 \\
49403735\end{array}$ & 1 & 0 & 854 & 16273 & MELO3C024861 & \\
\hline [] & GO:0004814 & \begin{tabular}{|l|} 
arginine-tRNA \\
ligase activity
\end{tabular} & $\begin{array}{l}\text { MOLECULAR } \\
\text { FUNCTION }\end{array}$ & \begin{tabular}{|l|}
0.658697966 \\
5414314
\end{tabular} & $\begin{array}{l}0.049918262 \\
49403735\end{array}$ & 1 & 0 & 854 & 16273 & MELO3C015422 & \\
\hline [] & GO:0070205 & \begin{tabular}{|l|} 
2-succinyl-6- \\
hydroxy-2,4- \\
cyclohexadien \\
e-1- \\
carboxylate \\
synthase \\
activity \\
\end{tabular} & $\begin{array}{l}\text { MOLECULAR } \\
\text { FUNCTION }\end{array}$ & \begin{tabular}{|l|}
0.658697966 \\
5414314
\end{tabular} & $\begin{array}{l}0.049918262 \\
49403735\end{array}$ & 1 & 0 & 854 & 16273 & MELO3C012178 & \\
\hline [] & GO:0047560 & $\begin{array}{l}\text { 3- } \\
\text { dehydrosphing } \\
\text { anine } \\
\text { reductase } \\
\text { activity }\end{array}$ & $\begin{array}{l}\text { MOLECULAR } \\
\text { FUNCTION }\end{array}$ & \begin{tabular}{|l|}
0.658697966 \\
5414314
\end{tabular} & $\begin{array}{l}0.049918262 \\
49403735\end{array}$ & 1 & 0 & 854 & 16273 & MELO3C006553 & \\
\hline [] & GO:0003977 & $\begin{array}{l}\text { UDP-N- } \\
\text { acetylglucosa } \\
\text { mine } \\
\text { diphosphoryla } \\
\text { se activity } \\
\end{array}$ & $\begin{array}{l}\text { MOLECULAR } \\
\text { FUNCTION }\end{array}$ & \begin{tabular}{|l|}
0.658697966 \\
5414314
\end{tabular} & $\begin{array}{l}0.049918262 \\
49403735\end{array}$ & 1 & 0 & 854 & 16273 & MELO3C007022 & \\
\hline [] & GO:0003978 & \begin{tabular}{|l|} 
UDP-glucose 4- \\
epimerase \\
activity
\end{tabular} & $\begin{array}{l}\text { MOLECULAR } \\
\text { FUNCTION }\end{array}$ & \begin{tabular}{|l|}
0.658697966 \\
5414314
\end{tabular} & $\begin{array}{l}0.044214600 \\
976853265\end{array}$ & 2 & 5 & 853 & 16268 & $\begin{array}{l}\text { MELO3C007043; } \\
\text { MELO3C016300 }\end{array}$ & $\begin{array}{l}\text { MELO3C019557; } \\
\text { MELO3C005769; } \\
\text { MELO3C026841; } \\
\text { MELO3C021813; } \\
\text { MELO3C005640 }\end{array}$ \\
\hline [] & GO:0080007 & $\begin{array}{l}\text { S- } \\
\text { nitrosoglutathi } \\
\text { one reductase } \\
\text { activity }\end{array}$ & $\begin{array}{l}\text { MOLECULAR } \\
\text { FUNCTION }\end{array}$ & \begin{tabular}{|l|}
0.658697966 \\
5414314
\end{tabular} & $\begin{array}{l}0.049918262 \\
49403735\end{array}$ & 1 & 0 & 854 & 16273 & MELO3C022399 & \\
\hline [] & GO:0010487 & $\begin{array}{l}\text { thermospermi } \\
\text { ne synthase } \\
\text { activity }\end{array}$ & $\begin{array}{l}\text { MOLECULAR } \\
\text { FUNCTION }\end{array}$ & \begin{tabular}{|l|}
0.658697966 \\
5414314
\end{tabular} & $\begin{array}{l}0.049918262 \\
49403735\end{array}$ & 1 & 0 & 854 & 16273 & MELO3C011484 & \\
\hline[] & GO:0034020 & \begin{tabular}{|l} 
neoxanthin \\
synthase \\
activity \\
\end{tabular} & $\begin{array}{l}\text { MOLECULAR } \\
\text { FUNCTION }\end{array}$ & \begin{tabular}{|l|}
0.658697966 \\
5414314
\end{tabular} & $\begin{array}{l}0.049918262 \\
49403735\end{array}$ & 1 & 0 & 854 & 16273 & MELO3C017963 & \\
\hline [] & GO:0000026 & $\begin{array}{l}\text { alpha-1,2- } \\
\text { mannosyltrans } \\
\text { ferase activity }\end{array}$ & $\begin{array}{l}\text { MOLECULAR } \\
\text { FUNCTION }\end{array}$ & \begin{tabular}{|l|}
0.658697966 \\
5414314
\end{tabular} & $\begin{array}{l}0.049918262 \\
49403735\end{array}$ & 1 & 0 & 854 & 16273 & MELO3C025637 & \\
\hline
\end{tabular}




\begin{tabular}{|c|c|c|c|c|c|c|c|c|c|c|c|}
\hline [] & GO:0010297 & $\begin{array}{l}\text { heteropolysac } \\
\text { charide } \\
\text { binding }\end{array}$ & $\begin{array}{l}\text { MOLECULAR } \\
\text { FUNCTION }\end{array}$ & $\begin{array}{l}0.658697966 \\
5414314\end{array}$ & $\begin{array}{l}0.049918262 \\
49403735\end{array}$ & 1 & 0 & 854 & 16273 & MELO3C019135 & \\
\hline[] & GO:0005751 & $\begin{array}{l}\text { mitochondrial } \\
\text { respiratory } \\
\text { chain complex } \\
\text { IV }\end{array}$ & $\begin{array}{l}\text { CELLULAR_C } \\
\text { OMPONENT }\end{array}$ & $\begin{array}{l}0.658697966 \\
5414314\end{array}$ & $\begin{array}{l}0.044214600 \\
976853265\end{array}$ & 2 & 5 & 853 & 16268 & $\begin{array}{l}\text { MELO3C004105; } \\
\text { MELO3C011044 }\end{array}$ & $\begin{array}{l}\text { MELO3C015922; } \\
\text { MELO3C003366; } \\
\text { MELO3C016926; } \\
\text { MELO3C014946; } \\
\text { MELO3C016120 }\end{array}$ \\
\hline [] & GO:0034099 & \begin{tabular}{|l} 
luminal \\
surveillance \\
complex
\end{tabular} & $\begin{array}{l}\text { CELLULAR_C } \\
\text { OMPONENT }\end{array}$ & $\begin{array}{l}0.658697966 \\
5414314\end{array}$ & $\begin{array}{l}0.049918262 \\
49403735\end{array}$ & 1 & 0 & 854 & 16273 & MELO3C005757 & \\
\hline[] & GO:0005662 & \begin{tabular}{|l|} 
DNA \\
replication \\
factor A \\
complex \\
\end{tabular} & $\begin{array}{l}\text { CELLULAR_C } \\
\text { OMPONENT }\end{array}$ & $\begin{array}{l}0.658697966 \\
5414314\end{array}$ & $\begin{array}{l}0.049918262 \\
49403735\end{array}$ & 1 & 10 & 854 & 16273 & MELO3C017228 & \\
\hline[] & GO:0009842 & cyanelle & $\begin{array}{l}\text { CELLULAR_C } \\
\text { OMPONENT }\end{array}$ & $\begin{array}{l}0.658697966 \\
5414314\end{array}$ & $\begin{array}{l}0.049918262 \\
49403735\end{array}$ & 1 & 0 & 854 & 16273 & MELO3C016140 & \\
\hline [] & GO:0009930 & $\begin{array}{l}\text { longitudinal } \\
\text { side of cell } \\
\text { surface }\end{array}$ & $\begin{array}{l}\text { CELLULAR_C } \\
\text { OMPONENT }\end{array}$ & $\begin{array}{l}0.658697966 \\
5414314\end{array}$ & $\begin{array}{l}0.049918262 \\
49403735\end{array}$ & 1 & 0 & 854 & 16273 & MELO3C017242 & \\
\hline[] & GO:0042555 & MCM complex & $\begin{array}{l}\text { CELLULAR_C } \\
\text { OMPONENT }\end{array}$ & $\begin{array}{l}0.658697966 \\
5414314 \\
\end{array}$ & $\begin{array}{l}0.049918262 \\
49403735\end{array}$ & 1 & 0 & 854 & 16273 & MELO3C020001 & \\
\hline [] & GO:0008305 & $\begin{array}{l}\text { integrin } \\
\text { complex }\end{array}$ & $\begin{array}{l}\text { CELLULAR_C } \\
\text { OMPONENT }\end{array}$ & \begin{tabular}{|l|}
0.658697966 \\
5414314
\end{tabular} & $\begin{array}{l}0.049918262 \\
49403735\end{array}$ & 1 & 0 & 854 & 16273 & MELO3C007690 & \\
\hline [] & GO:0031672 & A band & $\begin{array}{l}\text { CELLULAR_C } \\
\text { OMPONENT }\end{array}$ & \begin{tabular}{|l}
0.658697966 \\
5414314 \\
\end{tabular} & $\begin{array}{l}0.049918262 \\
49403735 \\
\end{array}$ & 1 & 10 & 854 & 16273 & MELO3C011193 & \\
\hline$[$ [] & GO:0031429 & \begin{tabular}{|l}
$\begin{array}{l}\text { box H/ACA } \\
\text { snoRNP } \\
\text { complex }\end{array}$ \\
\end{tabular} & $\begin{array}{l}\text { CELLULAR_C } \\
\text { OMPONENT }\end{array}$ & $\begin{array}{l}0.658697966 \\
5414314\end{array}$ & $\begin{array}{l}0.049918262 \\
49403735\end{array}$ & 1 & 0 & 854 & 16273 & MELO3C008801 & \\
\hline[] & GO:0018444 & $\begin{array}{l}\text { translation } \\
\text { release factor } \\
\text { complex }\end{array}$ & $\begin{array}{l}\text { CELLULAR_C } \\
\text { OMPONENT }\end{array}$ & $\begin{array}{l}0.658697966 \\
5414314\end{array}$ & $\begin{array}{l}0.049918262 \\
49403735\end{array}$ & 1 & 0 & 854 & 16273 & MELO3C002118 & \\
\hline [] & GO:0009316 & $\begin{array}{l}\text { 3- } \\
\text { isopropylmalat } \\
\text { e dehydratase } \\
\text { complex }\end{array}$ & $\begin{array}{l}\text { CELLULAR_C } \\
\text { OMPONENT }\end{array}$ & $\begin{array}{l}0.658697966 \\
5414314\end{array}$ & $\begin{array}{l}0.049918262 \\
49403735\end{array}$ & 1 & 0 & 854 & 16273 & MELO3C005518 & \\
\hline$[$ [] & GO:0034663 & \begin{tabular}{|l} 
endoplasmic \\
reticulum \\
chaperone \\
complex
\end{tabular} & $\begin{array}{l}\text { CELLULAR_C } \\
\text { OMPONENT }\end{array}$ & $\begin{array}{l}0.658697966 \\
5414314\end{array}$ & $\begin{array}{l}0.049918262 \\
49403735\end{array}$ & 1 & 0 & 854 & 16273 & MELO3C005757 & \\
\hline [] & GO:0009349 & $\begin{array}{l}\text { riboflavin } \\
\text { synthase } \\
\text { complex }\end{array}$ & $\begin{array}{l}\text { CELLULAR_C } \\
\text { OMPONENT }\end{array}$ & $\begin{array}{l}0.658697966 \\
5414314\end{array}$ & $\begin{array}{l}0.049918262 \\
49403735\end{array}$ & 1 & 0 & 854 & 16273 & MELO3C011439 & \\
\hline [] & GO:0010005 & \begin{tabular}{|l} 
cortical \\
microtubule, \\
transverse to \\
long axis \\
\end{tabular} & $\begin{array}{l}\text { CELLULAR_C } \\
\text { OMPONENT }\end{array}$ & $\begin{array}{l}0.658697966 \\
5414314\end{array}$ & $\begin{array}{l}0.049918262 \\
49403735\end{array}$ & 1 & 0 & 854 & 16273 & MELO3C012361 & \\
\hline [] & GO:0005960 & $\begin{array}{l}\text { glycine } \\
\text { cleavage } \\
\text { complex }\end{array}$ & $\begin{array}{l}\text { CELLULAR_C } \\
\text { OMPONENT }\end{array}$ & $\begin{array}{l}0.658697966 \\
5414314\end{array}$ & $\begin{array}{l}0.044214600 \\
976853265\end{array}$ & 2 & 5 & 853 & 16268 & $\begin{array}{l}\text { MELO3C021742; } \\
\text { MELO3C021247 }\end{array}$ & $\begin{array}{l}\text { MELO3C003386; } \\
\text { MELO3C003734; } \\
\text { MELO3C002444; } \\
\text { MELO3C006734; } \\
\text { MELO3C010754 }\end{array}$ \\
\hline [] & GO:0033162 & $\begin{array}{l}\text { melanosome } \\
\text { membrane }\end{array}$ & $\begin{array}{l}\text { CELLULAR_C } \\
\text { OMPONENT } \\
\end{array}$ & \begin{tabular}{|l|}
0.658697966 \\
5414314 \\
\end{tabular} & $\begin{array}{l}0.049918262 \\
49403735 \\
\end{array}$ & 1 & 0 & 854 & 16273 & MELO3C006926 & \\
\hline [] & GO:0005923 & \begin{tabular}{|l|} 
bicellular tight \\
junction
\end{tabular} & $\begin{array}{l}\text { CELLULAR_C } \\
\text { OMPONENT }\end{array}$ & $\begin{array}{l}0.658697966 \\
5414314\end{array}$ & $\begin{array}{l}0.049918262 \\
49403735\end{array}$ & 1 & 0 & 854 & 16273 & MELO3C022488 & \\
\hline
\end{tabular}




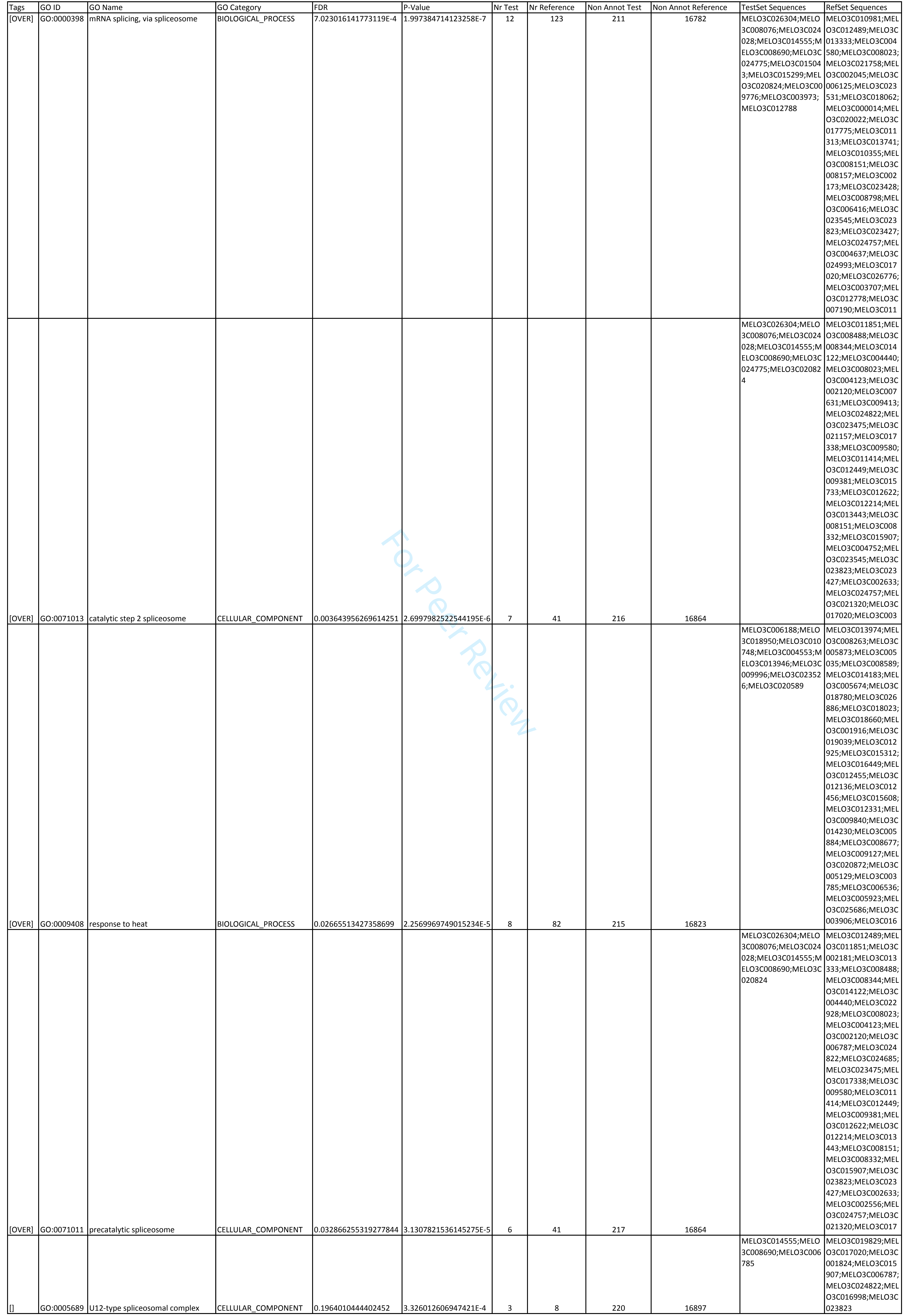



016946:MELO3C013 16758 O3C016946;MELO3C

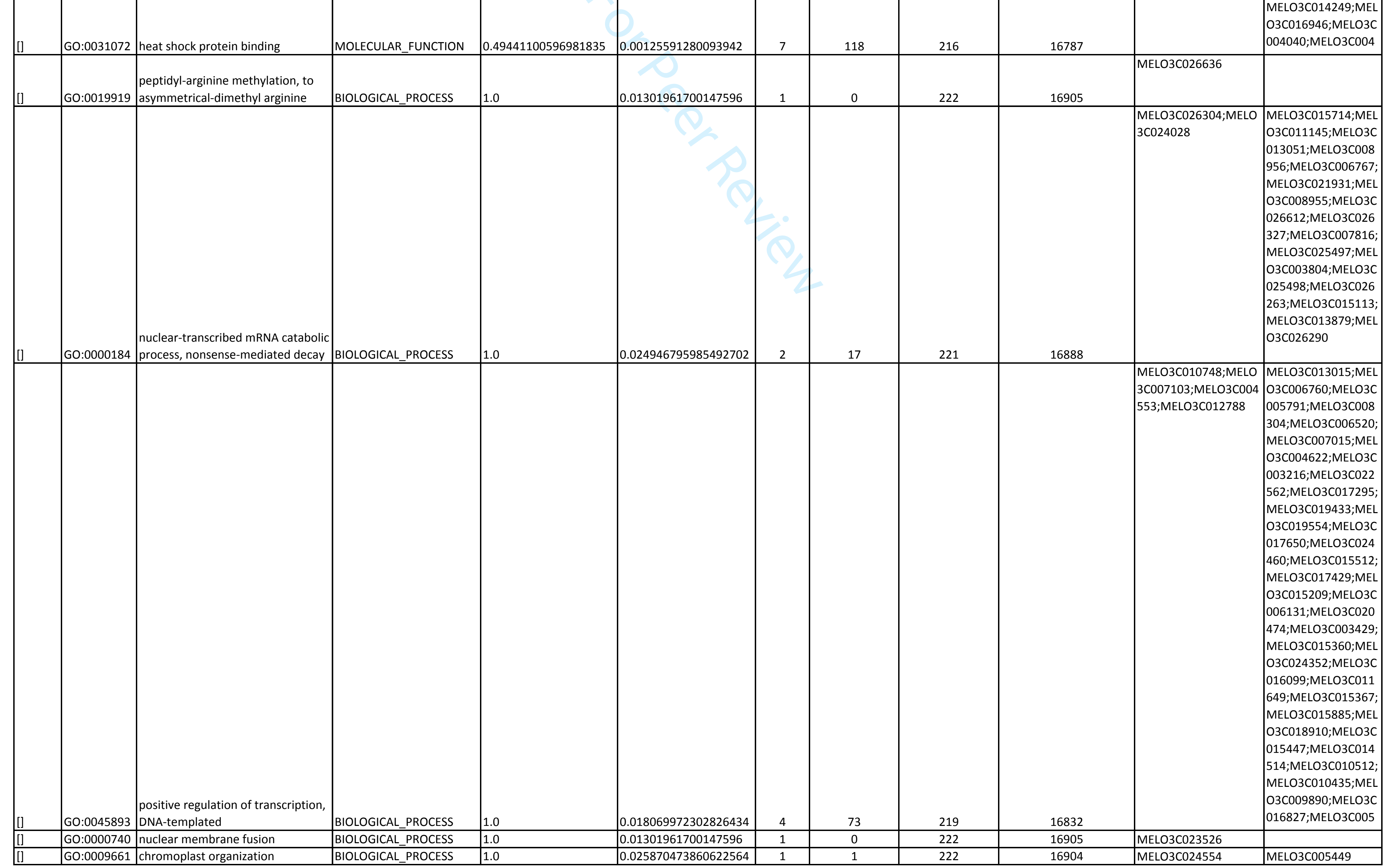




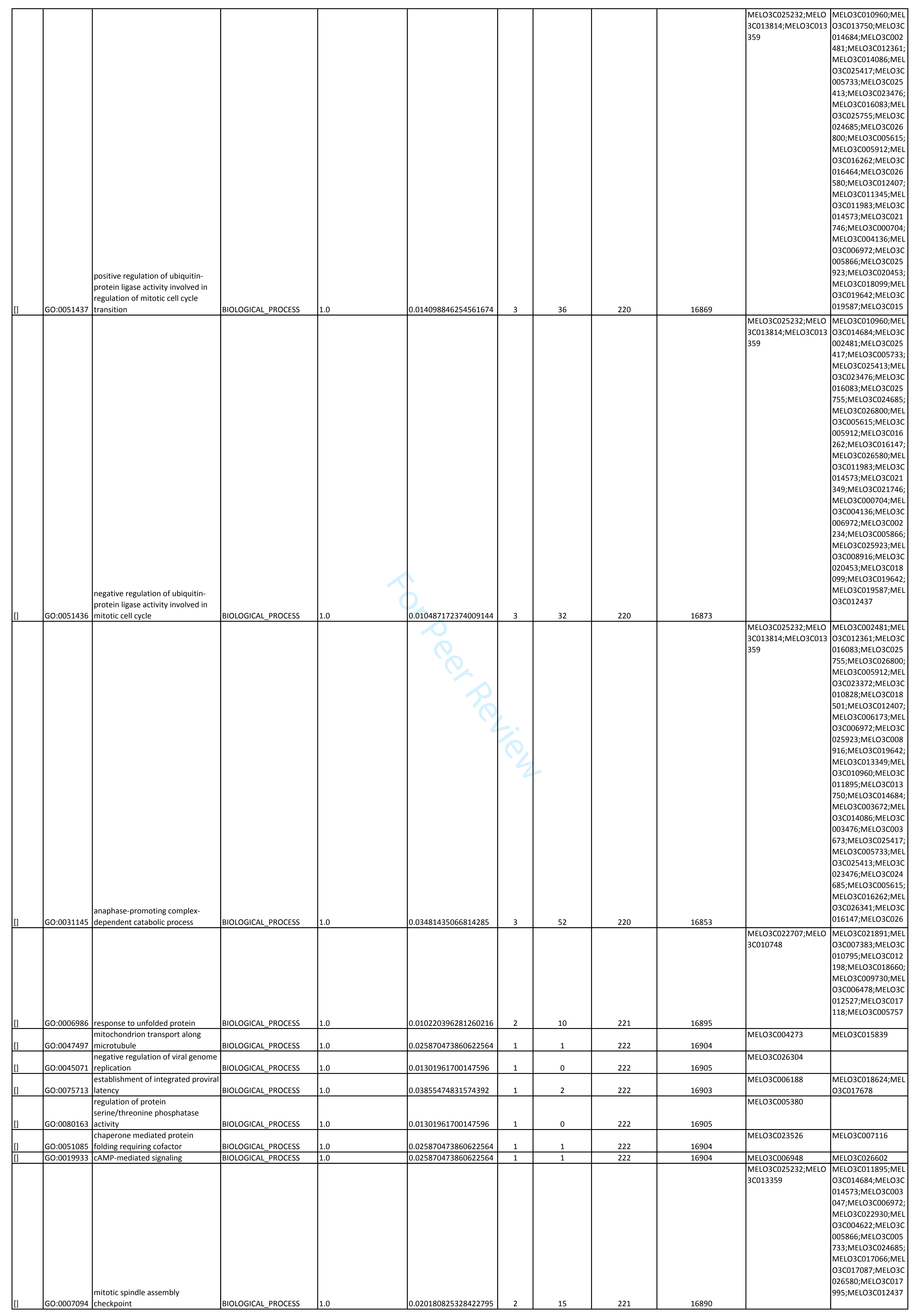




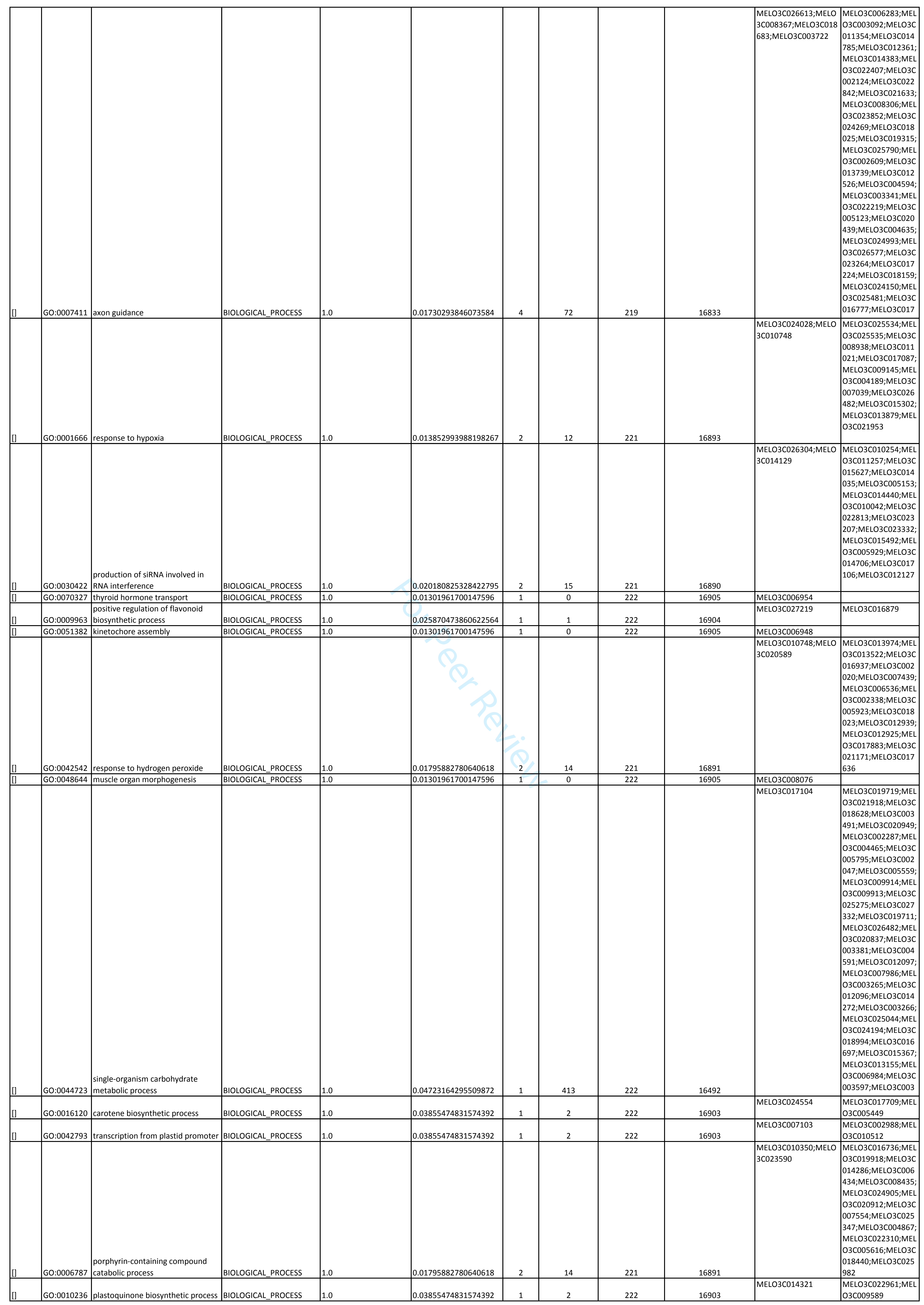




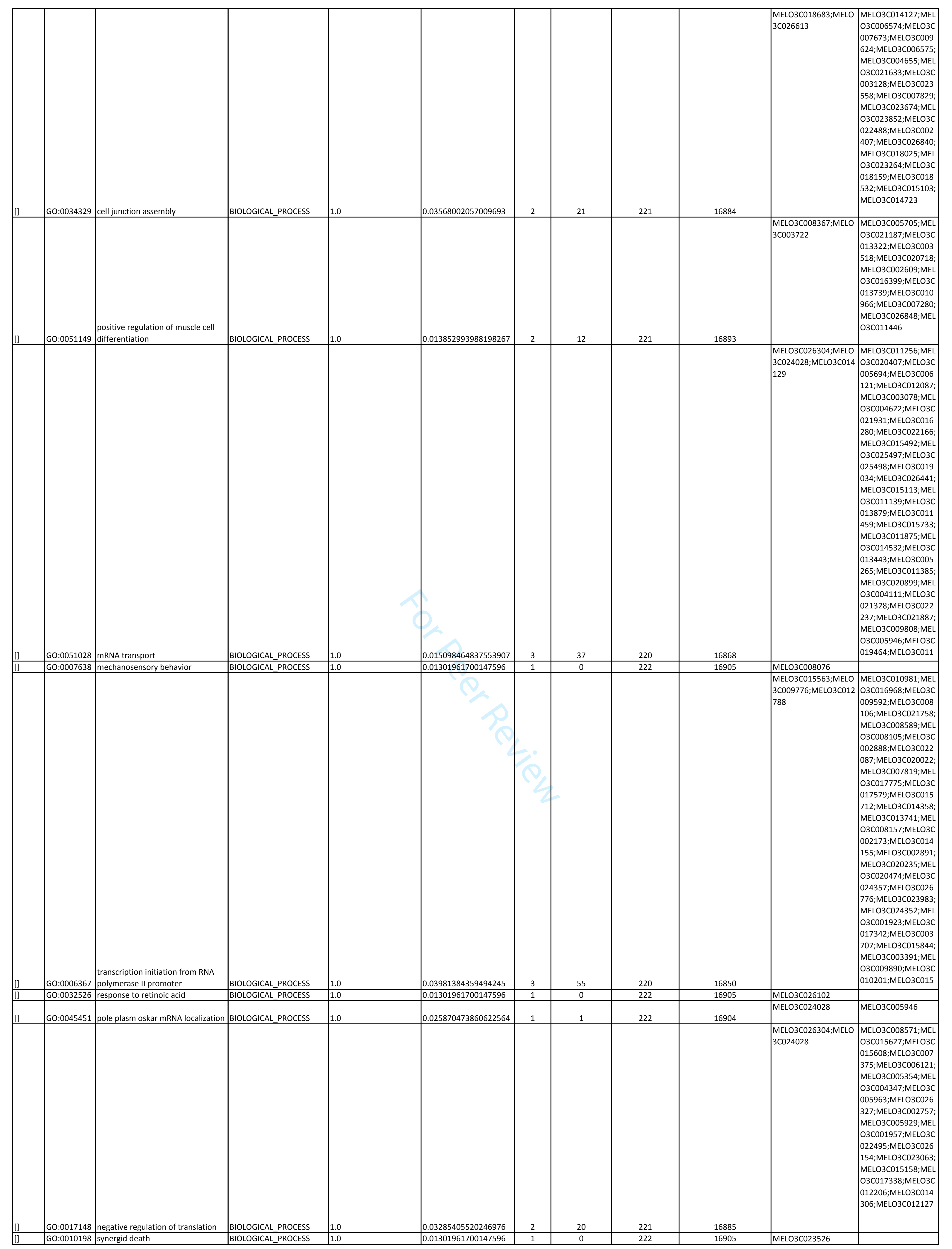




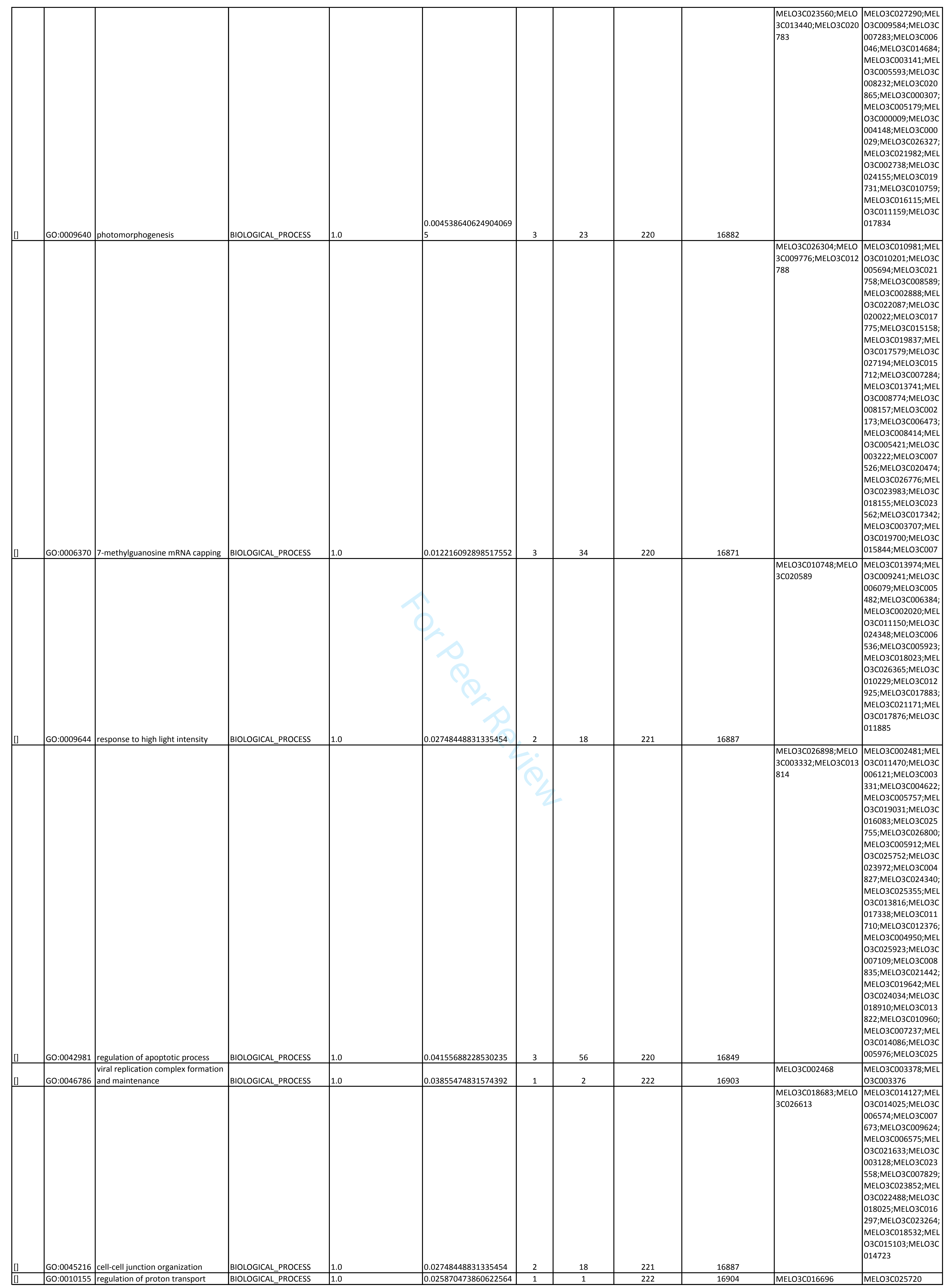




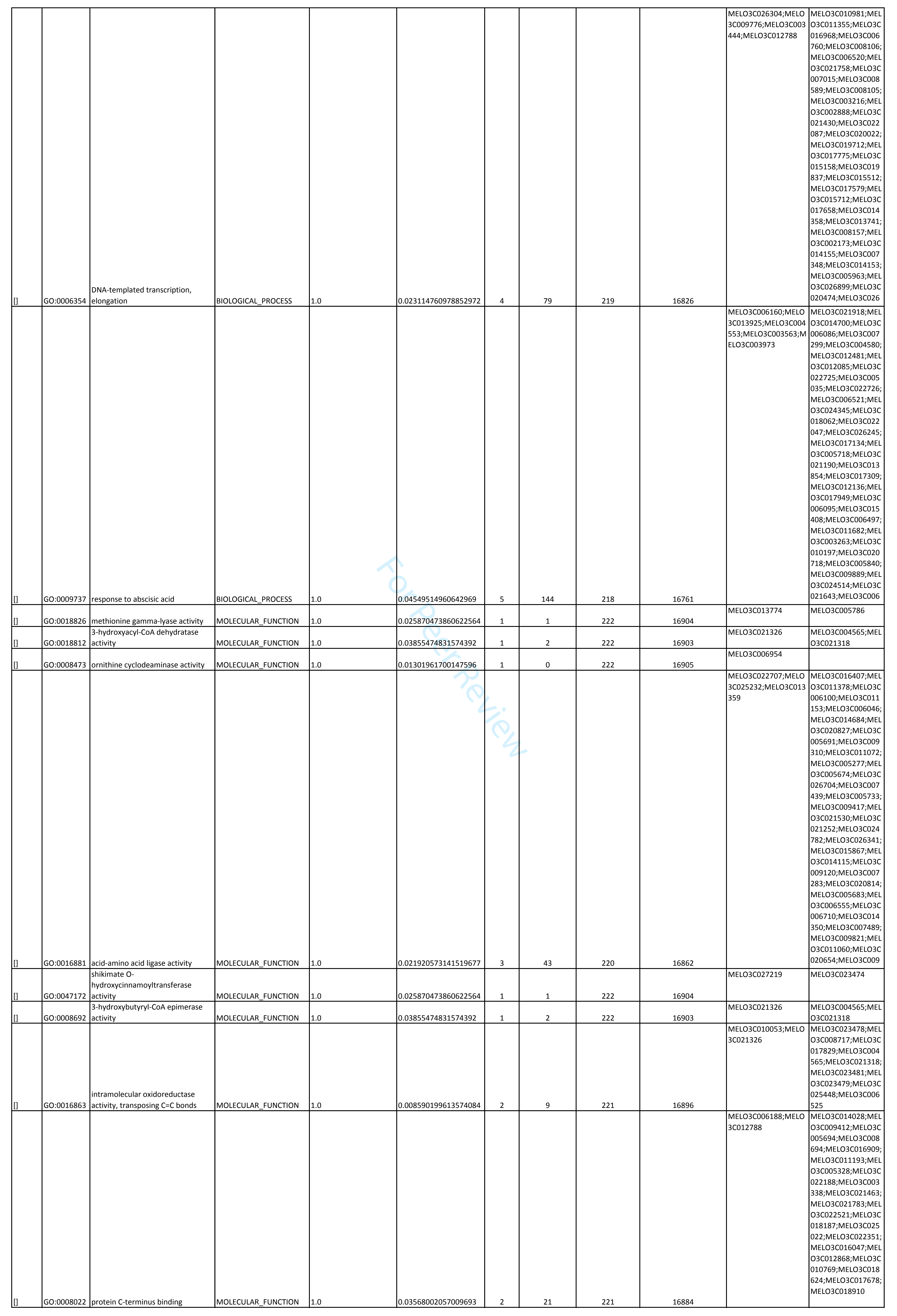




\begin{tabular}{|c|c|c|c|c|c|c|c|c|c|c|c|}
\hline [] & GO:0008270 & zinc ion binding & MOLECULAR_FUNCTION & 1.0 & 0.039097915237540186 & 25 & 1252 & 198 & 15653 & 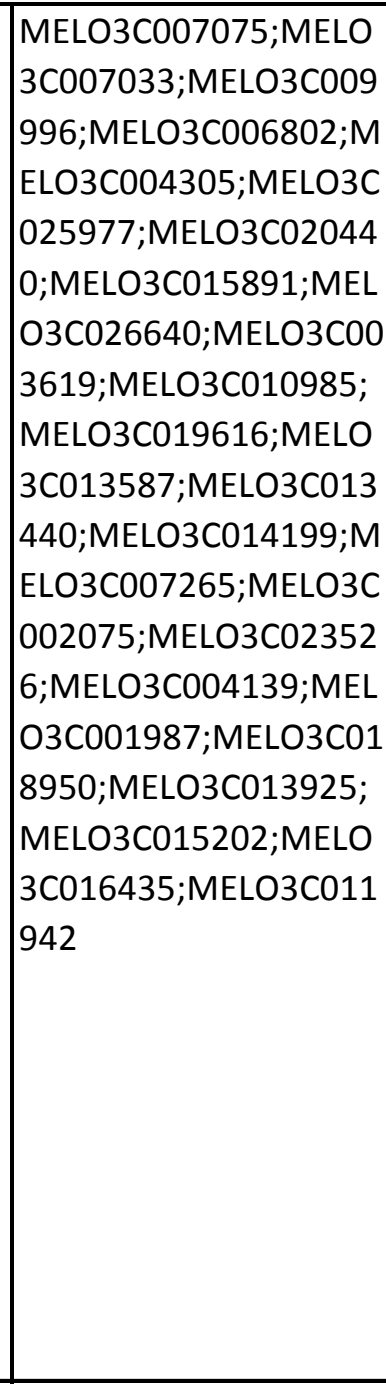 & 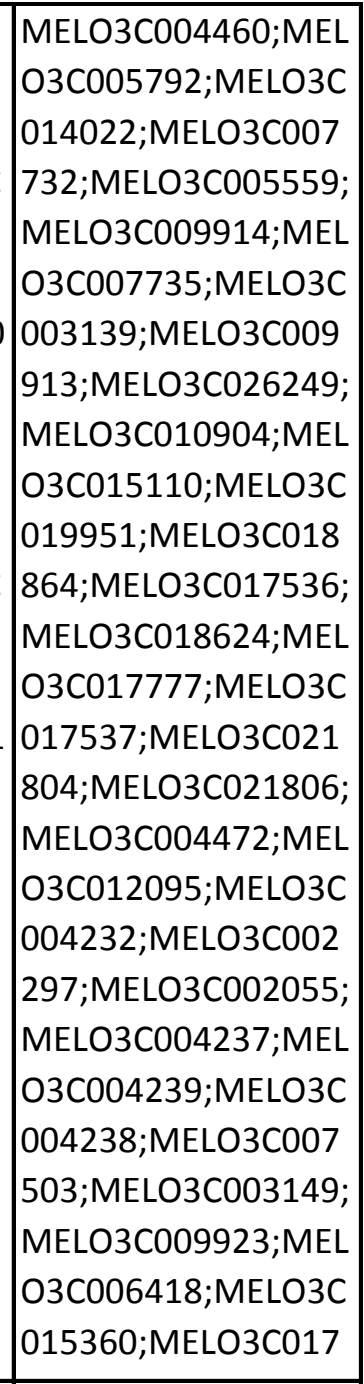 \\
\hline [] & GO:0051741 & $\begin{array}{l}\text { 2-methyl-6-phytyl-1,4- } \\
\text { benzoquinone methyltransferase } \\
\text { activity }\end{array}$ & MOLECULAR_FUNCTION & 1.0 & 0.01301961700147596 & 1 & 0 & 222 & 16905 & MELO3C014321 & \\
\hline [] & GO:0016301 & kinase activity & MOLECULAR_FUNCTION & 1.0 & 0.03698824387283609 & 10 & 1411 & 213 & 15494 & $\begin{array}{l}\text { MELO3CO08076;MELO } \\
\text { 3C008367;MELO3C007 } \\
\text { O47;MELO3CO07711;M } \\
\text { ELO3CO09967;MELO3C } \\
\text { O03487;MELO3CO1775 } \\
\text { 6;MELO3CO16139;MEL } \\
\text { O3C023889;MELO3CO0 } \\
\text { 37222 } \\
\\
\end{array}$ & 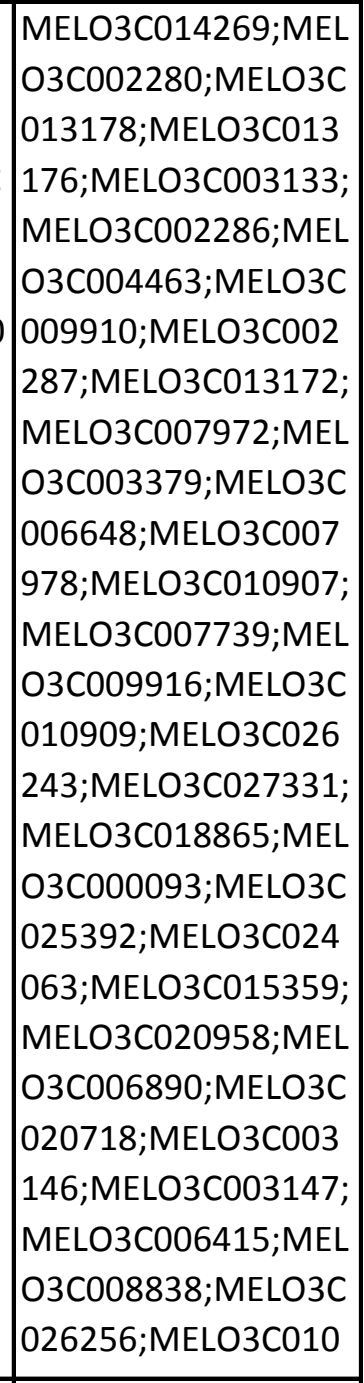 \\
\hline [] & GO:0003962 & $\begin{array}{l}\text { cystathionine gamma-synthase } \\
\text { activity }\end{array}$ & MOLECULAR_FUNCTION & 1.0 & 0.03855474831574392 & 1 & 2 & 222 & 16903 & MELO3C013774 & \begin{tabular}{|l|} 
MELO3C009845;MEL \\
O3C005786
\end{tabular} \\
\hline [] & G0:0070324 & thyroid hormone binding & MOLECULAR_FUNCTION & 1.0 & 0.01301961700147596 & 1 & 0 & 222 & 16905 & MELO3C006954 & \\
\hline [] & GO:0019888 & $\begin{array}{l}\text { protein phosphatase regulator } \\
\text { activity }\end{array}$ & MOLECULAR_FUNCTION & 1.0 & 0.03568002057009693 & 2 & 21 & 221 & 16884 & $\begin{array}{l}\text { MELO3C005380;MELO } \\
\text { 3C026288 }\end{array}$ & 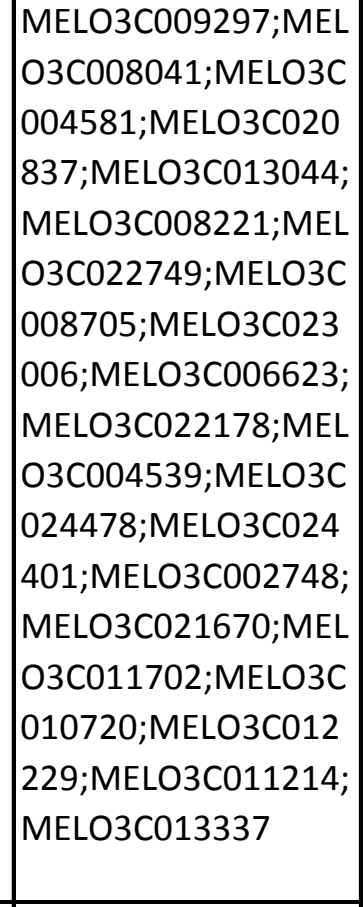 \\
\hline [] & GO:0005366 & $\begin{array}{l}\begin{array}{l}\text { myo-inositol:proton symporter } \\
\text { activity }\end{array} \\
\end{array}$ & MOLECULAR_FUNCTION & 1.0 & 0.01301961700147596 & 1 & 0 & 222 & 16905 & MELO3C017757 & \\
\hline [] & GO:0004158 & dihydroorotate oxidase activity & MOLECULAR_FUNCTION & 1.0 & 0.03855474831574392 & 1 & 2 & 222 & 16903 & MELO3C005067 & \begin{tabular}{|l|} 
MELO3C015352;MEL \\
O3C002149
\end{tabular} \\
\hline [] & GO:0047205 & $\begin{array}{l}\text { quinate O- } \\
\text { hydroxycinnamoyltransferase } \\
\text { activity }\end{array}$ & MOLECULAR_FUNCTION & 1.0 & 0.01301961700147596 & 1 & 0 & 222 & 16905 & MELO3C027219 & \\
\hline [] & GO:0005212 & structural constituent of eye lens & MOLECULAR_FUNCTION & 1.0 & 0.01301961700147596 & 1 & 0 & 222 & 16905 & MELO3C006954 & \\
\hline [] & GO:0080124 & pheophytinase activity & MOLECULAR_FUNCTION & 1.0 & 0.01301961700147596 & 1 & 0 & 222 & 16905 & MELO3C023590 & \\
\hline [] & GO:0035242 & $\begin{array}{l}\text { protein-arginine omega- } \mathrm{N} \\
\text { asymmetric methyltransferase } \\
\text { activity }\end{array}$ & MOLECULAR_FUNCTION & 1.0 & 0.03855474831574392 & 1 & 2 & 222 & 16903 & MELO3C026636 & \begin{tabular}{|l|} 
MELO3C005078;MEL \\
O3C015447
\end{tabular} \\
\hline [] & GO:0035241 & \begin{tabular}{|l} 
protein-arginine omega-N \\
monomethyltransferase activity
\end{tabular} & MOLECULAR_FUNCTION & 1.0 & 0.03855474831574392 & 1 & 2 & 222 & 16903 & MELO3C026636 & \begin{tabular}{|l|} 
MELO3C005078;MEL \\
O3C015447
\end{tabular} \\
\hline$[1]$ & GO:0008420 & CTD phosphatase activity & MOLECULAR_FUNCTION & 1.0 & 0.025870473860622564 & 1 & 1 & 222 & 16904 & MELO3C010744 & MELO3C007164 \\
\hline [] & GO:0008793 & $\begin{array}{l}\text { aromatic-amino-acid:2- } \\
\text { oxoglutarate aminotransferase } \\
\text { activity }\end{array}$ & MOLECULAR_FUNCTION & 1.0 & 0.025870473860622564 & 1 & 1 & 222 & 16904 & MELO3C005597 & MELO3C006840 \\
\hline [] & GO:0016034 & $\begin{array}{l}\text { maleylacetoacetate isomerase } \\
\text { activity }\end{array}$ & MOLECULAR_FUNCTION & 1.0 & 0.03855474831574392 & 1 & 2 & 222 & 16903 & MELO3C006189 & $\begin{array}{l}\text { MELO3C023224;MEL } \\
\text { O3C023220 }\end{array}$ \\
\hline [] & GO:0016277 & $\begin{array}{l}\text { [myelin basic protein]-arginine N- } \\
\text { methyltransferase activity }\end{array}$ & MOLECULAR_FUNCTION & 1.0 & 0.01301961700147596 & 1 & 0 & 222 & 16905 & MELO3C026636 & \\
\hline [] & GO:0004726 & $\begin{array}{l}\text { non-membrane spanning protein } \\
\text { tyrosine phosphatase activity }\end{array}$ & MOLECULAR_FUNCTION & 1.0 & $|0.025870473860622564|$ & 1 & 1 & 222 & 16904 & MELO3C021441 & MELO3C009840 \\
\hline
\end{tabular}




\begin{tabular}{|c|c|c|c|c|c|c|c|c|c|c|c|}
\hline [] & GO:0005747 & $\begin{array}{l}\text { mitochondrial respiratory chain } \\
\text { complex I }\end{array}$ & CELLULAR_COMPONENT & 1.0 & 0.038596267237901986 & 2 & 22 & 221 & 16883 & 列 & 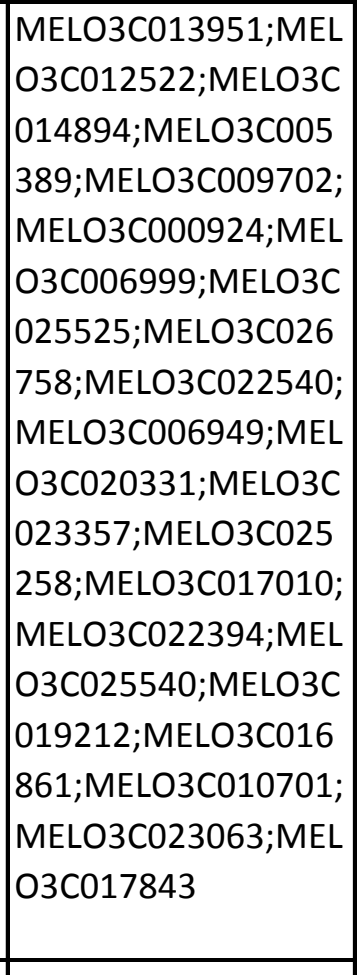 \\
\hline [] & GO:0005846 & nuclear cap binding complex & CELLULAR_COMPONENT & 1.0 & 0.01301961700147596 & 1 & 0 & 222 & 16905 & MELO3C026304 & \\
\hline [] & GO:0000783 & nuclear telomere cap complex & CELLULAR_COMPONENT & 1.0 & 0.03855474831574392 & 1 & 2 & 222 & 16903 & MELO3C006188 & \begin{tabular}{|l|} 
MELO3C017678;MEL \\
O3C023972
\end{tabular} \\
\hline [] & GO:0043564 & Ku70:Ku80 complex & CELLULAR_COMPONENT & 1.0 & 0.025870473860622564 & 1 & 1 & 222 & 16904 & MELO3C006188 & MELO3C017678 \\
\hline [] & GO:0009706 & chloroplast inner membrane & CELLULAR_COMPONENT & 1.0 & $\mid 0.019488870688019518$ & 3 & 41 & 220 & 16864 & $\begin{array}{l}\text { MELO3C016354;MELO } \\
\text { 3C014321;MELO3C014 } \\
\text { 379 }\end{array}$ & 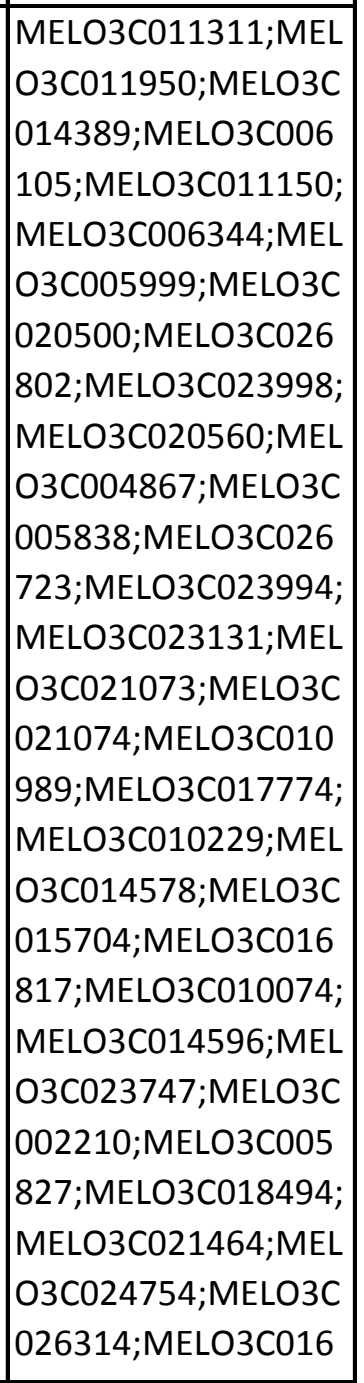 \\
\hline [] & GO:0000811 & GINS complex & CELLULAR_COMPONENT & 1.0 & 0.03855474831574392 & 1 & 2 & 222 & 16903 & MELO3C006096 & \begin{tabular}{|l|} 
MELO3C024840;MEL \\
O3C023404
\end{tabular} \\
\hline [] & GO:0000243 & commitment complex & CELLULAR_COMPONENT & 1.0 & 0.01301961700147596 & 1 & 0 & 222 & 16905 & MELO3C026304 & \\
\hline
\end{tabular}


Supplementary Table 1.E Gene Ontology terms (GO terms) of the cluster 1.4 obtained considering the comparison between lines over time fruit during storage at $20.5^{\circ} \mathrm{C}$ and $88 \%$ relative humidity for $18 \mathrm{~d}$ the near-isogenic line SC10-2 and its parental "Piel de Sapo" obtained according to the differentially expressed gene pattern (Supl. Table 1.A)

\begin{tabular}{|c|c|c|c|c|c|c|c|c|c|c|c|}
\hline Tags & GO ID & GO Name & GO Category & FDR & P-Value & Nr Test & Nr Reference & $\begin{array}{l}\text { Non Annot } \\
\text { Test }\end{array}$ & $\begin{array}{l}\text { Non Annot } \\
\text { Reference }\end{array}$ & \begin{tabular}{|l|} 
TestSet \\
Sequences
\end{tabular} & RefSet Sequences \\
\hline [] & GO:0043622 & \begin{tabular}{|l} 
cortical \\
microtubule \\
organization
\end{tabular} & BIOLOGICAL_PROCESS & 1.0 & \begin{tabular}{|l|}
0.045285367 \\
2488166
\end{tabular} & 1 & 4 & 157 & 16966 & MELO3C002054 & $\begin{array}{l}\text { MELO3C007829; } \\
\text { MELO3C014496; } \\
\text { MELO3C002717; } \\
\text { MELO3C023374 }\end{array}$ \\
\hline [] & GO:0044314 & $\begin{array}{l}\text { protein K27- } \\
\text { linked } \\
\text { ubiquitination }\end{array}$ & BIOLOGICAL_PROCESS & 1.0 & $\begin{array}{l}0.009224661 \\
372776416\end{array}$ & 1 & 0 & 157 & 16970 & MELO3C026341 & \\
\hline[] & GO:0042780 & $\begin{array}{l}\text { tRNA 3'-end } \\
\text { processing }\end{array}$ & BIOLOGICAL_PROCESS & 1.0 & \begin{tabular}{|l|}
0.036394355 \\
10742036
\end{tabular} & 1 & 3 & 157 & 16967 & MELO3C014447 & $\begin{array}{l}\text { MELO3C020738; } \\
\text { MELO3C010582; } \\
\text { MELO3C024265 }\end{array}$ \\
\hline[] & GO:0010017 & $\begin{array}{l}\text { red or far-red } \\
\text { light signaling } \\
\text { pathway }\end{array}$ & BIOLOGICAL_PROCESS & 1.0 & $\begin{array}{l}0.043416625 \\
286064055\end{array}$ & 2 & 34 & 156 & 16936 & $\begin{array}{l}\text { MELO3C026502; } \\
\text { MELO3C014459 }\end{array}$ & $\begin{array}{l}\text { MELO3C009692; } \\
\text { MELO3C006046; } \\
\text { MELO3C014684; } \\
\text { MELO3C012221; } \\
\text { MELO3C006142; } \\
\text { MELO3C014386; } \\
\text { MELO3C011250; } \\
\text { MELO3C003075; } \\
\text { MELO3C006225; } \\
\text { MELO3C021715; } \\
\text { MELO3C003574; } \\
\text { MELO3C024805; } \\
\text { MELO3C026506; } \\
\text { MELO3C004148; } \\
\text { MELO3C002742; } \\
\text { MELO3C005738; } \\
\text { MELO3C002705; } \\
\text { MELO3C005939; } \\
\text { MELO3C019731; } \\
\text { MELO3C010769; } \\
\text { MELO3C025191; } \\
\text { MELO3C014699; } \\
\text { MELO3C008213; } \\
\text { MELO3C009202; } \\
\text { MELO3C005120; } \\
\text { MELO3C002793; } \\
\text { MELO3C006717; }\end{array}$ \\
\hline [] & GO:0010375 & \begin{tabular}{|l} 
stomatal \\
complex \\
patterning
\end{tabular} & BIOLOGICAL_PROCESS & 1.0 & \begin{tabular}{|l|}
0.027421072 \\
152703972
\end{tabular} & 1 & 2 & 157 & 16968 & MELO3C002054 & $\begin{array}{l}\text { MELO3C021111; } \\
\text { MELO3C020718 }\end{array}$ \\
\hline [] & GO:0006974 & $\begin{array}{l}\text { cellular } \\
\text { response to } \\
\text { DNA damage } \\
\text { stimulus }\end{array}$ & BIOLOGICAL_PROCESS & 1.0 & $\begin{array}{l}0.023182426 \\
598434357\end{array}$ & 8 & 367 & 150 & 16603 & $\begin{array}{l}\text { MELO3C025853; } \\
\text { MELO3C009561; } \\
\text { MELO3C013630; } \\
\text { MELO3C002271; } \\
\text { MELO3C018500; } \\
\text { MELO3C006136; } \\
\text { MELO3C022742; } \\
\text { MELO3C010378 }\end{array}$ & $\begin{array}{l}\text { MELO3C006760; } \\
\text { MELO3C020706; } \\
\text { MELO3C014386; } \\
\text { MELO3C014021; } \\
\text { MELO3C014020; } \\
\text { MELO3C004226; } \\
\text { MELO3C023535; } \\
\text { MELO3C008825; } \\
\text { MELO3C004227; } \\
\text { MELO3C019281; } \\
\text { MELO3C008829; } \\
\text { MELO3C02680;; } \\
\text { MELO3C027337; } \\
\text { MELO3C017096; } \\
\text { MELO3C018187; } \\
\text { MELO3C020022; } \\
\text { MELO3C017775; } \\
\text { MELO3C010901; } \\
\text { MELO3C018624; } \\
\text { MELO3C014703; } \\
\text { MELO3C011434; } \\
\text { MELO3C006096; } \\
\text { MELO3C018518; } \\
\text { MELO3C013741; } \\
\text { MELO3C013740; } \\
\text { MELO3C008157; } \\
\text { MELO3C002173; }\end{array}$ \\
\hline[] & GO:0009959 & $\begin{array}{l}\text { negative } \\
\text { gravitropism }\end{array}$ & BIOLOGICAL_PROCESS & 1.0 & $\begin{array}{l}0.036394355 \\
10742036\end{array}$ & 1 & 3 & 157 & 16967 & MELO3C008088 & $\begin{array}{l}\text { MELO3C025310; } \\
\text { MELO3C008847; } \\
\text { MELO3C024155 }\end{array}$ \\
\hline [] & GO:0006659 & $\begin{array}{l}\text { phosphatidylseri } \\
\text { ne biosynthetic } \\
\text { process }\end{array}$ & BIOLOGICAL_PROCESS & 1.0 & $\begin{array}{l}0.018364762 \\
00573981\end{array}$ & 1 & 1 & 157 & 16969 & MELO3C005332 & MELO3C012286 \\
\hline
\end{tabular}




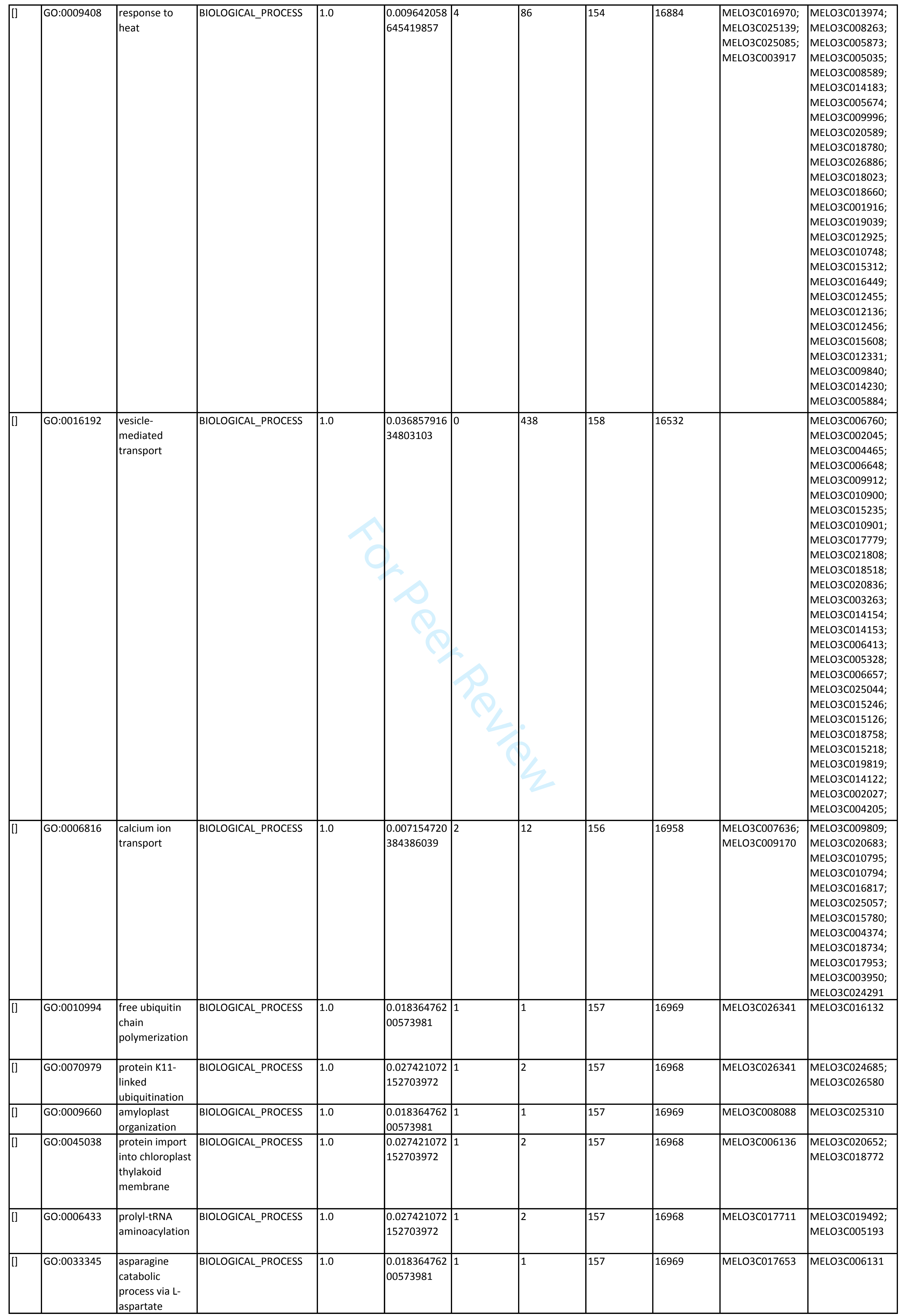




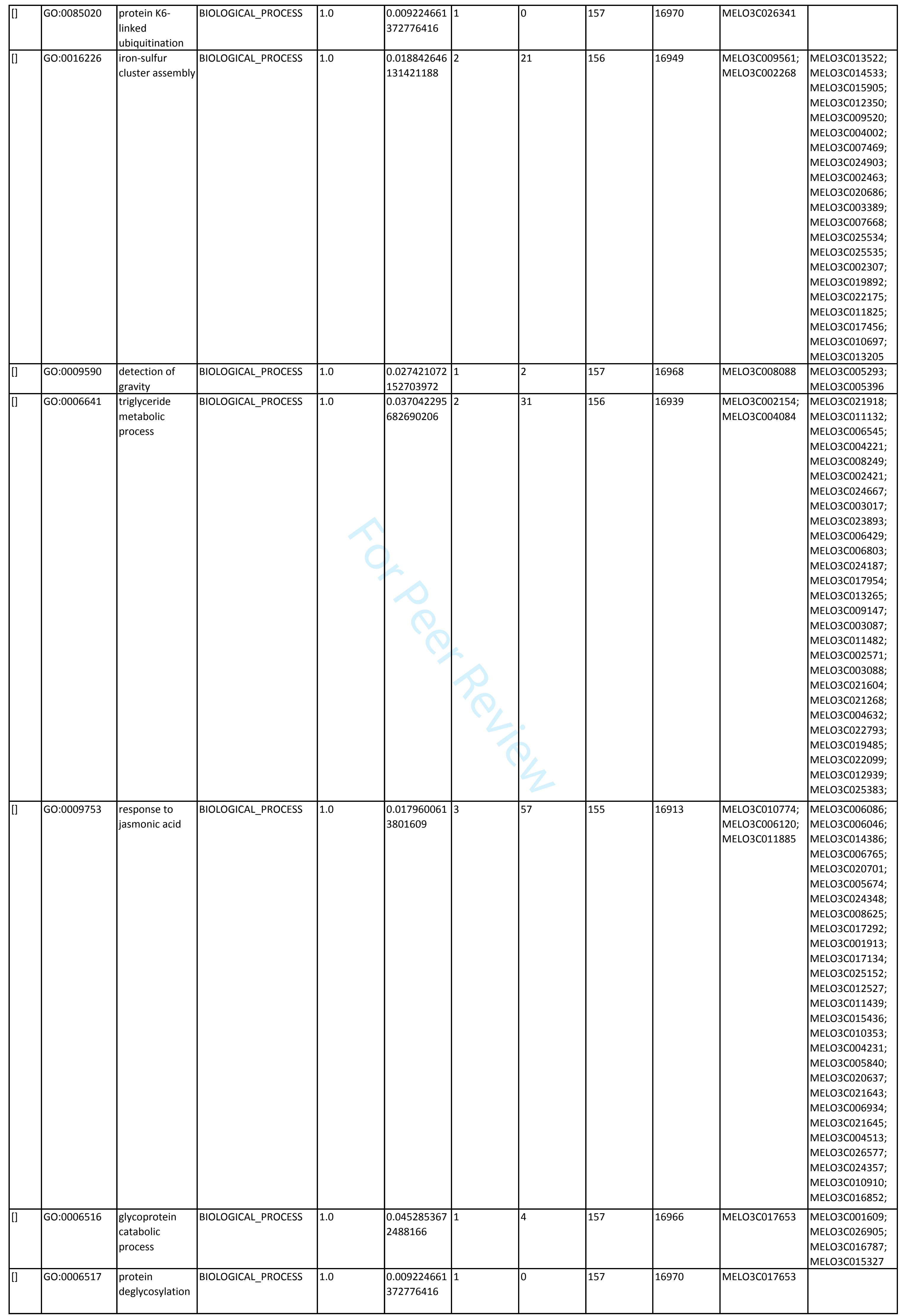




\begin{tabular}{|c|c|c|c|c|c|c|c|c|c|c|}
\hline [] & GO:0035519 & \begin{tabular}{|l|} 
protein K29- \\
linked \\
ubiquitination
\end{tabular} & BIOLOGICAL_PROCESS & 1.0 & \begin{tabular}{|l|}
0.009224661 \\
372776416
\end{tabular} & 1 & 157 & 16970 & MELO3C026341 & \\
\hline [] & GO:0051604 & \begin{tabular}{|l|} 
protein \\
maturation
\end{tabular} & BIOLOGICAL_PROCESS & 1.0 & \begin{tabular}{|l|}
0.037042295 \\
682690206 \\
\end{tabular} & 2 & 156 & 16939 & $\begin{array}{l}\text { MELO3C017653; } \\
\text { MELO3C016970 }\end{array}$ & 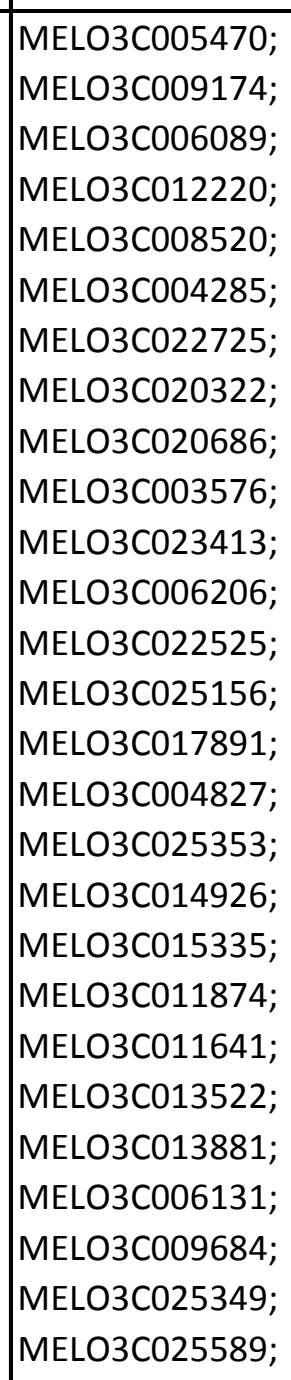 \\
\hline [] & GO:0010458 & \begin{tabular}{|l|}
$\begin{array}{l}\text { exit from } \\
\text { mitosis }\end{array}$ \\
\end{tabular} & BIOLOGICAL_PROCESS & 1.0 & \begin{tabular}{|l|}
0.018364762 \\
00573981
\end{tabular} & 1 & & 16969 & MELO3C026341 & MELO3C002182 \\
\hline [1] & GO:0070887 & \begin{tabular}{|l|} 
cellular \\
response to \\
chemical \\
stimulus
\end{tabular} & BIOLOGICAL_PROCESS & 1.0 & \begin{tabular}{|l|}
.047293101 \\
23083138
\end{tabular} & 1 & 603 & 16367 & MELO3C010774 & 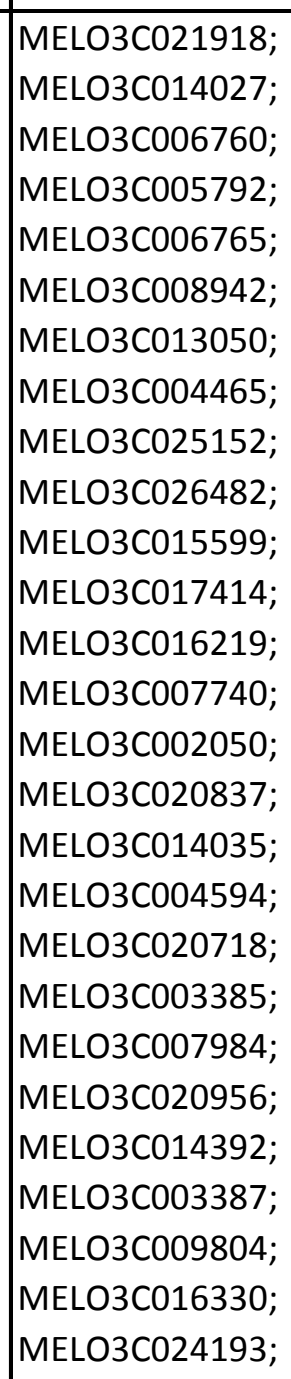 \\
\hline []] & GO:0035071 & \begin{tabular}{|l} 
salivary gland \\
cell autophagic \\
cell death
\end{tabular} & BIOLOGICAL_PROCESS & 1.0 & \begin{tabular}{|l|}
0.045285367 \\
2488166
\end{tabular} & 1 & & 16966 & MELO3C015523 & \begin{tabular}{|l} 
MELO3C026898; \\
MELO3C022308; \\
MELO3C007891; \\
MELO3C025809
\end{tabular} \\
\hline [] & GO:0032515 & \begin{tabular}{|l|} 
negative \\
regulation of \\
phosphoprotein \\
phosphatase \\
activity
\end{tabular} & BIOLOGICAL_PROCESS & 1.0 & \begin{tabular}{|l|}
0.009224661 \\
372776416
\end{tabular} & 1 & & 16970 & MELO3C018500 & \\
\hline []] & GO:0010224 & \begin{tabular}{|l|} 
response to UV- \\
B
\end{tabular} & BIOLOGICAL_PROCESS & 1.0 & \begin{tabular}{|l|}
0.015829085 \\
893849265
\end{tabular} & 2 & 156 & 16951 & $\begin{array}{l}\text { MELO3C025853; } \\
\text { MELO3CO08944 }\end{array}$ & 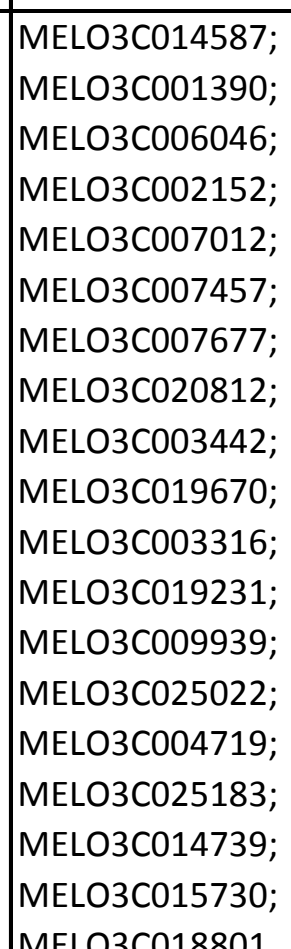 \\
\hline
\end{tabular}




\begin{tabular}{|c|c|c|c|c|c|c|c|c|c|c|c|c|}
\hline $\begin{array}{l}2 \\
3 \\
4\end{array}$ & & & & & & & & & & & & \\
\hline $\begin{array}{l}5 \\
6 \\
7 \\
8 \\
9 \\
10 \\
11 \\
12 \\
13 \\
14 \\
15 \\
16 \\
17 \\
18 \\
19\end{array}$ & [] & GO:0007030 & $\begin{array}{l}\text { Golgi } \\
\text { organization }\end{array}$ & BIOLOGICAL_PROCESS & 1.0 & $\begin{array}{l}0.007154720 \\
384386039\end{array}$ & 2 & 12 & 156 & 16958 & $\begin{array}{l}\text { MELO3C008088; } \\
\text { MELO3C004825 }\end{array}$ & $\begin{array}{l}\text { MELO3C021320; } \\
\text { MELO3C005918; } \\
\text { MELO3C008037; } \\
\text { MELO3C025044; } \\
\text { MELO3C008782; } \\
\text { MELO3C024284; } \\
\text { MELO3C021946; } \\
\text { MELO3C022038; } \\
\text { MELO3C006811; } \\
\text { MELO3C015756; } \\
\text { MELO3C016416; } \\
\text { MELO3C022305 }\end{array}$ \\
\hline $\begin{array}{l}20 \\
21 \\
22 \\
23 \\
24 \\
25 \\
26 \\
27 \\
28 \\
29 \\
30 \\
31 \\
32 \\
33 \\
34 \\
35 \\
36 \\
37 \\
38 \\
39 \\
40 \\
41 \\
42 \\
43 \\
44 \\
45 \\
46 \\
47 \\
48 \\
49 \\
50 \\
51\end{array}$ & {[]} & GO:0006979 & \begin{tabular}{|l} 
response to \\
oxidative stress
\end{tabular} & BIOLOGICAL_PROCESS & 1.0 & $\begin{array}{l}0.043773117 \\
73428759\end{array}$ & 5 & 203 & 153 & 16767 & $\begin{array}{l}\text { MELO3C015857; } \\
\text { MELO3C019503; } \\
\text { MELO3C025085; } \\
\text { MELO3C024505; } \\
\text { MELO3C011885 }\end{array}$ & $\begin{array}{l}\text { MELO3C013974; } \\
\text { MELO3C012522; } \\
\text { MELO3C010344; } \\
\text { MELO3C021914; } \\
\text { MELO3C004220; } \\
\text { MELO3C009112; } \\
\text { MELO3C002164; } \\
\text { MELO3C003377; } \\
\text { MELO3C005674; } \\
\text { MELO3C007618; } \\
\text { MELO3C017096; } \\
\text { MELO3C016564; } \\
\text { MELO3C011313; } \\
\text { MELO3C020719; } \\
\text { MELO3C010351; } \\
\text { MELO3C020718; } \\
\text { MELO3C020711; } \\
\text { MELO3C007984; } \\
\text { MELO3C007868; } \\
\text { MELO3C008835; } \\
\text { MELO3C009924; } \\
\text { MELO3C006536; } \\
\text { MELO3C025724; } \\
\text { MELO3C002978; } \\
\text { MELO3C018077; } \\
\text { MELO3C011328; } \\
\text { MELO3C018756; }\end{array}$ \\
\hline $\begin{array}{l}53 \\
54 \\
55\end{array}$ & {[]} & GO:0031648 & $\begin{array}{l}\text { protein } \\
\text { destabilization }\end{array}$ & BIOLOGICAL_PROCESS & 1.0 & $\begin{array}{l}0.009224661 \\
372776416\end{array}$ & 1 & 0 & 157 & 16970 & MELO3C015523 & \\
\hline $\begin{array}{l}56 \\
57 \\
58 \\
59 \\
60\end{array}$ & {[]} & GO:0048510 & $\begin{array}{l}\text { regulation of } \\
\text { timing of } \\
\text { transition from } \\
\text { vegetative to } \\
\text { reproductive } \\
\text { phase }\end{array}$ & BIOLOGICAL_PROCESS & 1.0 & $\begin{array}{l}0.045285367 \\
2488166\end{array}$ & 1 & 4 & 157 & 16966 & MELO3C022388 & $\begin{array}{l}\text { MELO3C027290; } \\
\text { MELO3C019330; } \\
\text { MELO3C013793; } \\
\text { MELO3C009465 }\end{array}$ \\
\hline & {[]} & GO:0070534 & \begin{tabular}{|l} 
protein K63- \\
linked \\
ubiquitination
\end{tabular} & BIOLOGICAL_PROCESS & 1.0 & \begin{tabular}{|l|}
0.027421072 \\
152703972
\end{tabular} & 1 & 2 & 157 & 16968 & MELO3C026341 & $\begin{array}{l}\text { MELO3C022725; } \\
\text { MELO3C020654 }\end{array}$ \\
\hline & {[]} & GO:0002949 & $\begin{array}{l}\text { tRNA } \\
\text { threonylcarbam } \\
\text { oyladenosine } \\
\text { modification }\end{array}$ & BIOLOGICAL_PROCESS & 1.0 & \begin{tabular}{l|}
0.009224661 \\
372776416
\end{tabular} & 1 & 0 & 157 & 16970 & MELO3C006202 & \\
\hline & {[]} & GO:0015976 & $\begin{array}{l}\text { carbon } \\
\text { utilization }\end{array}$ & BIOLOGICAL_PROCESS & 1.0 & \begin{tabular}{|l|}
0.036394355 \\
10742036
\end{tabular} & 1 & 3 & 157 & 16967 & MELO3C018056 & $\begin{array}{l}\text { MELO3C011997; } \\
\text { MELO3C011095; } \\
\text { MELO3C009958 }\end{array}$ \\
\hline & [] & GO:0016404 & $\begin{array}{l}15- \\
\text { hydroxyprostagl } \\
\text { andin } \\
\text { dehydrogenase } \\
(N A D+) \text { activity }\end{array}$ & MOLECULAR_FUNCTION & 1.0 & \begin{tabular}{|l|}
0.009224661 \\
372776416
\end{tabular} & 1 & 0 & 157 & 16970 & MELO3C019503 & \\
\hline & [] & GO:0004144 & $\begin{array}{l}\text { diacylglycerol O- } \\
\text { acyltransferase } \\
\text { activity }\end{array}$ & MOLECULAR_FUNCTION & 1.0 & \begin{tabular}{|l|}
0.027421072 \\
152703972
\end{tabular} & 1 & 2 & 157 & 16968 & MELO3C013703 & $\begin{array}{l}\text { MELO3C004632; } \\
\text { MELO3C018460 }\end{array}$ \\
\hline & [] & GO:0004416 & \begin{tabular}{|l} 
hydroxyacylglut \\
athione \\
hydrolase \\
activity
\end{tabular} & MOLECULAR_FUNCTION & 1.0 & \begin{tabular}{|l|}
0.027421072 \\
152703972
\end{tabular} & 1 & 2 & 157 & 16968 & MELO3C020172 & $\begin{array}{l}\text { MELO3C017798; } \\
\text { MELO3C025595 }\end{array}$ \\
\hline & {[]} & GO:0003846 & $\begin{array}{l}\text { 2-acylglycerol } 0 \\
\text { acyltransferase } \\
\text { activity }\end{array}$ & MOLECULAR_FUNCTION & 1.0 & $\begin{array}{l}0.009224661 \\
372776416\end{array}$ & 1 & 0 & 157 & 16970 & MELO3C013703 & \\
\hline & [] & GO:0042781 & $\begin{array}{l}\text { 3'-tRNA } \\
\text { processing } \\
\text { endoribonuclea } \\
\text { se activity }\end{array}$ & MOLECULAR_FUNCTION & 1.0 & \begin{tabular}{|l|}
0.027421072 \\
152703972
\end{tabular} & 1 & 2 & 157 & 16968 & MELO3C014447 & $\begin{array}{l}\text { MELO3C020738; } \\
\text { MELO3C024265 }\end{array}$ \\
\hline & [] & GO:0004827 & $\begin{array}{l}\text { proline-tRNA } \\
\text { ligase activity }\end{array}$ & MOLECULAR_FUNCTION & 1.0 & \begin{tabular}{|l|}
0.027421072 \\
152703972 \\
\end{tabular} & 1 & 2 & 157 & 16968 & MELO3C017711 & $\begin{array}{l}\text { MELO3C019492; } \\
\text { MELO3C005193 }\end{array}$ \\
\hline
\end{tabular}




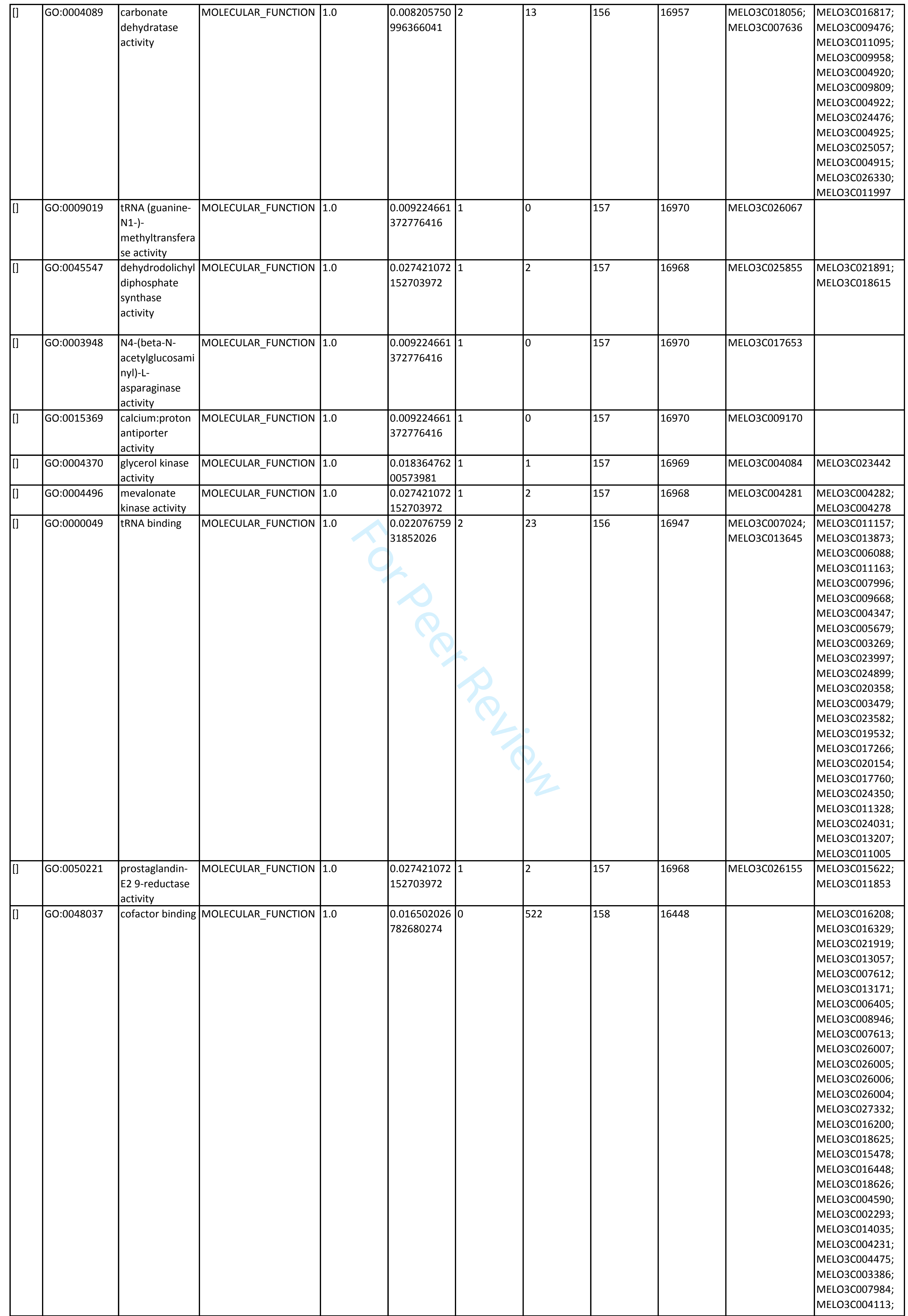




\begin{tabular}{|c|c|c|c|c|c|c|c|c|c|c|c|}
\hline$[$ [] & GO:0033743 & \begin{tabular}{|l|} 
peptide- \\
methionine (R)- \\
S-oxide \\
reductase \\
activity \\
\end{tabular} & MOLECULAR_FUNCTION & 1.0 & \begin{tabular}{|l|}
0.018364762 \\
00573981
\end{tabular} & 1 & 1 & 157 & 16969 & MELO3C018569 & MELO3C016676 \\
\hline [] & GO:0008270 & zinc ion binding & MOLECULAR_FUNCTION & 1.0 & $\begin{array}{l}0.045573585 \\
11781277\end{array} \mid$ & 19 & 1258 & 139 & 15712 & 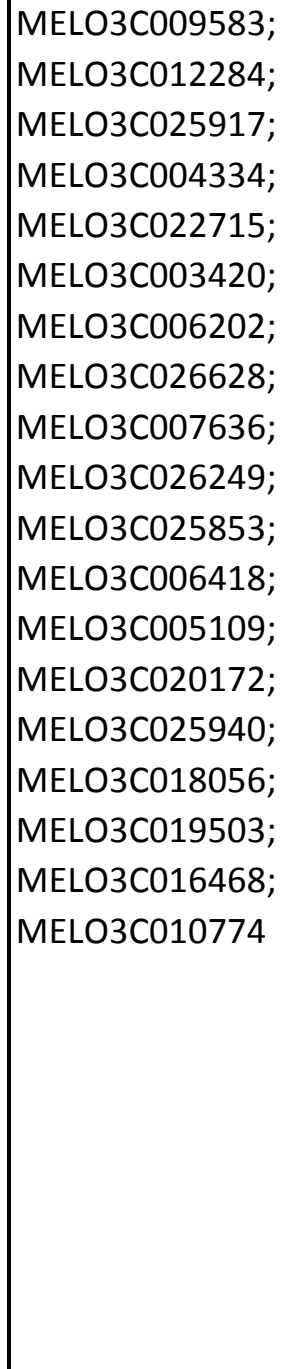 & 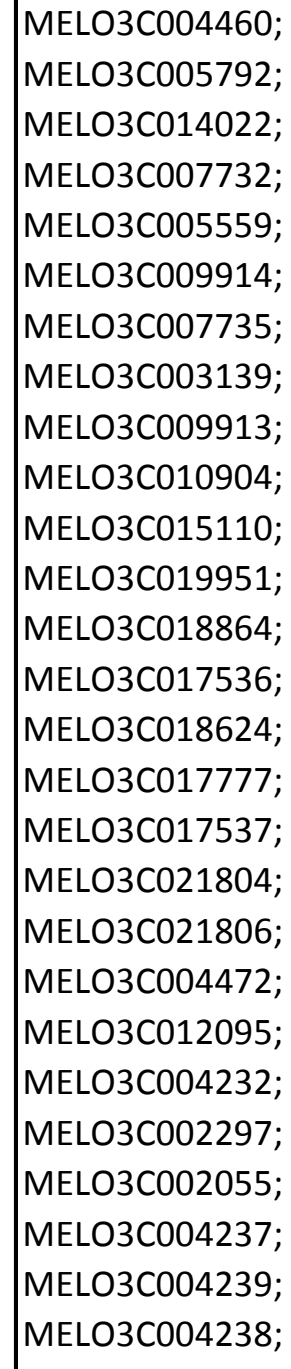 \\
\hline [] & GO:0008834 & $\begin{array}{l}\text { di-trans,poly-cis- } \\
\text { decaprenylcistra } \\
\text { nsferase activity }\end{array}$ & MOLECULAR_FUNCTION & 1.0 & \begin{tabular}{|l|}
0.027421072 \\
152703972 \\
\end{tabular} & 1 & 2 & 157 & 16968 & MELO3C025855 & $\begin{array}{l}\text { MELO3C021891; } \\
\text { MELO3C018615 }\end{array}$ \\
\hline [] & GO:0008798 & $\begin{array}{l}\text { beta-aspartyl- } \\
\text { peptidase } \\
\text { activity }\end{array}$ & MOLECULAR_FUNCTION & 1.0 & \begin{tabular}{|l|}
0.045285367 \\
2488166
\end{tabular} & 1 & 4 & 157 & 16966 & MELO3C017653 & $\begin{array}{l}\text { MELO3C001609; } \\
\text { MELO3C016787; } \\
\text { MELO3CO06131; } \\
\text { MELO3C015327 }\end{array}$ \\
\hline[] & GO:0004557 & $\begin{array}{l}\text { alpha- } \\
\text { galactosidase } \\
\text { activity }\end{array}$ & MOLECULAR_FUNCTION & 1.0 & \begin{tabular}{|l|}
0.045285367 \\
2488166
\end{tabular} & 1 & 4 & 157 & 16966 & MELO3C020139 & $\begin{array}{l}\text { MELO3C020138; } \\
\text { MELO3C011771; } \\
\text { MELO3C010698; } \\
\text { MELO3C011770 }\end{array}$ \\
\hline[] & GO:0003882 & \begin{tabular}{|l|} 
CDP- \\
diacylglycerol- \\
serine O- \\
phosphatidyltra \\
nsferase activity
\end{tabular} & MOLECULAR_FUNCTION & 1.0 & \begin{tabular}{|l|}
0.018364762 \\
00573981
\end{tabular} & 1 & 1 & 157 & 16969 & MELO3C005332 & MELO3C012286 \\
\hline [] & GO:0004965 & \begin{tabular}{|l|} 
G-protein \\
coupled GABA \\
receptor activity
\end{tabular} & MOLECULAR_FUNCTION & 1.0 & \begin{tabular}{|l|}
0.045285367 \\
2488166
\end{tabular} & 1 & 4 & 157 & 16966 & MELO3C019256 & $\begin{array}{l}\text { MELO3C019258; } \\
\text { MELO3C017043; } \\
\text { MELO3CO07977; } \\
\text { MELO3C019260 }\end{array}$ \\
\hline [] & GO:0009705 & $\begin{array}{l}\text { plant-type } \\
\text { vacuole } \\
\text { membrane }\end{array}$ & CELLULAR_COMPONENT & 1.0 & \begin{tabular}{|l|}
0.007154720 \\
384386039 \\
\end{tabular} & 2 & 12 & 156 & 16958 & $\begin{array}{l}\text { MELO3C008088; } \\
\text { MELO3C009170 }\end{array}$ & 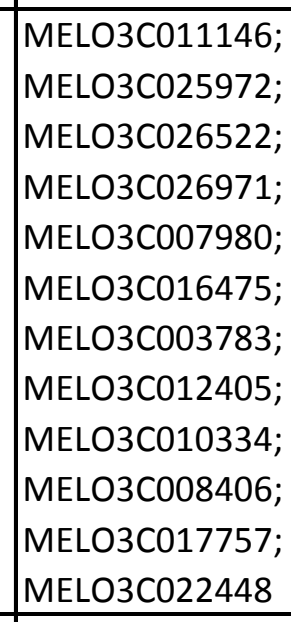 \\
\hline [] & GO:0005764 & lysosome & CELLULAR_COMPONENT & 1.0 & $\begin{array}{l}0.018842646 \\
131421188\end{array}$ & 2 & 21 & 156 & 16949 & $\begin{array}{l}\text { MELO3C017653; } \\
\text { MELO3C015523 }\end{array}$ & 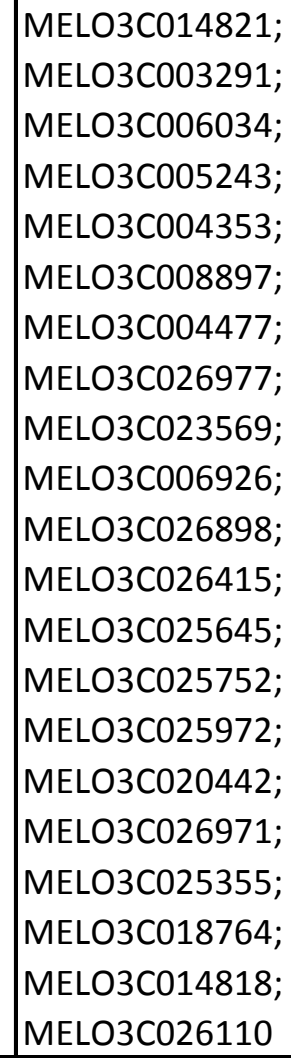 \\
\hline
\end{tabular}




\begin{tabular}{|c|c|c|c|c|c|c|c|c|c|c|}
\hline[] & GO:0031983 & vesicle lumen & \begin{tabular}{|l|} 
CELLULAR_COMPONENT \\
\end{tabular} & 1.0 & \begin{tabular}{|l|}
0.009224661 \\
372776416
\end{tabular} & To & 157 & 16970 & MELO3C015523 & \\
\hline [] & GO:0042718 & yolk granule & CELLULAR_COMPONENT & 1.0 & \begin{tabular}{|l|}
0.009224661 \\
372776416
\end{tabular} & 0 & 157 & 16970 & MELO3C015523 & \\
\hline [] & GO:0030529 & \begin{tabular}{|l} 
intracellular \\
ribonucleoprote \\
in complex
\end{tabular} & CELLULAR_COMPONENT & 1.0 & \begin{tabular}{|l|}
0.022544929 \\
6878492
\end{tabular} & 666 & 157 & 16304 & MELO3C024747 & 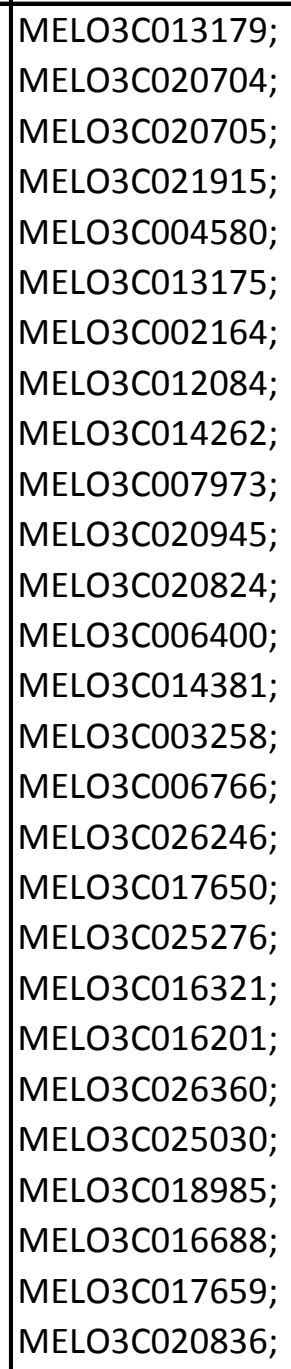 \\
\hline [] & GO:0016602 & \begin{tabular}{l|} 
CCAAT-binding \\
factor complex
\end{tabular} & CELLULAR_COMPONENT & 1.0 & \begin{tabular}{|l|}
0.045285367 \\
2488166
\end{tabular} & 4 & 157 & 16966 & MELO3C023161 & $\begin{array}{l}\text { MELO3C009551; } \\
\text { MELO3C015320; } \\
\text { MELO3C023554; } \\
\text { MELO3C007077 }\end{array}$ \\
\hline [] & GO:0045169 & fusome & CELLULAR_COMPONENT & 1.0 & \begin{tabular}{|l|}
0.027421072 \\
152703972
\end{tabular} & 2 & 157 & 16968 & MELO3C015523 & $\begin{array}{l}\text { MELO3C006951; } \\
\text { MELO3C024150 }\end{array}$ \\
\hline [] & GO:0005680 & \begin{tabular}{|l} 
anaphase- \\
promoting \\
complex
\end{tabular} & CELLULAR_COMPONENT & 1.0 & \begin{tabular}{|l|}
0.036394355 \\
10742036
\end{tabular} & 3 & 157 & 16967 & MELO3C026341 & $\begin{array}{l}\text { MELO3C002234; } \\
\text { MELO3C024685; } \\
\text { MELO3C026580 }\end{array}$ \\
\hline [] & GO:0009570 & $\begin{array}{l}\text { chloroplast } \\
\text { stroma }\end{array}$ & CELLULAR_COMPONENT & 1.0 & $\begin{array}{l}0.009235598 \\
713916146\end{array}$ & 374 & 149 & 16596 & $\begin{array}{l}\text { MELO3C007024; } \\
\text { MELO3C014178; } \\
\text { MELO3C016970; } \\
\text { MELO3CO08932; } \\
\text { MELO3CO24086; } \\
\text { MELO3CO04223; } \\
\text { MELO3CO24701; } \\
\text { MELO3C018569; } \\
\text { MELO3C013645 }\end{array}$ & 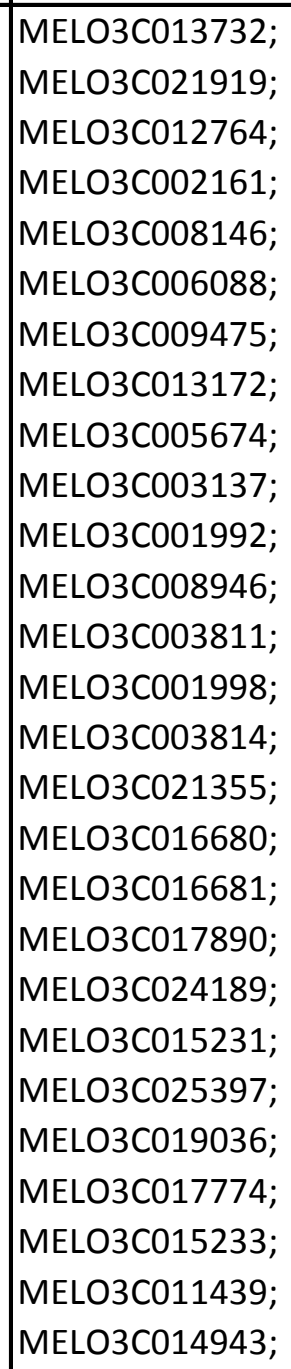 \\
\hline
\end{tabular}


Supplementary Table 1.F Gene Ontology terms (GO terms) of the cluster 1.5 obtained considering the comparison between lines over time fruit during storage at $20.5^{\circ} \mathrm{C}$ and $88 \%$ relative humidity for $18 \mathrm{~d}$ the near-isogenic line SC10-2 and its parental "Piel de Sapo" obtained according to the differentially expressed gene pattern (Supl. Table 1.A)

\begin{tabular}{|c|c|c|c|c|c|c|c|c|c|c|c|}
\hline Tags & GO ID & GO Name & GO Category & FDR & P-Value & Nr Test & $\begin{array}{l}\mathrm{Nr} \\
\text { Reference }\end{array}$ & $\begin{array}{l}\text { Non Annot } \\
\text { Test }\end{array}$ & $\begin{array}{l}\text { Non Annot } \\
\text { Reference }\end{array}$ & $\begin{array}{l}\text { TestSet } \\
\text { Sequences }\end{array}$ & \begin{tabular}{|l} 
RefSet \\
Sequences
\end{tabular} \\
\hline[] & GO:0004008 & $\begin{array}{l}\text { copper-exporting } \\
\text { ATPase activity }\end{array}$ & $\begin{array}{l}\text { MOLECULAR_F } \\
\text { UNCTION }\end{array}$ & $\begin{array}{l}0.367489802 \\
3835551\end{array}$ & \begin{tabular}{|l|}
3.473735973 \\
$621571 \mathrm{E}-4$
\end{tabular} & 4 & 8 & 518 & 16598 & $\begin{array}{l}\text { MELO3C019011; } \\
\text { MELO3C016915; } \\
\text { MELO3C019010; } \\
\text { MELO3C019012 }\end{array}$ & $\begin{array}{l}\text { MELO3C000189; } \\
\text { MELO3C018643; } \\
\text { MELO3C005665; } \\
\text { MELO3C007027; } \\
\text { MELO3C008846; } \\
\text { MELO3C020853; } \\
\text { MELO3C008845; } \\
\text { MELO3C003996 }\end{array}$ \\
\hline [] & GO:0006342 & chromatin silencing & $\begin{array}{l}\text { BIOLOGICAL_P } \\
\text { ROCESS }\end{array}$ & $\begin{array}{l}0.738251046 \\
6190081\end{array}$ & $\begin{array}{l}0.001194365 \\
0135181208\end{array}$ & 5 & 22 & 517 & 16584 & $\begin{array}{l}\text { MELO3C022443; } \\
\text { MELO3C013879; } \\
\text { MELO3C023986; } \\
\text { MELO3C014035; } \\
\text { MELO3C007648 }\end{array}$ & $\begin{array}{l}\text { MELO3C011256; } \\
\text { MELO3C015649; } \\
\text { MELO3C010882; } \\
\text { MELO3C007894; } \\
\text { MELO3C008048; } \\
\text { MELO3C009773; } \\
\text { MELO3C014440; } \\
\text { MELO3C014153; } \\
\text { MELO3C003583; } \\
\text { MELO3C024942; } \\
\text { MELO3C023217; } \\
\text { MELO3C004615; } \\
\text { MELO3C022562; } \\
\text { MELO3C021585; } \\
\text { MELO3C021442; } \\
\text { MELO3C023752; } \\
\text { MELO3C021058; } \\
\text { MELO3C015560; } \\
\text { MELO3C027223; } \\
\text { MELO3C012615; } \\
\text { MELO3C014706; } \\
\text { MELO3C017558 }\end{array}$ \\
\hline [] & GO:0008270 & zinc ion binding & $\begin{array}{l}\text { MOLECULAR_F } \\
\text { UNCTION }\end{array}$ & $\begin{array}{l}0.738251046 \\
6190081\end{array}$ & $\begin{array}{l}0.001250213 \\
4574411653\end{array}$ & 59 & 1218 & 463 & 15388 & $\begin{array}{l}\text { MELO3C009276; } \\
\text { MELO3C010584; } \\
\text { MELO3C005035; } \\
\text { MELO3C026803; } \\
\text { MELO3C009914; } \\
\text { MELO3C026804; } \\
\text { MELO3C005754; } \\
\text { MELO3C022568; } \\
\text { MELO3C004944; } \\
\text { MELO3C002767; } \\
\text { MELO3C019470; } \\
\text { MELO3C018461; } \\
\text { MELO3C024385; } \\
\text { MELO3C018265; } \\
\text { MELO3C018264; } \\
\text { MELO3C017131; } \\
\text { MELO3C017571; } \\
\text { MELO3C015277; } \\
\text { MELO3C011317; } \\
\text { MELO3C027373; } \\
\text { MELO3C015477; } \\
\text { MELO3C017657; } \\
\text { MELO3C017813; } \\
\text { MELO3C011640; } \\
\text { MELO3C002690; } \\
\text { MELO3C004279; } \\
\text { MELO3C004239; }\end{array}$ & $\begin{array}{l}\text { MELO3C004460; } \\
\text { MELO3C005792; } \\
\text { MELO3C014022; } \\
\text { MELO3C007732; } \\
\text { MELO3C005559; } \\
\text { MELO3C007735; } \\
\text { MELO3C003139; } \\
\text { MELO3C009913; } \\
\text { MELO3C026249; } \\
\text { MELO3C010904; } \\
\text { MELO3C015110; } \\
\text { MELO3C019951; } \\
\text { MELO3C018864; } \\
\text { MELO3C017536; } \\
\text { MELO3C018624; } \\
\text { MELO3C017777; } \\
\text { MELO3C017537; } \\
\text { MELO3C021804; } \\
\text { MELO3C021806; } \\
\text { MELO3C004472; } \\
\text { MELO3C012095; } \\
\text { MELO3C004232; } \\
\text { MELO3C002297; } \\
\text { MELO3C002055; } \\
\text { MELO3C004237; } \\
\text { MELO3C004238; } \\
\text { MELO3C007503; }\end{array}$ \\
\hline [] & GO:0050826 & response to freezing & \begin{tabular}{|l|} 
BIOLOGICAL_P \\
ROCESS \\
\end{tabular} & \begin{tabular}{|l|}
0.827121087 \\
427183 \\
\end{tabular} & \begin{tabular}{|l|}
0.002724961 \\
000167915 \\
\end{tabular} & 2 & 1 & 520 & 16605 & $\begin{array}{l}\text { MELO3C021253; } \\
\text { MELO3C005035 }\end{array}$ & MELO3C022358 \\
\hline [] & GO:0004345 & $\begin{array}{l}\text { glucose-6-phosphate } \\
\text { dehydrogenase } \\
\text { activity }\end{array}$ & $\begin{array}{l}\text { MOLECULAR_F } \\
\text { UNCTION }\end{array}$ & $\begin{array}{l}0.827121087 \\
427183\end{array}$ & $\mid \begin{array}{l}0.002724961 \\
000167915\end{array}$ & 2 & 1 & 520 & 16605 & $\begin{array}{l}\text { MELO3C008986; } \\
\text { MELO3C008985 }\end{array}$ & MELO3C003280 \\
\hline [] & GO:0047938 & $\begin{array}{l}\text { glucose-6-phosphate } \\
\text { 1-epimerase activity }\end{array}$ & $\begin{array}{l}\text { MOLECULAR_F } \\
\text { UNCTION }\end{array}$ & $\begin{array}{l}0.827121087 \\
427183\end{array}$ & $\begin{array}{l}0.002724961 \\
000167915\end{array}$ & 2 & 1 & 520 & 16605 & $\begin{array}{l}\text { MELO3C016314; } \\
\text { MELO3C006055 }\end{array}$ & MELO3C004001 \\
\hline
\end{tabular}




\begin{tabular}{|c|c|c|c|c|c|c|c|c|c|c|c|}
\hline [] & GO:0008138 & $\begin{array}{l}\text { protein } \\
\text { tyrosine/serine/threo } \\
\text { nine phosphatase } \\
\text { activity }\end{array}$ & $\begin{array}{l}\text { MOLECULAR_F } \\
\text { UNCTION }\end{array}$ & $\begin{array}{l}0.827121087 \\
427183\end{array}$ & $\begin{array}{l}0.002295845 \\
7581069703\end{array}$ & 4 & 15 & 518 & 16591 & $\begin{array}{l}\text { MELO3C004603; } \\
\text { MELO3C023830; } \\
\text { MELO3C003222; } \\
\text { MELO3C013921 }\end{array}$ & $\begin{array}{l}\text { MELO3C012378; } \\
\text { MELO3C009960; } \\
\text { MELO3C014446; } \\
\text { MELO3C007580; } \\
\text { MELO3C009840; } \\
\text { MELO3C021405; } \\
\text { MELO3C004587; } \\
\text { MELO3C020114; } \\
\text { MELO3C002645; } \\
\text { MELO3C026602; } \\
\text { MELO3C019894; } \\
\text { MELO3C018474; } \\
\text { MELO3C026166; } \\
\text { MELO3C011936; } \\
\text { MELO3C026163 }\end{array}$ \\
\hline []] & GO:0016262 & $\begin{array}{l}\text { protein } \mathrm{N}- \\
\text { acetylglucosaminyltra } \\
\text { nsferase activity }\end{array}$ & \begin{tabular}{|l|} 
MOLECULAR_F \\
UNCTION
\end{tabular} & $\begin{array}{l}0.827121087 \\
427183\end{array}$ & \begin{tabular}{|l|}
0.002724961 \\
000167915
\end{tabular} & 2 & 1 & 520 & 16605 & $\begin{array}{l}\text { MELO3C010102; } \\
\text { MELO3C024563 }\end{array}$ & MELO3C013249 \\
\hline []] & GO:0009736 & $\begin{array}{l}\text { cytokinin-activated } \\
\text { signaling pathway }\end{array}$ & $\left|\begin{array}{l}\text { BIOLOGICAL_P } \\
\text { ROCESS }\end{array}\right|$ & $\begin{array}{l}0.952227450 \\
3872248\end{array}$ & $\begin{array}{l}0.003868107 \\
1564413854\end{array} \mid$ & 3 & 8 & 519 & 16598 & $\begin{array}{l}\text { MELO3C014230; } \\
\text { MELO3C024563; } \\
\text { MELO3C024357 }\end{array}$ & $\begin{array}{l}\text { MELO3C004183; } \\
\text { MELO3C016937; } \\
\text { MELO3C003198; } \\
\text { MELO3C025617; } \\
\text { MELO3C016973; } \\
\text { MELO3C010714; } \\
\text { MELO3C017877; } \\
\text { MELO3C024439 }\end{array}$ \\
\hline [] & GO:0042256 & $\begin{array}{l}\text { mature ribosome } \\
\text { assembly }\end{array}$ & \begin{tabular}{|l} 
BIOLOGICAL_P \\
ROCESS
\end{tabular} & 1.0 & \begin{tabular}{|l|}
0.005339884 \\
106746404
\end{tabular} & 2 & 2 & 520 & 16604 & $\begin{array}{l}\text { MELO3C010584; } \\
\text { MELO3C013183 }\end{array}$ & $\begin{array}{l}\text { MELO3C023215; } \\
\text { MELO3C015319 }\end{array}$ \\
\hline [] & GO:0000184 & $\begin{array}{l}\text { nuclear-transcribed } \\
\text { mRNA catabolic } \\
\text { process, nonsense- } \\
\text { mediated decay }\end{array}$ & $\begin{array}{l}\text { BIOLOGICAL_P } \\
\text { ROCESS }\end{array}$ & 1.0 & \begin{tabular}{|l|}
0.018962465 \\
976286615
\end{tabular} & 3 & 16 & 519 & 16590 & $\begin{array}{l}\text { MELO3C026263; } \\
\text { MELO3C013879; } \\
\text { MELO3C013051 }\end{array}$ & $\begin{array}{l}\text { MELO3C015714; } \\
\text { MELO3C011145; } \\
\text { MELO3C008956; } \\
\text { MELO3C006767; } \\
\text { MELO3C021931; } \\
\text { MELO3C008955; } \\
\text { MELO3C026304; } \\
\text { MELO3C026612; } \\
\text { MELO3C026327; } \\
\text { MELO3C007816; } \\
\text { MELO3C024028; } \\
\text { MELO3C025497; } \\
\text { MELO3C003804; } \\
\text { MELO3C025498; } \\
\text { MELO3C015113; } \\
\text { MELO3C026290 }\end{array}$ \\
\hline [] & GO:0032446 & $\begin{array}{l}\text { protein modification } \\
\text { by small protein } \\
\text { conjugation }\end{array}$ & \begin{tabular}{|l|} 
BIOLOGICAL_P \\
ROCESS
\end{tabular} & 1.0 & \begin{tabular}{|l|}
0.044054389 \\
12341239
\end{tabular} & 10 & 163 & 512 & 16443 & $\begin{array}{l}\text { MELO3C008494; } \\
\text { MELO3C018461; } \\
\text { MELO3C011882; } \\
\text { MELO3C005490; } \\
\text { MELO3C018720; } \\
\text { MELO3C016464; } \\
\text { MELO3C005035; } \\
\text { MELO3C005971; } \\
\text { MELO3C013879; } \\
\text { MELO3C009879 }\end{array}$ & $\begin{array}{l}\text { MELO3C003490; } \\
\text { MELO3C011153; } \\
\text { MELO3C013694; } \\
\text { MELO3C020827; } \\
\text { MELO3C012361; } \\
\text { MELO3C003650; } \\
\text { MELO3C020149; } \\
\text { MELO3C022725; } \\
\text { MELO3C006520; } \\
\text { MELO3C022726; } \\
\text { MELO3C007214; } \\
\text { MELO3C026528; } \\
\text { MELO3C007339; } \\
\text { MELO3C021512; } \\
\text { MELO3C008669; } \\
\text { MELO3C026527; } \\
\text { MELO3C019281; } \\
\text { MELO3C005717; } \\
\text { MELO3C026800; } \\
\text { MELO3C023372; } \\
\text { MELO3C022280; } \\
\text { MELO3C012406; } \\
\text { MELO3C018501; } \\
\text { MELO3C012407; } \\
\text { MELO3C014436; } \\
\text { MELO3C009880; } \\
\text { MELO3C008310; }\end{array}$ \\
\hline [] & GO:0006968 & $\begin{array}{l}\text { cellular defense } \\
\text { response }\end{array}$ & $\begin{array}{l}\text { BIOLOGICAL_P } \\
\text { ROCESS }\end{array}$ & 1.0 & \begin{tabular}{|l|}
0.030476412 \\
893692188
\end{tabular} & 1 & 0 & 521 & 16606 & MELO3C006765 & \\
\hline [] & GO:0001833 & $\begin{array}{l}\text { inner cell mass cell } \\
\text { proliferation }\end{array}$ & \begin{tabular}{|l} 
BIOLOGICAL_P \\
ROCESS \\
\end{tabular} & 1.0 & \begin{tabular}{|l|}
0.030476412 \\
893692188 \\
\end{tabular} & 1 & 0 & 521 & 16606 & MELO3C010584 & \\
\hline
\end{tabular}




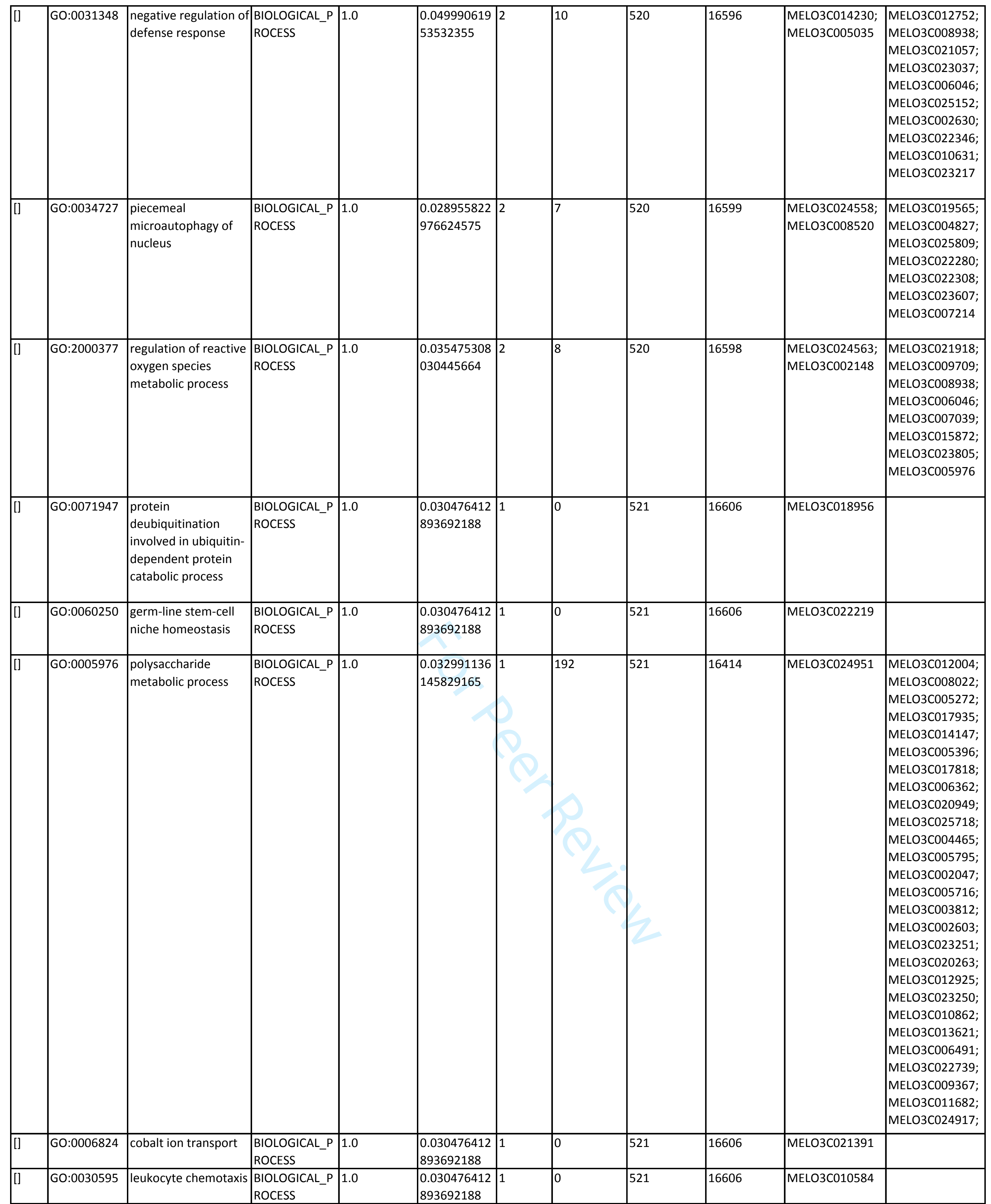




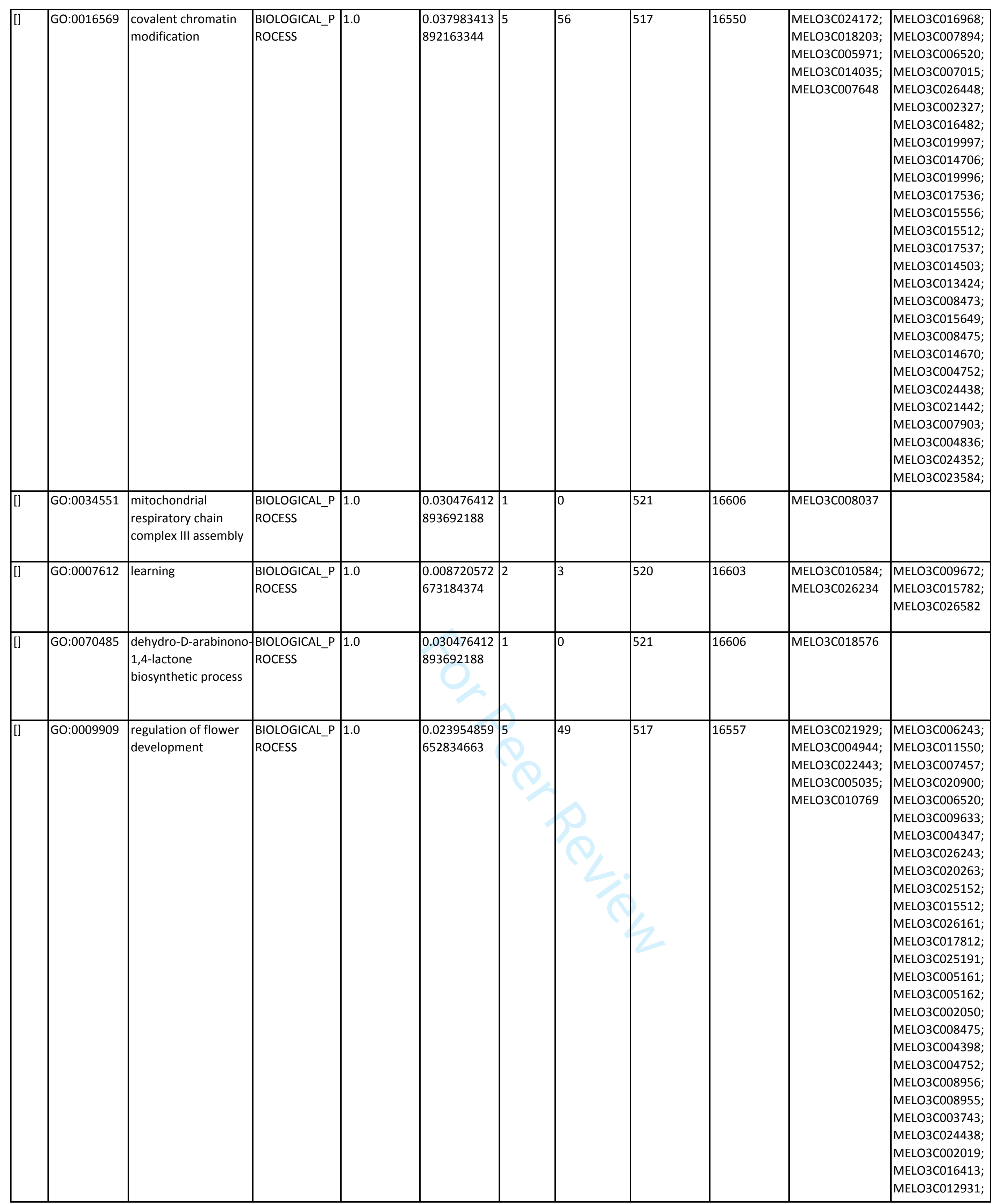




\begin{tabular}{|c|c|c|c|c|c|c|c|c|c|c|}
\hline [] & GO:0007623 & circadian rhythm & \begin{tabular}{|l} 
BIOLOGICAL_P \\
ROCESS
\end{tabular} & 1.0 & \begin{tabular}{|l|}
0.011067071 \\
885078236
\end{tabular} \mid 4 & 25 & 518 & 16581 & $\begin{array}{l}\text { MELO3C013369; } \\
\text { MELO3C025049; } \\
\text { MELO3C010769; } \\
\text { MELO3C001962 } \\
\end{array}$ & 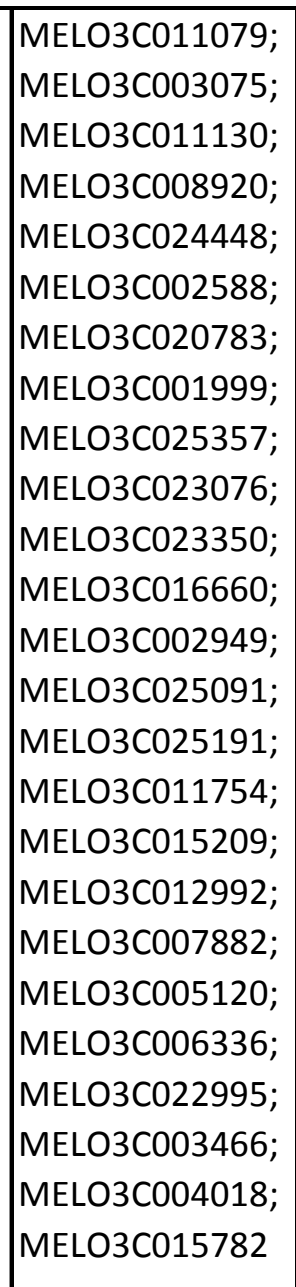 \\
\hline[] & GO:0000422 & mitophagy & \begin{tabular}{|l} 
BIOLOGICAL_P \\
ROCESS
\end{tabular} & 1.0 & \begin{tabular}{|l|}
0.017585801 \\
731193642
\end{tabular} & 5 & 520 & 16601 & $\begin{array}{l}\text { MELO3C024558; } \\
\text { MELO3C008520 }\end{array}$ & $\begin{array}{l}\text { MELO3C022280; } \\
\text { MELO3C023607; } \\
\text { MELO3CO07214; } \\
\text { MELO3C019565; } \\
\text { MELO3C025809 }\end{array}$ \\
\hline [] & GO:0006777 & $\begin{array}{l}\text { Mo-molybdopterin } \\
\text { cofactor biosynthetic } \\
\text { process }\end{array}$ & \begin{tabular}{|l|} 
BIOLOGICAL_P \\
ROCESS
\end{tabular} & 1.0 & \begin{tabular}{|l|}
0.035475308 \\
030445664
\end{tabular} & 8 & 520 & 16598 & $\begin{array}{l}\text { MELO3C022174; } \\
\text { MELO3C019661 }\end{array}$ & $\begin{array}{l}\text { MELOOC014007; } \\
\text { MELO3C014535; } \\
\text { MELO3CO09365; } \\
\text { MELO3C01418; } \\
\text { MELO3C023505; } \\
\text { MELO3C01559;; } \\
\text { MELO3C024139; } \\
\text { MELO3C007668 }\end{array}$ \\
\hline [0 & GO:0044765 & $\begin{array}{l}\text { single-organism } \\
\text { transport }\end{array}$ & \begin{tabular}{|l} 
BIOLOGICAL_P \\
ROCESS
\end{tabular} & 1.0 & \begin{tabular}{|l|}
0.029403874 \\
525730116
\end{tabular} & 735 & 509 & 15871 & $\begin{array}{l}\text { MELO3C016308; } \\
\text { MELO3C020836; } \\
\text { MELO3C014230; } \\
\text { MELO3CO22501; } \\
\text { MELO3CO0499; } \\
\text { MELO3C021783; } \\
\text { MELO3C003629; } \\
\text { MELO3C01535; } \\
\text { MELO3C018720; } \\
\text { MELO3C011139; } \\
\text { MELO3C016149; } \\
\text { MELO3C009480; } \\
\text { MELO3C013437 }\end{array}$ & 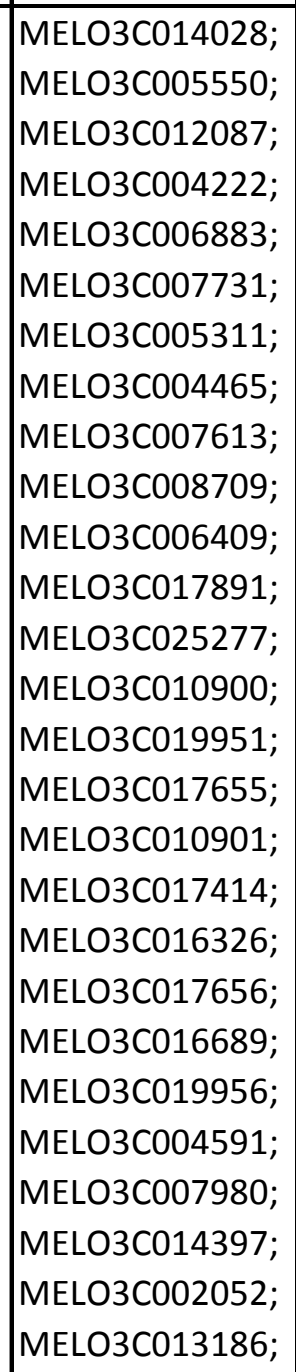 \\
\hline
\end{tabular}




\begin{tabular}{|c|c|c|c|c|c|c|c|c|c|c|c|}
\hline [] & GO:0006406 & $\begin{array}{l}\text { mRNA export from } \\
\text { nucleus }\end{array}$ & $\begin{array}{l}\text { BIOLOGICAL_P } \\
\text { ROCESS }\end{array}$ & $\mid 1.0$ & $\begin{array}{l}0.009771068 \\
479987946\end{array}$ & 4 & 24 & 518 & 16582 & $\begin{array}{l}\text { MELO3C003078; } \\
\text { MELO3C013879; } \\
\text { MELO3C012713; } \\
\text { MELO3C015733 }\end{array}$ & $\begin{array}{l}\text { MELO3C011256 } \\
\text { MELO3C014532 } \\
\text { MELO3C013443 } \\
\text { MELO3C020407 } \\
\text { MELO3C005265 } \\
\text { MELO3C005694 } \\
\text { MELO3C004622 } \\
\text { MELO3C021931 } \\
\text { MELO3C021887 } \\
\text { MELO3C026304 } \\
\text { MELO3C016280 } \\
\text { MELO3C022166 } \\
\text { MELO3C009808 } \\
\text { MELO3C005946 } \\
\text { MELO3C015492 } \\
\text { MELO3C025497 } \\
\text { MELO3C025498 } \\
\text { MELO3C019034 } \\
\text { MELO3C026441 } \\
\text { MELO3C011924 } \\
\text { MELO3C015113 } \\
\text { MELO3C015447 } \\
\text { MELO3C016756 } \\
\text { MELO3C014129 }\end{array}$ \\
\hline [] & GO:0010228 & \begin{tabular}{|l|} 
vegetative to \\
reproductive phase \\
transition of \\
meristem
\end{tabular} & $\begin{array}{l}\text { BIOLOGICAL_P } \\
\text { ROCESS }\end{array}$ & 1.0 & \begin{tabular}{|l|}
0.044368614 \\
5552901
\end{tabular} & 4 & 40 & 8 & 16566 & $\begin{array}{l}\text { MELO3C013369; } \\
\text { MELO3C022443; } \\
\text { MELO3C005971; } \\
\text { MELO3C010769 }\end{array}$ & $\begin{array}{l}\text { MELO3C012301 } \\
\text { MELO3C014401 } \\
\text { MELO3C009890, } \\
\text { MELO3C005593 } \\
\text { MELO3C005310 } \\
\text { MELO3C006520 } \\
\text { MELO3C026009, } \\
\text { MELO3C002588 } \\
\text { MELO3C022205 } \\
\text { MELO3C006888 } \\
\text { MELO3C019330 } \\
\text { MELO3C002327 } \\
\text { MELO3C017295 } \\
\text { MELO3C015173 } \\
\text { MELO3C025211 } \\
\text { MELO3C019731 } \\
\text { MELO3C015158 } \\
\text { MELO3C021171 } \\
\text { MELO3C019813 } \\
\text { MELO3C012645 } \\
\text { MELO3C025191 } \\
\text { MELO3C013458 } \\
\text { MELO3C007681 } \\
\text { MELO3C012312 } \\
\text { MELO3C007085 } \\
\text { MELO3C008232 } \\
\text { MELO3C020854 }\end{array}$ \\
\hline [] & GO:0009696 & $\begin{array}{l}\text { salicylic acid } \\
\text { metabolic process }\end{array}$ & $\begin{array}{l}\text { BIOLOGICAL_P } \\
\text { ROCESS }\end{array}$ & 1.0 & \begin{tabular}{|l|}
0.008720572 \\
673184374
\end{tabular} & 2 & 3 & 520 & 16603 & $\begin{array}{l}\text { MELO3C005035; } \\
\text { MELO3C002148 }\end{array}$ & $\begin{array}{l}\text { MELO3C027349, } \\
\text { MELO3C002099, } \\
\text { MELO3C016008 }\end{array}$ \\
\hline [] & GO:0048582 & $\begin{array}{l}\text { positive regulation of } \\
\text { post-embryonic } \\
\text { development }\end{array}$ & $\begin{array}{l}\text { BIOLOGICAL_P } \\
\text { ROCESS }\end{array}$ & 1.0 & \begin{tabular}{|l|}
0.021814414 \\
1696528
\end{tabular} & 3 & 17 & 519 & 16589 & $\begin{array}{l}\text { MELO3C004944; } \\
\text { MELO3C022443; } \\
\text { MELO3C009101 }\end{array}$ & $\begin{array}{l}\text { MELO3C011221 } \\
\text { MELO3C011771 } \\
\text { MELO3C011299, } \\
\text { MELO3C002050 } \\
\text { MELO3C004398, } \\
\text { MELO3C007457, } \\
\text { MELO3C006520 } \\
\text { MELO3C022995 } \\
\text { MELO3C004632 } \\
\text { MELO3C023325 } \\
\text { MELO3C009627 } \\
\text { MELO3C020484 } \\
\text { MELO3C016660 } \\
\text { MELO3C024265 } \\
\text { MELO3C025183, } \\
\text { MELO3C026161 } \\
\text { MELO3C011104 }\end{array}$ \\
\hline [] & GO:0046167 & $\begin{array}{l}\text { glycerol-3-phosphate } \\
\text { biosynthetic process }\end{array}$ & \begin{tabular}{|l} 
BIOLOGICAL_P \\
ROCESS
\end{tabular} & 1.0 & \begin{tabular}{|l|}
0.030476412 \\
893692188
\end{tabular} & 1 & 0 & 521 & 16606 & MELO3C020157 & \\
\hline [] & GO:2000070 & \begin{tabular}{|l} 
regulation of \\
response to water \\
deprivation
\end{tabular} & $\begin{array}{l}\text { BIOLOGICAL_P } \\
\text { ROCESS }\end{array}$ & 1.0 & $\begin{array}{l}0.030476412 \\
893692188\end{array}$ & 1 & 0 & 521 & 16606 & MELO3C005035 & \\
\hline
\end{tabular}




\begin{tabular}{|c|c|c|c|c|c|c|c|c|c|c|}
\hline [] & GO:0042127 & $\begin{array}{l}\text { regulation of cell } \\
\text { proliferation }\end{array}$ & $\begin{array}{l}\text { BIOLOGICAL_P } \\
\text { ROCESS }\end{array}$ & 1.0 & \begin{tabular}{|l|}
0.035729304 \\
18181029
\end{tabular} & 55 & 517 & 16551 & $\begin{array}{l}\text { MELO3C023475; } \\
\text { MELO3C010640; } \\
\text { MELO3C007648; } \\
\text { MELO3C015733; } \\
\text { MELO3C026068 }\end{array}$ & 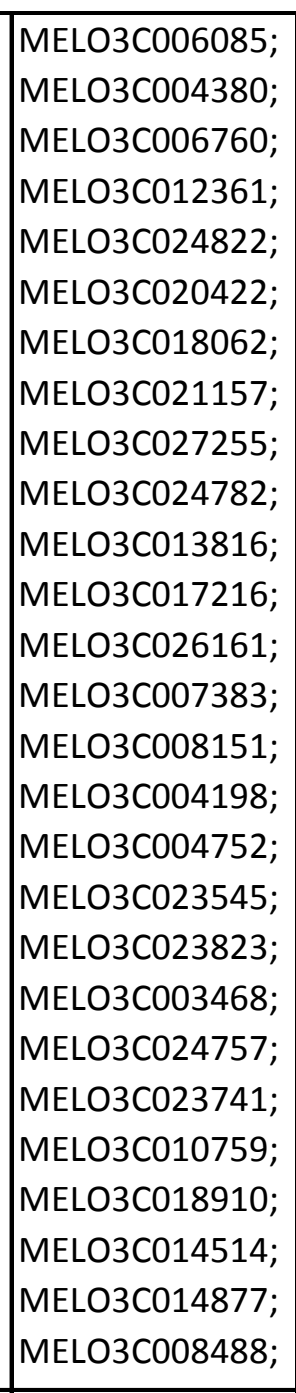 \\
\hline [] & GO:0000463 & $\begin{array}{l}\text { maturation of LSU- } \\
\text { rRNA from tricistronic } \\
\text { rRNA transcript (SSU- } \\
\text { rRNA, 5.8S rRNA, LSU- } \\
\text { rRNA) }\end{array}$ & $\begin{array}{l}\text { BIOLOGICAL_P } \\
\text { ROCESS }\end{array}$ & 1.0 & \begin{tabular}{|l|l|}
0.049990619 & 2 \\
53532355
\end{tabular} & 10 & 520 & 16596 & $\begin{array}{l}\text { MELO3C013183; } \\
\text { MELO3C023306 }\end{array}$ & $\begin{array}{l}\text { MELO3C018272; } \\
\text { MELO3C024586; } \\
\text { MELO3C022134; } \\
\text { MELO3CO08999; } \\
\text { MELO3CO03340; } \\
\text { MELO3C015907; } \\
\text { MELO3CO04873; } \\
\text { MELO3C023052; } \\
\text { MELO3C027392; } \\
\text { MELO3C017744 }\end{array}$ \\
\hline [] & GO:0018315 & \begin{tabular}{|l} 
molybdenum \\
incorporation into \\
molybdenum- \\
molybdopterin \\
complex
\end{tabular} & \begin{tabular}{|l} 
BIOLOGICAL_P \\
ROCESS
\end{tabular} & 1.0 & \begin{tabular}{|l|}
0.030476412 \\
893692188
\end{tabular} & 0 & 521 & 16606 & MELO3C022174 & \\
\hline [] & GO:0044724 & \begin{tabular}{|l|} 
single-organism \\
carbohydrate \\
catabolic process
\end{tabular} & \begin{tabular}{|l} 
BIOLOGICAL_P \\
ROCESS
\end{tabular} & 1.0 & \begin{tabular}{|l|}
0.023430499 \\
533914177
\end{tabular} & 138 & 522 & 16468 & & 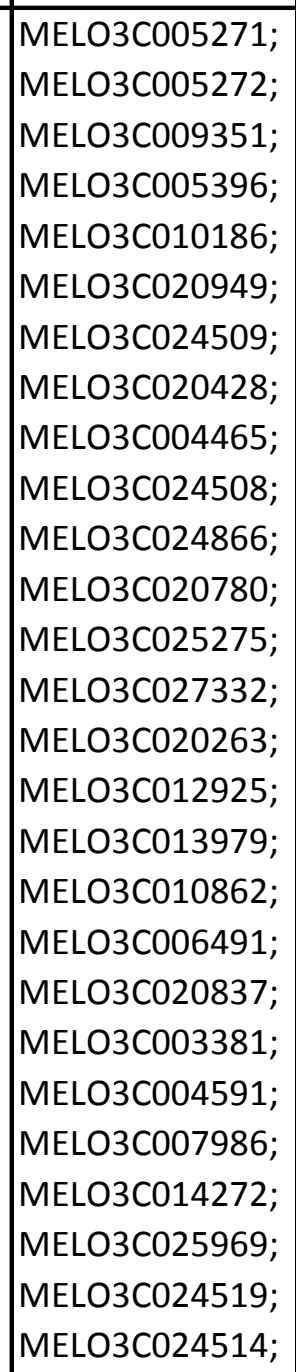 \\
\hline
\end{tabular}




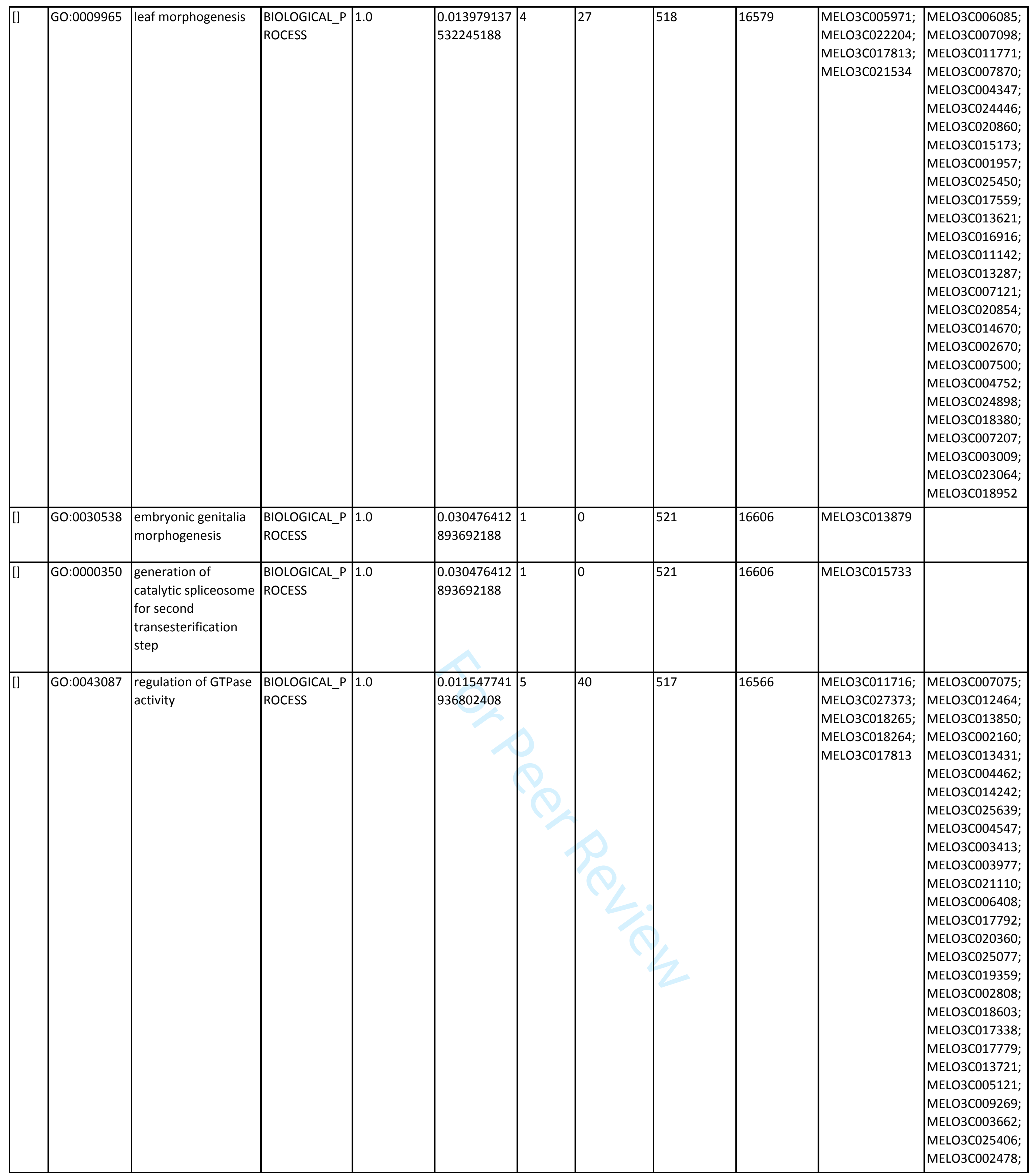




\begin{tabular}{|c|c|c|c|c|c|c|c|c|c|c|}
\hline [] & GO:0016246 & RNA interference & \begin{tabular}{|l|} 
BIOLOGICAL_P \\
ROCESS
\end{tabular} & 1.0 & $\left|\begin{array}{l}0.032811319 \\
96060644\end{array}\right| 4$ & 36 & 518 & 16570 & $\begin{array}{l}\text { MELO3C020836; } \\
\text { MELO3C013879; } \\
\text { MELO3C014035; } \\
\text { MELO3C007648 }\end{array}$ & 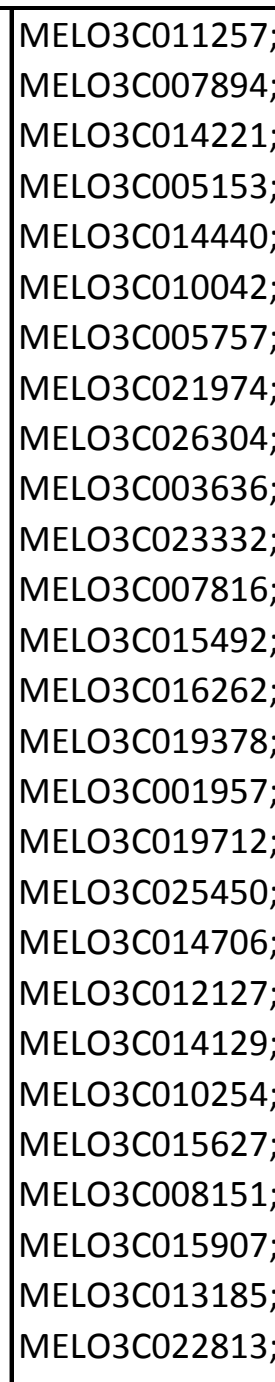 \\
\hline [] & GO:0010389 & $\begin{array}{l}\text { regulation of } \mathrm{G} 2 / \mathrm{M} \\
\text { transition of mitotic } \\
\text { cell cycle }\end{array}$ & \begin{tabular}{|l|} 
BIOLOGICAL_P \\
ROCESS
\end{tabular} & 1.0 & $\left.$\begin{tabular}{|l|l}
0.018962465 \\
976286615
\end{tabular}\right|$^{3}$ & 16 & 519 & 16590 & $\begin{array}{l}\text { MELO3C023986; } \\
\text { MELO3C007648; } \\
\text { MELO3C005971 }\end{array}$ & 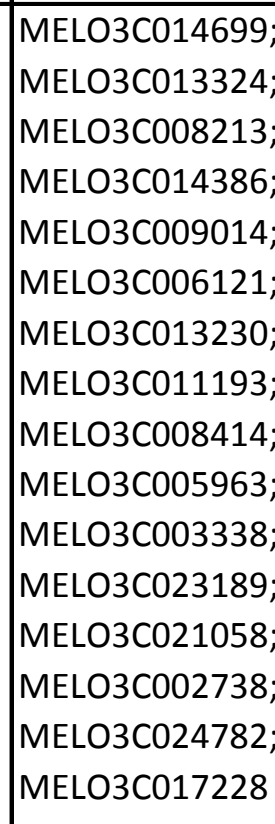 \\
\hline[] & GO:0022900 & $\begin{array}{l}\begin{array}{l}\text { electron transport } \\
\text { chain }\end{array} \\
\end{array}$ & \begin{tabular}{|l|} 
BIOLOGICAL_P \\
ROCESS
\end{tabular} & 1.0 & $\left|\begin{array}{l}0.023004593 \\
432375684\end{array}\right| 1$ & 204 & 521 & 16402 & MELO3C025023 & 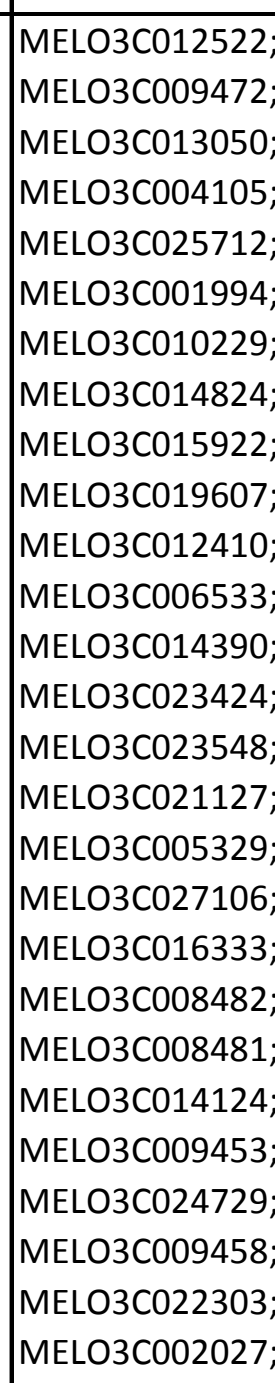 \\
\hline [] & GO:0048539 & $\begin{array}{l}\text { bone marrow } \\
\text { development }\end{array}$ & \begin{tabular}{|l|} 
BIOLOGICAL_P \\
ROCESS
\end{tabular} & 1.0 & \begin{tabular}{|l|l|}
0.030476412 & 1 \\
893692188 &
\end{tabular} & 10 & 521 & 16606 & MELO3C010584 & \\
\hline [] & GO:0043097 & \begin{tabular}{|l} 
pyrimidine \\
nucleoside salvage
\end{tabular} & \begin{tabular}{|l|} 
BIOLOGICAL_P \\
ROCESS
\end{tabular} & 1.0 & \begin{tabular}{|l|l|}
0.030476412 & 1 \\
893692188 & \\
\end{tabular} & 0 & 521 & 16606 & MELO3C022212 & \\
\hline [] & GO:0006419 & $\begin{array}{l}\text { alanyl-tRNA } \\
\text { aminoacylation }\end{array}$ & \begin{tabular}{|l|} 
BIOLOGICAL_P \\
ROCESS
\end{tabular} & 1.0 & $\left.\begin{array}{l}0.028955822 \\
976624575\end{array}\right|^{2}$ & 7 & 520 & 16599 & $\begin{array}{l}\text { MELO3C000756; } \\
\text { MELO3C000425 }\end{array}$ & $\begin{array}{l}\text { MELO3C012870; } \\
\text { MELO3CO10097; } \\
\text { MELO3C024031; } \\
\text { MELO3C018624; } \\
\text { MELO3C027271; } \\
\text { MELO3C027308; } \\
\text { MELO3C013645 }\end{array}$ \\
\hline
\end{tabular}




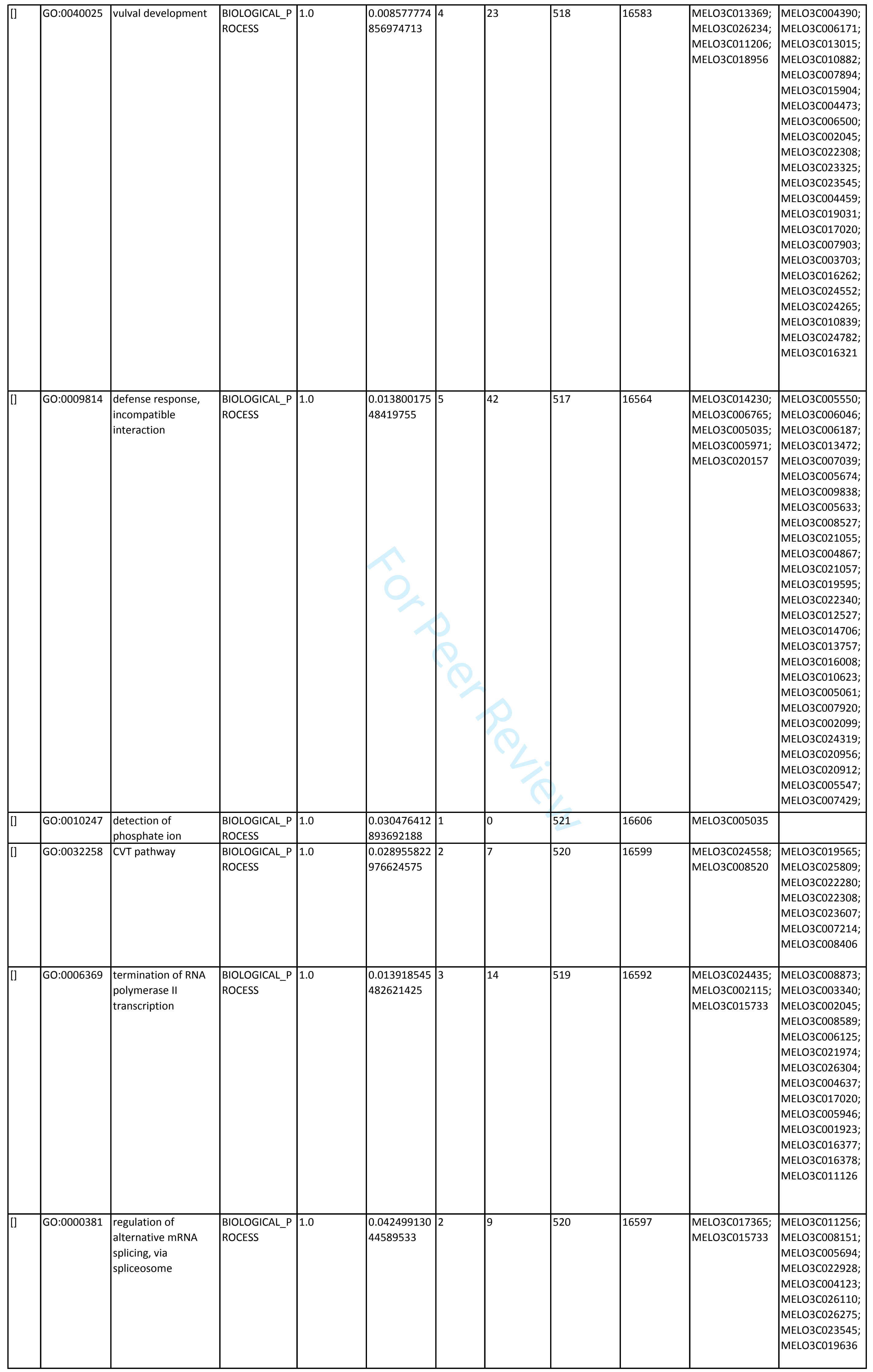




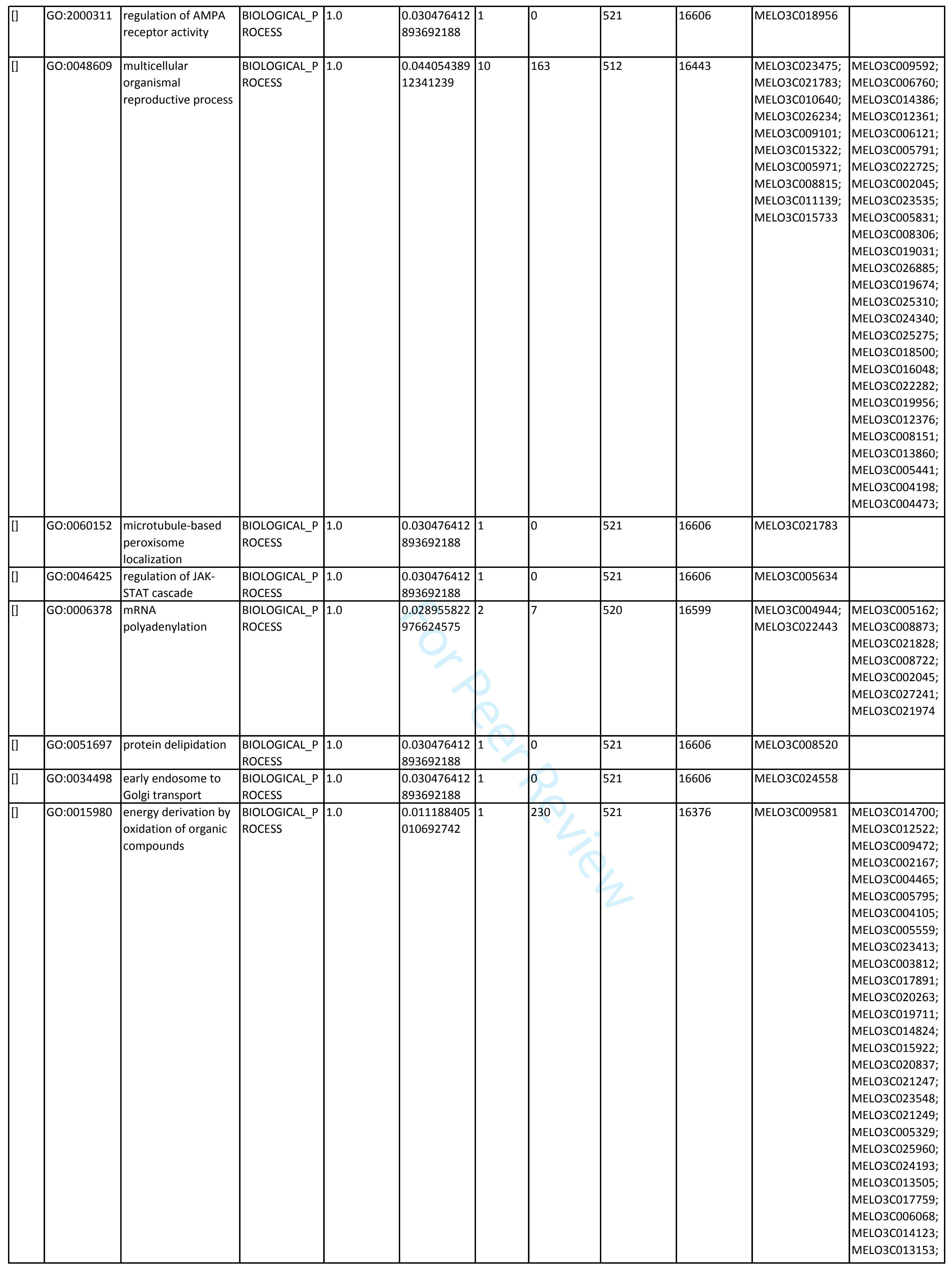




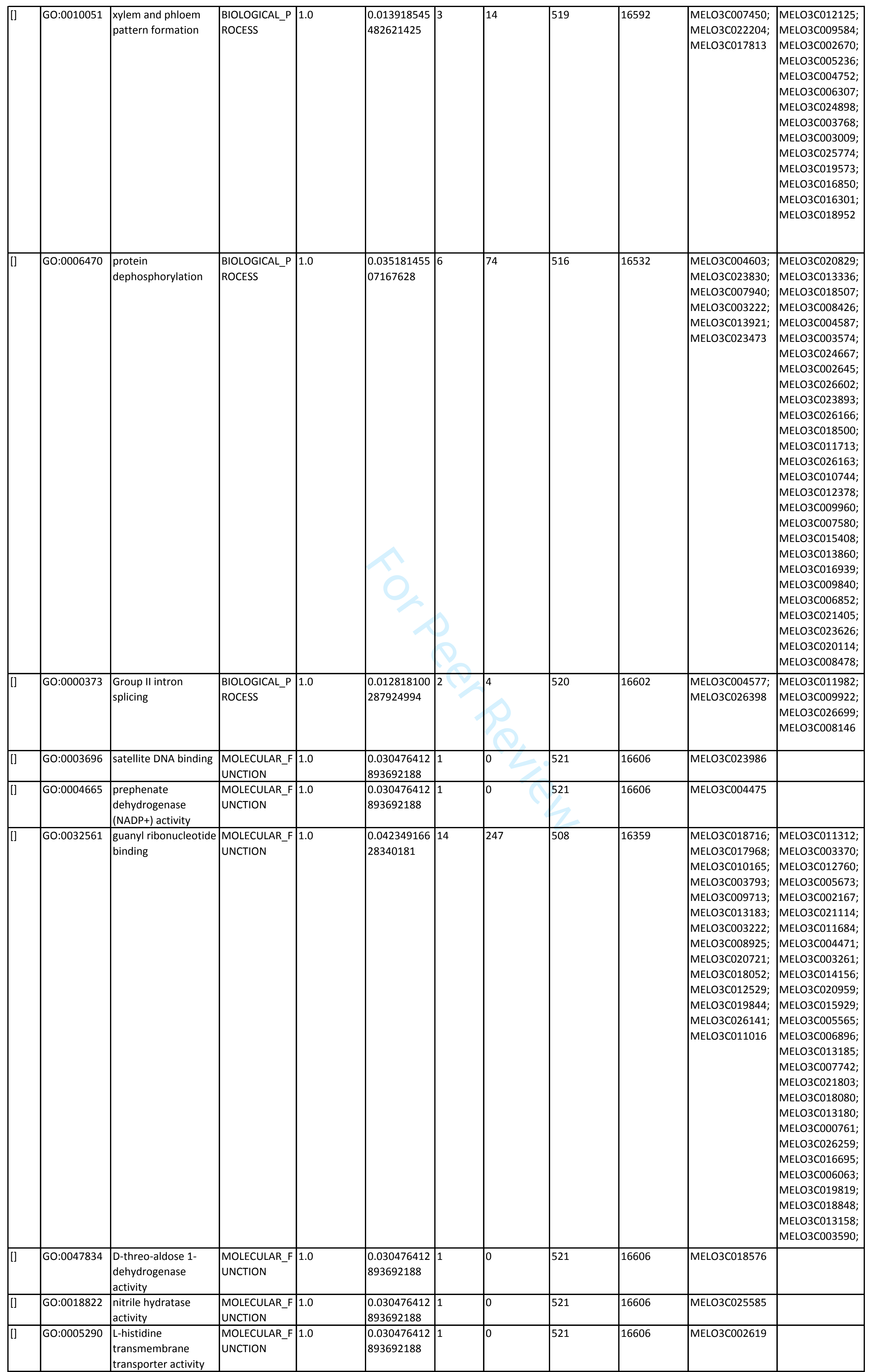




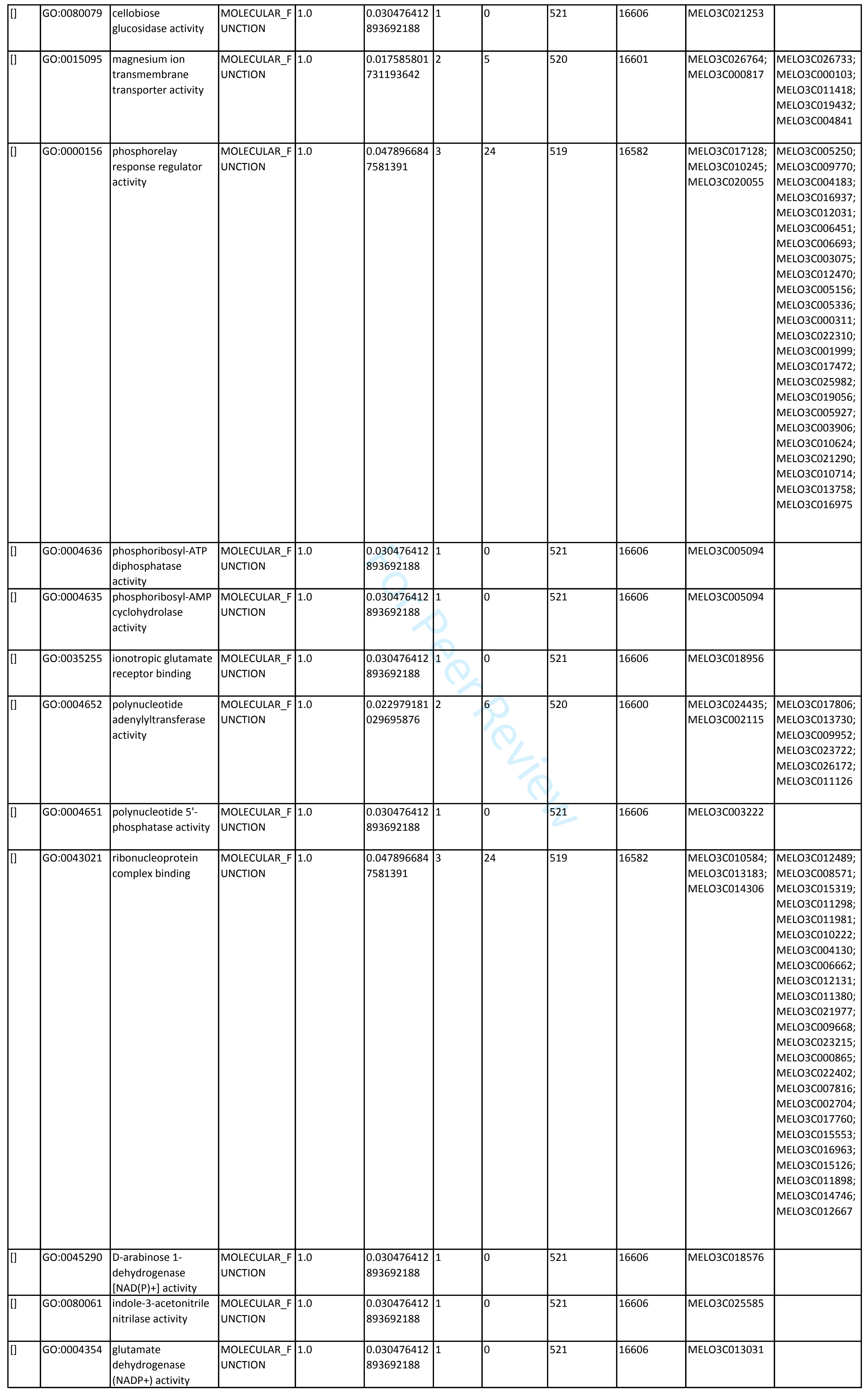




\begin{tabular}{|c|c|c|c|c|c|c|c|c|c|c|c|}
\hline [] & GO:0019151 & \begin{tabular}{|l|} 
galactose 1- \\
dehydrogenase \\
activity
\end{tabular} & \begin{tabular}{|l|} 
MOLECULAR_F \\
UNCTION
\end{tabular} & 1.0 & \begin{tabular}{|l|}
0.030476412 \\
893692188
\end{tabular} & 1 & 0 & 521 & 16606 & MELO3C018576 & \\
\hline [] & GO:0019154 & \begin{tabular}{|l} 
glycolate \\
dehydrogenase \\
activity
\end{tabular} & \begin{tabular}{|l|} 
MOLECULAR_F \\
UNCTION
\end{tabular} & 1.0 & \begin{tabular}{|l|}
0.030476412 \\
893692188
\end{tabular} & 1 & 0 & 521 & 16606 & MELO3C009581 & \\
\hline [] & GO:0008949 & \begin{tabular}{|l} 
oxalyl-CoA \\
decarboxylase \\
activity
\end{tabular} & $\begin{array}{l}\text { MOLECULAR_F } \\
\text { UNCTION }\end{array}$ & 1.0 & \begin{tabular}{|l|}
0.030476412 \\
893692188
\end{tabular} & 1 & 0 & 521 & 16606 & MELO3C010381 & \\
\hline [] & GO:0010349 & \begin{tabular}{|l} 
L-galactose \\
dehydrogenase \\
activity
\end{tabular} & \begin{tabular}{|l|} 
MOLECULAR_F \\
UNCTION
\end{tabular} & 1.0 & \begin{tabular}{|l|}
0.030476412 \\
893692188
\end{tabular} & 1 & 0 & 521 & 16606 & MELO3C018576 & \\
\hline [] & GO:0005096 & $\begin{array}{l}\text { GTPase activator } \\
\text { activity }\end{array}$ & \begin{tabular}{|l|} 
MOLECULAR_F \\
UNCTION
\end{tabular} & 1.0 & \begin{tabular}{|l|}
0.012640048 \\
13944158 \\
\end{tabular} & 5 & 41 & 517 & 16565 & $\begin{array}{l}\text { MELO3C011716; } \\
\text { MELO3C027373; } \\
\text { MELO3C018265; } \\
\text { MELO3C018264; } \\
\text { MELO3C017813 }\end{array}$ & $\begin{array}{l}\text { MELO3C007075; } \\
\text { MELO3C012464; } \\
\text { MELO3C013850; } \\
\text { MELO3C002160; } \\
\text { MELO3C013431; } \\
\text { MELO3C004462; } \\
\text { MELO3C014242; } \\
\text { MELO3C004980; } \\
\text { MELO3C025639; } \\
\text { MELO3C006548; } \\
\text { MELO3C004547; } \\
\text { MELO3C003413; } \\
\text { MELO3C003977; } \\
\text { MELO3C021110; } \\
\text { MELO3C006408; } \\
\text { MELO3C022488; } \\
\text { MELO3C017792; } \\
\text { MELO3C020360; } \\
\text { MELO3C025077; } \\
\text { MELO3C019359; } \\
\text { MELO3C015111; } \\
\text { MELO3C002808; } \\
\text { MELO3C017654; } \\
\text { MELO3C017779; } \\
\text { MELO3C013721; } \\
\text { MELO3C005121; } \\
\text { MELO3C009269; }\end{array}$ \\
\hline [] & GO:0047499 & $\begin{array}{l}\text { calcium-independent } \\
\text { phospholipase A2 } \\
\text { activity }\end{array}$ & \begin{tabular}{|l|} 
MOLECULAR_F \\
UNCTION
\end{tabular} & 1.0 & \begin{tabular}{|l|}
0.030476412 \\
893692188 \\
\end{tabular} & 1 & 0 & 521 & 16606 & MELO3C020721 & \\
\hline [] & GO:0047816 & \begin{tabular}{|l|}
-arabinose 1- \\
dehydrogenase (NAD) \\
activity
\end{tabular} & \begin{tabular}{|l|} 
MOLECULAR_F \\
UNCTION
\end{tabular} & 1.0 & \begin{tabular}{|l|}
0.030476412 \\
893692188
\end{tabular} & 1 & 0 & 521 & 16606 & MELO3C018576 & \\
\hline [] & GO:0015220 & $\begin{array}{l}\text { choline } \\
\text { transmembrane } \\
\text { transporter activity }\end{array}$ & \begin{tabular}{|l|} 
MOLECULAR_F \\
UNCTION
\end{tabular} & 1.0 & \begin{tabular}{|l|}
0.030476412 \\
893692188
\end{tabular} & 1 & 0 & 521 & 16606 & MELO3C020808 & \\
\hline [] & GO:0080048 & $\begin{array}{l}\text { GDP-D-glucose } \\
\text { phosphorylase } \\
\text { activity }\end{array}$ & \begin{tabular}{|l|} 
MOLECULAR_F \\
UNCTION
\end{tabular} & 1.0 & \begin{tabular}{|l|}
0.030476412 \\
893692188
\end{tabular} & 1 & 0 & 521 & 16606 & MELO3C013136 & \\
\hline [] & GO:0004572 & $\begin{array}{l}\text { mannosyl- } \\
\text { oligosaccharide 1,3- } \\
\text { 1,6-alpha- } \\
\text { mannosidase activity }\end{array}$ & \begin{tabular}{|l|} 
MOLECULAR_F \\
UNCTION
\end{tabular} & 1.0 & \begin{tabular}{|l|}
0.030476412 \\
893692188
\end{tabular} & 1 & 0 & 521 & 16606 & MELO3C009914 & \\
\hline [] & GO:0008139 & $\begin{array}{l}\text { nuclear localization } \\
\text { sequence binding }\end{array}$ & $\begin{array}{l}\text { MOLECULAR_F } \\
\text { UNCTION }\end{array}$ & 1.0 & \begin{tabular}{|l|}
0.030476412 \\
893692188 \\
\end{tabular} & 1 & 0 & 521 & 16606 & MELO3C020836 & \\
\hline [] & GO:0003918 & \begin{tabular}{|l|} 
DNA topoisomerase \\
type II (ATP- \\
hydrolyzing) activity
\end{tabular} & \begin{tabular}{|l|} 
MOLECULAR_F \\
UNCTION
\end{tabular} & 1.0 & \begin{tabular}{|l|}
0.049990619 \\
53532355
\end{tabular} & 2 & 10 & 520 & 16596 & $\begin{array}{l}\text { MELO3C023986; } \\
\text { MELO3C011386 }\end{array}$ & $\begin{array}{l}\text { MELO3C019163; } \\
\text { MELO3C022420; } \\
\text { MELO3C003071; } \\
\text { MELO3C019089; } \\
\text { MELO3C014153; } \\
\text { MELO3C024924; } \\
\text { MELO3C014639; } \\
\text { MELO3C019090; } \\
\text { MELO3C024756; } \\
\text { MELO3C024755 }\end{array}$ \\
\hline [] & GO:0016807 & $\begin{array}{l}\text { cysteine-type } \\
\text { carboxypeptidase } \\
\text { activity }\end{array}$ & \begin{tabular}{|l|} 
MOLECULAR_F \\
UNCTION
\end{tabular} & 1.0 & \begin{tabular}{|l|}
0.030476412 \\
893692188
\end{tabular} & 1 & 0 & 521 & 16606 & MELO3C008520 & \\
\hline [] & GO:0008865 & fructokinase activity & \begin{tabular}{|l|} 
MOLECULAR_F \\
UNCTION
\end{tabular} & 1.0 & \begin{tabular}{|l|}
0.022979181 \\
029695876
\end{tabular} & 2 & 6 & 520 & 16600 & $\begin{array}{l}\text { MELO3C015750; } \\
\text { MELO3C009032 }\end{array}$ & $\begin{array}{l}\text { MELO3C022452; } \\
\text { MELO3C015749; } \\
\text { MELO3C020278; } \\
\text { MELO3C003755; } \\
\text { MELO3C005395; } \\
\text { MELO3C009200 }\end{array}$ \\
\hline [] & GO:0047427 & $\begin{array}{l}\text { cyanoalanine nitrilase } \\
\text { activity }\end{array}$ & \begin{tabular}{|l|} 
MOLECULAR_F \\
UNCTION
\end{tabular} & 1.0 & \begin{tabular}{|l|}
0.030476412 \\
893692188 \\
\end{tabular} & 1 & 0 & 521 & 16606 & MELO3C025585 & \\
\hline
\end{tabular}




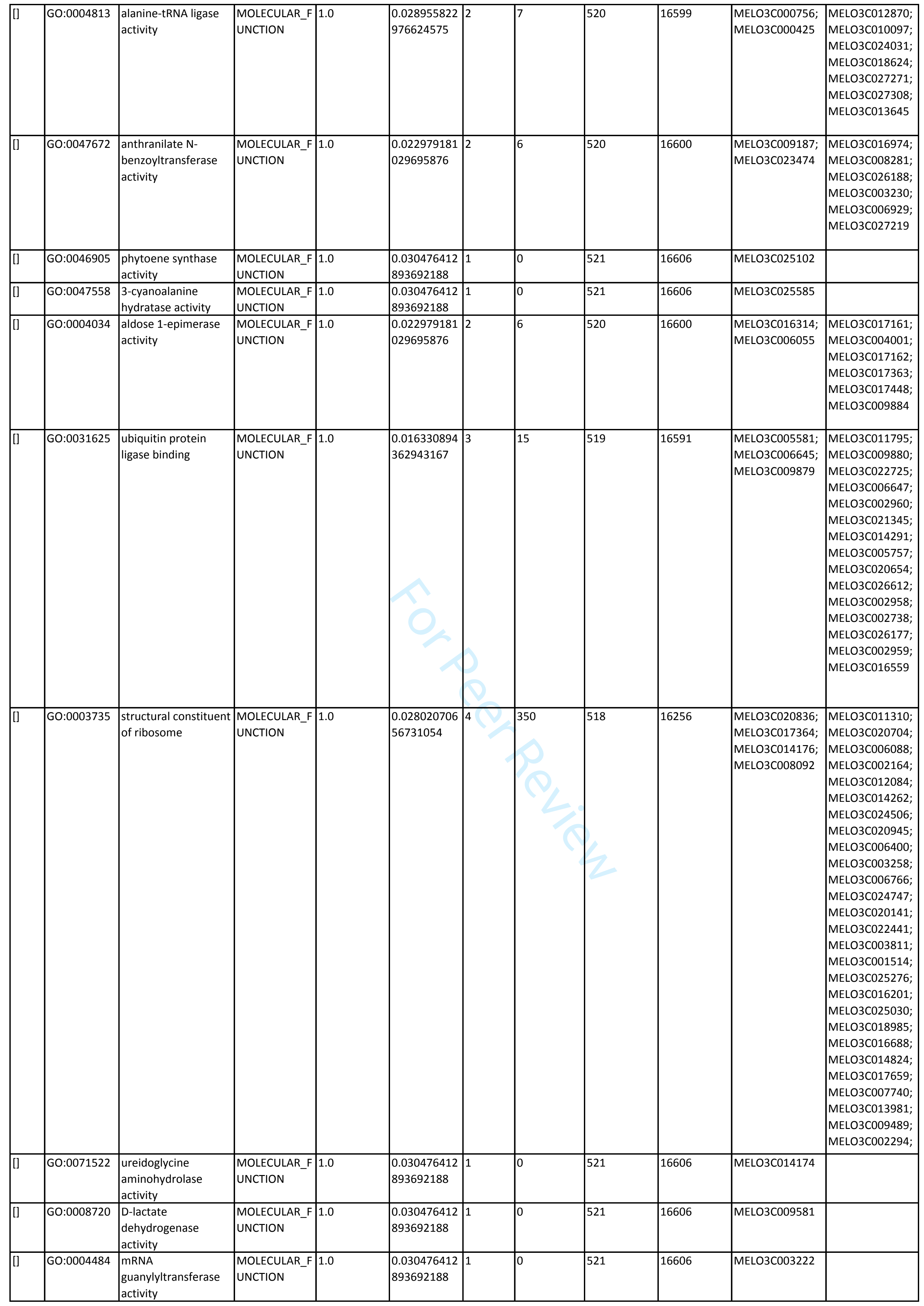




\begin{tabular}{|c|c|c|c|c|c|c|c|c|c|c|c|}
\hline [] & GO:0004004 & \begin{tabular}{|l|} 
ATP-dependent RNA \\
helicase activity
\end{tabular} & $\begin{array}{l}\text { MOLECULAR_F } \\
\text { UNCTION }\end{array}$ & 1.0 & \begin{tabular}{|l|l}
0.047896684 \\
7581391
\end{tabular} & 3 & 24 & 519 & 16582 & $\begin{array}{l}\text { MELO3C013879; } \\
\text { MELO3CO06765; } \\
\text { MELO3C003842 }\end{array}$ & 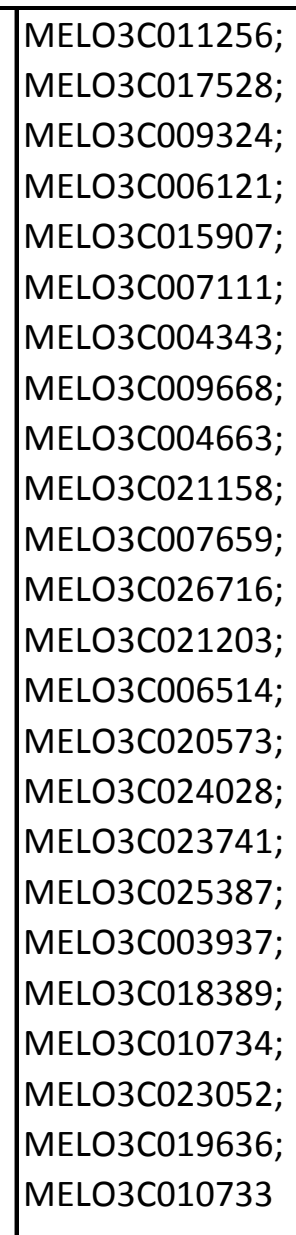 \\
\hline$[$ [] & GO:0004137 & $\begin{array}{l}\text { deoxycytidine kinase } \\
\text { activity }\end{array}$ & $\begin{array}{l}\text { MOLECULAR_F } \\
\text { UNCTION }\end{array}$ & 1.0 & \begin{tabular}{|l|}
0.030476412 \\
893692188
\end{tabular} & 1 & 0 & 521 & 16606 & MELO3C022212 & \\
\hline [] & GO:0030515 & snoRNA binding & $\begin{array}{l}\text { MOLECULAR_F } \\
\text { UNCTION }\end{array}$ & 1.0 & \begin{tabular}{|l|}
0.042499130 \\
44589533
\end{tabular} & 2 & 9 & 520 & 16597 & $\begin{array}{l}\text { MELO3C017440; } \\
\text { MELO3C011408 }\end{array}$ & $\begin{array}{l}\text { MELO3C001556; } \\
\text { MELO3CO26228; } \\
\text { MELO3CO0206; } \\
\text { MELO3CO02835; } \\
\text { MELO3CO24126; } \\
\text { MELO3CO1765; } \\
\text { MELO3CO13185; } \\
\text { MELO3CO1588; } \\
\text { MELO3CO08801 }\end{array}$ \\
\hline [] & GO:0050235 & \begin{tabular}{|l} 
pyridoxal 4- \\
dehydrogenase \\
activity
\end{tabular} & $\begin{array}{l}\text { MOLECULAR_F } \\
\text { UNCTION }\end{array}$ & 1.0 & \begin{tabular}{|l|}
0.030476412 \\
893692188
\end{tabular} & & 0 & 521 & 16606 & MELOЗC018576 & \\
\hline [] & GO:0033730 & \begin{tabular}{|l}
$\begin{array}{l}\text { arogenate } \\
\text { dehydrogenase } \\
\text { (NADP+) activity }\end{array}$ \\
\end{tabular} & $\begin{array}{l}\text { MOLECULAR_F } \\
\text { UNCTION }\end{array}$ & 1.0 & \begin{tabular}{|l|}
0.030476412 \\
893692188
\end{tabular} & 1 & 0 & 521 & 16606 & MELO3C004475 & \\
\hline$[$ [] & GO:0042624 & $\begin{array}{l}\text { ATPase activity, } \\
\text { uncoupled }\end{array}$ & $\begin{array}{l}\text { MOLECULAR_F } \\
\text { UNCTION }\end{array}$ & 1.0 & \begin{tabular}{|l|}
0.007038772 \\
705743546
\end{tabular} & 5 & 35 & 517 & 16571 & $\begin{array}{l}\text { MELO3C021783; } \\
\text { MELO3COO3629; } \\
\text { MELO3C017365; } \\
\text { MELO3C015733; } \\
\text { MELO3CO03842 }\end{array}$ & 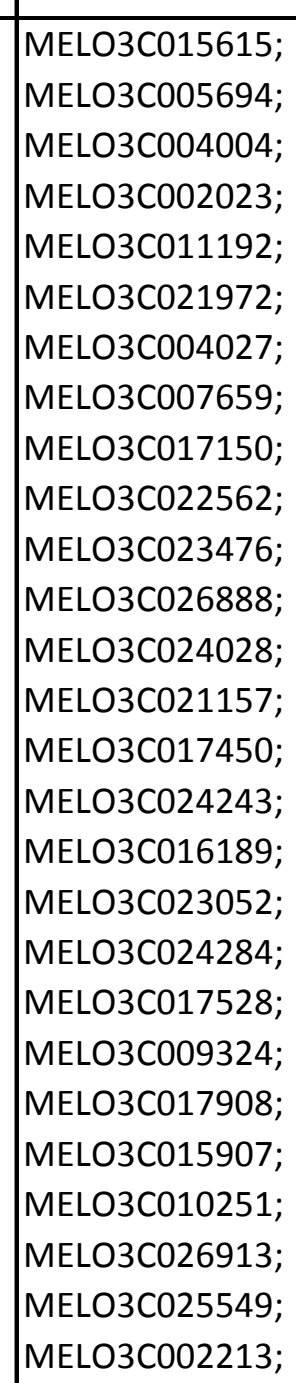 \\
\hline [] & GO:0008672 & \begin{tabular}{|l|} 
2-dehydro-3- \\
deoxyglucarate \\
aldolase activity
\end{tabular} & $\begin{array}{l}\text { MOLECULAR_F } \\
\text { UNCTION }\end{array}$ & 1.0 & \begin{tabular}{|l|}
0.030476412 \\
893692188
\end{tabular} & & 10 & 521 & 16606 & MELO3C013779 & \\
\hline [] & GO:0015087 & \begin{tabular}{|l|} 
cobalt ion \\
transmembrane \\
transporter activity
\end{tabular} & $\begin{array}{l}\text { MOLECULAR_F } \\
\text { UNCTION }\end{array}$ & 1.0 & \begin{tabular}{|l|}
0.030476412 \\
893692188
\end{tabular} & & 0 & 521 & 16606 & MELO3C021391 & \\
\hline
\end{tabular}




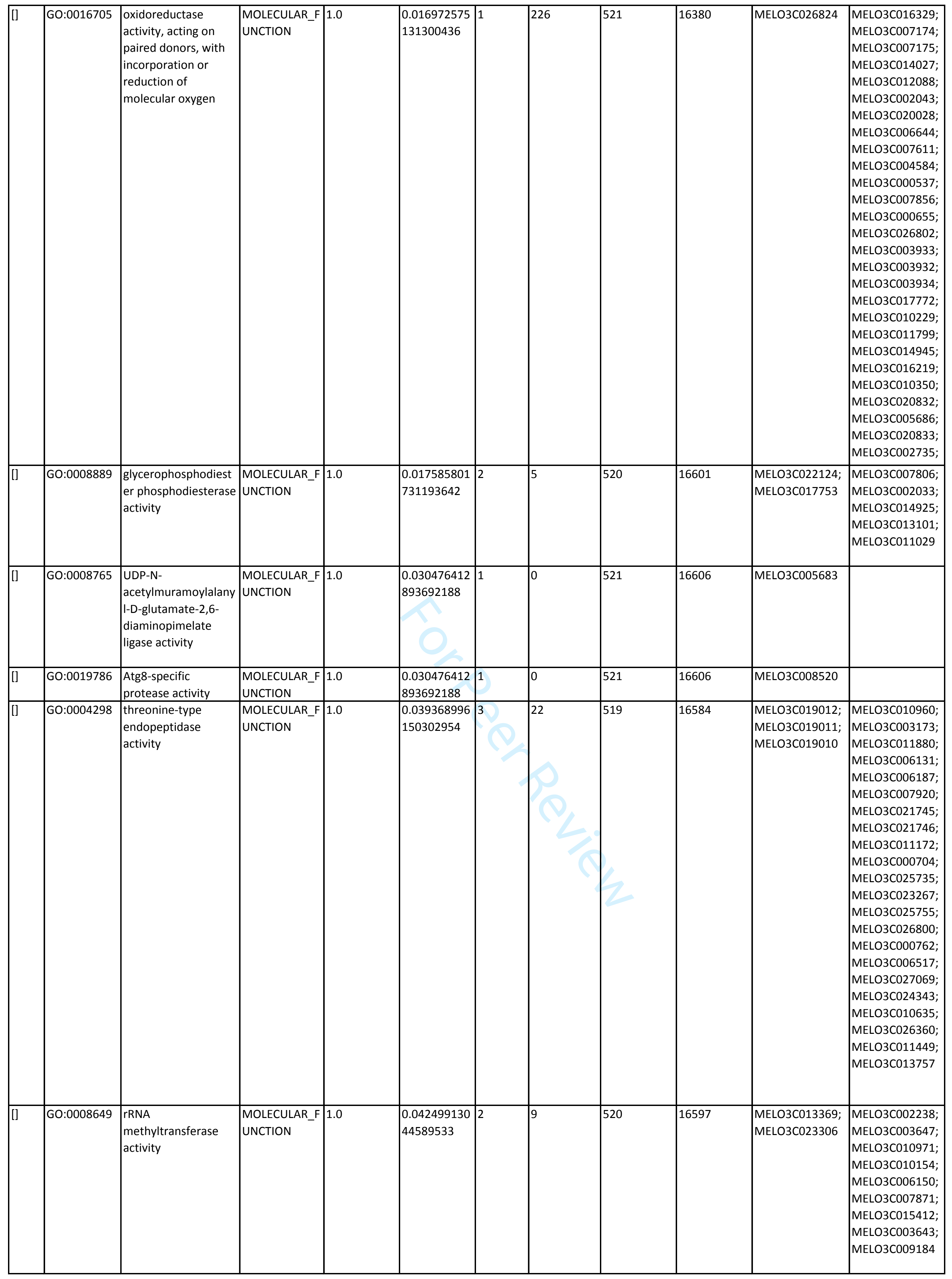




\begin{tabular}{|c|c|c|c|c|c|c|c|c|c|c|c|}
\hline[] & GO:0004725 & $\begin{array}{l}\text { protein tyrosine } \\
\text { phosphatase activity }\end{array}$ & $\begin{array}{l}\text { MOLECULAR_F } \\
\text { UNCTION }\end{array}$ & 1.0 & $\begin{array}{l}0.008577774 \\
856974713\end{array}$ & 4 & 23 & 518 & 16583 & $\begin{array}{l}\text { MELO3C004603; } \\
\text { MELO3C023830; } \\
\text { MELO3C003222; } \\
\text { MELO3C013921 }\end{array}$ & $\begin{array}{l}\text { MELO3C018606; } \\
\text { MELO3C014446; } \\
\text { MELO3C007580; } \\
\text { MELO3C011186; } \\
\text { MELO3C013860; } \\
\text { MELO3C009840; } \\
\text { MELO3C021405; } \\
\text { MELO3C010061; } \\
\text { MELO3C020114; } \\
\text { MELO3C002645; } \\
\text { MELO3C023674; } \\
\text { MELO3C026602; } \\
\text { MELO3C021441; } \\
\text { MELO3C018474; } \\
\text { MELO3C026166; } \\
\text { MELO3C019876; } \\
\text { MELO3C018159; } \\
\text { MELO3C019734; } \\
\text { MELO3C011936; } \\
\text { MELO3C019875; } \\
\text { MELO3C026163; } \\
\text { MELO3C024481; } \\
\text { MELO3C014438 }\end{array}$ \\
\hline[] & GO:0030140 & $\begin{array}{l}\text { trans-Golgi network } \\
\text { transport vesicle }\end{array}$ & \begin{tabular}{|l} 
CELLULAR_CO \\
MPONENT
\end{tabular} & 1.0 & $\begin{array}{l}0.022979181 \\
029695876\end{array}$ & 2 & 6 & 520 & 16600 & $\begin{array}{l}\text { MELO3C003242; } \\
\text { MELO3C017813 }\end{array}$ & $\begin{array}{l}\text { MELO3C025730; } \\
\text { MELO3C013605; } \\
\text { MELO3C024167; } \\
\text { MELO3C007298; } \\
\text { MELO3C005579; } \\
\text { MELO3C008306 }\end{array}$ \\
\hline [] & GO:0044445 & cytosolic part & $\begin{array}{l}\text { CELLULAR_CO } \\
\text { MPONENT }\end{array}$ & 1.0 & $\begin{array}{l}0.037633646 \\
104854285\end{array}$ & 4 & 337 & 518 & 16269 & $\begin{array}{l}\text { MELO3C015796; } \\
\text { MELO3C017364; } \\
\text { MELO3C014176; } \\
\text { MELO3C008092 }\end{array}$ & $\begin{array}{l}\text { MELO3C011310; } \\
\text { MELO3C006088; } \\
\text { MELO3C013175; } \\
\text { MELO3C012084; } \\
\text { MELO3C014262; } \\
\text { MELO3C024506; } \\
\text { MELO3C020141; } \\
\text { MELO3C022441; } \\
\text { MELO3C026800; } \\
\text { MELO3C001514; } \\
\text { MELO3C016201; } \\
\text { MELO3C026360; } \\
\text { MELO3C025030; } \\
\text { MELO3C018985; } \\
\text { MELO3C017659; } \\
\text { MELO3C013981; } \\
\text { MELO3C009489; } \\
\text { MELO3C002294; } \\
\text { MELO3C006412; } \\
\text { MELO3C025609; } \\
\text { MELO3C006411; } \\
\text { MELO3C024514; } \\
\text { MELO3C023666; } \\
\text { MELO3C005446; } \\
\text { MELO3C006657; } \\
\text { MELO3C024994; } \\
\text { MELO3C025168; }\end{array}$ \\
\hline [] & GO:0071007 & $\begin{array}{l}\text { U2-type catalytic step } \\
\text { 2 spliceosome }\end{array}$ & $\begin{array}{l}\text { CELLULAR_CO } \\
\text { MPONENT }\end{array}$ & 1.0 & $\begin{array}{l}0.030476412 \\
893692188\end{array}$ & 1 & 0 & 521 & 16606 & MELO3C015733 & \\
\hline [] & GO:0000172 & $\begin{array}{l}\text { ribonuclease MRP } \\
\text { complex }\end{array}$ & $\begin{array}{l}\text { CELLULAR_CO } \\
\text { MPONENT }\end{array}$ & 1.0 & $\begin{array}{l}0.030476412 \\
893692188\end{array}$ & 1 & 0 & 521 & 16606 & MELO3C009318 & \\
\hline [] & GO:0009571 & proplastid stroma & \begin{tabular}{|l} 
CELLULAR_CO \\
MPONENT
\end{tabular} & 1.0 & $\begin{array}{l}0.030476412 \\
893692188\end{array}$ & 1 & 0 & 521 & 16606 & MELO3C023469 & \\
\hline [] & GO:0005655 & $\begin{array}{l}\text { nucleolar } \\
\text { ribonuclease P } \\
\text { complex }\end{array}$ & \begin{tabular}{|l} 
CELLULAR_CO \\
MPONENT
\end{tabular} & 1.0 & $\begin{array}{l}0.030476412 \\
893692188\end{array}$ & 1 & 0 & 521 & 16606 & MELO3C009318 & \\
\hline
\end{tabular}




\begin{tabular}{|c|c|c|c|c|c|c|c|c|c|c|c|}
\hline[] & GO:0005654 & nucleoplasm & \begin{tabular}{|l} 
CELLULAR_CO \\
MPONENT
\end{tabular} & 1.0 & \begin{tabular}{|l|}
0.026283398 \\
317527608
\end{tabular} & 14 & 238 & 508 & 16368 & 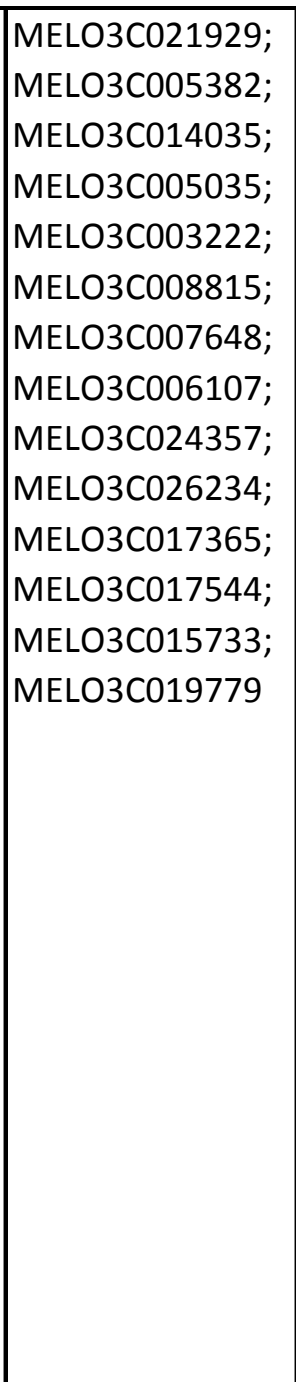 & 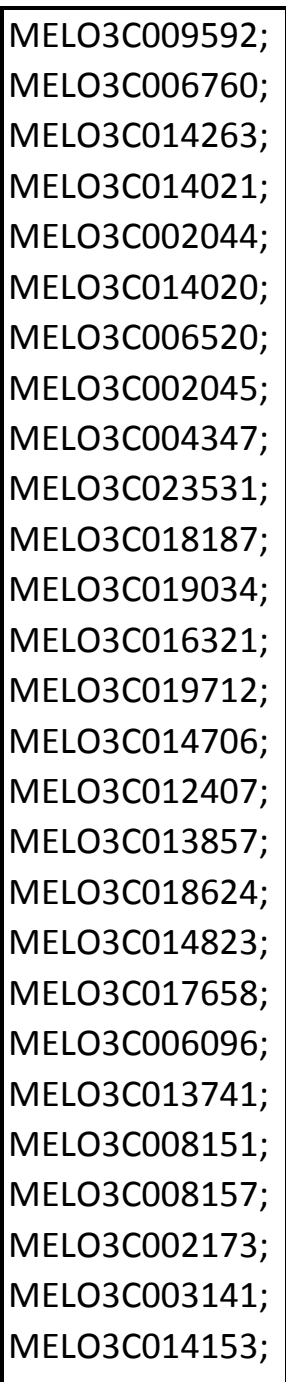 \\
\hline[] & GO:0008623 & CHRAC & \begin{tabular}{|l|} 
CELLULAR_CO \\
MPONENT
\end{tabular} & 1.0 & \begin{tabular}{|l|}
0.030476412 \\
893692188
\end{tabular} & 1 & 0 & 521 & 16606 & MELO3C023986 & \\
\hline[] & GO:0000325 & plant-type vacuole & \begin{tabular}{|l} 
CELLULAR_CO \\
MPONENT
\end{tabular} & 1.0 & \begin{tabular}{|l|}
0.022276131 \\
344187322
\end{tabular} & 5 & 48 & 517 & 16558 & $\begin{array}{l}\text { MELO3C016475; } \\
\text { MELO3C011601; } \\
\text { MELO3C025627; } \\
\text { MELO3C010334; } \\
\text { MELO3C013437 }\end{array}$ & 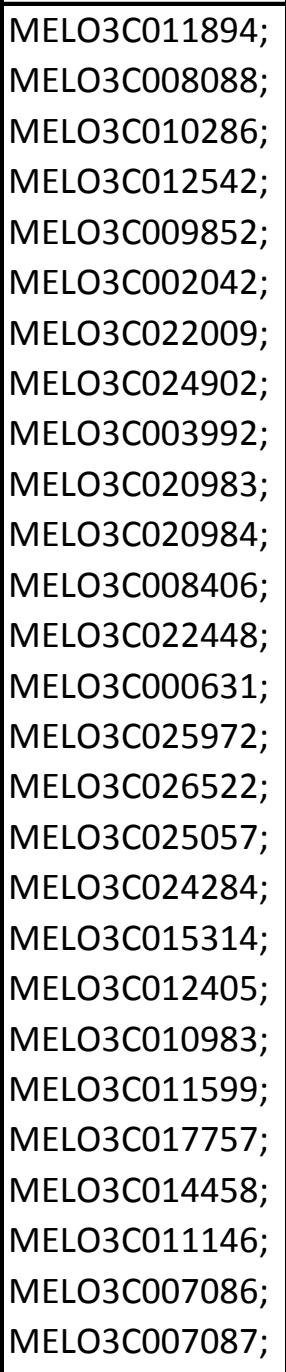 \\
\hline[] & GO:0009508 & plastid chromosome & \begin{tabular}{|l} 
CELLULAR_CO \\
MPONENT
\end{tabular} & 1.0 & \begin{tabular}{|l|}
0.035475308 \\
030445664
\end{tabular} & 2 & 8 & 520 & 16598 & $\begin{array}{l}\text { MELO3C026221; } \\
\text { MELO3C010512 }\end{array}$ & $\begin{array}{l}\text { MELO3C019372; } \\
\text { MELO3C006321; } \\
\text { MELO3C017774; } \\
\text { MELO3C00239; } \\
\text { MELO3C007103; } \\
\text { MELO3C022303; } \\
\text { MELO3C003216; } \\
\text { MELO3C025888 }\end{array}$ \\
\hline
\end{tabular}




\begin{tabular}{|c|c|c|c|c|c|c|c|c|c|c|c|}
\hline [] & GO:0005840 & ribosome & $\begin{array}{l}\text { CELLULAR_CO } \\
\text { MPONENT }\end{array}$ & 1.0 & $\begin{array}{l}0.034208601 \\
081400294\end{array}$ & 6 & 435 & 516 & 16171 & $\begin{array}{l}\text { MELO3C014381; } \\
\text { MELO3C015796; } \\
\text { MELO3C020836; } \\
\text { MELO3C017364; } \\
\text { MELO3C014176; } \\
\text { MELO3C008092 }\end{array}$ & $\begin{array}{l}\text { MELO3C020704; } \\
\text { MELO3C020705; } \\
\text { MELO3C013175; } \\
\text { MELO3C002164; } \\
\text { MELO3C012084; } \\
\text { MELO3C014262; } \\
\text { MELO3C020945; } \\
\text { MELO3C006400; } \\
\text { MELO3C003258; } \\
\text { MELO3C006766; } \\
\text { MELO3C025276; } \\
\text { MELO3C016201; } \\
\text { MELO3C026360; } \\
\text { MELO3C025030; } \\
\text { MELO3C018985; } \\
\text { MELO3C016688; } \\
\text { MELO3C017659; } \\
\text { MELO3C007740; } \\
\text { MELO3C002294; } \\
\text { MELO3C006412; } \\
\text { MELO3C006411; } \\
\text { MELO3C007869; } \\
\text { MELO3C005446; } \\
\text { MELO3C006657; } \\
\text { MELO3C025168; } \\
\text { MELO3C018512; } \\
\text { MELO3C015489; }\end{array}$ \\
\hline [] & GO:0030692 & $\begin{array}{l}\begin{array}{l}\text { Noc4p-Nop14p } \\
\text { complex }\end{array} \\
\end{array}$ & \begin{tabular}{|l} 
CELLULAR_CO \\
MPONENT \\
\end{tabular} & 1.0 & \begin{tabular}{|l|}
0.030476412 \\
893692188
\end{tabular} & 1 & 0 & 521 & 16606 & MELO3C011408 & \\
\hline [] & GO:0035102 & PRC1 complex & \begin{tabular}{|l|} 
CELLULAR_CO \\
MPONENT
\end{tabular} & 1.0 & \begin{tabular}{|l|}
0.030476412 \\
893692188
\end{tabular} & 1 & 0 & 521 & 16606 & MELO3C007648 & \\
\hline [] & GO:0005801 & cis-Golgi network & \begin{tabular}{|l} 
CELLULAR_CO \\
MPONENT
\end{tabular} & 1.0 & \begin{tabular}{|l|}
0.022979181 \\
029695876
\end{tabular} & 2 & 6 & 520 & 16600 & $\begin{array}{l}\text { MELO3C017833; } \\
\text { MELO3C007195 }\end{array}$ & $\begin{array}{l}\text { MELO3C004163; } \\
\text { MELO3C004336; } \\
\text { MELO3C005845; } \\
\text { MELO3C003263; } \\
\text { MELO3C022890; } \\
\text { MELO3C022888 }\end{array}$ \\
\hline [] & GO:0010168 & ER body & \begin{tabular}{|l|} 
CELLULAR_CO \\
MPONENT
\end{tabular} & 1.0 & \begin{tabular}{|l|}
0.030476412 \\
893692188
\end{tabular} & 1 & 0 & 521 & 16606 & MELO3C012716 & \\
\hline
\end{tabular}


Supplementary Table 1.G Gene Ontology terms (GO terms) of the cluster 1.6 obtained considering the comparison between lines over time fruit during storage at $20.5^{\circ} \mathrm{C}$ and $88 \%$ relative humidity for $18 \mathrm{~d}$ the near-isogenic line SC10-2 and its parental "Piel de Sapo" obtained according to the differentially expressed gene pattern (Supl. Table 1.A)

\begin{tabular}{|c|c|c|c|c|c|c|c|c|c|c|c|}
\hline Tags & GO ID & GO Name & GO Category & FDR & P-Value & Nr Test & Nr Reference & \begin{tabular}{|l|} 
Non Annot \\
Test
\end{tabular} & $\begin{array}{l}\text { Non Annot } \\
\text { Reference }\end{array}$ & \begin{tabular}{|l} 
TestSet \\
Sequences
\end{tabular} & \begin{tabular}{|l} 
RefSet \\
Sequences
\end{tabular} \\
\hline [] & GO:0008104 & $\begin{array}{l}\text { protein } \\
\text { localization }\end{array}$ & \begin{tabular}{|l|} 
BIOLOGICAL_ \\
PROCESS
\end{tabular} & 1.0 & \begin{tabular}{|l|}
0.034025958 \\
0381457
\end{tabular} & 6 & 568 & 391 & 16163 & $\begin{array}{l}\text { MELO3C017705; } \\
\text { MELO3C007010; } \\
\text { MELO3C021328; } \\
\text { MELO3C015113; } \\
\text { MELO3C003249; } \\
\text { MELO3C021274 }\end{array}$ & $\begin{array}{l}\text { MELO3C014028; } \\
\text { MELO3C004581; } \\
\text { MELO3C014143; } \\
\text { MELO3C012087; } \\
\text { MELO3C014382; } \\
\text { MELO3C004347; } \\
\text { MELO3C004589; } \\
\text { MELO3C009912; } \\
\text { MELO3C017891; } \\
\text { MELO3C026482; } \\
\text { MELO3C018988; } \\
\text { MELO3C016326; } \\
\text { MELO3C018518; } \\
\text { MELO3C020836; } \\
\text { MELO3C004471; } \\
\text { MELO3C020837; } \\
\text { MELO3C003263; } \\
\text { MELO3C003141; } \\
\text { MELO3C004111; } \\
\text { MELO3C005445; } \\
\text { MELO3C007742; } \\
\text { MELO3C006532; } \\
\text { MELO3C021803; } \\
\text { MELO3C005328; } \\
\text { MELO3C013180; } \\
\text { MELO3C009808; } \\
\text { MELO3C025044; }\end{array}$ \\
\hline[] & GO:0042254 & $\begin{array}{l}\text { ribosome } \\
\text { biogenesis }\end{array}$ & \begin{tabular}{|l|} 
BIOLOGICAL_ \\
PROCESS
\end{tabular} & 1.0 & \begin{tabular}{|l|}
0.011069894 \\
197904951
\end{tabular} & 0 & 222 & 397 & 16509 & & $\begin{array}{l}\text { MELO3C010584; } \\
\text { MELO3C010582; } \\
\text { MELO3C013051; } \\
\text { MELO3C003135; } \\
\text { MELO3C006766; } \\
\text { MELO3C020141; } \\
\text { MELO3C022441; } \\
\text { MELO3C018062; } \\
\text { MELO3C023654; } \\
\text { MELO3C019034; } \\
\text { MELO3C017650; } \\
\text { MELO3C016321; } \\
\text { MELO3C010355; } \\
\text { MELO3C013185; } \\
\text { MELO3C025609; } \\
\text { MELO3C013183; } \\
\text { MELO3C023545; } \\
\text { MELO3C023306; } \\
\text { MELO3C006657; } \\
\text { MELO3C021484; } \\
\text { MELO3C009808; } \\
\text { MELO3C003946; } \\
\text { MELO3C025168; } \\
\text { MELO3C015489; } \\
\text { MELO3C005096; } \\
\text { MELO3C003474; } \\
\text { MELO3C008920; }\end{array}$ \\
\hline [] & GO:0006287 & \begin{tabular}{|l|} 
base-excision \\
repair, gap- \\
filling
\end{tabular} & \begin{tabular}{|l|} 
BIOLOGICAL_ \\
PROCESS
\end{tabular} & 1.0 & $\left|\begin{array}{l}0.023178421 \\
297923826\end{array}\right|$ & 1 & 0 & 396 & 16731 & MELO3C024596 & \\
\hline$[$ [] & GO:0071375 & $\begin{array}{l}\text { cellular } \\
\text { response to } \\
\text { peptide } \\
\text { hormone } \\
\text { stimulus }\end{array}$ & \begin{tabular}{|l|} 
BIOLOGICAL_ \\
PROCESS
\end{tabular} & 1.0 & \begin{tabular}{|l|}
0.039915987 \\
19037741
\end{tabular} & 5 & 76 & 392 & 16655 & $\begin{array}{l}\text { MELO3C017705; } \\
\text { MELO3C012073; } \\
\text { MELO3C007740; } \\
\text { MELO3C026210; } \\
\text { MELO3C009659 }\end{array}$ & $\begin{array}{l}\text { MELO3C014700; } \\
\text { MELO3C013334; } \\
\text { MELO3C012361; } \\
\text { MELO3C002124; } \\
\text { MELO3C002766; } \\
\text { MELO3C025555; } \\
\text { MELO3C018463; } \\
\text { MELO3C020380; } \\
\text { MELO3C025790; } \\
\text { MELO3C013815; } \\
\text { MELO3C010983; } \\
\text { MELO3C010992; } \\
\text { MELO3C012376; } \\
\text { MELO3C011365; } \\
\text { MELO3C010151; } \\
\text { MELO3C009840; } \\
\text { MELO3C004594; } \\
\text { MELO3C010790; } \\
\text { MELO3C011040; } \\
\text { MELO3C023626; } \\
\text { MELO3C006454; } \\
\text { MELO3C009887; } \\
\text { MELO3C021246; } \\
\text { MELO3C004635; } \\
\text { MELO3C026614; } \\
\text { MELO3C004439; } \\
\text { MELO3C024039; }\end{array}$ \\
\hline
\end{tabular}




\begin{tabular}{|c|c|c|c|c|c|c|c|c|c|c|c|}
\hline [] & GO:0034622 & $\begin{array}{l}\text { cellular } \\
\text { macromolec } \\
\text { ular complex } \\
\text { assembly }\end{array}$ & \begin{tabular}{|l|} 
BIOLOGICAL_ \\
PROCESS
\end{tabular} & 1.0 & \begin{tabular}{|l|}
0.024473600 \\
19157677
\end{tabular} & 1 & 280 & 396 & 16451 & MELO3C018406 & $\begin{array}{l}\text { MELO3C010584; } \\
\text { MELO3C012522; } \\
\text { MELO3C020706; } \\
\text { MELO3C014386; } \\
\text { MELO3C005673; } \\
\text { MELO3C026801; } \\
\text { MELO3C004227; } \\
\text { MELO3C021472; } \\
\text { MELO3C016321; } \\
\text { MELO3C012648; } \\
\text { MELO3C014823; } \\
\text { MELO3C019956; } \\
\text { MELO3C008151; } \\
\text { MELO3C008037; } \\
\text { MELO3C014156; } \\
\text { MELO3C004473; } \\
\text { MELO3C010592; } \\
\text { MELO3C013185; } \\
\text { MELO3C013183; } \\
\text { MELO3C024877; } \\
\text { MELO3C022335; } \\
\text { MELO3C005328; } \\
\text { MELO3C003700; } \\
\text { MELO3C023427; } \\
\text { MELO3C006657; } \\
\text { MELO3C024757; } \\
\text { MELO3C015363; }\end{array}$ \\
\hline [] & GO:0006297 & \begin{tabular}{|l|} 
nucleotide- \\
excision \\
repair, DNA \\
gap filling
\end{tabular} & \begin{tabular}{|l|} 
BIOLOGICAL_- \\
PROCESS
\end{tabular} & 1.0 & $\begin{array}{l}0.005528841 \\
9775967506\end{array}$ & 3 & 13 & 394 & 16718 & $\begin{array}{l}\text { MELO3C018521; } \\
\text { MELO3C024596; } \\
\text { MELO3C026949 }\end{array}$ & $\begin{array}{l}\text { MELO3C014021; } \\
\text { MELO3C014020; } \\
\text { MELO3C008864; } \\
\text { MELO3C025879; } \\
\text { MELO3C003638; } \\
\text { MELO3C010507; } \\
\text { MELO3C001937; } \\
\text { MELO3C025142; } \\
\text { MELO3C014529; } \\
\text { MELO3C017228; } \\
\text { MELO3C014528; } \\
\text { MELO3C017458; } \\
\text { MELO3C010631 }\end{array}$ \\
\hline [] & GO:0006972 & $\begin{array}{l}\text { hyperosmoti } \\
\text { c response }\end{array}$ & \begin{tabular}{|l|} 
BIOLOGICAL_- \\
PROCESS
\end{tabular} & 1.0 & $\begin{array}{l}0.035308944 \\
45099476\end{array}$ & 2 & 11 & 395 & 16720 & $\begin{array}{l}\text { MELO3C024239; } \\
\text { MELO3C025349 }\end{array}$ & $\begin{array}{l}\text { MELO3C004428; } \\
\text { MELO3C009971; } \\
\text { MELO3C010211; } \\
\text { MELO3C006142; } \\
\text { MELO3C006721; } \\
\text { MELO3C012085; } \\
\text { MELO3C008666; } \\
\text { MELO3C004127; } \\
\text { MELO3C008815; } \\
\text { MELO3C002752; } \\
\text { MELO3C013249 }\end{array}$ \\
\hline [] & GO:0032790 & $\begin{array}{l}\text { ribosome } \\
\text { disassembly }\end{array}$ & $\begin{array}{l}\text { BIOLOGICAL_} \\
\text { PROCESS }\end{array}$ & 1.0 & $\begin{array}{l}0.023178421 \\
297923826\end{array} \mid$ & 1 & 0 & 396 & 16731 & MELO3C005565 & \\
\hline [] & GO:0042276 & \begin{tabular}{|l|} 
error-prone \\
translesion \\
synthesis \\
\end{tabular} & \begin{tabular}{|l|} 
BIOLOGICAL_ \\
PROCESS
\end{tabular} & 1.0 & \begin{tabular}{|l|}
0.045820925 \\
34215229
\end{tabular} & 1 & 1 & 396 & 16730 & MELO3C007012 & MELO3C005496 \\
\hline [] & GO:0071281 & \begin{tabular}{|l|} 
cellular \\
response to \\
iron ion
\end{tabular} & \begin{tabular}{|l|} 
BIOLOGICAL_- \\
PROCESS
\end{tabular} & 1.0 & $\begin{array}{l}0.013679577 \\
378288468\end{array}$ & 2 & 6 & 395 & 16725 & $\begin{array}{l}\text { MELO3C015210; } \\
\text { MELO3C014099 }\end{array}$ & $\begin{array}{l}\text { MELO3C014230; } \\
\text { MELO3C019550; } \\
\text { MELO3C015531; } \\
\text { MELO3C026952; } \\
\text { MELO3C016340; } \\
\text { MELO3C003906 }\end{array}$ \\
\hline [] & GO:0010891 & $\begin{array}{l}\text { negative } \\
\text { regulation of } \\
\text { sequestering } \\
\text { of } \\
\text { triglyceride }\end{array}$ & \begin{tabular}{|l|} 
BIOLOGICAL_- \\
PROCESS
\end{tabular} & 1.0 & \begin{tabular}{|l|}
0.023178421 \\
297923826
\end{tabular} & 1 & 0 & 396 & 16731 & MELO3C003537 & \\
\hline
\end{tabular}




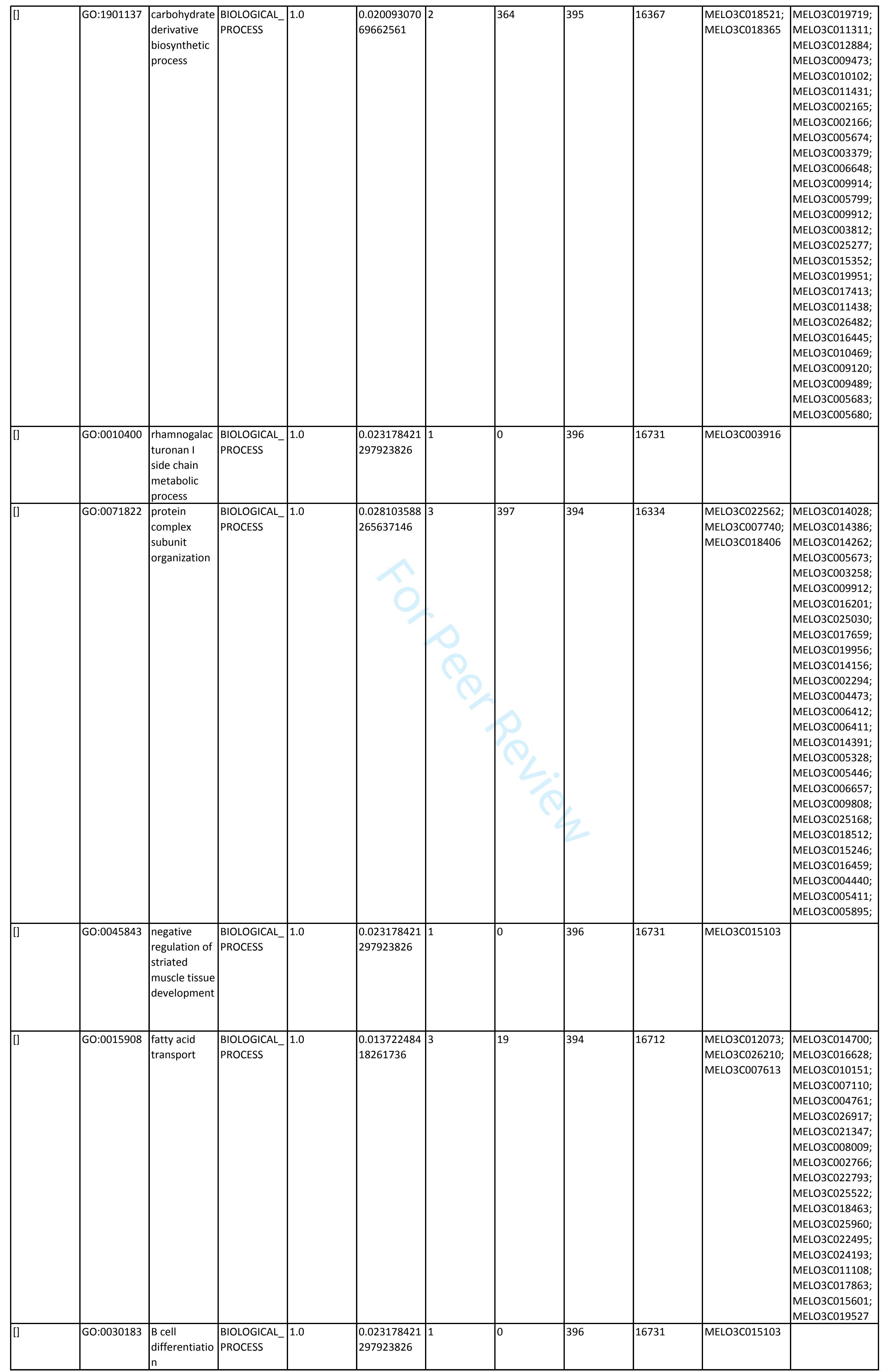




\begin{tabular}{|c|c|c|c|c|c|c|c|c|c|c|c|}
\hline [] & GO:0006772 & $\begin{array}{l}\text { thiamine } \\
\text { metabolic } \\
\text { process }\end{array}$ & $\begin{array}{l}\text { BIOLOGICAL } \\
\text { PROCESS }\end{array}$ & 1.0 & \begin{tabular}{|l|}
0.009087864 \\
176985786
\end{tabular} & 3 & 16 & 394 & 16715 & $\begin{array}{l}\text { MELO3C016014; } \\
\text { MELO3C022609; } \\
\text { MELO3C022605 }\end{array}$ & $\begin{array}{l}\text { MELO3C014401; } \\
\text { MELO3C014400; } \\
\text { MELO3C011230; } \\
\text { MELO3C022607; } \\
\text { MELO3C006346; } \\
\text { MELO3C025408; } \\
\text { MELO3C021005; } \\
\text { MELO3C021375; } \\
\text { MELO3C020562; } \\
\text { MELO3C018097; } \\
\text { MELO3C017499; } \\
\text { MELO3C016136; } \\
\text { MELO3C024240; } \\
\text { MELO3C014967; } \\
\text { MELO3C014965; } \\
\text { MELO3C024092 }\end{array}$ \\
\hline [] & GO:0048577 & \begin{tabular}{|l|} 
negative \\
regulation of \\
short-day \\
photoperiodi \\
sm, flowering
\end{tabular} & $\begin{array}{l}\text { BIOLOGICAL_ } \\
\text { PROCESS }\end{array}$ & 1.0 & \begin{tabular}{|l|}
0.023178421 \\
297923826
\end{tabular} & 1 & 0 & 396 & 16731 & MELO3C019813 & \\
\hline [] & GO:0010106 & \begin{tabular}{|l|} 
cellular \\
response to \\
iron ion \\
starvation
\end{tabular} & \begin{tabular}{|l} 
BIOLOGICAL \\
PROCESS
\end{tabular} & 1.0 & \begin{tabular}{|l|}
0.045820925 \\
34215229
\end{tabular} & 1 & 1 & 396 & 16730 & MELO3C017320 & MELO3C017166 \\
\hline [] & GO:0071329 & \begin{tabular}{|l|} 
cellular \\
response to \\
sucrose \\
stimulus
\end{tabular} & \begin{tabular}{|l|} 
BIOLOGICAL \\
PROCESS
\end{tabular} & 1.0 & \begin{tabular}{|l|}
0.023178421 \\
297923826
\end{tabular} & 1 & 0 & 396 & 16731 & MELO3C022310 & \\
\hline[] & GO:0090306 & \begin{tabular}{|l} 
spindle \\
assembly \\
involved in \\
meiosis
\end{tabular} & \begin{tabular}{|l|} 
BIOLOGICAL \\
PROCESS
\end{tabular} & 1.0 & \begin{tabular}{|l|}
0.045820925 \\
34215229
\end{tabular} & 1 & 1 & 396 & 16730 & MELO3C015113 & MELO3C008594 \\
\hline [] & GO:0048579 & \begin{tabular}{|l|} 
negative \\
regulation of \\
long-day \\
photoperiodi \\
sm, flowering
\end{tabular} & \begin{tabular}{|l} 
BIOLOGICAL \\
PROCESS
\end{tabular} & 1.0 & \begin{tabular}{|l|}
0.023178421 \\
297923826
\end{tabular} & 1 & 0 & 396 & 16731 & MELO3C019813 & \\
\hline [] & GO:0007043 & \begin{tabular}{|l} 
cell-cell \\
junction \\
assembly
\end{tabular} & $\begin{array}{l}\text { BIOLOGICAL } \\
\text { PROCESS }\end{array}$ & 1.0 & \begin{tabular}{|l|}
0.021322474 \\
207042183
\end{tabular} & 2 & 8 & 395 & 16723 & $\begin{array}{l}\text { MELO3C006574; } \\
\text { MELO3C015103 }\end{array}$ & $\begin{array}{l}\text { MELO3C007829; } \\
\text { MELO3C026613; } \\
\text { MELO3C022488; } \\
\text { MELO3C007673; } \\
\text { MELO3C018025; } \\
\text { MELO3C018532; } \\
\text { MELO3C006575; } \\
\text { MELO3C014723 }\end{array}$ \\
\hline [] & GO:0034517 & ribophagy & $\begin{array}{l}\text { BIOLOGICAL } \\
\text { PROCESS }\end{array}$ & 1.0 & \begin{tabular}{|l|}
0.023178421 \\
297923826
\end{tabular} & 1 & 0 & 396 & 16731 & MELO3C011731 & \\
\hline [] & GO:0034757 & \begin{tabular}{|l|} 
negative \\
regulation of \\
iron ion \\
transport \\
\end{tabular} & \begin{tabular}{|l|} 
BIOLOGICAL \\
PROCESS
\end{tabular} & 1.0 & \begin{tabular}{|l|}
0.045820925 \\
34215229
\end{tabular} & 1 & 1 & 396 & 16730 & MELO3C022310 & MELO3C025982 \\
\hline \begin{tabular}{|l}
{[]} \\
\end{tabular} & GO:0010201 & \begin{tabular}{|l} 
response to \\
continuous \\
far red light \\
stimulus by \\
the high- \\
irradiance \\
response \\
system
\end{tabular} & \begin{tabular}{|l|} 
BIOLOGICAL \\
PROCESS
\end{tabular} & 1.0 & \begin{tabular}{|l|}
0.023178421 \\
297923826
\end{tabular} & 1 & 0 & 396 & 16731 & MELO3C009542 & \\
\hline[] & GO:0016926 & \begin{tabular}{|l|} 
protein \\
desumoylatio \\
$\mathrm{n}$
\end{tabular} & \begin{tabular}{|l|} 
BIOLOGICAL_ \\
PROCESS
\end{tabular} & 1.0 & \begin{tabular}{|l|}
0.023178421 \\
297923826
\end{tabular} & 1 & 0 & 396 & 16731 & MELO3C021328 & \\
\hline [] & GO:0045004 & \begin{tabular}{|l|} 
DNA \\
replication \\
proofreading
\end{tabular} & \begin{tabular}{|l|} 
BIOLOGICAL_ \\
PROCESS
\end{tabular} & 1.0 & \begin{tabular}{|l|}
0.023178421 \\
297923826
\end{tabular} & 1 & 0 & 396 & 16731 & MELO3C024596 & \\
\hline \begin{tabular}{|l}
{[]} \\
\end{tabular} & GO:0032049 & \begin{tabular}{|l|} 
cardiolipin \\
biosynthetic \\
process
\end{tabular} & \begin{tabular}{|l|} 
BIOLOGICAL_ \\
PROCESS
\end{tabular} & 1.0 & \begin{tabular}{|l|}
0.045820925 \\
34215229
\end{tabular} & 1 & 1 & 396 & 16730 & MELO3C007010 & MELO3C013700 \\
\hline
\end{tabular}




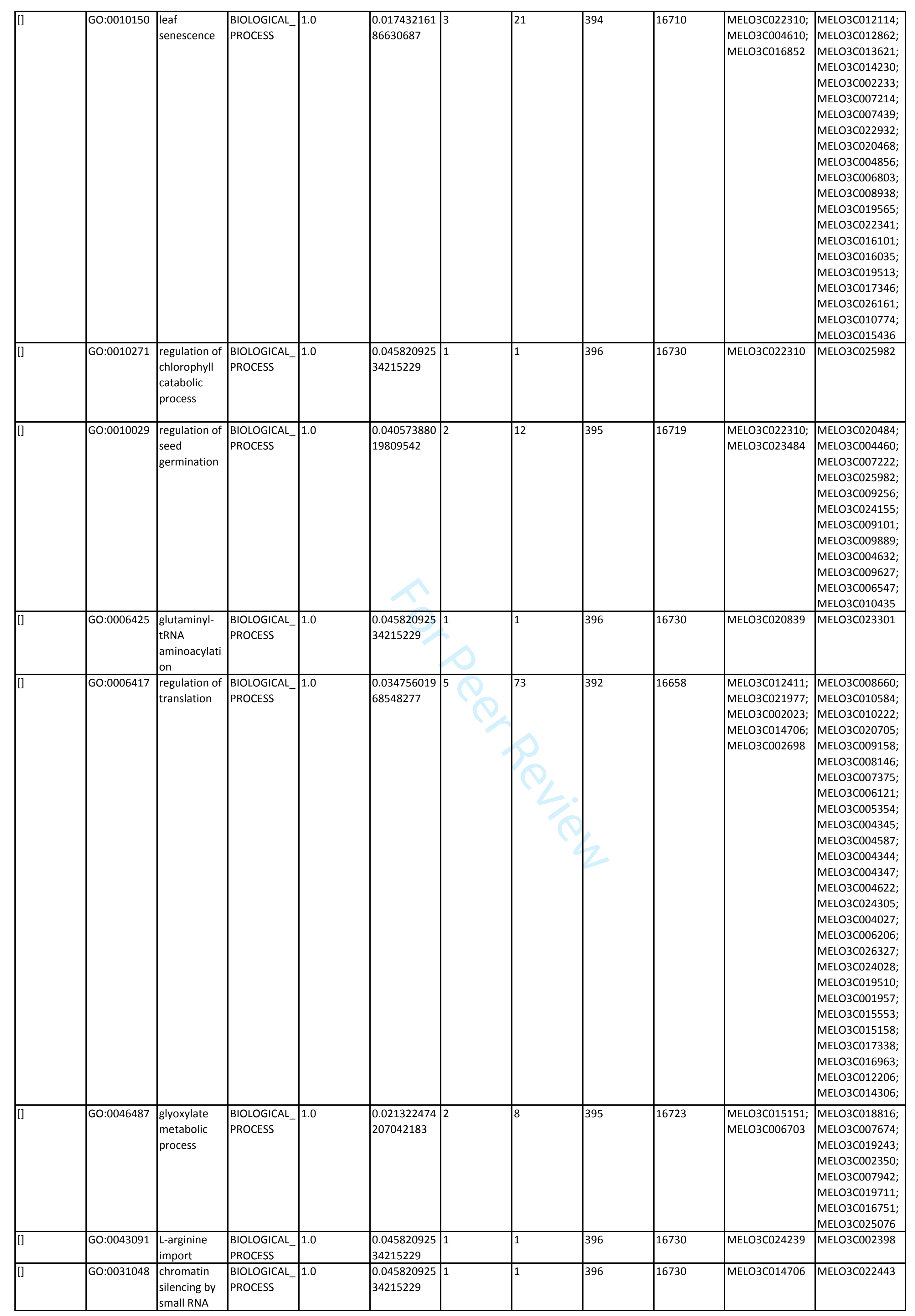




\begin{tabular}{|c|c|c|c|c|c|c|c|c|c|c|c|}
\hline[] & GO:0009074 & $\begin{array}{l}\text { aromatic } \\
\text { amino acid } \\
\text { family } \\
\text { catabolic } \\
\text { process }\end{array}$ & $\begin{array}{l}\text { BIOLOGICAL_ } \\
\text { PROCESS }\end{array}$ & 1.0 & \begin{tabular}{|l|}
0.040365369 \\
900272056
\end{tabular} & 3 & 30 & 394 & 16701 & $\begin{array}{l}\text { MELO3C022961; } \\
\text { MELO3C025613; } \\
\text { MELO3C012201 }\end{array}$ & $\begin{array}{l}\text { MELO3C014227; } \\
\text { MELO3C014226; } \\
\text { MELO3C014225; } \\
\text { MELO3C014224; } \\
\text { MELO3C014223; } \\
\text { MELO3C006189; } \\
\text { MELO3C014222; } \\
\text { MELO3C010461; } \\
\text { MELO3C007433; } \\
\text { MELO3C023775; } \\
\text { MELO3C023373; } \\
\text { MELO3C016089; } \\
\text { MELO3C019811; } \\
\text { MELO3C017998; } \\
\text { MELO3C017811; } \\
\text { MELO3C017810; } \\
\text { MELO3C014229; } \\
\text { MELO3C014228; } \\
\text { MELO3C015944; } \\
\text { MELO3C008136; } \\
\text { MELO3C019809; } \\
\text { MELO3C013583; } \\
\text { MELO3C017809; } \\
\text { MELO3C025786; } \\
\text { MELO3C023224; } \\
\text { MELO3C024595; } \\
\text { MELO3C023220; }\end{array}$ \\
\hline [] & GO:0019428 & \begin{tabular}{|l|} 
allantoin \\
biosynthetic \\
process
\end{tabular} & $\begin{array}{l}\text { BIOLOGICAL } \\
\text { PROCESS }\end{array}$ & 1.0 & \begin{tabular}{|l|}
0.045820925 \\
34215229
\end{tabular} & 1 & 1 & 396 & 16730 & MELO3C012335 & MELO3C006233 \\
\hline [] & GO:0009083 & $\begin{array}{l}\text { branched- } \\
\text { chain amino } \\
\text { acid catabolic } \\
\text { process }\end{array}$ & \begin{tabular}{|l|} 
BIOLOGICAL \\
PROCESS
\end{tabular} & 1.0 & \begin{tabular}{|l|}
0.012054887 \\
373608313
\end{tabular} & 3 & 18 & 394 & 16713 & $\begin{array}{l}\text { MELO3C011705; } \\
\text { MELO3C010776; } \\
\text { MELO3C006679 }\end{array}$ & $\begin{array}{l}\text { MELO3C008063; } \\
\text { MELO3C004297; } \\
\text { MELO3C006792; } \\
\text { MELO3C006434; } \\
\text { MELO3C007664; } \\
\text { MELO3C004545; } \\
\text { MELO3C021247; } \\
\text { MELO3C006734; } \\
\text { MELO3C025757; } \\
\text { MELO3C007705; } \\
\text { MELO3C002813; } \\
\text { MELO3C018583; } \\
\text { MELO3C018456; } \\
\text { MELO3C026065; } \\
\text { MELO3C015564; } \\
\text { MELO3C015796; } \\
\text { MELO3C013913; } \\
\text { MELO3C024190 }\end{array}$ \\
\hline [] & GO:0033169 & \begin{tabular}{|l|} 
histone H3- \\
K9 \\
demethylatio \\
n
\end{tabular} & \begin{tabular}{|l|} 
BIOLOGICAL_ \\
PROCESS
\end{tabular} & 1.0 & \begin{tabular}{|l|}
0.003117472 \\
032681783
\end{tabular} & 2 & 2 & 395 & 16729 & $\begin{array}{l}\text { MELO3C025053; } \\
\text { MELO3C019813 }\end{array}$ & $\begin{array}{l}\text { MELO3C002327; } \\
\text { MELO3C004836 }\end{array}$ \\
\hline [] & GO:0010189 & \begin{tabular}{|l|} 
vitamin E \\
biosynthetic \\
process
\end{tabular} & \begin{tabular}{|l|} 
BIOLOGICAL_ \\
PROCESS
\end{tabular} & 1.0 & \begin{tabular}{|l|}
0.013679577 \\
378288468
\end{tabular} & 2 & 6 & 395 & 16725 & $\begin{array}{l}\text { MELO3C022961; } \\
\text { MELO3C025613 }\end{array}$ & $\begin{array}{l}\text { MELO3C011150; } \\
\text { MELO3C017176; } \\
\text { MELO3C004006; } \\
\text { MELO3C014321; } \\
\text { MELO3C024736; } \\
\text { MELO3C014712 }\end{array}$ \\
\hline [] & GO:0035103 & \begin{tabular}{|l} 
sterol \\
regulatory \\
element \\
binding \\
protein \\
cleavage
\end{tabular} & $\begin{array}{l}\text { BIOLOGICAL_ } \\
\text { PROCESS }\end{array}$ & 1.0 & \begin{tabular}{|l|}
0.023178421 \\
297923826
\end{tabular} & 1 & 0 & 396 & 16731 & MELO3C025349 & \\
\hline [] & GO:0009294 & \begin{tabular}{|l|} 
DNA \\
mediated \\
transformati \\
on
\end{tabular} & \begin{tabular}{|l|} 
BIOLOGICAL_ \\
PROCESS
\end{tabular} & 1.0 & \begin{tabular}{|l|}
0.013679577 \\
378288468
\end{tabular} & 2 & 6 & 395 & 16725 & $\begin{array}{l}\text { MELO3C013472; } \\
\text { MELO3C015103 }\end{array}$ & $\begin{array}{l}\text { MELO3C023256; } \\
\text { MELO3C020854; } \\
\text { MELO3C019754; } \\
\text { MELO3C026234; } \\
\text { MELO3C004806; } \\
\text { MELO3C017558 }\end{array}$ \\
\hline[] & GO:0001570 & $\begin{array}{l}\text { vasculogenes } \\
\text { is }\end{array}$ & \begin{tabular}{|l|} 
BIOLOGICAL_- \\
PROCESS
\end{tabular} & 1.0 & \begin{tabular}{|l|}
0.023178421 \\
297923826
\end{tabular} & 1 & 0 & 396 & 16731 & MELO3C015103 & \\
\hline
\end{tabular}




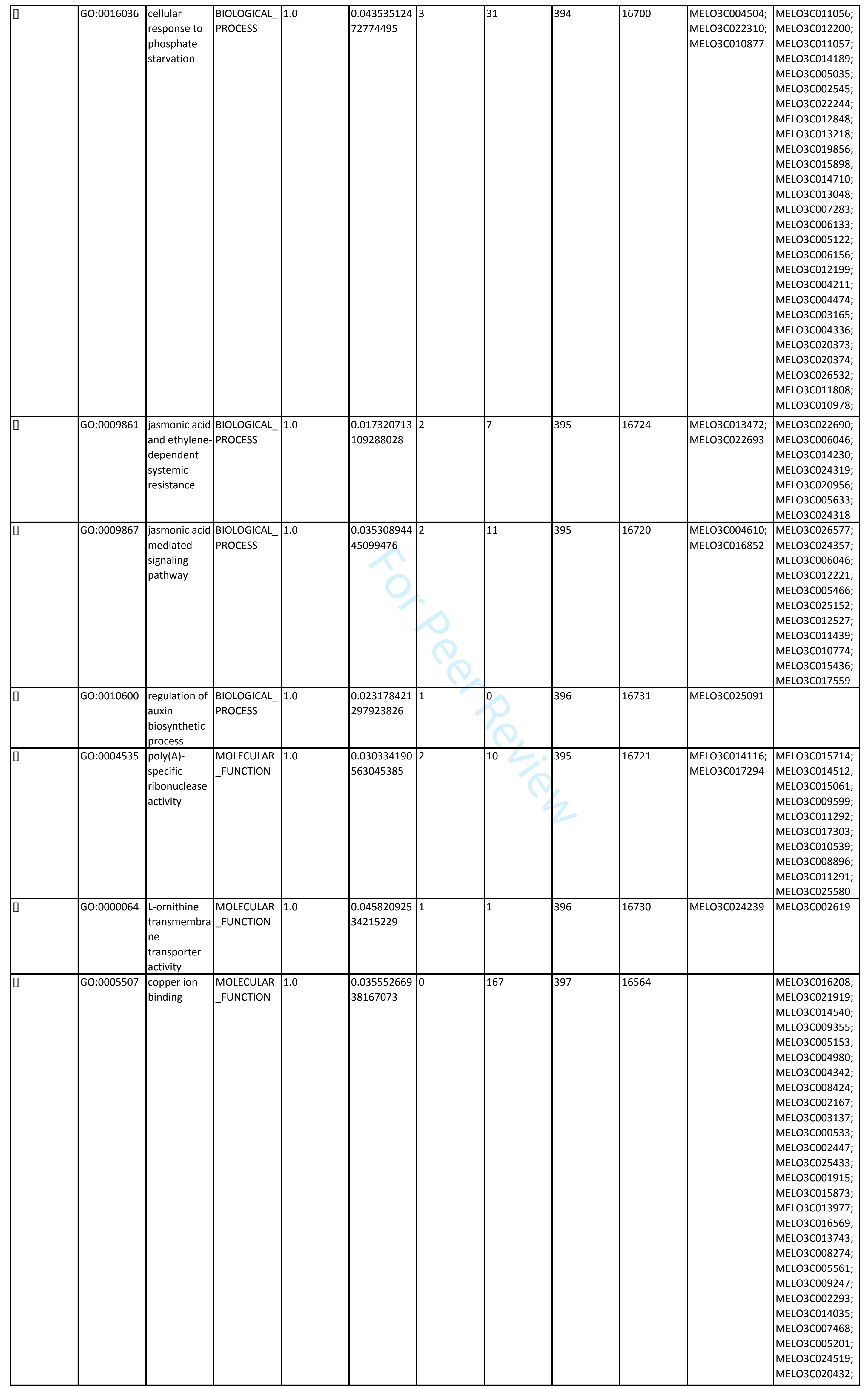




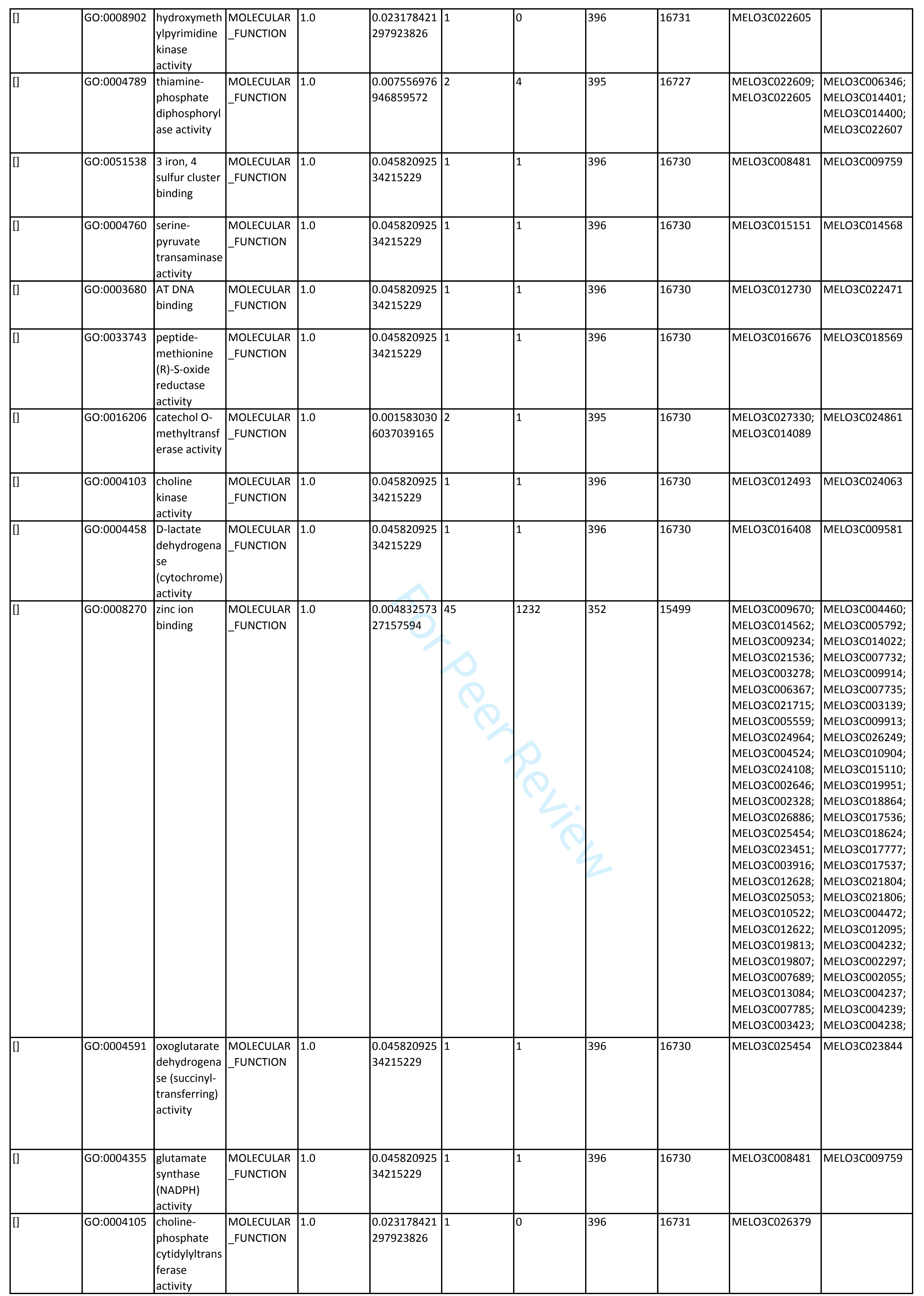




\begin{tabular}{|c|c|c|c|c|c|c|c|c|c|c|c|}
\hline[] & |GO:0016778 & \begin{tabular}{|l} 
diphosphotra \\
nsferase \\
activity
\end{tabular} & \begin{tabular}{|l|} 
MOLECULAR \\
-FUNCTION
\end{tabular} & 1.0 & \begin{tabular}{|l|}
0.040573880 \\
19809542
\end{tabular} & & 12 & 395 & 16719 & $\begin{array}{l}\text { MELO3C024869; } \\
\text { MELO3C016014 }\end{array}$ & 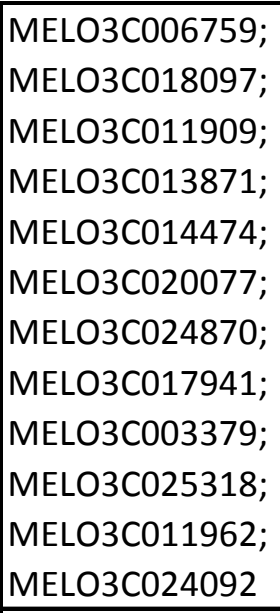 \\
\hline[] & \begin{tabular}{|l|} 
GO:0046969 \\
\end{tabular} & \begin{tabular}{|l} 
NAD- \\
dependent \\
histone \\
deacetylase \\
activity (H3- \\
K9 specific)
\end{tabular} & \begin{tabular}{|l|} 
MOLECULAR \\
FUNCTION
\end{tabular} & 1.0 & \begin{tabular}{|l|}
0.010418338 \\
915837976
\end{tabular} & 2 & 5 & 395 & 16726 & $\begin{array}{l}\text { MELO3C013472; } \\
\text { MELO3C015103 }\end{array}$ & $\begin{array}{l}\text { MELO3C021442; } \\
\text { MELO3C008064; } \\
\text { MELO3C026267; } \\
\text { MELO3C02557; } \\
\text { MELO3C016865 }\end{array}$ \\
\hline[] & GO:0047936 & \begin{tabular}{|l|} 
glucose 1- \\
dehydrogena \\
se [NAD(P)] \\
activity
\end{tabular} & $\begin{array}{l}\text { MOLECULAR } \\
\text { FUNCTION }\end{array}$ & 1.0 & \begin{tabular}{|l|}
0.023178421 \\
297923826
\end{tabular} & 1 & 0 & 396 & 16731 & MELO3C025347 & \\
\hline[] & \begin{tabular}{|l|}
$G O: 0051753$ \\
\end{tabular} & $\begin{array}{l}\text { mannan } \\
\text { synthase } \\
\text { activity }\end{array}$ & \begin{tabular}{|l|} 
MOLECULAR \\
FUNCTION
\end{tabular} & 1.0 & \begin{tabular}{|l|}
0.023178421 \\
297923826
\end{tabular} & 1 & 0 & 396 & 16731 & MELO3C021458 & \\
\hline[] & GO:0051990 & \begin{tabular}{|l} 
(R)-2- \\
hydroxygluta \\
rate \\
dehydrogena \\
se activity
\end{tabular} & \begin{tabular}{|l|} 
MOLECULAR \\
FUNCTION
\end{tabular} & 1.0 & \begin{tabular}{|l|}
0.023178421 \\
297923826
\end{tabular} & 1 & 0 & 396 & 16731 & MELO3C016408 & \\
\hline[] & \begin{tabular}{|l|}
$G O: 0046970$ \\
\end{tabular} & $\begin{array}{l}\text { NAD- } \\
\text { dependent } \\
\text { histone } \\
\text { deacetylase } \\
\text { activity (H4- } \\
\text { K16 specific) }\end{array}$ & \begin{tabular}{|l|} 
MOLECULAR \\
FUNCTION
\end{tabular} & 1.0 & \begin{tabular}{|l|}
0.010418338 \\
915837976
\end{tabular} & 2 & 5 & 395 & 16726 & $\begin{array}{l}\text { MELO3C013472; } \\
\text { MELO3C015103 }\end{array}$ & $\begin{array}{l}\text { MELO3C021442; } \\
\text { MELO3CO0806; } \\
\text { MELO3C026267; } \\
\text { MELO3C025575; } \\
\text { MELO3C016865 }\end{array}$ \\
\hline []] & GO:0016929 & \begin{tabular}{|l|} 
SUMO- \\
specific \\
protease \\
activity
\end{tabular} & \begin{tabular}{|l|} 
MOLECULAR \\
-FUNCTION
\end{tabular} & 1.0 & \begin{tabular}{|l|}
0.023178421 \\
297923826
\end{tabular} & 1 & 0 & 396 & 16731 & MELO3C021328 & \\
\hline[] & GO:0050281 & \begin{tabular}{|l|} 
serine- \\
glyoxylate \\
transaminase \\
activity
\end{tabular} & \begin{tabular}{|l|} 
MOLECULAR \\
FUNCTION
\end{tabular} & 1.0 & \begin{tabular}{|l|}
0.045820925 \\
34215229
\end{tabular} & 1 & 1 & 396 & 16730 & MELO3C015151 & MELO3C014568 \\
\hline[] & GO:0004147 & $\begin{array}{l}\text { dihydrolipoa } \\
\text { mide } \\
\text { branched } \\
\text { chain } \\
\text { acyltransfera } \\
\text { se activity }\end{array}$ & \begin{tabular}{|l|} 
MOLECULAR \\
FUNCTION
\end{tabular} & 1.0 & \begin{tabular}{|l|}
0.023178421 \\
297923826
\end{tabular} & 1 & 0 & 396 & 16731 & MELO3C006679 & \\
\hline$\left.\right|^{0}$ & GO:0032041 & \begin{tabular}{|l|} 
NAD- \\
dependent \\
histone \\
deacetylase \\
activity (H3- \\
K14 specific)
\end{tabular} & \begin{tabular}{|l|} 
MOLECULAR \\
FUNCTION
\end{tabular} & 1.0 & \begin{tabular}{|l|}
0.010418338 \\
915837976
\end{tabular} & 2 & 5 & 395 & 16726 & $\begin{array}{l}\text { MELO3C013472; } \\
\text { MELO3C015103 }\end{array}$ & $\begin{array}{l}\text { MELO3C021442; } \\
\text { MELO3C008064; } \\
\text { MELO3C026267; } \\
\text { MELO3C025575; } \\
\text { MELO3C016865 }\end{array}$ \\
\hline [] & GO:0003735 & \begin{tabular}{|l} 
structural \\
constituent \\
of ribosome
\end{tabular} & \begin{tabular}{|l|} 
MOLECULAR \\
FUNCTION
\end{tabular} & 1.0 & \begin{tabular}{|l|}
0.019217811 \\
351406117
\end{tabular} & 2 & 352 & 395 & 16379 & $\begin{array}{l}\text { MELO3C007740; } \\
\text { MELO3C022017 }\end{array}$ & 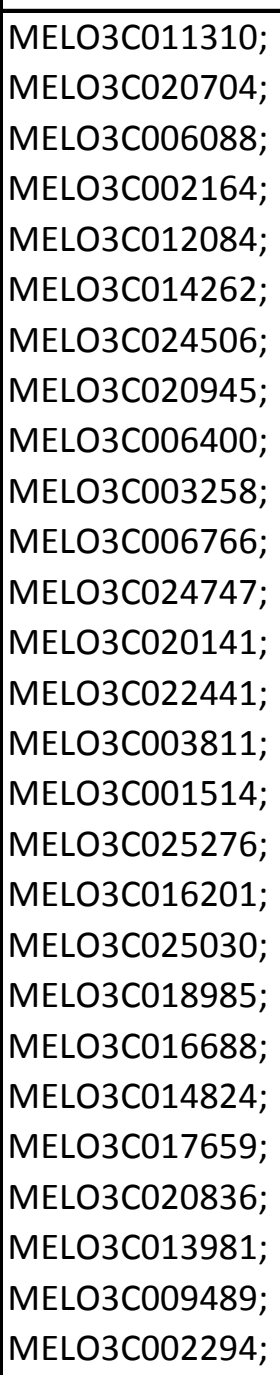 \\
\hline |[] & GO:0004370 & \begin{tabular}{|l|} 
glycerol \\
kinase \\
activity
\end{tabular} & \begin{tabular}{|l|} 
MOLECULAR \\
FUNCTION
\end{tabular} & 1.0 & \begin{tabular}{|l|}
0.045820925 \\
34215229 \\
\end{tabular} & 1 & 1 & 396 & 16730 & MELO3C023442 & MELO3C004084 \\
\hline
\end{tabular}




\begin{tabular}{|c|c|c|c|c|c|c|c|c|c|c|c|}
\hline[] & GO:0008972 & $\begin{array}{l}\text { phosphomet } \\
\text { hylpyrimidine } \\
\text { kinase } \\
\text { activity } \\
\end{array}$ & $\begin{array}{l}\text { MOLECULAR } \\
\text { FUNCTION }\end{array}$ & 1.0 & $\begin{array}{l}0.001583030 \\
6037039165\end{array}$ & 2 & 1 & 395 & 16730 & $\begin{array}{l}\text { MELO3C022609; } \\
\text { MELO3C022605 }\end{array}$ & MELO3C022607 \\
\hline [] & GO:0008780 & $\begin{array}{l}\text { acyl-[acyl- } \\
\text { carrier- } \\
\text { protein]-UDP- } \\
\text { N- } \\
\text { acetylglucosa } \\
\text { mine O- } \\
\text { acyltransfera } \\
\text { se activity } \\
\end{array}$ & $\begin{array}{l}\text { MOLECULAR } \\
\text { FUNCTION }\end{array}$ & 1.0 & $\begin{array}{l}0.045820925 \\
34215229\end{array}$ & 1 & 1 & 396 & 16730 & MELO3C015455 & MELO3C007369 \\
\hline [] & GO:0003887 & $\begin{array}{l}\text { DNA-directed } \\
\text { DNA } \\
\text { polymerase } \\
\text { activity }\end{array}$ & $\begin{array}{l}\text { MOLECULAR } \\
\text { FUNCTION }\end{array}$ & 1.0 & $\begin{array}{l}0.009324061 \\
419061221\end{array}$ & 4 & 32 & 393 & 16699 & $\begin{array}{l}\text { MELO3C018521; } \\
\text { MELO3C016434; } \\
\text { MELO3C024596; } \\
\text { MELO3C007012 }\end{array}$ & $\begin{array}{l}\text { MELO3C011257; } \\
\text { MELO3C016705; } \\
\text { MELO3C007194; } \\
\text { MELO3C005496; } \\
\text { MELO3C014021; } \\
\text { MELO3C002044; } \\
\text { MELO3C014020; } \\
\text { MELO3C009536; } \\
\text { MELO3C021434; } \\
\text { MELO3C021435; } \\
\text { MELO3C005956; } \\
\text { MELO3C020860; } \\
\text { MELO3C005957; } \\
\text { MELO3C001935; } \\
\text { MELO3C010507; } \\
\text { MELO3C001936; } \\
\text { MELO3C025396; } \\
\text { MELO3C001937; } \\
\text { MELO3C025395; } \\
\text { MELO3C014529; } \\
\text { MELO3C011159; } \\
\text { MELO3C014503; } \\
\text { MELO3C002892; } \\
\text { MELO3C022319; } \\
\text { MELO3C004237; } \\
\text { MELO3C004239; } \\
\text { MELO3C004238; }\end{array}$ \\
\hline [] & GO:0008429 & $\begin{array}{l}\text { phosphatidyl } \\
\text { ethanolamin } \\
\text { e binding }\end{array}$ & $\begin{array}{l}\text { MOLECULAR } \\
\text {-FUNCTION }\end{array}$ & 1.0 & $\begin{array}{l}0.045820925 \\
34215229\end{array}$ & 1 & 1 & 396 & 16730 & MELO3C009465 & MELO3C009141 \\
\hline[] & GO:0051219 & $\begin{array}{l}\text { phosphoprot } \\
\text { ein binding }\end{array}$ & $\begin{array}{l}\text { MOLECULAR } \\
\text {-FUNCTION }\end{array}$ & 1.0 & $\begin{array}{l}0.017320713 \\
109288028\end{array}$ & 2 & 7 & 395 & 16724 & $\begin{array}{l}\text { MELO3C009316; } \\
\text { MELO3C005002 }\end{array}$ & $\begin{array}{l}\text { MELO3C008473; } \\
\text { MELO3C025740; } \\
\text { MELO3C01578; } \\
\text { MELO3C009317; } \\
\text { MELO3C016377; } \\
\text { MELO3C016378; } \\
\text { MELO3C014260 }\end{array}$ \\
\hline [] & GO:0034256 & $\begin{array}{l}\text { chlorophyll(i } \\
\text { de) b } \\
\text { reductase } \\
\text { activity } \\
\end{array}$ & $\begin{array}{l}\text { MOLECULAR } \\
\text { FUNCTION }\end{array}$ & 1.0 & \begin{tabular}{|l}
0.045820925 \\
34215229
\end{tabular} & 1 & 1 & 396 & 16730 & MELO3C025347 & MELO3C019918 \\
\hline[] & GO:0046556 & \begin{tabular}{|l} 
alpha-L- \\
arabinofuran \\
osidase \\
activity \\
\end{tabular} & $\begin{array}{l}\text { MOLECULAR } \\
\text {-FUNCTION }\end{array}$ & 1.0 & $\begin{array}{l}0.045820925 \\
34215229\end{array}$ & 1 & 1 & 396 & 16730 & MELO3C021281 & MELO3C009722 \\
\hline[] & GO:0004044 & \begin{tabular}{|l} 
amidophosph \\
oribosyltrans \\
ferase \\
activity \\
\end{tabular} & $\begin{array}{l}\text { MOLECULAR } \\
\text { FUNCTION }\end{array}$ & 1.0 & $\begin{array}{l}0.045820925 \\
34215229\end{array}$ & 1 & 1 & 396 & 16730 & MELO3C020749 & MELO3C025646 \\
\hline[] & GO:0003868 & \begin{tabular}{|l}
$4-$ \\
hydroxyphen \\
ylpyruvate \\
dioxygenase \\
activity \\
\end{tabular} & $\begin{array}{l}\text { MOLECULAR } \\
\text {-FUNCTION }\end{array}$ & 1.0 & $\begin{array}{l}0.045820925 \\
34215229\end{array}$ & 1 & 1 & 396 & 16730 & MELO3C022961 & MELO3C013690 \\
\hline [] & GO:0004838 & $\begin{array}{l}\text { L-tyrosine:2- } \\
\text { oxoglutarate } \\
\text { aminotransfe } \\
\text { rase activity }\end{array}$ & $\begin{array}{l}\text { MOLECULAR } \\
\text { FUNCTION }\end{array}$ & 1.0 & $\begin{array}{l}0.045820925 \\
34215229\end{array}$ & 1 & 1 & 396 & 16730 & MELO3C025613 & MELO3C013583 \\
\hline[] & GO:0010293 & \begin{tabular}{|l} 
abscisic \\
aldehyde \\
oxidase \\
activity \\
\end{tabular} & $\begin{array}{l}\text { MOLECULAR } \\
\text {-FUNCTION }\end{array}$ & 1.0 & $\begin{array}{l}0.045820925 \\
34215229\end{array}$ & 1 & 1 & 396 & 16730 & MELO3C014719 & MELO3C014717 \\
\hline[] & GO:0004846 & $\begin{array}{l}\text { urate oxidase } \\
\text { activity }\end{array}$ & $\begin{array}{l}\text { MOLECULAR } \\
\text {-FUNCTION }\end{array}$ & 1.0 & $\begin{array}{l}0.023178421 \\
297923826\end{array}$ & 1 & 0 & 396 & 16731 & MELO3C012335 & \\
\hline
\end{tabular}




\begin{tabular}{|c|c|c|c|c|c|c|c|c|c|c|}
\hline [] & GO:0019899 & $\begin{array}{l}\text { enzyme } \\
\text { binding }\end{array}$ & \begin{tabular}{|l|} 
MOLECULAR \\
_FUNCTION
\end{tabular} & 1.0 & \begin{tabular}{|l|l}
0.041298933 \\
08657816
\end{tabular} \mid 6 & 103 & 391 & 16628 & $\begin{array}{l}\text { MELO3C022310; } \\
\text { MELO3C015113; } \\
\text { MELO3C014488; } \\
\text { MELO3C021345; } \\
\text { MELO3C010964; } \\
\text { MELO3C011138 }\end{array}$ & 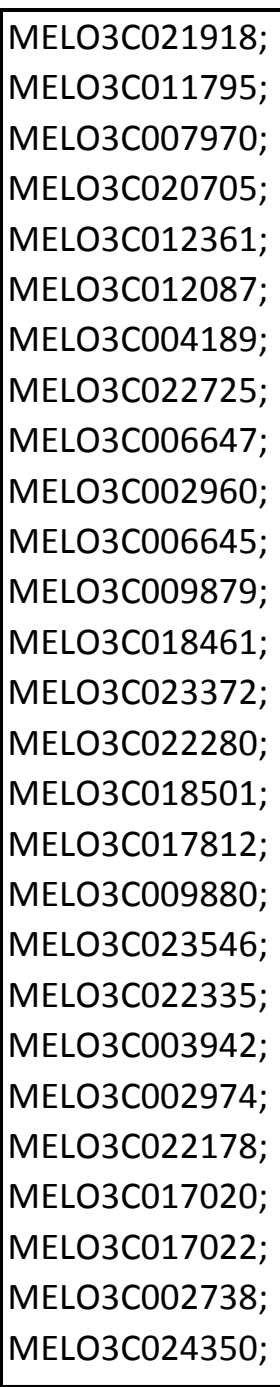 \\
\hline [] & GO:0034990 & $\begin{array}{l}\text { nuclear } \\
\text { mitotic } \\
\text { cohesin } \\
\text { complex } \\
\end{array}$ & $\begin{array}{l}\text { CELLULAR_C } \\
\text { OMPONENT }\end{array}$ & 1.0 & $\begin{array}{l}0.023178421 \\
297923826\end{array} \mid 1$ & 0 & 396 & 16731 & MELO3C012730 & \\
\hline [] & GO:0044445 & cytosolic part & \begin{tabular}{|l} 
CELLULAR_C \\
OMPONENT
\end{tabular} & 1.0 & $\left.$\begin{tabular}{|l|l}
0.027077262 \\
658931633
\end{tabular}\right|$^{2}$ & 339 & 395 & 16392 & $\begin{array}{l}\text { MELO3C013175; } \\
\text { MELO3C017023 }\end{array}$ & 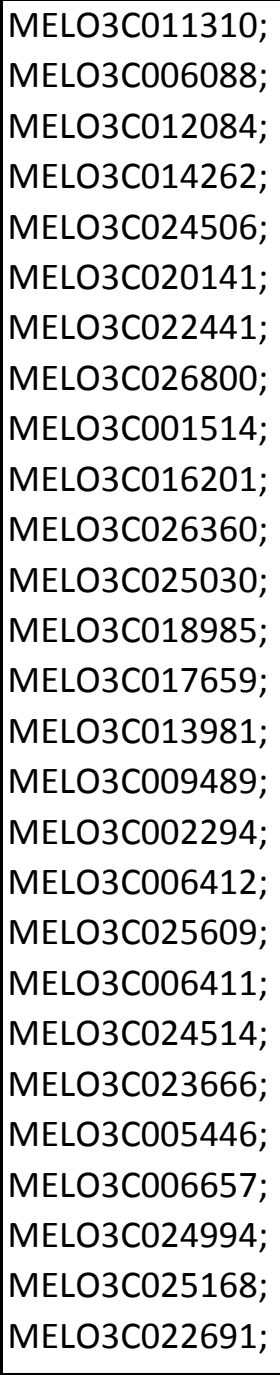 \\
\hline [] & GO:0005882 & \begin{tabular}{|l|} 
intermediate \\
filament
\end{tabular} & \begin{tabular}{|l|} 
CELLULAR_C \\
OMPONENT \\
\end{tabular} & 1.0 & \begin{tabular}{|l|l}
0.023178421 & 1 \\
297923826
\end{tabular} & 0 & 396 & 16731 & MELO3C019500 & \\
\hline [] & GO:0030057 & desmosome & \begin{tabular}{|l|} 
CELLULAR_C \\
OMPONENT \\
\end{tabular} & 1.0 & \begin{tabular}{|l|l}
0.023178421 & 1 \\
297923826 & \\
\end{tabular} & 0 & 396 & 16731 & MELO3C019500 & \\
\hline [] & GO:0031934 & \begin{tabular}{|l|} 
mating-type \\
region \\
heterochrom \\
atin
\end{tabular} & $\begin{array}{l}\text { CELLULAR_C } \\
\text { OMPONENT }\end{array}$ & 1.0 & \begin{tabular}{|l|}
0.023178421 \\
297923826
\end{tabular} & 0 & 396 & 16731 & MELO3C022562 & \\
\hline
\end{tabular}




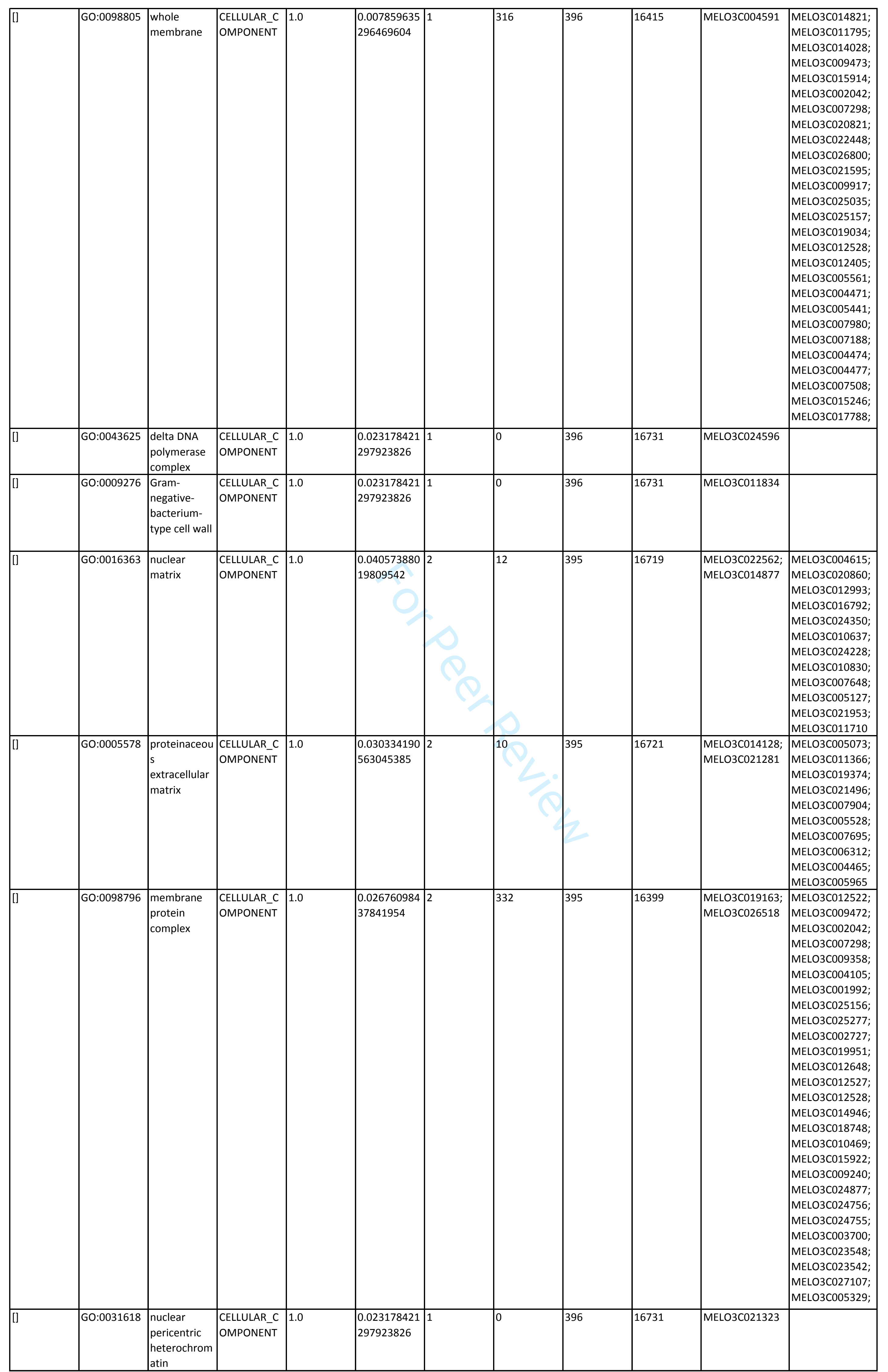




\begin{tabular}{|c|c|c|c|c|c|c|c|c|c|c|}
\hline [] & GO:0080008 & \begin{tabular}{|l} 
Cul4-RING E3 \\
ubiquitin \\
ligase \\
complex
\end{tabular} & \begin{tabular}{|l} 
CELLULAR_C \\
OMPONENT
\end{tabular} & 1.0 & \begin{tabular}{|l|}
0.042933993 \\
40984404
\end{tabular} & 53 & 393 & 16678 & $\begin{array}{l}\text { MELO3C003537; } \\
\text { MELO3C017705; } \\
\text { MELO3CO09659; } \\
\text { MELO3C007899 }\end{array}$ & 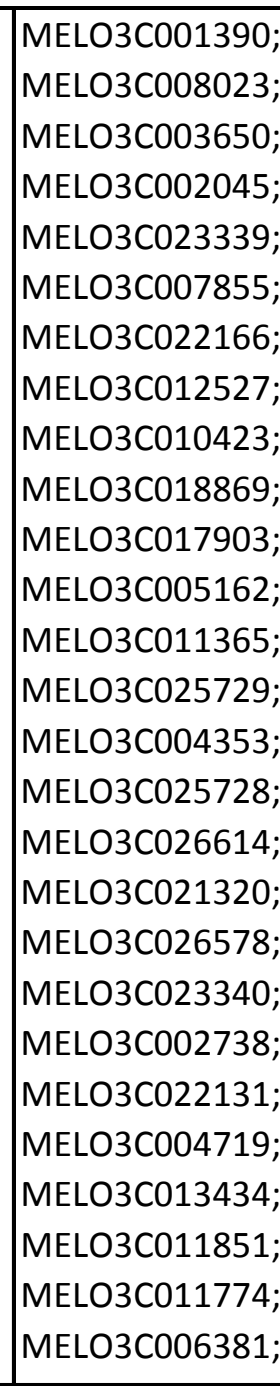 \\
\hline[] & GO:0015934 & \begin{tabular}{|l|} 
large \\
ribosomal \\
subunit
\end{tabular} & $\begin{array}{l}\text { CELLULAR_C } \\
\text { OMPONENT }\end{array}$ & 1.0 & \begin{tabular}{|l|}
0.036391198 \\
16595434
\end{tabular} & 172 & 397 & 16559 & & 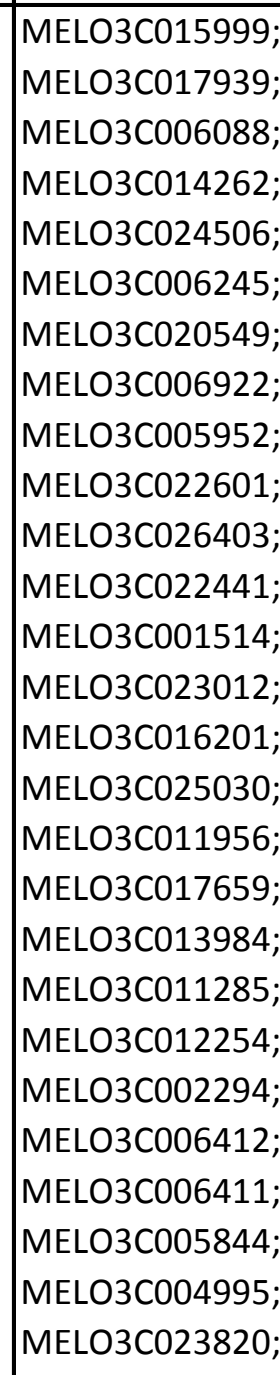 \\
\hline [] & GO:0005947 & \begin{tabular}{|l|} 
mitochondria \\
I alpha- \\
ketoglutarate \\
dehydrogena \\
se complex
\end{tabular} & \begin{tabular}{|l} 
CELLULAR_C \\
OMPONENT
\end{tabular} & 1.0 & \begin{tabular}{|l|}
0.045820925 \\
34215229
\end{tabular} & 1 & 396 & 16730 & MELO3C006679 & MELO3C015564 \\
\hline [] & \begin{tabular}{|l|l}
$G 0: 0005956$ \\
\end{tabular} & \begin{tabular}{|l|l} 
protein \\
kinase CK2 \\
complex
\end{tabular} & \begin{tabular}{|l|} 
CELLULAR_C \\
OMPONENT
\end{tabular} & 1.0 & \begin{tabular}{|l|}
0.045820925 \\
34215229
\end{tabular} & 1 & 396 & 16730 & MELO3C003341 & MELO3C020365 \\
\hline
\end{tabular}




\begin{tabular}{|c|c|c|c|c|c|c|c|c|c|c|c|}
\hline[] & GO:0031410 & \begin{tabular}{|l|} 
cytoplasmic \\
vesicle
\end{tabular} & $\begin{array}{l}\text { CELLULAR_C } \\
\text { OMPONENT }\end{array}$ & 1.0 & \begin{tabular}{|l|}
0.033590926 \\
224202275
\end{tabular} & 27 & 1672 & 370 & 15059 & $\begin{array}{l}\text { MELO3C014027; } \\
\text { MELO3C012201; } \\
\text { MELO3C016608; } \\
\text { MELO3C002381; } \\
\text { MELO3C005252; } \\
\text { MELO3C005511; } \\
\text { MELO3C022208; } \\
\text { MELO3C007613; } \\
\text { MELO3C026689; } \\
\text { MELO3C026723; } \\
\text { MELO3C003659; } \\
\text { MELO3C025255; } \\
\text { MELO3C014925; } \\
\text { MELO3C011731; } \\
\text { MELO3C014215; } \\
\text { MELO3C013125; } \\
\text { MELO3C016939; } \\
\text { MELO3C014353; } \\
\text { MELO3C006594; } \\
\text { MELO3C013261; } \\
\text { MELO3C013260; } \\
\text { MELO3C005245; } \\
\text { MELO3C004732; } \\
\text { MELO3C023644; } \\
\text { MELO3C007206; } \\
\text { MELO3C026594; } \\
\text { MELO3C011227 }\end{array}$ & $\begin{array}{l}\text { MELO3C015119; } \\
\text { MELO3C002280; } \\
\text { MELO3C012088; } \\
\text { MELO3C002286; } \\
\text { MELO3C012085; } \\
\text { MELO3C026249; } \\
\text { MELO3C026243; } \\
\text { MELO3C017775; } \\
\text { MELO3C015599; } \\
\text { MELO3C025391; } \\
\text { MELO3C003142; } \\
\text { MELO3C003144; } \\
\text { MELO3C003146; } \\
\text { MELO3C004477; } \\
\text { MELO3C014270; } \\
\text { MELO3C005328; } \\
\text { MELO3C020951; } \\
\text { MELO3C008838; } \\
\text { MELO3C017300; } \\
\text { MELO3C019962; } \\
\text { MELO3C019939; } \\
\text { MELO3C020928; } \\
\text { MELO3C014249; } \\
\text { MELO3C012068; } \\
\text { MELO3C014246; } \\
\text { MELO3C003595; } \\
\text { MELO3C012061; }\end{array}$ \\
\hline[] & GO:0000800 & $\begin{array}{l}\text { lateral } \\
\text { element }\end{array}$ & $\begin{array}{l}\text { CELLULAR_C } \\
\text { OMPONENT }\end{array}$ & 1.0 & \begin{tabular}{|l|}
0.023178421 \\
297923826
\end{tabular} & 1 & 0 & 396 & 16731 & MELO3C014687 & \\
\hline
\end{tabular}


Supplementary Table 1.H Gene Ontology terms (GO terms) of the cluster 1.7 obtained considering the comparison between lines over time fruit during storage at $20.5^{\circ} \mathrm{C}$ and $88 \%$ relative humidity for $18 \mathrm{~d}$ the near-isogenic line SC10-2 and its parental "Piel de Sapo" obtained according to the differentially expressed gene pattern (Supl. Table 1.A)

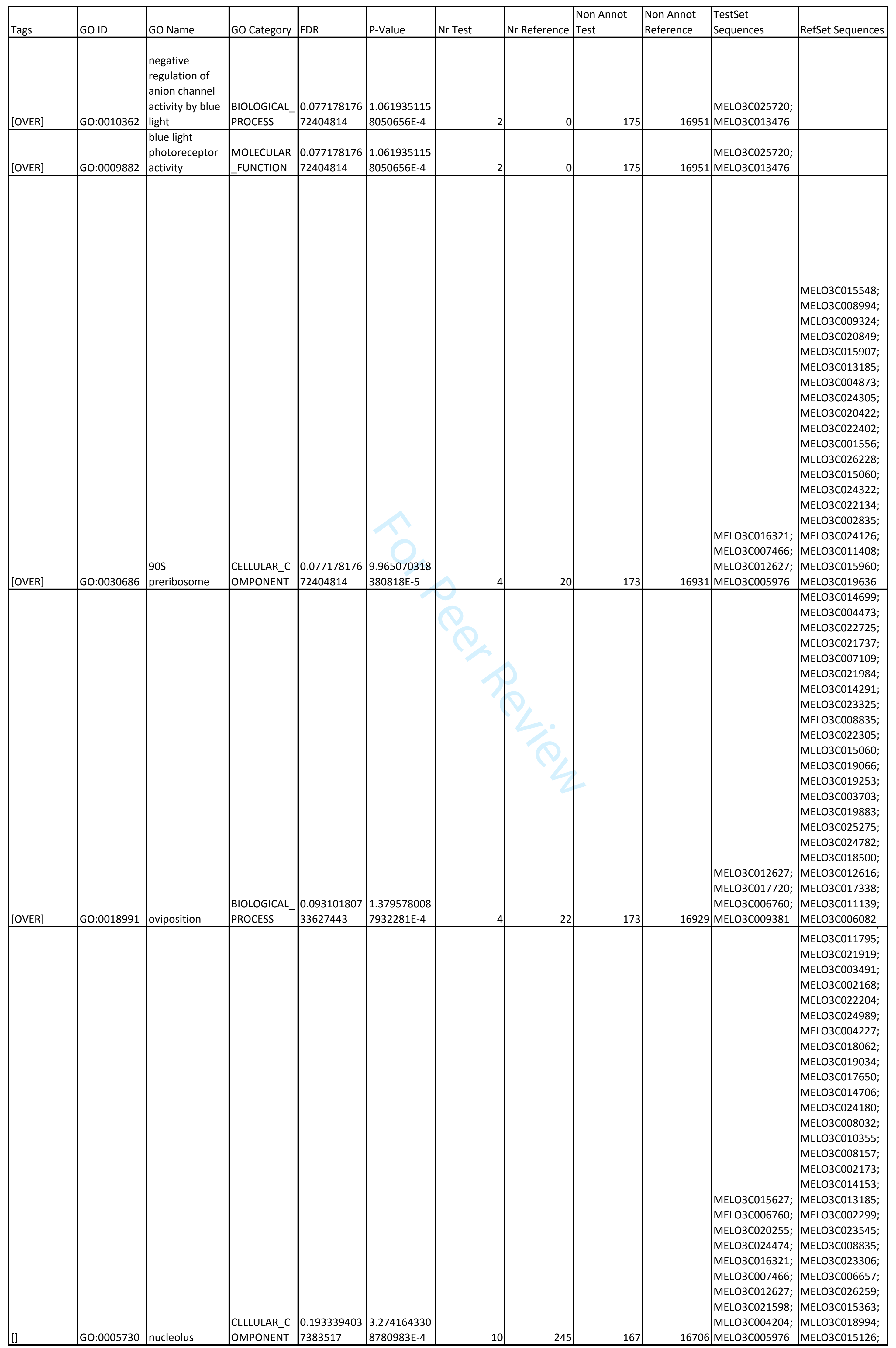




\begin{tabular}{|c|c|c|c|c|c|c|c|c|c|c|c|}
\hline [] & GO:0042026 & $\begin{array}{l}\text { protein } \\
\text { refolding }\end{array}$ & $\begin{array}{l}\text { BIOLOGICAL } \\
\text { PROCESS }\end{array}$ & $\begin{array}{l}0.319871748 \\
92373604\end{array}$ & $\begin{array}{l}0.001040397 \\
5368459023 \\
\end{array}$ & 2 & 3 & 175 & 16948 & $\begin{array}{l}\text { MELO3C012180; } \\
\text { MELO3C026919 }\end{array}$ & $\begin{array}{l}\text { MELO3C023058; } \\
\text { MELO3CO21741; } \\
\text { MELO3C024388 }\end{array}$ \\
\hline [] & GO:0009638 & phototropism & $\begin{array}{l}\text { BIOLOGICAL } \\
\text { PROCESS }\end{array}$ & $\begin{array}{l}0.532788278 \\
1604056 \\
\end{array}$ & $\begin{array}{l}0.002155253 \\
2981376532 \\
\end{array}$ & 2 & 5 & 175 & 16946 & $\begin{array}{l}\text { MELO3C025720; } \\
\text { MELO3C013476 }\end{array}$ & $\begin{array}{l}\text { MELO3CO24837; } \\
\text { MELO3CO18590; } \\
\text { MELO3CO09692; } \\
\text { MELO3CO22995; } \\
\text { MELO3C016232 }\end{array}$ \\
\hline [l] & GO:0015995 & (1) & $\begin{array}{l}\text { BIOLOGICAL } \\
\text { PROCESS }\end{array}$ & $\begin{array}{l}0.846589907 \\
8972182\end{array}$ & $\begin{array}{l}0.003942628 \\
69892862\end{array}$ & 3 & 28 & 174 & 16923 & $\begin{array}{l}\text { MELO3C016714; } \\
\text { MELO3C023131; } \\
\text { MELO3C018572 }\end{array}$ & 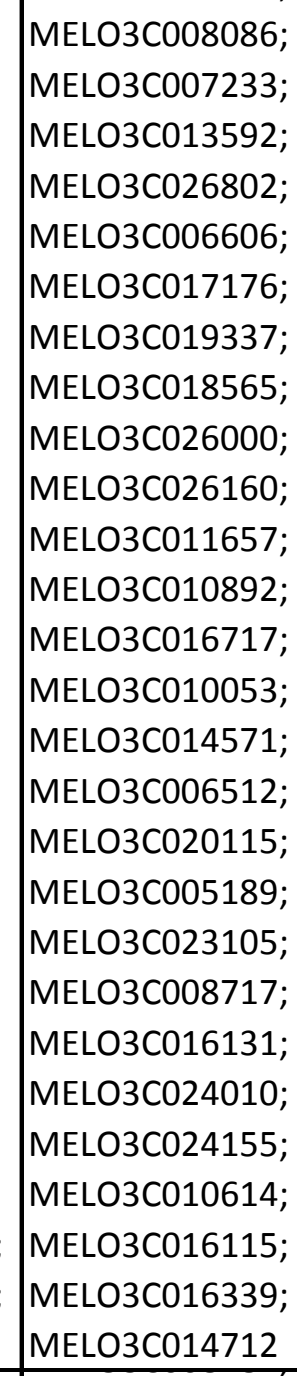 \\
\hline [] & GO:0010181 & FMN binding & $\begin{array}{l}\text { MOLECULAR } \\
\text { _FUNCTION }\end{array}$ & $\begin{array}{l}0.849954170 \\
1733855\end{array}$ & $\begin{array}{l}0.004317778 \\
572168493\end{array}$ & 3 & 29 & 174 & 16922 & $\begin{array}{l}\text { MELO3C021944; } \\
\text { MELO3C025720; } \\
\text { MELO3C013476 }\end{array}$ & 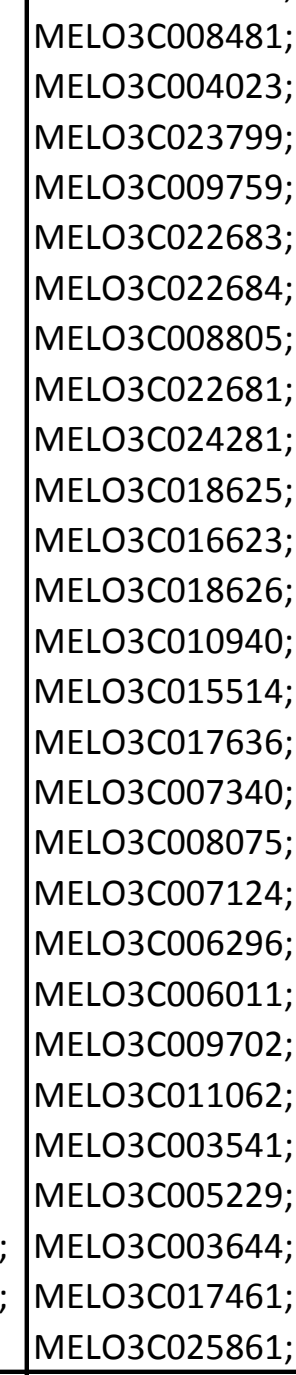 \\
\hline [] & GO:0051091 & \begin{tabular}{|l} 
positive \\
regulation of \\
sequence- \\
specific DNA \\
binding \\
transcription \\
factor activity
\end{tabular} & $\begin{array}{l}\text { BIOLOGICAL_ } \\
\text { PROCESS }\end{array}$ & \begin{tabular}{|l|l}
0.923487244 \\
6965384
\end{tabular} & $\begin{array}{l}0.006547341 \\
533695067\end{array}$ & 2 & 10 & 175 & 16941 & $\begin{array}{l}\text { MELO3C006760; } \\
\text { MELO3C018099 }\end{array}$ & $\begin{array}{l}\text { MELO3CO16083; } \\
\text { MELO3C01198; } \\
\text { MELO3C005912; } \\
\text { MELO3C019642; } \\
\text { MELO3C002481; } \\
\text { MELO3C019932; } \\
\text { MELO3C025417; } \\
\text { MELO3C023325; } \\
\text { MELO3CO05424; } \\
\text { MELO3C025767 }\end{array}$ \\
\hline
\end{tabular}




\begin{tabular}{|c|c|c|c|c|c|c|c|c|c|c|c|}
\hline [] & 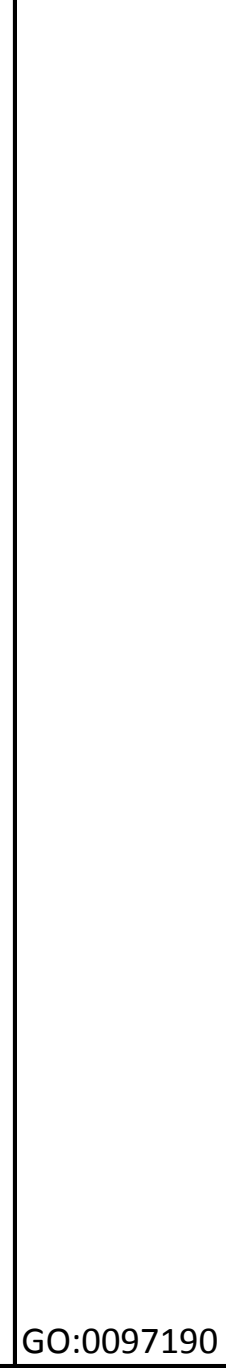 & 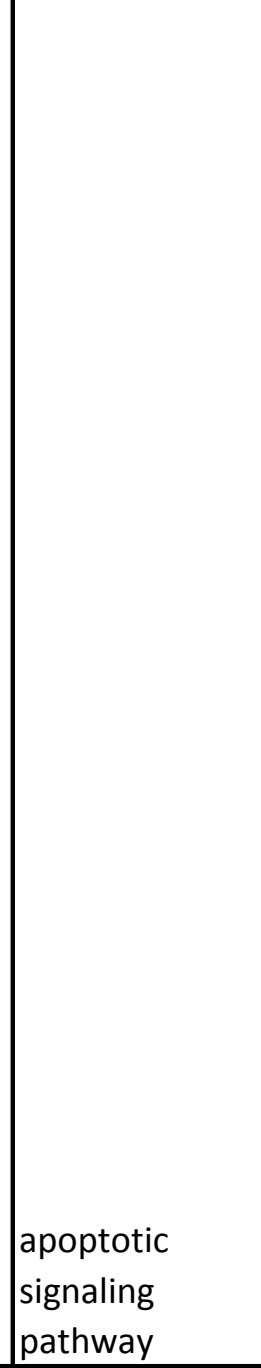 & $\begin{array}{l}\text { BIOLOGICAL_ } \\
\text { PROCESS }\end{array}$ & $-\begin{array}{l}0.923487244 \\
6965384\end{array}$ & $\begin{array}{l}0.008097951 \\
336000693\end{array}$ & 3 & 37 & 174 & 16914 & \begin{tabular}{|l} 
\\
MELO3C011641; \\
MELO3C005976; \\
MELO3C018099
\end{tabular} & $\begin{array}{l}\text { MELO3C013754; } \\
\text { MELO3C011354; } \\
\text { MELO3C012146; } \\
\text { MELO3C002481; } \\
\text { MELO3C003572; } \\
\text { MELO3C020322; } \\
\text { MELO3C025417; } \\
\text { MELO3C025413; } \\
\text { MELO3C016083; } \\
\text { MELO3C003536; } \\
\text { MELO3C005912; } \\
\text { MELO3C015493; } \\
\text { MELO3C016340; } \\
\text { MELO3C019157; } \\
\text { MELO3C019315; } \\
\text { MELO3C025010; } \\
\text { MELO3C018726; } \\
\text { MELO3C011421; } \\
\text { MELO3C013522; } \\
\text { MELO3C011983; } \\
\text { MELO3C020816; } \\
\text { MELO3C011041; } \\
\text { MELO3C015928; } \\
\text { MELO3C007243; } \\
\text { MELO3C003562; } \\
\text { MELO3C002032; } \\
\text { MELO3C025009; }\end{array}$ \\
\hline [] & GO:2000011 & \begin{tabular}{|l|} 
regulation of \\
adaxial/abaxial \\
pattern \\
formation
\end{tabular} & \begin{tabular}{|l} 
BIOLOGICAL \\
PROCESS
\end{tabular} & $-\begin{array}{l}0.923487244 \\
6965384\end{array}$ & $\begin{array}{l}0.010333956 \\
095153881\end{array}$ & 1 & 0 & 176 & 16951 & MELO3C015627 & \\
\hline [] & GO:0009903 & \begin{tabular}{|l} 
chloroplast \\
avoidance \\
movement
\end{tabular} & \begin{tabular}{|l} 
BIOLOGICAL_ \\
PROCESS
\end{tabular} & $-\begin{array}{l}0.923487244 \\
6965384\end{array}$ & $\begin{array}{l}0.010333956 \\
095153881\end{array}$ & 1 & 0 & 176 & 16951 & MELO3C025720 & \\
\hline [] & GO:0032929 & \begin{tabular}{|l|} 
negative \\
regulation of \\
superoxide \\
anion \\
generation \\
\end{tabular} & $\begin{array}{l}\text { BIOLOGICAL_ } \\
\text { PROCESS }\end{array}$ & $-\begin{array}{l}0.923487244 \\
6965384\end{array}$ & $\begin{array}{l}0.010333956 \\
095153881\end{array}$ & 1 & 0 & 176 & 16951 & MELO3C005976 & \\
\hline [] & GO:0018298 & \begin{tabular}{|l} 
\\
\\
\\
protein- \\
chromophore \\
linkage
\end{tabular} & 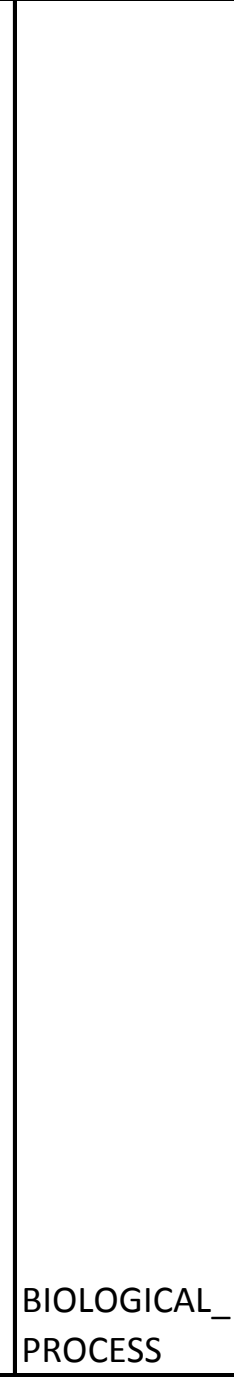 & (⿸丆口 & $\begin{array}{l} \\
\\
0.008671871 \\
761091517\end{array}$ & 3 & 38 & 174 & 16913 & $\begin{array}{l}\text { MELO3C012912; } \\
\text { MELO3C025720; } \\
\text { MELO3C013476 }\end{array}$ & $\begin{array}{l}\text { MELO3C012066; } \\
\text { MELO3C007154; } \\
\text { MELO3C001595; } \\
\text { MELO3C001992; } \\
\text { MELO3C026506; } \\
\text { MELO3C024206; } \\
\text { MELO3C003238; } \\
\text { MELO3C017180; } \\
\text { MELO3C026502; } \\
\text { MELO3C000131; } \\
\text { MELO3C002727; } \\
\text { MELO3C002705; } \\
\text { MELO3C015076; } \\
\text { MELO3C016660; } \\
\text { MELO3C010807; } \\
\text { MELO3C000173; } \\
\text { MELO3C019677; } \\
\text { MELO3C015536; } \\
\text { MELO3C015998; } \\
\text { MELO3C008358; } \\
\text { MELO3C020213; } \\
\text { MELO3C004214; } \\
\text { MELO3C022995; } \\
\text { MELO3C001664; } \\
\text { MELO3C004018; } \\
\text { MELO3C003644; } \\
\text { MELO3C000500; }\end{array}$ \\
\hline [] & GO:0033262 & \begin{tabular}{|l} 
regulation of \\
nuclear cell \\
cycle DNA \\
replication \\
\end{tabular} & $\begin{array}{l}\text { BIOLOGICAL_ } \\
\text { PROCESS }\end{array}$ & $\begin{array}{l}0.923487244 \\
6965384\end{array}$ & $\begin{array}{l}0.010333956 \\
095153881\end{array}$ & 1 & 0 & 176 & 16951 & MELO3C006760 & \\
\hline [] & GO:0034462 & $\begin{array}{l}\text { small-subunit } \\
\text { processome } \\
\text { assembly }\end{array}$ & $\begin{array}{l}\text { BIOLOGICAL_ } \\
\text { PROCESS }\end{array}$ & $\begin{array}{l}0.923487244 \\
6965384\end{array}$ & $\begin{array}{l}0.010333956 \\
095153881\end{array}$ & 1 & 0 & 176 & 16951 & MELO3C016321 & \\
\hline [] & GO:0090045 & \begin{tabular}{|l} 
positive \\
regulation of \\
deacetylase \\
activity
\end{tabular} & $\begin{array}{l}\text { BIOLOGICAL_ } \\
\text { PROCESS }\end{array}$ & $\begin{array}{l}0.923487244 \\
6965384\end{array}$ & $\begin{array}{l}0.010333956 \\
095153881\end{array}$ & 1 & 0 & 176 & 16951 & MELO3C017720 & \\
\hline [] & GO:0090044 & \begin{tabular}{|l} 
positive \\
regulation of \\
tubulin \\
deacetylation \\
\end{tabular} & $\begin{array}{l}\text { BIOLOGICAL_ } \\
\text { PROCESS }\end{array}$ & $\begin{array}{l}0.923487244 \\
6965384\end{array}$ & $\begin{array}{l}0.010333956 \\
095153881\end{array}$ & 1 & 0 & 176 & 16951 & MELO3C017720 & \\
\hline
\end{tabular}




\begin{tabular}{|c|c|c|c|c|c|c|c|c|c|c|c|}
\hline [] & GO:0000447 & $\begin{array}{l}\text { endonucleolytic } \\
\text { cleavage in ITS1 } \\
\text { to separate SSU- } \\
\text { rRNA from 5.8S } \\
\text { rRNA and LSU- } \\
\text { rRNA from } \\
\text { tricistronic rRNA } \\
\text { transcript (SSU- } \\
\text { rRNA, 5.8S } \\
\text { rRNA, LSU- } \\
\text { rRNA) }\end{array}$ & $\begin{array}{l}\text { BIOLOGICAL } \\
\text { PROCESS }\end{array}$ & $\begin{array}{l}0.923487244 \\
6965384\end{array}$ & $\left|\begin{array}{l}0.010206693 \\
282011533\end{array}\right|$ & 2 & 13 & 175 & 16938 & $\begin{array}{l}\text { MELO3C016321; } \\
\text { MELO3C012627 }\end{array}$ & $\begin{array}{l}\text { MELO3C008994; } \\
\text { MELO3C006662; } \\
\text { MELO3C020849; } \\
\text { MELO3C024305; } \\
\text { MELO3C020422; } \\
\text { MELO3C015060; } \\
\text { MELO3C002835; } \\
\text { MELO3C024126; } \\
\text { MELO3C017650; } \\
\text { MELO3C011408; } \\
\text { MELO3C015663; } \\
\text { MELO3C019636; } \\
\text { MELO3C015997 }\end{array}$ \\
\hline [] & GO:0009866 & \begin{tabular}{|l} 
induced \\
systemic \\
resistance, \\
ethylene \\
mediated \\
signaling \\
pathway
\end{tabular} & $\begin{array}{l}\text { BIOLOGICAL } \\
\text { PROCESS }\end{array}$ & $\begin{array}{l}0.923487244 \\
6965384\end{array}$ & $\begin{array}{l}0.010333956 \\
095153881\end{array} \mid$ & 1 & 0 & 176 & 16951 & MELO3C026577 & \\
\hline [] & GO:0070984 & \begin{tabular}{|l} 
SET domain \\
binding
\end{tabular} & $\begin{array}{l}\text { MOLECULAR } \\
\text { FUNCTION }\end{array}$ & $\begin{array}{l}0.923487244 \\
6965384\end{array}$ & $\begin{array}{l}0.010333956 \\
095153881\end{array}$ & 1 & 0 & 176 & 16951 & MELO3C006760 & \\
\hline [] & GO:0042286 & \begin{tabular}{|l} 
glutamate-1- \\
semialdehyde \\
$2,1-$ \\
aminomutase \\
activity \\
\end{tabular} & $\begin{array}{l}\text { MOLECULAR } \\
\text { FUNCTION }\end{array}$ & $\begin{array}{l}0.923487244 \\
6965384\end{array}$ & \begin{tabular}{|l|}
0.010333956 \\
095153881
\end{tabular} & 1 & 0 & 176 & 16951 & MELO3C018572 & \\
\hline [] & GO:0043522 & $\begin{array}{l}\text { leucine zipper } \\
\text { domain binding }\end{array}$ & $\begin{array}{l}\text { MOLECULAR } \\
\text { FUNCTION }\end{array}$ & \begin{tabular}{|l}
0.923487244 \\
6965384 \\
\end{tabular} & \begin{tabular}{|l|}
0.010333956 \\
095153881 \\
\end{tabular} & 1 & 0 & 176 & 16951 & MELO3C005976 & \\
\hline [] & GO:0051116 & $\begin{array}{l}\text { cobaltochelatas } \\
\text { e activity }\end{array}$ & $\begin{array}{l}\text { MOLECULAR } \\
\text { FUNCTION }\end{array}$ & $\begin{array}{l}0.923487244 \\
6965384\end{array}$ & \begin{tabular}{|l|}
0.010333956 \\
095153881
\end{tabular} & 1 & 0 & 176 & 16951 & MELO3C023131 & \\
\hline [] & GO:0016514 & $\begin{array}{l}\text { SWI/SNF } \\
\text { complex }\end{array}$ & $\begin{array}{l}\text { CELLULAR_C } \\
\text { OMPONENT }\end{array}$ & $\begin{array}{l}0.923487244 \\
6965384\end{array}$ & $\begin{array}{l}0.010333956 \\
095153881\end{array}$ & & 0 & 176 & 16951 & MELO3C006760 & \\
\hline [] & GO:0071564 & npBAF complex & $\begin{array}{l}\text { CELLULAR_C } \\
\text { OMPONENT }\end{array}$ & $\begin{array}{l}0.923487244 \\
6965384\end{array}$ & \begin{tabular}{|l|}
0.010333956 \\
095153881
\end{tabular} & 1 & 0 & 176 & 16951 & MELO3C006760 & \\
\hline [] & GO:0071565 & nBAF complex & $\begin{array}{l}\text { CELLULAR_C } \\
\text { OMPONENT }\end{array}$ & $\begin{array}{l}0.923487244 \\
6965384\end{array}$ & $\begin{array}{l}0.010333956 \\
095153881\end{array}$ & 1 & 0 & 176 & 16951 & MELO3C006760 & \\
\hline [] & 更 & 更 & $\begin{array}{l}\text { CELLULAR_C } \\
\text { OMPONENTT }\end{array}$ & $\begin{array}{l}0.923487244 \\
6965384\end{array}$ & \begin{tabular}{|l|} 
\\
\\
0.010206693 \\
282011533
\end{tabular} & 2 & 13 & 175 & 16938 & $\begin{array}{l}\text { MELO3C026433; } \\
\text { MELO3C022928 }\end{array}$ & $\begin{array}{l}\text { MELO3C012871; } \\
\text { MELO3C004440; } \\
\text { MELO3C023809; } \\
\text { MELO3C017908; } \\
\text { MELO3C006422; } \\
\text { MELO3C005127; } \\
\text { MELO3C004018; } \\
\text { MELO3C008801; } \\
\text { MELO3C019034; } \\
\text { MELO3C017365; } \\
\text { MELO3C017696; } \\
\text { MELO3C014706; } \\
\text { MELO3C022370 }\end{array}$ \\
\hline [] & GO:0016586 & RSC complex & $\begin{array}{l}\text { CELLULAR_C } \\
\text { OMPONENT }\end{array}$ & $\begin{array}{l}0.923487244 \\
6965384 \\
\end{array}$ & \begin{tabular}{|l|}
0.010333956 \\
095153881 \\
\end{tabular} & 1 & 0 & 176 & 16951 & MELO3C006760 & \\
\hline [] & GO:0007254 & JNK cascade & 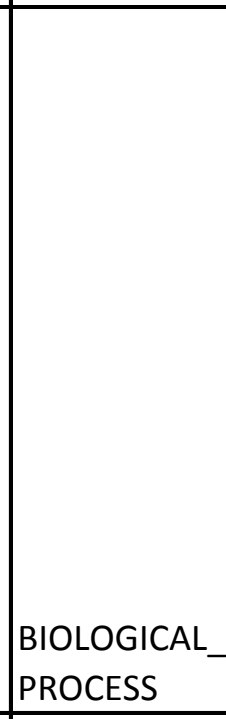 & 更 & $\begin{array}{l}0.011586177 \\
649073121 \\
\end{array}$ & 2 & 14 & 175 & 16937 & $\begin{array}{l}\text { MELO3C018099; } \\
\text { MELO3C026577 }\end{array}$ & $\begin{array}{l}\text { MELO3C011983; } \\
\text { MELO3C002150; } \\
\text { MELO3C002481; } \\
\text { MELO3C004594; } \\
\text { MELO3C025417; } \\
\text { MELO3C005328; } \\
\text { MELO3C004635; } \\
\text { MELO3C016083; } \\
\text { MELO3C026602; } \\
\text { MELO3C005912; } \\
\text { MELO3C021442; } \\
\text { MELO3C019642; } \\
\text { MELO3C025790; } \\
\text { MELO3C015497 }\end{array}$ \\
\hline (] & GO:0008587 & $\begin{array}{l}\text { imaginal disc- } \\
\text { derived wing } \\
\text { margin } \\
\text { morphogenesis } \\
\end{array}$ & $\begin{array}{l}\text { BIOLOGICAL_ } \\
\text { PROCESS }\end{array}$ & 1.0 & \begin{tabular}{|l|}
0.020561718 \\
679675958
\end{tabular} \mid & 1 & 1 & 176 & 16950 & MELO3C006760 & MELO3C006520 \\
\hline & GO:0008586 & $\begin{array}{l}\text { imaginal disc- } \\
\text { derived wing } \\
\text { vein } \\
\text { morphogenesis }\end{array}$ & $\begin{array}{l}\text { BIOLOGICAL_ } \\
\text { PROCESS }\end{array}$ & 1.0 & $\begin{array}{l}0.020561718 \\
679675958\end{array}$ & 1 & 1 & 176 & 16950 & MELO3C006760 & MELO3C006520 \\
\hline
\end{tabular}




\begin{tabular}{|c|c|c|c|c|c|c|c|c|c|c|c|}
\hline [] & GO:0000187 & $\begin{array}{l}\text { activation of } \\
\text { MAPK activity }\end{array}$ & $\begin{array}{l}\text { BIOLOGICAL_} \\
\text { PROCESS }\end{array}$ & 1.0 & \begin{tabular}{|l|}
0.023301891 \\
810173274
\end{tabular} & 2 & 21 & 175 & 16930 & \begin{tabular}{|l|} 
\\
MELO3C018099; \\
MELO3C026577
\end{tabular} & 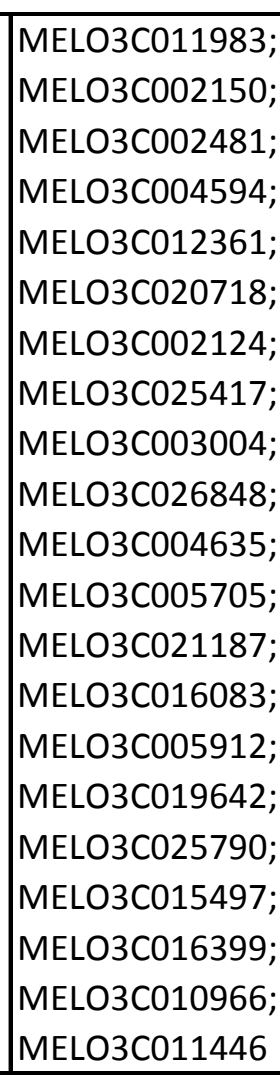 \\
\hline [] & GO:0005986 & \begin{tabular}{|l|} 
sucrose \\
biosynthetic \\
process
\end{tabular} & \begin{tabular}{|l|} 
BIOLOGICAL_- \\
PROCESS
\end{tabular} & 1.0 & \begin{tabular}{|l|}
0.040702992 \\
789935
\end{tabular} & 1 & 3 & 176 & 16948 & MELO3C011682 & $\begin{array}{l}\text { MELO3C025149; } \\
\text { MELO3C012320; } \\
\text { MELO3C009570 }\end{array}$ \\
\hline [] & GO:0002119 & $\begin{array}{l}\text { nematode larval } \\
\text { development }\end{array}$ & \begin{tabular}{|l} 
BIOLOGICAL \\
PROCESS
\end{tabular} & 1.0 & $\begin{array}{l}0.035479345 \\
38526353\end{array}$ & 7 & 291 & 170 & 16660 & \begin{tabular}{|l|} 
\\
\\
\\
\\
MELO3C006760; \\
MELO3C024474; \\
MELO3C016321; \\
MELO3C007466; \\
MELO3C012627; \\
MELO3C017720; \\
MELO3C009381 \\
\end{tabular} & 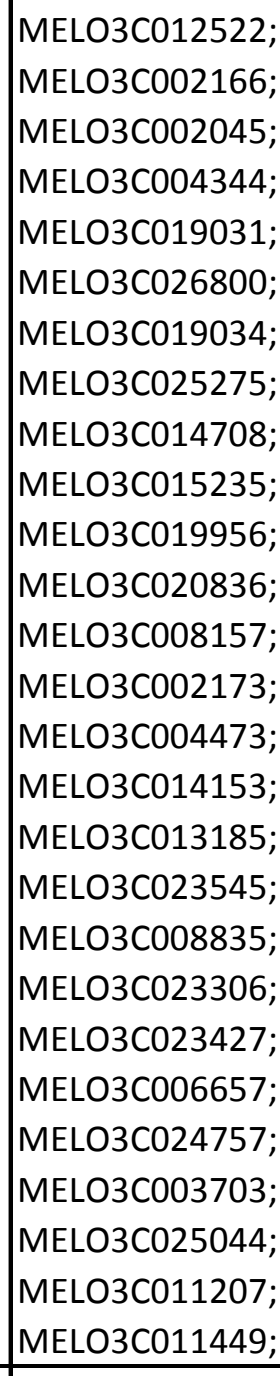 \\
\hline [] & GO:0030388 & \begin{tabular}{|l|} 
fructose 1,6- \\
bisphosphate \\
metabolic \\
process
\end{tabular} & \begin{tabular}{|l} 
BIOLOGICAL \\
PROCESS
\end{tabular} & 1.0 & \begin{tabular}{|l|}
0.020561718 \\
679675958
\end{tabular} & 1 & 1 & 176 & 16950 & MELO3C011682 & MELO3C003214 \\
\hline [] & GO:0048011 & $\begin{array}{l}\text { neurotrophin } \\
\text { TRK receptor } \\
\text { signaling } \\
\text { pathway }\end{array}$ & $\begin{array}{l}\text { BIOLOGICAL } \\
\text { PROCESS }\end{array}$ & 1.0 & \begin{tabular}{|l|}
0.035020126 \\
629563865
\end{tabular} & 4 & 115 & 173 & 16836 & $\begin{array}{l}\text { MELO3C011641; } \\
\text { MELO3C026577; } \\
\text { MELO3C005976; } \\
\text { MELO3C018099 }\end{array}$ & 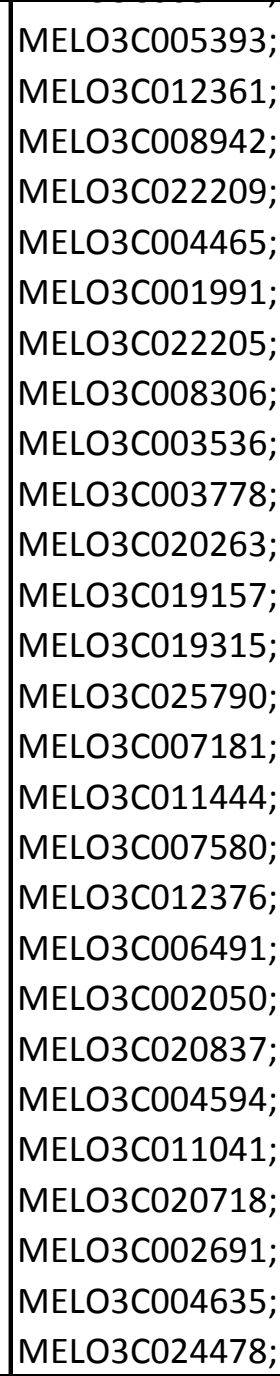 \\
\hline [] & GO:0007474 & $\begin{array}{l}\text { imaginal disc- } \\
\text { derived wing } \\
\text { vein } \\
\text { specification }\end{array}$ & \begin{tabular}{|l} 
BIOLOGICAL \\
PROCESS
\end{tabular} & 1.0 & \begin{tabular}{|l|}
0.020561718 \\
679675958
\end{tabular} & 1 & 1 & 176 & 16950 & MELOЗC006760 & MELO3C015173 \\
\hline []] & GO:0000282 & $\begin{array}{l}\begin{array}{l}\text { cellular bud site } \\
\text { selection }\end{array} \\
\text { al }\end{array}$ & \begin{tabular}{|l|} 
BIOLOGICAL_ \\
PROCESS
\end{tabular} & 1.0 & \begin{tabular}{|l|}
.030684372 \\
877273734
\end{tabular} & 1 & 2 & 176 & 16949 & & $\begin{array}{l}\text { MELO3C013504; } \\
\text { MELO3C015367 }\end{array}$ \\
\hline [] & GO:0070983 & \begin{tabular}{|l}
$\begin{array}{l}\text { dendrite } \\
\text { guidance }\end{array}$ \\
\end{tabular} & \begin{tabular}{|l|} 
BIOLOGICAL \\
PROCESS \\
\end{tabular} & 1.0 & \begin{tabular}{|l|}
0.020561718 \\
679675958 \\
\end{tabular} & 1 & & 176 & & MELO3C006760 & MELO3C017295 \\
\hline
\end{tabular}




\begin{tabular}{|c|c|c|c|c|c|c|c|c|c|c|c|}
\hline [] & GO:1902582 & $\begin{array}{l}\text { single-organism } \\
\text { intracellular } \\
\text { transport }\end{array}$ & $\begin{array}{l}\text { BIOLOGICAL } \\
\text { PROCESS }\end{array}$ & 1.0 & $\begin{array}{l}0.049266416 \\
36975816 \\
\end{array}$ & 4 & 129 & 173 & 16822 & $\begin{array}{l} \\
\text { MELO3C004761; } \\
\text { MELO3C019156; } \\
\text { MELO3C004204; } \\
\text { MELO3C020533 }\end{array}$ & 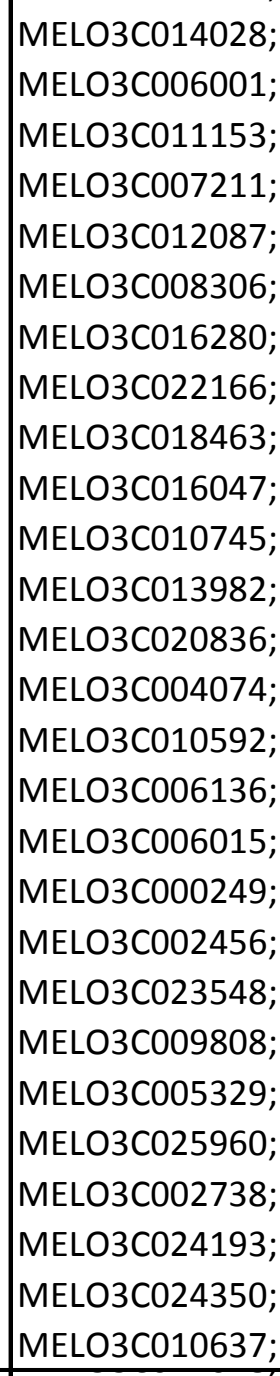 \\
\hline [] & GO:0072594 & $\begin{array}{l}\text { astablishment } \\
\text { of protein } \\
\text { localization to } \\
\text { organelle }\end{array}$ & $\begin{array}{l}\text { BIOLOGICAL } \\
\text { PROCESS }\end{array}$ & 1.0 & $\begin{array}{l}0.048157211 \\
38180463\end{array}$ & 4 & 128 & 173 & 16823 & $\begin{array}{l}\text { MELO3C019156; } \\
\text { MELO3CO04204; } \\
\text { MELO3C020533; } \\
\text { MELO3C013766 }\end{array}$ & 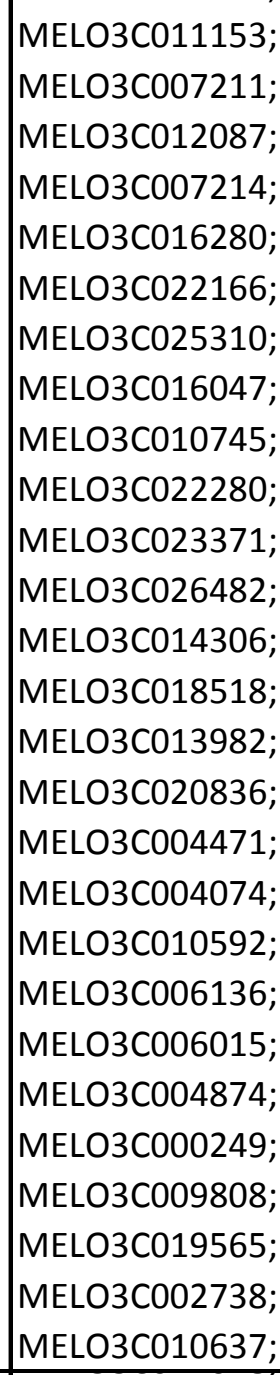 \\
\hline [] & GO:0017038 & protein import & 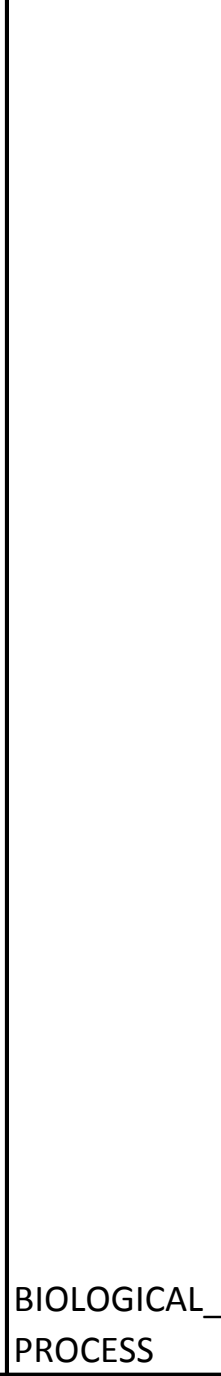 & 1.0 & \begin{tabular}{|l}
0.048496417 \\
87186457
\end{tabular} & 3 & 76 & 174 & 16875 & \begin{tabular}{|l} 
\\
MELO3C019156; \\
MELO3C004204; \\
MELO3C020533
\end{tabular} & 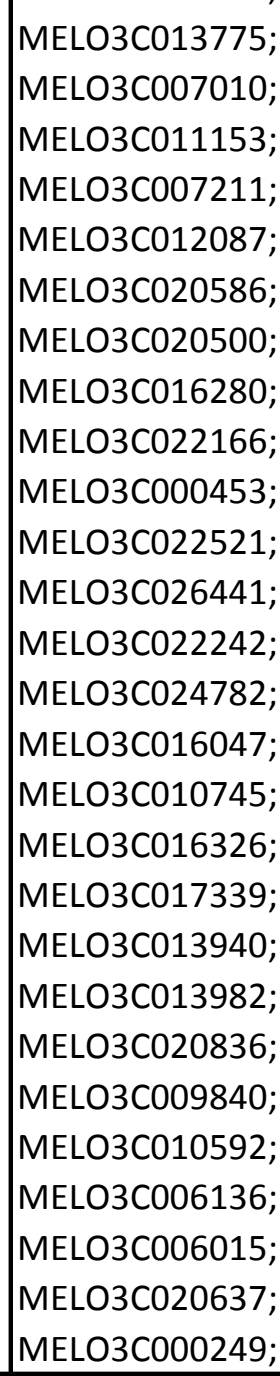 \\
\hline
\end{tabular}




\begin{tabular}{|c|c|c|c|c|c|c|c|c|c|c|c|}
\hline [] & GO:0040035 & $\begin{array}{l}\text { hermaphrodite } \\
\text { genitalia } \\
\text { development }\end{array}$ & \begin{tabular}{|l} 
BIOLOGICAL_ \\
PROCESS
\end{tabular} & 1.0 & \begin{tabular}{|l}
0.013933141 \\
239127323 \\
\end{tabular} & 5 & 132 & 172 & & \begin{tabular}{|l} 
MELO3C016321; \\
MELO3C007466; \\
MELO3C017720; \\
MELO3CO06760; \\
MELO3C009381
\end{tabular} & 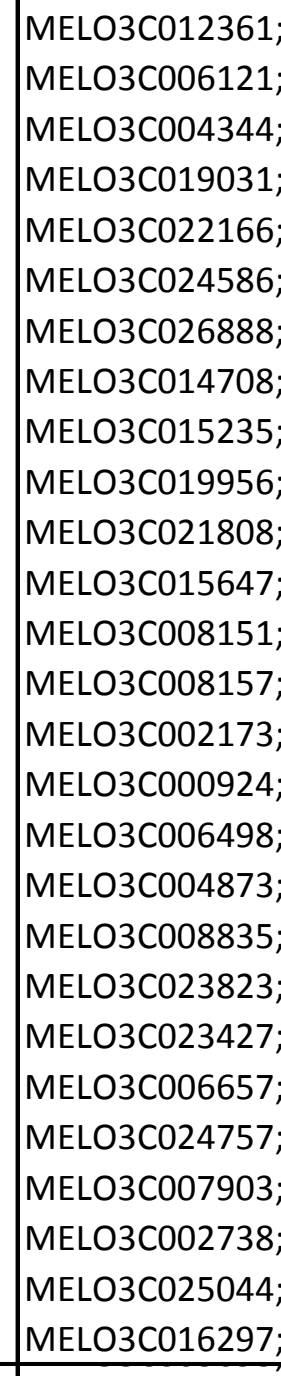 \\
\hline [] & GO:0007173 & $\begin{array}{l}\text { epidermal } \\
\text { growth factor } \\
\text { receptor } \\
\text { signaling } \\
\text { pathway } \\
\end{array}$ & $\begin{array}{l}\text { BIOLOGICAL_ } \\
\text { PROCESS }\end{array}$ & 1.0 & $\begin{array}{l}0.045482947 \\
61164327 \\
\end{array}$ & 2 & 31 & 175 & 16920 & $\begin{array}{l}\text { MELO3C018099; } \\
\text { MELO3C026577 }\end{array}$ & 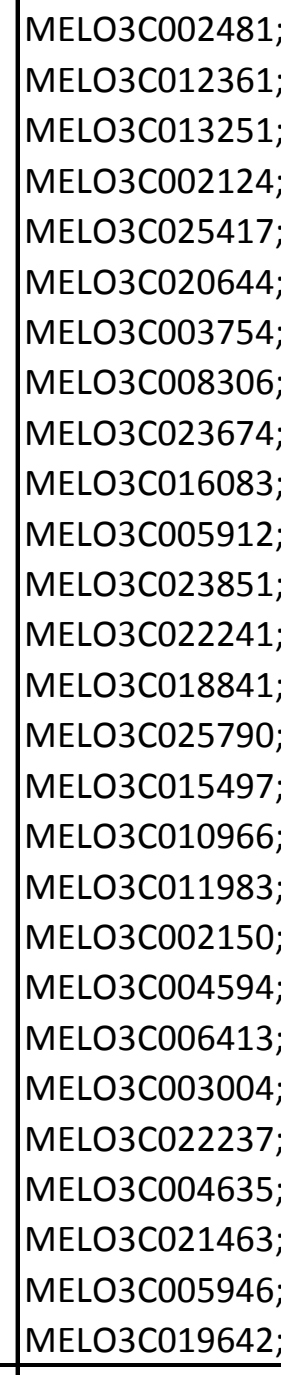 \\
\hline [] & GO:0007179 & $\begin{array}{l}\text { transforming } \\
\text { growth factor } \\
\text { beta receptor } \\
\text { signaling } \\
\text { pathway } \\
\end{array}$ & \begin{tabular}{|l} 
BIOLOGICAL_ \\
PROCESS
\end{tabular} & 1.0 & $\begin{array}{l}0.040702992 \\
789935\end{array}$ & 1 & 3 & 176 & 16948 & MELO3C017720 & $\begin{array}{l}\text { MELOЗC015782; } \\
\text { MELOЗC016377; } \\
\text { MELOЗC016378 }\end{array}$ \\
\hline [] & GO:0009904 & \begin{tabular}{|l|} 
chloroplast \\
accumulation \\
movement
\end{tabular} & \begin{tabular}{|l} 
BIOLOGICAL_ \\
PROCESS
\end{tabular} & 1.0 & \begin{tabular}{|l}
0.020561718 \\
679675958
\end{tabular} & 1 & 1 & 176 & 16950 & MELO3C025720 & MELO3C003878 \\
\hline [] & GO:2000034 & $\begin{array}{l}\text { regulation of } \\
\text { seed maturation }\end{array}$ & $\begin{array}{l}\text { BIOLOGICAL_ } \\
\text { PROCESS }\end{array}$ & 1.0 & $\begin{array}{l}0.030684372 \\
877273734\end{array}$ & 1 & 2 & 176 & 16949 & | MELОЗСО20484 & $\begin{array}{l}\text { MELOЗСО05929; } \\
\text { MELOЗСОo9101 }\end{array}$ \\
\hline [] & GO:0009691 & \begin{tabular}{|l|} 
cytokinin \\
biosynthetic \\
process \\
\end{tabular} & \begin{tabular}{|l} 
BIOLOGICAL_ \\
PROCESS
\end{tabular} & 1.0 & \begin{tabular}{|l}
0.040702992 \\
789935
\end{tabular} & 1 & 3 & 176 & 16948 & MELO3C015852 & $\begin{array}{l}\text { MELO3C009521 } \\
\text { MELO3C007417; } \\
\text { MELO3C016881 }\end{array}$ \\
\hline [] & GO:0090630 & \begin{tabular}{|l} 
activation of \\
GTPase activity
\end{tabular} & $\begin{array}{l}\begin{array}{l}\text { BIOLOGICAL__ } \\
\text { PROCESS }\end{array} \\
\end{array}$ & 1.0 & $\begin{array}{l}0.020561718 \\
679675958\end{array}$ & 1 & 1 & 176 & 16950 & MELOЗC018195 & MELO3C013721 \\
\hline
\end{tabular}




\begin{tabular}{|c|c|c|c|c|c|c|c|c|c|c|c|}
\hline [] & GO:0044281 & $\begin{array}{l}\text { small molecule } \\
\text { metabolic } \\
\text { process }\end{array}$ & $\begin{array}{l}\text { BIOLOGICAL_ } \\
\text { PROCESS }\end{array}$ & 1.0 & \begin{tabular}{|l|}
0.016383216 \\
252722998
\end{tabular} & 7 & 1506 & 170 & 15445 & \begin{tabular}{|l} 
\\
\\
MELO3C023599; \\
MELO3CO12088; \\
MELO3CO11682; \\
MELO3CO1300; \\
MELO3CO11270; \\
MELO3CO18027; \\
MELO3CO04761
\end{tabular} & 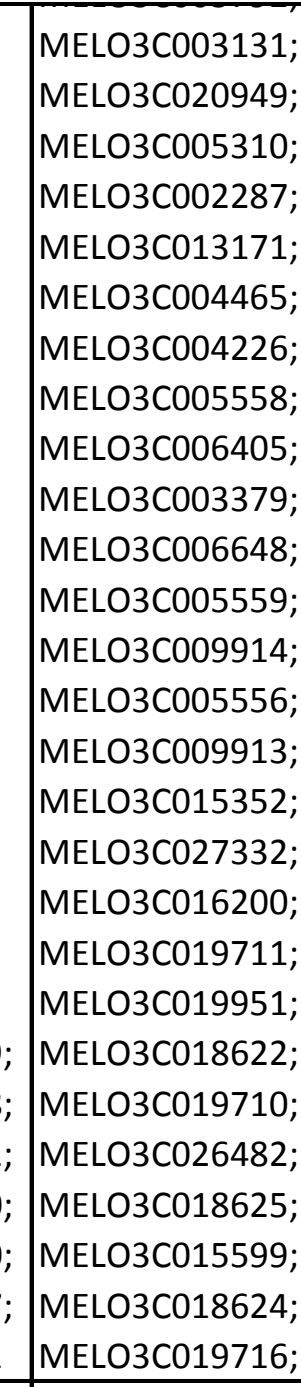 \\
\hline [] & GO:0010030 & \begin{tabular}{|l} 
positive \\
regulation of \\
seed \\
germination
\end{tabular} & $\begin{array}{l}\begin{array}{l}\text { BIOLOGICAL } \\
\text { PROCESS }\end{array} \\
\text { POES }\end{array}$ & 1.0 & \begin{tabular}{|l|}
0.040702992 \\
789935
\end{tabular} & 1 & 3 & 176 & 16948 & MELO3C020484 & $\begin{array}{l}\text { MELO3C004632; } \\
\text { MELO3C009627; } \\
\text { MELO3C009101 }\end{array}$ \\
\hline [] & GO:0006337 & $\begin{array}{l}\text { nucleosome } \\
\text { disassembly }\end{array}$ & \begin{tabular}{|l} 
BIOLOGICAL \\
PROCESS
\end{tabular} & 1.0 & $\begin{array}{l}0.020561718 \\
679675958\end{array}$ & 1 & 1 & 176 & 16950 & MELO3C006760 & MELO3C007085 \\
\hline [] & GO:0045944 & \begin{tabular}{|l} 
\\
\\
positive \\
regulation of \\
transcription \\
from RNA \\
polymerase II \\
promoter
\end{tabular} & $\begin{array}{l}\text { BIOLOGICAL_ } \\
\text { PROCESS }\end{array}$ & 1.0 & \begin{tabular}{|l|}
0.029336667 \\
88391751
\end{tabular} & 2 & 24 & 175 & 16927 & \begin{tabular}{|} 
MELO3CO06760; \\
MELO3CO05976
\end{tabular} & 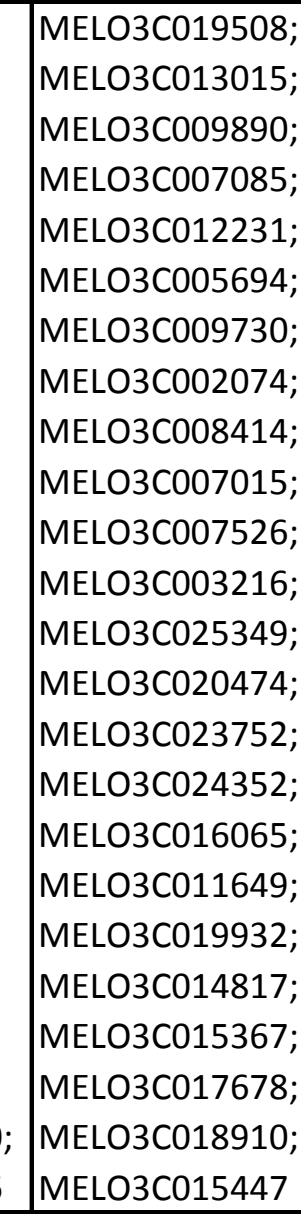 \\
\hline [] & GO:0006302 & $\begin{array}{l}\text { double-strand } \\
\text { break repair }\end{array}$ & $\begin{array}{l}\text { BIOLOGICAL_ } \\
\text { PROCESS }\end{array}$ & 1.0 & $\begin{array}{l}0.038512363 \\
9460339\end{array}$ & 3 & 69 & 174 & 16882 & $\begin{array}{l}\text { MELO3C003108; } \\
\text { MELO3COOG6760; } \\
\text { MELO3CO16674 }\end{array}$ & 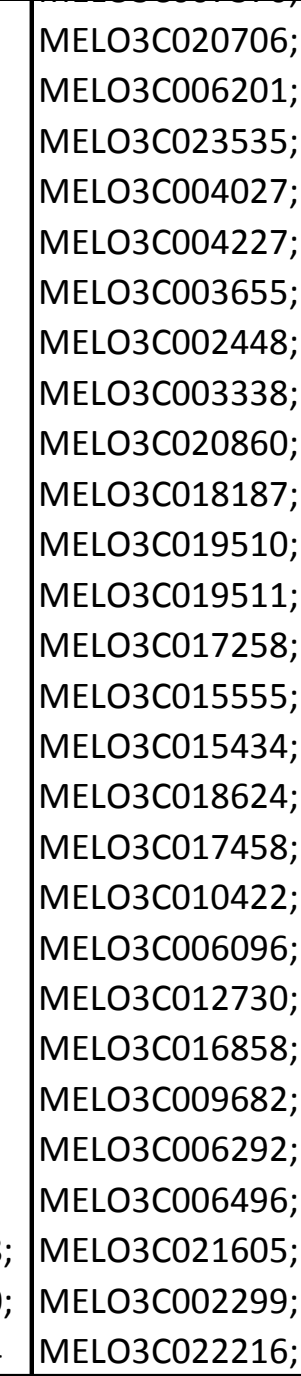 \\
\hline[] & GO:0031055 & \begin{tabular}{|l} 
chromatin \\
remodeling at \\
centromere
\end{tabular} & $\begin{array}{l}\text { BIOLOGICAL_ } \\
\text { PROCESS }\end{array}$ & 1.0 & $\begin{array}{l}0.040702992 \\
789935\end{array}$ & 1 & 3 & 176 & & MELOЗC006760 & $\begin{array}{l}\text { MELO3C005059; } \\
\text { MELO3C01758; } \\
\text { MELO3C023986 }\end{array}$ \\
\hline
\end{tabular}




\begin{tabular}{|c|c|c|c|c|c|c|c|c|c|c|c|}
\hline [] & GO:0010118 & $\begin{array}{l}\text { stomatal } \\
\text { movement }\end{array}$ & \begin{tabular}{|l} 
BIOLOGICAL \\
PROCESS
\end{tabular} & 1.0 & \begin{tabular}{|l}
0.013042579 \\
502851575
\end{tabular} & 2 & 15 & 175 & 16936 & $\begin{array}{l}\text { MELOЗС025720; } \\
\text { MELOЗC013476 }\end{array}$ & 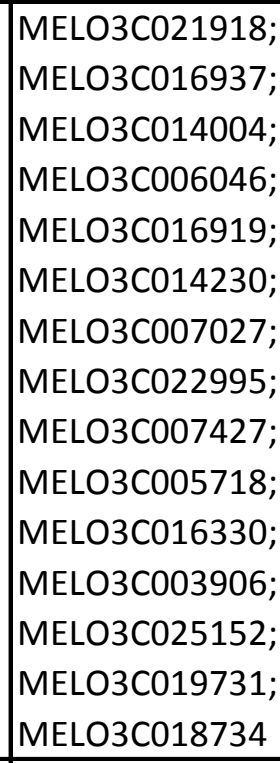 \\
\hline [] & GO:0031053 & \begin{tabular}{|l} 
primary miRNA \\
processing
\end{tabular} & $\mid \begin{array}{l}\text { BIOLOGICAL } \\
\text { PROCESS }\end{array}$ & 1.0 & $\begin{array}{l}0.040702992 \\
789935\end{array}$ & 1 & 3 & 176 & 16948 & MELOЗС015627 & $\begin{array}{l}\text { MELOO3C026304; } \\
\text { MELO3CO05929; } \\
\text { MELO3C015158 }\end{array}$ \\
\hline [] & GO:0031293 & \begin{tabular}{|l} 
membrane \\
protein \\
intracellular \\
domain \\
proteolysis \\
\end{tabular} & \begin{tabular}{|l} 
BIOLOGICAL_ \\
PROCESS
\end{tabular} & 1.0 & $\begin{array}{l}0.040702992 \\
789935\end{array}$ & 1 & 3 & 176 & 16948 & MELO3C011641 & $\begin{array}{l}\text { MELO3C002032; } \\
\text { MELO3C020322; } \\
\text { MELO3C019157 }\end{array}$ \\
\hline [] & GO:0039692 & \begin{tabular}{|l} 
single stranded \\
viral RNA \\
replication via \\
double stranded \\
DNA \\
intermediate \\
\end{tabular} & \begin{tabular}{|l|} 
BIOLOGICAL_- \\
PROCESS
\end{tabular} & 1.0 & \begin{tabular}{|l|}
0.020561718 \\
679675958 \\
\end{tabular} & 1 & 1 & 176 & 16950 & MELOЗС006760 & MELO3C008414 \\
\hline [] & GO:0007220 & $\begin{array}{l}\text { Notch receptor } \\
\text { processing }\end{array}$ & \begin{tabular}{|l|} 
BIOLOGICAL_ \\
PROCESS
\end{tabular} & 1.0 & \begin{tabular}{|l|}
0.040702992 \\
789935
\end{tabular} & 1 & 3 & 176 & 16948 & MELOЗС011641 & $\begin{array}{l}\text { MELO3C002032; } \\
\text { MELO3CO20322; } \\
\text { MELO3C019157 }\end{array}$ \\
\hline [] & GO:0000389 & $\begin{array}{l}\text { mRNA 3'-splice } \\
\text { site recognition }\end{array}$ & $\begin{array}{l}\text { BIOLOGICAL_ } \\
\text { PROCESS }\end{array}$ & 1.0 & \begin{tabular}{|l|}
0.040702992 \\
789935 \\
\end{tabular} & 1 & 3 & 176 & 16948 & MELOЗС009381 & $\begin{array}{l}\text { MELO3C008488; } \\
\text { MELO3C023427; } \\
\text { MELO3C008151 }\end{array}$ \\
\hline [] & GO:0006921 & \begin{tabular}{|l} 
\\
cellular \\
component \\
disassembly \\
involved in \\
execution phase \\
of apoptosis
\end{tabular} & \begin{tabular}{|l} 
BIOLOGICAL \\
PROCESS
\end{tabular} & 1.0 & $\begin{array}{l}0.027262180 \\
07250078\end{array}$ & 2 & 23 & 175 & 16928 & $\begin{array}{l}\text { MELO3C026577; } \\
\text { MELO3C017720 }\end{array}$ & 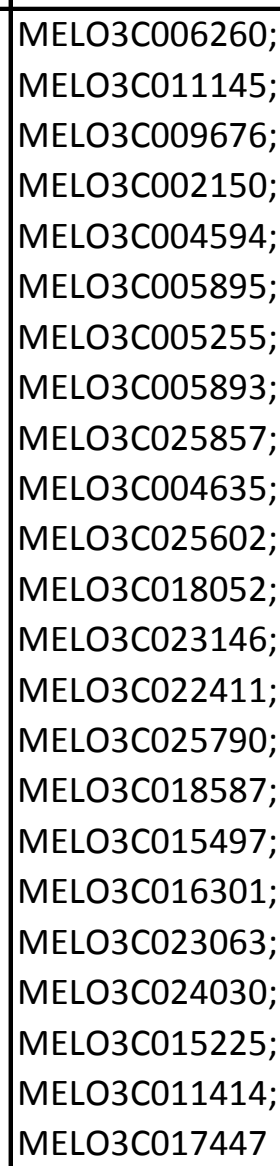 \\
\hline [] & GO:0018343 & $\begin{array}{l}\text { protein } \\
\text { farnesylation }\end{array}$ & \begin{tabular}{|l|} 
BIOLOGICAL \\
PROCESS \\
\end{tabular} & 1.0 & \begin{tabular}{|l|}
0.020561718 \\
679675958 \\
\end{tabular} & 1 & 1 & 176 & 16950 & MELOЗС017720 & MELOЗC009957 \\
\hline [] & GO:0018344 & \begin{tabular}{|l|} 
protein \\
geranylgeranyla \\
tion
\end{tabular} & $\begin{array}{l}\text { BIOLOGICAL } \\
\text { PROCESS }\end{array}$ & 1.0 & \begin{tabular}{|l}
0.030684372 \\
877273734
\end{tabular} & 1 & 2 & 176 & 16949 & MELOЗC017720 & $\begin{array}{l}\text { MELO3C013721; } \\
\text { MELO3CO21654 }\end{array}$ \\
\hline [] & GO:0051103 & \begin{tabular}{|l|} 
DNA ligation \\
involved in DNA \\
repair \\
\end{tabular} & \begin{tabular}{|l} 
BIOLOGICAL \\
PROCESS
\end{tabular} & 1.0 & $\begin{array}{l}0.040702992 \\
789935\end{array}$ & 1 & 3 & 176 & 16948 & MELOЗС010375 & $\begin{array}{l}\text { MELO3C018624; } \\
\text { MELO3C015121; } \\
\text { MELO3C026949 }\end{array}$ \\
\hline [] & GO:0070423 & \begin{tabular}{|l} 
nucleotide- \\
binding \\
oligomerization \\
domain \\
containing \\
signaling \\
pathway
\end{tabular} & $\begin{array}{l}\text { BIOLOGICAL } \\
\text { PROCESS }\end{array}$ & 1.0 & $\begin{array}{l}0.019603395 \\
228084016\end{array}$ & 2 & 19 & 175 & 16932 & $\begin{array}{l}\text { MELO3C018099; } \\
\text { MELO3C026577 }\end{array}$ & 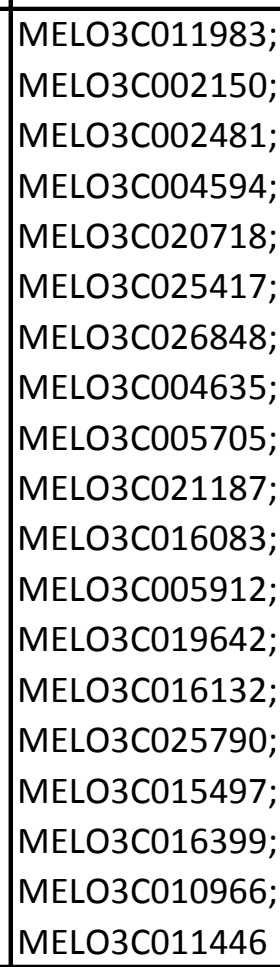 \\
\hline [] & GO:0010155 & \begin{tabular}{|l} 
regulation of \\
proton \\
transport \\
\end{tabular} & \begin{tabular}{|l|} 
BIOLOGICAL_ \\
PROCESS
\end{tabular} & 1.0 & \begin{tabular}{|l|}
0.020561718 \\
679675958
\end{tabular} & 1 & 1 & 176 & 16950 & MELOЗC025720 & MELO3C016696 \\
\hline
\end{tabular}




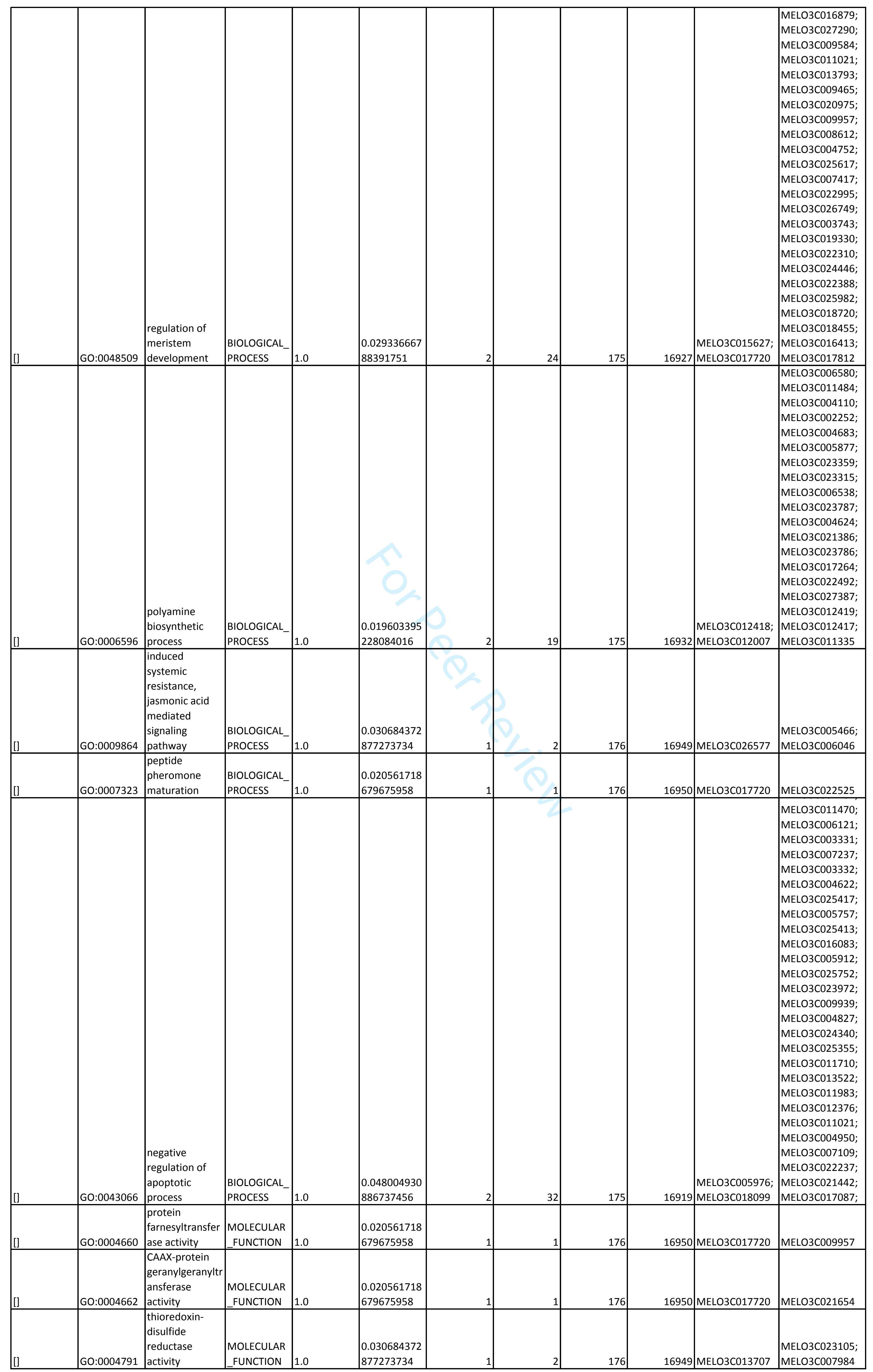




\begin{tabular}{|c|c|c|c|c|c|c|c|c|c|c|c|}
\hline [] & GO:0000156 & \begin{tabular}{|l} 
phosphorelay \\
response \\
regulator \\
activity \\
\end{tabular} & $\begin{array}{l}\text { MOLECULAR } \\
\text { FUNCTION }\end{array}$ & 1.0 & $\begin{array}{l}0.031472058 \\
45062728\end{array}$ & 2 & 25 & 175 & 16926 & \begin{tabular}{|l} 
MELO3C009770; \\
MELO3C019056 \\
\end{tabular} & 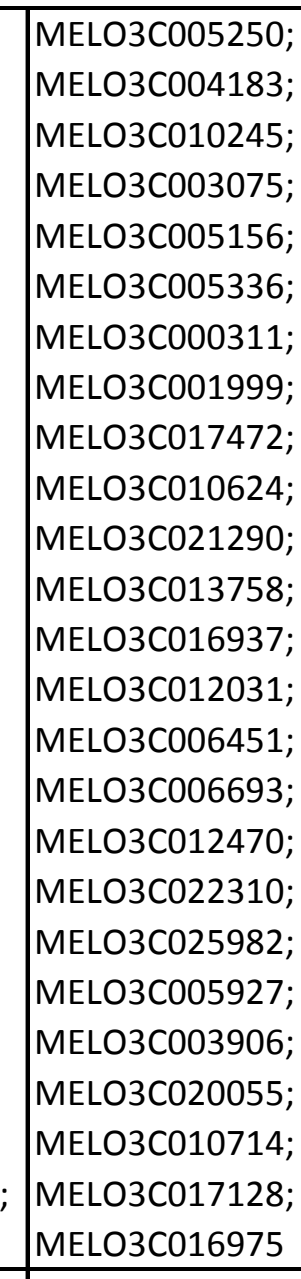 \\
\hline [] & GO:0043014 & $\begin{array}{l}\text { alpha-tubulin } \\
\text { binding }\end{array}$ & $\begin{array}{l}\text { MOLECULAR } \\
\text { FUNCTION }\end{array}$ & 1.0 & $\begin{array}{l}0.030684372 \\
877273734\end{array}$ & 1 & 2 & 176 & 16949 & MELO3C017720 & $\begin{array}{l}\text { MELO3C006171; } \\
\text { MELO3C011830 }\end{array}$ \\
\hline [] & GO:0004402 & \begin{tabular}{|l} 
\\
\\
histone \\
acetyltransferas \\
e activity
\end{tabular} & $\begin{array}{c}\text { MOLECULAR } \\
\text { FUNCTION }\end{array}$ & 1.0 & $\begin{array}{l}0.040593699 \\
43336301\end{array}$ & 2 & 29 & 175 & 16922 & \begin{tabular}{|l} 
\\
MELO3C026433; \\
MELO3C017030 \\
\end{tabular} & 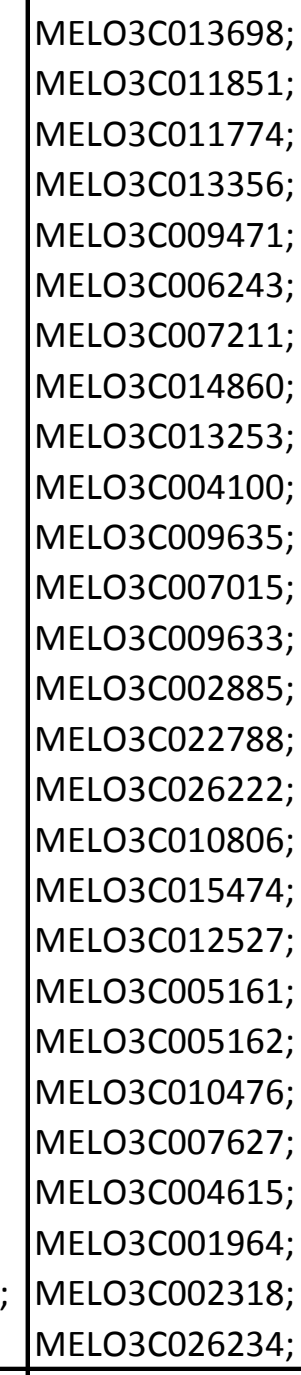 \\
\hline[] & GO:0000170 & \begin{tabular}{|l|} 
sphingosine \\
hydroxylase \\
activity
\end{tabular} & $\begin{array}{l}\text { MOLECULAR } \\
\text { FUNCTION }\end{array}$ & 1.0 & \begin{tabular}{|l}
0.020561718 \\
679675958
\end{tabular} & 1 & 1 & 176 & 16950 & MELO3C012088 & MELOЗС008543 \\
\hline [] & GO:0000774 & \begin{tabular}{|l|} 
adenyl- \\
nucleotide \\
exchange factor \\
activity
\end{tabular} & $\begin{array}{l}\text { MOLECULAR } \\
\text { FUNCTION }\end{array}$ & 1.0 & \begin{tabular}{|l}
0.030684372 \\
877273734
\end{tabular} & 1 & 2 & 176 & 16949 & MELO3C019156 & $\begin{array}{l}\text { MELOЗС014179; } \\
\text { MELO3C011373 }\end{array}$ \\
\hline [] & GO:0002039 & p53 binding & $\begin{array}{l}\text { MOLECULAR } \\
\text { FUNCTION }\end{array}$ & 1.0 & $\begin{array}{l}0.040702992 \\
789935\end{array}$ & 1 & 3 & 176 & 16948 & MELOЗC006760 & $\begin{array}{l}\text { MELO3C024469; } \\
\text { MELO3C011731; } \\
\text { MELO3C015367 }\end{array}$ \\
\hline [] & GO:0004473 & $\begin{array}{l}\text { malate } \\
\text { dehydrogenase } \\
\text { (decarboxylatin } \\
\text { g) (NADP+) } \\
\text { activity }\end{array}$ & $\begin{array}{l}\text { MOLECULAR } \\
\text { FUNCTION }\end{array}$ & 1.0 & $\begin{array}{l}0.040702992 \\
789935\end{array}$ & 1 & 3 & 176 & 16948 & MELO3C013000 & $\begin{array}{l}\text { MELO3C011129; } \\
\text { MELO3C015407; } \\
\text { MELO3C024441 }\end{array}$ \\
\hline
\end{tabular}




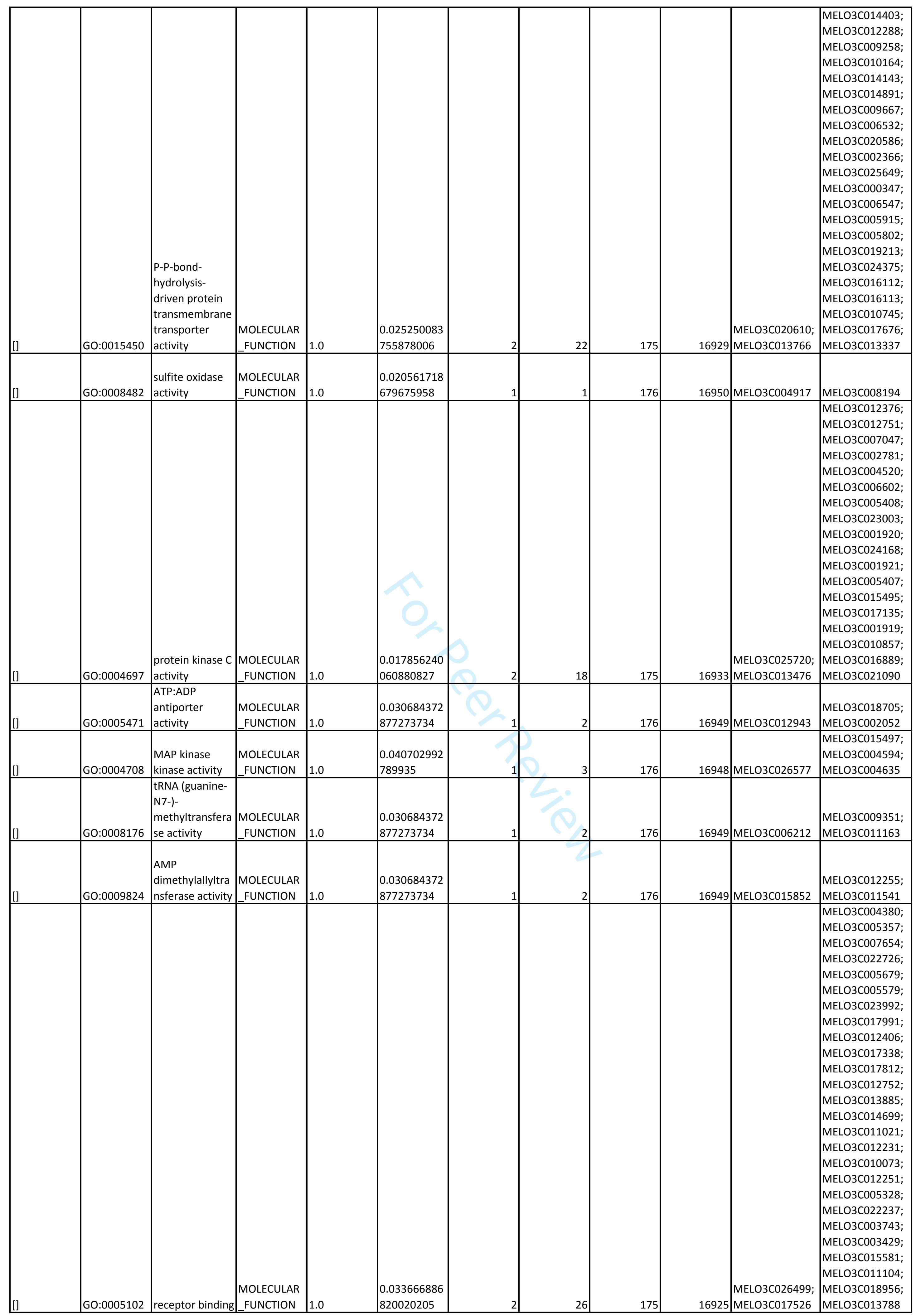




\begin{tabular}{|c|c|c|c|c|c|c|c|c|c|c|c|}
\hline [] & GO:0003712 & \begin{tabular}{|l|} 
transcription \\
cofactor activity
\end{tabular} & $\begin{array}{c}\text { MOLECULAR } \\
\text { FUNCTION }\end{array}$ & 1.0 & \begin{tabular}{|l}
0.024152859 \\
110298835
\end{tabular} & 3 & 57 & 174 & 16894 & $\begin{array}{l}\text { MELO3C005286; } \\
\text { MELO3CO21598; } \\
\text { MELO3C024474 }\end{array}$ & 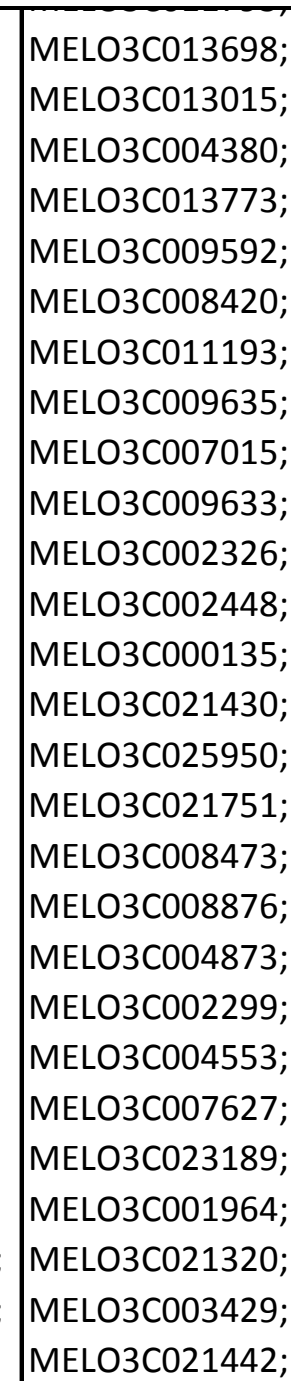 \\
\hline [] & GO:0070330 & \begin{tabular}{|l} 
aromatase \\
activity
\end{tabular} & $\begin{array}{l}\text { MOLECULAR } \\
\text { FUNCTION }\end{array}$ & 1.0 & $\begin{array}{l}0.031472058 \\
45062728\end{array}$ & 2 & 25 & 175 & 16926 & $\begin{array}{l}\text { MELO3CO21845; } \\
\text { MELO3C021846 }\end{array}$ & 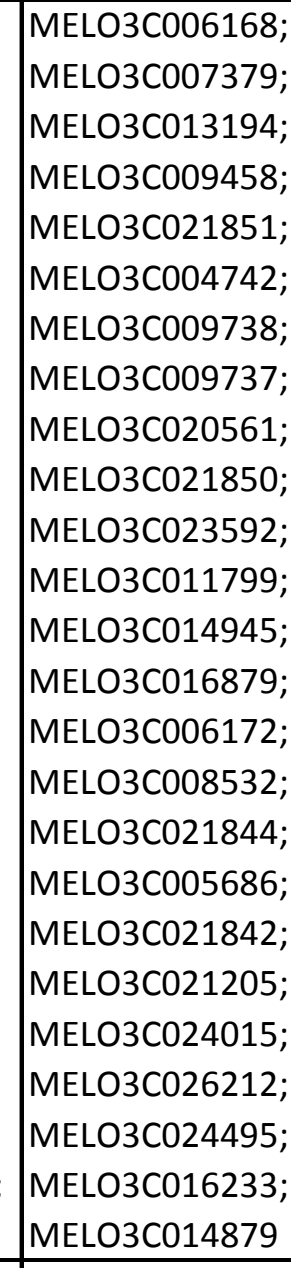 \\
\hline [] & GO:0003838 & \begin{tabular}{|l|} 
sterol 24-C- \\
methyltransfera \\
se activity
\end{tabular} & $\begin{array}{l}\text { MOLECULAR } \\
\text { FUNCTION }\end{array}$ & 1.0 & $\begin{array}{l}0.020561718 \\
679675958\end{array}$ & 1 & 1 & 176 & 16950 & MELO3C023989 & MELОЗС023990 \\
\hline [] & GO:0030750 & \begin{tabular}{|l|} 
putrescine N- \\
methyltransfera \\
se activity
\end{tabular} & $\begin{array}{c}\text { MOLECULAR } \\
\text { FUNCTION } \\
\end{array}$ & 1.0 & $\begin{array}{l}0.040702992 \\
789935\end{array}$ & 1 & 3 & 176 & 16948 & MELO3C012007 & $\begin{array}{l}\text { MELO3C008477; } \\
\text { MELO3C002252; } \\
\text { MELO3C005877 }\end{array}$ \\
\hline [] & GO:0052658 & \begin{tabular}{|l} 
inositol-1,4,5- \\
trisphosphate 5- \\
phosphatase \\
activity
\end{tabular} & $\begin{array}{l}\text { MOLECULAR } \\
\text { FUNCTION }\end{array}$ & 1.0 & \begin{tabular}{|l}
0.040702992 \\
789935
\end{tabular} & 1 & 3 & 176 & 16948 & MELO3CO09908 & $\begin{array}{l}\text { MELOЗС005242; } \\
\text { MELOЗCO13872; } \\
\text { MELO3C014293 }\end{array}$ \\
\hline [] & GO:0016851 & \begin{tabular}{|l|} 
magnesium \\
chelatase \\
activity
\end{tabular} & $\begin{array}{l}\text { MOLECULAR } \\
\text { FUNCTION }\end{array}$ & 1.0 & \begin{tabular}{|l|l|}
0.030684372 \\
877273734
\end{tabular} & 1 & 2. & 176 & 16949 & MELO3C023131 & $\begin{array}{l}\text { MELO3C007233; } \\
\text { MELO3C014571 }\end{array}$ \\
\hline [] & GO:0052659 & \begin{tabular}{|l} 
inositol-1,3,4,5- \\
tetrakisphospha \\
te 5- \\
phosphatase \\
activity \\
\end{tabular} & $\begin{array}{c}\text { MOLECULAR } \\
\text { FUNCTION }\end{array}$ & 1.0 & \begin{tabular}{|l|}
0.040702992 \\
789935 \\
\end{tabular} & 1 & 3 & 176 & 16948 & MELO3C009908 & $\begin{array}{l}\text { MELOЗС005242; } \\
\text { MELOЗC013872; } \\
\text { MELOЗC014293 }\end{array}$ \\
\hline [[] & GO:0016711 & \begin{tabular}{|l|} 
flavonoid 3'- \\
monooxygenase \\
activity
\end{tabular} & $\begin{array}{l}\text { MOLECULAR } \\
\text { FUNCTION }\end{array}$ & 1.0 & $\begin{array}{l}0.040702992 \\
789935\end{array}$ & 1 & 3 & 176 & 16948 & MELOЗ С022246 & $\begin{array}{l}\text { MELO3C005572; } \\
\text { MELO3C009741; } \\
\text { MELO3C017219 }\end{array}$ \\
\hline
\end{tabular}




\begin{tabular}{|c|c|c|c|c|c|c|c|c|c|c|c|}
\hline [] & GO:0000155 & \begin{tabular}{|l|l} 
& \\
\end{tabular} & $\begin{array}{l}\text { MOLECULAR } \\
\text { FUNCTION }\end{array}$ & 1.0 & $\begin{array}{l}0.031472058 \\
45062728 \\
\end{array}$ & 2 & 25 & 175 & 16926 & $\begin{array}{l}\text { MELO3C025720; } \\
\text { MELO3C013476 }\end{array}$ & $\begin{array}{l}\text { MELO3C005250; } \\
\text { MELO3C004183; } \\
\text { MELO3C012066; } \\
\text { MELO3C026506; } \\
\text { MELO3C021931; } \\
\text { MELO3C026502; } \\
\text { MELO3C024168; } \\
\text { MELO3C006749; } \\
\text { MELO3C002705; } \\
\text { MELO3C016660; } \\
\text { MELO3C013758; } \\
\text { MELO3C016937; } \\
\text { MELO3C005265; } \\
\text { MELO3C006451; } \\
\text { MELO3C005266; } \\
\text { MELO3C003205; } \\
\text { MELO3C006717; } \\
\text { MELO3C022310; } \\
\text { MELO3C025982; } \\
\text { MELO3C003906; } \\
\text { MELO3C020055; } \\
\text { MELO3C024196; } \\
\text { MELO3C015961; } \\
\text { MELO3C024195; } \\
\text { MELO3C011940 }\end{array}$ \\
\hline [] & GO:0050366 & $\begin{array}{l}\text { tyramine } \mathrm{N} \text { - } \\
\text { feruloyltransfer } \\
\text { ase activity }\end{array}$ & $\begin{array}{l}\text { MOLECULAR } \\
\text { FUNCTION }\end{array}$ & 1.0 & $\begin{array}{l}0.020561718 \\
679675958 \\
\end{array}$ & 1 & 1 & 176 & 16950 & MELO3C012418 & MELO3C012417 \\
\hline [] & GO:0016829 & Iyase activity & 列 & 1.0 & $\begin{array}{l}0.023729517 \\
07526035\end{array}$ & 0 & 406 & 177 & 16545 & & $\begin{array}{l}\text { MELO3C013057; } \\
\text { MELO3C003491; } \\
\text { MELO3C006520; } \\
\text { MELO3C002288; } \\
\text { MELO3C003259; } \\
\text { MELO3C026007; } \\
\text { MELO3C026005; } \\
\text { MELO3C026006; } \\
\text { MELO3C026004; } \\
\text { MELO3C024189; } \\
\text { MELO3C018622; } \\
\text { MELO3C014039; } \\
\text { MELO3C004110; } \\
\text { MELO3C007744; } \\
\text { MELO3C014392; } \\
\text { MELO3C004355; } \\
\text { MELO3C009809; } \\
\text { MELO3C019840; } \\
\text { MELO3C025168; } \\
\text { MELO3C016693; } \\
\text { MELO3C018994; } \\
\text { MELO3C010910; } \\
\text { MELO3C015368; } \\
\text { MELO3C016339; } \\
\text { MELO3C013152; } \\
\text { MELO3C011095; } \\
\text { MELO3C012184; }\end{array}$ \\
\hline [] & GO:0008897 & $\begin{array}{l}\text { holo-[acyl- } \\
\text { carrier-protein] } \\
\text { synthase } \\
\text { activity }\end{array}$ & $\begin{array}{l}\text { MOLECULAR } \\
\text { FUNCTION }\end{array}$ & 1.0 & $\begin{array}{l}0.020561718 \\
679675958 \\
\end{array}$ & 1 & 1 & 176 & 16950 & MELO3C012351 & MELO3C011647 \\
\hline [] & GO:0000254 & $\begin{array}{l}\text { C-4 } \\
\text { methylsterol } \\
\text { oxidase activity }\end{array}$ & $\begin{array}{l}\text { MOLECULAR } \\
\text { FUNCTION }\end{array}$ & 1.0 & \begin{tabular}{|l}
0.040702992 \\
789935 \\
\end{tabular} & 1 & 3 & 176 & 16948 & MELO3C012088 & $\begin{array}{l}\text { MELO3C008543; } \\
\text { MELO3C013991; } \\
\text { MELO3C020602 }\end{array}$ \\
\hline [] & GO:0003999 & $\begin{array}{l}\text { adenine } \\
\text { phosphoribosylt } \\
\text { ransferase } \\
\text { activity }\end{array}$ & $\begin{array}{l}\text { MOLECULAR } \\
\text { FUNCTION } \\
\end{array}$ & 1.0 & $\begin{array}{l}0.040702992 \\
789935\end{array}$ & 1 & 3 & 176 & 16948 & MELO3C011270 & $\begin{array}{l}\text { MELO3C010812; } \\
\text { MELO3C013962; } \\
\text { MELO3C005556 }\end{array}$ \\
\hline [] & GO:0071001 & U4/U6 snRNP & \begin{tabular}{|l|} 
CELLULAR_C \\
OMPONENT
\end{tabular} & 1.0 & \begin{tabular}{|l|l|}
0.020561718 \\
679675958 \\
\end{tabular} & 1 & 1 & 176 & 16950 & MELO3C026433 & MELO3C012489 \\
\hline [] & GO:0005965 & $\begin{array}{l}\text { protein } \\
\text { farnesyltransfer } \\
\text { ase complex }\end{array}$ & $\begin{array}{l}\text { CELLULAR_C } \\
\text { OMPONENT }\end{array}$ & 1.0 & $\begin{array}{l}0.020561718 \\
679675958\end{array}$ & 1 & 1 & 176 & 16950 & MELO3C017720 & MELO3C009957 \\
\hline [] & GO:0035060 & \begin{tabular}{|l|} 
brahma \\
complex
\end{tabular} & $\begin{array}{l}\text { CELLULAR_C } \\
\text { OMPONENT }\end{array}$ & 1.0 & $\begin{array}{l}0.020561718 \\
679675958\end{array}$ & 1 & 1 & 176 & 16950 & MELO3C006760 & MELO3C017295 \\
\hline & GO:0010445 & $\begin{array}{l}\text { nuclear dicing } \\
\text { body }\end{array}$ & \begin{tabular}{|l|} 
CELLULAR_C \\
OMPONENT \\
\end{tabular} & 1.0 & \begin{tabular}{|l|}
0.020561718 \\
679675958 \\
\end{tabular} & 1 & 1 & 176 & 16950 & MELO3C015627 & MELO3C005929 \\
\hline
\end{tabular}




\begin{tabular}{|c|c|c|c|c|c|c|c|c|c|c|c|}
\hline [] & GO:0009707 & 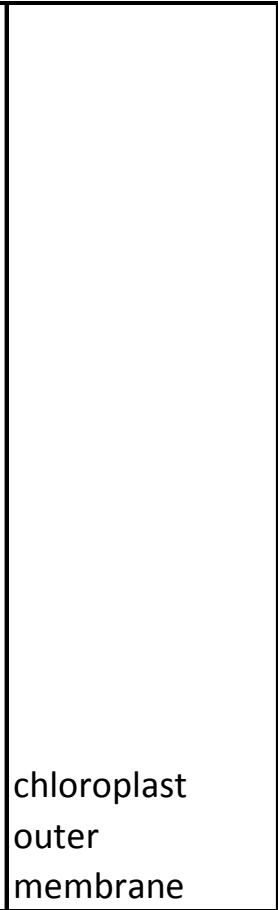 & 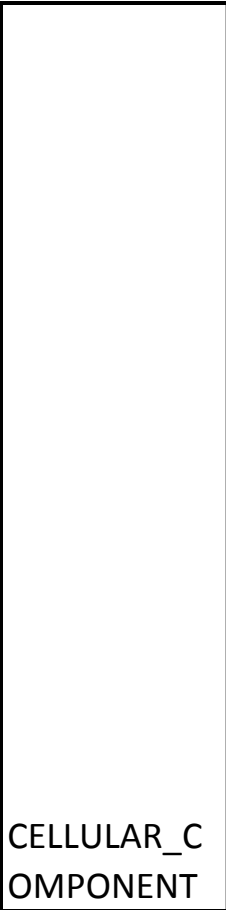 & 1.0 & $\begin{array}{l}0.017856240 \\
060880827\end{array}$ & 2 & 18 & 175 & 16933 & $\begin{array}{l}\text { MELO3C020610; } \\
\text { MELO3C013766 }\end{array}$ & $\begin{array}{l}\text { MELO3C015416; } \\
\text { MELO3C017968; } \\
\text { MELO3C006133; } \\
\text { MELO3C004471; } \\
\text { MELO3C016719; } \\
\text { MELO3C004591; } \\
\text { MELO3C004474; } \\
\text { MELO3C006974; } \\
\text { MELO3C002387; } \\
\text { MELO3C024587; } \\
\text { MELO3C021253; } \\
\text { MELO3C017360; } \\
\text { MELO3C018772; } \\
\text { MELO3C019874; } \\
\text { MELO3C014409; } \\
\text { MELO3C014605; } \\
\text { MELO3C011502; } \\
\text { MELO3C011415 }\end{array}$ \\
\hline [] & GO:0010007 & $\begin{array}{l}\text { magnesium } \\
\text { chelatase } \\
\text { complex }\end{array}$ & $\begin{array}{l}\text { CELLULAR_C } \\
\text { OMPONENT }\end{array}$ & 1.0 & $\begin{array}{l}0.020561718 \\
679675958 \\
\end{array}$ & 1 & 1 & 176 & 16950 & MELO3C023131 & MELO3C014571 \\
\hline [] & 更 & . & 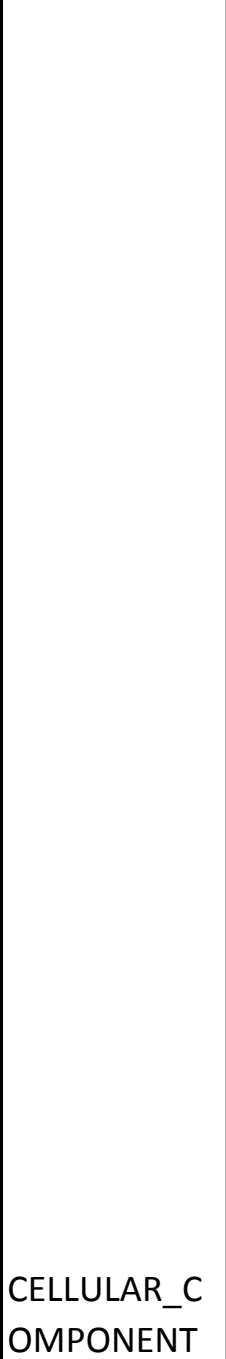 & 1.0 & $\begin{array}{l}0.038229114 \\
226002024\end{array}$ & 2 & 28 & 175 & $16923 \mid$ & $\begin{array}{l} \\
\text { MELO3C015627; } \\
\text { MELO3C022928 }\end{array}$ & $\begin{array}{l}\text { MELO3C012489; } \\
\text { MELO3C008488; } \\
\text { MELO3C004440; } \\
\text { MELO3C014086; } \\
\text { MELO3C005035; } \\
\text { MELO3C024822; } \\
\text { MELO3C002326; } \\
\text { MELO3C024028; } \\
\text { MELO3C016008; } \\
\text { MELO3C014823; } \\
\text { MELO3C005382; } \\
\text { MELO3C015826; } \\
\text { MELO3C013781; } \\
\text { MELO3C023809; } \\
\text { MELO3C017908; } \\
\text { MELO3C023427; } \\
\text { MELO3C024757; } \\
\text { MELO3C021320; } \\
\text { MELO3C017365; } \\
\text { MELO3C026275; } \\
\text { MELO3C019500; } \\
\text { MELO3C016115; } \\
\text { MELO3C012616; } \\
\text { MELO3C010637; } \\
\text { MELO3C019702; } \\
\text { MELO3C014459; } \\
\text { MELO3C012579 }\end{array}$ \\
\hline [] & GO:0005953 & \begin{tabular}{|l|} 
CAAX-protein \\
geranylgeranyltr \\
ansferase \\
complex
\end{tabular} & $\begin{array}{l}\text { CELLULAR_C } \\
\text { OMPONENT }\end{array}$ & 1.0 & $\begin{array}{l}0.020561718 \\
679675958\end{array}$ & 1 & 1 & 176 & 16950 & MELO3C017720 & MELO3C021654 \\
\hline [] & GO:0005925 & focal adhesion & $\begin{array}{l}\text { CELLULAR_C } \\
\text { OMPONENT }\end{array}$ & 1.0 & $\begin{array}{l}0.030684372 \\
877273734\end{array}$ & 1 & 2 & 176 & 16949 & MELO3C005976 & $\begin{array}{l}\text { MELO3C005382; } \\
\text { MELO3C007007 }\end{array}$ \\
\hline
\end{tabular}


Supplementary Table 2.A List of the Differentially Expressed Genes (2068 DEGs) only considering the postharvest ripening time in melon fruit during postharvest storage at $20.5^{\circ} \mathrm{C}$ and $88 \%$ relative humidity for $18 \mathrm{~d}$ of the near-isogenic line SC10-2 and its parental control 'Piel de Sapo' (PS). Gene Ontology terms (GO terms) of the six different clusters (named 2.x) obtained according to the differentially expressed gene.

\begin{tabular}{|c|c|c|c|}
\hline Gene ID & pval & $\overline{\text { qval }}$ & Description \\
\hline MELO3C010748 & $5,90 \mathrm{E}-12$ & $1,16 \mathrm{E}-07$ & Heat shock transcription factor \\
\hline MELO3C005540 & $1,88 \mathrm{E}-11$ & $1,86 \mathrm{E}-07$ & $14 \mathrm{kDa}$ proline-rich protein dc2.15 \\
\hline MELO3C023889 & $7,79 \mathrm{E}-11$ & $5,13 \mathrm{E}-07$ & Ultraviolet-B receptor UVR8 \\
\hline MELO3C003298 & $6,24 \mathrm{E}-10$ & $2,94 \mathrm{E}-06$ & high mobility group B protein 1 \\
\hline MELO3C018265 & $7,45 E-10$ & $2,94 \mathrm{E}-06$ & ADP-ribosylation factor GTPase-activating protein \\
\hline MELO3C015733 & $1,56 \mathrm{E}-09$ & $5,14 \mathrm{E}-06$ & Pre-mRNA-splicing factor ATP-dependent RNA helicase \\
\hline MELO3C003282 & $3,84 \mathrm{E}-09$ & $1,08 \mathrm{E}-05$ & cleft lip and palate transmembrane protein 1 homolog \\
\hline MELO3C008669 & $1,05 \mathrm{E}-08$ & $2,60 \mathrm{E}-05$ & RING-type E3 ubiquitin transferase \\
\hline MELO3C026279 & $1,50 \mathrm{E}-08$ & $3,29 \mathrm{E}-05$ & cell division cycle protein 48 homolog \\
\hline MELO3C018038 & $2,96 \mathrm{E}-08$ & $4,66 \mathrm{E}-05$ & proline iminopeptidase \\
\hline MELO3C007022 & $3,77 \mathrm{E}-08$ & $4,66 \mathrm{E}-05$ & UDP-N-acetylglucosamine diphosphorylase 2-like \\
\hline MELO3C022036 & $3,28 \mathrm{E}-08$ & $4,66 \mathrm{E}-05$ & protein LONGIFOLIA 2 \\
\hline MELO3C015789 & $2,62 \mathrm{E}-08$ & $4,66 \mathrm{E}-05$ & sucrose-binding protein-like \\
\hline MELO3C023342 & $2,79 \mathrm{E}-08$ & $4,66 \mathrm{E}-05$ & kinesin-related protein 11-like \\
\hline MELO3C002468 & $3,61 \mathrm{E}-08$ & $4,66 \mathrm{E}-05$ & Tobamovirus multiplication protein 1 \\
\hline MELO3C003752 & $3,53 \mathrm{E}-08$ & $4,66 \mathrm{E}-05$ & gibberellin-regulated protein 11-like \\
\hline MELO3C003570 & $4,19 \mathrm{E}-08$ & 4,87E-05 & Protein ROOT PRIMORDIUM DEFECTIVE 1 \\
\hline MELO3C006028 & $5,64 \mathrm{E}-08$ & $5,45 \mathrm{E}-05$ & cyclic nucleotide-gated ion channel 1 \\
\hline MELO3C021108 & $5,62 \mathrm{E}-08$ & $5,45 \mathrm{E}-05$ & Peptidylprolyl isomerase \\
\hline MELO3C002508 & $5,38 \mathrm{E}-08$ & $5,45 \mathrm{E}-05$ & thioredoxin-like protein CXXS1 \\
\hline MELO3C024028 & $5,80 \mathrm{E}-08$ & $5,45 \mathrm{E}-05$ & DEAD-box ATP-dependent RNA helicase \\
\hline MELO3C004023 & $7,06 \mathrm{E}-08$ & $6,18 \mathrm{E}-05$ & NADPH--cytochrome $\mathrm{P} 450$ reductase \\
\hline MELO3C019973 & $7,20 \mathrm{E}-08$ & $6,18 \mathrm{E}-05$ & 2-C-methyl-D-erythritol 4-phosphate cytidylyltransferase \\
\hline MELO3C016073 & $8,21 \mathrm{E}-08$ & $6,49 \mathrm{E}-05$ & Tudor/PWWP/MBT superfamily protein \\
\hline MELO3C001317 & $8,13 \mathrm{E}-08$ & $6,49 E-05$ & Coiled-coil protein (DUF572) \\
\hline MELO3C003905 & $9,86 \mathrm{E}-08$ & 7,31E-05 & glycine--tRNA ligase, mitochondrial 1 \\
\hline MELO3C003444 & $9,99 E-08$ & 7,31E-05 & transcription factor IWS1 \\
\hline MELO3C022213 & $1,09 \mathrm{E}-07$ & $7,43 \mathrm{E}-05$ & Myb family transcription factor family protein \\
\hline MELO3C022533 & $1,07 \mathrm{E}-07$ & $7,43 \mathrm{E}-05$ & shaggy-related protein kinase theta \\
\hline MELO3C023449 & $1,40 \mathrm{E}-07$ & $9,21 \mathrm{E}-05$ & UPF0481 plant-like protein \\
\hline MELO3C016475 & $1,70 \mathrm{E}-07$ & 0,000104363 & Chloride channel protein \\
\hline MELO3C024278 & $1,74 \mathrm{E}-07$ & 0,000104363 & Mitochondrial carrier protein \\
\hline MELO3C012356 & $1,74 \mathrm{E}-07$ & 0,000104363 & Aspartyl aminopeptidase \\
\hline MELO3C023146 & $2,03 \mathrm{E}-07$ & 0,000104556 & villin-2-like \\
\hline MELO3C025783 & $1,81 \mathrm{E}-07$ & 0,000104556 & receptor-like serine/threonine-protein kinase NCRK \\
\hline MELO3C010244 & $2,06 \mathrm{E}-07$ & 0,000104556 & Temperature-induced lipocalin \\
\hline MELO3C017271 & $2,05 E-07$ & 0,000104556 & Acetyl-coenzyme A synthetase \\
\hline MELO3C017213 & $1,95 \mathrm{E}-07$ & 0,000104556 & UDP-glucose 6-dehydrogenase \\
\hline MELO3C003563 & $1,86 \mathrm{E}-07$ & 0,000104556 & No data found \\
\hline MELO3C014061 & $2,13 \mathrm{E}-07$ & 0,000105194 & WPP domain interacting protein, putative \\
\hline MELO3C004433 & $2,20 \mathrm{E}-07$ & 0,000106054 & calcium uptake protein 1 , mitochondrial-like isoform X1 \\
\hline MELO3C013868 & $2,32 \mathrm{E}-07$ & 0,000107032 & Cytochrome P450 family ent-kaurenoic acid oxidase \\
\hline MELO3C019616 & $2,33 \mathrm{E}-07$ & 0,000107032 & E3 ubiquitin-protein ligase RGLG2 \\
\hline MELO3C006120 & $2,88 \mathrm{E}-07$ & 0,000129143 & MYB-related transcription factor \\
\hline MELO3C004799 & $3,03 \mathrm{E}-07$ & 0,000132974 & splicing factor U2af small subunit B-like \\
\hline MELO3C017380 & $3,28 \mathrm{E}-07$ & 0,000141013 & zinc finger $\mathrm{CCHC}$ domain-containing protein 7 isoform $\mathrm{X} 3$ \\
\hline MELO3C006297 & $3,64 \mathrm{E}-07$ & 0,000148781 & RNA recognition motif (RRM) containing protein \\
\hline MELO3C012728 & $3,67 \mathrm{E}-07$ & 0,000148781 & Vacuolar sorting-associated protein 2-like protein \\
\hline MELO3C023559 & $3,89 \mathrm{E}-07$ & 0,000148781 & 40 S ribosomal protein $\mathrm{S} 27$ \\
\hline MELO3C003812 & $3,77 \mathrm{E}-07$ & 0,000148781 & Glucose-1-phosphate adenylyltransferase \\
\hline MELO3C012788 & $3,82 \mathrm{E}-07$ & 0,000148781 & Transcription initiation factor IIF alpha subunit family protein \\
\hline MELO3C012930 & $3,92 \mathrm{E}-07$ & 0,000148781 & Zinc knuckle family protein \\
\hline MELO3C013136 & $4,05 E-07$ & 0,000150962 & GDP-L-galactose phosphorylase 1 \\
\hline MELO3C015720 & $4,16 \mathrm{E}-07$ & 0,000152121 & Smr (Small MutS-related) domain protein \\
\hline MELO3C022416 & $4,70 \mathrm{E}-07$ & 0,000168621 & Ubiquitin family protein \\
\hline MELO3C003875 & $5,08 \mathrm{E}-07$ & 0,000179224 & Protein phosphatase $2 c$, putative \\
\hline MELO3C016754 & $6,18 \mathrm{E}-07$ & 0,000214273 & Cold regulated gene 27 , putative isoform 3 \\
\hline MELO3C004194 & $6,44 \mathrm{E}-07$ & 0,000215458 & calreticulin \\
\hline MELO3C022408 & $6,40 \mathrm{E}-07$ & 0,000215458 & UV-stimulated scaffold protein A homolog \\
\hline MELO3C013622 & $6,73 \mathrm{E}-07$ & 0,000217727 & mannan endo-1,4-beta-mannosidase 2 \\
\hline MELO3C011379 & $6,66 \mathrm{E}-07$ & 0,000217727 & Chaperone protein dnaJ \\
\hline MELO3C024626 & $7,24 \mathrm{E}-07$ & 0,000230696 & Protein LIKE COV 1 \\
\hline MELO3C000452 & $8,00 \mathrm{E}-07$ & 0,000243073 & Coiled-coil protein (DUF572) \\
\hline MELO3C021903 & $7,87 \mathrm{E}-07$ & 0,000243073 & Alpha/beta hydrolase-3 \\
\hline MELO3C002485 & $7,94 \mathrm{E}-07$ & 0,000243073 & Transmembrane protein, putative \\
\hline MELO3C022066 & $8,16 \mathrm{E}-07$ & 0,000244276 & Zinc finger $\mathrm{CCHC}$ domain-containing protein 8 \\
\hline MELO3C003448 & $8,87 \mathrm{E}-07$ & 0,000261442 & Methyltransferase-related family protein \\
\hline MELO3C003331 & $9,74 \mathrm{E}-07$ & 0,0002828 & BAX inhibitor-1 \\
\hline MELO3C012149 & $1,01 \mathrm{E}-06$ & 0,000288475 & PLASMODESMATA CALLOSE-BINDING PROTEIN 3 \\
\hline MELO3C013183 & $1,07 E-06$ & 0,00029567 & elongation factor-like GTPase 1 \\
\hline MELO3C006997 & $1,05 \mathrm{E}-06$ & 0,00029567 & galactan beta-1,4-galactosyltransferase GALS3-like \\
\hline MELO3C021100 & $1,08 \mathrm{E}-06$ & 0,00029567 & Heat shock $70 \mathrm{kDa}$ protein \\
\hline MELO3C024188 & $1,12 \mathrm{E}-06$ & 0,000299785 & ribulose-1,5 bisphosphate carboxylase/oxygenase large subunit $\mathrm{N}$-methyltransferase, chloroplastic \\
\hline MELO3C009091 & $1,15 \mathrm{E}-06$ & 0,000299785 & $50 \mathrm{~S}$ ribosomal protein $\mathrm{L} 17$ \\
\hline
\end{tabular}




\begin{tabular}{|c|c|c|c|}
\hline MELO3C014359 & 1,17E-06 & 0,000299785 & Calcium-binding EF-hand family protein \\
\hline MELO3C007735 & 1,15E-06 & 0,000299785 & E3 ubiquitin-protein ligase RING1 \\
\hline MELO3C022511 & $1,15 \mathrm{E}-06$ & 0,000299785 & synaptotagmin-5 \\
\hline MELO3C013988 & $1,23 \mathrm{E}-06$ & 0,000311097 & Myelodysplasia-myeloid leukemia factor 1-interacting protein \\
\hline MELO3C013712 & $1,34 \mathrm{E}-06$ & 0,000334119 & Fact complex subunit spt16 \\
\hline MELO3C006921 & 1,37E-06 & 0,000338067 & No data found \\
\hline MELO3C014555 & 1,47E-06 & 0,000358476 & splicing factor 3B subunit 6-like protein \\
\hline MELO3C018149 & 1,51E-06 & 0,000364822 & Beta-glucosidase, putative \\
\hline MELO3C002104 & $1,54 \mathrm{E}-06$ & 0,000366033 & Receptor-like kinase \\
\hline MELO3C018525 & $1,56 \mathrm{E}-06$ & 0,00036677 & Myosin heavy chain-like protein \\
\hline MELO3C005069 & $1,60 \mathrm{E}-06$ & 0,00037138 & GEM-like protein 4 \\
\hline MELO3C026068 & $1,65 \mathrm{E}-06$ & 0,000379411 & Transcriptional adapter ADA2b-like protein \\
\hline MELO3C017897 & 1,77E-06 & 0,000398202 & Plant/protein \\
\hline MELO3C015804 & 1,77E-06 & 0,000398202 & heat stress transcription factor $\mathrm{A}-6 \mathrm{~b}$-like isoform $\mathrm{X} 1$ \\
\hline MELO3C022210 & $1,96 \mathrm{E}-06$ & 0,000434452 & regulator of nonsense transcripts UPF3-like \\
\hline MELO3C007072 & $2,12 \mathrm{E}-06$ & 0,000465214 & Caffeoylshikimate esterase \\
\hline MELO3C008069 & $2,15 \mathrm{E}-06$ & 0,000466976 & Phosphoinositide phosphatase family protein \\
\hline MELO3C005382 & $2,26 \mathrm{E}-06$ & 0,000478687 & luc7-like protein 3 \\
\hline MELO3C024270 & $2,28 \mathrm{E}-06$ & 0,000478687 & Terpene cyclase/mutase family member \\
\hline MELO3C020612 & 2,30E-06 & 0,000478687 & Unknown protein \\
\hline MELO3C018644 & $2,26 \mathrm{E}-06$ & 0,000478687 & serine/arginine-rich splicing factor SC35 \\
\hline MELO3C001976 & $2,35 \mathrm{E}-06$ & 0,000483125 & Pentatricopeptide repeat-containing family protein \\
\hline MELO3C023857 & $2,40 \mathrm{E}-06$ & 0,000488711 & GATA zinc finger domain-containing protein 10 -like isoform $X 2$ \\
\hline MELO3C002674 & 2,57E-06 & 0,000518413 & BnaA04g24650D protein \\
\hline MELO3C015067 & $2,65 \mathrm{E}-06$ & 0,000527841 & transcription factor LHW \\
\hline MELO3C021462 & $2,70 \mathrm{E}-06$ & 0,000532957 & DDT domain-containing protein PTM \\
\hline MELO3C024775 & $2,85 \mathrm{E}-06$ & 0,000552422 & Bud13 \\
\hline MELO3C002056 & 2,83E-06 & 0,000552422 & O-fucosyltransferase family protein \\
\hline MELO3C005952 & $3,12 \mathrm{E}-06$ & 0,000597548 & $60 \mathrm{~S}$ acidic ribosomal protein $\mathrm{P} 1$-like \\
\hline MELO3C026898 & $3,24 \mathrm{E}-06$ & 0,000614621 & Cathepsin B-like cysteine protease \\
\hline MELO3C022204 & $3,44 \mathrm{E}-06$ & 0,000647494 & SART-1 family protein DOT2 isoform X2 \\
\hline MELO3C009579 & $3,54 \mathrm{E}-06$ & 0,000647539 & Myosin-binding protein 7 \\
\hline MELO3C025257 & $3,54 \mathrm{E}-06$ & 0,000647539 & KH domain-containing family protein \\
\hline MELO3C005301 & $3,52 \mathrm{E}-06$ & 0,000647539 & At1g70780 \\
\hline MELO3C014083 & $3,77 \mathrm{E}-06$ & 0,000680058 & Transmembrane protein, putative \\
\hline MELO3C025488 & $3,79 \mathrm{E}-06$ & 0,000680058 & transcription initiation factor TFIID subunit 1-like \\
\hline MELO3C025502 & $3,82 \mathrm{E}-06$ & 0,000680058 & Pentatricopeptide repeat-containing protein \\
\hline MELO3C015322 & $3,94 \mathrm{E}-06$ & 0,000689115 & cyclin-L1-1 \\
\hline MELO3C011284 & $3,94 \mathrm{E}-06$ & 0,000689115 & Aspartate aminotransferase \\
\hline MELO3C017093 & $4,18 \mathrm{E}-06$ & 0,000723327 & Kinesin light chain \\
\hline MELO3C009482 & 4,24E-06 & 0,000725607 & Rho GTPase-activating protein \\
\hline MELO3C023408 & $4,26 \mathrm{E}-06$ & 0,000725607 & Lactoylglutathione lyase \\
\hline MELO3C009461 & $4,48 \mathrm{E}-06$ & 0,000737581 & Protein LITTLE ZIPPER 4 \\
\hline MELO3C020244 & $4,48 \mathrm{E}-06$ & 0,000737581 & UTP--glucose-1-phosphate uridylyltransferase \\
\hline MELO3C002302 & $4,42 \mathrm{E}-06$ & 0,000737581 & Kinase family protein \\
\hline MELO3C019941 & $4,38 \mathrm{E}-06$ & 0,000737581 & T-complex protein 11 \\
\hline MELO3C007207 & $4,57 \mathrm{E}-06$ & 0,000746373 & Kinase family protein \\
\hline MELO3C013630 & $4,62 \mathrm{E}-06$ & 0,000748551 & switch 2 isoform X1 \\
\hline MELO3C006383 & $5,10 \mathrm{E}-06$ & 0,000812025 & acid phosphatase 1-like \\
\hline MELO3C006836 & 5,09E-06 & 0,000812025 & Activating transcription factor 7-interacting 2 \\
\hline MELO3C016101 & 5,14E-06 & 0,000812025 & UBP1-associated protein $2 \mathrm{C}$ \\
\hline MELO3C017908 & $5,32 \mathrm{E}-06$ & 0,000827253 & No data found \\
\hline MELO3C020989 & $5,28 \mathrm{E}-06$ & 0,000827253 & phosphatidylinositol 4-kinase alpha 1 \\
\hline MELO3C010295 & $5,46 \mathrm{E}-06$ & 0,000841847 & Armadillo-like helical \\
\hline MELO3C017364 & $5,64 \mathrm{E}-06$ & 0,000856673 & Ribosomal protein L19 \\
\hline MELO3C023986 & $5,61 \mathrm{E}-06$ & 0,000856673 & DNA topoisomerase 2 \\
\hline MELO3C002700 & $5,84 \mathrm{E}-06$ & 0,000880994 & Serine/threonine-protein kinase PLK4 \\
\hline MELO3C016366 & $5,94 \mathrm{E}-06$ & 0,000888531 & Phosphatidylcholine transfer protein \\
\hline MELO3C018948 & $5,99 \mathrm{E}-06$ & 0,000889151 & Alpha-1,4 glucan phosphorylase \\
\hline MELO3C024262 & $6,09 E-06$ & 0,000897106 & activating signal cointegrator 1 complex subunit 1 \\
\hline MELO3C014178 & $6,21 \mathrm{E}-06$ & 0,000905978 & Trigger factor \\
\hline MELO3C007432 & $6,24 \mathrm{E}-06$ & 0,000905978 & Phosphatidate cytidylyltransferase \\
\hline MELO3C007139 & $6,36 \mathrm{E}-06$ & 0,000909299 & la-related protein $6 \mathrm{~A}$ \\
\hline MELO3C007499 & $6,35 \mathrm{E}-06$ & 0,000909299 & protein DA1-related 1-like \\
\hline MELO3C021534 & $6,54 \mathrm{E}-06$ & 0,000909299 & homeobox-leucine zipper protein HAT5-like \\
\hline MELO3C024447 & $6,54 \mathrm{E}-06$ & 0,000909299 & Protein SEC13 like \\
\hline MELO3C012136 & $6,51 \mathrm{E}-06$ & 0,000909299 & Nodulin-related protein 1 \\
\hline MELO3C020875 & $6,61 \mathrm{E}-06$ & 0,000909299 & CLK4-associating serine/arginine-rich protein \\
\hline MELO3C022342 & $6,63 \mathrm{E}-06$ & 0,000909299 & NAC domain-containing protein 83 \\
\hline MELO3C002148 & $6,45 \mathrm{E}-06$ & 0,000909299 & NF-X1-type zinc finger protein NFXL1 \\
\hline MELO3C006107 & $6,91 \mathrm{E}-06$ & 0,000940831 & RNA polymerase I-specific transcription initiation factor RRN3 \\
\hline MELO3C009423 & 7,01E-06 & 0,000948429 & Uridylate kinase \\
\hline MELO3C003915 & $7,08 \mathrm{E}-06$ & 0,000950539 & Pentatricopeptide repeat-containing protein \\
\hline MELO3C013703 & $7,12 \mathrm{E}-06$ & 0,000950539 & diacylglycerol O-acyltransferase 2-like \\
\hline MELO3C009276 & 7,30E-06 & 0,00096137 & Acyl-CoA N-acyltransferase with RING/FYVE/PHD-type zinc finger domain-containing protein \\
\hline MELO3C005731 & $7,29 \mathrm{E}-06$ & 0,00096137 & Intracellular protein transport protein USO1-like protein \\
\hline MELO3C017657 & $7,42 \mathrm{E}-06$ & 0,000970741 & branchpoint-bridging protein \\
\hline MELO3C008933 & 7,84E-06 & 0,001018808 & Zinc finger family protein \\
\hline MELO3C004591 & $8,11 \mathrm{E}-06$ & 0,001046869 & Phosphotransferase \\
\hline MELO3C008910 & $8,25 \mathrm{E}-06$ & 0,001058188 & transcription termination factor MTEF18, mitochondrial-like \\
\hline
\end{tabular}


MELO3C009591

MELO3C006959

MELO3C014174

MELO3C006602

MELO3C017677

MELO3C025526

MELO3C01111

MELO3C024862

MELO3C014649

MELO3C023188

MELO3C009187

MELO3C005211

MELO3C011825

MELO3C01174

MELO3C005262

MELO3C007075

MELO3C013128

MELO3C022707

MELO3C008520

MELO3C017856

MELO3C007069

MELO3C025855

MELO3C017521

MELO3C010119

MELO3C012196

MELO3C011271

MELO3C01392

MELO3C018053

MELO3C016877

MELO3C013369

MELO3C02545

MELO3C01302

MELO3C004278

MELO3C020701

MELO3C013123

MELO3C025079

MELO3C026613

MELO3C02506

MELO3C023417

MELO3C000817

MELO3C006260

MELO3C009296

MELO3C023513

MELO3C006926

MELO3C025977

MELO3C007177

MELO3C017474

MELO3C021398

MELO3C01654

MELO3C02484

MELO3C025256

MELO3C019633

MELO3C026235

MELO3C014575

MELO3C00708

MELO3C025282

MELO3C022499

MELO3C011324

MELO3C002677

MELO3C014530

MELO3C004492

MELO3C020109

MELO3C015263

MELO3C015197

MELO3C025345

MELO3C026090

MELO3C008330

MELO3C014749

MELO3C007033

MELO3C013248

MELO3C023526

MELO3C001987

MELO3C02662

MELO3C00222

MELO3C015277

MELO3C016380

MELO3C003506

MELO3C013366

MELO3C010997

MELO3C020444
8,33E-06

$8,45 \mathrm{E}-06$

$8,41 \mathrm{E}-06$

$8,54 \mathrm{E}-06$

$8,66 \mathrm{E}-06$

$8,87 \mathrm{E}-06$

$8,88 \mathrm{E}-06$

$9,68 \mathrm{E}-06$

9,79E-06

$9,81 \mathrm{E}-06$

$1,01 \mathrm{E}-05$

$1,00 \mathrm{E}-05$

$1,02 \mathrm{E}-05$

$1,01 \mathrm{E}-05$

$1,04 \mathrm{E}-05$

$1,06 \mathrm{E}-05$

$1,09 \mathrm{E}-05$

$1,09 \mathrm{E}-05$

1,13E-05

$1,13 \mathrm{E}-05$

$1,12 \mathrm{E}-05$

1,12E-05

$1,13 \mathrm{E}-05$

1,15E-05

$1,15 \mathrm{E}-05$

$1,18 \mathrm{E}-05$

1,20E-05

$1,21 \mathrm{E}-05$

$1,22 \mathrm{E}-05$

$1,24 \mathrm{E}-05$

$1,23 \mathrm{E}-05$

$1,24 \mathrm{E}-05$

$1,25 \mathrm{E}-05$

$1,24 \mathrm{E}-05$

$1,27 \mathrm{E}-05$

$1,27 \mathrm{E}-05$

$1,26 \mathrm{E}-05$

$1,31 \mathrm{E}-05$

$1,31 \mathrm{E}-05$

$1,30 \mathrm{E}-05$

$1,32 \mathrm{E}-05$

$1,35 \mathrm{E}-05$

$1,35 \mathrm{E}-05$

$1,39 \mathrm{E}-05$

1,44E-05

$1,45 \mathrm{E}-05$

$1,47 \mathrm{E}-05$

$1,49 \mathrm{E}-05$

$1,55 \mathrm{E}-05$

1,52E-05

$1,54 \mathrm{E}-05$

$1,54 \mathrm{E}-05$

$1,54 \mathrm{E}-05$

$1,61 \mathrm{E}-05$

1,61E-05

$1,61 \mathrm{E}-05$

$1,59 \mathrm{E}-05$

$1,61 \mathrm{E}-05$

$1,63 \mathrm{E}-05$

$1,68 \mathrm{E}-05$

$1,69 \mathrm{E}-05$

$1,68 \mathrm{E}-05$

$1,67 \mathrm{E}-05$

$1,68 \mathrm{E}-05$

$1,68 \mathrm{E}-05$

$1,71 \mathrm{E}-05$

$1,75 \mathrm{E}-05$

1,81E-05

$1,81 \mathrm{E}-05$

$1,85 \mathrm{E}-05$

$1,82 \mathrm{E}-05$

$1,84 \mathrm{E}-05$

$1,84 \mathrm{E}-05$

$1,86 \mathrm{E}-05$

$1,88 \mathrm{E}-05$

1,90E-05

$1,91 \mathrm{E}-05$

$1,94 \mathrm{E}-05$

$1,97 \mathrm{E}-05$

2,03E-05
0,001061017

0,001062493

0,001062493

0,001066875

0,001075323

0,001089274

0,001089274

0,001180217

0,001181894

0,001181894

0,001194634

0,001194634

0,001194634

0,001194634

0,001220292

0,001234544

0,001251957

0,001251957

0,001262069

0,001262069

0,001262069

0,001262069

0,001262069

0,001270454

0,001273125

0,001298592

0,001311679

0,001311679

0,001313028

0,001313028

0,001313028

0,001313028

0,00131307

0,00131307

0,001314742

0,001314742

0,001314742

0,001334848

0,001334848

0,001334848

0,001336381

0,00135638

0,00135638

0,001386896

0,001425409

0,001426946

0,001441788

0,00145805

0,001475351

0,001475351

0,001475351

0,001475351

0,001475351

0,001502947

0,001502947

0,001502947

0,001502947

0,001502947

0,001510878

0,001520089

0,001520089

0,001520089

0,001520089

0,001520089

0,001520089

0,001534494

0,001565386

0,001606901

0,001606901

0,00160794

0,00160794

0,00160794

0,00160794

0,00161177

0,001621496

0,001633533

0,001636029

0,001654622

0,001670415

0,00171292

domain-containing protein IQM6

Reticulon-like protein

(S)-ureidoglycine aminohydrolase

serine/threonine-protein kinase RUNKEL

itinase-like protein 1

ollen Ole e 1 allergen/extensin

receptor-like protein kinase HSL1

ant peroxidase

etal tolerance protein $\mathrm{C} 2$

-galactosidase

Transferase family protein

Zinc finger, $\mathrm{CCCH}$-type

ifU-like protein 2, chloroplastic

Protein FAR1-RELATED SEQUENCE 5

PLATZ transcription factor family protein, putative

ADP-ribosylation factor GTPase-activating protein AGD12

Golgin family A protein

biquitin-conjugating enzyme family protein

Cysteine protease

(lensin-like

Protein phosphatase 2c, putative

Alkyl transferase

data found

U11/U12 small nuclear ribonucleoprotein $48 \mathrm{kDa}$ protein

Phospholipid-transporting ATPase

$4 \mathrm{kDa}$ proline-rich protein DC2.15

Myb transcription factor

Costars family protein At4g 33640

Beta-fructofuranosidase, insoluble isoenzyme CWINV1

Methyltransferase-like protein

Actin-depolymerizing factor family protein

Serine/threonine-protein kinase ATM

mevalonate kinase-like

Myb transcription factor

Syntaxin-51

universal stress protein A-like protein

Tubulin alpha chain

$V$-type proton ATPase subunit $C$

DUF4050 family protein

Magnesium transporter MRS2-like protein

Dynamin, putative

Energy-coupling factor transporter ATP-binding EcfA 1

Metal tolerance protein C2

Divalent ion symporter

OBERON-like protein

Transcription factor GTE12

Zinc finger protein

serine incorporator 3

Peroxisomal membrane $22 \mathrm{kDa}$ (Mpv17/PMP22) family protein 
MELO3C017620

MELO3C024407

MELO3C020780

MELO3C014678

MELO3C006313

MELO3C021113

MELO3C025859

MELO3C007825

MELO3C02578

MELO3C021782

MELO3C004305

MELO3C011160

MELO3C016552

MELO3C011089

MELO3C016074

MELO3C025887

MELO3C009595

MELO3C022721

MELO3C017346

MELO3C007673

MELO3C013383

MELO3C024514

MELO3C021587

MELO3C014507

MELO3C004641

MELO3C013104

MELO3C017749

MELO3C007609

MELO3C001175

MELO3C017813

MELO3C015327

MELO3C00238

MELO3C002115

MELO3C011422

MELO3C015734

MELO3C022647

MELO3C013870

MELO3C007265

MELO3C006929

MELO3C026502

MELO3C015439

MELO3C011285

MELO3C006736

MELO3C011144

MELO3C02057O

MELO3C014009

MELO3C025111

MELO3C004669

MELO3C006493

MELO3C00455

MELO3C008046

MELO3C016002

MELO3C013664

MELO3C022436

MELO3C02610

MELO3C008690

MELO3C011843

MELO3C010136

MELO3C014019

MELO3C00558

MELO3C021786

MELO3C025956

MELO3C017095

MELO3C009339

MELO3C008596

MELO3C021119

MELO3C011003

MELO3C019730

MELO3C009827

MELO3C019522

MELO3C026239

MELO3C012737

MELO3C02078

MELO3C009967

MELO3C006705

MELO3C02120

MELO3C024863

MELO3C009222

MELO3C015594

MELO3C015603
2,06E-05

2,10E-05

2,10E-05

2,13E-05

2,12E-05

2,11E-05

2,14E-05

2,17E-05

2,16E-05

2,16E-05

2,19E-05

2,21E-05

$2,23 \mathrm{E}-05$

2,22E-05

2,29E-05

$2,29 \mathrm{E}-05$

2,32E-05

$2,32 \mathrm{E}-05$

2,34E-05

2,35E-05

$2,37 \mathrm{E}-05$

2,42E-05

$2,42 \mathrm{E}-05$

$2,49 \mathrm{E}-05$

2,56E-05

2,57E-05

2,58E-05

$2,56 \mathrm{E}-05$

$2,58 \mathrm{E}-05$

2,62E-05

$2,61 \mathrm{E}-05$

$2,71 \mathrm{E}-05$

2,75E-05

$2,75 \mathrm{E}-05$

2,76E-05

$2,77 \mathrm{E}-05$

2,80E-05

$2,80 \mathrm{E}-05$

$2,82 \mathrm{E}-05$

2,83E-05

2,90E-05

2,89E-05

2,95E-05

2,96E-05

3,03E-05

3,07E-05

3,05E-05

3,06E-05

3,10E-05

3,16E-05

$3,18 \mathrm{E}-05$

3,17E-05

3,17E-05

3,14E-05

3,14E-05

3,24E-05

$3,28 \mathrm{E}-05$

$3,28 \mathrm{E}-05$

$3,42 \mathrm{E}-05$

3,42E-05

$3,41 \mathrm{E}-05$

$3,48 \mathrm{E}-05$

3,49E-05

$3,54 \mathrm{E}-05$

3,57E-05

3,61E-05

3,62E-05

3,74E-05

3,85E-05

3,87E-05

$3,88 \mathrm{E}-05$

$3,94 \mathrm{E}-05$

3,95E-05

3,99E-05

$4,03 \mathrm{E}-05$

$4,04 \mathrm{E}-05$

$4,14 \mathrm{E}-05$

$4,25 \mathrm{E}-05$

$4,32 \mathrm{E}-05$

4,37E-05
0,001732538

0,001750958

0,001750958

0,001751257

0,001751257

0,001751257

0,001755197

0,001755992

0,001755992

0,001755992

0,001765171

0,001773889

0,001775985

0,001775985

0,001811186

0,001811186

0,001817184

0,001817184

0,001823593

0,001827806

0,001839283

0,0018583

0,0018583

0,001907765

0,001939773

0,001939773

0,001939773

0,001939773

0,001939773

0,00195107

0,00195107

0,00201456

0,002026023

0,002026023

0,002026925

0,002028876

0,002034696

0,002034696

0,002036511

0,002036891

0,002077194

0,002077194

0,002099278

0,002099278

0,002147341

0,002148255

0,002148255

0,002148255

0,002161953

0,002169827

0,002169827

0,002169827

0,002169827

0,002169827

0,002169827

0,002208703

0,002219494

0,002219494

0,002291067

0,002291067

0,002291067

0,00232373

0,002323867

0,00234295

0,002359948

0,00237836

0,00237836

0,002443832

0,002508953

0,002510923

0,002510923

0,002539535

0,002539535

0,002557116

0,00257263

0,00257263

0,002631109

0,002692175

0,002725898

0,002745529

BnaA10g17500D protein

Charged multivesicular body 1

Triosephosphate isomerase

Kelch repeat-containing F-box family protein

My family transcription factor APL

sparagine--tRNA ligase, cytoplasmic 2

zinc finger $\mathrm{CCCH}$ domain-containing protein 25

andom slug protein 5-like

Zinc finger (C3HC4-type RING finger) family protein

lpha-glucosidase

re-mRNA-splicing factor SLU7

2-on-2 hemoglobin

expressed protein

-fucosyltransferase family protein

data found

No data found

serine/arginine-rich SC35-like splicing factor SCL33

inja-family protein AFP3

lpha-dioxygenase 2

tubulin alpha chain-like

Calcium-binding EF hand family protein

enolase

政

F-box family protein

Protein BREVIS RADIX

Upstream activation factor subunit spp27

BAG family molecular chaperone regulator 6-like

Subtilisin-like protease

Glutathione s-transferase

ADP-ribosylation factor GTPase-activating protein AGD3

ADPaspartyl peptidase/L-asparaginase

Coiled-coil domain-containing protein SCD2

Poly(A) polymerase

Pentatricopeptide repeat-containing protein No data found

UDP-glycosyltransferase 89A2-like

Chloroplast $40 \mathrm{kDa}$ outer membrane envelope protein

Protein DEHYDRATION-INDUCED 19

BAHD acyltransferase DCR

Phytochrome

Protein PLASTID MOVEMENT IMPAIRED 1-RELATED 1

$60 S$ ribosomal protein L39

Beta-1,3-galactosyltransferase-like protein

Translation initiation factor IF-2

Maternal effect embryo arrest protein

- 5 -like protein $1 \mathrm{~A}$, chloroplastic

CoA ligase

Pentatricopeptide repeat-containing protein

ATP sulfurylase 
MELO3C016303

MELO3C011196

MELO3C002041

MELO3C016262

MELO3C010761

MELO3C013810

MELO3C017946

MELO3C022162

MELO3C02177

MELO3C026234

MELO3C010675

MELO3C01775

MELO3C024316

MELO3C019225

MELO3C023419

MELO3C020132

MELO3C021228

MELO3C018502

MELO3C025812

MELO3C010297

MELO3C024005

MELO3C007562

MELO3C024545

MELO3C021979

MELO3C022786

MELO3C016019

MELO3C01303

MELO3C009776

MELO3C011731

MELO3C022901

MELO3C014309

MELO3C01666

MELO3C012529

MELO3C007450

MELO3C025278

MELO3C015764

MELO3C020957

MELO3C010102

MELO3C025265

MELO3C016917

MELO3C024435

MELO3C022773

MELO3C017683

MELO3C009495

MELO3C02421

MELO3C012242

MELO3C021273

MELO3C002469

MELO3C025779

MELO3C009272

MELO3C005094

MELO3C005565

MELO3C021232

MELO3C010548

MELO3C02373

MELO3C02000

MELO3C019844

MELO3C002885

MELO3C024385

MELO3C025853

MELO3C020626

MELO3C022998

MELO3C018749

MELO3C008321

MELO3C014016

MELO3C017700

MELO3C023018

MELO3C00435

MELO3C005945

MELO3C005293

MELO3C003561

MELO3C008367

MELO3C01412

MELO3C023560

MELO3C021923

MELO3C009879

MELO3C005405

MELO3C016468

MELO3C016354

MELO3C026097
4,47E-05

$4,53 \mathrm{E}-05$

$4,55 \mathrm{E}-05$

4,65E-05

$4,65 \mathrm{E}-05$

4,76E-05

$4,74 \mathrm{E}-05$

$4,69 \mathrm{E}-05$

4,76E-05

$4,74 \mathrm{E}-05$

$4,74 \mathrm{E}-05$

$4,84 \mathrm{E}-05$

$4,83 \mathrm{E}-05$

$4,84 \mathrm{E}-05$

$4,93 \mathrm{E}-05$

4,94E-05

$4,91 \mathrm{E}-05$

$4,91 \mathrm{E}-05$

5,00E-05

$5,05 \mathrm{E}-05$

$5,08 \mathrm{E}-05$

$5,21 \mathrm{E}-05$

$5,20 \mathrm{E}-05$

$5,20 \mathrm{E}-05$

$5,23 \mathrm{E}-05$

$5,23 \mathrm{E}-05$

$5,23 \mathrm{E}-05$

$5,30 \mathrm{E}-05$

$5,41 \mathrm{E}-05$

$5,40 \mathrm{E}-05$

$5,53 \mathrm{E}-05$

$5,43 \mathrm{E}-05$

$5,49 \mathrm{E}-05$

$5,49 \mathrm{E}-05$

$5,51 \mathrm{E}-05$

$5,52 \mathrm{E}-05$

$5,52 \mathrm{E}-05$

$5,50 \mathrm{E}-05$

$5,59 \mathrm{E}-05$

$5,63 \mathrm{E}-05$

$5,62 \mathrm{E}-05$

$5,68 \mathrm{E}-05$

$5,78 \mathrm{E}-05$

$5,81 \mathrm{E}-05$

$5,88 \mathrm{E}-05$

$5,88 \mathrm{E}-05$

$5,86 \mathrm{E}-05$

$5,86 \mathrm{E}-05$

$5,92 \mathrm{E}-05$

$5,94 \mathrm{E}-05$

$6,04 \mathrm{E}-05$

$6,03 \mathrm{E}-05$

$6,15 \mathrm{E}-05$

$6,17 \mathrm{E}-05$

$6,23 \mathrm{E}-05$

$6,20 \mathrm{E}-05$

$6,22 \mathrm{E}-05$

$6,28 \mathrm{E}-05$

$6,38 \mathrm{E}-05$

6,51E-05

$6,59 \mathrm{E}-05$

$6,69 \mathrm{E}-05$

$6,69 \mathrm{E}-05$

$6,73 \mathrm{E}-05$

6,77E-05

$6,77 \mathrm{E}-05$

$6,87 \mathrm{E}-05$

$6,85 \mathrm{E}-05$

$6,86 \mathrm{E}-05$

$6,82 \mathrm{E}-05$

$6,86 \mathrm{E}-05$

$6,97 \mathrm{E}-05$

$7,03 \mathrm{E}-05$

$7,00 \mathrm{E}-05$

$7,03 \mathrm{E}-05$

$7,24 \mathrm{E}-05$

$7,26 \mathrm{E}-05$

$7,29 \mathrm{E}-05$

$7,36 \mathrm{E}-05$

7,37E-05
0,002801259

0,002833395

0,002835312

0,002880684

0,002880684

0,002891896

0,002891896

0,002891896

0,002891896

0,002891896

0,002891896

0,002913268

0,002913268

0,002913268

0,002938396

0,002938396

0,002938396

0,002938396

0,002963418

0,002983067

0,00299654

0,003030419

0,003030419

0,003030419

0,003030419

0,003030419

0,003030419

0,003063321

0,003103925

0,003103925

0,003104434

0,003104434

0,003104434

0,003104434

0,003104434

0,003104434

0,003104434

0,003104434

0,003125624

0,003130049

0,003130049

0,003148872

0,00319616

0,003205231

0,003207012

0,003207012

0,003207012

0,003207012

0,003220471

0,003223467

0,003257855

0,003257855

0,003309755

0,003313352

0,003315726

0,003315726

0,003315726

0,003331834

0,003375474

0,003437938

0,003471635

0,003506

0,003506

0,003517413

0,003517802

0,003517802

0,00352199

0,00352199

0,00352199

0,00352199

0,00352199

0,003567809

0,003567921

0,003567921

0,003567921

0,003665313

0,003665515

0,003671413

0,003696337

0,003696337

ATP-dependent zinc metalloprotease FtsH

ABC1 family protein, expressed

HOMBOID-like protein 12, mitochondrial

$26 \mathrm{~S}$ proteasome non-ATPase regulatory subunit 8

upstream element-binding protein 1

t3g49990

bifunctional epoxide hydrolase 2-like

mannan endo-1,4-beta-mannosidase 1-like

xocyst complex component EXO84C

complex protein 3

ATP-citrate synthase alpha chain protein

NAC domain-containing protein

ate embryogenesis abundant protein, LEA-14

ormin-like protein

methyl-CpG-binding domain-containing protein 11

Potassium transporter

tRNA pseudouridine synthase

-binding filament-like protein

ATP-dependent zinc metalloprotease FtsH

Purple acid phosphatase

Acyl-CoA--sterol O-acyltransferase 1

Lipase

Transmembrane 9 superfamily member

Glutaredoxin family protein, putative

Phd finger protein, putative

heat shock factor-binding protein 1

Glutamate dehydrogenase, putative

DNA-directed RNA polymerase II, putative

Ubiquitin carboxyl-terminal hydrolase

Alpha/beta-Hydrolases superfamily protein, putative

Magnesium transporter MRS2-like protein

Flavin-binding kelch domain $\mathrm{F}$ box protein

Ras-related Rab7

No data found

Emp24/gp25L/p24 family/GOLD family protein

F-box/LRR-repeat protein 17

GDSL esterase/lipase 5-like

O-linked $\mathrm{n}$-acetylglucosamine transferase, ogt, putative

Unknown protein

Damaged dna-binding 2, putative isoform 1

Poly(A) polymerase

Transmembrane protein, putative

Clathrin interactor EPSIN 2

CASP-like protein

Cytochrome b561 and domon domain-containing protein

Chylene-responsive transcription factor ERF118

Seed maturation protein PM36

BnaC01g13120D protein

Nucleotide/sugar transporter family protein 
MELO3C008752

MELO3C005656

MELO3C003147

MELO3C026470

MELO3C022501

MELO3C026141

MELO3C009203

MELO3C014114

MELO3C026614

MELO3C017384

MELO3C005812

MELO3C011099

MELO3C025903

MELO3C004610

MELO3C007571

MELO3C021116

MELO3C019012

MELO3C021529

MELO3C019120

MELO3C021999

MELO3C012410

MELO3C015590

MELO3C010165

MELO3C009350

MELO3C023590

MELO3C015374

MELO3C020589

MELO3C013736

MELO3C007483

MELO3C021258

MELO3C016360

MELO3C023979

MELO3C004308

MELO3C011085

MELO3C005490

MELO3C013455

MELO3C025582

MELO3C012378

MELO3C007153

MELO3C015704

MELO3C024344

MELO3C014150

MELO3C008197

MELO3C025034

MELO3C009895

MELO3C009354

MELO3C017560

MELO3C025468

MELO3C023685

MELO3C026975

MELO3C003471

MELO3C005467

MELO3C003508

MELO3C013591

MELO3C005035

MELO3C010760

MELO3C010705

MELO3C027060

MELO3C019722

MELO3C003733

MELO3C014175

MELO3C023808

MELO3C015339

MELO3C026764

MELO3C013214

MELO3C000826

MELO3C009076

MELO3C02146

MELO3C010245

MELO3C026804

MELO3C02316

MELO3C024366

MELO3C01018

MELO3C006645

MELO3C013254

MELO3C016245

MELO3C024450

MELO3C004999

MELO3C00956

MELO3C004593
$7,44 \mathrm{E}-05$

$7,42 \mathrm{E}-05$

$7,53 \mathrm{E}-05$

$7,55 \mathrm{E}-05$

$7,65 \mathrm{E}-05$

$7,65 \mathrm{E}-05$

$7,74 \mathrm{E}-05$

$7,85 \mathrm{E}-05$

$7,87 \mathrm{E}-05$

$7,90 \mathrm{E}-05$

$8,05 \mathrm{E}-05$

$8,05 \mathrm{E}-05$

$8,07 \mathrm{E}-05$

$8,12 \mathrm{E}-05$

$8,15 \mathrm{E}-05$

$8,14 \mathrm{E}-05$

$8,19 \mathrm{E}-05$

$8,20 \mathrm{E}-05$

$8,32 \mathrm{E}-05$

$8,29 \mathrm{E}-05$

$8,35 \mathrm{E}-05$

$8,37 \mathrm{E}-05$

$8,36 \mathrm{E}-05$

$8,41 \mathrm{E}-05$

$8,49 \mathrm{E}-05$

$8,52 \mathrm{E}-05$

$8,72 \mathrm{E}-05$

$8,76 \mathrm{E}-05$

$8,91 \mathrm{E}-05$

$8,91 \mathrm{E}-05$

$8,94 \mathrm{E}-05$

$8,96 \mathrm{E}-05$

$9,02 \mathrm{E}-05$

$9,09 \mathrm{E}-05$

9,19E-05

$9,18 \mathrm{E}-05$

9,26E-05

$9,28 \mathrm{E}-05$

$9,32 \mathrm{E}-05$

9,36E-05

$9,35 \mathrm{E}-05$

$9,44 \mathrm{E}-05$

$9,44 \mathrm{E}-05$

$9,44 \mathrm{E}-05$

9,61E-05

$9,65 \mathrm{E}-05$

$9,71 \mathrm{E}-05$

9,70E-05

$9,76 \mathrm{E}-05$

9,80E-05

9,87E-05

0,000100246

0,000100674

0,000102175

0,000102449

0,000102713

0,000102939

0,000103272

0,000103716

0,000103809

0,000105084

0,000105023

0,000107667

0,000108528

0,00010835

0,000108179

0,000109346

0,00010932

0,000109483

0,000109889

0,000110585

0,000111579

0,000113033

0,000115046

0,000115548

0,000115714

0,000115488

0,000117465

0,000118756

0,000118147
0,003709205

0,003709205

0,003744722

0,003744722

0,003777968

0,003777968

0,003814164

0,003854576

0,00385474

0,003863001

0,003913918

0,003913918

0,003917532

0,003927962

0,003927962

0,003927962

0,00393172

0,00393172

0,003965589

0,003965589

0,003965589

0,003965589

0,003965589

0,003972539

0,004002215

0,004004007

0,004091828

0,004101537

0,004151361

0,004151361

0,004153313

0,004155775

0,004171791

0,004195778

0,004222227

0,004222227

0,004241924

0,004243844

0,004249426

0,004249426

0,004249426

0,004256592

0,004256592

0,004256592

0,004324758

0,004329302

0,004337109

0,004337109

0,00435218

0,00436109

0,004378557

0,004438907

0,004447917

0,00450416

0,004506164

0,004507639

0,004507639

0,004512221

0,004515705

0,004515705

0,004551125

0,004551125

0,004652752

0,004659375

0,004659375

0,004659375

0,004669937

0,004669937

0,004669937

0,00467717

0,004696648

0,004728701

0,004780062

0,004851885

0,004851885

0,004851885

0,004851885

0,004914861

0,004916778

0,004916778

TATA box-binding protein-associated factor RNA polymerase I subunit B

Protein LURP-one-related 11

OW QUALITY PROTEIN: L-type lectin-domain containing receptor kinase S.4-like

otassium transporter

SNARE-interacting protein KEULE

DP-ribosylation factor-like

tubulin beta chain-like

PM1-interacting protein 4

WD repeat-containing protein 55

tRNA pseudouridine synthase

ARM repeat superfamily protein

Universal stress protein A-like protein

t5g59460

zinc finger $\mathrm{CCCH}$ domain-containing protein 20-like

OTU domain-containing protein

No data found

No data found

Homain-containing protein, putative

$\mathrm{R} 3 \mathrm{H}$ domain-containing protein 1-like isoform $\mathrm{X}$

protein

thioredoxin-like 3-2, chloroplastic isoform X8

protochlorophyllide-dependent translocon component 52, chloroplastic-like

Cyclic nucleotide-gated ion channel-like protein

TSL-kinase interacting protein 1

Pheophytinase, chloroplastic

Superoxide dismutase [Cu-Zn]

$17.5 \mathrm{kDa}$ class I heat shock protein

asic endochitinase $C$

$A B C$ transporter family protein

flowering time control protein FCA isoform X2

Transferring glycosyl group transferase

Cellulose synthase

Photosystem II reaction center PsbP family protein

Argininosuccinate lyase

U-box domain-containing protein 35 -like isoform X2

Calcium ion-binding protein

Ribosomal protein S5/S7

phosphoglucan phosphatase DSP4, amyloplastic

No data found

Protein trigalactosyldiacylglycerol 2, chloroplastic

Promily protein (DUF1677)

Fimbrin, putative

Chloride channel protein

peroxiredoxin $\mathrm{Q}$, chloroplastic

Actin cross-linking protein

Gamma-glutamylcysteinyltransferase 1

Succinate dehydrogenase [ubiquinone] flavoprotein subunit, mitochondrial

$A B C$ transporter B family protein

alcohol dehydrogenase-like

40 S ribosomal protein $\mathrm{S} 15 \mathrm{a}$

protein SAWADEE HOMEODOMAIN HOMOLOG 1-like isoform X2 
MELO3C00665

MELO3C020294

MELO3C017104

MELO3C025594

MELO3C017653

MELO3C015496

MELO3C003911

MELO3C003294

MELO3C015230

MELO3C002075

MELO3C000994

MELO3C012076

MELO3C005797

MELO3C01160

MELO3C013346

MELO3C008986

MELO3C015186

MELO3C026522

MELO3C003146

MELO3C018455

MELO3C023484

MELO3C021940

MELO3C011044

MELO3C016601

MELO3C011868

MELO3C016298

MELO3C024396

MELO3C019451

MELO3C006854

MELO3C003186

MELO3C022443

MELO3C025606

MELO3C007082

MELO3C012173

MELO3C013046

MELO3C009338

MELO3C024885

MELO3C019139

MELO3C015930

MELO3C003332

MELO3C024004

MELO3C001948

MELO3C008469

MELO3C013883

MELO3C025307

MELO3C010053

MELO3C006853

MELO3C016126

MELO3C007653

MELO3C010913

MELO3C009782

MELO3C019435

MELO3C017248

MELO3C010769

MELO3C02620

MELO3C003975

MELO3C006594

MELO3C026248

MELO3C017226

MELO3C019470

MELO3C018956

MELO3C011444

MELO3C022658

MELO3C015111

MELO3C006344

MELO3C004542

MELO3C005967

MELO3C00917

MELO3C017884

MELO3C011588

MELO3C010214

MELO3C023475

MELO3C01844

MELO3C024138

MELO3C011408

MELO3C009063

MELO3C021279

MELO3C022345

MELO3C020557

MELO3C013527
0,000118406

0,00011852

0,000118047

0,000119502

0,000120136

0,000120135

0,000121139

0,000123184

0,000123277

0,000125653

0,000126324

0,000126973

0,000127327

0,000128223

0,000129469

0,000129552

0,000128867

0,000129123

0,000130195

0,000130274

0,000131214

0,000131077

0,000133374

0,000133904

0,000134717

0,00013613

0,000135867

0,000136453

0,000137541

0,000138025

0,000137848

0,000138545

0,000138903

0,000141571

0,000141781

0,000142572

0,000144879

0,000144203

0,00014451

0,000143831

0,000144864

0,000145692

0,000146222

0,000147575

0,000147486

0,000148287

0,000149737

0,000149702

0,000149933

0,000149696

0,000149106

0,000151452

0,000150882

0,000151363

0,000152382

0,000152984

0,000152844

0,000153686

0,00015485

0,000156016

0,000156177

0,000156102

0,000156684

0,000157133

0,000157815

0,000158277

0,000160086

0,000160419

0,000161451

0,000161995

0,00016233

0,000163152

0,000163123

0,000164805

0,000164884

0,000165244

0,000165964

0,000165703

0,000166158

0,000166518
0,004916778

0,004916778

0,004916778

0,004937323

0,004942865

0,004942865

0,004973739

0,005040585

0,005040585

0,005127117

0,005143843

0,005159631

0,005163393

0,005189103

0,005200251

0,005200251

0,005200251

0,005200251

0,00520807

0,00520807

0,00522447

0,00522447

0,005299798

0,005310178

0,005331706

0,005366137

0,005366137

0,005368136

0,005397731

0,005397731

0,005397731

0,005407372

0,005410626

0,005501034

0,005501034

0,005520885

0,005555765

0,005555765

0,005555765

0,005555765

0,005555765

0,0055761

0,00558555

0,005615542

0,005615542

0,005631773

0,005640063

0,005640063

0,005640063

0,005640063

0,005640063

0,005664813

0,005664813

0,005664813

0,005688818

0,00568979

0,00568979

0,005705154

0,005737567

0,005754372

0,005754372

0,005754372

0,005762284

0,005768053

0,00578234

0,005788536

0,005843894

0,005845212

0,005871985

0,005880967

0,005882308

0,005890462

0,005890462

0,005931329

0,005931329

0,005933466

0,005933924

0,005933924

0,005933924

0,005936027

No data found

DNA-directed RNA polymerase subunit beta

rehalose-6-phosphate synthase, putative

2 domain-containing protein

doptidase/L-asparaginase

lant/F27B13-30 protein

protein BONZAI 3

acuolar cation/proton exchanger 3

ectin acetylesterase

wall-associated receptor kinase 2 -like

UDP-galactose/UDP-glucose transporter family protein

known protein

No data found

Phospholipase-like protein (PEARLI 4) family protein

No data found

Sulfite reductase

Sulfite-sensing protein 2-like

-type lectin-domain containing receptor kinase IV.1-like

thioredoxin M3, chloroplastic

AP2-like ethylene-responsive transcription factor At1g16060

Cold acclimation protein

cytochrome $\mathrm{c}$ oxidase subunit $6 \mathrm{~b}$-1-like

No data found

Rubber elongation factor protein (REF)

Ribosome maturation factor

Cytochrome p450

nknown protein

transcription factor bHLH118-like

Proteasome inhibitor-related

flowering time control protein FPA

Unknown protein

No data found

DNA-directed RNA polymerase

Kinase family protein

S ribosomal protein L12

ATP-dependent DNA helicase

Nascent polypeptide-associated complex subunit alpha-like protein

Translation initiation factor IF-2

BAX inhibitor-1

No data found

Short-chain dehydrogenase, putative

Kinase family protein

BOI-related E3 ubiquitin-protein ligase 1

Transmembrane 19

Isopentenyl diphosphate isomerase

receptor-like serine/threonine-protein kinase isoform X2

Pathogen-related protein

Protein PHLOEM PROTEIN 2-LIKE A10 
MELO3C022196

MELO3C013841

MELO3C007611

MELO3C022514

MELO3C014719

MELO3C014557

MELO3C014128

MELO3C021064

MELO3C001849

MELO3C003393

MELO3C003842

MELO3C018437

MELO3C017242

MELO3C018195

MELO3C027373

MELO3C027375

MELO3C002689

MELO3C009539

MELO3C024910

MELO3C008879

MELO3C023255

MELO3C015373

MELO3C011622

MELO3C003977

MELO3C016696

MELO3C008440

MELO3C010877

MELO3C004551

MELO3C009996

MELO3C009429

MELO3C012572

MELO3C00314

MELO3C005245

MELO3C025848

MELO3C010275

MELO3C015450

MELO3C007624

MELO3C017898

MELO3C005252

MELO3C020394

MELO3C019462

MELO3C010565

MELO3C012201

MELO3C004732

MELO3C025049

MELO3C002382

MELO3C010235

MELO3C007086

MELO3C006974

MELO3C01400

MELO3C012525

MELO3C024444

MELO3C00215

MELO3C008332

MELO3C01043

MELO3C024850

MELO3C024975

MELO3C010798

MELO3C011507

MELO3C02082

MELO3C011905

MELO3C005517

MELO3C025798

MELO3C024644

MELO3C000425

MELO3C016288

MELO3C011107

MELO3C01977

MELO3C018478

MELO3C002566

MELO3C015502

MELO3C020924

MELO3C01872

MELO3C016213

MELO3C010512

MELO3C005808

MELO3C000237

MELO3C022212

MELO3C010478

MELO3C008925
0,000167781

0,000168271

0,000168612

0,000168701

0,000171133

0,000171945

0,000172955

0,000173027

0,000172465

0,000171685

0,000172433

0,000173525

0,000174787

0,000175905

0,000176765

0,000177846

0,000177821

0,000181792

0,000182037

0,000182842

0,000182737

0,000184106

0,000184591

0,000188808

0,000188295

0,000188691

0,000191246

0,00019215

0,000192459

0,000194464

0,000194952

0,000194632

0,000195037

0,000195176

0,000194973

0,000195936

0,00019699

0,000199382

0,000199914

0,000201726

0,000200747

0,000201305

0,000200408

0,000201502

0,000202498

0,000203267

0,000203253

0,000204456

0,000205083

0,000205522

0,000206484

0,000208262

0,000208341

0,000209733

0,000212163

0,000213884

0,000215317

0,000215009

0,000215859

0,000216965

0,00021792

0,000218944

0,00022376

0,000224718

0,000225399

0,000228158

0,000229497

0,000230207

0,000231655

0,000232154

0,000232658

0,00023482

0,00023523

0,000235725

0,000236489

0,000237926

0,000237808

0,000238624

0,000239375

0,000239951
0,005970265

0,005970729

0,005970729

0,005970729

0,006045972

0,006048

0,006048

0,006048

0,006048

0,006048

0,006048

0,006054682

0,006087962

0,006116118

0,00613522

0,006151116

0,006151116

0,006274078

0,006274078

0,006279914

0,006279914

0,006312352

0,006318019

0,006428914

0,006428914

0,006428914

0,006500733

0,00651951

0,00651951

0,006544185

0,006544185

0,006544185

0,006544185

0,006544185

0,006544185

0,006558527

0,006582664

0,006651329

0,006657858

0,006662025

0,006662025

0,006662025

0,006662025

0,006662025

0,006676334

0,006679412

0,006679412

0,006707317

0,006716729

0,00671996

0,00674026

0,006778458

0,006778458

0,006812512

0,006880133

0,00692458

0,006948193

0,006948193

0,006954317

0,006978582

0,006997901

0,007019354

0,007162141

0,007181155

0,007191293

0,007267571

0,007298438

0,007309266

0,007343421

0,007347439

0,007351626

0,007408081

0,007409188

0,007412948

0,007425151

0,007446607

0,007446607

0,007456609

0,007468269

0,007474446

Kinase family protein

ucleolar protein 12

rolyl 4-hydroxylase alpha-like protein

UTP:RNA uridylyltransferase 1

dehyde oxidase, putative

Peptidyl-prolyl cis-trans isomerase

Calcium-dependent protein kinase

alonyl-CoA:anthocyanidin 5-O-glucoside-6"-O-malonyltransferase No data found

Plant intracellular ras group-related LRR protein

Dead box ATP-dependent RNA helicase, putative

Pentatricopeptide repeat-containing protein At5g39710

rotein COBRA-like

Arf GTPase activating protein

AD-ribosylation factor GTPase-activating protein AGD12-like

Pectinesterase

serine/threonine-protein kinase STY8-like

comain-containing protein

RIGIDA-like protein

Triosephosphate isomerase

DNA-directed RNA polymerase subunit beta-beta protein, putative (DUF506)

40 S ribosomal protein $\mathrm{S} 25$

eceptor-like kinase

Microtubule-associated family protein

PROTON PUMP INTERACTOR 1 family protein

wall-associated receptor kinase 2-like

Phosphatidate phosphatase PAH2

guanine-nucleotide exchange factor GNL2

dnaJ protein homolog

peroxiredoxin-2E-2, chloroplastic

CTP synthase

Cyclin d, putative

Xyloglucan endotransglucosylase/hydrolase

Peptidyl-prolyl cis-trans isomerase

Serine-rich protein-like protein

Pollen-specific protein SF21

Acyl-CoA N-acyltransferases (NAT) superfamily protein

-box family protein

Ankyrin repeat-containing protein, putative

Phospholipid-transporting ATPase

Membralin, putative

No data found

Isoprenylcysteine alpha-carbonyl methylesterase ICME protein

Alpha/beta-Hydrolases superfamily protein

transcription factor TCP21-like

Kinase family protein

No data found

$\mathrm{ABC}$ transporter $\mathrm{C}$ family member 12 -like

Plastid division protein PDV1

Superoxide dismutase 1 
MELO3C020386

MELO3C021292

MELO3C017799

MELO3C005882

MELO3C021339

MELO3C012651

MELO3C011395

MELO3C00354

MELO3C00769

MELO3C003242

MELO3C008236

MELO3C010329

MELO3C00782

MELO3CO09994

MELO3C018718

MELO3C015431

MELO3C022114

MELO3C015929

MELO3C025940

MELO3C019503

MELO3C018203

MELO3C008295

MELO3C007421

MELO3C023830

MELO3C017572

MELO3C006175

MELO3C01670

MELO3C002609

MELO3C016016

MELO3C023291

MELO3C026966

MELO3C02004

MELO3C002223

MELO3C003658

MELO3C023667

MELO3C005673

MELO3C00410

MELO3C022406

MELO3C002332

MELO3C015185

MELO3C026398

MELO3C016868

MELO3C026803

MELO3C023464

MELO3C026155

MELO3C003695

MELO3C003768

MELO3C010928

MELO3C017784

MELO3C00575

MELO3C023306

MELO3C002328

MELO3C024596

MELO3C011402

MELO3C024192

MELO3C006413

MELO3C026221

MELO3C011090

MELO3C025449

MELO3C02454

MELO3C013188

MELO3C018461

MELO3C003817

MELO3C010524

MELO3C016542

MELO3C016140

MELO3C026030

MELO3C00523

MELO3C016717

MELO3C011640

MELO3C009566

MELO3C014089

MELO3C02602

MELO3C021759

MELO3C003825

MELO3C001954

MELO3C009550

MELO3C015514

MELO3C01125

MELO3C011173
0,000241205

0,000240838

0,000243307

0,000246426

0,000248214

0,00024916

0,000249164

0,00025058

0,000252707

0,000252682

0,000253956

0,00025456

0,00025592

0,0002553

0,000255613

0,000257438

0,000259465

0,000260097

0,000260202

0,000260858

0,000262

0,000262691

0,000263489

0,000263933

0,000267737

0,000268353

0,000269787

0,000270664

0,000273434

0,000273551

0,000275157

0,000275744

0,000275412

0,000275946

0,000277469

0,000278018

0,000280336

0,000280149

0,000279544

0,000280098

0,00028134

0,000285092

0,00028349

0,000284025

0,000284337

0,000284827

0,000285069

0,000285792

0,000287022

0,000287719

0,000286822

0,000287751

0,000288618

0,000288957

0,00029184

0,000291842

0,00029224

0,000292398

0,000294384

0,000294894

0,000296733

0,000296828

0,000296781

0,000297643

0,000298955

0,000300483

0,000300216

0,000301079

0,000303125

0,0003039

0,000305243

0,00030836

0,00030857

0,000308348

0,000312163

0,000313336

0,000314264

0,000314106

0,00031477

0,000316606
0,007489865

0,007489865

0,007543272

0,007628007

0,007671325

0,007676646

0,007676646

0,007708259

0,007749561

0,007749561

0,00777579

0,007782212

0,007787613

0,007787613

0,007787613

0,007821755

0,00786941

0,00786941

0,00786941

0,00787719

0,007899594

0,00790837

0,00792031

0,007921598

0,008023584

0,008029865

0,008060539

0,008074539

0,008136071

0,008136071

0,008158176

0,008158176

0,008158176

0,008158176

0,008190939

0,0081949

0,008214178

0,008214178

0,008214178

0,008214178

0,008231374

0,008267658

0,008267658

0,008267658

0,008267658

0,008267658

0,008267658

0,00827581

0,008283948

0,008283948

0,008283948

0,008283948

0,008294506

0,008294506

0,00834476

0,00834476

0,00834476

0,00834476

0,008389297

0,00839174

0,008410413

0,008410413

0,008410413

0,008421422

0,008446441

0,008465387

0,008465387

0,008470097

0,008515536

0,008525163

0,008550704

0,008607261

0,008607261

0,008607261

0,008695208

0,008715584

0,00871686

0,00871686

0,008718633

0,008757205

E3 ubiquitin-protein ligase LAP

Protein DEHYDRATION-INDUCED 19

2P16.20 protein, putative isoform 1

Eukaryotic translation initiation factor 3 subunit 10

ribosomal protein L13a, putative

RIGIDA-like protein

ydroxyproline O-arabinosyltransferase 1

AD(P)H-hydrate epimerase

Auxin-responsive protein

in light chain

-adenosyl-L-methionine-dependent methyltransferases superfamily protein isoform 1

methyl-CpG-binding domain-containing protein 11-like

1 small nuclear ribonucleoprotein $70 \mathrm{kDa}$

MD-2-related lipid recognition domain-containing protein / ML domain-containing protein

$2 \mathrm{~g} 42760$

OS ribosomal protein $\mathrm{S} 30$

RmIC-like cupins superfamily protein

Chotein SAR1A

CNSTANS-like zinc finger protein

-binding alcohol dehydrogenase domain-containing protein 2

ysine-specific histone demethylase 1-like protein

ABC1-like protein

6-phosphate 1-phosphotransferase subunit beta

rotein-tyrosine-phosphatase MKP1 isoform X1

transcription factor TGA1 isoform X1

ankyrin repeat and zinc finger domain-containing protein 1

Choline transporter-like protein 2

serine/threonine-protein kinase HT1-like

xyloglucan 6-xylosyltransferase 1

glycine-rich RNA-binding protein 1-like

2-oxoglutarate (2OG) and Fe(II)-dependent oxygenase superfamily protein

Ribosomal protein $\mathrm{S} 5 / \mathrm{S} 7$

hydroxyproline O-galactosyltransferase GALT6

Protein MARD1

Tetratricopeptide repeat-containing protein

thulin beta chain-like

Cytochrome c oxidase subunit

(osphatidylinositol 4-kinase gamma 4

Telomere length regulation TEL2

MP domain-containing protein

APO protein 1 , chloroplastic

serine/threonine-protein kinase EDR1

S-acyltransferase

sister chromatid cohesion protein PDS5 homolog B-B-like

(+)-neomenthol dehydrogenase

ethylene-responsive transcription factor SHINE 2-like

Auxin response factor

PPPDE putative thiol peptidase family protein

Pentatricopeptide repeat-containing family protein 
MELO3C014658

MELO3C014394

MELO3C007492

MELO3C008785

MELO3C009886

MELO3C00443

MELO3C016314

MELO3C023996

MELO3C02058

MELO3C017165

MELO3C006813

MELO3C009670

MELO3C016015

MELO3C00558

MELO3C009624

MELO3C006418

MELO3C015563

MELO3C014619

MELO3C022488

MELO3C020622

MELO3C010714

MELO3C015375

MELO3C000065

MELO3C019816

MELO3C008494

MELO3C016573

MELO3C00112

MELO3C013921

MELO3C019404

MELO3C008018

MELO3C021608

MELO3C001996

MELO3C000201

MELO3C023990

MELO3C002872

MELO3C022068

MELO3C017755

MELO3C02487

MELO3C009836

MELO3C022005

MELO3C002767

MELO3C022359

MELO3C012956

MELO3C007909

MELO3C026835

MELO3C025349

MELO3C019688

MELO3C00288

MELO3C017480

MELO3C02445

MELO3C015290

MELO3C026263

MELO3C011208

MELO3C004100

MELO3C014565

MELO3C006950

MELO3C021280

MELO3C012670

MELO3C01241

MELO3C011773

MELO3C025346

MELO3C003659

MELO3C005971

MELO3C022868

MELO3C012283

MELO3C003078

MELO3C018487

MELO3C01077

MELO3C023409

MELO3C024982

MELO3C007441

MELO3C025102

MELO3C015627

MELO3C011110

MELO3C008732

MELO3C019813

MELO3C017753

MELO3C007398

MELO3C023163

MELO3C020808
0,000321415

0,000321565

0,000321389

0,000320693

0,000320267

0,000323167

0,000324657

0,000324312

0,000326013

0,000325784

0,000326594

0,000328256

0,000327885

0,000329536

0,000330877

0,000334017

0,000337528

0,000339532

0,000339917

0,000339005

0,000340222

0,000341602

0,000343344

0,000343724

0,000344946

0,000346012

0,000345871

0,000347074

0,000348394

0,000347799

0,000348624

0,000350501

0,000355846

0,00035901

0,000359765

0,000360452

0,000361737

0,000362308

0,000363929

0,000367548

0,000371429

0,00037247

0,000374173

0,000376025

0,000376753

0,000377451

0,000378015

0,000379322

0,000379509

0,00038239

0,000386293

0,000387563

0,000387115

0,000389113

0,000391002

0,000391236

0,000394794

0,000397299

0,000397107

0,000399887

0,000403884

0,000407065

0,00040998

0,000409725

0,000409036

0,000410585

0,000411533

0,000415371

0,000416908

0,000417648

0,000419687

0,000419977

0,00042005

0,000418692

0,000421822

0,000425661

0,000427866

0,000427556

0,000427278

0,000427603
0,008832526

0,008832526

0,008832526

0,008832526

0,008832526

0,00886421

0,00888041

0,00888041

0,008892853

0,008892853

0,008896413

0,008917104

0,008917104

0,008939583

0,008963643

0,009036314

0,009118805

0,009141554

0,009141554

0,009141554

0,009141554

0,00916616

0,009198122

0,009198122

0,009218319

0,009221845

0,009221845

0,009237694

0,009241571

0,009241571

0,009241571

0,009278878

0,009407762

0,009478731

0,009485971

0,009491434

0,009512585

0,009514927

0,009544809

0,00962694

0,009715694

0,009730034

0,009761609

0,00979698

0,009803012

0,00980825

0,009810011

0,009822965

0,009822965

0,009884581

0,009972425

0,00997912

0,00997912

0,010005992

0,01003445

0,01003445

0,010112568

0,010150395

0,010150395

0,010203324

0,010291994

0,010359693

0,010393714

0,010393714

0,010393714

0,010395704

0,01040636

0,010489987

0,010515345

0,01052057

0,010527378

0,010527378

0,010527378

0,010527378

0,010558394

0,010640976

0,010642221

0,010642221

0,010642221

0,010642221

Peroxidase

Ribosomal $\mathrm{N}$-lysine methyltransferase 5

ongation of fatty acids protein 3-like

TB/POZ domain protein

cysteine oxidase 2

Serine-rich protein-like protein

Aldose 1-epimerase, putative

RNA polymerase sigma factor sigD, chloroplastic

No data found

VA22-like protein

monocopper oxidase-like protein SKS1

HD-finger protein

AA-type ATPase family protein

No data found

Actin family protein

Ankyrin repeat-containing protein

TATA-box-binding protein

-interacting protein-like

ubulin-folding cofactor $D$

Rab5-interacting family protein

two-component response regulator ARR11

Sodium/hydrogen exchanger

-type proton ATPase proteolipid subunit

Amino acid transporter family protein

E3 ubiquitin-protein ligase

Serine/threonine-protein kinase

Aspartate aminotransferase

Protein-tyrosine phosphatase mitochondrial 1-like protein

phytosulfokine receptor 2

Like-COV protein

inc finger protein CONSTANS-LIKE 6

NAC domain-containing protein 100 -like

dof zinc finger protein DOF1.5-like

Methyltransferase

Short-chain dehydrogenase/reductase family protein

proline-rich receptor-like protein kinase PERK8

zinc finger protein CONSTANS-LIKE 15-like

serine/threonine-protein phosphatase 4 regulatory subunit 2 isoform X1

Saccharopine dehydrogenase-like protein

Phosphate transporter PHO1-like protein

Nuclear-interacting partner of ALK

F3H9.20 protein

Protein MARD1

Armadillo repeat only protein

Pentatricopeptide repeat-containing protein At2g30100, chloroplastic

Subtilisin-like protease SBT6.1

Peroxisomal and mitochondrial division factor 2

Alpha-taxilin

Xyloglucan endotransglucosylase/hydrolase

ATP-dependent zinc metalloprotease FtsH 
MELO3C013429

MELO3C005147

MELO3C012447

MELO3C011986

MELO3C008209

MELO3C011212

MELO3C019630

MELO3C011139

MELO3C017711

MELO3C021670

MELO3C003312

MELO3C013779

MELO3C013139

MELO3C01361

MELO3C008959

MELO3C027064

MELO3C015385

MELO3C00605

MELO3C00624

MELO3C025549

MELO3C007767

MELO3C00793

MELO3C021742

MELO3C014297

MELO3C003017

MELO3C019820

MELO3C02083

MELO3C020835

MELO3C017432

MELO3C018510

MELO3C007248

MELO3C02072

MELO3C003313

MELO3C012873

MELO3C024951

MELO3C00575

MELO3C013974

MELO3C018052

MELO3C011576

MELO3C024558

MELO3C009133

MELO3C005703

MELO3C012960

MELO3C011675

MELO3C009250

MELO3C004186

MELO3C006280

MELO3C015098

MELO3C014358

MELO3C01063

MELO3C011793

MELO3C001962

MELO3C007833

MELO3C009797

MELO3C00809

MELO3C005518

MELO3C005679

MELO3C013315

MELO3C02602

MELO3C007495

MELO3C00025

MELO3C016915

MELO3C026443

MELO3C025885

MELO3C003880

MELO3C009562

MELO3C009408

MELO3C01754

MELO3C023481

MELO3C023412

MELO3C013645

MELO3C015350

MELO3C01360

MELO3C013757

MELO3C012622

MELO3C015860

MELO3C021858

MELO3C025585

MELO3C009758

MELO3C017878
0,000430502

0,00043159

0,000430274

0,000431246

0,000430571

0,000430422

0,000435381

0,000437758

0,000441544

0,000443308

0,000443736

0,000446718

0,000447584

0,000447902

0,000451143

0,000453055

0,00045326

0,000453925

0,000457096

0,000456881

0,000462486

0,000462652

0,000463278

0,000466624

0,000469162

0,000469204

0,000469883

0,000472388

0,000475584

0,000475893

0,000478865

0,000478166

0,000478841

0,00047936

0,000481456

0,000481084

0,000483505

0,000482496

0,000483087

0,00048545

0,00048648

0,000487478

0,000487669

0,000489723

0,000492684

0,000492599

0,000492176

0,000493039

0,000494212

0,00049758

0,000502871

0,00050303

0,000504272

0,000504618

0,000506949

0,000507675

0,000509158

0,000512098

0,00051243

0,000515516

0,00051635

0,000518351

0,000520272

0,000521179

0,000522313

0,000524514

0,000526576

0,000525788

0,000527659

0,000526748

0,000527767

0,000528832

0,000530899

0,000533573

0,000536651

0,000536727

0,000536048

0,000538898

0,000540825

0,000541271
0,010654347 kinesin-related protein 11

0,010654347 NADPH:QUINONE OXIDOREDUCTASE family protein

0,010654347 Scarecrow-like 3

0,010654347 Polygalacturonase

0,010654347 Ankyrin

0,010654347 Photosystem II reaction center PsbP family protein

0,010734505 ribosomal RNA large subunit methyltransferase I

Nuclear pore complex protein NUP85

0,010859341 Proline--tRNA ligase

$0,01088614 \quad$ Nuclear inhibitor of protein phosphatase 1

$0,01088614 \quad$ Protein kinase superfamily protein

0,010945695 2-keto-3-deoxy-L-rhamnonate aldolase

0,010947544 prohibitin-1, mitochondrial

0,010947544 E3 ubiquitin-protein ligase RMA1H1

0,011013129 1-acyl-sn-glycerol-3-phosphate acyltransferase

0,011037513 Pentatricopeptide repeat-containing protein

0,011037513 Pentatricopeptide repeat-containing protein

BTB/POZ domain-containing protein POB1

0,011089916 protein NRT1/ PTR FAMILY 7.3-like

0,011089916 vesicle-fusing ATPase

0,011197199 Lipoxygenase y domain-containing protein 1

0,011197199 Protein preY, mitochondrial, putative

011198624 Glycine cleavage system $\mathrm{H}$, mitochondria

0,011265711 type I inositol polyphosphate 5-phosphatase 4

$0,01130037 \quad$ Alpha/beta-Hydrolases superfamily protein

0,01130037 Glycerol-3-phosphate dehydrogenase

0,011302944 BZIP transcription factor, putative (DUF630 and DUF632)

0,011349391 Unknown protein

$0,011405846 \quad$ At1g16840/F17F16.27

0,011405846 somatic embryogenesis receptor kinase 1-like

0,011433429 Transcription factor VOZ1

0,011433429 triacylglycerol lipase SDP1

$0,011433429 \quad$ Membrane steroid-binding protein

0,011433429 NAC domain protein

0,011455744 cellulose synthase-like protein E6

0,011463072

0,011463072

0,011463072

0,01149538

0,011505988

0,011506551

0,011506551

0,011541219

0,011564161

0,011564161

0,011564161

0,011564161

0,01157792

0,011643018

0,011742725

0,011742725

0,011751999

0,011751999

0,011792393

0,011795371

0,011815931

0,011863977

0,011863977

0,011921451

0,011926777

0,011959004

0,011989319

0,011996228

0,012008341

0,012044923

0,01204955

0,01204955

0,01204955

0,01204955

0,01204955

0,012059945

0,012093112

0,012140022

0,012169726

0,012169726

0,012169726

0,012204926

0,012230611

0,012230611

(lind heat shock protein 70

Hsp70-Hsp90 organizing protein 3

inamin-related protein 3A-like

zinc finger protein CONSTANS-LIKE 6

sorting nexin 1

Receptor-like kinase

Beta-carotene 3-hydroxylase

BZIP protein, putative

$\mathrm{Cmp}$-sialic acid transporter, putative

Laccase

Unknown protein

$60 S$ ribosomal protein $\mathrm{L} 37 \mathrm{a}$

Tubulin beta chain

transcription initiation factor TFIID subunit 6-like

$40 \mathrm{~S}$ ribosomal protein $\mathrm{S} 8$

transcription termination factor MTERF9, chloroplastic-like

protein REVEILLE 2-like

ATPase family AAA domain-containing protein 3

HSP20-like chaperones superfamily protein

eukaryotic translation initiation factor 2D

3-isopropylmalate dehydratase small subunit 3-like

Tyrosine--tRNA ligase

O-methyltransferase, putative

PRA1 family protein

protein LYK5-like

uxin response facto

copper-transporting ATPase PAA2, chloroplastic isoform X1

ras-related protein Rab11C

Histone deacetylase complex subunit

Pentatricopeptide repeat-containing protein

Tetratricopeptide repeat (TPR)-like superfamily protein

DNA-directed RNA polymerase III subunit RPC5

nudix hydrolase 3-like

O-fucosyltransferase family protein

Alanine--tRNA ligase

Sugar transporter ERD6-like protein

O-methyltransferase, putative

Proteasome subunit alpha type

peptidyl-prolyl cis-trans isomerase CYP59

Calcium permeable stress-gated cation channel 1

WEB family protein At3g02930, chloroplastic

bifunctional nitrilase/nitrile hydratase NIT4A

Amino acid transporter family protein

Protein LURP-one-related 17 
MELO3C014176

MELO3C005869

MELO3C017320

MELO3C00632O

MELO3C010964

MELO3C005520

MELO3C013437

MELO3C025989

MELO3C010052

MELO3C013946

MELO3C015198

MELO3C025813

MELO3C004597

MELO3C002817

MELO3C005300

MELO3C009966

MELO3C002816

MELO3C021588

MELO3C009038

MELO3C011138

MELO3C004437

MELO3C00305

MELO3C012628

MELO3C021552

MELO3C019948

MELO3C003916

MELO3C019527

MELO3C019163

MELO3C017347

MELO3C006802

MELO3C004505

MELO3C014849

MELO3C003120

MELO3C011752

MELO3C022609

MELO3C021408

MELO3C002159

MELO3C005439

MELO3C011193

MELO3C017300

MELO3C017091

MELO3C013710

MELO3C023725

MELO3C014306

MELO3C003945

MELO3C023492

MELO3C012100

MELO3C003726

MELO3C003326

MELO3C002819

MELO3C009101

MELO3C008052

MELO3C022930

MELO3C025637

MELO3C017968

MELO3C010651

MELO3C006948

MELO3C010273

MELO3C009380

MELO3C015796

MELO3C023200

MELO3C008088

MELO3C002470

MELO3C008775

MELO3C009735

MELO3C023114

MELO3C013343

MELO3C01473

MELO3C016464

MELO3C013440

MELO3C005745

MELO3C009779

MELO3C01746

MELO3C010693

MELO3C006268

MELO3C003638

MELO3C017902

MELO3C014090

MELO3C024333

MELO3C023776
0,00054293

0,000544101

0,000543557

0,000545691

0,000547068

0,000548142

0,000550127

0,00055226

0,000552388

0,000560347

0,000560559

0,000559301

0,000562498

0,000562464

0,000565577

0,000573553

0,000574467

0,000578257

0,000579828

0,000579399

0,000581551

0,000585005

0,000585702

0,000587182

0,000586876

0,000590103

0,000589502

0,00058932

0,000589846

0,000591331

0,000592869

0,000597937

0,000600664

0,00060038

0,000601905

0,000599518

0,000601837

0,000603103

0,00060643

0,000607829

0,000607555

0,000611069

0,000616646

0,000615127

0,000616214

0,000614151

0,000616737

0,000614743

0,000619344

0,000620421

0,000623036

0,000624482

0,000627924

0,000630045

0,000629519

0,000630188

0,000634012

0,000635076

0,000638714

0,000639351

0,000639914

0,000639383

0,00064277

0,000644342

0,000646709

0,000646794

0,000648394

0,000652227

0,000649795

0,000651079

0,000652429

0,000650797

0,00065854

0,000663173

0,000670702

0,000670016

0,000676531

0,000679738

0,000689727

0,000692692
0,0122525

0,0122525

0,0122525

0,01227432

0,012291283

0,012301423

0,012331966

0,012354603

0,012354603

0,012494896

0,012494896

0,012494896

0,012509883

0,012509883

0,012564217

0,012727086

0,012733052

0,01280268

0,01280876

0,01280876

0,012832462

0,012894272

0,012895245

0,012899069

0,012899069

0,012905799

0,012905799

0,012905799

0,012905799

0,012918363

0,012937653

0,013033838

0,013048329

0,013048329

0,013048329

0,013048329

0,013048329

0,013059956

0,013117619

0,013119132

0,013119132

0,013174678

0,013210354

0,013210354

0,013210354

0,013210354

0,013210354

0,013210354

0,013251824

0,013260494

0,013301987

0,013318459

0,013377414

0,013382351

0,013382351

0,013382351

0,013449086

0,013457201

0,013501767

0,013501767

0,013501767

0,013501767

0,013547571

0,013566207

0,013588861

0,013588861

0,013608008

0,013620321

0,013620321

0,013620321

0,013620321

0,013620321

0,01373337

0,013815404

0,013942832

0,013942832

0,014049225

0,014100991

0,014293207

0,014335035

OS ribosomal protein S6

Bidirectional sugar transporter SWEET

Zinc finger protein, putative

Type 1 membrane protein, putative isoform 1

yclin-T1-like protein

Ribosomal protein L15

-type proton ATPase subunit a

nase superfamily protein

Basic leucine zipper/W2 domain protein

heat shock protein, chloroplastic

entatricopeptide repeat-containing protein

Polyadenylate-binding protein-interacting protein 3

WUF1005 family protein (DUF1005)

No data found

BnaC07g20870D protein

ADP-ribosylation factor-like

obg-like ATPase 1

(CRS1/YhbY (CRM) domain protein

adenylosuccinate synthetase, chloroplastic

Ultraviolet-B receptor UVR8

Methyltransferase-related family protein

Phosphatidylinositol-4-phosphate 5-kinase, putative

OOl-related E3 ubiquitin-protein ligase 1-like

universal stress protein A-like protein

Oxidoreductase family protein

Cellulose synthase

CBS domain-containing protein CBSX1, chloroplastic

ABC transporter $F$ family-like protein

Actin-interacting protein 1-2

Protein DEHYDRATION-INDUCED 19

Prosomal protein L31

Alpha/beta hydrolase-3

E3 ubiquitin ligase BIG BROTHER-related

No data found

Protein trichome birefringence-like 10

Nascent polypeptide-associated complex subunit beta

NO-associated protein 1, chloroplastic/mitochondrial

COP9 signalosome complex subunit $5 b$

AT-rich interactive domain-containing protein $5 \mathrm{~A}$, putative

Pleckstrin homology domain-containing family $\mathrm{M}$ member 3

Auxin efflux carrier

Protein ROOT PRIMORDIUM DEFECTIVE 1

signal recognition particle $9 \mathrm{kDa}$ protein

Protein trichome birefringence

Receptor kinase

ribonuclease 3-like

Methyltransferase

Ataxin-10

eukaryotic translation initiation factor 3 subunit A-like 
MELO3C017757

MELO3C016033

MELO3C011944

MELO3C023442

MELO3C008149

MELO3C01148

MELO3C021436

MELO3C019873

MELO3C01725

MELO3C015750

MELO3C007472

MELO3C01445

MELO3C007690

MELO3C002180

MELO3C018494

MELO3C017385

MELO3C018120

MELO3C014722

MELO3C019369

MELO3C016139

MELO3C009005

MELO3C022074

MELO3C018732

MELO3C016152

MELO3C013338

MELO3C007743

MELO3C02330

MELO3C011482

MELO3C007152

MELO3C01713

MELO3C008233

MELO3C021404

MELO3C023047

MELO3C027308

MELO3C011759

MELO3C026198

MELO3C013173

MELO3C007494

MELO3C017115

MELO3C020978

MELO3C002034

MELO3C005942

MELO3C016383

MELO3C012713

MELO3C001997

MELO3C016562

MELO3C024241

MELO3C026760

MELO3C021368

MELO3C00828

MELO3C026027

MELO3C001994

MELO3C00958

MELO3C005199

MELO3C01542

MELO3C025799

MELO3C002271

MELO3C009961

MELO3C021384

MELO3C009148

MELO3C011847

MELO3C017833

MELO3C018360

MELO3C014230

MELO3C004480

MELO3C01042

MELO3C022059

MELO3C020963

MELO3C012715

MELO3C009771

MELO3C006154

MELO3C023367

MELO3C01358

MELO3C017416

MELO3C002651

MELO3C01354

MELO3C002314

MELO3C017945

MELO3C00718

MELO3C019564
0,000693923

0,000693729

0,00069511

0,000695857

0,000702007

0,000702629

0,000709823

0,000712436

0,0007278

0,000730788

0,000737797

0,000743376

0,000741216

0,000743002

0,000741174

0,000742664

0,000740158

0,000746062

0,000746633

0,000748004

0,000746916

0,000747376

0,000744619

0,000750497

0,00075512

0,000754472

0,000754129

0,000757284

0,000760376

0,000764993

0,000766033

0,000767416

0,000770707

0,000770119

0,000772506

0,000771969

0,000780326

0,000780392

0,000781959

0,000785857

0,000785617

0,000788048

0,00078938

0,000788594

0,000788804

0,000790211

0,000794484

0,000794199

0,00080597

0,000806924

0,000808771

0,000811478

0,000814919

0,000814794

0,000812644

0,000814018

0,000822787

0,000827069

0,000828633

0,00083221

0,000834449

0,000835285

0,000837062

0,000837903

0,000841204

0,000847224

0,000847733

0,000849397

0,00085277

0,000855185

0,000856674

0,000856226

0,000858523

0,000858187

0,000861593

0,000864234

0,000873986

0,000876911

0,000880809

0,000883614
0,014335035

0,014335035

0,01434455

0,014344959

0,014454388

0,014454388

0,014587203

0,01462568

0,014925576

0,014971299

0,015099226

0,015119403

0,015119403

0,015119403

0,015119403

0,015119403

0,015119403

0,015120083

0,015120083

0,015120083

0,015120083

0,015120083

0,015120083

0,015154969

0,015201698

0,015201698

0,015201698

0,01522973

0,015276373

0,015353508

0,015358769

0,015370886

0,015405551

0,015405551

0,015410315

0,015410315

0,01553626

0,01553626

0,015551777

0,015597885

0,015597885

0,015605062

0,015605062

0,015605062

0,015605062

0,015605875

0,015658951

0,015658951

0,015869496

0,015872456

0,015892961

0,015930293

0,015934492

0,015934492

0,015934492

0,015934492

0,016072425

0,01614011

0,016154657

0,016208388

0,016235996

0,01623626

0,016254804

0,016255158

0,016303183

0,016397526

0,016397526

0,016413642

0,016462712

0,01648973

0,01648973

0,01648973

0,016493166

0,016493166

0,016536046

0,016570635

0,016741364

0,016781123

0,016839406

0,01687668

nositol transporter 1

glutathione S-transferase-like

Beta-1,4-mannosyl-glycoprotein 4-beta-N-acetylglucosaminyltransferase

glycerol kinase

作 10 DISEASE-RESISTANCE LOCUS RECEPTOR-LIKE PROTEIN KINASE-like 1.4 isoform X

ImG homolog protein 2, chloroplastic

$60 \mathrm{~S}$ acidic ribosomal protein $\mathrm{P} 2 \mathrm{~B}$-like

-oxoacyl-[acyl-carrier-protein] synthase-like protein

Sterile alpha motif domain-containing family protein

data found

RR receptor-like serine/threonine-protein kinase GSO1 isoform X1

Receptor-like protein kinase

o data found

mRNA splicing factor, Cwf18

Protein TIC 21, chloroplastic

pollen receptor-like kinase 1

Purple acid phosphatase

Purtor RAP2-11

50 S ribosomal protein $\mathrm{L} 9$

kinase

Unknown protein

Tryptophan synthase-related

Histone-lysine $\mathrm{N}$-methyltransferase, $\mathrm{H} 3$ lysine-36 specific

Gibberellin 2-oxidase

RNA-binding protein 39 isoform $X 1$

Polyadenylate-binding 2

Isoflavone reductase like

ATP-citrate synthase beta chain protein 2-like

Leucine-rich receptor-like protein kinase family protein

General negative regulator of transcription subunit 4

Thylakoid lumenal $15 \mathrm{kDa}$ protein 1, chloroplastic

Heavy metal-associated isoprenylated plant protein 21

glutamyl-tRNA(Gln) amidotransferase subunit B, chloroplastic/mitochondria

alanine--tRNA ligase-like

transcription factor DIVARICATA

amma carbonic anhydrase-like 1, mitochondrial

NAC domain-containing protein 17-like

Hexosyltransferase

succinate dehydrogenase subunit 7B, mitochondrial-like

Adenylyl cyclase-associated protein

ninja-family protein 6 -like isoform $X$

Kinase family protein

RING-type E3 ubiquitin transferase

Zinc finger, $\mathrm{CCCH}$-type

Galactoside 2-alpha-L-fucosyltransferase

At4g33560

Kinesin-like protein

Arabinogalactan protein

DnaJ subfamily B member 14

eukaryotic translation initiation factor 3 subunit $F$ 
MELO3C012722

MELO3C007800

MELO3C007986

MELO3C022783

MELO3C003344

MELO3C004366

MELO3C004577

MELO3C010042

MELO3C010456

MELO3C020789

MELO3C017960

MELO3C003222

MELO3C006412

MELO3C02525

MELO3C014273

MELO3C013965

MELO3C022639

MELO3C009914

MELO3C020591

MELO3C015748

MELO3C019253

MELO3C019858

MELO3C014611

MELO3C009455

MELO3C002363

MELO3C003671

MELO3C00420

MELO3C008058

MELO3C018476

MELO3C013769

MELO3C004279

MELO3C014018

MELO3C007047

MELO3C026518

MELO3C010055

MELO3C016246

MELO3C025917

MELO3C016469

MELO3C002741

MELO3C003375

MELO3C013406

MELO3C01950

MELO3C010686

MELO3C003973

MELO3C009172

MELO3C017703

MELO3C007010

MELO3C010353

MELO3C022144

MELO3C01485

MELO3C009334

MELO3C021622

MELO3C002464

MELO3C014604

MELO3C009663

MELO3C014161

MELO3C010286

MELO3C027408

MELO3C014346

MELO3C018950

MELO3C016923

MELO3C015867

MELO3C008092

MELO3C010601

MELO3C010958

MELO3C015747

MELO3C015692

MELO3C013076

MELO3C006189

MELO3C020825

MELO3C015518

MELO3C008773

MELO3C00876

MELO3C014895

MELO3C022014

MELO3C000075

MELO3C015304

MELO3C018768

MELO3C013175

MELO3C017440
0,000891105

0,00089249

0,000892438

0,000891729

0,000890474

0,000900021

0,000902227

0,000901754

0,00090483

0,000905325

0,000907356

0,0009085

0,000910434

0,00091322

0,000917455

0,000916457

0,000915894

0,000918184

0,000923743

0,00092849

0,000930162

0,000933305

0,0009373

0,000940284

0,00094684

0,000947369

0,00095

0,000949138

0,000953839

0,000956211

0,00096026

0,000957996

0,000959447

0,000958725

0,000964219

0,000969134

0,000968892

0,000970779

0,000973931

0,000975079

0,000976852

0,000978735

0,000979008

0,000982277

0,000986526

0,000986533

0,000985587

0,000986326

0,000992835

0,000995059

0,000999425

0,001003418

0,001003114

0,001005214

0,001009648

0,001009339

0,001008161

0,001010878

0,001017641

0,001016798

0,001022046

0,001022134

0,001020377

0,001025788

0,001025271

0,001032087

0,001037635

0,001042091

0,001045776

0,001046103

0,001051409

0,001052661

0,001057254

0,001064445

0,001066831

0,00107158

0,001074501

0,001075167

0,00108687

0,001088108
0,016964184

0,016964184

0,016964184

0,016964184

0,016964184

0,017090883

0,017099894

0,017099894

0,017125726

0,017125726

0,017147725

0,017152936

0,01717303

0,017209147

0,017236905

0,017236905

0,017236905

0,017236905

0,01732478

0,017397286

0,017412104

0,017454393

0,017512516

0,017551679

0,017650564

0,017650564

0,017666245

0,017666245

0,01772094

0,017748314

0,017756725

0,017756725

0,017756725

0,017756725

0,01781324

0,017870612

0,017870612

0,01788425

0,017925603

0,01793001

0,017945908

0,017952113

0,017952113

0,017995345

0,018006515

0,018006515

0,018006515

0,018006515

0,018104803

0,018128608

0,01819137

0,01823046

0,01823046

0,01824629

0,018276383

0,018276383

0,018276383

0,018281896

0,018370564

0,018370564

0,018401207

0,018401207

0,018401207

0,018433383

0,018433383

0,018529709

0,018612402

0,018675371

0,018713312

0,018713312

0,018791208

0,01879657

0,018861521

0,018972668

0,018998061

0,01906543

0,019094858

0,019094858

0,019285347

0,019289993

Pentatricopeptide repeat-containing family protein

Receptor protein kinase, putative

istidine phosphatase family (Branch 1) protein

transmembrane protein $50 \mathrm{~A}$

-isopropylmalate dehydratase large subunit

renylyltransferase superfamily protein

RS2-associated factor 1, chloroplastic

doribonuclease Dicer homolog 2 isoform $X_{2}$

hosphatidylinositol-4-phosphate 5-kinase, putative

Pucleoporin NUP188 like

NADH dehydrogenase [ubiquinone] iron-sulfur protein 5-B

mRNA-capping enzyme

S ribosomal protein L14, putative

Zinc transporte

(a)

vin-containing monooxygenase

No data found

Alpha-mannosidase

Glycosyltransferase

iption factor EMB1444

thioredoxin domain-containing protein 9 homolog

No data found

e-mRNA-splicing factor CWC22

BZIP transcription factor family protein

TITAN-like protein

SPOC domain/transcription elongation factor S-II, putative

Importin subunit alpha

mitogen-activated protein kinase kinase kinase YODA isoform $X$

cyclin-D3-3

Seven transmembrane receptor

RING zinc finger family protein

Pentatricopeptide repeat-containing protein family

Protein kinase

Photosystem II reaction center PsbP family protein

4-hydroxy-4-methyl-2-oxoglutarate aldolase

Kinase family protein

CAX-interacting protein 4

zinc finger $\mathrm{CCCH}$ domain-containing protein 5

Protein TIFY 5A

Two-component response regulator-like protein APRR2

RNA-dihydrouridine (47) synthase $[N A D(P)(+)]$

Meiosis arrest female protein 1-like protein

Alanine aminotransferase 2

SNW/SKI-interacting protein-like

RING finger protein

disease resistance protein RGA2-like

phosphatidate cytidylyltransferase, mitochondrial isoform X1

protein NRT1/ PTR FAMILY 6.2-like

$\mathrm{TMV}$ resistance protein $\mathrm{N}$-like 
MELO3C024226

MELO3C009261

MELO3C014027

MELO3C025232

MELO3C015946

MELO3C023413

MELO3C002757

MELO3C017149

MELO3C018617

MELO3C011484

MELO3C011088

MELO3C006088

MELO3C012391

MELO3C020942

MELO3C006409

MELO3C016775

MELO3C010517

MELO3C007103

MELO3C005918

MELO3C020806

MELO3C017223

MELO3C021345

MELO3C010985

MELO3C020834

MELO3C004448

MELO3C013773

MELO3C022477

MELO3C002144

MELO3C006294

MELO3C004015

MELO3C007433

MELO3C008815

MELO3C008313

MELO3C014315

MELO3C027330

MELO3C00880

MELO3C005079

MELO3C005332

MELO3C021456

MELO3C003305

MELO3C004439

MELO3C005367

MELO3C014091

MELO3C017255

MELO3C021620

MELO3C005651

MELO3C004142

MELO3C016826

MELO3C002219

MELO3C00631

MELO3C024505

MELO3C006160

MELO3C018758

MELO3C005704

MELO3C015202

MELO3C026288

MELO3C017581

MELO3C009583

MELO3C017358

MELO3C02422

MELO3C023338

MELO3C022494

MELO3C009234

MELO3C008598

MELO3C017939

MELO3C022819

MELO3C012004

MELO3C01382

MELO3C005849

MELO3C008947

MELO3C016623

MELO3C006145

MELO3C02630

MELO3C013750

MELO3C003826

MELO3C018434

MELO3C013489

MELO3C010223

MELO3C014447

MELO3C022094
0,00109041

0,001093328

0,001103763

0,001102913

0,001106583

0,001105341

0,001104571

0,001102634

0,001106334

0,001104414

0,001108133

0,001117354

0,001117266

0,001115702

0,001125418

0,001123501

0,001125381

0,001130481

0,001128976

0,001131583

0,001130983

0,001136978

0,001137691

0,00113984

0,001143358

0,001144942

0,001146898

0,001148916

0,001154872

0,001156853

0,001159232

0,001162718

0,00116263

0,001169638

0,001170708

0,001169091

0,001166811

0,001165013

0,001170444

0,001167299

0,001173596

0,001174443

0,001178037

0,001182788

0,001185964

0,001186684

0,001189887

0,001188294

0,001190259

0,001193205

0,001194936

0,001197871

0,001202

0,001205731

0,001210885

0,001212665

0,001216996

0,001222917

0,001226322

0,00122875

0,001234763

0,001235308

0,001237827

0,001240561

0,00123777

0,001239145

0,001240427

0,001245009

0,001247648

0,001248718

0,001251995

0,001255715

0,001256932

0,001262209

0,001266017

0,001269051

0,001281695

0,00128181

0,001297412

0,001296764
0,019313455

0,019347793

0,019442977

0,019442977

0,019442977

0,019442977

0,019442977

0,019442977

0,019442977

0,019442977

0,019452898

0,019562608

0,019562608

0,019562608

0,019651524

0,019651524

0,019651524

0,019689543

0,019689543

0,019689543

0,019689543

0,019761011

0,019761011

0,019780936

0,019824557

0,019834607

0,019851081

0,019868609

0,019954119

0,019970875

0,019994482

0,02001964

0,02001964

0,020034938

0,020034938

0,020034938

0,020034938

0,020034938

0,020034938

0,020034938

0,02006408

0,02006408

0,020108075

0,020171749

0,02020329

0,02020329

0,020211881

0,020211881

0,020211881

0,020244514

0,02025648

0,020288819

0,020341295

0,020386969

0,02045661

0,020469162

0,02052472

0,020606981

0,020646745

0,020670004

0,020744986

0,020744986

0,020744988

0,020744988

0,020744988

0,020744988

0,020744988

0,020801755

0,020828226

0,020828498

0,020865521

0,02090987

0,020912504

0,020982623

0,021028241

0,021060915

0,021236976

0,021236976

0,021444979

0,021444979

Sulfate transporter 3.1

Peptide transporter family protein

tochrome P450 family protein

Ubiquitin-conjugating enzyme, E2

transcription factor-like protein

gnal peptidase I

signal recognition particle 14 kDa protein

entatricopeptide repeat-containing protein

Pentatricopeptide repeat-containing protein

rmidine synthase

mechanosensitive ion channel protein 3, chloroplastic-like

$50 \mathrm{~S}$ ribosomal protein $\mathrm{L} 5$, chloroplastic

domain-containing protein 16

Q-loop repeat family protein / transmembrane family protein

(ronsfer protein domain-containing protein

ucine-rich repeat receptor-like protein kinase family

WAT1-related protein

Protein plastid transcriptionally active 12

PL4 family protein

Peptidyl-prolyl cis-trans isomerase

Mitochondrial carrier protein, putative

cullin-1

B-box zinc finger protein 20-like

Unknown protein

DNA helicase

Mediator of RNA polymerase II transcription subunit

Protein nuclear fusion defective 4

eceptor-like kinase 1

Dna repair helicase $\mathrm{xpb1}$

Pentatricopeptide repeat-containing family protein

fumarylacetoacetase

ucleolar MIF4G domain-containing protein 1

--glucose-1-phosphate uridylyltransferase 3 , chloroplastic

Bifunctional DNA-directed RNA polymerase subunit beta-beta

Caffeic acid O-methyltransferase

/ACA ribonucleoprotein complex subunit

protein ASPARTIC PROTEASE IN GUARD CELL 2

CDP-diacylglycerol--serine O-phosphatidyltransferase 1

histone deacetylase HDT1-like

Protein CDI

Protein phosphatase $2 \mathrm{c}$, putative

dehydration-responsive element-binding protein 1D-like

caffeic acid 3-O-methyltransferase-like

BSD domain-containing protein

transcription initiation factor TFIID subunit 7

30S ribosomal S1

Unknown protein

At4g33800

HIPL1 protein-like isoform $X 1$

$60 S$ ribosomal protein L13a, putative 
MELO3C005201

MELO3C006420

MELO3C007127

MELO3C01430

MELO3C008076

MELO3C014258

MELO3C005913

MELO3C017730

MELO3C016550

MELO3C002227

MELO3C009467

MELO3C023985

MELO3C01213

MELO3C018180

MELO3C005779

MELO3C004645

MELO3C013149

MELO3C007899

MELO3C026058

MELO3C005214

MELO3C026194

MELO3C018688

MELO3C010731

MELO3C010640

MELO3C005711

MELO3C021185

MELO3C016149

MELO3C015695

MELO3C019011

MELO3C006759

MELO3C013780

MELO3C00165

MELO3C005389

MELO3C018264

MELO3C003423

MELO3C007413

MELO3C021308

MELO3C013745

MELO3C013760

MELO3C01468

MELO3C021360

MELO3C002687

MELO3C004084

MELO3C006202

MELO3C01950

MELO3C017880

MELO3C025272

MELO3C004833

MELO3C008116

MELO3C011498

MELO3C021563

MELO3C010599

MELO3C002717

MELO3C005939

MELO3C022399

MELO3C004475

MELO3C000251

MELO3C00282

MELO3C004467

MELO3C005926

MELO3C005821

MELO3C022448

MELO3C013702

MELO3C016558

MELO3C007269

MELO3C020749

MELO3C018985

MELO3C019053

MELO3C015954

MELO3C021295

MELO3C003394

MELO3C012730

MELO3C002092

MELO3C017167

MELO3C023251

MELO3C020927

MELO3C019661

MELO3C022124

MELO3C016608

MELO3C005331
0,001297623

0,001299336

0,001302118

0,001311889

0,001313603

0,00131955

0,001328415

0,001329905

0,001332312

0,001334843

0,001336203

0,001340004

0,001342593

0,001343365

0,001350039

0,001354559

0,001355635

0,001357906

0,001357441

0,001360416

0,001361813

0,001360022

0,001361834

0,00136367

0,001366829

0,001367208

0,001369639

0,001377374

0,001378575

0,001385022

0,001388796

0,001392959

0,001395363

0,001399616

0,001400854

0,001405347

0,001405962

0,001413439

0,001414678

0,001418108

0,001419955

0,001420383

0,001428955

0,00143051

0,001430486

0,001423996

0,001429508

0,001425696

0,001429946

0,001426771

0,001441577

0,001442786

0,001445458

0,001448732

0,00145033

0,001453822

0,001453758

0,001454245

0,001460846

0,001468715

0,001467018

0,001467626

0,001471309

0,001475647

0,001475218

0,001473578

0,001478932

0,001482362

0,001488106

0,001489848

0,001489988

0,001497104

0,001496421

0,001501045

0,001504394

0,001505539

0,00150409

0,001507441

0,001511362

0,001510473
0,021444979

0,021455341

0,021483309

0,021626452

0,021636651

0,021716499

0,021844183

0,021850495

0,021871848

0,021895188

0,021899309

0,021943403

0,021962024

0,021962024

0,022052878

0,022099308

0,022099308

0,022099308

0,022099308

0,022099308

0,022099308

0,022099308

0,022099308

0,022110937

0,022131961

0,022131961

0,022153147

0,022260026

0,022261231

0,022347062

0,022389659

0,022438453

0,022458857

0,022508972

0,022510545

0,02255593

0,02255593

0,02265747

0,022658937

0,022695105

0,022695105

0,022695105

0,022709919

0,022709919

0,022709919

0,022709919

0,022709919

0,022709919

0,022709919

0,022709919

0,022867225

0,022868044

0,022892023

0,022925487

0,022932398

0,022939204

0,022939204

0,022939204

0,02302494

0,023093668

0,023093668

0,023093668

0,023116053

0,023129006

0,023129006

0,023129006

0,023162115

0,023197442

0,023261476

0,023261476

0,023261476

0,023335688

0,023335688

0,023378654

0,023393301

0,023393301

0,023393301

0,023404444

0,023428483

0,023428483

multicopper oxidase LPR1-like

Cotton fiber protein

-cis-epoxycarotenoid dioxygenase

RA1 family protein

data found

-domain 1

Auxin-responsive family protein

Pentatricopeptide repeat-containing family protein

Unknown protein

known protein

Alpha/beta-Hydrolases superfamily protein

RNA/RNP complex-1-interacting phosphatase, putative

ukaryotic translation initiation factor $5 \mathrm{~A}$-like

RNA (guanine(37)-N1)-methyltransferase

Metal-dependent protein hydrolase

40 S ribosomal S3-like protein

UPF0505 protein C16orf62 homolog isoform X1

repeat-containing protein 82

Non-specific serine/threonine protein kinase

Defensin

No data found

dynamin-like protein ARC5

clin-dependent kinase $\mathrm{G}-2$ isoform $\mathrm{X} 1$

zinc finger $\mathrm{CCCH}$ domain-containing protein 1

heavy metal-associated isoprenylated plant protein 3-like

Peptidase M48

Cationic amino acid transporter, putative

copper-transporting ATPase PAA1, chloroplastic isoform X1

Ca2+-activated RelA/SpoT-like protein

Early nodulin-like protein 1

Unknown protein

NADH dehydrogenase [ubiquinone] 1 alpha subcomplex subunit 12

No data found

Zinc finger $\mathrm{CCCH}$ domain protein

Core-2/I-branching beta-1,6-N-acetylglucosaminyltransferase family protein

Multiple organellar RNA editing factor 8, chloroplastic/mitochondria

Phosphatase $2 \mathrm{C}$ family protein

$26 \mathrm{~S}$ proteasome non-ATPase regulatory subunit 4 homolog

Structural maintenance of chromosomes protein

Pentatricopeptide repeat-containing family protein

dynamin-related protein 4C-like

No data found

Peptidase M22 domain-containing protein

Transmembrane 53

ilucan endo-1,3-beta-glucosidase

AT3g54190/F24B22_150

crooked neck-like protein 1

Magnesium transporter NIPA

Splicing factor u2af large subunit, putative 
MELO3C009587

MELO3C005136

MELO3C025590

MELO3C009872

MELO3C003416

MELO3C004564

MELO3C016884

MELO3C004273

MELO3C01072

MELO3C023503

MELO3C018573

MELO3C019724

MELO3C014888

MELO3C01254

MELO3C003249

MELO3C004944

MELO3C021217

MELO3C009149

MELO3C024337

MELO3C003487

MELO3C019026

MELO3C00413

MELO3C023358

MELO3C011654

MELO3C007617

MELO3C006316

MELO3C020369

MELO3C006870

MELO3C018106

MELO3C00428

MELO3C024916

MELO3C01933

MELO3C011011

MELO3C00725

MELO3C005103

MELO3C016315

MELO3C020836

MELO3C026715

MELO3C005511

MELO3C007328

MELO3C004321

MELO3C00740

MELO3C025308

MELO3C008847

MELO3C00216

MELO3C017207

MELO3C005109

MELO3C010057

MELO3C012445

MELO3C002118

MELO3C002390

MELO3C022228

MELO3C020733

MELO3C018306

MELO3C00950

MELO3C025490

MELO3C025725

MELO3C025872

MELO3C009188

MELO3C013738

MELO3C002312

MELO3C002646

MELO3C021845

MELO3C026143

MELO3C023540

MELO3C011172

MELO3C006949

MELO3C01259

MELO3C002016

MELO3C026657

MELO3C006136

MELO3C009119

MELO3C009318

MELO3C023310

MELO3C018521

MELO3C019818

MELO3C009278

MELO3C009019

MELO3C023309

MELO3C009949
0,001521811

0,001521725

0,001526407

0,001525706

0,001529192

0,001534372

0,001539685

0,001542611

0,001542628

0,001549719

0,001550384

0,001557475

0,001567833

0,00156905

0,001569429

0,001569178

0,001571244

0,001572553

0,001576201

0,00157823

0,001585064

0,001588934

0,001586845

0,001589152

0,001591437

0,001593731

0,001597715

0,001595901

0,001596976

0,001605072

0,001604299

0,001606757

0,001610571

0,001613304

0,00161323

0,001620821

0,001625795

0,001625262

0,001628068

0,001631145

0,001634236

0,001634885

0,001637045

0,001648475

0,001652239

0,001652461

0,00165439

0,001655678

0,001657268

0,001660133

0,00166271

0,001662029

0,001666156

0,001665461

0,00166957

0,001670243

0,001669696

0,001673066

0,001675463

0,00167882

0,00167825

0,001687107

0,001689148

0,001694488

0,001697192

0,001697815

0,001709312

0,00171077

0,00170755

0,001709578

0,00171365

0,001718851

0,001727588

0,001728325

0,001726606

0,001724792

0,0017382

0,001741661

0,001739777

0,00174184
0,023553483

0,023553483

0,023587651

0,023587651

0,023612208

0,023673689

0,023737111

0,023745402

0,023745402

0,023827649

0,023827649

0,023918018

0,024026857

0,024026857

0,024026857

0,024026857

0,024036012

0,024037418

0,024074551

0,024086905

0,024172538

0,024178861

0,024178861

0,024178861

0,024194985

0,024211227

0,024215862

0,024215862

0,024215862

0,024290084

0,024290084

0,02429697

0,024336007

0,024340055

0,024340055

0,024434804

0,024472427

0,024472427

0,024487975

0,024515588

0,024534462

0,024534462

0,024548219

0,024700867

0,024723069

0,024723069

0,024733189

0,024733726

0,024738761

0,024762816

0,024763845

0,024763845

0,024777792

0,024777792

0,024782586

0,024782586

0,024782586

0,024805848

0,02482275

0,02483522

0,02483522

0,024939126

0,02495062

0,025010794

0,025022503

0,025022503

0,025138388

0,025138388

0,025138388

0,025138388

0,025161981

0,025219605

0,02528348

0,02528348

0,02528348

0,02528348

0,025405911

0,025405911

0,025405911

0,025405911

S-adenosyl-L-methionine-dependent methyltransferases superfamily protein

Protein EARLY RESPONSIVE TO DEHYDRATION 15

F-kappa-B inhibitor-like protein 2 isoform 2

Gibberellin-regulated family protein

nown protein

protein NUCLEAR FUSION DEFECTIVE 6, chloroplastic/mitochondrial-like isoform X2

Methyltransferase

Mitochondrial Rho GTPase

3-oxoacyl-[acyl-carrier-protein] reductase 4

known protein

Inorganic pyrophosphatase

Triacylglycerol lipase 2 , putative

$\mathrm{rdC}$ domain-containing protein, mitochondrial isoform $\mathrm{X} 2$

phosphatidylinositol 4-kinase alpha 1-like

target of Myb protein 1 isoform X1

polyadenylation and cleavage factor homolog 4 isoform $X 2$

MACPF domain-containing CAD1-like protein

Copper chaperone SCO1/SenC

transmembrane protein 87B isoform X2

receptor-like protein kinase HAIKU2

Phosphate transporter

leucine aminopeptidase 1-like

Ubiquitin fusion degradation 1 protein

RING zinc finger protein-like

At5g67390

cytochrome b5

$60 S$ ribosomal protein L37a

列

$A B C$ transporter $B$ family protein

mevalonate kinase-like

Agenet domain-containing protein / bromo-adjacent domain-containing protein, putative

DUF679 domain membrane protein

(1) superfamily protein

guanylate-binding protein 2

Cytochrome b-c1 complex subunit Rieske, mitochondrial

Unknown protein

transportin-1

Tetratricopeptide repeat (TPR)-like superfamily protein

palmitoyl-protein thioesterase 1-like

Wound-responsive family protein

Vat protein

peroxisomal membrane protein PMP22

auxin-responsive protein IAA16-like

M50 family peptidase

Succinate--CoA ligase [ADP-forming] subunit alpha, mitochondrial

YR motif-containing protein 4

alpha-mannosidase

WRKY transcription factor SUSIBA2-like isoform X2

ATP synthase subunit $d$, mitochondrial 
MELO3C009275

MELO3C006005

MELO3C006569

MELO3C016483

MELO3C016879

MELO3C005299

MELO3C012967

MELO3C017043

MELO3C02223

MELO3C021047

MELO3C021370

MELO3C003456

MELO3C004139

MELO3C018495

MELO3C017838

MELO3C005286

MELO3C019947

MELO3C020860

MELO3C002240

MELO3C009032

MELO3C013353

MELO3C00708

MELO3C014099

MELO3C022021

MELO3C025593

MELO3C016931

MELO3C01746

MELO3C008248

MELO3C006943

MELO3C005446

MELO3C01695

MELO3C01195

MELO3C008389

MELO3C003593

MELO3C002589

MELO3C010716

MELO3C00636

MELO3C023469

MELO3C008040

MELO3C017808

MELO3C026053

MELO3C010350

MELO3C023473

MELO3C007011

MELO3C01305

MELO3C006598

MELO3C016379

MELO3C011705

MELO3C022411

MELO3C01385

MELO3C013331

MELO3C01748

MELO3C007612

MELO3C018999

MELO3C026015

MELO3C015999

MELO3C011885

MELO3C027092

MELO3C023885

MELO3C025699

MELO3C014825

MELO3C011716

MELO3C017836

MELO3C011206

MELO3C019899

MELO3C004391

MELO3C011977

MELO3C00668

MELO3C007737

MELO3C019326

MELO3C002372

MELO3C026247

MELO3C002897

MELO3C011109

MELO3C017786

MELO3C010755

MELO3C009159

MELO3C014488

MELO3C021929

MELO3C026782
0,001749203

0,001751647

0,001747599

0,001744253

0,001748919

0,001746783

0,00175148

0,001755354

0,001755155

0,001762342

0,001762177

0,001761414

0,00176555

0,001768672

0,001777557

0,001783225

0,001782242

0,001789058

0,001807554

0,001813381

0,001817417

0,001821971

0,001824298

0,001827571

0,001834197

0,001837696

0,001838808

0,001836651

0,001845063

0,001846912

0,001854951

0,001855104

0,001855419

0,001853717

0,001869205

0,001872575

0,001875238

0,001878526

0,001881556

0,001885382

0,001887507

0,001886706

0,001895232

0,001898043

0,001897813

0,001903585

0,00190291

0,001905403

0,001916373

0,001926227

0,001924304

0,001926725

0,001929109

0,001934757

0,001938149

0,001937128

0,001937355

0,0019373

0,001943686

0,001945414

0,001948929

0,001953014

0,001960071

0,001960649

0,001972807

0,001978193

0,001982034

0,001986267

0,001987191

0,001990879

0,002008671

0,002012254

0,002014896

0,002016465

0,002018683

0,002023602

0,002035301

0,002035415

0,002034457

0,002039701
0,02541754

0,02541754

0,02541754

0,02541754

0,02541754

0,02541754

0,02541754

0,025433964

0,025433964

0,025479124

0,025479124

0,025479124

0,025506839

0,025533265

0,025642781

0,025687028

0,025687028

0,025752271

0,025999557

0,026064389

0,026103398

0,026149788

0,026164165

0,026192087

0,026267986

0,026276854

0,026276854

0,026276854

0,026347185

0,026354528

0,02639962

0,02639962

0,02639962

0,02639962

0,026576627

0,026605377

0,026624071

0,026651592

0,026675408

0,026702269

0,026702269

0,026702269

0,02679237

0,026793748

0,026793748

0,026833623

0,026833623

0,026840092

0,026975379

0,027063223

0,027063223

0,027063223

0,027077453

0,02710801

0,02710801

0,02710801

0,02710801

0,02710801

0,027166205

0,027171132

0,027200989

0,027238758

0,027306672

0,027306672

0,027456636

0,027512209

0,027546231

0,027579088

0,027579088

0,027610857

0,027838061

0,027868159

0,027885197

0,027887376

0,027898511

0,027946928

0,028051229

0,028051229

0,028051229

0,028090688

No data found

Bifunctional fucokinase/fucose pyrophosphorylase

ytochrome P450 family protein

phosphoribosylamine--glycine ligase

Cytochrome P45, putative

2 domain-containing protein

At5g57230

Autamate receptor

Corepressor

olin-like

AX-interacting protein 1

Alpha/beta-Hydrolases superfamily protein

biquitin carboxyl-terminal hydrolase, putative

Carbohydrate esterase, putative (DUF303)

biquitin system component Cue

Mediator of RNA polymerase II transcription subunit 23

Oxidoreductase family protein

A polymerase

RING-type E3 ubiquitin transferase

fructokinase-like 2, chloroplastic

heavy metal-associated isoprenylated plant protein 26-like

SIT4 phosphatase-associated family protein

77690 protein

Lysine--trna ligase

Pentatricopeptide repeat-containing family protein

Hydroxyproline-rich glycoprotein

(DL)-glycerol-3-phosphatase 2

GRF1-interacting factor 3

Keratin-associated protein, putative (DUF819)

$60 \mathrm{~S}$ ribosomal protein $\mathrm{L} 13$

lutathione peroxidase

UDP-galactose:fucoside alpha-3-galactosyltransferase

RNA-binding protein 2

Bromo-adjacent-like (BAH) domain protein

At2g47960/T9J23.10

Protein HHL1, chloroplastic

histone-lysine N-methyltransferase family member SUVH9

Protein LOW PSII ACCUMULATION 3, chloroplastic

RING-type E3 ubiquitin transferase

Protein NEOXANTHIN-DEFICIENT 1

Promily protein At2g38370

heme oxygenase 1 , chloroplastic

Phosphatase $2 \mathrm{C}$ family protein

No data found

Dead box ATP-dependent RNA helicase, putative

serine/arginine-rich SC35-like splicing factor SCL28

Ultraviolet-B receptor UVR8

Pyruvate dehydrogenase E1 component subunit alpha

No data found

CAAX amino terminal protease 
MELO3C011984

MELO3C003852

MELO3C003861

MELO3C01956

MELO3C017979

MELO3C012218

MELO3C018406

MELO3C006528

MELO3C002785

MELO3C024673

MELO3C019810

MELO3C00351

MELO3C012160

MELO3C010420

MELO3C001323

MELO3C017259

MELO3C003545

MELO3C022500

MELO3C003292

MELO3C020906

MELO3C021441

MELO3C00968

MELO3C023402

MELO3C026403

MELO3C014272

MELO3C009328

MELO3C010600

MELO3C013822

MELO3C017142

MELO3C025073

MELO3C023201

MELO3C018368

MELO3C008318

MELO3C022425

MELO3C023799

MELO3C023753

MELO3C008985

MELO3C015941

MELO3C016253

MELO3C018365

MELO3C021777

MELO3C01515

MELO3C010737

MELO3C014860

MELO3C018042

MELO3C012695

MELO3C007232

MELO3C010695

MELO3C026636

MELO3C00675

MELO3C000756

MELO3C025854

MELO3C023370

MELO3C011263

MELO3C004533

MELO3C009284

MELO3C005759

MELO3C002058

MELO3C018569

MELO3C01859

MELO3C01245

MELO3C014588

MELO3C00446

MELO3C007580

MELO3C012642

MELO3C007613

MELO3C008074

MELO3C02671

MELO3C026250

MELO3C016308

MELO3C011105

MELO3C022382

MELO3C00342

MELO3C024108

MELO3C017571

MELO3C012634

MELO3C011989

MELO3C01941

MELO3C016970

MELO3C026046
0,002046965

0,00205307

0,002053843

0,002059087

0,002059733

0,00205906

0,002057861

0,002063123

0,002062723

0,002067343

0,002067002

0,002064632

0,002082866

0,002085005

0,002089158

0,002087757

0,002089665

0,002094619

0,002110998

0,002112654

0,002119311

0,002121381

0,002122362

0,002124813

0,002126328

0,00213544

0,002138232

0,002143379

0,002151301

0,002156608

0,002162151

0,002174462

0,002183195

0,002187971

0,002193667

0,002198867

0,002200413

0,002203298

0,002213095

0,002215524

0,002218623

0,002226652

0,002225506

0,002232141

0,002230883

0,002239626

0,00224746

0,002255474

0,002257047

0,0022639

0,00226318

0,002276317

0,002278528

0,002281069

0,00230233

0,002314987

0,002315355

0,002320837

0,002321785

0,002323123

0,002324859

0,002329523

0,002328875

0,002330184

0,002340501

0,0023421

0,002345054

0,002357921

0,002366285

0,00236964

0,002372222

0,002378877

0,002381324

0,002390668

0,002393379

0,002392484

0,002396412

0,002402097

0,002408407

0,002406738
0,028171092

0,028226406

0,028226406

0,028228772

0,028228772

0,028228772

0,028228772

0,028235108

0,028235108

0,028235108

0,028235108

0,028235108

0,028427444

0,028436991

0,02844162

0,02844162

0,02844162

0,02848941

0,028692428

0,028695185

0,028765823

0,028767693

0,028767693

0,028781161

0,028781935

0,028885477

0,028903446

0,028953215

0,029040357

0,029092111

0,029146973

0,029292935

0,029390538

0,029434771

0,029491311

0,029541101

0,029541772

0,029560419

0,029671694

0,029684113

0,029705479

0,02977262

0,02977262

0,029805646

0,029805646

0,029885381

0,029969679

0,030056245

0,030056923

0,030107586

0,030107586

0,030252349

0,030261358

0,03027475

0,030536406

0,030667975

0,030667975

0,030709077

0,030709077

0,030709077

0,030711465

0,030720166

0,030720166

0,030720166

0,030835587

0,030836086

0,030854414

0,031003045

0,031092325

0,031115699

0,031128911

0,031195509

0,031206873

0,031302545

0,031302545

0,031302545

0,031321469

0,031367529

0,031367529

0,031367529

yrrolidone-carboxylate peptidase

DNA ligase-like protein

/ACA ribonucleoprotein complex non-core subunit NAF1-like isoform X1

At1g78995

ional protein FolD

Two-component response regulator

Cytochrome $\mathrm{c}$ biogenesis protein

At1g05410/T25N20_5

epimerase family protein SDR39U1 homolog, chloroplastic-like

transcription factor

Adenosine kinase

No data found

Protein BIG GRAIN 1-like E

DUF581 family protein, putative (DUF581)

transmembrane protein $184 \mathrm{C}$

Protoheme IX farnesyltransferase, mitochondrial

$28 \mathrm{kDa}$ ribonucleoprotein

acuolar protein sorting 26

protein $\mathrm{N}$-methyltransferase NNT1 isoform X1

60S ribosomal protein L26-1-like

Low molecular weight protein-tyrosine-phosphatase, putative No data found

carboxyl carrier protein of acetyl-CoA carboxylase

60 S ribosomal protein $\mathrm{L} 27$

Pyruvate kinase

ectin receptor kinase-like protein

DNA ligase 1 isoform X3

nknown protein

tRNA (Ile)-lysidine synthase

GRIP and coiled-coil domain-containing protein 1

No data found

omega-hydroxypalmitate O-feruloyl transferase

Drotein $2 \mathrm{C}$

$15 \mathrm{kDa}$ selenoprotein

12-oxophytodienoate reductase 3

(2-0x0627400D protein

Glucose-6-phosphate 1-dehydrogenase

Long cell-linked locus protein, putative, expressed

Zinc finger protein, putative

GDP-Man:Man(3)GICNAc(2)-PP-Dol alpha-1,2-mannosyltransferase

vesicle-associated membrane protein, putative

Alanine:glyoxylate aminotransferase

RING-type E3 ubiquitin transferase

Transcription factor GTE8

Phosphoglycerate mutase-like protein 1

a-related protein $6 \mathrm{~B}$ isoform $\mathrm{X}$

spermidine coumaroyl-CoA acyltransferase No data found

Protein arginine N-methyltransferase 
MELO3C003278

MELO3C022568

MELO3C017274

MELO3C019815

MELO3C012361

MELO3C023445

MELO3C008977

MELO3C009290

MELO3C020988

MELO3C015664

MELO3C004459

MELO3C014877

MELO3C006546

MELO3C010794

MELO3C01987

MELO3C006362

MELO3C006224

MELO3C016382

MELO3C025893

MELO3C002644

MELO3C009581

MELO3C018344

MELO3C008115

MELO3C006942

MELO3C017094

MELO3C02435

MELO3C011216

MELO3C009748

MELO3C016056

MELO3C026427

MELO3C025897

MELO3C01260

MELO3C01066

MELO3C016031

MELO3C016574

MELO3C007940

MELO3C01432

MELO3C011104

MELO3C006158

MELO3C011769

MELO3C025550

MELO3C020003

MELO3C016706

MELO3C020533

MELO3C015210

MELO3C006348

MELO3C006055

MELO3C001354

MELO3C014730

MELO3C00610

MELO3C023317

MELO3C011045

MELO3C022715

MELO3C006785

MELO3C00577

MELO3C021348

MELO3C011550

MELO3C006555

MELO3C006817

MELO3C018616

MELO3C003793

MELO3C015779

MELO3C003924

MELO3C015791

MELO3C004163

MELO3C011320

MELO3C003135

MELO3C026799

MELO3C022000

MELO3C007601

MELO3C011236

MELO3C012844

MELO3C009572

MELO3C016114

MELO3C017128

MELO3C003532

MELO3C004441

MELO3C014706

MELO3C022174

MELO3C023622
0,002403885

0,002409466

0,002405103

0,002415556

0,002418655

0,00242176

0,00242354

0,002430501

0,002436848

0,00244087

0,002445923

0,002454349

0,00245917

0,00245781

0,002461341

0,002466494

0,002471833

0,002470672

0,002470417

0,002480634

0,002484269

0,00248821

0,002491333

0,002496694

0,002497905

0,002500774

0,002506002

0,002525227

0,002528753

0,002527159

0,002525532

0,002533219

0,002534744

0,002536861

0,00253857

0,002540917

0,002547808

0,002547373

0,002565104

0,002564335

0,002564713

0,002561197

0,002573575

0,002573546

0,002582759

0,002586612

0,002590276

0,002590061

0,002592731

0,002599318

0,002598954

0,002596188

0,002602778

0,002604786

0,002606831

0,002612184

0,002612382

0,00261696

0,002620451

0,002618082

0,002620709

0,002624553

0,00263039

0,002629404

0,002635886

0,002636044

0,002638042

0,002650628

0,002660118

0,00266637

0,002666451

0,002668541

0,00267166

0,002679947

0,002678091

0,0026795

0,002681757

0,002688709

0,002686306

0,002688572
0,031367529

0,031367529

0,031367529

0,031426095

0,0314457

0,031465348

0,031467785

0,031537432

0,031599023

0,031630406

0,031675097

0,031763391

0,031784128

0,031784128

0,031791379

0,031837123

0,031843597

0,031843597

0,031843597

0,03193614

0,03196211

0,031991964

0,032011282

0,032054016

0,032054016

0,032069996

0,032116174

0,032323842

0,032323842

0,032323842

0,032323842

0,032358543

0,032358543

0,032364646

0,032365535

0,032374558

0,032420527

0,032420527

0,032556707

0,032556707

0,032556707

0,032556707

0,032622291

0,032622291

0,032717708

0,032745518

0,032749907

0,032749907

0,032759975

0,03278029

0,03278029

0,03278029

0,032802982

0,032807344

0,03281218

0,032840187

0,032840187

0,032861199

0,032861199

0,032861199

0,032861199

0,032888516

0,032919884

0,032919884

0,032948877

0,032948877

0,032952998

0,033089282

0,033186779

0,033223806

0,033223806

0,033228888

0,033246763

0,033286969

0,033286969

0,033286969

0,033288505

0,033291105

0,033291105

0,033291105

HD finger protein At1g33420

protein indeterminate-domain 5, chloroplastic

patch domain-containing protein TGH

Receptor-like protein kinase

control protein 2 homolog A

-box/LRR-repeat protein 14

in-like protein

Posphatidate phosphatase PAH1

Unknown protein

-type 33 ubiquitin transferase

ctin-related protein 6

NA/RNA-binding protein KIN17

kinase inhibitor 1-like

No data found

ytol kinase

Beta-amylase

HOPM interactor 7

mall nuclear ribonucleoprotein Sm D2-like

chaperone protein DnaJ

Transcription factor

D-lactate dehydrogenase, putative

No data found

xonuclease

Receptor-like protein kinase, putative

No data found

transcription initiation factor TFIID subunit $12 b$

Photosynthetic NDH subcomplex B 3

Pentatricopeptide repeat-containing protein At1g80270, mitochondria

monothiol glutaredoxin-S11

B-cell receptor-associated 31-like protein

pumilio homolog 1-like

Phosphoglycerate kinase

glutathione S-transferase-like

Serine/threonine-protein kinase

Protein phosphatase $2 \mathrm{c}$, putative

2-methyl-6-phytyl-1,4-hydroquinone methyltransferase, chloroplastic

Glucose-6-phosphate isomerase

lysophospholipid acyltransferase LPEAT2 isoform X1

Pyruvate kinase

No data found

40 S ribosomal protein $\mathrm{S} 26$

E3 ubiquitin-protein ligase RMA3

Ran-binding protein 1

ETHYLENE INSENSITIVE 3-like 3 protein

HD finger protein ING

Aldose 1-epimerase

Kinase family protein

$\mathrm{N}$ utilization substance $B$ 
MELO3C02345

MELO3C026897

MELO3C013125

MELO3C013852

MELO3C024701

MELO3C024086

MELO3C022386

MELO3C014100

MELO3C013699

MELO3C017906

MELO3C009047

MELO3C017993

MELO3C025710

MELO3C00508

MELO3C016434

MELO3C027219

MELO3C008932

MELO3C021604

MELO3C002736

MELO3C017478

MELO3C010631

MELO3C018156

MELO3C018637

MELO3C014008

MELO3C007572

MELO3C018716

MELO3C01419

MELO3C013444

MELO3C016186

MELO3C016198

MELO3C009755

MELO3C01033

MELO3C020848

MELO3C014298

MELO3C011496

MELO3C019673

MELO3C005244

MELO3C027082

MELO3C014560

MELO3C011106

MELO3C023727

MELO3C017963

MELO3C027349

MELO3C006867

MELO3C02440

MELO3C006795

MELO3C007935

MELO3C011717

MELO3C021242

MELO3C01948

MELO3C021247

MELO3C007934

MELO3C023224

MELO3C013878

MELO3C01056

MELO3C005260

MELO3C013221

MELO3C020139

MELO3C022885

MELO3C00887

MELO3C021452

MELO3C017319

MELO3C01326

MELO3C013879

MELO3C007001

MELO3C020574

MELO3C024984

MELO3C006957

MELO3C019021

MELO3C024729

MELO3C016890

MELO3C010886

MELO3C027370

MELO3C025196

MELO3C022180

MELO3C019044

MELO3C024869

MELO3C01456

MELO3C012284

MELO3C018849
0,002686991

0,002693668

0,002696375

0,002701952

0,002722685

0,002730488

0,002729666

0,002733928

0,002735738

0,002748047

0,002752363

0,002756806

0,002766025

0,002768466

0,002785114

0,002778258

0,002780255

0,00278398

0,002774689

0,002785704

0,002785354

0,002778243

0,002795115

0,002799344

0,002797667

0,002801119

0,002811106

0,002818216

0,002820951

0,002821729

0,002830707

0,002831112

0,002843447

0,002848395

0,002862369

0,002864285

0,00287285

0,002872432

0,002886753

0,002886027

0,002889946

0,00289547

0,002897783

0,002901755

0,00290197

0,002905902

0,002914473

0,002914359

0,002915878

0,002921813

0,002929612

0,002950461

0,002950867

0,002957395

0,002957726

0,002957489

0,002962542

0,00296188

0,002964338

0,002966193

0,002968697

0,002971065

0,002980565

0,002977994

0,002983521

0,002982642

0,002982609

0,002986868

0,002990581

0,002999388

0,003010612

0,003017045

0,00302186

0,003020505

0,003023713

0,003030012

0,003033633

0,003046338

0,003044031

0,00304664
0,033291105

0,03333161

0,033344218

0,033392271

0,033627463

0,033681705

0,033681705

0,033703091

0,033704361

0,033834902

0,033866931

0,033900477

0,033992675

0,034001512

0,034043854

0,034043854

0,034043854

0,034043854

0,034043854

0,034043854

0,034043854

0,034043854

0,03413774

0,034147159

0,034147159

0,034147712

0,034248325

0,034313781

0,03431424

0,03431424

0,034386002

0,034386002

0,034514594

0,034553407

0,034701612

0,034703532

0,034764654

0,034764654

0,034890143

0,034890143

0,034907366

0,034952714

0,034959267

0,034967061

0,034967061

0,034993084

0,035049105

0,035049105

0,035049105

0,035099083

0,035171372

0,035383526

0,035383526

0,035401287

0,035401287

0,035401287

0,035416003

0,035416003

0,035416035

0,035416771

0,03542525

0,0354321

0,035473547

0,035473547

0,035473547

0,035473547

0,035473547

0,035491975

0,035514727

0,035597902

0,035709654

0,035764474

0,035778621

0,035778621

0,035779094

0,035832154

0,035853509

0,035942705

0,035942705

0,035942705

RCA1-associated protein

thioredoxin-like 1-1, chloroplastic

ipid-binding serum glycoprotein family protein, putative

Plant/protein

ts, mitochondrial

$\mathrm{kDa}$ heat shock protein

S ribosomal protein $\mathrm{L} 14$

meavy metal-associated isoprenylated plant protein 21

Pectinesterase

in thioesterase 2

ndonuclease/exonuclease/phosphatase family protein

OS ribosomal protein $\mathrm{S} 29$

felear factor related to kappa-B-binding protein

3 ubiquitin-protein ligase

STICHE

Hydroxycinnamoyl-CoA shikimate/quinate hydroxycinnamoyltransferase

thioredoxin-like 3-1, chloroplastic

lycerol-3-phosphate dehydrogenase $[\mathrm{NAD}(+)]$

Calcyclin-binding protein

Xyloglucan endotransglucosylase/hydrolase

replication factor $C$ subunit 5

HEAT repeat-containing 8

Outer envelope protein 64 , mitochondrial

Adenosine monophosphate-protein transferase and cysteine protease ibpA

AP2-like ethylene-responsive transcription factor TOE3

ras-related protein Rab11D

3 ubiquitin-protein ligase RING1-like

o data found

No data found

No data found

Sigma factor binding protein 1 , chloroplastic

Non-specific serine/threonine protein kinase

-like ethylene-responsive transcription factor TOE3 isoform X1

CST complex subunit STN1

BRCT domain DNA repair protein

BOl-related E3 ubiquitin-protein ligase 1-like

$A B C$ transporter $F$ family member 3

transcription factor bHLH149-like

Phospholipase A2

Pentatricopeptide repeat-containing protein

CDT1-like protein a, chloroplastic

Lycopene beta-cyclase

MACPF domain NSL1-like protein

ATP-dependent Clp protease ATP-binding subunit clpX

Tudor/PWWP/MBT superfamily protein

-box protein SKIP16

Peroxidase

Protein kinase-like protein

YTH domain family protein 2 
MELO3C01478

MELO3C008481

MELO3C011261

MELO3C007950

MELO3C023110

MELO3C023308

MELO3C007661

MELO3C025139

MELO3C015862

MELO3C012787

MELO3C006983

MELO3C003418

MELO3C025651

MELO3C00211

MELO3C01755

MELO3C011955

MELO3C007043

MELO3C024047

MELO3C003323

MELO3C004576

MELO3C013476

MELO3C011323

MELO3C014607

MELO3C013949

MELO3C013891

MELO3C024474

MELO3CO05504

MELO3C022307

MELO3C002719

MELO3C016702

MELO3C012906

MELO3C01743

MELO3C016083

MELO3C020055

MELO3C013359

MELO3C012180

MELO3C016846

MELO3C003619

MELO3C007340

MELO3C02322

MELO3C022442

MELO3C024172

MELO3C022903

MELO3C003874

MELO3C004825

MELO3C009653

MELO3C009569

MELO3C013245

MELO3C007947

MELO3C026640

MELO3C006187

MELO3C013411

MELO3C01765

MELO3C011897

MELO3C00260

MELO3C002313

MELO3C017221

MELO3C020002

MELO3C011279

MELO3C010865

MELO3C010744

MELO3C003368

MELO3C00465

MELO3C013243

MELO3C013737

MELO3C026387

MELO3C015852

MELO3C02135

MELO3C012869

MELO3C011787

MELO3C019429

MELO3C006925

MELO3C00652

MELO3C014459

MELO3C002381

MELO3C015616

MELO3C006846

MELO3C01170

MELO3C008489

MELO3C026175
0,003053533

0,003067925

0,003069643

0,003073946

0,003077357

0,003076209

0,003080611

0,003083892

0,003094223

0,003098021

0,003103923

0,00312099

0,00312838

0,003129288

0,003135065

0,003151726

0,003158388

0,003169067

0,003174141

0,003176483

0,003178234

0,003180577

0,003194901

0,003200132

0,003198512

0,003199865

0,0031908

0,003194397

0,003197284

0,003207778

0,003217846

0,003244779

0,003254038

0,003260646

0,003263656

0,00326865

0,003282629

0,003300998

0,003313583

0,003316953

0,003319357

0,003325012

0,003325963

0,003325589

0,00333147

0,003335391

0,003341995

0,003345101

0,003346602

0,003346185

0,003373939

0,003359761

0,003365623

0,003364709

0,003368718

0,003361391

0,003372724

0,003354814

0,003373493

0,003371209

0,003353091

0,003371584

0,003388972

0,003386839

0,00339013

0,003383352

0,003381614

0,003385027

0,003384461

0,003395586

0,00339882

0,003401941

0,003406663

0,003412741

0,003410916

0,003409437

0,003415532

0,003416767

0,003421744

0,003426007
0,03600252 AT5g13970/MAC12_6

0,036149299 Glutamate synthase 1 [nadh], chloroplastic

0,036149299 Peroxidase

0,03617543 Thiol-disulfide oxidoreductase LTO1

$0,03617543 \quad$ Alkaline alpha galactosidase

$0,03617543 \quad$ F-box protein At2g16365

0,036192143 Transmembrane protein, putative

No data found

0,036308865 Unknown protein

0,036331842 Sterol 3-beta-glucosyltransferase

0,036379454 Protein phosphatase 2C

0,036557785 PLATZ transcription factor family protein

0,036611552

0,036611552

0,036657431

0,036830441

0,036886457

0,036989311

0,037026649

0,037030643

0,037030643

0,037036095

0,037110629

0,037110629

0,037110629

0,037110629

0,037110629

0,037110629

0,037110629

0,037177474

0,037272286

0,037562214

0,037647333

0,037701701

0,037714419

0,037750045

0,037889324

0,038079098

0,038201954

0,038218502

0,0382239

0,038233085

0,038233085

0,038233085

0,038274119

0,03829688

0,038336453

0,038336453

0,038336453

0,038336453

0,038382447

0,038382447

0,038382447

0,038382447

0,038382447

0,038382447

0,038382447

0,038382447

0,038382447

0,038382447

0,038382447

0,038382447

0,038411752

0,038411752

0,038411752

0,038411752

0,038411752

0,038411752

0,038411752

0,038451502

0,038466072

0,038479344

0,038510695

0,038513275

0,038513275

0,038513275

0,038514691

0,038514691

0,03854879

0,03857481

Protein transport protein USO1, putative

-complex protein 1 subunit delta

entricopertecontaining protein

tratricopeptide-like helical

UDP-glucose 4-epimerase, putative

repeat-containing F-box family protein

Myosin heavy chain-like protein

transport protein 14-like

phototropin-2

No data found

Zinc transporter ZTP29

Ribosomal protein $\mathrm{L} 20$

AT5g47090/K14A3 4

inc finger protein 593

Disease resistance family protein

Pentatricopeptide repeat-containing family protein

$60 \mathrm{~S}$ ribosomal protein $\mathrm{L} 18 \mathrm{a}$

translation initiation factor IF-1, chloroplastic

CDT1-like protein a, chloroplastic

Fiber protein $\mathrm{Fb} 15$

histidine kinase 4-like

Ubiquitin-conjugating enzyme, E2

Phosphoribosyl-AMP cyclohydrolase

serine/arginine-rich splicing factor RSZ22A

$N A D(P) H$ dehydrogenase (Quinone) FQR1-like

$A B C$ transporter-like protein

At1g08760

Histone deacetylase

data found

cytochrome P450 734A1-like

Ser/thr-rich protein T10 in DGCR region-like protein

transcription factor bHLH48

Gb|AAF02136.1

$40 \mathrm{~S}$ ribosomal protein S11-like

Proteasome subunit beta type

Receptor-like kinase

F-box protein SKIP14

Serine/threonine-protein kinase AFC2

Histone deacetylase

Protein IDA

Hydroxyproline O-arabinosyltransferase 1

Calcium-binding EF-hand family protein, putative

CTD small phosphatase-like protein 2

Intron maturase, type II family protein

DENN (AEX-3) domain-containing protein

ruBisCO large subunit-binding protein subunit beta, chloroplastic-like

Ankyrin repeat domain-containing protein, chloroplastic

Mitogen-activated protein kinase kinase kinase 1 isoform 1

NADP dependent sorbitol 6-phosphate dehydrogenase family protein

Sulfate/thiosulfate import ATP-binding protein cysA, putative 
MELO3C011882

MELO3C021636

MELO3C012052

MELO3C018126

MELO3C020666

MELO3C013892

MELO3C002722

MELO3C025912

MELO3C01873

MELO3C019827

MELO3C007008

MELO3C007106

MELO3C009713

MELO3C009747

MELO3C025091

MELO3C005532

MELO3C01513

MELO3C02706

MELO3C011942

MELO3C016296

MELO3C014270

MELO3C004534

MELO3C007344

MELO3C014260

MELO3C006765

MELO3C008503

MELO3C01669

MELO3C012088

MELO3C022354

MELO3C015093

MELO3C010763

MELO3C008037

MELO3C002653

MELO3C007055

MELO3C015718

MELO3C002891

MELO3C00298

MELO3C012083

MELO3C010745

MELO3C017766

MELO3C020908

MELO3C022417

MELO3C012428

MELO3C022004

MELO3C004466

MELO3C024980

MELO3C020601

MELO3C013403

MELO3C007784

MELO3C026293

MELO3C026249

MELO3C026102

MELO3C024088

MELO3C015825

MELO3C007635

MELO3C017844

MELO3C023195

MELO3C025023

MELO3C016913

MELO3C010482

MELO3C024352

MELO3C005089

MELO3C019497

MELO3C023221

MELO3C025053

MELO3C011399

MELO3C004637

MELO3C01685

MELO3C021385

MELO3C020532

MELO3C015523

MELO3C018576

MELO3C01002

MELO3C007547

MELO3C003813

MELO3C015455

MELO3C009308

MELO3C024282

MELO3C021366

MELO3C012881
0,003434884

0,003446073

0,003452074

0,003462355

0,003464992

0,003475055

0,003474179

0,003482485

0,003480823

0,00348579

0,003505922

0,003505337

0,00351416

0,003531004

0,003530344

0,003527148

0,003533209

0,003535623

0,003539061

0,003541354

0,003548068

0,003544491

0,003547741

0,00356425

0,003561252

0,003567236

0,00356367

0,003568551

0,00356635

0,003557723

0,003555075

0,003570997

0,003573311

0,003581872

0,003581143

0,003585267

0,003585848

0,003592586

0,003593846

0,003610937

0,003610566

0,003611989

0,003616813

0,003620939

0,003624832

0,003638813

0,00364269

0,003646581

0,003649542

0,003656398

0,003662455

0,003665971

0,003673788

0,00367858

0,003691045

0,003701289

0,003703179

0,003700209

0,003713995

0,003712349

0,003714595

0,0037201

0,003727132

0,003736333

0,003746829

0,003746213

0,003756844

0,003760096

0,003760539

0,003759449

0,003757061

0,003753217

0,003766895

0,003777406

0,003780437

0,003791053

0,003803179

0,003804855

0,00380155

0,003803284
0,038652717

0,038756546

0,038801943

0,038895368

0,038902854

0,038971525

0,038971525

0,03901055

0,03901055

0,039025439

0,039206378

0,039206378

0,039276258

0,039397631

0,039397631

0,039397631

0,039399968

0,039404641

0,03942071

0,039424015

0,039432076

0,039432076

0,039432076

0,039481967

0,039481967

0,039481967

0,039481967

0,039481967

0,039481967

0,039481967

0,039481967

0,039486906

0,039490386

0,039540431

0,039540431

0,039540431

0,039540431

0,039584422

0,039584422

0,039717805

0,039717805

0,039717805

0,03974872

0,039771924

0,039792552

0,039923839

0,039944192

0,039964669

0,039974931

0,040027832

0,040071928

0,040088183

0,040151428

0,04018157

0,040295438

0,040360967

0,040360967

0,040360967

0,040418482

0,040418482

0,040418482

0,040456085

0,040510255

0,040587924

0,040657217

0,040657217

0,040671892

0,040671892

0,040671892

0,040671892

0,040671892

0,040671892

0,040718344

0,040809619

0,040820042

0,040912299

0,040971692

0,040971692

0,040971692

0,040971692

RING-type E3 ubiquitin transferase No data found

naJ homolog subfamily B member 1

Autophagy-related protein $18 \mathrm{f}$

A

alcineurin B-like protein

3-oxoacyl-[acyl-carrier-protein] synthase

rotein kinase

PI-anchored protein LORELE

2BNB-like protein

ytochrome c6, chloroplastic

Remorin family protein

translation factor GUF1 homolog, chloroplastic

Zinc finger (C3HC4-type RING finger) family protein, putative

rotein REVEILLE 1-like isoform $X$

ectinesterase

Pentatricopeptide repeat-containing family protein

PLA transcription factor family protein

3 ubiquitin-protein ligase

Chlororespiratory reduction 31

No data found

Glycine-rich RNA-binding protein, putative

No data found

Interactor of constitutive active ROPs-like protein

DEAD-box ATP-dependent RNA helicase 50

Protein ABIL2

VQ motif-containing protein

Q

Light-regulated protein, putative

Fasciclin-like arabinogalactan protein 16

vacular-processing enzyme-like

Ubiquinol-cytochrome $\mathrm{C}$ chaperone family protein

No data found

reticuline oxidase-like protein

ATP binding protein

Pseudouridine synthase, RsuA/RluB/C/D/E/F

transmembrane emp24 domain-containing protein p24delta9-like

Actin-binding LIM protein 1, putative

mitochondrial import receptor subunit TOM20-like

Ras family

(lycosyl hydrolase family 43 protein

RNA-binding protein 24-B

mRNA-decapping enzyme subunit 2-like

SPX domain protein

Alpha-galactosidase

No data found

Protein phosphatase $2 \mathrm{C}$

auxin-responsive protein SAUR36-like

Unknown protein 
MELO3C024947

MELO3C004524

MELO3C005600

MELO3C02476

MELO3C019691

MELO3C010338

MELO3C010688

MELO3C024739

MELO3C009560

MELO3C004555

MELO3C024926

MELO3C002209

MELO3C004423

MELO3C01606

MELO3C022388

MELO3C021783

MELO3C026222

MELO3C023969

MELO3C021407

MELO3C021846

MELO3C007841

MELO3C010378

MELO3C005360

MELO3C005850

MELO3C002789

MELO3C024960

MELO3C026392

MELO3C008056

MELO3C019135

MELO3C021253

MELO3C011386

MELO3C022742

MELO3C022225

MELO3C015024

MELO3C002556

MELO3C003039

MELO3C013929

MELO3C019002

MELO3C012683

MELO3C024468

MELO3C007936

MELO3C005650

MELO3C010427

MELO3C007195

MELO3C002292

MELO3C004334

MELO3C012231

MELO3C026332

MELO3C003917

MELO3C013792

MELO3C006643

MELO3C01038

MELO3C024538

MELO3C011908

MELO3C012400

MELO3C017187

MELO3C023569

MELO3C009178

MELO3C012716

MELO3C009268

MELO3C021818

MELO3C020579

MELO3C00751

MELO3C014384

MELO3C026567

MELO3C022309

MELO3C022301

MELO3C003173

MELO3C017343

MELO3C017925

MELO3C015891

MELO3C023098

MELO3C009370

MELO3C006875

MELO3C014035

MELO3C017623

MELO3C005067

MELO3C02437

MELO3C011742

MELO3C018549
0,003815395

0,003821849

0,003831717

0,003834984

0,003841997

0,003842544

0,003849725

0,003857873

0,003869597

0,003869804

0,003865295

0,003866952

0,00387519

0,003886455

0,003895212

0,003899657

0,003897988

0,003899371

0,0039026

0,00390593

0,003917636

0,003926868

0,00392708

0,003925023

0,003938719

0,003956688

0,003958903

0,003957199

0,003951906

0,003960223

0,003948698

0,003952049

0,003974648

0,003977944

0,003983689

0,003992429

0,004016596

0,00402459

0,004034378

0,004043191

0,004053995

0,004052481

0,004077216

0,004077084

0,004074355

0,004082556

0,004086041

0,004092545

0,004095247

0,004109432

0,004132745

0,00413255

0,004127031

0,004132723

0,004137771

0,004145526

0,004145485

0,004153711

0,0041542

0,004161762

0,004163213

0,004171474

0,004177658

0,004183069

0,004192935

0,004191627

0,004197551

0,004207307

0,004218144

0,004233382

0,004238724

0,004242721

0,004260693

0,004249928

0,00425177

0,004256934

0,004263392

0,004262261

0,004252653

0,004260993
0,041062803

0,041109857

0,041193563

0,041206251

0,041242606

0,041242606

0,041297244

0,041362184

0,041400198

0,041400198

0,041400198

0,041400198

0,041435366

0,041533337

0,041584413

0,041584413

0,041584413

0,041584413

0,041593331

0,041606376

0,041708569

0,041741601

0,041741601

0,041741601

0,041842795

0,041913419

0,041913419

0,041913419

0,041913419

0,041913419

0,041913419

0,041913419

0,04204356

0,042055892

0,0420941

0,042163897

0,042396446

0,042458132

0,042538673

0,042608845

0,042677153

0,042677153

0,042853085

0,042853085

0,042853085

0,042886386

0,042900167

0,042945629

0,042951162

0,043077059

0,043229655

0,043229655

0,043229655

0,043229655

0,043259309

0,043294547

0,043294547

0,043339304

0,043339304

0,043387488

0,043387488

0,043450658

0,043492126

0,043525516

0,043582254

0,043582254

0,043607279

0,043685655

0,043775159

0,043910224

0,043942551

0,04396091

0,043990453

0,043990453

0,043990453

0,043990453

0,043990453

0,043990453

0,043990453

0,043990453

Inner centromere protein, ARK-binding region protein

zinc finger $\mathrm{CCCH}$ domain-containing protein 39-like

hosphate translocator-related family protein

GDSL esterase/lipase At2g30310-like

osyltransferase

Outward-rectifying potassium channel 4-like protein

F-box protein At1g70590

rowth-regulating factor 4-like

Polyketide cyclase/dehydrase/lipid transport superfamily protein

wo-pore potassium channel 1 isoform $X 1$

Homeobox leucine zipper family protein

rotease 2

Mitochondrial processing peptidase beta subunit

Pre-mRNA-processing factor 39

Peroxisome biogenesis protein 1

Transcription factor GTE10

nknown protein

Stem-specific protein TSJT1

cytochrome P450 89A2-like

Bacteriophage N4 adsorption B

No data found

mediator of RNA polymerase II transcription subunit 36a-like

S-type anion channel SLAH2

BnaCnng71930D protein

pentatricopeptide repeat-containing protein At5g66520-like

RING/U-box superfamily protein

$\mathrm{AC}$ domain-containing protein 82

4-alpha-glucanotransferase DPE2

Beta-glucosidase

DNA gyrase subunit $A$

ATP-dependent DNA helicase, RecQ family protein, expressed

protein NRT1/ PTR FAMILY 5.10-like

RING-type E3 ubiquitin transferase

heterogeneous nuclear ribonucleoprotein U-like protein 1

No data found

Unknown protein

Pentatricopeptide repeat-containing protein, mitochondrial

Thioredoxin, putative

sspartyl protease family protein 1-like

Dead box ATP-dependent RNA helicase, putative

aspartate-semialdehyde dehydrogenase

ER lumen protein-retaining receptor

transcription factor MYB86-like

ERAD-associated E3 ubiquitin-protein ligase HRD1B-like

Homologous-pairing protein 2-like protein

DNA helicase INO80-like protein

Heat shock $70 \mathrm{kDa}$ protein

Ribosomal protein $\mathrm{L} 28$ 
MELO3C009480

MELO3C013951

MELO3C007097

MELO3C018374

MELO3C010135

MELO3C019168

MELO3C017365

MELO3C011234

MELO3C00700

MELO3C019226

MELO3C023373

MELO3C019907

MELO3C010243

MELO3C02200

MELO3C022530

MELO3C022219

MELO3C020562

MELO3C014562

MELO3C003447

MELO3C015857

MELO3C002619

MELO3C014283

MELO3C007793

MELO3C023173

MELO3C005182

MELO3C00542

MELO3C02532

MELO3C020541

MELO3C024896

MELO3C007660

MELO3C002171

MELO3C00611

MELO3C010522

MELO3C016498

MELO3C00798

MELO3C024434

MELO3C007689

MELO3C008214

MELO3C018008

MELO3C012753

MELO3C011357

MELO3C020113

MELO3C002044

MELO3C015727

MELO3C013370

MELO3C004636

MELO3C018966

MELO3C026199

MELO3C019056

MELO3C01195

MELO3C014991

MELO3C016801

MELO3C016795

MELO3C009312

MELO3C02045

MELO3C013215

MELO3C007647

MELO3C000279

MELO3C008114

MELO3C01876

MELO3C004603

MELO3C005627

MELO3C00540

MELO3C018500

MELO3C025210

MELO3C02617

MELO3C008513

MELO3C01382O

MELO3C011375

MELO3C010991

MELO3C00345

MELO3C00382

MELO3C004992

MELO3C017563

MELO3C015861

MELO3C01884

MELO3C012033

MELO3C002083

MELO3C023385

MELO3C004333
0,004270564

0,004268865

0,004276916

0,004278249

0,0042796

0,004293298

0,004301125

0,004308579

0,00431774

0,004317006

0,004327562

0,004348841

0,004352503

0,00436531

0,004370793

0,00437392

0,004381523

0,004383797

0,004388805

0,004393219

0,004397541

0,004405154

0,004410314

0,004408955

0,004412094

0,004410409

0,00440425

0,004415679

0,004425602

0,00442696

0,004433773

0,004436391

0,004443462

0,004450952

0,004451051

0,004448586

0,00446891

0,004470553

0,004484931

0,004495256

0,004510552

0,004517785

0,004521056

0,004533629

0,004542785

0,004562754

0,004563283

0,0045674

0,004577074

0,004581695

0,004592244

0,004595981

0,004616259

0,004637357

0,004640894

0,004637275

0,004642262

0,004642023

0,004641918

0,004631049

0,004650444

0,004658441

0,004661356

0,004666952

0,004670139

0,004684672

0,004697722

0,004711581

0,00471355

0,004708747

0,004711087

0,004712946

0,004716173

0,004720256

0,00473689

0,00474106

0,004754153

0,00475552

0,004763871

0,00477455
0,044018463

0,044018463

0,044042639

0,044042639

0,044042639

0,04416059

0,044218068

0,044271653

0,044319683

0,044319683

0,044397416

0,044592553

0,044606949

0,044714989

0,044747951

0,044756755

0,044811338

0,044811393

0,044839378

0,044861266

0,044882192

0,044891527

0,044891527

0,044891527

0,044891527

0,044891527

0,044891527

0,044904867

0,044973267

0,044973267

0,04501932

0,045022754

0,045071359

0,045078873

0,045078873

0,045078873

0,045229993

0,045229993

0,045352224

0,045433371

0,045564646

0,045614382

0,045624084

0,045727594

0,045796557

0,045956283

0,045956283

0,045974302

0,046048208

0,046071233

0,046153801

0,046167865

0,046347994

0,046443786

0,046443786

0,046443786

0,046443786

0,046443786

0,046443786

0,046443786

0,046502086

0,046558473

0,046564044

0,04659638

0,046604633

0,046726052

0,046832565

0,046872055

0,046872055

0,046872055

0,046872055

0,046872055

0,046874535

0,046891514

0,047033107

0,047050848

0,047146974

0,047146974

0,047206067

0,047288161

$A B C$ transporter family protein

ADH dehydrogenase [ubiquinone] 1 beta subcomplex subunit 8 , mitochondrial

box protein SKIP19

DUF248-1

repeat-containing protein family

Metacaspase- 9

DEAD-box ATP-dependent RNA helicase 42

TP-binding nuclear protein

atanin p80 WD40 repeat-containing subunit B1 homolog

cription factor ILR3-like

Aspartate aminotransferase

OS ribosomal protein $\mathrm{S} 17$

PMP-like protein YKT61

transmembrane emp24 domain-containing protein p24delta9

Armadillo/beta-catenin repeat family protein

$\mathrm{SPla} / \mathrm{RYanodine}$ receptor (SPRY) domain-containing protein No data found

3 ubiquitin-protein ligase ICPO

Major facilitator superfamily transporter

data found

Mitochondrial carrier protein, expressed

BIG SEEDS 1

Cytochrome P450 CYP736A12-like

sister chromatid cohesion 1 protein 3

Short-chain dehydrogenase/reductase family protein

GTF2H2

Leucine-rich repeat extensin-like protein 4

Omegatty acid desaturase

Calcium-dependent lipid-binding (CaLB domain) family protein

DUF21 domain-containing-like protein

OW QUALITY PROTEIN: probable metal-nicotianamine transporter YSL5

Remorin

Zinc knuckle family protein

MACPF domain-containing protein At4g24290

Plant intracellular ras group-related LRR protein

protein IQ-DOMAIN 14-like

protein RMD5 homolog A

Emb|CAB62340.1

Glycyl-tRNA synthetase beta subunit

Unknown protein

serine/arginine-rich splicing factor SC35-like

ADP,ATP carrier protein

DNA polymerase alpha subunit B

Maternal effect embryo arrest 60

translocase of chloroplast 120, chloroplastic-like

-complex protein 1 subunit beta

ATP-dependent 6-phosphofructokinase

Transmembrane protein, putative

two-component response regulator ARR8-like 


\begin{tabular}{|c|c|c|c|}
\hline MELO3C003581 & 0,004779227 & 0,047310758 & signal peptide peptidase \\
\hline MELO3C005964 & 0,004786932 & 0,047363283 & Late cornified envelope protein $1 \mathrm{E}$ \\
\hline MELO3C010772 & 0,004792676 & 0,047396378 & clathrin interactor EPSIN 1 \\
\hline MELO3C013364 & 0,004797805 & 0,047423351 & MYB-related transcription factor \\
\hline MELO3C011490 & 0,004807445 & 0,047494858 & Myosin \\
\hline MELO3C011715 & 0,004821563 & 0,047586732 & CDP-diacylglycerol--glycerol-3-phosphate 3-phosphatidyltransferase 2 \\
\hline MELO3C025943 & 0,004819407 & 0,047586732 & RPM1-interacting protein 4 \\
\hline MELO3C024211 & 0,004835288 & 0,047626989 & BTB/POZ domain-containing protein At5g48130 \\
\hline MELO3C018935 & 0,004829019 & 0,047626989 & Unknown protein \\
\hline MELO3C005513 & 0,004833075 & 0,047626989 & E3 ubiquitin-protein ligase HOS1 \\
\hline MELO3C025354 & 0,004832401 & 0,047626989 & Late embryogenesis abundant protein \\
\hline MELO3C010709 & 0,004851085 & 0,047758765 & telomere-associated protein RIF1-like isoform X2 \\
\hline MELO3C007871 & 0,00485956 & 0,047818358 & rRNA adenine N(6)-methyltransferase \\
\hline MELO3C004179 & 0,004867492 & 0,047872556 & RNA polymerase II-associated protein 3 \\
\hline MELO3C007325 & 0,00487634 & 0,047934223 & Sugar transporter, putative \\
\hline MELO3C015459 & 0,004878616 & 0,047934223 & Cytidine deaminase \\
\hline MELO3C021353 & 0,004882791 & 0,047951391 & Chromatin remodeling factor, putative \\
\hline MELO3C021565 & 0,004892331 & 0,048017693 & DExH-box ATP-dependent RNA helicase DExH17-like \\
\hline MELO3C011062 & 0,004894406 & 0,048017693 & Serine hydrolase FSH \\
\hline MELO3C015103 & 0,004900855 & 0,04805709 & Histone deacetylase \\
\hline MELO3C014540 & 0,004906707 & 0,048066291 & blue copper protein-like \\
\hline MELO3C006050 & 0,004905261 & 0,048066291 & Phosphatidylinositol-4-phosphate 5-kinase family protein \\
\hline MELO3C009984 & 0,004909095 & 0,048066291 & Zinc finger $\mathrm{CCCH}$ domain-containing protein 41 \\
\hline MELO3C009686 & 0,004920878 & 0,048110111 & Pleiotropic drug resistance $A B C$ transporter \\
\hline MELO3C022845 & 0,004920763 & 0,048110111 & RING-type E3 ubiquitin transferase \\
\hline MELO3C002054 & 0,00491912 & 0,048110111 & Movement protein binding protein $2 \mathrm{C}$ \\
\hline MELO3C016817 & 0,004932507 & 0,048184439 & Calcium-transporting ATPase \\
\hline MELO3C010960 & 0,00493336 & 0,048184439 & Proteasome subunit alpha type \\
\hline MELO3C005578 & 0,004939104 & 0,048216689 & Amine oxidase \\
\hline MELO3C020276 & 0,004949485 & 0,048270311 & Tobamovirus multiplication 1 \\
\hline MELO3C018564 & 0,004948426 & 0,048270311 & Protein trichome birefringence-like 12 \\
\hline MELO3C011383 & 0,004955995 & 0,04830994 & DNA-directed RNA polymerase subunit beta \\
\hline MELO3C004152 & 0,004959362 & 0,04831891 & $40 S$ ribosomal protein S23-1 \\
\hline MELO3C022348 & 0,004994168 & 0,048634032 & Protein TIFY 9 \\
\hline MELO3C006574 & 0,005016799 & 0,048830338 & guanylate kinase 3 , chloroplastic-like \\
\hline MELO3C007071 & 0,005035167 & 0,048946423 & WPP domain associated protein \\
\hline MELO3C004988 & 0,00503616 & 0,048946423 & Protein SIEL \\
\hline MELO3C011016 & 0,005035252 & 0,048946423 & tubulin beta chain \\
\hline MELO3C005710 & 0,005041507 & 0,048948249 & heavy metal-associated isoprenylated plant protein 3 \\
\hline MELO3C017291 & 0,005040338 & 0,048948249 & $40 \mathrm{~S}$ ribosomal protein $\mathrm{S} 28$ \\
\hline MELO3C008219 & 0,005046262 & 0,048948249 & glyceraldehyde-3-phosphate dehydrogenase \\
\hline MELO3C019779 & 0,005045138 & 0,048948249 & U4/U6 small nuclear ribonucleoprotein PRP4-like protein \\
\hline MELO3C002071 & 0,005049346 & 0,048954117 & single-stranded DNA-binding protein, mitochondrial \\
\hline MELO3C024964 & 0,005065109 & 0,049058772 & E3 ubiquitin-protein ligase \\
\hline MELO3C010806 & 0,005063283 & 0,049058772 & DNA excision repair protein ERCC-8 \\
\hline MELO3C026554 & 0,005078 & 0,049159522 & alcohol dehydrogenase-like 7 \\
\hline MELO3C002931 & 0,00508282 & 0,049182075 & Paired amphipathic helix SIN3-like protein \\
\hline MELO3C014724 & 0,00509845 & 0,049274668 & Mitochondrial carrier family \\
\hline MELO3C014519 & 0,005095436 & 0,049274668 & BEL1-like homeodomain protein 1 \\
\hline MELO3C012470 & 0,00510131 & 0,049274668 & two-component response regulator ORR9-like \\
\hline MELO3C002405 & 0,00510237 & 0,049274668 & 50 S ribosomal protein $\mathrm{L} 7 / \mathrm{L} 12$ \\
\hline MELO3C006249 & 0,005116632 & 0,049340898 & No data found \\
\hline MELO3C007711 & 0,005112354 & 0,049340898 & U-box domain-containing protein kinase family protein, putative \\
\hline MELO3C002385 & 0,005116723 & 0,049340898 & Transcription factor Inducer of CBF expression 1 \\
\hline MELO3C011167 & 0,00512558 & 0,049402188 & basic leucine zipper 9 \\
\hline MELO3C013378 & 0,005138683 & 0,049422864 & Unknown protein \\
\hline MELO3C013419 & 0,005140238 & 0,049422864 & 30 S ribosomal protein S31, mitochondrial \\
\hline MELO3C014215 & 0,005140167 & 0,049422864 & No data found \\
\hline MELO3C002515 & 0,005137092 & 0,049422864 & Eukaryotic translation initiation factor-like protein \\
\hline MELO3C024016 & 0,005132207 & 0,049422864 & Metal-dependent phosphohydrolase \\
\hline MELO3C005696 & 0,005146382 & 0,049457856 & E3 ubiquitin-protein ligase RHA1B \\
\hline MELO3C015130 & 0,005149257 & 0,04946142 & polygalacturonase-like \\
\hline MELO3C014379 & 0,005165809 & 0,049596282 & Protein RETICULATA-RELATED 1, chloroplastic \\
\hline MELO3C016899 & 0,005169158 & 0,049604328 & Mitochondrial carrier protein \\
\hline MELO3C006092 & 0,005178299 & 0,04966489 & Glycoside hydrolase family 28 protein/polygalacturonase family protein \\
\hline MELO3C003072 & 0,005180499 & 0,04966489 & Thioredoxin-like protein AAED1, chloroplastic \\
\hline MELO3C009552 & 0,005195047 & 0,049780192 & Mads box protein, putative \\
\hline MELO3C000885 & 0,005198453 & 0,049788673 & auxin-responsive protein SAUR71-like \\
\hline MELO3C008811 & 0,005201536 & 0,049794056 & importin-5 \\
\hline MELO3C009970 & 0,005205874 & 0,04981144 & polygalacturonase At1g48100 \\
\hline MELO3C014419 & 0,005215486 & 0,049855095 & Unknown protein \\
\hline MELO3C017659 & 0,005213556 & 0,049855095 & Ribosomal protein L15 \\
\hline MELO3C018769 & 0,005218299 & 0,049857856 & Wound-responsive family protein \\
\hline MELO3C019780 & 0,005232823 & 0,049972446 & protein kinase $2 \mathrm{~B}$, chloroplastic-like \\
\hline
\end{tabular}

0,047310758

47363283

0,047423351

0,047494858

0,047586732

0,047626989

0,047626989

0,047626989

0,047934223

0,047934223

0,048017693

066291

0,048066291

0,048110111

0,048184439

0,048184439

0,048216689

048270311

0,048634032

0,048946423

0,048946423

0,04894824

0,048948249

0,049182075

0,049274668

0,049274668

04934089

0,049457856

0,04946142

0,04966489

0,049972446
ESIN

DP-diacylglycerol--glycerol-3-phosphate 3-phosphatidyltransferase

\section{nown protein}

lemere-associated protein RIF1-like isoform X2

omatin remodeling factor, putative

per protein-like

finger $\mathrm{CCCH}$ domain-containing protein 41

otropic drug resistance $A B C$ transporte

NA-directed RNA polymerase subunit beta

protein $523-1$

guanylate kinase 3 , chloroplastic-like

tubulin beta chain

metal-associated isoprenylated plant protein 3

4/U6 small nuclear ribonucleoprotein PRP4-like protein

mitochondrial

Mitochondrial carrier family

$50 S$ ribosomal protein $\mathrm{L} 7 / \mathrm{L} 12$

No data found

basic leucine zipper 9

Eukaryotic translation initiation factor-like protein

polygalacturonase-like

Mitochondrial carrier protein

Glycoside hydrolase family 28 protein/polygalacturonase family protein

Mads box protein, putative

Ribosomal protein L15

protein kinase $2 \mathrm{~B}$, chloroplastic-like 
Supplementary Table 2.B Gene Ontology terms (GO terms) of the cluster 2.1 obtained considering the comparison between lines the postharvest ripening time fruit during storage at $20.5^{\circ} \mathrm{C}$ and $88 \%$ relative humidity for $18 \mathrm{~d}$ the near-isogenic line SC10-2 and its parental "Piel de Sapo" obtained according to the differentially expressed gene pattern (Supl. Table 2.A)

\begin{tabular}{|c|c|c|c|c|c|c|c|c|c|c|}
\hline GOID & GO Name & GO Category & FDR & P-Value & sc10-2 & PS & Non Annot Test & \begin{tabular}{|l} 
Non Annot \\
Reference
\end{tabular} & \begin{tabular}{|l} 
Testset \\
Sequences
\end{tabular} & \begin{tabular}{|l}
$\begin{array}{l}\text { Refset } \\
\text { Sequences }\end{array}$ \\
\end{tabular} \\
\hline \begin{tabular}{|l|} 
GO:0006415 \\
\end{tabular} & translational termination & BIOLOGICAL_PROCESS & $7,88 \mathrm{E}-13$ & $4,17 \mathrm{E}-16$ & 35 & 146 & 592 & 16355 & 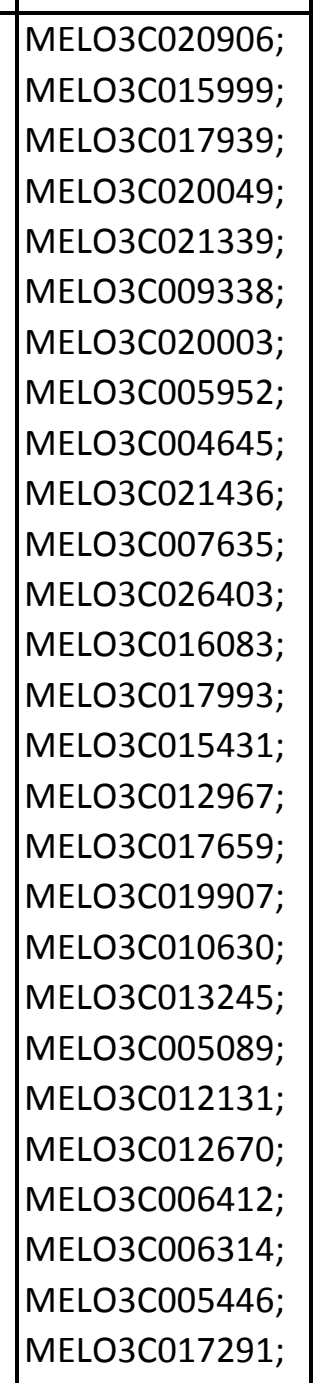 & 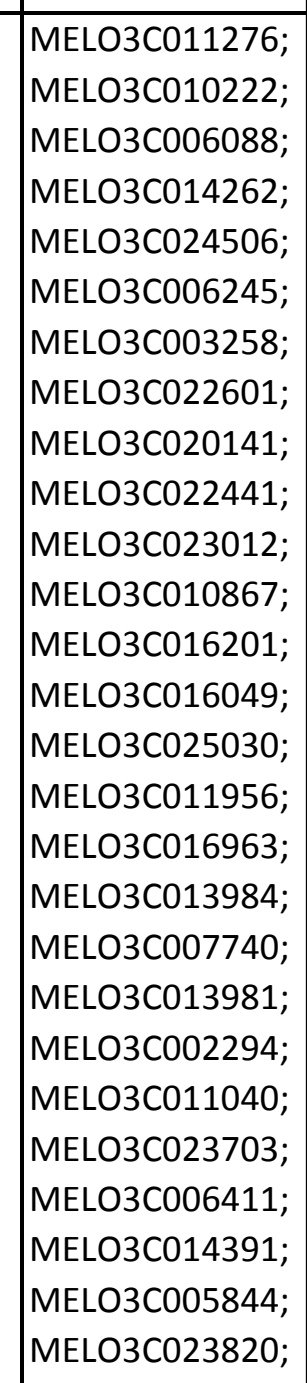 \\
\hline GO:0006414 & translational elongation & BIOLOGICAL_PROCESS & $1,61 \mathrm{E}-12$ & $1,02 \mathrm{E}-15$ & 36 & 161 & 591 & 16340 & 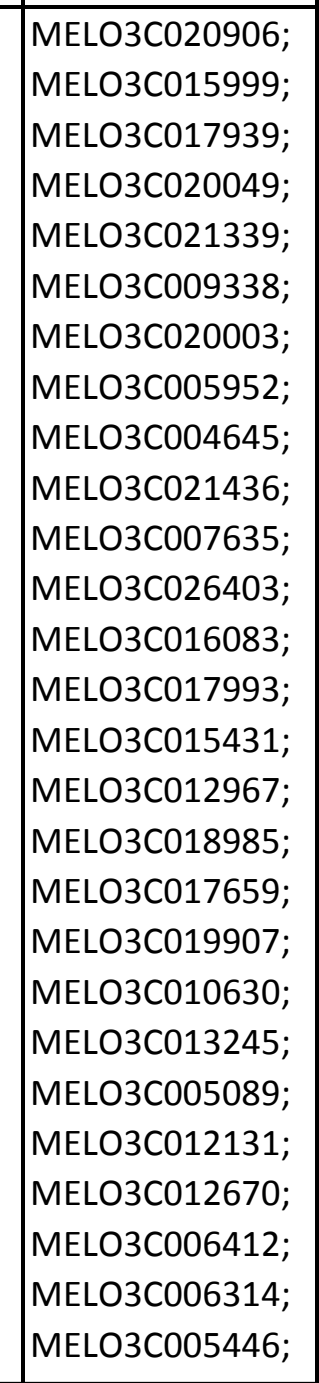 & 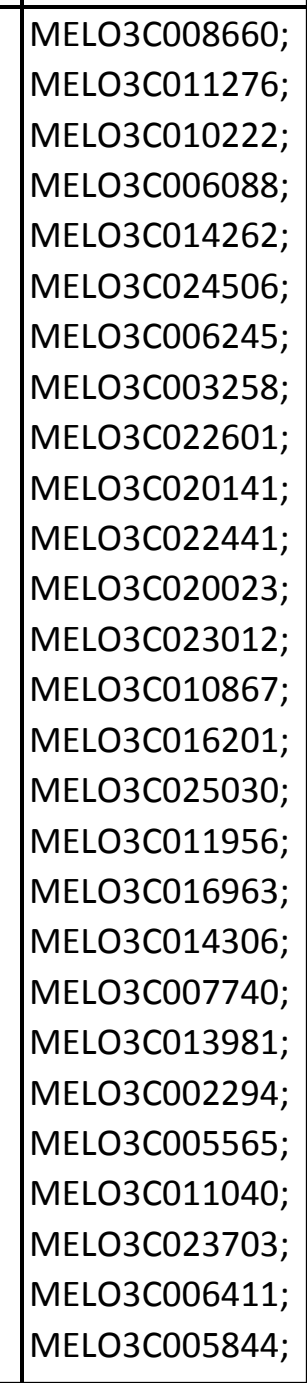 \\
\hline \begin{tabular}{|l|} 
GO:0003735 \\
\end{tabular} & structural constituent of ribosome & MOLECULAR_FUNCTION & $7,01 \mathrm{E}-10$ & $1,19 \mathrm{E}-12$ & 44 & 310 & 583 & 16191 & 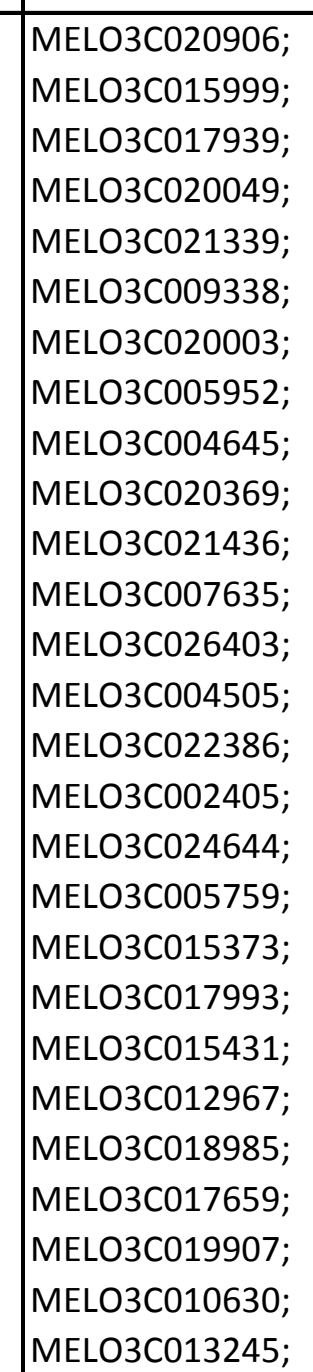 & 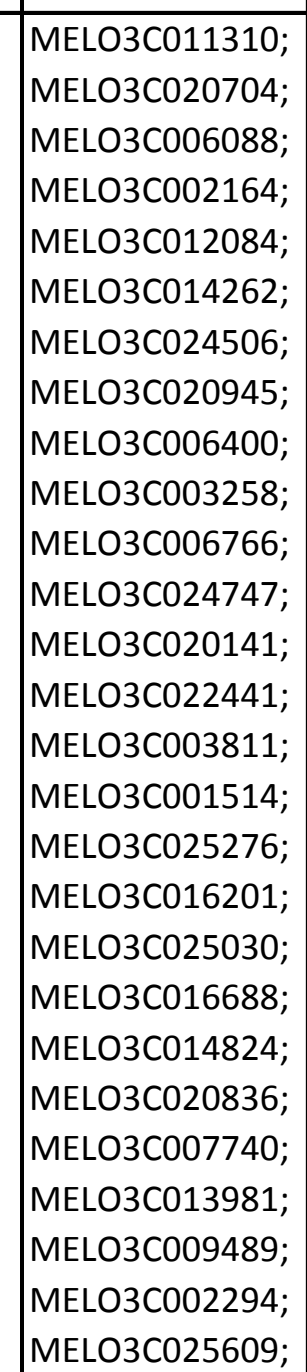 \\
\hline \begin{tabular}{|l}
$G O: 0022625$ \\
\end{tabular} & cytosolic large ribosomal subunit & CELLULAR_COMPONENT & $3,67 \mathrm{E}-09$ & $7,00 E-12$ & 27 & 124 & 600 & 16377 & 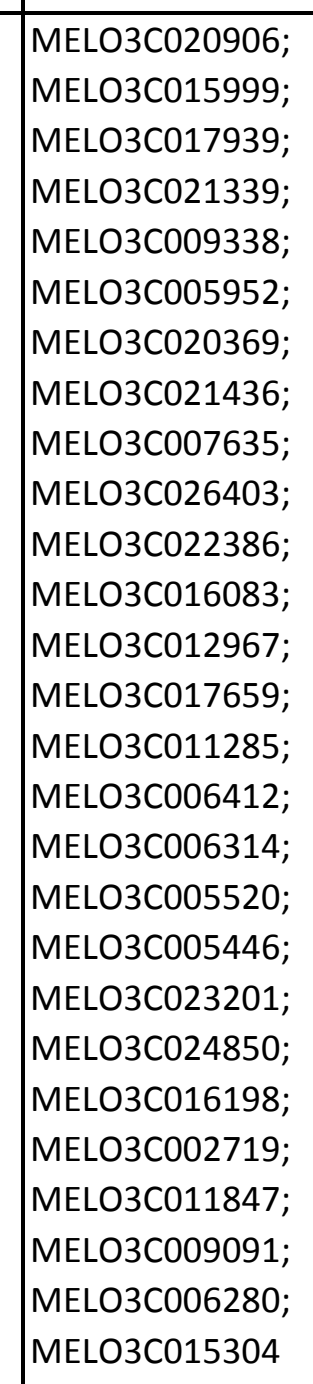 & 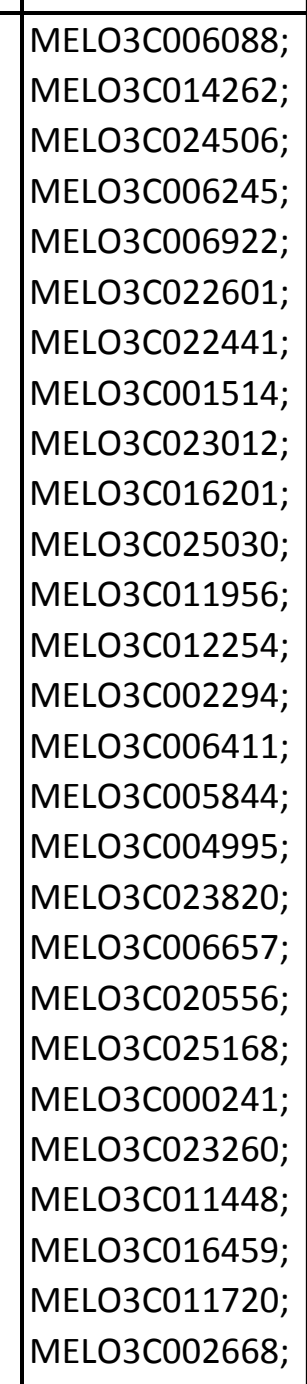 \\
\hline
\end{tabular}




\begin{tabular}{|c|c|c|c|c|c|c|c|c|c|c|}
\hline $60: 0046686$ & response to cadmium ion & BIOLOGICAL_PROCESS & $1,84-04$ & $8,95 E-07$ & 22 & 160 & 605 & 16341 & 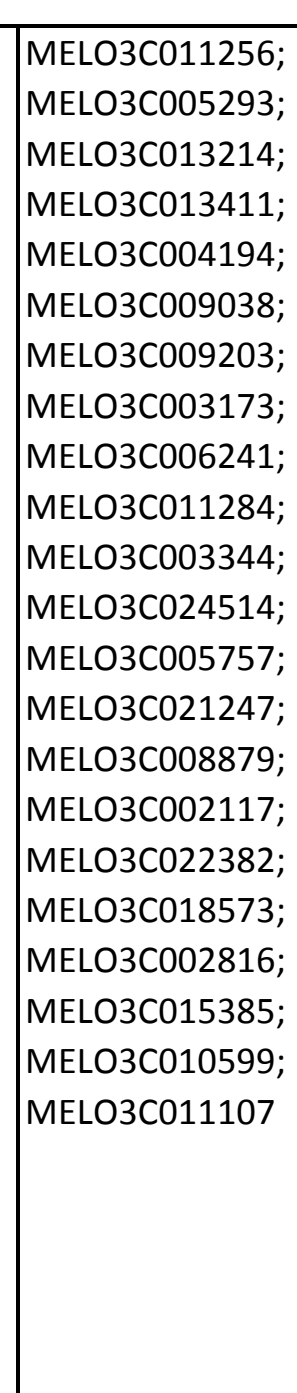 & 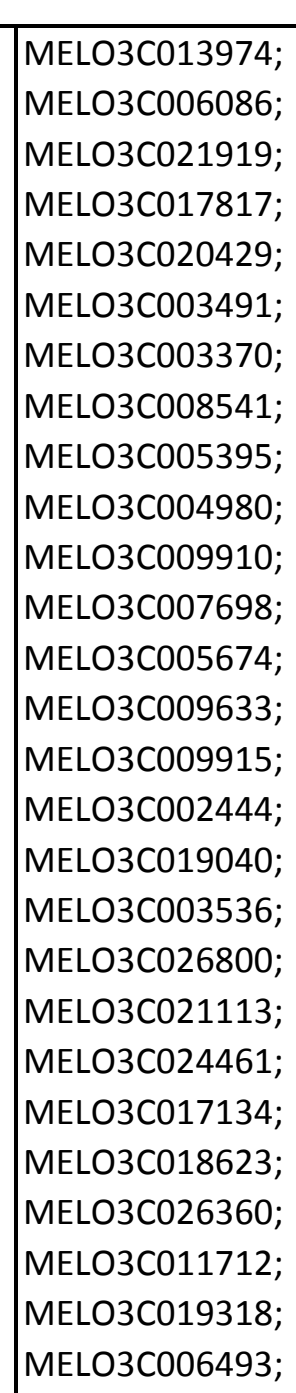 \\
\hline GO:0016071 & mRNA metabolic process & BIOLOGICAL_PROCESS & $4,59 \mathrm{E}-04$ & $b$ & 38 & 415 & 589 & 16086 & 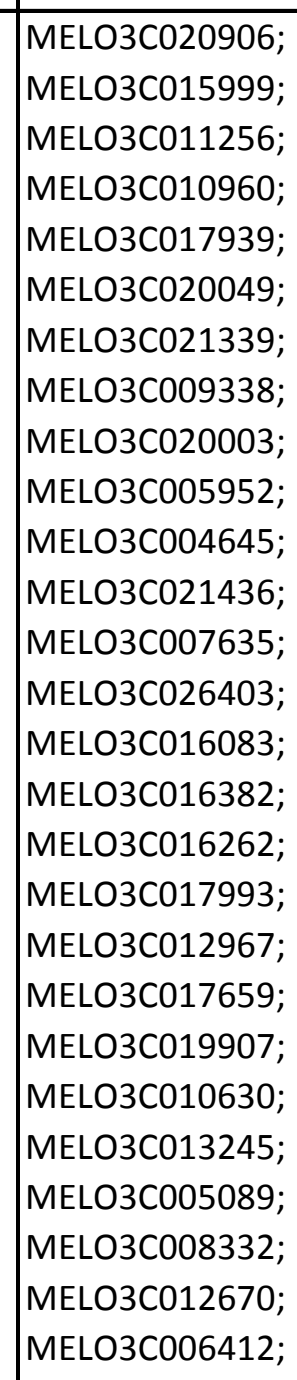 & 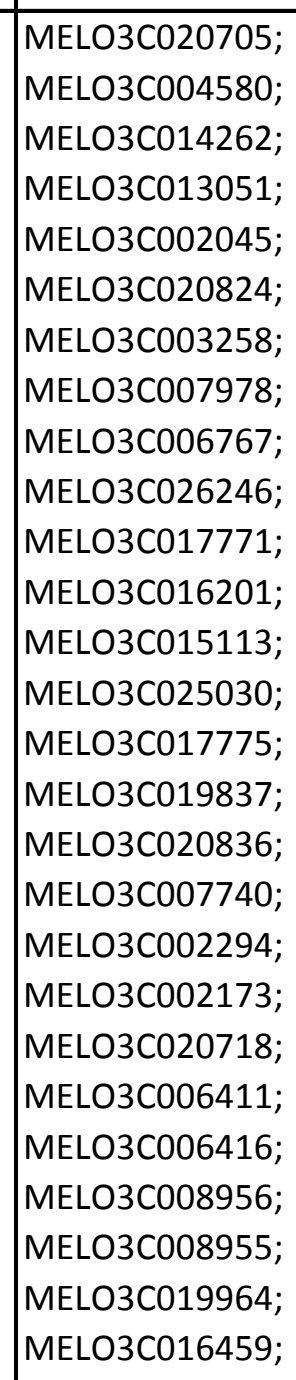 \\
\hline 60:0000287 & magnesium ion binding & MOLECULAR_FUNCTION & 0 & $2,248 \mathrm{E}-05$ & 21 & 186 & 606 & 16315 & 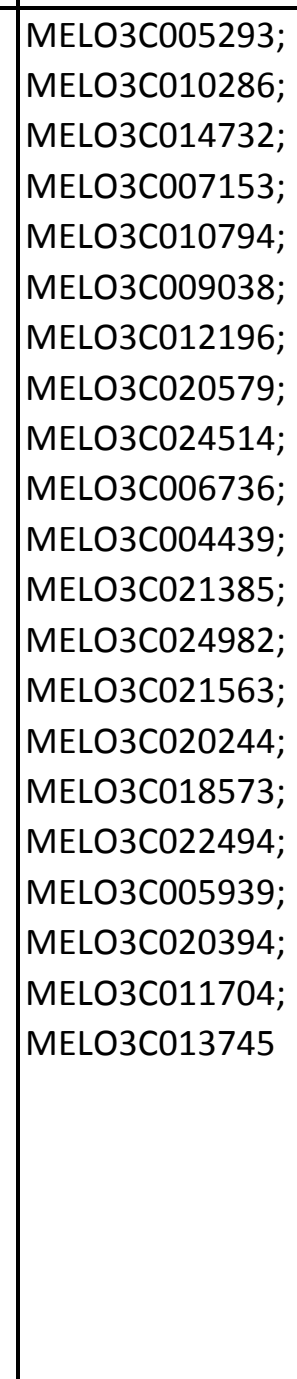 & 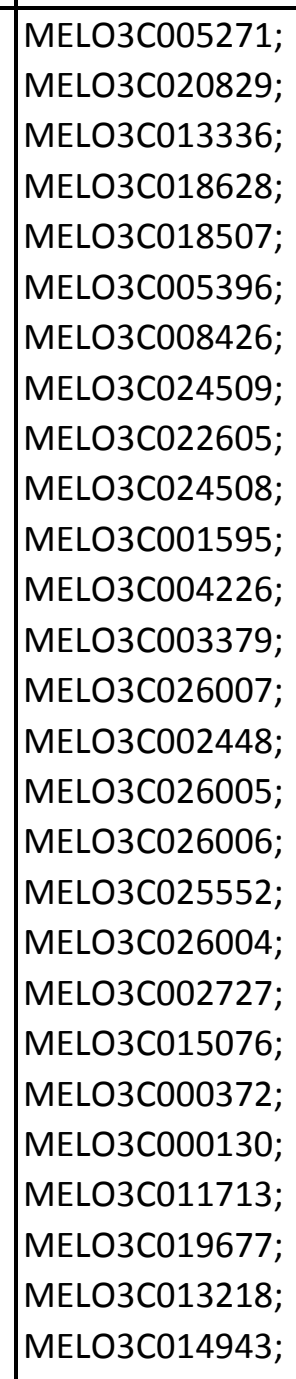 \\
\hline GO:0004775 & $\begin{array}{l}\text { succinate-CoA ligase (ADP-forming) } \\
\text { activity }\end{array}$ & MOLECULAR_FUNCTION & 0,002863815 & $2,52 \mathrm{E}-05$ & 4 & $\overline{2}$ & 623 & 16499 & $\begin{array}{l}\text { MELO3CO02167; } \\
\text { MELOSCO22382; } \\
\text { MELO3CO1067; } \\
\text { MELO3CO11482 }\end{array}$ & $\begin{array}{l}\text { MELO3C021268; } \\
\text { MELO3C015245 }\end{array}$ \\
\hline GO:0009651 & response to salt stress & BIOLOGICAL_PROCESS & 0,00360577 & $3,28 E-05$ & 18 & 146 & 609 & 16355 & 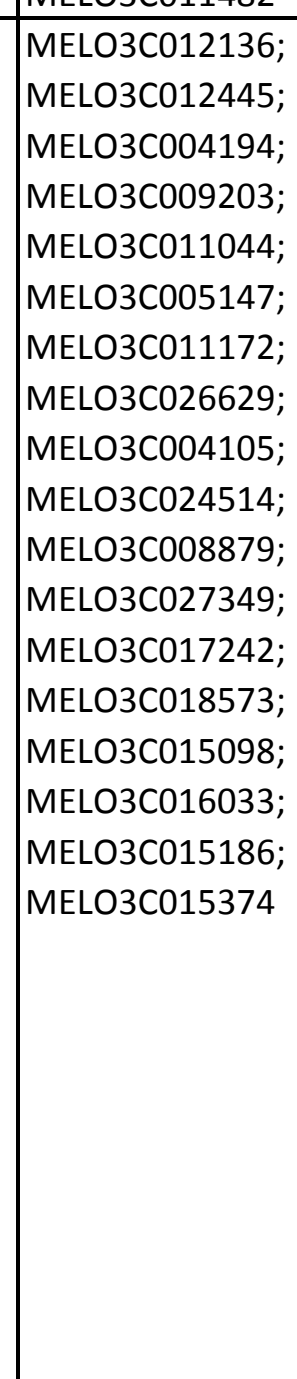 & 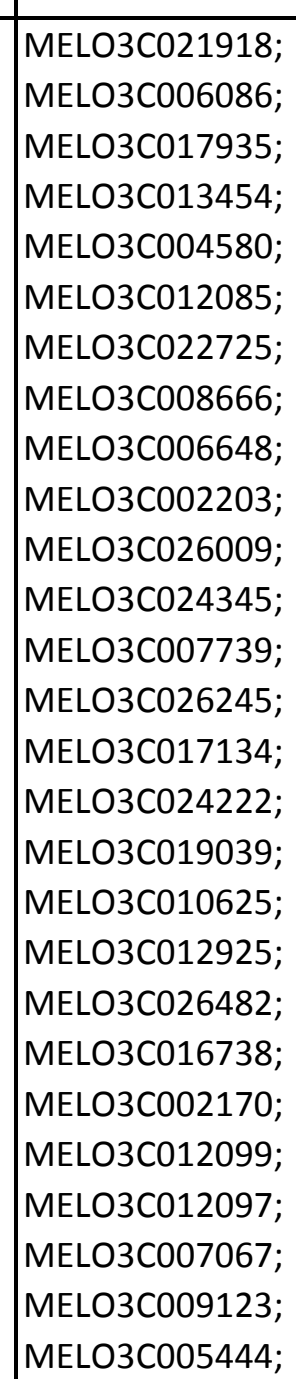 \\
\hline
\end{tabular}




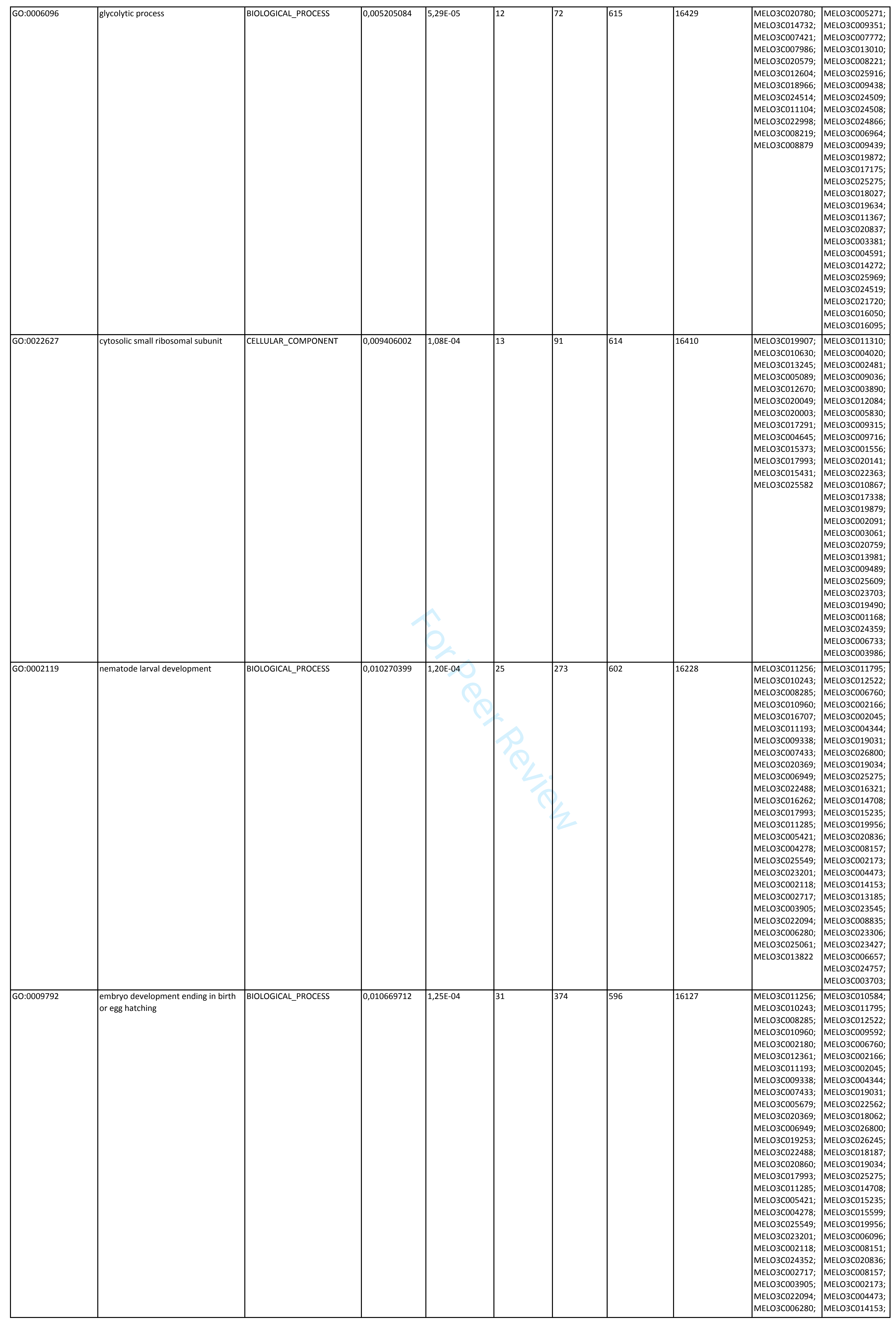




\begin{tabular}{|c|c|c|c|c|c|c|c|c|c|c|}
\hline 60:0006099 & |tricarboxylic acid cycle & BIOLOGICAL_PROCESS & 0,029273395 & $4,00 E-04$ & 7 & 31 & 620 & 16470 & $\begin{array}{l}\text { MELO3CO21563; } \\
\text { MELO3CO22382; } \\
\text { MEEOC_017560; } \\
\text { MELOSCO11482; } \\
\text { MELO3CO02167; } \\
\text { MELO3CO21247; } \\
\text { MELO3C022998; }\end{array}$ & 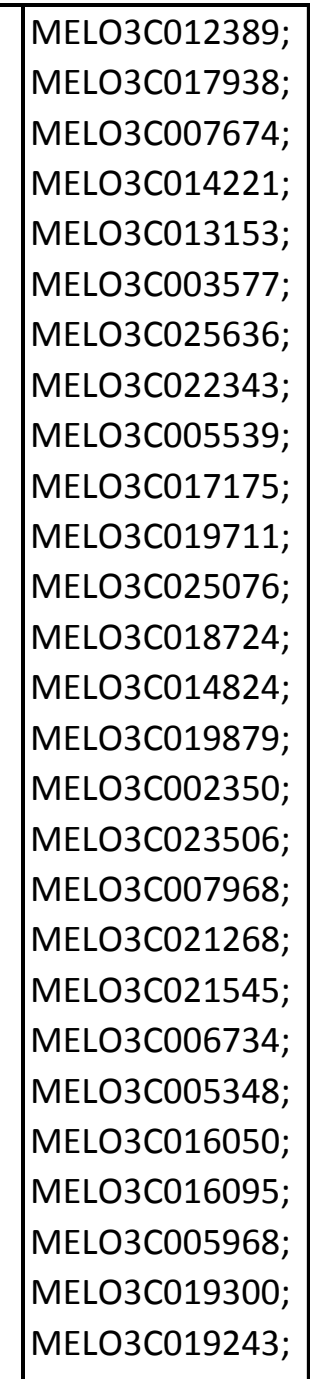 \\
\hline 60:0005618 & cell wall & CELLULAR_COMPONENT & 0,030634705 & O & 28 & 351 & 599 & 16150 & 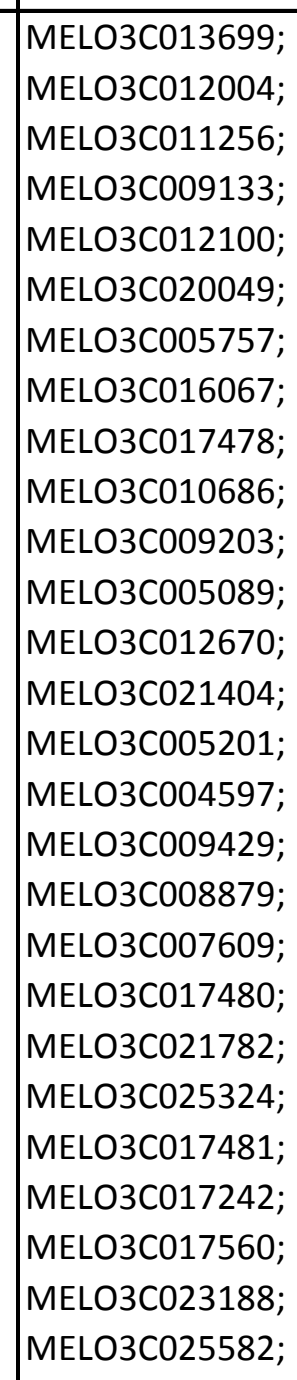 & 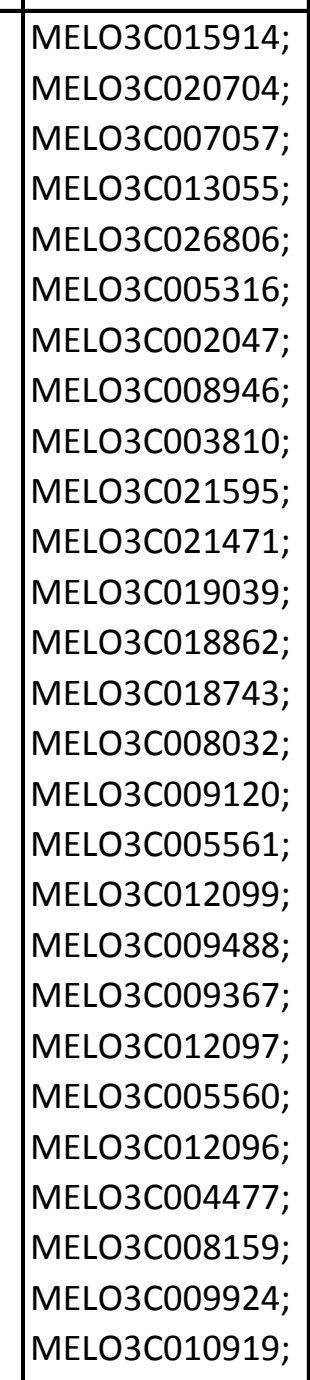 \\
\hline 60:0003878 & ATP citrate synthase activity & MOLECULAR_FUNCTION & 0,031860458 & $4,462-04$ & 3 & 2 & 624 & 16499 & 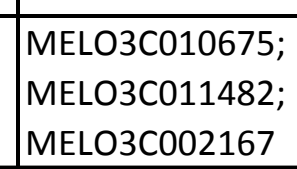 & $\begin{array}{l}\text { MELO3C021268; } \\
\text { MELO3C015245 }\end{array}$ \\
\hline 60:0008340 & determination of adult lifespan & \begin{tabular}{|l|l|} 
BIOLOGICAL_PROCESS \\
\end{tabular} & 0,032223247 & $4,81 \mathrm{E}-04$ & 11 & 80 & 616 & 16421 & 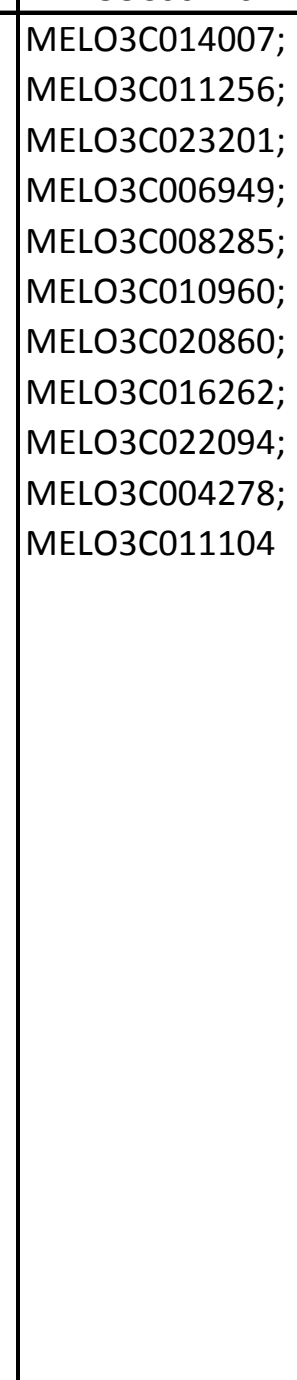 & 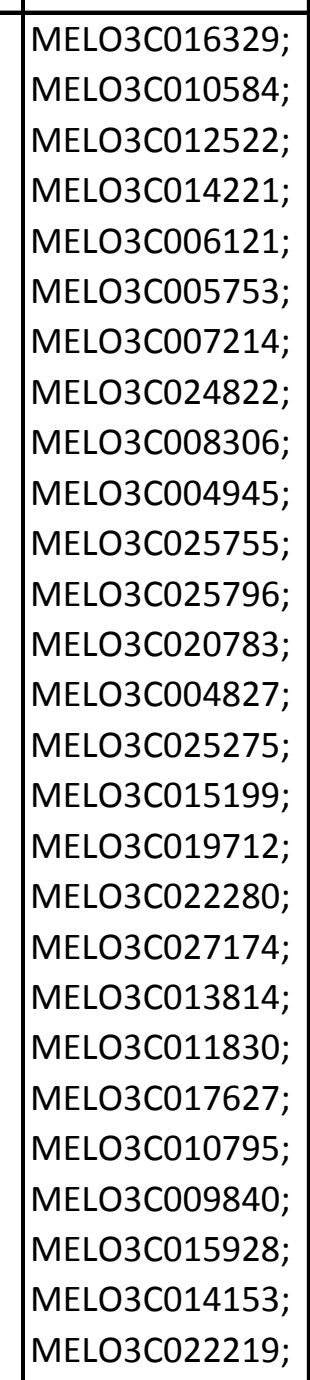 \\
\hline GO:0031018 & endocrine pancreas development & \begin{tabular}{|l|} 
BIOLOGICAL_PROCESS \\
\end{tabular} & 0,032223247 & $4,78 \mathrm{E}-04$ & 4 & 7 & 623 & 16494 & $\begin{array}{l}\text { MELOЗ_023201; } \\
\text { MELOЗCO17993; } \\
\text { MELOЗCO09338; } \\
\text { MELO3C017291 }\end{array}$ & 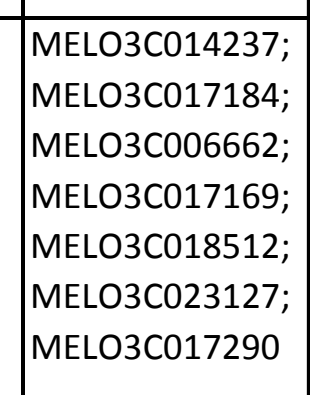 \\
\hline GO:0010181 & FMN binding & MOLECULAR_FUNCTION & 0,057904252 & $9,44 \mathrm{E}-04$ & 6 & 26 & 621 & 16475 & $\begin{array}{l}\text { MELOЗСС007340; } \\
\text { MELOC_CO11062; } \\
\text { MELO3CO03541; } \\
\text { MELO3CO23799; } \\
\text { MELO3CO16623; } \\
\text { MELO3C015514 }\end{array}$ & 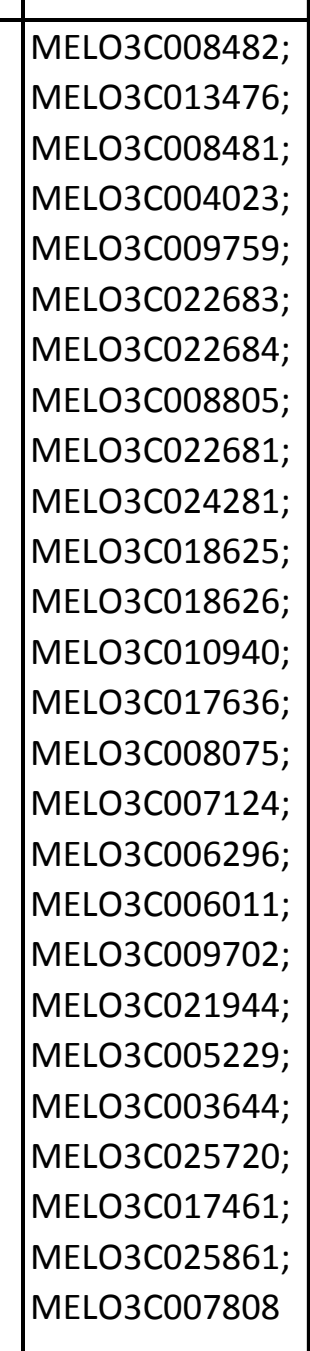 \\
\hline
\end{tabular}




\begin{tabular}{|c|c|c|c|c|c|c|c|c|c|c|}
\hline 60:0004776 & $\begin{array}{l}\text { succinate-COA ligase (GDP-forming) } \\
\text { activity }\end{array}$ & MOLECULAR_FUNCTION & 0,076614304 & 0,001337993 & 2 & 0 & 625 & 16501 & $\begin{array}{l}\text { MELO3C022382; } \\
\text { MELO3C002167 }\end{array}$ & \\
\hline 60:0003861 & \begin{tabular}{|l|}
$\begin{array}{l}\text { 3-isopropylmalate dehydratase } \\
\text { activity }\end{array}$ \\
\end{tabular} & MOLECULAR_EUNCTION & 0,076614304 & 0,001337993 & 2 & 0 & 625 & 16501 & 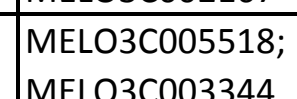 & \\
\hline 60:0040002 & $\begin{array}{l}\text { collagen and cuticulin-based cuticle } \\
\text { development }\end{array}$ & BIOLOGICAL_PROCESS & 0,086570236 & 0,001530189 & 3 & 4 & 624 & 16497 & $\begin{array}{l}\text { MELOSCOC16707; } \\
\text { MELO3C019253; } \\
\text { MELO3C012361 }\end{array}$ & \begin{tabular}{|l} 
MELO3C016321; \\
MELO3C023052; \\
MELO3CO08151; \\
MELO3C015126
\end{tabular} \\
\hline 60:0048046 & apoplast & CELLULAR_COMPONENT & 0,08752412 & 0,001574841 & 18 & 203 & 609 & 16298 & 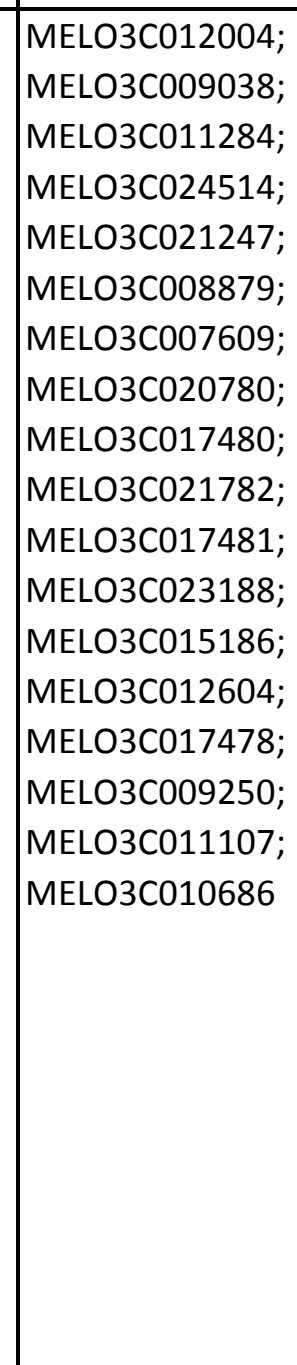 & 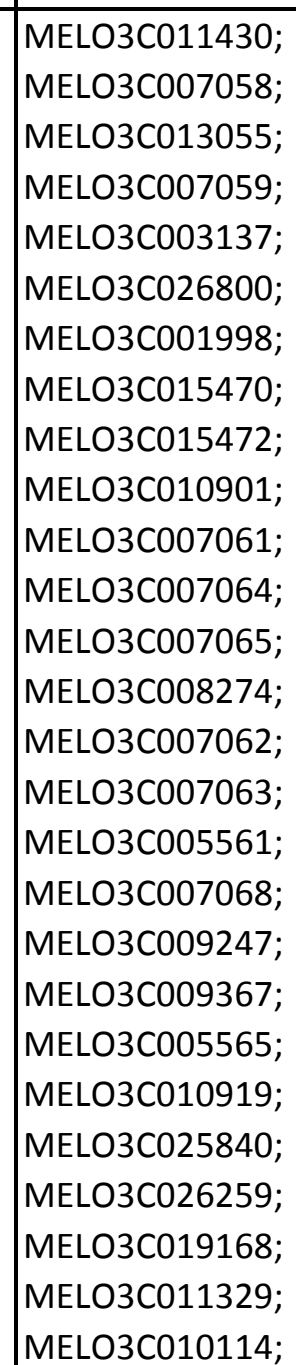 \\
\hline 60:0004298 & threonine-type endopeptidase activity & MOLECULAR_FUNCTION & 0,099790014 & Pro & 5 & 20 & 622 & 16481 & 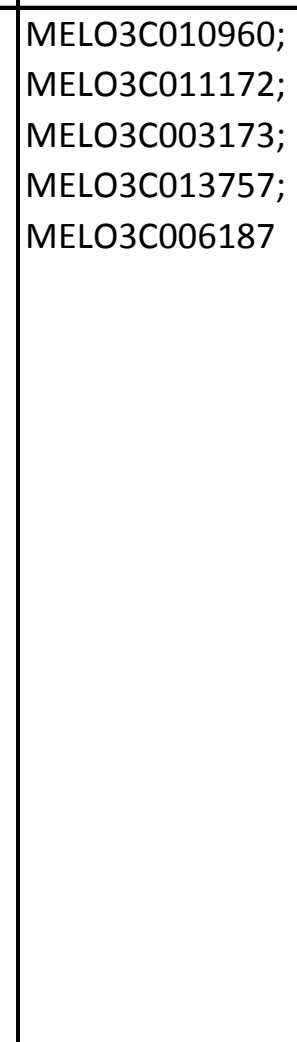 & 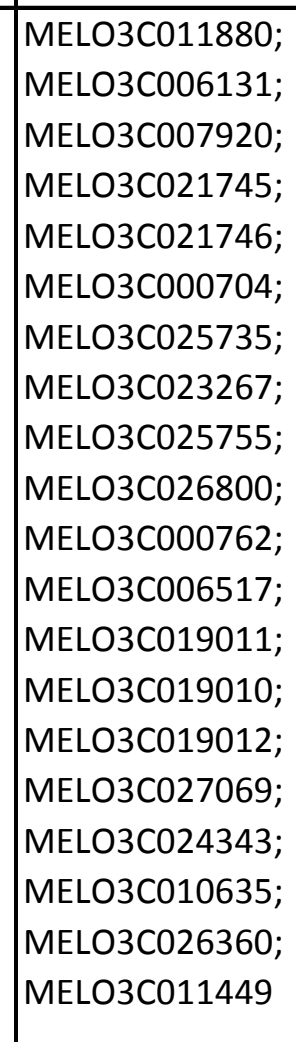 \\
\hline GO:0015631 & tubulin binding & MOLECULAR_FUNCTION & 0,099790014 & 0,001869478 & 5 & 20 & 622 & 16481 & $\begin{array}{l}\text { MELO3C022488; } \\
\text { MELO3C013702; } \\
\text { MELO3CO02717; } \\
\text { MELO3C017221; } \\
\text { MELO3C006602 }\end{array}$ & 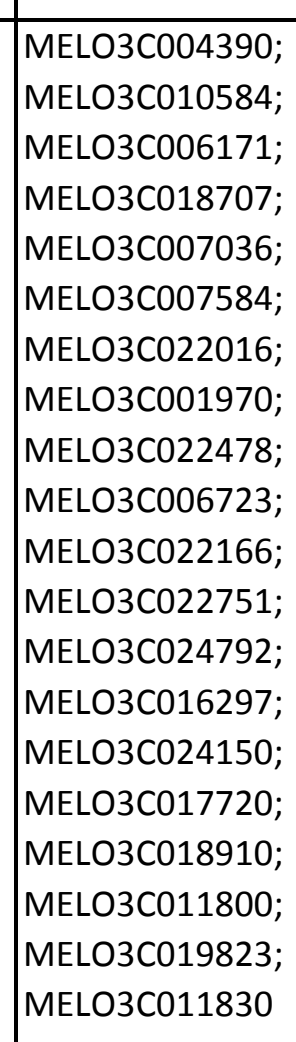 \\
\hline G0:0000084 & mitotic S phase & BIOLOGICAL_PROCESS & 0,102607716 & 0,001954846 & 99 & 68 & 618 & 16433 & 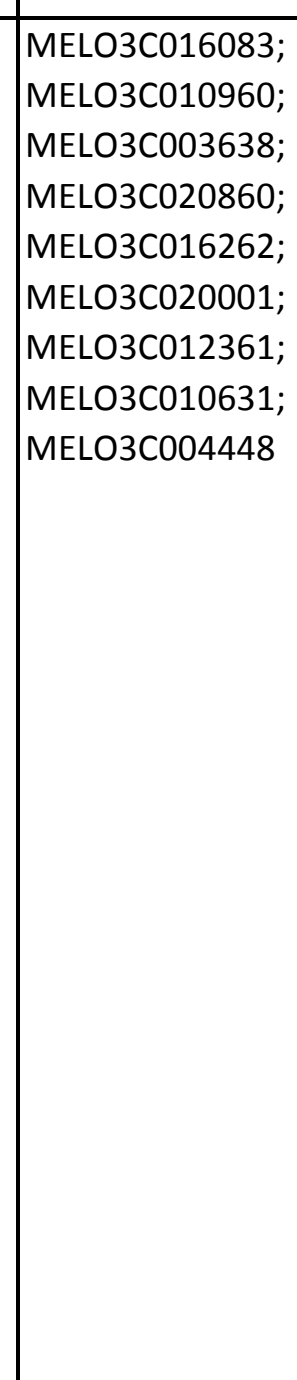 & 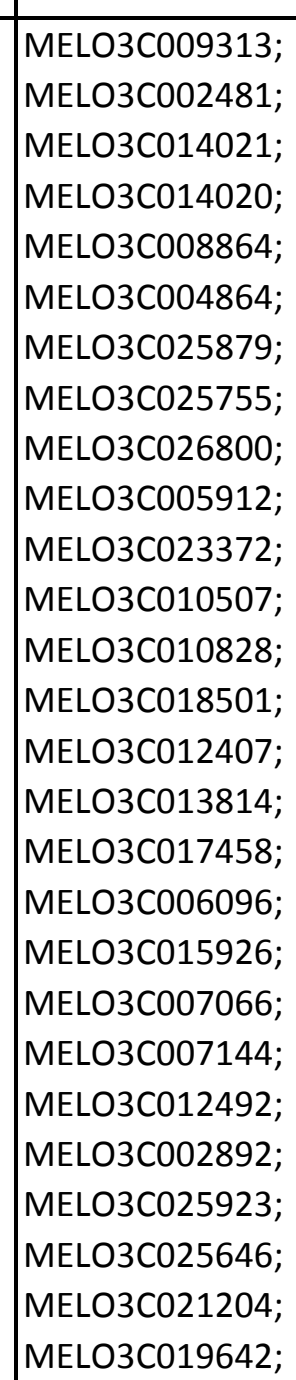 \\
\hline
\end{tabular}




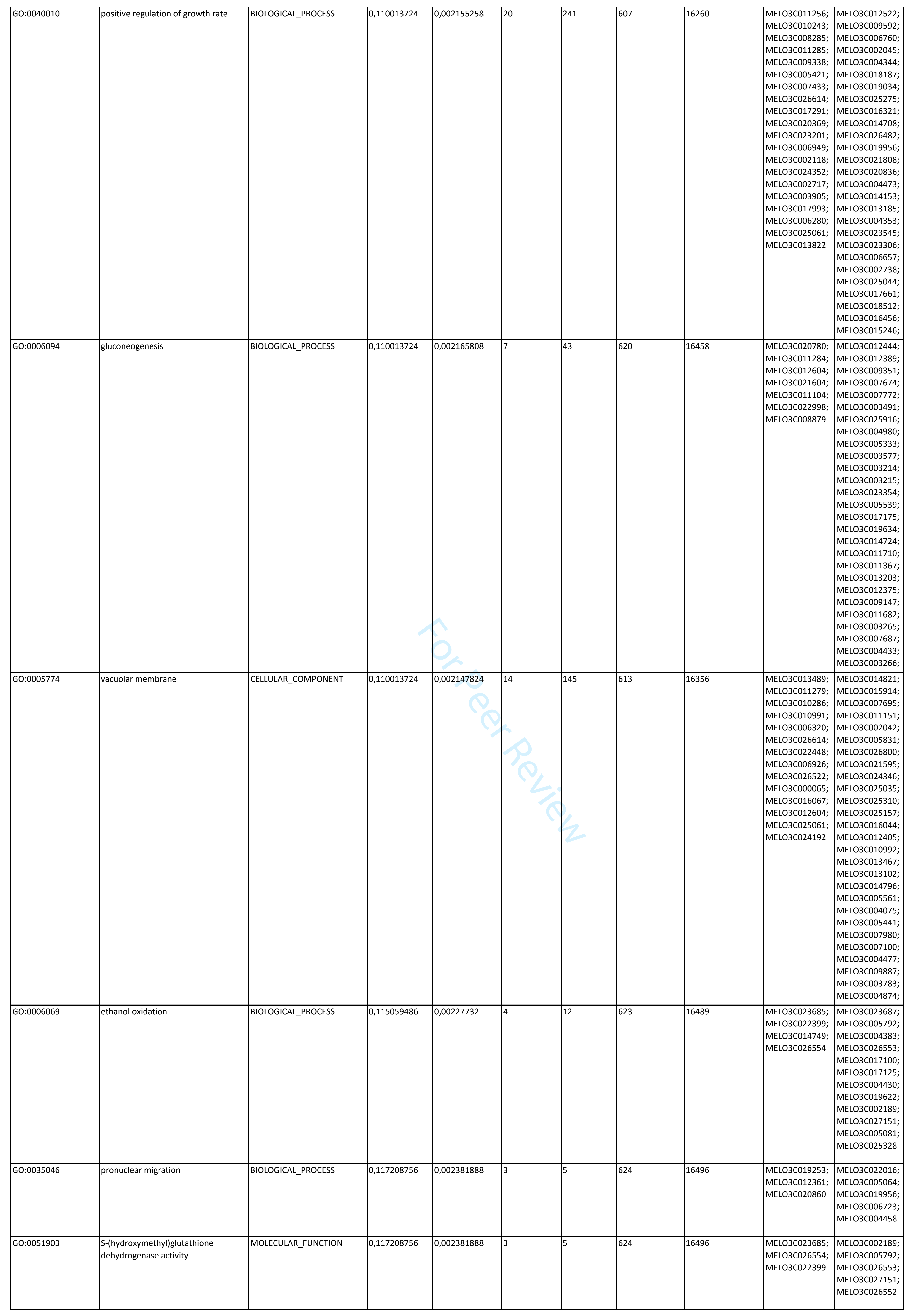




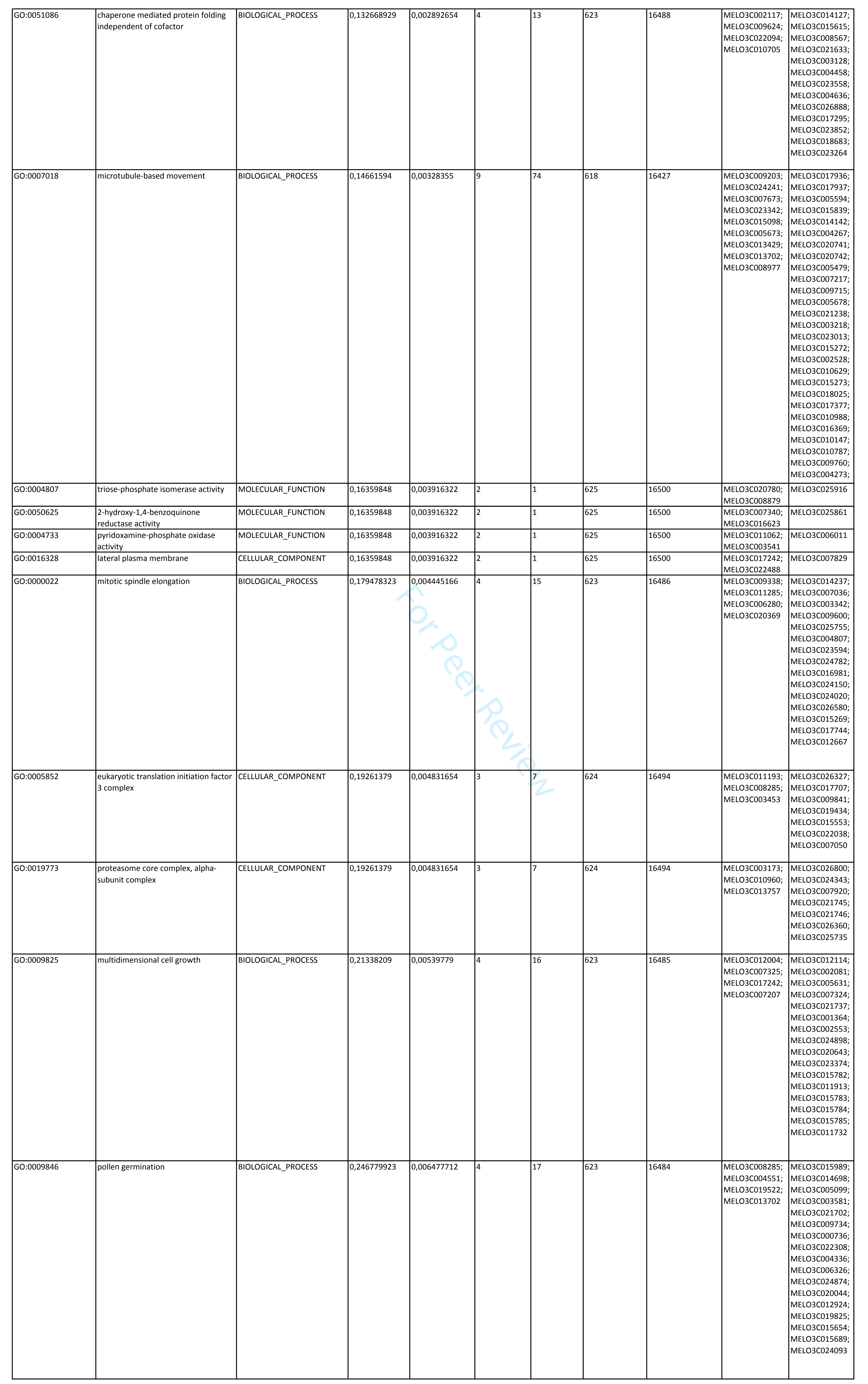




\begin{tabular}{|c|c|c|c|c|c|c|c|c|c|c|}
\hline 60:0042450 & $\begin{array}{l}\text { argininin biosynthetic process via } \\
\text { ornithine }\end{array}$ & BIOLOGICAL_PROCESS & 0,267436664 & 0,007642665 & 2 & 2 & 625 & 16499 & $\begin{array}{l}\text { MELO3C011085; } \\
\text { MELOЗCO11208 }\end{array}$ & $\begin{array}{l}\text { MELOOC023629; } \\
\text { MELOЗСО03523 }\end{array}$ \\
\hline 60:0018279 & \begin{tabular}{|l} 
protein N-linked glycosylation via \\
asparagagine
\end{tabular} & BIOLOGICAL_PROCESS & 0,267436664 & 0,007321952 & 7 & 55 & 620 & 16446 & 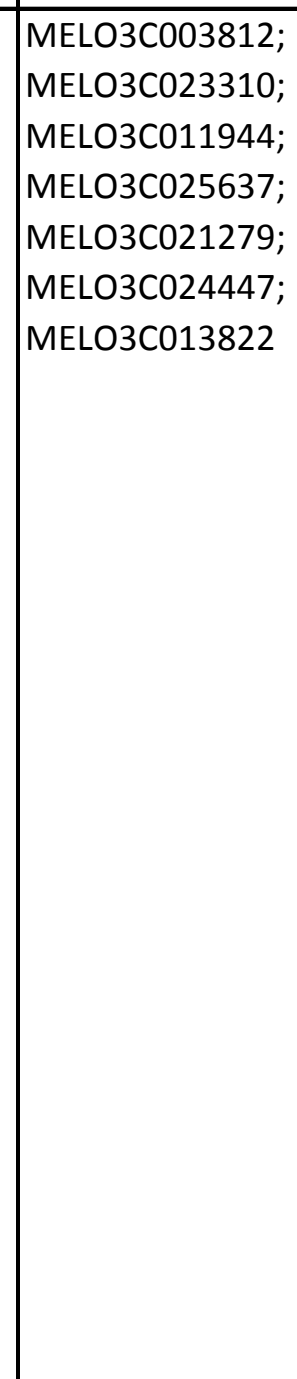 & 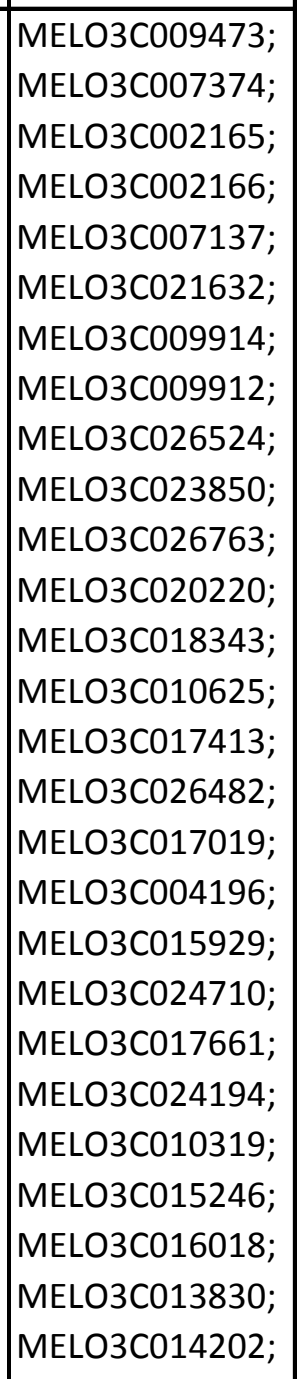 \\
\hline 60:0006272 & |eading strand elongation & BIOLOGICAL_PROCESS & 0,267436664 & 0,007642665 & 2 & 2 & 625 & 16499 & $\begin{array}{l}\text { MELO3C010631; } \\
\text { MELO3C020860 }\end{array}$ & $\begin{array}{l}\text { MELOOCO14021; } \\
\text { MELO3C010507 }\end{array}$ \\
\hline 60:0004634 & phosphopyruvate hydratase activity & MOLECULAR_FUNCTION & 0,267436664 & 0,007642665 & 2 & 2 & 625 & 16499 & $\begin{array}{l}\text { MELO3C020579; } \\
\text { MELO3C024514 }\end{array}$ & \begin{tabular}{|l|} 
MELO3C017268; \\
MELO3C026748
\end{tabular} \\
\hline 60:0004133 & glycogen debranching enzyme activity & MOLECULAR_FUNCTION & 0,267436664 & 0,007642665 & 2 & 2 & 625 & 16499 & $\begin{array}{l}\text { MELO3C015861; } \\
\text { MELOSC019135 }\end{array}$ & $\begin{array}{l}\text { MELOOCO017391; } \\
\text { MELO3C020949 }\end{array}$ \\
\hline 60:0008541 & $\begin{array}{l}\text { proteasome regulatory particle, lid } \\
\text { subcomplex }\end{array}$ & CELLULAR_COMPONENT & 0,267436664 & 0,007642665 & 2 & 2 & 625 & 16499 & $\begin{array}{l}\text { MELO3C016262; } \\
\text { MELOSC011193 }\end{array}$ & \begin{tabular}{|l} 
MELOOCOC25923; \\
MELOSCO13814
\end{tabular} \\
\hline 60:0000015 & phosphopyruvate hydratase complex & CELLULAR_COMPONENT & 0,267436664 & 0,007642665 & 2 & 2 & 625 & 16499 & $\begin{array}{l}\text { MELO3C020579; } \\
\text { MELO3C024514 }\end{array}$ & \begin{tabular}{|l} 
MELOOCO017268; \\
MELO3C026748
\end{tabular} \\
\hline 60:0034515 & proteasome storage granule & $\begin{array}{l}\text { CELLULAR_COMPONENT } \\
\end{array}$ & 0,285040264 & 0,008387087 & ${ }^{3}$ & $\frac{9}{9}$ & 624 & 16492 & $\begin{array}{l}\text { MELO3C016262; } \\
\text { MELOSCO11193; } \\
\text { MELO3C010960 }\end{array}$ & 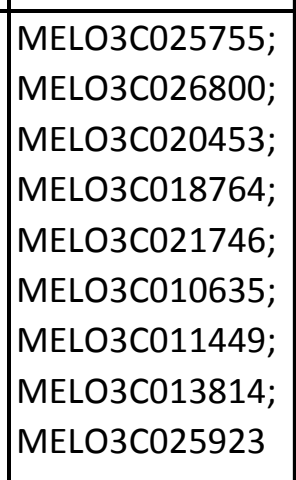 \\
\hline 60:0030170 & pyridoxal phosphate binding & MOLECULAR_FUNCTION & 0,330546323 & 0,010250854 & 11 & 123 & 616 & 16378 & 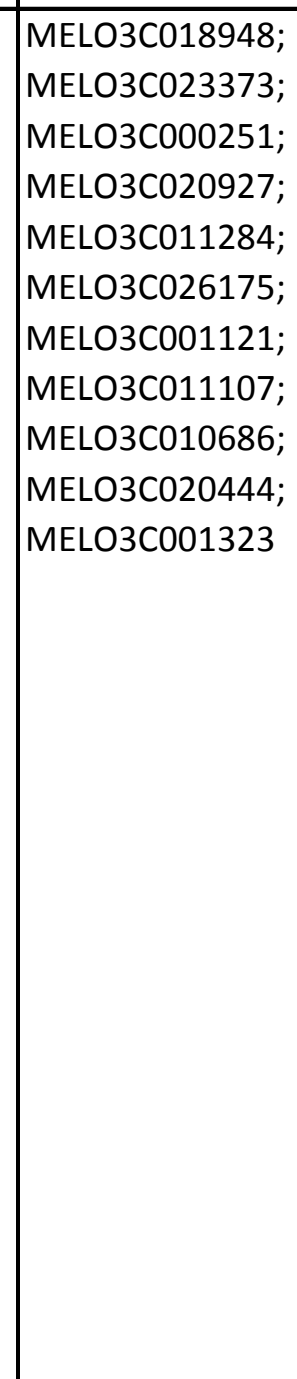 & 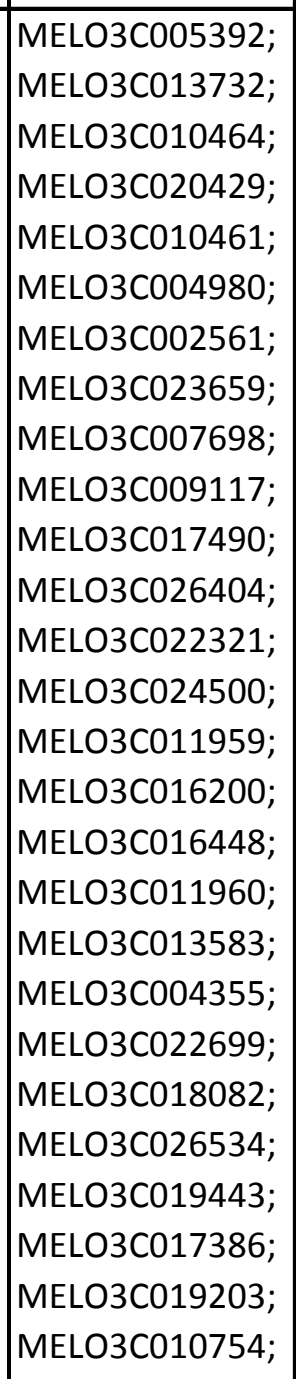 \\
\hline 60:0005509 & calcium ion binding & MOLECULAR_FUNCTION & 0,358334546 & 0,011491889 & 16 & 211 & 611 & 16290 & 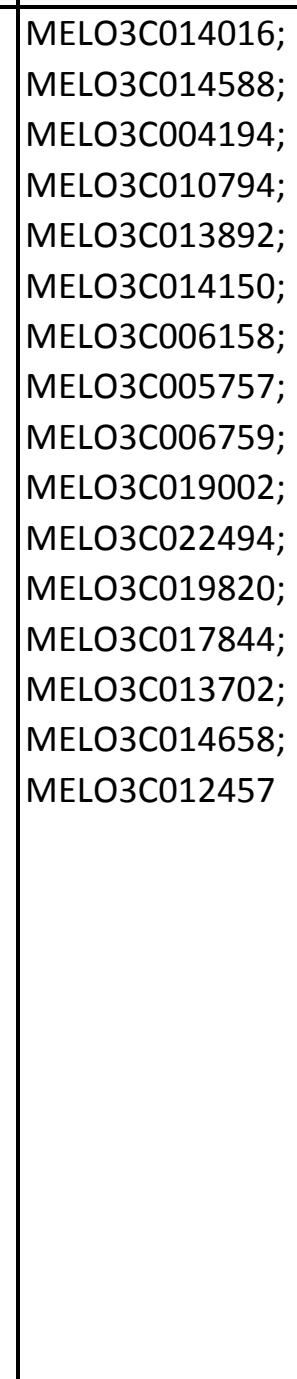 & 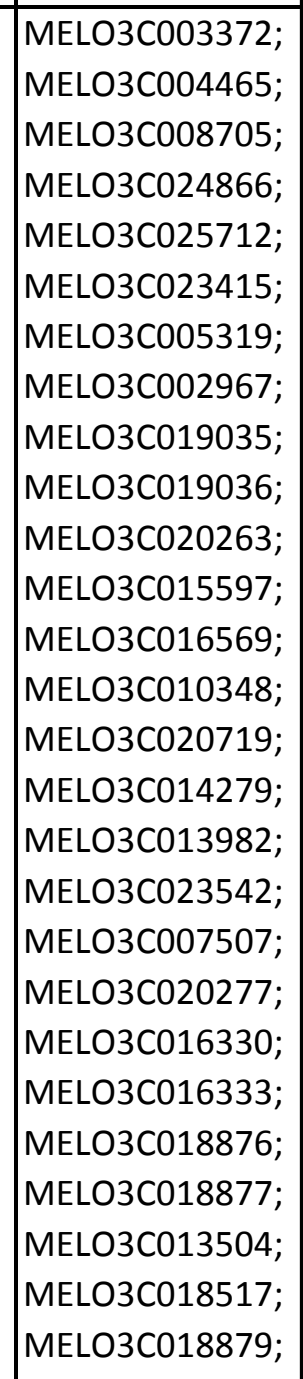 \\
\hline
\end{tabular}




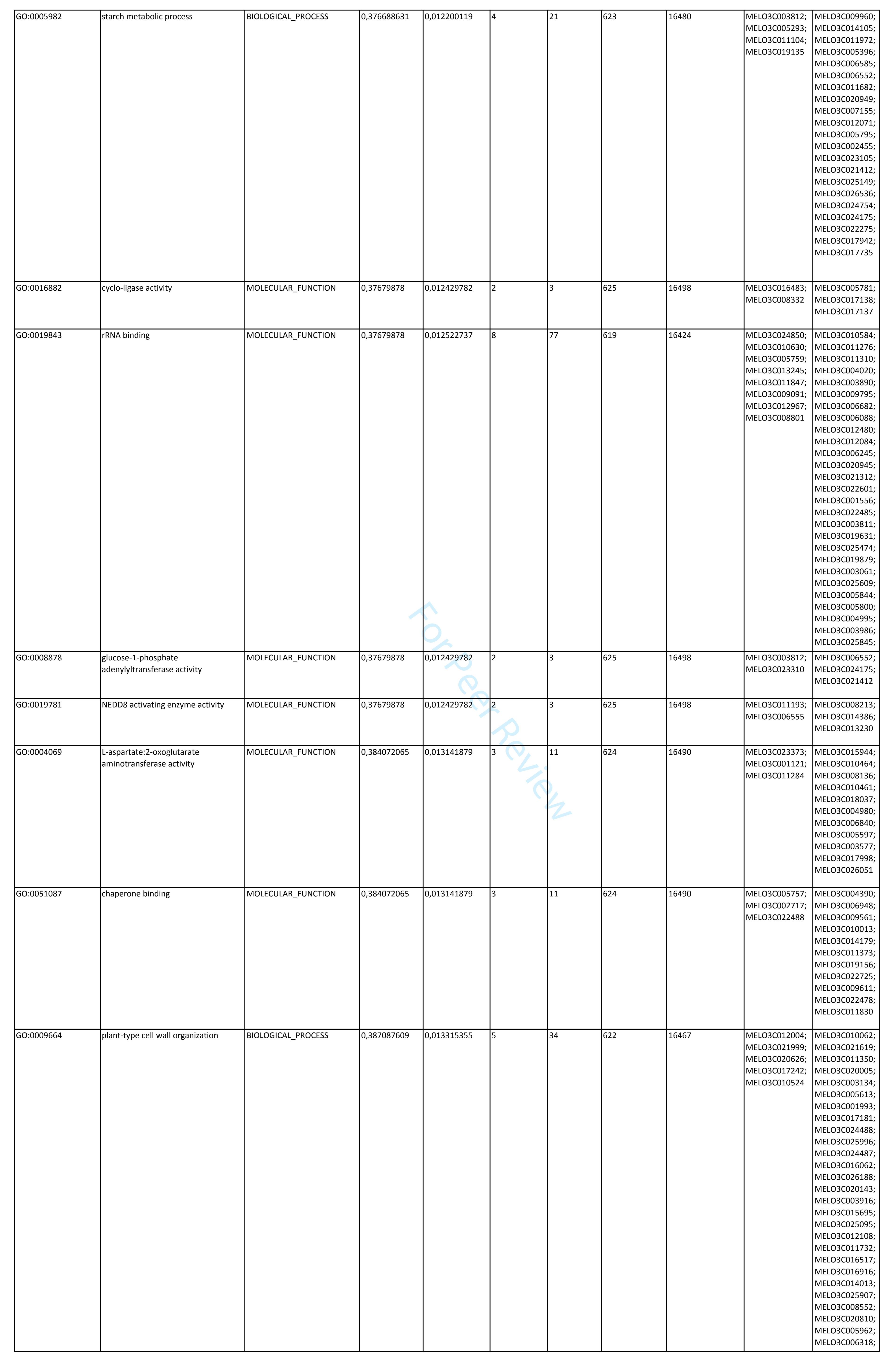




\begin{tabular}{|c|c|c|c|c|c|c|c|c|c|c|}
\hline 60:0005978 & gIlycogen biosynthetic process & BIOLOGICAL_PROCESS & 0,003293101 & 0,014009997 & 4 & 22 & 623 & 16479 & 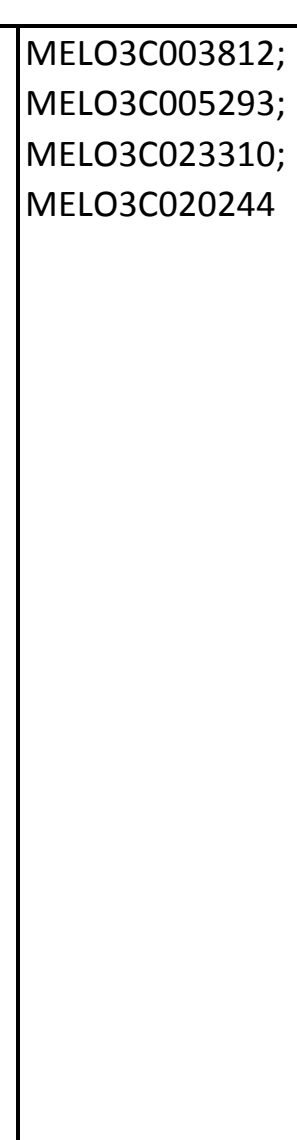 & 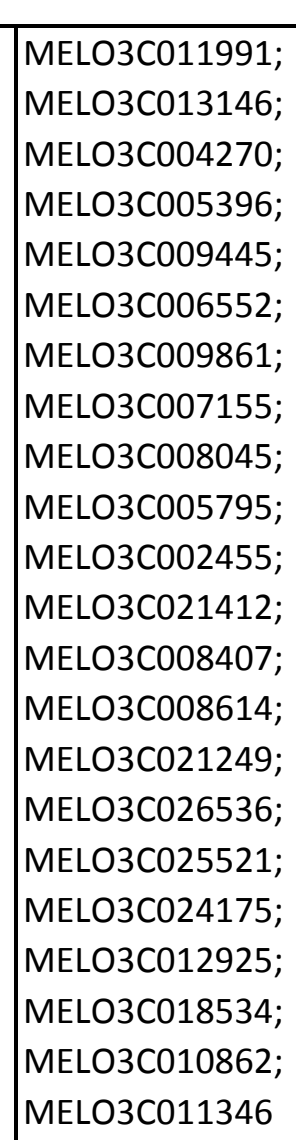 \\
\hline G0:0043161 & \begin{tabular}{|l} 
proteasome-mediated ubiquitin- \\
dependent protein catabolic process
\end{tabular} & \begin{tabular}{|l|} 
BIOLOGICAL_PROCESS \\
\end{tabular} & 0,425423227 & 0,015219417 & 8 & 80 & 619 & 16421 & 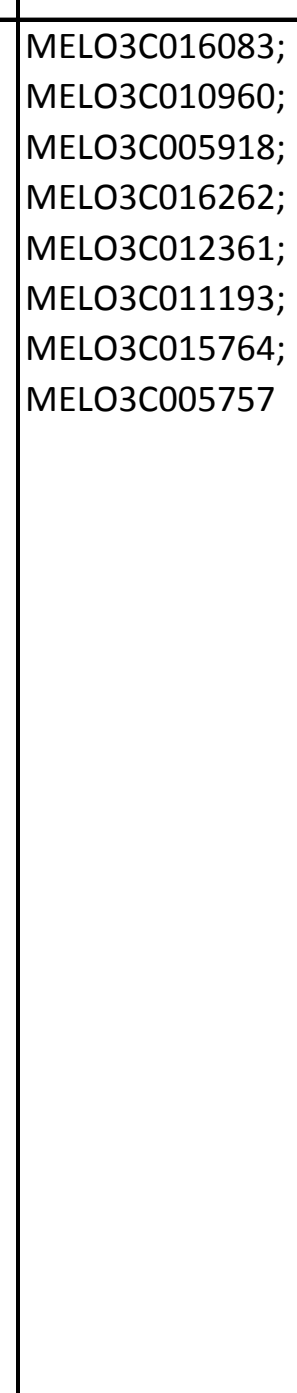 & 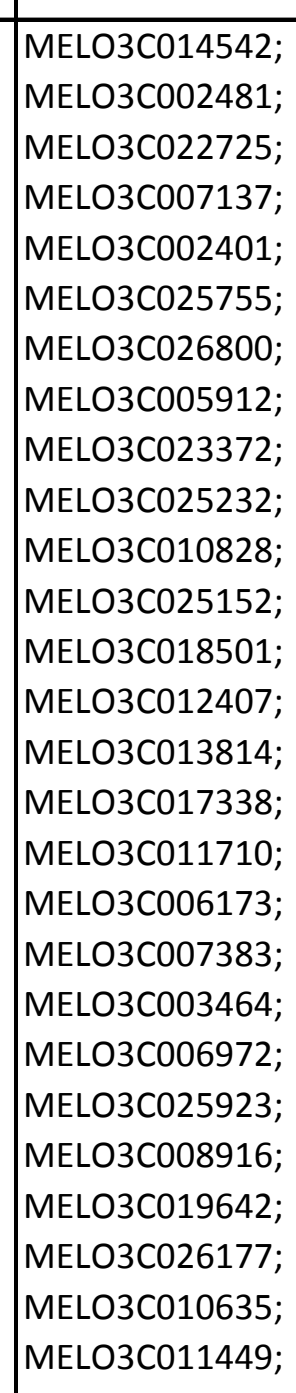 \\
\hline 60:0006351 & transcription, DNA-templated & BIOLOGICAL_PROCESS & 0,432026982 & 0 & 43 & 1606 & 584 & 14895 & 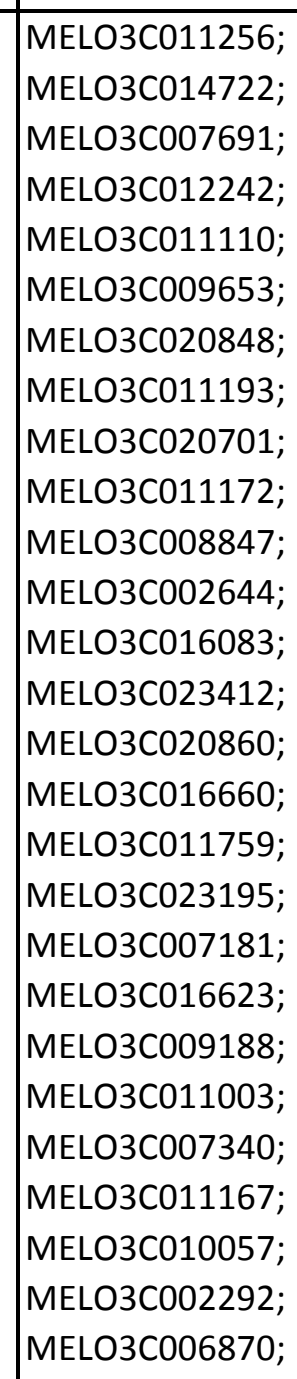 & 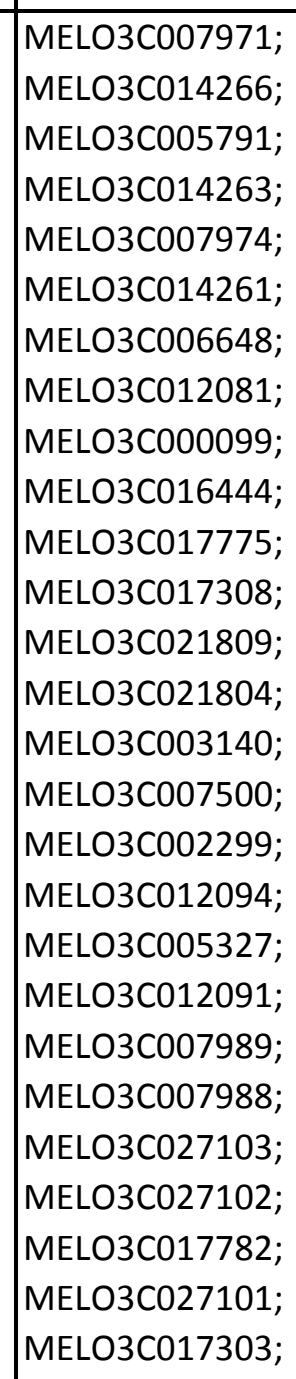 \\
\hline 60:0005506 & iron ion binding & MOLECULAR_FUNCTION & 0,432026982 & 0,015575442 & 4 & 320 & 623 & 16181 & 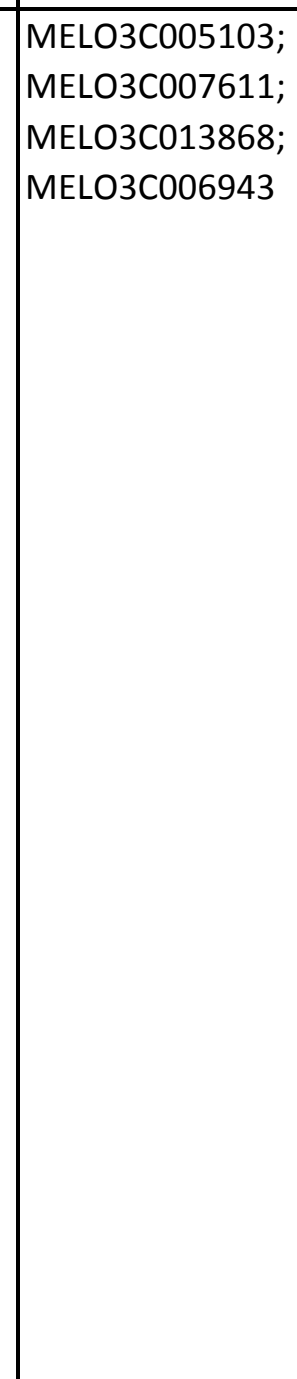 & 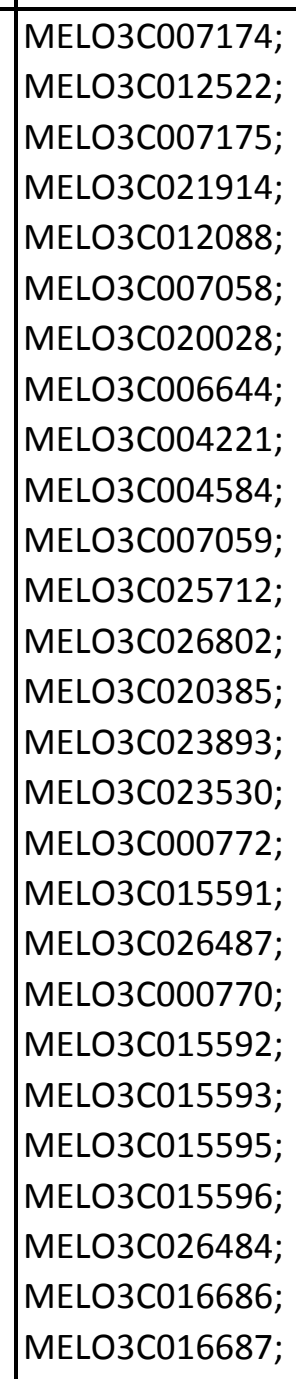 \\
\hline
\end{tabular}




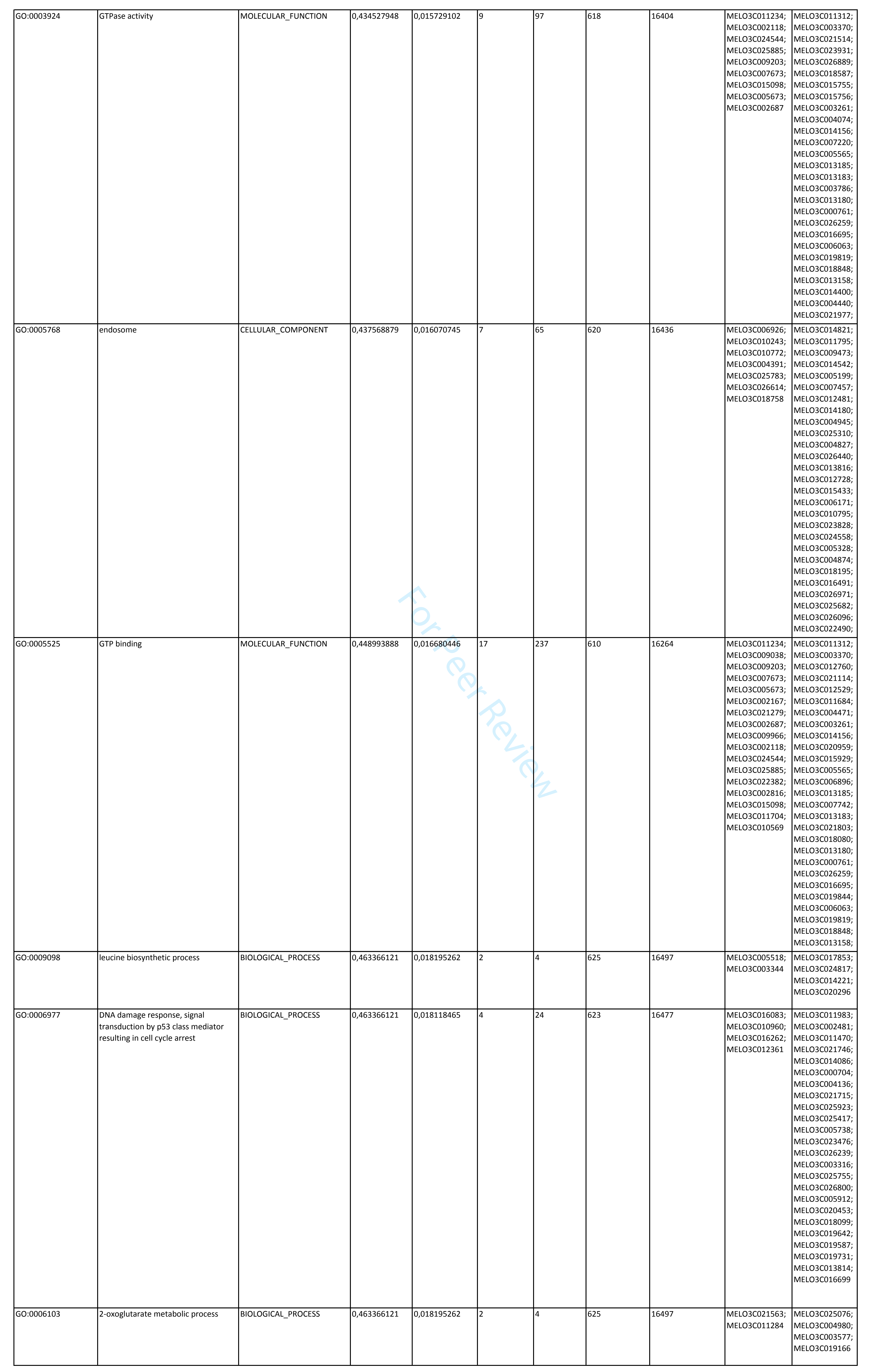




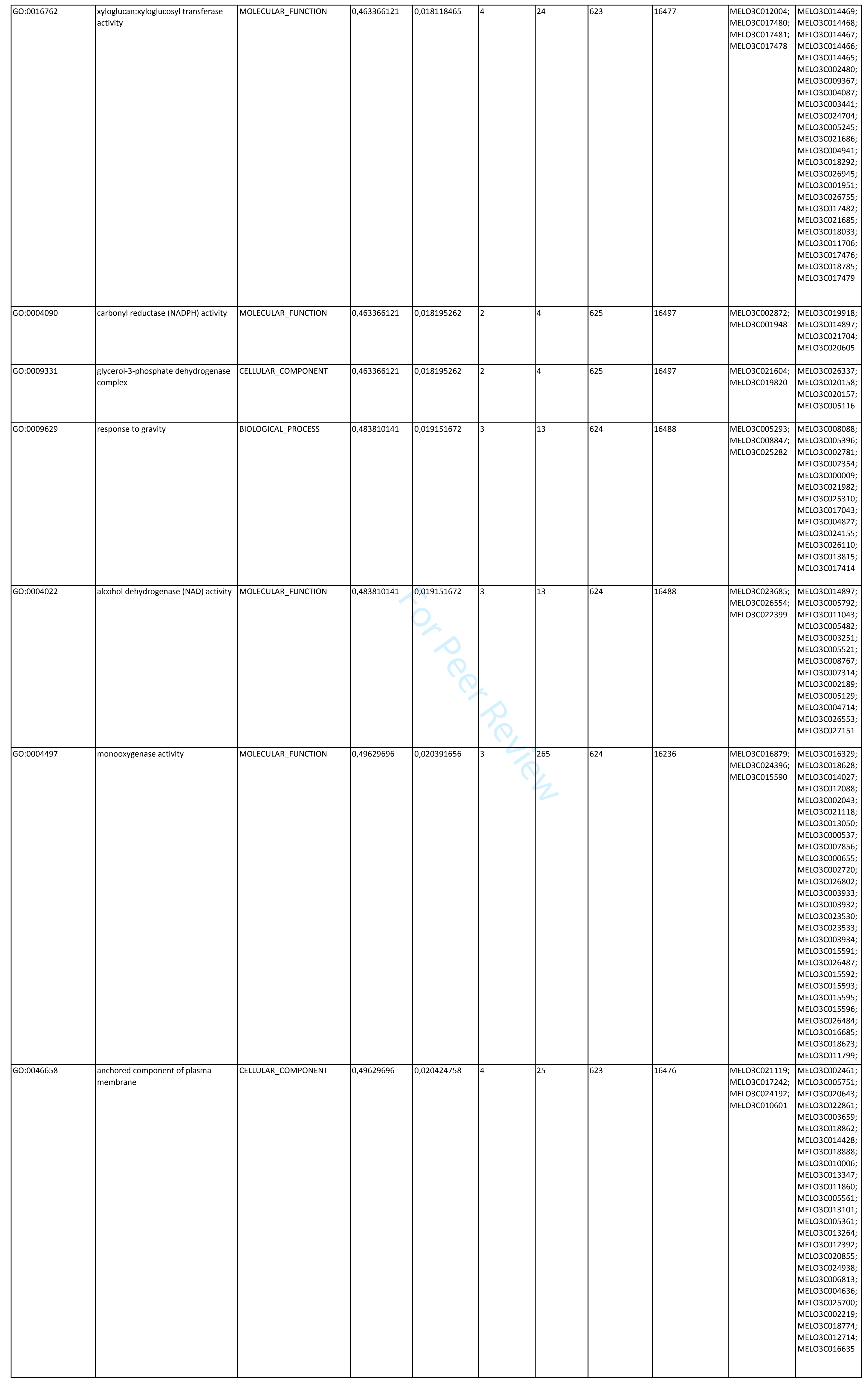




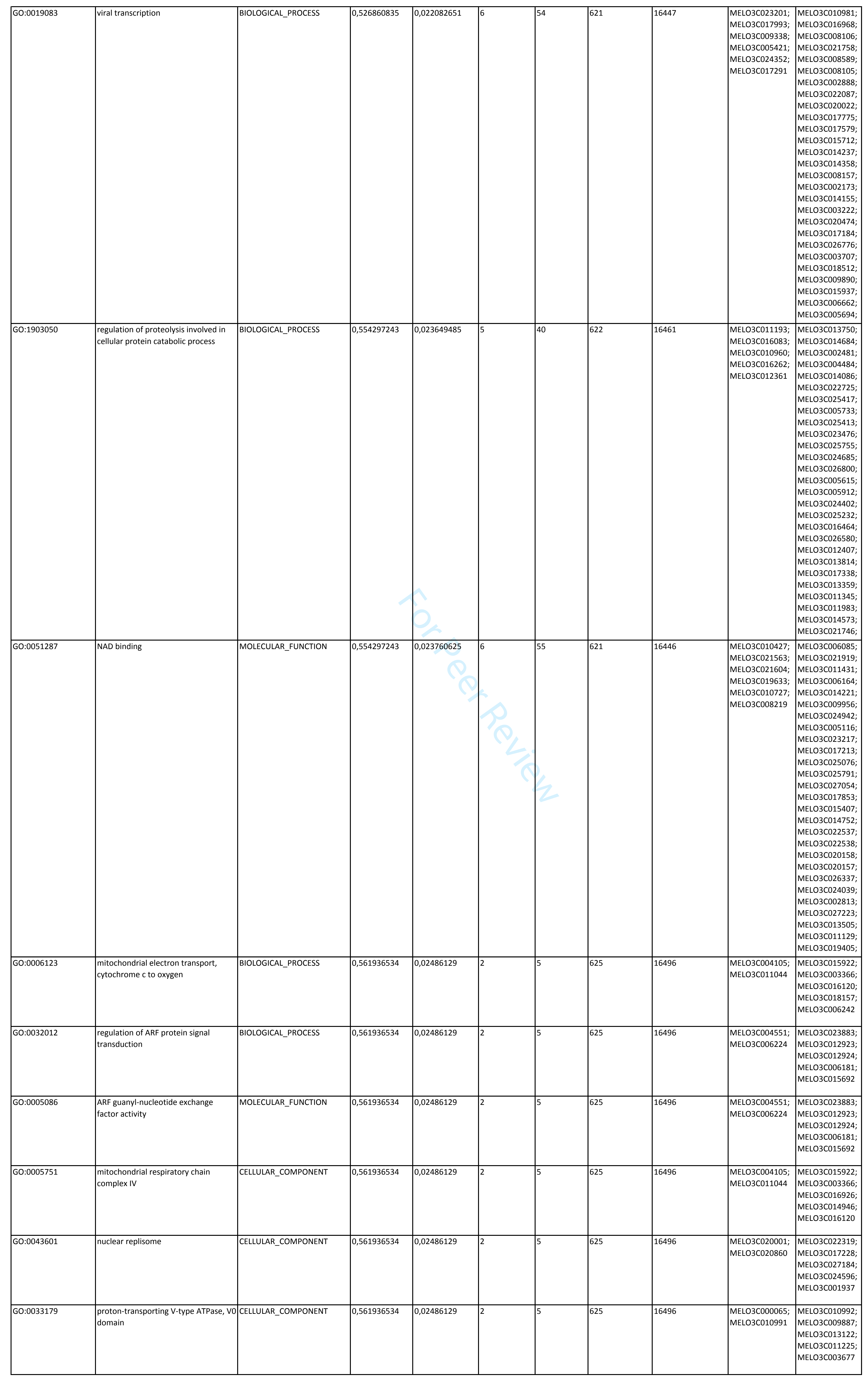




\begin{tabular}{|c|c|c|c|c|c|c|c|c|c|c|}
\hline 60:0005960 & glycine cleavage complex & CELLULAR_COMPONENT & 0,561936534 & 0,02486129 & $2^{2}$ & 5 & 625 & 16496 & \begin{tabular}{|l|} 
MELO3C021247; \\
MELO3C021742
\end{tabular} & $\begin{array}{l}\text { MELO3C003386; } \\
\text { MELO3C003734; } \\
\text { MELO3CO02444; } \\
\text { MELO3CO06734; } \\
\text { MELO3C010754 }\end{array}$ \\
\hline 60:0008237 & metallopeptidase activity & MOLECULAR_FUNCTION & 0,569490972 & 0,025353183 & 10 & 114 & 617 & 16387 & 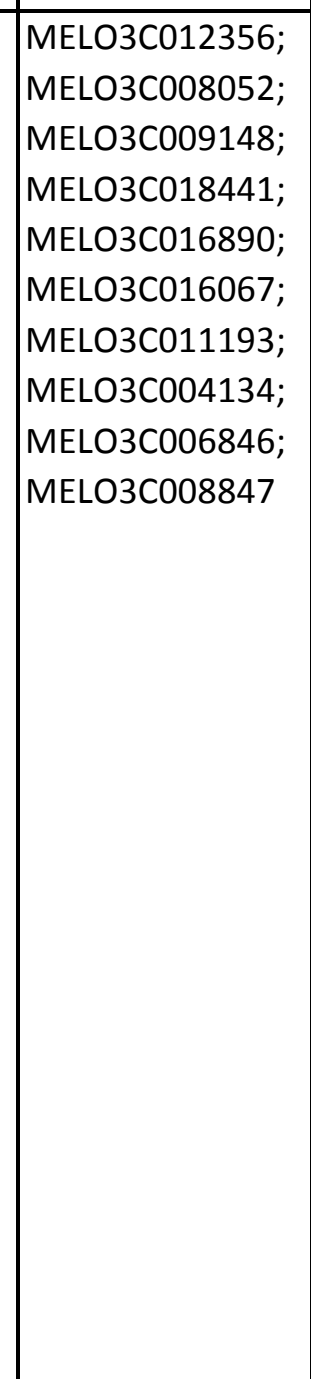 & 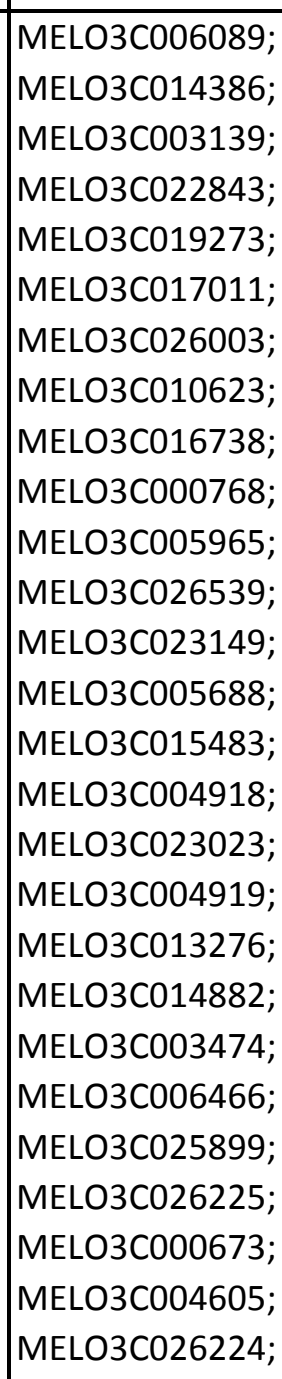 \\
\hline 60:0005351 & sugar:proton symporter activity & MOLECULAR_FUNCTION & 0,571343664 & r & 5 & 41 & 622 & 16460 & 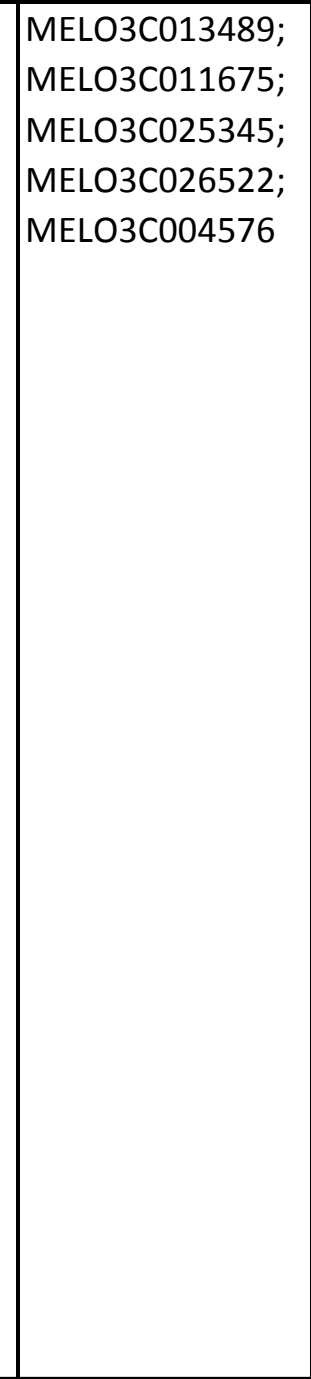 & 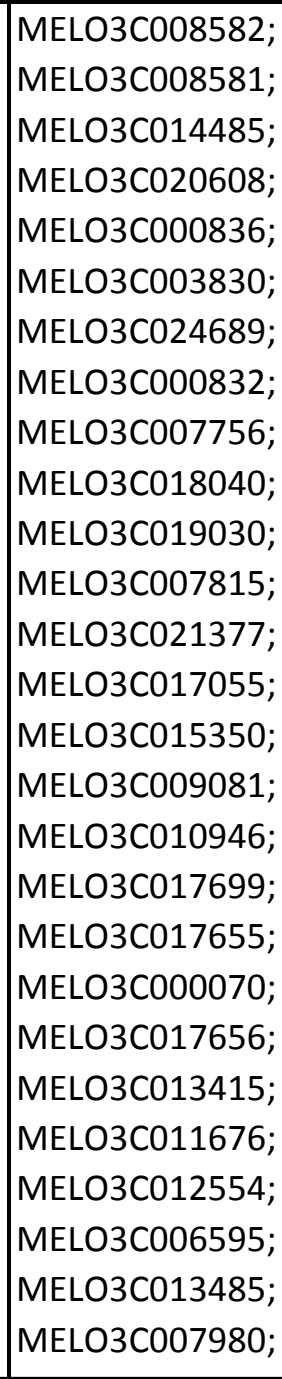 \\
\hline 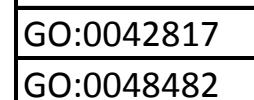 & \begin{tabular}{|l} 
pyridoxal metabolic process \\
plant tovule morphogenesis
\end{tabular} & 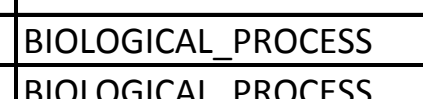 & $\begin{array}{l}0,5832383857 \\
058232357\end{array}$ & $\begin{array}{l}0,036606726 \\
03607526\end{array}$ & $\frac{1}{11}$ & $\frac{0}{0}$ & $\frac{626}{626}$ & $\frac{16501}{16501}$ & 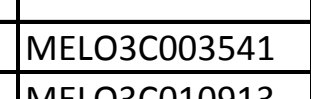 & \\
\hline 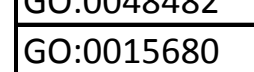 & 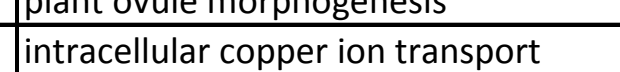 & 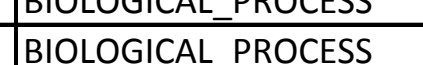 & 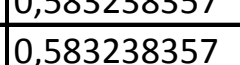 & $\frac{1,050007626}{0.03607726}$ & $\frac{1}{1}$ & $\frac{0}{0}$ & $\frac{1026}{1626}$ & $\frac{10301}{16501}$ & \begin{tabular}{|l|} 
MELLO3C01091313 \\
MELO3C014007
\end{tabular} & 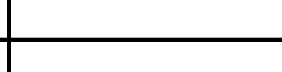 \\
\hline 60:0046087 & cytidine metabolic process & BIOLOGICAL_PROCESS & 0,583238357 & 0,036606726 & & 0 & 626 & 16501 & MELO3C015459 & \\
\hline 60:0006898 & receptor-mediated endocytosis & BIOLOGICAL_PROCESS & 0,583238357 & 0,03304935 & 10 & 125 & 617 & 16376 & 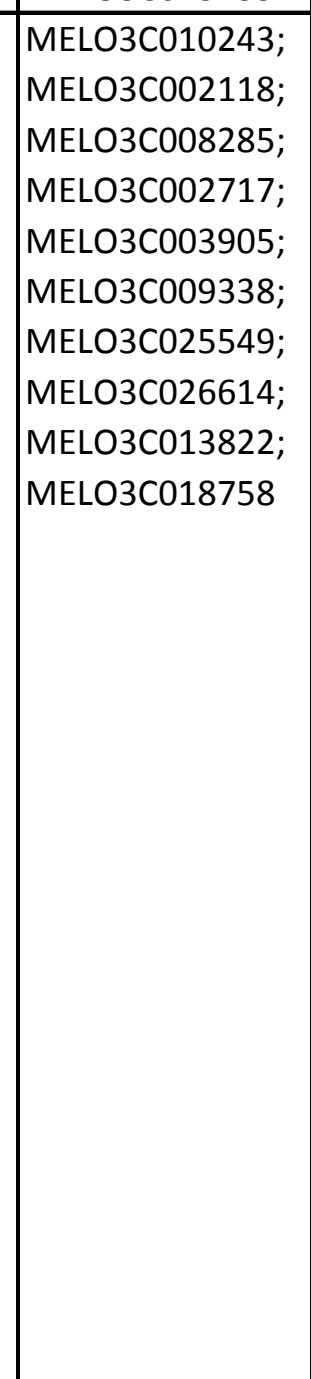 & 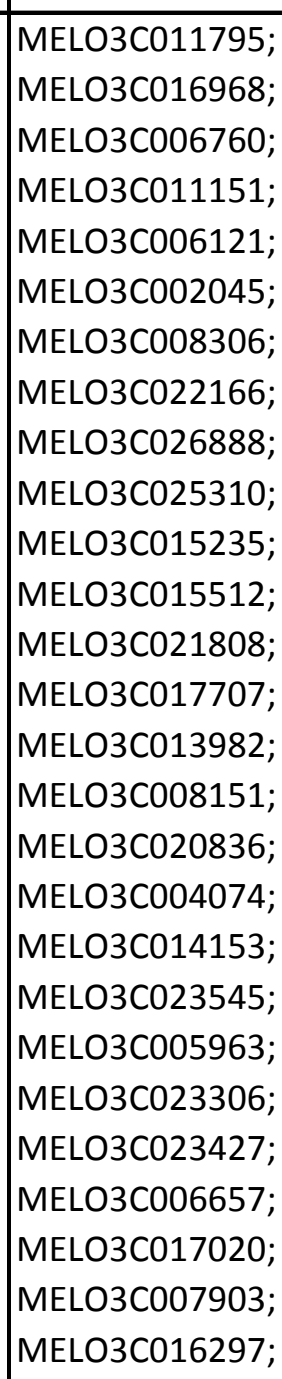 \\
\hline GO:0006616 & $\begin{array}{l}\text { SRP-dependent cotranslational proteir } \\
\text { targeting to membrane, translocation }\end{array}$ & BIOLOGICAL_PROCESS & 0,583238357 & 0,036606726 & & 5 & 626 & 16501 & MELO3C005757 & \\
\hline G0:0030950 & $\begin{array}{l}\text { establishment or maintenance of actin } \\
\text { cytoskeleton polarity }\end{array}$ & BIOLOGICAL_PROCESS & 0,583238357 & 0,036606726 & & 0 & 626 & 16501 & MELO3C026614 & \\
\hline \begin{tabular}{|l}
$60: 0046292$ \\
$G 0: 0010812$ \\
\end{tabular} & $\begin{array}{l}\text { formaldehydde metabolic process } \\
\text { foegative regulation of cell-substrate } \\
\text { achesion }\end{array}$ & $\begin{array}{l}\text { BIOLOGICALPROCESS } \\
\text { BIOLOGICALPROCESS }\end{array}$ & $\begin{array}{l}0,583238357 \\
0,583238357\end{array}$ & $\begin{array}{l}0,036606726 \\
0,036606726\end{array}$ & & $\frac{0}{0}$ & $\frac{626}{626}$ & $\frac{16501}{16501}$ & \begin{tabular}{|l|} 
MELO3C022399 \\
MELO3C022488
\end{tabular} & \\
\hline
\end{tabular}




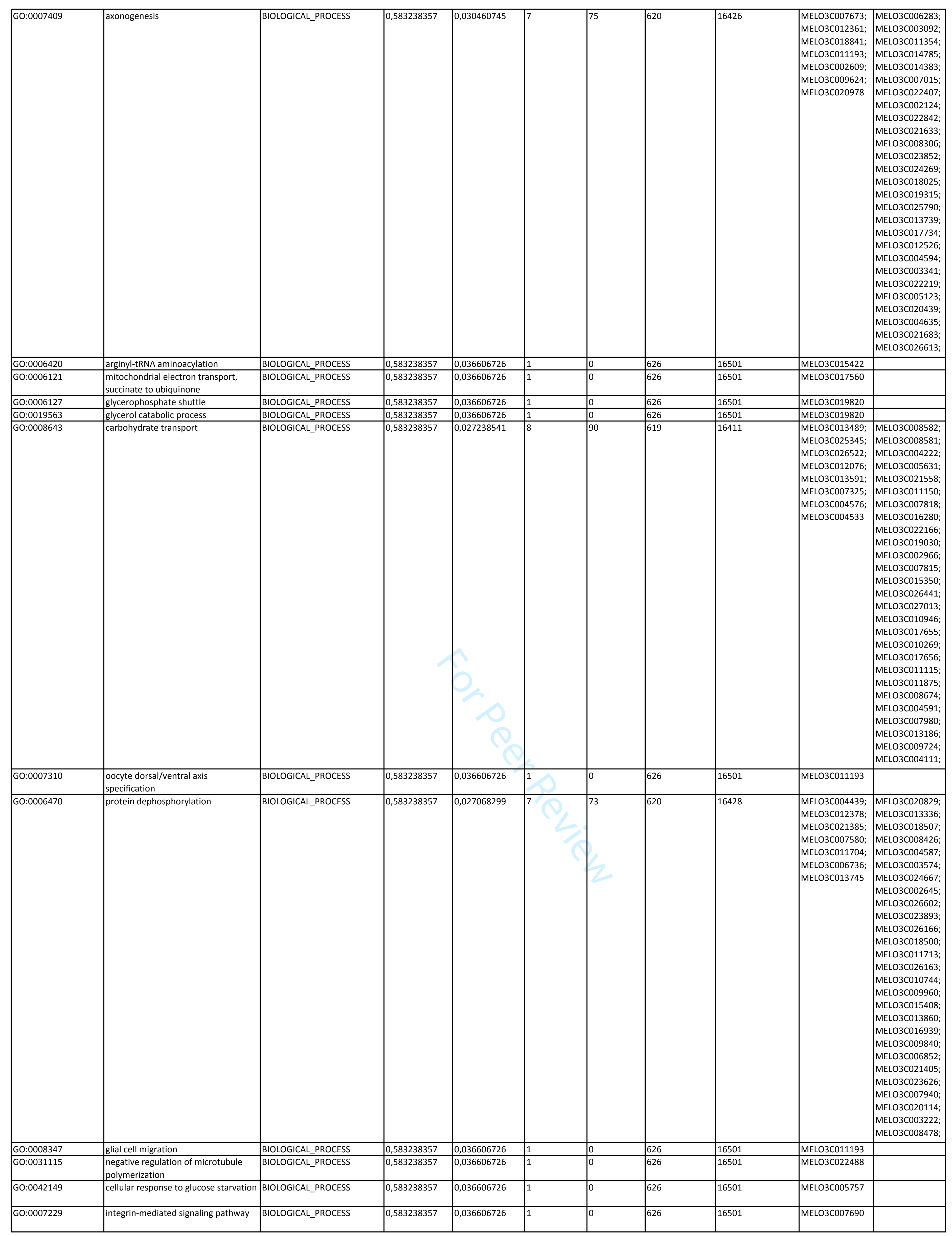




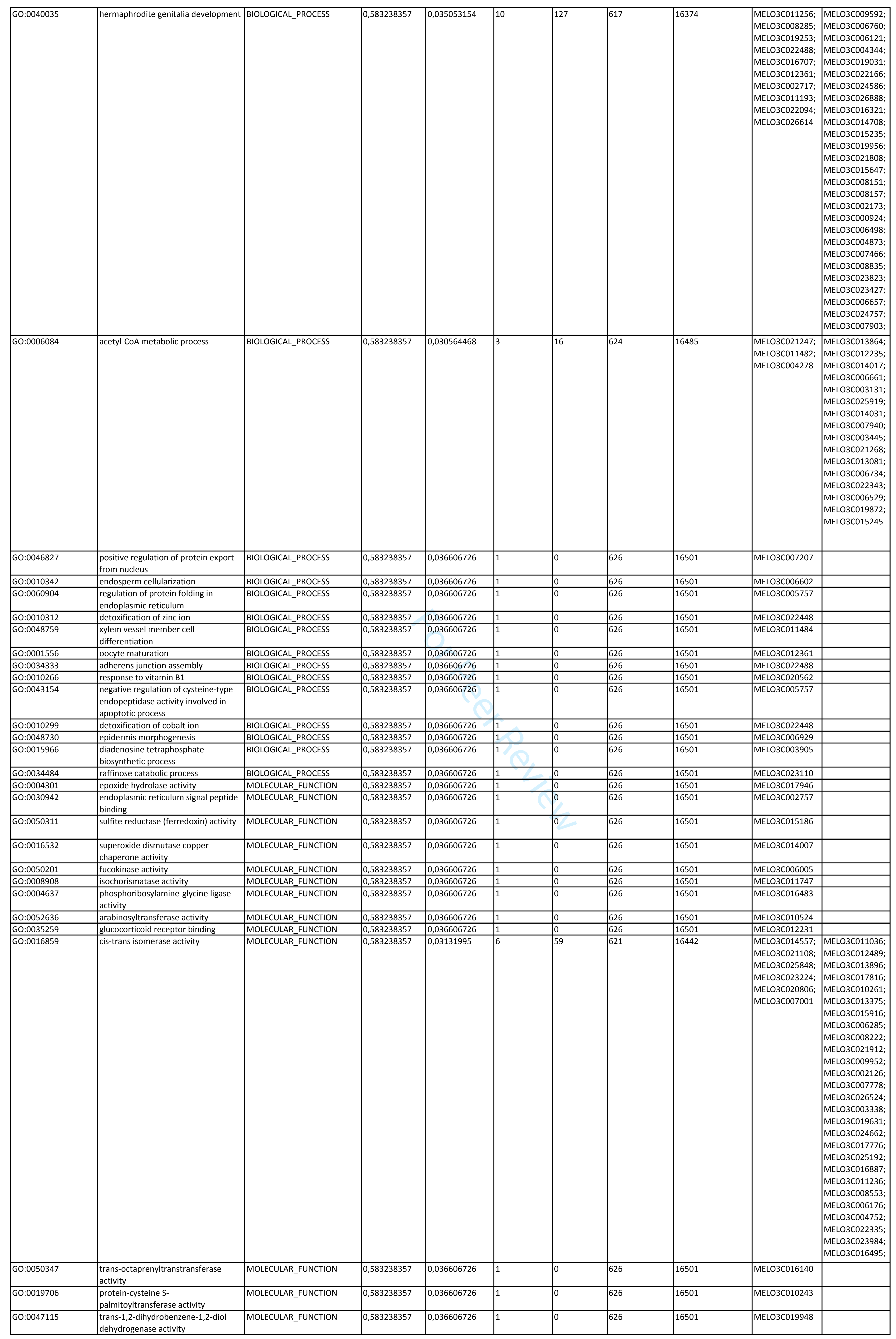




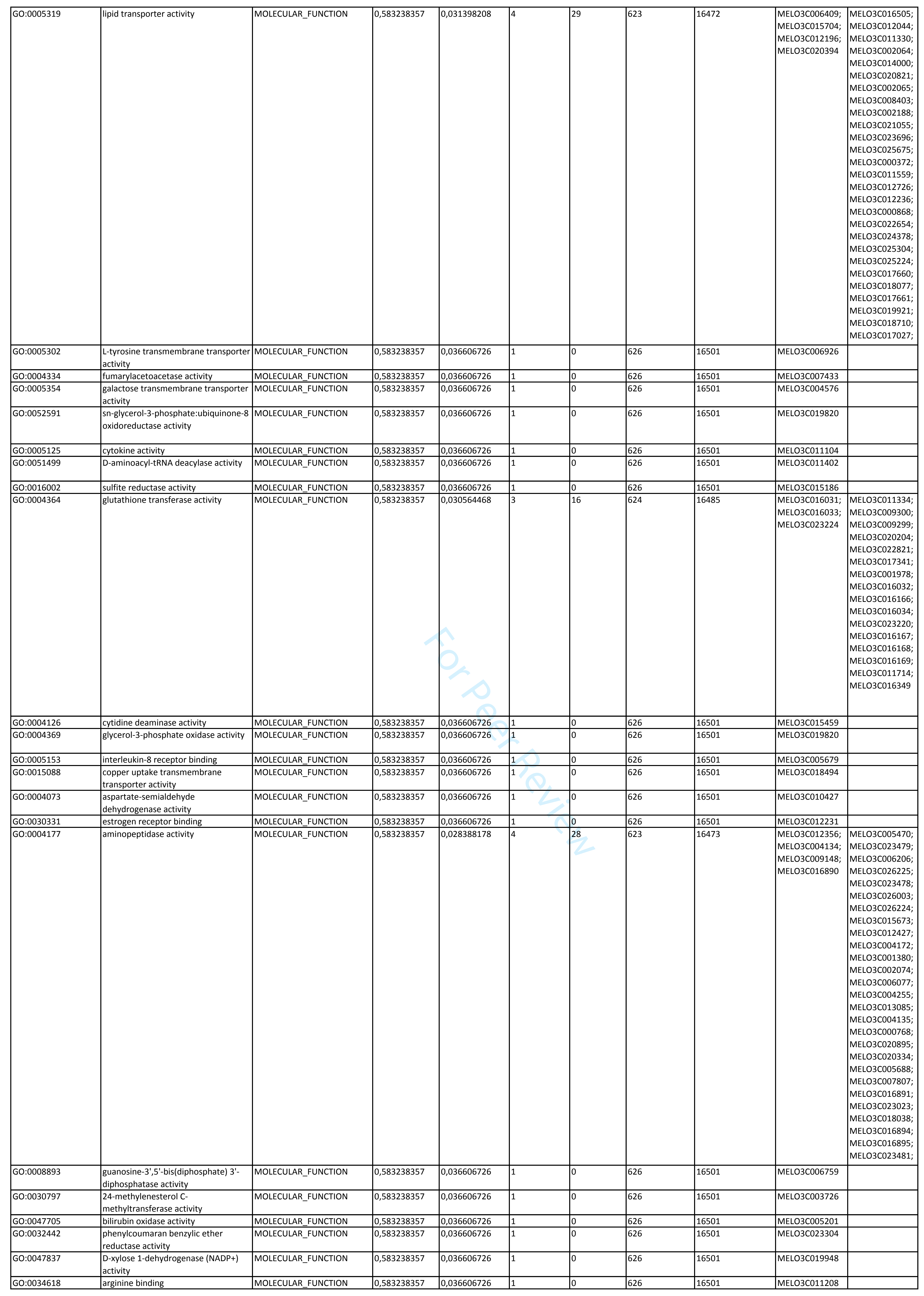




\begin{tabular}{|c|c|c|c|c|c|c|c|c|c|}
\hline $60: 0043565$ & sequence-specific DNA binding & MOLECULAR_FUNCTION & 0,583238357 & 0,036287843 & 324 & 622 & 16177 & 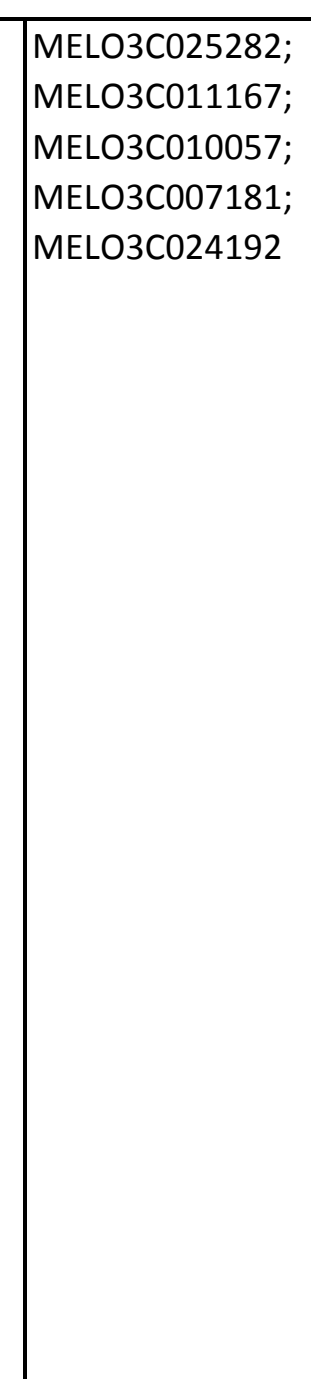 & 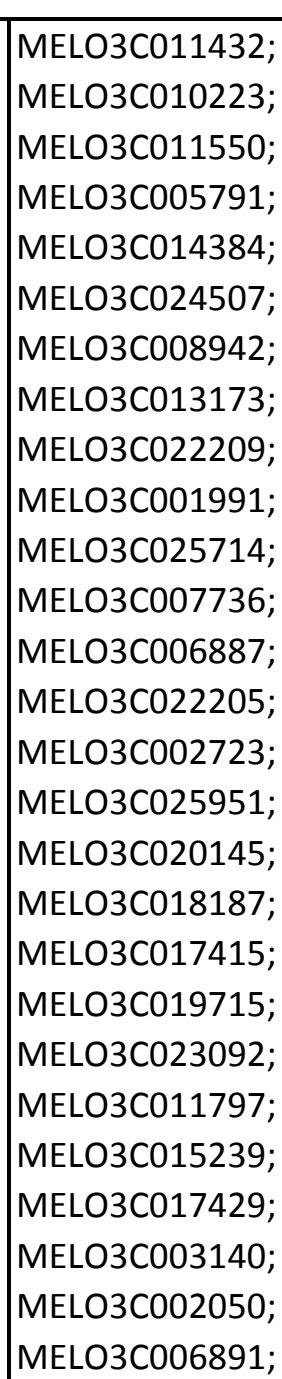 \\
\hline 60:0003841 & $\begin{array}{l}\text { 1-acylgycerol-3-phosphate O- } \\
\text { acyltransferase activity }\end{array}$ & MOLECULAR_FUNCTION & 0,583238357 & 0,032354424 & 6 & 625 & 16495 & $\begin{array}{l}\text { MELO3C009663; } \\
\text { MELO3C008959 }\end{array}$ & 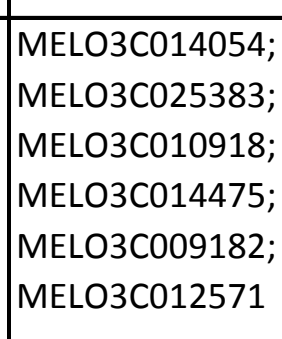 \\
\hline 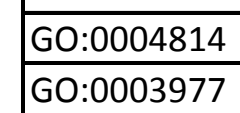 & 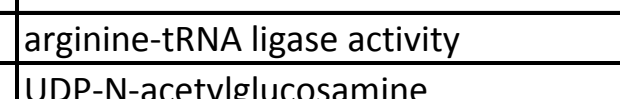 & $\begin{array}{l}\text { MOLECULAR_FUNCTION } \\
\text { MOLICUUAR UENCTION }\end{array}$ & $\begin{array}{l}0,5823238357 \\
0.58323357 \\
\end{array}$ & $\begin{array}{l}0,036606726 \\
0,036606726\end{array}$ & $\frac{0}{10}$ & $\frac{626}{6626}$ & $\frac{16501}{16501}$ & \begin{tabular}{|l|l|} 
MELOSCOC15422 \\
ME103007022
\end{tabular} & \\
\hline & $\begin{array}{l}\text { UDP-N-acetylygucosamine } \\
\text { diphosphorylase activity }\end{array}$ & & & & & & & & \\
\hline 60:0080007 & S-nitrosoglutathione reductase activiti & $\bar{y} \mid$ MOLECULAR_FUNCTION & 0,5882338357 & 0,036606726 & 0 & 626 & 16501 & MELO3C022399 & \\
\hline 60:0010487 & thermospermine synthase activity & MOLECULAR_FUNCTION & 0,583238357 & 0,036606726 & 0 & 626 & 16501 & MELO3C011484 & \\
\hline$\overline{60.0034020}$ & nooxanthin synthase activity & MOLECULAR FUNCTION & 0.583238357 & 0.036006726 & 0 & 1626 & 16501 & ME0З3С017963 & $x_{-1}$ \\
\hline 60:0000026 & \begin{tabular}{|l} 
alphha-1,2-mannosyltransferase \\
activity
\end{tabular} & MOLECULAR_FUNCTION & 0,583238357 & 0,036606726 & 0 & 626 & 16501 & MELO3C025637 & \\
\hline 60:0016830 & carbon-carbon Iyase activity & MOLECULAR_FUNCTION & 0,583238357 & 0,035904578 & 110 & 627 & 16391 & & 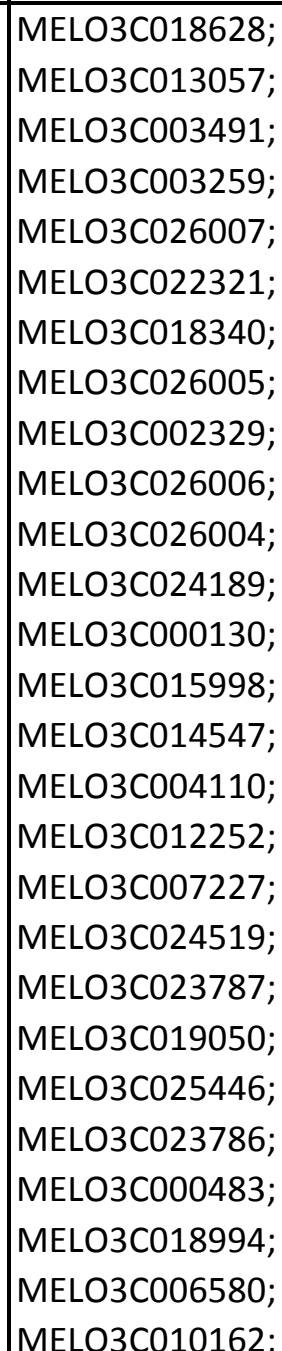 \\
\hline \begin{tabular}{|l|}
$60: 0010297$ \\
$60: 0005875$ \\
\end{tabular} & \begin{tabular}{|l} 
heteropolysaccharide binding \\
microtubule associated complex
\end{tabular} & \begin{tabular}{|l} 
MOLECULAR_EUNCTION \\
CEELULAR_COMPONENT
\end{tabular} & $\begin{array}{l}0,583238357 \\
0,583238357\end{array}$ & $\begin{array}{l}0,036606726 \\
0,033428346\end{array}$ & $\frac{0}{60}$ & $\frac{626}{621}$ & $\frac{16501}{16441}$ & 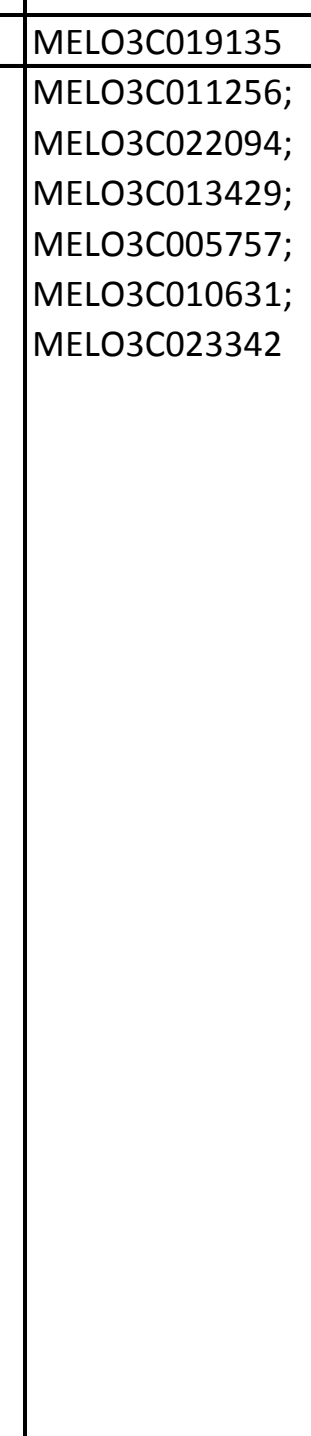 & 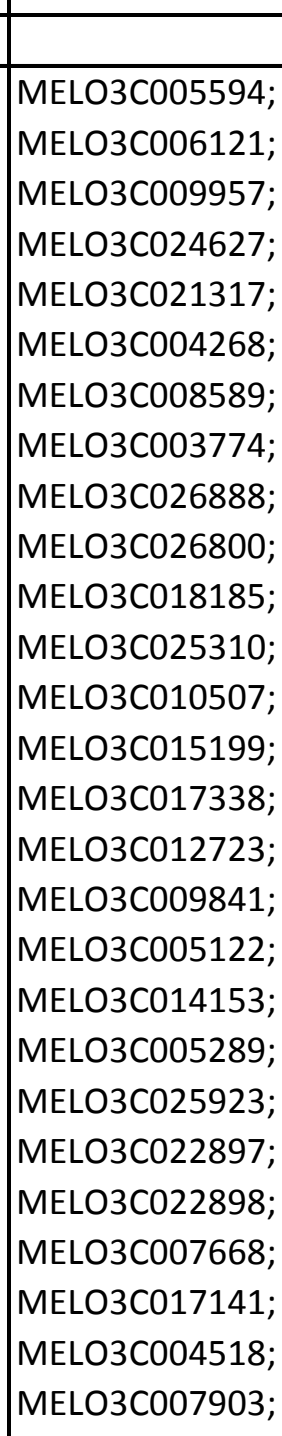 \\
\hline 60:0034099 & |uminal surveillance complex & CELLULAR_S & 0,583238357 & 0,036606726 & & 626 & 16501 & MELO3C005757 & \\
\hline 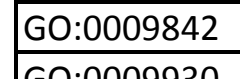 & 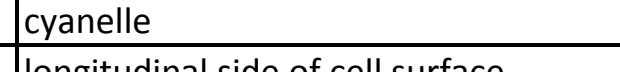 & \begin{tabular}{|l} 
CELLULAR_COMPONENT \\
CFUD
\end{tabular} & $0,0,583238357$ & 0,036606726 & & $\frac{626}{626}$ & $\frac{16501}{16501}$ & MELO3C016140 & \\
\hline$\frac{60: 0009930}{60: 0042555}$ & $\begin{array}{l}\text { longitudinal side of cell surface } \\
\text { MCM complex }\end{array}$ & \begin{tabular}{|l} 
CELLUUAR_COMPNENT \\
CELLLLARCOMPONENT
\end{tabular} & $00 \frac{0,5832323357}{0,58328357}$ & $\frac{0,036600726}{0,036606726}$ & & $\frac{626}{626}$ & $\frac{16501}{16501}$ & \begin{tabular}{|l|l|} 
MELOOCOCO1/242 \\
MELOOCO20001
\end{tabular} & \\
\hline 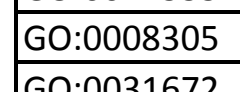 & $\begin{array}{l}\text { integrin complex } \\
\text { Ashin d }\end{array}$ & $\begin{array}{l}\text { CELLULAR_COMPONENT } \\
\text { CLIUA_ }\end{array}$ & $\begin{array}{l}0,583238357 \\
158232357\end{array}$ & 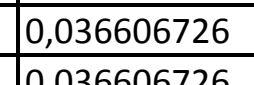 & & $\frac{626}{66}$ & $\frac{16501}{16501}$ & MELOЗС007690 & \\
\hline
\end{tabular}




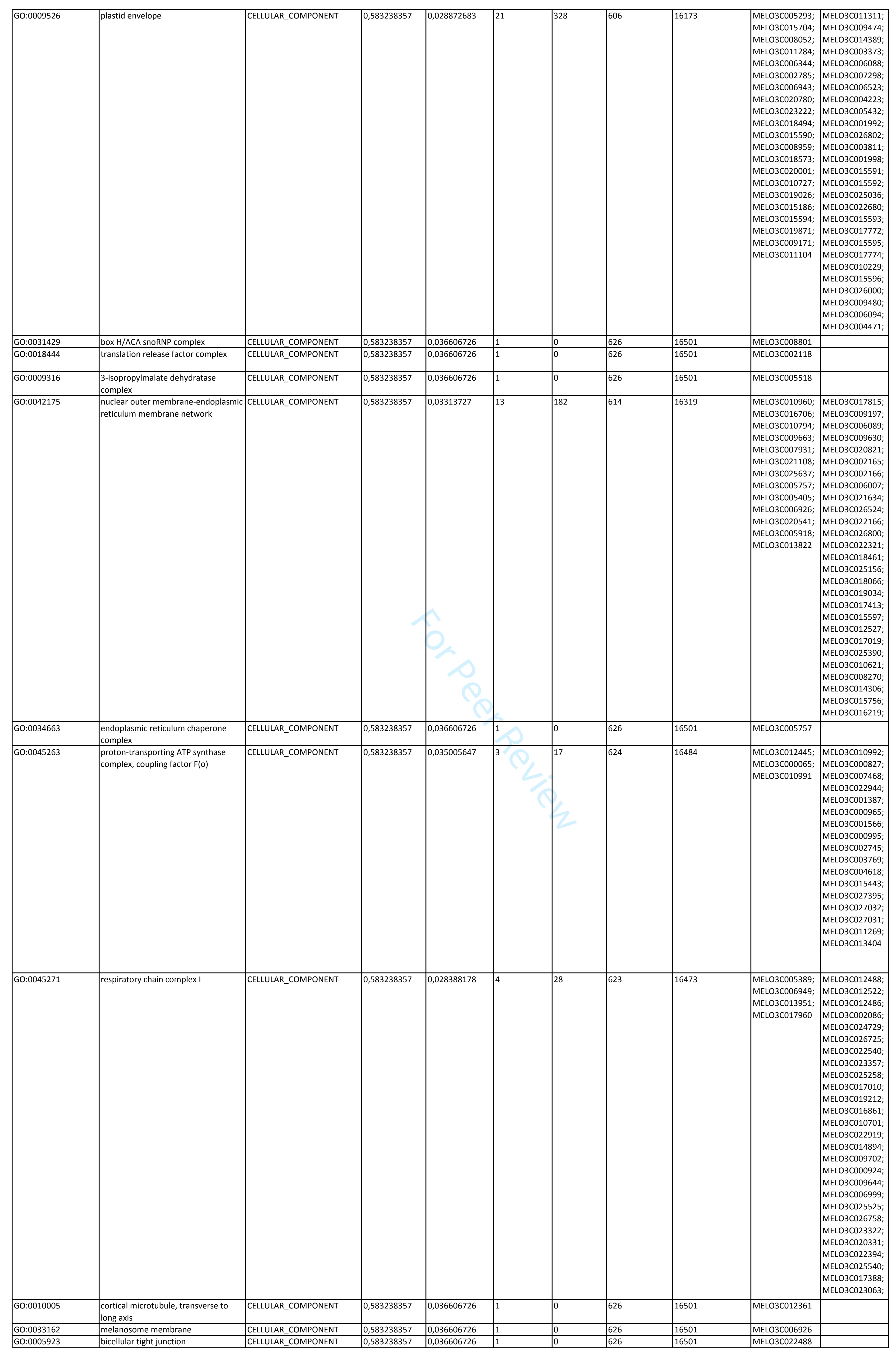




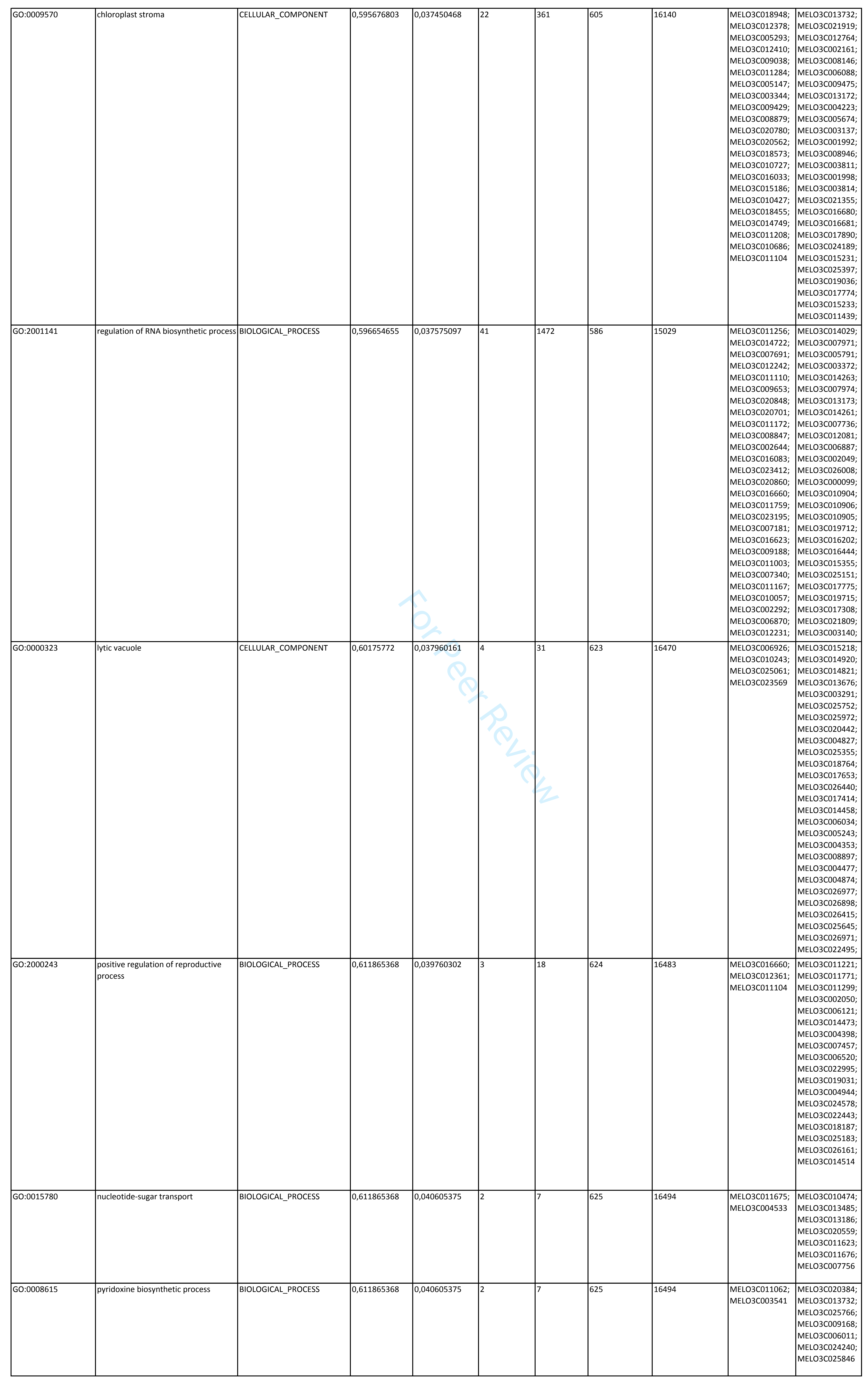




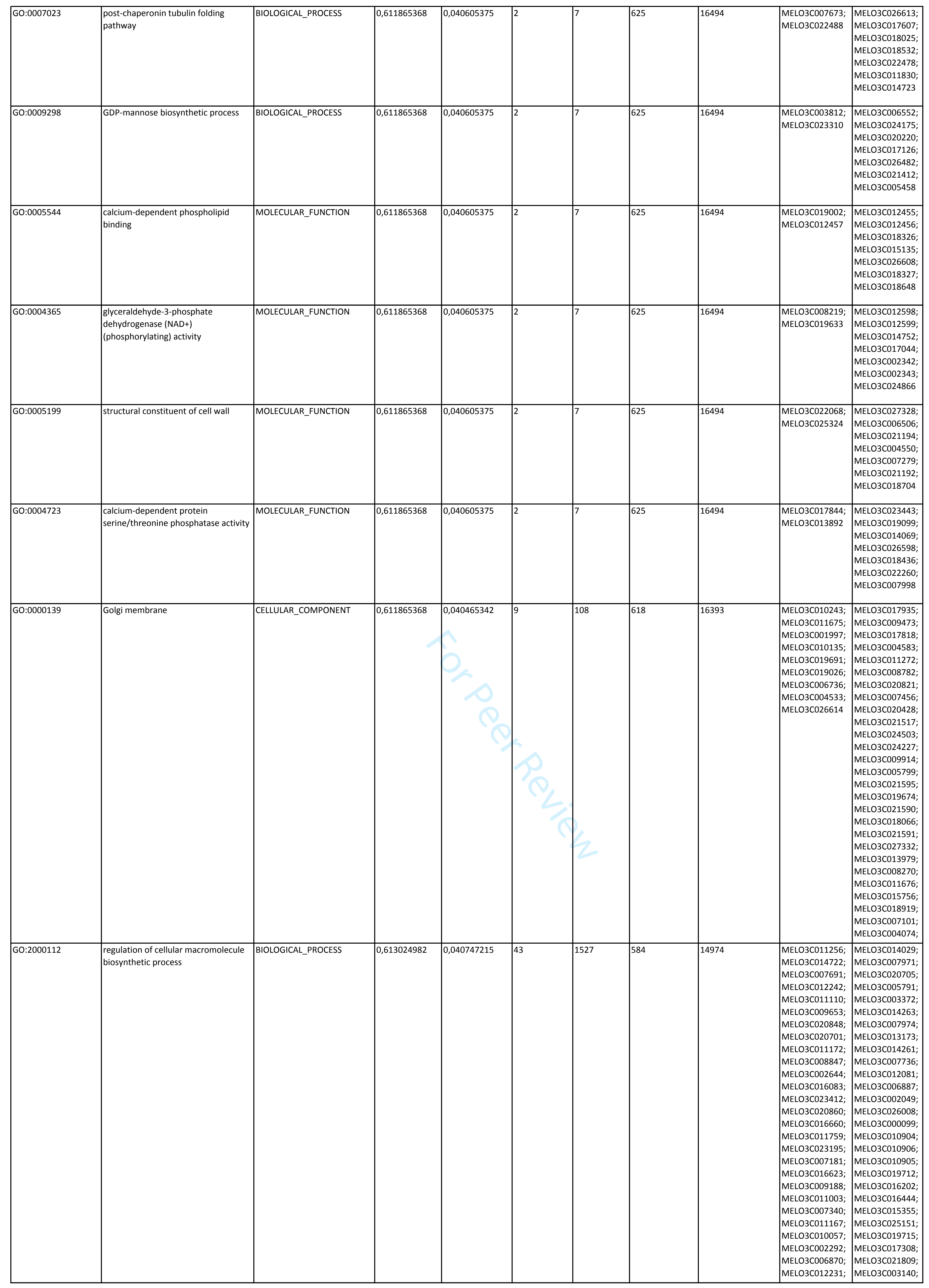




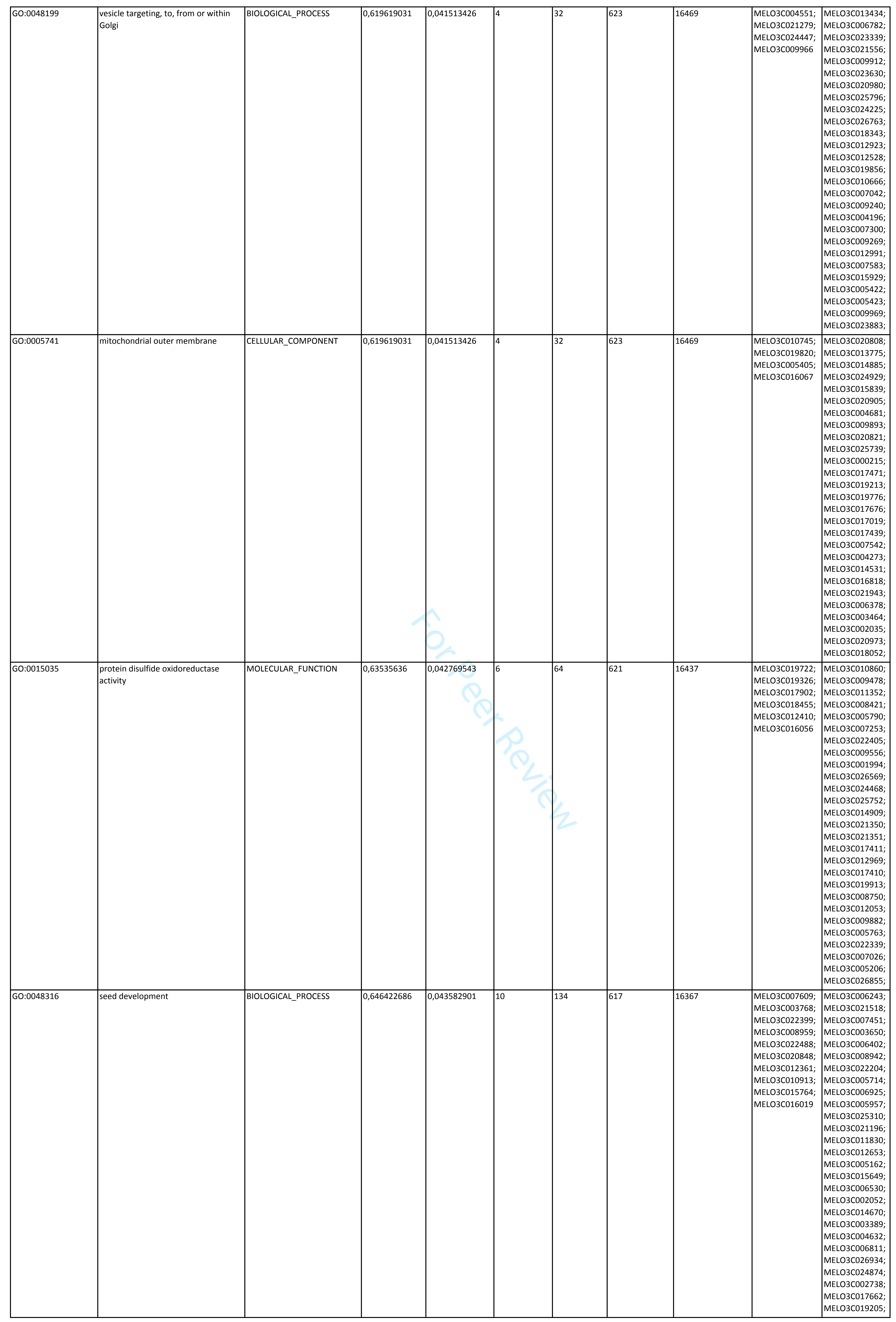




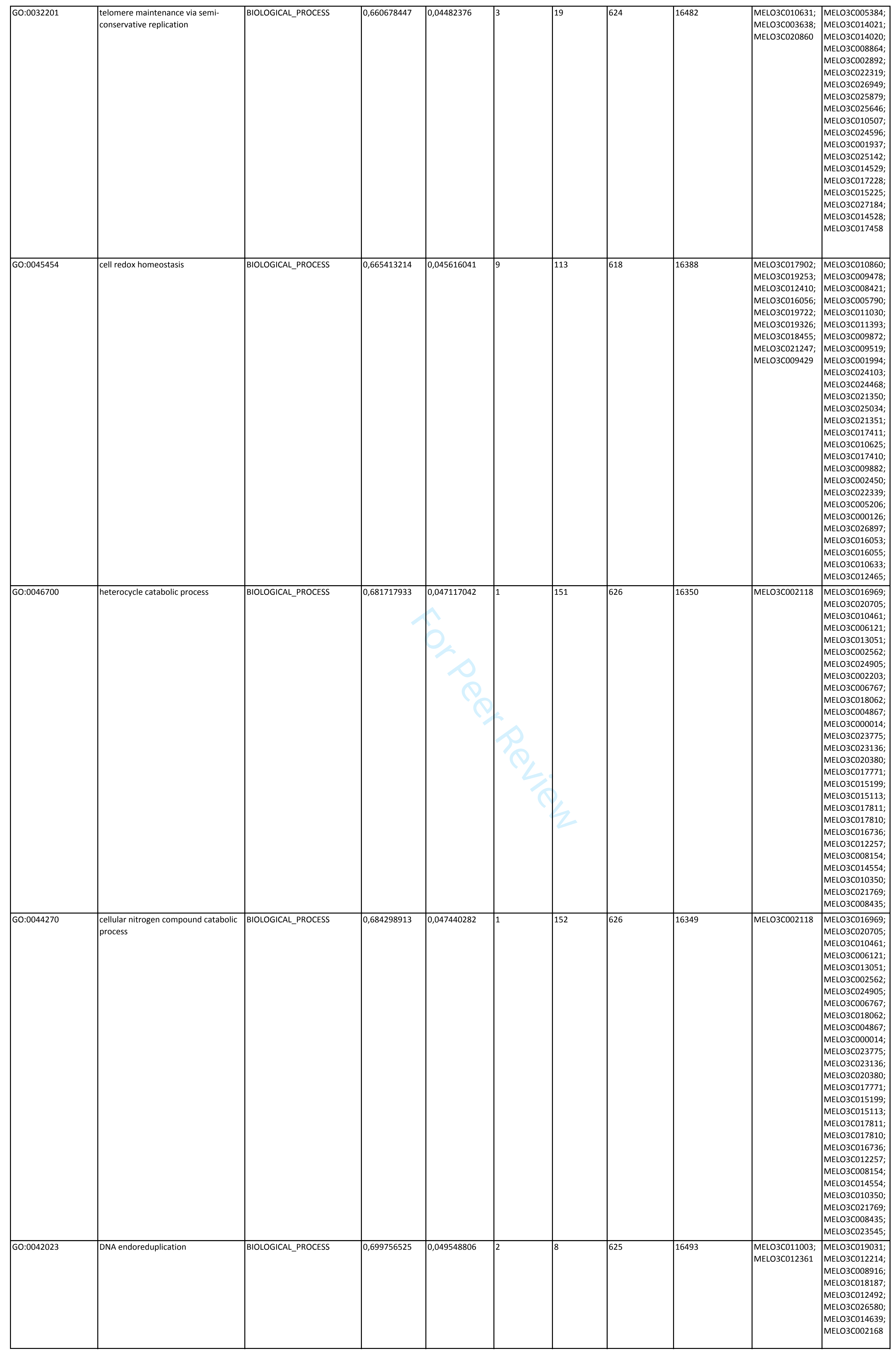




\begin{tabular}{|c|c|c|c|c|c|c|c|c|c|c|}
\hline 60:0009695 & jasmonic acid biosynthetic process & BIOLOGICAL_PROCESS & 0,699756525 & 0,049548806 & 2 & 8 & 625 & 16493 & $\begin{array}{l}\text { MELO3C002470; } \\
\text { MELO3C015514 }\end{array}$ & $\begin{array}{l}\text { MELOOC004955; } \\
\text { MELO3C00936; } \\
\text { MELO3C01238; } \\
\text { MELO3CO10910; } \\
\text { MELO3CO04565; } \\
\text { MELO3CO08062; } \\
\text { MELO3C018734; } \\
\text { MELO3CO02346 }\end{array}$ \\
\hline \begin{tabular}{|l|} 
GO:0007043 \\
\end{tabular} & cell-cell junction assembly & BIOLOGICAL_PROCESS & 0,699756525 & 0,049548806 & 2 & 8 & 625 & 16493 & $\begin{array}{l}\text { MELO3C007673; } \\
\text { MELO3C022488 }\end{array}$ & 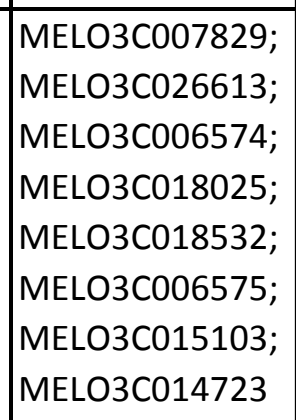 \\
\hline \begin{tabular}{|l|}
$G O: 0000578$ \\
\end{tabular} & embryonic axis specification & BIOLOGICAL_PROCESS & 0,699756525 & 0,049548806 & 2 & 8 & 625 & 16493 & $\begin{array}{l}\text { MELO3C003768; } \\
\text { MELO3C011193 }\end{array}$ & $\begin{array}{l}\text { MELOOCO25389; } \\
\text { MELO3C024028; } \\
\text { MELO3CO05946; } \\
\text { MELO3CO24874; } \\
\text { MELO3CO05099; } \\
\text { MELO3CO07036; } \\
\text { MELO3C024782; } \\
\text { MELO3C019956 }\end{array}$ \\
\hline \begin{tabular}{|l|} 
GO:0046474 \\
\end{tabular} & \begin{tabular}{|l|} 
Ilycerophospholipid biosynthetic \\
process
\end{tabular} & BIOLOGICAL_PROCESS & 0,699756525 & 0,049164821 & 4 & 34 & 623 & 16467 & $\begin{array}{l}\text { MELO3C007931; } \\
\text { MELO3CO08959; } \\
\text { MELO3C025637; } \\
\text { MELO3C012545 }\end{array}$ & 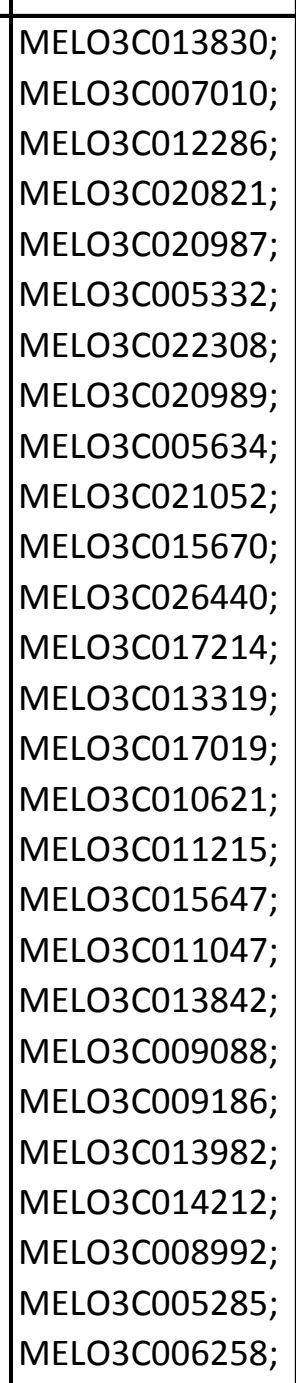 \\
\hline \begin{tabular}{|l|} 
GO:0016161 \\
\end{tabular} & beta-amylase activity & MOLECULAR_FUNCTION & 0,699756525 & 0,049548806 & 2 & 8 & 625 & 16493 & $\begin{array}{l}\text { MELO3C006362; } \\
\text { MELO3C016213 }\end{array}$ & $\begin{array}{l}\text { MELO3C014105; } \\
\text { MELO3CO22740; } \\
\text { MELO3CO22739; } \\
\text { MELO3CO23067; } \\
\text { MELO3CO18794; } \\
\text { MELOOCO221362; } \\
\text { MELO3C021214; } \\
\text { MELO3C013887 }\end{array}$ \\
\hline
\end{tabular}


Supplementary Table $2 . \mathrm{CG}$ Gene Ontology terms $\left(G \mathrm{O}\right.$ terms) of the cluster 2.2 obtained considering the comparison between lines the postharvest ripening time fruit during storage at $20.5^{\circ} \mathrm{C}$ and $88 \%$ relative humidity for $18 \mathrm{~d}$ the near-isogenic line SC10-2 and its parental "Piel de Sapo"

\begin{tabular}{|c|c|c|c|c|c|c|c|c|c|c|}
\hline GO ID & GO Name & GO Category & FDR & P-Value & SC10-2 & PS & \begin{tabular}{|l|l|} 
Non Annot Test \\
\end{tabular} & $\begin{array}{l}\text { Non Annot } \\
\text { Reference }\end{array}$ & \begin{tabular}{|l}
$\begin{array}{l}\text { TestSet } \\
\text { Sequences }\end{array}$ \\
\end{tabular} & \begin{tabular}{|l}
$\begin{array}{l}\text { RefSet } \\
\text { Sequences }\end{array}$ \\
\end{tabular} \\
\hline 60:0004370 & glycerol kinase activity & MOLECULAR_FUNCTION & 0,459917717 & $4,87 E-05$ & 2 & 0 & 118 & 17008 & \begin{tabular}{|l|} 
MELO3C023442; \\
MELO3CO04084
\end{tabular} & \\
\hline 60:0043069 & negative regulation of programmed cell death & BIOLOGICAL_PROCESS & 1 & 0,030471862 & 2 & 37 & 118 & 16971 & $\begin{array}{l}\text { MELOSCO03331; } \\
\text { MELO3C017623 } \\
\end{array}$ & 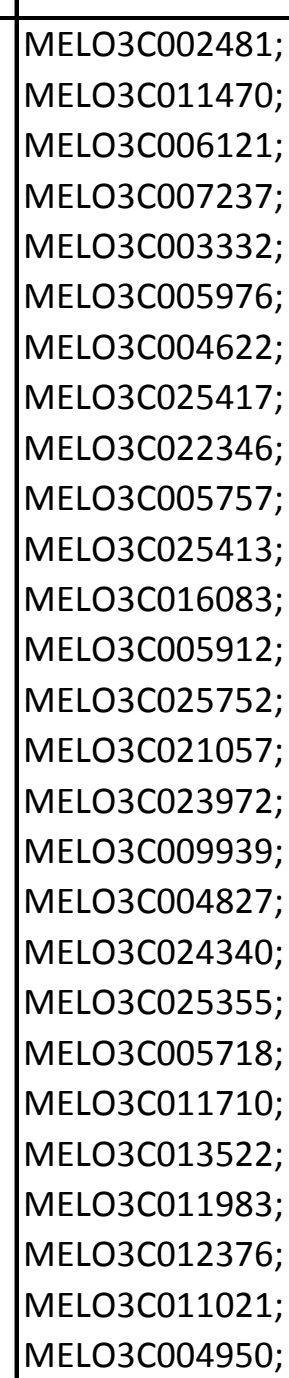 \\
\hline 60:0043622 & cortical microtubule organization & BIOLOGICAL_PROCESS & 1 & 0,034546914 & 1 & 4 & 119 & 17004 & MELO3C002054 & $\begin{array}{l}\text { MELO3C007829; } \\
\text { MELO3CO14496; } \\
\text { MELO3CO02717; } \\
\text { MELO3C023374 }\end{array}$ \\
\hline 60:0006954 & inflammatory response & BIOLOGICAL_PROCESS & 1 & 0,041312923 & 1 & 5 & 119 & 17003 & MELO3C026027 & $\begin{array}{l}\text { MELO3C026028; } \\
\text { MELO3C015103; } \\
\text { MELO3C017426; } \\
\text { MELO3C018756; } \\
\text { MELO3C022821 }\end{array}$ \\
\hline 60:0042780 & tRNA 3'-end processing & BIOLOGICAL_PROCESS & 1 & 0,027733554 & 1 & 3 & 119 & 17005 & MELO3C014447 & $\begin{array}{l}\text { MELO3CO202738; } \\
\text { MELO3CO10582; } \\
\text { MELO3C024265 }\end{array}$ \\
\hline G0:0010017 & |red or far-red light signaling pathway & ( & 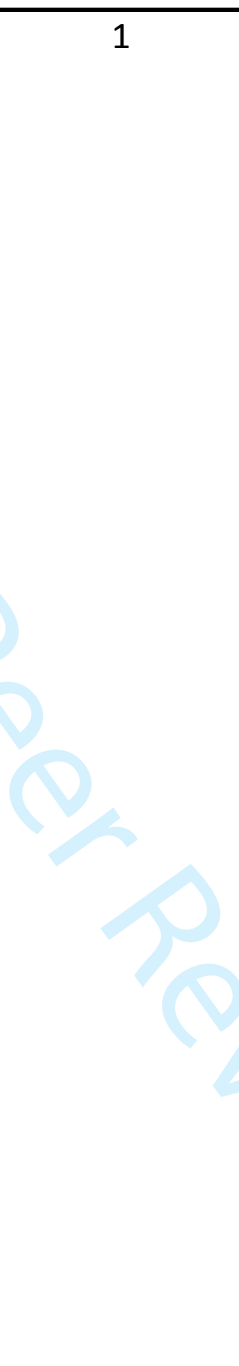 & $0^{\circ}$ & 2 & 34 & 118 & 16974 & $\begin{array}{l}\text { MELO3C026502; } \\
\text { MELO3C014459 }\end{array}$ & 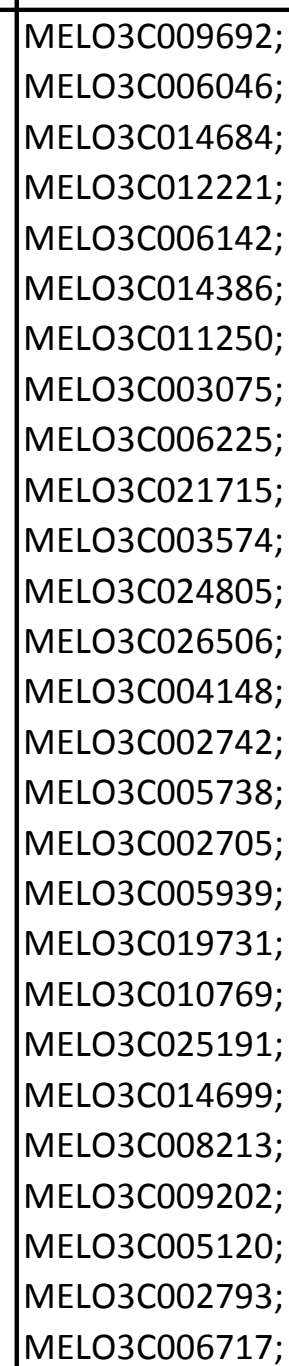 \\
\hline G0:0010497 & plasmodesmata-mediated intercellular transport & BIOLOGICAL_PROCESS & 1 & 0,041312923 & 1 & 5 & 119 & 17003 & MELO3C002054 & $\begin{array}{l}\text { MELO3C018455; } \\
\text { MELO3COO2933; } \\
\text { MELO3C014025; } \\
\text { MELO3C010974; } \\
\text { MELO3C012843 }\end{array}$ \\
\hline 60:0010375 & stomatal complex patterning & BIOLOGICAL_PROCESS & 1 & 0,020872515 & 1 & 2 & 119 & 17006 & MELO3C002054 & $\begin{array}{l}\text { MELO3C021111; } \\
\text { MELO3C020718 }\end{array}$ \\
\hline 60:0070838 & divalent metal ion transport & BIOLOGICAL_PROCESS & 1 & 0,019826524 & 2 & 29 & 118 & 16979 & $\begin{array}{l}\text { MELO3C016817; } \\
\text { MELOЗC014607 }\end{array}$ & 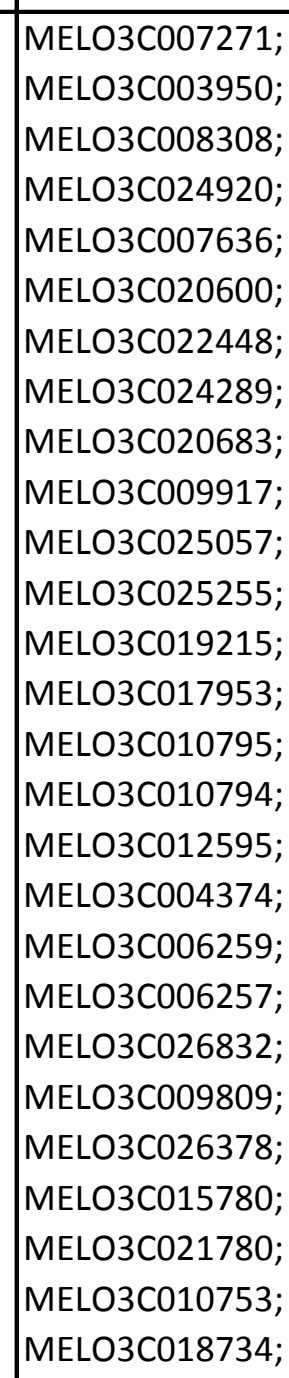 \\
\hline 60:0009959 & negative gravitropism & BIOLOGICAL_PROCESS & 1 & 0,027733554 & 1 & 3 & 119 & 17005 & MELO3C008088 & $\begin{array}{l}\text { MELO3C025310; } \\
\text { MELO3CO088847; } \\
\text { MELO3C024155 }\end{array}$ \\
\hline 60:0006659 & phosphatidylserine biosynthetic process & BIOLOGICAL_PROCESS & 1 & 0,013963465 & 1 & 1 & 119 & 17007 & MELO3C005332 & MELO3C012286 \\
\hline
\end{tabular}




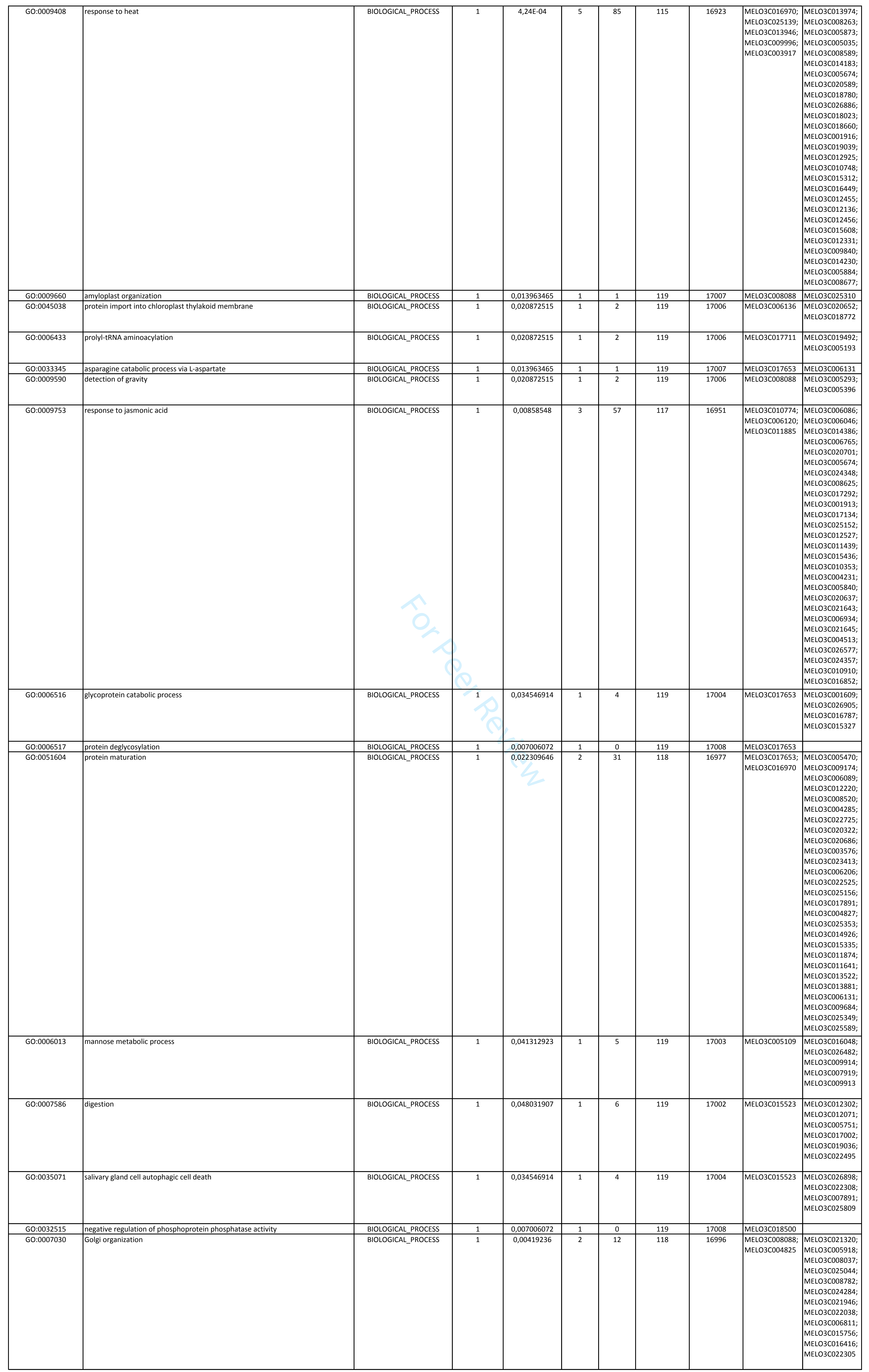




\begin{tabular}{|c|c|c|c|c|c|c|c|c|c|c|}
\hline 60:0017006 & protein-tetrapyrrole linkage & BIOLOGICAL_PROCESS & 1 & 0,048031907 & 1 & 6 & 119 & 17002 & MELO3C026502 & $\begin{array}{l}\text { MELO3C006717; } \\
\text { MELO3C024196; } \\
\text { MELO3C024195; } \\
\text { MELO3C026506; } \\
\text { MELO3CO02705; } \\
\text { MELO3C015204 }\end{array}$ \\
\hline 60:0051865 & protein autoubiquitination & BIOLOGICAL_PROCESS & 1 & 0,041312923 & 1 & 5 & 119 & 17003 & MELO3C025853 & $\begin{array}{l}\text { MELO3C011752; } \\
\text { MELO3C022725; } \\
\text { MELO3C010168; } \\
\text { MELO3C011153; } \\
\text { MELO3C023546 }\end{array}$ \\
\hline 60:0006072 & glycerol-3-phosphate metabolic process & BIOLOGICAL_PROCESS & 1 & 0,00132592 & 2 & 6 & 118 & 17002 & $\begin{array}{l}\text { MELO3C023442; } \\
\text { MELO3COO4084 }\end{array}$ & $\begin{array}{l}\text { MELO3C026337; } \\
\text { MELO3C021604; } \\
\text { MELO3C020158; } \\
\text { MELO3C019820; } \\
\text { MELO3C020157; } \\
\text { MELO3CO05116 }\end{array}$ \\
\hline 60:0031648 & protein destabilization & BIOLOGICAL_PROCESS & 1 & 0,007006072 & 1 & 0 & 119 & 17008 & MELO3C015523 & \\
\hline 60:0048510 & regulation of timing of transition from vegetative to reproductive phase & BIOLOGICAL_PROCESS & 1 & 0,034546914 & 1 & 4 & 119 & 17004 & MELO3C022388 & $\begin{array}{l}\text { MELO3C027290; } \\
\text { MELOSCO1930; } \\
\text { MELO3C013793; } \\
\text { MELO3C009465 }\end{array}$ \\
\hline 60:0002949 & tRNA threonylcarbamoyladenosine modification & BIOLOGICAL_PROCESS & 1 & 0,007006072 & 1 & 0 & 119 & 17008 & MELO3C006202 & \\
\hline 60:0016404 & 15-hydroxyprostaglandin dehydrogenase (NAD+) activity & MOLECULAR_FUNCTION & 1 & 0,007006072 & 1 & 0 & 119 & 17008 & MELO3C019503 & \\
\hline 60:0004144 & diacylgIycerol O-acyltransferase activity & MOLECULAR_FUNCTION & 1 & 0,020872515 & 1 & 2 & 119 & 17006 & MELO3C013703 & $\begin{array}{l}\text { MELO3C004632; } \\
\text { MELO3C018460 }\end{array}$ \\
\hline 60:0003846 & 2-acy|glycerol O-acyltransferase activity & MOLECULAR_FUNCTION & 1 & 0,007006072 & 1 & 0 & 119 & 17008 & MELO3C013703 & \\
\hline GO:0042781 & 3'-tRNA processing endoribonuclease activity & MOLECULAR_FUNCTION & 1 & 0,020872515 & 1 & 2 & 119 & 17006 & MELO3C014447 & $\begin{array}{l}\text { MELO3C020738; } \\
\text { MELO3C024265 }\end{array}$ \\
\hline 60:0046872 & metal ion binding & ( & 1 & 0,031268561 & 28 & 2666 & 92 & 14342 & 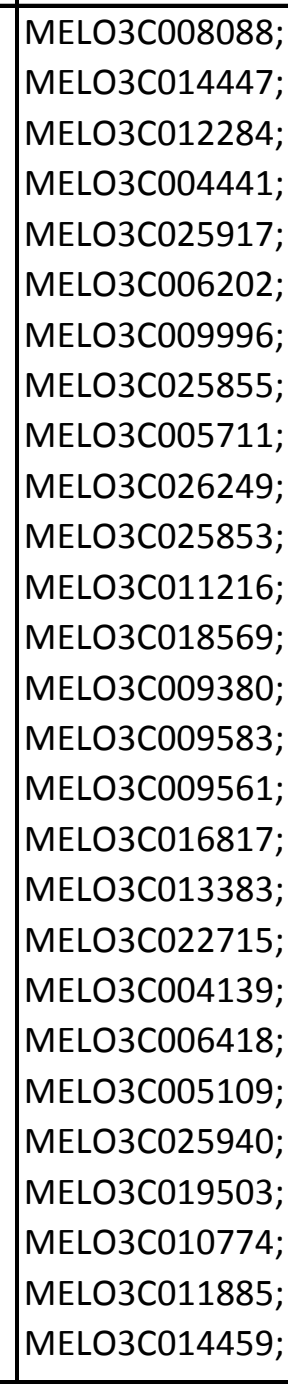 & 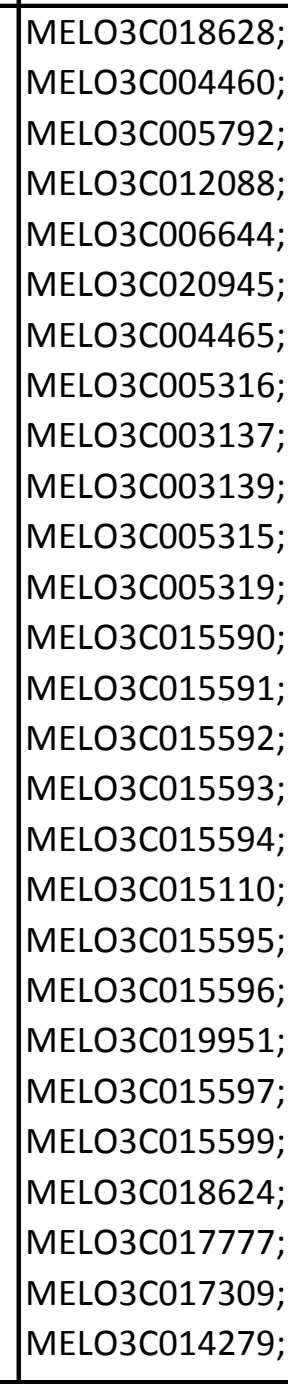 \\
\hline 60:0004827 & proline-tRNA ligase activity & MOLECULAR_FUNCTION & 1 & 0,020872515 & 1 & 2 & 119 & 17006 & MELO3C017711 & $\begin{array}{l}\text { MELO3CO19492; } \\
\text { MELO3C005193 }\end{array}$ \\
\hline 60:0045547 & dehydrodolichyl diphosphate synthase activity & MOLECULAR_FUNCTION & 1 & 0,020872515 & 1 & 2 & 119 & 17006 & MELO3C025855 & $\begin{array}{l}\text { MELO3CO21891; } \\
\text { MELO3C018615 }\end{array}$ \\
\hline G0:0050178 & phenylpyruvate tautomerase activity & MOLECULAR_FUNCTION & 1 & 0,007006072 & 1 & 0 & 119 & 17008 & MELO3C026027 & \\
\hline 60:0003948 & N4-(beta-N-acetylglucosaminyl)-L-asparaginase activity & MOLECULAR_FUNCTION & 1 & 0,007006072 & 1 & 0 & 119 & 17008 & MELO3C017653 & \\
\hline 60:0050221 & prostaglandin-E2 9-reductase activity & MOLECULAR_FUNCTION & 1 & 0,020872515 & 1 & 2 & 119 & 17006 & MELO3C026155 & $\begin{array}{l}\text { MELO3C015622; } \\
\text { MELO3C011853 }\end{array}$ \\
\hline $60: 0033743$ & peptide-methionine (R)-S-oxide reductase activity & MOLECULAR_FUNCTION & 1 & 0,013963465 & 1 & 1 & 119 & 17007 & MELO3C018569 & MELO3C016676 \\
\hline 60:0008020 & \begin{tabular}{|l} 
G-protein coupled photoreceptor activity \\
\end{tabular} & MOLECULAR_FUNCTION & 1 & 0,041312923 & 1 & 5 & 119 & 17003 & MELO3C026502 & $\begin{array}{l}\text { MELO3C006717; } \\
\text { MELO3C024196; } \\
\text { MELO3C024195; } \\
\text { MELO3C026506; } \\
\text { MELO3C002705 }\end{array}$ \\
\hline GO:0004197 & cysteine-type endopeptidase activity & MOLECULAR_FUNCTION & 1 & 0,017468633 & 2 & 27 & 118 & 16981 & $\begin{array}{l}\text { MELO3C015523; } \\
\text { MELO3C017623 }\end{array}$ & 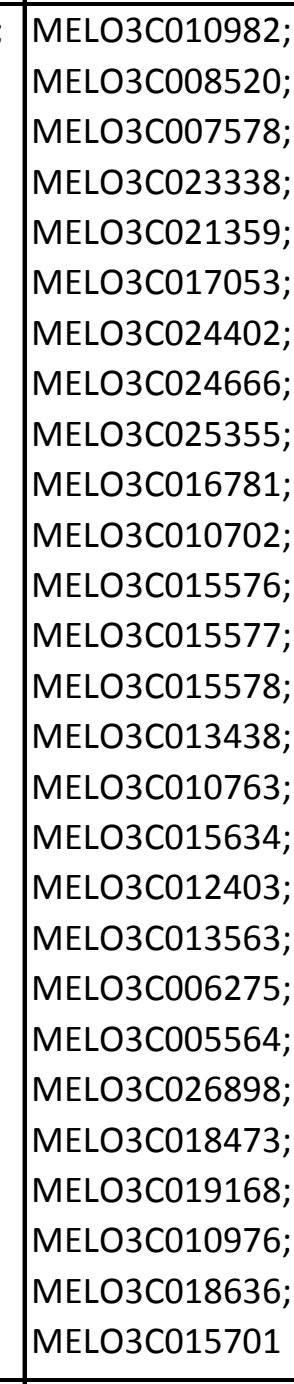 \\
\hline 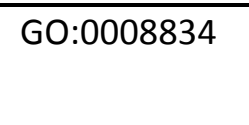 & di-trans, poly-cis-decaprenylcistransferase activity & MOLECULAR_FUNCTION & 1 & 0,020872515 & 1 & 2 & 119 & 17006 & MELO3C025855 & $\begin{array}{l}\text { MELO3C021891; } \\
\text { MELO3C018615 }\end{array}$ \\
\hline $\begin{array}{lll}\text { GO:0008798 } \\
\end{array}$ & beta-aspartyl-peptidase activity & MOLECULAR_FUNCTION & 1 & 0,034546914 & 1 & 4 & 119 & 17004 & MELO3C017653 & $\begin{array}{l}\text { MELOЗCO001609; } \\
\text { MELOЗCO16787; } \\
\text { MELOSCO06131; } \\
\text { MELO3C015327 }\end{array}$ \\
\hline G0:0004557 & \begin{tabular}{|l|l|} 
alpha-galactosidase activity \\
\end{tabular} & MOLECULAR_FUNCTION & 1 & 0,034546914 & 1 & 4 & 119 & 17004 & MELO3C020139 & 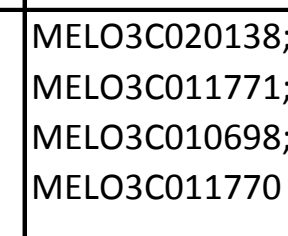 \\
\hline G0:0005262 & calcium channel activity & MOLECULAR_FUNCTION & 1 & 0,034546914 & 1 & 4 & 119 & 17004 & MELO3C016817 & $\begin{array}{l}\text { MELO3C017043; } \\
\text { MELO3CO22020; } \\
\text { MELO3CO18734; } \\
\text { MELO3C007977 }\end{array}$ \\
\hline G0:0003882 & CDP-diacylglycerol-serine O-phosphatidyltransferase activity & MOLECULAR_FUNCTION & 1 & 0,013963465 & 1 & 1 & 119 & 17007 & MELO3C005332 & MELO3C012286 \\
\hline 60:0004605 & phosphatidate cytidylytransferase activity & MOLECULAR_FUNCTION & 1 & 0,027733554 & 1 & 3 & 119 & 17005 & MELO3C007432 & $\begin{array}{l}\text { MELOЗСС2020525; } \\
\text { MELOSCO21695; } \\
\text { MELO3C0211445 }\end{array}$ \\
\hline
\end{tabular}




\begin{tabular}{|c|c|c|c|c|c|c|c|c|c|c|}
\hline G0:0047268 & galactinol-raffinose galactosyltransferase activity & MOLECULAR_FUNCTION & 1 & 0,048031907 & 1 & 6 & 119 & 17002 & MELO3C026247 & $\begin{array}{l}\text { MELOЗCO15912; } \\
\text { MELO3COOO997; } \\
\text { MELO3CO02287; } \\
\text { MELO3CO25599; } \\
\text { MELO3CO23110; } \\
\text { MELO3CO10314 }\end{array}$ \\
\hline 60:0034511 & U3 snoRNA binding & MOLECULAR_FUNCTION & 1 & 0,013963465 & 1 & 1 & 119 & 17007 & MELO3C017440 & MELO3C013185 \\
\hline 60:0005764 & |ysosome & CELLULAR_COMPONENT & 1 & 0,011186588 & 2 & 21 & 118 & 16987 & $\begin{array}{l}\text { MELO3CO17653 } \\
\text { MELO3C015523 }\end{array}$ & 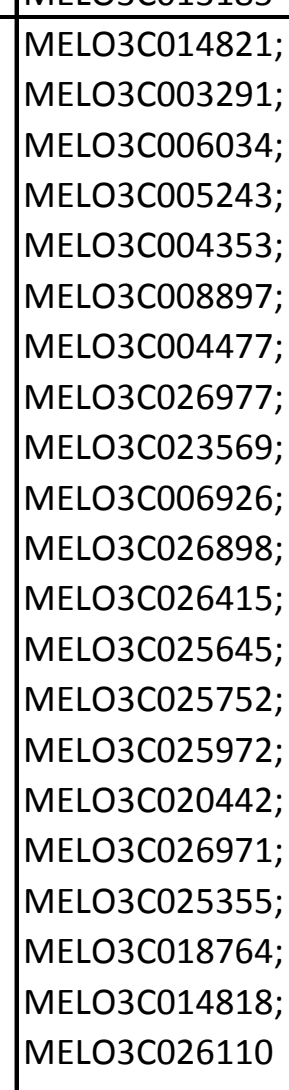 \\
\hline 60:0031983 & vesicle lumen & CELLULAR_COMPONENT & 1 & 0,007006072 & 1 & 0 & 119 & 17008 & MELO3C015523 & \\
\hline 60:0042718 & yolk granule & CELLULAR_COMPONENT & 1 & 0,007006072 & 1 & 0 & 119 & 17008 & MELO3C015523 & \\
\hline 60:0016602 & CCAAT-binding factor complex & CELLULAR_COMPONENT & 1 & 0,034546914 & 1 & 4 & 119 & 17004 & MELO3C023161 & $\begin{array}{l}\text { MELO3CO09551; } \\
\text { MELO3CO15320; } \\
\text { MELO3CO23554; } \\
\text { MELO3CO07077 }\end{array}$ \\
\hline 60:0045169 & fusome & $\begin{array}{l}\text { CELLULAR_COMPONENT } \\
\end{array}$ & 1 & 0,020872515 & 1 & 2 & 119 & 17006 & MELO3C015523 & \begin{tabular}{|l} 
MELO3CO06951; \\
MELO3C024150
\end{tabular} \\
\hline 60:0005783 & endoplasmic reticulum & CELLULAR_COMPONENT & 1 & 0,011232603 & 8 & 425 & 112 & 16583 & 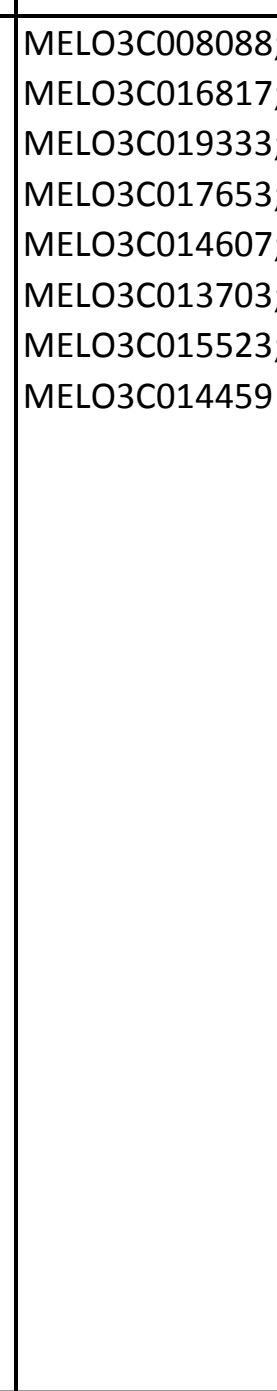 & 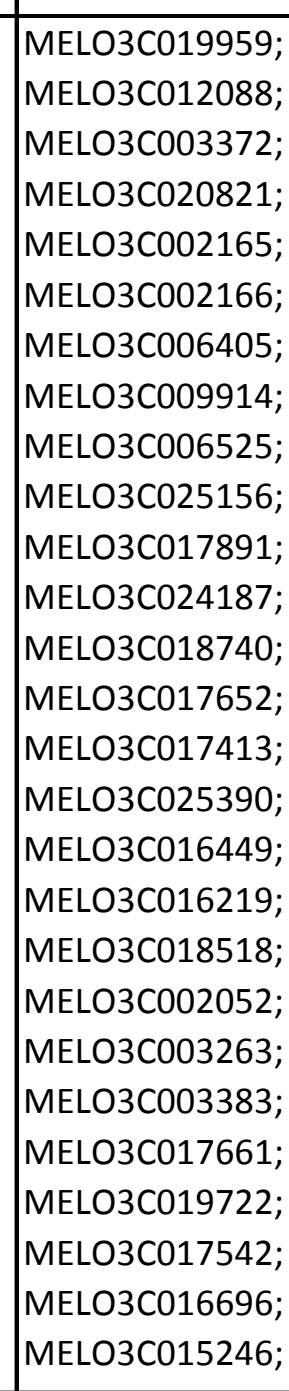 \\
\hline
\end{tabular}




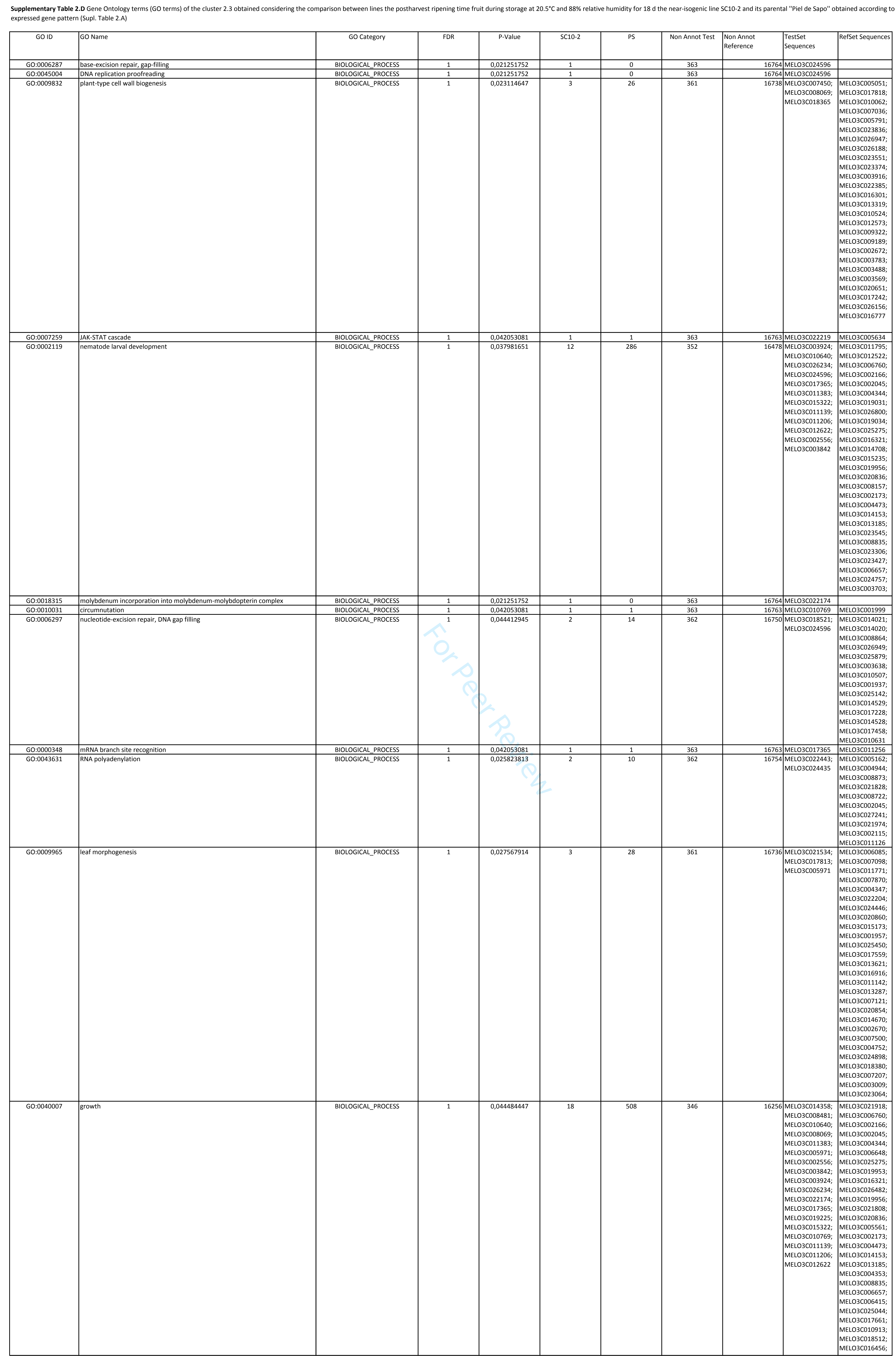




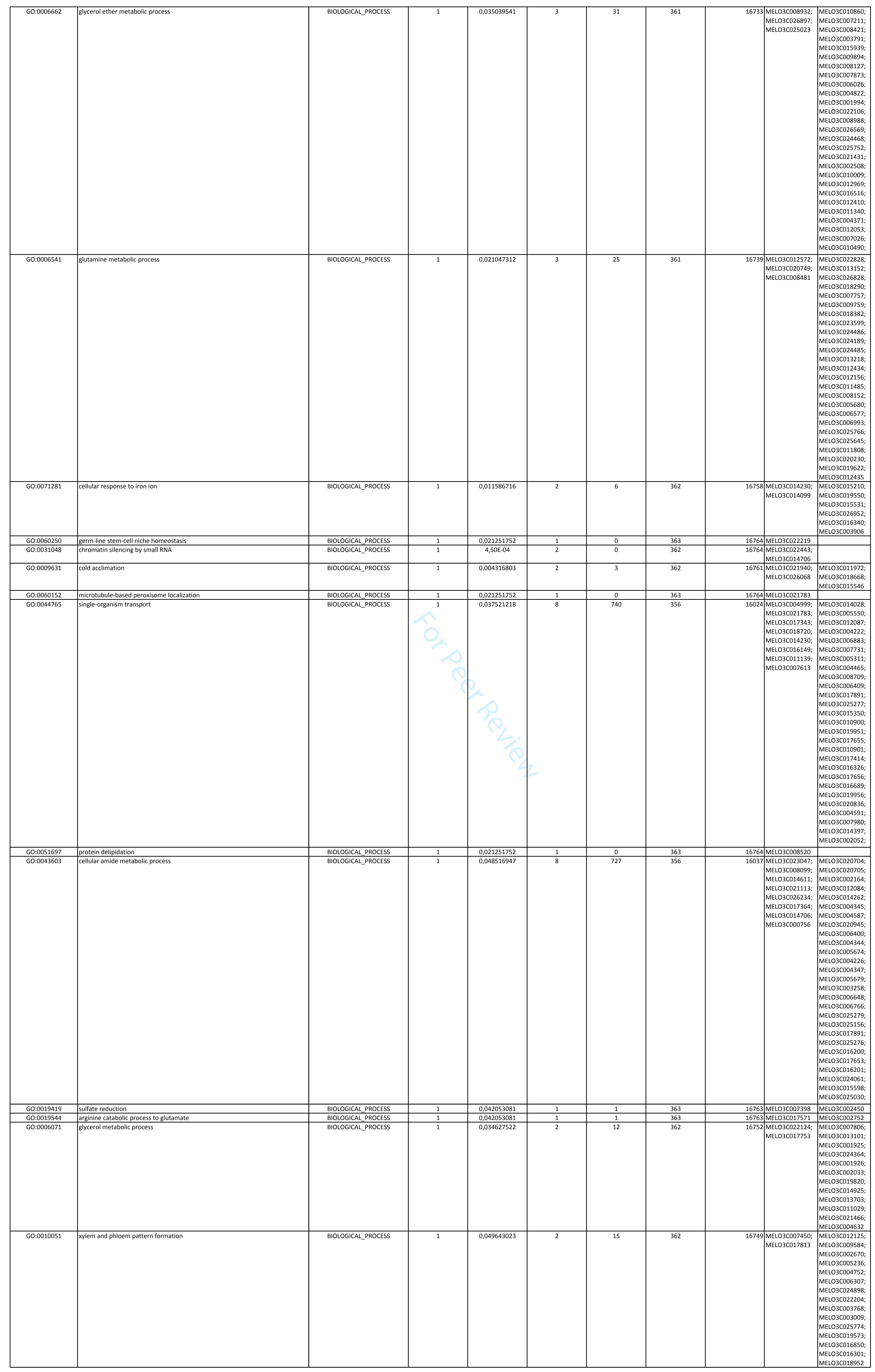




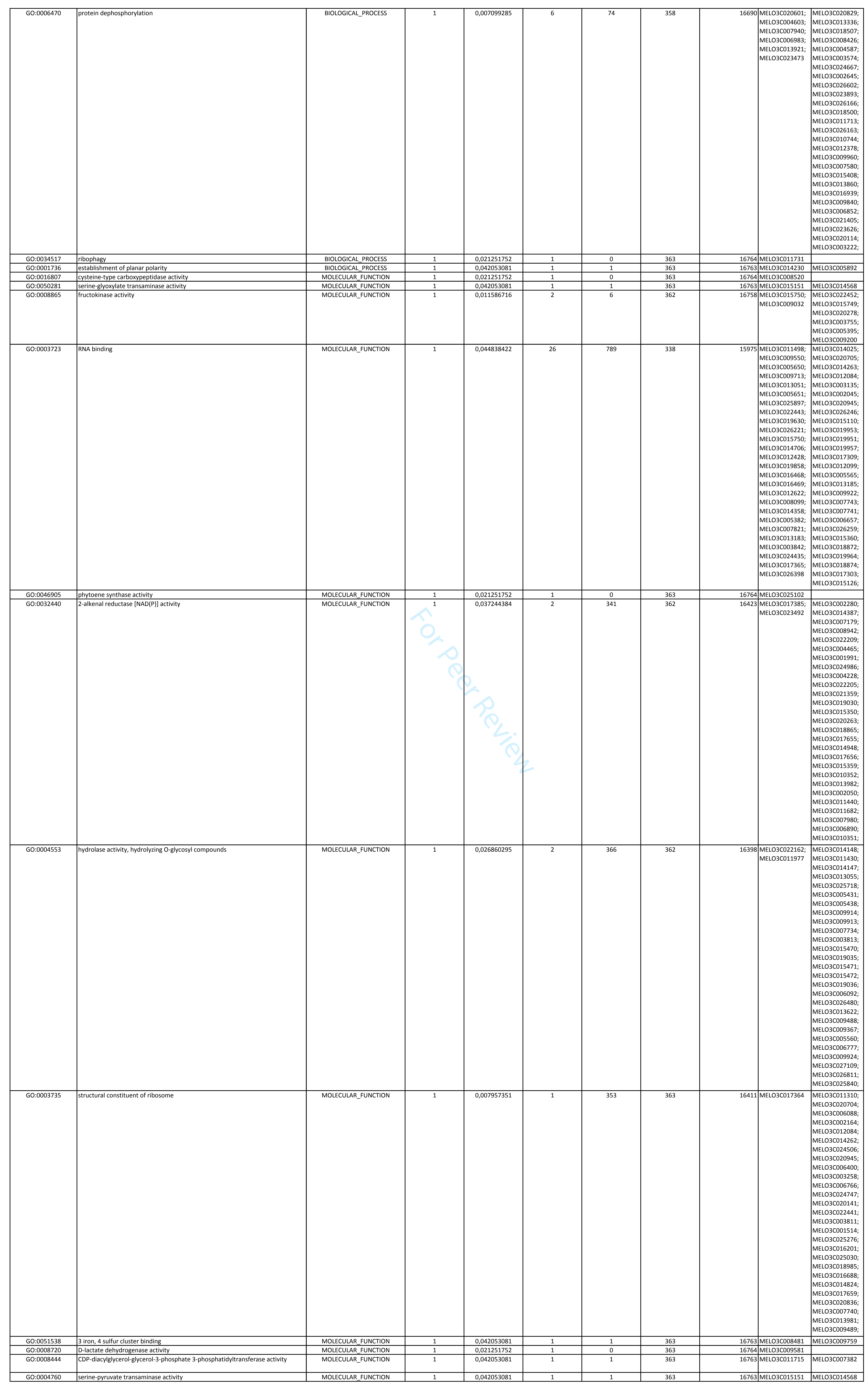




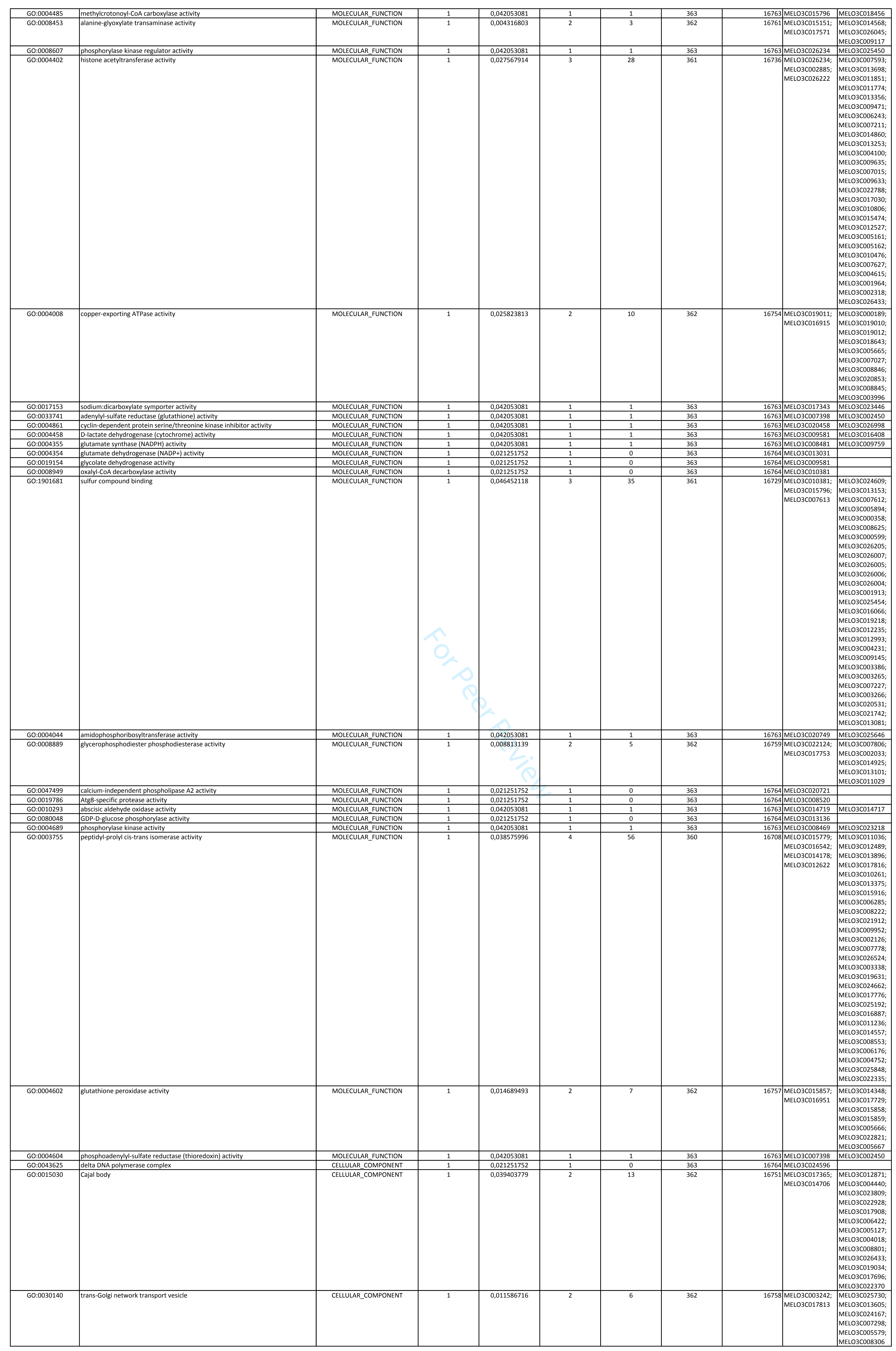




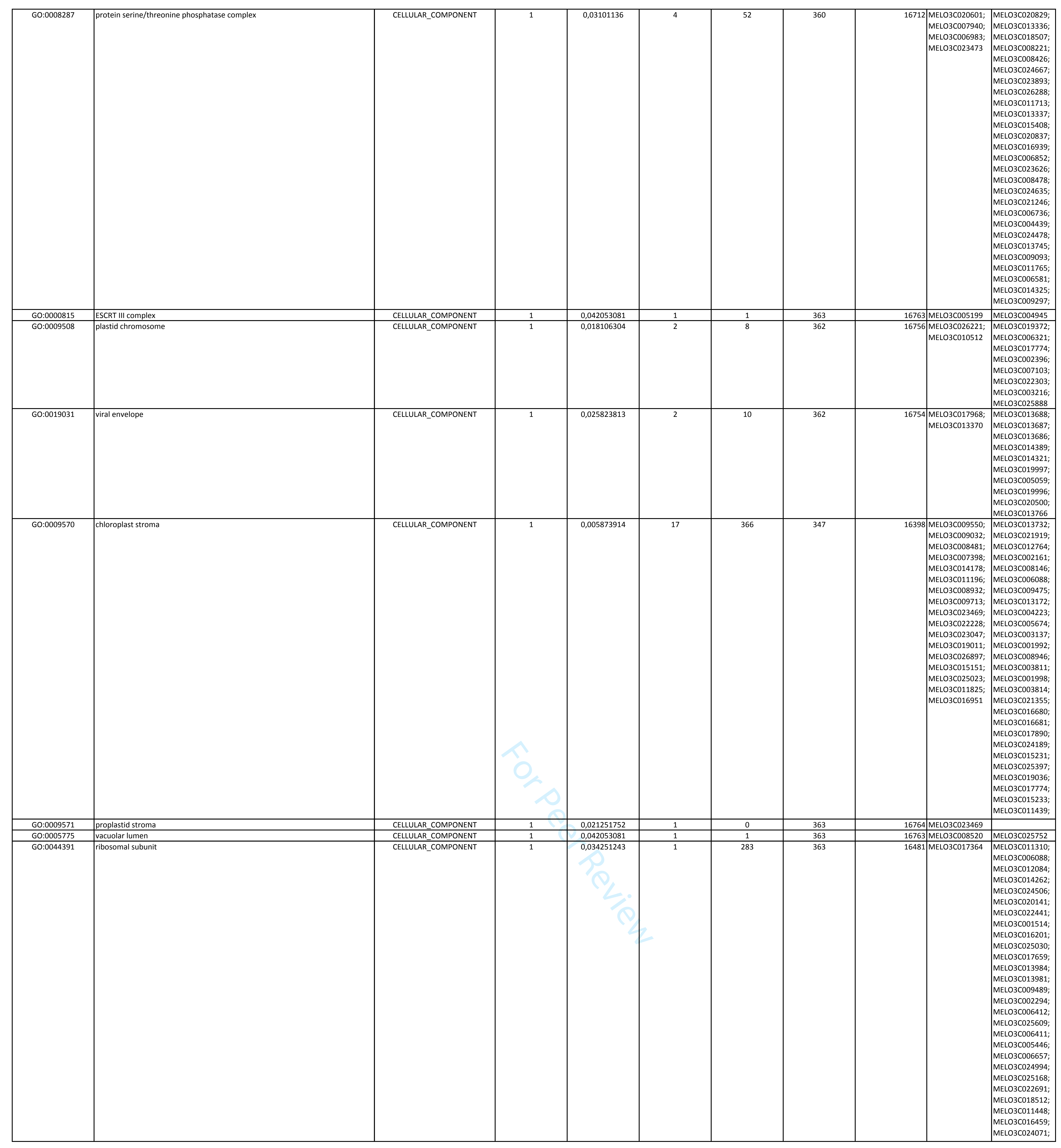


Supplementary Table 2.E Gene Ontology terms (GO terms) of the cluster 2.4 obtained considering the comparison between lines the postharvest ripening time fruit during storage at $20.5^{\circ} \mathrm{C}$ and $88 \%$ relative humidity for $18 \mathrm{~d}$ the near-isogenic line SC10-2 and its parental "Piel de Sapo" obtained according to the differentially expressed gene pattern (Supl. Table 2.A)

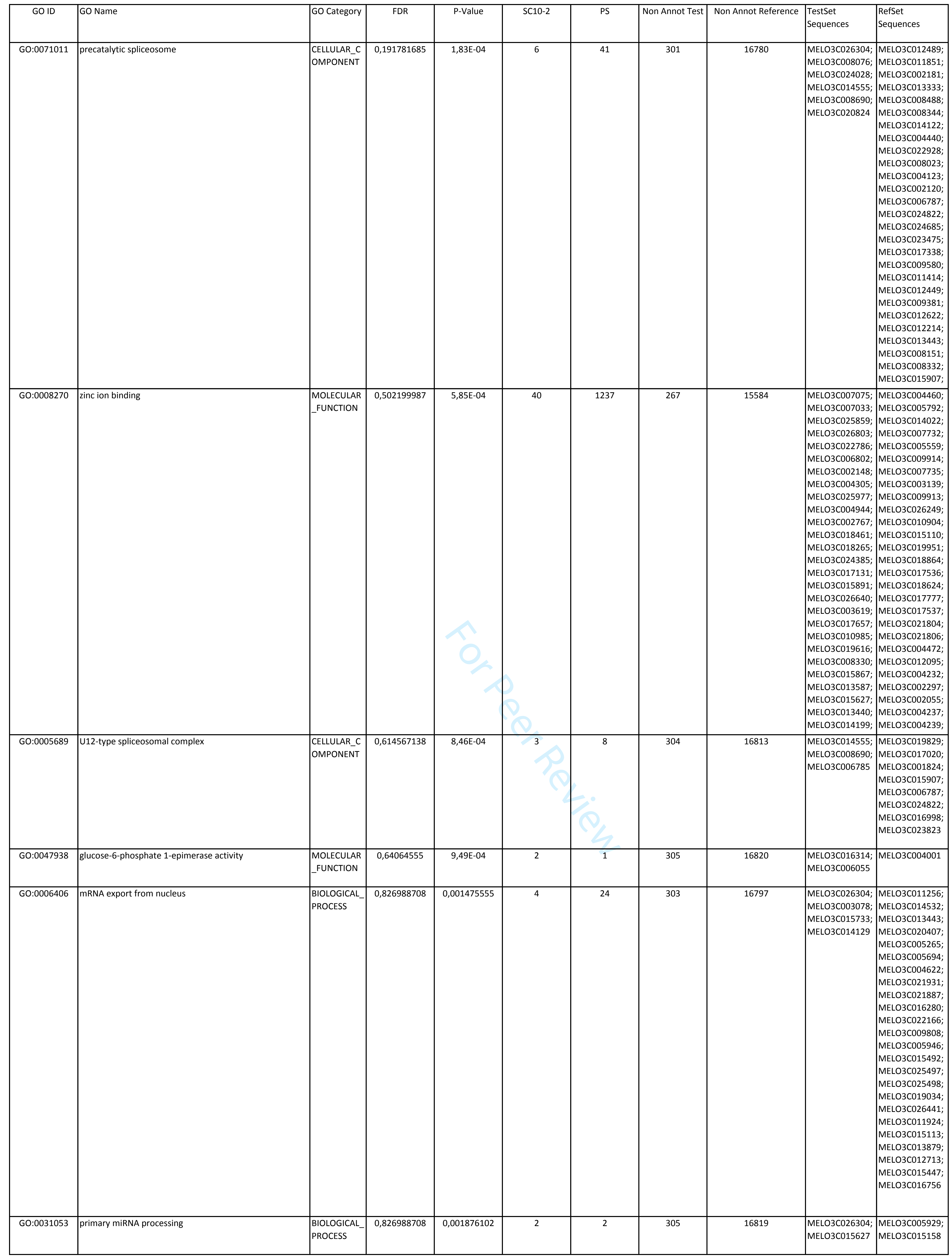




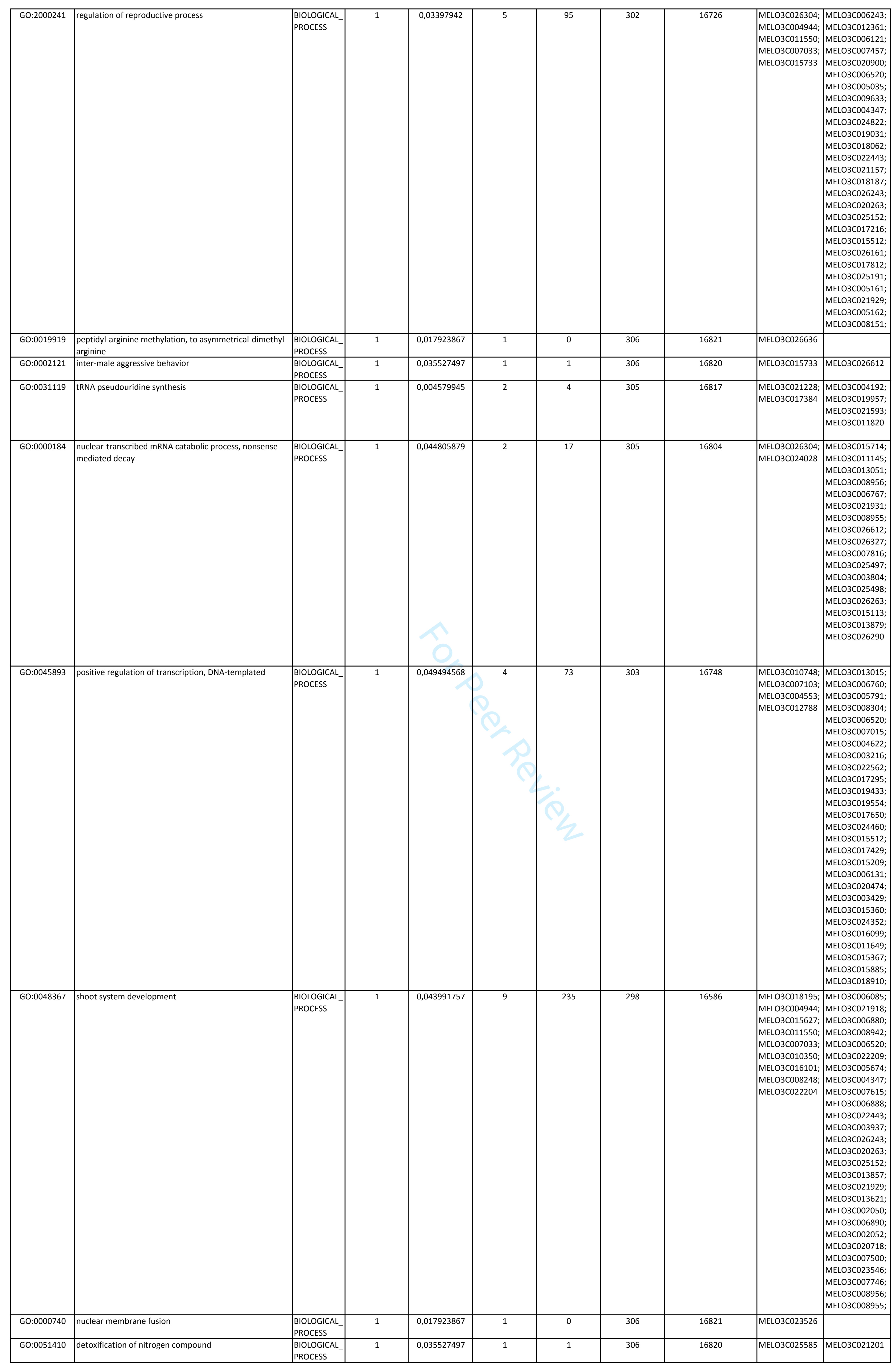




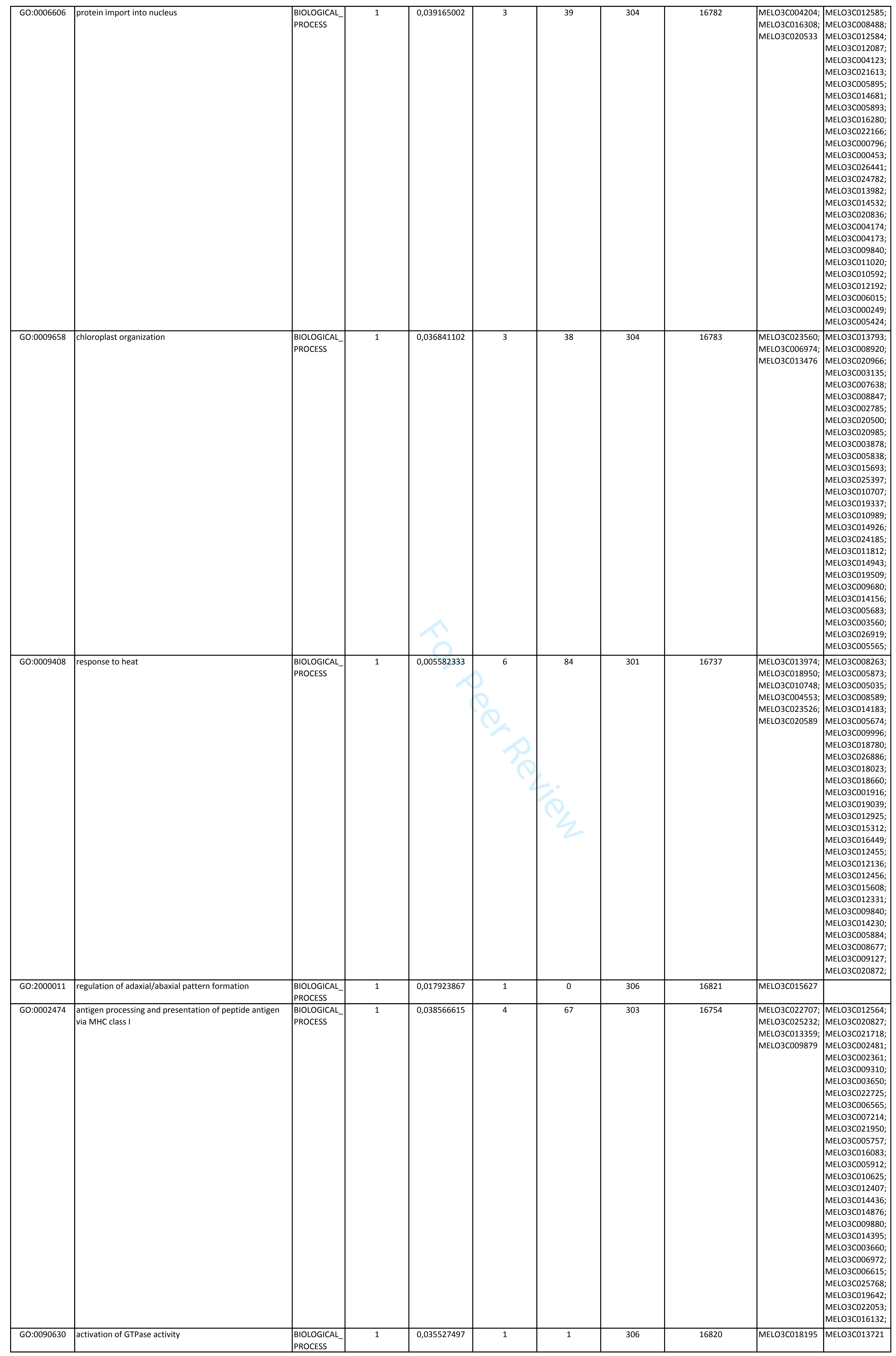




\begin{tabular}{|c|c|c|c|c|c|c|c|c|c|c|}
\hline GO:0006986 & |response to unfolded protein & \begin{tabular}{|l|} 
BIOLOGICAL_ \\
PROCESS
\end{tabular} \mid & 1 & 0,018771079 & 2 & 10 & 305 & 16811 & $\begin{array}{l}\text { MELO3C022707; } \\
\text { MELO3C010748 }\end{array}$ & 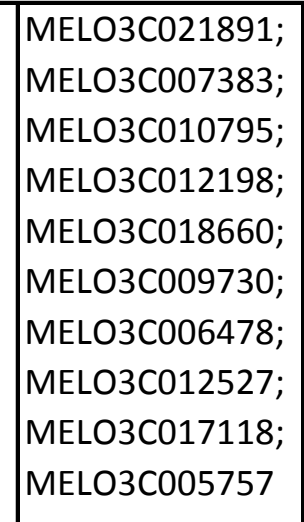 \\
\hline GO:0047497 & mitochondrion transport along microtubule & $\begin{array}{l}\begin{array}{l}\text { BIOLOGICAL_- } \\
\text { PROCESS }\end{array} \\
\text { PRS }\end{array}$ & 1 & 0,035527497 & 1 & 1 & 306 & 16820 & MELO3C004273 & MELO3C015839 \\
\hline GO:0045071 & negative regulation of viral genome replication & $\left|\begin{array}{l}\text { BIOLOGICAL_- } \\
\text { PROCESS }\end{array}\right|$ & 1 & 0,017923867 & 1 & 0 & 306 & 16821 & MELO3C026304 & \\
\hline GO:0051085 & chaperone mediated protein folding requiring cofactor & \begin{tabular}{|l|} 
BIOLOGICAL_- \\
PROCESS
\end{tabular} & 1 & 0,035527497 & 1 & 1 & 306 & 16820 & MELO3C023526 & MELO3C007116 \\
\hline GO:0031124 & mRNA 3'-end processing & \begin{tabular}{|l|}
$\begin{array}{l}\text { BIOLOGICAL_- } \\
\text { PROCESS }\end{array}$ \\
\end{tabular} & 1 & 0,005969919 & 3 & 18 & 304 & 16803 & $\begin{array}{l}\text { MELO3C026304; } \\
\text { MELO3CO04944; } \\
\text { MELO3C015733 }\end{array}$ & 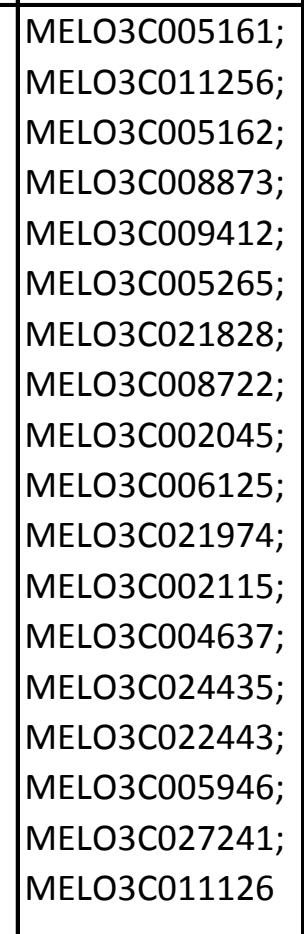 \\
\hline GO:0019933 & cAMP-mediated signaling & \begin{tabular}{|l|}
$\begin{array}{l}\text { BIOLOGICAL__ } \\
\text { PROCESS }\end{array}$ \\
\end{tabular} & 1 & 0,035527497 & 1 & 1 & 306 & 16820 & MELO3C006948 & MELO3C026602 \\
\hline GO:0007094 & mitotic spindle assembly checkpoint & \begin{tabular}{|l|} 
BIOLOGICAL_- \\
PROCESS
\end{tabular} & 1 & 0,036476381 & 2 & 15 & 305 & 16806 & $\begin{array}{l}\text { MELO3C025232; } \\
\text { MELO3C013359 }\end{array}$ & 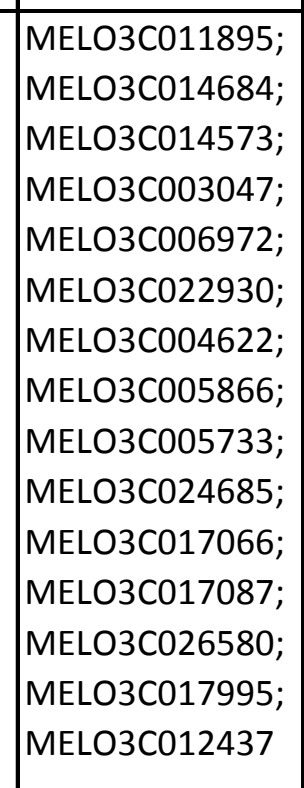 \\
\hline GO:0001666 & \begin{tabular}{|l} 
response to hypoxia \\
\end{tabular} & \begin{tabular}{|l|} 
BIOLOGICAL_- \\
PROCESS
\end{tabular} & 1 & 0,025279833 & 2 & 12 & 305 & 16809 & $\begin{array}{l}\text { MELO3C024028; } \\
\text { MELO3C010748 }\end{array}$ & 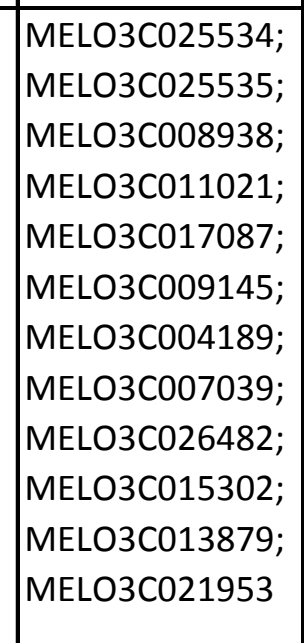 \\
\hline GO:0055062 & phosphate ion homeostasis & \begin{tabular}{|l|}
$\begin{array}{l}\text { BIOLOGICAL__ } \\
\text { PROCESS }\end{array}$ \\
\end{tabular} & 1 & 0,035527497 & 1 & 1 & 306 & 16820 & MELO3C010297 & MELO3C007283 \\
\hline GO:0009963 & positive regulation of flavonoid biosynthetic process & \begin{tabular}{|l|}
$\begin{array}{l}\text { BIOLOGICAL_ } \\
\text { PROCESS }\end{array}$ \\
\end{tabular} & 1 & 0,035527497 & 1 & 1 & 306 & 16820 & MELO3C027219 & MELO3C016879 \\
\hline GO:0051382 & kinetochore assembly & $\mid \begin{array}{l}\text { BIOLOGICAL_- } \\
\text { PROCESS }\end{array}$ & 1 & 0,017923867 & 1 & 0 & 306 & 16821 & MELO3C006948 & \\
\hline GO:0042542 & response to hydrogen peroxide & \begin{tabular}{|l|} 
BIOLOGICAL_ \\
PROCESS
\end{tabular} & 1 & 0,002685504 & 3 & 13 & 304 & 16808 & $\begin{array}{l}\text { MELOЗC013974; } \\
\text { MELOЗC010748; } \\
\text { MELO3C020589 }\end{array}$ & 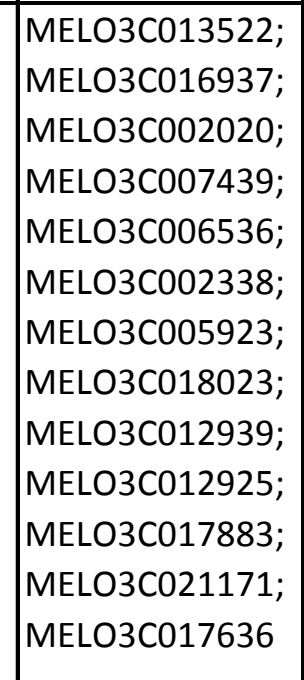 \\
\hline GO:0048644 & muscle organ morphogenesis & \begin{tabular}{|l|}
$\begin{array}{l}\text { BIOLOGICAL_ } \\
\text { PROCESS }\end{array}$ \\
\end{tabular} & 1 & 0,017923867 & 1 & 0 & 306 & 16821 & MELO3C008076 & \\
\hline GO:0006206 & pyrimidine nucleobase metabolic process & \begin{tabular}{|l|} 
BIOLOGICAL__ \\
PROCESS
\end{tabular} & 1 & 0,04055957 & 2 & 16 & 305 & 16805 & \begin{tabular}{|l|} 
MELO3C005067; \\
MELO3C022212
\end{tabular} & 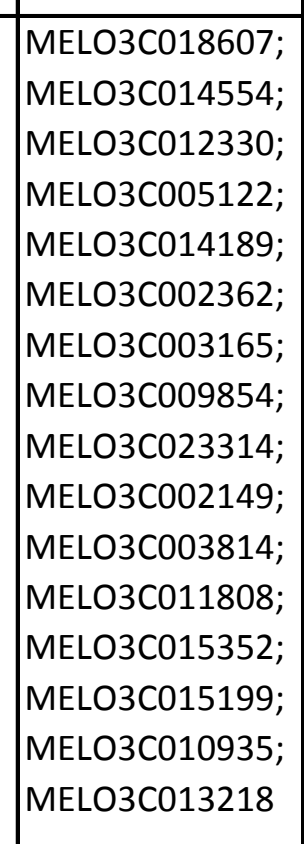 \\
\hline GO:0000350 & \begin{tabular}{|l}
$\begin{array}{l}\text { generation of catalytic spliceosome for second } \\
\text { transesterification step }\end{array}$ \\
\end{tabular} & \begin{tabular}{|l|}
$\begin{array}{l}\text { BIOLOGICAL_ } \\
\text { PROCESS }\end{array}$ \\
\end{tabular} & 1 & 0,017923867 & 1 & 0 & 306 & 16821 & MELO3C015733 & \\
\hline GO:0010267 & production of ta-siRNAs involved in RNA interference & \begin{tabular}{|l|} 
BIOLOGICAL_ \\
PROCESS
\end{tabular} & 1 & 0,013103927 & 2 & 8 & 305 & 16813 & \begin{tabular}{|c|} 
MELO3C015627; \\
MELO3C014129
\end{tabular} & $\begin{array}{l}\text { MELO3C010254; } \\
\text { MELO3CO11257; } \\
\text { MELO3CO15492; } \\
\text { MELO3COOS929; } \\
\text { MELOOCO10042; } \\
\text { MELOOCO222813; } \\
\text { MELO3C023207; } \\
\text { MELO3C012127 }\end{array}$ \\
\hline GO:0010362 & negative regulation of anion channel activity by blue 1 & \begin{tabular}{|l|} 
BIOLOGICAL \\
PROCESS
\end{tabular} & 1 & 0,035527497 & 1 & 1 & 306 & 16820 & MELO3C013476 & MELO3C025720 \\
\hline
\end{tabular}




\begin{tabular}{|c|c|c|c|c|c|c|c|c|c|c|}
\hline GO:0006787 & porphyrin-containing compound catabolic process & \begin{tabular}{|l|} 
BIOLOGICAL \\
PROCESS
\end{tabular} & 1 & 0,032563635 & 2 & 14 & 305 & 16807 & $\begin{array}{l}\text { MELO3C010350; } \\
\text { MELO3C023590 }\end{array}$ & 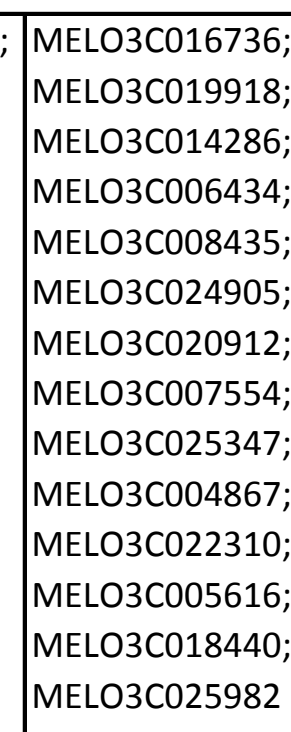 \\
\hline GO:0043097 & pyrimidine nucleoside salvage & $\begin{array}{l}\text { BIOLOGICAL } \\
\text { PROCESS }\end{array}$ & 1 & 0,017923867 & 1 & 0 & 306 & 16821 & MELO3C022212 & \\
\hline GO:0006419 & alanyl-tRNA aminoacylation & \begin{tabular}{|l|} 
BIOLOGICAL_ \\
PROCESS
\end{tabular} & 1 & 0,010607789 & 2 & 7 & 305 & 16814 & $\begin{array}{l}\text { MELO3CO00425; } \\
\text { MELO3C027308 }\end{array}$ & $\begin{array}{l}\text { MELO3C012870; } \\
\text { MELO3C010097; } \\
\text { MELO3C024031; } \\
\text { MELO3C00075; } \\
\text { MELO3C01862; } \\
\text { MELO3C02727; } \\
\text { MELO3C013645 }\end{array}$ \\
\hline GO:0040022 & feminization of hermaphroditic germ-line & \begin{tabular}{|l|} 
BIOLOGICAL \\
PROCESS
\end{tabular} & 1 & 0,008348753 & 2 & 6 & 305 & 16815 & $\begin{array}{l}\text { MELO3CO26304; } \\
\text { MELO3C015733 }\end{array}$ & 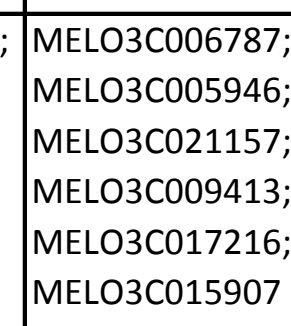 \\
\hline GO:0000209 & protein polyubiquitination & \begin{tabular}{|l|} 
BIOLOGICAL_ \\
PROCESS
\end{tabular} & 1 & 0,009420097 & 5 & 67 & 302 & 16754 & $\begin{array}{l}\text { MELO3C018461; } \\
\text { MELO3CO22707; } \\
\text { MELO3C025232; } \\
\text { MELO3CO1335; } \\
\text { MELO3C009879 } \\
\end{array}$ & 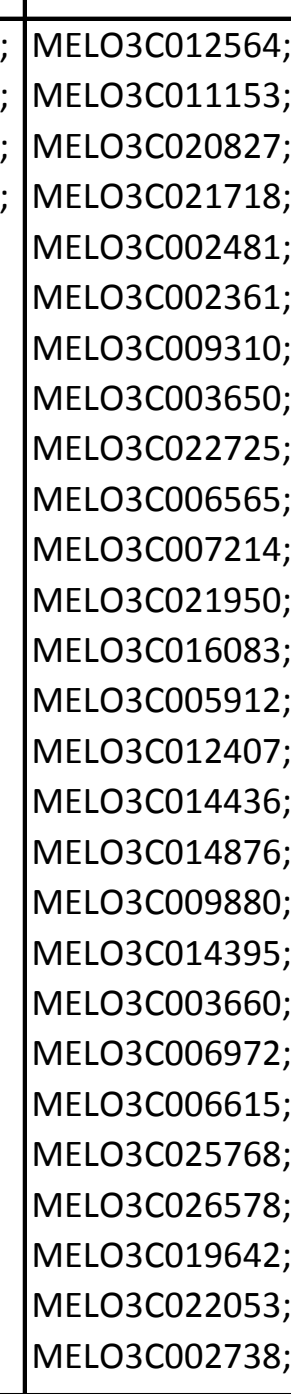 \\
\hline GO:0007525 & somatic muscle development & \begin{tabular}{|l|} 
BIOLOGICAL \\
PROCESS
\end{tabular} & 1 & 0,035527497 & 1 & 1 & 306 & 16820 & MELO3C018265 & MELO3C025387 \\
\hline GO:0007638 & mechanosensory behavior & \begin{tabular}{|l|} 
BIOLOGICAL \\
PROCESS
\end{tabular} & 1 & 0,017923867 & 1 & 0 & 306 & 16821 & MELO3C008076 & \\
\hline GO:0006369 & termination of RNA polymerase II transcription & \begin{tabular}{|l|} 
BIOLOGICAL_ \\
PROCESS
\end{tabular} & 1 & 0,036476381 & 2 & 2 & 305 & 16806 & $\begin{array}{l}\text { MELO3CO26304; } \\
\text { MELO3C015733 }\end{array}$ & 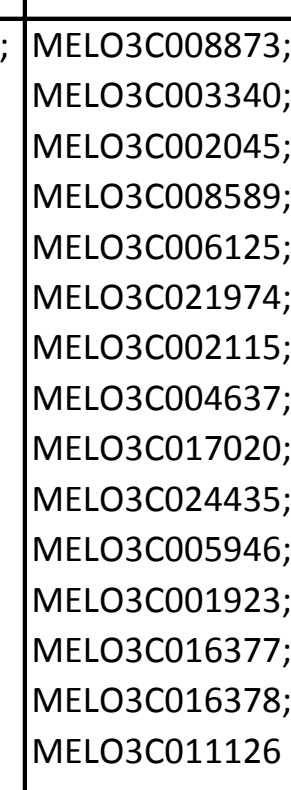 \\
\hline GO:0032526 & response to retinoic acid & \begin{tabular}{|l|} 
BIOLOGICAL_ \\
PROCESS
\end{tabular} & 1 & 0,017923867 & 1 & 0 & 306 & 16821 & MELO3C026102 & \\
\hline GO:0045451 & pole plasm oskar mRNA localization & \begin{tabular}{|l|} 
BIOLOGICAL \\
PROCESS
\end{tabular} & 1 & 0,035527497 & 1 & 1 & 306 & 16820 & MELO3C024028 & MELO3C005946 \\
\hline GO:0010198 & synergid death & $\begin{array}{l}\text { BIOLOGICAL_ } \\
\text { PROCESS }\end{array}$ & 1 & 0,017923867 & 1 & 0 & 306 & 16821 & MELO3C023526 & \\
\hline GO:0009640 & photomorphogenesis & \begin{tabular}{|l|} 
BIOLOGICAL_ \\
PROCESS
\end{tabular} & 1 & 0,010927009 & 3 & 23 & 304 & 16798 & $\begin{array}{l}\text { MELO3C023560; } \\
\text { MELO3C013440; } \\
\text { MELO3C020783 }\end{array}$ & 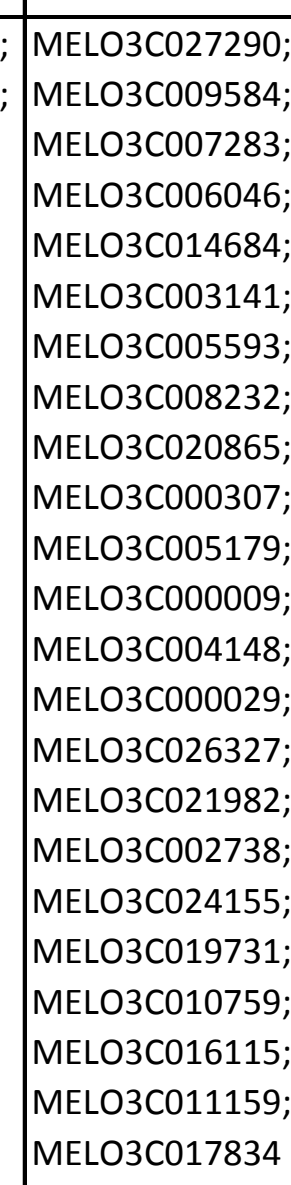 \\
\hline
\end{tabular}




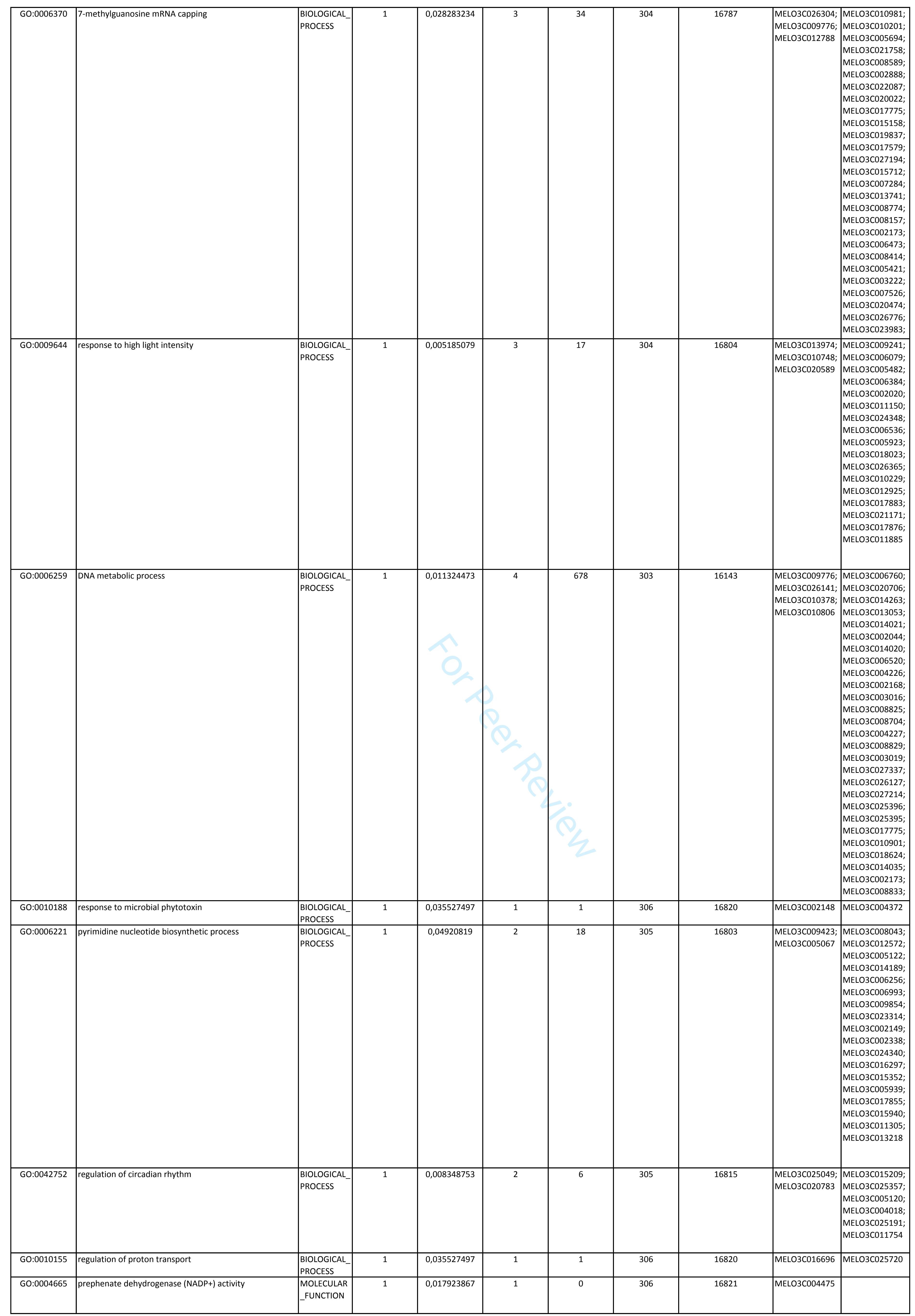




\begin{tabular}{|c|c|c|c|c|c|c|c|c|c|c|}
\hline GO:0016758 & transferase activity, transferring hexosyl groups & $\begin{array}{l}\text { MOLECULAR } \\
\text { FUNCTION }\end{array}$ & 1 & 0,011308174 & 1 & 391 & 306 & 16430 & \begin{tabular}{|c} 
MELO3C017104 \\
\\
\end{tabular} & 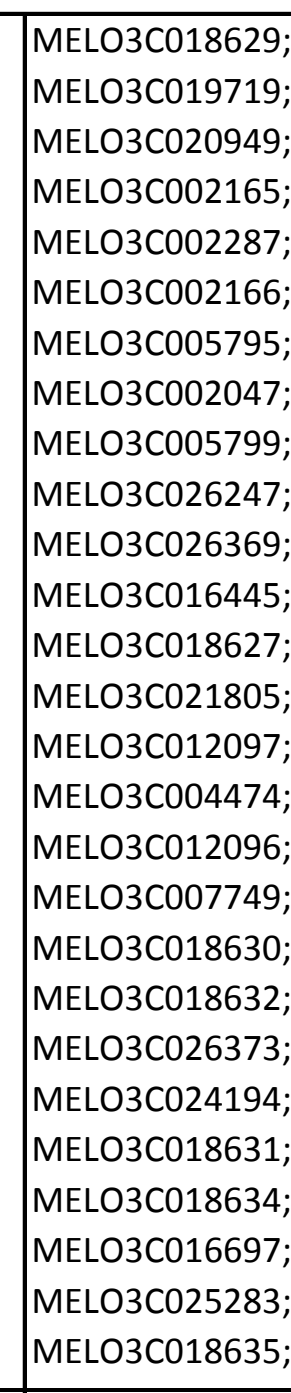 \\
\hline GO:0016881 & acid-amino acid ligase activity & \begin{tabular}{|l|} 
MOLECULAR \\
FUNCTION
\end{tabular} & 1 & 1 & 4 & 42 & 303 & 16779 & $\begin{array}{l}\text { MELOЗСС15867; } \\
\text { MELOЗCO22707; } \\
\text { MELO3CO25232; } \\
\text { MELO3C013359 }\end{array}$ & 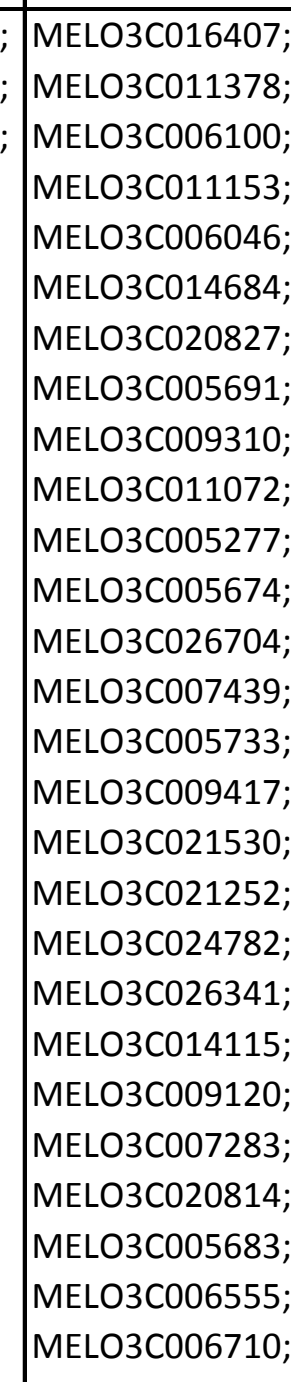 \\
\hline GO:0018822 & nitrile hydratase activity & \begin{tabular}{|l|} 
MOLECULAR \\
FUNCTION
\end{tabular} & 1 & 0,017923867 & 1 & 0 & 306 & 16821 & MELO3C025585 & \\
\hline GO:0080079 & cellobiose glucosidase activity & $\begin{array}{l}\text { MOLECULAR } \\
\text { FUNCTION }\end{array}$ & 1 & 0,017923867 & $0^{1}$ & 0 & 306 & 16821 & MELO3C021253 & \\
\hline GO:0047172 & shikimate O-hydroxycinnamoyltransferase activity & \begin{tabular}{|l|} 
MOLECULAR \\
FUNCTION
\end{tabular} & 1 & 0,035527497 & 1 & 1 & 306 & 16820 & MELO3C027219 & MELO3C023474 \\
\hline GO:0004402 & histone acetyltransferase activity & \begin{tabular}{|l|} 
MOLECULAR \\
FUNCTION
\end{tabular} & 1 & 0,017693064 & 3 & 28 & 304 & 16793 & $\begin{array}{l}\text { MELO3C014860; } \\
\text { MELO3C004100; } \\
\text { MELO3C010806 }\end{array}$ & 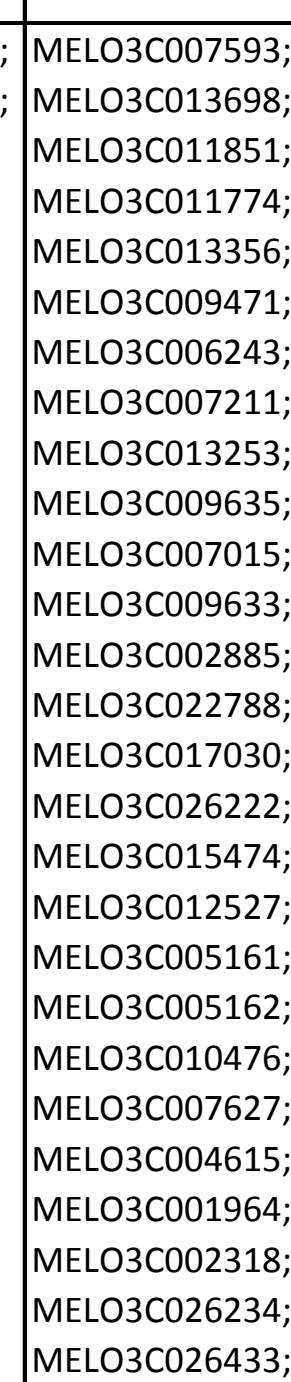 \\
\hline GO:0000170 & sphingosine hydroxylase activity & \begin{tabular}{|l|} 
MOLECULAR \\
FUNCTION
\end{tabular} & 1 & 0,035527497 & 1 & 1 & 306 & 16820 & MELO3C012088 & MELO3C008543 \\
\hline GO:0033862 & UMP kinase activity & \begin{tabular}{|l|} 
MOLECULAR \\
FUNCTION
\end{tabular} & 1 & 0,035527497 & 1 & 1 & 306 & 16820 & MELO3C009423 & MELO3C008043 \\
\hline GO:0050638 & taxadien-5-alpha-ol O-acetyltransferase activity & \begin{tabular}{|l|} 
MOLECULAR \\
_FUNCTION
\end{tabular} & 1 & 0,035527497 & 1 & 1 & 306 & 16820 & MELO3C018368 & MELO3C025311 \\
\hline GO:0080061 & indole-3-acetonitrile nitrilase activity & \begin{tabular}{|l|} 
MOLECULAR \\
FUNCTION
\end{tabular} & 1 & 0,017923867 & 1 & 0 & 306 & 16821 & MELO3C025585 & \\
\hline
\end{tabular}




\begin{tabular}{|c|c|c|c|c|c|c|c|c|c|c|}
\hline GO:0005096 & GTPase activator activity & $\begin{array}{l}\text { MOLECULAR } \\
\text { FUNCTION }\end{array}$ & 1 & 0,009142966 & 4 & 42 & 303 & 16779 & $\begin{array}{l}\text { MELO3C018195; } \\
\text { MELO3CO07075; } \\
\text { MELO3017176; } \\
\text { MELO3C018265 }\end{array}$ & 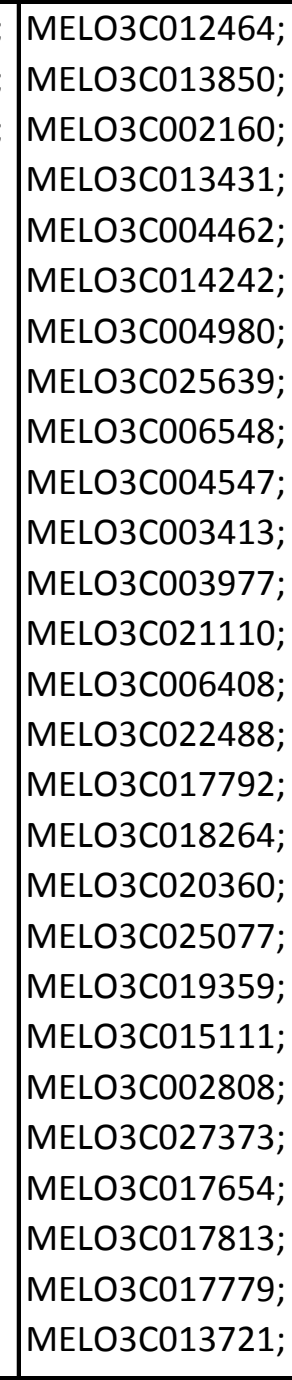 \\
\hline GO:0051741 & $\begin{array}{l}\text { 2-methyl-6-phytyl-1,4-benzoquinone methyltransferase } \\
\text { activity }\end{array}$ & $\begin{array}{l}\text { MOLECULAR } \\
\text { FUNCTION }\end{array}$ & 1 & 0,017923867 & 1 & 0 & 306 & 16821 & MELO3C014321 & \\
\hline GO:0015220 & choline transmembrane transporter activity & $\begin{array}{l}\text { MOLECULAR } \\
\text { FUNCTION }\end{array}$ & 1 & 0,017923867 & 1 & 0 & 306 & 16821 & MELO3C020808 & \\
\hline GO:0051082 & unfolded protein binding & $\begin{array}{l}\text { MOLECULAR } \\
\text { FUNCTION }\end{array}$ & 1 & 0,021826864 & 7 & 148 & 300 & 16673 & $\begin{array}{l}\text { MELO3CO04636; } \\
\text { MELO3CO13792; } \\
\text { MELO3CO08871; } \\
\text { MELO3CO18950; } \\
\text { MELO3COOO4908; } \\
\text { MELO3CO23526; } \\
\text { MELO3C011379 }\end{array}$ & 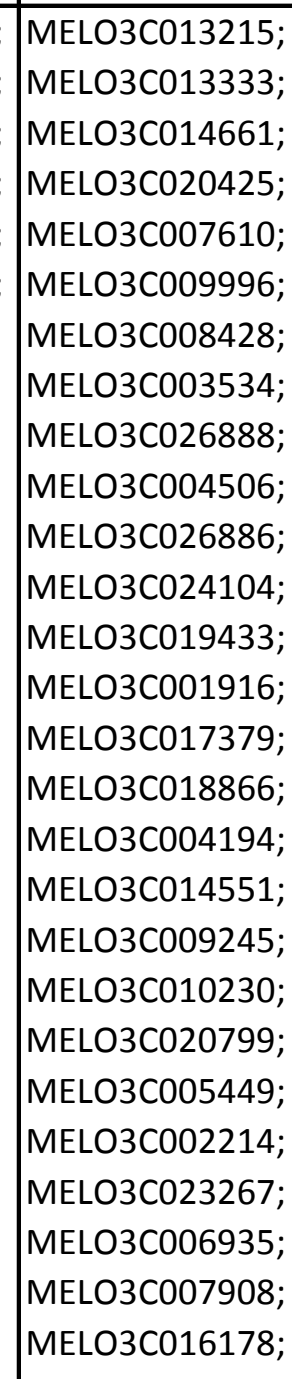 \\
\hline GO:0004697 & protein kinase C activity & \begin{tabular}{|l|} 
MOLECULAR \\
FUNCTION
\end{tabular} & 1 & 0,04920819 & 2 & 18 & 305 & 16803 & $\begin{array}{l}\text { MELO3C007047; } \\
\text { MELO3C013476 }\end{array}$ & 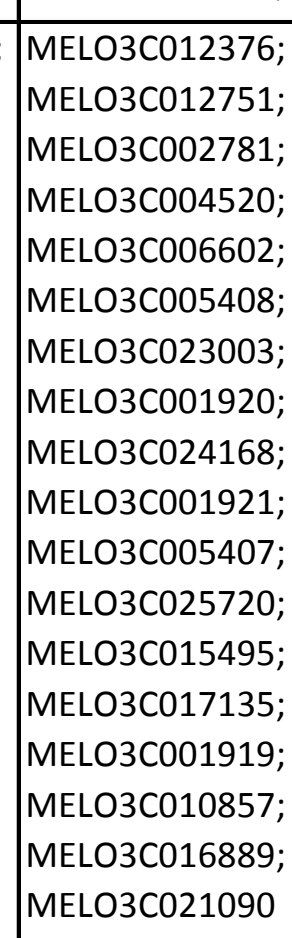 \\
\hline GO:0050662 & coenzyme binding & \begin{tabular}{|l|} 
MOLECULAR \\
FUNCTION
\end{tabular} & 1 & 0,048984136 & 1 & 314 & 306 & 16507 & MELO3C008985 & 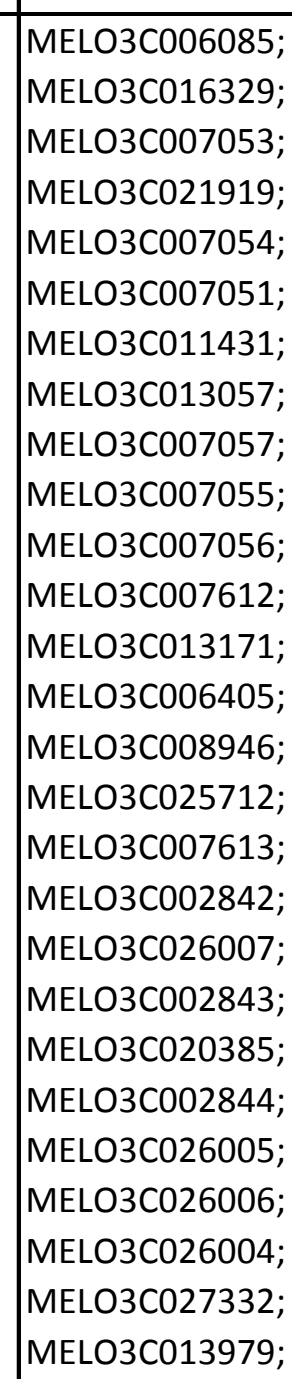 \\
\hline GO:0047427 & cyanoalanine nitrilase activity & $\begin{array}{l}\text { MOLECULAR } \\
\text { FUNCTION }\end{array}$ & 1 & 0,017923867 & 1 & 0 & 306 & 16821 & MELO3C025585 & \\
\hline GO:0004813 & alanine-tRNA ligase activity & $\begin{array}{l}\text { MOLECULAR } \\
\text { FUNCTION }\end{array}$ & 1 & 0,010607789 & 2 & 7 & 305 & 16814 & $\begin{array}{l}\text { MELO3C000425; } \\
\text { MELO3C027308 }\end{array}$ & $\begin{array}{l}\text { MELO3CO12870; } \\
\text { MELO3CO10097; } \\
\text { MELO3C024031; } \\
\text { MELO3CO00756; } \\
\text { MELO3C018624; } \\
\text { MELO3C027271; } \\
\text { MELO3C013645 }\end{array}$ \\
\hline GO:0047558 & 3-cyanoalanine hydratase activity & $\begin{array}{l}\text { MOLECULAR } \\
\text { FUNCTION }\end{array}$ & 1 & 0,017923867 & 1 & 0 & 306 & 16821 & MELO3C025585 & \\
\hline
\end{tabular}




\begin{tabular}{|c|c|c|c|c|c|c|c|c|c|c|}
\hline GO:0004034 & aldose 1-epimerase activity & $\begin{array}{l}\text { MOLECULAR } \\
\text { FUNCTION }\end{array}$ & 1 & 0,008348753 & 2 & 6 & 305 & 16815 & $\begin{array}{l}\text { MELO3C016314; } \\
\text { MELO3C006055 }\end{array}$ & 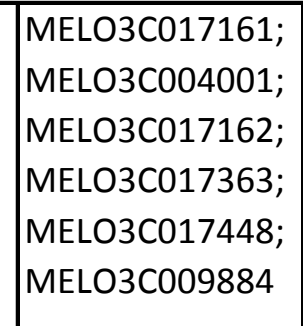 \\
\hline GO:0005366 & myo-inositol:proton symporter activity & $\begin{array}{l}\text { MOLECULAR } \\
\text { FUNCTION }\end{array}$ & 1 & 0,017923867 & 1 & 0 & 306 & 16821 & MELO3C017757 & \\
\hline GO:0031624 & ubiquitin conjugating enzyme binding & $\begin{array}{l}\text { MOLECULAR } \\
\text { FUNCTION }\end{array}$ & 1 & 0,035527497 & 1 & 1 & 306 & 16820 & MELO3C018461 & MELO3C023546 \\
\hline GO:0071522 & ureidoglycine aminohydrolase activity & $\begin{array}{l}\text { MOLECULAR } \\
\text { FUNCTION }\end{array}$ & 1 & 0,017923867 & 1 & 0 & 306 & 16821 & MELO3C014174 & \\
\hline GO:0047205 & quinate O-hydroxycinnamoyltransferase activity & $\begin{array}{l}\text { MOLECULAR } \\
\text { FUNCTION }\end{array}$ & 1 & 0,017923867 & 1 & 0 & 306 & 16821 & MELO3C027219 & \\
\hline GO:0080124 & pheophytinase activity & $\begin{array}{l}\text { MOLECULAR } \\
\text { FUNCTION }\end{array}$ & 1 & 0,017923867 & 1 & 0 & 306 & 16821 & MELO3C023590 & \\
\hline GO:0004137 & deoxycytidine kinase activity & $\begin{array}{l}\text { MOLECULAR } \\
\text { FUNCTION }\end{array}$ & 1 & 0,017923867 & 1 & $\overline{0}$ & 306 & 16821 & MELO3C022212 & \\
\hline GO:0008420 & CTD phosphatase activity & $\begin{array}{l}\text { MOLECULAR } \\
\text { FUNCTION }\end{array}$ & 1 & 0,035527497 & 1 & 1 & 306 & 16820 & MELO3C010744 & MELO3C007164 \\
\hline GO:0033730 & arogenate dehydrogenase $(\mathrm{NADP}+)$ activity & $\begin{array}{l}\text { MOLECULAR } \\
\text { FUNCTION }\end{array}$ & 1 & 0,017923867 & 1 & 0 & 306 & 16821 & MELO3C004475 & \\
\hline GO:0042624 & ATPase activity, uncoupled & $\begin{array}{l}\text { MOLECULAR } \\
\text { FUNCTION }\end{array}$ & 1 & 0,034590522 & 3 & 37 & 304 & 16784 & $\begin{array}{l}\text { MELO3C024028; } \\
\text { MELO3C015733; } \\
\text { MELO3C017908 }\end{array}$ & 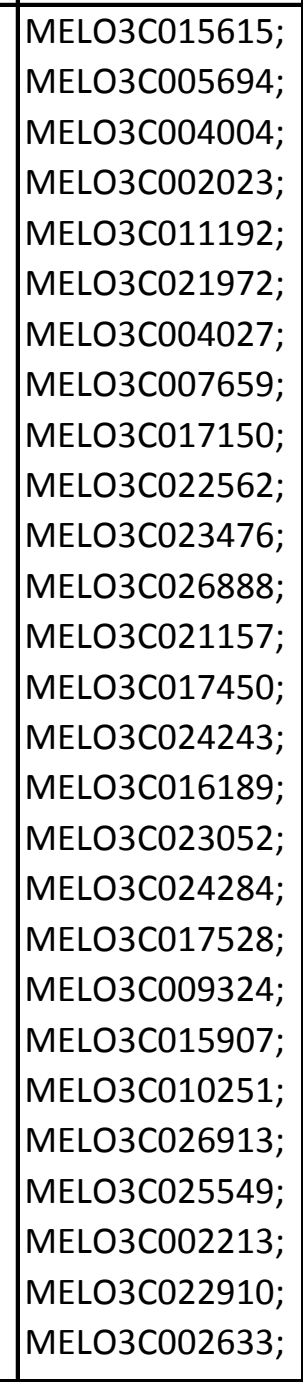 \\
\hline GO:0009882 & blue light photoreceptor activity & $\begin{array}{l}\text { MOLECULAR } \\
\text { FUNCTION }\end{array}$ & 1 & 0,035527497 & 1 & 1 & 306 & 16820 & MELO3C013476 & MELO3C025720 \\
\hline GO:0016277 & $\begin{array}{l}\text { [myelin basic protein]-arginine N-methyltransferase } \\
\text { activity }\end{array}$ & $\begin{array}{l}\text { MOLECULAR } \\
\text { FUNCTION }\end{array}$ & 1 & 0,017923867 & 1 & 0 & 306 & 16821 & MELO3C026636 & \\
\hline GO:0004842 & ubiquitin-protein transferase activity & \begin{tabular}{|l|} 
MOLECULAR \\
FUNCTION
\end{tabular} & 1 & 0,01225715 & 7 & 131 & 300 & 16690 & $\begin{array}{l}\text { MELO3C018461; } \\
\text { MELO3CO11882; } \\
\text { MELO3CO0549; } \\
\text { MELO3CO22707; } \\
\text { MELO3C025232; } \\
\text { MELO3CO07711; } \\
\text { MELO3C003563 }\end{array}$ & 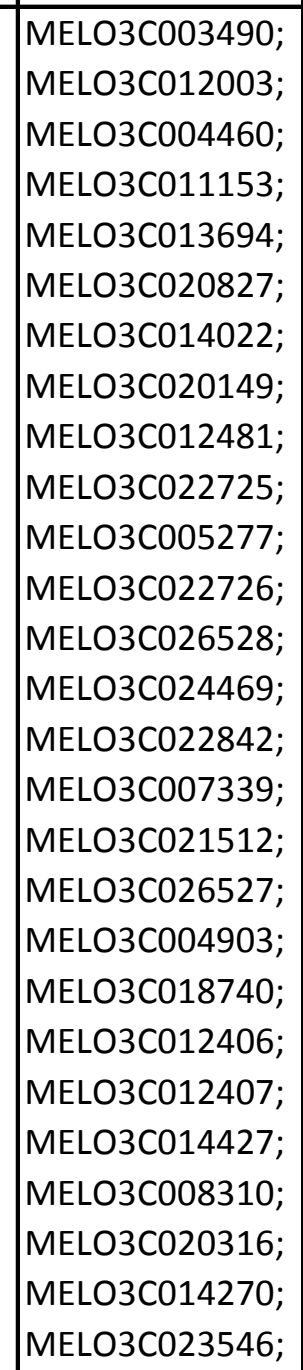 \\
\hline GO:0004726 & $\begin{array}{l}\text { non-membrane spanning protein tyrosine phosphatase } \\
\text { activity }\end{array}$ & $\begin{array}{l}\text { MOLECULAR } \\
\text { FUNCTION }\end{array}$ & 1 & 0,035527497 & 1 & 1 & 306 & 16820 & MELO3C021441 & MELO3C009840 \\
\hline GO:0071007 & U2-type catalytic step 2 spliceosome & \begin{tabular}{|l|} 
CELLULAR_C \\
OMPONENT
\end{tabular} & 1 & 0,017923867 & 1 & $\overline{0}$ & 306 & 16821 & MELO3C015733 & \\
\hline
\end{tabular}




\begin{tabular}{|c|c|c|c|c|c|c|c|c|c|c|}
\hline 60:0005730 & nucleolus & $\begin{array}{l}\text { CELLULAR_C C } \\
\text { OMPONENT }\end{array}$ & 1 & 0,026607121 & 10 & 245 & 297 & 16576 & 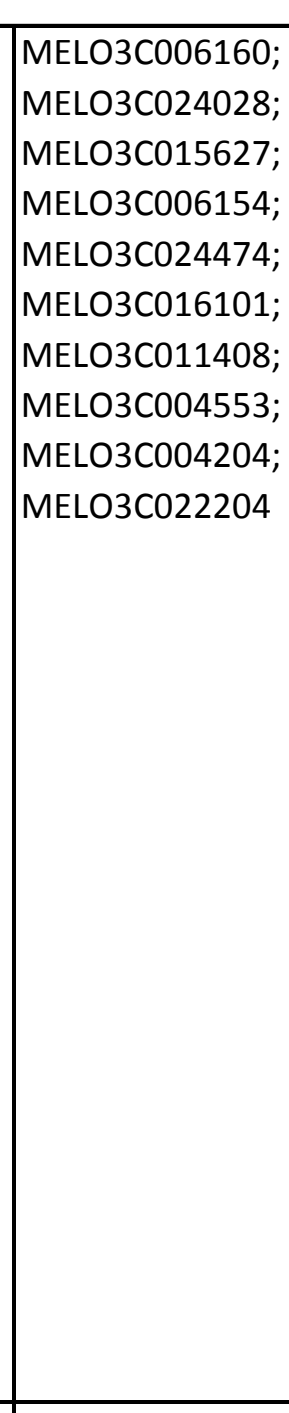 & 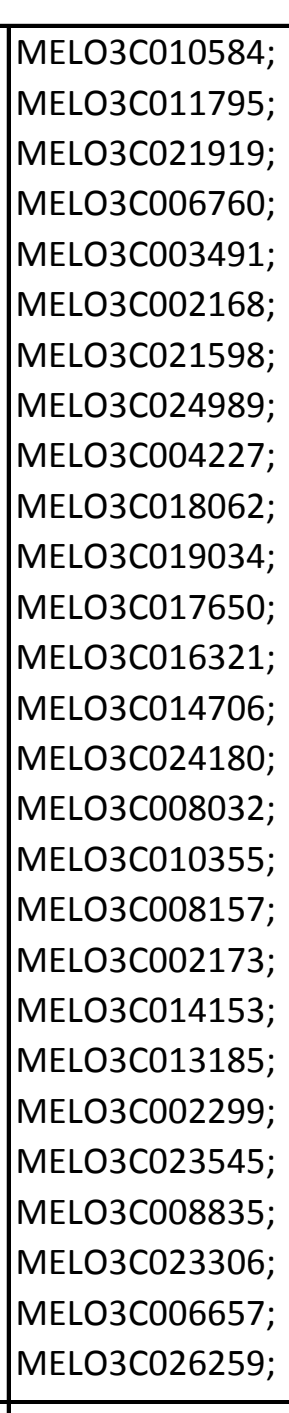 \\
\hline GO:0005846 & 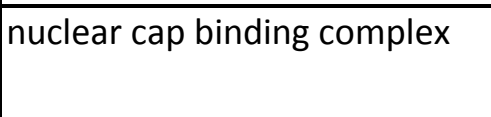 & \begin{tabular}{|l} 
CELLULAR_C \\
OMPONENT
\end{tabular} & 1 & $\begin{array}{ll}0,017923867 \\
\end{array}$ & 1 & 0 & 306 & 16821 & MELO3C026304 & \\
\hline GO:0005783 & endoplasmic reticulum & \begin{tabular}{|l} 
CEELULAR_C \\
OMPONENT
\end{tabular} & 1 & 0,014998725 & 15 & 418 & 292 & 16403 & 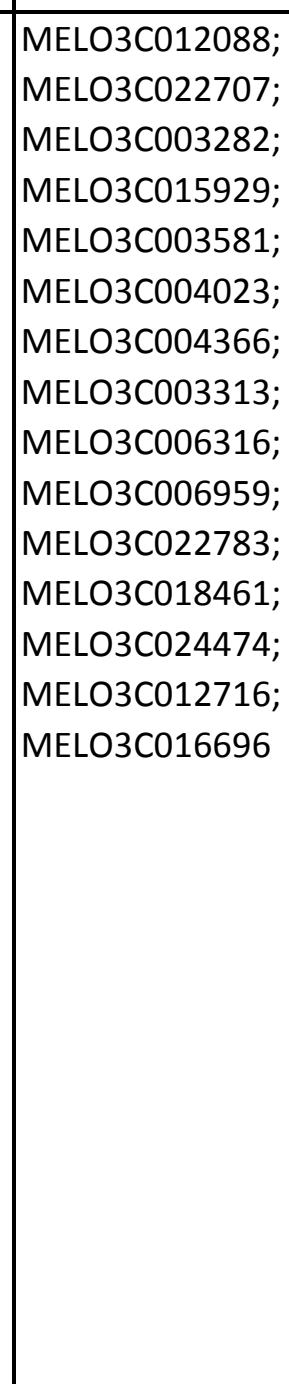 & 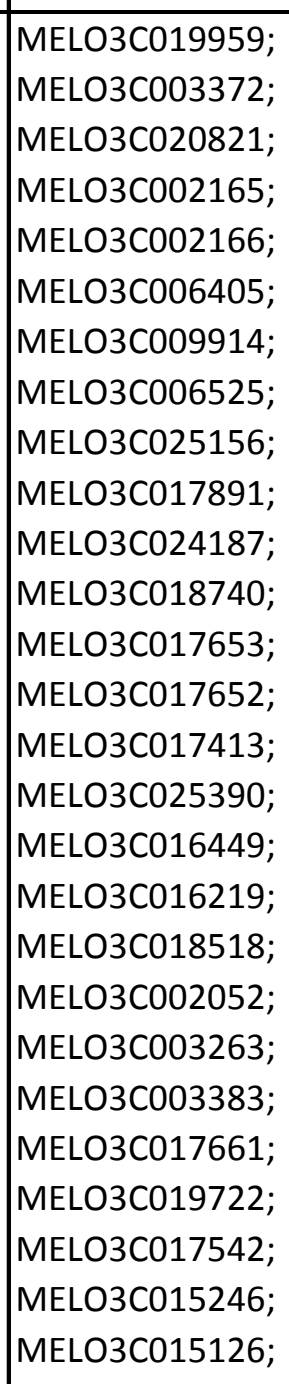 \\
\hline GO:0010445 & nuclear dicing body & \begin{tabular}{|l} 
CELUULAR_C \\
OMPONENT
\end{tabular} & 1 & $\begin{array}{ll}0,035527497 \\
\end{array}$ & 1 & 1 & 306 & 16820 & MELO3C015627 & MELO3C005929 \\
\hline 60:0009707 & chloroplast outer membrane & $\begin{array}{l}\text { CELLULAR_C } \\
\text { OMPONENT }\end{array}$ & 1 & 0,04920819 & 2 & 2 & 305 & 16803 & \begin{tabular}{|l|} 
MELLOSCO21253; \\
MELOOCOCO69774
\end{tabular} & 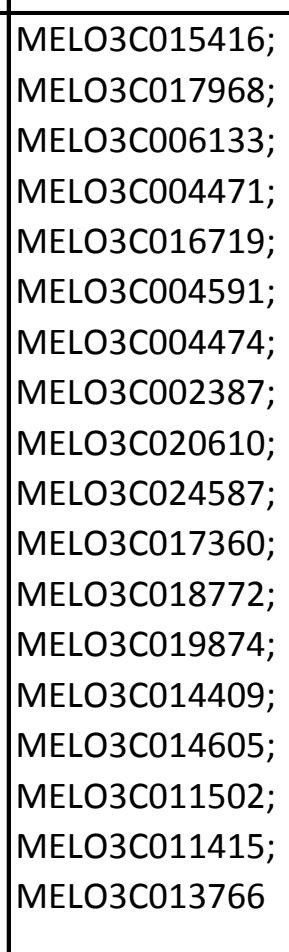 \\
\hline 60:0009706 & chloroplast inner membrane & $\begin{array}{l}\text { CELLULAR_C } \\
\text { OMPONENT }\end{array}$ & 1 & 0,044030549 & 3 & 41 & 304 & 16780 & \begin{tabular}{|l} 
MELO3C016354; \\
MELOSCO14321; \\
MELOSCO14379
\end{tabular} & 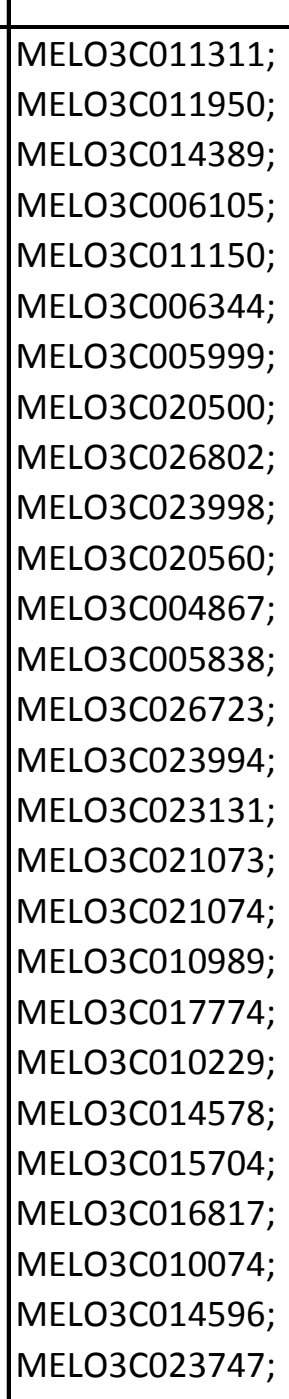 \\
\hline GO:0009705 & plant-type vacuole membrane & \begin{tabular}{|l|l} 
CELLULAR_C \\
OMPONENT
\end{tabular} & 1 & 0,025279833 & 2 & 12 & 305 & 16809 & $\begin{array}{l}\text { MELO3C016475; } \\
\text { MELO3C017757 }\end{array}$ & 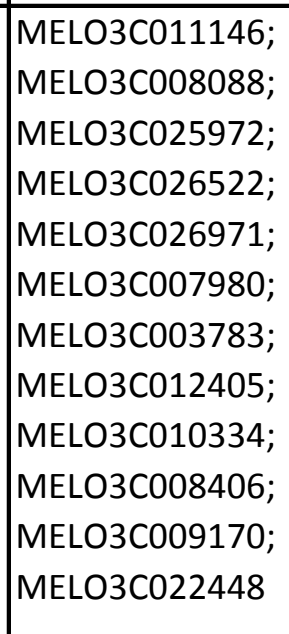 \\
\hline
\end{tabular}




\begin{tabular}{|c|c|c|c|c|c|c|c|c|c|c|}
\hline GO:0016607 & nuclear speck & \begin{tabular}{|l|} 
CELLULAR_C \\
OMPONENT
\end{tabular} & 1 & 0,016191216 & 3 & 27 & 304 & 16794 & $\begin{array}{l}\text { MELO3C024028; } \\
\text { MELO3C015627; } \\
\text { MELO3C017908 } \\
\end{array}$ & 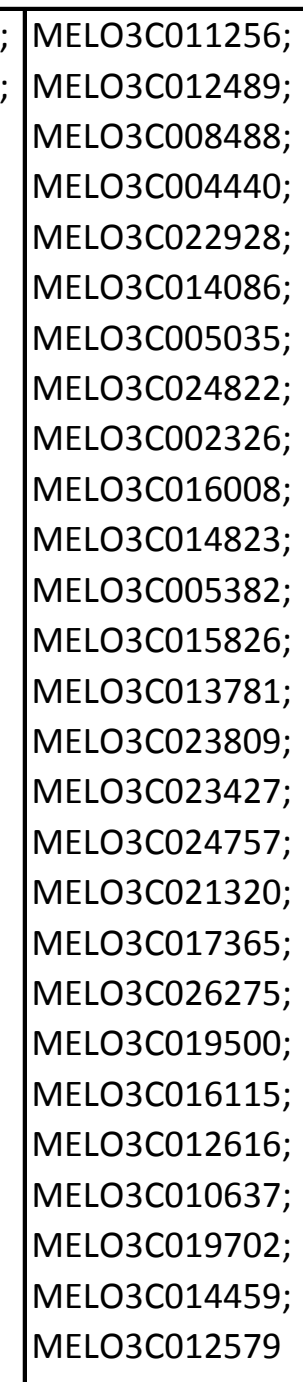 \\
\hline GO:0000151 & |ubiquitin ligase complex & $\begin{array}{l}\text { CELLULAR_C } \\
\text { OMPONENT }\end{array}$ & 1 & 1 & 6 & 132 & 301 & 16689 & \begin{tabular}{|} 
MELO3C006948; \\
MELO3CO07711; \\
MELO3CO11882; \\
MELO3CO05490; \\
MELO3CO14129; \\
MELO3CO09879
\end{tabular} & 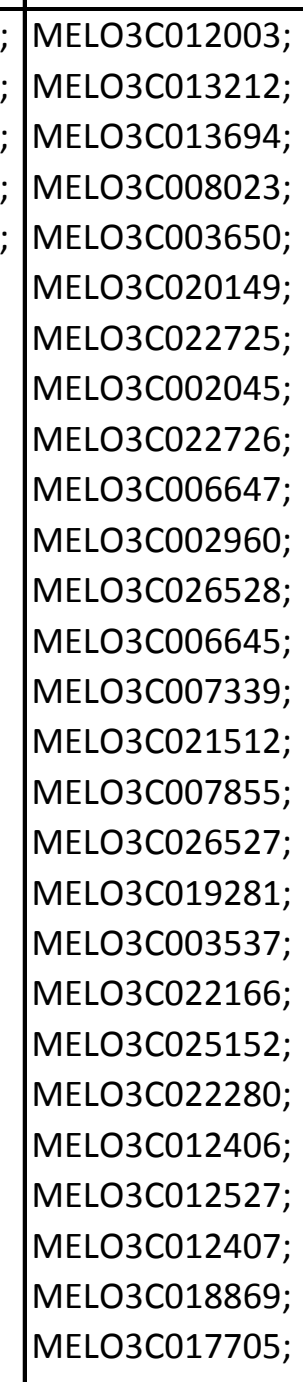 \\
\hline GO:0030692 & Noc4p-Nop14p complex & $\begin{array}{l}\text { CELLULAR_C } \\
\text { OMPONENT }\end{array}$ & 1 & 0,017923867 & 1 & 0 & 306 & 16821 & MELO3C011408 & \\
\hline GO:0000243 & commitment complex & $\begin{array}{l}\text { CELLULAR_C } \\
\text { OMPONENT }\end{array}$ & 1 & 0,017923867 & $0^{1}$ & 0 & 306 & 16821 & MELO3C026304 & \\
\hline GO:0010168 & ER body & \begin{tabular}{|l|} 
CELLULAR_C \\
OMPONENT
\end{tabular} & 1 & 0,017923867 & 1 & 0 & 306 & 16821 & MELO3C012716 & \\
\hline
\end{tabular}


Supplementary Table 2.F Gene Ontology terms (GO terms) of the cluster 2.5 obtained considering the comparison between lines the postharvest ripening time fruit during storage at $20.5^{\circ} \mathrm{C}$ and $88 \%$ relative humidity for $18 \mathrm{~d}$ the near-isogenic line $\mathrm{SC} 10-2$ and its parental "Piel de Sapo" obtained according to the differentially expressed gene pattern (Supl. Table 2.A)

\begin{tabular}{|c|c|c|c|c|c|c|c|c|c|c|}
\hline GOID & GO Name & GO Category & $\begin{array}{l}\text { FDR } \\
\end{array}$ & 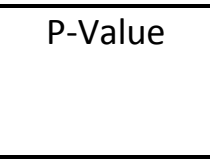 & SC10-2 & PS & $\begin{array}{l}\text { Non } \\
\text { Annot } \\
\text { Test } \\
\end{array}$ & $\begin{array}{l}\text { Non Annot } \\
\text { Reference }\end{array}$ & $\begin{array}{l}\begin{array}{l}\text { TestSet } \\
\text { Sequences }\end{array} \\
\end{array}$ & $\begin{array}{l}\text { RefSet } \\
\text { Sequences }\end{array}$ \\
\hline GO:0016308 & 1-phosphatidylinositol-4-phosphate 5-kinase activity & MOLECULAR_FUNCTION & 0,304197153 & $2,25 \mathrm{E}-04$ & 3 & 7 & 214 & 16904 & $\begin{array}{l}\text { MELOЗCO10456; } \\
\text { MELO3CO06050; } \\
\text { MELO3CO03057 }\end{array}$ & $\begin{array}{l}\text { MELO3CO19075; } \\
\text { MELO3COOO333; } \\
\text { MELO3CO12475; } \\
\text { MELO3CO14374; } \\
\text { MELO3CO05148; } \\
\text { MELO3CO09382; } \\
\text { MELO3C010116 }\end{array}$ \\
\hline GO:0055114 & oxidation-reduction process & BIOLOGICAL_PROCESS & 0,340910349 & $3,28 \mathrm{E}-04$ & 7 & 1688 & 210 & 15223 & $\begin{array}{l}\text { MELO3C024222; } \\
\text { MELO3CO13829; } \\
\text { MELO3CO19404; } \\
\text { MELO3COO9886; } \\
\text { MELO3CO2398; } \\
\text { MELO3CO11601; } \\
\text { MELO3C001994 }\end{array}$ & 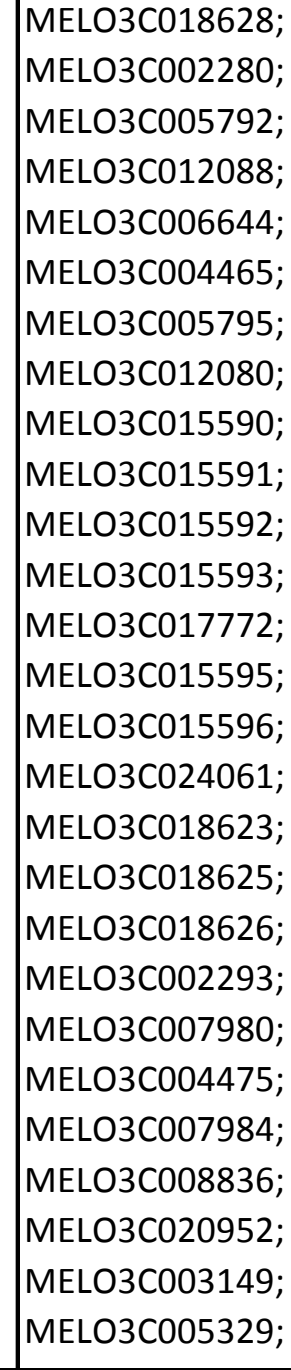 \\
\hline GO:0033169 & histone $\mathrm{H3}-\mathrm{K} 9$ demethylation & BIOLOGICAL_PROCESS & 0,404853209 & $9,43 \mathrm{E}-04$ & 2 & 2 & 215 & 16909 & $\begin{array}{l}\text { MELOSC025053; } \\
\text { MELO3C019813 }\end{array}$ & $\begin{array}{l}\text { MELO3CO02327; } \\
\text { MELO3CO04836 }\end{array}$ \\
\hline GO:0008270 & zinc ion binding & MOLECULAR_FUNCTION & 0,404853209 & $(2)$ & 31 & 1246 & 186 & 15665 & 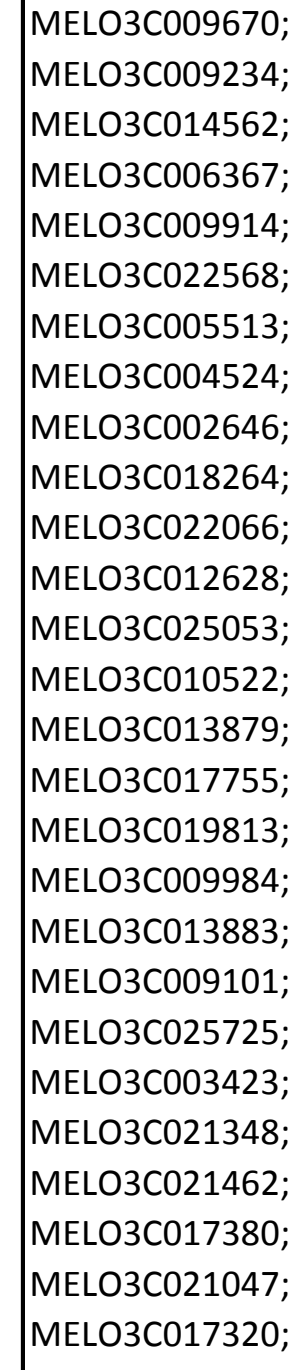 & 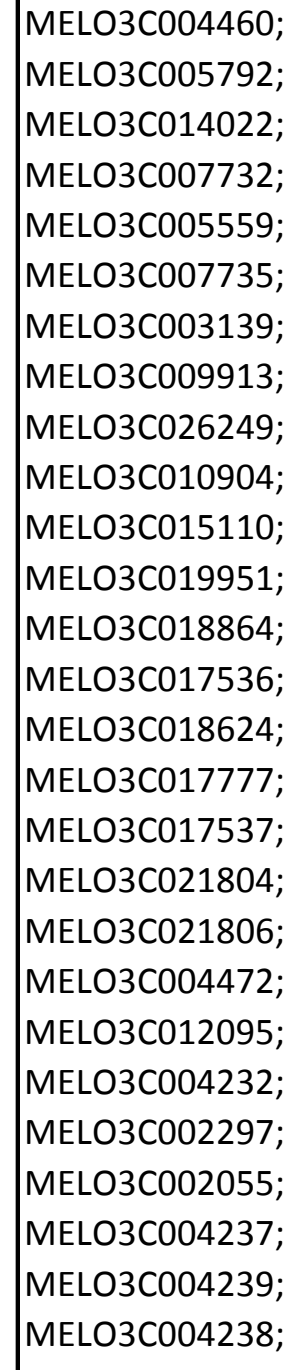 \\
\hline GO:0031491 & nucleosome binding & MOLECULAR_FUNCTION & 0,404853209 & $9,43 \mathrm{E}-04$ & 2 & 2 & 215 & 16909 & $\begin{array}{l}\text { MELO3C021353; } \\
\text { MELO3CO04459 }\end{array}$ & $\begin{array}{l}\text { MELO3C021352; } \\
\text { MELO3CO09890 }\end{array}$ \\
\hline GO:0005524 & ATP binding & MOLECULAR_FUNCTION & 0,705878947 & 0,00310521 & 41 & 2020 & 176 & 14891 & 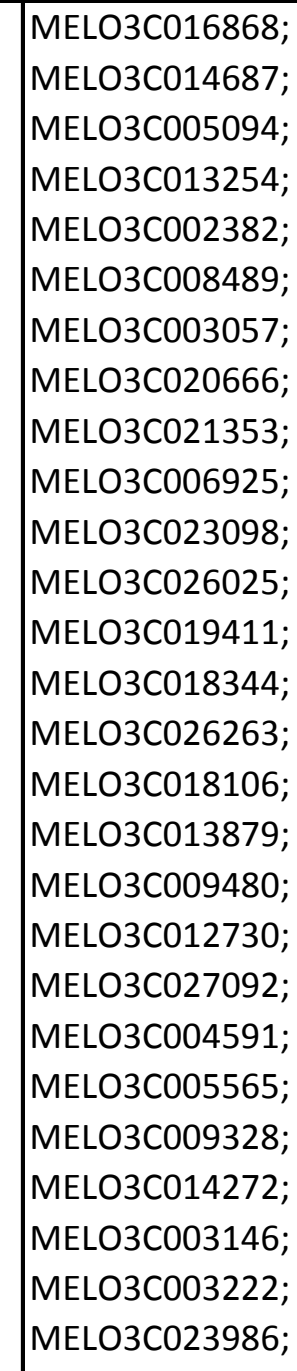 & 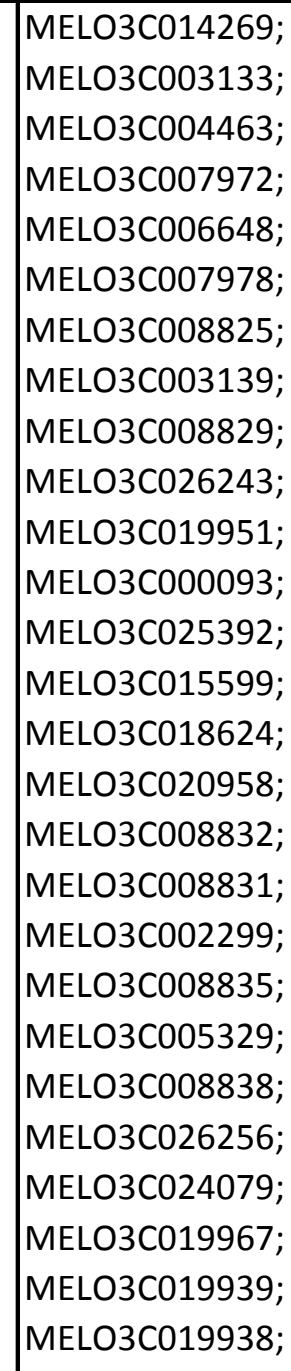 \\
\hline
\end{tabular}




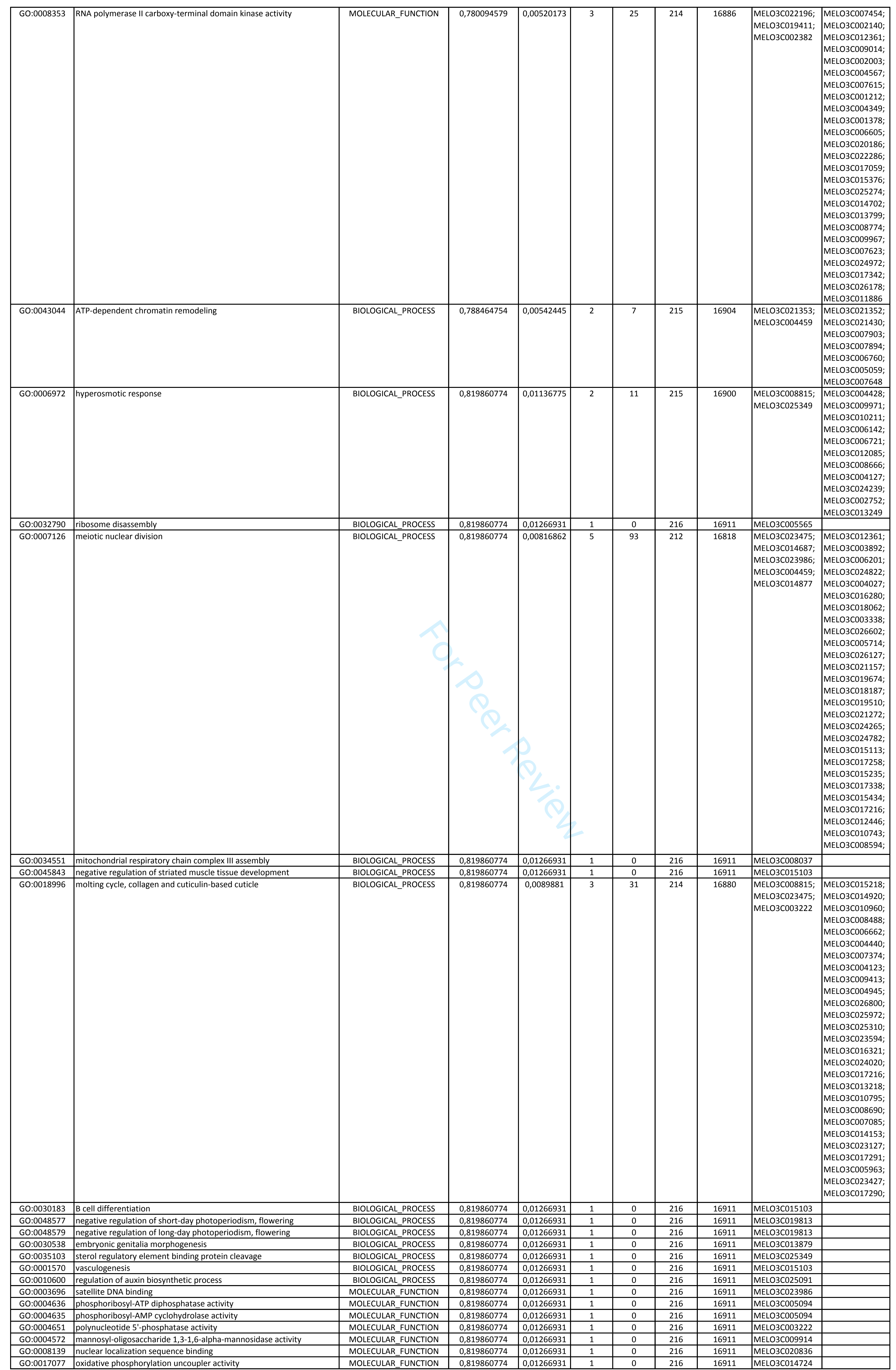




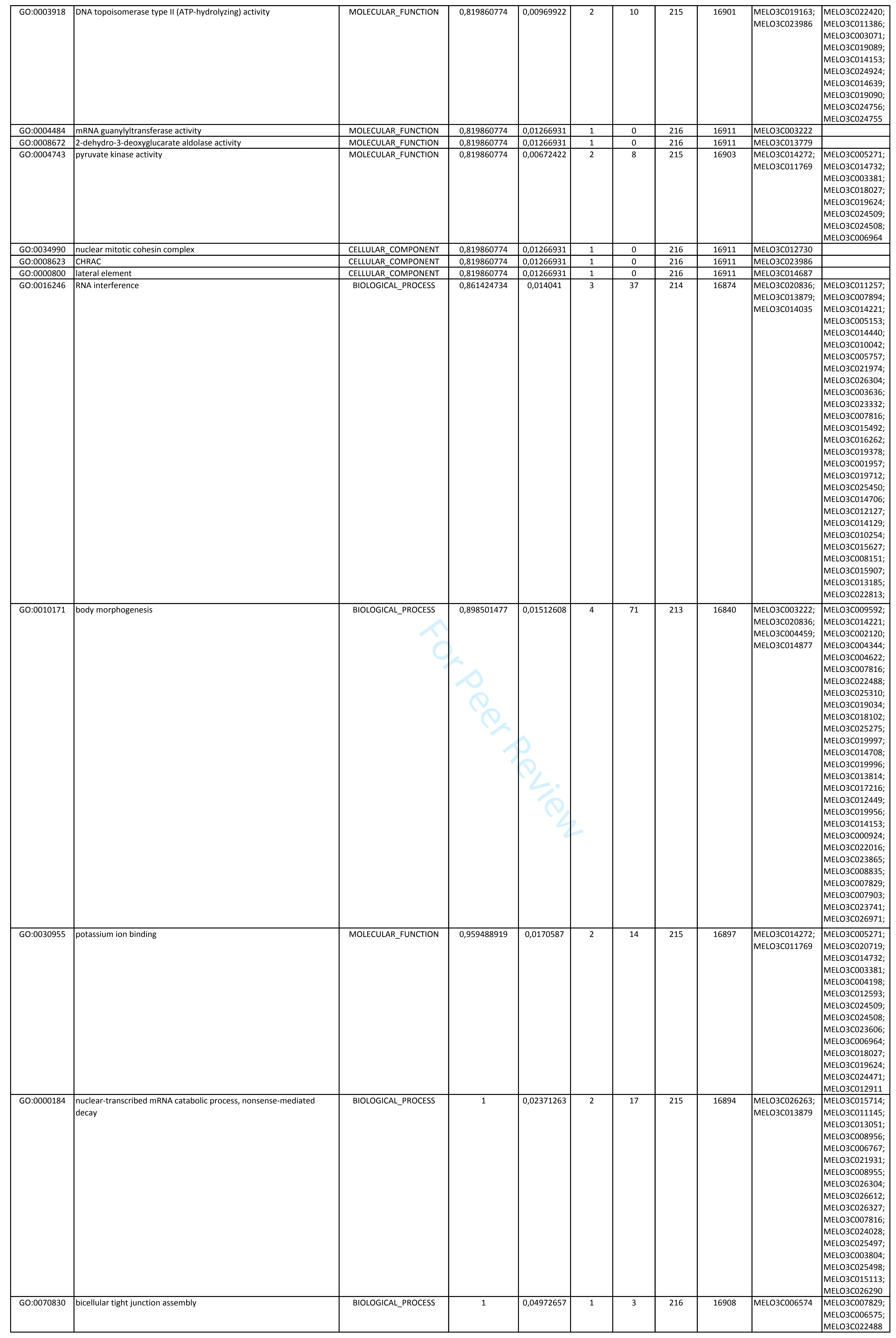




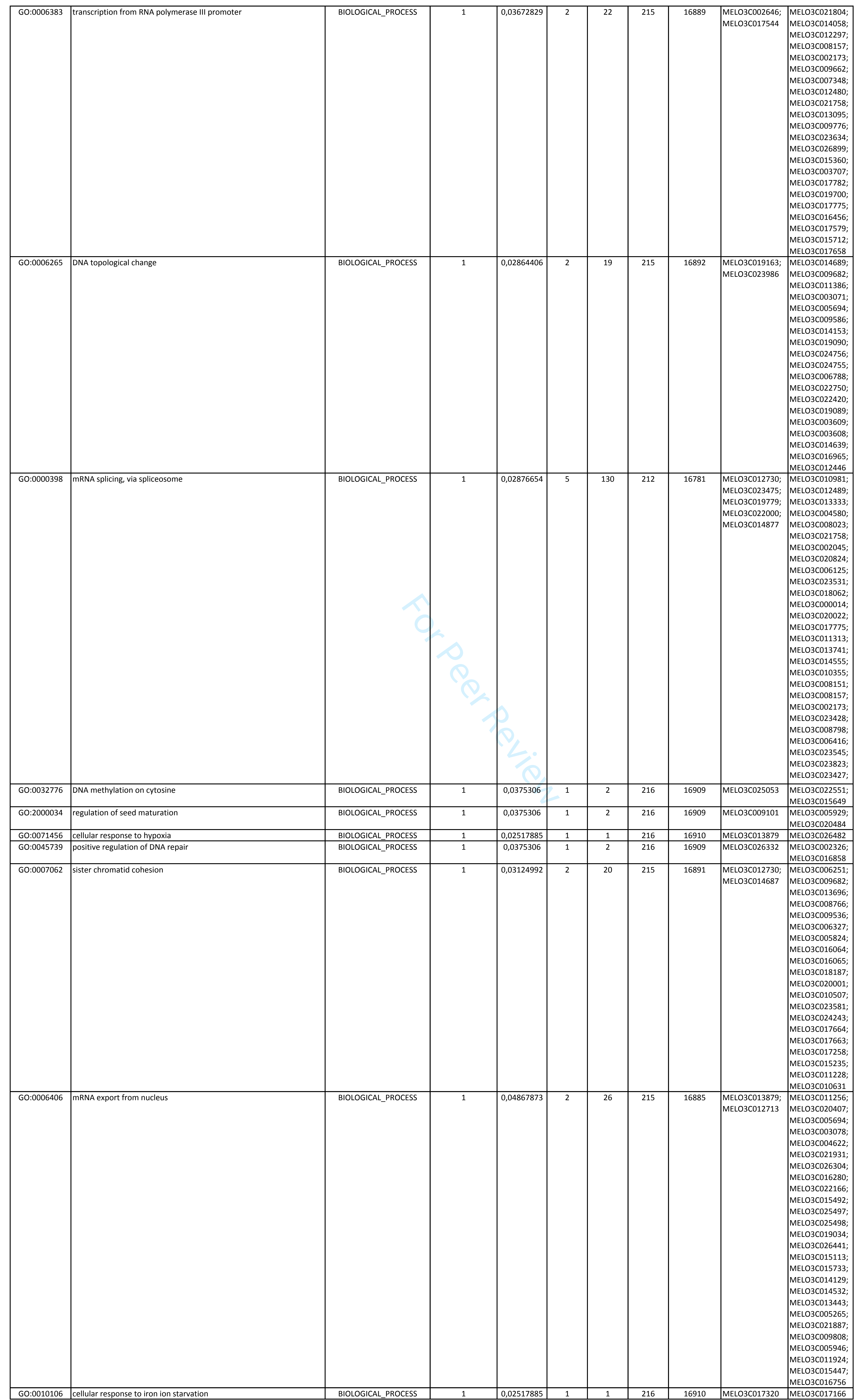




\begin{tabular}{|c|c|c|c|c|c|c|c|c|c|c|}
\hline GO:0007091 & metaphase/anaphase transition of mitotic cell cycle & BIOLOGICAL_PROCESS & 1 & 0,02864406 & 2 & 19 & 215 & 16892 & $\begin{array}{l}\text { MELO3C012730; } \\
\text { MELO3C022930 }\end{array}$ & 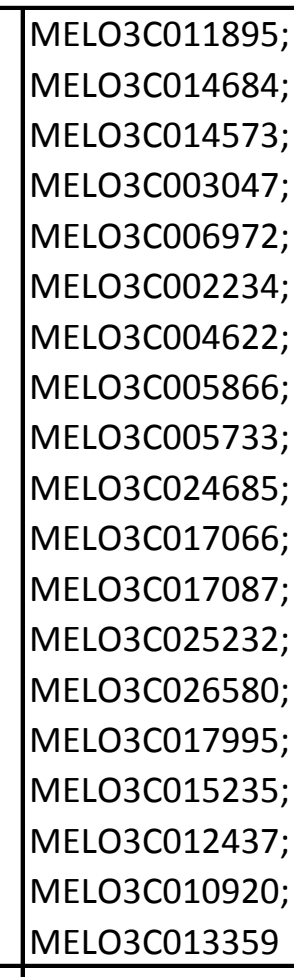 \\
\hline G0:0032049 & cardiolipin biosynthetic process & BIOLOGICAL_PROCESS & 1 & 0,02517885 & 1 & 1 & 216 & 16910 & MELO3C007010 & MELO3C013700 \\
\hline GO:0010030 & positive regulation of seed germination & BIOLOGICAL_PROCESS & 1 & 0,04972657 & 1 & 3 & 216 & 16908 & MELO3C009101 & $\begin{array}{l}\text { MELO3C020484; } \\
\text { MELO3C004632; } \\
\text { MELO3C009627 }\end{array}$ \\
\hline GO:0006569 & tryptophan catabolic process & BIOLOGICAL_PROCESS & 1 & 0,04972657 & 1 & 3 & 216 & 16908 & MELO3C012201 & $\begin{array}{l}\text { MELO3CO23775; } \\
\text { MELO3CO10461; } \\
\text { MELO3C024595 }\end{array}$ \\
\hline GO:0031055 & chromatin remodeling at centromere & BIOLOGICAL_PROCESS & 1 & 0,04972657 & 1 & 3 & 216 & 16908 & MELO3C023986 & $\begin{array}{l}\text { MELOZCO06760; } \\
\text { MELO3COO5059; } \\
\text { MELO3CO17558 }\end{array}$ \\
\hline GO:0016233 & telomere capping & BIOLOGICAL_PROCESS & 1 & \begin{tabular}{|l|}
0,0375306 \\
\end{tabular} & 1 & 2 & 216 & 16909 & MELO3C014298 & $\begin{array}{l}\text { MELO3CO18187; } \\
\text { MELO3C006292 }\end{array}$ \\
\hline GO:0010495 & long-distance posttranscriptional gene silencing & BIOLOGICAL_PROCESS & 1 & 0,02517885 & 1 & 1 & 216 & 16910 & MELO3C014035 & MELO3C017106 \\
\hline GO:0046488 & phosphatidylinositol metabolic process & BIOLOGICAL_PROCESS & 8 & 0,0422135 & 3 & 58 & 214 & 16853 & $\begin{array}{l}\text { MELO3C010456; } \\
\text { MELO3C006050; } \\
\text { MELO3C003057 }\end{array}$ & 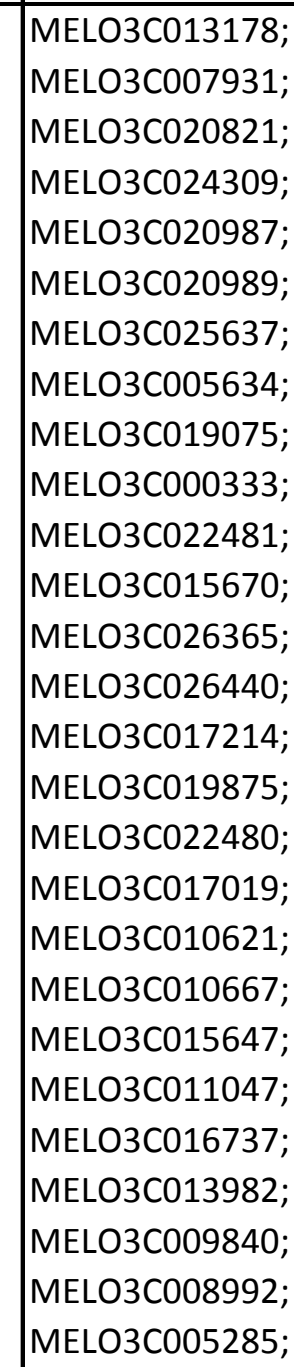 \\
\hline GO:0019318 & hexose metabolic process & BIOLOGICAL_PROCESS & 1 & \begin{tabular}{|r}
0,04860452 \\
\\
\end{tabular} & 5 & 151 & 212 & 16760 & $\begin{array}{l}\text { MELO3C014272; } \\
\text { MELO3C011769; } \\
\text { MELO3COO9914; } \\
\text { MELO3CO14724; } \\
\text { MELO3C013829 }\end{array}$ & 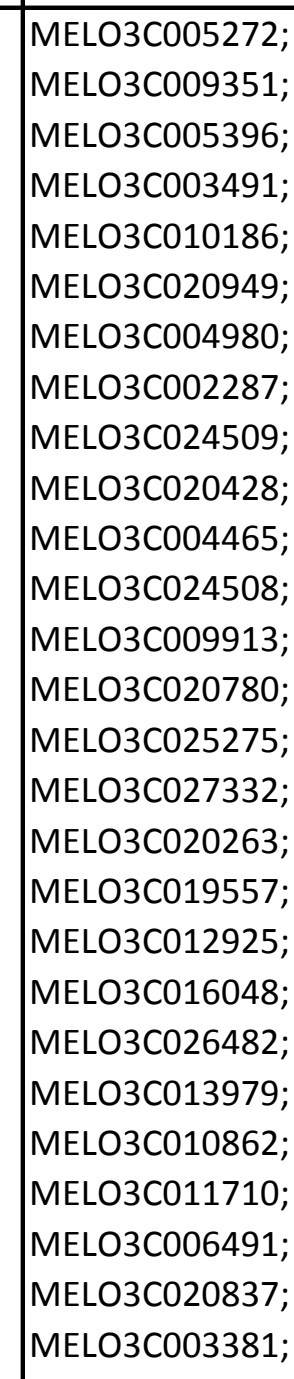 \\
\hline GO:0030466 & chromatin silencing at silent mating-type cassette & BIOLOGICAL_PROCESS & 1 & \begin{tabular}{|l|}
0,04972657 \\
\end{tabular} & 1 & 3 & 216 & 16908 & MELO3C013879 & $\begin{array}{l}\text { MELO3CO22562; } \\
\text { MELO3CO09773; } \\
\text { MELO3C0108882 }\end{array}$ \\
\hline GO:0071044 & histone mRNA catabolic process & BIOLOGICAL_PROCESS & 1 & \begin{tabular}{|l|}
0,0375306 \\
\end{tabular} & 1 & 2 & 216 & 16909 & MELO3C013879 & $\begin{array}{l}\text { MELO3C011748; } \\
\text { MELO3C017087 }\end{array}$ \\
\hline GO:0042078 & germ-line stem cell division & BIOLOGICAL_PROCESS & 1 & 0,02517885 & 1 & 1 & 216 & 16910 & MELO3C008815 & MELO3C008594 \\
\hline GO:0006346 & methylation-dependent chromatin silencing & BIOLOGICAL_PROCESS & 1 & 0,02517885 & 1 & 1 & 216 & 16910 & MELO3C014035 & MELO3C017558 \\
\hline GO:0005506 & iron ion binding & MOLECULAR_FUNCTION & 1 & 0,03808894 & 0 & 324 & 217 & 16587 & & 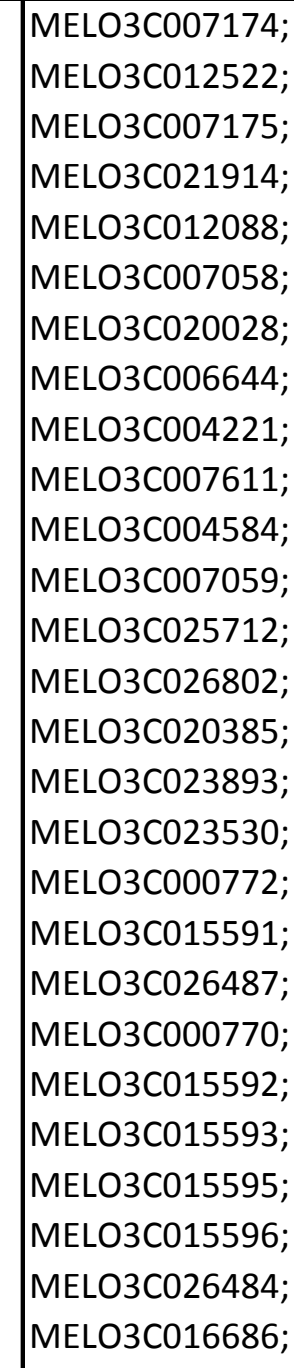 \\
\hline GO:0000182 & rDNA binding & MOLECULAR_FUNCTION & 1 & 0,0375306 & 1 & 2 & 216 & 16909 & C023986 & $\begin{array}{l}\text { MELOЗ3C012480; } \\
\text { MELOЗC020235 }\end{array}$ \\
\hline
\end{tabular}




\begin{tabular}{|c|c|c|c|c|c|c|c|c|c|c|}
\hline GO:0000156 & phosphorelay response regulator activity & MOLECULAR_FUNCTION & 1 & 0,04557323 & 2 & 25 & 215 & 16886 & $\begin{array}{l}\text { MELO3C017128; } \\
\text { MELO3C020055 }\end{array}$ & 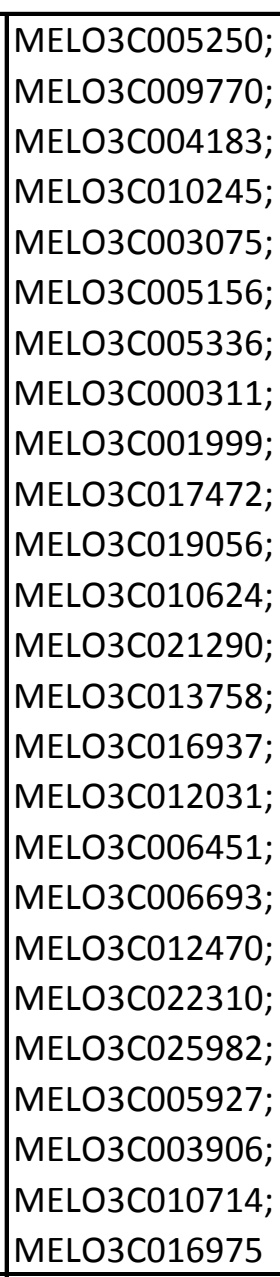 \\
\hline GO:0003680 & AT DNA binding & MOLECULAR_FUNCTION & 1 & 0,02517885 & 1 & 1 & 216 & 16910 & MELO3C012730 & MELO3C022471 \\
\hline GO:0051721 & protein phosphatase $2 \mathrm{~A}$ binding & MOLECULAR_FUNCTION & 1 & 0,04972657 & 1 & 3 & 216 & 16908 & MELO3C013879 & 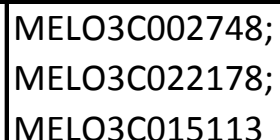 \\
\hline GO:0047800 & cysteamine dioxygenase activity & MOLECULAR_FUNCTION & 1 & \begin{tabular}{|l|}
0,04972657 \\
\end{tabular} & 1 & 3 & 216 & 16908 & MELO3C009886 & $\begin{array}{l}\text { MELO3C017267; } \\
\text { MELO3CO08836; } \\
\text { MELO3C013579 }\end{array}$ \\
\hline GO:0016435 & rRNA (guanine) methyltransferase activity & MOLECULAR_FUNCTION & 1 & \begin{tabular}{|l|}
0,0375306 \\
\end{tabular} & 1 & 2 & 216 & 16909 & MELO3C023306 & $\begin{array}{l}\text { MELO3C002238; } \\
\text { MELO3C015412 }\end{array}$ \\
\hline GO:0000400 & four-way junction DNA binding & MOLECULAR_FUNCTION & 1 & 0,02517885 & 1 & 1 & 216 & 16910 & MELO3C023986 & MELO3C015928 \\
\hline GO:0008138 & protein tyrosine/serine/threonine phosphatase activity & MOLECULAR_FUNCTION & 1 & 0,02371263 & 2 & 17 & 215 & 16894 & $\begin{array}{l}\text { MELO3C023830; } \\
\text { MELO3CO03222 }\end{array}$ & 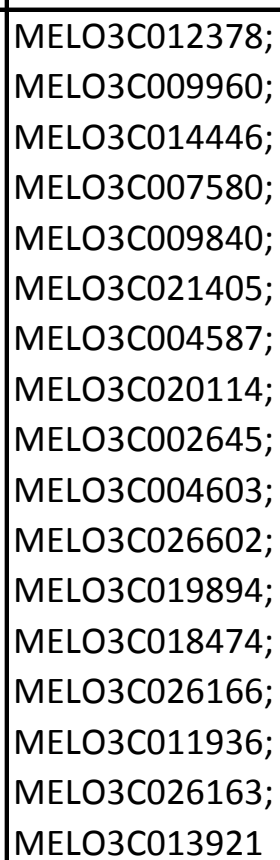 \\
\hline GO:0004385 & \begin{tabular}{|l} 
guanylate kinase activity \\
\end{tabular} & MOLECULAR_FUNCTION & 1 & 0,04972657 & 1 & 3 & 216 & 16908 & MELO3C006574 & 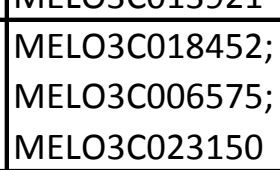 \\
\hline GO:0003729 & mRNA binding & MOLECULAR_FUNCTION & & 0,01917371 & 2 & 15 & 215 & 16896 & $\begin{array}{l}\text { MELO3C012411; } \\
\text { MELO3C023986 }\end{array}$ & 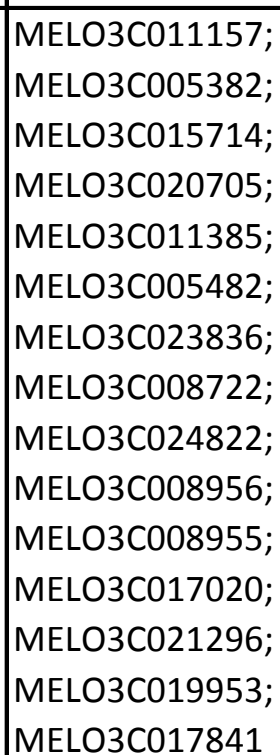 \\
\hline GO:0003955 & NAD(P)H dehydrogenase (quinone) activity & MOLECULAR_FUNCTION & 1 & \begin{tabular}{|l|}
0,0375306 \\
\end{tabular} & 1 & 2 & 216 & 16909 & MELO3C007950 & $\begin{array}{l}\text { MELO3CO05147; } \\
\text { MELO3C012476 }\end{array}$ \\
\hline GO:0008780 & $\begin{array}{l}\text { acyl-[acyl-carrier-protein]-UDP-N-acetylglucosamine O-acyltransferase } \\
\text { activity }\end{array}$ & MOLECULAR_FUNCTION & 1 & 0,02517885 & 1 & 1 & 216 & 16910 & MELO3C015455 & MELO3C007369 \\
\hline GO:0009884 & cytokinin receptor activity & MOLECULAR_FUNCTION & 1 & 0,04972657 & 1 & 3 & 216 & 16908 & MELO3C020055 & $\begin{array}{l}\text { MELO3CO05250; } \\
\text { MELO3C022310; } \\
\text { MELO3C025982 }\end{array}$ \\
\hline GO:0004864 & protein phosphatase inhibitor activity & MOLECULAR_FUNCTION & 1 & 0,04972657 & 1 & 3 & 216 & 16908 & MELO3C021670 & $\begin{array}{l}\text { MELO3C002748; } \\
\text { MELO3C022178; } \\
\text { MELO3C005380 }\end{array}$ \\
\hline GO:0003743 & translation initiation factor activity & MOLECULAR_FUNCTION & 1 & 0,04989364 & 4 & 105 & 213 & 16806 & $\begin{array}{l}\text { MELO3C002646; } \\
\text { MELO3CO02819; } \\
\text { MELO3CO02817; } \\
\text { MELO3C006107 }\end{array}$ & 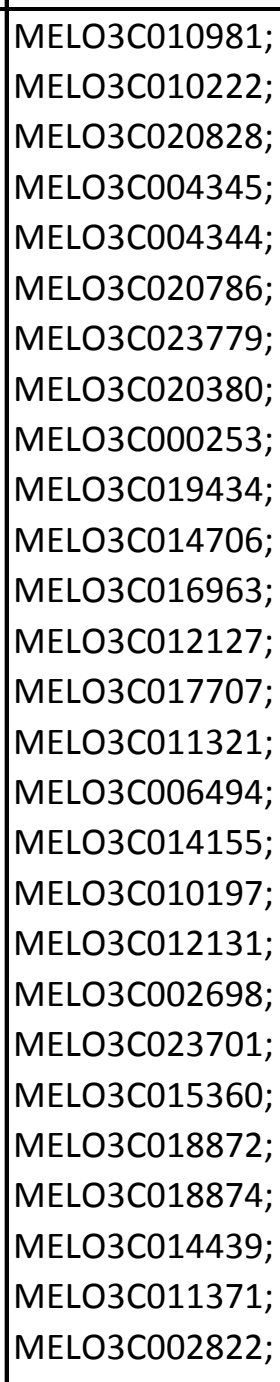 \\
\hline GO:0008650 & rRNA (uridine-2'-O---methyltransferase activity & MOLECULAR_FUNCTION & 1 & 0,02517885 & $\begin{array}{l}1 \\
\end{array}$ & 1 & 216 & 16910 & 3306 & MELO3C009184 \\
\hline
\end{tabular}




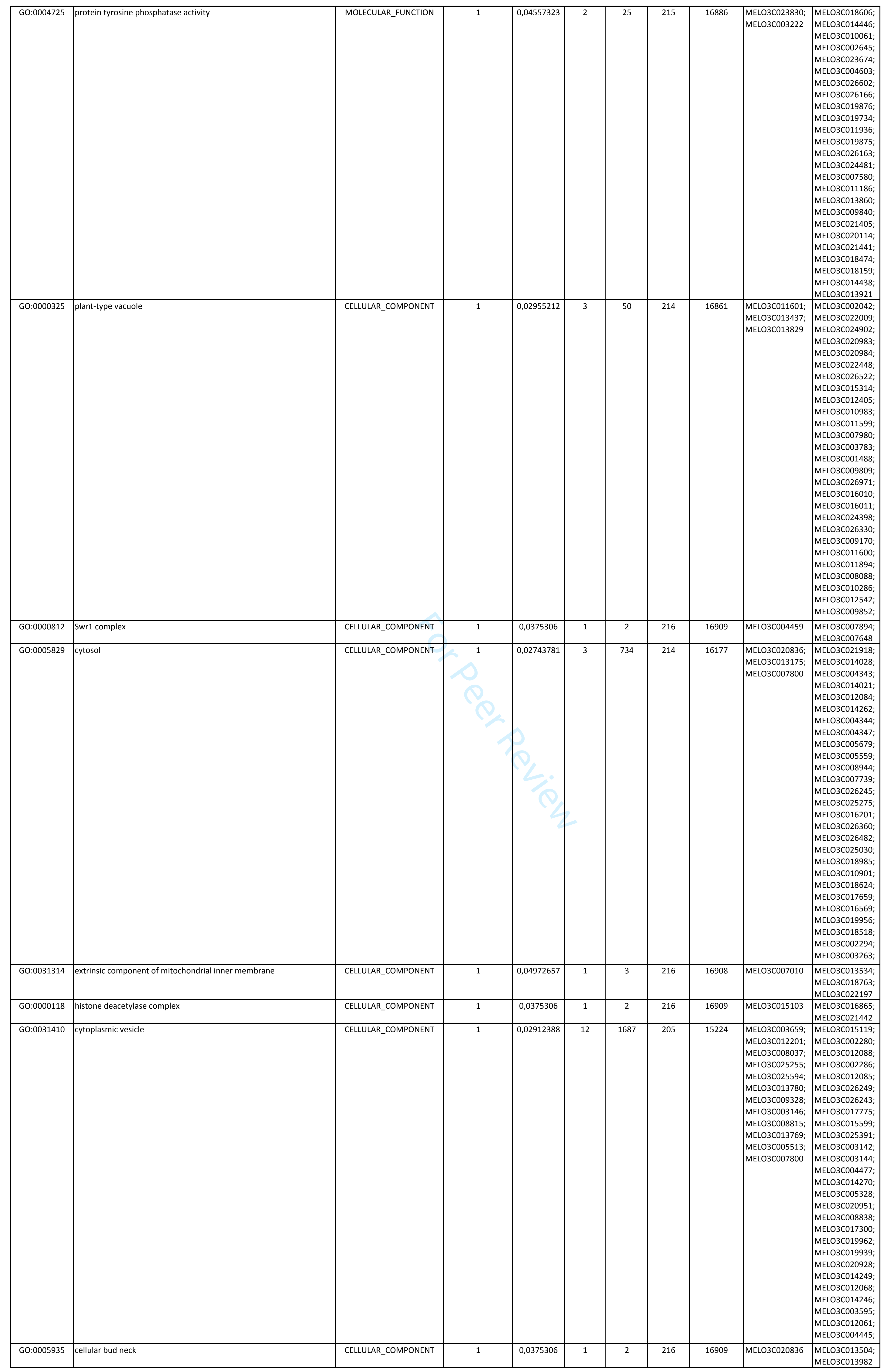


Supplementary Table 2.G Gene Ontology terms (GO terms) of the cluster 2.6 obtained considering the comparison between lines the postharvest ripening time fruit during storage at $20.5^{\circ} \mathrm{C}$ and $88 \%$ relative humidity for $18 \mathrm{~d}$ the near-isogenic line SC10-2 and its parental "Piel de Sapo" obtained according to the differentially expressed gene pattern (Supl. Table 2.A)

\begin{tabular}{|c|c|c|c|c|c|c|c|c|c|c|}
\hline GOID & GO Name & GO Category & FDR & P-Value & SC10-2 & PS & $\begin{array}{c}\text { Non } \\
\text { Annot } \\
\text { Test } \\
\end{array}$ & $\begin{array}{l}\text { Non Annot } \\
\text { Reference }\end{array}$ & \begin{tabular}{|l} 
TestSet \\
Sequences
\end{tabular} & \begin{tabular}{|l|} 
RefSet \\
Sequences
\end{tabular} \\
\hline GO:0016206 & catechol O-methyltransferase activity & MOLECULAR_FUNCTION & 0,281888732 & $5,97 \mathrm{E}-05$ & 2 & 1 & 75 & 17050 & $\begin{array}{l}\text { MELO3C027330; } \\
\text { MELO3C014089 }\end{array}$ & MELO3C024861 \\
\hline GO:0042446 & hormone biosynthetic process & BIOLOGICAL_PROCESS & 0,510918541 & $1,62 \mathrm{E}-04$ & 4 & 57 & 73 & 16994 & $\begin{array}{l}\text { MELOZC027330; } \\
\text { MELO3C027370; } \\
\text { MELO3C014089; } \\
\text { MELO3C013315 }\end{array}$ & 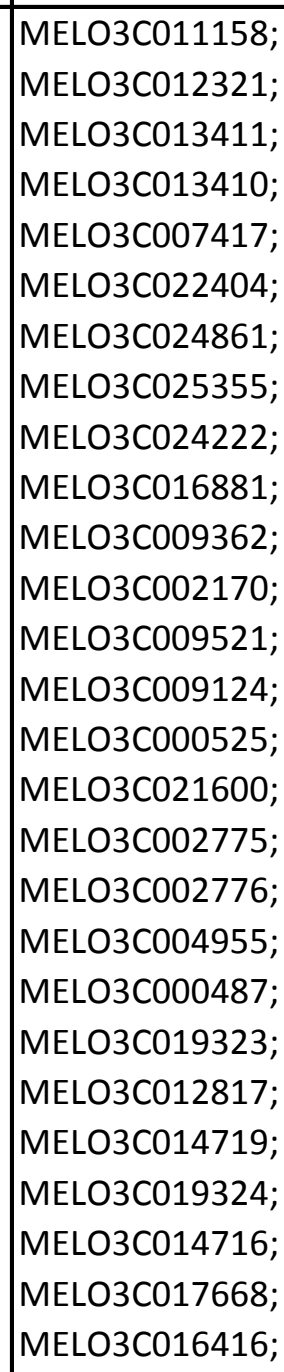 \\
\hline GO:0015095 & $\begin{array}{l}\text { magnesium ion transmembrane transporter } \\
\text { activity }\end{array}$ & MOLECULAR_FUNCTION & 0,659113425 & $4,13 \mathrm{E}-04$ & 2 & 5 & 75 & 17046 & $\begin{array}{l}\text { MELO3C026764; } \\
\text { MELO3C000817 }\end{array}$ & $\begin{array}{l}\text { MELO3C026733; } \\
\text { MELO3CO00103; } \\
\text { MELO3C011418; } \\
\text { MELO3C019432; } \\
\text { MELO3C004841 }\end{array}$ \\
\hline GO:0006968 & cellular defense response & BIOLOGICAL_PROCESS & 0,94386839 & 0,004495563 & 1 & 0 & 76 & 17051 & MELO3C006765 & \\
\hline GO:0071395 & cellular response to jasmonic acid stimulus & BIOLOGICAL_PROCESS & 0,94386839 & 0,002016649 & 2 & 13 & 75 & 17038 & $\begin{array}{l}\text { MELOSC006765; } \\
\text { MELO3C024357 }\end{array}$ & 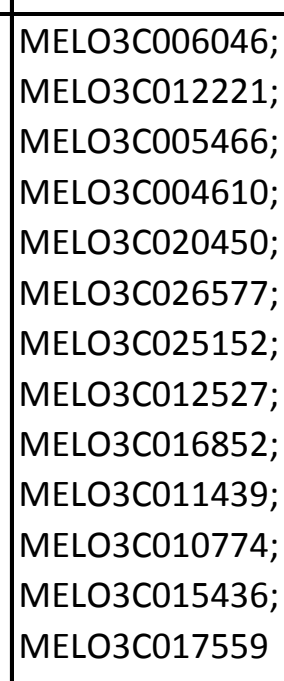 \\
\hline GO:0071947 & $\begin{array}{l}\text { protein deubiquitination involved in ubiquitin- } \\
\text { dependent protein catabolic process }\end{array}$ & BIOLOGICAL_PROCESS & 0,94386839 & 0,004495563 & 1 & 0 & 76 & 17051 & MELO3C018956 & \\
\hline GO:0010247 & detection of phosphate ion & BIOLOGICAL_PROCESS & 0,94386839 & 0,004495563 & 1 & 0 & 76 & 17051 & MELO3C005035 & \\
\hline GO:0010400 & $\begin{array}{l}\text { rhamnogalacturonan I side chain metabolic } \\
\text { process }\end{array}$ & BIOLOGICAL_PROCESS & 0,94386839 & 0,004495563 & 1 & 0 & 76 & 17051 & MELO3C003916 & \\
\hline GO:0070485 & $\begin{array}{l}\text { dehydro-D-arabinono-1,4-lactone } \\
\text { biosynthetic process }\end{array}$ & BIOLOGICAL_PROCESS & 0,94386839 & 0,004495563 & 1 & 0 & 76 & 17051 & MELO3C018576 & \\
\hline GO:2000311 & regulation of AMPA receptor activity & BIOLOGICAL_PROCESS & 0,94386839 & 0,004495563 & 1 & 0 & 76 & 17051 & MELO3C018956 & \\
\hline GO:0034498 & early endosome to Golgi transport & BIOLOGICAL_PROCESS & 0,94386839 & 0,004495563 & 1 & 0 & 76 & 17051 & MELO3C024558 & \\
\hline GO:2000070 & regulation of response to water deprivation & BIOLOGICAL_PROCESS & 0,94386839 & 0,004495563 & 1 & 0 & 76 & 17051 & MELO3C005035 & \\
\hline GO:0047834 & D-threo-aldose 1-dehydrogenase activity & MOLECULAR_FUNCTION & 0,94386839 & 0,004495563 & 1 & 0 & 76 & 17051 & MELO3C018576 & \\
\hline GO:0005290 & $\begin{array}{l}\text { L-histidine transmembrane transporter } \\
\text { activity }\end{array}$ & MOLECULAR_FUNCTION & 0,94386839 & 0,004495563 & 1 & 0 & 76 & 17051 & MELO3C002619 & \\
\hline GO:0035255 & ionotropic glutamate receptor binding & MOLECULAR_FUNCTION & 0,94386839 & 0,004495563 & 1 & 0 & 76 & 17051 & MELO3C018956 & \\
\hline GO:0050235 & pyridoxal 4-dehydrogenase activity & MOLECULAR_FUNCTION & 0,94386839 & 0,004495563 & 1 & 0 & 76 & 17051 & MELO3C018576 & \\
\hline GO:0045290 & $\begin{array}{l}\text { D-arabinose 1-dehydrogenase }[\mathrm{NAD}(\mathrm{P})+] \\
\text { activity }\end{array}$ & MOLECULAR_FUNCTION & 0,94386839 & 0,004495563 & 1 & 0 & 76 & 17051 & MELO3C018576 & \\
\hline GO:0019151 & galactose 1-dehydrogenase activity & MOLECULAR_FUNCTION & 0,94386839 & 0,004495563 & 1 & 0 & 76 & 17051 & MELO3C018576 & \\
\hline GO:0010349 & L-galactose dehydrogenase activity & MOLECULAR_FUNCTION & 0,94386839 & 0,004495563 & 1 & 0 & 76 & 17051 & MELO3C018576 & \\
\hline GO:0047816 & D-arabinose 1-dehydrogenase (NAD) activity & MOLECULAR_FUNCTION & 0,94386839 & 0,004495563 & 1 & 0 & 76 & 17051 & MELO3C018576 & \\
\hline GO:0000172 & ribonuclease MRP complex & CELLULAR_COMPONENT & 0,94386839 & 0,004495563 & 1 & 0 & 76 & 17051 & MELOЗC009318 & \\
\hline GO:0005655 & nucleolar ribonuclease $\mathrm{P}$ complex & CELLULAR_COMPONENT & 0,94386839 & 0,004495563 & 1 & 0 & 76 & 17051 & MELOSCOOOS18 & \\
\hline GO:0007098 & centrosome cycle & BIOLOGICAL_PROCESS & 1 & 0,026675887 & 1 & 5 & 76 & 17046 & MELO3C013369 & $\begin{array}{l}\text { MELO3C015782; } \\
\text { MELO3CO24150; } \\
\text { MELO3CO22094; } \\
\text { MELO3C019034; } \\
\text { MELO3C010920 }\end{array}$ \\
\hline GO:0016925 & protein sumoylation & BIOLOGICAL_PROCESS & 1 & 0,026675887 & 1 & 5 & 76 & 17046 & MELO3C005035 & $\begin{array}{l}\text { MELO3C005717; } \\
\text { MELO3CO25617; } \\
\text { MELO3C014183; } \\
\text { MELO3C023325; } \\
\text { MELO3C024782 }\end{array}$ \\
\hline GO:0046854 & phosphatidylinositol phosphorylation & BIOLOGICAL_PROCESS & 1 & 0,0397492 & 1 & 8 & 76 & 17043 & MELO3C020989 & $\begin{array}{l}\text { MELO3C012544; } \\
\text { MELO3CO0805; } \\
\text { MELO3CO13045; } \\
\text { MELO3CO20987; } \\
\text { MELO3CO26141; } \\
\text { MELO3CO12545; } \\
\text { MELO3CO106667; } \\
\text { MELO3C021931 }\end{array}$ \\
\hline GO:0030259 & lipid glycosylation & BIOLOGICAL_PROCESS & 1 & 0,044068338 & 1 & 9 & 76 & 17042 & MELO3C015339 & $\begin{array}{l}\text { MELO3C011311; } \\
\text { MELO3CO10079; } \\
\text { MELO3CO20997; } \\
\text { MELO3CO04474; } \\
\text { MELO3CO1008; } \\
\text { MELO3CO02112; } \\
\text { MELO3CO02113; } \\
\text { MELO3C012787; } \\
\text { MELO3C008339 }\end{array}$ \\
\hline
\end{tabular}




\begin{tabular}{|c|c|c|c|c|c|c|c|c|c|c|}
\hline GO:0034727 & piecemeal microautophagy of nucleus & BIOLOGICAL_PROCESS & 1 & 0,0397492 & 1 & 8 & 76 & 17043 & MELO3C024558 & $\begin{array}{l}\text { MELO3CO19565; } \\
\text { MELO3CO04827; } \\
\text { MELO3CCO08520; } \\
\text { MELO3CO25809; } \\
\text { MELO3CO22280; } \\
\text { MELO3CO22308; } \\
\text { MELO3CO23607; } \\
\text { MELO3C007214 }\end{array}$ \\
\hline GO:0030001 & metal ion transport & $\begin{array}{l}\text { BIOLOGICAL_PROCESS } \\
\end{array}$ & 1 & 0,016770116 & 4 & 214 & 73 & 16837 & $\begin{array}{l}\text { MELO3C026764; } \\
\text { MELO3C014309; } \\
\text { MELO3COCO0817; } \\
\text { MELO3C002372 }\end{array}$ & 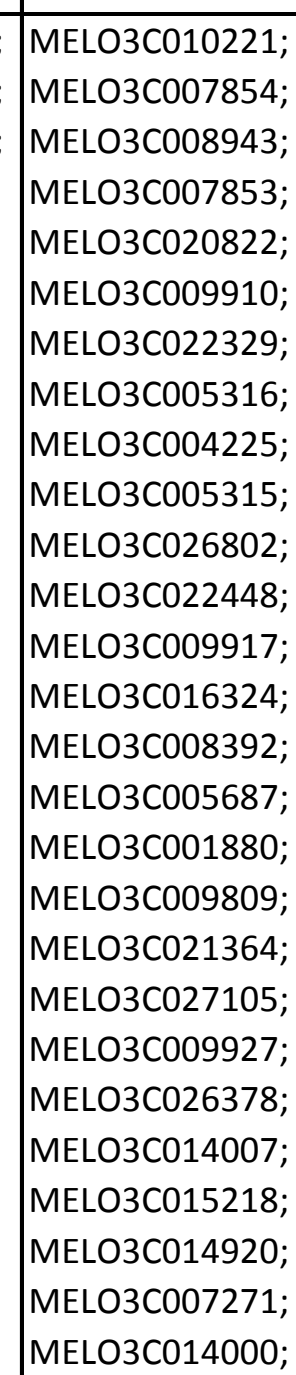 \\
\hline GO:0010113 & $\begin{array}{l}\text { negative regulation of systemic acquired } \\
\text { resistance }\end{array}$ & BIOLOGICAL_PROCESS & 1 & 0,008971177 & 1 & 1 & 76 & 17050 & MELO3C005035 & MELO3C002630 \\
\hline GO:0040025 & vulval development & BIOLOGICAL_PROCESS & 1 & 0,006510321 & 2 & 25 & 75 & 17026 & $\begin{array}{l}\text { MELO3C013369; } \\
\text { MELO3C018956 }\end{array}$ & 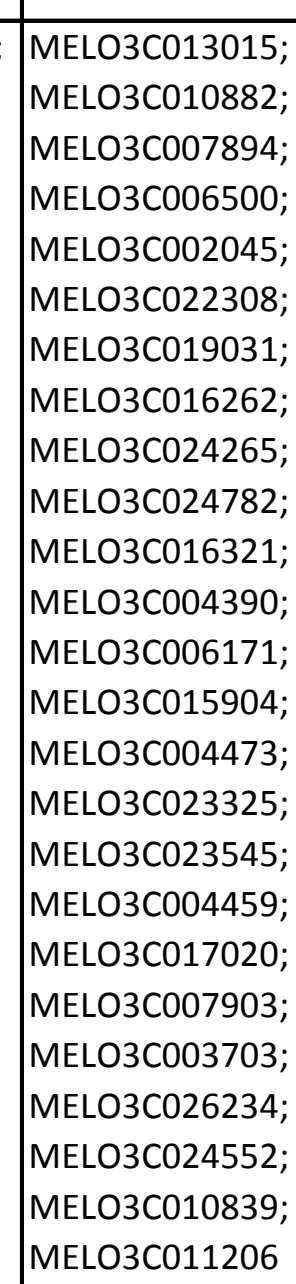 \\
\hline GO:0000050 & urea cycle & BIOLOGICAL_PROCESS & 1 & 0,035410803 & 1 & 7 & 76 & 17044 & MELO3C002619 & $\begin{array}{l}\text { MELO3C003814; } \\
\text { MELO3CO11085; } \\
\text { MELO3COO2352; } \\
\text { MELO3COO2353; } \\
\text { MELO3CO13218; } \\
\text { MELO3CO26229; } \\
\text { MELO3CO03523 }\end{array}$ \\
\hline GO:0019853 & L-ascorbic acid biosynthetic process & BIOLOGICAL_PROCESS & 1 & 0,013426929 & 1 & 2 & 76 & 17049 & MELO3C018576 & $\begin{array}{l}\text { MELO3CO26482; } \\
\text { MELO3CO04377 }\end{array}$ \\
\hline GO:0032258 & CVT pathway & BIOLOGICAL_PROCESS & 1 & 0,0397492 & 1 & 8 & 76 & 17043 & MELO3C024558 & $\begin{array}{l}\text { MELO3CO19565; } \\
\text { MELO3COOS520; } \\
\text { MELO3CO25809; } \\
\text { MELO3CO2228; } \\
\text { MELO3CO22308; } \\
\text { MELO3CC23607; } \\
\text { MELO3CO007214; } \\
\text { MELO3CO088406 }\end{array}$ \\
\hline GO:0010187 & negative regulation of seed germination & BIOLOGICAL_PROCESS & 1 & 0,026675887 & 1 & 5 & 76 & 17046 & MELO3C023484 & $\begin{array}{l}\text { MELO3C009889; } \\
\text { MELO3CO07222; } \\
\text { MELO3CO24155; } \\
\text { MELO3COO6547; } \\
\text { MELO3C010435 }\end{array}$ \\
\hline GO:0045053 & protein retention in Golgi apparatus & BIOLOGICAL_PROCESS & 1 & 0,017862908 & 1 & 3 & 76 & 17048 & MELO3C018052 & $\begin{array}{l}\text { MELO3C013753; } \\
\text { MELO3C015420; } \\
\text { MELO3C006811 }\end{array}$ \\
\hline GO:0010337 & regulation of salicylic acid metabolic process & BIOLOGICAL_PROCESS & 1 & 0,017862908 & 1 & 3 & 76 & 17048 & MELO3C005035 & $\begin{array}{l}\text { MELO3C027349; } \\
\text { MELO3C002099; } \\
\text { MELO3C016008 }\end{array}$ \\
\hline GO:0016559 & peroxisome fission & BIOLOGICAL_PROCESS & 1 & 0,035410803 & 1 & 7 & 76 & 17044 & MELO3C018052 & $\begin{array}{l}\text { MELO3C022531; } \\
\text { MELO3CO19045; } \\
\text { MELO3CO10926; } \\
\text { MELO3C011193; } \\
\text { MELO3COO6147; } \\
\text { MELO3C015136; } \\
\text { MELO3COO6810 }\end{array}$ \\
\hline GO:0000422 & mitophagy & BIOLOGICAL_PROCESS & 1 & 0,03105306 & 1 & 6 & 76 & 17045 & MELO3C024558 & $\begin{array}{l}\text { MELO3C022280; } \\
\text { MELO3CO23607; } \\
\text { MELO3CO07214; } \\
\text { MELO3C019565; } \\
\text { MELO3CO08520; } \\
\text { MELO3C025809 }\end{array}$ \\
\hline
\end{tabular}




\begin{tabular}{|c|c|c|c|c|c|c|c|c|c|c|}
\hline GO:0006379 & mRNA cleavage & BIOLOGICAL_PROCESS & 1 & 0,0397492 & 1 & 8 & 76 & 17043 & MELO3C009318 & $\begin{array}{l}\text { MELOЗCO05162; } \\
\text { MELO3COO8873; } \\
\text { MELO3COOS929; } \\
\text { MELO3CO21828; } \\
\text { MELO3COO8722; } \\
\text { MELO3COO2045; } \\
\text { MELO3CO27241; } \\
\text { MELO3CO21974 }\end{array}$ \\
\hline GO:0071446 & cellular response to salicylic acid stimulus & BIOLOGICAL_PROCESS & 1 & 0,0397492 & 1 & 8 & 76 & 17043 & MELO3C006765 & 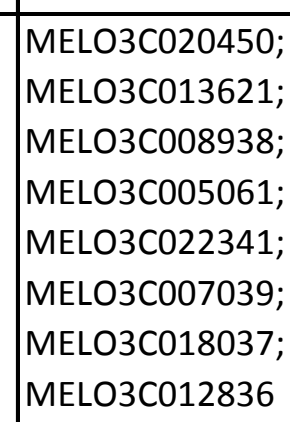 \\
\hline GO:0010104 & $\begin{array}{l}\text { regulation of ethylene-activated signaling } \\
\text { pathway }\end{array}$ & BIOLOGICAL_PROCESS & 1 & 0,026675887 & 1 & 5 & 76 & 17046 & MELO3C024357 & $\begin{array}{l}\text { MELO3C007039; } \\
\text { MELO3CO16937; } \\
\text { MELO3CO15961; } \\
\text { MELO3CO03906; } \\
\text { MELO3C016866 }\end{array}$ \\
\hline GO:0006065 & UDP-glucuronate biosynthetic process & BIOLOGICAL_PROCESS & 1 & 0,026675887 & 1 & 5 & 76 & 17046 & MELO3C017213 & $\begin{array}{l}\text { MELO3C016068; } \\
\text { MELO3CO03703; } \\
\text { MELO3C011431; } \\
\text { MELO3C020244; } \\
\text { MELO3C011346 }\end{array}$ \\
\hline GO:0060625 & regulation of protein deneddylation & BIOLOGICAL_PROCESS & 1 & 0,008971177 & 1 & 1 & 76 & 17050 & MELO3C016464 & MELO3C014386 \\
\hline GO:0009736 & cytokinin-activated signaling pathway & BIOLOGICAL_PROCESS & 1 & 0,048368299 & 1 & 10 & 76 & 17041 & MELO3C024357 & $\begin{array}{l}\text { MELO3CO04183; } \\
\text { MELO3CO16937; } \\
\text { MELO3CO24563; } \\
\text { MELO3CO14230; } \\
\text { MELO3CCO3198; } \\
\text { MELO3CO25617; } \\
\text { MELO3CO16973; } \\
\text { MELOCOC10114; } \\
\text { MELO3CO17877; } \\
\text { MELO3CO244339 }\end{array}$ \\
\hline GO:0045900 & $\begin{array}{l}\text { negative regulation of translational } \\
\text { elongation }\end{array}$ & BIOLOGICAL_PROCESS & 1 & 0,008971177 & 1 & 1 & 76 & 17050 & MELO3C014306 & MELO3C002757 \\
\hline GO:0006614 & $\begin{array}{l}\text { SRP-dependent cotranslational protein } \\
\text { targeting to membrane }\end{array}$ & BIOLOGICAL_PROCESS & 1 & 0,048368299 & 1 & 10 & 76 & 17041 & MELO3C014306 & 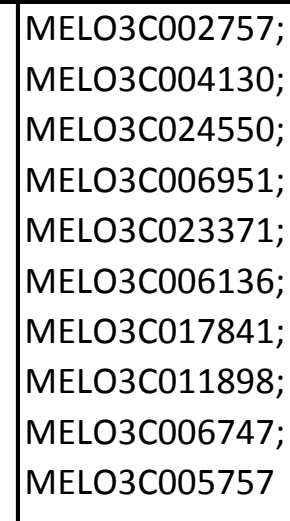 \\
\hline GO:0050826 & response to freezing & BIOLOGICAL_PROCESS & 1 & 0,013426929 & 1 & 2 & 76 & 17049 & MELO3C005035 & $\begin{array}{l}\text { MELO3C021253; } \\
\text { MELO3C022358 }\end{array}$ \\
\hline GO:0008988 & $\begin{array}{l}\text { rRNA (adenine-N6-)-methyltransferase } \\
\text { activity }\end{array}$ & MOLECULAR_FUNCTION & 1 & 0,017862908 & 1 & 3 & 76 & 17048 & MELO3C013369 & $\begin{array}{l}\text { MELO3CO10154; } \\
\text { MELO3CO0787; } \\
\text { MELO3C006150 }\end{array}$ \\
\hline GO:0000064 & $\begin{array}{l}\text { L-ornithine transmembrane transporter } \\
\text { activity }\end{array}$ & MOLECULAR_FUNCTION & 1 & 0,008971177 & 1 & 1 & 76 & 17050 & MELO3C002619 & MELO3C024239 \\
\hline GO:0008234 & cysteine-type peptidase activity & MOLECULAR_FUNCTION & 1 & 0,034577805 & 2 & 63 & 75 & 16988 & $\begin{array}{l}\text { MELO3C019168; } \\
\text { MELO3C018956 }\end{array}$ & 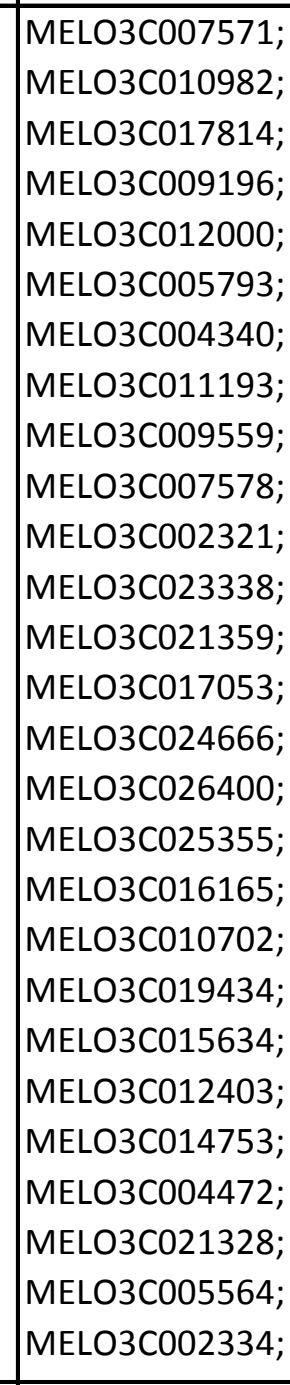 \\
\hline GO:0004430 & 1-phosphatidylinositol 4-kinase activity & MOLECULAR_FUNCTION & 1 & 0,013426929 & 1 & 2 & 76 & 17049 & MELO3C020989 & $\begin{array}{l}\text { MELO3C008054; } \\
\text { MELO3C012545 }\end{array}$ \\
\hline GO:0008239 & dipeptidyl-peptidase activity & $\begin{array}{l}\text { MOLECULAR_FUNCTION } \\
\end{array}$ & 1 & 0,017862908 & 1 & 3 & 76 & 17048 & MELO3C023481 & $\begin{array}{l}\text { MELO3C015446; } \\
\text { MELO3CO23479; } \\
\text { MELO3C023478 }\end{array}$ \\
\hline GO:0003979 & UDP-glucose 6-dehydrogenase activity & MOLECULAR_FUNCTION & 1 & 0,013426929 & 1 & 2 & 76 & 17049 & MELO3C017213 & $\begin{array}{l}\text { MELO3C016068; } \\
\text { MELO3C011431 }\end{array}$ \\
\hline GO:0032266 & phosphatidylinositol-3-phosphate binding & $\begin{array}{l}\text { MOLECULAR_FUNCTION } \\
\end{array}$ & 1 & 0,017862908 & 1 & 3 & 76 & 17048 & MELO3C024558 & $\begin{array}{l}\text { MELO3CO26612; } \\
\text { MELO3CO2015; } \\
\text { MELO3C003655 }\end{array}$ \\
\hline GO:0016633 & galactonolactone dehydrogenase activity & MOLECULAR_FUNCTION & 1 & 0,013426929 & 1 & 2 & 76 & 17049 & MELO3C018576 & $\begin{array}{l}\text { MELO3C020737; } \\
\text { MELO3C020736 }\end{array}$ \\
\hline GO:0004652 & 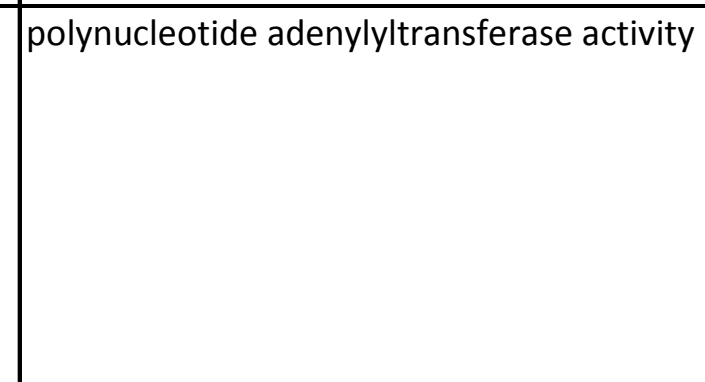 & MOLECULAR_FUNCTION & 1 & 0,035410803 & 1 & 7 & 76 & 17044 & MELO3C002115 & $\begin{array}{l}\text { MELO3C017806; } \\
\text { MELO3CO240235; } \\
\text { MELO3CO13730; } \\
\text { MELO3CO09952; } \\
\text { MELO3CO23722; } \\
\text { MELO3CO26172; } \\
\text { MELO3C011126 }\end{array}$ \\
\hline
\end{tabular}




\begin{tabular}{|c|c|c|c|c|c|c|c|c|c|c|}
\hline GO:0004526 & ribonuclease $\mathrm{P}$ activity & MOLECULAR_FUNCTION & 1 & 0,022279198 & 1 & 4 & 76 & 17047 & MELO3C009318 & $\begin{array}{l}\text { MELOO3C016319; } \\
\text { MELO3C023673; } \\
\text { MELO3C025708; } \\
\text { MELO3C010071 }\end{array}$ \\
\hline GO:0008312 & 7S RNA binding & MOLECULAR_FUNCTION & 1 & 0,035410803 & 1 & 7 & 76 & 17044 & MELO3C014306 & $\begin{array}{l}\text { MELO3C018272; } \\
\text { MELO3COO2757; } \\
\text { MELO3CO2455; } \\
\text { MELO3COO6136; } \\
\text { MELO3CO17841; } \\
\text { MELO3CO1189; } \\
\text { MELO3C006747 }\end{array}$ \\
\hline GO:0005047 & signal recognition particle binding & MOLECULAR_FUNCTION & 1 & 0,022279198 & 1 & 4 & 76 & 17047 & MELO3C014306 & $\begin{array}{l}\text { MELO3CO11981; } \\
\text { MELO3CO04130; } \\
\text { MELO3CO15126; } \\
\text { MELO3CO11898 }\end{array}$ \\
\hline GO:0047763 & caffeate O-methyltransferase activity & MOLECULAR_FUNCTION & 1 & 0,013426929 & 1 & 2 & 76 & 17049 & MELO3C014089 & $\begin{array}{l}\text { MELO3C024861; } \\
\text { MELO3C009403 }\end{array}$ \\
\hline GO:0033843 & xyloglucan 6-xylosyltransferase activity & MOLECULAR_FUNCTION & 1 & 0,022279198 & 1 & 4 & 76 & 17047 & MELO3C007647 & $\begin{array}{l}\text { MELO3CO06664; } \\
\text { MELO3CO17190; } \\
\text { MELO3CO17191; } \\
\text { MELO3C000841 }\end{array}$ \\
\hline GO:0004564 & beta-fructofuranosidase activity & MOLECULAR_FUNCTION & 1 & 0,048368299 & 1 & 10 & 76 & 17041 & MELO3C016877 & 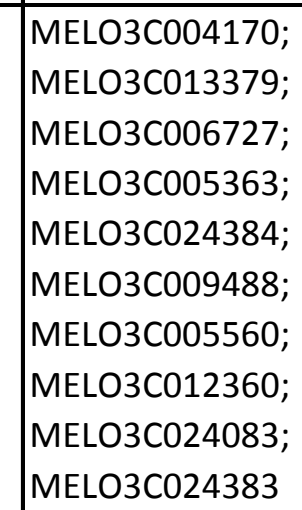 \\
\hline GO:0005524 & ATP binding & $\begin{array}{l}\text { MOLECULAR_FUNCTION } \\
\end{array}$ & 1 & 0,021994726 & 3 & 2058 & 74 & 14993 & $\begin{array}{l}\text { MELO3C006765; } \\
\text { MELO3C013076; } \\
\text { MELO3C016434 }\end{array}$ & 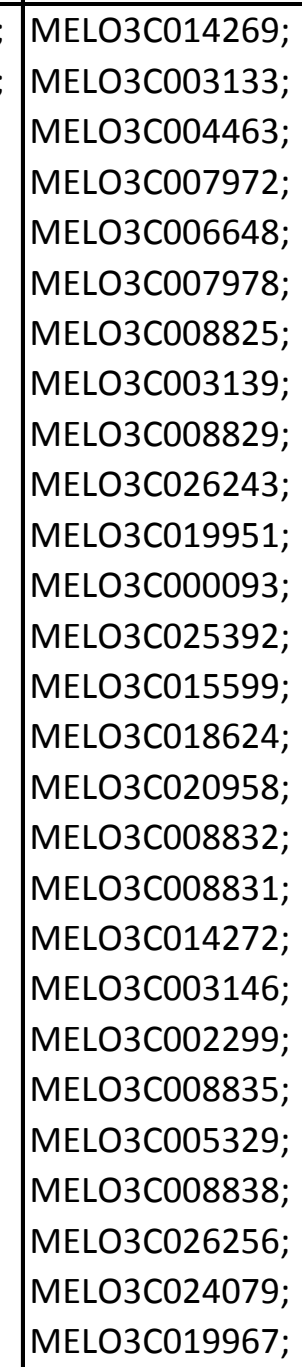 \\
\hline GO:0019789 & SUMO transferase activity & MOLECULAR_FUNCTION & 1 & 0,013426929 & 1 & 2 & 76 & 17049 & MELO3C005035 & $\begin{array}{l}\text { MELO3C025617; } \\
\text { MELO3C024782 }\end{array}$ \\
\hline GO:0015189 & L-lysine transmembrane transporter activity & MOLECULAR_FUNCTION & 1 & 0,008971177 & 1 & 1 & 76 & 17050 & MELO3C002619 & MELO3C002398 \\
\hline GO:0004452 & $\begin{array}{l}\text { isopentenyl-diphosphate delta-isomerase } \\
\text { activity }\end{array}$ & MOLECULAR_FUNCTION & 1 & 0,022279198 & 1 & 4 & 76 & 17047 & MELO3C023481 & $\begin{array}{l}\text { MELO3CO23478; } \\
\text { MELO3COO8717; } \\
\text { MELO3CO23479; } \\
\text { MELO3C010053 }\end{array}$ \\
\hline GO:0015181 & arginine transmembrane transporter activity & MOLECULAR_FUNCTION & 1 & 0,008971177 & 1 & 1 & 76 & 17050 & MELO3C002619 & MELO3C002398 \\
\hline GO:0004577 & $\begin{array}{l}\mathrm{N} \text {-acetylglucosaminyldiphosphodolichol } \mathrm{N}- \\
\text { acetylglucosaminyltransferase activity }\end{array}$ & MOLECULAR_FUNCTION & 1 & 0,008971177 & 1 & 1 & 76 & 17050 & MELO3C015339 & MELO3C013839 \\
\hline GO:0044297 & cell body & CELLULAR_COMPONENT & 1 & 0,035410803 & 1 & 7 & 76 & 17044 & MELO3C018956 & $\begin{array}{l}\text { MELO3CO08571; } \\
\text { MELO3COO9840; } \\
\text { MELO3CO1699; } \\
\text { MELO3COO9668; } \\
\text { MELO3COO5662; } \\
\text { MELO3CO18534; } \\
\text { MELO3C017338 }\end{array}$ \\
\hline GO:0043541 & $\begin{array}{l}\text { UDP-N-acetylglucosamine transferase } \\
\text { complex }\end{array}$ & CELLULAR_COMPONENT & 1 & 0,008971177 & 1 & 1 & 76 & 17050 & MELO3C015339 & MELO3C013839 \\
\hline GO:0009507 & chloroplast & CELLULAR_COMPONENT & 1 & 0,046022234 & 2 & 1593 & 75 & 15458 & $\begin{array}{l}\text { MELO3C006765; } \\
\text { MELO3C003916 }\end{array}$ & 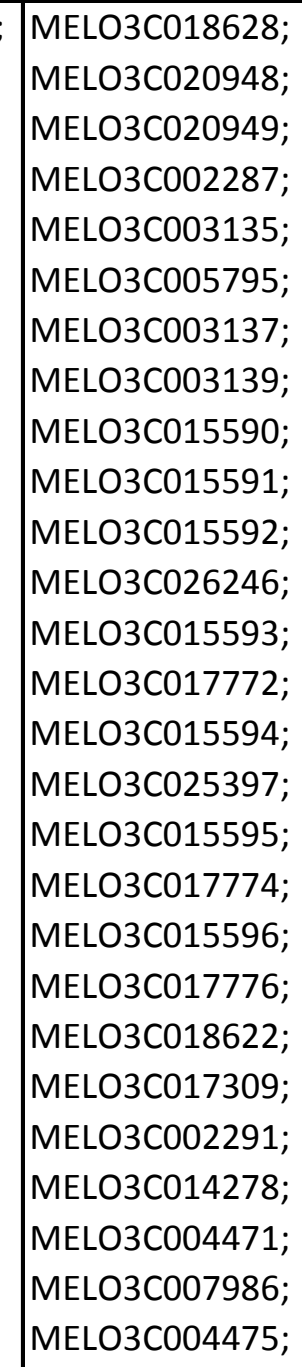 \\
\hline
\end{tabular}




\begin{tabular}{|c|c|c|c|c|c|c|c|c|c|c|}
\hline G0:0005669 & transcription factor TFIII complex & $\begin{array}{l}\text { CELLULAR_COMPONENT } \\
\end{array}$ & 1 & 0,035410803 & 1 & 7 & 76 & 17044 & MELO3C024357 & 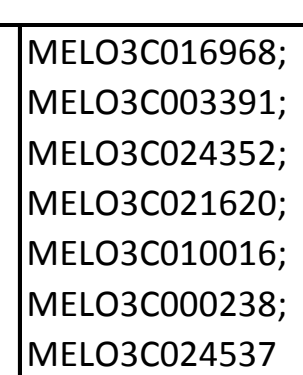 \\
\hline G0:0009360 & DNA polymerase III complex & CELLULAR_COMPONENT & 1 & 0,013426929 & 1 & 2 & 76 & 17049 & MELO3C016434 & $\begin{array}{l}\text { MELO3C007194; } \\
\text { MELO3C014529 }\end{array}$ \\
\hline G0:0005768 & endosome & CELLULAR_COMPONENT & 1 & 0,041648667 & 2 & 70 & 75 & 16981 & \begin{tabular}{|l} 
MELOO3C024558; \\
MELO3C018956
\end{tabular} & 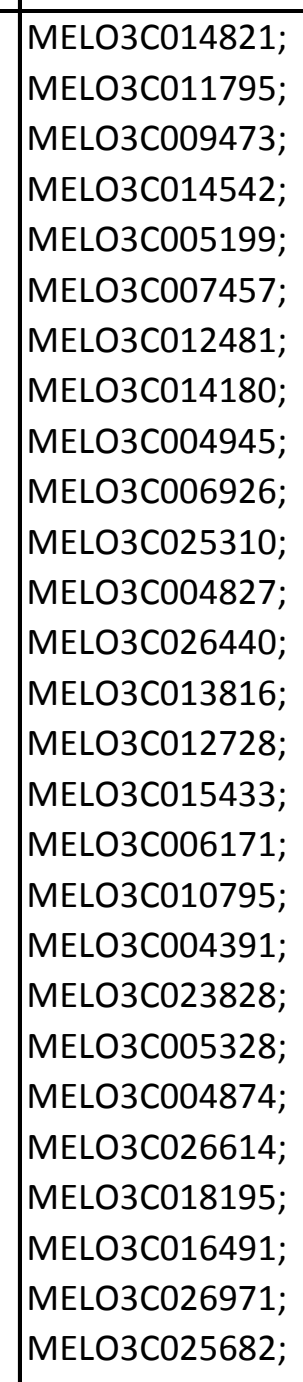 \\
\hline 60:0042406 & $\begin{array}{l}\text { extrinsic component of endoplasmic } \\
\text { reticulum membrane }\end{array}$ & $\begin{array}{l}\text { CELLULAR_COMPONENT } \\
\end{array}$ & 1 & 0,013426929 & 1 & 2 & 76 & 17049 & MELO3C015339 & $\begin{array}{l}\text { MELO3CO11649; } \\
\text { MELOSC018518 }\end{array}$ \\
\hline G0:0000407 & pre-autophagosomal structure & CELLULAR_COMPONENT & 1 & 0,03105306 & 1 & 6 & 76 & 17045 & MELO3C024558 & $\begin{array}{l}\text { MELO3C007534; } \\
\text { MELOSCO22308; } \\
\text { MELOSCO23607; } \\
\text { MELOSCO07214; } \\
\text { MELO3CO19565; } \\
\text { MELO3C025809 }\end{array}$ \\
\hline G0:0005785 & signal recognition particle receptor complex & CELLULAR_COMPONENT & 1 & 0,008971177 & 1 & 1 & 76 & 17050 & MELO3C014306 & MELO3C004130 \\
\hline G0:0005786 & $\begin{array}{l}\text { signal recognition particle, endoplasmic } \\
\text { reticulum targeting }\end{array}$ & CELLULAR_COMPONENT & & 0,035410803 & 1 & 7 & 76 & 17044 & MELO3C014306 & 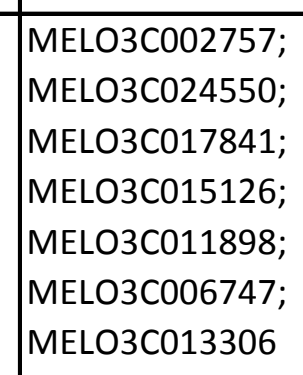 \\
\hline
\end{tabular}

.


Supplementary Table 3. List of the Differentially Expressed Genes (909 DEGs) exclusive of considering the comparison between lines over time in melon fruit of the near-isogenic line SC10-2 and its parental "Piel de sapo" during storage at $20.5^{\circ} \mathrm{C}$ and $88 \%$ relative humidity for $18 \mathrm{~d}$.

\begin{tabular}{|c|c|c|c|}
\hline Gene ID & pval & qval & Description \\
\hline MELO3C018099 & $6.98878999116914 \mathrm{e}-09$ & $8.62635084597496 \mathrm{e}-06$ & No data found \\
\hline MELO3C021514 & $8.24147254885332 \mathrm{e}-08$ & $3.77341577131167 \mathrm{e}-05$ & No data found \\
\hline MELO3C012331 & $1.44328094586754 \mathrm{e}-07$ & $5.37799158489398 \mathrm{e}-05$ & dnaJ protein homolog \\
\hline MELO3C007204 & $2.70307014549331 \mathrm{e}-07$ & $8.47348131799166 \mathrm{e}-05$ & $60 \mathrm{~S}$ ribosomal protein L11-like \\
\hline MELO3C023537 & $5.4337361676815 \mathrm{e}-07$ & 0.00012691733086263 & zinc finger A20 and AN1 domain-containing stress-associated protein 8-like \\
\hline MELO3C012146 & $7.17645318282223 \mathrm{e}-07$ & 0.000149187130429007 & 14-3-3 protein, putative \\
\hline MELO3C020880 & $2.09783662086238 \mathrm{e}-06$ & 0.000312158869772302 & Pathogenesis-related protein 1 \\
\hline MELO3C026045 & $2.71888340064308 \mathrm{e}-06$ & 0.000365273661763947 & alanine--glyoxylate aminotransferase 2 homolog 2, mitochondrial-like \\
\hline MELO3C012074 & $3.70429519958027 \mathrm{e}-06$ & 0.000425326313351806 & Ubiquitin-conjugating enzyme, E2 \\
\hline MELO3C007827 & $4.27775306732769 \mathrm{e}-06$ & 0.00047169877077526 & pyridine nucleotide-disulfide oxidoreductase domain-containing protein 2-like \\
\hline MELO3C013000 & $4.45895326350865 \mathrm{e}-06$ & 0.000478586239136045 & Malic enzyme \\
\hline MELO3C016805 & 4.5614759661694e-06 & 0.000480700689972296 & No data found \\
\hline MELO3C000673 & $4.68826114552456 \mathrm{e}-06$ & 0.000482679936310805 & ATP-dependent zinc metalloprotease FtsH \\
\hline MELO3C026738 & $6.6257926438773 \mathrm{e}-06$ & 0.000608617576390385 & Acyl-CoA N-acyltransferase (NAT) superfamily protein \\
\hline MELO3C022065 & $7.89187488892118 \mathrm{e}-06$ & 0.0006773432768121 & Nuclear matrix constituent protein 1 \\
\hline MELO3C011963 & $7.81226541701763 \mathrm{e}-06$ & 0.0006773432768121 & anthranilate phosphoribosyltransferase \\
\hline MELO3C026410 & $8.27649747914272 \mathrm{e}-06$ & 0.000689673201331601 & transcription factor PIF4-like \\
\hline MELO3C026689 & $1.01759043160676 \mathrm{e}-05$ & 0.000788974717069347 & LRR receptor-like kinase, putative \\
\hline MELO3C007614 & $1.15006113516891 \mathrm{e}-05$ & 0.00086458369524327 & Leucine-rich repeat family protein \\
\hline MELO3C026749 & $1.20811670227816 \mathrm{e}-05$ & 0.000896958524559827 & DNA helicase \\
\hline MELO3C009315 & $1.23509541338773 \mathrm{e}-05$ & 0.00090491340308527 & Ribosomal protein S12 \\
\hline MELO3C016855 & $1.24174151722167 \mathrm{e}-05$ & 0.00090491340308527 & CRG16 \\
\hline MELO3C017720 & $1.5829999835848 \mathrm{e}-05$ & 0.00104557413631492 & protein farnesyltransferase/geranylgeranyltransferase type-1 subunit alpha \\
\hline MELO3C013093 & $1.62023783316645 \mathrm{e}-05$ & 0.0010508747649051 & glyoxylate/succinic semialdehyde reductase 2 , chloroplastic-like \\
\hline MELO3C021107 & $1.93475282579403 \mathrm{e}-05$ & 0.00118295459927574 & Peptidylprolyl isomerase \\
\hline MELO3C012243 & $2.02363198715805 \mathrm{e}-05$ & 0.00121105176104195 & tetraketide alpha-pyrone reductase 2 -like \\
\hline MELO3C012748 & $2.83333883075443 \mathrm{e}-05$ & 0.00148385868132083 & Lysine-specific demethylase \\
\hline MELO3C024292 & $3.02042248422341 \mathrm{e}-05$ & 0.00153552012010767 & O-fucosyltransferase family protein \\
\hline MELO3C013470 & $3.44618684419151 \mathrm{e}-05$ & 0.00168046281446761 & Ankyrin repeat domain-containing 2 -like protein \\
\hline MELO3C024586 & $3.89204117599018 \mathrm{e}-05$ & 0.00180760621925962 & Ribosomal protein $\mathrm{S} 8 \mathrm{e} /$ ribosomal biogenesis NSA2 \\
\hline MELO3C018394 & $4.6087660756533 \mathrm{e}-05$ & 0.00201368409796631 & Abscisic acid receptor \\
\hline MELO3C015043 & $4.71004272699638 \mathrm{e}-05$ & 0.00204436557836157 & DnaJ-like protein \\
\hline MELO3C025391 & $4.83084956899305 \mathrm{e}-05$ & 0.00207353939040731 & No data found \\
\hline MELO3C025056 & $4.8402534760128 \mathrm{e}-05$ & 0.00207353939040731 & zinc finger $\mathrm{CCCH}$ domain-containing protein 53 -like \\
\hline MELO3C024376 & $4.90235715564502 \mathrm{e}-05$ & 0.00208656576437141 & Eukaryotic initiation factor 4A (ATP-dependent RNA helicase elF4A) \\
\hline MELO3C009292 & $5.42517615724769 \mathrm{e}-05$ & 0.00222633555573646 & $A B C$ transporter $\mathrm{G}$ family member 20 \\
\hline MELO3C022693 & $5.60978884305285 \mathrm{e}-05$ & 0.00227958271319858 & GDSL esterase/lipase 5 \\
\hline MELO3C021332 & $6.05753663890374 \mathrm{e}-05$ & 0.00238547596635887 & $40 S$ ribosomal protein $\mathrm{S} 21$ \\
\hline MELO3C005992 & $6.3408310252755 \mathrm{e}-05$ & 0.00246211971008436 & homeobox-leucine zipper protein ATHB-7 \\
\hline MELO3C020255 & $6.65873213148149 \mathrm{e}-05$ & 0.00253378229026258 & 3-phosphoinositide-dependent protein kinase-1 \\
\hline MELO3C025026 & $7.38424094659518 \mathrm{e}-05$ & 0.00271924112679749 & abscisic stress-ripening protein 2-like \\
\hline MELO3C013084 & $7.63203350274688 \mathrm{e}-05$ & 0.0027671089367998 & Aminopeptidase \\
\hline MELO3C025654 & $8.41080056708243 \mathrm{e}-05$ & 0.00292498547025566 & Transmembrane protein \\
\hline MELO3C005828 & $8.65793669142523 \mathrm{e}-05$ & 0.00296335514244293 & SKP1-like protein 21 \\
\hline MELO3C005872 & $8.92453339496058 \mathrm{e}-05$ & 0.00303555374175946 & Polypyrimidine tract binding protein, putative \\
\hline MELO3C006670 & $9.20264952706251 \mathrm{e}-05$ & 0.00307398449987238 & Translationally-controlled tumor protein homolog \\
\hline MELO3C012943 & $9.46720039655125 \mathrm{e}-05$ & 0.00313704262804514 & ADP,ATP carrier protein \\
\hline MELO3C010021 & $9.6358700537813 e-05$ & 0.00317694153075337 & ER membrane protein complex subunit 10 \\
\hline MELO3C021901 & 0.000102486479856445 & 0.00328012097486915 & Alpha/beta hydrolase-3 \\
\hline MELO3C013261 & 0.000104022481609345 & 0.00329094438804991 & No data found \\
\hline MELO3C003906 & 0.000107750928353134 & 0.00338431427849777 & Ethylene receptor \\
\hline MELO3C002085 & 0.000113224239913556 & 0.00346676823884159 & ethylene-responsive transcription factor ERF071 \\
\hline MELO3C024324 & 0.000116198570528248 & 0.00352495494601283 & Chaperone protein DnaJ \\
\hline MELO3C022837 & 0.000116753774780598 & 0.00352564265770953 & Cysteine-rich repeat secretory protein 60 \\
\hline MELO3C010776 & 0.000116715602386908 & 0.00352564265770953 & Branched-chain-amino-acid aminotransferase \\
\hline MELO3C001165 & 0.000124820210286147 & 0.00368471499692246 & eukaryotic translation initiation factor 3 subunit $\mathrm{M}$ \\
\hline MELO3C016924 & 0.000125729655771067 & 0.00369128273352684 & pentatricopeptide repeat-containing protein At4g33170-like \\
\hline MELO3C023989 & 0.000126739273406784 & 0.00369715496382656 & Methyltransferase \\
\hline MELO3C007246 & 0.000136437561226055 & 0.00384148920539927 & $28 \mathrm{kDa}$ heat/acid-stable phosphoprotein-like protein \\
\hline MELO3C023033 & 0.000141418977736452 & 0.00392810603560785 & heterogeneous nuclear ribonucleoprotein 1-like \\
\hline MELO3C019087 & 0.000146130882510365 & 0.00398610331311767 & UDP-glycosyltransferase 76E2-like \\
\hline MELO3C013563 & 0.000146093610227327 & 0.00398610331311767 & Metacaspase-5 \\
\hline MELO3C005692 & 0.000146809861828023 & 0.00399910063619536 & E3 ubiquitin-protein ligase At3g02290-like \\
\hline MELO3C024861 & 0.000151226502948631 & 0.00407445048667466 & Caffeic acid O-methyltransferase \\
\hline MELO3C018160 & 0.000152143725675513 & 0.00407691511311494 & factor of DNA methylation 1-like \\
\hline MELO3C008974 & 0.000155525458351624 & 0.00413387924224256 & $60 S$ ribosomal protein L35a \\
\hline MELO3C002163 & 0.000164730021437798 & 0.00426283287985112 & E3 ubiquitin-protein ligase RING1-like \\
\hline MELO3C014217 & 0.000167148322577915 & 0.00427592256812336 & Telomere repeat-binding protein 4 \\
\hline MELO3C005001 & 0.000170562545132369 & 0.00434637381137955 & dof zinc finger protein DOF5.3-like \\
\hline MELO3C020583 & 0.000173737441671706 & 0.0044017019494474 & Neutral ceramidase \\
\hline MELO3C014799 & 0.000175285251514445 & 0.00442673712552274 & TRANSPORT INHIBITOR RESPONSE 1 protein, putative \\
\hline MELO3C022562 & 0.00017556921672901 & 0.00442824579972059 & protein CHROMATIN REMODELING 19 isoform X1 \\
\hline MELO3C006239 & 0.000194745937404539 & 0.0048075468972528 & Receptor protein kinase, putative \\
\hline MELO3C022684 & 0.000194616340531306 & 0.0048075468972528 & peroxisomal (S)-2-hydroxy-acid oxidase GLO4-like \\
\hline MELO3C024902 & 0.000197626941677576 & 0.00484235046053404 & $A B C$ subfamily $C$ transporter \\
\hline MELO3C022540 & 0.000216005163480082 & 0.00519433149706969 & NADH dehydrogenase [ubiquinone] 1 beta subcomplex subunit 7 \\
\hline MELO3C019629 & 0.000220238525441285 & 0.00524667145831115 & ALA-interacting subunit 3-like \\
\hline MELO3C019921 & 0.000224478914295334 & 0.00530926236936354 & Phospholipid-transporting ATPase \\
\hline MELO3C011682 & 0.00023316957210473 & 0.00547828218461161 & Fructose-1,6-bisphosphatase \\
\hline MELO3C005111 & 0.00023446163867713 & 0.00548351994895583 & Coiled-coil protein \\
\hline MELO3C014614 & 0.000235860026803869 & 0.00549292413838397 & Leucine-rich repeat receptor-like protein kinase family \\
\hline MELO3C017008 & 0.000239417539566356 & 0.00552722123260604 & Ubiquitin family protein \\
\hline MELO3C024688 & 0.000240582824529545 & 0.00554407258066976 & TATA-binding protein-associated factor $2 \mathrm{~N}$ \\
\hline MELO3C009127 & 0.000241609568591317 & 0.00556124402110712 & WRKY family transcription factor family protein \\
\hline MELO3C007425 & 0.000247283160818346 & 0.0056205337601488 & 1-aminocyclopropane-1-carboxylate oxidase 1 \\
\hline MELO3C002351 & 0.000259527766881318 & 0.00580454571703188 & Receptor protein kinase-like protein \\
\hline
\end{tabular}


MEL03C013766 MELO3C022679 MELO3C005002 MELO3C012349 MELO3C008097 MELO3C022353 MELO3C020850 MELO3C003771 MELO3C007084 MELO3C012335 MELO3C002298 MELO3C004818 MELO3C025628 MELO3C017698 MELO3C021510 MELO3C007119 MELO3C026919 MELO3C006096 MELO3C006726 MELO3C023350 MELO3C00586 MELO3C020610 MELO3C021139 MELO3C005875 MELO3C012171 MELO3C008086 MELO3C025753 MELO3C006289 MELO3C023876 MELO3C009542 MELO3C009490 MELO3C026433 MELO3C026780 MELO3C019735 MELO3C02670 MELO3C013938 MELO3C011070 MELO3C013436 MELO3C012073 MELO3C008245 MELO3C023998 MELO3C006430 MELO3C008866 MELO3C005720 MELO3C008491 MELO3C009346 MELO3C007391 MELO3C025062 MELO3C003567 MELO3C012178 MELO3C022260 MELO3C008806 MELO3C005706 MELO3C002714 MELO3C024760 MELO3C011805 MELO3C018462 MELO3C005899 MELO3C011873 MELO3C017030 MELO3C015270 MELO3C018027 MELO3C012452 MELO3C001971 MELO3C007012 MELO3C000881 MELO3C013007 MELO3C008775 MELO3C007667 MELO3C009128 MELO3C019498 MELO3C014062 MELO3C00458 MELO3C017561 MELO3C003678 MELO3C003086 MELO3C021326 MELO3C01523 MELO3C015686 MELO3C002662 MELO3C02364 MELO3C00728 MELO3C021170 MELO3C015844 MELO3C01376 MELO3C026224 MELO3C011018 MELO3C016522 MELO3C007143 MELO3C001947
0.000277047881957371 0.000277183104936407 0.000281040859585691 0.000282115086382206 0.000282891183413736 0.000283476774613156 0.000287415273795277 0.000289095891857327 0.000290601122250878 0.00030093175099899 0.00030226452811033 0.000313659812932854 0.000319630051430653 0.00034384648671526 0.000345680560706607 0.000364741286312031 0.000385875008708902 0.000388869747816378 0.000393613047360519 0.000393498277305726 0.000394326202309614 0.000397760016506599 0.000416728063664884 0.000415414077330256 0.000417125825128095 0.00042505111070712 0.000438646630847916 0.000446332049387155 0.000456434521640348 0.00046017704841339 0.000477551177120339 0.000478847432524576 0.000482363906618488 0.000492040262321525 0.000490305274858382 0.000499419243035093 0.000500007962388027 0.000502109941717843 0.000503847273066516 0.000504765109731187 0.000507430810900544 0.000517174720864766 0.000517893399529346 0.000530338114349549 0.000531797074916351 0.000538853443754816 0.000540671539647652 0.000544142102876677 0.000545821213981501 0.000548063155305423 0.000550317736865757 0.000551102874571718 0.000567796577401691 0.000579702326297893 0.000592163910059229 0.000592819805662836 0.000600683577282934 0.000607220982714818 0.000621061425400415 0.000630415357957714 0.000642051019905554 0.000645454282273139 0.000667682946013937 0.000668754302383112 0.000677563644158052 0.000697534212565021 0.000705481661930962 0.000711395060906339 0.000719402723322826 0.000720765197783857 0.000730368821527216 0.000737302663945227 0.000738241873302403 0.000741241733906883 0.000746920851982136 0.000755050317633899 0.000766461351612402 0.000766447720925711 0.000773924241273871 0.000774471299118229 0.0007827561517204 0.000784187854190543 0.00078421110262572 0.00079612896994774 0.000797345327828602 0.000803412442861307 0.000806198958957616 0.000812005118265402 0.000815966088231534 0.000819606583127408 0.000841859856859473
0.00602295331887094 Translocase of chloroplast

0.00602295331887094 oligouridylate-binding protein 1B-like

0.00607916312810274 Interactor of constitutive active ROPs-like protein

0.00609572302074638 replication protein A $14 \mathrm{kDa}$ subunit B-like

0.00610581200135287 Polyprotein

$0.0061117716395581 \quad$ tRNA dimethylallyltransferase

$0.00616304477978603 \quad$ Filament-like plant protein 7

0.00616328919688229 Mitochondrial 39S ribosomal protein L53

$0.00617769813060558 \quad$ BTB/POZ domain-containing protein

0.00634386020921778 Uricase

0.00635973082156247 Methylthioribose-1-phosphate isomerase

0.00651363579980119 S-adenosyl-L-methionine-dependent methyltransferase superfamily protein

0.00660290155408364 Aspartyl/glutamyl-tRNA (Asn/GIn) amidotransferase subunit B

0.00697905885523091 Vesicle transport protein GOT1

0.00699471864077334 Ferredoxin-thioredoxin reductase catalytic chain

0.00730555341113216 E3 ubiquitin-protein ligase arkadia-A, putative

0.00765125054918887 RuBisCO large subunit-binding protein subunit alpha, chloroplastic

0.00769517900764094 DNA replication complex GINS protein SLD5

0.00775021343202681 Galactokinase

0.00775021343202681 GATA transcription factor

0.00775296869722632 Hexosyltransferase

0.00776020694699844 protein TOC75-3, chloroplastic

$0.00798238170586701 \quad$ No data found

0.00798238170586701 Curved DNA-binding protein

0.00798238170586701 phosphatidylinositol 4-kinase gamma 5

0.00809656108130136 Delta-aminolevulinic acid dehydratase

0.0082897916867134 elongation factor 1-alpha

$0.00840287096601232 \quad$ Unknown protein

0.00855230110804101 glycerol-3-phosphate acyltransferase 5-like

0.00859657502875971 BEL1-like homeodomain protein 1

0.00880593669182967 Ribosomal protein S11

0.00882160256056703

0.00886692143153668

0.00894906064744133

0.00894906064744133

0.00902351976145818

0.00902351976145818

0.00903935208658677

0.00906236775572916

0.00906236922916473

0.00908544975927003

0.00920981385244208 0.00921430337595049 0.0093264892433564 0.00934382600758276

0.00942651619399203 0.00944931171371813 0.0094740663502676 0.00948892883355692 0.00951115927427662 0.0095251752720086 0.00953041214528622 0.00971699705988388 0.00988647775652598 0.00997836609194515 0.0099809022523745 0.0100436026132101 0.0101086526857278 0.0102808654980692 0.0103750607535891 0.0104878954442637 0.0105173899510002 0.0107902195406569 0.0107902195406569 0.0108878799092574 0.0111004054504002 0.0111962038589772 0.0112574848219866 0.0113207046875717 0.0113240985608858 0.0113934074694637 0.0114563259718759 0.0114619015376173 0.0114728845422048 0.0115331820999181 0.0116405064191662 0.0117888202749169 0.0117888202749169 0.0118566152606867 0.0118566152606867 0.0119187750503673 0.0119225443154391 0.0119225443154391 0.0120388598985436 0.012048028216746 0.0120892909496463 0.0120946682916889 0.0121487038489571 0.0121714739300738 0.0121977471063928 0.0124352208774254

U4/U6 small nuclear ribonucleoprotein PRP4-like protein

Ribosomal protein $\mathrm{S} 19$

1-aminocyclopropane-1-carboxylate oxidase

esicle-associated membrane protein, putative

rotein transport protein Sec16B

hamnogalacturonate lyase

DNA helicase

SNF1-related kinase regulatory subunit gamma 1

Delta-1-pyrroline-5-carboxylate synthetase

Protein RETICULATA-RELATED 4, chloroplastic

thylene-responsive transcription factor $1 B$

Plasma-membrane choline transporter family protein

Late embryogenesis abundant protein

Zinc finger protein CONSTANS

translin 
ME103C016408 MELO3C024287 MELO3C022319 MELO3C020710 MELO3C003439 MELO3C013707 MELO3C01868 MELO3C010903 MELO3C025720 MELO3C010183 MELO3C003139 MELO3C020172 MELO3C007144 MELO3C018046 MELO3C025347 MELO3C009770 MELO3C015926 MELO3C007917 MELO3C004539 MELO3C005046 MELO3C007646 MELO3C012016 MELO3C005915 MELO3C025944 MELO3C016811 MELO3C015770 MELO3C007467 MELO3C007316 MELO3C001943 MELO3C024239 MELO3C01095 MELO3C014116 MELO3C021598 MELO3C01082 MELO3C01382 MELO3C000254 MELO3C013964 MELO3C019125 MELO3C00213 MELO3C00812 MELO3C020616 MELO3C004713 MELO3C019140 MELO3C006995 MELO3C02551 MELO3C003420 MELO3C024554 MEL03C018981 MELO3C026230 MELO3C02688 MELO3C007914 MELO3C007724 MELO3C003675 MELO3C02200 MELO3C010375 MELO3C026013 MELO3C008252 MELO3C014398 MELO3C016882 MELO3C023961 MELO3C011040 MELO3C006507 MELO3C014190 MELO3C016300 MELO3C012715 MELO3C005294 MELO3C021556 MELO3C01229 MELO3C01540 MELO3C003846 MELO3C009873 MELO3C015398 MELO3C008375 MELO3C017272 MELO3C006587 MELO3C027346 MELO3C016965 MELO3C017133 MELO3C008096 MELO3C019524 MELO3C011834 MELO3C022932 MELO3C015477 MELO3C014281 MELO3C021914 MELO3C007497 MELO3C00241 MELO3C021536 MELO3C003457 MELO3C021148 MELO3C025035
0.000849878202600962 0.000858057790619982 0.000857819419544437 0.000855996771340739 0.000864308651512991 0.000869260816383277 0.000892082156492835 0.00089679269942633 0.00090037014311295 0.000904336723108301 0.000913198703413887 0.000915802238327545 0.000921378557700914 0.000926412561525591 0.000928175455473412 0.000931788216151919 0.000935266432297022 0.000939995495577994 0.000946307413841607 0.000947141386938188 0.000954499819987076 0.00097897348711995 0.000986201687010468 0.000985917354598187 0.000987087449194402 0.000999619597627954 0.00100905049094524 0.00101829843398527 0.00103568118847042 0.0010406075041931 0.00104181627537914 0.0010524633137019 0.0010608705730426 0.00106381826751312 0.0010657321313921 0.00107265318236205 0.00107467152470697 0.00107877740647 0.0010914121549846 0.0010950939938712 0.00110160610411703 00111704118586142 0.00112109638180102 0.0011295194113708 0.00116227454348072 0.0011601994899415 0.00117317342071144 0.00117475910333109 0.0011757436050832 0.00121509032211076 0.00122356487409925 0.00123610608361802 .00123674154707298 0.0012575131063919 0.00126258066098428 00126215947958241 00126698624011023 0.0012717098365631 0.00128643995485178 0.00128738672658923 0.00129103487176252 0.00129529568976838 0.00130600334466013 0.00132248461135576 0.00132243173147539 0.00132249068593726 .0013237026370145 0.00133013796895898 0.00134065781250059 0.00135074087660314 00136459747634987 0.0013691153760095 0.00138251768956899 0.00138518236716068 0.0013871485080218 0.013986380991619 0.0014064642461353 0.00141800169024686 0.00142424626433679 0.0014304129339574 0.0014366188125860 0.0014593798909456 0.00146228225489542 0.0014702360035872 0.00146823354847903 0.0014780779626572 0.00148622342396076 0.00151639341105281 0.00152415156525021 0.0015313685935086 0.00153071881752509
0.0124919222927065 0.0125439869644173 0.0125439869644173 0.0125439869644173 0.0126158400286253 0.0126787532221221 0.0129257010334387 0.0129749150336781 0.0129920969393496 0.0129920969393496 0.013059204340131 0.0130774970388508 0.0131286472842968 0.0131718658585809 0.0131874367411111 0.0132197453166553 0.0132500550727646 0.013286163190543 0.0133322132934015 0.0133322132934015 0.013397595554317 0.0136153150684027 0.0136581326204556 0.0136581326204556 0.0136608199258166 0.0137301972062592 0.0138099363448909 0.0139089177661161 0.0140667073781726 0.0140953069960971 0.014102007966047 0.0142168932847461 0.0142852160059244 0.0143018018823121 0.014308051572307 0.014391187295155 0.0144084778964277 0.0144537143828874 0.0145872662731249 0.0146128454628124 0.014689816981908 0.0148355389237238 0.0148595083563484 0.0149353473849775 0.0152506151645319 0.0152506151645319 0.0153437098580332 0.0153529891038268 0.0153529891038268 0.0157562828439694 0.015796148569604 0.0158993585323115 0.0158993585323115 0.0160950267907553 0.0161389679441932 0.0161389679441932 0.016174344703256 0.0162085217333131 0.016327700943681 0.0163292231621135 0.0163649856755058 0.0163979452418178 0.0165017658692853 0.0166461877352294 0.0166461877352294 0.0166461877352294 0.0166508301773249 0.016678663332679 0.016746774914025 0.0168514097106983 0.0169920779069569 0.017026863703283 0.0171288217385809 0.0171403299304863 0.0171431763985747 0.0171921631272379 0.0172416278069067 0.0172971682400774 0.0173305233976508 0.017373447129597 0.017427386320492 0.017595417256585 0.0176089099097132 0.0176508758874429 0.0176508758874429 0.017723474003956 0.017777968746094 0.018029652904805 0.018078359917193 0.0181146196894978 0.0181146196894978

D-2-hydroxyglutarate dehydrogenase, mitochondrial-like GATA transcription factor 24-like

DNA primase large subunit

THO complex subunit $4 A$

$N A D(P)$-binding rossmann-fold protein

Thioredoxin reductase

nuclear transcription factor $Y$ subunit $C-2$

thylakoidal processing peptidase 1 , chloroplastic-like

phototropin-1

glycine-rich cell wall structural protein 2-like

ATP-dependent zinc metalloprotease FtsH

Hydroxyacylglutathione hydrolase

DNA helicase

Zinc transport ZntB

Short-chain dehydrogenase/reductase

two-component response regulator ORR9-like 
MELO3C020427 MELO3C022392 MELO3C011439 MELO3C010197 MELO3C010403 MELO3C024313 MELO3C014944 MELO3C016193 MELO3C022042 MELO3C019491 MELO3C007099 MELO3C020159 MELO3C023852 MELO3C013753 MELO3C024001 MELO3C003770 MELO3C009090 MELO3C019490 MELO3C005455 MELO3C008195 MELO3C016494 MELO3C017294 MELO3C015470 MELO3C021264 MELO3C018459 MELO3C02514 MELO3C002604 MELO3C008786 MELO3C026593 MELO3C012493 MELO3C012420 MELO3C012852 MELO3C015177 MELO3C008032 MELO3C018413 MELO3C023862 MELO3C025744 MELO3C020588 MELO3C008516 MELO3C019814 MELO3C006934 MELO3C007228 MELO3C014417 MELO3C01878 MELO3C011972 MELO3C009600 MELO3C013539 MELO3C010198 MELO3C002268 MELO3C026887 MELO3C011763 MELO3C021153 MELO3C025491 MELO3C015457 MELO3C002598 MELO3C00948 MELO3C003496 MELO3C01104 MELO3C019361 MELO3C006212 MELO3C009757 MELO3C008970 MELO3C015292 MELO3C005976 MELO3C007466 MELO3C007270 MELO3C008100 MELO3C022165 MELO3C018492 MELO3C006106 MELO3C016952 MELO3C017817 MELO3C003722 MELO3C013034 MELO3C019725 MELO3C023404 MELO3C02152 MELO3C026286 MELO3C027184 MELO3C00476 MELO3C018797 MELO3C009908 MELO3C011264 MELO3C009639 MELO3C010493 MELO3C019827 MELO3C019500 MELO3C018683 MELO3C011084 MELO3C004381 MELO3C023844
0.00153376154089113 0.00153951311588807 0.00154415748059011 0.00157227802397697 0.00157447526897514 0015826012621280 0.00158975633025693 0.00158896575333467 0.00159143253219463 0.00160887864625414 0.0016085255194175 0.0016070314789058 0.00164327417852994 0.00168358970314475 0.00169134873987065 0.0016973381454457 0.00173121000396637 0.00173728752931601 0.00173406833228484 0.00173319093407187 0.00174848962342988 0.00174730515676302 0.00175565135908395 0.00177097699103468 .00179830009973014 0.00180918499272109 0.00183522225955302 0.00185244710486099 0.00186878067212692 0.0018729599387148 0.00187233804376197 0.00188039613797097 0.00188263980533365 0.00188467890401967 0.00188895876601003 0.0019068491527422 0.00190635644741377 0.00196327140921926 .0019668560492823 0.0020127419677088 0.00202201822291359 .00202308834498344 0.00204144586045041 0.0020542658700694 0.0020804500498798 0.00209252401614957 0.00209583349110565 0.00210011636188545 0.00211346018252012 0.0021211655104229 0.0021263565296451 0.00213199409674547 0.00215106029913348 0.0021621262145803 0.00217096035433062 0.0021885277242305 000219345078663657 0.00220196138837392 0.00220806422194508 0.00222542135216774 0.00222821468022771 0.0022326445144933 0.0022520797474736 0.00225640094494073 0.00225882808818756 0.00226366852216031 .00229727735813157 0.00231035379912992 0.0023123247278860 0.00232061450614851 0.00234128339180883 0.0023669444389276 0.00237262336257049 0.00238546539224549 0.00239709679529321 0.00240231684114978 0.0024069868686340 0.00240990356136972 0.00242276758035198 0.00243983236026302 .0024383704580994 0.0024512492327091 0.00245758550501518 0.00246576674180576 0.0246180150676134 0.0024672049874503 0.00249021021586082 0.00249307436724266 0.00250020007177665 0.00251245552795387 0.00252223327356038
0.018127023740909 0.018173248371592 0.0182171840407253 0.0184785490735071 0.0184865113477943 0.0185349689647406 0.0185423015211278 0.0185423015211278 0.0185423015211278 0.0186904378734547 0.0186904378734547 0.0186904378734547 0.0189672833148964 0.0193016055803 0.0193637369644669 0.019401769103248 0.0196392051611116 0.0196392051611116 0.0196392051611116 0.0196392051611116 0.019709430121642 0.019709430121642 0.0197675933241442 0.0198834704922933 0.0201216026456491 0.0202090466183534 0.0204536142234269 0.0206223099627394 0.0207573394228541 0.0207687174787643 0.0207687174787643 0.020820323247398 0.0208292736781705 0.020840158832858 0.020856911657507 0.0210027680620675 0.0210027680620675 0.0214806908923386 0.0214960930366782 0.0218719472185081 0.021892587246618 0.021892587246618 0.0220188499716194 0.0220967846775603 0.0223176578137305 0.022398513167988 0.0224217852740224 0.0224554401899706 0.0225736750376365 0.022643728467753 0.0226746301857241 0.0227224778287244 0.0228516889981641 0.0229445623921263 0.0230012317798688 0.0231377055812785 0.0231525705960907 0.0232175843347552 0.023269509241832 0.0233900193102506 0.0234069211275623 0.023440986983907 0.0236199272081026 0.0236526869753898 0.0236530201026597 0.023678596209822 0.0239287608363609 0.0239890521445934 0.0239969001844565 0.0240576461322451 0.0241830573770045 0.024333568831015 0.0243792605553614 0.0244603094659689 0.0245540791546917 0.0245947927920513 0.024604339373009 0.0246086791279682 0.024688976751481 0.0248134188898108 0.0248134188898108 0.024902119905747 0.024928020615585 0.0249496524561083 0.0249496524561083 0.024949652456108 0.0251170385868414 0.0251330912091247 0.0251920669477128 0.025251137008428 0.0253107646948902

DEAD-box ATP-dependent RNA helicase 42-like

Cytochrome oxidase complex assembly protein

6,7-dimethyl-8-ribityllumazine synthase

Tetratricopeptide repeat (TPR)-like superfamily protei

OW QUALITY PROTEIN: LEAF RUST 10 DISEASE-RESISTANCE LOCUS RECEPTOR-LIKE PROTEIN KINASE-like 1.4 AT-rich interactive domain-containing protein 4-like

homeobox-leucine zipper protein HAT22-like

Lysine-specific histone demethylase 1

DUF2039 family protein

Nucleolar complex protein 2 homolog

Nucleoporin protein Ndc1-Nup

transmembrane protein 87A

Actin family protein

Vacuolar protein sorting-associated protein 35

MADS-box transcription factor

TLD-domain nucleolar protein

Kinase family protein 
MEL03C019704 MELO3C018460 MELO3C016014 MELO3C002277 MELO3C026572 MELO3C003818 MELO3C024244 MELO3C017158 MELO3C00778 MELO3C009532 MELO3C021618 MELO3C027119 MELO3C02690 MELO3C004385 MELO3C004519 MELO3C006439 MELO3C022497 MELO3C014925 MELO3C023599 MELO3C013603 MELO3C00615 MELO3C017228 MELO3C007857 MELO3C012418 MELO3C025494 MELO3C013665 MELO3C015682 MELO3C012175 MELO3C020157 MELO3C018408 MELO3C006576 MELO3C012268 MELO3C022246 MELO3C002232 MELO3C015807 MELO3C023406 MELO3C017624 MELO3C022694 MELO3C012527 MELO3C026077 MELO3C019004 MELO3C026638 MELO3C017687 MELO3C022482 MELO3C011123 MELO3C006635 MELO3C01221 MELO3C013275 MELO3C019546 MELO3C006945 MELO3C025916 MELO3C006833 MELO3C006596 MELO3C02062 MELO3C026935 MELO3C003790 MELO3C016435 MELO3C026949 MELO3C017665 MELO3C020958 MELO3C026019 MELO3C013566 MELO3C006900 MELO3C011141 MELO3C011641 MELO3C017682 MELO3C011626 MELO3C01088 MELO3C015845 MELO3C014696 MELO3C024476 MELO3C01145 MELO3C013981 MELO3C021429 MELO3C022717 MELO3C012390 MELO3C003646 MELO3C007740 MELO3C015670 MELO3C009826 MELO3C020709 MELO3C003618 MELO3C003537 MELO3C005597 MELO3C012627 MELO3C008749 MELO3C017141 MELO3C011443 MELO3C019807 MELO3C016321 MELO3C013068
0.00252200355091303 0.00253286875232273 0.0025571448957098 0.0025908406468802 0.00260563742446895 0.00260420294403418 0.0026150850948101 0.00262413446791454 0.00263134950686683 0.0026377958393965 0.0026352378493771 0.00263408267344634 0.00263783859288103 0.00265207979831772 0.00267496625574049 0.00270856493483707 0.00270918512667995 0.00272905227228559 0.00273464015593183 0.0027441539772568 0.0027544389401850 0.00276531793823609 0.00277499196625031 0.00277589428653835 0.0027840452237947 0.0027884348833464 0.00283388627394909 00285815822128543 0.00286972839969413 0.00286762071520663 0.0028719926008340 0.00290928855179373 0.00291399362481704 0.029214818791248 0.00294721274408638 0.00294286704306013 0.0029620275572394 0.00296775156879869 0.0029725011849468 0.0029907075838328 0.00299954481518294 0.0030153881996442 0.00303163409125018 0.00303891540561274 0.00303966505635012 0.00304572063851438 0.00304978606683126 00307285130009638 0.0030815256375955 0.00308380034190114 0.00309672394330129 0.00310508976483725 0.00312259521677238 0.00314969349944749 0.00315856654564306 00316375085180232 0.00318004727588184 0.00318437434441865 0.0031976396211168 0.0032001673855715 0.00321334455151734 0.00323029250749773 0.00323377434712158 0.00324029178816565 0.00326082986585996 0.00329068344277839 .0032905557500575 0.00328785247888019 0.00329570830198256 00330563892729141 0.00332258243518369 0.00333358893825408 0.00335602505490162 0.00335193882671869 0.00338495688318097 0.0033925587246355 0.00339160336545463 0.00339467117104308 .00339843699782583 0.0033996930658422 0.00340145935932612 0.00340400756003645 0.00340619799978614 0.00341005728531718 000344637204195242 0.00344966036879268 0.00345887223368579 0.00346914766687134 0.00348962571938949 0.00351307924928468 0.00352314090533479
0.0253107646948902 .025365935593114 0.0255184712205016 0.0257506350957408 0.0258067871092464 0.0258067871092464 0.0258485062749775 0.0258938185759979 0.0259431666044565 0.025943562933669 0.025943562933669 0.025943562933669 0.025943562933669 0.0260253671353869 0.0261783491499598 0.026447699983589 0.0264476999835899 0.026570628428043 0.0265885133692798 0.0266573029497514 0.0267440583233598 0.0267576011573859 0.0268074015965016 0.0268074015965016 0.0268729761117898 0.0269021985887685 0.0272607988427767 0.0274274862546968 0.0274591329505104 0.0274591329505104 0.0274668193093808 0.0277428969625178 0.0277743533284323 0.0278188744603845 0.0279586801462572 0.0279586801462572 0.0280830927642443 0.0281103720538155 0.0281418628482816 0.0282602117218581 0.0282948581551635 0.0283981409417135 0.0285138619671975 0.0285394966468777 0.0285394966468777 0.0285701369470703 0.028581418257601 0.0287238455479359 0.0287740188259455 0.0287816507335566 0.0288575686588257 0.0288905872097688 0.0290066476651166 0.029162352049033 0.0292034319802925 0.0292240016708344 0.0293470811455095 0.0293732876823559 0.0294681217346882 0.0294776612395762 0.0295852408148792 0.0296859221640636 0.0297040974796763 0.0297363022883287 0.0298692620690484 0.0300590690617162 0.0300590690617162 0.0300590690617162 0.030079897053516 0.0301121140106449 0.030226028325222 0.0302968467287528 0.030418811552392 0.030418811552392 0.0305947430141606 0.030635410266496 0.030635410266496 0.0306404757572806 0.0306437874748141 0.0306437874748141 0.0306457212077242 0.030654694620683 0.0306604395158507 0.0306784743320635 0.030951524082091 0.0309669739196758 0.03102146582337 0.0310853889623603 0.0312405341487865 0.0313935756082005 0.0314579681657778

Serine/threonine protein phosphatase 7 long form 0 -acyltransferase WSD1-like

thiamine pyrophosphokinase 1

Glutathione transport system permease protein gsiD

Proline transporter

Tubby-like F-box protein

Ribosomal protein L37

Serine/threonine-protein kinase atr

RING-H2 zinc finger protein RHA2a

NDR1/HIN1-like protein 12

ribosomal RNA large subunit methyltransferase E-like

1-aminocyclopropane-1-carboxylate oxidase 2

$\mathrm{HNH}$ endonuclease

pathogenesis-related protein PR-4-like

$\mathrm{NADH}$-cytochrome b5 reductase-like protein

1-aminocyclopropane-1-carboxylate oxidase homolog 1-like 
MELO3C008002 MELO3C006293 MELO3C006679 MELO3C018634 MELO3C002187 MELO3C002921 MELO3C011266 MELO3C009118 MELO3C003452 MELO3C027277 MELO3C012303 MELO3C006470 MELO3C013634 MELO3C018700 MELO3C009973 MELO3C019010 MELO3C010566 MELO3C026594 MELO3C019954 MELO3C018086 MELO3C00236 MELO3C007651 MELO3C017041 MELO3C011580 MELO3C011306 MELO3C00936 MELO3C021694 MELO3C012147 MELO3C020756 MELO3C00931 MELO3C019256 MELO3C023561 MELO3C025595 MELO3C024527 MELO3C02107 MELO3C022135 MELO3C009659 MELO3C025844 MELO3C005590 MELO3C023131 MELO3C015877 MELO3C018872 MELO3C007206 MELO3C007781 MELO3C026665 MELO3C016629 MELO3C004813 MELO3C002951 MELO3C022756 MELO3C016972 MELO3C005658 MELO3C002698 MELO3C017496 MELO3C007029 MELO3C012831 MELO3C017322 MELO3C026504 MELO3C008158 MELO3C019983 MELO3C016939 MELO3C008944 MELO3C026495 MELO3C013044 MELO3C007919 MELO3C022017 MELO3C011127 MELO3C02128 MELO3C018648 MELO3C00695 MELO3C026373 MELO3C007648 MELO3C019798 MELO3C009105 MELO3C026103 MELO3C016780 MELO3C006678 MELO3C011227 MELO3C023474 MELO3C011726 MELO3C026210 MELO3C006891 MELO3C002258 MELO3C00960 MELO3C006406 MELO3C024610 MELO3C011869 MELO3C019649 MELO3C016540 MELO3C024466 MELO3C015915 MELO3C013444
0.0035312711957558 0.00354341023305915 0.00354132679588104 0.00353843304329915 0.00354604168811656 00354961794420117 0.0035569037029529 0.00356566194604402 0.00357083843738115 0.00357454085905751 .0035787590215314 0.0035942224934137 0.00359595157280856 0.00361828856415125 0.00361824768775709 0.00362104834108679 0.00362510291757867 0.00365773426356975 0.00367499471328736 0.00367393222298185 0.0036832238019022 0.00368605120965426 0.00369325977155832 0.00369339856859607 .00370901783915945 0.0037233074899027 0.003723971676308 0.00373986616904487 0.0037457596254104 0.0037490548129930 0.0037571145519466 0.00376053621892014 0.00377304170472659 00377219600226342 0.00376902665796097 0.00377269415701442 0.00378297423157448 0.00378430346700454 .00378620329661872 0.00380245973358373 0.00381685302485724 0.00382096715178948 0.00382538871009896 0.0038398486676372 0.0038477634826739 0.00385657378659299 0.0038570066897727 0.00386183647055627 0.0038593975305655 0.0038804751937977 0.00387972951477389 0.00388183824560484 0.0038742185144211 0.0038845447000714 0.0038908092763863 0.00389583227761392 0039082746315698 0.0039303127626537 0.0039298946854792 0.00394970593609312 0.00394846519055947 0.0039448975667013 0.0039479270356819 0.0039553660213749 0.00395691777946994 0.00395892010502719 .00397525249759023 0.00397329808233615 0.0039929747580305 0.0039887085281346 00039869412751354 0.00398754437598248 0.0040092906562702 0.00401371508088511 0.00401640311312934 0.0040206956388575 0.0040244727681475 0.00402423910770855 0.00404263312343378 0.00404664750198225 .0040552911346800 0.00406375095139522 0.00408256489346259 .00409087920567841 0.004090155111938 0.0040885893571098 0.00408633114963042 0.00409820484929724 0.00409776864969524 0.00409647381032929 0.0041087192936025
0.0314849096365604 0.0314952662272863 0.0314952662272863 0.0314952662272863 0.0315028237960477 0.0315062493393388 0.0315425645395677 0.0316060402928291 0.0316377246746704 0.031648946287679 0.0316511025151031 0.0317736347459395 0.0317746969178507 0.0319105985709065 0.0319105985709065 0.0319107914717193 0.0319266674182015 0.032133716179376 0.0321994102008483 0.0321994102008483 0.0322572003830452 0.0322676530760027 0.032289034232494 0.032289034232494 0.0324112359759115 0.032484415475003 0.032484415475003 0.0325742299591907 0.032588108741071 0.0326024145758694 0.032643755075404

0.03265460151583 0.0326903479823329 0.0326903479823329 0.0326903479823329 0.0326903479823329 0.0327359654708158 0.0327359654708158 0.0327380599408595 0.0328498588270101 0.0329453804142944 0.0329664833030539 0.0329902190549102 0.0331004676286194 0.0331397649451927 0.0331759691273178 0.0331759691273178 0.0331771225999734 0.0331771225999734 0.0332591859056182 0.0332591859056182 0.0332591859056182 0.0332591859056182 0.0332679415792332 0.0332927176773627 0.0333068362123798 0.0333732318117167 0.0334712146397797 0.0334712146397797 0.0335715480937751 0.0335715480937751 0.0335715480937751 0.0335715480937751 0.033581937785454 0.0335819377854541 0.0335844987775695 0.0336507765001755 0.0336507765001755 0.0336852876960037 0.0336852876960037 0.0336852876960037 0.0336852876960037 0.0337845085349958 0.0338024985639232 0.0338107182784277 0.0338324321141021 0.0338353821618331 0.033835382161833 0.033945725909317 0.0339639785451115 0.0340076197956673 0.0340640991252564 0.034175454074849 0.034175454074849 0.034175454074849 0.034175454074849 0.034175454074849 0.0341932604853279 0.0341932604853279 0.034193260485327 0.0342665106965191

serine/threonine-protein kinase D6PKL2

Werner Syndrome-like exonuclease

Dihydrolipoamide acetyltransferase component of pyruvate dehydrogenase complex

7 -deoxyloganetin glucosyltransferase-like

Plant/protein (DUF789)

Cysteine proteinase inhibito

Histone acetyltransferase

TOM1-like protein 2

Synaptonemal complex protein 1

Polygalacturonase

Trichome birefringence-like protein

-loop nucleoside triphosphate hydrolase superfamily protein

DNA-directed RNA polymerase subunit beta

UDP-glycosyltransferase 74F2-like

Dead box ATP-dependent RNA helicase, putative

No data found

Topoisomerase II-associated protein PAT1 
MEL03C011320 MELO3C009370 MELO3C024404 MELO3C015314 MELO3C022568 MELO3C018374 MELO3C024936 MELO3C005710 MELO3C005504 MELO3C021144 MELO3C014591 MELO3C022382 MELO3C003323 MELO3C021407 MELO3C004316 MELO3C017094 MELO3C011908 MELO3C00208 MELO3C017520 MELO3C027370 MELO3C01179 MELO3C021944 MELO3C013419 MELO3C012361 MELO3C027137 MELO3C004239 MELO3C019295 MELO3C013835 MELO3C017989 MELO3C00978 MELO3C004382 MELO3C022236 MELO3C019833 MELO3C025454 MELO3C01724 MELO3C01298 MELO3C017756 MELO3C022535 MELO3C011419 MELO3C011719 MELO3C025492 MELO3C007317 MELO3C006341 MELO3C026519 MELO3C013004 MELO3C012080 MELO3C016733 MEL03C017857 MELO3C00281 MELO3C018333 MELO3C017023 MELO3C018884 MELO3C024747 MELO3C011270 MELO3C021302 MELO3C00261 MELO3C022766 MELO3C013309 MELO3C007896 MELO3C022208 MELO3C002241 MELO3C02145 MELO3C01496 MELO3C007108 MELO3C025122 MELO3C00225
MELO3C018609 MELO3C010982 MELO3C010869 MELO3C008126 MELO3C02446 MELO3C020620 MELO3C00738 MELO3C017526 MELO3C010860 MELO3C025264 MELO3C024359 MELO3C021766 MELO3C010205 MELO3C026379 MELO3C004128 MELO3C006557 MELO3C014503 MELO3C004504 MELO3C019979 MELO3C021380 MELO3C009865 MELO3C011936 MELO3C014848 MELO3C004616
0.00411998198635644 0.0041279603538038 0.00412659958826311 0.0041261201706035 0.00413550035043642 0.00413637456871707 0.00413994951308028 0.00414841548622957 0.00415565745621238 0.00415874744190292 .0041709464678263 0.00417453238090104 0.00417463696282061 .00418955137017907 00419185506808106 0.00419379751734894 0.00419762735150164 0.00420015070006774 0.00420329844082712 0.00420544322376737 0.00420786589404742 0.00421779613002127 0.00423115977641308 0.00422999190468754 .0042317638638140 0.00424349429512305 0.00426894623574081 .00427577875347029 0.00430088886261248 0.00431780204327548 0.0043395798835370 0.00435478022543923 0.00436921025270542 0043807402067110 0.004395158508533 0.0044040002206789 0.0044125107954931 0.00441084542698378 .0044137281287506 0.0044188927835399 0.00442331481104608 0.00443550104810231 0.00444064861850657 0.0044430026080279 0.00444391127554433 0.00444939745034345 0.0044536961431249 0.00447660665509919 0.0044895890993457 0.00451435304406078 0.00452304563686967 0.00453160110978612 0.00453016901239933 0.00455362792746339 0.0045788351918522 00459515873505412 0.0045942119727837 0.00459622213447552 0.00461226279515592 0.00461243236161057 0.00461235112080338 0.00460760544686867 0.00461777456584467 0.00462684788511114 0.00462896316894279 0.00469406507564141 .0046904699441916 0.00468423052131772 0.00469150142351815 0.00470255932622954 0.0047120532693638 0.0047202723733617 0.00474164902427332 0.00476193222621446 0.00476558186294107 0047696110190803 0.00480324345966632 0.00479821584987294 0.00480031640730605 0.00480220260665098 .0048189230823675 0.00482450500558018 0.00482848374613742 000484227068846665 0.004853594233164 0.00486300975469733 0.00487514380395182 0.00488061309291565 0.00491136386101831 0.00492368631958517 0.0049210016286798
0.0343459367870634 0.0343689245477535 0.0343689245477535 0.0343689245477535 0.0344099668734598 0.0344099668734598 0.03442520544582 0.0344810847801127 0.0345267476242063 0.0345378903406816 0.0346116311413704 0.0346116311413704 0.0346116311413704 0.0347207091941529 0.0347252289175893 0.0347267535304504 0.0347438988117376 0.0347502204338659 0.0347617005476946 0.0347648799607291 0.034770352946252 0.0348378317740652 0.0349094003953484 0.0349094003953484 0.0349094003953484 0.0349623566267773 0.0351280913373522 0.0351696603924552 0.0353173613919892 0.0354267854394048 0.035551505038091 0.0356231832101503 0.0356854976346896 0.0357648773635127 0.0358381442547557 0.0358543790682549 0.0358838655392167 0.0358838655392167 0.0358838655392167 0.035895190189408 0.0359046626400941 0.0359466902553953 0.0359683622052152 0.0359683622052152 0.0359683622052152

0.03598327200935 0.0360032931357245 0.0361736926479353 0.0362341214233668 0.0364042295905089 0.0364365717273029 0.0364688632099291 0.0364688632099291 0.0366013829627491 0.0367591122780037 0.036793997135694 0.036793997135694 0.036793997135694 0.0368639930026092 0.0368639930026092 0.0368639930026092 0.0368639930026092 0.036884580916089 0.0369343649486903 0.0369363206559399 0.0372301209290144 0.037230120929014 0.0372301209290144 0.037230120929014 0.0372675939541361 0.0373278539978607 0.037348183901565 0.0374679317075795 0.0375724900381318 0.0375860528000093 0.0376028135791688 0.0377390862236877 0.0377390862236877 0.0377390862236877 0.0377390862236877 0.0378405216515612 0.037869296246106 0.0378854690117076 0.0379785559279301 0.038052255859768 0.0381054504915579 0.038156374088363 0.0381563740883631 0.038307474285644 0.0383581385110405 0.0383581385110405

NAD(P)-binding rossmann-fold protein ACT domain-containing protein Tudor/PWWP/MBT superfamily protein protein SRC2-like

protein indeterminate-domain 5 , chloroplastic DUF248-1

Cleavage and polyadenylation specificity factor subunit heavy metal-associated isoprenylated plant protein 3

Disease resistance family protein

squamosa promoter-binding-like protein 8

cyclin-dependent protein kinase inhibitor SMR3-like

Succinate--CoA ligase [ADP-forming] subunit beta, mitochondrial

Myosin heavy chain-like protein

Stem-specific protein TSJT1

Prefoldin chaperone subunit family protein, putative 
MELO3C013809 MELO3C023525 MELO3C005683 MELO3C014666 MELO3C022914 MELO3C002200 MELO3C005243 MELO3C026734 MELO3C016831 MELO3C024452 MELO3C018867 MELO3C020617 MELO3C002030 MELO3C009465 MELO3C018257 MELO3C002891 MELO3C006299 MELO3C022027 MELO3C021713 MELO3C012854 MELO3C017092 MELO3C018572 MELO3C002679 MELO3C013472 MELO3C019542 MELO3C016667 MELO3C012583 MELO3C021691 MELO3C015299 MELO3C018610 MELO3C014936 MELO3C022426 MELO3C012084 MELO3C007190 MELO3C005803 MELO3C014288 MELO3C016221 MELO3C004917 MELO3C006182 MELO3C022310 MELO3C005215 MELO3C019137 MELO3C007409 MELO3C006221 MELO3C006760 MELO3C010941 MELO3C008118 MELO3C003627 MELO3C003690 MELO3C007688 MELO3C020748 MELO3C001920 MELO3C015481 MELO3C005634 MELO3C023004 MELO3C012295 MELO3C012912 MELO3C002072 MELO3C01495 MELO3C020489 MELO3C005380 MELO3C009151 MELO3C016700 MELO3C024248 MELO3C015113 MELO3C007235 MELO3C01755 MELO3C017169 MELO3C002293 MELO3C009170 MELO3C016881 MELO3C022605 MELO3C019156 MELO3C015423 MELO3C026577 MELO3C02296 MELO3C009329 MELO3C024348 MELO3C005298 MELO3C003554 MELO3C009549 MELO3C006093 MELO3C006553 MELO3C009753 MELO3C005559 MELO3C02171 MELO3C007563 MELO3C013581 MELO3C008152 MELO3C014876 MELO3C016778
0.00492199748758904 0.0049377167399449 0.0049435184544383 0.00496701622528295 0.0049684827506102 00497295451208024 0.00498172170978672 0.00499084688857632 0.00499389552060658 0.00499963757377242 .0049992695907844 0.00500844294115677 0.00501632420632747 0.00502843552261645 0.00503800739884341 0.00504688773087425 0.00505397492352333 0.0050556821683212 0.00505438158441507 0.00505464637579456 0.00509335428043201 0.00509558321440995 0.00510933008744363 0.0051198180085511 0.00513228821363398 0.00514137660805702 0.00513599158009104 0.00514153474524282 0.00514189399425591 0.0051432171743353 0.0051599163782603 0.0051671505635198 0.00517341272002758 0051842803465876 0.0051916759460539 0.00520148075471183 0.00520810517208448 0.00522269622442229 .0052315405757021 0.00524944366594116 0.0052570899040556 0.00526112589863548 0.0052749225490653 0.00527834117750325 0.0052793425328869 0.00528496183711913 0.0052918874227319 0.00529445457556355 0.0052942519993075 0.00531587170426806 0.00533361546979561 0.00533117758364088 .0053335447014657 0.0053363824587568 0.00534674243286837 0.00536354190955324 00536579815994709 0.00536839161606895 0.0053885175648908 0.00538680953554949 0.00539842275126157 00540405465087668 0.0054129560468902 0.00541360366330046 0.00542243994015512 0.00543615744316983 0.00544379027512432 0.0054419971112214 0.0054466767774377 0.00545100219701766 0.00546445639647308 0.00548519315338203 0.0054933644681418 0.00549259467745444 0.00549584987134866 0054992772192019 0.0055054093650102 0.00550384303802409 0.00551011550099556 0.00551021715769784 .0055192664066752 0.0055487512471621 0.00555487297960233 00556782329136662 0055675905840990 0.0055844146050554 0.00564058020111158 0.00563795363412223 0.00563998177954761 0.00565538385941067 0.00565933442381517
0.0383581385110405 0.038445784202726 0.0384671181862498 0.0385853581760918 0.0385853581760918 0.0386049051332833 0.038657768977044 0.0386837337375921 0.0386903771333979 0.0386903771333979 0.0386903771333979 0.0387281674412314 0.0387739282781844 0.0388523369077278 0.038911070832913 0.0389644197799201 0.038971376714354 0.038971376714354 0.0389713767143541 0.0389713767143541 0.0391808196243162 0.0391808196243162 0.0392470478012152 0.0393052524011742 0.0393774514106673 0.0393847987498828 0.0393847987498828 0.0393847987498828 0.0393847987498828 0.0393847987498828 0.0394820567819693 0.0395220977842573 0.0395393683466814 0.0396070996382051 0.039632937092624 0.0396924433635255 0.0397171785628582 0.0397791184372191 0.0398141405894191 0.0399196237807747 0.0399623820304827 0.0399776742486156 0.0400516898967673 0.0400544508958836 0.0400544508958836 0.040066299931388 0.0400863298080399 0.0400863298080399 0.040086329808039 0.0402080238558368 0.0402651268780556 0.0402651268780556 0.0402651268780556 0.040270621772254 0.040294608949124 0.0403829924406279 0.0403845837884127 0.0403887108669507 0.0405092628051124 0.0405092628051124 0.0405682842141038 0.0405797244487314 0.040618695791854 0.0406186957918544 0.0406559477517553 0.0407433295427556 0.0407695920149526 0.040769592014952 0.0407757466556548 0.0407926647930662 0.0408778596113435 0.0410019226291225 0.0410039815297562 0.0410039815297562 0.0410039815297562 0.0410140580823336 0.041028803603617 0.0410288036036176 0.0410336646483313 0.0410336646483313 0.0410855605975985 0.0412118421888699 0.0412417994263784 0.0412913789640253 0.0412913789640253 0.0413988753886035 0.0416900517933206 0.0416900517933206 0.0416900517933206 0.041752589098879 0.0417661418295686

calcium permeable stress-gated cation channel 1-like $60 S$ ribosomal protein $\mathrm{L} 6$

UDP-N-acetylmuramoyl-L-alanyl-D-glutamate--2, 6-diaminopimelate ligase Dirigent protein

$50 \mathrm{~S}$ ribosomal protein $\mathrm{L} 15$

$A B C$ transporter A family protein

purple acid phosphatase 4-like

lamin-like protein

RING-type E3 ubiquitin transferase

No data found

6-like protein

Haloacid dehalogenase-like hydrolase

Glycine-rich RNA-binding protein

Terminal flower 1

No data found

Pseudouridine synthase, RsuA/RluB/C/D/E/F

RNA-directed DNA polymerase (Reverse transcriptase)-related family protein 
MEL03C004117 MELO3C00763 MELO3C020997 MELO3C026499 MELO3C013692 MELO3C002261 MELO3C009193 MELO3C019997 MELO3C014390 MELO3C006151 MELO3C016676 MELO3C003123 MELO3C025152 MELO3C009325 MELO3C016723 MELO3C01235 MELO3C016017 MELO3C002023 MELO3C007814 MELO3C021695 MELO3C01435 MELO3C011890 MELO3C025877 MELO3C007133 MELO3C024909 MELO3C006765 MELO3C018802 MELO3C012708 MELO3C004011 MELO3C006191 MELO3C009201 MELO3C006188 MELO3C016108 MELO3C019145 MELO3C002690 MELO3C003396 MELO3C024777 MELO3C009446 MELO3C026748 MELO3C01223 MELO3C000960 MELO3C012636 MELO3C007305 MELO3C01743 MELO3C011968 MELO3C020484 MELO3C019453 MELO3C012929 MELO3C021977 MELO3C005419 MELO3C008275 MELO3C018119 MELO3C012855 MELO3C00402 MELO3C024178 MELO3C023608 MELO3C021934 MELO3C006919 MELO3C00274 MELO3C002036 MELO3C007762 MELO3C021058 MELO3C01515 MELO3C027004 MELO3C022302 MELO3C020793 MELO3C012054 MELO3C01388 MELO3C024563 MELO3C014872 MELO3C007513 MELO3C01308 MELO3C01270 MELO3C018356 MELO3C021329 MELO3C026388 MELO3C01015 MELO3C00938 MELO3C018174 MELO3C00435 MELO3C018412 MELO3C025627 MELO3C025613 MELO3C006253 MELO3C00334 MELO3C009195 MELO3C014214 MELO3C010810 MELO3C013814 MELO3C002275
0.00566929710292929 0.0056726351900258 0.00568704454460378 00568866895933029 0.00569296709952316 00570743449773126 0.0057141543213195 0.0057189492179438 0.00572324775500266 0.0057406825704176 0.00574342477656464 0.00574566882149075 0.00576102443116722 0.00577467309870794 0.00577229566189108 0.0057778707165629 0.00578589705892163 0.00580928505066813 0.00582928347011036 0.0058292778713428 0.0058439083088799 0.00585525734603176 0.00585523358807216 0.0058645615389016 0.005876180212674 0.0058769949613295 0.00587443787186281 00588417269765351 0.00590548212715403 0.0059058181355653 0.00591891723251758 0.00592525493769747 0.0059234494023328 0.00591396721573012 0.0059132905934770 0.00592368884294037 0.00593479565849098 0.00593804692995192 0.0059455675826553 0.0059950859167329 0.00601217131829979 0.00604022024987105 0.00604570718491382 0.00605024264847209 0.00606024825596196 0.0060595841623905 0.00606717259072709 0060867702258559 0.00610215935596248 0.00610228848337935 0.00609738000015136 0.00610157934889466 0.0061085656130438 0.0061196128433687 0.00612424666004419 0.00613091710727787 00613720039845989 0.0061455830638144 0.0061672737271172 0.0062265195025024 0.00623705673597619 0.00623574434668006 0.0062386245880182 0.00624716628925381 .00627652237112752 0.00628178082278441 .0062932694991421 0.0063018219586894 0.00631506277400962 .00632247490555671 00632190735505112 0.00633709589246012 0.0063692231190719 0.00636756099341584 0.00636951373363392 0063753740350731 0.0063763488913524 0.00639888795602483 0.00642353608518753 0.00642233138082371 .0064282645913686 0.00643238898121734 0.00644076471782506 0.00644431749613228 00646031208615239 0.0064614141997918 0.00646569829267873 00646880382342496 0.00646732888793111 0.00648131394496065 0.00649370496098678
0.0418240375367018 0.041833036731822 0.0419199713723186 0.0419199713723186 0.0419359967357266 0.042026891832846 0.0420606909026235 0.0420803010824039 0.0420962457778575 0.0421920901889014 0.0421920901889014 0.0421920901889014 0.0422639195732249 0.0423191774837995 0.0423191774837995 0.0423246175005202 0.0423519944464949 0.0425074362599648 0.0426221840989298 0.0426221840989298 0.0426975009959562 0.0427329923602296 0.0427329923602296 0.0427776942902953 0.0427809706934379 0.0427809706934379 0.0427809706934379 0.042817437953559 0.0429352413293379 0.0429352413293379 0.0429423338585641 0.0429423338585641 0.0429423338585641 0.0429423338585641 0.042942333858564 0.0429423338585641 0.0429799337952102 0.0429877158429694 0.043026388490238 0.0433154505707741 0.0433812098520652 0.0435676806846981 0.0435754274433807 0.0435922079768973 0.0436165607897204 0.0436165607897204 0.0436504887046518 0.043759601452649 0.043807377411217 0.0438073774112173 0.0438073774112173 0.0438073774112173 0.043836505193315 0.0438679615403588 0.0438852493792499 0.0439171135116542 0.0439352662012157 0.0439743188142285 0.0440975701798835 0.0444568089858714 0.0444628643048621 0.044462864304862 0.044462864304862 0.0445033742420186 0.044636312678213 0.0446576275986931 0.0447075308950235 0.044751773413217 0.044829681784297 0.0448500563612929 0.0448500563612929 0.044921502792604 0.0451003657442134 0.0451003657442134 0.0451003657442134 0.0451026197189537 0.045102619718953 0.0452296486197332 0.045371392756211 0.0453713927562119 0.045385226863187 0.0453852268631873 0.0454066095911773 0.0454066095911773 0.0454115548155478 0.0454115548155478 0.0454150041623959 0.045415004162395 0.0454150041623959 0.0454866627928315 0.0455574349110223

Carboxypeptidase

Calcium-transporting ATPase

Sterol 3-beta-glucosyltransferase

Zinc finger HIT domain-containing protein 3

Transmembrane protein, putative

serine/threonine-protein kinase HT1-like

Transmembrane protein

Histone $\mathrm{H} 4$

mavicyanin-like

protein-ribulosamine 3-kinase, chloroplastic

Peptide methionine sulfoxide reductase

E3 ubiquitin-protein ligase XB3-like

Coronatine-insensitive 1

POLAR LOCALIZATION DURING ASYMMETRIC DIVISION AND protein

serpin-ZX-like

L-aminoadipate-semialdehyde dehydrogenase-phosphopantetheinyl transferase-like 
MELO3C004648 MELO3C014127 MELO3C013774 MELO3C017394 MELO3C011399 MELO3C009226 MELO3C006727 MELO3C014592 MELO3C02538 MELO3C003629 MELO3C025085 MELO3C01711 MELO3C000210 MELO3C002863 MELO3C010710 MELO3C01410 MELO3C020940 MELO3C011317 MELO3C023313 MELO3C002412 MELO3C021730 MELO3C023315 MELO3C016714 MELO3C024515 MELO3C002102 MELO3C006703 MELO3C005600 MELO3C022928 MELO3C015597 MELO3C018604 MELO3C026067 MELO3C007325 MELO3C023845 MELO3C018418 MELO3C010532 MELO3C026628 MELO3C022323 MELO3C007871 MELO3C022636 MELO3C015424 MELO3C026546 MELO3C014262 MELO3C020440 MELO3C006100 MELO3C014870 MELO3C010475 MELO3C007024 MELO3C025262 MELO3C003085 MELO3C02202 MELO3C020840 MELO3C025870 MELO3C002552 MELO3C00823 MELO3C021323 MELO3C026341 MELO3C006306 MELO3C02143 MELO3C007597 MELO3C017791 MELO3C024179 MELO3C018056 MELO3C024853 MELO3C022802 MELO3C009638 MELO3C014057 MELO3C003108 MELO3C025150 MELO3C021391 MELO3C021274 MELO3C012904 MELO3C010939 MELO3C026824 MELO3C021328 MELO3C004223 MELO3C017562 MELO3C008420 MELO3C009316 MELO3C016448 MELO3C019257 MELO3C005288 MELO3C00603 MELO3C012007 MELO3C010766 MELO3C019260 MELO3C017705 MELO3C012421 MELO3C01058 MELO3C005980 MELO3C020839 MELO3C008610
0.00650643547640417 0.00651810160220068 0.00651526241483036 0.00652352679417967 0.00652419783353908 0065368918836370 0.00654713755056613 0.00655080438696443 0.00655608608069769 0.00657160924631839 0.00657351860449662 0.00658733845633164 0.00659371367780071 0.00659694398511679 0.0066031101351280 0.0066143732940664 0.00661550757875262 0.00660728708017455 0.00665769860589904 0.00666469864478825 0.00666911679857307 0.00668561297068715 0.00672515339221591 0.00672876682696899 .00673479236241348 0.00674630940843268 0.0067735201512592 0.00677352836102607 0.00677974577639429 0.00677908066685062 0.00678676319927907 0.00679902181931435 0.00680785721675858 0.00681891427759185 0.0068468528991985 0.00686069658808242 0.00686333010384244 0.00687289009524761 .0068811341802448 0.0069071556206503 0.00693441822564089 0.00696264092237953 0.00696854568206295 0.00695734585909258 0.00695033903996145 0.00694843697490344 0.00695655689989738 000696807649833175 0.00696185243230085 0.00693627676482245 .00696181741775093 0.0069539047423004 .0069344143119494 0.0069637931369588 0.00699292881807612 0.00704211024903056 00704624370381213 0.00709534752098828 0.0071254243029672 0.00713147184175345 0.00715903843773669 0071568643555988 0.00717709886898835 0.00718337005107827 0.00720397515405002 0.00719121069185757 .0072021846449362 0.00720791735611159 0.00719687947412662 0.0072083283046094 00719324434856972 0.00722100799204228 0.00723804249067217 0.00724702951271161 0.00725452501643375 0.0072538699027948 0.0072686274147804 0.0072779413909807 0.00729807390629156 0.00729310653565496 0.00730395227585312 0.00731675729151005 0.00732642441238118 0.00733014592886683 00733326272891488 0.0073471073381503 0.0073514013120205 0.00736507254652774 0.00738295761679109 0.00739116720083399 0.00743112678605029
0.045614339447464 0.0456522271398093 0.0456522271398093 0.0456578253063655 0.0456578253063655 0.0457291104621977 0.0457697060127895 0.0457791351161219 0.04579983870099 0.0458729395477752 0.0458729395477752 0.045953142767253 0.0459813740900022 0.046014404396134 0.0460196052035173 0.0460196052035173 0.0460196052035173 0.0462804962224218 0.0463128548683755 0.046327255594449 0.0463928919740339 0.0466181306924788 0.0466268126546704 0.046652197251948 0.0467155906406511 0.046871202383288 0.046871202383288 0.0468813723172307 0.0468813723172307 0.0469134709214429 0.046981764139132 0.0470263631247867 0.0470862720518047 0.0472626696631497 0.0473416830601117 0.0473433133848356 0.0473761628240995 0.0474164406579397 0.0475625580028675 0.0476860043919131 0.047686004391913 0.047686004391913 0.047686004391913 0.047686004391913 0.047686004391913 0.0476860043919131 0.047686004391913 0.047686004391913 0.0476860043919131 0.047686004391913 0.0476860043919131 0.047686004391913 0.047686004391913 0.0478197199543578 0.0480894312960251 0.048101025546694 0.0484027696690838 0.0485743888710046 0.048598839683502 0.0487239522278649 0.0487239522278649 0.048818278277645 0.0488345525434578 0.0488443099327942 0.0488443099327942 0.0488443099327942 0.0488443099327942 0.0488443099327942 0.0488443099327942 0.0488443099327942 0.0488884768031687 0.048987012045334 0.0490182548751763 0.0490313533708249 0.049031353370824 0.0491098606960309 0.0491559728216409 0.049197335726012 0.0491973357260124 0.049213836061352 0.0492664983805087 0.0492894286514099 0.0492894286514099 0.0492936030065827 0.049369861456662 0.0493819130990112 0.049456925440794 0.0495433333245012 0.0495801838855691 0.0497819955555316

Stress-response A/B barrel domain-containing protein UP3 Actin-related protein 8

Cystathionine gamma-synthase

No data found

LOW QUALITY PROTEIN: transcription factor BIM2

serine/threonine-protein kinase ATG1c

Neutral/alkaline invertase

heterogeneous nuclear ribonucleoprotein $\mathrm{F}$

Polyadenylate-binding protein-interacting protein 4

Plasma membrane ATPase

Class I heat shock protein

$39 S$ ribosomal protein $L 47$, mitochondrial

Unknown protein

patellin-4

Short-chain dehydrogenase/reductase

Unknown protein

Pentatricopeptide repeat-containing family protein 
MELO3C021306

MELO3C017009

MELO3C022759

0.00743815264091596

0.00744176012404185

0.00746050443032131

0.00746559537964697
0.0497952801713388 0.0498025485224339

0.0498941760902186 ethylene-responsive transcription factor RAP2-3-like 4-coumarate--CoA ligase family protein

MOB kinase activator-like $1 \mathrm{~A}$

U3 small nucleolar RNA-associated protein $14 \mathrm{~B}$ isoform 3 
Supplementary Table 4. Functionality of differentially expressed genes transcripts from RNA-seq (23 DEGs) that were exclusive to the effect of introgression over time in the near-isogenic line (NIL) SC10-2 fruit $(\mathrm{n}=3)$ of melon and its parental control 'Piel de Sapo' (PS) during postharvest ripening at $20.5_{-}^{\circ} \mathrm{C}$ and $88 \%$ relative humidity for $18 \mathrm{~d}$.

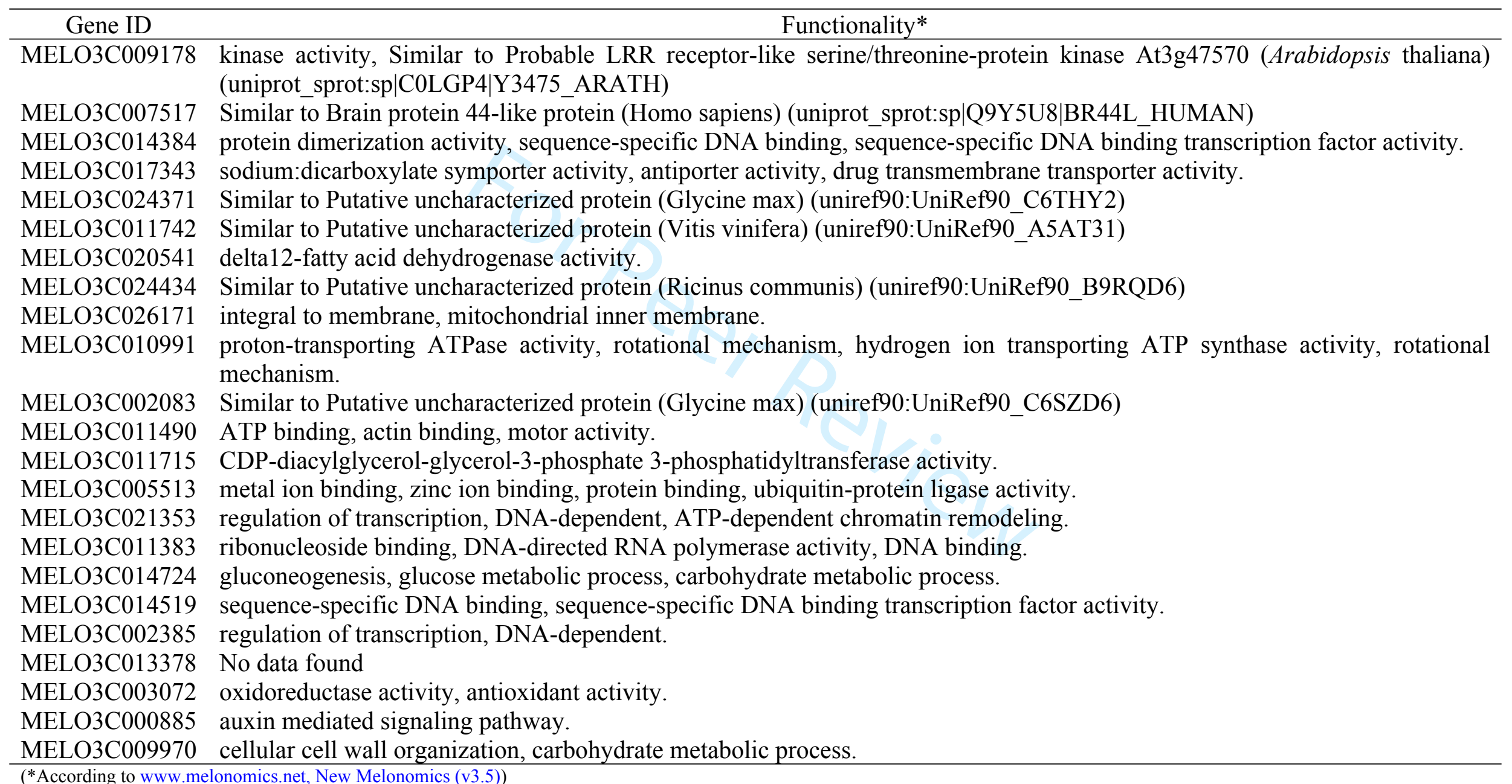

(*According to www.melonomics.net, New Melonomics (v3.5)) 
Supplementary Table 5. Expression levels of differentially expressed genes in melon fruit of the near-isogenic line SC10-2 and its parental control Piel de Sapo (PS) during postharvest storage at $20.5^{\circ} \mathrm{C}$ and $88 \%$ relative humidity for 18 d. *,**;***,****: significance at $\mathrm{P} \leq 0.05,0.01,0.001$ or 0.0001 , respectively. Gene code according with Melonomics 4.0

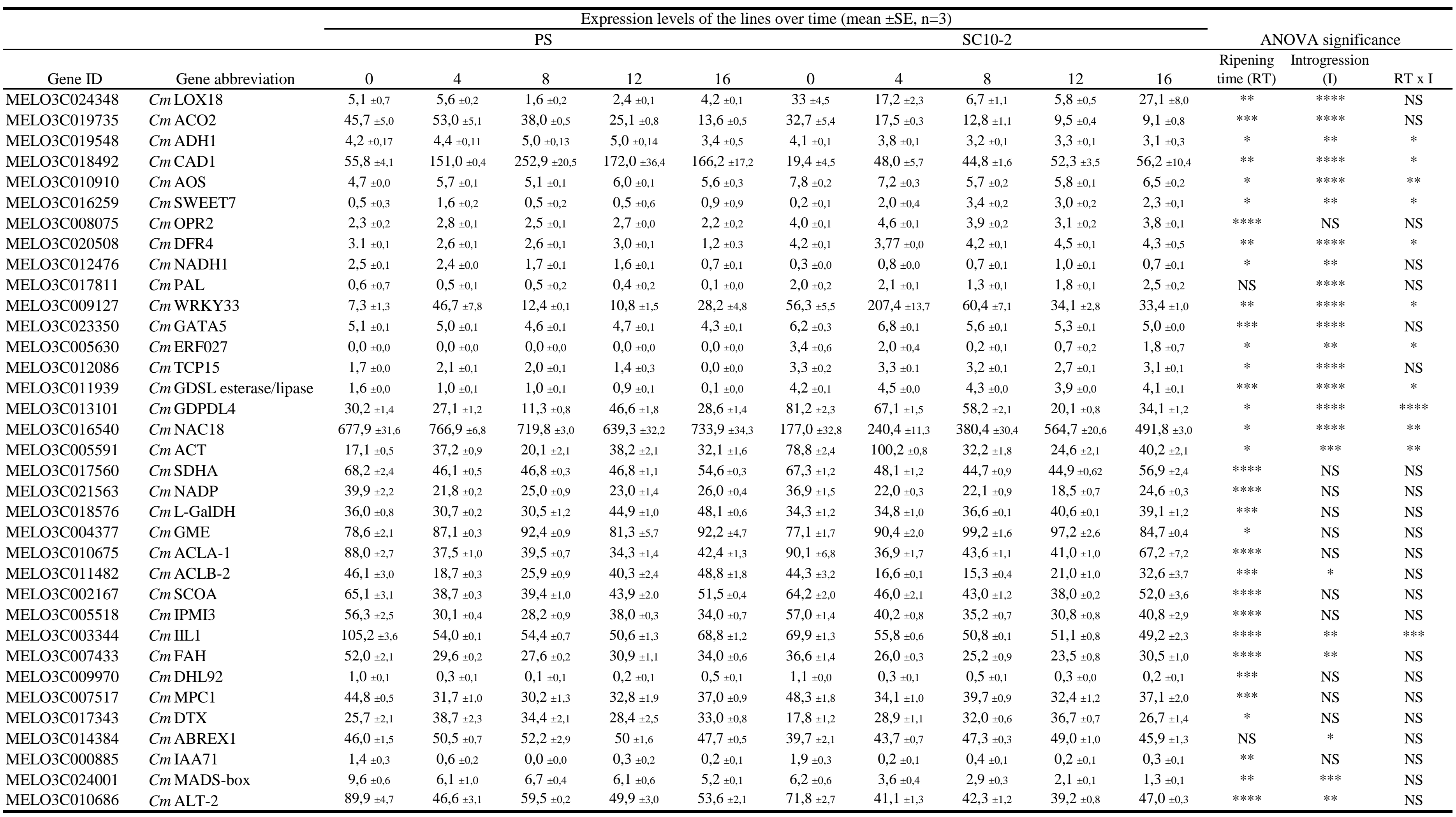


Supplementary Table 6. Clustes obtained the heatmap and hierarchical clustering (using Euclidean distance as the similarity measure and Ward's linkage) applied to thirty-seven differentially expressed genes (Table 1) and the quality traits measured

(textural traits and VOCs) over postharvest ripening time (columns represent samples and rows variables). Original data were scaled and log-transformed. Predominant trend in the heatmap was represented in red or blue color and the main melon line (NIL SC10-2 or the parental PS).

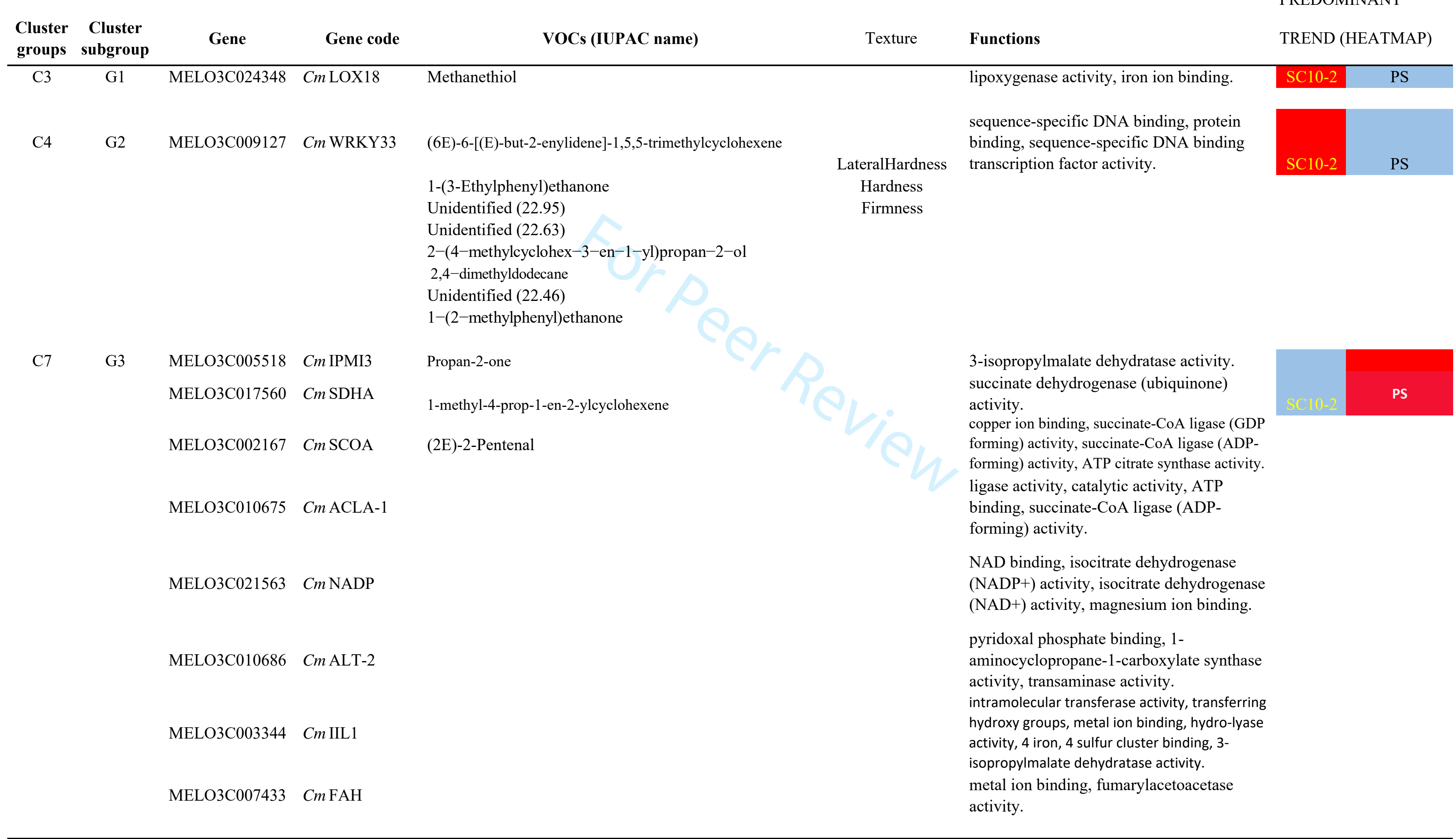


C7 G3 (cont) MELO3C011482 Cm ACLB-2

$$
\begin{aligned}
& \text { Ethyl 2-methylbutanoate } \\
& \text { Methyl acetate } \\
& \text { Ethyl-2-methylpropanoate } \\
& \text { 2-Methylbutyl-acetate }
\end{aligned}
$$

C7 $\quad$ G4 $\quad$ MELO3C019735 Cm ACO2

MELO3C024001

$$
\text { Cm MADS-box 2-Methoxybenzenethiol }
$$

2-Decanone

2-Octanylpropionate

\section{2-methylpropyl acetate}

Hexyl acetate

2,3-Butanediyl diacetate

2-Methyl-2-propen-1-yl acetate

2,3-Butanediyl diacetate-r (isomer)

S-Methyl propanethioate

\section{C8 G7 MELO3C016540 Cm NAC18 1-(4-Methylphenyl)ethanone}

Cyclohexanone

S-Methyl 2-methylpropanethioate

S-Methyl 3-methylbutanethioate

Isopropyl propionate
Lyase activity, acyltransferase activity, binding succinate-CoA ligase (ADP-forming) activity, ATP citrate synthase activity. Necessary for the generation of the cytosolic pool of acetyl-CoA

metal ion binding, L-ascorbic acid binding, 1 aminocyclopropane-1-carboxylate oxidase activity.

sequence-specific DNA binding, protein binding sequence-specific DNA binding transcription factor activity.

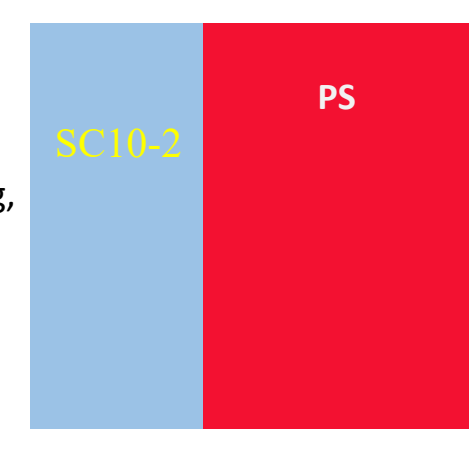

cinnamyl-alcohol dehydrogenase activity, zinc iol binding.

D-arabinose 1-dehydrogenase (NAD) activity, D arabinose 1-dehydrogenase [NAD $(\mathrm{P})+]$ activity, oxidoreductase activity, protein binding,

pyridoxal 4-dehydrogenase activity, L-galactose dehydrogenase activity.

DNA binding.

ATP binding, protein binding, receptor activity, two-component response regulator activity, twocomponent sensor activity. 